Species composition and distribution of the dipterans (Insecta: Diptera) in Bulgaria

Zdravko Hubenov 



\section{Species composition and distribution of the dipterans (Insecta: Diptera)}

in Bulgaria

\section{Zdravko Hubenov}

\section{Pensoft}

National Museum of Natural History, Sofia

Bulgarian Academy of Sciences 
Species composition and distribution of the dipterans (Insecta: Diptera) in Bulgaria

\author{
Zdravko Hubenov
}

Photo on the front cover: Tachina fera, juniper heath in the Kornberg nature reserve near Gruibingen,

Germany. (Photo by Pjt56 taken on 19 August 2018).

First published 2021

ISBN 978-619-248-050-9 (paperback)

ISBN 978-619-248-051-6 (e-book)

Pensoft Series Faunistica No 223

Hubenov Z. 2021. Species composition and distribution of the dipterans (Insecta: Diptera) in Bulgaria. Pensoft \& National Museum of Natural History, Sofia, 276 pp.

(c) PENSOFT Publishers

(c) National Museum of Natural History, Sofia

Pensoft Publishers

Prof. Georgi Zlatarski 12, Sofia 1700, Bulgaria

Fax: +359-2-870-42-82

info@pensoft.net

www.pensoft.net 


\section{Contents}

Contents 5

Introduction 7

Material and methods .......................................................

Abbreviations used .................................................... 11

Results and Discussion ................................................ 12

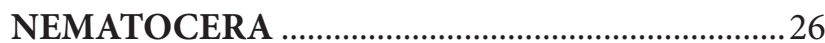

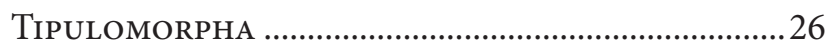

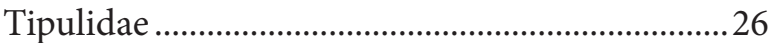

Limoniidae...............................................................29

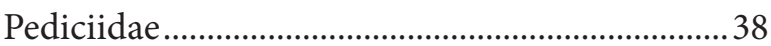

Cylindrotomidae ............................................................

BLEPHARICEROMORPHA................................................ 39

Blephariceridae …………………………………........ 39

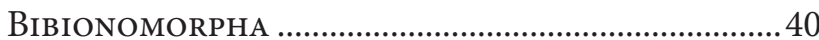

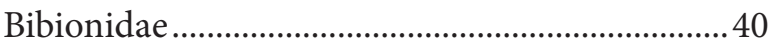

Mycetophilidae (Fungivoridae) ...............................40

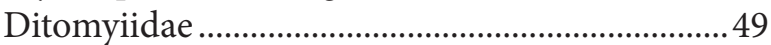

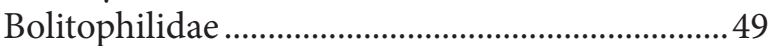

Diadocidiidae .............................................................. 50

Keroplatidae (+ Macroceridae) ..................................50

Sciaridae................................................................ 51

Cecidomyiidae............................................................. 54

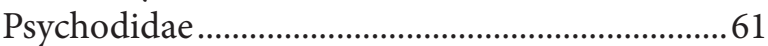

Trichoceridae (Petauristidae)..................................64

Anisopodidae (Rhyphidae, Phryneidae) ...............64

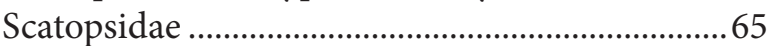

Ptychopteridae (Liriopidae) ......................................65

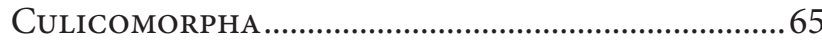

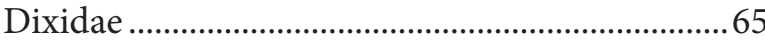

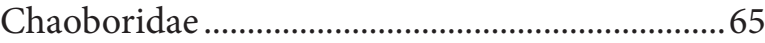

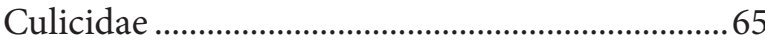

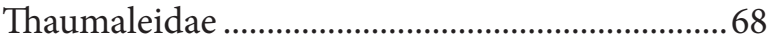

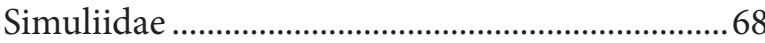

Ceratopogonidae (Heleidae) .....................................72

Chironomidae ............................................................

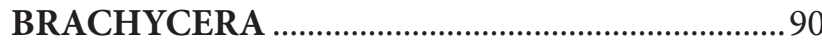

ORTHORRHAPHA …...................................................90

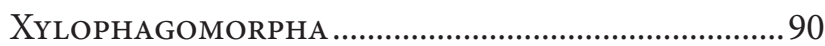

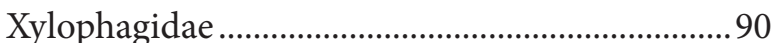

Cenomyiidae ..........................................................90

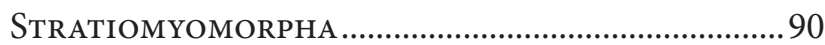

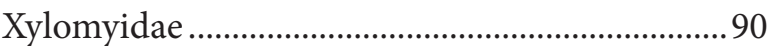

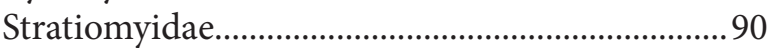

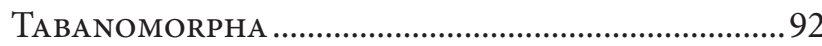

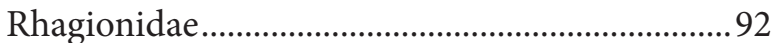

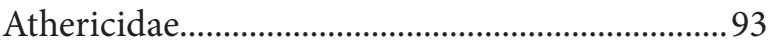

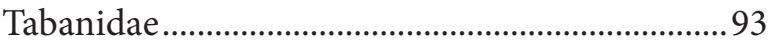

Vermileonidae .........................................................99

Nemestrinidae _........................................................99

Acroceridae (Cyrtidae, Oncodidae, Ogcodidae) ………………………………........99

Bombyliidae..............................................................99

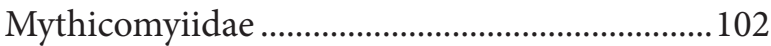

Therevidae.................................................................... 102

Scenopinidae (Omphralidae) ................................102

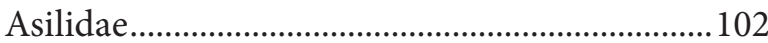

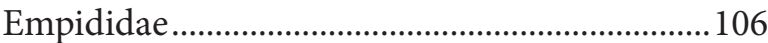

Hybotidae ..............................................................111

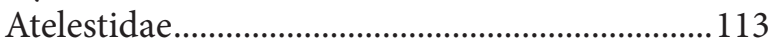

Microphoridae .............................................................113

Dolichopodidae.......................................................114

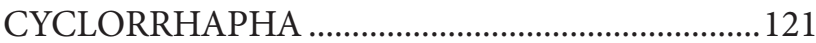

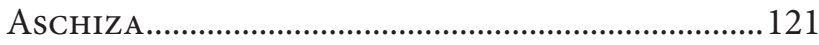

Platypezidae (Clythiidae)........................................121

Lonchopteridae (Muscidoridae) ..........................121

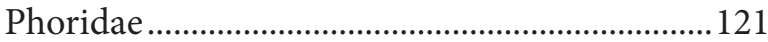

Pipunculidae (Dorylaidae) .....................................127

Syrphidae (Microdontidae) ..................................127

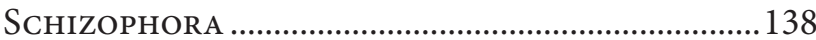

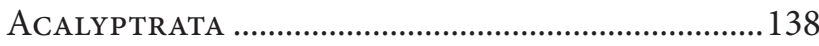

Micropezidae (Tylidae) .........................................138

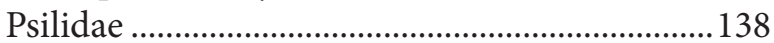

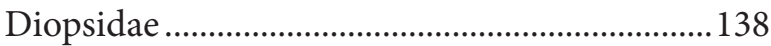

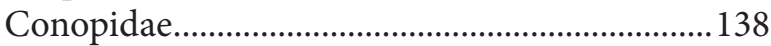

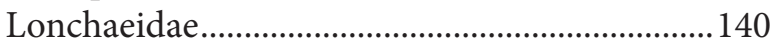

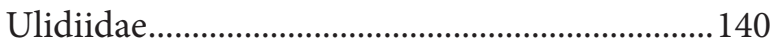

Otitidae (Ortalidae) .................................................. 140

Platystomatidae (Platystomidae) ...........................140

Tephritidae (Trypetidae)........................................140

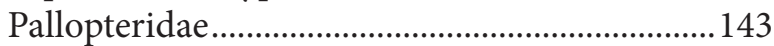

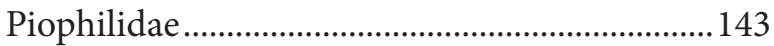

Lauxaniidae (Sapromyzidae) ...................................143

Cremifaniidae............................................................. 144

Chamaemyiidae (Ochthiphilidae) ........................ 144

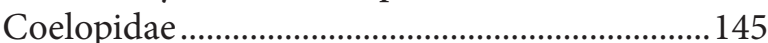

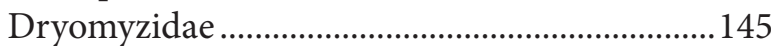

Sciomyzidae (Tetanoceridae) ..................................145

Phaeomyiidae ........................................................146

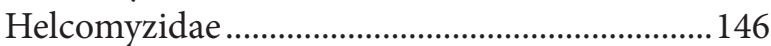

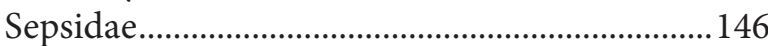

Acartophthalmidae ................................................146

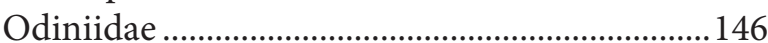

Agromyzidae .......................................................146

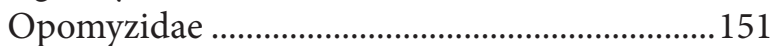

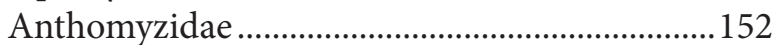

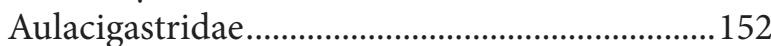

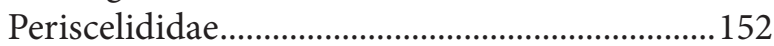

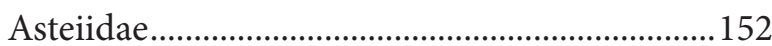

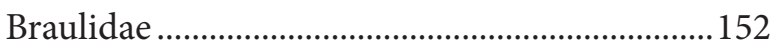

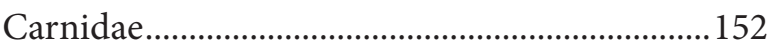




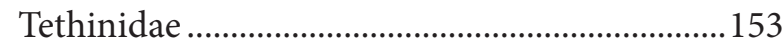

Canacidae (Canaceidae) ........................................... 153

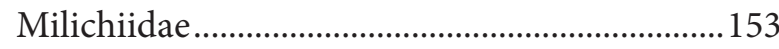

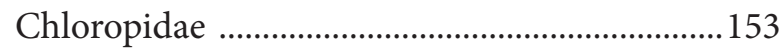

Siphonellopsidae .....................................................161

Heleomyzidae (Helomyzidae) .................................161

Trixoscelididae (Trichoscelidae) ..............................163

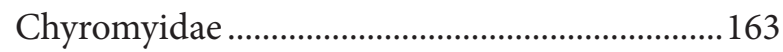

Sphaeroceridae (Borboridae) .................................163

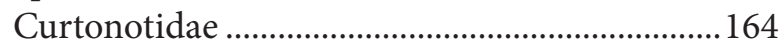

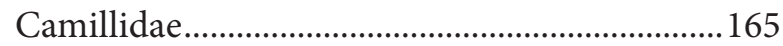

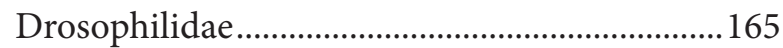

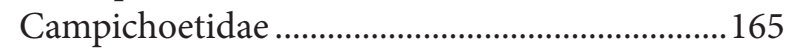

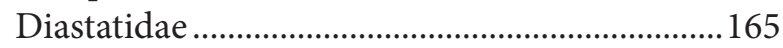

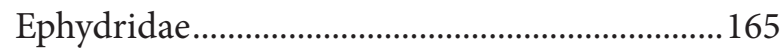

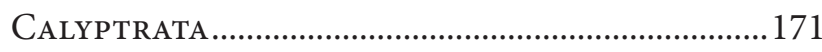

Hippoboscidae
Streblidae

171

Nycteribiidae .............................................................171

Scathophagidae (Cordyluridae, Scatomyzidae, Scopeumatidae) ................................................172

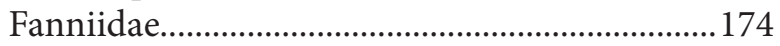

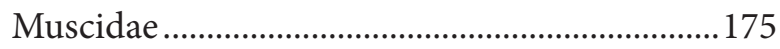

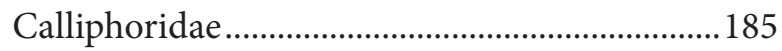

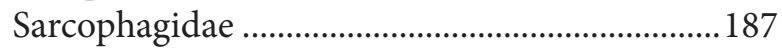

Rhinophoridae ………………………………....192

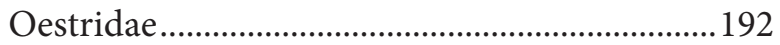

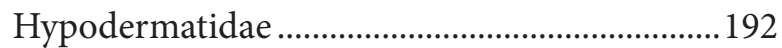

Gasterophilidae ....................................................193

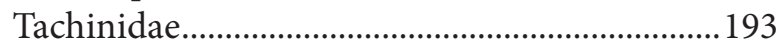

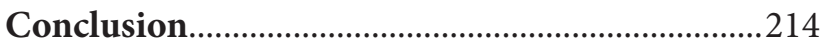

Acknowledgements......................................................215

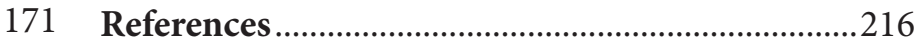




\section{Introduction}

The Bulgarian dipteran fauna has been studied for 160 years (Löw 1862). Since then, a vast material of faunistic data concerning the territory of Bulgaria has been accumulated. During the last 70 years, different parts of the country are under landscape changes and anthropogenic impact. Changes in the natural communities are caused by some alien species, introduced in the last 100 years. The economic importance of the biodiversity, the dynamic character of the fauna and its protection necessiate a periodic updating of the data concerning the faunistic diversity of the separate taxonomic groups.

The first data on Diptera from Bulgaria were reported by Löw $(1862,1863)$, Meunier (1897) and Joakimoff (1899). Nedelkov reported new families and reviews the dipteran fauna of Bulgaria (Nedelkov 1909, 1910, 1912). Various publications have been written by other authors (Kovachev 1905; Vimmer 1916; Drenowsky 1920a, 1920b, 1921a, 1922b, 1922c, 1923a, 1923b, 1923c, 1929a, 1929b, 1931, 1936, 1937, 1939; Enderlein 1921, 1924, 1926, 1930; Komárek \& Vimmer 1921, 1922, 1934; Konsuloff 1921a, 1921b, 1922a, 1922b, 1922c, 1923a, 1923b; Buresch 1924, 1926a, 1926b, 1928, 1930; Konsuloff \& Paspalev 1924, 1925; Drensky 1926, 1928, 1931a, 1931b, 1932a, 1932c, 1934c, 1936, 1939b, 1940, 1942; Drensky \& Drensky 1928; Czerný 1930; Szilády 1934; Zilahi 1934; Jacentkovsky 1936, 1937, 1939; Lindner 1936; Lackschewitz 1940a, 1940b; Buhr 1941; Valkanov 1941; Delkeskamp 1942). Studies on the separate families were reported by Drensky (1929, 1933, 1934a, 1939a, 1943). Numerous data on the plant pests and species of medical significance are available in the applied entomological literature. The number of publications increased rapidly after the Second World War. Volumes from the series Fauma of Bulgaria and catalogues of the separate families have been published (Beschovski 1985, 2009, 2013b; Lavčiev 2003; Bechev 2006, 2010; Kechev et al. 2020). Taxonomic and faunistic studies were performed by Bulgarian and foreign authors. The hydrobiological research are important for elucidating the species composition of the water-related dipterans. The investigations of the cave fauna contribute to the study of separate families. In many monographs and catalogues related to Diptera of the various geographical areas, taxa from Bulgaria without accurate localities are mentioned. In 1700 publications there are data related to Diptera in Bulgaria.

In 1968 an overview (unpublished) of the Diptera families, reported from Bulgaria, was made by Beschovski - 1952 species. Later Beschovski (1976b, 1993, 2001) reported new families and presented a list of the families in Bulgaria. Data on the vertical distribution of Diptera refer to separate families. There are data in some publications (Buresch \& Arndt 1926; Beron 1969; Hubenov 1993), in the volumes of the series Fauna of Bulgaria (Beschovski 1985, 2009), in the catalogues (Lavčiev 2003; Bechev 2006, 2010; Beschovski 2013b) and in some dissertations (Dimitrova 1989; Dzhambazov 2000; Langourov 2001; Bechev 2007; Kechev 2007; Pavlova 2020b). In the publications on Diptera from the Pirin, Rila, Vitosha and Vrachanska Planina Mts., the vertical distribution of 2275 species of 81 families is analyzed (Hubenov 2015b, 2016, 2017, 2018, 2019a, 2019b).

The aim of this work is to present the fauna, distribution according to the vegetation belts and zoogeography of the order Diptera in Bulgaria.

\section{Material and methods}

All species reported from Bulgaria are included. The processing of the literary data has reviewed all data that refer to the Bulgarian Diptera. Due to the big number of literature sources and the character of the publications, the cited literature does not include all studies. Attention is paid to the publications that indicate the localities of the species. The first reports, generalized works and the most important literary sources are given. The names of the taxa are updated according to the newest electronic publications. The classification of Diptera is based on the works of Soós \& Pap (1984-1993), McAlpine (1989), Wood \& Borkend (1989), Woodley (1989), Nagatomi (1996), Papp \& Darvas (1997, 1998, 2000a, 2000b), Yeates \& Wiegmann (1999), Nartshuk (2003), Ziegler (2003), Oosterbroek (2006), Yeates et al. (2007) and Pape et al. (2011).

Weaknesses in the literature data which limit the obtaining of an equivalent information when comparing territories and systematic groups include: different levels of study of the individual taxa; insufficient research of many families in the corresponding areas; a lack of exact localities for the part of the recorded species; insufficient data for the localities and vertical distribution; existence of rich synonymy; outdated data; a lack of generalized investigations; significant differences in the number of taxa in the separate areas; unexplored territories; pro- 
longed periods of data accumulation for most regions; predominance of the ecological studies versus those of the fauna; priority research on groups of economic and medical significance. These weaknesses lead to 6 problems: 1) Continuous supplementation of an existing historical list of the fauna. As a result, species diversity in a given area is higher than in reality. 2) Incomparability of data in terms of time periods. Data comparisons between two areas very often cover different periods as it is not possible to study all taxonomic groups and territories simultaneously. 3) Inaccurate zoogeographical characteristic of some taxa due to the lack of information on their vertical distribution. 4) Incomplete reporting of the anthropogenic impact, successional and landscape changes on the communities in the separate regions. Thus, a number of well-studied areas in the past have already been significantly changed. 5) Prioritization of research in areas under monitoring or environmental protection legislation. 6) Workload of the specialists with environmental, medical and other applied investigations that do not allow them to devote time to faunistic research.

A system of natural territories is used to represent the species distribution in Bulgaria (Hubenov 1997, 2021). This is a hierarchical system (Fig. 1, Table 1) which allows using larger or smaller number of territorial units. The first letter in the abbreviations corresponds to the region; the second - to the subregion; and the figure - to a smaller territorial unit.

The vertical distribution is presented according to the vegetation belts. The vegetation of Bulgaria is differentiated in a system of six vegetation belts (Stojanov, 1966; Velchev et al., 1982, 1989; Velchev, Tonkov, 1986; Bondev, 1991, 1997, 2002; Velchev, 1997, 2002): 1) Xerothermic oak forests (sub-Mediterranean vegetation) up to 600-700 m a.s.l.; 2) Mesophylic and xeromesophylic mixed (oak-hornbeam) forests - from 600-700 $\mathrm{m}$ to 900-1000 m a.s.l.; 3) Beech forests - from 900-1000 m to 1500-1600 m a.s.l. ; 4) Coniferous forests - from 1500 (1300)-1600 m to 2000-2200 m a.s.l.; 5) Subalpine vegetation - from 2000-2200 m to $2500 \mathrm{~m}$ a.s.l.; 6) Alpine vegetation - over 2400-2500 m a.s.l. The boundaries between the vegetation belts are not defined clearly and depending on the relief, climate, exposure and human activities there are mixed zones up to $200-300 \mathrm{~m}$ a.s.l.

The classification of the areas is based on the available literature and recent electronic issues. A zoogeographical analysis for the taxa categorization was used. This method allows obtaining data information about species complexes with different zoogeographical character based on the published data regarding species distribution and results of the faunistic research. These complexes contain zoogeographical information about the taxonomic groups which, combined with the origin of the ranges, determines the zoogeographical character of the fauna. The distribution of the species according to the zoogeographical categories in the different vegetation belts and the distribution of the zoogeographical categories in each belt are scrutinized. The classification of the areas is based on the works of Geptner (1936), Darlington (1957), Kryzhanovsky (1965, 1976, 2002), de Lattin (1967), Müller (1974, 1980), Udvardi (1975), Crosskey \& White (1977), Malicky et al. (1983), Gorodkov (1984a), Grehan (1988, 1993), Vigna Taglianti et al. (1999), Procheş \& Ramdhani (2012), Holt et al. (2013), Ficetola et al.

Table 1. Distribution of Diptera in the natural geographic territorial units of Bulgaria

\begin{tabular}{|c|c|c|c|c|}
\hline \multirow[b]{2}{*}{ The system of the natural geographic territorial units } & \multirow[b]{2}{*}{ Abbreviations } & \multicolumn{3}{|c|}{ Number of species } \\
\hline & & $\begin{array}{c}\text { Nematocera } \\
\text { (1672) }\end{array}$ & $\begin{array}{c}\text { Brachycera } \\
(3348)\end{array}$ & $\begin{array}{c}\text { Total } \\
(5020)\end{array}$ \\
\hline DANUBIAN PLAIN & D & $328(19.6)$ & $566(16.9)$ & $894(17.8)$ \\
\hline Western Danubian Plain & DW & $219(13.1)$ & $227(6.8)$ & $446(8.9)$ \\
\hline Middle Danubian Plain & DM & $210(12.6)$ & $218(6.5)$ & $428(8.5)$ \\
\hline Eastern Danubian Plain & $\mathrm{DE}$ & $191(11.4)$ & $367(11.0)$ & $558(11.1)$ \\
\hline Popovo-Provadiya district & E1 & $154(9.2)$ & $184(5.5)$ & $338(6.7)$ \\
\hline Loudogorie-Dobroudzha district & E2 & $144(8.6)$ & $271(8.1)$ & $415(8.3)$ \\
\hline STARA PLANINA RANGE SYSTEM & $\mathbf{S}$ & $719(43.0)$ & $1026(30.7)$ & $1745(34.8)$ \\
\hline Predbalkan & SP & $329(19.7)$ & $426(12.7)$ & $755(15.0)$ \\
\hline Western Predbalkan & P1 & $247(14.8)$ & $234(7.0)$ & $481(9.6)$ \\
\hline Middle Predbalkan & $\mathrm{P} 2$ & $198(11.8)$ & $262(7.8)$ & $432(8.6)$ \\
\hline Eastern Predbalkan & P3 & $19(1.1)$ & $24(0.7)$ & $43(0.8)$ \\
\hline Stara Planina (Balkan) Mts. & SB & $542(32.4)$ & $884(26.4)$ & $1426(28.4)$ \\
\hline Western Stara Planina (Balkan) Mts. & B1 & $457(27.3)$ & $515(15.4)$ & $972(19.4)$ \\
\hline Middle Stara Planina (Balkan) Mts. & B2 & $198(11.8)$ & $429(12.8)$ & $627(12.5)$ \\
\hline
\end{tabular}




\begin{tabular}{|c|c|c|c|c|}
\hline Eastern Stara Planina (Balkan) Mts. & B3 & $23(1.4)$ & $246(7.3)$ & $269(5.4)$ \\
\hline TRANSITIONAL REGION & $T$ & $781(46.7)$ & $1893(56.6)$ & 2674 (53.3) \\
\hline Kraishte-Konyavo district & TK & $114(6.8)$ & $143(4.3)$ & $257(5.1)$ \\
\hline Rouy Mt. & K1 & & $1(0.03)$ & $1(0.02)$ \\
\hline Trun Basin (Znepole) & $\mathrm{K} 2$ & & $17(0.5)$ & $17(0.3)$ \\
\hline Strazha-Cherna Gora-Rudini Mts. & K3 & $29(1.7)$ & $1(0.03)$ & $30(0.6)$ \\
\hline Golo Burdo Mt. & K4 & $8(0.5)$ & $15(0.4)$ & $23(0.5)$ \\
\hline Verila Mts. & K5 & $2(0.1)$ & & $2(0.04)$ \\
\hline Kraishte & K6 & & $41(1.2)$ & $41(0.8)$ \\
\hline Zemenska Planina Mt. & K7 & $38(2.3)$ & $1(0.03)$ & $39(0.8)$ \\
\hline Konyavska Planina Mt. & K8 & $53(3.2)$ & $23(0.7)$ & $76(1.5)$ \\
\hline Kyustendil Basin & K9 & $71(4.2)$ & $77(2.3)$ & $148(2.9)$ \\
\hline Vitosha district & TV & $561(33.6)$ & $1431(42.8)$ & $1992(39.7)$ \\
\hline Sofia Basin & V1 & $257(15.4)$ & $822(24.6)$ & $1079(21.5)$ \\
\hline Zavalska-Viskyar Mts. & V2 & $1(0.06)$ & $2(0.06)$ & $3(0.06)$ \\
\hline Lyulin Mt. & V3 & $18(1.1)$ & $66(2.0)$ & $84(1.7)$ \\
\hline Vitosha Mt. & V4 & $329(19.7)$ & $972(29.0)$ & $1301(25.9)$ \\
\hline Plana Mts. & V5 & $81(4.8)$ & $70(2.1)$ & $151(3.0)$ \\
\hline Srednogorie-Podbalkan subregion & TS & $192(11.5)$ & $645(19.3)$ & $837(16.7)$ \\
\hline Podbalkan Basins & S1 & $107(6.4)$ & $397(11.9)$ & $504(10.0)$ \\
\hline Sredna Gora Mts. & S2 & $101(6.0)$ & $352(10.5)$ & $453(9.0)$ \\
\hline Ihtimanska Sredna Gora Mts. & S21 & $90(5.4)$ & $206(6.1)$ & $296(5.9)$ \\
\hline Lozenska Planina Mt. & S211 & $65(3.9)$ & $158(4.7)$ & $223(4.4)$ \\
\hline Sushtinska Sredna Gora Mts. & $\mathrm{S} 22$ & $19(1.1)$ & $77(2.3)$ & $96(1.9)$ \\
\hline Surnena Sredna Gora Mts. & $\mathrm{S} 23$ & $8(0.5)$ & $95(2.8)$ & $103(2.0)$ \\
\hline Thracian Lowland & $\mathrm{TL}$ & $200(12.0)$ & $528(15.8)$ & $728(14.5)$ \\
\hline Toundzha-Strandzha subregion & TT & $192(11.5)$ & $282(8.4)$ & $474(9.4)$ \\
\hline Sakar-Toundzha district & $\mathrm{T} 1$ & $87(5.2)$ & $53(1.6)$ & $140(2.8)$ \\
\hline Sakar Mt. & $\mathrm{T} 11$ & $7(0.4)$ & $39(1.2)$ & $46(0.9)$ \\
\hline Bakadzhik-Bourgas district & T2 & $28(1.7)$ & $66(2.0)$ & $94(1.9)$ \\
\hline Strandzha-Dervent district & T3 & $119(7.1)$ & $236(7.0)$ & $355(7.1)$ \\
\hline Strandzha Mts. & T31 & $119(7.1)$ & $236(7.0)$ & $355(7.1)$ \\
\hline RILA-RHODOPE MASSIF & $\mathbf{R}$ & $813(48.6)$ & $1871(55.9)$ & $2684(53.5)$ \\
\hline Osogovo-Belasitsa group & RO & $248(14.8)$ & $593(17.7)$ & $841(16.8)$ \\
\hline Osogovska Planina Mts. & 01 & $4(0.2)$ & $25(0.7)$ & $29(0.6)$ \\
\hline Vlahina Planina Mts. & 02 & & $2(0.06)$ & $2(0.04)$ \\
\hline Maleshevska Planina Mts. & 03 & & $22(0.6)$ & $22(0.4)$ \\
\hline Ograzhden Mts. & 04 & & $47(1.4)$ & $47(0.9)$ \\
\hline Belasitsa Mts. & 05 & $14(0.8)$ & $201(6.0)$ & $215(4.3)$ \\
\hline Srednostroumska Valley & 06 & $193(11.5)$ & $515(15.4)$ & $708(14.1)$ \\
\hline Boboshevo-Simitli Valley & 061 & $159(9.5)$ & $172(5.1)$ & $331(6.6)$ \\
\hline Kroupnik-Sandanski-Petrich Valley & 062 & $140(8.4)$ & $432(12.9)$ & $572(11.4)$ \\
\hline Rila-Pirin group & RP & $525(31.4)$ & $1117(33.4)$ & $1642(32.7)$ \\
\hline Rila Mts. & $\mathrm{R} 1$ & $333(19.9)$ & $716(21.4)$ & $1049(20.9)$ \\
\hline Pirin Mts. & $\mathrm{R} 2$ & $233(13.9)$ & $608(18.2)$ & $841(16.7)$ \\
\hline Slavyanka Mt. & R3 & $14(0.8)$ & $231(6.9)$ & $245(4.9)$ \\
\hline Sturgach Mt. & R4 & $1(0.06)$ & $42(1.2)$ & $43(0.8)$ \\
\hline Mesta Valley & R5 & $114(6.8)$ & $107(3.2)$ & $221(4.4)$ \\
\hline Rhodope Mts. & RR & $449(26.8)$ & $1184(35.4)$ & $1633(32.5)$ \\
\hline Western Rhodope Mts. & RW & $385(23.0)$ & $1018(30.4)$ & $1403(27.9)$ \\
\hline Eastern Rhodope Mts. & $\mathrm{RE}$ & $153(9.1)$ & $383(11.4)$ & $536(10.7)$ \\
\hline BLACK SEA COAST & B & $367(21.9)$ & $1025(30.6)$ & $1392(27.7)$ \\
\hline Northern Black Sea Coast & BN & $285(17.0)$ & $710(21.2)$ & $995(19.8)$ \\
\hline Southern Black Sea Coast & BS & $165(9.9)$ & $646(19.3)$ & $811(16.2)$ \\
\hline
\end{tabular}




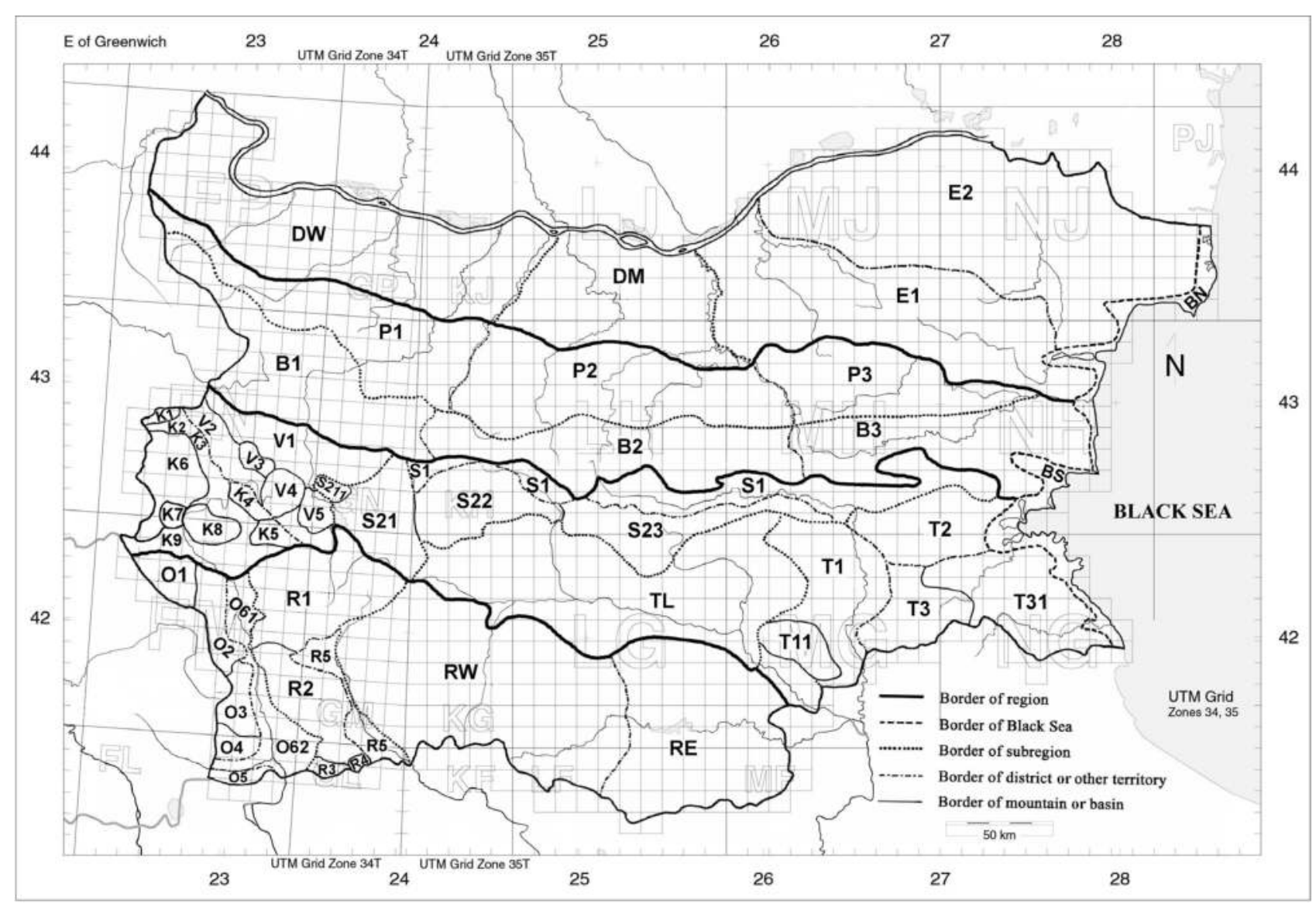

Fig. 1. Natural geographical territorial units of Bulgaria

Abbreviations used: B - Black Sea Coast; B1 - Western Stara Planina (Balkan) Mts.; B2 - Middle Stara Planina (Balkan) Mts.; B3 - Eastern Stara Planina (Balkan) Mts.; BN - Northern Black Sea Coast; BS - Southern Black Sea Coast; D - Danubian Plain; DM - Middle Danubian Plain; DW - Western Danubian Plain; E1 - PopovoProvadiya district; E2 - Loudogorie-Dobroudzha district; P1 - Western Predbalkan; K1 - Rouy Mt.; K2 - Trun Basin (Znepole); K3 - Strazha-Cherna Gora-Rudini Mts.; K4 - Golo Burdo Mt.; K5 - Verila Mts.; K6 - Kraishte; K7 - Zemenska Planina Mt.; K8 - Konyavska Planina Mt.; K9 - Kyustendil Basin; 01 - Osogovska Planina Mts.; O2 - Vlahina Planina Mts.; O3 - Maleshevska Planina Mts.; O4 - Ograzhden Mts.; 05 - Belasitsa Mts.; $\mathbf{O 6}$ Srednostroumska Valley; 061 - Boboshevo-Simitli Valley; 062 - Kroupnik-Sandanski-Petrich Valley; P2 - Middle Predbalkan; P3 - Eastern Predbalkan; R - Rila-Rhodope Massif; R1 - Rila Mts.; R2 - Pirin Mts.; R3 - Slavyanka Mt.; R4 - Sturgach Mt.; R5 - Mesta Valley; RE - Eastern Rhodope Mts.; RO - Osogovo-Belasitsa group; RP - RilaPirin group; RR - Rhodope Mts.; RW - Western Rhodope Mts.; S - Stara Planina Range System; S1 - Podbalkan Basins; S2 - Sredna Gora Mts.; S21 - Ihtimanska Sredna Gora Mts.; S211 - Lozenska Planina Mt.; S22 - Sushtinska Sredna Gora Mts.; S23 - Surnena Sredna Gora Mts.; SB - Stara Planina (Balkan) Mts.; SP - Predbalkan; T - Transitional Region; T1 - Sakar-Toundzha district; T11 - Sakar Mt.; T2 - Bakadzhik-Bourgas district; T3 Strandzha-Dervent district; T31 - Strandzha Mts.; TK - Kraishte-Konyavo district; TL - Thracian Lowland; TS Srednogorie-Podbalkan subregion; TT - Toundzha-Strandzha subregion; TV - Vitosha district; V1 - Sofia Basin; V2 - Zavalska-Viskyar Mts.; V3 - Lyulin Mt.; V4 - Vitosha Mt.; V5 - Plana Mts.

(2017) and Emeljanov (2018). The traditional nomenclature of the areas and the border between the Western and Eastern Palaearctic along the Yenisei River is accepted. To compare the fauna, Czekanowski-Dice-Sørensen coefficient of similarity is used (Czekanowski 1909, Dice 1945, Sørensen 1948).

For each species are given: 1) a recent scientific name, synonyms and names under which it is reported from Bulgaria; 2) distribution in the Bulgarian territory; 3) altitude at which it is established; 4) vegetation belts 
which it inhabits; 5) aerographical characteristic (general distribution); 6) references. In various foreign publications species without localities, reported from Bulgaria, are included. In such cases, the sign „" (lack of data) is used. In some species more than one aerographical category is given. This is due to the differences in the taxa distribution, presented in the separated literature sources. In the introduced taxa, the natural range is indicated first and then is the secondary (anthropogenic) range. Unfortunately, in many publications recent ranges without information about the anthropogenic and natural ranges are given. The zoogeographical analysis includes only the first aerographical categories. Before the following categories, the question mark "?" is used (in case of differences between the literature sources) or the letter " $i$ " (in case of data for introduction).

\section{Abbreviations used}

[ ] - names and synonyms under which the species are reported from Bulgaria; $\bullet$ - species without precise locality; - species, presented everywhere, without precise locality; $\boldsymbol{\Lambda}$ - species of human or veterinary medical significance; - - enemy for forestry or agriculture; - lack of data; - outdated information; +++ - species, reported for the first time and localities, from which species are reported for the first time.

Vegetation belts: 1 - Xerothermic oak forests, 2 - Mesophylic and xeromesophylic mixed (oak-hornbeam) forests, 3 - Beech forests, 4 - Coniferous forests, 5 - Subalpine vegetation, 6 - Alpine vegetation.

Range types: ? - probable category, aa - Arctoalpine, am - Arctomontane, atm - Afrotropical-Mediterranean, ba - Boreoalpine, ban - Balkan-Anatolian, bc - Balkan-Caucasian, bci - Balkan-Caucasian-Iranian, bct Balkan-Caucasian-Turanian, bm - Boreomontane, cee - Central and East European, ceean - Central and East European-Anatolian, ceet - Central and East European-Turanian, cse - Central and South European, csean Central and South European-Anatolian, ceet - Central and East European-Turanian, cse - Central and South European, csean - Central and South European-Anatolian, cseanna - Central and South European-AnatolianNorth African, csee - Central and Southeast European, cseean - Central and Southeast European-Anatolian, cseei - Central and Southeast European-Iranian, cseel - Central and Southeast European-Lebanonian, cseet - Central and Southeast European-Turanian, csei - Central and South European-Iranian, cseit - Central and South European-Iran-Turanian, csel - Central and South European-Lebanonian, csena - Central and South European-North African, csess - Central and South European and South Siberian, cset - Central (Middle) and South European-Turanian, csewca - Central and South European-West Central Asian, des - Disjunct Eurosiberian, dp - Disjunct Palaearctic, dpat - Disjunct Palaearctic-Afrotropical, dpo - Disjunct PalaearcticOriental, e - European, ean - European-Anatolian, eanca - European-Anatolian-Central Asian, eani - European-Anatolian-Iranian, eanit - European-Anatolian-Iran-Turanian, eanna - European-Anatolian-North African, eant - European-Anatolian-Turanian, Eb - Balkan endemic, Ebg - Bulgarian endemic, Ebs - Balkan subendemic, eca - European-Central Asian, ee - East European, eeca - East European-Central Asian, ees - East European-Siberian, eet - East European-Turanian, ei - European-Iranian, eit - European-Iran-Turanian, em East Mediterranean, emca - East Mediterranean-Central Asian, emi - East Mediterranean-Iranian, emit - East Mediterranean-Iran-Turanian, en - European-Neotropical, ena - European-North African, eno - EuropeanNeotropical-Oriental, eo - European-Oriental, Er - Regional endemic, esan - Eurosiberian-Anatolian, esanca - Eurosiberian-Anatolian-Central Asian, esca - Eurosiberian-Central Asian, esit - Eurosiberian-Iran-Turanian, ess - European and South Siberian, eswa - European-Southwest Asian, et - European-Turanian, ewca - EuropeanWest Central Asian, $\mathbf{h}$ - Holarctic, $\mathbf{h}^{\star}$ - species introduced in North America, ha - Holarctic-Australian, hat - Holarctic-Afrotropical, hata - Holarctic-Afrotropical-Australian, hn - Holarctic-Neotropical, hna - HolarcticNeotropical-Australian, hnat - Holarctic-Neotropical-Afrotropical, hno - Holarctic-Neotropical-Oriental, ho - Holarctic-Oriental, hoa - Holarctic-Oriental-Australian, hoes - Holoeurosiberian, hom - Holomediterranean, hop - Holopalaearctic, hpt - Holarctic-Paleotropical, hpta - Holarctic-Paleotropical-Australian, hptn - Holarctic-Paleotropical-Neotropical, $\mathbf{i}$ - introduced species (immigrants), $\mathbf{k}$ - Cosmopolitan, $\mathbf{m}$ - montane, mca - Mediterranean-Central Asian, mfe - Mediterranean-Far East, mi - Mediterranean-Iranian, mit Mediterranean-Iran-Turanian, $\mathbf{m m}$ - montane-Mediterranean, mss - Mediterranean and South Siberian, mt - Mediterranean-Turanian, mwca - Mediterranean-West Central Asian, nem - Northeast Mediterranean, nemi - Northeast Mediterranean-Iranian, nemit - Northeast Mediterranean-Iran-Turanian, nemwca - Northeast Mediterranean-West Central Asian; nm - North Mediterranean, nmca - North Mediterranean-Central Asian, nmi - North Mediterranean-Iranian, nmsfe - North Mediterranean and South Far East, nmsws - North 
Mediterranean and Southwest Siberian, nmwca - North Mediterranean-West Central Asian, oem - OrientalEast Mediterranean, om - Oriental-Mediterranean, pa - Palaearctic-Australian, pat - Palaearctic-Afrotropical, pata - Palaearctic-Afrotropical-Australian, patn - Palaearctic-Afrotropical-Neotropical, pn - PalaearcticNeotropical, po - Palaearctic-Oriental, poa - Palaearctic-Oriental-Australian, ppt - Palaearctic-Paleotropical, ppta - Palaearctic-Paleotropical-Australian, pptn - Palaearctic-Paleotropical-Neotropical, ptm - PaleotropicalMediterranean, se - South European, see - Southeast European, seean - Southeast European-Anatolian, seeani - Southeast European-Anatolian-Iranian, seeanna - Southeast European-Anatolian-North African, seeca Southeast European-Central Asian, seei - Southeast European-Iranian, seem - Southeast European montane, seena - Southeast European-North African, seesfe - Southeast European and South Far East, seess - Southeast European and South Siberian, seet - Southeast European-Turanian, seewca - Southeast European-West Central Asian, sena - South European-North African, sesfe - South European and South Far East, sess - South European and South Siberian, set - South European-Turanian, sk - Semicosmopolitan, sk ${ }^{\star}$ - species introduced, sp - South Palaearctic, spat - South Palaearctic-Afrotropical, spo - South Palaearctic-Oriental, sppt - South Palaearctic-Paleotropical, sppta - South Palaearctic-Paleotropical-Australian, swp - Southwest Palaearctic, swpat - Southwest Palaearctic-Afrotropical, swpnata - Southwest Palaearctic-Neotropical-Afrotropical-Australian, swpo - Southwest Palaearctic-Oriental, swppt - Southwest Palaearctic-Paleotropical, tes - Transeurosiberian, tp - Transpalaearctic, wces - West and Central Eurosiberian, wcp - West and Central Palaearctic, wes - West Eurosiberian, wesan - West Eurosiberian-Anatolian, wesanca - West Eurosiberian-Anatolian-Central Asian, wesani - West Eurosiberian-Anatolian-Iranian, wesant - West Eurosiberian-Anatolian-Turanian, wesca - West Eurosiberian-Central Asian, wesit - West Eurosiberian-Iran-Turanian, west - West Eurosiberian-Turanian, weswca - West Eurosiberian-West Central Asian, wp - West Palaearctic, wpat - West Palaearctic-Afrotropical, wpn - West Palaearctic-Neotropical, wpo - West Palaearctic-Oriental, wppt - West Palaearctic-Paleotropical.

\section{Results and Discussion}

A total of 5038 species of Diptera - 26.3\% of the European species (Nematocera - 1672 species, $22.4 \%$; Brachycera -3366 species, $28.8 \%$ ) that belong to 110 families has been established in Bulgaria so far. The families Tachinidae ( 425 species), Chironomidae ( 327 species), Syrphidae (303 species), Muscidae ( 268 species), Mycetophilidae (263 species), Cecidomyiidae (262 species), Phoridae (229 species), Limoniidae (221 species) and Dolichopodidae (217 species) are the most numerous. The other families contain less than 200 species (Table 2).

Of all 132 families known from Europe (Oosterbroek 2006), twenty-two families have not been found in Bulgaria. Of Nematocera (32 families), six families (Axymyiidae, Pachyneuridae, Pleciidae, Mycetobiidae, Synneuridae and Canthyloscelidae) have not been found and of Brachycera (100 families), sixteen families (Rachiceridae, Hilarimorphidae, Brachystomatidae, Mydidae, Opetiidae, Pseudopomyzidae, Tanypezidae, Strongylophthalmyiidae, Megamerinidae, Heterocheilidae, Pyrgotidae, Stenomicridae, Xenasteiidae, Cryptochetidae, Chiropteromyzidae and Cnemospathidae) have not been established. These families (with the exception of Mycetobiidae, Brachystomatidae and Mydidae - 4 to 13 species) are represented by 1 to 3 species in Europe.

Usually the families studied in Bulgaria include over 25\% of the European species. Of the largest families (over 800 species in Europe) - Mycetophilidae (945 species), Cecidomyiidae (1640 species), Chironomidae (1190 species), Empididae (810 species), Syrphidae (830 species), Agromysidae (910 species) and Tachinidae (880 species), in Bulgaria are found 27.8\%, 16.0\%, 27.5\%, 22.6\%, 36.5\%, 20.9\% and 48.3\% of the European species. The families Limoniidae, Tabanidae, Syrphidae, Conopidae, Calliphoridae and Sarcophagidae are well represented - from 36.5\% to $48.2 \%$ of the European taxa. In Bulgaria the families Culicidae, Chloropidae, Ephydridae and Muscidae have been studied for the longest time and most systematically, which includes from $40.3 \%$ to $46.4 \%$ of the European species. The families Scatopsidae, Thaumaleidae, Platypezidae, Micropezidae, Lonchaeidae, Pallopteridae, Trixoscelididae and Chyromyidae are the most poorly studied in Bulgaria. Of these families, from $1.7 \%$ to $5.3 \%$ of the European taxa are known. Of the larger families, Ceratopogonidae, Sciaridae and Anthomyiidae are poorly studied, of which from $11.0 \%$ to $13.5 \%$ of the European species are known in Bulgaria. Of the family Bombyliidae, a total of 89 species (26.2\% of the European forms) has been established but thirty-two of them have been reported for Bulgaria based on their distribution in the neighboring countries. Actually, 57 species (16.8\% of the European taxa) have been established in the territory of Bulgaria and the family can be considered poorly studied. Some old data about the Chironomidae family, collected in the hydrobiological stud- 
Table 2. The distribution of Diptera according to the vegetation belts of Bulgaria

\begin{tabular}{|c|c|c|c|c|c|c|c|}
\hline \multirow[b]{2}{*}{ Families } & \multirow[b]{2}{*}{$\begin{array}{c}\text { Total } \\
\text { number }\end{array}$} & \multicolumn{6}{|c|}{ Vegetation belts of Bulgaria } \\
\hline & & 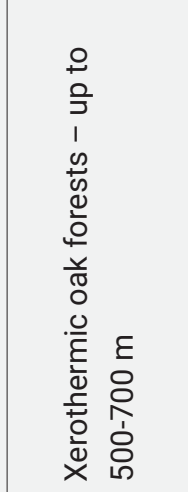 & 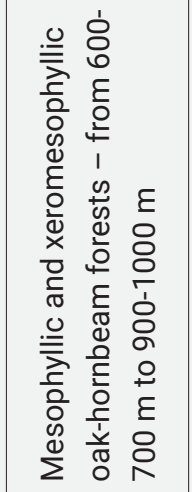 & 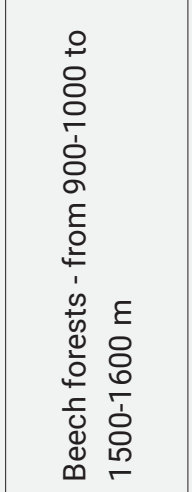 & 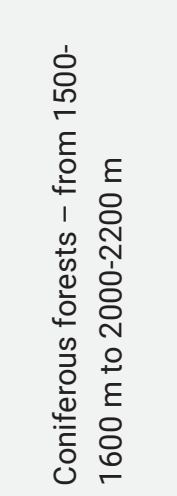 & 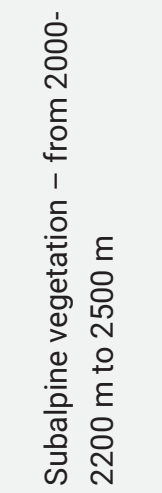 & 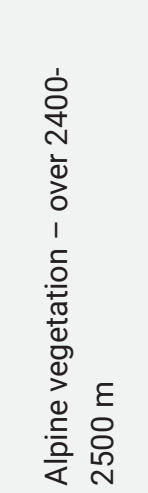 \\
\hline NEMATOCERA (7456) & $1672(22.4)$ & $1087(65.0)$ & $932(55.7)$ & 756 (45.2) & $314(18.8)$ & $84(5.0)$ & $14(0.8)$ \\
\hline Tipulomorpa (1146) & $343(29.9)$ & $171(15.7)$ & $144(15.4)$ & $157(20.8)$ & $84(26.7)$ & $27(32.1)$ & $4(28.6)$ \\
\hline Tipulidae (470) & $89(18.9)$ & $50(4.6)$ & $38(4.1)$ & $18(2.4)$ & $6(1.9)$ & $4(4.8)$ & $1(7.1)$ \\
\hline Limoniidae (560) & $221(39.5)$ & $117(10.8)$ & $100(10.7)$ & $118(15.6)$ & $66(21.0)$ & $21(25.0)$ & $3(21.4)$ \\
\hline Pediciidae (60) & $25(41.7)$ & $3(0.3)$ & $3(0.3)$ & $16(2.1)$ & $10(3.2)$ & $2(2.4)$ & \\
\hline Cylindrotomidae (6) & $1(16.6)$ & & & $1(0.1)$ & $1(0.3)$ & & \\
\hline Trichoceridae (50) & $7(14.0)$ & $1(0.09)$ & $3(0.3)$ & $3(0.4)$ & $1(0.3)$ & & \\
\hline Blephariceromorpha (38) & $8(21.0)$ & & $5(0.5)$ & $3(0.4)$ & $1(0.3)$ & & \\
\hline Blephariceridae (38) & $8(21.0)$ & & $5(0.5)$ & $3(0.4)$ & $1(0.3)$ & & \\
\hline Bibionomorpha (3409) & $687(20.1)$ & $396(36.4)$ & $441(47.3)$ & $373(49.3)$ & $113(36.0)$ & $7(8.3)$ & \\
\hline Bibionidae (47) & $13(27.6)$ & $10(0.9)$ & $9(1.0)$ & $2(0.3)$ & $1(0.3)$ & & \\
\hline Hesperinidae (1) & $1(100.0)$ & & $1(0.1)$ & $1(0.1)$ & & & \\
\hline Mycetophilidae (945) & $263(27.8)$ & $135(12.4)$ & $192(20.6)$ & $172(22.7)$ & $58(18.5)$ & $3(3.6)$ & \\
\hline Ditomyiidae (3) & $3(100.0)$ & $2(0.2)$ & $2(0.2)$ & $2(0.3)$ & & & \\
\hline Bolitophilidae (36) & $10(27.8)$ & $4(0.4)$ & $7(0.7)$ & $7(0.9)$ & $5(1.6)$ & & \\
\hline Diadocidiidae (5) & $3(60.0)$ & $1(0.09)$ & $2(0.2)$ & $3(0.4)$ & $2(0.6)$ & & \\
\hline Keroplatidae (110) & $48(43.6)$ & $38(3.5)$ & $31(3.3)$ & $23(3.0)$ & $16(5.1)$ & & \\
\hline Sciaridae (620) & $84(13.5)$ & $38(3.5)$ & $52(5.6)$ & $40(5.3)$ & $3(1.0)$ & & \\
\hline Cecidomyiidae (1640) & $262(16.0)$ & $168(15.5)$ & $145(15.6)$ & $123(16.3)$ & $28(8.9)$ & $4(4.8)$ & \\
\hline Psychodomorpha (617) & $109(17.7)$ & $75(6.9)$ & $48(5.1)$ & $35(4.6)$ & $14(4.5)$ & $1(1.2)$ & \\
\hline Psychodidae (500) & $102(20.4)$ & $71(6.5)$ & $45(4.8)$ & $33(4.4)$ & $14(4.5)$ & $1(1.2)$ & \\
\hline Anisopodidae (10) & $3(30.0)$ & $1(0.09)$ & $3(0.3)$ & $1(0.1)$ & & & \\
\hline Scatopsidae (100) & $4(4.0)$ & $3(0.3)$ & & $1(0.1)$ & & & \\
\hline Ptychopteromorpha (15) & $5(33.3)$ & $3(0.3)$ & $2(0.2)$ & & $1(0.3)$ & & \\
\hline Ptychopteridae (15) & $5(33.3)$ & $3(0.3)$ & $2(0.2)$ & & $1(0.3)$ & & \\
\hline Culicomorpha (2231) & $521(23.3)$ & $442(40.7)$ & $292(31.3)$ & $188(24.9)$ & $101(32.2)$ & $49(58.3)$ & $10(71.4)$ \\
\hline Dixidae (32) & $2(6.2)$ & $2(0.2)$ & $1(0.1)$ & & & & \\
\hline Chaoboridae (9) & $1(11.1)$ & $1(0.09)$ & $1(0.1)$ & $1(0.1)$ & & & \\
\hline Culicidae (105) & $47(44.8)$ & $46(4.2)$ & $22(2.4)$ & $15(2.0)$ & $3(1.0)$ & $1(1.2)$ & \\
\hline Thaumaleidae (75) & $4(5.3)$ & $1(0.09)$ & $2(0.2)$ & $1(0.1)$ & $1(0.3)$ & & \\
\hline Simuliidae (230) & $7432.2)$ & $40(3.7)$ & $47(5.0)$ & $42(5.5)$ & $33(10.5)$ & $20(23.8)$ & \\
\hline Ceratopogonidae (590) & $66(11.2)$ & $61(5.6)$ & $35(3.7)$ & $7(0.9)$ & $3(1.0)$ & $2(2.4)$ & $1(7.1)$ \\
\hline Chironomidae (1190) & $327(27.5)$ & $291(26.8)$ & $184(19.7)$ & $122(16.1)$ & $61(19.4)$ & $26(31.0)$ & $9(64.3)$ \\
\hline BRACHYCERA (11701) & $3366(28.7)$ & $2275(68.0)$ & $1666(49.8)$ & $1516(45.3)$ & $809(24.2)$ & $264(7.9)$ & $43(1.3)$ \\
\hline Orthorrhapha (1552) & $379(24.4)$ & $276(12.1)$ & $195(11.7)$ & $134(8.8)$ & $72(8.9)$ & $15(5.7)$ & $6(13.9)$ \\
\hline Xylophagomorpha (6) & $2(33.3)$ & $1(0.04)$ & $1(0.06)$ & $1(0.06)$ & & & \\
\hline Xylophagidae (5) & $1(20.0)$ & $1(0.04)$ & $1(0.06)$ & & & & \\
\hline Coenomyiidae (1) & $1(100.0)$ & & & $1(0.06)$ & & & \\
\hline Stratiomyomorpha (148) & $51(34.5)$ & $43(1.9)$ & $23(1.4)$ & $12(0.8)$ & $3(0.4)$ & & \\
\hline
\end{tabular}




\begin{tabular}{|c|c|c|c|c|c|c|c|}
\hline \multirow[b]{2}{*}{ Families } & \multirow[b]{2}{*}{$\begin{array}{c}\text { Total } \\
\text { number }\end{array}$} & \multicolumn{6}{|c|}{ Vegetation belts of Bulgaria } \\
\hline & & 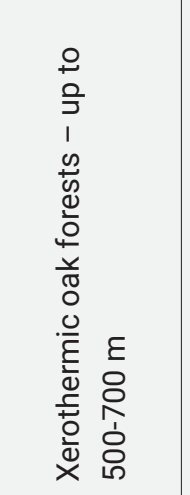 & 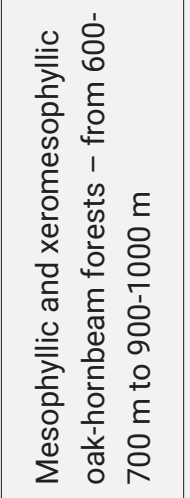 & 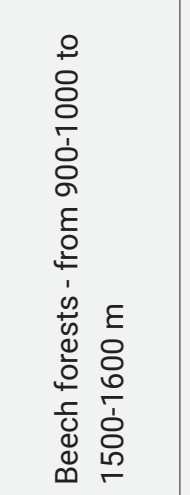 & 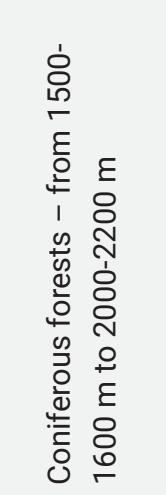 & 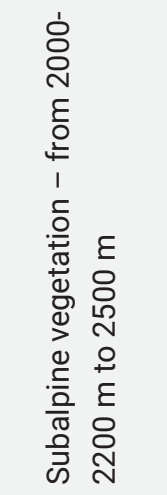 & 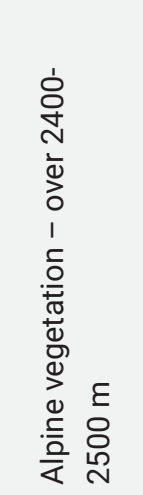 \\
\hline Xylomyidae (8) & $1(12.5)$ & $1(0.04)$ & & & & & \\
\hline Stratiomyidae (140) & $50(35.7)$ & $42(1.8)$ & $23(1.4)$ & $12(0.8)$ & $3(0.4)$ & & \\
\hline Tabanomorpha (1398) & $326(23.3)$ & $232(10.2)$ & $171(10.2)$ & $121(8.0)$ & $69(8.5)$ & $15(5.7)$ & $6(13.9)$ \\
\hline Rhagionidae (85) & $18(21.2)$ & $10(0.4)$ & $8(0.5)$ & $10(0.6)$ & $4(0.5)$ & $1(0.4)$ & \\
\hline Athericidae (10) & $2(20.0)$ & & $2(0.1)$ & & & & \\
\hline Tabanidae $(220)$ & $85(38.6)$ & $73(3.2)$ & $59(3.5)$ & $44(2.9)$ & $31(3.8)$ & $6(2.3)$ & $4(9.3)$ \\
\hline Vermileonidae (9) & $1(11.1)$ & $1(0.04)$ & & & & & \\
\hline Nemestrinidae (13) & $1(7.7)$ & $1(0.04)$ & & & & & \\
\hline Acroceridae (35) & $2(5.7)$ & $1(0.04)$ & & & $1(0.1)$ & $1(0.4)$ & $1(2.3)$ \\
\hline Bombyliidae (340) & $89(26.2)$ & $48(2.1)$ & $34(2.0)$ & $9(0.6)$ & $4(0.5)$ & & \\
\hline Mythicomyiidae (30) & $2(6.7)$ & $2(0.09)$ & & & & & \\
\hline Therevidae $(100)$ & $10(10.0)$ & $6(0.3)$ & $4(0.2)$ & $5(0.3)$ & & & \\
\hline Scenopinidae (16) & $2(12.5)$ & $2(0.09)$ & $1(0.06)$ & & & & \\
\hline Asilidae $(540)$ & $114(21.1)$ & $88(3.9)$ & $63(3.8)$ & $53(3.5)$ & $29(3.6)$ & $7(2.6)$ & $1(2.3)$ \\
\hline Eremoneura (2045) & $494(24.1)$ & $274(12.0)$ & $167(10.0)$ & $209(13.8)$ & $108(13.3)$ & $17(6.4)$ & $1(2.3)$ \\
\hline Empididae (810) & $183(22.6)$ & $63(2.8)$ & $68(4.1)$ & $106(7.0)$ & $60(7.4)$ & $8(3.0)$ & $1(2.3)$ \\
\hline Hybotidae (440) & $87(19.8)$ & $47(2.1)$ & $32(1.9)$ & $36(2.4)$ & $18(2.2)$ & $1(0.4)$ & \\
\hline Atelestidae (4) & $2(50.0)$ & & & $2(0.1)$ & & & \\
\hline Microphoridae (16) & $5(31.2)$ & $2(0.09)$ & $4(0.2)$ & $3(0.2)$ & & & \\
\hline Dolichopodidae (775) & $217(27.0)$ & $162(7.1)$ & $63(3.8)$ & $62(4.1)$ & $30(3.7)$ & $8(3.0)$ & \\
\hline Cyclorrhapha (8104) & $2490(30.7)$ & $1725(75.8)$ & $1304(78.3)$ & $1173(77.4)$ & $629(77.7)$ & $232(87.9)$ & $37(86.0)$ \\
\hline ASCHIZA (1693) & $564(33.3)$ & $315(13.8)$ & $323(19.4)$ & $349(23.0)$ & $164(20.3)$ & $82(31.1)$ & $3(7.0)$ \\
\hline Platypezidae (45) & $2(4.4)$ & & $2(0.1)$ & $1(0.06)$ & & & \\
\hline Lonchopteridae (13) & $4(30.8)$ & $4(0.2)$ & $3(0.2)$ & $3(0.2)$ & $2(0.2)$ & $1(0.4)$ & \\
\hline Phoridae (605) & $229(37.8)$ & $87(3.8)$ & $123(7.4)$ & $160(10.5)$ & $92(11.4)$ & $64(24.2)$ & \\
\hline Pipunculidae (200) & $26(13.0)$ & $18(0.8)$ & $13(0.8)$ & $8(0.5)$ & $7(0.9)$ & $1(0.4)$ & $1(2.3)$ \\
\hline Syrphidae (830) & $303(36.5)$ & $206(9.0)$ & $182(10.9)$ & $177(11.7)$ & $63(7.8)$ & $16(6.1)$ & $2(4.6)$ \\
\hline SCHIZOPHORA (6411) & $1925(30.0)$ & $1410(62.0)$ & $981(58.9)$ & $824(54.3)$ & $465(57.5)$ & $150(56.8)$ & $34(79.1)$ \\
\hline Acalyptratae (3696) & $941(25.5)$ & $719(31.6)$ & $417(25.0)$ & $335(22.1)$ & $200(24.7)$ & $90(34.1)$ & $21(48.8)$ \\
\hline Micropezidae (22) & $1(4.5)$ & $1(0.04)$ & $1(0.06)$ & & & & \\
\hline Psilidae (50) & $7(14.0)$ & $6(0.3)$ & $1(0.06)$ & $1(0.06)$ & $1(0.1)$ & & \\
\hline Diopsidae (1) & $1(100.0)$ & $1(0.04)$ & & & & & \\
\hline Conopidae (85) & $41(48.2)$ & $33(1.4)$ & $26(1.6)$ & $21(1.4)$ & $7(0.9)$ & $1(0.4)$ & \\
\hline Lonchaeidae (100) & $5(5.0)$ & $4(0.2)$ & $1(0.06)$ & & $1(0.1)$ & & \\
\hline Ulidiidae (29) & $3(10.3)$ & $2(0.09)$ & $1(0.06)$ & $1(0.06)$ & $1(0.1)$ & & \\
\hline Otitidae (76) & $13(17.1)$ & $10(0.4)$ & $1(0.06)$ & & & & \\
\hline Platystomatidae (20) & $4(20.0)$ & $3(0.1)$ & $1(0.06)$ & & & & \\
\hline Tephritidae (270) & $79(29.2)$ & $61(2.7)$ & $29(1.7)$ & $17(1.1)$ & $9(1.1)$ & $4(1.5)$ & \\
\hline Pallopteridae (23) & $1(4.3)$ & $1(0.04)$ & & & & & \\
\hline Piophilidae (30) & $3(10.0)$ & $3(0.1)$ & $2(0.1)$ & $1(0.06)$ & $1(0.1)$ & & \\
\hline Lauxaniidae (160) & $21(13.1)$ & $9(0.4)$ & $2(0.1)$ & $1(0.06)$ & & & \\
\hline
\end{tabular}




\begin{tabular}{|c|c|c|c|c|c|c|c|}
\hline \multirow[b]{2}{*}{ Families } & \multirow[b]{2}{*}{$\begin{array}{c}\text { Total } \\
\text { number }\end{array}$} & \multicolumn{6}{|c|}{ Vegetation belts of Bulgaria } \\
\hline & & 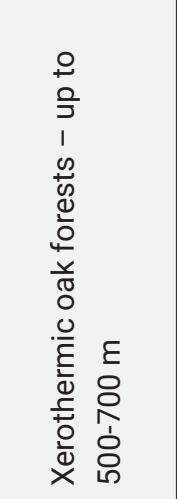 & 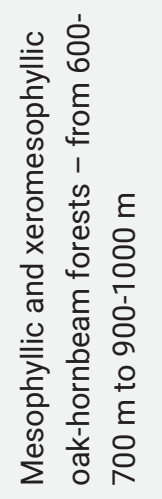 & 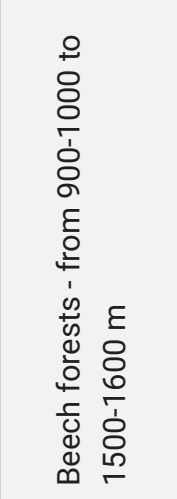 & 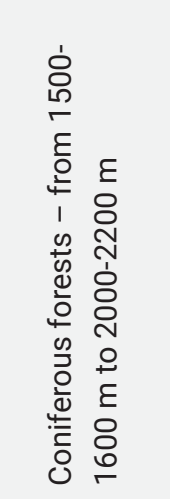 & 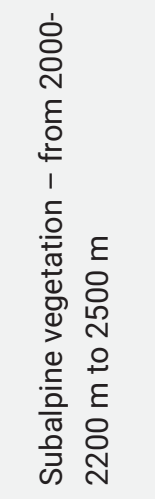 & 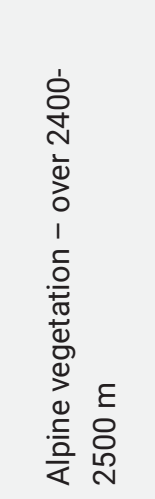 \\
\hline Cremifaniidae (2) & $1(50.0)$ & & & & & $1(0.4)$ & \\
\hline Chamaemyiidae (110) & $29(26.4)$ & $22(1.0)$ & $15(0.9)$ & $15(1.0)$ & $9(1.1)$ & $5(1.9)$ & $1(2.3)$ \\
\hline Coelopidae (3) & $3(100.0)$ & $2(0.09)$ & & & & & \\
\hline Dryomyzidae (4) & $2(50.0)$ & $2(0.09)$ & $2(0.1)$ & $2(0.1)$ & $1(0.1)$ & & \\
\hline Sciomyzidae (140) & $36(25.7)$ & $24(1.0)$ & $10(0.6)$ & $1(0.06)$ & & & \\
\hline Phaeomyiidae (3) & $1(33.3)$ & & $1(0.06)$ & & & & \\
\hline Helcomyzidae (2) & $1(50.0)$ & $1(0.04)$ & & & & & \\
\hline Sepsidae (50) & $11(22.0)$ & $7(0.3)$ & $3(0.2)$ & $2(0.1)$ & $1(0.1)$ & & \\
\hline Acartophthalmidae (3) & $2(66.7)$ & $1(0.04)$ & $1(0.06)$ & & $1(0.1)$ & & \\
\hline Odiniidae (14) & $2(14.3)$ & $2(0.09)$ & & & & & \\
\hline Agromyzidae (910) & $190(20.9)$ & $119(5.2)$ & $82(4.9)$ & $74(4.9)$ & $28(2.5)$ & $11(4.2)$ & $2(4.6)$ \\
\hline Opomyzidae (33) & $7(21.2)$ & $6(0.3)$ & $6(0.4)$ & $6(0.4)$ & $4(0.5)$ & $3(1.1)$ & $1(2.3)$ \\
\hline Anthomyzidae (30) & $5(16.7)$ & $3(0.1)$ & & & & & \\
\hline Aulacigastridae (4) & $1(25.0)$ & $1(0.04)$ & & & & & \\
\hline Periscelididae (4) & $1(25.0)$ & $1(0.04)$ & $1(0.06)$ & $1(0.06)$ & & & \\
\hline Asteiidae (18) & $3(16.7)$ & $3(0.1)$ & $1(0.06)$ & & & & \\
\hline Braulidae (3) & $3(100.0)$ & $1(0.04)$ & & & & & \\
\hline Carnidae (40) & $8(20.0)$ & $4(0.2)$ & $5(0.3)$ & $5(0.3)$ & $6(0.7)$ & $2(0.7)$ & $1(2.3)$ \\
\hline Tethinidae (35) & $7(20.0)$ & $7(0.3)$ & & & & & \\
\hline Canacidae (4) & $1(25.0)$ & $1(0.04)$ & & & & & \\
\hline Milichiidae (45) & $8(17.8)$ & $7(0.3)$ & $4(0.2)$ & $2(0.1)$ & $1(0.1)$ & $1(0.4)$ & \\
\hline Chloropidae (395) & $166(42.0)$ & $150(6.6)$ & $109(6.5)$ & $92(6.1)$ & $67(8.3)$ & $31(11.7)$ & $5(11.6)$ \\
\hline Siphonellopsidae (3) & $2(66.7)$ & $2(0.09)$ & $1(0.06)$ & & & & \\
\hline Heleomyzidae (150) & $51(34.0)$ & $32(1.4)$ & $26(1.6)$ & $25(1.6)$ & $15(1.8)$ & $7(2.6)$ & \\
\hline Trixoscelididae (25) & $1(4.0)$ & $1(0.04)$ & & & & & \\
\hline Chyromyidae (60) & $1(1.7)$ & $1(0.04)$ & $1(0.06)$ & & & & \\
\hline Sphaeroceridae (260) & $60(23.1)$ & $36(1.6)$ & $12(0.7)$ & $11(0.7)$ & $9(1.1)$ & $3(1.1)$ & \\
\hline Curtonotidae (1) & $1(100.0)$ & $1(0.04)$ & & & & & \\
\hline Camillidae (8) & $1(12.5)$ & $1(0.04)$ & $1(0.06)$ & & & & \\
\hline Drosophilidae (120) & $17(14.2)$ & $14(0.6)$ & $6(0.4)$ & $3(0.2)$ & $2(0.2)$ & $1(0.4)$ & $1(2.3)$ \\
\hline Campichoetidae (7) & $2(28.6)$ & $3(0.1)$ & $1(0.06)$ & & & & \\
\hline Diastatidae (9) & $4(44.4)$ & $3(0.1)$ & $2(0.1)$ & $2(0.1)$ & & & \\
\hline Ephydridae (335) & $135(40.3)$ & $127(5.6)$ & $62(3.7)$ & $52(3.4)$ & $36(4.4)$ & $20(7.6)$ & $10(23.2)$ \\
\hline Calyptratae (2715) & $985(36.3)$ & $691(30.4)$ & $564(33.8)$ & $489(32.2)$ & $265(32.7)$ & $60(22.7)$ & $13(30.2)$ \\
\hline Hippoboscidae (30) & $12(40.0)$ & $10(0.4)$ & $6(0.4)$ & $4(0.3)$ & $3(0.4)$ & $3(1.1)$ & \\
\hline Streblidae (1) & $1(100.0)$ & $1(0.04)$ & $1(0.06)$ & & & & \\
\hline Nycteribiidae (15) & $7(46.7)$ & $7(0.3)$ & $7(0.4)$ & $4(0.3)$ & & & \\
\hline Scathophagidae (160) & $11(6.9)$ & $4(0.2)$ & $3(0.2)$ & $5(0.3)$ & $1(0.1)$ & & $1(2.3)$ \\
\hline Anthomyiidae (480) & $53(11.0)$ & $34(1.5)$ & $25(1.5)$ & $22(1.4)$ & $13(1.6)$ & & \\
\hline Fanniidae (82) & $27(32.9)$ & $22(1.0)$ & $18(1.1)$ & $18(1.2)$ & $8(1.0)$ & $2(0.7)$ & $1(2.3)$ \\
\hline Muscidae (575) & $267(46.4)$ & $190(8.3)$ & $162(9.7)$ & $167(11.0)$ & $132(16.3)$ & $30(11.4)$ & $7(16.3)$ \\
\hline
\end{tabular}




\begin{tabular}{|c|c|c|c|c|c|c|c|}
\hline \multirow[b]{2}{*}{ Families } & \multirow[b]{2}{*}{$\begin{array}{c}\text { Total } \\
\text { number }\end{array}$} & \multicolumn{6}{|c|}{ Vegetation belts of Bulgaria } \\
\hline & & 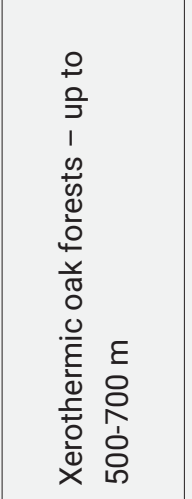 & 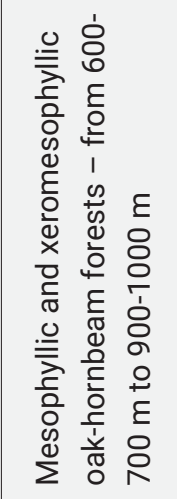 & 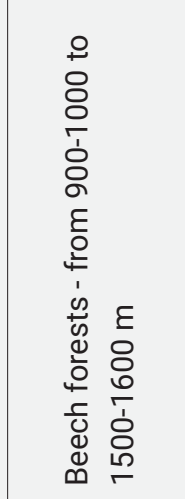 & 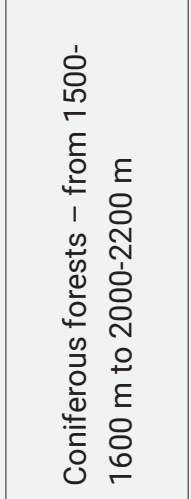 & 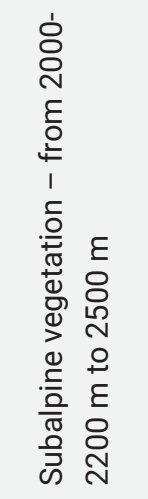 & 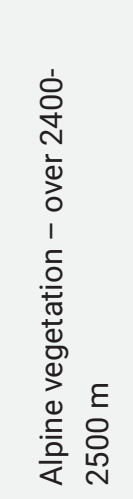 \\
\hline Calliphoridae (115) & $41(35.6)$ & $36(1.6)$ & $20(1.2)$ & $14(0.9)$ & $9(1.1)$ & $1(0.4)$ & \\
\hline Sarcophagidae (310) & $122(39.3)$ & $107(4.7)$ & $47(2.8)$ & $39(2.6)$ & $32(3.9)$ & $9(3.4)$ & \\
\hline Rhinophoridae (45) & $8(17.8)$ & $6(0.3)$ & $2(0.1)$ & $1(0.06)$ & & & \\
\hline Oestridae (8) & $2(25.0)$ & $2(0.09)$ & $1(0.06)$ & & & & \\
\hline Hypodermatidae (8) & $4(50.0)$ & $4(0.2)$ & $2(0.1)$ & $1(0.06)$ & & & \\
\hline Gasterophilidae (6) & $4(66.7)$ & $3(0.1)$ & $3(0.2)$ & $3(0.2)$ & & & \\
\hline Tachinidae (880) & $425(48.3)$ & $265(11.6)$ & $267(16.0)$ & $211(13.9)$ & $67(8.3)$ & $15(5.7)$ & $4(9.3)$ \\
\hline DIPTERA: 19157 & $5038(26.3)$ & $3362(66.7)$ & $2598(51.6)$ & $2272(45.1)$ & $1123(22.3)$ & $348(6.9)$ & $57(1.1)$ \\
\hline
\end{tabular}

Note. After the families the number of species established in Europe is given in brackets according to OsterBROEK (2006) with changes in Ulidiidae and Chloropidae. The percentages for the vegetation belts are calculated separately for Nematocera and Brachycera (and not total for Diptera).

ies, are problematic (in some cases incorrect identification is not excluded as an accurate determination of the larvae by species is not always possible). In the last 10 years, two new families have been found for the Bulgarian fauna - Cremifaniidae (Papp 2010) and Diopsidae (Kutsarov \& Hubenov 2019).

The infraorders of Nematocera, represented in Bulgaria, include from 17.7\% (Psychodomorpha) to 33.3\% (Ptychopteromorpha) of the European taxa. Of the infraorders of Brachycera, Orthorrhapha includes 24.4\%, Eremoneura $-24.0 \%$ and Cyclorrhapha $-30.7 \%$ of the European species (Acalyptratae $-25.4 \%$ and Calyptratae $-36.3 \%)$.

Of the established in Bulgaria species of the order Diptera, 75 species have a medical significance and 61 species are pests on the vegetation. Of the suborder Nematocera, 26 species have a human or veterinary medical significance and 40 species are pests on the forestry or agriculture. Most representatives of medical significance includes the family Culicidae (13), followed by Ceratopogonidae (7), Psychodidae (5) and Simuliidae (1). The main part of the vegetation pests belongs to the family Cecidomyiidae (35). Only 5 species are shared out among Tipulidae (2), Bibionidae (2) and Sciaridae (1). Of the suborder Brachycera, 49 species have a human or veterinary medical significance and 20 species are pests on the forestry or agriculture. Most representatives of medical significance include the families Muscidae (11) and Tabanidae (9), followed by Sarcophagidae (5), Calliphoridae (4), Hypodermatidae (4), Gastrophilidae (4), Fanniidae (3) Hippoboscidae (3), Oestridae (2), Piophilidae (2), Braulidae (1) and Anthomyiidae (1). The main part of the vegetation pests belongs to Anthomyiidae (6), Tephritidae (4) and Chloropidae (4). With one species each are presented Psilidae, Lonchaeidae, Agromyzidae, Opomyzidae, Heleomyzidae, Drosophilidae and Ephydridae. The medical significance of Culicidae, Ceratopogonidae, Oestridae, Hypodermatidae, Gasterophilidae and the separate species of Simuliidae, Tabanidae, Piophilidae, Hippoboscidae, Calliphoridae and Sarcophagidae are connected with the predominant distribution in the first 2-3 vegetation belts where the main part of the population is concentrated.

The distribution of Diptera on the territory of Bulgaria (Table 1) is related to the specific natural conditions of the respective regions, the peculiarities of the families and their study. The wide distribution of Diptera assumes a similar fauna of the different regions after a good research. Most dipterans have vast ranges and the endemics are poorly represented [ 128 species $-2.5 \%$ (Nematocera $-3.6 \%$ and Brachycera $-2.0 \%$ )]. Often these are newly described taxa or rare species with unclear range. The taxa presence is connected with the exploration of the 
corresponding parts of the country. This is evident when comparing the established species with regard to the separate regions in Bulgaria. In Nematocera there are no data for the mountains Rouy, Vlahina, Maleshevska, Ograzhden and the Trun Basin. For the fifteen natural territories (1-29 species known) there are single reports (Eastern Predbalkan, Eastern Stara Planina Mts., the mountains of Kraishte, Viskyar and Zavalska, Sushtinska and Surnena Sredna Gora Mts., Sakar Mt., Bakadzhik-Bourgas district, Osogovska Planina Mts., Belasitsa Mts., Slavyanka Mt. and Sturgach Mt.). In Brachycera there are no data for Verila Mts. One-two species each are known from the mountains Rouy, Strazha, Cherna Gora, Rudini, Zemenska, Zavalska, Viskyar and Vlahina. For the five natural territories (below 30 species found) there are single reports (Eastern Predbalkan, Golo Burdo, Konyavska, Osogovska and Maleshevska Planina Mountains).

Several areas with better research of the Diptera fauna are outlined. These are natural areas located near research centers, included in the national and nature parks, or areas subject of dissertation works for separate families. Among the territories with better study of the fauna with respect to more systematic groups (represented by over 14-15\% of the species found in Bulgaria) are the Western Predbalkan, Western Stara Planina Mts., Sofia Basin, Vitosha Mt., Thracian Lowland, Rila, Pirin and Western Rhodope Mts. and the Black Sea Coast (from $14.8 \%$ to $27.3 \%$ of Nematocera and $15.4 \%$ до $30.4 \%$ of Brachycera, established in Bulgaria). In the Western Stara Planina Mts., Vitosha Mt., Thracian Lowland, Srednostroumska Valley, Western Rhodope Mts. and the Black Sea Coast, dissertation works have been performed on the families Mycetophilidae, Cecidomyiidae, Tabanidae, Empididae, Dolichopodidae, Phoridae and Tachinidae. There is a decreasing tendency of the level of research from west to east and from north to south, which is followed in the Stara Planina Range system, the Rila-Rhodope massif and the Black Sea coast. An exception of this tendency are the mountains in the western part of the Transitional Region (west of Sofia) which are traditionally neglected by the most zoologists and are poorly studied. Investigations on the synbovil and synanthropic forms of the families Tabanidae, Fanniidae, Muscidae, Calliphoridae and Sarcophagidae have enriched the faunistic composition of the Brachycera in the Eastern Danubian Plain, Sofia Basin, Surnena Sredna Gora Mts., Thracian Lowland and the Black Sea Coast.

Regarding to all Diptera it is impressive the close species diversity between the Transitional Region and the Rila-Rhodope massif (53.3-53.5\% of all species established). There are small differences in the suborders. For Nematocera, the species diversity is closer between the Danubian Plain and the Black Sea coast (19.6-21.9\% of the species established) on the one hand and the Stara Planina Range system, the Transitional Region and the Rila-Rhodope massif (43.0-48.6\% of the species established) from another. For Brachycera, the species diversity is closer between the Transitional Region and the Rila-Rhodope massif (55.9-56.6\% of the species established) on the one hand and the Stara Planina Range system and the Black Sea coast (30.6-30.7\% of the species established) from another. The most visited and explored territories such as Sofia Basin, Vitosha Mt., Struma River Valley and the Rila, Pirin and Western Rhodope Mts. are in the Transitional Region and the Rila-Rhodope massif. For Sredna Gora Mts. it can be noted that almost half of the taxa (223 species - 49.2\%) are found in the Lozenska Planina Mt. (a small part of the Ihtimanska Sredna Gora Mts., located near Sofia) and the Sushtinska (21.2\% of the species) and Surnena Sredna Gora Mts. (22.7\% of the species) are poorly studied. The situation is similar in the Toundzha-Strandzha subregion where the most species (74.9\%) are found in the Strandzha Mts. (all species of the Strandzha-Dervent district). Of the mountains of the Osogovo-Belasitsa group most species are known from Belasitsa. The Rila-Pirin group is dominated by the taxa found in the Rila (63.9\%) and Pirin (51.2\%) Mts., while there are few reports for Slavyanka and Sturgach Mt. The difference in the number of species between the valleys of the rivers Struma (708 species - 14.1\%) and Mesta (221 species - 4.4\%) is determined both by the specific natural conditions and the many studies of the Sandanski-Petrich Valley and Kresna Gorge compared to the limited research in the valley of the Mesta River. The difference between the Western (1403 species - 27.9\%) and Eastern Rhodope Mts. (536 species - 10.7\%) is also related to the natural conditions and prevailing interest of the specialists in the Western Rhodope Mts. Vitosha Mt. is the most well-studied Bulgarian mountain with a taxonomic diversity (1301 species - 25.9\%) comparable to that of the Stara Planina Mts., Rila and Western Rhodope Mts. despite its smaller area. In the better studied families (Limoniidae, Simuliidae and Tachinidae) the differences among the Vitosha, Rila and Pirin Mts. are not big (Hubenov 2019b). Further studies of the Pirin Mts. would like increase the number of the dipterans and it might exceed most of the Bulgarian mountains. This is related to the wide variety of natural habitats, as well as the geographical location which the mountain occupies in Southwest Bulgaria. The Sofia Basin is the best studied area (1079 species - $21.5 \%$ of the species established in Bulgaria). A comparison of Brachycera with Nematocera shows differences among the separate territorial units (Table 1) which reach to $12.3 \%$ in the Stara Planina Range System. 
The greatest number of species $(3362-66.7 \%)$ has been established in the xerothermic oak forests belt (Table 2). This is due to the specificity of the separate families and the position of the most localities below $1000 \mathrm{~m}$ a.s.l. In the next two belts - the mesophilic and xeromesophilic mixed forests $(2598$ species $-51.6 \%)$ and beech forests (2272 species $-45.1 \%$ ), the number of species decreases with $15.2 \%$ and $21.7 \%$. In the other vegetation belts the number of species decreases almost triple in the coniferous belt (1123 species - 22.4\%) and sharply in the subalpine and alpine vegetation belts (348 species $-6.9 \%$ and 57 species $-1.1 \%$ ). There is an exception in the families Limoniidae, Pediciidae, Trichoceridae, Mycetophilidae, Bolitophilidae, Sciaridae, Cecidomyiidae, Chironomidae, Simuliidae, Phoridae, Chloropidae, Ephydridae, Muscidae, Sarcophagidae and Tachinidae (well represented from the beech to the subalpine vegetation belts), which are studied and well represented in the mountainous areas. In Nematocera this explains the percentage increase of Tipulomorpha $(20.8 \%-32.1 \%)$ and Culicomorpha (24.9\% - 58.3\%) from the beech to the subalpine vegetation belts. In Bibionomorpha, the percentage increase is greatest in the beech forests belt (49.3\%). This is related to the research of the superfamily Sciaroidea in the Vitosha and Western Stara Planina Mts. Most hydrobiological studies are concentrated in the first 2-3 vegetation belts (mainly below $1300 \mathrm{~m}$ a.s.l.) and are connected mainly with the families Tipulidae, Simuliidae and Chironomidae. In Brachycera, Tabanidae, Phoridae, Chloropidae, Ephydridae, Muscidae, Sarcophagidae and Tachinidae determine the increase of Tabanomorpha (8.5\% - 13.9\%), Aschiza (23.0\% - 31.1\%), Schizophora $(54.3 \%-79.1 \%)$, Acalyptratae $(22.1 \%-48.8 \%)$ and the strong presence of Calyptratae $(22.7 \%-32.7 \%)$ from the beech to the alpine vegetation belts. The infraorder Cyclorrhapha (from $75.8 \%$ to $87.9 \%$ ) predominates in all belts and determines the faunistic diversity. Often there are open spaces in which species from the surrounding valleys penetrate and the fauna of the first two vegetation belts is similar. The families Cylindrotomidae, Blephariceridae, Hesperinidae, Coenomyiidae, Athericidae, Atelestidae and Platypezidae have not been established in the belt of the xerothermic oak forests. In Nematocera, most families (24 each) are represented in the belts of the mesophilic and xeromesophilic mixed forests and beech forests. In the coniferous belts (314 species - 18.8\%), 20 families have been found and the species composition is determined by the larger ones - Limoniidae, Mycetophilidae, Keroplatidae, Cecidomyiidae, Simuliidae and Chironomidae. In Brachycera, most families (80 and 64) are represented in the belts of the xerothermic oak forests and the mesophilic and xeromesophilic mixed forests. In the next 3 belts the diversity decreases with 10 families each (from 50 in the beech belt to 30 in the subalpine belt) and the species composition is determined by the families Tabanidae, Asilidae, Empididae, Dolichopodidae, Phoridae, Syrphidae, Agromyzidae, Chloropidae, Ephydridae, Muscidae, Sarcophagidae and Tachinidae. The upper limit of the coniferous forests gradually passes into the subalpine vegetation with wide mixing zones. Thus, some of the species are common to both vegetation belts and the number of taxa established in the subalpine belt increases. Forty families are presented, of which Limoniidae, Simuliidae, Chironomidae, Phoridae, Syrphidae, Chloropidae, Ephydridae, Muscidae and Tachinidae determine the faunistic diversity. Of the species found in the alpine vegetation belt ( 14 species $-0.8 \%$ of Nematocera and 43 species $-1.3 \%$ of Brachycera), three species are typical only for it [Micropsectra radialis Goet. - Palaearctic-Oriental species of Chironomidae; Didea alneti (Fallén, 1817) - Holarctic species of Syrphidae; Eudorylas jenkinsoni Coe, 1966 - European species of Pipunculidae]. The other species have been established in the subalpine belt and most of them in other vegetation belts as well. Twenty-one families have been found in the alpine zone, as the families Chironomidae, Tabanidae, Chloropidae, Ephidridae, Muscidae and Tachinidae mainly determine the faunistic diversity.

When comparing the generalized data for the Vrachanska Planina Mts., Vitosha, Rila and Pirin Mts. (Hubenov 2019b) it is seen that the fauna of the subalpine belt of the Vitosha Mt. is richer than the fauna of the Rila and Pirin Mts. This is due to the lower height of the Vitosha Mt. and the lack of a clear coniferous belt in the southern parts of the mountain. With the exception of some families, the investigations in this part of the Rila and Pirin Mts. are insufficient and fragmentary. The degree of similarity between the vegetation belts of the Rila and Pirin Mts. is low. It is the largest (46.6\%) in the second vegetation belt and is completely lacking in the alpine zone (Table 3).

Regarding to the hypsometric belts, there are significant variations in the maximum number of species among the families in the separate mountains. In the Vrachanska Planina Mts., the maximum number of species was recorded between 300 and $600 \mathrm{~m}$ a.s.l. In the Vitosha and Pirin Mts. this number is located between 900 and $1300 \mathrm{~m}$ a.s.l., and in the Rila Mts. - between 1000 and $1500 \mathrm{~m}$ a.s.l. In general, for the whole country maximum number of species was established between 400 and $1000 \mathrm{~m}$ a.s.l. as there are differences in the mountains of $\pm 200 \mathrm{~m}$ (Hubenov 1993, 2019b). In some cases, the finding of species at certain altitude is accidental. The lack of systematic research on many families, the unclear boundaries among the vegetation belts and the fragmen- 
Table 3. Similarity of the Diptera fauna in percentages according to the vegetation belts of the Rila and Pirin mountains

\begin{tabular}{|c|c|c|c|c|c|c|}
\hline Vegetation belts of & \multicolumn{5}{|c|}{ Vegetation belts of the Rila Mts. } \\
\cline { 2 - 6 } the Pirin Mts. & $\mathbf{1}$ & $\mathbf{2}$ & $\mathbf{3}$ & $\mathbf{4}$ & $\mathbf{5}$ & \\
\hline $\mathbf{1}$ & $40.8(108)$ & & & & \\
\hline $\mathbf{2}$ & & $46.6(163)$ & & & & \\
\hline $\mathbf{3}$ & & & $40.9(234)$ & & & \\
\hline $\mathbf{4}$ & & & & $30.7(83)$ & & \\
\hline $\mathbf{5}$ & & & & & & \\
\hline $\mathbf{6}$ & & & & & & \\
\hline
\end{tabular}

Note. 1 - Xerothermic oak forests, 2 - Mesophyllic and xeromesophyllic oak-hornbeam forests, 3 - Beech forests, 4 - Coniferous forests, 5 - Subalpine vegetation, 6 - Alpine vegetation; Common species are given in brackets.

tary data for most dipterans do not allow explicit conclusions about the adherence of the taxa to one or another vegetation zone to be made. The distribution of species in groups according to their presence in the vegetation belts has a relative character and depends on the specific features of taxa and research areas, as well as on the duration of the research. There is a correlation between the horizontal and vertical distribution of Diptera. The species with wide vertical distribution usually comprise large areas of the European, Eurosiberian, Palaearctic, Super Palaearctic and Cosmopolitan type. The dipterans found in the subalpine and alpine zones of the Rila and Pirin Mts. have Holarctic-Oriental, Holarctic, Transpalaearctic, West and Central Palaearctic, West Palaearctic, European-North African, Holoeurosiberian, West and Central Eurosiberian, West Eurosiberian, Disjunct Eurosiberian and European ranges (Hubenov 2017, 2019b).

The zoogeographical categorization of the species was made on the basis of current data about their distribution. Thus, the dipterans are divided into 150 categories, combined into three main groups and six complexes (Table 4).

Species distributed in the Palaearctic and beyond it. This group (1199 species - 23.9\%) includes 45 categories, of which 32 combine species of northern type (widely distributed in the Holarctic and Palaearctic) and 13 - species of southern type (distributed only in the southern parts of the Palaearctic). The difference between the separate vegetation belts reaches $18.1 \%$ and varies from $25.8 \%$ (beech forests) to $43.9 \%$ (alpine vegetation). Of the other areographical categories, this difference is the highest in the Cosmopolitan (6.0\%) and the Holarctic (6.7\%) species. The establishment of other species of the group of the northern type in the subalpine and the alpine vegetation belts is very likely, owing to their distribution and insufficient studies of the higher parts of the mountains. It is known that the species of the northern type have vast areas and ecological flexibility. In the Super-Palaearctic group, the Holarctic species prevail: [ 532 species - 10.6\% (from 10.8\% in the xerothermic oak forests to $17.5 \%$ in the alpine belt)]. Of the other areographical types, the Palaearctic-Oriental [155 species - 3.1\% (from 3 to 132 species, $2.6 \%$ to $5.3 \%$ in the separate belts)] and the Holarctic-Oriental [123 species - 2.4\% (from 10 to 104 species, $2.9 \%$ to $4.2 \%$ )] forms are better presented. The species of the southern type (58 species - 1.2\%) are represented mainly in the first two (three) vegetation belts (two species are found in the coniferous forests belt). The group is not important for the zoogeographical characteristic because of the small number of species (2-48 species, $0.2-1.4 \%)$. Usually the Super-Palaearctic group is scantly presented and is not determinant for the zoogeographical characteristic of taxa in the Bulgarian terrestrial fauna (with the exception of the coastal fauna). Only in a highly mobile forms (such as Diptera), the complex is well presented and could reach $20 \%$ (Hubenov 2015a). It is best represented in percentages in the alpine belt and less in the other vegetation belts (Table 4). In the two-winged insects significant numbers of synanthropic and synbovil forms with cosmopolitan or subcosmopolitan distribution occur. They have anthropogenic areas, structured with the development of the human civilisation (before the contemporary studies).

Species distributed only in the Palaearctic but in more than one subregion (Palaearctic type). Taxa, whose areas include more than one Palaearctic subregion in latitudinal direction belong to this group. They are well represented in the high mobile groups and comprise about $20-25 \%$ of the species composition. A total of 32 areographical categories, including 1200 species (23.9\%) of the Bulgarian Diptera fauna, has been registered (Table 4). The character of the Palaearctic complex is determined by the Transpalaearctic [234 species $-4.7 \%$ 
Table 4. Zoogeographical characteristic of Diptera according to the vegetation belts in Bulgaria

\begin{tabular}{|c|c|c|c|c|c|c|c|}
\hline \multirow[b]{2}{*}{ Classification of the areas } & \multirow[b]{2}{*}{ 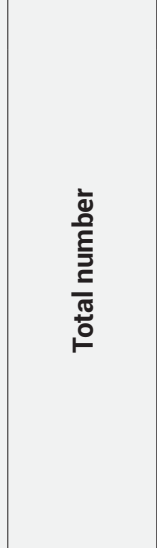 } & \multicolumn{6}{|c|}{ Vegetation belts } \\
\hline & & 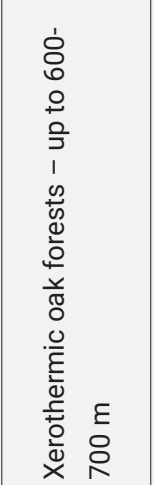 & 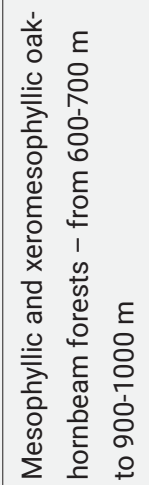 & 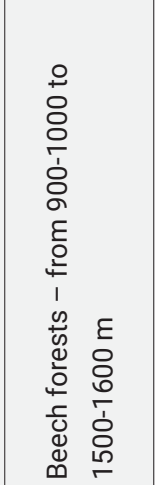 & 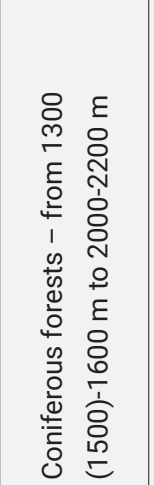 & 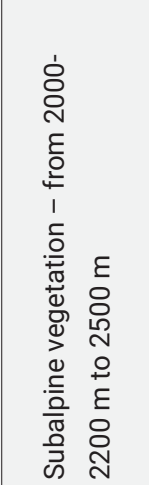 & 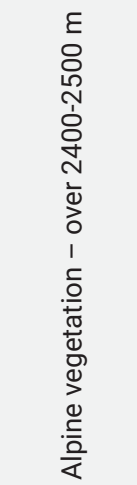 \\
\hline Species distributed in Palaearctic and out of it & $1199(23.9)$ & $911(27.1)$ & $700(26.9)$ & $587(25.8)$ & $301(26.8)$ & $101(29.0)$ & 25 (43.9) \\
\hline North type & $1141(22.7)$ & $863(25.7)$ & $680(26.2)$ & $577(25.4)$ & $299(26.6)$ & $101(29.0)$ & $25(43.8)$ \\
\hline Cosmopolitan & $46(0.9)$ & $42(1.2)$ & $28(1.1)$ & $23(1.0)$ & $15(1.3)$ & $9(2.6)$ & $4(7.0)$ \\
\hline Semicosmopolitan & $26(0.5)$ & $22(0.6)$ & $13(0.5)$ & $12(0.5)$ & $7(0.6)$ & $2(0.6)$ & $1(1.7)$ \\
\hline Holarctic-Paleotropical-Neotropical & $5(0.1)$ & $3(0.09)$ & $4(0.1)$ & $3(0.1)$ & $2(0.2)$ & $1(0.3)$ & \\
\hline Holarctic-Paleotropical-Australian & $10(0.2)$ & $10(0.3)$ & $5(0.2)$ & $3(0.1)$ & $2(0.2)$ & & \\
\hline Holarctic-Paleotropical & $7(0.1)$ & $7(0.2)$ & $3(0.1)$ & $3(0.1)$ & $2(0.2)$ & $1(0.3)$ & \\
\hline Holarctic-Neotropical-Afrotropical & $3(0.06)$ & $10(0.3)$ & $10(0.4)$ & $7(0.3)$ & $4(0.4)$ & $2(0.6)$ & $1(1.7)$ \\
\hline Holarctic-Neotropical-Oriental & $18(0.4)$ & $11(0.3)$ & $10(0.4)$ & $8(0.3)$ & $5(0.4)$ & $1(0.3)$ & $1(1.7)$ \\
\hline Holarctic-Neotropical-Australian & $5(0.1)$ & $3(0.09)$ & $3(0.1)$ & $2(0.09)$ & $1(0.09)$ & & \\
\hline Holarctic-Afrotropical-Australian & $2(0.04)$ & $2(0.06)$ & & & & & \\
\hline Holarctic-Oriental-Australian & $8(0.2)$ & $7(0.2)$ & $5(0.2)$ & $4(0.2)$ & & & \\
\hline Holarctic-Afrotropical & $12(0.2)$ & $8(0.2)$ & $8(0.3)$ & $5(0.2)$ & $3(0.3)$ & $2(0.6)$ & $1(1.7)$ \\
\hline Holarctic-Oriental & $123(2.4)$ & $104(3.1)$ & $86(3.3)$ & $77(3.4)$ & $47(4.2)$ & $10(2.9)$ & \\
\hline Holarctic-Neotropical & $21(0.4)$ & $19(0.6)$ & $13(0.5)$ & $10(0.4)$ & $7(0.6)$ & $5(1.4)$ & $1(1.7)$ \\
\hline Holarctic-Australian & $13(0.3)$ & $10(0.3)$ & $7(0.3)$ & $4(0.2)$ & $1(0.09)$ & & \\
\hline Holarctic & $532(10.6)$ & $365(10.8)$ & $314(12.1)$ & $278(12.2)$ & $139(12.4)$ & $49(14.1)$ & $10(17.5)$ \\
\hline Palaearctic-Paleotropical-Neotropical & $1(0.02)$ & $1(0.03)$ & & & & & \\
\hline Palaearctic-Paleotropical-Australian & $12(0.2)$ & $1(0.03)$ & $6(0.2)$ & $5(0.2)$ & $2(0.2)$ & $1(0.3)$ & \\
\hline Palaearctic-Afrotropical-Neotropical & $1(0.02)$ & $1(0.03)$ & $1(0.04)$ & $1(0.04)$ & $1(0.09)$ & & \\
\hline Palaearctic-Afrotropical-Australian & $2(0.04)$ & $2(0.06)$ & & & & & \\
\hline Palaearctic-Oriental-Australian & $2(0.04)$ & $2(0.06)$ & $2(0.08)$ & $2(0.09)$ & $1(0.09)$ & & \\
\hline Palaearctic-Paleotropical & $42(0.8)$ & $38(1.1)$ & $19(0.7)$ & $15(0.7)$ & $6(0.5)$ & $4(1.1)$ & $1(1.7)$ \\
\hline Palaearctic-Afrotropical & $19(0.4)$ & $16(0.5)$ & $11(0.4)$ & $8(0.4)$ & $3(0.3)$ & $3(0.9)$ & $1(1.7)$ \\
\hline Palaearctic-Oriental & $155(3.1)$ & $132(3.9)$ & $92(3.5)$ & $81(3.6)$ & $44(3.9)$ & $9(2.6)$ & $3(5.3)$ \\
\hline Palaearctic-Neotropical & $1(0.02)$ & & & & & & \\
\hline Palaearctic-Australian & $1(0.02)$ & $1(0.03)$ & $1(0.04)$ & $1(0.04)$ & $1(0.09)$ & $1(0.3)$ & $1(1.7)$ \\
\hline West Palaearctic-Paleotropical & $1(0.02)$ & $1(0.03)$ & $1(0.04)$ & $1(0.04)$ & & & \\
\hline West Palaearctic-Afrotropical & $18(0.4)$ & $6(0.2)$ & $8(0.3)$ & $7(0.3)$ & $2(0.2)$ & & \\
\hline West Palaearctic-Oriental & $25(0.5)$ & $20(0.6)$ & $15(0.6)$ & $10(0.4)$ & $1(0.09)$ & & \\
\hline West Palaearctic-Neotropical & $1(0.02)$ & $1(0.03)$ & $1(0.04)$ & $1(0.04)$ & $1(0.09)$ & & \\
\hline Disjunct Palaearctic-Afrotropical & $1(0.02)$ & $1(0.03)$ & & & & & \\
\hline Disjunct Palaearctic-Oriental & $24(0.5)$ & $14(0.4)$ & $13(0.5)$ & $6(0.3)$ & $2(0.2)$ & $1(0.3)$ & \\
\hline European-Oriental & $4(0.08)$ & $3(0.09)$ & $1(0.04)$ & & & & \\
\hline South type & $58(1.2)$ & $48(1.4)$ & $20(0.8)$ & $10(0.4)$ & $2(0.2)$ & & \\
\hline South Palaearctic-Paleotropical-Australian & 4() $.08)$ & $4(0.1)$ & $2(0.08)$ & $2(0.09)$ & & & \\
\hline $\begin{array}{l}\text { Southwest Palaearctic-Neotropical- } \\
\text { Afrotropical-Australian }\end{array}$ & $1(0.02)$ & $1(0.03)$ & & & & & \\
\hline South Palaearctic-Paleotropical & $11(0.2)$ & $10(0.3)$ & $4(0.1)$ & $2(0.09)$ & & & \\
\hline Southwest Palaearctic-Paleotropical & $2(0.04)$ & & $1(0.04)$ & & & & \\
\hline South Palaearctic-Afrotropical & $2(0.04)$ & $2(0.06)$ & $1(0.04)$ & $1(0.04)$ & & & \\
\hline Southwest Palaearctic-Afrotropical & $6(0.1)$ & $5(0.1)$ & $2(0.08)$ & & & & \\
\hline
\end{tabular}




\begin{tabular}{|c|c|c|c|c|c|c|c|}
\hline \multirow[b]{2}{*}{ Classification of the areas } & \multirow[b]{2}{*}{ 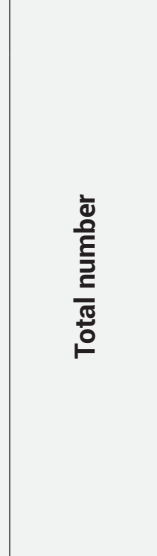 } & \multicolumn{6}{|c|}{ Vegetation belts } \\
\hline & & 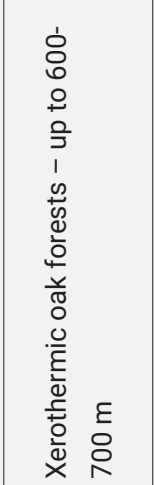 & 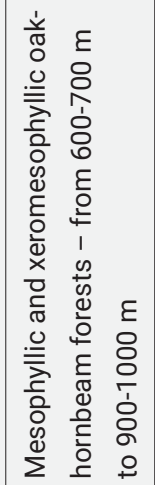 & 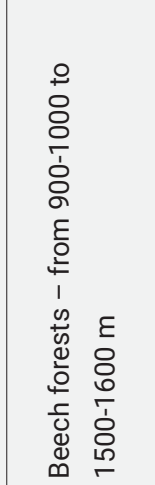 & 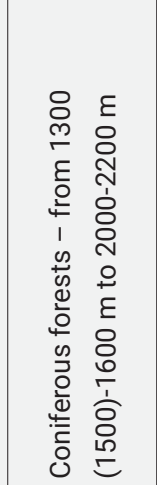 & 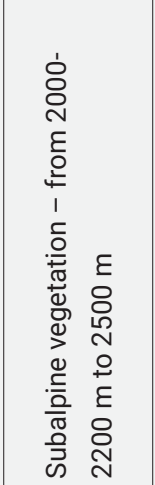 & 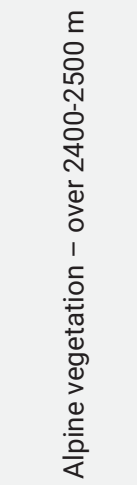 \\
\hline South Palaearctic-Oriental & $11(0.2)$ & $9(0.3)$ & $5(0.2)$ & $3(0.1)$ & $2(0.2)$ & & \\
\hline Southwest Palaearctic-Oriental & $3(0.06)$ & $3(0.09)$ & $1(0.04)$ & $1(0.04)$ & & & \\
\hline Paleotropical-Mediterranean & $3(0.06)$ & $1(0.03)$ & & & & & \\
\hline Afrotropical-Mediterranean & $11(0.2)$ & $9(0.3)$ & $3(0.1)$ & & & & \\
\hline Oriental-Mediterranean & $1(0.02)$ & $1(0.03)$ & $1(0.04)$ & $1(0.04)$ & & & \\
\hline Oriental-East Mediterranean & $2(0.04)$ & $2(0.06)$ & & & & & \\
\hline European-Neotropical-Oriental & $1(0.02)$ & $1(0.03)$ & & & & & \\
\hline Species with Palaearctic distribution & $3821(76.1)$ & $2451(72.9)$ & $1898(73.1)$ & $1685(74.2)$ & $822(73.2)$ & $247(71.0)$ & $32(56.1)$ \\
\hline Palaearctic type & $1200(23.9)$ & $954(28.4)$ & $714(27.5)$ & $545(24.0)$ & $261(23.2)$ & $86(24.7)$ & $11(19.3)$ \\
\hline Holopalaearctic & $26(0.5)$ & $23(0.7)$ & $24(0.9)$ & $18(0.8)$ & $10(0.9)$ & $5(1.4)$ & $3(5.3)$ \\
\hline Transpalaearctic & $234(4.7)$ & $192(5.7)$ & $154(5.9)$ & $119(5.2)$ & $57(5.1)$ & $14(4.0)$ & $2(3.5)$ \\
\hline West and Central Palaearctic & $123(2.4)$ & $109(3.2)$ & $81(3.1)$ & $60(2.6)$ & $28(2.5)$ & $10(2.9)$ & $2(3.5)$ \\
\hline West Palaearctic & $192(3.8)$ & $164(4.9)$ & $109(4.2)$ & $84(3.7)$ & $46(4.1)$ & $11(3.2)$ & \\
\hline Disjunct Palaearctic & $92(1.8)$ & $63(1.9)$ & $53(2.0)$ & $37(1.6)$ & $17(1.5)$ & $8(2.3)$ & $3(5.3)$ \\
\hline South Palaearctic & $2(0.04)$ & $2(0.06)$ & $2(0.08)$ & $1(0.04)$ & & & \\
\hline Southwest Palaearctic & $14(0.3)$ & $10(0.3)$ & $6(0.2)$ & $4(0.2)$ & & & \\
\hline Eurosiberian-Anatolian-Central Asian & $15(0.3)$ & $12(0.4)$ & $9(0.3)$ & $7(0.3)$ & $4(0.4)$ & $1(0.3)$ & \\
\hline Eurosiberian-Central Asian & $82(1.6)$ & $54(1.6)$ & $44(1.7)$ & $36(1.6)$ & $17(1.5)$ & $4(1.1)$ & $1(1.7)$ \\
\hline Eurosiberian-Anatolian (esan) & $3(0.06)$ & $2(0.06)$ & $2(0.08)$ & $2(0.09)$ & $1(0.09)$ & $1(0.3)$ & \\
\hline West Eurosiberian-Anatolian-Central Asian & $3(0.06)$ & $3(0.09)$ & $1(0.04)$ & $1(0.04)$ & $1(0.09)$ & & \\
\hline West Eurosiberian-Central Asian & $11(0.2)$ & $11(0.3)$ & $9(0.3)$ & $6(0.3)$ & $4(0.4)$ & $3(0.9)$ & \\
\hline West Eurosiberian-West Central Asian & $5(0.1)$ & $4(0.1)$ & $2(0.08)$ & & & & \\
\hline West Eurosiberian-Iran-Turanian & $3(0.06)$ & $2(0.06)$ & $2(0.08)$ & $5(0.2)$ & $1(0.09)$ & $1(0.3)$ & \\
\hline West Eurosiberian-Anatolian-Iranian & $1(0.02)$ & $1(0.03)$ & $1(0.04)$ & $1(0.04)$ & $1(0.09)$ & $1(0.3)$ & \\
\hline West Eurosiberian-Anatolian-Turanian & $3(0.06)$ & $3(0.09)$ & $2(0.08)$ & $1(0.04)$ & $1(0.09)$ & & \\
\hline West Eurosiberian-Turanian & $5(0.1)$ & $5(0.1)$ & $4(0.1)$ & $2(0.09)$ & & & \\
\hline West Eurosiberian-Anatolian & $9(0.2)$ & $7(0.2)$ & $4(0.1)$ & $4(0.2)$ & $3(0.3)$ & $1(0.3)$ & \\
\hline European-Anatolian-North African & $45(0.9)$ & $38(1.1)$ & $28(1.1)$ & $20(0.9)$ & $10(0.9)$ & $5(1.4)$ & \\
\hline European-North African & $156(3.1)$ & $116(3.4)$ & $82(3.2)$ & $68(3.0)$ & $32(2.8)$ & $12(3.4)$ & \\
\hline European-Anatolian-Central Asian & $14(0.3)$ & $11(0.3)$ & $7(0.3)$ & $3(0.1)$ & $2(0.2)$ & & \\
\hline European-Central Asian & $22(0.4)$ & $17(0.5)$ & $8(0.3)$ & $8(0.3)$ & $5(0.4)$ & $1(0.3)$ & \\
\hline European-West Central Asian & $27(0.5)$ & $21(0.6)$ & $13(0.5)$ & $8(0.3)$ & $4(0.4)$ & $2(0.6)$ & \\
\hline European-Southwest Asian & $39(0.8)$ & $29(0.9)$ & $21(0.8)$ & $22(1.0)$ & $11(1.0)$ & $4(1.1)$ & \\
\hline European-Anatolian-Iran-Turanian & $8(0.2)$ & $6(0.2)$ & $5(0.2)$ & $3(0.1)$ & & & \\
\hline European-Iran-Turanian & $4(0.08)$ & $2(0.06)$ & $3(0.1)$ & $1(0.04)$ & & & \\
\hline European-Anatolian-Iranian & $19(0.4)$ & $14(0.4)$ & $11(0.4)$ & $8(0.3)$ & $3(0.3)$ & $2(0.6)$ & \\
\hline European-Anatolian-Turanian & $4(0.08)$ & $4(0.1)$ & $2(0.08)$ & & & & \\
\hline European-Iranian & $18(0.4)$ & $10(0.3)$ & $10(0.4)$ & $9(0.4)$ & $1(0.09)$ & & \\
\hline European-Turanian & $17(0.3)$ & $16(0.5)$ & $14(0.5)$ & $7(0.3)$ & $2(0.2)$ & & \\
\hline East European-Central Asian & $3(0.06)$ & $2(0.06)$ & $1(0.04)$ & & & & \\
\hline East European-Turanian & $1(0.02)$ & $1(0.03)$ & & & & & \\
\hline Species distributed in one subregion & $2621(52.2)$ & $1497(44.5)$ & $1184(45.6)$ & $1140(50.2)$ & $561(49.9)$ & $161(46.3)$ & $21(36.8)$ \\
\hline Eurosiberian type & $2035(40.5)$ & $1101(32.7)$ & $1028(39.6)$ & $1015(44.7)$ & $496(44.2)$ & $144(41.4)$ & $18(31.6)$ \\
\hline Holoeurosiberian & $104(2.1)$ & $60(1.8)$ & $70(2.7)$ & $75(3.3)$ & $36(3.2)$ & $12(3.4)$ & $2(3.5)$ \\
\hline
\end{tabular}




\begin{tabular}{|c|c|c|c|c|c|c|c|}
\hline \multirow[b]{2}{*}{ Classification of the areas } & \multirow[b]{2}{*}{ 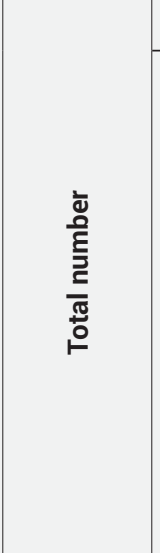 } & \multicolumn{6}{|c|}{ Vegetation belts } \\
\hline & & 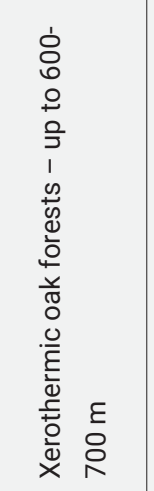 & 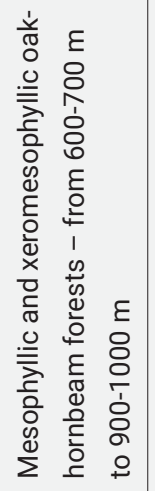 & 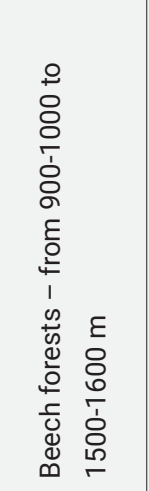 & 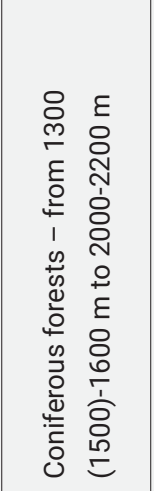 & 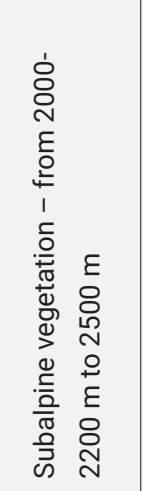 & 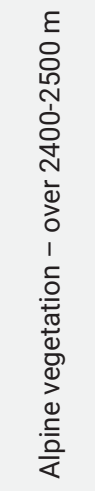 \\
\hline Transeurosiberian & $67(1.3)$ & $44(1.3)$ & $40(1.5)$ & $42(1.8)$ & $22(2.0)$ & $3(0.9)$ & \\
\hline West and Central Eurosiberian & $109(2.2)$ & $71(2.1)$ & $65(2.5)$ & $62(2.7)$ & $25(2.2)$ & $8(2.3)$ & $3(5.3)$ \\
\hline West Eurosiberian & $118(2.3)$ & $65(1.9)$ & $71(2.7)$ & $61(2.7)$ & $33(2.9)$ & $5(1.4)$ & $1(1.7)$ \\
\hline Disjunct Eurosiberian & $194(3.9)$ & $103(3.1)$ & $99(3.8)$ & $96(4.2)$ & $55(4.9)$ & $13(3.7)$ & $2(3.5)$ \\
\hline European and South Siberian & $12(0.2)$ & $8(0.2)$ & $7(0.3)$ & $6(0.3)$ & $2(0.2)$ & $1(0.3)$ & \\
\hline European-Anatolian & $85(1.7)$ & $54(1.6)$ & $48(1.8)$ & $41(1.8)$ & $19(1.7)$ & $3(0.9)$ & \\
\hline European & $1117(22.3)$ & $572(17.0)$ & $519(20.0)$ & $546(24.0)$ & $254(22.6)$ & $81(23.3)$ & $5(8.8)$ \\
\hline East European-Siberian & $8(0.2)$ & $3(0.09)$ & $1(0.04)$ & $2(0.09)$ & $1(0.09)$ & $1(0.3)$ & \\
\hline East European & $21(0.4)$ & $16(0.5)$ & $9(0.3)$ & $5(0.2)$ & $3(0.3)$ & $1(0.3)$ & $1(1.7)$ \\
\hline Central and East European-Turanian & $1(0.02)$ & $1(0.03)$ & $1(0.04)$ & $1(0.04)$ & $1(0.09)$ & & \\
\hline Central and East European-Anatolian & $2(0.04)$ & $1(0.03)$ & $1(0.04)$ & $2(0.09)$ & & & \\
\hline Central and East European & $13(0.3)$ & $8(0.2)$ & $8(0.3)$ & $3(0.1)$ & $1(0.09)$ & & \\
\hline Central and South European and South Siberian & $1(0.02)$ & & $1(0.04)$ & $1(0.04)$ & & & \\
\hline Central and South European-Anatolian & $14(0.3)$ & $12(0.4)$ & $11(0.4)$ & $8(0.3)$ & $2(0.2)$ & $1(0.3)$ & $1(1.7)$ \\
\hline Central and Southeast European-Anatolian & $6(0.1)$ & $5(0.1)$ & $4(0.1)$ & $2(0.09)$ & $1(0.09)$ & $1(0.3)$ & \\
\hline Central and South European & $82(1.6)$ & $45(1.3)$ & $40(1.5)$ & $38(1.7)$ & $22(2.0)$ & $7(2.0)$ & $3(5.3)$ \\
\hline Central and Southeast European & $81(1.6)$ & $33(1.0)$ & $33(1.3)$ & $24(1.1)$ & $19(1.7)$ & $7(2.0)$ & \\
\hline Mediterranean type & $458(9.1)$ & $324(9.6)$ & $133(5.1)$ & $93(4.1)$ & $34(3.0)$ & $12(3.4)$ & $2(3.5)$ \\
\hline Mediterranean and South Siberian & $2(0.04)$ & $1(0.03)$ & $2(0.08)$ & $1(0.04)$ & & & \\
\hline Mediterranean-Far East & $1(0.02)$ & & & & & & \\
\hline North Mediterranean and South Far East & $1(0.02)$ & $1(0.03)$ & $1(0.04)$ & & & & \\
\hline South European and South Far East & $2(0.04)$ & $1(0.03)$ & $1(0.04)$ & & & & \\
\hline Southeast European and South Far East & $1(0.02)$ & & & & & & \\
\hline Mediterranean-Central Asian & $15(0.3)$ & $10(0.3)$ & $6(0.2)$ & $2(0.09)$ & $1(0.09)$ & $1(0.3)$ & $1(1.7)$ \\
\hline Mediterranean-West Central Asian & $21(0.4)$ & $18(0.5)$ & $6(0.2)$ & $3(0.1)$ & & & \\
\hline Mediterranean-Iran-Turanian & $12(0.2)$ & $1(0.03)$ & $5(0.2)$ & $2(0.09)$ & $1(0.09)$ & & \\
\hline Mediterranean-Iranian & $19(0.4)$ & $14(0.4)$ & $7(0.3)$ & $1(0.04)$ & & & \\
\hline Mediterranean-Turanian & $8(0.2)$ & $4(0.1)$ & $2(0.08)$ & $2(0.09)$ & & & \\
\hline East Mediterranean-Central Asian & $3(0.06)$ & $2(0.06)$ & $1(0.04)$ & & & & \\
\hline East Mediterranean-Iran-Turanian & $1(0.02)$ & $1(0.03)$ & & & & & \\
\hline East Mediterranean-Iranian & $1(0.02)$ & $1(0.03)$ & & & & & \\
\hline North Mediterranean-Central Asian & $3(0.06)$ & $1(0.03)$ & $1(0.04)$ & $1(0.04)$ & & & \\
\hline North Mediterranean-West Central Asian & $9(0.2)$ & $8(0.2)$ & $4(0.1)$ & & & & \\
\hline North Mediterranean-Iranian & $8(0.2)$ & $6(0.2)$ & $3(0.1)$ & $2(0.09)$ & & & \\
\hline Northeast Mediterranean-West Central Asian & $2(0.04)$ & $2(0.06)$ & $1(0.04)$ & $1(0.04)$ & & & \\
\hline Northeast Mediterranean-Iran-Turanian & $1(0.02)$ & $1(0.03)$ & & & & & \\
\hline Northeast Mediterranean-Iranian & $2(0.04)$ & $1(0.03)$ & $1(0.04)$ & $1(0.04)$ & & & \\
\hline South European and South Siberian & $2(0.04)$ & $1(0.03)$ & $1(0.04)$ & & & & \\
\hline Southeast European and South Siberian & $1(0.02)$ & $1(0.03)$ & $1(0.04)$ & $1(0.04)$ & & & \\
\hline Southeast European-Central Asian & $5(0.1)$ & $2(0.06)$ & & & & $1(0.3)$ & \\
\hline Southeast European-West Central Asian & $2(0.04)$ & $2(0.06)$ & & & & & \\
\hline $\begin{array}{l}\text { Central and South European-West Central } \\
\text { Asian }\end{array}$ & $1(0.02)$ & $1(0.03)$ & $1(0.04)$ & $1(0.04)$ & & & \\
\hline Central and South European-Iran-Turanian & $3(0.06)$ & $3(0.09)$ & $2(0.08)$ & $2(0.09)$ & & & \\
\hline
\end{tabular}




\begin{tabular}{|c|c|c|c|c|c|c|c|}
\hline \multirow[b]{2}{*}{ Classification of the areas } & \multirow[b]{2}{*}{ 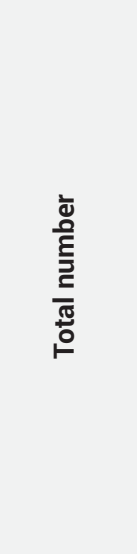 } & \multicolumn{6}{|c|}{ Vegetation belts } \\
\hline & & 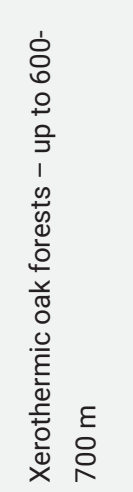 & 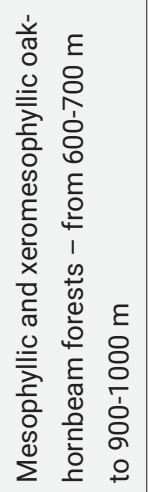 & 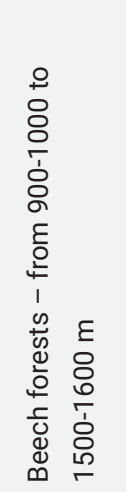 & 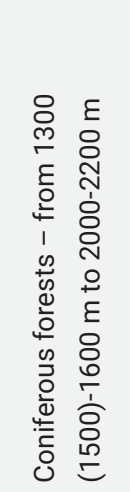 & 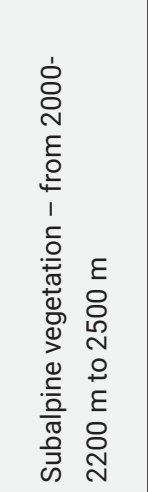 & 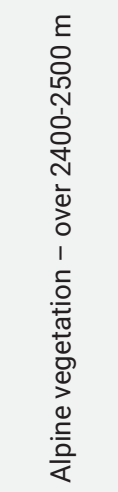 \\
\hline Central (Middle) and South European-Iranian & $7(0.1)$ & $4(0.1)$ & $3(0.1)$ & $3(0.1)$ & $1(0.09)$ & $1(0.3)$ & \\
\hline Central and Southeast European-Iranian & $2(0.04)$ & $2(0.06)$ & $1(0.04)$ & $1(0.04)$ & & & \\
\hline Central (Middle) and South European-Turanian & $4(0.08)$ & $4(0.1)$ & $2(0.08)$ & & & & \\
\hline Central and Southeast European-Turanian & $1(0.02)$ & $1(0.03)$ & $1(0.04)$ & $1(0.04)$ & & & \\
\hline Central and South European-Lebanonian & $1(0.02)$ & $1(0.03)$ & & & & & \\
\hline Central and Southeast European-Lebanonian & $2(0.04)$ & $2(0.06)$ & $1(0.04)$ & $1(0.04)$ & & & \\
\hline $\begin{array}{l}\text { Central and South European-Anatolian-North } \\
\text { African }\end{array}$ & $1(0.02)$ & $1(0.03)$ & & & & & \\
\hline Central and South European-North African & $14(0.3)$ & $9(0.3)$ & $4(0.1)$ & $4(0.2)$ & $4(0.4)$ & $1(0.3)$ & \\
\hline South European-North African & $37(0.7)$ & $29(0.9)$ & $9(0.3)$ & $5(0.2)$ & $3(0.3)$ & $1(0.3)$ & $1(1.7)$ \\
\hline Southeast European-Anatolian-North African & $2(0.04)$ & & & $1(0.04)$ & & & \\
\hline Southeast European-North African & $2(0.04)$ & & & $1(0.04)$ & $1(0.09)$ & & \\
\hline Southeast European-Anatolian-Iranian & $1(0.02)$ & & $1(0.04)$ & $1(0.04)$ & & & \\
\hline Southeast European-Iranian & $3(0.06)$ & $2(0.06)$ & & & & & \\
\hline South European-Turanian & $2(0.04)$ & $1(0.03)$ & & & & & \\
\hline Southeast European-Turanian & $2(0.04)$ & $2(0.06)$ & & & & & \\
\hline Holomediterranean & $42(0.8)$ & $34(1.0)$ & $13(0.5)$ & $8(0.3)$ & $5(0.4)$ & $1(0.3)$ & \\
\hline East Mediterranean & $15(0.3)$ & $8(0.2)$ & $1(0.04)$ & $2(0.09)$ & $1(0.09)$ & $1(0.3)$ & \\
\hline North Mediterranean & $26(0.5)$ & $18(0.5)$ & $13(0.5)$ & $5(0.2)$ & $1(0.09)$ & & \\
\hline South European & $60(1.2)$ & $38(1.1)$ & $12(0.5)$ & $14(0.6)$ & $7(0.6)$ & $1(0.3)$ & \\
\hline Northeast Mediterranean & $4(0.08)$ & $4(0.1)$ & $2(0.08)$ & & & & \\
\hline Southeast European-Anatolian & $18(0.4)$ & $12(0.4)$ & $6(0.2)$ & $8(0.3)$ & & & \\
\hline Southeast European & $57(1.1)$ & $42(1.2)$ & $12(0.5)$ & $13(0.6)$ & $3(0.3)$ & $1(0.3)$ & \\
\hline Balkan-Caucasian-Iranian & $1(0.02)$ & $1(0.03)$ & & & & & \\
\hline Balkan-Caucasian-Turanian & $1(0.02)$ & $1(0.03)$ & & & & & \\
\hline Balkan-Caucasian & $7(0.1)$ & $4(0.1)$ & $1(0.04)$ & $1(0.04)$ & $1(0.09)$ & $1(0.3)$ & \\
\hline Balkan-Anatolian & $20(0.4)$ & $15(0.4)$ & $5(0.2)$ & $7(0.3)$ & $5(0.4)$ & $2(0.6)$ & \\
\hline Endemics & $128(2.5)$ & $72(2.1)$ & $24(0.9)$ & $35(1.5)$ & $22(2.0)$ & $6(1.7)$ & $1(1.7)$ \\
\hline Balkan subendemic & $4(0.08)$ & $3(0.09)$ & $1(0.04)$ & $1(0.04)$ & $1(0.09)$ & & \\
\hline Balkan endemic & $39(0.8)$ & $25(0.7)$ & $5(0.2)$ & $8(0.3)$ & $3(0.3)$ & & \\
\hline Bulgarian endemic & $64(1.3)$ & $34(1.0)$ & $12(0.5)$ & $19(0.8)$ & $15(1.3)$ & $4(1.1)$ & $1(1.7)$ \\
\hline Regional endemic & $21(0.4)$ & $10(0.3)$ & $6(0.2)$ & $7(0.3)$ & $3(0.3)$ & $2(0.6)$ & \\
\hline Total Diptera & 5038 & $\begin{array}{l}3362 \\
(66.7)\end{array}$ & $\begin{array}{l}2598 \\
(51.6)\end{array}$ & $\begin{array}{l}2272 \\
(45.1)\end{array}$ & $\begin{array}{l}1123 \\
(22.3)\end{array}$ & $348(6.9)$ & $57(1.1)$ \\
\hline
\end{tabular}

(3.5\% to 5.9\% in the separate vegetation belts)], West Palaearctic [192 species - 3.8\% (3.2\% to 4.9\%)], EuropeanNorth African [156 species - 3.1\% (2.8\% to 3.4\%)] and West and Central Palaearctic [123 species - 2.4\% (2.5\% to 3.5\%)] species. The Disjunct Palaearctic (92 species), Eurosiberian-Central Asian (67 species) and EuropeanAnatolian-North-African (45 species) taxa are well presented. In Nematocera, the complex is represented only by Culicoides pictipennis (Staeger, 1839) in the alpine belt - a West and Central Palaearctic species of the family Ceratopogonidae, distributed in all vegetation belts. The correlation of the mentioned categories is kept in the separate vegetation belts and varies from $0.9 \%$ to $5.9 \%$ (2 to 192 species). Ninety-two species (1.8\%) have a lon- 
gitudinal disjunction of the areas with regard to Siberia and Central Asia - from 3 to 63 species in the separate vegetation belts. Probably some of these species are presented with sparse populations and will be studied in more detail as a result of further research. Most often a latitudinal disjunction of the areas of this complex is lacking (Gorodkov 1984, Josifov 1988, Hubenov 2015a). Rarely single boreomontane forms are presented. A significant part of the species with wide vertical distribution (above 23\%) belong to the Palaearctic group. The difference between the separate vegetation belts (from 11 to 954 species) reaches $78.9 \%$ and varies from $0.9 \%$ (alpine vegetation) to 79.5\% (xerothermic oak forests) of the species. For the different areographical categories this difference is the largest in the Holopalaearctic species (4.8\%). The vast areas and wide vertical distribution of the taxa of this group are an indication of the greater ecological flexibility of its species. From the mountains for which there are generalized studies on Diptera, the Palaearctic group (like the Super Palaearctic one) is best represented in the Vrachanska Planina Mts. (where it comprises $30.4 \%$ of the established species) and poorly represented in the Vitosha, Rila and Pirin Mts. (where it comprises from 23.7\% to 27.5\% of the known species). This is probably related to the insufficient studies of the Vrachanska Planina Mts. Thus, owing to the lack of sufficient research and the non-systematic sampling, more common and widespread species have been collected (Hubenov 2019b).

Species distributed within one subregion of the Palaearctic. This group (2621 species - 52.2\%) includes from 21 to 1497 species ( $36.8 \%$ to 50.2\%) in the separate vegetation belts. The group combines species with Eurosiberian and Mediterranean type of distribution. Endemics are also included in this group (73 categories total). The Mediterranean-Central Asian species are also included here according to Kryzhanovsky $(1965,2002)$ and Lopatin (1989), who combine the Mediterranean and Central Asian subregions. The species with Mediterranean type of distribution are accepted in a general way and include Submediterranean, Subiranian and Pontian faunistic elements that could be also considered separately from the Mediterranean ones (Gruev \& Kusmanov 1994, 1999; Gruev 1995; Gruev \& Bechev 2000).

The Eurosiberian species include 18 areographical categories (2035 species or 40.5\%). These are from 18 to 1101 (31.6\% to $44.7 \%)$ species in the separate vegetation belts (Table 4). The European [1117 species (22.3\%) - from 5 to 572 species (8.8\% to 24.0\%) in the separate belts], Disjunct Eurosiberian [194 species (3.9\%) - from 2 to 103 species (3.1\% to $4.9 \%)$ ], West Eurosiberian [118 species (2.3\%) - from 1 to 71 species (1.4\% to $2.9 \%)$ ], West and Central Eurosiberian [109 species (2.2\%) - from 3 to 71 species (2.1\% to 5.3\%)] and Holoeurosiberian [104 species (2.1\%) - from 2 to 75 species (1.8\% to 3.5\%)] taxa are the most numerous. The European-Anatolian, Central and South European, Central and Southeast European and Transeurosiberian species are well represented. The ratio of these categories is different for the separate families (the Holoeurosiberian, Disjunct Eurosiberian and European species are almost equal in number as the Eurosiberian forms are of about $50 \%$ in total, while in other families the Central and South European species are better represented). The number of taxa of these categories per vegetation belt varies from $0.03 \%$ to $24.0 \%$ (1-572 species) and increases in percentage with height to $2000 \mathrm{~m}$ a.s.l. For the different areographical categories this difference is the largest in the European species (15.2\%). The greatest number of Eurosiberian species (as a percentage) are found in the beech forests belt (1015 species $-44.7 \%$ ). In the the coniferous ( 496 species $-44.7 \%$ ) and the subalpine (144 species $-41.4 \%$ ) belts these species predominate over the other zoogeographical categories. In the alpine belt taxa with Super Palaearctic areas dominate $(25$ species $-43.8 \%)$. The Eurosiberian species are poorly represented (18 species $-31.6 \%)$ and include 9 areographical categories. When compared to the mountains, from which the data on Diptera are generalized, there is a small difference (Hubenov 2019b). In the subalpine belt of the Vitosha (57.3\%) and Rila (40.4\%) Mts., the Eurosiberian species predominate over the other zoogeographical categories while in the Pirin Mts. they are poorly represented (35.4\%). In the alpine belt of the Rila Mts., the Eurosiberian species (42.\%) are better represented than in the Pirin Mts. (31.0\%). The Eurosiberian complex includes a number of disjunctive areas - a longitudinal disjunction for Siberia and Central Asia and latitudinal disjunction with boreomontane, boreoalpine and arctic-alpine distribution (Gorodkov 1984; Josifov 1988; Hubenov 2015a). Of interest is the significant presence of Eurosiberian species in the first two vegetation belts (32.7\% and $39.6 \%)$. This could be explained in three ways: 1) it is possible a part of these species to have unclear Palaearctic distribution; 2) the humid mountain valleys characterised with cooler climate, have facilitated the migration of the above-mentioned forms to the lowlands; 3 ) predominant research of the lower parts of the mountain compared to the higher ones. Eurosiberian boreomontane forms at low altitudes have also been found for other groups as Heteroptera, Cerambycidae (Coleoptera) and Tachinidae (Diptera) (Josifov 1963, 1976; Georgiev \& Hubenov 2006; Hubenov 2008b). For Cerambycide this fact is due to the large afforestations of conifers in the first two vegetation belts. 
Probably because of this, many boreomontane and montane species that feed on conifers, go down below 1000 $m$ a.s.l. Probably, under further research of the Diptera fauna in the high parts of the mountains, the number of the Eurosiberian species will increase.

The Mediterranean species include 51 areographical categories (458 species or 9.1\%). These are from 2 to $324(3.0 \%$ to $9.1 \%)$ species in the separate vegetation belts (Table 4). They are presented mainly in the first two (three) vegetation belts and their number rapidly decreases with the altitude. The Mediterranean species, established in one or two vegetation belts, prevail. The significant percentage of these species in the lower vegetation belts (70.7\% in the first and $29.0 \%$ in the second belt) and their relatively scarce populations are due to the lower ecological flexibility of the Mediterranean forms in comparison with the previous ones. Because of the big variety of these areas, the group is divided into many subgroups with different origin, distribution and ecological peculiarities of the taxa. This complexity contributes to establishing of various zoogeographical classifications for Bulgaria (Josifov, 1981, 1986, 1988, 1999; Gruev 1988, 1995, 2000a, 2000b, 2000c, 2002; Heiss \& Josifov, 1990; Gruev \& Kusmanov, 1994; Hubenov 1996, 2008a; Gruev \& Bechev, 2000; Popov, 2002). For the different areographical categories the difference between the vegetation belts is the largest in the MediterraneanCentral Asian species (1.6\%). The South European (60 species - 1.2\%), Southeast European (57 species - 1.1\%), Holomediterranean (42 species 0.8\%) and South European-North African (37 species - 0.7\%) \%) taxa are the most numerous. In the subalpine belt twelve species have been found ( 3 of Nematocera and 9 of Brachycera), part of which could be Montane Mediterranean forms. In the alpine zone two species of Brachycera have been established. There are no significant differences in the distribution of the well presented aerographical categories in the Mediterranean species of the mountains. When comparing with the Vitosha, Rila and Pirin Mts., it makes an impression the higher percentage $(3.6 \%-4.4 \%-5.5 \%)$ of the Mediterranean taxa southwards (HubENov $2019 \mathrm{~b})$. This is related to the natural conditions and the geographical location of the mountains. This does not apply to the Vrachanska Planina Mts. (5.0\%), which is connected with the karst terrain, xerothermic habitats and lower altitude of the mountain.

Endemics. This category includes taxa, which are not distributed outside the Balkan Peninsula. The percentage of endemism in Diptera is low (128 species or 2.5\%). The endemism is differently presented in Nematocera (60 species or $3.6 \%$ ) and Brachycera (68 species or $2.0 \%$ ). The Bulgarian (64 species - 50.0\%) and Balkan (39 species - 30.5\%) endemic forms prevail. The main part of the endemic species is related to the xerothermic oak forests $(72$ species $-56.2 \%)$. In the next two vegetation belts their number significantly decreases ( 24 and 35 species $-18.7 \%$ and $27.3 \%$ ). Some of the endemics in the coniferous forests (22 species) and the subalpine belt (6 species) are Eurosiberian forms and can be considered as postglacial neoendemics. The endemics established in the first vegetation belt of the Vrachanska Planina and Pirin Mts. probably do not belong to this category (Hubenov 2019b). In the alpine belt, one Bulgarian endemic (Molophilus lautereri Stary, 1974 of the family Limoniidae) has been reported from the Rila Mts. Local endemics have not been established among Diptera. The endemic Diptera are often newly described taxa or rare species with unclear range. 


\section{NEMATOCERA}

\section{TIPULOMORPHA}

\section{Tipulidae}

Ctenophora (Cnemoncosis) fastuosa Loew, 1871 - BN; 0-50 m; 1; tp, ? h; Loew 1871; Theowald \& Oosterbroek 1986; Oosterbroek \& Theowald 1992; Popov 1999; Bechev 2009; Oosterbroek 2017.

Ctenophora (Cnemoncosis) ornata Meigen, 1818 - S2; 1, 2, 3; wp; Nedelkov 1912; Theowald \& Oosterbroek 1986; Popov 1999; Bechev 2009; Oosterbroek 2017.

Ctenophora (Ctenophora) elegans Meigen, 1818 - B1; 350-370 m; 1; cse; Nedelkov 1912; Popov 1999; Bechev 2009; Oosterbroek 2017.

Ctenophora (Ctenophora) flaveolata (Fabricius, 1794) - RW; 300-350 m; 1; e; Bechev 2009; Oosterbroek 2017.

Dictenidia bimaculata (Linnaeus, 1760) [Ctenophora] - B1, V1, S21; 300-700 m; 1, 2; tp, ? h; Meunier 1897; Nedelkov 1912; Theowald \& Oosterbroek 1986; Popov 1999; Bechev 2009; Oosterbroek 2017.

Dolichopeza (Dolichopeza) nitida Mik, 1874 [D. graeca Mannheims, 1954] - B1, R2, T31; 200-1200 m; 1, 2, 3; ean; Theowald \& Oosterbroek 1986; Oosterbroek \& Theowald 1992; Popov 1999; Oosterbroek \& Lantsov 2011; Oosterbroek 2017.

Nephrotoma aculeata (Loew, 1871) - T31; 10-30 m; 1; tp; Theowald \& Oosterbroek 1986; Oosterbroek \& Theowald 1992; Popov 1999; Oosterbroek 2017.

Nephrotoma analis (Schummel, 1833) - \$; tp; Theowald \& Oosterbroek 1986; Oosterbroek \& Theowald 1992; Popov 1999; Oosterbroek 2017.

Nephrotoma appendiculata (Pierre, 1919) [N. maculata (Meigen, 1804); N. maculosa (Meigen, 1818)] - B1, V1, V4, T31; 10-880 m; 1, 2; wp; Nedelkov 1912; Theowald \& Oosterbroek 1986; Oosterbroek \& Theowald 1992; Popov 1999; Oosterbroek 2017.

Nephrotoma cornicina (Linnaeus, 1758) [Pachyrhina] - B1, V1, R1; 300-1200 m; 1, 2, 3; ho, ? hpt; Nedelkov 1912; Szilady 1934; Popov 1999; Oosterbroek 2009a, 2017.

Nephrotoma crocata (Linnaeus, 1758) - B1, V1, T31, RR; 10-600 m; 1, 2; tp; Nedelkov 1912; Popov 1999; Beschovski 2006; Oosterbroek 2009b, 2017.

Nephrotoma croceiventris lindneri (Mannheims, 1951) - V1; 600 m; 2; ean; Oosterbroek \& Theowald 1992; Popov 1999; Oosterbroek 2017.

Nephrotoma dorsalis (Fabricius, 1781) - \$ dp, ? des; Theowald \& Oosterbroek 1986; Oosterbroek \& Theowald 1992; Popov 1999; Oosterbroek 2017.

Nephrotoma flavescens (Linnaeus, 1758) - RW; 600 m; 1, 2; h; Theowald \& Oosterbroek 1986; Oosterbroek \& Theowald 1992; Popov 1999; Oosterbroek 2017.

Nephrotoma guestfalica (Westhoff, 1879) - \$; ean; Theowald \& Oosterbroek 1986; Oosterbroek \& Theowald 1992; Popov 1999; Oosterbroek 2017.

Nephrotoma lunulicornis (Schummel, 1833) - esca, ? tp, ? h; Theowald \& Oosterbroek 1986; Oosterbroek \& Theowald 1992; Popov 1999; Oosterbroek 2017.

? Nephrotoma pratensis (Linnaeus, 1758) [Pachyrhina] - E2, V1, V4, RR; 225-830 m; 1, 2; wes; ? Nedelkov 1912; Drensky 1955; ? Beschovski 2006; Oosterbroek 2017. [according to Oosterbroek (2017) need confirmation].

Nephrotoma quadrifaria (Meigen, 1804) [Pachyrhina] - B1, V1, RW; 500-600 m; 1, 2; eani, ? eswa; Nedelkov 1912; Oosterbroek \& Theowald 1992; Popov 1999; Oosterbroek 2017.

Nephrotoma scalaris (Meigen, 1818) [Pachyrhina] - B1, DM, TL; 50-320 m; 1; wp, ? wpo; Nedelkov 1912; Szilady 1934; Oosterbroek \& Theowald 1992; Popov 1999; Oosterbroek 2017.

Nephrotoma scurra (Meigen, 1818) - V1; 590 m; tp, ? po; Nedelkov 1912; Oosterbroek \& Theowald 1992; Popov 1999; Oosterbroek 2017.

Nephrotoma tenuipes (Riedel, 1910) - esca; Oosterbroek \& Theowald 1992; Popov 1999; Oosterbroek 2017.

Nigrotipula nigra (Linnaeus, 1758) - TL, \$; 206 m; 1; tp, ? h; Nedelkov 1912; Szilady 1934; Oosterbroek \& Theowald 1992; Popov 1999; Oosterbroek 2017. 
Tanyptera (Tanyptera) atrata (Linnaeus, 1758) [Xiphura atrata L., Ctenophora] - B1, V1, R1; 300-1200 m; 1, 2, 3; tp; Joakimoff 1899; Nedelkov 1912; Oosterbroek \& Theowald 1992; Popov 1999; Bechev 2009; Oosterbroek 2009b, 2017.

Tipula (Acutipula) balcanica Vermoolen, 1983 [T. gigantea Schrank, 1776; T. maxima Poda, 1761; Acutipula maxima balcanica Vermoolen, 1983] - E2, B1, B2, V4, S2, R1; 200-1300 m; 1, 2, 3; cseean; Meunier 1897; Joakimoff 1899; Nedelkov 1909, 1912; Drensky 1955; Oosterbroek \& Theowald 1992; Popov 1999; Oosterbroek 2017.

Tipula (Acutipula) bosnica Strobl, 1898 - T31; 10-30 m; 1; csee; Oosterbroek 2017.

Tipula (Acutipula) fulvipennis De Geer, 1776 [Acutipula] - \$; esca; Theowald \& Oosterbroek 1986; Oosterbroek \& Theowald 1992; Popov 1999; Oosterbroek 2017.

Tipula (Acutipula) latifurca Vermoolen, 1983 [T. transcaucasica Savchenko, 1961; Acutipula transcaucasica latifurca Vermoolen, 1983] - V1, R1, RW, T31; 100-2200 m; 1, 2, 3, 4, 5; ban, ? seean; Vermoolen, 1983; Oosterbroek \& Theowald 1992; Popov 1999; Oosterbroek 2017.

Tipula (Acutipula) tenuicornis Schummel, 1833 - B1; 500 m; 1; e; Oosterbroek 2009b, 2017.

Tipula (Beringotipula) unca Wiedemann, 1817 [Beringotipula] - esca; Theowald \& Oosterbroek 1986; Oosterbroek \& Theowald 1992; Popov 1999; Oosterbroek 2017.

Tipula (Dendrotipula) flavolineata Meigen, 1804 - B1, RW; 500-900 m; 1, 2; eanca; Oosterbroek \& Theowald 1992; Oosterbroek 2009b, 2017.

Tipula (Emodotipula) obscuriventris Strobl, 1900 [Emodotipula] - P1, 520-580 m; 1; wp; Theowald \& Oosterbroek 1986; Oosterbroek \& Theowald 1992; Popov 1999; Oosterbroek 2017.

Tipula (Emodotipula) saginata Bergroth, 1891 [Emodotipula] - e; Theowald \& Oosterbroek 1986; Popov 1999.

Tipula (Lunatipula) affinis Schummel, 1833 [Lunatipula] - V1, RW, TL; 220-600 m; 1, 2; west; Nedelkov 1909, 1912; Popov 1999, Beschovski 2006; Oosterbroek 2017.

Tipula (Lunatipula) antichasia Theischinger, 1979 [Lunatipula] - ^; Eb; Theowald \& Oosterbroek 1986; Oosterbroek \& Theowald 1992; Popov 1999.

Tipula (Lunatipula) bispina Loew, 1873 [Lunatipula] - s; see, ? Ebs; Theowald \& Oosterbroek 1986; Oosterbroek \& Theowald 1992; Popov 1999; Oosterbroek 2017.

Tipula (Lunatipula) borysthenica Savchenko, 1954 [Lunatipula] - O61, O62; 130-400 m; cseean; Tomov 1975; Popov 1999; Oosterbroek 2017.

Tipula (Lunatipula) cretis Mannheims, 1965 [Lunatipula] - \$; ? se; Theowald \& Oosterbroek 1986; Oosterbroek \& Theowald 1992; Popov 1999; Oosterbroek 2017.

Tipula (Lunatipula) fascingulata Mannheims, 1966 [Lunatipula] - \$; cse; Theowald \& Oosterbroek 1986; Oosterbroek \& Theowald 1992; Popov 1999; Oosterbroek 2017.

Tipula (Lunatipula) fascipennis Meigen, 1818 - B1, V1; 400-600 m; 1, 2; e; ? Oosterbroek \& Theowald 1992; Oosterbroek 2017.

Tipula (Lunatipula) furcula Mannheims, 1954 [Lunatipula] - R2; 600 m; ban; Tomov 1975; Popov 1999; Oosterbroek 2017.

Tipula (Lunatipula) graecolivida Mannheims, 1954 [Lunatipula] - \$; Eb; ? Theowald \& Oosterbroek 1986; Oosterbroek \& Theowald 1992; Popov 1999; Oosterbroek 2017.

Tipula (Lunatipula) helvola Loew, 1873 [Lunatipula] - K4, T11; 200-900 m; 1, 2; ean; Tomov 1975; Theowald \& Oosterbroek 1986; Oosterbroek \& Theowald 1992; Popov 1999; Oosterbroek 2017.

Tipula (Lunatipula) hera Theischinger, 1979 [Lunatipula] - \$; Ebs; Theowald \& Oosterbroek 1986; Oosterbroek \& Theowald 1992; Popov 1999; Oosterbroek 2017.

Tipula (Lunatipula) heros Egger, 1863 [Lunatipula] - R1, RW; 885-2200 m; 2, 3, 4, 5; csee; ? Oosterbroek \& Theowald 1992; Oosterbroek 2009b, 2017.

Tipula (Lunatipula) istriana Erhan and Theowald, 1961 [Lunatipula] - T11; 250-270 m; 1; seean; Tomov 1975; Oosterbroek \& Theowald 1992; Popov 1999; Oosterbroek 2017.

Tipula (Lunatipula) laetabilis Zetterstedt, 1838 [Lunatipula] - w; wces; Theowald \& Oosterbroek 1986; Popov 1999; Oosterbroek 2017.

Tipula (Lunatipula) limitata Schummel, 1833 [Lunatipula] - \$; wces; Oosterbroek \& Theowald 1992; Popov 1999; Oosterbroek 2017.

Tipula (Lunatipula) lunata Linnaeus, 1758 [Lunatipula] - B1, V1, TL, R1, RE; 180-1300 m; 1, 2, 3; hoes; Joakimoff 1899; Nedelkov 1909, 1912; Popov 1999; Oosterbroek 2017. 
Tipula (Lunatipula) macropeliostigma Mannheims, 1954 [Lunatipula] - O62, R2; 130-600 m; 1; Eb; Tomov 1975; Popov 1999; Oosterbroek 2017.

Tipula (Lunatipula) mellea Schummel, 1833 [Lunatipula] - SB, V1, V4, TL; 200-900 m; 1, 2; e; Nedelkov 1912; Popov 1999; Oosterbroek 2017.

Tipula (Lunatipula) peliostigma Schummel, 1833 [Lunatipula] - S2, S22; 550 m; 1, 2; wp; Nedelkov 1909, 1912; Popov 1999; Oosterbroek 2017.

Tipula (Lunatipula) savtschenkoi Simova, 1960 [Lunatipula] - E1, B2; 250-400 m; Eb, ? ban; Tomov 1975; Theowald \& Oosterbroek 1986; Oosterbroek \& Theowald 1992; Popov 1999; Oosterbroek 2017.

Tipula (Lunatipula) soosi Mannheims, 1954 [Lunatipula] - K4, O62, R2; 150-1500 m; 1, 2, 3, 4; ceean; Tomov 1975; Theowald \& Oosterbroek 1986; Oosterbroek \& Theowald 1992; Popov 1999; Oosterbroek 2017.

Tipula (Lunatipula) tibonella Theischinger, 1977 - B1; 300 m; 1; ban; Oosterbroek 2017.

Tipula (Lunatipula) truncata Loew, 1873 [Lunatipula truncata truncata Loew, 1873] - B2, K4; 400-1000 m; 1, 2; cse, ? csean; Tomov 1975; Theowald \& Oosterbroek 1986; Oosterbroek \& Theowald 1992; Popov 1999; Oosterbroek 2017.

Tipula (Lunatipula) tyche Mannheims, 1966 - T11; 350-400 m; 1; Eb; Oosterbroek 2009b, 2017.

Tipula (Lunatipula) vernalis Meigen, 1804 [Lunatipula] - V1, V4, R1; 550-2100 m; 1, 2, 3, 4; e; Joakimoff 1899; Nedelkov 1912; Popov 1999; Oosterbroek 2017.

Tipula (Lunatipula) verrucosa Pierre, 1919 [Tipula (Lunatipula) brunneinervis Pierre, 1921] - P2, B1, B3, V1, K4, O62, R2; 180-1000 m; 1, 2; e, ? csean; Tomov 1975; Oosterbroek \& Theowald 1992; Popov 1999; Oosterbroek 2017.

Tipula (Mediotipula) sarajevensis Strobl, 1898 [Mediotipula] - RW; 900 m; 2; e; Theowald \& Oosterbroek 1986; Oosterbroek \& Theowald 1992; Popov 1999; Oosterbroek 2017.

Tipula (Mediotipula) stigmatella Schummel, 1833 [Mediotipula] - ^; ean; Theowald \& Oosterbroek 1986; Oosterbroek \& Theowald 1992; Popov 1999; Oosterbroek 2017.

Tipula (Platytipula) luteipennis Meigen, 1830 - R1; 800-1000 m; 2; esca; ? Oosterbroek \& Theowald 1992; Oosterbroek 2017.

Tipula (Pterelachisus) glacialis (Pokorny, 1887) [Pterelachisus] - R1, RW; 900 m; 2; cse, m; Theowald \& Oosterbroek 1986; Oosterbroek \& Theowald 1992; Popov 1999; Oosterbroek 2017.

Tipula (Pterelachisus) irrorata Macquart, 1826 [Pterelachisus] - wces; Theowald \& Oosterbroek 1986; Oosterbroek \& Theowald 1992; Popov 1999; Oosterbroek 2017.

Tipula (Pterelachisus) pabulina Meigen, 1818 - B1, RW; 500-600 m; 1; e; Oosterbroek \& Theowald 1992; Oosterbroek 2009b, 2017.

Tipula (Pterelachisus) plitviciensis Simova, 1962 [Pterelachisus] - 1; csee; Theowald \& Oosterbroek 1986; Oosterbroek \& Theowald 1992; Popov 1999; Oosterbroek 2017.

Tipula (Pterelachisus) pseudovariipennis Czizek, 1912 [Pterelachisus] - RW; 900 m; 2; e, ? ean; Theowald \& Oosterbroek 1986; Oosterbroek \& Theowald 1992; Popov 1999; Oosterbroek 2017.

Tipula (Pterelachisus) truncorum Meigen, 1830 [Oreomyza, Pterelachisus] - SB; wes; Szilady 1934; Theowald \& Oosterbroek 1986; Oosterbroek \& Theowald 1992; Popov 1999; Oosterbroek 2017.

Tipula (Savtshenkia) alpium Bergroth, 1888 [Savtshenkia] - s; h; Theowald \& Oosterbroek 1986; Oosterbroek \& Theowald 1992; Popov 1999; Oosterbroek 2017.

Tipula (Savtshenkia) cheethami Edwards, 1924 [Savtshenkia] - \$; e, ? h; Theowald \& Oosterbroek 1986; Oosterbroek \& Theowald 1992; Popov 1999; Oosterbroek 2017.

Tipula (Savtshenkia) rufina Meigen, 1818 [Savtshenkia] - V1; 550 m; wcp; Nedelkov 1912; Russev 1961; Popov 1999; Oosterbroek 2017.

Tipula (Savtshenkia) subnodicornis Zetterstedt, 1838 [Savtshenkia] - RW; 1500 m; 3; wces; Theowald \& Oosterbroek 1986; Oosterbroek \& Theowald 1992; Popov 1999; Oosterbroek 2017.

Tipula (Savtshenkia) subsignata Lackschewitz, 1933 [Savtshenkia] - RW; 1500 m; 3; e; Oosterbroek \& Theowald 1992; Popov 1999; Oosterbroek 2017.

Tipula (Schummelia) variicornis Schummel, 1833 [Schummelia] - tp, ? h; Theowald \& Oosterbroek 1986; Oosterbroek \& Theowald 1992; Popov 1999; Oosterbroek 2017.

Tipula (Tipula) italica errans Theowald, 1984 - RW (Uhlovitsa cave); 885-1500 m; 2, 3; ? nm; Theowald \& Oosterbroek 1986; Oosterbroek \& Theowald 1992; Popov 1999; Oosterbroek 2017. 
Tipula (Tipula) oleracea Linnaeus, 1758 - • - - DE, V1, V4, TL; 150-1000 m; 1, 2; ena (hn, i); Malkov 1907; Nedelkov 1912; Tschorbadjiev 1932; Nikolova 1949a, 1962; Drensky 1955; Buresch \& Lazarov 1956; Popov 1956; Popov \& Nikolova 1958; Grigorov 1972, 1976; Oosterbroek \& Theowald 1992; Popov 1999; Oosterbroek 2017.

Tipula (Tipula) orientalis Lackschewitz, 1930 - pat, ? wpat; Theowald \& Oosterbroek 1986; Oosterbroek \& Theowald 1992; Popov 1999; Oosterbroek 2017.

Tipula (Tipula) paludosa Meigen, 1830 - •, -; E2, V1, B; 0-550 m; 1; wp, ? tp (h, i); Nikolova 1949, 1962; Drensky 1955; Popov \& Nikolova 1958; Grigorov 1972, 1976; Oosterbroek \& Theowald 1992; Popov 1999; Oosterbroek 2017.

Tipula (Vestiplex) excisa Schummel, 1833 [Vestiplex excisa excisa Schummel, 1833] - B2, T31, R1; 200-2900 m; 1, 4, 5, 6; hoes, m; Nedelkov 1912; Szilady 1934; Theowald \& Oosterbroek 1986; Oosterbroek \& Theowald 1992; Popov 1999; Oosterbroek 2017.

Tipula (Vestiplex) montana Curtis, 1834 [Vestiplex] - \$; e, m; Theowald \& Oosterbroek 1986; Oosterbroek \& Theowald 1992; Popov 1999; Oosterbroek 2017.

Tipula (Vestiplex) nubeculosa Meigen, 1804 [Vestiplex] - B1, R1; 450-1350 m; 1, 2, 3; wces, ? esca; Nedelkov 1912; Theowald \& Oosterbroek 1986; Popov 1999; Oosterbroek 2017.

Tipula (Vestiplex) pallidicosta Pierre, 1924 [Vestiplex] - R2; 1200 m; 3; ean; Theowald \& Oosterbroek 1986; Oosterbroek \& Theowald 1992; Popov 1999; Oosterbroek 2017.

Tipula (Vestiplex) scripta Meigen, 1830 [Vestiplex] - V1, V4, R1; 500-2100 m; 1, 2, 3, 4; eca, ? hes; Joakimoff 1899; Nedelkov 1912; Theowald \& Oosterbroek 1986; Popov 1999; Oosterbroek 2017.

Tipula (Yamatotipula) caesia Schummel, 1833 [Yamatotipula] - B1, V1, V4; 250-1000 m; 1, 2; ean; Nedelkov 1912; Popov 1999; Oosterbroek 2009, 2017.

Tipula (Yamatotipula) couckei Tonnoir, 1921 [Yamatotipula] - wces; Theowald \& Oosterbroek 1986; Popov 1999; Oosterbroek 2017.

Tipula (Yamatotipula) lateralis Meigen, 1804 [Yamatotipula] - B1, B3, V1, V4, T31, R1, RR; 80-1000 m; 1, 2, 3; wcp; Nedelkov 1912; Szilady 1934; Theowald \& Oosterbroek 1986; Beschovski 2006; Popov 1999; Oosterbroek 2017.

Tipula (Yamatotipula) marginella Theowald, 1980 [Yamatotipula, T. marginata Meigen, 1818] - V1, V4; 6001000 m; 1, 2; wes; Nedelkov 1912; Popov 1999; Oosterbroek 2017.

? Tipula (Yamatotipula) montium Egger, 1863 [Yamatotipula] - 2; V4, R1; 800-1300 m; 2, 3; wces; Nedelkov 1912; Popov 1999 [according to Oosterbroek (2017) need confirmation].

Tipula (Yamatotipula) pruinosa Wiedemann, 1817 [Yamatotipula] - V4; hoes; Nedelkov 1912; Popov 1999; Oosterbroek 2017.

Tipula (Yamatotipula) riedeli Mannheims, 1952 - csee; Oosterbroek \& Theowald 1992; Oosterbroek 2017.

\section{Limoniidae}

Phyllolabis alexanderi Lackschewitz, 1940 - RE; 300 m; 1; Eb; Krzemiński 1984; Krzemiński \& Starý 1989; Savchenko et al. 1992; Oosterbroek 2017.

Phyllolabis pubipennis Lackschewitz, 1940 - R1; 2389 m; 5; csee, ? cse; Lackschewitz, 1940b; Krzemiński \& Starý 1989; Savchenko et al. 1992; Oosterbroek 2017.

Paradelphomyia (Oxyrhiza) czizekiana Stary, 1971 - V5, S211; 710-730 m; 2; ei; Krzemiński \& Starý 1989; Savchenko et al. 1992; Oosterbroek 2017.

Paradelphomyia (Oxyrhiza) ecalcarata (Edwards, 1938) - P1; 525 m; e; Oosterbroek 2017.

Paradelphomyia (Oxyrhiza) fuscula (Loew, 1873) - R1; 1147; 3; ei, ? eit; Krzemiński \& Starý 1989; Savchenko et al. 1992; Oosterbroek 2017.

Paradelphomyia (Oxyrhiza) senilis (Haliday, 1833) - E1, B1, B2, T31, BN, BS; 100-700 m; 1, 2; eanca; Krzemiński \& Starý 1989; Savchenko et al. 1992; Oosterbroek 2017.

Austrolimnophila (Archilimnophila) unica (Osten Sacken, 1869) - R1, R2, RW; 1300-1850 m; 3, 4; h; Krzemiński \& Starý 1989; Savchenko et al. 1992; Beschovski 2006; Oosterbroek 2017.

Austrolimnophila (Austrolimnophila) ochracea (Meigen, 1804) - E1, B2, V4, V5, S211, R2, BN, BS; 0-1250; 1, 2, 3; eani, ? eit; Krzemiński \& Starý 1989; Savchenko et al. 1992; Oosterbroek 2017. 
Dactylolabis (Dactylolabis) sexmaculata (Macquart, 1826) - V4; 1400-1420 m; 3; Krzemiński \& Starý 1989; Savchenko et al. 1992; Oosterbroek 2017.

Dactylolabis (Dactylolabis) symplectoidea Egger, 1863 - V4; 890-950 m; 2; ? sena; Nedelkov 1912; Krzemiński 1984; Oosterbroek 2017.

Dactylolabis (Dactylolabis) transversa (Meigen, 1804) [D. tergestina Egger, 1863] - V1, V4, R2, B; 0-1700 m; 1, 2, 3, 4; e; Nedelkov 1912; Krzemiński 1984; Krzemiński \& Starý 1989; Savchenko et al. 1992; Oosterbroek 2017.

Epiphragma (Epiphragma) ocellare (Linnaeus, 1760) - V4, V5, S211, R1, R2, BN; 0-1250 m; 1, 2, 3; h; Mendl 1986; Krzemiński \& Starý 1989; Savchenko et al. 1992; Hubenov 2015, 2016; Oosterbroek 2017.

Eloeophila apicata (Loew, 1871) - R2; 1000 m; eit; Savchenko et al. 1992; Oosterbroek 2017.

Eloeophila maculata (Meigen, 1804) - V4, V5, S211, R1, R2, T31, BN; 0-1700 m; 1, 2, 3, 4; et; Mendl 1986; Krzemiński \& Starý 1989; Savchenko et al. 1992; Oosterbroek 2017.

Eloeophila miliaria (Egger, 1863) - B2; 700-800 m; 2; ean; Krzemiński \& Starý 1989; Savchenko et al. 1992; Oosterbroek 2017.

Eloeophila mundata (Loew, 1871) - R1, R2; 1230-2389 m; 3, 4, 5; Krzemiński 1984; Krzemiński \& Starý 1989; Savchenko et al. 1992; Oosterbroek 2017.

Eloeophila sparsipunctum Starý, 2009 - V4, R1, R2; 500-1300 m; 1, 2, 3; Ebg; Starý 2009b; Oosterbroek 2017.

Eloeophila submarmorata (Verrall, 1887) - V4; 2, 3; eani, ? eit; Krzemiński 1984; Savchenko et al. 1992; Oosterbroek 2017.

Eloeophila trimaculata (Zetterstedt, 1838) - R1; 2389 m; 5; e; Lackschewitz 1940b; Krzemiński 1984; Savchenko et al. 1992; Oosterbroek 2017.

Eloeophila verralli (Bergroth, 1912) - B2, V5, S211, TL, RW, B; 0-1100 m; 1, 2, 3; ena; Mendl 1986; Krzemiński \& Starý 1989; Savchenko et al. 1992; Beschovski 2006; Oosterbroek 2017.

Euphylidorea (Euphylidorea) aperta (Verrall, 1887) - B2; 650-750 m; 1, 2; ean; Krzemiński \& Starý 1989; Savchenko et al. 1992; Oosterbroek 2017.

Euphylidorea (Euphylidorea) lineola (Meigen, 1804) [Limnophila, Phylidorea] - V4, R1, RW; 800-2389 m; 2, 3, 4, 5; wp; Nedelkov 1912; Lackschewitz 1940b; Savchenko \& Tomov 1975; Krzemiński 1984; Krzemiński \& Starý 1989; Savchenko et al. 1992; Beschovski 2006; Oosterbroek 2017.

Idioptera pulchella (Meigen, 1830) [Limnobia, Limnophila] - V1; 570-600 m; 1, 2; tes, ? hoes; Nedelkov 1912; Krzemiński 1984; Krzemiński \& Starý 1989; Savchenko et al. 1992; Starý 2007; Oosterbroek 2017.

Limnophila (Limnophila) pictipennis (Meigen, 1818) [Poecilostola] - V4; 750-850 m; 2; hoes, ? tes; Nedelkov 1912; Krzemiński 1984; Krzemiński \& Starý 1989; Savchenko et al. 1992; Oosterbroek 2017.

Limnophila (Limnophila) schranki Oosterbroek, 1992 [L. punctata Schrank, 1781] - V4, TL, RW; 70-1000 m; 1, 2; eant, ? et; Mendl 1986; Krzemiński \& Starý 1989; Savchenko et al. 1992; Beschovski 2006; Oosterbroek 2017.

Dicranophragma (Brachylimnophila) nemorale (Meigen, 1818) [Limnophila leucophaea (Meigen, 1818), Brachylimnophila, Neolimnomyia, Pilaria] - B2, V1, V5, S211, T31, R1, R2, RW, BS; 0-2389 m; 1, 2, 3, 4, 5; tp; Nedelkov 1912; Szilady 1934; Lackschewitz 1940b; Starý 1974b; Savchenko \& Tomov 1975; Krzemiński 1984; Mendl 1986; Krzemiński \& Starý 1989; Savchenko et al. 1992; Beschovski 2006; Starý \& Reusch 2009; Oosterbroek 2017.

Dicranophragma (Brachylimnophila) separatum (Walker, 1848) - R2; 2000 m; 4, 5; e; Starý \& Reusch 2009; Oosterbroek 2017.

Neolimnomyia batava (Edwards, 1938) [Limnophila leucophaea (Meigen, 1818] - V1, T31; 100-700 m; 1, 2; e; Nedelkov 1912; Krzemiński 1984; Mendl 1986; Krzemiński \& Starý 1989; Savchenko et al. 1992; Oosterbroek 2017.

Phylidorea (Macrolabina) alexanderi (Stary, 1974) - R2, RW; 1200-1750 m; 3, 4; Eb; Starý 1974b; Savchenko \& Tomov 1975; Krzemiński 1984; Mendl 1986; Krzemiński \& Starý 1989; Savchenko et al. 1992; Oosterbroek 2017.

Phylidorea (Paraphylidorea) fulvonervosa (Schummel, 1829) [Euphylidorea] - R1; 1876 m; 5; des; Krzemiński \& Starý 1989; Savchenko et al. 1992; Oosterbroek 2017.

Phylidorea (Phylidorea) ferruginea (Meigen, 1818) [Limnophila] - V1, V4, TL, R1, RE; 200-1700 m; 1, 2, 3, 4; ? wcp, ? esanca; Nedelkov 1912; Starý 1973a; Savchenko \& Tomov 1975; Krzemiński 1984; Krzemiński \& Starý 1989; Savchenko et al. 1992; Oosterbroek 2017. 
Pilaria discicollis (Meigen, 1818) [Limnophila] - V1,V5, S211, T31, RE; 50-720 m; 1, 2; eani; Nedelkov 1912; Mendl 1986; Krzemiński \& Starý 1989; Savchenko et al. 1992; Oosterbroek 2017.

Pilaria fuscipennis (Meigen, 1818) - B1, V5, S211, T31, R2, BS; 0-1914 m; 1, 2, 3, 4, 5, 6; des; Mendl 1986; Krzemiński \& Starý 1989; Savchenko et al. 1992; Oosterbroek 2017.

Prionolabis cognata (Lackschewitz, 1940) - R2; 1200-1700m; Eb; Starý \& Krzemiński 1993; Oosterbroek 2017.

Prionolabis hospes (Egger, 1863) [Limnophila platyptera (Macquart, 1834)] - V4, R1, R2, RW; 1300-2389 m; 3, 4, 5; ean; Lackschewitz 1940b; Krzemiński 1984; Mendl 1986; Krzemiński \& Starý 1989; Savchenko et al. 1992; Oosterbroek 2017.

Pseudolimnophila (Pseudolimnophila) lucorum (Meigen, 1818) [Lipsothrix] - B1, B2, V1, V5, S211, R1, B; 0-1200 m; 1, 2, 3; esca; Szilady 1934; Krzemiński 1984; Krzemiński \& Starý 1989; Savchenko et al. 1992; Oosterbroek 2017.

Pseudolimnophila (Pseudolimnophila) sepium (Verrall, 1886) - B1, B2, V5, S211, T31, R1, R2, BS; 0-2100 m; 1, 2, 3, 4; wp; Mendl 1986; Krzemiński \& Starý 1989; Savchenko et al. 1992; Oosterbroek 2017.

Hexatoma (Cladolipes) simplex (Loew, 1865) - R5; 500 m; 1; ban; Krzemiński \& Starý 1989; Savchenko et al. 1992; Oosterbroek 2017.

Hexatoma (Coreozelia) cimicoides (Scopoli, 1763) - B2; 700-1450m; cee; Starý \& Krzemiński 1993b ; Oosterbroek 2017.

Hexatoma (Eriocera) chirothecata (Scopoli, 1763) [Penthoptera] - B1, B2, B3, T31, V1, V4, B; 0-1100 m; 1, 2, 3; csean; Nedelkov 2012; Krzemiński 1984; Mendl 1986; Krzemiński \& Starý 1989; Savchenko et al. 1992; Oosterbroek 2017.

Hexatoma (Eriocera) grisea (Riedel, 1914) - T31; 200 m; 1; see; Mendl 1986; Krzemiński \& Starý 1989; Savchenko et al. 1992; Oosterbroek 2017.

Hexatoma (Hexatoma) bicolor (Meigen, 1818) - B1, B2; R1; 300-1200 m; 1, 2, 3; eanna; Krzemiński \& Starý 1989; Savchenko et al. 1992; Oosterbroek 2017.

Chionea (Sphaeconophilus) lutescens Lundstrom, 1907 - R1; 2000 m; 4; e; Czerny 1930; Kantardzhieva-Minkova 1957; Burghele-Bălăcesko 1969; Krzemiński 1984; Krzemiński \& Starý 1989; Savchenko et al. 1992; Osterbroek \& Reusch 2008; Oosterbroek 2017.

Crypteria (Crypteria) limnophiloides Bergroth, 1913 - V4, R1; 1230-1390 m; 3; e; Krzemiński 1984; Krzemiński \& Starý 1989; Savchenko et al. 1992; Oosterbroek 2017.

Neolimnophila carteri (Tonnoir, 1921) - V4, R1; 1400-2389 m; 3, 4, 5; e; Lackschewitz, 1940b; Starý 1973a; Krzemiński 1984; Krzemiński \& Starý 1989; Savchenko et al. 1992; Oosterbroek 2017.

Arctoconopa melampodia (Loew, 1873) - R5; 500 m; 1; wces; Krzemiński \& Starý 1989; Savchenko et al. 1992; Osterbroek 2017.

Baeoura malickyi Mendl and Tjeder, 1976 - S23; 420 m; 1; see; Ujvárosi 2005b; Oosterbroek 2017.

Erioptera (Erioptera) divisa (Walker, 1848) - R1; 1700-2389 m; 4, 5; e; Lackschewitz, 1940a; Krzemiński 1984; Savchenko et al. 1992; Oosterbroek 2017.

Erioptera (Erioptera) flavata (Westhoff, 1882) [E. gemina Tjeder, 1967] - V5, S211, T31, R1, BN; 200-1700 m; 1, 2, 3, 4; wes; Starý 1973a; Mendl 1986; Krzemiński \& Starý 1989; Savchenko et al. 1992; Oosterbroek 2017.

Erioptera (Erioptera) fusculenta Edwards, 1938 - V5, S211, TL, T31, R2, RW, RE; 200-1000 m; 1, 2; eant, ? wp; Mendl 1986; Krzemiński 1984; Krzemiński \& Starý 1989; Savchenko et al. 1992; Beschovski 2006; Oosterbroek 2017.

Erioptera (Erioptera) griseipennis Meigen, 1838 - R2; 1000 m; 1, 2; e; Krzemiński \& Starý 1989; Savchenko et al. 1992; Oosterbroek 2017.

Erioptera (Erioptera) limbata Loew, 1873 - E2, BN; 300-350 m; 1; e; Krzemiński \& Starý 1989; Savchenko et al. 1992; Oosterbroek 2017.

Erioptera (Erioptera) longicauda Loew, 1871 [E. flavissima Stary, 1972] - BS, 0-20 m; 1; e; Starý 1972a, 2006a; Krzemiński 1984; Krzemiński \& Starý 1989; Savchenko et al. 1992; Oosterbroek 2017.

Erioptera (Erioptera) lutea Meigen, 1804 - B2, V4, R1, R2, RW; 650-1900 m; 2, 3, 4; wcp; Krzemiński 1984; Krzemiński \& Starý 1989; Savchenko et al. 1992; Beschovski 2006; Oosterbroek 2017.

Erioptera (Mesocyphona) bivittata (Loew, 1873) [Molophilus, Ormosia] - DW, TL, R1; 30-2389 m; 1, 2, 3, 4, 5; tp; Szilady 1934; Lackschewitz, 1940a; Krzemiński 1984; Savchenko et al. 1992; Oosterbroek 2017.

Gonempeda flava (Schummel, 1829) - R2; 1000 m; 2, 3; ean; Krzemiński \& Starý 1989; Savchenko et al. 1992; Oosterbroek 2017. 
Scleroprocta balcanica Starý, 1976 - R1, R2, RW; 1147-1450 m; 3; ban; Starý 1976a; Krzemiński 1984; Mendl 1986; Krzemiński \& Starý 1989; Savchenko et al. 1992; Beschovski 2006; Oosterbroek 2017.

Scleroprocta krzeminskii Stary, 2008 - R1, R2; 1150-1500 m; 3, 4; Ebg; Starý 2008; Oosterbroek 2017.

Scleroprocta pentagonalis (Loew, 1873) - V4; 800-1000 m; 2, 3; ewca; Mendl 1986; Krzemiński \& Starý 1989; Savchenko et al. 1992; Oosterbroek 2017.

Symplecta (Psiloconopa) pusilla (Schiner, 1865) [Psiliconopa] - T31; 60 m; 1; e; Mendl 1986; Krzemiński \& Starý 1989; Savchenko et al. 1992; Oosterbroek 2017.

Symplecta (Psiloconopa) stictica (Meigen, 1818) [Symplectomorpha] - V1, V4, T31, RW, BN, BS; 0-1500 m; 1, 2, 3; wp (? ho); Slípka 1959; Savchenko \& Tomov, 1975; Krzemiński \& Starý 1989; Savchenko et al. 1992; Beschovski 2006; Oosterbroek 2017.

Symplecta (Symplecta) hybrida (Meigen, 1804) [S. punctipennis (Meigen, 1818)] - B1, V1, V4, T31, O61, R1, R2, R5, RW, BS; 0-1800 m; ho; Nedelkov 1912; Starý 1973a; Savchenko \& Tomov 1975; Krzemiński 1984; Mendl 1986; Krzemiński \& Starý 1989; Savchenko et al. 1992; Beschovski 2006; Oosterbroek 2017.

Cheilotrichia (Cheilotrichia) imbuta (Meigen, 1818) - V1; 580-600 m; 1, 2; des; Nedelkov 1912; Krzemiński 1984; Krzemiński \& Starý 1989; Savchenko et al. 1992; Oosterbroek 2017.

Cheilotrichia (Cheilotrichia) meridiana Mendl, 1974 - O62; 200-600 m; 1; nm; Starý \& Krzemiński 1993b; Oosterbroek 2017.

Cheilotrichia (Empeda) cinerascens (Meigen, 1804) - O62; 230-300 m; 1; wp; Starý 1987; Krzemiński \& Starý 1989; Savchenko et al. 1992; Oosterbroek 2017.

Cheilotrichia (Empeda) minima (Strobl, 1898) - O62; 230-300 m; 1; ? mwca; Starý 1987; Krzemiński \& Starý 1989; Savchenko et al. 1992; Oosterbroek 2017.

Cheilotrichia (Empeda) staryi Mendl, 1973 - R1, R2; 1230-2300 m; 3, 4, 5; e; Krzemiński 1984; Krzemiński \& Starý 1989; Savchenko et al. 1992; Oosterbroek 2017.

Erioconopa diuturna (Walker, 1848) - TL, RE; 230-280 m; 1; eanna; Krzemiński 1984; Krzemiński \& Starý 1989; Savchenko et al. 1992; Oosterbroek 2017.

Erioconopa symplectoides (Kuntze, 1914) - V4, R2, RW; 1000-2100 m; 3, 4; ? hom; Starý 1976b; Mendl 1986; Krzemiński \& Starý 1989; Savchenko et al. 1992; Beschovski 2006; Oosterbroek 2017.

Erioconopa trivialis (Meigen, 1818) [Erioptera] - R1, R2; 1700-2389 m; 4, 5; eani; Lackschewitz 1940a; Krzemiński 1984; Krzemiński \& Starý 1989; Savchenko et al. 1992; Hubenov 2015, 2016; Oosterbroek 2017.

Hoplolabis (Eurasicesa) idiophallus (Savchenko, 1973) - O62; 230-300 m; 1; csee; Krzemiński \& Starý 1989; Savchenko et al. 1992; Oosterbroek 2017.

Hoplolabis (Parilisia) longior Stary, 2006 - O62; 100-300 m; 1; em; Starý 2006f; Oosterbroek 2017.

Hoplolabis (Parilisia) obtusiapex (Savchenko, 1982) [Ilisia punctigera punctigera Lackschewitz, 1940] - TL, T31; 150-400 m; 1; csena; Starý 2006f; Oosterbroek 2017.

Hoplolabis (Parilisia) punctigera (Lackschewitz, 1940) - O62, TL, T31; 150-400 m; 1; mwca, ? mca; Mendl 1986; Krzemiński \& Starý 1989; Savchenko et al. 1992; Oosterbroek 2017.

Hoplolabis (Parilisia) subalpina (Bangerter, 1947) [Ilisia] - O62, T31; 230-400 m; 1; e; Mendl 1986; Krzemiński \& Starý 1989; Savchenko et al. 1992; Oosterbroek 2017.

Hoplolabis (Parilisia) vicina (Tonnoir, 1920) - E2, B2, R5, BN; 120-700 m; 1, 2; e; Krzemiński \& Starý 1989; Savchenko et al. 1992; Oosterbroek 2017.

Hoplolabis (Parilisia) yezoana (Alexander, 1924) - V5, S211, O62, R1; 230-1250 m; 1, 2, 3; esca; Krzemiński \& Starý 1989; Savchenko et al. 1992; Oosterbroek 2017.

Ilisia maculata (Meigen, 1804) - B2, V5, S211, T31, R2, RW, BN; 0-1000 m; 1, 2; wp; Mendl 1986; Krzemiński \& Starý 1989; Savchenko et al. 1992; Beschovski 2006; Hubenov 2015; Oosterbroek 2017.

Ilisia occoecata Edwards, 1936 - V5, S211; 700-750 m; 2; e; Krzemiński \& Starý 1989; Savchenko et al. 1992; Oosterbroek 2017.

Molophilus (Molophilus) aduncus Starý, 1978 - R2; 900-1000 m; 2, 3; nmca; Starý 1978; Krzemiński \& Starý 1989; Savchenko et al. 1992; Oosterbroek 2017.

Molophilus (Molophilus) appendiculatus (Staeger, 1840) - R1, R2; 1230-1950 m; 3, 4; wces; Krzemiński 1984; Krzemiński \& Starý 1989; Savchenko et al. 1992; Oosterbroek 2017.

Molophilus (Molophilus) ater (Meigen, 1804) - V4; 1350-1400 m; 3; wces; Krzemiński \& Starý 1989; Savchenko et al. 1992; Oosterbroek 2017. 
Molophilus (Molophilus) balcanicus Kolcsár, 2015 - B1; 1100-1200 m; 3; Ebg; Kolcsár et al., 2015a; Oosterbroek 2017.

Molophilus (Molophilus) bifidus Goetghebuer, 1920 - B1, R1; 350-1147 m; 1, 2; 3; ei; Krzemiński \& Starý 1989; Savchenko et al. 1992; Oosterbroek 2017.

Molophilus (Molophilus) bihamatus de Meijere, 1918 - •; ; e; Savchenko et al. 1992; Oosterbroek 2017.

Molophilus (Molophilus) brevihamatus Bangerter, 1947 - B2, V1, V4, R2; 700-1230 m; 2, 3; csee; Mendl 1986; Krzemiński \& Starý 1989; Savchenko et al. 1992; Oosterbroek 2017.

Molophilus (Molophilus) cinereifrons de Meijere, 1920 - B2; 1468 m; 3; e; Kolcsár et al., 2015a; Oosterbroek 2017.

Molophilus (Molophilus) corniger de Meijere, 1920 - V4, R2, RW; 1000-2000 m; 3, 4; e; Savchenko \& Tomov 1975; Krzemiński 1984; Krzemiński \& Starý 1989; Savchenko et al. 1992; Beschovski 2006; Oosterbroek 2017.

Molophilus (Molophilus) crassipygus de Meijere, 1918 - R1; 1147-1250 m; 3; e; Krzemiński 1984; Krzemiński \& Starý 1989; Savchenko et al. 1992; Oosterbroek 2017.

Molophilus (Molophilus) curvatus Tonnoir, 1920 - B2, V4, R2; 700-1250 m; 2, 3; e; Mendl 1986; Krzemiński \& Starý 1989; Savchenko et al. 1992; Oosterbroek 2017.

Molophilus (Molophilus) czizeki Lackschewitz, 1931 - V4; ^; e; Krzemiński \& Starý 1989; Savchenko et al. 1992; Oosterbroek 2017.

Molophilus (Molophilus) directidens Starý, 1976 - R1, R2, RW; 1147-2000 m; 3, 4; ban; Savchenko \& Tomov 1975 ?; Starý 1976a; Krzemiński 1984; Krzemiński \& Starý 1989; Savchenko et al. 1992; Beschovski 2006; Oosterbroek 2017.

Molophilus (Molophilus) flagellatus Starý, 1976 - R1, R2; 1230-2000 m; 3, 4; Er; Starý 1976; Krzemiński 1984; Krzemiński \& Starý 1989; Savchenko et al. 1992; Oosterbroek 2017.

Molophilus (Molophilus) griseus (Meigen, 1804) - V5, S211, R2, RE; 400-1000 m; 1, 2, 3; eanna; Starý \& Krzemiński 1993b; Oosterbroek 2017.

Molophilus (Molophilus) lackschewitzianus Alexander, 1953 - R1; ^; e; Krzemiński 1984; Krzemiński \& Starý 1989; Savchenko et al. 1992; Hubenov 2016; Oosterbroek 2017.

Molophilus (Molophilus) lanceolatus Starý, 1971 - R2; 1400 m; 3; Er; Starý 1971; Krzemiński 1984; Krzemiński \& Starý 1989; Savchenko et al. 1992; Oosterbroek 2017.

Molophilus (Molophilus) lautereri Stary, 1974 - R1, RW; 1450-2666 m; 3, 4, 5, 6; Ebg; Starý 1974; Krzemiński 1984; Mendl 1986; Krzemiński \& Starý 1989; Savchenko et al. 1992; Beschovski 2006; Oosterbroek 2017.

Molophilus (Molophilus) medius de Meijere, 1918 - V5, S211, R1, R2; 720-1700 m; 2, 3, 4; e; Starý 1973; Krzemiński 1984; Krzemiński \& Starý 1989; Savchenko et al. 1992; Oosterbroek 2017.

Molophilus (Molophilus) obscurus (Meigen, 1818) - V5, S211, O62, R1, R2, RE, BS; 0-1700 m; 1, 2, 3, 4; eanna; Szilady 1934; Starý 1973; Krzemiński 1984; Krzemiński \& Starý 1989; Savchenko et al. 1992; Oosterbroek 2017.

Molophilus (Molophilus) obsoletus Lackschewitz, 1940 - V4, R1, R2, RW; 1200-2389 m; 3, 4, 5; ban, ? seean; Lackschewitz 1940a; Krzemiński 1984; Mendl 1986; Krzemiński \& Starý 1989; Savchenko et al. 1992; Beschovski 2006; Oosterbroek 2017.

Molophilus (Molophilus) ochraceus (Meigen, 1818) - V4, R2; 1000 m; 2, 3; ean; Krzemiński 1984; Krzemiński \& Starý 1989; Savchenko et al. 1992; Oosterbroek 2017.

Molophilus (Molophilus) priapoides Stary, 1971 - R2; 1000-1850 m; 3, 4; e; Krzemiński 1984; Krzemiński \& Starý 1989; Savchenko et al. 1992; Oosterbroek 2017.

Molophilus (Molophilus) propinquus (Egger, 1863) [M. gladius de Meijere, 1920] - B1, B2, TL, T31, O62, R1, R2, RW; BS; 0-1700 m; 1, 2, 3, 4; tp; Szilady 1934; Starý 1973a; Savchenko \& Tomov 1975; Krzemiński 1984; Mendl 1986; Krzemiński \& Starý 1989; Savchenko et al. 1992; Beschovski 2006; Oosterbroek 2017.

Molophilus (Molophilus) scutellatus Goetghebuer, 1929 - R1; 2389 m; 5; e, ? cse; Lackschewitz 1940; Starý 1970, 1973a; Krzemiński 1984; Krzemiński \& Starý 1989; Savchenko et al. 1992; Oosterbroek 2017.

Molophilus (Molophilus) spinifer Lackschewitz, 1940 - O1; 1600 m; 3, 4; see, ? Ebs; Szilady 1934; Lackschewitz, 1940a; Krzemiński 1984; Krzemiński \& Starý 1989; Savchenko et al. 1992; Oosterbroek 2017.

Molophilus (Molophilus) stroblianus Nielsen, 1953 - R2; 1000 m; 2, 3; csee, ? e; Starý \& Krzemiński 1993b; Oosterbroek 2017.

Molophilus (Molophilus) tjederi Stary, 1968 - O62; 185-2000 m; 1, 2, 3, 4; cse; Starý \& Krzemiński 1993b; Oosterbroek 2017. 
Ormosia (Ormosia) albitibia Edwards, 1921 - R1; 1230-1390 m; 3; e; Krzemiński 1984; Krzemiński \& Starý 1989; Savchenko et al. 1992; Hubenov 2016; Oosterbroek 2017.

Ormosia (Ormosia) amicorum Savchenko et Tomov, 1975 - V4, RW; 1200-1400 m; 3; Ebg; Savchenko \& Tomov 1975; Krzemiński 1984; Krzemiński \& Starý 1989; Savchenko et al. 1992; Beschovski 2006; Oosterbroek 2017.

Ormosia (Ormosia) bifida (Lackschewitz, 1940) - V4, R2; 1400-2000 m; 3, 4; e; Krzemiński 1984; Krzemiński \& Starý 1989; Savchenko et al. 1992; Oosterbroek 2017.

Ormosia (Ormosia) clavata (Tonnoir, 1920) - R1; 1230-1390 m; 3; e; Krzemiński 1984; Krzemiński \& Starý 1989; Savchenko et al. 1992; Oosterbroek 2017.

Ormosia (Ormosia) fascipennis (Zetterstedt, 1838) - R1, R2; 1200-2389 m; 3, 4, 5; h; Lackschewitz, 1940a; Starý 1973; Krzemiński 1984; Krzemiński \& Starý 1989; Savchenko et al. 1992; Oosterbroek 2017.

Ormosia (Ormosia) hederae (Curtis, 1835) - RW; 1200 m; 3; eanca; Savchenko \& Tomov 1975; Krzemiński 1984; Krzemiński \& Starý 1989; Savchenko et al. 1992; Beschovski 2006; Oosterbroek 2017.

Ormosia (Ormosia) lineata (Meigen, 1804) - V4; e; Krzemiński \& Starý 1989; Savchenko et al. 1992; Oosterbroek 2017.

Ormosia (Ormosia) microstyla Savchenko, 1973 - V4; 1400-1420 m; 3; see; Krzemiński \& Starý 1989; Savchenko et al. 1992; Oosterbroek 2017.

Ormosia (Ormosia) pirinensis Stary, 1971 - R1, R2; 1000-1700 m; 3, 4; Ebg; Starý 1971a; Krzemiński 1984; Krzemiński \& Starý 1989; Savchenko et al. 1992; Oosterbroek 2017.

Ormosia (Ormosia) staegeriana Alexander, 1953 - R1, R2; 1230-1800 m; 3, 4; e; Krzemiński 1984; Krzemiński \& Starý 1989; Savchenko et al. 1992; Oosterbroek 2017.

Rhypholophus bifurcatus Goetghebuer, 1920 - R2; 2000 m; 4; ean; Krzemiński 1984; Krzemiński \& Starý 1989; Savchenko et al. 1992; Oosterbroek 2017.

Rhypholophus haemorrhoidalis (Zetterstedt, 1838) - R1, R2; 1147-2000 m; 3, 4; e; Szilady 1934; Krzemiński \& Starý 1989; Savchenko et al. 1992; Oosterbroek 2017.

Rhypholophus obtusistyla (Stary, 1976) [Ormosia] - R1; 1147-1850 m; 3, 4; Er; Starý 1976a; Krzemiński 1984; Krzemiński \& Starý 1989; Savchenko et al. 1992; Hubenov 2016; Oosterbroek 2017.

Rhypholophus phryganopterus Kolenati, 1860 - V4, R2; 1700-2400 m; 4, 5; e, ? cse; Krzemiński 1984; Krzemiński \& Starý 1989; Savchenko et al. 1992; Oosterbroek 2017.

Tasiocera (Dasymolophilus) fuscescens (Lackschewitz, 1940) - R2; 1200-1250 m; 3; e; Starý \& Krzemiński 1993b; Oosterbroek 2017.

Tasiocera (Dasymolophilus) murina (Meigen, 1818) - V4; 900-1400; 2, 3; eanna; Mendl 1986; Krzemiński \& Starý 1989; Savchenko et al. 1992; Oosterbroek 2017.

Tasiocera (Dasymolophilus) robusta (Bangerter, 1947) - T31, BS; 0-200 m; 1; e; Mendl 1986; Krzemiński \& Starý 1989; Savchenko et al. 1992; Oosterbroek 2017.

Dicranoptycha cinerascens (Meigen, 1818) - V1, V4, R2; 600-2000 m; 2, 3, 4; e; Nedelkov 1912; Krzemiński 1984; Krzemiński \& Starý 1989; Savchenko et al. 1992; Oosterbroek 2017.

Dicranoptycha fuscescens (Schummel, 1829) - E2, B1, B2, T31, R1, RW, BN, BS; 0-1350 m; 1, 2, 3; wcp; Savchenko \& Tomov 1975; Krzemiński 1984; Mendl 1986; Krzemiński \& Starý 1989; Savchenko et al. 1992; Oosterbroek 2017.

Dicranoptycha livescens Loew, 1871 [Dicranomyia] - R1, R2; 1147-2000 m; 3, 4; e; Szilady 1934; Krzemiński 1984; Krzemiński \& Starý 1989; Savchenko et al. 1992; Oosterbroek 2017.

Dicranoptycha paralivescens Stary, 1972 - V1, R1, R2; 500-2000 m; 2, 3, 4; e, ? cse; Krzemiński 1984; Krzemiński \& Starý 1989; Savchenko et al. 1992; Oosterbroek 2017.

Ellipteroides (Ellipteroides) lateralis (Macquart, 1835) - B1, V5, S211; 380-750 m; 1, 2; eanna; Krzemiński \& Starý 1989; Savchenko et al. 1992; Oosterbroek 2017.

Ellipteroides (Protogonomyia) alboscutellatus (von Roser, 1840) - P1, R2; 500-550 m; 1, 2; eanna; Savchenko et al. 1992; Starý \& Krzemiński 1993; Oosterbroek 2017.

Ellipteroides (Protogonomyia) limbatus (von Roser, 1840) - T31; 200-250 m; 1; ean; Mendl 1986; Krzemiński \& Starý 1989; Savchenko et al. 1992; Oosterbroek 2017.

Gnophomyia viridipennis (Gimmerthal, 1847) - R2; 300-400 m; 1; wes; Starý \& Krzemiński 1993b; Oosterbroek 2017. 
Gonomyia (Gonomyia) abscondita Lackschewitz, 1935 - R2; 300-1000 m; 1, 2; ena; Starý 2011a; Oosterbroek 2017.

Gonomyia (Gonomyia) conoviensis Barnes, 1924 - V5, S211, R2; 700-1350 m; 2, 3; eanit; Krzemiński \& Starý 1989; Savchenko et al. 1992; Oosterbroek 2017.

Gonomyia (Gonomyia) hippocampi Stubbs and Geiger, 1993 [G. ingrica Lackschewitz, 1964] - B2; 700-750 m; 2; e; Krzemiński \& Starý 1989; Oosterbroek 2017.

Gonomyia (Gonomyia) lucidula de Meijere, 1920 - B2, V5, S211, T31, R2; 200-1350 m; 1, 2, 3; ean; Mendl 1986; Krzemiński \& Starý 1989; Savchenko et al. 1992; Starý 2011 a; Oosterbroek 2017.

Gonomyia (Gonomyia) recta Tonnoir, 1920 - B1, B2, V5, S211, T31; 200-750 m; 1, 2; ean; Mendl 1986; Krzemiński \& Starý 1989; Savchenko et al. 1992; Oosterbroek 2017.

Gonomyia (Gonomyia) securiformis Starý, 2011 - V4; ? e; Starý 2011a; Oosterbroek 2017.

Gonomyia (Gonomyia) simplex Tonnoir, 1920 - V4; 1400-1420 m; 3; e; Krzemiński \& Starý 1989; Savchenko et al. 1992; Oosterbroek 2017.

Gonomyia (Gonomyia) tenella (Meigen, 1818) - R2; 900-2000 m; 2, 3, 4; eanna; Krzemiński 1984; Krzemiński \& Starý 1989; Savchenko et al. 1992; Oosterbroek 2017.

Gonomyia (Idiocerodes) concinna Lackschewitz, 1940 - O62; 95-130 m; 1; se; Starý \& Krzemiński 1993b; Oosterbroek 2017.

Gonomyia (Prolipophleps) abbreviata Loew, 1873 - O62, R2; 230-350 m; 1; ei; Starý \& Krzemiński 1993b; Oosterbroek 2017.

Gonomyia (Teuchogonomyia) edwardsi Lackschewitz, 1925 - R2; 1200 m; 3; ess; Krzemiński \& Starý 1989; Savchenko et al. 1992; Oosterbroek 2017.

Idiocera (Idiocera) hasta Stary, 1982 - T31; 80-400 m; 1; Er; Starý 1982; Mendl 1986; Krzemiński \& Starý 1989; Savchenko et al. 1992; Oosterbroek 2017.

Idiocera (Idiocera) pulchripennis (Loew, 1856) - V1, R2; RW; 550-1000 m; 1, 2; wp; Starý 1974b; Krzemiński 1984; Krzemiński \& Starý 1989; Savchenko et al. 1992; Beschovski 2006; Oosterbroek 2017.

Idiocera (Idiocera) punctata (Edwards, 1938) - B1, T31, BS; 35-380 m; 1; ? ewca, ? wp; Krzemiński \& Starý 1989; Savchenko et al. 1992; Oosterbroek 2017.

Idiocera (Idiocera) sziladyi (Lackschewitz, 1940) [Gonomyia, Ptilostena] - B1, T31; 50-450 m; 1; wpat, ? wp; Szilady 1934; Krzemiński 1984; Mendl 1986; Krzemiński \& Starý 1989; Savchenko et al. 1992; Oosterbroek 2017.

Lipsothrix errans (Walker, 1848) - R1, R2; 1147-1350 m; 3; e; Krzemiński \& Starý 1989; Savchenko et al. 1992; Oosterbroek 2017.

Lipsothrix nobilis Loew, 1873 - R2; 500-700 m; 1, 2; ean; Starý \& Krzemiński 1993; Starý 2007; Oosterbroek 2017.

Lipsothrix remota (Walker, 1848) - SB, R1, R2; 1147-1350 m; 3; e; Szilady 1934; Krzemiński 1984; Krzemiński \& Starý 1989; Savchenko et al. 1992; Oosterbroek 2017.

Rhabdomastix (Lurdia) falcata Starý, 2003 - R2; 1700-1900 m; e; 3, 4; Starý 2003b; Oosterbroek 2017.

Rhabdomastix (Lurdia) lurida (Loew, 1873) - R2; 1200 m; 3; e; Starý 2003b; Oosterbroek 2017.

Rhabdomastix (Rhabdomastix) corax Starý, 2004 - R2; 500-1200 m; 1, 2, 3; Eb; Starý 2004a; Oosterbroek 2017.

Rhabdomastix (Rhabdomastix) edwardsi Tjeder, 1967 - R2; 360-650 m; 1; e; Starý 2004a; Oosterbroek 2017.

Rhabdomastix (Rhabdomastix) eugeni Starý, 2004 - O62, R2, RW; 180-450 m; 1; e; Starý 2004a; Oosterbroek 2017.

Rhabdomastix (Rhabdomastix) filata Starý, 2004 - T31; 200-700 m; 1, 2; nem; Starý 2004a; Oosterbroek 2017.

Rhabdomastix (Rhabdomastix) hirticornis (Lackschewitz, 1940) - O62, R2; 150-450 m; 1; ena; Starý 2004a; Oosterbroek 2017.

Rhabdomastix (Rhabdomastix) japonica Alexander, 1924 [R. laeta (Loew, 1873)] - B2, T31, O62; 150-700 m; 1, 2; tp; Starý 2004a; Oosterbroek 2017.

Rhabdomastix (Rhabdomastix) laeta (Loew, 1873) - B2, T31, O62, R2; 150-700 m; 1, 2; wces; Mendl 1986; Krzemiński \& Starý 1989; Savchenko et al. 1992; Starý 2004a; Oosterbroek 2017.

Rhabdomastix (Rhabdomastix) laetoidea Starý, 2004 - O62, R2, RW; 150-1000 m; 1, 2; csee; Starý 2004a; Oosterbroek 2017.

Rhabdomastix (Rhabdomastix) subparva Stary, 1971 [R. schistacea (Schummel, 1829)] - DW, B, T31, R2; 35-550 m; 1; e; Szilady 1934; Starý 2004a; Mendl 1986; Krzemiński \& Starý 1989; Savchenko et al. 1992; Oosterbroek 2017. 
Helius (Helius) calviensis Edwards, 1928 - BS; 0-20 m; 1; hom; Krzemiński \& Starý 1989; Savchenko et al. 1992; Oosterbroek 2017.

Helius (Helius) flavus (Walker, 1856) - B1, B2, V5, S211; 350-700 m; 1, 2; des; Krzemiński \& Starý 1989; Savchenko et al. 1992; Oosterbroek 2017.

Helius (Helius) longirostris (Meigen, 1818) - V5, S211, T31, BS; 0-700 m; 1, 2; eanna; Krzemiński \& Starý 1989; Savchenko et al. 1992; Oosterbroek 2017.

Antocha (Antocha) vitripennis (Meigen, 1830) - B1, V5, S211, T31, R2; 350-1810 m; 1, 2, 3, 4; wcp; Krzemiński 1984; Mendl 1986; Krzemiński \& Starý 1989; Savchenko et al. 1992; Oosterbroek 2017.

Antocha (Orimargula) alpigena (Mik, 1883) - R1, R2; 1147-2000 m; 3, 4; e; Krzemiński 1984; Krzemiński \& Starý 1989; Savchenko et al. 1992; Oosterbroek 2017.

Elliptera hungarica Madarassy, 1881 - B1; 360 m;1; cse; Krzemiński \& Starý 1989; Savchenko et al. 1992; Hubenov 2015, 2016; Oosterbroek 2017.

Elliptera omissa Schiner, 1863 - RW; 800 m; 2; e; Mendl 1986; Krzemiński \& Starý 1989; Savchenko et al. 1992; Beschovski 2006; Oosterbroek 2017.

Orimarga (Orimarga) attenuata (Walker, 1848) - B1, T31, R2; 0-2200 m; 1, 2, 3, 4; wp; Mendl 1986; Krzemiński \& Starý 1989; Savchenko et al. 1992; Oosterbroek 2017.

Orimarga (Orimarga) juvenilis (Zetterstedt, 1851) - R2; 1000 m; 2, 3; e; Krzemiński \& Starý 1989; Savchenko et al. 1992; Oosterbroek 2017.

Thaumastoptera (Thaumastoptera) calceata Mik, 1866 - B1, B2, V5, S211, T31, O62; 50-700 m; 1, 2; ? wp; Mendl 1986; Krzemiński \& Starý 1989; Savchenko et al. 1992; Oosterbroek 2017.

Achyrolimonia decemmaculata (Loew, 1873) - R2; 1200 m; 3; eani; Starý \& Krzemiński, 1993b; Oosterbroek 2017.

Achyrolimonia neonebulosa (Alexander, 1924) - R5; 500 m; 1; h; Mendl 1986; Krzemiński \& Starý 1989; Savchenko et al. 1992; Oosterbroek 2017.

Atypophthalmus (Atypophthalmus) inustus (Meigen, 1818) - T31, BS; 0-200 m; 1; des, ? hoes; Mendl 1986; Krzemiński \& Starý 1989; Savchenko et al. 1992; Oosterbroek 2017.

Dicranomyia (Dicranomyia) autumnalis (Staeger, 1840) - V4; wpo; Starý \& Krzemiński, 1993b; Oosterbroek 2017.

Dicranomyia (Dicranomyia) chorea (Meigen, 1818) - V1, T31, R2; 400-1700 m; 1, 2, 3, 4; h; Nedelkov 1912; Krzemiński 1984; Mendl 1986; Krzemiński \& Starý 1989; Savchenko et al. 1992; Oosterbroek 2017.

Dicranomyia (Dicranomyia) conchifera (Strobl, 1900) - B1, T31, R2, RW; 200-1350 m; 1, 2, 3; e; Mendl 1986; Krzemiński \& Starý 1989; Savchenko et al. 1992; Beschovski 2006; Oosterbroek 2017.

Dicranomyia (Dicranomyia) didyma (Meigen, 1804) - E1, B1, RE; 200-400 m; 1; wcp; Szilady 1934; Krzemiński 1984; Mendl 1986; Krzemiński \& Starý 1989; Savchenko et al. 1992; Oosterbroek 2017.

Dicranomyia (Dicranomyia) fuscinota Starý, 2009 [D. luteipennis Goetghebuer, 1920] - R1, R2; 1147-2600 m; 3, 4, 5, 6; cse; Krzemiński \& Starý 1989; Starý 2009a; Oosterbroek 2017.

Dicranomyia (Dicranomyia) imbecilla Lackschewitz, 1941 - E2; 117 m; 1; e; Starý et Stubbs 2015; Oosterbroek 2017.

Dicranomyia (Dicranomyia) kamakensis Starý, 1993 - RE; 200 m; 1; ban; Starý 1993; Oosterbroek 2017.

Dicranomyia (Dicranomyia) longipennis (Schummel, 1829) - E1, V1, RW; 10-800 m; 1, 2; ho; Krzemiński \& Starý 1989; Savchenko et al. 1992; Beschovski 2006; Oosterbroek 2017.

Dicranomyia (Dicranomyia) lucida de Meijere, 1918 - B1, V5, S211, T31; 200-700 m; 1, 2; ean; Mendl 1986; Krzemiński \& Starý 1989; Savchenko et al. 1992; Oosterbroek 2017.

Dicranomyia (Dicranomyia) mitis (Meigen, 1830) - B1, V4, O62, T31, R1, R2, RW; 350-1900 m; 1, 2, 3, 4; wp; Savchenko \& Tomov, 1975; Krzemiński 1984; Mendl 1986; Krzemiński \& Starý 1989; Savchenko et al. 1992; Beschovski 2006; Oosterbroek 2017.

Dicranomyia (Dicranomyia) modesta (Meigen, 1818) - E1, V1, V5, S211, T31, O62, RW; 10-720 m; 1, 2; h; Nedelkov 1912; Krzemiński 1984; Krzemiński \& Starý 1989; Savchenko et al. 1992; Beschovski 2006; Oosterbroek 2017.

Dicranomyia (Dicranomyia) ornata (Meigen, 1818) - R2; 350-1000 m; 1, 2; ean; Starý \& Krzemiński 1993; Oosterbroek 2017.

Dicranomyia (Dicranomyia) pallidinota Starý, 2009 - BN; 0-20 m; 1; em; Starý 2009a; Oosterbroek 2017.

Dicranomyia (Dicranomyia) patricia Starý, 1982 - BS; 0-15 m; 1; hom; Starý 1982; Savchenko et al. 1992; Oosterbroek 2017. 
Dicranomyia (Dicranomyia) quadra (Meigen, 1838) - O62, RE; 200-400 m; 1; ena; Starý \& Sttubbs 2015; Oosterbroek 2017.

Dicranomyia (Dicranomyia) sera (Walker, 1848) - BS; 0-10 m; 1; h; Krzemiński \& Starý 1989; Savchenko et al. 1992; Oosterbroek 2017.

Dicranomyia (Dicranomyia) signatella Stary and Freidberg, 2007 [D. signata Lackschewitz, 1941] - V4, R2; 800-1000 m; 2, 3; em; Mendl 1986; Krzemiński \& Starý 1989; Savchenko et al. 1992; Oosterbroek 2017.

Dicranomyia (Dicranomyia) ventralis (Schummel, 1829) - T31; 100-200 m; 1; po; Mendl 1986; Savchenko et al. 1992; Oosterbroek 2017.

Dicranomyia (Glochina) sericata (Meigen, 1830) - BS; 0-15 m; 1; eanna; Krzemiński \& Starý 1989; Savchenko et al. 1992; Oosterbroek 2017.

Dicranomyia (Glochina) transsilvanica Lackschewitz, 1928 - B1; 490-500 m; 1; ean; Starý \& Krzemiński 1993b; Oosterbroek 2017.

Dicranomyia (Glochina) tristis (Schummel, 1829) - B1, V4; 400-900 m; 1, 2; hop, ? ho; Mendl 1986; Krzemiński \& Starý 1989; Savchenko et al. 1992; Oosterbroek 2017.

Dicranomyia (Idiopyga) nigristigma Nielsen, 1919 - V4; ^; e; Starý \& Krzemiński 1993b; Oosterbroek 2017.

Dicranomyia (Melanolimonia) caledonica Edwards, 1926 - R2, RW; 1200-1800 m; 3, 4; hoes; Mendl 1986; Krzemiński \& Starý 1989; Savchenko et al. 1992; Beschovski 2006; Oosterbroek 2017.

Dicranomyia (Melanolimonia) morio (Fabricius, 1787) - V1, R2; 350-550 m; 1, 2; wcp; Starý \& Krzemiński 1993b; Oosterbroek 2017.

Dicranomyia (Numantia) fusca (Meigen, 1804) - R2; 1900-2000 m; 4; h; Krzemiński 1984; Krzemiński \& Starý 1989; Savchenko et al. 1992; Oosterbroek 2017.

Dicranomyia (Sivalimnobia) aquosa Verrall, 1886 - R2; 1270 m; 3; e; Krzemiński \& Starý 1989; Savchenko et al. 1992; Oosterbroek 2017.

Discobola annulata (Linnaeus, 1758) - R1; 1147-1250 m; 3; hoa; Krzemiński \& Starý 1989; Savchenko et al. 1992; Oosterbroek 2017.

Geranomyia caloptera Mik, 1867 - B1, O62; 150-400 m; 1; eanna; Krzemiński \& Starý 1989; Savchenko et al. 1992; Oosterbroek 2017.

Limonia flavipes (Fabricius, 1787) - B1, B2, V5, S211, R1, R2, RW, BS; 0-1810 m; 1, 2, 3, 4; ena; Krzemiński 1984; Krzemiński \& Starý 1989; Savchenko et al. 1992; Beschovski 2006; Oosterbroek 2017.

Limonia hercegovinae (Strobl, 1898) - V4, R2, RW; 800-1200 m; 2, 3; ? wp; Savchenko \& Tomov 1975; Krzemiński 1984; Mendl 1986; Krzemiński \& Starý 1989; Beschovski 2006; Savchenko et al. 1992; Oosterbroek 2017.

Limonia macrostigma (Schummel, 1829) - E2, B1, B2, V4, T31, RW, BS; 0-1700 m; 1, 2, 3, 4; po; Mendl 1986; Krzemiński \& Starý 1989; Savchenko et al. 1992; Beschovski 2006; Oosterbroek 2017.

? Limonia maculipennis (Meigen, 1818) - RW; 1200 m; 3; ? cse; Savchenko \& Tomov 1975; Krzemiński 1984; Beschovski 2006. [According to Oosterbroek (2017) the presence of the species needs confirmation. According to Krzemiński \& Starý (1989) it is probably refers to L. splendens (Kuntze, 1920)].

Limonia nigropunctata (Schummel, 1829) - B2, V4, R2; 700-1800 m; 2, 3, 4; ean; Krzemiński \& Starý 1989; Savchenko et al. 1992; Oosterbroek 2017.

Limonia nubeculosa Meigen, 1804 - P2, B1, B3, V2, V4, T31, O62, R2, RW; 200-2000 m; 1, 2, 3, 4; h; subtroglophile; Buresch 1926, 1936; Czerny 1930; Buresch et al. 1949; Guéorguiev \& Beron 1962; Beron \& Guéorguiev 1967; Savchenko \& Tomov 1975; Krzemiński 1984; Mendl 1986; Krzemiński \& Starý 1989; Savchenko et al. 1992; Beron 1994, 2015, 2016; Beschovski 2006; Beron et al. 2011; Oosterbroek 2017.

Limonia pannonica (Kowarz, 1868) - SB, V4, T31, O62, R2, RW, RE, BS; 0-1350 m; 1, 2, 3; csean; Savchenko \& Tomov 1975; Krzemiński 1984; Krzemiński \& Starý 1989; Savchenko et al. 1992; Beschovski 2006; Oosterbroek 2017.

Limonia phragmitidis (Schrank, 1781) [L. tripunctata (Fabricius, 1781)] - E1, E2, SB, B2, V1, V4, V5, S211, T31, O62, R1, R2, RW, BN, BS; 0-1350 m; 1, 2, 3; wp; Nedelkov 1912; Krzemiński 1984; Mendl 1986; Krzemiński \& Starý 1989; Savchenko et al. 1992; Beschovski 2006; Oosterbroek 2017.

Limonia splendens Kuntze, 1920 - O62, RE; 150-400 m; 1; csean; Krzemiński 1984; Savchenko et al. 1992; Beschovski 2006; Oosterbroek 2017.

Limonia stigma (Meigen, 1818) - R2; 1000-2300 m; 3, 4, 5; e; Krzemiński \& Starý 1989; Savchenko et al. 1992; Hubenov 2015; Oosterbroek 2017. 
Limonia sylvicola (Schummel, 1829) - R1, R2; 1147-1850 m; 3, 4; wces; Krzemiński 1984; Krzemiński \& Starý 1989; Savchenko et al. 1992; Beschovski 2006; Oosterbroek 2017.

Limonia taurica (Strobl, 1895) - R2, RW; 1200-1800 m; 3, 4; ean; Savchenko \& Tomov 1975; Krzemiński 1984; Krzemiński \& Starý 1989; Savchenko et al. 1992; Beschovski 2006; Oosterbroek 2017.

Limonia trivittata (Schummel, 1829) [Limnobia] - V1, R1, RW; 600-1390 m; 2, 3; des, ? esanca; Nedelkov 1912; Krzemiński 1984; Krzemiński \& Starý 1989; Savchenko et al. 1992; Beschovski 2006; Oosterbroek 2017.

Metalimnobia (Metalimnobia) bifasciata (Schrank, 1781) [M. xanthoptera (Meigen, 1804)] - RR; ; tp; Nedelkov 1912; Krzemiński 1984; Beschovski 2006; Oosterbroek 2017.

Metalimnobia (Metalimnobia) zetterstedti (Tjeder, 1968) - V4, R1, R2, RW; 1200-1876 m; 3, 4; hoes; Mendl 1986; Krzemiński \& Starý 1989; Savchenko et al. 1992; Beschovski 2006; Hubenov 2016; Oosterbroek 2017.

Neolimonia dumetorum (Meigen, 1804) - B2, V1, V4, S21, T31, R1, R2, BN, BS; 0-1700 m; 1, 2, 3, 4; ean; Mendl 1986; Krzemiński \& Starý 1989; Savchenko et al. 1992; Oosterbroek 2017.

Rhipidia (Rhipidia) ctenophora Loew, 1871 - B2; 700 m; 2; e; Krzemiński \& Starý 1989; Savchenko et al. 1992; Oosterbroek 2017.

Rhipidia (Rhipidia) maculata Meigen, 1818 - R1, R2, T31; 200-1900 m; 1, 2, 3, 4; ho; Krzemiński 1984; Mendl 1986; Krzemiński \& Starý 1989; Savchenko et al. 1992; Oosterbroek 2017.

Rhipidia (Rhipidia) uniseriata Schiner, 1864 - V1; O62; 160-800 m; 1, 2; hoes; Krzemiński \& Starý 1989; Savchenko et al. 1992; Oosterbroek 2017.

\section{Pediciidae}

Ula (Ula) mollissima Haliday, 1833 - V4, R1, RW; 1200-1390 m; 3; ean; Savchenko \& Tomov 1975; Krzemiński 1984; Savchenko et al. 1992; Beschovski 2006; Oosterbroek 2017.

Ula (Ula) sylvatica (Meigen, 1818) - V4; 1400 m; 3; h; Krzemiński \& Starý 1989; Savchenko et al. 1992; Oosterbroek 2017.

Dicranota (Dicranota) bimaculata (Schummel, 1829) - R1; 1876 m; 4; wes; Krzemiński \& Starý 1989; Savchenko et al. 1992; Oosterbroek 2017.

Dicranota (Ludicia) lucidipennis (Edwards, 1921) - V1, R1, R2; 550-1876 m; 2, 3, 4; ean; Krzemiński 1984; Krzemiński \& Starý 1989; Savchenko et al. 1992; Starý 2007; Oosterbroek 2017.

Dicranota (Paradicranota) auripontium Stary and Krzeminski, 1993 - Ebg; Stary \& Krzeminski, 1993a; Oosterbroek 2017.

Dicranota (Paradicranota) brevicornis Bergroth, 1891 - R1; 1876 m; 4; cse, ? e; Krzemiński \& Starý 1989; Savchenko et al. 1992; Oosterbroek 2017.

Dicranota (Paradicranota) candelisequa Stary, 1981 - V4; \$; ena; Krzemiński \& Starý 1989; Savchenko et al. 1992; Oosterbroek 2017.

Dicranota (Paradicranota) cinerascens Lackschewitz, 1940 [D. pallens f. cinerascens Lackschewitz, 1940] - R2; 1200 m; 3; csee; Lackschewitz, 1940b; Starý 2004b; Oosterbroek 2017.

Dicranota (Paradicranota) flammatra Stary, 1981 - R1; 1147 m; 3; ean; Krzemiński \& Starý 1989; Savchenko et al. 1992; Oosterbroek 2017.

Dicranota (Paradicranota) fuscipennis Lackschewitz, 1940 - V4; 800-1000 m; 2; csean; Mendl 1986; Krzemiński \& Starý 1989; Savchenko et al. 1992; Oosterbroek 2017.

Dicranota (Paradicranota) landrocki Czizek, 1931 [Dicranomyia] - O62, RW; 360-640 m; 1; wp; Szilady 1934; Krzemiński 1984; Krzemiński \& Starý 1989; Savchenko et al. 1992; Starý \& Krzemiński 1993b; Beschovski 2006; Oosterbroek 2017.

Dicranota (Paradicranota) pallens Lackschewitz, 1940 - R1; 1876-2389 m; 4, 5; e, ? cse; Krzemiński 1984; Krzemiński \& Starý 1989; Savchenko et al. 1992; Oosterbroek 2017.

Dicranota (Paradicranota) schistacea Lackschewitz, 1940 - R2; 470-540 m; 1; cseean; Starý \& Krzemiński 1993b; Oosterbroek 2017.

Dicranota (Paradicranota) simulans Lackschewitz, 1940 - R1; 1147 m; 3; e; Krzemiński \& Starý 1989; Savchenko et al. 1992; Starý 2004b; Oosterbroek 2017.

Dicranota (Paradicranota) subtilis Loew, 1871 - V4, R2; 1200-1400 m; 3; ean; Krzemiński \& Starý 1989; Savchenko et al. 1992; Oosterbroek 2017. 
Pedicia (Amalopis) fusca Ujvárosi and Bálint, 2012 - R1; 1890 m; 4; csee; Ujvárosi \& Bálint 2012; Oosterbroek 2017.

Pedicia (Amalopis) occulta (Meigen, 1830) - R1, R2, R4, RW; 1147-2389 m; 3, 4, 5; ean; Lackschewitz, 1940b; Krzemiński 1984; Krzemiński \& Starý 1989; Savchenko et al. 1992; Ujvárosi et al. 2009; Ujvárosi et al. 2010; Ujvárosi \& Bálint 2012; Oosterbroek 2017.

Pedicia (Crunobia) littoralis (Meigen, 1804) - R1; 1147 m; 3; ean; Krzemiński \& Starý 1989; Savchenko et al. 1992; Oosterbroek 2017.

Pedicia (Crunobia) nielseni (Slipka, 1955) [P. riedeli nielseni (Slipka, 1955)] - R1; 1250 m; 3; e; Krzemiński \& Starý 1989; Savchenko et al. 1992; Oosterbroek 2017.

Pedicia (Crunobia) spinifera Starý, 1974 - T31, R1, RW; 200-1876 m; 1, 2, 3, 4; Ebs; Starý, 1974b; Krzemiński 1984; Mendl 1986; Krzemiński \& Starý 1989; Savchenko et al. 1992; Beschovski 2006; Kolcsar et al. 2012; Oosterbroek 2017.

Pedicia (Pedicia) rivosa (Linnaeus, 1758) - R1, R2; 1230-1750 m; 3, 4; wes; Starý, 1973; Krzemiński 1984; Krzemiński \& Starý 1989; Savchenko et al. 1992; Oosterbroek 2017.

Tricyphona (Tricyphona) immaculata (Meigen, 1804) - V4, R2, RW; 1200-2200 m; 3,4; wp; Savchenko \& Tomov 1975; Krzemiński 1984; Mendl 1986; Krzemiński \& Starý 1989; Savchenko et al. 1992; Beschovski 2006; Oosterbroek 2017.

Tricyphona (Tricyphona) livida Madarassy, 1881 - R1, R2; 12330-2000 m; 3, 4; e; Krzemiński 1984; Krzemiński \& Starý 1989; Savchenko et al. 1992; Oosterbroek 2017.

Tricyphona (Tricyphona) schummeli Edwards, 1921 - RW; 1200 m; 3; e; Savchenko \& Tomov 1975; Krzemiński 1984; Krzemiński \& Starý 1989; Savchenko et al. 1992; Beschovski 2006; Oosterbroek 2017.

Tricyphona (Tricyphona) zwicki Mendl, 1973 - R2; 1200 m; 3; nmi; Starý 2007; Oosterbroek 2017.

\section{Cylindrotomidae}

Cylindrotoma distinctissima (Meigen, 1818) [C. d. distinctissima (Meigen, 1818)] - R1, RW; 1320-1860 m; 3, 4; hoes; Ujvárosi et al. 2011; Oosterbroek 2017.

\section{BLEPHARICEROMORPHA}

\section{Blephariceridae}

Blepharicera fasciata (Westwood, 1842) - V4, R1, R5, RW, RE; 750-1400 m; 2, 3; eani; Komárek \& Vimmer 1921, 1922, 1934; Arndt 1943; Buresch 1953a; Russev 1961, 1964; Gulička 1966; Russev \& Janeva 1975; Zwick 1992; Beron 2004; Beschovski 2006; Uzunov et al. 2011; Varadinova et al. 2013.

Liponeura bilobata Loew, 1869 - ; Eb; Zwick 1992.

Liponeura bischoffi Edwards, 1928 [L. minor Bischoff, 1925] - RR; se; Gulička 1966; Russev \& Janeva 1975.

Liponeura brevirostris Loew, 1877 - R5; 500-900 m; 2; e; Uzunov et al. 2011; Varadinova et al. 2013.

Liponeura cinerascens Loew, 1844 [L. cinerascens subsp. jugoslavica Komarek \& Vimmer, 1934; L. cinerascens subsp. komareki Bischoff, 1925; ? L. komareki Vimmer, 1916] - V4, R1, R5, RW; 800-1100 m (? 2000 m); 2, 3, ? 4; ean; Komárek \& Vimmer 1934; Buresch 1953a; Zwick 1992; Beschovski 2006; Uzunov et al. 2011; Varadinova et al. 2013.

Liponeura cordata Vimmer, 1916 - B2, RW; 700-1150 m; 2, 3; e; Vimmer 1916; Buresch 1953a; Gulička 1966; Russev \& Janeva 1975; Russev et al. 1984b; Zwick 1992; Beschovski 2006.

Liponeura klapaleki Vimmer, 1916 [L. vimmeri Mannheims, 1954] - V4, RW; 750-1000 m; 2; csee; Vimmer 1916; Komárek \& Vimmer 1921, 1934; Buresch 1953a; Gulička 1966; Russev \& Janeva 1975; Zwick 1992, 2007.

Liponeura komareki Vimmer, 1916 - V4; 750-850 m; 2; Er; Vimmer 1916; Komárek \& Vimmer 1922, 1934; Buresch 1953a; Zwick 1992. 


\section{BIBIONOMORPHA}

\section{Bibionidae $^{1}$}

Bibio clavipes Meigen, 1818 - V1; 550 m; 1; hoes, ? tes; Nedelkov 1912; Krivosheina 1986.

Bibio consanguineus Loew, 1869 [B. pomonae var. consanguineus Loew, 1869] - B2; \$; ees; Szilády 1934.

Bibio fulviventris Meigen, 1818 - V1, P1, P2; 120-600 m; 1, 2; ? e; Nedelkov 1912; Nikolova \& Natskova 1965. Bibio graecus Duda, 1930 [B. hortulanus var. graecus Duda, 1930] - \$; ? -; Eb; Nikolova \& Natskova 1965.

Bibio hortulanus (Linnaeus, 1758) - -; ; DW, E1, E2, V1, TL, R1; 20-1200 m; 1, 2, 3; wp; Meunier 1897; Joakimoff 1899; Kovachev 1905; Nedelkov 1912; Szilády 1934; Drensky 1955; Popov \& Nikolova 1958; Grigorov 1972.

Bibio johannis (Linnaeus, 1767) - BN; 0-30 m; 1; h, ? ho; Loew 1862.

Bibio lanigerus Meigen, 1818 - R1; 550-2100 m; 2, 3, 4; e, ? des; Joakimoff 1899.

Bibio marci (Linnaeus, 1758) - DM, E1, E2, V1, K9, R1, TL; 25-1200 m; 1, 2, 3; ena; Meunier 1897; Kovachev 1905; Nedelkov 1912; Drensky 1928; Popov \& Nikolova 1958; Nikolova \& Natskova 1965; Tsolova \& Koleva 2018.

Bibio pomonae (Fabricius, 1775) - P1, P2, SB, V1, O1, R1, RR; 150-1000 m; 1, 2; des, ? dp; Meunier 1897; Nedelkov 1912; Drenowsky 1936; Nikolova \& Natskova 1965.

Bibio reticulatus Loew, 1846 - V1; 550-600 m; 1, 2; e; Nedelkov 1912.

Bibio varipes Meigen, 1830 - V1; 550-600 m; 1, 2; wces; Nedelkov 1912.

Dilophus febrilis (Linnaeus, 1758) [D. vulgaris Meigen, 1818] - V1, S1, S23; 550-800 m; 1, 2; ewca; Meunier 1897; Nedelkov 1912; Nikolova \& Natskova 1965.

Dilophus femoratus Meigen, 1804 - K9; 520-550 m; 1, 2; wcp, ? h; Nedelkov 1912.

Hesperinidae

Hesperinus imbecillus (Loew, 1858) - B1, RW; 500-1266 m; 2, 3; seean, ? csean, m; Bechev 1991b; Popova 2006.

\section{Mycetophilidae (Fungivoridae) ${ }^{2}$}

Mycomya (Cymomya) circumdata (Stæger, 1840) - P1, B1, B2, T31; 200-1600 m; 1, 2, 3; hoes; Väisänen 1984; Bechev 1985a, 1997, 2000, 2002a, 2010; Bechev \& Pavlova 2016.

Mycomya (Mycomya) bicolor (Dziedzicki, 1885) - B1; 1700 m; 4; h; Väisänen 1984; Bechev 1985a, 1997, 2000, 2002a, 2010.

Mycomya (Mycomya) cinerascens (Macquart, 1826) - P1, B1, B2, O62, R1, R2, RW; 170-1740 m; 1, 2, 3, 4; ho; Väisänen 1984; Bechev 1985a, 1997, 2000, 2002a, 2006b, 2010; Bechev \& Pavlova 2016; Pavlova \& Stojanova 2020; Pavlova 2020b.

Mycomya (Mycomya) denmax Vaisanen, 1979 - B1; 775 m; 2; h; Väisänen 1984; Bechev 1985a, 1997, 2000, 2002a, 2010.

Mycomya (Mycomya) digitifera Edwards, 1925 - B1; 775 m; 2; e; Väisänen 1984; Bechev 1985a, 1997, 2000, 2002, 2010.

Mycomya (Mycomya) disa Vaisanen, 1984 - R1, R2; 1450-1740 m; 3, 4; e; Bechev 1996a, 1997, 2000, 2002a, 2010.

Mycomya (Mycomya) flavicollis (Zetterstedt, 1852) - P1, B1, B2, T31, R2; 100-1400 m; 1, 2, 3; e; Väisänen 1984; Bechev 1985a, 1997, 2000, 2002a, 2010.

Mycomya (Mycomya) griseovittata (Zetterstedt, 1852) [M. fasciata Zetterstedt, 1838] - B2; 700-800 m; 2; h; Bechev 1989a, 1997, 2000, 2002a, 2010.

Mycomya (Mycomya) marginata (Meigen, 1818) - P1, B1, B2, O62, R2, RW; 146-1740 m; 1, 2, 3, 4; dp; Väisänen 1984; Bechev 1985a, 1997, 2000, 2002a, 2006b, 2010; Bechev \& Pavlova 2016 Pavlova \& Stojanova 2020; Pavlova 2020a, 2020b.

Mycomya (Mycomya) neohyalinata Väisänen, 1984 [M. hyalinata (Meigen, 1830)] - B1, R2; 440-600 m; 1, 2; h; Väisänen 1984; Bechev 1985a, 1989a, 1997, 2000, 2002a, 2010; Pavlova 2020 b.

$1 \quad \dagger$ Plecia sp. [Langourov M., Pavlova A., Hubenov Z., Bozukov V. \& Simov N. 2021. First record of fossil Diptera (Insecta) in Miocene deposits in Bulgaria. Comptes rendus de l’Académie bulgare des Sciences 74 (2): 233-240.]

$2 \dagger$ Mycetophila aff. pulchella Heer. (Dipt.) [Drensky P. \& Stefanoff At. 1939. Das erste fossile Insekt aus Bulgarien. Mitteilungen der Bulgarischen Entomologischen Gesellschaft in Sofia 10: 51-54. (In Bulgarian with German summary).] 
Mycomya (Mycomya) occultans (Winnertz, 1863) - B1; 350 m; 1; po; Bechev 1989a, 1997, 2000, 2002a, 2010; Bechev \& Pavlova 2016.

Mycomya (Mycomya) parva (Dziedzicki, 1885) - B1; 800-1250 m; 2, 3; des; Bechev 1989a, 1997, 2000, 2002a, 2010; Bechev \& Pavlova 2016.

Mycomya (Mycomya) prominens (Lundstrom, 1913) - O62, R2, RW; 146-950 m; 1, 2, 3; e, ? wes; Väisänen 1984; Bechev 1997, 2002a, 2006b, 2010; Pavlova \& Stojanova 2020; Pavlova 2020a, 2020 b.

Mycomya (Mycomya) ruficollis (Zetterstedt, 1852) - R1; 1450 m; 3; h; Bechev 1991a, 1997, 2002a, 2010.

Mycomya (Mycomya) sigma Johannsen, 1910 - B1, B2; 700-800 m; 2; h; Bechev 1989a, 1997, 2000, 2002a, 2010.

Mycomya (Mycomya) tenuis (Walker, 1856) - P1, B1, B2, O62, R1, R2, RW; 170-1740 m; 1, 2, 3, 4; ? wes, ? h; Väisänen 1984; Bechev 1985a, 1997, 2000, 2002a, 2010; Bechev \& Pavlova 2016; Pavlova \& Stojanova 2020; Pavlova 2020b.

Mycomya (Mycomya) tridens (Lundstrom, 1911) - B1, B2; 350-1250 m; 1, 2, 3; e; Bechev 1989a, 1997, 2000, 2002a, 2010; Bechev \& Pavlova 2016.

Mycomya (Mycomya) vittiventris (Zetterstedt, 1852) - B1; 1700 m; 4; des; Väisänen 1984; Bechev 1985a, 1997, 2000, 2002a, 2010.

Mycomya (Mycomya) wankowiczii (Dziedzicki, 1885) - B1, B2; 775-1100 m; 2, 3; h; Väisänen 1984; Bechev 1985a, 1997, 2000, 2002a, 2010.

Mycomya (Mycomya) winnertzi (Dziedzicki, 1885) - B1, RW; 350-900 m; 1, 2; dpo; Bechev 1989a, 1997, 2000, 2002a, 2010; Bechev \& Pavlova 2016; Pavlova 2020b.

Mycomya (Mycomyopsis) penicillata (Dziedzicki, 1885) - BN; 20 m; 1; wes, ? e; Bechev 1994, 1997, 2002a, 2010.

Mycomya (Mycomyopsis) trilineata (Zetterstedt, 1838) [M. neolittoralis Väisänen, 1984] - B1, B2, O62, R2; 203-1400 m; 1, 2, 3; des; Bechev 1989a, 1997, 2000, 2002a, 2010; Bechev \& Pavlova 2016; Pavlova 2020b.

Mycomya (Neomycomya) fimbriata (Meigen, 1818) - T31; 5-100 m; 1; ho; Bechev 1996a, 1997, 2002a, 2010.

Neoempheria bimaculata (von Roser, 1840) - B1, B2; 200-900 m; 1, 2; e; Bechev 1990a, 1997, 2000, 2002a, 2010.

Neoempheria lineola (Meigen, 1818) - B1, O62, R2; 146-770 m; 1, 2; des; Bechev 1986b, 1997, 2000, 2002a, 2010; Pavlova 2020a, 2020b.

Neoempheria pictipennis (Haliday, 1833) - P1; 550 m; 1; des; Bechev 1989a, 1997, 2000, 2002a, 2010.

Neoempheria proxima (Winnertz, 1863) - B1; 350-900 m; 1, 2; des; Bechev 1986a, 1997, 2000, 2002a, 2010; Bechev \& Pavlova 2016.

Neoempheria striata (Meigen, 1818) - B1, V1, TL, O62, RE; 150-775 m; 1, 2; hoes; Nedelkov 1912; Bechev 1985a, 1997, 2000, 2002a, 2004a, 2010; Bechev \& Pavlova 2016; Pavlova 2020b.

Apolephthisa subincana (Curtis, 1837) - RW; 1185-1300 m; 3; e; Bechev 1994, 1997, 2002a, 2006b, 2010.

Boletina anderschi Stannius, 1881 - P1, B1, RW; 380-800 m; 1, 2; csee; Bechev 1986b, 1997, 2000, 2002a, 2006b, 2010; Bechev \& Pavlova 2016.

Boletina basalis (Meigen, 1818) - B1; 1250 m; 3; e, ? po; Bechev 1989a, 1997, 2000, 2002a, 2010; Bechev \& Pavlova 2016.

Boletina gripha Dziedzicki, 1885 [B. dispecta Dziedzicki, 1885] - P1, B1, B2, O62, R1, R2, RE; 146-2390 m; 1, 2, 3, 4, 5; hoes; Bechev 1986a, 1986b, 1997, 2000, 2002a, 2004a, 2010; Bechev \& Pavlova 2016; Pavlova \& Stojanova 2020; Pavlova 2020a, 2020b.

Boletina lundstroemi Landrock, 1912 - B1, RW; 775-1500 m; 2, 3; wes; Bechev 1986b, 1997, 2000, 2002a, 2006b, 2010; Bechev \& Pavlova 2016.

Boletina nigricoxa Stæger, 1840 - P1, B1, O62, R2, RW, RE; 170-1700 m; 1, 2, 3, 4; des; Bechev 2000, 2002a, 2004a, 2006b, 2010; Bechev \& Pavlova 2016; Pavlova \& Stojanova 2020; Pavlova 2020b.

Boletina nitida Grzegorzek, 1885 - P1, B1, R2; 250-700 m; 1, 2; des; Bechev 1989a, 1997, 2000, 2002a, 2010; Bechev \& Pavlova 2016; Pavlova 2020b.

Boletina pallidula Edwards, 1925 - B2, RW; 800-1500 m; 2, 3; e; Bechev 1994, 1997, 2000, 2002a, 2006b, 2010.

Boletina plana Walker, 1856 - R2, RW; 500-1740 m; 1, 2, 3, 4; des; Bechev 1994, 1997, 2002a, 2006b, 2010.

Boletina sciarina Stæger, 1840 - P1, B1, B2, O62, R1, R2, RW, RE; 170-1740 m; 1, 2, 3, 4; h; Bechev 1994, 1997, 2000, 2002a, 2004a, 2010; Bechev \& Pavlova 2016; Pavlova 2020b.

Boletina trispinosa Edwards, 1913 - B1; 775 m; 2; ? dpo, ? e; Bechev 1989a, 1997, 2000, 2002, 2010.

Boletina trivittata (Meigen, 1818) - B1, B2, RW; 900-1700 m; 3, 4; des; Bechev 1986a, 1997, 2000, 2002a, 2006b, 2010; Bechev \& Pavlova 2016. 
Coelosia flava (Stæger, 1840) - B1, O62, R2; 170-1250 m; 1, 2, 3; e; Bechev 1986b, 1997, 2000, 2002a, 2010; Bechev \& Pavlova, 2016; Pavlova \& Stojanova 2020; Pavlova 2020b.

Coelosia fusca Bezzi, 1892 - O62, R2, RW; 146-510 m; 1; eswa; Pavlova, 2020a, 2020b.

Ectrepesthoneura ledenikiensis Bechev, 1988 - B1, O62; 170-800 m; 1, 2, 3; Eb; Bechev 1988a, 1997, 2000, 2002a, 2010; Bechev \& Pavlova 2016; Pavlova 2020b, 2020c.

Grzegorzekia collaris (Meigen, 1818) - B1; 203-1250 m; 1, 2, 3; ? e, ? wes; Bechev 1986b, 1997, 2000, 2002a, 2010; Bechev \& Pavlova 2016; Pavlova 2020b.

Palaeodocosia vittata (Coquillett, 1901) [P. janickii Diziedzicki, 1923] - B1; 1250 m; 3; h; Bechev 1986b, 1997, 2000, 2002a, 2010; Bechev \& Pavlova 2016.

Saigusaia flaviventris (Strobl, 1894) - B2; 1000 m; 3; des; Bechev 1994, 1997, 2000, 2002a, 2010.

Synapha fasciata Meigen, 1818 - RW, RE, BN; 10-500 m; 1; e; Bechev 1991a, 1997, 2000, 2002a, 2004, 2006b, 2010.

Synapha vitripennis (Meigen, 1818) - P1, B1, B2; 350-1300 m; 1, 2, 3; h; Bechev 1986b, 1997, 2000, 2002a, 2010; Bechev \& Pavlova 2016.

Tetragoneura ambigua (Grzegorzek, 1885) - B1, B2; 700-1300 m; 2, 3; e; Bechev 1986b, 1994, 1997, 2000, 2002a, 2010; Bechev \& Pavlova 2016.

Tetragoneura sylvatica (Curtis, 1837) - B1, RW; 600-1700 m; 2, 3, 4; e; Bechev 1986b, 1997, 2000, 2002a, 2006b, 2010.

Acnemia amoena Winnertz, 1863 - T31, O62; 146-550 m; 1; des; Bechev 2001, 2002a, 2010; Pavlova 2020 b.

Acnemia angusta Zaitzev, 1982 - P1; 200 m; 1; e; Bechev 2001, 2002a, 2010.

Acnemia falcata Zaitzev, 1982 - B1; 800 m; 2, 3; e, ? wes; Bechev 1986a, 1997, 2000, 2002a, 2010; Bechev \& Pavlova, 2016.

Acnemia longipes Winnertz, 1863 - P1, B1; 350-550 m; 1; des; Bechev 1986a, 1997, 2000, 2002a, 2010; Bechev \& Pavlova, 2016.

Acnemia nitidicollis (Meigen, 1818) - P1, B1, R2, BS; 40-1400 m; 1, 2, 3; des; Bechev 1986a, 1997, 2000, 2002a, 2010; Bechev \& Pavlova 2016.

Acnemia vratzatica Bechev, 1985 - B1; 550-650 m; 1, 2; Er; Bechev 1985b, 1997, 2000, 2002a, 2010; Bechev \& Pavlova 2016.

Anaclileia beshovskii Bechev, 1990 - B1, B2, V4; 775-1600 m; 2, 3; e; Bechev 1990b, 1997, 2000, 2002a, 2010; Bechev \& Pavlova 2016.

Azana (Azana) anomala (Stæger, 1840) - T3, T31, O62, R2, RW; 203-1185 m; 1, 2, 3; ena; Bechev 1991a, 1997, 2002a, 2006b, 2010; Pavlova 2020b.

Azana (Azana) flavohalterata Strobl in Czerny \& Strobl, 1909 [A. bulgarense Coher, 1995] - T3, O62; 146-270 m; 1; nm, ? hom; Coher 1995; Bechev 2002a, 2010; Pavlova 2020a, 2020b, 2020c.

Azana (Jugazana) nigricoxa Strobl, 1898 - E1, BN; 50-220 m; 1; Eb; Bechev 2003, 2010.

Megalopelma nigroclavatum (Strobl, 1910) - B1; 320-1250 m; 1, 2, 3; h; Bechev 1990a, 1997, 2000, 2002a, 2010; Bechev \& Pavlova 2016.

Monoclona rufilatera (Walker, 1837) - B1, R2, RW; 350-700 m; 1, 2; h; Bechev 1986b, 1997, 2000, 2002a, 2006b, 2010; Bechev \& Pavlova 2016; Pavlova 2020b.

Neuratelia minor (Lundström, 1912) - P1, B1, T31, RE; 100-1250 m; 1, 2, 3; et; Bechev 1986a, 1997, 2000, 2002a, 2004a, 2010; Bechev \& Pavlova 2016.

Neuratelia nemoralis (Meigen, 1818) - B1, B2, RW; 775-1700 m; 2, 3, 4; h; Bechev 1986b, 1997, 2000, 2002a, 2006b, 2010; Bechev \& Pavlova 2016.

Paratinia sciarina Mik, 1874 - RW; 1185 m; 3; e; Bechev 1991a, 1997, 2000, 2002a, 2006b, 2010.

Phthinia humilis Winnertz, 1863 - B1, B2; 400-1250 m; 1, 2, 3; des; Bechev 1986a, 1997, 2000, 2002a, 2010; Bechev \& Pavlova 2016.

Phthinia winnertzi Mik, 1869 - B1, T31; 180-600 m; 1, 2; e; Bechev 1986b, 1997, 2000, 2002a, 2010.

Polylepta guttiventris (Zetterstedt, 1852) - B1, B2, R2, RW; 775-1740 m; 2, 3, 4; h; Bechev 1985a, 1990c, 1997, 2000, 2002a, 2006b, 2010.

Polylepta zonata Zetterstedt, 1852 [P. meridionalis Bechev, 1990] - T31, R3; 200-1300 m; 1, 2, 3; e; Bechev 1990c, 1997, 2002a, 2003, 2010; Kurina 2003.

Sciophila baltica Zaitzev, 1982 - P1, B1; 550-1250 m; 1, 2, 3; e; Bechev 1986a, 1997, 2000, 2002a, 2010; Bechev \& Pavlova 2016.

Sciophila fenestella Curtis, 1837 - RW; 400 m; 1; h; Bechev 2001, 2002a, 2006b, 2010. 
Sciophila hirta Meigen, 1818 - B1, O62, R2; 203-775 m; 1, 2; h; Bechev 1986a, 1997, 2000, 2002a, 2010; Pavlova 2020b.

Sciophila lutea Macquart, 1826 - B1, B2, O62, R2; 146-1300 m; 1, 2, 3; tp; Bechev 1986a, 1989b, 1997, 1999a, 2000, 2002a, 2010; Bechev \& Pavlova 2016; Pavlova 2020b.

Sciophila nonnisilva Hutson, 1979 - B1; 350 m; 1; h; Bechev 1986a, 1997, 2000, 2002a, 2010; Bechev \& Pavlova 2016.

Sciophila rufa Meigen, 1830 - B1, O62, R2; 170-1700 m; 1, 2, 3, 4; esca; Bechev 1986b, 1986c, 1989b, 1997, 1999a, 2000, 2002a, 2010; Kolarov \& Bechev 1995; Pavlova \& Stojanova 2020; Pavlova 2020b.

Sciophila thoracica Stæger, 1840 - P1, B1; 250-550 m; 1; e; Bechev 1986a, 1997, 2000, 2002a, 2003, 2010; Bechev \& Pavlova 2016.

Sciophila zaitzevi Bechev, 1988 - B1; 775; 2; Er; Bechev 1988b, 1997, 2000, 2002a, 2010.

Speolepta leptogaster (Winnertz, 1863) - P1, B1, B2, RW; 400-1200 m; 1, 2, 3; e, ? ho; troglophile; Burghele-Bălăcesko 1966; Beron \& Guéorguiev 1967; Hazelton 1970; Bechev 1985a, 1997, 2000, 2002a, 2006b, 2010; Beron et al. 2011; Beron 2015; Bechev \& Pavlova 2016.

Clastobasis alternans (Winnertz, 1863) - BN; 10-20 m; 1; eca ?; Bechev 1994, 1997, 2002a, 2010.

Docosia gilvipes (Walker, 1856) - P1, B1, O62, R2, RW, RE; 146-1500 m; 1, 2, 3; tp; Bechev 1989a, 1997, 2000, 2002a, 2004, 2006b, 2010; Bechev \& Pavlova 2016; Pavlova \& Stojanova 2020; Pavlova 2020a, 2020b.

Docosia lastovkai Chandler, 1994 - B1, O62, R2; 146-800 m; 1, 2, 3; eswa; Bechev \& Pavlova 2016; Pavlova 2020a, 2020b.

Docosia moravica Landrock, 1916 - P1, B1, B2, RW, RE; 380-900 m; 1, 2; e ?; Bechev 1989a, 1997, 2000, 2002a, 2004a, 2006b, 2010; Bechev \& Pavlova 2016.

Docosia muranica Kurina \& Ševčik, 2011 - B1, O62, R2; 203-800 m; 2, 3; csee; Bechev \& Pavlova 2016; Pavlova 2020b.

Docosia nigra Landrock, 1928 - B1; 600-650 m; 1, 2; csee; Bechev \& Pavlova 2016.

Docosia rohaceki Sevcik, 2006 - O62, R2, RW; 203-510 m; 1; csee; Pavlova 2020b, 2020c.

Greenomyia mongolica Laštovka et Matile, 1974 - P1, O62; 170-550 m; 1; esca; Bechev 1989a, 1997, 2000, 2002a, 2010; Pavlova 2020b.

Greenomyia tomovi Bechev \& Pavlova, 2012 - RE; 150-200 m; 1; Er; Bechev \& Pavlova 2012.

Leia bimaculata (Meigen, 1804) [Neoglaphyroptera fasciola Meigen, 1818] - P1, B1, B2, O62, R2, RW, RE; 146 510 m; 1, 2; wcp; Nedelkov 1912; Bechev 1985a, 1989b, 1997, 1999a, 2000, 2002a, 2004a, 2006b, 2010; Bechev \& Pavlova 2016; Pavlova \& Stojanova 2020; Pavlova 2020a, 2020b.

Leia cylindrica (Winnertz, 1863) - P1, B1, RW, RE; 250-1500 m; 1, 2, 3; e; Bechev 1986b, 1997, 2000, 2002a, 2004a, 2006b, 2010; Bechev \& Pavlova 2016.

Leia graeca Bechev, 1997 - O62, R2; 170-510 m; 1; Eb; Pavlova 2020b, 2020c.

Leia picta Meigen, 1818 - RW; 1500 m; 3; e; Bechev 1986a, 1997, 2000, 2002a, 2006b, 2010.

Leia winthemii Lehmann, 1822 - B1, O62, R1, R2; 146-1300 m; 1, 2, 3, 4; ho; Bechev 1986b, 1997, 2000, 2002a, 2010; Bechev \& Pavlova 2016; Pavlova 2020a, 2020b.

Megophthalmidia crassicornis (Curtis, 1837) - O62, BS; 20-200 m; 1; e; Bechev 2003, 2010; Pavlova 2020b.

Novakia scatopsiformis Strobl, 1893 - B1, R1, RW; 800-1500 m; 2, 3, 4; ena; Bechev 2000, 2002a, 2006b, 2010; Bechev \& Pavlova 2016.

Rondaniella dimidiata (Meigen, 1804) - B1, RW; 600-1500 m; 2, 3, 4; h, ? hn; Bechev 1986b, 1997, 2000, 2002a, 2006b, 2010; Bechev \& Pavlova 2016.

Allodia (Allodia) anglofennica Edwards, 1921 - B1; 1700 m; 4; h; Bechev 1997, 1998, 2000, 2002a, 2010.

Allodia (Allodia) lugens (Wiedemann, 1817) - P1, B1, B2, O62; 170-1700 m; 1, 2, 3; h; Bechev 1997, 1998, 2000, 2002a, 2010; Bechev \& Pavlova 2016; Pavlova \& Stojanova 2020.

Allodia (Allodia) ornaticollis (Meigen, 1818) - P1, B1, B2, O62, R2, BN; 10-800 m; 1, 2, 3; h; Bechev 1997, 1998, 2000, 2002a, 2010; Bechev \& Pavlova 2016; Pavlova 2020b.

Allodia (Allodia) truncata Edwards, 1921 - B1, B2; 700-1300 m; 2, 3; h; Bechev 1997, 1998, 2000, 2002a, 2010.

Allodia (Brachycampta) alternans (Zetterstedt, 1838) - P1, B1, O62; 170-1250 m; 1, 2, 3; h; Bechev 1997, 1998, 2000, 2002a, 2010; Bechev \& Pavlova 2016; Pavlova \& Stojanova 2020; Pavlova 2020 b.

Allodia (Brachycampta) barbata (Lundstrom, 1909) - B1; 350 m; 1; h; Bechev 1997, 1998, 2000, 2002a, 2010; Bechev \& Pavlova 2016. 
Allodia (Brachycampta) foliifera (Strobl, 1910) [A. triangularis (Strobl, 1895)] - B1; 350-700 m; 1, 2; h; Bechev 1997, 1998, 2000, 2002a, 2003, 2010; Bechev \& Pavlova 2016.

Allodia (Brachycampta) grata (Meigen, 1830) - P1, B1, B2; 350-1300 m; 1, 2, 3; dp; Bechev 1997, 1998, 2000, 2002a, 2010; Bechev \& Pavlova 2016.

Allodia (Brachycampta) neglecta Edwards, 1925 - B1; 350-600 m; 1, 2; e; Bechev 1997, 1998, 2000, 2002a, 2010; Bechev \& Pavlova 2016.

Allodia (Brachycampta) pistillata (Lundstrom, 1911) - B1, O62; 170-1250 m; 1, 2, 3; h; Bechev 1997, 1998, 2000, 2002a, 2010; Bechev \& Pavlova 2016; Pavlova \& Stojanova 2020; Pavlova 2020b.

Allodia (Brachycampta) silvatica (Landrock, 1912) - B1; 350 m; 1; dp; Bechev 1997, 1998, 2000, 2002a, 2010; Bechev \& Pavlova 2016.

Allodia (Brachycampta) triangularis (Strobl, 1895) [A. retracta (Plassmann, 1977)] - B1; 350 m; 1; tes; Bechev 1997, 1998, 2000, 2002a, 2003, 2010; Bechev \& Pavlova 2016.

Allodia (Brachycampta) westerholti Caspers, 1980 - P1, B1; 550-800 m; 1, 2, 3; e; Bechev 2003, 2010; Bechev \& Pavlova 2016.

Allodiopsis domestica (Meigen, 1830) - B1, B2, O62, R2; 170-1250 m; 1, 2, 3; h; Bechev 1990a, 1997, 2000, 2002a, 2010; Bechev \& Pavlova 2016; Pavlova \& Stojanova 2020; Pavlova 2020 b.

Allodiopsis pseudodomestica (Lackschewitz, 1937) - B2; 1000 m; 2, 3; tes, ? des; Bechev 1990a, 1997, 2000, 2002a, 2010.

Allodiopsis rustica (Edwards, 1941) - B1, B2, T31, RW; 100-1500 m; 1, 2, 3, 4; tp; Bechev 1991a, 1997, 2000, 2002a, 2006b, 2010; Bechev \& Pavlova 2016.

Anatella ciliata Winnertz, 1863 - B1, RW; 1185-1700 m; 3, 4; h; Bechev 1989a, 1997, 2000, 2002a, 2006b, 2010.

Anatella lenis Dziedzicki, 1923 - B2; 1000 m; 3; des; Bechev 1990a, 1997, 2000, 2002a, 2010.

Anatella minuta (Stæger, 1840) - B1, B2; 700-1200 m; 2, 3; h; Bechev 1989a, 1997, 2000, 2002a, 2010.

Anatella novata Dziedzicki, 1923 - B1; 1700 m; 4; des; Bechev 1990a, 1997, 2000, 2002a, 2010.

Anatella simpatica Dziedzicki, 1923 - B1; 600 m; 2; h; Bechev 1989a, 1997, 2000, 2002a, 2010; Bechev \& Pavlova, 2016.

Anatella turi Dziedzicki, 1923 - B2, O62; 203-1000 m; 1, 2, 3; des; Bechev 1990a, 1997, 2000, 2002a, 2010; Pavlova 2020b.

Brevicornu fissicauda (Lundstrom, 1911) - B1, B2, O62, R2; 146-1250 m; 1, 2, 3; h; Bechev 1991a, 1997, 2000, 2002a, 2010; Bechev \& Pavlova 2016; Pavlova \& Stojanova 2020; Pavlova 2020a, 2020b.

Brevicornu griseicolle (Stæger, 1840) - P1, B1, B2, O62, R2; 350-1250 m; 1, 2, 3; h; Bechev 1991a, 1997, 2000, 2002a, 2010; Bechev \& Pavlova 2016; Pavlova 2020b.

Brevicornu ruficorne (Meigen, 1838) - B1; 600-1700 m; 2, 3, 4; h; Bechev 1991a, 1997, 2000, 2002a, 2010.

Brevicornu sericoma (Meigen, 1830) - P1, B1, B2, O62, R2, RW; 170-1750 m; 1, 2, 3, 4; h; Bechev 1991a, 1997, 2000, 2002a, 2006b, 2010; Bechev \& Pavlova 2016; Pavlova 2020b.

Brevicornu spathulatum (Lundström, 1911) - B1; 600 m; 2; csee; Bechev 1991a, 1997, 2000, 2002a, 2010.

Brevicornu verralli (Edwards, 1925) - O62, R2; 170-510 m; 1; wp; Pavlova $2020 \mathrm{~b}$.

Stigmatomeria crassicornis (Stannius, 1831) - O62; 170-240 m; 1; h; Pavlova \& Stojanova 2020.

Cordyla brevicornis (Stæger, 1840) - B1, V1, O62, R2, RW; 170-1500 m; 1, 2, 3, 4; wces; Nedelkov 2012; Bechev 1985a, 1997, 2000, 2002a, 2006b, 2010; Pavlova \& Stojanova 2020; Pavlova 2020 b.

Cordyla crassicornis Meigen, 1818 - P1, B1, T31, O62, BN, BS; 5-600 m; 1, 2; des; Bechev 1996a, 1997, 2000, 2002a, 2010; Bechev \& Pavlova 2016; Pavlova 2020b.

Cordyla fasciata Meigen, 1830 - RW; 1500 m; 3, 4; des; Bechev 1996a, 1997, 2002a, 2010.

Cordyla fissa Edwards, 1925 - P1, B1, O62, R2, RW, BS; 5-1500 m; 1, 2, 3, 4; wes; Bechev 1996a, 1997, 2000, 2002a, 2006b, 2010; Bechev \& Pavlova 2016; Pavlova \& Stojanova 2020; Pavlova 2020b.

Cordyla flaviceps (Stæger, 1840) - B1; 800 m; 2, 3; wes; Bechev 1996a, 1997, 2000, 2002a, 2010.

Cordyla fusca Meigen, 1804 - B1, O62, R2; 146-1100 m; 1, 2, 3; wces; Bechev 1996a, 1997, 1999a, 2000, 2002a, 2010; Bechev \& Pavlova 2016; Pavlova 2020a, 2020b.

Cordyla murina Winnertz, 1863 - B1, T31, R1, R2, R3; 350-2200 m; 1, 2, 3, 4, 5; hoes; Bechev 1996a, 1997, 2000, 2002a, 2010; Bechev \& Pavlova 2016; Pavlova 2020b.

Cordyla nitens Winnertz, 1863 - B1, O62; 170-1100 m; 1, 2, 3; wces; Bechev 1996a, 1997, 1999a, 2000, 2002a, 2010; Bechev \& Pavlova 2016; Pavlova \& Stojanova 2020; Pavlova 2020b. 
Cordyla nitidula Edwards, 1925 - B1, O62, R2, RW; 146-1500 m; 1, 2, 3, 4; wes; Bechev 1996a, 1997, 1999a, 2000, 2002a, 2006b, 2010; Pavlova 2020a, 2020b.

Cordyla semiflava (Stæger, 1840) - B1; 800 m; 2; tes; Bechev 1996a, 1997, 2000, 2002a, 2010.

Exechia bicincta (Stæger, 1840) - P1, B1, B2, RE; 250-1250 m; 1, 2, 3; h; Bechev 1989a, 1989b, 1997, 1999a, 2000, 2002a, 2004a, 2010; Bechev \& Pavlova 2016.

Exechia dizona Edwards, 1924 - P1; 320 m; 1; tes, ? hoes; Bechev 1990a, 1997, 2000, 2002a, 2010.

Exechia dorsalis (Stæger, 1840) - P1, B1, B2, O62, R2; 170-1000 m; 1, 2, 3; hoes; Bechev 1989a, 1997, 2000, 2002a, 2010; Bechev \& Pavlova 2016; Pavlova 2020b.

Exechia exigua Lundström, 1909 - B1, B2, RE; 300-1250 m; 1, 2, 3; tes; Bechev 1989a, 1997, 2000, 2002a, 2004a, 2010; Bechev \& Pavlova 2016.

Exechia festiva Winnertz, 1863 - B2, R2; 440-900 m; 1, 2, 3; ena; Bechev 1989a, 1997, 2000, 2002a, 2010; Pavlova $2020 \mathrm{~b}$.

Exechia fulva Santos Abreu, 1920 [E. peyerimhoffi Burghele-Balacesco, 1966] - B1, B2, RW; 700-1750 m; 2, 3, 4; ena; Bechev 1986a, 1997, 1999a, 2000, 2002a, 2006b, 2010; Bechev \& Pavlova 2016.

Exechia fusca (Meigen, 1804) [E. lateralis Meigen, 1818] - P1, B1, B2, T31, O62, R1, R2, RW, RE; 100-1700 m; 1, 2, 3, 4; h; Joakimov 1899; Bechev 1985a, 1989b, 1997, 1999a, 2000, 2002a, 2004a, 2006b, 2010; Bechev \& Pavlova 2016; Pavlova \& Stojanova 2020; Pavlova 2020a, 2020b.

Exechia lundstroemi Landrock, 1923 - B1, RW; 800-1700 m; 3, 4; hoes; Bechev 1989a, 1997, 1999a, 2000, 2002a, 2006b, 2010; Bechev \& Pavlova 2016.

Exechia macula Chandler, 2001 [E. maculipennis Stannius, 1831] - P1; 550 m; 1; esca; Bechev 1994, 1997, 2000, 2002a, 2003, 2010.

Exechia parva Lundstrom, 1909 - P1, B1, B2, R2, RE; 150-1700 m; 1, 2, 3, 4; hoes; Bechev 1989a, 1997, 2000, 2002a, 2004a, 2010; Pavlova 2020b.

Exechia pseudocincta Strobl, 1910 - B1, B2; 600-1750 m; 2, 3, 4; hoes; Bechev 1989a, 1997, 2000, 2002a, 2010.

Exechia separata Lundstrom, 1912 - B1, RE; 300-1250 m; 1, 2, 3; tp; Bechev 1989a, 1989b, 1997, 1999a, 2000, 2002a, 2004a, 2010; Bechev \& Pavlova 2016.

Exechia seriata (Meigen, 1830) - P1, B1, B2, R2, RE; 350-1250 m; 1, 2, 3; hoes, ? h; Bechev 1986a, 1989b, 1997, 1999a, 2000, 2002a, 2004a, 2010; Bechev \& Pavlova 2016; Pavlova 2020b.

Exechiopsis (Exechiopsis) clypeata (Lundstrom, 1911) - B2, R1; 1000-2390 m; 3, 4, 5; e; Bechev 1990a, 1997, 2000, 2002a, 2010.

Exechiopsis (Exechiopsis) dumitrescae (Burghele-Balacesco, 1972) - B2; 1000 m; 3; wces; Bechev 1990a, 1997, 2000, 2002a, 2010.

Exechiopsis (Exechiopsis) furcata (Lundstrom, 1911) [Exechia] - B1; 800-1250 m; 2, 3; e; subtroglophile; Burghele-Balacesco 1966; Beron \& Gueoruiev 1967; Bechev 1985a, 1997, 2000, 2002a, 2010; Beron 2015, 2016; Bechev \& Pavlova 2016.

Exechiopsis (Exechiopsis) indecisa (Walker, 1856) [Exechia] - B1; $800 \mathrm{~m}$; 2, 3; hoes; subtroglophile; Burghele-Balacesco 1966; Beron \& Gueoruiev 1967; Bechev 1985a, 1997, 2000, 2002a, 2010; Beron 2015, 2016; Bechev \& Pavlova 2016.

Exechiopsis (Exechiopsis) intersecta (Meigen, 1818) [Exechia] - B1; 800 m; 2, 3; e; subtroglophile; Burghele-Balacesco 1966; Beron \& Gueoruiev 1967; Bechev 1985a, 1997, 2000, 2002a, 2010; Beron 2015, 2016; Bechev \& Pavlova 2016.

Exechiopsis (Exechiopsis) lackschewitziana (Stackelberg, 1948) - RW; 1500 m; 3, 4; wes; Bechev 1996a, 1997, 2002a, 2006b, 2010.

Exechiopsis (Exechiopsis) magnicauda (Lundstrom, 1911) - B1, O62; 170-1250 m; 1, 2, 3; des; Bechev 1986a, 1997, 2000, 2002a, 2010; Bechev \& Pavlova 2016; Pavlova \& Stojanova 2020; Pavlova 2020b.

Exechiopsis (Exechiopsis) subulata (Winnertz, 1863) - B2; 700-1000 m; 2, 3; e; Bechev 1989a, 1997, 2000, 2002a, 2010.

Exechiopsis (Exechiopsis) unguiculata (Lundstrom, 1911) - B1; 1700 m; 4; e; Bechev 1989a, 1997, 2000, 2002a, 2010.

Exechiopsis (Exechiopsis) vizzavonensis (Edwards, 1928) [Exechia] - B1, B2, RW; 800-1350 m; 2, 3; ena; subtroglophile; Burghele-Balacesco 1966; Beron \& Gueoruiev 1967; Bechev 1985a, 1997, 2000, 2002a, 2006b, 2010; Beron 2015, 2016; Bechev \& Pavlova 2016. 
Exechiopsis (Xenexechia) crucigera (Lundstrom, 1909) - P1, B1, RE; 300-700 m; 1, 2; e; Bechev 1989a, 1997, 2000, 2002a, 2004a, 2010.

Exechiopsis (Xenexechia) davatchii (Matile, 1969) - B1, 800 m; 2; dp; Bechev 1989a, 1997, 2000, $2002 \mathrm{a}, 2010$. Exechiopsis (Xenexechia) leptura (Meigen, 1830) - B2; 1000 m; 3; des; Bechev 1991a, 1997, 2000, 2002a, 2010. Notolopha cristata (Stæger, 1840) [Allodiopsis] - B1, B2, O62; 170-1700 m; 1, 2, 3, 4; h; Bechev 1991a, 1997, 2000, 2002a, 2010; Pavlova 2020.

Pseudexechia trisignata (Edwards, 1913) - B1; 1700 m; 4; dp; Bechev 1989a, 1997, 2000, 2002a, 2010.

Pseudobrachypeza helvetica (Walker, 1856) - B1, B2; 600-1200 m; 2, 3; ei; Bechev 1994, 1997, 2000, 2002a, 2010; Bechev \& Pavlova 2016.

Pseudorymosia fovea (Dziedzicki, 1910) - B1, B2; 700-1200 m; 2, 3; wes; Bechev 1990a, 1997, 2000, 2002a, 2010. Rymosia affinis Winnertz, 1863 [R. gracilipes Dziedzicki, 1910] - P1, B1, B2, RE; 150-1250 m; 1, 2, 3; wp; subtroglophile; Burghele-Balacesco 1966; Beron \& Gueoruiev 1967; Bechev 1985a, 1997, 2000, 2002a, 2004a, 2010; Beron 2015; Bechev \& Pavlova 2016.

Rymosia fasciata (Meigen, 1804) [R. festiva Winnertz, 1863] - P1, B1, B2, T31, RE; 100-1000 m; 1, 2; e, ? pn; Bechev 1989a, 1997, 2000, 2002a, 2004a, 2010; Bechev \& Pavlova 2016.

Rymosia placida Winnertz, 1863 - B2; 700-1000 m; 2; des; Bechev 1990a, 1997, 2000, 2002a, 2010.

Rymosia setiger Dziedzicki, 1910 - B1; 650 m; 2; e; Bechev 1990a, 1997, 2000, 2002a, 2010.

Rymosia spinipes Winnertz, 1863 - P1, B1, RE; 150-800 m; 1, 2, 3; wp; Bechev 1990a, 1997, 2000, 2002a, 2004a, 2010; Bechev \& Pavlova 2016.

Rymosia virens Dziedzicki, 1910 - B1, B2, O62, R2; 203-1700 m; 2, 3, 4; e; Bechev 1990a, 1997, 2000, 2002a, 2010; Pavlova 2020b.

Stigmatomeria crassicornis (Stannius, 1931) - O62, R2, RW; 146-510 m; 1; h; Pavlova 2020a, 2020b.

Synplasta exclusa (Dziedzicki, 1910) - B1; 600-650 m; 2; cee; Bechev \& Pavlova, 2016.

Synplasta gracilis (Winnertz, 1863) [Allodiopsis excogitata (Dziedzicki, 1910), S. exogitata (Dziedzicki, 1910)] B1, B2, O62; 170-1250 m; 1, 2, 3; e, ? des; Bechev 1991a, 1997, 2000, 2002a, 2003, 2010; Bechev \& Pavlova 2016; Pavlova \& Stojanova 2020; Pavlova 2020b.

Synplasta sintenisi (Lackschewitz, 1937) [Allodiopsis] - B1; 600-650 m; 2; e; Bechev 1991a, 1997, 2000, 2002a, 2010.

Tarnania dziedzickii (Edwards, 1941) [Rhymosia] - P1, T31, RE; 180-300 m; 1; ena; subtroglophile; Burghele-Balacesco 1966; Beron \& Gueoruiev 1967; Bechev 1985a, 1997, 2000, 2002a, 2004a, 2010; Beron 2015, 2016.

Tarnania fenestralis (Meigen, 1838) [Rhymosia] - P1, B1, B3, T31, O62, RW, RE; 100-1250 m; 1, 2, 3, 4; e, ? pn; subtroglophile; Burghele-Balacesco 1966; Beron \& Gueoruiev 1967; Bechev 1985a, 1997, 2000, 2002a, 2004a, 2006b, 2010; Beron 2015, 2016; Bechev \& Pavlova 2016; Pavlova \& Stojanova 2020; Pavlova 2020b.

Tarnania nemoralis (Edwards, 1941) - B1, O62; 146-1200 m; 1, 2, 3; e; Bechev 1991a, 1997, 2000, 2002a, 2010; Pavlova \& Stojanova 2020; Pavlova 2020a, 2020b.

Dynatosoma fuscicorne (Meigen, 1818) - P1, B1, O62, RE; 170-1700 m; 1, 2, 3, 4; h; Bechev 1991a, 1997, 2000, 2002a, 2004a, 2010; Bechev \& Pavlova 2016; Pavlova \& Stojanova 2020; Pavlova 2020b.

Dynatosoma majus Landrock, 1912 - B1, B2, R3, RW, RE; 350-1300 m; 1, 2, 3; hoes; Bechev 1986b, 1997, 2000, 2002a, 2004a, 2006b, 2010; Bechev \& Pavlova 2016.

Dynatosoma nigromaculatum Lundstrom, 1913 - BN; 10 m; 1; tes; Bechev 1994, 1997, 2002a, 2010.

Epicypta torquata Matile, 1977 - B1, O62, RW; 170-500 m; 1; csei; Bechev 1994, 1997, 2000, 2002a, 2006b, 2010; Bechev \& Pavlova 2016; Pavlova 2020b.

Mycetophila abiecta (Laštovka, 1963) - B1; 600-800 m; 2, 3; e; Bechev 1991a, 1997, 2000, 2002a, 2010.

Mycetophila adumbrata Mik, 1884 - B2; 1000 m; 3; e; Bechev 1991a, 1997, 2000, 2002a, 2010.

Mycetophila alea Laffoon, 1965 - P1, B1, B2, RW, RE, BN; 10-1500 m; 1, 2, 3; h; Bechev 1989b, 1991a, 1997, 1999a, 2000, 2002a, 2004a, 2006b, 2010; Bechev \& Pavlova 2016.

Mycetophila bialorussica Dziedzicki, 1884 - B1; 800 m; 2, 3; dp, ? tp; Bechev 1991a, 1997, 2000, 2002a, 2010; Bechev \& Pavlova 2016.

Mycetophila blanda Winnertz, 1863 - B1, R2, RW, RE; 150-2000 m; 1, 2, 3, 4; esca; Bechev 1989b, 1991a, 1997, 1999a, 2000, 2002a, 2004a, 2006b, 2010; Bechev \& Pavlova 2016.

Mycetophila britannica Lastovka \& Kidd, 1975 - O62; 203-400 m; 1; ? e, ? ena; Pavlova 2020b.

Mycetophila caudata Stæger, 1840 - RW, RE; 650-1100 m; 1, 2, 3; h; Bechev 2001, 2002a, 2006b, 2010. 
Mycetophila confluens Dziedzicki, 1884 - P1, B1, B2, R2, RW; 550-2000 m; 1, 2, 3, 4; po; Bechev 2000, 2002a, 2006b, 2010; Bechev \& Pavlova 2016.

Mycetophila czizekii Landrock, 1911 - P1, B1, R2, RE; 300-2000 m; 1, 2, 3, 4; e; Bechev 1991a, 1997, 2000, 2002a, 2010; Bechev \& Pavlova 2016.

Mycetophila devioides Bechev, 1988 - B1 ; 775 m; 2, 3; e; Bechev 1988a, 1997, 2000, 2002a, 2010.

Mycetophila distigma Meigen, 1830 - B1; 350-775 m; 1, 2; e; Bechev 1991a, 1997, 2000, 2002a, 2010; Bechev \& Pavlova, 2016.

Mycetophila edwardsi Lundstrom, 1913 - B1, B2, O62, R2; 170-1700 m; 1, 2, 3, 4; e; Bechev 1994, 1997, 2000, 2002a, 2010; Bechev \& Pavlova 2016; Pavlova 2020b.

Mycetophila evanida Lastovka, 1972 - O62, R2; 146-510 m; 1; ? wces; Pavlova 2020b.

Mycetophila formosa Lundstrom, 1911 - B2; 1000 m; 3; wesit; Bechev 1991a, 1997, 2000, 2002a, 2010.

Mycetophila fraterna Winnertz, 1863 - B1; 775-1250 m; 2, 3; e; Bechev 1991a, 1997, 2000, 2002a, 2010.

Mycetophila fungorum (De Geer, 1776) [M. punctata (Meigen, 18004)] - P1, B1, B2, O62, R2, RW, RE; 150-1700 m; 1, 2, 3, 4; hno, ? ho; Nedelkov 1912; Bechev 1985a, 1989b, 1997, 1999a, 2000, 2002a, 2004a, 2006b, 2010; Bechev \& Pavlova 2016; Pavlova \& Stojanova 2020; Pavlova 2020b.

Mycetophila gibbula Edwards, 1925 - B2; 1000 m; 3; dp; Bechev 1991a, 1997, 2000, 2002a, 2010.

Mycetophila hyrcania Laštovka \& Matile, 1964 - B1, BN; 10-700 m; 1, 2; ei; Bechev 1991a, 1997, 2000, 2002a, 2010.

Mycetophila lamellata Lundstrom, 1911 - B1; 775 m; 2; e; Bechev 1991a, 1997, 2000, 2002a, 2010.

Mycetophila lastovkai Caspers, 1984 - B1, B2; 800-1000 m; 2, 3; e; Bechev 1991a, 1997, 2000, 2002a, 2010.

Mycetophila luctuosa Meigen, 1830 - B1, RW, RE; 150-1600 m; 1, 2, 3, 4; ho, ? h; Bechev 1989b, 1991a, 1997, 1999a, 2000, 2002a, 2004a, 2006b, 2010; Bechev \& Pavlova 2016.

Mycetophila lunata Meigen, 1804 - BN; 10 m; 1; tes; Bechev 1994, 1997, 2002a, 2010.

Mycetophila marginata Winnertz, 1863 - B1, B2, O62, R2, R3, RW, RE; 170-2000 m; 1, 2, 3, 4; e; Bechev 1985a, 1997, 2000, 2002a, 2004a, 2006b, 2010; Bechev \& Pavlova 2016; Pavlova \& Stojanova 2020; Pavlova 2020b.

Mycetophila mitis (Johannsen, 1912) - DW; 60 m; 1; h; Bechev 1997, 2000, 2002a, 2010.

Mycetophila morosa Winnertz, 1863 - B1; 775-1250 m; 2, 3; h; Bechev 1994, 1997, 2000, 2002a, 2010; Bechev \& Pavlova 2016.

Mycetophila occultans Lundström, 1913 - B1; 775 m; 2 ; e; Bechev 1994, 1997, 2000, 2002a, 2010.

Mycetophila ocellus Walker, 1848 - B1, B2, O62, R2, RW, RE; 170-2000 m; 1, 2, 3, 4; ho, ? h ; Bechev 1991a, 1997, 2000, 2002a, 2004a, 2006b, 2010; Bechev \& Pavlova 2016; Pavlova \& Stojanova 2020; Pavlova 2020b.

Mycetophila ornata Stephens, 1846 - B1, B2, R3, RW, RE; 350-1400 m; 1, 2, 3, 4; des; Burghele-Balacesco 1966; Beron \& Gueoruiev 1967; Bechev 1985a, 1997, 2000, 2002a, 2004a, 2006b, 2010; Beron 2015, 2016; Bechev \& Pavlova 2016.

Mycetophila pumila Winnertz, 1863 - P1, B1, B2; 350-1000 m; 1, 2, 3; dp; Bechev 1994, 1997, 2000, 2002a, 2010; Bechev \& Pavlova 2016.

Mycetophila scotica Edwards, 1941 - B1; 700 m; 2; h; Bechev 1991a, 1997, 2000, 2002a, 2010.

Mycetophila sigillata Dziedzicki, 1884 - B2, RW; 1000-1500 m; 3; h; Bechev 1991a, 1997, 2000, 2002a, 2006b, 2010.

Mycetophila signata Meigen, 1830 [Fungivora] - P1, B1, B2, V1, O62, RW; 170-1100 m; 1, 2, 3; des; Nedelkov 2012; Bechev 1985a, 1997, 2000, 2002a, 2006b, 2010; Pavlova 2020 b.

Mycetophila signatoides Dziedzicki, 1884 - B1; 550-775 m; 2; h; Bechev 1991a, 1997, 2000, 2002a, 2010; Bechev \& Pavlova, 2016.

Mycetophila spectabilis Winnertz, 1863 - P1, B1, B2, R3, RW, RE; 300-1500 m; 1, 2, 3, 4; e; Bechev 1991a, 1997, 2000, 2002a, 2004a, 2006b, 2010; Bechev \& Pavlova, 2016.

Mycetophila stolida Walker, 1856 - B1; 800 m; 2, 3; h; Bechev 1991a, 1997, 2000, 2002a, 2010; Bechev \& Pavlova, 2016.

Mycetophila stylata (Dziedzicki, 1884) - B1, R2; 650-2000 m; 2, 3, 4; tes; Bechev 1991a, 1997, 2000, 2002a, 2010.

Mycetophila tridentata Lundstrom, 1911 - B2; 1000 m; 2, 3; e; Bechev 1991a, 1997, 2000, 2002a, 2010.

Mycetophila trinotata Stæger, 1840 - B1; 350-1250 m; 1, 2, 3; h; Bechev 1994, 1997, 2000, 2002a, 2010; Bechev \& Pavlova 2016.

Mycetophila unguiculata Lundstrom, 1913 - B1; 600-775 m; 2; wes; Bechev 1994, 1997, 2000, 2002a, 2010.

Mycetophila vittipes Zetterstedt, 1852 - B2; 1000 m; 3; hoes; Bechev 1991a, 1997, 2000, 2002a, 2010. 
Mycetophila v-nigrum Lundstrom, 1913 - B1, B2; 775-1000 m; 2, 3; e; Bechev 1994, 1997, 2000, 2002a, 2010. Phronia basalis Winnertz, 1863 - T31, O62, R2; 100-510 m; 1; ena; Bechev 1999b, 2002a, 2010; Pavlova \& Stojanova 2020; Pavlova 2020b.

Phronia biarcuata (Becker, 1908) [Ph. nitidiventris van der Wulp, 1859] - B1, B2, O62, R2; 146-1200 m; 1, 2, 3; dp, ? tp; Bechev 1999b, 2000, 2002a, 2010; Bechev \& Pavlova 2016; Pavlova \& Stojanova 2020; Pavlova 2020a, 2020b.

Phronia cinerascens Winnertz, 1863 - B1, T31; 150-1200 m; 1, 2, 3; h; Bechev 1986a, 1997, 1999b, 2000, 2002a, 2010; Bechev \& Pavlova 2016.

Phronia conformis (Walker, 1856) - B1, T31; 100-700 m; 1, 2; h; Bechev 1999b, 2000, 2002a, 2010; Bechev \& Pavlova, 2016.

Phronia egregia Dziedzicki, 1889 - B1, B2; 400-900 m; 1, 2, 3; h; Bechev 1999b, 2000, 2002a, 2010; Bechev \& Pavlova, 2016.

Phronia forcipata Winnertz, 1863 - B1, B2; 700-900 m; 2, 3; wces; Bechev 1999b, 2000, 2002a, 2010.

Phronia mutabilis Dziedzicki, 1889 - B1; 800 m; 2, 3; h; Bechev 1999b, 2000, 2002a, 2010.

Phronia nitidiventris (van der Wulp, 1859) [Ph. vitiosa Winnertz, 1863] - B1, B2; 550-900 m; 2, 3; hoes; Bechev 1999b, 2000, 2002a, 2010; Bechev \& Pavlova 2016.

Phronia notata Dziedzicki, 1889 - B1, T31; 150-900 m; 1, 2; wes; Bechev 1999b, 2000, 2002a, 2010.

Phronia obtusa Winnertz, 1863 - B1; 350-800 m; 1, 2, 3; h; Bechev 1999b, 2000, 2002a, 2010; Bechev \& Pavlova, 2016.

Phronia signata Winnertz, 1863 [Ph. austriaca Winnertz, 1863] - B1; 550-800 m; 2, 3; hoes; Bechev 1999b, 2000, 2002a, 2010; Bechev \& Pavlova 2016.

Phronia strenua Winnertz, 1863 [Ph. flavicollis Winnertz, 1863] - B1; 550 m; 2; h; Bechev 1999b, 2000, 2002a, 2010; Bechev \& Pavlova 2016.

Phronia tenuis Winnertz, 1863 - B1, B2; 300-1000 m; 1, 2, 3; ho; Bechev 1999b, 2000, 2002a, 2010.

Platurocypta punctum (Stannius, 1831) - DW, B1, B2, RW; 60-1500 m; 1, 2, 3, 4; h; Bechev 1994, 1997, 2000, 2002a, 2006b, 2010.

Sceptonia cryptocauda Chandler, 1991 - P1, B1, O62; 170-850 m; 1, 2, 3; e; Bechev 1994, 1995, 1997, 2000, 2002a, 2010; Bechev \& Pavlova 2016; Pavlova \& Stojanova 2020; Pavlova $2020 \mathrm{~b}$.

Sceptonia flavipuncta Edwards, 1925 - P1, B1, B2, K7, T31, O62, BS; 10-1000 m; 1, 2, 3; e; Bechev 1994, 1995, 1997, 2000, 2002a, 2010; Pavlova \& Stojanova 2020; Pavlova 2020b.

Sceptonia humerella Edwards, 1925 - P1, B1; 300-900 m; 1, 2, 3; e; Bechev 1994, 1995, 1997, 2000, 2002a, 2010; Bechev \& Pavlova, 2016.

Sceptonia membranacea Edwards, 1925 - P1, B1, T31, O62, R1, RW, BN; 10-1500 m; 1, 2, 3, 4; e; Bechev 1994, 1995, 1997, 2000, 2002a, 2006b, 2010; Bechev \& Pavlova, 2016; Pavlova \& Stojanova 2020; Pavlova 2020b.

Sceptonia nigra (Meigen, 1804) - B1; 350-1250 m; 1, 2, 3; h; Bechev 1994, 1995, 1997, 2000, 2002a, 2010; Bechev \& Pavlova, 2016.

Sceptonia pilosa Bukowski, 1934 - B1, R1; 350-1200 m; 1, 2, 3; e; Bechev 1994, 1995, 1997, 2000, 2002a, 2010; Bechev \& Pavlova, 2016.

Sceptonia pughi Chandler, 1991 - B1; 700 m; 2; e; Bechev 1994, 1995, 1997, 2000, 2002a, 2010.

Sceptonia tenuis Edwards, 1925 - P1, B1; 250-1250 m; 1, 2, 3; e; Bechev 1994, 1995, 1997, 2000, 2002a, 2010; Bechev \& Pavlova 2016.

Trichonta apicalis Strobl, 1898 - P1, R2; 280-510 m; 1; e; Bechev 1990a, 1997, 2000, 2002a, 2010; Pavlova 2020b.

Trichonta beata Gagne, 1981 - B1; 600 m; 1, 2; h; Bechev 1996a, 1997, 2000, 2002a, 2010; Bechev \& Pavlova, 2016.

Trichonta clavigera Lundström, 1913 - O62, RW; 146-1185 m; 1, 2, 3; dp; Bechev 1991a, 1997, 2002a, 2006b, 2010; Pavlova 2020a, 2020b.

Trichonta conjungens Lundström, 1909 - B1; 1700 m; 4; e; Bechev 1990a, 1997, 2000, 2002a, 2010.

Trichonta falcata Lundström, 1911 - B1, B2; 350-1250 m; 1, 2, 3; h; Bechev 1990a, 1997, 2000, 2002a, 2010; Bechev \& Pavlova 2016.

Trichonta fragilis Gagne, 1981 - B1; 350-650 m; 1, 2; h; Bechev 1990a, 1997, 2000, 2002a, 2010; Bechev \& Pavlova, 2016.

Trichonta fusca Landrock, 1918 - B1; 775 m; 2, 3; e; Bechev 1990a, 1997, 2000, 2002a, 2010.

Trichonta girschneri Landrock, 1912 - P1, B1; 550-775 m; 2; h; Bechev 1990a, 1997, 2000, 2002a, 2010.

Trichonta melanura (Stæger, 1840) - B1, T31; 100-800 m; 1, 2, 3; h; Bechev 1990a, 1997, 2000, 2002a, 2010. 
Trichonta subfusca Lundström, 1909 - B1, RW; 775-1500 m; 2, 3, 4; h; Bechev 1990a, 1997, 2000, 2002a, 2006b, 2010.

Trichonta submaculata (Stæger, 1840) - B1; 400-800 m; 2, 3; hoes; Bechev 1990a, 1997, 2000, 2002a, 2010; Bechev \& Pavlova, 2016.

Trichonta terminalis (Walker, 1856) - P1, B1, B2, RW; 350-1450 m; 1, 2, 3, 4; h; Bechev 1990a, 1997, 2000, 2002a, 2006b, 2010; Bechev \& Pavlova, 2016.

Trichonta venosa (Stæger, 1840) - B2, O62, R2; 170-1000 m; 1, 2, 3; h; Bechev 1990a, 1997, 2000, 2002a, 2010; Pavlova \& Stojanova 2020; Pavlova 2020b.

Trichonta vitta (Meigen, 1830) - P1, B1, B2, T31, O62, R2; 100-1000 m; 1, 2, 3; h; Bechev 1990a, 1997, 2000, 2002a, 2010; Bechev \& Pavlova 2016; Pavlova \& Stojanova 2020; Pavlova 2020 b.

Trichonta vulgaris Loew, 1869 - B1, B2, R2; 440-1250 m; 1, 2, 3; h; Bechev 1990a, 1997, 2000, 2002a, 2010; Bechev \& Pavlova 2016; Pavlova 2020b.

Zygomyia humeralis (Wiedemann, 1817) - B1; 1250 m; 3; e (? i New Zealand); Bechev 1996a, 1997, 2000, 2002a, 2010; Bechev \& Pavlova, 2016.

Zygomyia pseudohumeralis Caspers, 1980 - P1, O62, R2, RW; 170-510 m; 1; e; Bechev 1996a, 1997, 2000, 2002a, 2006b, 2010; Pavlova 2020b.

Zygomyia semifusca (Meigen, 1818) - P1, B2, R2, RW; 440-1500 m; 1, 2, 3, 4; e; Bechev 1996a, 1997, 2000, 2002a, 2006b, 2010; Pavlova 2020b.

Zygomyia valida Winnertz, 1863 - RW; 1350 m; 3; wes; Bechev 1994, 1997, 2002a, 2006b, 2010.

\section{Ditomyiidae}

Ditomyia fasciata (Meigen, 1818) - P1, B1, RW, RE; 200-1250 m; 1, 2, 3; csei, ? ei; Bechev 1985a, 1989b, 1997, 1999a, 2000, 2002a, 2004b, 2006b, 2006c; Bechev \& Pavlova 2016.

Ditomyia macroptera (Winnertz, 1852) - O61; 350-400 m; 1; des; Kurina \& Chandler 2018.

Symmerus annulatus (Meigen, 1830) - P1, B2, T31, BN; 10-900 m; 1, 2, 3; wes; Bechev 1986b, 1997, 2000, 2002a, 2004b, 2006c.

\section{Bolitophilidae}

Bolitophila (Bolitophila) basicornis (Mayer, 1951) - B1, B2; 770-1000 m; 2, 3; des, ? tes; Bechev 1989a, 1997, 2000, 2002a, 2006c; Bechev \& Chandler 2011.

Bolitophila (Bolitophila) cinerea Meigen, 1818 - P1, B1, B2, R1, R2, R3, RW, RE; 300-1500 m; 1, 2, 3, 4; h; Bechev 1986a, 1997, 2000, 2002a, 2004a, 2004b, 2006b, 2006c; Bechev \& Chandler 2011; Bechev \& Pavlova 2016.

Bolitophila (Bolitophila) saundersii (Curtis, 1836) [Messala] - B1, B2, B3, R1, R2, RW; 400-1800 m; 1, 2, 3, 4; tp; subtroglophile; Burghele-Balacesco 1966; Beron \& Gueoruiev 1967; Bechev 1985a, 1997, 2000, 2002a, 2004b, 2006b, 2006c; Plassmann 1988; Bechev \& Chandler 2011; Beron 2015; Bechev \& Pavlova 2016.

Bolitophila (Bolitophila) tenella Winnertz, 1863 - R1, RW; 1500-1700 m; 4; des; Bechev 2001, 2002a, 2006b, 2006c; Bechev \& Chandler 2011.

Bolitophila (Cliopisa) fumida Edwards, 1941 [B. glabrata Loew, 1869] - B1; 350-1300 m; 1, 2, 3; tes; Bechev 1989a, 1997, 2000, 2001, 2006c; Bechev \& Chandler 2011; Bechev \& Pavlova 2016.

Bolitophila (Cliopisa) hybrida (Meigen, 1804) [B. fusca Meigen, 1818; Macrocera] - B2, R1; 900 m; 2, 3; h; Nedelkov 1012; Bechev 1985a, 1997, 2000, 2002a, 2004b, 2006c; Bechev \& Chandler 2011.

Bolitophila (Cliopisa) maculipennis Walker, 1836 - B2; 700-1000 m; 2,3; tp; Bechev 1989a, 1997, 2000, 2002a, 2006c; Bechev \& Chandler 2011.

Bolitophila (Cliopisa) occlusa Edwards, 1913 - R2; 1740 m; 4; des; Bechev 1991a, 1997, 2002a, 2006c; Bechev \& Chandler 2011.

Bolitophila (Cliopisa) pseudohybrida Landrock, 1912 - P1, B1, R2, RW, RE, BS; 20-1450 m; 1, 2, 3; tes; Bechev 1989a, 2000, 1997, 2002a, 2004a, 2004b, 2006b, 2006c; Bechev \& Chandler 2011; Bechev \& Pavlova 2016.

Bolitophila (Cliopisa) rossica Landrock, 1912 - R2; 2000 m; 4; tes; Bechev 2001, 2002a, 2006c; Bechev \& Chandler 2011. 


\section{Diadocidiidae}

Diadocidia (Adidocidia) valida Mik, 1874 - B1, RW; 770-1600 m; 2,3,4; wes; Bechev 1986a, 1997, 2000, 2006b, 2006c; Bechev \& Chandler 2011.

Diadocidia (Diadocidia) ferruginosa (Meigen, 1830) - B1, B2, O5, RW, RE, BN; 5-1350 m; 1, 2, 3; h; Bechev 1986a, 1997, 2000, 2002a, 2004a, 2004b, 2006b, 2006c; Bechev \& Chandler 2011; Bechev \& Pavlova 2016.

Diadocidia (Diadocidia) spinosula Tollet, 1948 - B2, R1, R2, RW; 900-1740 m; 3, 4; des; Bechev 1989a, 1997, 2000, 2002a, 2004b, 2006b, 2006c; Bechev \& Chandler 2011.

\section{Keroplatidae (+ Macroceridae)}

Cerotelion racovitzai Matile \& Burghele-Balacesco, 1969 - B1, BS; 50-1250 m; 1, 2, 3; cseei; Bechev 1986b, 1997, 2000, 2002a, 2004b, 2006c; Bechev \& Pavlova 2016.

Cerotelion striatum (Gmelin, 1790) [Ceroplatus lineatus Fabricius, 1775] - B1, B2, V1, R3; 350-800 m; 1, 2; ei; Nedelkov 1012; Bechev 1985a, 1997, 2000, 2002a, 2004b, 2006c; Bechev \& Pavlova 2016.

Keroplatus reaumurii Dufour, 1839 - B1, BN; 20-400 m; 1; csena; Bechev 2000, 2001, 2002a, 2004b, 2006c; Bechev \& Pavlova 2016.

Keroplatus testaceus Dalman, 1818 - B1; 350-775 m; 1, 2; dp; Bechev 1986b, 1997, 2000, 2002a, 2006c; Bechev \& Pavlova 2016.

Keroplatus tipuloides Bosc, 1792 - O5; 700 m; 2; dp, ? tp; Bechev 1999a, 2002a, 2004b, 2006c.

Antlemon (Antlemonopsis) brevimanum (Loew, 1871) - B2, R1, RW; 1300-1900 m; 3, 4; csee; Bechev 1991a, 1997, 2000, 2002a, 2004b, 2006b, 2006c.

Isoneuromyia semirufa (Meigen, 1818) [Orfelia] - P1, B1, O5, BN; 100-770 m; 1, 2; des; Bechev 1985a, 1997, 2000, 2002a, 2004b, 2006c; Bechev \& Pavlova 2016.

Macrorrhyncha collarti (Tollet, 1955) [Asindulum exemplum Plassmann, 1978] - B2, RW; 800-1500 m; 2, 3, 4; e; Plassmann 1978; Bechev 1985a, 1992a, 1997, 2000, 2002a, 2006b, 2006c.

Macrorrhyncha flava Winnertz, 1846 - B1, O62, BN, BS; 10-700 m; 1, 2; e; Bechev 1985a, 1997, 2000, 2002a, 2004b, 2006c; Pavlova 2020b.

Macrorrhyncha veleka Bechev, 1992 - BS; 20 m; 1; Er; Bechev 1992b, 1997, 2002a, 2006c.

Monocentrota matilei Bechev, 1989 - B1, O62; 170-700 m; 1, 2; seena; Bechev 1989c, 1997, 2000, 2002a, 2006c; Bechev \& Pavlova 2016; Pavlova 2020b.

Neoplatyura modesta (Winnertz, 1863) - R2, BN; 5-510 m; 1; e; Bechev 2001, 2002a, 2006c; Pavlova 2020b.

Neoplatyura nigricauda (Strobl, 1893) - P1, B1, T31, RE; 200-350 m; 1; e; Bechev 1986b, 1997, 2000, 2002a, 2004b, 2006c; Bechev \& Pavlova 2016.

Orfelia bezzii (Strobl, 1910) - P2, B1, B2, O5; 350-850 m; 1, 2; e; Janeva \& Russev 1997; Bechev 2006a, 2006c; Bechev \& Pavlova 2016.

Orfelia bicolor (Macquart, 1826) - BN; 10 m; 1; e; Bechev 2006a, 2006c.

Orfelia discoloria (Meigen, 1818) - B1, T31; 200-770 m; 1, 2; h; Bechev 1985a, 2001, 2002a, 2006c.

Orfelia fasciata (Meigen, 1804) - B1, O62, RW; 300-1500 m; 1, 2, 3, 4; ho; Bechev 1986b, 1997, 2000, 2002a, 2004b, 2006b, 2006c.

Orfelia gruevi Bechev, 2002 - BN; 10-20 m; 1; csee; Bechev 2002b, 2006c.

Orfelia lugubris (Zetterstedt, 1851) [O. tristis (Lundström, 1911)] - B1, R2, RW; 350-1350 m; 1, 2, 3; e; Bechev 1990a, 1997, 2000, 2002a, 2004b, 2006b, 2006c; Bechev \& Pavlova 2016.

Orfelia nemoralis (Meigen, 1818) - DW, E1, E2, TL, O62, RW, BN, BS; 10-1400 m; 1, 2, 3, 4; h, ? e; Bechev 1986b, 2000, 2002a, 2004b, 2006b, 2006c.

Orfelia nigricornis (Fabricius, 1805) - B1; 350 m; 1; h, ? esca; Bechev 1990a, 1997, 2000, 2002a, 2004b, 2006c; Bechev \& Pavlova 2016.

Orfelia ochracea (Meigen, 1818) - B1; 350 m; 1; po, ? des; Bechev \& Pavlova 2016.

Orfelia unicolor (Staeger, 1840) [O. discoloria (Meigen, 1818), Platyura] - P1, B1, V1, TL, O5, R1, RW, BS; 10-1700 m; 1, 2, 3, 4; des, ? hoes; Nedelkov 1912; Bechev 1985a, 1997, 2000, 2002a, 2004b, 2006b, 2006c.

Platyura marginata Meigen, 1804 - B1, R1, R3, RW; 600-1820 m; 2, 3, 4; des; Bechev 1991a, 1997, 2000, 2002a, 2004b, 2006b, 2006c.

Pyratula oracula Chandler, 1994 - RE; 450 m; 1; cseel; Bechev 2004a, 2006c. 
Pyratula perpusilla (Edwards, 1913) [Orfelia] - B1; 370-750 m; 1, 2; wces; Bechev 1985a, 1996a, 1997, 2000, 2002a, 2006c; Bechev \& Pavlova 2016.

Pyratula subcanariae Chandler, 2001 - E1; 200 m; 1; csee; Bechev 2006a, 2006c.

Pyratula zonata (Zetterstedt, 1855) [Orfelia] - B1, K7, O5, O62, R2, R3, RW, RE; 146-1400 m; 1, 2, 3; e; Bechev 1989a, 1997, 2000, 2002a, 2004a, 2004b, 2006b, 2006c; Bechev \& Pavlova 2016; Pavlova 2020a, 2020b.

Urytalpa ochraceae (Meigen, 1918) - RW; 1620 m; 4; e; Bechev 2001, 2006b, 2006c.

Urytalpa rhapsodica Chandler, 1995 - RW; 2000 m; 4; csee; Bechev 2001, 2002a, 2006b, 2006c.

Macrocera anglica Edwards, 1925 - B1, B2, RW; 350-1000 m; 1, 2, 3; e; Bechev 1986b, 1997, 2000, 2002a, 2004b, 2006b, 2006c; Bechev \& Pavlova 2016.

Macrocera angulata Meigen, 1818 - B1; 350 m; 1; e; Bechev 1986b, 1997, 2000, 2002a, 2006c; Bechev \& Pavlova 2016.

Macrocera centralis Meigen, 1818 - B1, B2, R1, R2, RW, RE, BN; 150-1820 m; 1, 2, 3, 4; wes; Bechev 1985a, 1997, 2000, 2002a, 2004a, 2004b, 2006b, 2006c; Bechev \& Pavlova 2016.

Macrocera crassicornis Schiner, 1863 - B1; 350 m; 1; wp; Bechev 2000, 2002a, 2006b, 2006c; Bechev \& Pavlova 2016.

Macrocera fasciata Meigen, 1804 - P1, B1, T31, O5, O62, R3, RW, RE; 180-1700 m; 1, 2, 3, 4; dp; Bechev 1989a, 1997, 2000, 2002a, 2004a, 2004b, 2006b, 2006c; Bechev \& Pavlova 2016.

Macrocera fastuosa Loew, 1869 - B1, RW; 700-1100 m; 2, 3; des; Bechev 1986b, 1997, 2000, 2002a, 2006b, 2006c.

Macrocera gemagea Bechev, 1991 - P1, BN; 20-550 m; 1; see; Bechev 1991b, 1997, 2000, 2002a, 2006a, 2006c.

Macrocera grandis Lundström, 1912 - R1; 1150 m; 3; wes; Bechev 2006a, 2006c.

Macrocera inversa Loew, 1869 - E1, B1, O5, R1, RW, RE; 150-1740 m; 1, 2, 3, 4; wces; Bechev 1986b, 1997, 2000, 2002a, 2004a, 2004b, 2006b, 2006c.

Macrocera kerteszi Lundström, 1911 - B1, O5, RW; 350-720 m; 1, 2; see; Bechev 1986b, 1997, 2000, 2002a, 2006b, 2006c.

Macrocera lutea Meigen, 1804 - P1, B1, T31, O5, RW, RE, BN, BS; 10-1500 m; 1, 2, 3; des; Bechev 1986b, 1997, 2000, 2002a, 2004a, 2004b, 2006b, 2006c.

Macrocera nigricoxa Schiner, 1863 [M. tusca Loew, 1869] - B1, RW; 350-900 m; 1, 2, 3; wcp; Bechev 1989a, 1992a, 1997, 2000, 2002a, 2006b, 2006c; Bechev \& Pavlova 2016.

Macrocera parva Lundström, 1914 - B1, B2, R2; 600-1740 m; 2, 3, 4; wces; Bechev 1996a, 1997, 2000, 2002a, 2004b, 2006c; Bechev \& Pavlova 2016.

Macrocera phalerata Wiedemann in Meigen, 1818 - E1, E2, P1, P2, B1, T31, O5, R2, R3, RW, RE, BN, BS; 5-1450 m; 1, 2, 3, 4; wcp; Bechev 1986b, 1997, 2000, 2002a, 2004a, 2004b, 2006b, 2006c; Bechev \& Pavlova 2016; Pavlova 2020b.

Macrocera pilosa Landrock, 1917b - B1; 800 m; 2, 3; wcp, ? h; Bechev 1991a, 1997, 2000, 2002a, 2006c; Bechev \& Pavlova 2016.

Macrocera stigma Curtis, 1831 - B1, O62, R2, R3, RW; 170-1700 m; 1, 2, 3, 4; wces; Bechev 1986b, 1997, 2000, 2002a, 2004b, 2006b, 2006c; Bechev \& Pavlova 2016; Pavlova 2020b.

Macrocera stigmoides Edwards, 1925 - P1, R1, R2, RW, RE, BS; 5-2000 m; 1, 2, 3, 4; e; Bechev 1996a, 1997, 2000, 2002a, 2004b, 2004a, 2006b, 2006c.

Macrocera vittata Meigen, 1830 - P1, B1, O5, R3, RW; 550-1700 m; 1, 2, 3, 4; dp; Bechev 1985a, 1997, 2000, 2002a, 2004b, 2006b, 2006c; Bechev \& Pavlova 2016.

\section{Sciaridae}

Bradysia albanensis (Lengersdorf, 1926) - V1; 550-650 m; 1, 2; e; Mohrig et al. 1992.

Bradysia alpicola (Winnertz, 1867) [B. forcipata (Fabricius, 1775); B. morio (Fabricius, 1794); Sciara] - V1, BN; 20-650 m; 1, 2; e, ? po; Loew 1862; Nedelkov 1912.

Bradysia alutacea Dimitrova \& Mohrig, 1993 - BN; 10-20 m; 1; se; Dimitrova \& Mohrig 1993.

Bradysia angustipennis Winnertz, 1867 [B. campestris Mohrig \& Mamaev 1970] - V4; 1550 m; 3; wes; Dimitrova \& Mohrig 1993.

Bradysia bicolor (Meigen, 1818) - V4; 1550 m; 3; wes; Dimitrova \& Mohrig 1993.

Bradysia breviallata Mohrig \& Menzel, 1992 - V3, R2; 800-950 m; 2; e; Dimitrova \& Mohrig 1993.

Bradysia brevispina Tuomikoski, 1960 - V4, BN; 200-1000 m; 1, 2, 3; wes; Dimitrova \& Mohrig 1993. 
Bradysia conspersa Mohrig \& Dimitrova, 1993 - BN; 5-20 m; 1; Eb; Dimitrova \& Mohrig 1993.

Bradysia fenestralis (Zetterstedt, 1838) [Sciara, Neosciara] - -; •; 1, 2; ? e; Choleva 1964; Grigorov 1972.

Bradysia hirsutiseta Mohrig \& Krivosheina, 1989 - V1; 550-650 m; 1, 2; sesfe; Morhing et al. 1992.

Bradysia lobulifera Frey, 1948 - V4, BN; 20-1550 m; 1, 2, 3; wes; Dimitrova \& Mohrig 1993.

B. nitidicollis (Meigen, 1818) [Sciara] - V1; 550-650 m; 2; e, ? h; Nedelkov 1912.

Bradysia ocellaris (Comstock, 1882) [B. tritici (Coquillett, 1895)] - V4, BN; 200-1000 m; 1, 2, 3; sk; Dimitrova \& Mohrig 1993.

Bradysia pallidiventris (Winnertz, 1867) - BN; 20 m; 1; e; Dimitrova \& Mohrig 1993.

Bradysia pectoralis (Staeger, 1840) [B. castanea Mohrig \& Menzel, 1990] - V4; 650 m; 1, 2; e; Dimitrova \& Mohrig 1993.

Bradysia placida (Winnertz, 1867) [B. fimbricauda Tuomikoski, 1960] - V3, V4, BN; 20-950 m; 1, 2; et; Dimitrova \& Mohrig 1993.

Bradysia polonica (Lengersdorf, 1929) - V1; 550-650 m; 1, 2; e; Mohrig et al. 1992.

Bradysia praecox (Meigen, 1818) [Sciara] - V1; 550-650 m; 1, 2; e, ? h; Nedelkov 1912; Mohrig et al. 1992.

Bradysia regularis (Lengersdorf, 1934) - V4; 1100 m; 3; wes; Dimitrova \& Mohrig 1993.

Bradysia rufescens (Zetterstedt, 1852) - V1, V5; 550-1200 m; 2, 3; e, ? poa; Mohrig et al. 1992; Dimitrova \& Mohrig 1993.

Bradysia santorina Mohrig \& Menzel, 1992 - BN; 20 m; 1; Eb; Dimitrova \& Mohrig 1993.

Bradysia scabricornis Tuomikoski, 1960 - BN; 20 m; 1; des; Dimitrova \& Mohrig 1993.

Bradysia splendida Mohrig \& Krivosheina, 1989 - V1, BN; 20-650 m; 1, 2; et; Mohrig et al. 1992; Dimitrova \& Mohrig 1993.

Bradysia submoesta Mohrig \& Krivosheina, 1989 - V4; 1550 m; 3; e; Dimitrova \& Mohrig 1993.

Bradysia tilicola (Loew, 1850) [Sciara amoena Winnertz, 1871] - V1, V4; 550-1550 m; 2, 3; des, ? pa, ? i; Nedelkov 2012; Dimitrova \& Mohrig 1993.

Bradysia trivittata (Staeger, 1840) - V4, BN; 20-1550 m; 1, 2, 3; west; Dimitrova \& Mohrig 1993.

Bradysia vagans (Winnertz, 1868) [B. callicera Frey, 1948] - V3; 950 m; 2, 3; e; Dimitrova \& Mohrig 1993.

Camptochaeta bournei (Shaw, 1941) [Corynoptera subvivax Mohrig, 1985] - V4; 1100 m; 3; e; Dimitrova \& Mohrig 1993.

Claustropyga abblanda (Freeman, 1983) [Corynoptera] - V4; 900-1000 m; 3; h; Dimitrova \& Mohrig 1993.

Corynoptera acerrima Mohrig \& Dimitrova, 1992 - V4; 1000-1200 m; 3; csee; Mohrig \& Dimitrova 1992.

Corynoptera acuminata Mohrig \& Dimitrova, 1992 - BN; 20 m; 1; Ebg; Mohrig \& Dimitrova 1992.

Corynoptera alticola (Kieffer, 1919) [C. praepiniphila Mohrig \& Dimitrova, 1992; C. postpiniphila Mohrig \& Mamaev, 1992] - V1, V4; 550-1200 m; 2, 3; ena; Mohrig \& Dimitrova 1992; Mohrig et al. 1992.

Corynoptera applanata Mohrig \& Dimitrova, 1992 - V4; 800 m; 2; se; Mohrig \& Dimitrova 1992.

Corynoptera bispinulosa Mohrig \& Dimitrova, 1992 - BN; 20 m; 1; cse, ? e; Mohrig \& Dimitrova 1992.

Corynoptera bistrispina (Bukowski \& Lengersdorf, 1936) - V4; 800 m; 2; ? e, ? des; Dimitrova \& Mohrig 1993.

Corynoptera blanda (Winnertz, 1867) - V4; 1450 m; 3; h; Dimitrova \& Mohrig 1993.

Corynoptera bulgarica Mohrig \& Mamaev, 1992 - V1; 550-650 m; 2; csee; Mohrig et al. 1992.

Corynoptera deserta Heller \& Menzel, 2006 [C. minutula Bukowski \& Lengersdorf, 1936] - BN; 20 m; 1; e; Dimitrova \& Mohrig 1993.

Corynoptera dubitata Tuomikoski, 1960 - BN; 120 m; 1; e; Dimitrova \& Mohrig 1993.

Corynoptera flavicoxa Mohrig \& Mamaev, 1992 - V1; 550-650 m; 2; Ebg; Mohrig et al. 1992.

Corynoptera forcipata (Winnertz, 1867) - V4; 650 m; 1, 2; ena; Dimitrova \& Mohrig 1993.

Corynoptera furcifera Mohrig \& Krivosheina, 1987 - V1, BN; 200-650 m; 1, 2; e; Mohrig et al. 1992; Dimitrova \& Mohrig 1993.

Corynoptera hemiacantha Mohrig \& Mamaev, 1992 - V1; 550-650 m; 1, 2; cse; Mohrig et al. 1992.

Corynoptera hypopygialis (Lengersdorf, 1926) [C. piniphila (Lengersdorf, 1940)] - V1, V3; 550-950 m; 2, 3; e; Mohrig et al. 1992; Dimitrova \& Mohrig 1993.

Corynoptera irmgardis (Lengersdorf, 1930) - V1, V3, V4; 550-1100 m; 2, 3; wes; Mohrig et al. 1992; Dimitrova \& Mohrig 1993.

Corynoptera luteofusca (Bukowski \& Lengersdorf, 1936) - V4; 800 m; 2; e; Dimitrova \& Mohrig 1993.

Corynoptera magica Mohrig \& Dimitrova, 1992 - V3, V4; 900-1550 m; 2, 3; Ebg; Mohrig \& Dimitrova, 1992.

Corynoptera melanochaeta Mohrig \& Menzel, 1992 - V1; 550-650 m; 2; h; Mohrig et al. 1992. 
Corynoptera obscuripila Tuomikoski, 1960 - V4; 1000-1200 m; 3; e; Dimitrova \& Mohrig 1993.

Corynoptera praeparvula Mohrig \& Krivosheina, 1983 - BN; 150 m; 1; et; Dimitrova \& Mohrig 1993.

Corynoptera subparvula Tuomikoski, 1960 - V1, V4, BN; 20-1550 m; 1, 2, 3; et; Mohrig et al. 1992; Dimitrova \& Mohrig 1993.

Corynoptera subpiniphila Mohrig \& Mamaev, 1992 - V1; 550-650 m; 2; ban; Mohrig et al. 1992.

Corynoptera subtilis (Lengersdorf, 1929) [C. longicornis (Bukowski \& Lengersdorf, 1936)] - V4; 1100 m; 3; wes; Dimitrova \& Mohrig 1993.

Corynoptera trepida (Winnertz, 1867) [C. clinochaeta Tuomikoski, 1960] - V4; 1100 m; 3; h; Dimitrova \& Mohrig 1993.

Cratyna (Cratyna) ambigua (Lengersdorf, 1934) [Plastosciara latiforceps (Bukowski \& Lengersdorf, 1934)] V4, BN; 200-1100 m; 1, 2, 3; e, ? h, ? i; Dimitrova \& Mohrig 1993.

Cratyna (Cratyna) betulae (Mohrig \& Mamaev, 1992) [Plastosciara] - V1; 550-650 m; 2; e; Mohrig et al. 1992.

Cratyna (Spathobdella) falcifera (Lengersdorf, 1933) [Plastosciara] - V1; 550-650 m; 2; e; Mohrig et al. 1992.

Ctenosciara hyalipennis (Meigen, 1804) - V1, V4; 550-800 m; 1, 2; hpta, ? i; Mohrig et al. 1992; Dimitrova \& Mohrig 1993.

Ctenosciara lutea (Meigen, 1804) - V1; 550-650 m; 2; e; Mohrig et al. 1992.

Epidapus (Pseudoaptanogyna) anomalus Mohrig \& Dimitrova, 1993 - BN; 200 m; 1; csee; Mohrig \& Dimitrova, 1993.

Epidapus (Epidapus) antegracilis Mohrig \& Dimitrova, 1993 - V4; 1450-1550 m; 3; see; Mohrig \& Dimitrova, 1993.

Epidapus (Epidapus) atomarius (De Geer, 1778) - V1; 550-650 m; 2; wpo; Mohrig et al. 1992.

Epidapus (Epidapus) bipalpatus Mohrig, 1982 - V3, V4; 800-1500 m; 2, 3; cse; Dimitrova \& Mohrig 1993.

Epidapus (Epidapus) detriticola (Kratochvil, 1936) - V4; 800-1050 m; 2, 3; e, ? cse; Dimitrova \& Mohrig 1993.

Epidapus (Epidapus) gracilis (Walker, 1848) - V1, V3, V4; 550-1700 m; 2, 3, 4; wes; Mohrig et al. 1992; Dimitrova \& Mohrig 1993.

Epidapus (Epidapus) macrohalteratus Mohrig \& Menzel, 1992 - V3; 950 m; 2, 3; cse; Dimitrova \& Mohrig 1993. Epidapus (Epidapus) microthorax (Börner, 1903) [E. gracilicornis (Lengersdorf, 1926)] - V1, BN; 20-650 m; 1, 2; e; Mohrig et al. 1992; Dimitrova \& Mohrig 1993.

Epidapus (Epidapus) schillei (Börner, 1903) [E. titan Frey, 1948] - V3, V4, BN; 20-1550 m; 1, 2, 3; e; Dimitrova \& Mohrig 1993.

Epidapus (Pseudoaptanogyna) carpaticus (Mohrig \& Mamaev, 1985) - V4; 1450-1550 m; 3; csee; Dimitrova \& Mohrig 1993.

Leptosciarella (Leptosciarella) coarctata (Winnertz, 1867) [Trichosia] - V4; 1000-1550 m; 3; e; Dimitrova \& Mohrig 1993.

Leptosciarella (Leptosciarella) scutellata (Staeger, 1840) [Trichosia] - V4; 1000-1200 m; 3; des; Dimitrova \& Mohrig 1993.

Leptosciarella (Leptosciarella) viatica (Winnertz, 1867) [Trichosia] - V4; $850 \mathrm{~m} ; 2$; e; Dimitrova \& Mohrig 1993. Lycoriella (Lycoriella) castanescens (Lengersdorf, 1940) [L. fulcorum (Frey, 1948)] - V4, BN; 150-1450 m; 1, 2, 3; ha, ? i; Dimitrova \& Mohrig 1993.

Lycoriella (Lycoriella) ingenua (Dufour, 1839) [L. mali (Fitch, 1856); L. solani (Winnertz, 1871)] - BN; 50 m; 1; ha; Dimitrova \& Mohrig 1993.

Peyerimhoffia vagabunda (Winnertz, 1867) [Plastosciara brachyptera (Kieffer, 1903)] - V1; 550-650 m; 2; e; Mohrig et al. 1992.

Phytosciara (Phytosciara) halterata (Lengersdorf, 1926) [Lycoria] - R1; 1150-1200 m; 3; e; Buhr 1941.

Phytosciara (Phytosciara) macrotricha (Lengersdorf, 1926) [Psilomegalosphys] - R1; 1150-1200 m; 3; e; Buhr 1941.

Scatopsciara (Scatopsciara) atomaria (Zetterstedt, 1851) [S. vivida (Winnertz, 1867)] - V3, V4, BN; 20-1650 m; 1, 2, 3, 4; hno; Dimitrova \& Mohrig 1993.

Scatopsciara (Scatopsciara) edwardsi Freeman, 1983 - V4; 1100 m; 3; e; Dimitrova \& Mohrig 1993.

Scatopsciara (Scatopsciara) multispina (Bukowski \& Lengersdorf, 1936) - V1; 550-650 m; 2; wes; Mohrig et al. 1992.

Sciara analis Schiner, 1864 - V1, V4, R1; 550-2100 m; 2, 3, 4; e, ? wes; Joakimoff 1899; Nedelkov 1912.

Sciara helvola Winnertz, 1867 - BN; 20 m; 1; e; Dimitrova \& Mohrig 1993. 
Sciara hemerobioides (Scopoli, 1763) [S. thomae (Linnaeus, 1767)] - B1, V1, V4, R1; 220-1150 m; 1, 2, 3; po; Joakimoff 1899; Nedelkov 1912.

Xylosciara (Xylosciara) misella (Frey, 1948) - BN; 150 m; 1; e; Dimitrova \& Mohrig 1993.

\section{Cecidomyiidae}

Anaretella defecta (Winnertz, 1870) [A. spiraeina (Felt, 1907)] - V4;1100 m; 3; ? k; Dimitrova \& Mamaev 1993. Aprionus bidentatus (Kieffer, 1894) - V4; 1100 m; 3; e; Dimitrova \& Mamaev 1993.

Lestremia cinerea Macquart, 1826 - V4; 800 m; 2; hna; Dimitrova \& Mamaev 1993.

Polyardis adela Pritchard, 1947 [Monardia edwardsi (Kleesattel 1979)] - V4; 1000-1100m; 3; h; Dimitrova \& Mamaev 1993.

Monardia (Xylopriona) atra (Meigen, 1804) [Xylopriona] - V4; 1050-1500 m; 3; h; Dimitrova \& Mamaev 1993. Campylomyza flavipes Meigen, 1818 [C. pumila Winnertz 1870; C. strobli (Kieffer 1901)] - V4; 1400 m; 3; ha; Dimitrova \& Mamaev 1993.

Neurolyga verna (Mamaev, 1963) [Cordylomyia] - V4; 1150 m; 3; h; Dimitrova \& Mamaev 1993.

Peromyia ramosa (Edwards, 1938) - V4; 1100 m; 3; tes; Dimitrova \& Mamaev 1993.

Monepidosis bulgarica Mamaev \& Dimitrova 1992 - V4; 1550 m; 3; e; Mamaev \& Dimitrova 1992.

Neocolpodia paradoxa Mamaev, 1964 - V4; 1000 m; 2, 3; e; Dimitrova \& Mamaev 1993.

Porricondyla armata Spungis, 1981 - V4; 1400 m; 3; e; Dimitrova \& Mamaev 1993.

Porricondyla aurantiaca Panelius, 1965 - V4; 1500 m; 3; des; Dimitrova \& Mamaev 1993.

Porricondyla modesta Spungis, 1981 - V4; 1100 m; 3; des; Dimitrova \& Mamaev 1993.

Spungisomyia media (Spungis, 1981) [Porricondyla] - V4; 1100 m; 3; des; Dimitrova \& Mamaev 1993.

Porricondyla modesta Spungis, 1981 - V4; 1100 m; 3; e; Dimitrova \& Mamaev 1993.

Porricondyla nigripennis (Meigen, 1830) - V4; 1550 m; 3; h; Dimitrova \& Mamaev 1993.

Winnertzia curvata Panelius, 1965 - V4; 800 m; 2; e; Dimitrova \& Mamaev 1993.

Hilversidia autumnalis Mamaev, 1966 - V4; 1600 m; 4; ee; Dimitrova \& Mamaev 1993.

Hybolasioptera fasciata (Kieffer, 1904) [H.cerealis (Lindeman, 1880)] - -; V1; 510 m; 1; e; Popoff 1939a; Buresch \& Lazarov 1956; Skuhravá et al. 1991.

Lasioptera arundinis Schiner, 1854 - V4, BN; 10-730 m; 1, 2; e; Dimitrova 1989, 1990; Skuhravá et al. 1991.

Lasioptera carophila F. Löw, 1874 - TL, BN; 50-180 m; 1; wp; Skuhravá et al. 1991.

Lasioptera eryngii (Vallot, 1829) - V1, R1, R2, BN; 50-850 m; 1, 2; wp; Naidenov 1962; Skuhravá et al. 1991.

Lasioptera rubi (Schrank, 1803) - -; B1, B2, V1, V4, S1, S21, R1, TL, BN; 5-1500 m; 1, 2, 3; dp; Nikolova 1950; Buresch \& Lazarov 1956; Popoff 1956; Kovachevski et al. 1959; Lazarov et al. 1960; Grigorov 1962, 1972, 1976; Naidenov 1962, 1963; Dimitrova 1989; Skuhravá et al. 1991, 1992.

Ozirhincus anthemidis (Rübsaamen, 1916) - BN; 10-100 m; 1; hom; Skuhravá et al. 1991.

Apiomyia bergenstammi (Wachtl, 1882) - -; P2, V3, V4, TK, K4, K5, BN; 50-1000 m; 1, 2; wcp, ? eswa; Tschorbadjiew 1925a, 1925b, 1925c, 1926a, 1926b, 1927, 1928a, 1928b, 1932, 1933; Lazarov 1949a; Buresch \& Lazarov 1956; Naidenov 1962; Dimitrova 1989; Skuhravá et al. 1991, 1992.

Arnoldiola gemmae (Giraud, 1868) - B1, V4; 600-1080 m; 1, 2, 3; e; Dimitrova 1989; Skuhravá et al. 1991.

Arnoldiola libera (Kieffer, 1909) - B1, V4, BN; 40-750 m; 1, 2; e; Dimitrova 1989; Skuhravá et al. 1991.

Spurgia euphorbiae (Vallot, 1827) [Bayeria capitigena (Bremi, 1847); Dasineura subpatula (Bremi, 1847)] - B1, V1, V4, R1, R2, RW, BN; 20-2108 m; 1, 2, 3, 4, 5; ena, i, h; Naidenov 1962; Dimitrova 1987, 1989; Skuhravá et al. 1991, 1992; Beschovski 2006.

Bayeriola salicariae (Kieffer, 1888) - BN; 10-30 m; 1; e; Skuhravá et al. 1991, 1992.

Bayeriola thymicola (Kieffer, 1888) [Bayeria] - R1, R2, RW; 10-900 m; 1, 2; ena; Skuhravá et al. 1991, 1992; Beschovski 2006.

Bremiola onobrychidis (Bremi, 1847) - -; ewca; Donchev 1978; Skuhravá et al. 1991, 1992.

Coniophora nijveldti Dimitrova, 1992 - V4; 1150 m; 3; Er; Dimitrova 1989, 1992.

Craneiobia corni (Giraud, 1863) - V4, K8, TL, BN; 50-800 m; 1, 2; e; Naidenov 1962; Dimitrova 1987, 1989; Skuhravá et al. 1991, 1992; Beschovski 2006.

Cystiphora taraxaci (Kieffer, 1888) - V4, R2; 900-1050 m; 2, 3; wpo, i, ho; Dimitrova 1989; Skuhravá et al. 1991, 1992.

Dasineura acrophila (Winnertz, 1853) - R1, BN; 5-850 m; 1, 2; ena; Skuhravá et al. 1991, 1992. 
Dasineura affinis (Kieffer, 1886) - V1, BN; 50-650 m; 1; ena; Skuhravá et al. 1991, 1992.

Dasineura airae (Kieffer, 1897) - V4; 750 m; 2; ? e; Dimitrova 1989.

Dasineura alopecuri (Reuter, 1895) - •; e, i, ha; Lyubenov 1957; Skuhravá et al. 1991, 1992.

Dasineura aparines (Kieffer, 1889) - B1, BN; 5-650 m; 1, 2; ena; Skuhravá et al. 1991, 1992.

Dasineura asperulae (F. Löw, 1875) - V1, V4, R2, RW; 700-1550 m; 2, 3; cse; Dimitrova 1989; Skuhravá et al. 1991, 1992; Beschovski 2006.

Dasineura auritae Rübsaamen, 1916 - V4; 1000-1150 m; 3; e; Dimitrova 1987, 1989; Skuhravá et al. 1991, 1992.

Dasineura axillaris Kieffer, 1896 - BN; 50 m; 1; e; Skuhravá et al. 1991, 1992.

Dasineura bayeri Rübsaamen, 1914 - BN; 10-100 m; 1; e; Skuhravá et al. 1991, 1992.

Dasineura capsulae Kieffer, 1901 - BN; 10-100 m; 1; ena; Naidenov 1962; Skuhravá et al. 1991, 1992.

Dasineura corylina (Kieffer, 1913) - V4; 1200 m; 3; e; Dimitrova 1989.

Dasineura crataegi (Winnertz, 1853) [Cecidomyia] - -; DW, E2, P1, B1, B3, S1, S211, V1, V4, O61, O62, T1, TL, R1, R2, RW, RE, BN; 0-1300 m; 1, 2, 3; e; Malkov 1904a; Buresch \& Lazarov 1956; Naidenov 1962; Dimitrova 1987, 1989; Skuhravá et al. 1991, 1992; Beschovski 2006.

Dasineura dryophila Rübsaamen, 1917 - BN; 10-100 m; 1; e; Skuhravá et al. 1991, 1992.

Dasineura filipendulae (Kieffer, 1909) - V4; 1200 m; 3; e; Dimitrova 1989.

Dasineura fraxinea Kieffer, 1907 - B1, V1, V4, R1, BN; 5-1350 m; 1, 2, 3; e; Dimitrova 1989; Skuhravá et al. 1991, 1992.

Dasineura fraxini (Bremi, 1847) - V4, BN; 0-1350 m; 1, 2, 3; ena; Dimitrova 1989; Skuhravá et al. 1991, 1992.

Dasineura galiicola (F. Löw, 1880) - TL, R2; 160-1150 m; 1, 2, 3; e; Naidenov 1962; Skuhravá et al. 1991, 1992.

Dasineura glechomae (Kieffer, 1889) - B1, TL, BN; 10-650 m; 1; e, i, h; Naidenov 1962; Skuhravá et al. 1991, 1992.

Dasineura gleditchiae (Osten Sacken, 1866) - V1, TL; 150-550 m; 1; h, i; Dimitrova \& Pencheva 2004; Tomov et al. 2009.

Dasineura harrisoni (Bagnall, 1922) - V4; 750-1250 m; 2, 3; e; Dimitrova 1989, 1990; Skuhravá et al. 1991, 1992.

Dasineura helianthemi (Hardy, 1850) [Contarinia] - B1, V4; 750-1200 m; 2, 3; ena; Dimitrova 1989; Skuhravá et al. 1991, 1992.

Dasineura hyperici (Bremi, 1847) - B1, V1, V4, S21, TL, R1, R2, BN; 50-2000 m; 1, 2, 3, 4; e; Naidenov 1962; Dimitrova 1989; Skuhravá et al. 1991, 1992.

Dasineura irregularis (Bremi, 1847) [D. acercrispans Kieffer, 1888] - V4, R1; 830-1200 m; 2, 3; e, ? wpo; Dimitrova 1989; Skuhravá et al. 1991, 1992.

Dasineura kellneri (Henschel, 1875) [D. laricis Löw, 1878] - V1, V4; 700-1430 m; 2, 3; wes; Naidenov 1962; Dimitrova 1989; Skuhravá et al. 1991, 1992.

Dasineura lamii (Kieffer, 1909) - V1; 650 m; 1; e; Skuhravá et al. 1991, 1992.

Dasineura lathyri (Kieffer, 1909) - S21, BN; 10-900 m; 1, 2; e; Skuhravá et al. 1991, 1992.

Dasineura lathyricola (Rübsaamen, 1890) - TL; 160 m; 1; wes; Naidenov 1962; Skuhravá et al. 1991, 1992.

Dasineura leguminicola (Lintner, 1879) - V4; 750-1100 m; 2, 3; ena, i, h; Dimitrova 1989.

Dasineura lithospermi (Loew, 1850) - BN; 30-40 m; 1; e; Skuhravá et al. 1991, 1992.

Dasineura lupulinae (Kieffer, 1891) - BN, 50 m; 1; e; Skuhravá et al. 1991, 1992.

Dasineura mali (Kieffer, 1904) - -; B1, V1, K9, TL, BN; 0-1000 m; 1, 2; hoes, i, hna; Grigorov 1962, 1972; Naidenov 1962; Skuhravá et al. 1991, 1992.

Dasineura medicaginis (Bremi, 1847) - B1, TL, R1, RW, BN; 50-900 m; 1, 2; wes; Naidenov 1962; Skuhravá et al. 1991, 1992; Beschovski 2006.

Dasineura napi (Loew, 1850) [D. brassicae (Winnertz, 1853)] - -; \$ DM; 100-150 m; 1; e; Malkov 1903; Buresch \& Lazarov 1956; Kircheva 1991; Skuhravá et al. 1991, 1992.

Dasineura oxyacanthae Rübsaamen, 1914 - V4; 750 m; 2; e; Naidenov 1962; Dimitrova 1989; Skuhravá et al. 1991, 1992.

Dasineura papaveris (Winnertz, 1890) - B1, O61, R1, RW, BN; 10-850 m; 1, 2; eswa; Naidenov 1962; Skuhravá et al. 1991, 1992; Beschovski 2006.

Dasineura plicatrix (Loew, 1850) - B1, V4, TL, O61, R1, R2; 10-1430 m; 1, 2, 3; ena, i, h; Naidenov 1962; Dimitrova 1989, 1990; Skuhravá et al. 1991, 1992.

Dasineura populeti (Rübsaamen, 1889) - BN; 40 m; 1; e; Skuhravá et al. 1991, 1992.

Dasineura potentillae (Wachtl, 1885) - R2; 1300 m; 3; e; Skuhravá et al. 1991, 1992.

Dasineura pteridicola (Kieffer, 1901) - V4, R1; 850-880 m; 2; e; Dimitrova 1989; Skuhravá et al. 1991, 1992. 
Dasineura pteridis (Müller, 1871) [D. filicina (Kieffer, 1889)] - B1, K8, V4, R2; 550-1800 m; 1, 2, 3, 4; des, ? dp; Naidenov 1962; Dimitrova 1987, 1989; Skuhravá et al. 1991, 1992.

Dasineura pustulans (Rübsaamen, 1889) - V4; 750-1430 m; 2, 3; e; Dimitrova 1989, 1990; Skuhravá et al. 1991, 1992.

Dasineura pyri (Bouché, 1847) [Perrisia] - -; E1, E2, P1, V1, K9, TL, O61, R1, RW, BN, BS; 10-1000 m; 1, 2; e, i, ha; Malkov 1902; Tschorbadjiew 1939a; Lazarov 1949a; Buresch \& Lazarov 1956; Popoff 1956; Kovachevski et al. 1959; Naidenov 1962; Harizanov 1964; Grigorov 1972; Skuhravá et al. 1991, 1992; Beschovski 2006.

Dasineura ranunculi (Bremi, 1847) - V4; 750-800 m; 2; des; Dimitrova 1987, 1989; Skuhravá et al. 1991, 1992.

Dasineura ribis Barnes, 1940 - - ; ; e; Grigorov 1972; Ivanov 1981; Skuhravá et al. 1991, 1992.

Dasineura rosae (Bremi, 1847) [Wachtliella rosarium (Hardy, 1850)] - B1, V4, S21, TL, O61, R1, R2, RW; 10-1770 m; 1, 2, 3, 4; des, ? h; Naidenov 1962; Dimitrova 1987, 1989; Skuhravá et al. 1991, 1992; Beschovski 2006.

Dasineura rossi Rübsaamen, 1914 - R1; 850 m; 2; wes; Skuhravá et al. 1991, 1992.

Dasineura rubella Kieffer, 1896 - B1, V1, V4, BN; 10-850 m; 1, 2; e; Dimitrova 1989; Skuhravá et al. 1991, 1992.

Dasineura ruebsaameni (Kieffer, 1909) - B1, V4, S21, BN; 10-1200 m; 1, 2, 3; e; Dimitrova 1989; Skuhravá et al. 1991, 1992.

Dasineura schulzei Rübsaamen, 1917 - B1, V4, R1, R2; 500-1600 m; 1, 2, 3, 4; e; Dimitrova 1989; Skuhravá et al. 1991, 1992.

Dasineura serotina (Winnertz, 1853) - BN; 5-50 m; 1; e; Skuhravá et al. 1991, 1992.

Dasineura sisymbrii (Schrank, 1803) - B1, V1, V4, TL, R2, RW, BN; 5-1000 m; 1, 2; e; Naidenov 1962; Dimitrova 1989; Skuhravá et al. 1991, 1992; Beschovski 2006.

Dasineura spadicea Rübsaamen, 1917 - TL; 160 m; 1; wes; Naidenov 1962; Skuhravá et al. 1991, 1992.

Dasineura symphyti (Rübsaamen, 1892) - BN; 30-40 m; 1; e; Skuhravá et al. 1991, 1992.

Dasineura szepligetii (Kieffer, 1909) [Oligitrophus] - P1, V1, V4, BN; 50-1000 m; 1, 2; csee; Naidenov 1962; Dimitrova 1989; Skuhravá et al. 1991, 1992; Beschovski 2006.

Dasineura tetensi (Rübsaamen, 1892) - e; Gospodinov 1968; Skuhravá et al. 1991, 1992.

Dasineura thomasiana (Kieffer, 1888) - B1, R2, BN; 10-980 m; 1, 2; e; Skuhravá et al. 1991, 1992.

Dasineura tiliae (Schrank, 1803) [D. tiliamvolvens (Rübsaamen 1889)] - V4, BN; 50-1550 m; 1, 2, 3; des, ? dp; Naidenov 1962; Dimitrova 1989; Skuhravá et al. 1991, 1992.

Dasineura tortilis (Bremi, 1847) [D. alni (F. Löw, 1877)] - B1, R2; 550-1100 m; 1, 2, 3; e; Skuhravá et al. 1991, 1992.

Dasineura tortrix (F. Löw, 1877) - B1, K4, V1, V4, O61, R1, R2, RW, BN; 5-1300 m; 1, 2, 3; e; Naidenov 1962; Dimitrova 1989; Skuhravá et al. 1991, 1992; Beschovski 2006.

Dasineura trifolii (F. Löw, 1874) - B1, V1, V4, TL, R2, RW, BN; 50-1550 m; 1, 2, 3; wp, i, h; Naidenov 1962; Dimitrova 1989; Skuhravá et al. 1991, 1992; Beschovski 2006.

Dasineura tubularis (Kieffer, 1909) - B1, V4, BN; 30-1030 m; 1, 2, 3; cse; Dimitrova 1989; Skuhravá et al. 1991, 1992.

Dasineura tympani (Kieffer, 1909) - B1, V4, S21, BN; 10-1350 m; 1, 2, 3; e; Dimitrova 1987, 1989; Skuhravá et al. 1991, 1992.

Dasineura ulmaria (Bremi, 1847) - V4, R2; 1000-1430 m; 3; des; Naidenov 1962; Dimitrova 1987, 1989; Skuhravá et al. 1991, 1992.

Dasineura urticae (Perris, 1840) - B1, V1, V4, S21, TL, R1, R2, RW, BN; 10-1650 m; 1, 2, 3, 4; des; Naidenov 1962; Dimitrova 1987, 1989; Skuhravá et al. 1991, 1992.

Dasineura viciae (Kieffer, 1888) - B1, V1, V4, S21, TL, O61, R1, RW, BN; 5-1650 m; 1, 2, 3, 4; des, ? dp, tp; Naidenov 1962; Dimitrova 1987, 1989; Skuhravá et al. 1991, 1992; Beschovski 2006.

Rabdophaga clavifex (Kieffer, 1891) [Dasineura] - B1, V4; 950-1400 m; 2, 3; dp; Dimitrova 1989; Skuhravá et al. 1991, 1992; Georgiev et al. 2004.

Rabdophaga heterobia (Loew, 1850) [Dasineura] - B1, V4, R1, R2; 550-1300 m; 1, 2, 3; dp; Dimitrova 1989, 1990; Skuhravá et al. 1991, 1992.

Rabdophaga jaapi Rübsaamen, 1916 [Dasineura repentis Skuhravá, 1986] - V4, R1; 1400 m; 3; e; Naidenov 1962; Dimitrova 1989; Skuhravá et al. 1991, 1992.

Rabdophaga marginemtorquens (Bremi, 1847) [Dasineura] - V1, TL; 150-550 m; 1; dp, ? des; Naidenov 1962; Skuhravá et al. 1991, 1992.

Rabdophaga pierrei (Kieffer, 1896) [Dasineura] - V1; 550 m; 1; e; Naidenov 1962; Skuhravá et al. 1991, 1992. 
Rabdophaga rosaria (Loew, 1850) [Dasineura] - V4, S1, TL, BN; 10-1810 m; 1, 2, 3, 4; e, ? tp, h; Naidenov 1962; Dimitrova 1987, 1989; Skuhravá et al. 1991, 1992.

Rabdophaga salicis (Schrank, 1803) [Dasineura; R. karschi (Kieffer, 1891)] - V1, V4, TL, R2; 150-1400 m; 1, 2, 3; h; Naidenov 1962; Dimitrova 1987, 1989; Skuhravá et al. 1991, 1992.

Rabdophaga saliciperda (Dufour, 1841) [Dasineura] - -; B1, V1, V4, TK, TL, R2; 150-1300 m; 1, 2, 3; dp, ? tp; Malkov 1904b, 1906, 1907; Buresch \& Lazarov 1956; Naidenov 1962, 1963; Dimitrova 1987, 1989; Skuhravá et al. 1991, 1992; Georgiev \& Stojanova 2003.

Rabdophaga terminalis (Loew, 1850) [Dasineura] - B1, V4, R2, RW, BN; 10-1300 m; 1, 2, 3; dp, ? tp; Dimitrova 1987, 1989; Skuhravá et al. 1991, 1992; Beschovski 2006.

Didymomyia tiliacea (Bremi, 1847) [D. reaumuriana (Löw, 1878)] - V4, TL, RW, BN; 10-1350 m; 1, 2, 3; des, ? dp; Naidenov 1962; Dimitrova 1987, 1989; Skuhravá et al. 1991, 1992; Beschovski 2006.

Dryomyia circinans (Giraud, 1861) - ; ; DW, E2, B3, K9, V4, V5, S211, T31, R2, BN, BS; 10-1000 m; 1, 2; nmsfe, dp; Drensky 1955; Naidenov 1962, 1963; Zlatanov 1971; Dimitrova 1987, 1989; Skuhravá et al. 1991, 1992.

Dryomyia lichtensteinii (F. Löw, 1878) - -; ; 10-1000 m; 1, 2; sena; Zlatanov 1971; Skuhravá et al. 1991, 1992.

Euphorbomyia loewii (Mik, 1882) - E2, TL, O61, R1; 100-400 m; 1; cset; Naidenov 1962; Skuhravá et al. 1991, 1992.

Geocrypta braueri (Handlirsch, 1884) - BN; 40-50 m; 1; e; Skuhravá et al. 1991, 1992.

Geocrypta galii (Loew, 1850) - B1, K8, V1, V4, TL, R1, R2, BN; 10-2000 m; 1, 2, 3, 4; dp; Naidenov 1962; Dimitrova 1987, 1989; Skuhravá et al. 1991, 1992.

Gephyraulus raphanistri (Kieffer, 1886) - R1; 800 m; 2; e; Skuhravá et al. 1991, 1992.

Giraudiella inclusa (Frauenfeld, 1862) - BN; 10-100 m; 1; ? wcp; Skuhravá et al. 1991, 1992.

Acericecis vitrina (Kieffer, 1909) [Harrisomyia] - B1, V4; 550-1450 m; 1, 2, 3; e, ? cse; Dimitrova 1989, 1990; Skuhravá et al. 1991, 1992.

Hartigiola annulipes (Hartig, 1839) [Phegobia tornatella (Bremi, 1847)] - E1, B3, V1, V4, K4, S211, R1, RW; 250-1400 m; 1, 2, 3; ean, ? dp; Naidenov 1962; Dimitrova 1987, 1989; Skuhravá et al. 1991, 1992; Beschovski 2006.

Iteomyia capreae (Winnertz, 1853) - B1, V1, V4, R2; 550-1650 m; 1, 2, 3, 4; tp; Naidenov 1962; Dimitrova 1987, 1989; Skuhravá et al. 1991, 1992.

Jaapiella bryoniae (Bouché, 1847) - V1, R2, RW; 650-1000 m; 1, 2; ena; Skuhravá et al. 1991, 1992.

Jaapiella cirsiicola Rübsaamen, 1916 - BN; 10-30 m; 1; ewca; Skuhravá et al. 1991, 1992.

Jaapiella cucubali (Kieffer, 1909) - TL, R2, BN; 10-850 m; 1, 2; cse; Skuhravá et al. 1991, 1992.

Jaapiella floriperda (F. Löw, 1888) - B1, V4, BN; 50-1080 m; 1, 2, 3; e, ? ewca; Dimitrova 1989, 1990; Skuhravá et al. 1991, 1992.

Jaapiella genisticola (F. Löw, 1877) - ; wes; Naidenov 1962; Skuhravá et al. 1991, 1992.

Jaapiella hedickei Rübsaamen, 1921 - BN; 10-100 m; 1; e; Skuhravá et al. 1991, 1992.

Jaapiella jaapiana (Rübsaamen, 1914) - O61, R1; 350-400 m; 1; e; Skuhravá et al. 1991, 1992.

Jaapiella moraviae (Wachtl, 1883) - E2; 100 m; 1; csee; Skuhravá et al. 1991, 1992.

Jaapiella rubicundula (Rübsaamen, 1891) - V4; 1150 m; 3; e; Dimitrova 1989, 1990; Skuhravá et al. 1991, 1992.

Jaapiella schmidti (Rübsaamen, 1912) - B1, BN; 10-500 m; 1; e; Skuhravá et al. 1991, 1992.

Jaapiella veronicae (Vallot, 1827) - B1, V1, V4, S21, TL, R1, R2; 160-1550 m; 1, 2, 3; e; Naidenov 1962; Dimitrova 1989; Skuhravá et al. 1991, 1992.

Jaapiella viscariae (Kieffer, 1886) - E2; 100 m; 1; ? csee; Skuhravá et al. 1991, 1992.

Fabomyia medicaginis (Rübsaamen, 1912) [Jaapiella] - -; \$; V1, V4, TL, R2; 150-1120 m; 1, 2, 3; west, ? wes; Naidenov 1962; Grigorov 1972, 1976; Dimitrova 1989, 1990; Skuhravá et al. 1991, 1992.

Janetia cerris (Kollar, 1850) [Arnoldia] - DW, B1, V1, V4, RW; BN; 10-1030 m; 1, 2; dp; Naidenov 1962, 1963; Zlatanov 1971; Dimitrova 1987, 1989; Skuhravá et al. 1991, 1992; Beschovski 2006.

Janetia homocera (F. Löw, 1877) - DW, B1, BN, BS; 5-1050 m; 1, 2; cse; Naidenov 1962; Dimitrova 1989; Skuhravá et al. 1991, 1992.

Janetia nervicola (Kieffer, 1909) - B1, V4, BN; 100-750 m; 1, 2; csee; Dimitrova 1989; Skuhravá et al. 1991, 1992. Janetia pustularis (Kieffer, 1909) - V1, BN; 10-550 m; 1; csee; Skuhravá et al. 1991, 1992.

Janetia szepligetii Kieffer, 1896 - B1, V4, BN; 10-850 m; 1, 2; csean; Dimitrova 1989; Skuhravá et al. 1991, 1992. Janetiella fallax Kieffer, 1904 - E1, R1; 100-850 m; 1, 2; csee, ? e; Naidenov 1962; Skuhravá et al. 1991, 1992. 
Janetiella lemeei (Kieffer, 1904) - B1, R1, RW, BN; 10-600 m; 1; ean; Naidenov 1962; Skuhravá et al. 1991, 1992; Beschovski 2006.

Janetiella oenephila (Haimhoffen, 1875) [Cecidomyia, Vitisiella] - -; d dp; Dospevski 1908a; Buresch \& Lazarov 1956; Skuhravá et al. 1991, 1992.

Janetiella thymi (Kieffer, 1888) - V4, R2; 1100-1550 m; 3; e; Dimitrova 1989; Skuhravá et al. 1991, 1992.

Kaltenbachiola strobi (Winnertz, 1853) - -; RW; 1450-1900 m; 3, 4; e, ? bm; Tschorbadjiew 1928a, 1928b; Drensky 1928; Buresch \& Lazarov 1956; Skuhravá et al. 1991, 1992; Beschovski 2006.

Lathyromyza schlechtendali (Kieffer, 1886) - TL; 150-170; 1; ewca; Naidenov 1962; Skuhravá et al. 1991, 1992. Macrolabis heraclei Kaltenbach, 1862 - R1, R2, BN; 5-1140 m; 1, 2, 3; e; Skuhravá et al. 1991, 1992.

Macrolabis hieracii Rübsaamen, 1917 - B1, BN; 50-550 m; 1; e; Skuhravá et al. 1991, 1992.

Macrolabis lamii Rubsaamen, 1916 - B1, R2, BN; 10-980 m; 1, 2; e; Dimitrova 1989; Skuhravá et al. 1991, 1992. Macrolabis podagrariae (Loew, 1850) - V4; 750-950 m; 2, 3; e; Dimitrova 1987, 1989; Skuhravá et al. 1991, 1992. Macrolabis rhodophila (Hardy, 1850) [M. luceti Kieffer, 1898] - V4; 800-1810 m; 2, 3; e; Dimitrova 1987, 1989; Skuhravá et al. 1991, 1992.

Macrolabis ruebsaameni Hedicke, 1938 - V1; 550-650 m; 1, 2; e; Skuhravá et al. 1991, 1992.

Macrolabis stellariae (Liebel, 1889) - R2; 890 m; 2; e; Skuhravá et al. 1991, 1992.

Obolodiplosis robiniae (Haldeman, 1847) - ; 0-1000 m, 1, 2; h, i; Tomov \& Dimitrov 2009.

Mayetiola destructor (Say, 1817) - - ; DW, DM, E1, P1, V1, S1, T2, TL, BN; 0-1000 m; 1, 2; wp, i, ha; Malkov 1902b; Hitilov 1912a, 1912b; Drenowsky 1922a; Drenowsky \& Enderlein 1923; Tschorbadjiew 1925a, 1925c, 1926b, 1927, 1929a, 1932 1939a; Petkoff 1939; Lazarov 1935a, 1939, Buresch \& Lazarov 1956; Lyubenov 1956, 1960; Popoff 1956; Zamfirov 1958, 1961a; 1962a, 1962b, 1962c, 1963a; Makarov 1959; Kovachevski et al. 1959; Naidenov 1963; Grigorov 1972, 1976; Kontev et al. 1991; Skuhravá et al. 1991, 1992; Harizanov et al. 1996.

Mayetiola lanceolatae (Rübsaamen, 1895) - BN; 10-100 m; 1; e; Skuhravá et al. 1991, 1992.

Mayetiola graminis (Fourcroy, 1785) [M. poae (Bosc, 1817); Poomyia] - B1, V4, R1, RW; 600-1520 m; 2, 3; e; Naidenov 1963; Dimitrova 1987, 1989; Skuhravá et al. 1991, 1992; Beschovski 2006.

Mikomya coryli (Kieffer, 1901) - B1, V1, V4, S21, R1, BN; 10-1680 m; 1, 2, 3, 4; ean; Dimitrova 1989, 1990; Skuhravá et al. 1991, 1992.

Neomikiella beckiana (Mik, 1885) - BN; 5-30 m; 1; e; Skuhravá et al. 1991, 1992.

Oligotrophus juniperinus (Linnaeus, 1758) - V4, R1, R2, RW; 750-2500 m; 2, 3, 4, 5; e; Naidenov 1962; Dimitrova 1987, 1989; Skuhravá et al. 1991, 1992; Beschovski 2006.

Oligotrophus panteli Kieffer, 1898 - V4, R1, R2, RW; 350-1450 m; 1, 2, 3; ena; Naidenov 1962; Dimitrova 1989, 1990; Skuhravá et al. 1991, 1992; Beschovski 2006.

Phegomyia fagicola (Kieffer, 1901) - V1, V4, R2; 650-1200 m; 2, 3; e; Naidenov 1962; Dimitrova 1989; Skuhravá et al. 1991, 1992.

Physemocecis hartigi (Liebel, 1892) - V4, TL, BN; 180-1550 m; 1, 2, 3; ean; Naidenov 1962; Dimitrova 1987, 1989; Skuhravá et al. 1991, 1992.

Physemocecis ulmi (Kieffer, 1909) - B1, V1, V4, TL, R1, BN; 0-1150 m; 1, 2, 3; e; Dimitrova 1989; Skuhravá et al. 1991, 1992.

Rhopalomyia artemisiae (Bouché, 1834) - B1; 450-650 m; 1, 2; des; Skuhravá et al. 1991, 1992.

Rhopalomyia foliorum (Loew, 1850) - RW; 900 m; 2; des; Skuhravá et al. 1991, 1992; Beschovski 2006.

Rondaniola bursaria (Bremi, 1847) - B1, V1, TL, BN; 10-700 m; 1, 2; e; Skuhravá et al. 1991, 1992.

Sackenomyia reaumurii (Bremi, 1847) - K8, K9, V4, BN; 10-1400 m; 1, 2, 3; e; Naidenov 1962; Dimitrova 1989; Skuhravá et al. 1991, 1992.

Semudobia betulae (Winnertz, 1853) - B1, V1, V4, TL, R2, BN; 10-1450 m; 1, 2, 3; tp, i, h; Minchev \& Mincheva 1967; Dimitrova 1989; Skuhravá et al. 1991, 1992; Pelov 1999.

Semudobia skuhravae Roskam, 1977 - B1, V1, V4, R2; 500-1450 m; 1, 2, 3; h; Dimitrova 1989; Skuhravá et al. 1991, 1992.

Semudobia tarda Roskam, 1977 - V4; 830-1550 m; 2, 3; dp, i, h; Dimitrova 1989.

Wachtliella ericina (F. Löw, 1885) - B1; 600-650 m; 1, 2; ena; Skuhravá et al. 1991, 1992.

Wachtliella niebleri Rübsaamen, 1916 - V4, BN; 10-2000 m; 1, 2, 3, 4; wes; Dimitrova 1989; Skuhravá et al. 1991, 1992.

Wachtliella persicariae (Linnaeus, 1767) - TL; 150-170 m; 1; wp; Naidenov 1962; Skuhravá et al. 1991, 1992. 
Wachtliella stachydis (Bremi, 1847) - R1, R2; 1100-1150 m; 3; e; Skuhravá et al. 1991, 1992.

Zygiobia carpini (F. Löw, 1874) - B1, V4, S21, R1, R2, BN; 10-1200 m; 1, 2, 3; e; Dimitrova 1989; Skuhravá et al. 1991, 1992.

Mikiola fagi (Hartig, 1839) [Cecidimyia] - -; \$ SB, V4, S2, R1, R2, RR; 750-1900 m; 3; e, ? des; Drensky 1928; Russkoff 1928; Dimitrov 1934; Buresch \& Lazarov 1956; Naidenov 1962, 1963; Dimitrova 1987, 1989; Skuhravá et al. 1991, 1992; Beschovski 2006.

Mikiola orientalis Kieffer, 1908 - V4, T31, R1, RW; 100-200 m, 800-1400 m; 1, 3; ban; Naidenov 1962; Dimitrova 1989; Skuhravá et al. 1991, 1992; Beschovski 2006.

Kiefferia pericarpiicola (Bremi, 1847) [K. pimpinellae (F. Löw, 1874)] - V4, S21, TL; 160-1200 m; 1, 2, 3; des; Dimitrova 1989, 1990; Skuhravá et al. 1991, 1992.

Asphondylia baudysi Vimmer, 1937 - V4, BN; 10-1400 m; 1, 2, 3; e; Dimitrova 1989, 1990; Skuhravá et al. 1991, 1992.

Asphondylia calaminthae Kieffer, 1909 - B1; 600 m; 1; e; Skuhravá et al. 1991, 1992.

Asphondylia cytisi Frauenfeld, 1873 - R2, BN; 10-1300 m; 1, 2, 3; wes; Skuhravá et al. 1991, 1992.

Asphondylia dorycnii (Müller, 1870) - B1, BN; 10-600 m; 1; e; Skuhravá et al. 1991, 1992.

Asphondylia echii (Loew, 1850) - BN; 10-100 m; 1; cse; Skuhravá et al. 1991, 1992.

Asphondylia genistae (Loew, 1850) - BN; 10-100 m; 1; e; Skuhravá et al. 1991, 1992.

Asphondylia hornigi Wachtl, 1880 - R1, RW; 370-800 m; 1, 2; cee; Naidenov 1962; Skuhravá et al. 1991, 1992; Beschovski 2006.

Asphondylia massalongoi Rübsaamen, 1893 - B1; 600 m; 1; cse; Skuhravá et al. 1991, 1992.

Asphondylia melanopus Kieffer, 1890 - -; e; Donchev 1969; Skuhravá et al. 1991, 1992.

Asphondylia menthae Kieffer, 1902 - B1, V1, TL; 160-600 m; 1; cse, ? e; Naidenov 1962; Skuhravá et al. 1991, 1992.

Asphondylia miki Wachtl, 1880 - -; DM, V4; 150-1100 m; 1, 2, 3; wes; Donchev 1968; Grigorov 1972; Dimitrova 1989, 1990; Skuhravá et al. 1991, 1992.

Asphondylia ononidis F. Löw, 1873 - B1, V4; 600-800 m; 1, 2; ena; Dimitrova 1989; Skuhravá et al. 1991, 1992.

Asphondylia pruniperda Rondani, 1867 [A. prunorum Wachtl, 1880; Ischnonyx] - -; \$; V1, BN; 10-100 m; 1; e; Malkov 1906; Lazarov 1949a; Buresch \& Lazarov 1956; Nachev 1964; Grigorov 1972; Skuhravá et al. 1991, 1992.

Asphondylia scrophulariae Schiner, 1856 - B1, V4; 600-1020 m; 1, 2, 3; cse; Dimitrova 1989; Skuhravá et al. 1991, 1992.

Asphondylia verbasci (Vallot, 1827) - E2, B1, V4, BN; 10-2000 m; 1, 2, 3, 4, 5; ena; Dimitrova 1989, 1990; Skuhravá et al. 1991, 1992.

Placochela nigripes (F. Löw, 1877) [P. ligustri (Rübsaamen, 1899)] - B1, V1, V4, BN; 5-980 m; 1, 2; e; Dimitrova 1989; Skuhravá et al. 1991, 1992.

Schizomyia galiorum Kieffer, 1889 - V4, BN; 5-1350 m; 1, 2, 3; wp; Dimitrova 1989, 1990; Skuhravá et al. 1991, 1992.

Kochiomyia kochiae (Kieffer, 1909) - e; Skuhravá et al. 1991, 1992.

Feltiella acarisuga (Vallot, 1827) [Acaroletes tetranychi Kieffer 1908; Arthrocnodax] - TL; 160-180 m; 1; hoa; Naidenov 1962; Skuhravá et al. 1991.

Acodiplodis inulae (Loew, 1847) - B1, S21; 600-900 m; 1, 2; e; Skuhravá et al. 1991, 1992.

Ametrodiplosis auripes (H. Löw, 1888) - BN; 10-100 m; 1; e; Skuhravá et al. 1991, 1992.

Anabremia bellevoyei (Kieffer, 1896) - BN; 10-100 m; 1; e; Skuhravá et al. 1991, 1992.

Aphidoletes aphidimyza (Rondani, 1847) - V4; 1280 m; 3; hna; Grigorov 1982; Dimitrova 1989; Skuhravá et al. 1991.

Aschistonyx carpinicolus Rübsaamen, 1917 - B1, V4, R1, R2, BN; 5-1140 m; 1, 2, 3; e; Dimitrova 1989; Skuhravá et al. 1991, 1992.

Clinodiplosis cilicrus (Kieffer, 1889) - V1, V4, BN; 10-1500 m; 1, 2, 3, 4; wes; Dimitrova 1987, 1989; Skuhravá et al. 1991, 1992.

Contarinia anthobia (F. Löw, 1877) - R2; 1300 m; 3; e; Skuhravá et al. 1991, 1992.

Contarinia baeri (Prell, 1931) - R2; 850-1300 m; 2, 3; des, i, h; Skuhravá et al. 1991, 1992.

Contarinia barbichei (Kieffer, 1890) [C. barbichi Kieffer 1890] - B1, V4; 500-1550 m; 1, 2, 3; e; Dimitrova 1989; Skuhravá et al. 1991, 1992. 
Contarinia carpini Kieffer, 1897 - B1, V4, R1, BN; 10-1200 m; 1, 2, 3; e; Dimitrova 1989; Skuhravá et al. 1991, 1992.

Contarinia coryli (Kaltenbach, 1859) [C. corylina Löw 1878] - ; V1, V4, S21, R1; 500-1350 m; 1, 2, 3; des, ? esca; Naidenov 1962; Dimitrova 1989, 1990; Skuhravá et al. 1991, 1992.

Contarinia craccae Loew, 1850 - TL, R1; 160-420 m; 1; wes; Naidenov 1962; Skuhravá et al. 1991, 1992.

Contarinia fagi Rubsaamen, 1921 - B1, V4, R1, R2; 600-1350 m; 2, 3; e; Dimitrova 1989; Skuhravá et al. 1991, 1992.

Contarinia festucae Jones, 1940 - -; e; Lyubenov 1957; Skuhravá et al. 1991, 1992.

Contarinia floriperda Rübsaamen, 1917 - V4; 1150-1650 m; 3, 4; e; Dimitrova 1989, 1990; Skuhravá et al. 1991, 1992.

Contarinia gei Kieffer, 1909 [C. geicola Rübsaamen, 1917] - V1, V4, R1, BN; 5-1200 m; 1, 2, 3; e; Dimitrova 1989; Skuhravá et al. 1991, 1992.

Contarinia hypochoeridis (Rübsaamen, 1891) - V4, RW; 850-1350 m; 2, 3; e; Dimitrova 1989; Skuhravá et al. 1991, 1992; Beschovski 2006.

Contarinia jacobaeae (Loew, 1850) - BN; 5-30 m; 1; e; Skuhravá et al. 1991, 1992.

Contarinia lamii Kieffer, 1909 - V1, V4, R1; 600-1580 m; 2, 3, 4; cse; Dimitrova 1987, 1989; Skuhravá et al. 1991, 1992.

Contarinia lentis Aczél, 1944 [Diplosis pisi (Winnertz, 1854)] - -; \$; ? e; Malkov 1906; Skuhravá et al. 1991, 1992.

Contarinia loti (De Geer, 1776) - V4; 1500-1520 m; 4; e; Dimitrova 1987; Skuhravá et al. 1991, 1992.

Contarinia medicaginis Kieffer, 1895 - -; $\$$ V1, TL, BN; 10-600 m; 1, 2; ? h; Hristova, 1954; Popoff 1956; Hristova 1959; Kovachevski et al. 1959; Naidenov 1962; Donchev 1968; Grigorov 1972, 1976; Skuhravá et al. 1991, 1992; Harizanov et al. 1996.

Contarinia merceri Barnes, 1930 - -; e; Lyubenov 1957; Skuhravá et al. 1991, 1992.

Contarinia nasturtii (Kieffer, 1888) [C. torquens Meijere 1906] - -; DW, DM, P1, P2, B1, V1, V4, TL, R1, R2, RW; 100-1550 m; 1, 2, 3; ean, i, h; Nikolova 1943; Buresch \& Lazarov 1956; Popoff 1956; Popoff \& Nikolova 1958; Kovachevski et al. 1959; Naidenov 1963; Grigorov 1972, 1976; Dimitrova 1989; Skuhravá et al. 1991, 1992; Beschovski 2006.

Contarinia nicolayi (Rübsaamen, 1895) - BN; 10-100 m; 1; e; Skuhravá et al. 1991, 1992.

Contarinia onobrychidis Kieffer, 1895 - - ; wes; Donchev 1978; Skuhravá et al. 1991, 1992.

Contarinia petioli (Kieffer, 1898) - V4; 770-1650 m; 2, 3, 4; des; Naidenov 1962, 1963; Dimitrova 1987, 1989; Skuhravá et al. 1991, 1992.

Contarinia pisi (Loew, 1850) [Diplosis] - -; $\$$ E1, TL; 50-180 m; 1; wes; Malkov 1907; Buresch \& Lazarov 1956; Popoff 1956; Popoff \& Nikolova 1958; Grigorov 1972, 1976; Skuhravá et al. 1991, 1992; Harizanov et al. 1996.

Contarinia pyrivora (Riley, 1886) [Diplosis] - -; $\$$ V1, TL, RE; 150-650 m; 1, 2; e, i, ha; Malkov 1902c, 1906b; Vasilev 1931; Zhelev 1948b; Kovachevski et al. 1959; Lazarov 1949a; Buresch \& Lazarov 1956; Popoff 1956; Naidenov 1962; Sengalevich 1964; Grigorov 1972, 1976; Skuhravá et al. 1991, 1992.

Contarinia quercicola (Rübsaamen, 1899) - B1, V4, BN; 10-850 m; 1, 2; cse; Dimitrova 1989; Skuhravá et al. 1991, 1992.

Contarinia quercina (Rübsaamen, 1890) - B1, S21, BN; 5-900 m; 1, 2; e; Skuhravá et al. 1991, 1992.

Contarinia quinquenotata (F. Löw, 1888) - R2; 980 m; 2; e, i, ha; Skuhravá et al. 1991, 1992.

Contarinia scrophulariae Kieffer, 1896 - B1; 550 m; 1; e; Skuhravá et al. 1991, 1992.

Contarinia solani (Rübsaamen, 1892) - TL, BN; 10-180 m; 1; e; Skuhravá et al. 1991, 1992.

Contarinia steini (Karsch, 1881) - R1; 1140 m; 3; e; Skuhravá et al. 1991, 1992.

Contarinia subulifex Kieffer, 1897 - B1, BN; 20-700 m; 1, 2; cse; Skuhravá et al. 1991, 1992.

Contarinia tiliarum (Kieffer, 1890) - B1, V4; 650-1550 m; 2, 3; des, ? dp, ? h; Dimitrova 1987, 1989; Skuhravá et al. 1991, 1992.

Contarinia tragopogonis Kieffer, 1909 - BN; 30-40 m; 1; e; Skuhravá et al. 1991, 1992.

Contarinia tritici (Kirby, 1798) - -; DW, E2, P3, V1; 100-150 m; 1; ? h; Hitilov 1912b; Drenowsky 1922a; Tschorbadjiew 1939a; Buresch \& Lazarov 1956; Popoff 1956; Zamfirov 1961a; Grigorov 1972; Kontev et al. 1991; Skuhravá et al. 1991, 1992.

Contarinia vincetoxici Kieffer, 1909 - BN; 10-100 m; 1; e; Skuhravá et al. 1991, 1992.

Stenodiplosis geniculati Reuter, 1895 [Contarinia] - -; 1; e, i, ha; Lyubenov 1957; Skuhravá et al. 1991, 1992.

Stenodiplosis panici Plotnikov, 1926 [Contarinia] - DM; 120-200 m; 1; eet; Skuhravá et al. 1991, 1992. 
Diodaulus linariae (Winnertz, 1853) - BN; 10-100 m; 1; e; Skuhravá et al. 1991, 1992.

Drisina glutinosa Giard, 1893 - V1, V4, R1; 650-1450 m; 2, 3; e; Dimitrova 1989; Skuhravá et al. 1991, 1992.

Haplodiplosis marginata (von Roser, 1840) [H. equestris Wagner, 1871] - -; \$; DW, E2, P1, V1; 50-650 m; 1, 2; e; Popoff 1956; Makarov 1959; Kovachevski et al. 1959; Zamfirov 1961a; Grigorov 1972; Kontev et al. 1991; Skuhravá et al. 1991, 1992; Harizanov et al. 1996.

Harmandiola cavernosa (Rübsaamen, 1899) [Harmandia, Diplosis] - B1, V4, R1, RW; 600-1650 m; 2, 3, 4; ? wes, weswca; Naidenov 1962; Dimitrova 1987, 1989; Skuhravá et al. 1991, 1992; Beschovski 2006.

Harmandiola globuli (Rübsaamen, 1889) [Harmandia, Diplosis] - V4, R1; 770-1650 m; 2, 3, 4; ? wes, weswca; Naidenov 1962; Dimitrova 1987, 1989; Skuhravá et al. 1991, 1992.

Harmandiola tremulae (Winnertz, 1853) - V4, R1, RW; 770-2650 m; 2, 3, 4; wes, ? esca; Naidenov 1962; Dimitrova 1987, 1989; Skuhravá et al. 1991, 1992; Beschovski 2006.

Hygrodiplosis vaccinii (Kieffer, 1897) - V4; 1630-2290 m; 4, 5; e, bm; Dimitrova 1987, 1989, 1990; Skuhravá et al. 1991, 1992.

Macrodiplosis pustularis (Bremi, 1847) [M. dryobia Löw 1877] - B1, V1, V4, BN; 5-1000 m; 1, 2; ? et; Naidenov 1962; Dimitrova 1987, 1989; Skuhravá et al. 1991, 1992.

Macrodiplosis roboris (Hardy, 1854) [M. volvens Kieffer 1895] - B1, V1, TL, R1, BN; 5-1000 m; 1, 2; wesant; Naidenov 1962; Dimitrova 1989, 1990; Skuhravá et al. 1991, 1992.

Monarthropalpus flavus (Schrank, 1776) [buxi Laboulbène 1873] - BN; 10-100 m; 1; ean, i, h; Skuhravá et al. 1991, 1992.

Monodiplosis liebeli (Kieffer, 1889) - BN; 10-100 m; 1; e; Skuhravá et al. 1991, 1992.

Mycodiplosis coniophaga (Winnertz, 1853) - R1, R2; 1140-1300 m; 3; h; Skuhravá et al. 1991, 1992.

Mycodiplosis gymnosporangii Kieffer, 1904 - V4; 980-1080 m; 2, 3; se; Dimitrova 1989.

Mycodiplosis melampsorae (Rübsaamen, 1889) - V4; 1400 m; 3; e; Dimitrova 1989; Skuhravá et al. 1991, 1992.

Mycodiplosis saundersi Barnes, 1927 - R2, BN; 10-1000 m; 1, 2; e; Skuhravá et al. 1991, 1992.

Pterepidosis varimezovi Mamaev \& Dimitrova, 1998 - V4; 1600 m; 4; Ebg; Mamaev \& Dimitrova 1998.

Parallelodiplosis galliperda (F. Löw, 1889) - V4; 950; 2; e; Dimitrova 1989.

Putoniella pruni (Kaltenbach, 1872) [P. marsupialis Löw 1889] - -; E1, P1, B1, B2, V1, V4, K9, S1, R1, R2, RW; 50-1320 m; 1, 2, 3; e; Tschorbadjiew 1924a, 1925a, 1925b, 1925c, 1925d, 1926b, 1927, 1929a, 1930a, 1933; Lazarov 1949a; Buresch \& Lazarov 1956; Kovachevski et al. 1959; Lazarov et al. 1960, 1965; Grigorov 1972; Dimitrova 1987, 1989; Skuhravá et al. 1991, 1992; Beschovski 2006.

Resseliella ribis (Marikovskij, 1956) - \$; e; Ivanov 1981; Skuhravá et al. 1991, 1992.

Resseliella theobaldi (Barnes, 1927) [Thomasiniana] - -; B2, S21; \$; e; Lazarov et al. 1965; Stoyanov 1957; Skuhravá et al. 1991, 1992.

Sitodiplosis mosellana (Gehin, 1857) [Stenodiplosis] - -; $\$$; DW, E2; 100-225 m; 1, 2; tp, i, h; Popoff 1956; Makarov 1959; Zamfirov 1961a; Grigorov 1972; Kontev et al. 1991; Skuhravá et al. 1991, 1992.

Thecodiplosis brachyntera (Schwägrichen, 1835) [Cecidomyia] - -; V1, V4, S21, RW; 600-1215 m; 2, 3, 4; e; Dimitrov 1935; Buresch \& Lazarov 1956; Naidenov 1962; Ganchev 1981; Dimitrova 1989; Skuhravá et al. 1991, 1992; Mirchev 1993; Beschovski 2006.

Tricholaba trifolii Rübsaamen, 1917 - B1, V4; 600-1450 m; 2, 3; wes; Dimitrova 1989; Skuhravá et al. 1991, 1992.

Zeuxidiplosis giardi (Kieffer, 1896) - B1, V4, RW; 400-1550 m; 1, 2, 3, 4; e, i, hata; Naidenov 1962; Dimitrova 1989; Skuhravá et al. 1991, 1992; Beschovski 2006.

\section{Psychodidae}

Phlebotomus (Phlebotomus) papatasi (Scopoli, 1786) - $\mathbf{\Delta}$; DM, E1, E2, S1, TL,T1, O5, O62, RW, RE, BN, BS; 0-1400 m; 1, 2, 3; ppt; Chichkoff \& Konsuloff 1914; Drensky 1926, 1931, 1942, 1955; Drensky \& Drensky 1928; Kitanov 1943; Ganov 1949; Boychev 1950; Ježek et al. 2017, 2018, 2020.

Phlebotomus (Paraphlebotomus) alexandri Sinton, 1928 - sppt; Ježek et al. 2018.

Phlebotomus (Paraphlebotomus) caucasicus Marzinovsky, 1917 - seeca; Ježek et al. 2018.

Phlebotomus (Paraphlebotomus) sergenti Parrot, 1917 [Ph. sergenti simils Perfilev, 1963] - А; BS; 0-30 m; 1; sppt; Drensky \& Drensky 1928; Boychev 1950; Ježek et al. 2018, 2020.

Phlebotomus (Larroussius) perniciosus Newstead, 1911 - А ; BS; 0-30 m; 1; cseanna; Drensky \& Drensky 1928; Boychev 1950; Ježek et al. 2018, 2020. 
Phlebotomus (Adlerius) balcanicus Theodor, 1958 [Ph. chinensis Newstead, 1916] - $\mathbf{\Delta}$; DW; 30-40 m; 1; seei; Boychev 1950; Wagner 1990; Ježek et al. 2017, 2018, 2020.

Sergentomyia (Sergentomyia) minuta (Rondani, 1843) [Phlebotomus] - $\Delta$; S1, TL, BS; 0-350 m; 1; sena, ? om; Nedelkov 1909, 1912; Drensky \& Drensky 1928; Boychev 1950; Ježek et al. 2020.

Sycorax bicornua Krek, 1970 - P2, B1; 500-732 m; 1, 2; e; Ježek et al. 2020.

Sycorax popovi Ježek 1990 - O62; 250-350 m; 1; csee; Ježek 1990b; Ježek \& Goutner 1995; Ježek et al. 2020.

Sycorax silacea Haliday in Curtis, 1839 - B1; 710 m; 2; e; Ježek et al. 2020.

Sycorax tonnoiri Jung, 1954 - P2, B1; 710-827 m; 2; csee; Ježek 2004; Ježek et al. 2020.

Sycorax trifida Krek, 1970 - B3; 614 m; 1; Eb; Ježek et al. 2020.

Trichomyia kostovi Ježek, 1990 - O62; 250-350 m; 1; Ebg; Ježek 1990b; Wagner 2013; Ježek et al. 2020.

Berdeniella illiesi (Wagner, 1973) - B2, R1; 830-1200 m; 1, 2, 3; e, ? csee; Wagner \& Joost 1988; Wagner 2013, 2018; Ježek et al 2019, 2020.

Berdeniella kocii Ježek, 2006 - R1, R2, RW; 587-1800 m; 1, 2, 3, 4; csee; Ježek et al. 2020.

Berdeniella manicata (Tonnoir, 1919) [Pericoma] - V4, T31, R1, R2, RW; 150-1500 m; 1, 2, 3; cse; Arndt 1943; Wagner \& Joost 1988; Wagner 2013, 2018; Ježek et al 2019, 2020.

Berdeniella unispinosa (Tonnoir, 1919) - P1, B1, B2, B3, V4, S22, O62, R1, R2, RW; 200-2460 m; 1, 2, 3, 4, 5; e; Wagner \& Joost 1988; Wagner 2013, 2018; Ježek et al. 2020.

Berdeniella vimmeri Jezek, 1995 - RW; 1134 m; 3; csee; Ježek et al. 2020.

Saraiella carpatica Vaillant, 1981 - RW; 1300-1550 m; 3, 4; see; Wagner \& Joost 1988; Wagner 2013, 2018; Ježek et al. 2020.

Saraiella rotunda (Krek, 1970) - R1, R2; 900-1800 m; 2, 3, 4; e; Ježek et al. 2020.

Ulomyia bulgarica Wagner \& Joost, 1988 - B1, RW; 710-1300 m; 2, 3, 4; Ebg; Wagner \& Joost 1988; Wagner 2013.

Ulomyia fuliginosa (Meigen, 1818) - V1; 580-600 m; 1, 2; e; Nedelkov 1912.

Satchelliella canescens (Meigen, 1818) [Pneumia] - P2, B2, B3, R1, RW; 300-1134 m; 1, 2, 3; esanca; Ježek et al. 2020.

Satchelliella crispi (Freeman, 1953) [Pneumia] - R1, R2, RW; 900-1060 m; 2, 3; e; Ježek et al. 2020.

Satchelliella gracilis (Eaton, 1893) [Pneumia] - R1, RW; 590-980 m; 1, 2; e; Ježek et al. 2020.

Satchelliella mladeni (Ježek1 \& Oboňa, 2019) [Pneumia] - V4, R1, R2; 1800-2430 m; 4, 5; Ebg; Ježek1 \& Oboňa 2019; Ježek et al. 2020.

Satchelliella nubila (Meigen, 1818) [Pneumia] - P2, B1, B2, B3, S1, S22, T31, O62, R1, R2, RW; 200-2045 m; 1, 2, 3, 4; e; Wagner \& Joost 1988; Ježek \& Goutner 1995; Wagner 2013, 2018; Ježek et al. 2017, 2018, 2019, 2020.

Satchelliella palustris (Meigen, 1818) [Pneumia] - B1; $710 \mathrm{~m}$; 2; ean; Ježek et al. 2020.

Satchelliella pilularia (Tonnoir, 1940) [Pneumia] - S22, R1; 390-650 m; 1; ena, ? wp; Wagner \& Joost 1988; Ježek \& Goutner 1995; Wagner 2013, 2018; Ježek et al. 2020.

Satchelliella stammeri (Jung, 1954) [Pneumia] - P2, B2; 550 m; 1; des; Ježek et al. 2020.

Satchelliella trivialis (Eaton, 1893) [Pneumia] - P2, B1, B2; 550-810 m; 1, 2; e; Ježek et al. 2020.

Pericoma (Pericoma) bosnica Krek, 1967 - T31, RW; 200-1060 m; 1, 2, 3; bc; Wagner \& Joost 1988; Wagner 2013, 2018; Ježek et al. 2018, 2020.

Pericoma (Pericoma) bunae Krek, 1979 - RW; 1054 m; 3; bc; Ježek et al. 2020.

Pericoma (Pericoma) exquisita Eaton, 1893 - P2, B1, B2, B3, K9, T31, O62; 100-830 m; 1, 2; ena; Wagner \& Joost 1988; Ježek \& Goutner 1995; Wagner 2013, 2018; Ježek et al. 2017, 2018, 2020.

Pericoma (Pericoma) kariana Vaillant, 1978 - R2; 582 m; 1; ban; Ježek et al. 2020.

Pericoma (Pericoma) motasi Vaillant, 1978 - T31, R1, RW; 200-1200 m; 1, 2, 3; see; Wagner \& Joost 1988; Wagner 1990, 2013, 2018; Ježek et al. 2020.

Pericoma (Pericoma) pannonica Szabó, 1960 - B3; 490 m; 1; cse; Ježek et al. 2020.

Pericoma (Pericoma) pingarestica Vaillant, 1978 - T31; 200 m; 1; see; Wagner \& Joost 1988; Wagner 2013, 2018; Ježek et al. 2020.

Pericoma (Pericoma) pseudoexquisita Tonnoir, 1940 - V4, T11, T31, RW; 200-1500 m; 1, 2, 3; ena; Arndt 1943; Wagner \& Joost 1988; Ježek \& Goutner 1995; Wagner 2013; Ježek et al. 2019, 2020.

Pericoma (Pachypericoma) blandula Eaton, 1893 - P1, P2, B1, B2, B3, T31, O62, R1, RW; 150-1130 m; 1, 2, 3; eanna; Wagner \& Joost 1988; Ježek \& Goutner 1995; Wagner 2013, 2018; Ježek et al. 2017, 2018, 2019, 2020.

Pericoma (Pachypericoma) fallax Eaton, 1893 - P2, B2, O62, R1, R2; 200-590 m; 1; wes; Ježek et al. 2020.

Pericoma (Pachypericoma) nielseni Kvifte 2010 - P2, B1, R2, RW; 450-980 m; 1, 2; e; Ježek et al. 2020. 
Clytocerus (Boreoclytocerus) longicorniculatus Krek, 1987 - P2, B2, R1; 460-960 m; 1, 2; e; Ježek et al. 2020. Clytocerus (Boreoclytocerus) ocellaris (Meigen, 1818) - P2, B2, R1, R2; 460-1600 m; 3, 4; e; Wagner \& Joost 1988; Ježek \& Goutner 1995; Wagner 2013; Ježek \& et al. 2017, 2019, 2020.

Clytocerus (Boreoclytocerus) thracicus (Wagner \& Koç, 2013) - B1, B3; 290-710 m; 1, 2; ban; Ježek et al. 2020. Tonnoiriella pulchra (Eaton, 1893) - B1; 500-600 m; 1, 2; ena, ? e; Wagner \& Joost 1988; Wagner 2013, 2018; Ježek et al 2019, 2020.

Tonnoiriella sieberti Wagner, 1993 - P1, P2, B1, B2, B3, S1, O62, R1, R2, RW; 200-1060 m; 1, 2, 3; eswa; Ježek et al. 2020.

Bazarella subneglecta (Tonnoir, 1922) [Parabazarella] - R1, RW; 980-1270 m; 2, 3; ceean; Wagner \& Joost 1988; Wagner 2013, 2018; Ježek et al. 2020.

Tinearia alternata (Say, 1824) [Psychoda] - DM, E1, E2, P2, B3, K3, V1, S1, O61, R1, R2, R5, RW BN; 30-1200 m; 1, 2, 3; k; Nedelkov 1912; Islam et al. 1986; Russev et al. 1987; Wagner \& Joost 1988; Janeva 1989; Janeva \& Russev 1989, 1997; Soufi \& Uzunov 2008; Uzunov et al. 2011; Varadinova et al. 2013; Wagner 2013; Ježek et al. 2017, 2018, 2019; Ježek et al. 2020.

Tinearia lativentris (Berden, 1952) - B3, R2; 290-830 m; 1, 2; hn; Ježek et al. 2020.

Psychoda albipennis Zetterstedt, 1850 [Logima] - DM, P2, B1, B2, B3, V1, V4, O62, R1, RW; 20-1710 m; 1, 2, 3, 4; k; Nedelkov 1909, 1912; Wagner \& Joost 1988; Ježek \& Goutner 1995; Wagner 2013, 2018; Ježek et al. 2018, 2019, 2020.

Psychoda brevicornis Tonnoir, 1940 [Copropsychoda] - P2, B1; 420-809 m; 1, 2; wes; Ježek et al. 2020.

Psychoda cinerea Banks, 1894 [Psychodocha] - V4, R1, RW; 500-1400 m; 1, 2, 3; sk; Wagner \& Joost 1988; Ježek \& Goutner 1995; Wagner 2013, 2018; Ježek et al. 2017, 2018, 2019; Ježek et al. 2020.

Psychoda erminea Eaton, 1898 [Logima] - P2, B2; 420-800 m; 1, 2; dpo; Ježek et al. 2020.

Psychoda gemina (Eaton, 1904) [Psychodocha] - P2, B1, B2, B3, RW; 420-1140 m; 1, 2, 3; e; Ježek et al. 2020.

Psychoda grisescens Tonnoir, 1922 [Psycha] - B3, R1; 300-1200 m; 1, 2, 3; eanna; Wagner \& Joost 1988; Wagner 2013, 2018; Ježek et al. 2020.

Psychoda lobata Tonnoir, 1940 [Chodopsycha] - P2, B1, B2, B3, V4, O62, R1, R2, RW; 200-1400 m; 1, 2, 3, 4; e; Wagner \& Joost 1988; Ježek 1990a; Wagner 2013, 2018; Ježek et al. 2017, 2019, 2020.

Psychoda minuta Banks, 1894 [Psychodula] - B3; 292 m; 1; h; Wagner 2013, 2018; Ježek et al. 2017, 2019; Ježek et al. 2020.

Psychoda parthenogenetica Tonnoir, 1940 - T31, RW; 200-1600 m; 1, 2, 3, 4; ? k; Wagner \& Joost 1988; Wagner 2013, 2018.

Psychoda phalaenoides Linnaeus, 1758 - B2, R1; 800-1330 m; 2, 3; ha; Wagner 2018; Ježek et al. 2020.

Psychoda satchelli Quate, 1955 [Logima] - P2, B1, B2, B3, S1, R1, R2; 420-2050 m; 1, 2, 3, 4; h; Ježek et al. 2020.

Psychoda trinodulosa Tonnoir, 1922 [Psychomora] - P2, B1, B3, T11, R1; 200-830 m; 1, 2; h, ? k; Wagner \& Joost 1988; Ježek \& Goutner 1995; Wagner 2013, 2018; Ježek et al. 2017, 2019, 2020.

Psychoda uniformata Haseman, 1907 - P2, B2, B3; 300-800 m; 1, 2; h; Ježek et al. 2020.

Psychoda zetterstedti (Jezek, 1983) [P. albipennis Zetterstedt, 1850; Logima] - P2, B1, B2, B3, RW; 420-1140 m; 1, 2, 3; dp; Nedelkov 1909, 1912; Ježek et al. 2020.

Paramormia (Duckhousiella) ustulata (Walker, 1856) - P2, B2, B3, O62, R2, RW; 100-1054 m; 1, 2, 3; h; Wagner \& Joost 1988; Ježek \& Goutner 1995; Wagner 2013, 2018; Ježek et al. 2018, 2019, 2020.

Paramormia (Paramormia) polyascoidea (Krek, 1971) - P1, B2, B3; 380-809 m; 1, 2; wes; Ježek et al. 2020.

Paramormia (Phyllotelmatoscopus) acuta (Krek, 1971) - B2; 827 m; 2; e; ? cse; Ježek et al. 2020.

Panimerus denticulatus Krek, 1972 - P2, B2, B3, O62; 200-827 m; 1, 2; e; Ježek et al. 2019, 2020.

Panimerus elongatus Wagner, 1981 - B3, T11; 200-400 m; 1; Eb; Wagner \& Joost 1988; Wagner 2013; Ježek et al. 2019, 2020.

Panimerus kreki Vaillant, 1972 - cse; Ježek 2004; Ježek et al. 2019, 2020.

Lepimormia josanicana (Krek, 1972) - R2; 712 m; 2; Eb; Ježek et al. 2020.

Mormia (Mormia) curvistylis (Krek, 1971) - B1, V4, RW; 710-1200 m; 2, 3; Eb; Wagner \& Joost 1988; Wagner 2013; Ježek et al. 2020.

Mormia (Mormia) revisenda (Eaton, 1893) - RW; 1054 m; 3; ean; Ježek et al. 2020.

Mormia (Hemimormia) eatoni (Tonnoir, 1940) [Promormia] - P2, B1, RW; 419-900 m; 1, 2; e; Wagner \& Joost 1988; Ježek \& Goutner 1995; Wagner 2013; Ježek et al. 2020.

Mormia (Promormia) silesiensis (Jezek, 1983) - B1; 620-732 m; 1, 2; csee; Ježek et al. 2020. 
Mormia (Yomormia) furva (Tonnoir, 1940) - R1; 587 m; 1; e; Ježek et al. 2020.

Mormia (Yomormia) petrovi (Jezek, 1985) - bc; Wagner 2013; Ježek et al. 2020.

Jungiella (Parajungiella) bohdanecensis (Jezek \& Hájek, 2007) - P2; 457 m; 1; csee; Ježek et al. 2020.

Jungiella (Parajungiella) consors (Eaton, 1893) - B3; 292 m; 1; des; Ježek et al. 2020.

Jungiella (Parajungiella) longicornis (Tonnoir, 1919) - R1; 587 m; 1; wes; Ježek 2004; Ježek et al. 2020.

Jungiella (Parajungiella) serbica (Krek, 1985) - B3, O62; 200-300 m; 1; e; Ježek et al. 2020.

Jungiella (Jungiella) bohemica Jezek, 1979 - O62, R1, R2; 200-712 m; 1, 2; csee; Ježek et al. 2020.

Jungiella (Jungiella) hygrophila Jezek, 1983 - P2, B1; 620-1687 m; 1, 2, 3, 4; e; Ježek et al. 2020.

Jungiella (Jungiella) soleata (Walker, 1856) - B1; 620-1295 m; 1, 2, 3; ei; Ježek et al. 2020.

Jungiella (Jungiella) valachica (Vaillant, 1963) - B1, B2; 600-1687 m; 1, 2, 3, 4; e; Vaillant \& Joost 1983; Wagner 2018; Ježek et al. 2020.

Jungiella (Psychocha) acuminata (Szabó, 1960) - P2, B2, T31; 200-827 m; 1, 2; e; Wagner \& Joost 1988; Wagner 2013, 2018; Ježek et al. 2020.

Jungiella (Psychocha) furcillata Krek, 1979 - T11; 200-400 m; 1; Eb, ? see; Wagner \& Joost 1988; Wagner 2013, 2018; Ježek et al. 2020.

Jungiella (Psychocha) laminata (Szabó, 1960) - P2, B2, B3; 457-827 m; 1, 2; csee; Ježek et al. 2020.

Jungiella (Psychocha) monikae Wagner \& Joost, 1986 - \$; Ebg; Wagner 2013; 2018; Ježek et al. 2020.

Jungiella (Psychocha) procera Krek, 1971 - P2, B2, B3; 489-827 m; 1, 2; csee; Ježek et al. 2020.

Jungiella (Psychocha) stranzica Wagner \& Joost, 1988 [J. (Psychocha) ripicola (Bellier, 1967)] - P2, B1, B2,

B3, T11, T31, O62, R1, R2, RW; 200-983 m; 1, 2; Ebg; Ježek 2004; Wagner 2013, 2018; Ježek et al. 2020.

Lepiseodina rothschildi (Eaton, 1912) - P2; 457 m; 1; e; Ježek et al. 2020.

Seoda britteni (Tonnoir, 1940) - P2, B1; 620-827 m; 1, 2; e; Ježek et al. 2020.

Seoda morula (Eaton, 1893) - B2; 828 m; 2; e; Ježek et al. 2020.

Telmatoscopus bosnicus (Krek, 1977) - R1, RW; 1050-1270 m; 3; Eb, ? see; Wagner \& Joost 1988; Wagner 2013.

Peripsychoda auriculata (Curtis, 1839) - P2, T31; 200-457 m; 1; e; Wagner \& Joost 1988; Wagner 2013, 2018; Ježek et al. 2020.

Peripsychoda fusca (Macquart, 1826) - P2, B1, B2, B3, O62; 200-827 m; 1, 2; e; Ježek et al. 2020.

Threticus incurvus Krek, 1972 - RW; 1270 m; 3; e; Wagner \& Joost 1988; Wagner 2013, 2018; Ježek et al. 2020.

Threticus optabilis Krek, 1971 - R1; 1327 m; 3; Eb; Ježek et al. 2020.

Trichopsychoda hirtella (Tonnoir, 1919) - P2, B1, B2; 500-797 m; 1, 2; e; Ježek et al. 2020.

Feuerborniella obscura (Tonnoir, 1919) - B1, B2; 620-827 m; 1, 2; e; Ježek et al. 2020.

Philosepedon (Philosepedon humerale (Meigen, 1818) - V1; 580-600 m; 1, 2; ena; Nedelkov 1912; Ježek \& Goutner 1995; Ježek et al. 2020.

\section{Trichoceridae (Petauristidae)}

Trichocera (Metatrichocera) forcipula Nielsen, 1920 - B2; 1230 m; 3; e; Dahl 1992; Kolcsár et al. 2017.

Trichocera (Metatrichocera) unica Kolcsár, 1917 - B2, S23; 620-900 m; 2; Ebg; Kolcsár et al. 2017.

Trichocera (Trichocera) hiemalis (De Geer, 1776) [Petaurista] - V1, V4, R1; 650-2000 m; 2, 3, 4; h; Czerný 1930; Hubenov 2018.

? Trichocera (Trichocera) japonica Matsumura, 1915 - \$ h, ? bm; Dahl 1992.

Trichocera (Saltrichocera) parva Meigen, 1804 - e, ? h; Dahl 1992.

Trichocera (Saltrichocera) regelationis (Linnaeus, 1758) - V1, S21, RW, BS; 0-1330 m; 1, 2, 3; des, i, h; trogloxene; Manolov 1907; Nedelkov 1912; Czerný 1930; Guéorguiev \& Beron 1962; Beron 2015.

Trichocera (Saltrichocera) saltator (Harris, 1776) - des, ? h; Dahl 1992.

\section{Anisopodidae (Rhyphidae, Phryneidae)}

Sylvicola (Sylvicola) cinctus (Fabricius, 1787) - S23, WR; 292-1185 m; 1, 2, 3; ena, ? wp, ? h; Popova 2006; Dvořák et al. 2019.

Sylvicola (Sylvicola) fenestralis (Scopoli, 1763) - V1; 600-650 m; 2; e, ? ho; Nedelkov 1912.

Sylvicola (Anisopus) punctatus (Fabricius, 1787) - V1; 600-650 m; 2; e, ? h; Nedelkov 1912. 


\section{Scatopsidae}

Colobostema nigripenne (Meigen, 1830) [Scatopse bureschiana (Enderlein, 1926)] - R1; 1500 m; 3; ena; Enderlein 1926; Buresch 1928, 1930; Josifov 1957.

Reichertella pulicaria (Loew, 1846) [Scatopse] - V1; 550-600 m; 1; e; Nedelkov 1912.

Scatopse notata (Linnaeus, 1758) - V1, TL; 250-600 m; 1; ha, ? k; Nedelkov 1912.

Coboldia fuscipes (Meigen, 1830) - DW; 30-40 m; 1; hpta, sk; Szilády 1934.

\section{Ptychopteridae (Liriopidae)}

Ptychoptera (Ptychoptera) albimana (Fabricius, 1787) - R2, 500 m; 1; h; Bechev 1991b.

Ptychoptera (Ptychoptera) contaminata (Linnaeus, 1758) - TL, RW; 190-950 m; 1, 2; h; Bechev 1991b; Popova 2006.

Ptychoptera (Paraptychoptera) lacustris Meigen, 1830 - B1; 770; 2; e, ? h; Bechev 1991b.

Ptychoptera (Paraptychoptera) longicauda (Tonnoir, 1919) - T31; 300 m; 1; e; Bechev 1991b.

Ptychoptera (Ptychoptera) scutellaris Meigen, 1818 - R1; 2100 m; 4; h; Bechev 1991b.

\section{CULICOMORPHA}

\section{Dixidae}

Dixa maculata Meigen, 1818 - B2; 390-500 m; 1; e, ? ena; Arndt 1943.

Dixa submaculata Edwards, 1920 - R5; 500-800 m; 1, 2; e, ? ean; Varadinova et al. 2013.

\section{Chaoboridae}

Chaoborus (Chaoborus) crystallinus (De Geer, 1776) [Corethra plumicornis (Fabricius, 1787)] - DW, E1, E2, V1, RW, BN; 0-1423 m; 1, 2, 3; h; Chichkoff \& Konsuloff 1914; Kovachev \& Stoichev 1996; Stoichev 1998; Kovachev et al. 1999; Uzunov et al. 2001; Varadinova et al. 2011, 2012; Pavlova et al. 2012; Trichkova et al. 2013.

\section{Culicidae}

Anopheles (Anopheles) algeriensis Theobald, 1903 - P1, B1, O62, BN; 80-350 m; 1; mwca, ? wp; ? Drenowsky 1929; Drensky 1949, 1950, 1958; Dimchev et al. 1962; Avlavidov 1970; Christova 1980; Minár 1990; Bozhkov 1991; Mikov 2005.

Anopheles (Anopheles) atroparvus van Thiel, 1927 [A. maculipennis var. atroparvus van Thiel, 1927] - $\mathbf{\Delta}$; DW, DM, E1, E2, BN; 0-200 m; 1; ewca; Markov 1937; Drensky 1939a, 1949, 1950, 1958; Slivenski 1946; Avlavidov 1947, 1970; Boychev 1950b; Dimtchev et al. 1960, 1962; Bozhkov 1966a, 1991; Christova 1980; Mináŕ 1990; Mikov 2005; Kutsarov 2006.

Anopheles (Anopheles) claviger (Meigen, 1804) [A. bifurcatus Linnaeus, 1758] - DM, P3, B1, B2, B3, V1, V4, K9, TL, O61, O62; T2; T31, R5, RW, RE, BN, BS; 0-1300 m; 1, 2, 3; wcp; Chichkoff \& Konsuloff 1914; Konsuloff 1921, 1922a, 1922b; Markov 1925a, 1925b, 1929, 1937; Drenowsky 1929; Drensky 1931a, 1931b, 1932a; Slivenski 1935, 1956; Avlavidov 1947, 1959; Kozarov 1949; Drensky 1949, 1950, 1958; Paspalev 1950, 1951; Bozhkov 1961, 1967, 1974, 1991; Dimtchev et al. 1962; Bozhkov et al. 1969; Christova \& Todorova 1969; Christova et al. 1971; Russev \& Janeva 1975; Beron 2004; Mikov 2005, 2008a, 2008b; Beschovski 2006; Agushev 2014, 2018; Agushev \& Bileva 2014.

Anopheles (Anopheles) hyrcanus (Pallas, 1771) [A. hyrcanus var. pseudopictus Grassi, 1900; Myzorhynchus sinensis Wiedemann, 1828; M. pseudopictus (Grassi, 1900)] - DW, DM, E1, E2, P1, O62, TL; 10-200 m; 1; spo; Chichkoff \& Konsuloff 1914; Konsuloff 1921a, 1921b, 1922a, 1922c; Markov 1925a, 1929, 1937; Drenowsky 1929; Drensky 1931a; Slivenski 1935, 1946; Drensky 1949, 1950, 1958; Boychev 1950b; Dimtchev et al. 1962; Dorovski 1976; Bozhkov 1991; Mikov 2005; Kutsarov 2006; Agushev 2014, 2018; Agushev \& Bileva 2014. 
? Anopheles (Anopheles) labranchiae Falleroni, 1926 [A. maculipennis var. labranchiae Falleroni, 1926] - O62; 65-150 m; 1; hom; Markov 1937; Drensky 1939a, 1949, 1950, 1958; Schaffner et al. 2001.

Anopheles (Anopheles) maculipennis Meigen, 1818 - A ; DW, DM, E1, E2, B1, B2, B3, K9, V1, V4, S1, TL, T1, T2, T3, O62, T31, R1, R5, RW, RE, BN, BS; 0-2190 m; 1, 2, 3, 4; wcp; Manolov 1907, 1909a, 1909b; Theobald 1910; Nedelkov 1912; Chichkoff \& Konsuloff 1914; Konsuloff 1921a, 1922a, 1922b, 1922c, 1923a; Markov 1925a, 1925b, 1929, 1937; Drenowsky 1929; Markov \& Morov 1929; Drensky 1931a; Drensky 1939a, 1949, 1950, 1958; Arndt 1943; Slivenski 1946; Avlavidov 1947, 1948, 1958, 1959, 1961; Kozarov 1949; Boychev 1950b; Paspalev 1950, 1951; Bozhkov 1953, 1957, 1961, 1962, 1965, 1966b, 1991; Kozarov \& Bozhkov 1953; Stefanov 1955, 1956; Dimov 1957; Marinov 1957; Kovchazov 1958, 1961, 1976; Atanassov \& Christova 1960; Atanassov \& Petrov 1961; Dimtchev et al. 1962; Atanassov et al. 1962; Russev \& Janeva 1975; Mikov 2005, 2008a, 2011; Beron 2004; Beschovski 2006; Kutsarov 2006; Mikov et al. 2011; Agushev 2012, 2014, 2015, 2018; Agushev \& Bileva 2014.

Anopheles (Anopheles) marteri Senevet \& Prunelle, 1927 - O62, O5; 100-200 m; 1; mwca, ? mit; Drensky 1949, 1950, 1958; Christova 1980; Mináŕ 1990; Bozhkov 1991; Mikov 2005.

Anopheles (Anopheles) melanoon Hackett, 1934 [A. maculipennis var. melanoon Hackett, 1934] - DM, E1, E2, O62, RE, BS; 20-250 m; 1; nmi; Markov 1937; Drensky 1939a, 1949, 1950, 1958; Paspalev 1950, 1951; Dimchev et al. 1962; Dorovski 1976; Christova 1980; Mikov 2005, 2008a.

Anopheles (Anopheles) messeae Falleroni, 1926 [A. maculipennis var. messeae Falleroni, 1926] - $\mathbf{\Delta}$; DW, DM, E1, E2, V1, TL, T2, O62, RW, RE, BS; 0-800 m; 1, 2; esanca; Konsuloff 1921b; Markov 1937; Drensky 1939a, 1949, 1950, 1958; Slivenski 1946; Boychev 1950b; Paspalev 1950, 1951; Bozhkov 1953, 1961, 1991; Stefanov 1956; Dimchev et al. 1962; Mikov 2005, 2008; Kutsarov 2006; Agushev 2014, 2015, 2018; Agushev \& Bileva 2014.

Anopheles (Anopheles) plumbeus Stephens, 1828 - DM, P2, B1, B2, B3, V4, O62, BN, BS; 0-1200 m; 1, 2, 3; wp; ? Drenowsky 1929; Drensky 1949, 1950, 1958; Dimchev et al. 1962; Bozhkov 1966a, 1967, 1974, 1991; Bozhkov et al. 1969; Christova et al. 1971; Christova \& Bozhkov 1977; Mikov 2005.

Anopheles (Anopheles) sacharovi Favre, 1903 [A. maculipennis var. sacharovi Favre, 1903] - ム ; DW, DM, E1, E2, TL, T1, T2, O61, RE, BN, BS; 0-500 m; 1; mwca; Markov 1929, 1937; Markov \& Morov 1929; Slivenski 1935, 1946; Drensky 1939a, 1949, 1950, 1958; Avlavidov 1947, 1948, 1959, 1961; Bozhkov 1961, 1991; Atanassov \& Christova 1960; Atanassov et al. 1962; Mikov 2005, 2008.

Anopheles (Cellia) superpictus Grassi, 1899 [ A. (Myzomyia) superpictus Grassi, 1899] - $\mathbf{\Delta}$; DW, P1, B3, S1, TL, T1, T2, T3, O61, O62, R5, RE; 100-500 m; 1; swpo; Konsuloff 1921a, 1922a, 1923a; Markov 1925a, 1925b, 1929, 1937, 1950; Drenowsky 1929; Markov \& Morov 1929; Slivenski 1935, 1940, 1946; Avlavidov 1947, 1948; Paspalev 1950, 1951; Drensky 1949, 1950, 1958; Kozarov 1949; Boychev 1950b; Mondchadskiy 1951; Stefanov 1955; Bozhkov 1961, 1991; Russev \& Janeva 1975; Christova 1980; Mináŕ 1990; Beron 2004; Mikov 2005; Beschovski 2006; Agushev 2014, 2015, 2018; Agushev \& Bileva 2014.

Uranotaenia (Pseudoficalbia) unguiculata Edwards, 1913 - DM, V1, TL, O62, BS; 0-600 m; 1; swpo; Drenowsky 1929; Bozhkov et al. 1969; Bozhkov 1991; Christova et al. 1971; Dorovski 1976; Agushev 2014, 2015, 2018; Agushev \& Bileva 2014.

Orthopodomyia pulcripalpis (Rondani, 1872) - O62, BN, BS; 0-300 m; 1; eanna; Dimov 1959; Bozhkov et al. 1969; Christova et al. 1971; Christova \& Bozhkov 1977; Bozhkov 1991.

Culiseta (Culiseta) alaskaensis (Ludlow, 1906) - TL; 150-160 m; 1; h; Christova et al. 2000; Agushev 2012.

Culiseta (Culiseta) annulata (Schrank, 1776) [Theobaldia] - $\mathbf{\Delta}$; DW, DM, E1, E2, B1, V1, S22, TL, O62, R1, R5, RW, BN, BS; 0-1400 m; 1, 2, 3; wp; Nedelkov 1909, 1912; Theobald 1910; Chichkoff \& Konsuloff 1914; Konsuloff 1922b; Drenowsky 1929a, 1929b; Bozhkov 1958, 1959, 1961, 1962, 1965, 1967, 1991; Bozhkov et al. 1969; Christova \& Todorova 1969; Christova et al. 1971; Christova \& Dorovski 1972; Russev \& Janeva 1975; Dorovski 1976; Beschovski 2006; Kutsarov 2006; Mikov 2011; Agushev 2012, 2015, 2018.

Culiseta (Culiseta) glaphyroptera (Schiner, 1864) [Theobaldia] - TL, R1; 150-1400 m; 1, 2, 3; e; Bozhkov 1958, 1959, 1991; Beron 1969; Agushev 2014, 2015, 2018; Agushev \& Bileva 2014.

Culiseta (Culicella) fumipennis (Stephens, 1825) [Theobaldia; C. setivalva Maslov, 1936] - O62, BS; 0-150 m; 1; wp; Drenowsky 1929a; Christova \& Bozhkov 1966; Bozhkov et al. 1969; Christova et al. 1971; Bozhkov 1991.

Culiseta (Culicella) morsitans (Theobald, 1901) [Theobaldia] - TL, BN, BS; 0-160 m; 1; h; Christova \& Bozhkov 1966; Christova et al. 1971; Bozhkov 1991; Agushev 2014, 2015; Agushev \& Bileva 2014. 
Culiseta (Allotheobaldia) longiareolata (Macquart, 1838) [Theobaldia] - V1, S23, TL, O62, BN, BS; 0-600 m; 1, 2; ppt; Drenowsky 1929a; Bozhkov 1958, 1962, 1965, 1991; Agushev 2012, 2014, 2015; Agushev \& Bileva 2014, 2018.

Coquillettidia (Coquillettidia) richiardii (Ficalbi, 1889) [Mansonia] - $\mathbf{\Delta}$; DW, DM, E1, E2, BN, BS; 0-250 m; 1; wp; Chichkoff \& Konsuloff 1914; Mondchadskiy 1951; Bozhkov \& Christova 1965; Bozhkov et al. 1969; Dorovski 1976; Bozhkov 1991; Kutsarov 2006; Mikov et al. 2011.

Aedes (Stegomyia) albopictus (Skuse, 1894) - A; P1, TL, O61, BN, BS; 0-400 m; 1; sk, i; Mikov et al. 2013; Agushev 2015, 2018; Medlock et al. 2015.

Aedes (Aedes) cinereus Meigen, 1818 - DW, DM, E1, E2, TL, BS; 0-160 m; 1; h; Theobald 1907, 1910; Chichkoff \& Konsuloff 1914; Manolov 1907; Bozhkov 1961, 1991; Bozhkov \& Christova 1965; Christova \& Dorovski 1972; Dorovski 1976; Kutsarov 2006; Mikov 2011; Agushev 2014, 2015, 2018; Agushev \& Bileva 2014.

Aedes (Aedimorphus) vexans (Meigen, 1830) - DW, DM, E1, E2, P1, B1, V1, TL, O62, BN, BS; 0-600 m; 1, 2; hpta; Chichkoff \& Konsuloff 1914; Drenowsky 1929a; Bozhkov 1962, 1965, 1967, 1991; Bozhkov \& Christova 1965; Christova \& Todorova 1969; Bozhkov et al. 1969; Christova et al. 1971; Dorovski 1976; Kutsarov 2006; Mikov et al. 2011; Agushev \& Bileva 2014; Agushev 2015, 2018.

Aedes (Ochlerotatus) annulipes (Meigen, 1830) [Ochlerotatus] - DW, DM, E1, E2, V1, BN, BS; 0-700 m; 1, 2; ean; Nedelkov 1912; Chichkoff \& Konsuloff 1914; Bozhkov et al. 1969; Christova et al. 1971; Bozhkov 1991; Kutsarov 2006.

Aedes (Ochlerotatus) cantans (Meigen, 1818) [Ochlerotatus] - $\mathbf{\Delta}$; DW, DM, E1, E2, O62, BN, BS; 0-150 m; 1; h; Drenowsky 1929a; Bozhkov et al. 1969; Christova et al. 1971; Bozhkov 1991; Cutsarov 2006; Mikov 2011.

Aedes (Ochlerotatus) caspius (Pallas, 1771) [Ochlerotatus] - $\mathbf{\Delta}$; DM, P2, V1, TL, O62, RW, BN, BS; 0-750 m; 1, 2; hop, ? h; Caspers 1951; Bozhkov 1961, 1962, 1965, 1974a, 1974b, 1991; Christova \& Todorova 1969; Bozhkov et al. 1969; Dorovski 1976; Beschovski 2006; Mikov 2011; Agushev \& Bileva 2014; Agushev 2015, 2018.

Aedes (Ochlerotatus) cataphylla (Dyar, 1916) - B1, TL; 150-1500 m; 1, 2, 3; h; Bozhkov 1967, 1991; Agushev 2014, 2015, 2018; Agushev \& Bileva 2014.

Aedes (Ochlerotatus) communis (De Geer, 1776) - $\mathbf{\Delta}$; DW, DM, E1, E2, V4, R1, BS; 0-1450 m; 1, 2, 3; h; Theobald 1907, 1910; Chichkoff \& Konsuloff 1914; Bozhkov 1959, 1961, 1966b, 1991; Bozhkov \& Christova 1965; Cutsarov 2006.

Aedes (Ochlerotatus) detritus (Haliday, 1833) - BN, BS; 0-50 m; 1; tp; Christova \& Bozhkov 1966; Bozhkov et al. 1969; Christova et al. 1971; Bozhkov 1991.

Aedes (Ochlerotatus) dorsalis (Meigen, 1830) - DM, E1, E2, K9, V1, O62, BS; 0-600 m; 1, 2; h, ? ho; Manolov 1907; Theobald 1907, 1910; Chichkoff \& Konsuloff 1914; Drenowsky 1929a; Mondchadskiy 1951; Bozhkov 1961, 1991; Dorovski 1976.

Aedes (Ochlerotatus) excrucians (Walker, 1856) - O62; 150 m; 1; h; Drenowsky 1929a; Bozhkov 1991.

Aedes (Ochlerotatus) pulcritarsis (Rondani, 1872) - B1, B2, BN, BS; 0-1200 m; 1, 2, 3; mwca, ? mit; Dimov 1959; Bozhkov 1967, 1974a, 1991; Bozhkov et al. 1969; Christova et al. 1971.

Aedes (Ochlerotatus) pullatus (Coquillett, 1904) - V4, R1, RW; 1000-2390 m; 3, 4, 5; h, m; Bozhkov 1959, 1966b, 1991; Kalaydzhiev et al. 1960; Beron 1969; Russev \& Janeva 1975; Beschovski 2006.

Aedes (Ochlerotatus) punctor (Kirby, 1837) - DW, DM, E1, E2, V4, R1; 20-1400 m; 1, 2, 3; h; Bozhkov 1959, 1991; Minarzh \& Christova 1971; Kutsarov 2006.

Aedes (Ochlerotatus) sticticus (Meigen, 1838) - DM, V1, S22, O62, BN; 0-1243 m; 1, 2, 3; h; Chichkoff \& Konsuloff 1914; Drenowsky 1929a; Bozhkov 1962, 1991; Bozhkov \& Christova 1965; Bozhkov et al. 1969; Christova \& Todorova 1969; Christova et al. 1971; Dorovski 1976.

Aedes (Rusticoidus) rusticus (Rossi, 1790) [A. maculatus (Meigem, 1804)] - V1, S21, TL, O62, BN, BS; 0-700 m; 1, 2; eanna; Chichkoff \& Konsuloff 1914; Drenowsky 1929a; Bozhkov 1962, 1991; Bozhkov \& Christova 1965; Bozhkov et al. 1969; Christova et al. 1971; Agushev \& Bileva 2014; Agushev 2015, 2018.

Aedes (Finlaya) echinus Edwards, 1920 - BN, BS; 0-20 m; 1; hom; Dimov 1959; Bozhkov et al. 1969; Christova et al. 1971; Bozhkov 1991.

Aedes (Finlaya) geniculatus (Olivier, 1791) - DM, B1, B2, B3, V1, V4, TL, T1, T31, O62, R1, RW, BN, BS; 0-1700 m; 1, 2, 3; wp; Drenowsky 1929a; Bozhkov 1959, 1961, 1962, 1965, 1966b, 1967, 1974a, 1974b, 1991; Bozhkov et al. 1969; Christova et al. 1971; Christova \& Dorovski 1972; Dorovski 1976; Christova \& Bozhkov 1977; Beschovski 2006. 
Culex (Culex) mimeticus Noè, 1899 - O62; 150-160 m; 1; spo; Drenowsky 1929a, 1929b; Markov \& Morov 1929; Bozhkov 1991.

Culex (Culex) perexiguus Theobald, 1903 [? Culex univittatus Theobald, 1901] - O62; 100-150 m; 1; atm; Drenowsky 1929a; Bozhkov 1991.

Culex (Culex) pipiens Linnaeus, 1758 [C. pipiens var. ciliaris Linnaeus, 1758; C. pipiens var. molestus Forskål, 1775] - $\boldsymbol{\Delta} ;$; DW, DM, E1, E2, P2, B1, B2, B3, V1, V4, S22, S23, TL, T1, T31, O62, R1, RW, BN, BS; 0-2100 m; 1, 2, 3, 4; sk; trogloxene; Meunier 1897; Joakimoff 1899; Kovachev 1905; Manolov 1907; Nedelkov 1909, 1912; Chichkoff \& Konsuloff 1914; Konsuloff 1922b; Drenowsky 1929a; 1929b; Markov \& Morov 1929; Czerný 1930; Slivenski 1935, 1946; Drensky 1942; Caspers 1951; Bozhkov 1959, 1961, 1962, 1965, 1966b, 1991; Kalaydzhiev et al. 1960; Guéorguiev \& Beron 1962; Bozhkov \& Christova 1965; Russev 1966; Bozhkov et al. 1969; Christova \& Todorova 1969; Dorovski 1976; Beron 1994, 2015; Janeva \& Russev 1997; Kutsarov 2006; Mikov 2011; Agushev 2012, 2014, 2015, 2018; Agushev \& Bileva 2014.

Culex (Culex) theileri Theobald, 1903 - DM, B1, V1, V4, S23, TL, O62, BS; 0-1450 m; 1, 2, 3; sppt; Chichkoff \& Konsuloff 1914; Drenowsky 1929a; 1929b; Bozhkov 1961, 1965, 1966b, 1967, 1991; Russev 1966; Bozhkov et al. 1969; Christova \& Todorova 1969; Christova et al. 1971, 1972; Christova \& Dorovski 1972; Dorovski 1976; Christova \& Bozhkov 1977; Agushev 2015.

Culex (Culex) torrentium Martini, 1925 - TL; 150-160 m; 1; eswa; Agushev \& Bileva 2014; Agushev 2015, 2018.

Culex (Barraudius) modestus Ficalbi, 1890 - $\mathbf{\Delta}$; DW, DM, E1, E2, V1, TL; BN, BS; 0-600 m; 1; tp, ? wcp; Christova \& Todorova 1969; Bozhkov et al. 1969; Christova et al. 1971, 1972; Christova \& Dorovski 1972; Dorovski 1976; Bozhkov 1991; Kutsarov 2006; Mikov 2011.

Culex (Neoculex) territans Walker, 1856 [C. sergentii Theobald, 1903; C. apicalis Adams, 1903] - DW, DM, E2, P1, V1, TL; T3, T31, O62, R5, BN, BS; 0-600 m; 1, 2; h; Chichkoff \& Konsuloff 1914; Drenowsky 1929a; 1929b; Arndt 1943; Bozhkov 1961, 1962, 1965, 1967, 1991; Russev 1966; Bozhkov et al. 1969; Christova et al. 1971, 1972; Dorovski 1976; Beschovski 2006; Kutsarov 2006; Agushev 2014, 2015, 2018; Agushev \& Bileva 2014.

Culex (Maillotia) hortensis Ficalbi, 1889 - DW, E1, E2, V1, S23, T31, O62, R1, RW, BN, BS; 0-1390 m; 1, 2, 3; swpo; Chichkoff \& Konsuloff 1914; Drenowsky 1929a; 1929b; Bozhkov 1959, 1961, 1965, 1966b, 1967, 1991; Christova \& Todorova 1969; Bozhkov et al. 1969; Christova et al. 1971, 1972; Russev \& Janeva 1975; Dorovski 1976; Beschovski 2006; Kutsarov 2006.

\section{Thaumaleidae}

Thaumalea bezzii Edwards, 1929 - R1; 660 m; 1, 2; e; Joost 1978.

Thaumalea popovi Joost, 1978 - B2; 1600 m; 3; Ebg; Joost 1978.

Thaumalea testacea Ruthe, 1831 [Orphnephila] - V4; 700-1350 m; 2, 3; e; Arndt 1943.

Androprosopa larvata (Mik, 1888) - R1; 4; e; Joost 1978.

\section{Simuliidae}

Prosimulium (Prosimulium) fulvipes (Edwards, 1921) [P. rufipes var. fulvipes (Edwards, 1921)] - V4, R1, RW; 920-2400 m; 2, 3, 4; 5; cseean, ? des; Enderlein 1924; Konsuloff \& Paspalev 1925; Buresch 1938, 1953a; Russev et al. 1994; Kovachev 1976, 1985c, 2000; Adler \& Crosskey 2018.

Prosimulium (Prosimulium) hirtipes (Fries, 1824) - DM, P2, B1, B2, V4, R1, RW; 430-2450 m; 2, 3, 4, 5; tes; Nedelkov 1912; Russev 1961; Kovachev 1975, 1976, 1985a, 1985c, 2000; Uzunov et al. 1981, 2011; Janeva 1987, 1989; Russev et al. 1994; Janeva \& Russev 1997; Beschovski 2006; Sakelarieva et al. 2008; Moskova \& Uzunov 2011; Varadinova et al. 2013.

Prosimulium (Prosimulium) latimucro (Enderlein, 1925) - B1, V4, R1, RW; 620-2400 m; 2, 3, 4, 5; ena, ? e; Kovachev 1976, 1979, 1985a, 1985c, 1990, 2000; Uzunov et al. 1981, 2011; Islam et al. 1986; Russev et al. 1994; Varadinova et al. 2013; Adler \& Crosskey 2018.

Prosimulium (Prosimulium) petrosum Rubtsov, 1955 - V4, R1; 1100-2235 m; 3, 4, 5; em, mm; Kovachev 1973, 1990, 2000; Adler \& Crosskey 2018.

Prosimulium (Prosimulium) rachiliense Djafarov, 1954 - B1; 1200-1400 m; 3; seeanna; Kovachev 1969; Adler \& Crosskey 2018. 
Prosimulium (Prosimulium) rufipes (Meigen, 1830) [P. conistylum Rubtsov, 1956; P. fuscipes Knoz, 1965] - B2, V4, O1, O6, R1, R2; RW; 650-2300 m; 2, 3, 4, 5; eanna; Enderlein 1924; Konsuloff \& Paspalev 1925; Buresch 1938; Russev 1961; Kovachev 1975, 1976, 1979, 1985a, 1985c, 1990, 2000; Uzunov et al. 1981, 2011; Russev et al. 1984b, 1994; Islam et al. 1986; Beschovski 2006; Varadinova et al. 2013; Adler \& Crosskey 2018. Prosimulium (Prosimulium) tomosvaryi (Enderlein, 1921) [P. arvernense (Grenier, 1947); P. duodecimfiliatum Rubtsov, 1955; P. nigripes (Enderlein, 1925); P. balcanicum Enderlein, 1929] - DM, P2, B1, V1, V3, V4, V5, S211, O62, R1, RW; 50-2000 m; 2, 3, 4; des, ? dp; Enderlein 1930; Rubtsov 1956; Russev 1961, 1977; Kovachev 1969, 1975, 1976, 1979, 1985c, 1990, 2000; Janeva \& Russev 1985; Islam et al. 1986; Janeva 1989; Beschovski 2006; Uzunov et al. 2011; Varadinova et al. 2013; Adler \& Crosskey 2018.

Stegopterna trigonium (Lundström, 1911) [S. freyi (Enderlein, 1929)] - RE; 170 m; 1; h; Kovachev 1969.

Metacnephia lyra (Lundstrom, 1911) [M. trigonia Rubtsov, 1956] - wces; Russev et al. 1976.

Metacnephia uzunovi Kovachev, 1985 - T31; 60-200 m; 1; Er; Kovachev 1985b; Adler \& Crosskey 2018.

Simulium (Nevermannia) angustatum (Rubtsov, 1956) [Cnetha] - \$; des; Russev et al. 1976, 1994.

Simulium (Nevermannia) angustitarse (Lundström, 1911) [Chelocnetha; Eusimulium] - V4, TV, R1, RW; 7501700 m; 2, 3, 4; wcp; Kovachev 1975, 1976, 1979, 1990, 2000; Russev et al. 1976, 1994; Islam et al. 1986; Beschovski 2006.

Simulium (Nevermannia) beltukovae (Rubtsov, 1956) [S. carpathicum (Knoz, 1961); Cnetha; Eusimulus] - B1, B2, V4, R, R2, RW; 400-2000 m; 2, 3, 4; wces; Kovachev 1975, 1976, 1979, 1990, 2000; Russev et al. 1976, 1994; Beschovski 2006.

Simulium (Nevermannia) bertrandi Grenier \& Dorier, 1959 [Cnetha] - R1, R2; 620-2300 m; 2, 3, 4, 5; e; Kovachev 1976, 1985a, 2000; Russev et al. 1976; Uzunov et al. 1981; Adler \& Crosskey 2018.

Simulium (Nevermannia) brevidens (Rubtsov, 1956) [Cnetha; Eusimulium] - DM, P2, B1, B2, V4, R1, RW; 450-2350 m; 2, 3, 4, 5; ena; Kovachev 1975, 1976, 1979, 1985a, 1985c, 1990, 2000; Russev et al. 1976; Janeva \& Russev 1985, 1997; Janeva 1987; Russev et al. 1994; Beschovski 2006; Uzunov et al. 2011; Varadinova et al. 2013.

Simulium (Nevermannia) carthusiense Grenier \& Dorier, 1959 [Cnetha; Eusimulum] - V4, R1, RW; 620-2300 m; 2, 3, 4, 5; ena; Kovachev 1975, 1979, 1985a, 1990, 2000; Russev et al. 1976, 1994; Uzunov et al. 1981; Beschovski 2006.

Simulium (Nevermannia) codreanui (Sherban, 1958) [Cnetha; Eusimulum] - DM, P2, B1, B2; V4, R1, R2, RW; 890-2150 m; 2, 3, 4, 5; ean; Kovachev 1975, 1976, 1979, 1985a, 1085c, 1990, 2000; Russev et al. 1976, 1994; Uzunov et al. 1981; Janeva \& Russev 1997; Beschovski 2006; Sakelarieva et al. 2008; Uzunov et al. 2011; Varadinova et al. 2013; Adler \& Crosskey 2018.

Simulium (Nevermannia) costatum Friederichs, 1920 [Cnetha] - V4, R1; 780-2300 m; 2, 3, 4, 5; eanna; Kovachev 1976, 1990, 2000; Russev et al. 1976, 1994; Adler \& Crosskey 2018.

Simulium (Nevermannia) crenobium (Knoz, 1961) [Cnetha] - R1; 1400-2200 m; 3, 4, 5; csee; Kovachev 1976, 1979, 2000; Russev et al. 1976, 1994.

Simulium (Nevermannia) cryophilum (Rubtsov, 1959) [Cnetha; Eusimulium couverti Rubtsov, 1964] - P2, B1, B2, V4, O1, R1, R2, RW; 620-2200 m; 2, 3, 4, 5; eanna; Kovachev 1975, 1976, 1979, 1985a, 1985c, 2000; Islam et al. 1986; Janeva 1987; Russev et al. 1976, 1994; Russev 1977; Uzunov et al. 1981; Beschovski 2006; Uzunov et al. 2011; Varadinova et al. 2013; Adler \& Crosskey 2018.

Simulium (Nevermannia) curvans (Rubtsov \& Carlsson, 1965) [Eusimulium pygmaeum pungens: Rubtsov, 1956, not Meigen; Cnetha] - R1; 1200-2100 m; 3, 4, 5; hoes, bm; Kovachev 1969, 1973, 1976, 2000.

? Simulium (Nevermannia) delizhanensis (Rubzov, 1955) [Chelocnetha] - \$ ? see; Russev et al. 1976.

Simulium (Nevermannia) lundstromi (Enderlein, 1921) [Chelocnetha latigonium (Rubtsov, 1956); Eusimulium] - P1, P2, B1, V4, O62, R1, RW; 70-2000 m; 1, 2, 3, 4; wp; Enderlein 1924; Konsuloff \& Paspalev 1925; Buresch 1938; Kovachev 1975, 1976, 1985a, 1990, 2000; Russev 1977; Uzunov et al. 1981; Islam et al. 1986; Russev et al. 1994; Beschovski 2006; Adler \& Crosskey 2018.

Simulium (Nevermannia) vernum Macquart, 1826 - V5, R1; 620-890 m; 2; tp; Russev et al. 1976; Uzunov et al. 1981; Kovachev 1985a; Varadinova et al. 2013.

Simulium (Hellichiella) latipes (Meigen, 1804) [Cnetha; C. verna (Macquart, 1826); Eusimulium] - V1, V4, R1, R2, RW; 600-2070 m; 2, 3, 4, 5; tp; Nedelkov 2012; Kovachev 1975, 1976, 1979, 1985a, 1990, 2000; Russev et al. 1994; Beschovski 2006; Moskova \& Uzunov 2011; Uzunov et al. 2011; Stoyanova et al. 2013. 
Simulium (Byssodon) maculatum (Meigen, 1804) [Prosimulium vigintiquaterni (Enderlein, 1929); Titanopteryx) - V1, V4; 600-700 m; 2; h; Nedelkov 2012; Russev 1961.

Simulium (Simulium) alajense Rubtsov, 1938 [Tetisimulium] - B1, B2, V1, V3; 400-700 m; 1, 2; emca; Kovachev 1969; Adler \& Crosskey 2018.

Simulium (Simulium) argenteostriatum Strobl, 1898 [Cleitosimulium; S. alternans Enderlein, 1921; S. schoenbaueri Enderlein, 1921] - DW, P1, P2, B1, B2, V1, V4, O61, R1, R2, R5, RW; 50-2100 m; 1, 2, 3, 4; csena; Enderlein 1924; Konsuloff \& Paspalev 1925; Tschorbadjiew 1925e; Buresch 1938; Kovachev 1969, 1975, 1976, 1979, 1990, 2000; Russev et al. 1976; Islam et al. 1986; Janeva 1987; Russev et al. 1994; Beschovski 2006; Sakelarieva et al. 2008; Adler \& Crosskey 2018.

Simulium (Simulium) argyreatum Meigen, 1838 [Odagmia rheophilum (Knoz, 1961); S. obreptans Edwards, 1920] - DM, P1, P2, B1, B2, V4, TL, O62, R1, R5, RW, RE; 150-2400 m; 1, 2, 3, 4, 5; e; Kovachev 1975, 1976, 1979, 1985a, 1985c, 1990, 2000; Russev et al. 1976, 1994; Uzunov et al. 1981; Islam et al. 1986; Janeva 1987; Janeva \& Russev 1997; Janeva et al. 2001; Beschovski 2006; Uzunov et al. 2011; Varadinova et al. 2013; Adler \& Crosskey 2018.

Simulium (Simulium) baracorne Smart, 1944 - P1, P2, B2, V4; 400-1000 m; 1, 2, 3; cseet; Kovachev 1969, 1990; Russev et al. 1994; Adler \& Crosskey 2018.

Simulium (Simulium) bezzii (Corti, 1914) [Cnetha; Odagmia; Friesia tristrigata (Enderlein, 1921); F. tristrigata obscura (Enderlein, 1924); Tetisimulium kondici (Baranov, 1926); T. crinitum (Rubtsov, 1956)] - DW, P1, P2, B1, B2, K7, K8, K9, V3, O61, O62, R1, R5, RW, RE; 100-2200 m; 1, 2, 3, 4; wp, ? mvca; Enderlein 1924; Konsuloff \& Paspalev 1925; Buresch 1926, 1938; Kovachev 1969, 1975, 1976, 1979, 1985c, 1990, 2000; Russev et al. 1976, 1994; Russev 1977; Islam et al. 1986; Rubtsov \& Yankovsky 1988; Janeva 1989; Janeva et al. 2001; Beron 2004; Beschovski 2006; Varadinova et al. 2013; Adler \& Crosskey 2018.

Simulium (Simulium) bukovskii Rubtsov, 1940 [Gnus] - RW; 1200 m; 3; seean; Kovachev 1975; Russev et al. 1976; Beschovski 2006; Adler \& Crosskey 2018.

Simulium (Simulium) colombaschense (Scopoli, 1780) - $\mathbf{\Delta}$; DW, DM, E1, E2, P1, B1, V1, R1; 30-900 m; 1, 2; cee; Nedelkov 1912; Konsuloff 1923b; Markov 1923; Buresch 1924; Enderlein 1924; Konsuloff \& Paspalev 1924; Tschorbadjiew 1925e; Buresch 1938; Russev 1966b; Russev et al. 1976, 1994; Adler \& Crosskey 2018.

Simulium (Simulium) debacli Terteryan, 1952 [Odagmia] - P1; 120-150 m; 1; seean; Kovachev 1969; Adler \& Crosskey 2018.

Simulium (Simulium) degrangei Dorier \& Grenier, 1960 [Gnus; Paragnus] - P1, P2, B1, B2, K8, K9, V4, R1, R2, RW; 430-2200 m; 1, 2, 3, 4; csee, ? cse; Kovachev 1975, 1976, 1979, 2000; Islam et al. 1986; Janeva 1987, 1991; Russev et al. 1976, 1994; Beron 2004; Beschovski 2006; Adler \& Crosskey 2018.

Simulium (Simulium) deserticola Rubtsov, 1940 [Odagmia] - seeca; Russev et al. 1994.

Simulium (Simulium) desertorum Rubtsov, 1938 [Tetisimulium] - RE; 180-220 m; 1; seeca; Kovachev 1969; Adler \& Crosskey 2018.

Simulium (Simulium) fontanum Terteryan, 1952 [Odagmia] - nemi; Russev et al. 1994.

Simulium (Simulium) frigidum Rubtsov, 1940 [Odagmia] - 1; P1, P2; 1; wces; Russev et al. 1976, 1994.

Simulium (Simulium) ibariense Zivkovitch \& Grenier, 1959 [Gnus] - R1, R5, RW; 460-850 m; 1, 2; cee; Kovachev 1976, 1985c; Russev et al. 1076; Uzunov et al. 2011; Varadinova et al. 2013; Adler \& Crosskey 2018.

Simulium (Simulium) intermedium Roubaud, 1906 [Odagmia ornata var. nitidifrons Edwards, 1920] - DW, DM, P1, P2, B1, V1; 50-600 m; 1, 2; ena; Enderlein, 1924; Konsuloff \& Paspalev 1925; Tschorbadjiew 1925e; Buresch 1938; Russev et al. 1987, 1994; Janeva \& Russev 1997; Uzunov et al. 2011; Adler \& Crosskey 2018.

Simulium (Simulium) kerisorum (Rubtsov, 1956) [Tetisimulium] - RE; 180-220 m; 1; nemwca; Kovachev 1969; Adler \& Crosskey 2018.

Simulium kurense Rubtsov \& Dzhafarov, 1951 - O62, R5; 80-500 m; 1; bct; Kovachev 1976, 1985c; Russev et al. 1976; Islam et al. 1986.

Simulium (Simulium) kiritshenkoi Rubtsov, 1940 [S. caucasicum Rubtsov, 1940; Odagmia] - P1, P2, S21, S22, RW, RE; 300-1030 m; 1, 2, 3; nemwca; Kovachev 1969, 1975; Russev et al. 1994; Janeva \& Bancheva 2002; Beron 2004; Beschovski 2006; Adler \& Crosskey 2018.

Simulium (Simulium) latimentum (Rubtsov, 1956) [Tetisimulium] - RE; 200-300 m; 1; esca; Kovachev 1975; Beron 2004. 
Simulium (Simulium) maximum (Knoz, 1961) [Odagmia] - P1, P2, B1, V4, R1, R2, RW; 400-2000 m; 1, 2, 3, 4; e, cse; Kovachev 1976, 1979, 1985a, 1985c, 1990, 2000; Russev et al. 1976, 1994; Uzunov et al. 1981; Janeva 1987; Adler \& Crosskey 2018.

Simulium (Simulium) monticola Friederichs, 1920 [Odagmia] - B1, B2, V4, R1, R2, R5; 600-2200 m; 2, 3, 4, 5; ena; Enderlein 1924; Buresch 1938; Kovachev 1976, 1979, 1985a, 1990, 2000; Russev et al. 1976, 1994; Janeva \& Russev 1997; Uzunov et al. 2011; Stoyanova et al. 2013; Varadinova et al. 2013; Adler \& Crosskey 2018.

Simulium (Simulium) monticoloides (Rubtsov, 1956) [Odagmia] - seean; Russev et al. 1976, 1994; Adler \& Crosskey 2018.

Simulium (Simulium) morsitans Edwards, 1915 - DW, P1, R1, R5, RW, RE; 100-2000 m; 1, 2, 3, 4; tes; Russev et al. 1976, 1994; Kovachev 1975, 2000; Russev \& Janeva 1986; Beron 2004; Beschovski 2006; Uzunov et al. 2011; Varadinova et al. 2013.

Simulium (Simulium) noelleri Friederichs, 1920 [S. argyreatum: authors (incl. Rothfels, 1979), not Meigen] B1, B2, K3, V4, O62, R1, R5, RW; 180-1800 m; 1, 2, 3, 4; h; Kovachev 1985c, 1990, 2000; Islam et al. 1986; Russev et al. 1994; Uzunov et al. 2011; Varadinova et al. 2013.

Simulium (Simulium) ornatum Meigen, 1818 [Odagmia konsuloff (Enderlein, 1924); S. pratorum Friederichs, 1920] - DW, DM, E1, E2, P1, P2, P3, B1, B2, K3, K7, K8, V1, V4, V5, S1, S21, S22, S211, O61, O62, R1, R2, R5, RW, RE; 50-1100 m; 1, 2, 3; hop; Enderlein, 1924; Konsuloff \& Paspalev 1925; Tschorbadjiew 1925e; Buresch 1926, 1938; Rubtsov 1956; Kovachev 1975, 1976, 1985a, 1985c, 1990; Russev et al. 1976, 1984b, 1987, 1994; Russev 1977; Uzunov et al. 1981; Janeva \& Russev 1985, 1989, 1997; Islam et al. 1986; Janeva 1987, 1989, 1991; Rubtsov \& Yankovsky 1988; Janeva et al. 2001; Janeva \& Bancheva 2002; Beschovski 2006; Kenderov et al. 2008; Sakelarieva et al. 2008; Moskova \& Uzunov 2011; Borisova et al. 2013; Stoyanova et al. 2013; Varadinova et al. 2013; Adler \& Crosskey 2018.

Simulium (Simulium) paramorsitans Rubtsov, 1956 - ; tes; Rubtsov \& Yankovsky 1988; Adler \& Crosskey 2018.

Simulium (Simulium) reptans (Linnaeus, 1758) [S. galeratum Edwards, 1920; S. latimanus Enderlein, 1921; S. reptans var. galeratum Edwards, 1920] - P1, B1, V1, K3, K7, K8, V4, O62, R1, R5, RW; 400-2000 m; 1, 2, 3, 4; wcp, ? h, hoes; Nedelkov 1912; Enderlein, 1924; Konsuloff \& Paspalev 1925; Buresch 1938; Kovachev 1973, 1976, 1979, 1985a, 1985c, 1990, 2000; Russev et al. 1976, 1994; Uzunov et al. 1981, 2011; Islam et al. 1986; Varadinova et al. 2013; Adler \& Crosskey 2018.

Simulium (Simulium) rotundatum (Rubtsov, 1956) [Odagmia] - RE; 170-220 m; 1; e; Kovachev 1969; Adler \& Crosskey 2018.

Simulium (Simulium) simoff (Enderlein, 1924) - DW, P1, B1, V1; 50-600 m; 1, 2; Ebg; Enderlein, 1924; Konsuloff \& Paspalev 1925; Tschorbadjiew 1925e; Buresch 1926, 1938; Rubtsov 1956; Russev et al. 1994; Adler \& Crosskey 2018.

Simulium (Simulium) trifasciatum Curtis, 1839 [S. spinosum Doby \& Deblock, 1957; Odagmia; Wilhelmia] DW, DM, E1, E2, P1, P2, P3, V4, S1, R1, R2, R5, RW, RE; 100-950 m; 1, 2, 3; eanna; Kovachev 1975, 1976, 1985a, 1985c, 1990; Russev et al. 1976, 1987, 1994; Uzunov et al. 1981; Janeva \& Russev 1985, 1997; Islam et al. 1986; Janeva et al. 2001; Beschovski 2006; Uzunov et al. 2011; Borisova et al. 2013; Stoyanova et al. 2013; Varadinova et al. 2013.

Simulium (Simulium) tuberosum (Lundstrom, 1911) - DM, P2, B2, V4, R1; 300-2300 m; 1, 2, 3, 4, 5; h; Kovachev 1969, 1976, 1985a, 1990, 2000; Russev et al. 1976, 1994; Uzunov et al. 1981; Janeva \& Russev 1997; Adler \& Crosskey 2018.

Simulium (Simulium) variegatum Meigen, 1818 [Friesia bulgarica (Enderlein, 1921); Odagmia] - DW, DM, P1, P2, V4, S1, O61, R1, R2, R5, RW, RE; 300-2300 m; 1, 2, 3, 4, 5; wp; Enderlein, 1921; Kovachev 1975, 1976, 1979, 1985a, 1985c, 1990, 2000; Russev et al. 1976, 1994; Russev 1977; Uzunov et al. 1981, 2011; Janeva 1987; Janeva \& Russev 1997; Janeva et al. 2001; Janeva \& Bancheva 2002; Beron 2004; Beschovski 2006; Sakelarieva et al. 2008; Moskova \& Uzunov 2011; Stoyanova et al. 2013; Varadinova et al. 2013; Adler \& Crosskey 2018.

? Simulium (Simulium) verecundum Stone \& Jamnback, 1955 - B2, V4, R1, R5; 600-2000 m; 3, 4; ? h, bm; Kovachev 1976, 1990, 2000; Russev et al. 1976, 1994; Uzunov et al. 1981; Islam et al. 1986; Janeva \& Russev 1997; [according to Adler \& Crosskey (2018) not Old World (misidentified)].

Simulium (Eusimulium) aureum Fries, 1824 - DW, DM, P1, P2, V3, V4, V5, S1, S211, O62, R1, RW; 10-2000 m; 1, 2, 3, 4; wes, ? po; Kovachev 1969, 1975, 1976, 1979, 1990, 2000; Russev et al. 1976, 1994; Russev 
1977; Islam et al. 1986; Russev \& Janeva 1986; Janeva 1987, 1991; Janeva \& Russev 1989, 1997; Janeva et al. 2001; Janeva \& Bancheva 2002; Beschovski 2006; Adler \& Crosskey 2018.

Simulium (Eusimulium) krymense (Rubtsov, 1956) - ; see; Rubtsov \& Yankovsky 1988; Adler \& Crosskey 2018. Simulium (Eusimulium) rubzovianum (Sherban, 1961) [S. velutinum (Santos Abreu, 1922; S. serbicum Baranov, 1925; E. latinum (Rubtsov, 1962)] - DW, DM, P1, P2, K8, K9, V4, S1, O62, R1, R5, RW; 80-2200 m; 1, 2, 3, 4; wp; Kovachev 1976, 1979, 1985a, 1985c, 1990, 2000; Russev et al. 1976, 1994; Russev 1977; Uzunov et al. 1981; Islam et al. 1986; Janeva 1987, 1991; Janeva \& Russev 1997; Janeva \& Bancheva 2002; Uzunov et al. 2011; Varadinova et al. 2013.

Simulium (Eusimulium) angustipes Edwards, 1915 [E. latizonum (Rubtsov, 1956); E. securiforme (Rubtsov, 1956)] - DW, DM, E2, P1, P2, O62, R1, R2, RW; 20-2240 m; 1, 2, 3, 4, 5; wcp; Russev 1962, 1977; Kovachev 1976, 1979, 1985c, 2000; Russev et al. 1976, 1994; Janeva \& Russev 1997.

Simulium (Schoenbaueria) nigrum (Meigen, 1804) [S. behningi Enderlein, 1926] - P2, 140-357 m; 1; eca; Russev et al. 1976; Russev 1977.

Simulium (Schoenbaueria) pusillum Fries, 1824 [Eusimulium pygmaeum (Zetterstedt, 1838); ? E. pygmaeum: Rubtsov, 1956 (part); ? Simulium (Nevermannia) meigeni (Rubtsov \& Carlsson, 1965)] - RW; 1100 m; 3; tes; Kovachev 1975; Beschovski 2006.

Simulium (Wilhelmia) angustifurca (Rubtsov, 1956) - P2, K4, K5; 450-900 m; 1, 2; ees; Kovachev 1969; Russev et al. 1994; Adler \& Crosskey 2018.

Simulium (Wilhelmia) balcanicum (Enderlein, 1924) [W. balcanica severinense (Dinulescu, 1966)] - DW, DM, E1, E2, P1, P2, P3, B1, B2, K3, K7, K8, K9, V1, V4, S1, TL, T1, O61, O62, R1, R5, RR; 20-700 m; 1, 2; ean, ? eant; Enderlein, 1924; Tschorbadjiew 1925e; Buresch 1926b, 1938; Rubtsov 1956; Russev 1962, 1966b; Kovachev 1975, 1976, 1985a, 1985c, 1990; Russev et al. 1976, 1987, 1994; Uzunov et al. 1981, 2011; Janeva \& Russev 1985, 1997; Islam et al. 1986; Janeva 1987; Janeva \& Bancheva 2002; Borisova et al. 2013; Varadinova et al. 2013; Adler \& Crosskey 2018; Đuknić et al. 2019.

Simulium (Wilhelmia) equinum (Linnaeus, 1758) [W. secundum (Baranov, 1926); W. equina ivashentzovi Rubtsov, 1940] - DW, DM, P1, P2, B1, V1, V5, O62; 20-820 m; 1, 2; tp, ? po; Enderlein, 1924; Tschorbadjiew 1925e; Buresch 1938; Russev 1962, 1977; Kovachev 1969; Russev et al. 1976, 1994; Kenderov et al. 2008; Adler \& Crosskey 2018; Đuknić et al. 2019.

Simulium (Wilhelmia) lineatum (Meigen, 1804) [W. equina falcula Enderlein, 1921] - DW, DM, P1, P2, B1, B2, K7, V1, S1, S21, TL, R1, RE; 50-950 m; 1, 2; e; Enderlein, 1924; Konsuloff \& Paspalev 1925; Tschorbadjiew 1925e; Buresch 1938; Kovachev 1975, 1976, 1985a; Russev et al. 1976, 1984b, 1994; Russev 1977; Beron 2004; Beschovski 2006; Adler \& Crosskey 2018.

Simulium (Wilhelmia) paraequinum Puri, 1933 - DW, DM, P1, P2; 20-400 m; 1; oem, ? emit; Russev et al. 1994; Adler \& Crosskey 2018.

Simulium (Wilhelmia) pseudequinum Seguy, 1921 [W. fluminicola (Rivosecchi, 1972); W. stylatum (Baranov, 1926); S. mediterraneum Puri, 1925] - DW, DM, E1, E2, P1, P2, P3, K3, K7, K8, V4, S1, S21, S22, TL, T1, O61, O62, R1, R5, RW, RE; 100-900 m; 1, 2; spo; Kovachev 1976, 1985a, 1985c, 1990; Russev et al. 1976, 1984b, 1987, 1994; Russev 1977; Uzunov et al. 1981, 2011; Janeva \& Russev 1985, 1997; Islam et al. 1986; Janeva 1987, 1989, 1991; Rubtsov \& Yankovsky 1988; Janeva et al. 2001; Janeva \& Bancheva 2002; Borisova et al. 2013; Varadinova et al. 2013; Đuknić et al. 2019.

Simulium (Boophthora) erythrocephalum (De Geer, 1776) - P1, B1, V1, V4, S1, R1; 200-1000 m; 1, 2, 3; tp; Russev et al. 1976, 1994; Uzunov et al. 1981; Kovachev 1985a, 1990; Borisova et al. 2013; Georgieva et al. 2017.

Simulium (Trichodagmia) auricoma Meigen, 1818 [Obuchovia] - B1, B2, V4, R1; 1000-2400 m; 3, 4, 5; eanna; Enderlein, 1924; Konsuloff \& Paspalev 1925; Buresch 1938; Kovachev 1976, 1979, 1990, 2000; Russev et al. 1976, 1994; Adler \& Crosskey 2018.

Simulium (Trichodagmia) popowae Rubtsov, 1940 [Obuchovia] - V3; 700-800 m; 2; see; Kovachev 1969; Adler \& Crosskey 2018.

\section{Ceratopogonidae (Heleidae)}

Mallochohelea remota (Kieffer, 1919) [Sphaeromias nitida var. bulgarica (Zilahi-Sebess, 1934)] - E1; 250-300 m; 1; ? e; Zilahi 1934.

Palpomyia flavipes (Meigen, 1804) - V4; 700-800 m; 2; ? wp; Zilahi 1934. 
Palpomyia nana Zilahi-Sebess, 1934 - B1; 400-450 m; 1; Ebg; Zilahi 1934.

Ceratopogon niveipennis Meigen, 1818 [Psilohelea] - V1; 550 m; 1, 2; e; Nedelkov 1912.

Dasyhelea (Sebessia) acuminata Kieffer, 1919 [D. verticillata Kieffer, 1925] - B1; 400-450 m; 1; e; Zilahi 1934; Dominiak \& Szadziewski 2010.

Dasyhelea (Prokempia) flaviventris (Goetghebuer, 1910) - R1, R2; 400-1400 m; 1, 2. 3; dp; Dominiak \& Szadziewski 2010.

Dasyhelea (Dasyhelea) bilineata Goetghebuer, 1920 [D. geleiana Zilahi-Sebess, 1930] - V4, R1, R2, RW; 4002200 m; 1, 2, 3, 4, 5; eanna; Valkanov 1941; Dominiak \& Szadziewski 2010.

Dasyhelea (Dasyhelea) flavifrons (Guérin, 1833) - R1, R2; 400-1300 m; 1, 2, 3; h; Dominiak \& Szadziewski 2010.

Dasyhelea (Dasyhelea) halophila Kieffer, 1911 - BS; 0-20 m; 1; see, ? se; Valkanov 1954.

Dasyhelea (Pseudoculicoides) abhazica Remm, 1967 - R2; 400 m; 1; bc, m, ? see; Dominiak \& Szadziewski 2010.

Dasyhelea (Pseudoculicoides) arenivaga Macfie, 1943 - R2; 400 m; 1; ena; Dominiak \& Szadziewski 2010.

Dasyhelea (Pseudoculicoides) bicrenata Kieffer, 1923 - en ena; Dominiak \& Szadziewski 2010.

Dasyhelea (Pseudoculicoides) calycata Remm, 1972 - BS; 0-10 m; 1; eca; Dominiak \& Szadziewski 2010.

Dasyhelea (Pseudoculicoides) communis Kieffer, 1918 - BN; 0-50 m; 1; hom; Zilahi 1934.

Dasyhelea (Pseudoculicoides) fasciigera Kieffer, 1925 - R2; 400 m; 1; h; Dominiak \& Szadziewski 2010.

Dasyhelea (Pseudoculicoides) sericata (Winnertz, 1852) - V4; 700 m; 2; ewca; Zilahi 1934.

Dasyhelea (Dicryptoscena) modesta (Winnertz, 1852) - R1; 1580 m; 3, 4; tp; Dominiak \& Szadziewski 2010.

Dasyhelea (Dicryptoscena) thienemanni Spataru \& Damian-Georgescu, 1970 - \$; ee, ? e; Dominiak \& Szadziewski 2010.

Atrichopogon (Atrichopogon) fuscus (Meigen, 1804) [Kempia] - B1, BN; 0-400 m; 1; wp, ? ena; Zilahi 1934.

Atrichopogon (Atrichopogon) minutus (Meigen, 1830) - TL, RW; 180-380 m; 1; h; Zilahi 1934.

Atrichopogon (Atrichopogon) psilopterus Kieffer, 1919 - TL, RW; 220-380 m; 1; e; Zilahi 1934.

Atrichopogon (Atrichopogon) tritomus Kieffer, 1919 - RW; 380 m; 1; see; Zilahi 1934.

Atrichopogon (Lophomyidium) rostratus (Winnertz, 1852) [A. transversalis Kieffer, 1918] - B1, RW; 350-450 m; 1; eanna; Zilahi 1934.

Atrichopogon (Psammopogon) flavolineatus (Strobl, 1880) [A.trifasciatus Kieffer, 1918] - B1, RW; 370-380 m; 1; ? hom, ? csena; Zilahi 1934.

Forcipomyia (Lasiohelea) velox (Winnertz, 1852) - B1; 400-450 m; 1; wp; Zilahi 1934.

Forcipomyia (Euprojoannisia) bureschi (Zilahi-Sebess, 1934) - RW; 370-400 m; 1; Ebg; Zilahi 1934; Josifov 1957.

Forcipomyia (Forcipomyia) bipunctata (Linnaeus, 1767) - DW, V4, RW; 50-800 m; 1, 2; h; Zilahi 1934.

Forcipomyia (Forcipomyia) pallidipes Santos Abreu, 1918 [F. rustica (Kieffer, 1919)] - V1, TL, R1; 150-1400 m; 1, 2, 3; wp; Zilahi 1934.

Culicoides (Beltranmyia) circumscriptus Kieffer, 1918 [C. pulcher Zilahi-Sebess, 1934] - DW, E2, P2, TL, T1, T2, O61, RE; 20-370 m; 1; ppt; Zilahi 1934; Bobeva et al. 2013, 2014; Nedelchev 2013; Bobeva 2014; Pudar et al. 2018.

Culicoides (Beltranmyia) salinarius Kieffer, 1914 - DW, DM, E2, P1, P2, K9, TV, V1, TL, T1, T2, O61, RW, RE, BN, BS; 0-1000 m; 1, 2; tp; Bobeva et al. 2013; Nedelchev 2013; Pudar et al. 2018.

Culicoides (Culicoides) deltus Edwards, 1939 - E2, S1, TL, T1, T2, RW, BS ; 0-1000 m; 1, 2; des; Bobeva et al. 2013; Nedelchev 2013; Pudar et al. 2018.

Culicoides (Culicoides) fagineus Edwards, 1939 - DW, E1, E2, P1, P2, B1, K9, TV, V1, T1, T2, O61, RW, RE, BS; 20-1000 m; 1, 2; wcp; Bobeva et al. 2013; Nedelchev 2013; Pudar et al. 2018.

Culicoides (Culicoides) flavipulicaris Dhafarov, 1964 - E2, K9, TV, V1; 20-700 m; 1, 2; dp, ? nmca; Bobeva et al. 2013; Nedelchev 2013; Pudar et al. 2018.

Culicoides (Culicoides) grisescens Edwards, 1939 - E2, P2, TV, V1, T1, T2, RE, BS; 0-600 m, 1, 2; tp; Bobeva et al. 2013; Nedelchev 2013; Pudar et al. 2018.

Culicoides (Culicoides) impunctatus Goetghebuer, 1920 - DW, E2, P1, B1, TV, V1, O61, BN, BS; 0-700 m; 1, 2; wces; Bobeva et al. 2013; Nedelchev 2013; Pudar et al. 2018.

Culicoides (Culicoides) newsteadi Austen, 1921 [C. halophilus Kieffer, 1924] - $\mathbf{\Delta}$; DW, DM, E1, E2, P1, P2, B1, K9, TV, V1, S1, TL, T1, T2, O61, RE, BN, BS; 0-700 m; 1, 2; wp; Bobeva et al. 2013; Nedelchev 2013; Bobeva 2014; Pudar et al. 2018. 
Culicoides (Culicoides) pulicaris (Linnaeus, 1758) [Ceratopogon] - $\mathbf{\Delta}$; DW, DM, E1, E2, P1, P2, B1, K9, TV, V1, S1, T1, T2, TL, RW, RE, BN, BS; 0-1000 m; 1, 2; pat; Nedelkov 1912; Bobeva et al. 2013; Nedelchev 2013; Pudar et al. 2018.

Culicoides (Culicoides) punctatus (Meigen, 1804) - $\Delta$; DW, DM, E1, E2, P1, P2, B1, K9, TV, V1, S1, TL, T1, T2, O61, RE, RW, BN, BS; 0-1000 m; 1, 2; pat; Bobeva et al. 2013; Nedelchev 2013; Bobeva 2014; Pudar et al. 2018.

Culicoides (Avaritia) dewulfi Goetghebuer, 1936 - E2, P2; 20-250 m; 1; dp; Bobeva et al. 2013; Nedelchev 2013; Pudar et al. 2018.

Culicoides (Avaritia) obsoletus (Meigen, 1818) - $\boldsymbol{\Delta}$; DW, DM, E1, E2, P1, P2, B1, K9, V1, S1, TV, TL, T1, T2, RW, RE, BN, BS; 0-1000 m; 1, 2; h; Zilahi 1934; Bobeva et al. 2013; Nedelchev 2013; Bobeva 2014; Pudar et al. 2018.

Culicoides (Avaritia) scoticus Downes \& Kettle, 1952 - E2, RE; 20-250 m; 1; wcp; Bobeva et al. 2013; Nedelchev 2013; Pudar et al. 2018.

Culicoides (Silvaticulicoides) fascipennis (Staeger, 1839) - DW, DM, E1, E2, P1, P2, B1, B2, K9, V1, S1, TL, T1, T2, O61, RW, RE, BN, BS; 0-1000 m; 1, 2; tp; Bobeva et al. 2013; Nedelchev 2013; Pudar et al. 2018.

Culicoides (Silvaticulicoides) ostroushkoae Glukhova, 1989 - K9, TV, V1, O61; 300-700 m; 1; et, ? ewca; Bobeva et al. 2013; Nedelchev 2013; Pudar et al. 2018.

Culicoides (Silvaticulicoides) pallidicornis Kieffer, 1919 [C. pallidicornis var. bruneoscutellatus Zilahi-Sebess, 1934] - E1, E2, P2, K9, TV, V1, TL, T1, T2, O61, R1, RE, BN, BS; 0-1400 m; 1, 2, 3; h; Zilahi 1934; Bobeva et al. 2013; Nedelchev 2013; Pudar et al. 2018.

Culicoides (Silvaticulicoides) subfasciipennis Kieffer, 1919 - DM, E1, E2, P1, P2, B1, K9, TV, V1, TL, T1, T2, O61, RE, BN, BS; 0-700 m; 1, 2; wcp; Bobeva et al. 2013; Nedelchev 2013; Pudar et al. 2018.

Culicoides (Sensiculicoides) alazanicus Dzhafarov, 1961 - E2; 20 m; 1; ean; Bobeva 2014; Bobeva et al. 2014, 2015; Pudar et al. 2018.

Culicoides (Sensiculicoides) festivipennis Kieffer, 1914 - DM, E1, E2, P1, P2, B1, K9, V1, TL, T1, T2, O61, R1, BS; 0-600 m; 1, 2; hop; Bobeva et al. 2013, 2014; Nedelchev 2013; Bobeva 2014; Pudar et al. 2018.

Culicoides (Sensiculicoides) gejgelensis Dzhafarov, 1964 - E2, K9, TV, V1, S1, O61, RW, RE, BS; 0-1000 m; 1, 2; mca; Bobeva et al. 2013; Nedelchev 2013; Pudar et al. 2018.

Culicoides (Sensiculicoides) griseidorsum Kieffer, 1918 - E2; 20 m; 1; ena; Bobeva 2014; Szadziewski et al. 2016.

Culicoides (Sensiculicoides) kurensis Dzhafarov, 1960 - DW, P1, E1, E2, B1, V1, RW, RE; 30-1000 m; 1, 2; hom; Bobeva et al. 2013; Nedelchev 2013; Pudar et al. 2018.

Culicoides (Sensiculicoides) odiatus Austen, 1921 - DW, DM, E1, E2, P1, P2, B1, K9, V1, T1, T2, O61, RE, BS; 0-600 m; 1, 2; wcp; Bobeva et al. 2013; Nedelchev 2013; Pudar et al. 2018.

Culicoides (Sensiculicoides) pictipennis (Staeger, 1839) [C. arcuatus (Winnertz, 1852)] - DW, E2, P1, P2, B1, TV, V1, T1, T2, O61, R1, RW, RE; 30-2900 m; 1, 2, 3, 4, 5, 6; wcp; Zilahi 1934; Bobeva et al. 2013; Nedelchev 2013; Bobeva 2014; Pudar et al. 2018.

Culicoides (Sensiculicoides) shaklawensis Khalaf, 1957 - E2; 20 m; 1; mwca; Bobeva et al. 2013; Pudar et al. 2018.

Culicoides (Sensiculicoides) simulator Edwards, 1939 - DM, E1, E2, V1, S1, TL, T1, T2, BN, BS; 0-600 m; 1, 2; wcp; Bobeva et al. 2013; Nedelchev 2013; Pudar et al. 2018.

Culicoides (Oecacta) longipennis Khalaf, 1957 - E2, K9, V1, TV, S1, O61, RW, RE; 20-1000 nm; 1, 2; mwca; Bobeva et al. 2013; Nedelchev 2013; Pudar et al. 2018.

Culicoides (Oecacta) vexans (Staeger, 1839) - E1, E2, P2, TV, V1, S1, TL, T1, T2, RE, BN, BS; 0-700 m; 1, 2; esca; Bobeva et al. 2013; Nedelchev 2013; Pudar et al. 2018.

Culicoides (Monoculicoides) nubeculosus (Meigen, 1830) - $\mathbf{\Delta}$; DW, E1, E2, P1, P2, K9, V1, S1, O61, RE, BS; 0-600 m; 1, 2; des, ? tes; Bobeva et al. 2013; Nedelchev 2013; Bobeva 2014; Pudar et al. 2018.

Culicoides (Monoculicoides) parroti Kieffer, 1922 - DM, E2, P1, P2, TL, RE, BN, BS; 0-300 m; 1; wp; Bobeva et al. 2013; Nedelchev 2013; Pudar et al. 2018.

Culicoides (Monoculicoides) puncticollis (Becker, 1903) [C. impressus Kieffer, 1918] - $\mathbf{\Delta}$; DW, E1, E2, P1, K9, TV, V1, S1, TL, T1, T2, O61, RW, RE, BN, BS; 0-1000 m; 1, 2; sp; Zilahi 1934; Bobeva et al. 2013; Nedelchev 2013; Bobeva 2014; Pudar et al. 2018.

Culicoides (Monoculicoides) riethi Kieffer, 1914 - DM, E1, E2, P1, P2, B1, K9, V1, S1, TL, T1, T2, O61, RE, BN, BS; 0-600 m; 1, 2; po, ? ho; Bobeva et al. 2013; Nedelchev 2013; Bobeva 2014; Pudar et al. 2018. 
Culicoides (Monoculicoides) stigma (Meigen, 1818) - E2, P1, P2, B1, V1, TL, T1, T2, RW, RE, BS; 0-1000 m; 1, 2; wp; Bobeva et al. 2013; Nedelchev 2013; Pudar et al. 2018.

Culicoides (Wirthomyia) reconditus Campbell \& Pelham-Clinton, 1960 - DW, E2, P1, P2, B1, S1, TL, RE, BS; 0-450 m; 1; e; Bobeva et al. 2013; Nedelchev 2013; Pudar et al. 2018.

Culicoides (Pontoculicoides) saevus Kieffer, 1922 [C. drenskii Zilahi-Sebess, 1934] - E2, B1, V1; 20-600 m; 1, 2; wcp; Zilahi 1934; Bobeva et al. 2013; Nedelchev 2013; Pudar et al. 2018; Darlenski et al. 2020.

Culicoides (Pontoculicoides) sejfadinei Dzhafarov, 1958 - E2, K9, TV, V1, S1, T1, T2, O61, RE, RW; 20-1000 m; 1, 2; wcp; Bobeva et al. 2013; Nedelchev 2013; Pudar et al. 2018.

Culicoides (Pontoculicoides) tauricus Gutsevich, 1959 - DW, DM, E1, E2, P1, B1; 20-400 m; 1; ? cset; Bobeva et al. 2013; Nedelchev 2013; Pudar et al. 2018.

Culicoides (Remmia) schultzei (Enderlein), 1908 - $\Delta$; DW, DM, E1, E2, P1, P2, B1, TL, T1, T2; 20-450 m; 1; ppta; Bobeva et al. 2013; Nedelchev 2013; Pudar et al. 2018.

\section{Chironomidae}

Paraboreochlus minutissimus (Strobl, 1895) [Ablabesmyia pecteniphora Goetghebuer, 1934] - RW; 1100 m; 3; wp; Dimitrov 1962a, 1963a.

Clinotanypus nervosus (Meigen, 1818) - DM, E1, E2, P2, S1, T1, O61; 30-500 m; 1; ? tp, ? dp; Dimitrov 1957, 1963a; Russev et al. 1994; Stoichev 1994, 1996; Janeva \& Russev 1997.

Tanypus (Tanypus) kraatzi (Kieffer, 1912) [Pelopia] - E1, P2, TL, BS; 0-300 m; 1; tp; Mihailova-Neikova 1961; Dimitrov 1962b, 1963a; Cvetkov 1962; Russev et al. 1994; Stoichev 1994, 1996; Janeva \& Russev 1997.

Tanypus (Tanypus) punctipennis Meigen, 1818 [Pelopia; Protenthes punctipennis var. ferrugineus Kieffer, 1918] - DW, DM, E1, E2, P2, V1, V5, S211, S1, TL, RW, RE, BN, BS; 0-900 m; 1, 2; hno; Zilahi 1934; Cvetkov 1955a, 1955b, 1957, 1962; Valkanov 1957; Dimitrov 1957, 1960a, 1960b, 1962a, 1962b, 1962c, 1962d, 1963a, 1966, 1970, 1972, 1982; Mihailova-Neikova 1961; Russev 1966a, 1966b; Janeva 1987, 1991; Janeva \& Russev 1989; Russev et al. 1994; Stoichev 1994, 1996; Janeva \& Russev 1997.

Procladius (Holotanypus) choreus (Meigen, 1804) - DW, DM, E1, E2, P1, P2, K3, TL, T1, RW; 20-1500 m; 1, 2, 3, 4; po, ? ho; Michailova 1982, 2006; Islam et al. 1986; Russev et al. 1994; Stoichev 1994, 1996; Janeva \& Russev 1985, 1997; Janeva 1991.

Procladius (Holotanypus) ferrugineus (Kieffer, 1918) - DW, DM, E1, E2, P1, V4, T1; 20-800 m; 1, 2; tes, ? dp; Islam et al. 1986; Russev et al. 1994; Stoichev 1994, 1996; Janeva \& Russev 1985, 1997; Janeva 1991; Uzunov et al. 2011; Varadinova et al. 2013.

Procladius (Psilotanypus) imicola Kieffer, 1922 [P. nigriventris Kieffer, 1922] - DW, DM, T1; 132 m; 1; wces, ? wes; Janeva \& Russev 1985; Stoichev 1994, 1996.

Anatopynia plumipes (Fries, 1823) - DW, DM, E1, E2, P2, P3, S1, T1, R1, RW, RE, BN, BS; 0-2340 m; 1, 2, 3, 4, 5; dp, ? tp; Michailova 1982, 2006; Russev et al. 1994; Stoichev 1994, 1996, 2000a, 2002; Janeva \& Russev 1997.

Apsectrotanypus trifascipennis (Zetterstedt, 1838) [Anatopynia] - DW, E1, E2, B2, V5, R1; 20-1150 m; 1, 2, 3; ? wes; Dimitrov 1963a; Russev et al. 1987, 1994; Stoichev 1994, 1996; Dashinov 2017.

Macropelopia nebulosa (Meigen, 1804) - DW, DM, E1, V1, RW; 50-790 m; 1, 2; dpo; Janeva 1989; Russev et al. 1994; Stoichev 1996; Janeva \& Russev 1997; Michailova et al. 2014.

Psectrotanypus (Psectrotanypus) varius (Fabricius, 1787) [Anatopynia, Tanypus] - DW, V1, S22, TL, O61, RW; 30-1100 m; 1, 2, 3; po, ? ho; Nedelkov 1912; Dimitrov 1963a; Michailova 1982; Islam et al. 1986; Russev \& Janeva 1986; Russev et al. 1994.

Derotanypus sibiricus (Kruglova \& Chernovskij, 1940) [Anatopynia, Psectrotanypus] - S22; 350 m; 1; tes; Dimitrov 1963a.

Natarsia punctata (Fabricius, 1805) [Ablabesmyia fulva (Kieffer, 1918)] - DW, DM, E1, P2, TL, O61; 50-380 m; 1, des; Dimitrov 1969; Nachev 1983; Russev et al. 1984, 1991, 1994; Janeva \& Russev 1997.

Ablabesmyia (Ablabesmyia) longistyla Fittkau, 1962 - DW; 30-50 m; 1; dp, ? pat; Stoichev 1994, 1996.

Ablabesmyia (Ablabesmyia) monilis (Linnaeus, 1758) - DW, DM, E1, E2, P1, P2, V1, S1, TL, R1, RW, RE, BN; 0-1500 m; 1, 2, 3; hno; Cvetkov 1955a, 1962; Valkanov 1957a; Russev 1959, 1962, 1966a, 1966b; Dimitrov 1962b, 1963a, 1966; Russev \& Janeva 1975; Michailova 1982; Russev et al. 1984b, 1994; Stoichev 1994, 1996; Janeva \& Russev 1997; Soufi \& Uzunov 2008; Vidinova et al. 2008.

Conchapelopia intermedia Fittkau, 1962 - K9, O61;315-385 m; 1; e; Islam et al. 1986. 
Conchapelopia melanops (Meigen, 1818) - DW, DM, P1, P2, K7, K8, O61, O62; 20-450 m; 1, 2; dpo; Islam et al. 1986; Islam et al. 1986; Russev \& Janeva 1986; Russev et al. 1994; Janeva \& Russev 1997.

Conchapelopia pallidula (Meigen, 1818) - R5; 400-800 m; 1, 2; dp; Uzunov et al. 2011; Varadinova et al. 2013. Guttipelopia guttipennis (van der Wulp, 1861) [Ablabesmyia zavreli (Kieffer, 1918)] - R1; 2250-2324 m; 5; h; Stoichev 2000a.

Krenopelopia binotata (Wiedemann, 1817) [Ablabesmyia] - DM, P2, V5, S211, O61; 50-700 m; 1, 2; dp; Dimitrov 1963a; Nachev 1983; Russev et al. 1984, 1994; Islam et al. 1986.

Labrundinia longipalpis (Goetghebuer, 1921) - DW, DM, P1, P2; 670 m; 1, 2; e, ? h; Janeva 1987; Russev et al. 1994.

Larsia atrocincta (Goetghebuer, 1942) - R5; 1, 2; ena; Uzunov et al. 2011; Varadinova et al. 2013.

Larsia curticalcar (Kieffer, 1918) [Ablabesmyia] - DW, DM, E1, E2, P1, P2, B1, V1, V5, S1, S21, S22, S211, T1, R1, R2, R5, RW, RE, BN, BS; 20-2440 m; 1, 2, 3, 4, 5; wp; Russev 1959, 1962, 1966a, 1966b; Dimitrov 1962a, 1962d, 1963a, 1966; Russev \& Janeva 1975; Russev et al. 1984b, 1994; Janeva 1991; Stoichev 1994, 1996, 2002; Janeva \& Russev 1997; Janeva et al. 2001; Janeva \& Bancheva 2002; Moskova \& Uzunov 2011; Uzunov et al. 2011; Kenderov et al. 2012.

Monopelopia tenuicalcar (Kieffer, 1918) [Ablabesmyia] - V5, S211; 700 m; 2; h; Dimitrov 1962d; Russev et al. 1994.

Nilotanypus dubius (Meigen, 1804) - DW, P1, R1; 100-1960 m; 1, 2, 3, 4; dpo; Russev et al. 1994; Dashinov 2017. Rheopelopia maculipennis (Zetterstedt, 1838) - DW, P1; 210 m; 1; dp; Janeva 1991; Russev et al. 1994.

Telmatopelopia nemorum (Goetghebuer, 1921) - R5; 500-800 m; 1, 2; dp; Uzunov et al. 2011; Varadinova et al. 2013.

Thienemannimyia lentiginosa (Fries, 1823) - DW, P1, P2, B2, BN; 0-660 m; 1, 2; dp; Cvetkov 1955a; Valkanov 1957; Dimitrov 1963a; Russev 1966b; Janeva 1987; Russev et al. 1991, 1994; Stoichev 1994, 1996.

Trissopelopia flavida Kieffer, 1923 [Ablabesmyia] - V5, S211, TL, T1, RW, RE; 88-1000 m; 1, 2; et; Dimitrov 1962a, 1962c, 1962d, 1963a, 1966; Russev 1966a; Russev et al. 1984b, 1994.

Xenopelopia falcigera (Kieffer, 1911) [Ablabesmyia] - S1, O61, RW; 350-1100 m; 1, 2, 3; des; Dimitrov 1960a, 1962a, 1963a; Nachev 1983; Islam et al. 1986; Janeva 1989.

Zavrelimyia melanura (Meigen, 1804) [Ablabesmyia tretrastictus Kieffer, 1918] - DM, P1, P2, R1, RW; 40-1400 m; 1, 2, 3; wp; Dimitrov 1962d, 1963a; Janeva 1987; Russev et al. 1994; Stoichev 1994, 1996.

Zavrelimyia signatipennis (Kieffer, 1924) - DM, P2; e, ? cse; Russev et al. 1994.

Boreoheptagyia cinctipes (Edwards, 1928) - \$; ? se; Russev et al. 1994.

Boreoheptagyia legeri (Goetghebuer, 1933) [Heptagyia punctulata Goetghebuer, 1934] - O61, RW; 316-1260 m; 1, 2, 3; csena, ? ena; Russev \& Janeva 1975; Nachev 1983; Ashe \& Cranston 1990; Russev et al. 1994.

Diamesa (Diamesa) aberrata Lundbeck, 1898 - RW; 800 m; 2; ho; Michailova 1989, 2006.

? Diamesa (Diamesa) carpatica Botnariuc \& Cindea-Cure, 1954 [? Nomen dubium] - P2, RW; 40-1250 m; 1, 2, 3; see; Janeva 1987, 1989; Michailova 1989, 2006.

Diamesa (Diamesa) cinerella Meigen, 1835 - RW; 400-1300 m; 1, 2, 3; e; Michailova 1989.

Diamesa (Diamesa) insignipes Kieffer, 1908 [D. prolongata Kieffer, 1909] - DW, DM, P1, P2, K8, V4, V5, S1, S211, O61, R1, R5, RW, RE; 40-2440 m; 1, 2, 3, 4, 5; h; Dimitrov 1960a, 1962b, 1962c, 1962d, 1963a, 1966; Russev \& Janeva 1975; Nachev 1983; Russev et al. 1984b, 1991, 1994; Janeva \& Russev 1985, 1989, 1997; Islam et al. 1986; Janeva 1987, 1989; Michailova 1982, 1989, 2006; Stoichev 1994, 1996, 2000; Sakelarieva et al. 2008; Uzunov et al. 2011; Trichkova et al. 2013; Varadinova et al. 2013.

? Diamesa (Diamesa) heterodentata (Botnariuc et Cindea-Cure, 1954) [? Nomen dubium] - DW, DM, P1, P2, K7, K8, K9, T1, O61, O62; 27-510 m; 1; see; Nachev 1983; Janeva \& Russev 1985; Islam et al. 1986; Janeva 1987, 1991; Russev et al. 1991.

Diamesa (Diamesa) latitarsis (Goetghebuer, 1921) - P2; 400-500 m; 1; tp; Janeva 1987; Russev et al. 1994; Stoichev 1994, 1996; Janeva \& Russev 1997.

? Diamesa (Diamesa) pseudostylata (Chernovskij, 1949) [? Nomen dubium] - DW, DM, P1, P2; 30-250 m; 1; ee; Janeva 1987; Russev et al. 1994; Stoichev 1994, 1996; Janeva \& Russev 1997.

Diamesa (Diamesa) tonsa (Haliday, 1856) [D. thienemanni Kieffer, 1909] - RW; 1100-1200 m; 3; ena, ? wp; Michailova 1982, 1989, 2006.

Pagastia orientalis (Chernovskii, 1949) - TL; 150-180 m; 1; ho; Dimitrov 1962b, $1963 \mathrm{a}$. 
Potthastia gaedii (Meigen, 1838) [Diamesa, Polypedilum] - DW, DM, E1, P2, V5, S1, T1, R1; 40-1850 m; 1, 2, 3, 4; ho, ? h; Dimitrov 1962d; Russev et al. 1994; Stoichev 1994, 1996; Janeva \& Russev 1997; Dashinov 2017.

Potthastia longimanus Kieffer, 1922 [P. campestris Edwards, 1929] - DW, P1, P2, K9, O61, R1, R5; 50-1200 m; 1, 2, 3; ho; Nachev 1983; Islam et al. 1986; Janeva 1987; Russev et al. 1991, 1994; Uzunov et al. 2011; Varadinova et al. 2013; Dashinov 2017.

Potthastia montium (Edwards, 1929) - R1; 1370 m; 3; h; Dashinov 2017.

Pseudodiamesa (Pseudodiamesa) branickii (Nowicki, 1873) - R2, RW; 1100-1950 m; 3, 4; ho; Michailova 1989, 2006; Michailova \& Petrova 1989.

Pseudodiamesa (Pseudodiamesa) nivosa (Goetghebuer, 1928) [Syndiamesa] - DW, P1, R1; 30-1040 m; 1, 2; tp; Dimitrov 1962d; Russev et al. 1994.

Thalassomya frauenfeldi Schiner, 1856 - BN, BS; 0 m; 1; eno, ? en; Valkanov 1948, 1957a; Caspers 1951a, 1951b, 1951c; Strenzke 1951; Karnoschitzky 1952; Dimitrov 1963a; Beschovski 1965; Michailova 1976b, 1980b, 1989.

Monodiamesa bathyphila (Kieffer, 1918) [Prodiamesa, Syndiamesa] - P2; 210-345 m; 1; h; Janeva 1987; Russev et al. 1994.

Odontomesa fulva (Kieffer, 1919) [? O. dospatica Dimitrov, 1963; Ablabesmyia] - K7, K8, O61, O62, R5, RW; 590-1220 m; 1, 2, 3; h; Dimitrov 1963a, 1966; Nachev 1983; Michailova 1982; Islam et al. 1986; Uzunov et al. 2011; Varadinova et al. 2013.

Prodiamesa bureschi Michailova, 1977 - DW, P1, V1, RW; 130-1160 m; 1, 2, 3; see; Michailova 1977, 1982a, 1989, 2006.

Prodiamesa olivacea (Meigen, 1818) - DW, DM, P1, P2, V1, V5, S211, TL, O61, O62, R1, R5, RW, RE, BS; 0-2196 m; 1, 2, 3, 4; h; Belcheva 1959; Dimitrov 1962a, 1962d, 1963a, 1966; Michailova 1977; Nachev 1983; Russev et al. 1984, 1994; Islam et al. 1986; Janeva 1987, 1989, 1991; Michailova 1982a, 1989, 2006; Stoichev 1994, 1996; Janeva \& Russev 1989, 1997; Stoichev \& Chernev 2001; Uzunov et al. 2011; Kenderov et al. 2008, 2012; Vidinova et al. 2008; Varadinova et al. 2011, 2012, 2013; Dashinov 2017; Ihtimanska et al. 2018.

Prodiamesa rufovittata Goetghebuer, 1932 - DW, P1, V5, S211, RW; 50-1150 m; 1, 2, 3; wces; Dimitrov 1960a, 1962d, 1963a, 1966; Russev et al. 1994; Stoichev 1994, 1996.

Acricotopus lucens (Zetterstedt, 1850) [A. lucidus Staeger, 1839] - DW, E1, P1, P2, B1, V1, O61, O62, R1, R2, R5, RW, RE, BS; 0-1950 m; 1, 2, 3, 4; h; Michailova 1982a, 1989, 2006; Michailova \& Dimitrova 1984; Janeva 1987; Russev et al. 1994; Stoichev 1994, 1996; Janeva \& Russev 1997; Kenderov et al. 2008, 2012; Vidinova et al. 2008.

Brillia bifida (Kieffer, 1909) [B. modestus Meigen, 1830; ? B. immaculata Botnariuc \& Cure, 1956 (? nomina dubia); ? B. pallida Chernovskii, 1949 (? nomina dubia)] - DW, DM, E1, P2, B2, V4, O61, R1, R5, RW, BN; 0-2535 m; 1, 2, 3, 4, 5, 6; po, ? ho; Russev 1961; Dimitrov 1962d, 1963a, 1966; Nachev 1983; Janeva \& Russev 1985, 1997; Islam et al. 1986; Janeva 1987, 1991; Michailova 1989; Russev et al. 1991, 1994; Stoichev 1994, 1996, 2000a; Stoichev \& Chernev 2001; Varadinova et al. 2013; Dashinov 2017.

Brillia longifurca Kieffer, 1921 - DW, DM, P1, P2, B2, K7, K8, K9, V4, V5, S211, O61, R1, R5, RW, RE; 30-1400 m; 1, 2, 3; hop, ? tp; Nachev 1983; Russev et al. 1984, 1987, 1991, 1994; Janeva \& Russev 1985, 1989, 1997; Islam et al. 1986; Janeva 1989; Michailova 1989; Stoichev 1996; Vidinova et al. 2008; Uzunov et al. 2011; Varadinova et al. 2013; Dashinov 2017.

Bryophaenocladius subvernalis (Edwards, 1929) [B. subvernalis var. bulgarica Cure; B. subvernalis var. vitae Cure] - DW, DM; 25-30 m; 1; tp, ? dp; Janeva 1987; Russev et al. 1994.

Bryophaenocladius ictericus (Meigen, 1830) [Orthocladius bipunctellus (Zetterstedt, 1850)] - V1, R5, RW, BN, BS; 0-1100 m; 1, 2, 3; e; Michailova 1982a, 1982b, 1989, 2006.

Camptocladius stercorarius (De Geer, 1776) - DW, DM, P1, P2, V1, TL; 20-600 m; 1, 2; hoa; Nedelkov 1912; Dimitrov 1962b, 1963a, 1966; Janeva 1987; Russev et al. 1994.

Cardiocladius capucinus (Zetterstedt, 1850) - TL; 150-160 m; 1; dp; Dimitrov 1962b, $1963 \mathrm{a}$.

Chaetocladius (Chaetocladius) laminatus Brundin, 1947 - DM, P2; 50-360 m; 1; wces; Janeva 1987; Russev et al. 1994.

Chaetocladius (Chaetocladius) piger (Goetghebuer, 1913) - R1, BS; 20-1150 m; 1, 2, 3; h; Michailova 1982; Dashinov 2017.

Clunio marinus Haliday, 1855 - BN, BS; 0 m; 1; ena; Valkanov 1948, 1957a; Caspers 1951a, 1951b, 1951c; Strenzke 1951; Drensky 1959; Dimitrov 1963a; Beschovski 1965; Michailova 1973a. 
Clunio ponticus Michailova, 1980 - BN, BS; 0 m; 1; see; Michailova 1980a, 1989.

Corynoneura celeripes Winnertz, 1852 - DW, TL, O61, RW; 50-1150 m; 1, 2, 3; h; Dimitrov 1962a, 1962b, 1963a; Nachev 1983; Russev et al. 1991, 1994.

Corynoneura celtica Edwards, 1924 - DM, P2; 1; dp; Russev et al. 1994.

Corynoneura scutellata Winnertz, 1846 - DM, P2, K7, K8, K9, O61, O62; 50-480 m; 1; sk; Nachev 1983; Islam et al. 1986; Janeva 1987; Russev et al. 1994; Janeva \& Russev 1997.

Cricotopus (Cricotopus) algarum (Kieffer, 1911) - DW, DM, E1, E2, P1, P2, B1, V4, S1, S21, S22, TL, R1, R5, RW, RE, BN; 0-2394 m; 1, 2, 3, 4, 5; wces; Cvetkov 1955a; Valkanov 1957a; Dimitrov 1957, 1960a, 1962a, 1962b, 1962c, 1966; Russev 1959, 1961, 1966b, 1977; Russev \& Janeva 1975; Michailova 1978, 1982a, 1989, 1996; Janeva 1987, 1991; Russev et al. 1991, 1994; Stoichev 1994, 1996, 1998, 2000a, 2000b, 2002; Janeva \& Russev 1997; Janeva et al. 2001; Stoichev \& Chernev 2001; Uzunov et al. 2001, 2011; Janeva \& Bancheva 2002; Kenderov et al. 2008, 2012; Sakelarieva et al. 2008; Vidinova et al. 2008; Moskova \& Uzunov 2011; Varadinova et al. 2011, 2013; Borisova et al. 2013; Trichkova et al. 2013; Ihtimanska et al. 2018.

Cricotopus (Cricotopus) annulator Goetghebuer, 1927 [Eukiefferiella alpestris Goetghebuer, 1941] - DW, DM, E1, E2, P1, P2, P3, K7, K8, K9, V5, S1, T1, T31, O61, O62, R1, R2, R5, RW, RE, BS; 0-2365 m; 1, 2, 3, 4, 5; h; Dimitrov 1962d; Russev \& Janeva 1975; Michailova 1982; Nachev 1983; Russev et al. 1984, 1987, 1991, 1994; Janeva \& Russev 1985; Islam et al. 1986; Janeva 1987, 1989; Stoichev 1994, 1996; Stoichev \& Chernev 2001; Sakelarieva et al. 2008; Vidinova et al. 2008; Uzunov et al. 2011; Varadinova et al. 2013; Dashinov 2017.

Cricotopus (Cricotopus) bicinctus (Meigen, 1818) - DW, DM, E1, P1, P2, B2, K7, K8, K9, S1, TL, T1, T31, O61, O62, RW, RE, BS; 0-1030 m; 1, 2, 3; k; Michailova 1982, 1989, 2006; Nachev 1983; Russev et al. 1984, 1987, 1991, 1994; Janeva \& Russev 1985, 1989; Islam et al. 1986; Russev \& Janeva 1986; Janeva 1987, 1989, 1991; Stoichev 1996; Vidinova et al. 2008; Uzunov et al. 2011; Borisova et al. 2013; Varadinova et al. 2013.

Cricotopus (Cricotopus) curtus Hirvenoja, 1973 - DM, P2, K7, K8, K9, S1, TL, T1, O61, O62, R5, RW; 25-800 m; 1, 2; h; Nachev 1983; Russev et al. 1984, 1994; Janeva \& Russev 1985; Islam et al. 1986; Janeva 1987, 1989; Stoichev 1994, 1996; Uzunov et al. 2011; Varadinova et al. 2013.

Cricotopus (Cricotopus) festivellus (Kieffer, 1906) - V4, R1, R5; 700-1150 m; 1, 2, 3; h; Michailova 1989; Dashinov 2017.

Cricotopus (Cricotopus) flavocinctus (Kieffer, 1924) - DW, P1, P2, RW; 50-625 m; 1, 2; h; Janeva 1987, 1989; Janeva \& Russev 1989; Russev et al. 1994.

Cricotopus (Cricotopus) fuscus (Kieffer, 1909) [C. biformis Edwards, 1929] - DM, P1, P2, K9, S1, TL, O61, O62, R1, R5, RW, RE, BS; 0-1550 m; 1, 2, 3, 4; h; Dimitrov 1960a, 1962a, 1962b; 1963a; Russev 1964, 1066a; Michailova 1982, 1989, 2006; Russev et al. 1984b, 1994; Islam et al. 1986; Janeva 1987; Stoichev 1994, 1996; Janeva \& Russev 1997; Vidinova et al. 2008; Uzunov et al. 2011; Trichkova et al. 2013; Varadinova et al. 2013.

? Cricotopus (Cricotopus) latidentatus Chernovskii, 1949 [? Nomen dubium] - P2, RW; 200-250 m; 1; wces; Dimitrov 1963a, 1966; Janeva 1987.

Cricotopus (Cricotopus) pirifer Hirvenoja, 1973 - RW; 1100 m; 3; e; Michailova 1982, 2006.

Cricotopus (Cricotopus) tibialis (Meigen, 1804) - O62; $65 \mathrm{~m} ; 1$; h; Michailova 1982.

Cricotopus (Cricotopus) tremulus (Linnaeus, 1758) - DW, DM, P2, V5, T31, R1, BN, BS; 0-2250 m; 1, 2, 3, 4, 5; h; Janeva 1987; Michailova 1989; Russev et al. 1994; Stoichev 1996; Uzunov et al. 2011; Dashinov 2017.

Cricotopus (Cricotopus) triannulatus (Macquart, 1826) - DW, DM, P1, P2, K7, K8, K9, T1, O61, O62; 50-500 m; 1, 2; hn; Russev et al. 1984, 1991, 1994; Janeva \& Russev 1985; Islam et al. 1986; Janeva 1987.

Cricotopus (Cricotopus) trifascia Edwards, 1929 - DW, DM, E1, E2, P1, P2, B2, K7, K8, K9, S1, V5, O61, O62, R5; 20-800 m; 1, 2; ho; Nachev 1983; Janeva \& Russev 1985, 1997; Islam et al. 1986; Janeva 1987; Michailova 1989, 1998; Russev et al. 1994; Stoichev 1994, 1996; Uzunov et al. 2001, 2011; Varadinova et al. 2011, 1912, 2013; Borisova et al. 2013; Ihtimanska et al. 2018.

Cricotopus (Cricotopus) tristis Hirvenoja, 1973 - DW, SP, O61, R5, RW; 50-800 m; 1, 2; h; Nachev 1983; Janeva 1989; Janeva \& Russev 1989; Russev et al. 1994; Uzunov et al. 2011; Varadinova et al. 2013.

Cricotopus (Cricotopus) vierriensis Goetghebuer, 1935 - DW, DM, P1, P2, K7, K8, V4, S1, T1, O61, O62, R5; 30-800 m; 1, 2; h; Michailova 1982; Nachev 1983; Russev et al. 1984, 1991, 1994; Janeva \& Russev 1985; Islam et al. 1986; Russev \& Janeva 1986; Janeva 1987; Janeva \& Russev 1989; Stoichev 1996; Uzunov et al. 2011; Varadinova et al. 2013.

Cricotopus (Cricotopus) zavreli Szadziewski \& Hirvenoja, 1981 - S1, T1; 98-387 m; 1; ee; Borisova et al. 2013. 
Cricotopus (Isocladius) intersectus (Staeger, 1839) - DW, DM, E1, E2, P1, P2, K7, K8, K9, V4, S1, T1, O61, O62, R5, BN; 0-800 m; 1, 2; h; Nachev 1983; Islam et al. 1986; Michailova 1989, 1996; Russev et al. 1984, 1994; Janeva \& Russev 1985, 1989; Uzunov et al. 2011; Varadinova et al. 2013.

Cricotopus (Isocladius) laetus Hirvenoja, 1973 - DW, K9, T1; 30-600 m; 1, 2; h; Janeva \& Russev 1985, 1989; Islam et al. 1986; Russev et al. 1994.

Cricotopus (Isocladius) laricomalis Edwards, 1932 - O62; 65-70 m; 1; h; Michailova 1982.

Cricotopus (Isocladius) ornatus (Meigen, 1818) - DW, DM, E2, P1, P2, V1, RW, BN, BS; 0-1550 m; 1, 2, 3, 4; h, ? ho; Caspers 1951a; Strenzke 1951; Michailova 1976a, 1989, 1996, 1998, 2006; Janeva \& Russev 1989, 1997; Janeva 1991; Russev et al. 1991, 1994; Stoichev 1996, 1998; Kovachev et al. 1999; Uzunov et al. 2001; Varadinova et al. 2011, 1912; Ihtimanska et al. 2018.

Cricotopus (Isocladius) reversus Hirvenoja, 1973 - K8, R5; 800 m; 1, 2; des; Michailova 1982; Islam et al. 1986.

Cricotopus (Isocladius) suspiciosus Hirvenoja, 1973 - DW, DM, E1, P2, BN; 0-200 m; 1; e; Michailova 1982, 1996; Russev et al. 1984, 1994; Janeva \& Russev 1989.

Cricotopus (Isocladius) sylvestris (Fabricius, 1794) - DW, DM, E1, E2, P1, P2, B1, K9, V1, V4, V5, S1, S21, S22, S211, TL, T1, T31, O61, O62, R1, R2, R5, RW, RE, BN, BS; 0-2535 m; 1, 2, 3, 4, 5, 6; hno; Cvetkov 1955a, 1955b, 1962; Angelov 1956; Dimitrov 1957, 1960b, 1962a, 1962b, 1962c, 1962d, 1963a, 1963b, 1966, 1981, 1982; Valkanov 1957a; Belcheva 1959; Zaschev \& Angelov 1959; Mihailova-Neikova 1961; Russev 1961, 1966a; Russev \& Janeva 1975; Michailova 1976a, 1982a, 1989, 1996, 1998, 2006; Nachev 1983; Russev et al. 1984b, 1994; Islam et al. 1986; Janeva 1987, 1991; Janeva \& Russev 1989, 1997; Stoichev 1994, 1996, 1998, 2000a, 2000b, 2001a, 2002; Kovachev et al. 1999; Janeva et al. 2001; Stoichev \& Chernev 2001; Uzunov et al. 2001, 2011; Janeva \& Bancheva 2002; Vidinova et al. 2008; Kenderov et al. 2008, 2012; Moskova \& Uzunov 2011; Varadinova et al. 2011, 1912, 2013; Borisova et al. 2013; Trichkova et al. 2013; Ihtimanska et al. 2018.

Cricotopus (Isocladius) tricinctus (Meigen, 1818) - DW, DM, E1, E2, K9, O61, R5, BN, BS; 0-800 m; 1, 2; h; Michailova 1982; Nachev 1983; Islam et al. 1986; Russev \& Janeva 1986; Russev et al. 1987, 1994; Janeva \& Russev 1989, 1997; Janeva 1991; Uzunov et al. 2011; Varadinova et al. 2013.

Cricotopus (Isocladius) trifasciatus (Meigen, 1810) - DW, DM, E1, E2, P1, V4, O61, R5, RW; 50-1120 m; 1, 2, 3; ho; Michailova 1982a, 1988; Nachev 1983; Islam et al. 1986; Russev \& Janeva 1986; Russev et al. 1987, 1991, 1994; Janeva \& Russev 1997; Varadinova et al. 2013.

Epoicocladius ephemerae (Kieffer, 1924) [E. flavens Malloch, 1915; E. flavens Saether, 1969] - DW, P1, P2, V5, R1, RW; 180-1550 m; 1, 2, 3, 4; wces, ? h; Dimitrov 1957, 1963a, 1966; Belcheva 1959; Russev 1964; Russev et al. 1991, 1994; Janeva \& Russev 1997; Dashinov 2017.

? Eukiefferiella atrofasciata Goetghebuer, 1934 - RW; 900-1100 m; 1, 2, 3; cee; Michailova 1982.

Eukiefferiella brehmi Gowin, 1943 - O61; 316 m; 1; h; Nachev 1983.

Eukiefferiella brevicalcar (Kieffer, 1911) - DW, DM, P2, O61, R1; 30-1960 m; 1, 2, 3, 4; wp; Nachev 1983; Islam et al. 1986; Janeva 1987; Russev et al. 1991, 1994; Stoichev 1994, 1996; Stoichev \& Chernev 2001; Sakelarieva et al. 2008; Dashinov 2017.

Eukiefferiella claripennis (Lundbeck, 1898) [E. hospita Edwards, 1929] - DW, DM, P1, P2, K7, K8, S1, TL, O61, O62, R1, R5, RW; 180-1000 m; 1, 2; ho; Dimitrov 1963a; Russev 1964; Michailova 1982; Nachev 1983; Russev et al. 1984a, 1984b, 1991, 1994; Islam et al. 1986; Janeva 1987, 1989; Stoichev 1994, 1996; Uzunov et al. 2011; Varadinova et al. 2013; Dashinov 2017.

Eukiefferiella clypeata (Thienemann, 1919) [? E. clypeata (Kieffer, 1923)] - DW, DM, P1, S1, O61, R1, R5; 200-1550 m; 1, 2, 3; tp; Nachev 1983; Janeva \& Russev 1985; Islam et al. 1986; Russev et al. 1991, 1994; Stoichev 1994, 1996; Stoichev \& Chernev 2001; Sakelarieva et al. 2008; Uzunov et al. 2011; Trichkova et al. 2013; Varadinova et al. 2013.

Eukiefferiella coerulescens (Kieffer, 1926) - DW, DM, P2, S211, O61, RW; 200-800 m; 1, 2; h; Dimitrov 1962d, 1963a; Nachev 1983; Russev et al. 1984, 1991, 1994.

Eukiefferiella cyanea Thienemann, 1936 - O61; 316 m; 1; h; Nachev 1983; Islam et al. 1986.

Eukiefferiella fittkaui Lehmann, 1972 - O61; 316 m; 1; eanna, ? wp; Nachev 1983.

Eukiefferiella gracei (Edwards, 1929) [E. longicalcar Thienemann, 1926; E. potthasti Lehmann, 1972] - DW, DM, E1, E2, P1, B3, S1, TL, O61, R1, R5, RW, RE; 50-1200 m; 1, 2, 3; wcp, ? h; Dimitrov 1960a, 1962a, 1962d, 1963a, 1966; Russev 1966a, 1966b; Russev \& Janeva 1975; Michailova 1982; Nachev 1983; Islam et al. 1986; Janeva 1987, 1989; Russev et al. 1991, 1994; Stoichev 1994, 1996; Janeva \& Russev 1997; Ken- 
derov et al. 2008, 2012; Soufi \& Uzunov 2008; Vidinova et al. 2008; Moskova \& Uzunov 2011; Uzunov et al. 2011; Varadinova et al. 2011, 2012, 2013; Ihtimanska et al. 2018.

Eukiefferiella ilkleyensis (Edwards, 1929) [E. lutethorax Goetghebuer, 1949] - DW, DM, P1, O61, R1; 80-1400 m; 1, 2, 3; h; Nachev 1983; Islam et al. 1986; Janeva 1987; Stoichev 1994, 1996; Russev et al. 1994; Dashinov 2017.

Eukiefferiella lobifera Goetghebuer, 1934 - DW, P1, B1, K8, K9, S1, T31, O61, R5, RW, BS; 0-1200 m; 1, 2, 3; wp, ? h; Dimitrov 1963a, 1966; Nachev 1983; Islam et al. 1986; Stoichev 1994, 1996; Russev et al. 1984b, 1991, 1994; Uzunov et al. 2011; Varadinova et al. 2013.

? Eukiefferiella longipes Tshernovskij, 1949 [? Nomen dubium] - DW, P2, S1, TL, T1, O62, RE; 30-650 m; 1; ? wces; Dimitrov 1963a, 1966; Janeva 1987; Stoichev 1994, 1996.

? Eukiefferiella masordarjensis Pankratova, 1950 [? Nomen dubium] - DM, E1, O61, RW; 310-624 m; 1, 2; ? dp; Nachev 1983; Islam et al. 1986; Janeva 1989; Russev et al. 1994; Janeva \& Russev 1997.

? Eukiefferiella oxiana Pankratova, 1950 [? Nomen dubium] - DW, DM, P1, P2; 50-250 m; 1; ? seeca; Janeva 1987; Russev et al. 1994; Stoichev 1994, 1996.

? Eukiefferiella popovae Tshernovskij, 1949 [? Nomen dubium] - TL, O61, RW; 200-1000 m; 1, 2; ? see; Dimitrov 1963a; Michailova 1982; Nachev 1983.

Eukiefferiella similis Goetghebuer, 1939 [Crycotopus] - DW, DM, E1, E2, P1, P2, B1, V4, TL, R1, R5, RW; $20-2350$ m; 1, 2, 3, 4, 5; wcp, ? po; Russev 1959, 1966a, 1966b; Dimitrov 1962d, 1963a, 1966; Russev 1978; Janeva 1987; Stoichev 1994, 1996; Russev et al. 1994; Janeva \& Russev 1997; Stoichev \& Chernev 2001; Uzunov et al. 2001, 2011; Kenderov et al. 2008, 2012; Vidinova et al. 2008; Moskova \& Uzunov 2011; Varadinova et al. 2011, 2012, 2013; Ihtimanska et al. 2018.

? Eukiefferiella quadridentata Chernovskij, 1949 [? Nomen dubium] - DM, E1, P2, K8, S1, TL, O61, R1, RW; 200-1550 m; 1, 2, 3; seess; Dimitrov 1963a; Michailova 1982; Nachev 1983; Janeva \& Russev 1985, 1997; Islam et al. 1986; Janeva 1987, 1989; Russev et al. 1991; Stoichev 1994, 1996; Stoichev \& Chernev 2001.

Halocladius (Halocladius) millenarius (Santos Abreu, 1918) - BN; 0-15 m; 1; sena; Michailova 1989.

Halocladius (Halocladius) variabilis (Staeger, 1839) - BN, NS; 0 m; 1; h; Valkanov 1936, 1957; Caspers 1951a, 1951b; Dimitrov 1963a; Michailova 1989.

Halocladius (Halocladius) varians (Staeger, 1839) [Cricotopus] - BN, BS; 0 m; 1; h; Michailova 1975, 1989.

? Halocladius vitripennis (Meigen, 1818) [Cricotopus; ? Nomen dubium] - BN, BS; 0 m; 1; e; Strenzke 1951; Michailova 1975.

Hydrobaenus lugubris Fries, 1830 [Trissocladius griseipennis Goetghebuer, 1913] - BN; 0 m; 1; wces; Cvetkov 1955a, 1955b; Dimitrov 1963a.

Krenosmittia boreoalpina (Goetghebuer, 1944) - DM, P2; 1 ; h; Russev et al. 1994.

Krenosmittia camptophleps (Edwards, 1929) - O61; 316 m; 1; tp, ? h; Nachev 1983.

Lapposmittia parvibarba Edwards, 1939 - T1; RW; 120-1480 m; 1, 2, 3, 4; wces; Dimitrov 1963a; Russev \& Janeva 1975; Russev et al. 1984b.

Limnophyes asquamatus Andersen, 1937 [L. septentrionalis Goetghebuer, 1940] - DW, DM, E1, P2, S1, TL, O61, O62, RW, RE; 50-700 m; 1, 2; ho; Stoichev 1994, 1996.

? Limnophyes karelicus (Tshernovskij, 1949) [Nomen nudum] - DW, DM, V5, S211, T31, O61, BS; 0-100 m; 1; des; Nachev 1983; Islam et al. 1986; Russev et al. 1994; Stoichev 1996; Janeva \& Russev 1989, 1997.

Limnophyes minimus (Meigen, 1818) [L.pusillus Eaton, 1875] - DW, DM, E1, E2, P2, K3, TL, O61, R5, BN; 0-800 m; 1, 2; hptn, sk; Cvetkov 1955a, 1957; Valkanov 1957; Dimitrov 1962b, 1963a; Russev 1959, 1962, 1966a, 1966b; Nachev 1983; Russev et al. 1984, 1994; Islam et al. 1986; Uzunov et al 2011; Varadinova et al. 2013.

Limnophyes pentaplastus (Kieffer, 1921) [L. prolongatus Kieffer, 1921] - DW, DM, E1, P1, P2, P3, B3, V5, S211, TL, T1, T31, O61, O62, R5, RW, RE, BN, BS; 0-1000 m; 1, 2; ho; Cvetkov 1955a, 1955b; Valkanov 1957a; Dimitrov 1962d, 1963a, 1966; Russev 1977; Nachev 1983; Islam et al. 1986; Stoichev 1994, 1996, 2001a; Russev et al. 1994; Janeva \& Russev 1997; Uzunov et al 2011; Varadinova et al. 2013.

? Limnophyes pseudoprolongatus Botnariuc et Cindea-Cure, 1954 [? Nomen dubium] - O61; 316 m; 1; ? see; Nachev 1983; Islam et al. 1986.

? Limnophyes transcaucasicus Tshernovskij, 1949 [? Nomen dubium] - DM, V4; $20-800$ m; 1, 2; ? wces; Russev 1966b; Islam et al. 1986; Janeva \& Russev 1997. 
Metriocnemus (Metriocnemus) albolineatus (Meigen, 1818) [M. atratulus Zetterstedt, 1850] - DW, E1, E2, P2, V1, S1, RW; 20-1000 m; 1, 2; ho; Nedelkov 1912; Janeva \& Russev 1985; Islam et al. 1986; Janeva 1987; Michailova 1989, 2006; Russev et al. 1994.

Metriocnemus (Metriocnemus) cavicola Kieffer, 1921 [M. martinii Thienemann, 1921] - S1; 330-350 m; 1; e; Russev et al. 1984.

Metriocnemus (Metriocnemus) fuscipes (Meigen, 1818) - O61; 320 m; 1; h; Nachev 1983.

Metriocnemus (Metriocnemus) eurynotus (Holmgren, 1883) [M. hygropetricus Kieffer, 1912] - P1, P2, P3, B1, B3, S1, T1, O61, O62, R1, RE; 300-1000 m; 1, 2; ho; Arndt 1943; Dimitrov 1963a; Russev 1966b; Janeva 1987; Stoichev 1994, 1996; Russev et al. 1994; Kenderov et al. 2008, 2012.

Metriocnemus (Metriocnemus) picipes (Meigen, 1818) - DM, P2;

Metriocnemus (Metriocnemus) terrester Pagast, Thienemann \& Krueger, 1941 - P2; 185-380 m; 1; e; Russev et al. 1984.

Metriocnemus (Metriocnemus) ursinus (Holmgren, 1869) - S1; 350 m; 1; h; Janeva \& Russev 1985.

Nanocladius (Nanocladius) dichromus (Kieffer, 1906) [N. bicolor (Zetterstedt, 1938); Microcricotopus; Eukiefferiella] - DW, DM, E1, P1, P2, K3, K8, V5, S1, S211, T1, O61, RW; 50-1200 m; 1, 2, 3; wces; Russev \& Janeva 1975; Nachev 1983; Russev et al. 1984, 1987, 1991, 1994; Janeva \& Russev 1985, 1989, 1997; Islam et al. 1986; Janeva 1989, 1991.

Nanocladius (Nanocladius) parvulus (Kieffer, 1909) - DM, P2; $\$$; Russev et al. 1994.

? Orthocladius barbatus Kieffer, 1900 [? nomen dubium] - DM, P2, S1, TL, T1, T31, O61, O62, R5, RE, BS; 50-700 m; 1, 2; e; Janeva 1987; Russev et al. 1994; Stoichev 1994, 1996; Janeva \& Russev 1997.

? Orthocladius bulgarensis Goetghebuer, 1938 [? Nomen dubium] - BN; 0 m; 1; see; Dimitrov 1963a.

? Orthocladius calliginosa Pancratova, 1950 [invalid; Orthocladinae gen. ? calliginosa Pankr.] - DW, K7, K8, K9, O61, O62; 90-500 m; 1; seet; Islam et al. 1986; Janeva 1987.

? Orthocladius murvanidzei (Chernovskij, 1949) [? Nomen dubium] - DW, DM, P1, P2, V5, S211, TL, O61, O62, R1, R5, RW; 150-2440 m; 1, 2, 3, 4, 5, 6; wces; Dimitrov 1962d, 1963a, 1966; Russev et al. 1994; Stoichev 1994, 1996; Janeva \& Russev 1997.

Orthocladius (Eudactylocladius) fuscimanus (Kieffer, 1908) [O. bipunctellus auct.] - RW, BN; 0-1560 m; 1, 2, 3; wp; Michailova 1985, 1989, 2006.

Orthocladius (Eudactylocladius) olivaceus (Kieffer, 1911) - DW, DM, E1, P1, P2, P3, B3, T31, RW, RE, BS; 0-1100 m; 1, 2, 3; h; Dimitrov 1963a, 1966; Michailova 1985, 1989, 2006; Russev et al. 1994; Stoichev 1994, 1996; Janeva \& Russev 1997.

Orthocladius (Euorthocladius) abiskoensis Thienemann \& Krueger, 1937 - R5, RW; 800-1100 m; 2, 3; h; Michailova 1989, 2006.

Orthocladius (Euorthocladius) rivicola Kieffer, 1911 - P1, P2, TL; 150-220 m; 1; h, ? ho; Dimitrov 1963a, 1966; Russev et al. 1994.

Orthocladius (Euorthocladius) rivulorum Kieffer, 1909 - DW, DM, P2, K9, O61, R5, RW; 60-1020 m; 1, 2, 3; h; Nachev 1983; Islam et al. 1986; Janeva 1987, 1989; Russev et al. 1994; Janeva \& Russev 1997; Uzunov et al. 2011; Varadinova et al. 2013.

Orthocladius (Euorthocladius) saxosus (Tokunaga, 1939) - DM, E1, P2, O61; 50-330 m; 1; h; Nachev 1983; Russev et al. 1994; Stoichev 1996; Janeva \& Russev 1997.

Orthocladius (Euorthocladius) thienemanni Kieffer, 1906 - DW, DM, E1, P1, P2, K3, K7, K8, K9, V1, S1, O61, O62, R2, R5, RW; 30-1100 m; 1, 2, 3; h; Nachev 1983; Russev et al. 1984, 1987, 1991, 1994; Michailova 1985, 1989, 2006; Janeva \& Russev 1985; Islam et al. 1986; Janeva 1987, 1989, 1991; Stoichev 1994, 1996; Uzunov et al. 2011; Varadinova et al. 2013.

Orthocladius (Mesorthocladius) frigidus (Zetterstedt, 1838) - DW, DM, E1, P1, P2, K9, S1, T1, R1, RW, RE; 100-1500 m; 1, 2, 3; h, ? ho; Michailova 1982a, 1985, 1989, 2006; Islam et al. 1986; Janeva 1989; Russev et al. 1994; Stoichev 1994, 1996; Dashinov 2017.

Orthocladius (Orthocladius) oblidens (Walker, 1856) - R5, RW; 800-1000 m; 2; h; Michailova 1982a, 1985, 1989.

Orthocladius (Orthocladius) rubicundus (Meigen, 1818) [O. saxicola Kieffer, 1911] - DW, DM, E1, P1, P2, K3, K7, K8, K9, V4, S1, T1, O61, O62, R2, R5, RW, BN; 0-1560 m; 1, 2, 3, 4; h, ? hop; Cvetkov 1955a, 1955b; Valkanov 1957a; Dimitrov 1963a; Michailova 1982a, 1985, 1989, 2006; Russev et al. 1984, 1987, 1991, 1994; Janeva \& Russev 1985; Islam et al. 1986; Janeva 1987, 1989; Stoichev 1994, 1996; Uzunov et al. 2011; Varadinova et al. 2013. 
Orthocladius (Pogonocladius) consobrinus (Holmgren, 1869) - TL, BN; 0-200 m; 1; h, ? hoes, ? h; Michailova 1982a, 1989.

Orthocladius (Symposiocladius) lignicola Kieffer, 1914 [S. xylophila Botnarius et Cure, 1956] - E1, P2, TL, R1, R5; 150-1800 m; 1, 2, 3, 4; ho; Russev et al. 1994; Stoichev 1994, 1996; Janeva \& Russev 1997; Dashinov 2017.

Paracladius conversus (Walker, 1856) - DW, DM, P1, P2, K3, K9, V5, S1, O61, RW, BS; 0-1000 m; 1, 2, 3; h; Nachev 1983; Janeva \& Russev 1985; Islam et al. 1986; Janeva 1987, 1991; Michailova 1989, 2006; Russev et al. 1991, 1994; Dashinov 2017.

? Paracladius inaequalis Kieffer, 1926 [? Nomen dubium; Trichocladius] - P2, V5, S1, S211, TL, T1, R1, RW; 150-1200 m; 1, 2, 3; ? e; Dimitrov 1957, 1960a, 1962b, 1962d, 1963a, 1966; Cvetkov 1962; Russev 1966a; Russev et al. 1984b; Michailova 1989, 2006.

Paracricotopus niger (Kieffer, 1913) - DM, P2; 1; ena; Russev et al. 1994.

Parakiefferiella bathophila (Kieffer, 1912) [Orthocladius] - DW, DM, E1, E2, P1, P2, S1, TL, T1, O61, O62, R5, RW, RE; 20-700 m; 1, 2; h; Russev 1966b; Janeva 1987; Russev et al. 1994; Stoichev 1994, 1996; Michailova 1998; Uzunov et al. 2001, 2011; Varadinova et al. 2011, 2012, 2013; Ihtimanska et al. 2018.

Paralimnophyes longiseta (Thienemann, 1919) [P. hydrophilus Goetghebuer, 1921] - R5; 5-800 m; 1, 2; des; Uzunov et al. 2011; Varadinova et al. 2013.

Parametriocnemus stylatus (Spaerck, 1923) - DW, DM, P1, P2, K3, K8, V5, S1, T1, O61, O62, R1, R5, RW; 100-1970 m; 1, 2, 3, 4; po, ? ho; Nachev 1983; Janeva \& Russev 1985, 1997; Islam et al. 1986; Janeva 1987, 1989, 1991; Russev et al. 1991, 1994; Uzunov et al. 2011; Varadinova et al. 2013; Dashinov 2017.

Paratrichocladius rufiventris (Meigen, 1830) - K9, O61; 320-445 m; 1; ho; Nachev 1983; Islam et al. 1986.

Paratrissocladius excerptus (Walker, 1856) [Trissocladius fluviatilis Goetghebuer, 1937] - O61; 320 m; 1; ppt; Nachev 1983.

Parorthocladius nudipennis (Kieffer, 1908) [? Synorthocladius] - DW, DM, P1, P2, O61, R1, RW; 50-1200 m; 1, 2, 3; tp; Dimitrov 1962d, 1966; Nachev 1983; Islam et al. 1986; Janeva 1987; Stoichev 1994, 1996; Janeva \& Russev 1997; Stoichev \& Chernev 2001; Sakelarieva et al. 2008.

Propsilocerus lacustris Kieffer, 1923 [? P. orielica Chernovskij, 1949] - BN; 0 m; 1; cee; Thienemann 1936; Cvetkov 1955a, 1955b, 1957; Valkanov 1957a; Dimitrov 1963a.

Psectrocladius (Allopsectrocladius) obvius (Walker, 1856) [P. dilatatus van der Vulp, 1858] - DW, DM, E1, P1, P2, S1, TL, R5, RW, BN; 30-1200 m; 1, 2, 3; h; Cvetkov 1955a, 1962; Valkanov 1957a; Belcheva 1959; Dimitrov 1963a; Russev et al. 1984b, 1994; Janeva 1987; Michailova 1989, 2006; Uzunov et al. 2011; Borisova et al. 2013; Varadinova et al. 2013.

Psectrocladius (Allopsectrocladius) platypus (Edwards, 1929) - O61, O62; 75-320 m; 1; eanna; Nachev 1983; Islam et al. 1986.

Psectrocladius (Psectrocladius) barbimanus (Edwards, 1929) [P. ishimicus Chernovskij, 1949] - E2, V1, TL, O61; 20-550 m; 1, 2; h; Dimitrov 1962b, 1963a; Islam et al. 1986; Michailova 1989, 1998; Uzunov et al. 2001; Varadinova et al. 2011, 2012; Ihtimanska et al. 2018.

Psectrocladius (Psectrocladius) delatoris Zelentsov, 1980 - RW; 1000-1550 m; 2, 3, 4; wces; Michailova 1989, 2006.

Psectrocladius (Psectrocladius) psilopterus (Kieffer, 1906) - DW, DM, P2, TL, O61, O62, R1, R5, RW, BN; 302324 m; 1, 2, 3, 4, 5; h; Cvetkov 1955a; Valkanov 1957a; Belcheva 1959; Dimitrov 1960b, 1962a, 1963a; Janeva 1987; Russev et al. 1994; Stoichev 1994, 1996, 2000a; Varadinova et al. 2013.

Psectrocladius (Psectrocladius) simulans (Johannsen, 1937) - P2, TL, R1, RW, RE; 150-2324 m; 1, 2, 3, 4, 5; h; Dimitrov 1962a, 1962b, 1962c, 1963a; Janeva 1987; Russev et al. 1994; Stoichev 2000a.

Pseudosmittia angusta (Edwards, 1929) - P1, B1, O61; 320-600 m; 1; h; Nachev 1983; Islam et al. 1986; Russev et al. 1991, 1994.

Pseudosmittia gracilis (Goetghebuer, 1913) - O61, R5; 320-800 m; 1, 2; wces, h; Nachev 1983; Islam et al. 1986; Uzunov et al. 2011; Varadinova et al. 2013.

Pseudosmittia holsata Thienemann \& Strenzke, 1940 - O61; 230 m; 1; h; Nachev 1983; Islam et al. 1986;.

Rheocricotopus (Psilocricotopus) atripes (Kieffer, 1913) - BN; 0 m; 1; wcp; Caspers 1951a; Strenzke 1951.

Rheocricotopus (Psilocricotopus) chalybeatus (Edwards, 1929) - R5; 790-850 m; 2; tp; Michailova 1989.

Rheocricotopus (Rheocricotopus) effusus (Walker, 1856) [R. brunensis Goetghebuer, 1937; R. dorhieri Goetghebuer, 1932; Rheotanytarsus] - DW, DM, E1, P1, P2, B2, K3, K7, K8, K9, S1, T1, O61, O62, R2, R5, RW; 60-1950 m; 1, 2, 3, 4; ho; Nachev 1983; Russev et al. 1984, 1987, 1991, 1994; Janeva \& Russev 1985, 1989, 
1997; Islam et al. 1986; Russev \& Janeva 1986; Janeva 1989, 1991; Michailova 1989, 2006; Stoichev 1994, 1996; Uzunov et al. 2011; Varadinova et al. 2013.

? Rheosmittia delicatula Botnariuc et Cure, 1956 [? Invalid; Orthocladinae, Nomen nudum] - DM, P2, O61; 330 m 1; wces; Nachev 1983; Russev et al. 1994.

Smittia aterrima (Meigen, 1818) [Camptocladius] - V1; 550 m; 1; ho; ? hop; Nedelkov 1912.

Smittia contingens (Walker, 1856) [S. aquatilis Goetghebuer, 1921] - DM, TL, O61, O62, R5; 35-350 m; 1; wp, ? wes; Nachev 1983; Islam et al. 1986; Janeva 1987; Russev et al. 1994; Stoichev 1994, 1996; Janeva \& Russev 1997.

Smittia duplicata Strenzke, 1951 - BN; 0 m; 1; Ebg; Caspers 1951a, 1951b; Strenzke 1951; Pape \& Beuk 2017. Smittia nudipennis (Goetghebuer, 1913) [Camptocladius] - DW, DM, P1, P2, B1, S1, T1, O61, O62; 100-550 m; 1; tp; Stoichev 1994, 1996; Russev et al. 1991, 1994; Janeva \& Russev 1997.

? Smittia sedula Konstantinov, 1952 [? Nomen dubium] - DW, E1, DM, P2, TL, R5; 250-800 m; 1, 2; ? des; Janeva 1987; Russev et al. 1994; Stoichev 1994, 1996; Janeva \& Russev 1997.

? Smittia septentrionalis Chernovskij, 1949 [? Nomen dubium; Kieffer, 1922] - DW, DM, P2, P3, S1, TL, T1, O61, O62, BN; 100-500 m; 1; ? wces; Dimitrov 1962b, 1963a; Stoichev 1994, 1996; Janeva \& Russev 1997.

Symbiocladius rhithrogenae (Zavrel, 1924) - R1; 1120-1960 m; 3, 4; des; Dashinov 2017; Dashinov \& Vidinova 2018.

Synorthocladius semivirens (Kieffer, 1909) - DM, P1, P2, V5, S1, S211, O61, R1, R5, RW; 30-2250 m; 1, 2, 3, 4, 5; ho; Dimitrov 1962d, 1963a, 1966; Russev 1966a; Nachev 1981; Russev et al. 1984, 1991, 1994; Janeva \& Russev 1985, 1997; Islam et al. 1986; Janeva 1987, 1989; Stoichev 1994, 1996; Stoichev \& Chernev 2001; Uzunov et al. 2011; Varadinova et al. 2013; Dashinov 2017.

Thienemannia gracilis Kieffer, 1909 - R1, RW; 550-1850 m; 1, 2, 3, 4; e; Dimitrov 1962d, 1963a.

Thienemanniella acuticornis (Kieffer, 1912) [T. fusca Kieffer, 1925] - DM, P2, R1; 50-600 m; 1; po Dimitrov 1966; Russev 1966a; Janeva 1987; Russev et al. 1994; Stoichev 1994, 1996; Janeva \& Russev 1997.

Thienemanniella clavicornis (Kieffer, 1911) [Metriocnemus] - DW, DM, E1, E2, P1, P2, B2, K3, V5, T1, O61, R1; 20-1950 m; 1, 2, 3, 4; po Nachev 1983; Janeva \& Russev 1985, 1997; Islam et al. 1986; Janeva 1987, 1991; Russev et al. 1991, 1994; Stoichev 1994, 1996; Dashinov 2017.

? Thienemanniella flaviforceps Kieffer, 1925 [? Nomen dubium] - DW, S1, R1; 30-1850 m; 1, 2, 3, 4; wces; Dimitrov 1960a, 1962d, 1963a 1966; Russev 1966a; Russev et al. 1991.

? Thienemanniella nana Kieffer, 1911 [Corynoneura; ? Nomen dubium] - DM, P2; ? e; Russev et al. 1994.

? Trissocladius nudisquama Chernovskij, 1949 [? Nomen dubium] - R5; 800 m; 2; ee; Michailova 1982.

Tvetenia bavarica (Goetghebuer, 1934) [Eukiefferiella] - DW, DM, E1, P1, P2, K8, V5, S211, O61, R1, R5; 30-900 m; 1, 2; wp, ? h; Michailova 1982; Islam et al. 1986; Janeva 1987; Janeva \& Russev 1989, 1997; Russev et al. 1991, 1994; Stoichev 1994, 1996; Stoichev \& Chernev 2001; Sakelarieva et al. 2008; Varadinova et al. 2013.

Tvetenia calvescens (Edwards, 1929) [Eukiefferiella] - DW, DM, E1, E2, P1, P2, P3, B1, B3, K7, K8, S1, TL, T1, T31, O61, O62, R1, R5, RW, BS; 20-2250 m; 1, 2, 3, 4, 5; hat; Dimitrov 1962a, 1962d, 1963a, 1966; Russev 1964, 1966a; Russev \& Janeva 1975; Nachev 1983; Russev et al. 1984b, 1991, 1994; Janeva \& Russev 1985; Islam et al. 1986; Janeva 1987, 1989, 1991; Stoichev 1994, 1996; Stoichev \& Chernev 2001; Sakelarieva et al. 2008; Moskova \& Uzunov 2011; Uzunov et al. 2011; Kenderov et al. 2012; Varadinova et al. 2013; Dashinov 2017.

Tvetenia discoloripes (Goetghebuer \& Thienemann, 1936) [Eukiefferiella] - DW, DM, P1, P2, O61, R5; 30800 m; 1, 2; ? wp; Michailova 1982; Nachev 1983; Russev et al. 1991, 1994; Stoichev 1994, 1996; Janeva \& Russev 1997.

Tvetenia tshernovskii Pankratova, 1968 [Eukiefferiella] - DW, DM, P1, P2, K7, K8, K9, S1, O61; 35-590 m; 1, 2; h; Janeva \& Russev 1985; Islam et al. 1986; Janeva 1987, 1991; Russev et al. 1994; Stoichev 1994, 1996.

Tvetenia verralli (Edwards, 1929) - K3; 600-610 m; 1, 2; wcp, ? h; Islam et al. 1986.

Zalutschia mucronata (Brundin, 1949) [Orthocladius potamophila (Chernovskij, 1949); Trissocladius] - DW, DM, E1, E2, P1, P2, V5, S211, TL, R1, RW; 20-1050 m; 1, 2, 3; wces; Dimitrov 1962a, 1962d, 1963a, 1966; Russev 1966a, 1966b, 1978; Janeva 1987, 1991; Russev et al. 1994; Janeva \& Russev 1997.

Zalutschia tatrica (Pagast, 1935) [Orthocladius] - RE; 230-250 m; 1; h; Dimitrov 1963a; Russev 1964.

Baeotendipes noctivagus (Kieffer, 1911) [Halliella caspersi Strenzke, 1951] - BS; 0 m; 1; hom; Lenz 1950; Strenzke 1951a, 1951b; Caspers 1951a, 1951b, 1952, 1957; Valkanov 1957a; Dimitrov 1963a; Georgiev \& Nikolov 2010. 
Beckidia zabolotzkyi (Goetghebuer, 1938) [Cryptochironomus] - DM, E1, P1, P2, R5; 50-800 m; 1, 2; des, ? tes; Janeva 1987; Russev et al. 1994; Stoichev 1994, 1996; Janeva \& Russev 1997; Kenderov et al. 2012.

Chironomus (Chironomus) aberratus Keyl, 1961 - BN, BS; 0-20 m; 1; e; Michailova 1982, 1989.

Chironomus (Chironomus) acidophilus Keyl, 1960 - TL, RW; 185-1250 m; 1, 2, 3; e; Michailova 1982, 2006.

Chironomus alluaudi Kieffer, 1913 - V1; 520 m; 2; ? atm; Michailova 1982a.

Chironomus (Chironomus) anchialicus Michailova, 1974 - BS; 0 m; 1; see; Michailova 1973b, 1974, 1989.

Chironomus (Chironomus) annularius Meigen, 1818 - DW, E2, V1, TL, O61, R5, RW, BN; 0-1230 m; 1, 2, 3; h; Nedelkov 1912; Michailova 1982a, 1989, 1996, 1998; Uzunov et al. 2001; Varadinova et al. 2011, 2012; Ihtimanska et al. 2018; Michailova et al. 2018.

Chironomus (Chironomus) curabilis Belyanina, Sigareva \& Loginova, 1990 - E1; 15-20 m; 1; ee; Polukonova et al. 2005.

Chironomus (Chironomus) pallidivittatus Edwards, 1929 [Camptochironomus] - E1, E2; 15-20 m; 1; ho; Michailova 1989; Ihtimanska et al. 2018.

Chironomus (Chironomus) tentans Fabricius, 1805 [Camptochironomus] - BS; 0-20 m; 1; ho; Michailova 1989.

Chironomus (Chironomus) anthracinus Zetterstedt, 1860 [Tendipes bathophilus Kieffer, 1912] - P2, BS; 0-224 m; 1; h; Zaschev \& Angelov 1959; Janeva 1987.

Chironomus (Chironomus) aprilinus Meigen, 1818 [Ch. halophilus Kieffer, 1913] - V1, R1, BN, BS; 0-2250 m; 1, 2, 3, 4, 5; wp; Nedelkmov 1912; Caspers 1951a, 1951b, 1957; Strenzke 1951a; Michailova 1989, 1996; Stoichev 1996.

Chironomus (Chironomus) balatonicus Devai, Wuelker \& Scholl, 1983 - E2, TL, BN, BS; 0-180 m; 1; cee, ? e; Michailova 1989, 1996; Michailova \& Krastanov 2000; Gunderina et al. 2008; Ihtimanska et al. 2018.

Chironomus (Chironomus) bernensis Kloetzli, 1973 - TL, R5; 150-750 m; 1, 2; wp; Michailova 1989, 2006; Michailova et al. 2016.

Chironomus (Chironomus) bonus Shilova \& Dzhvarsheishvili, 1974 - BN; 0 m; 1; ? e; Michailova 1994, 1996.

Chironomus (Chironomus) acerbiphilus Tokunaga, 1939 [Ch. crassimanus Strenzke, 1959] - RW, BN; 0-1100 m; 1, 2, 3; des; Michailova 1982a, 1989.

Chironomus (Chironomus) heterodentatus Konstantinov, 1956 - DW, P1; 35-217 m; 1; ee; Janeva \& Russev 1989; Russev et al. 1991, 1994.

Chironomus (Chironomus) luridus Strenzke, 1959 - V1, TL, BN; 0-600 m; 1, 2; wp; Michailova 1989.

Chironomus (Chironomus) muratensis Ryser, Scholl \& Wuelker, 1983 - TL, RW, BS; 0-760 m; 1, 2; ? wes; Michailova 1989, 2006.

Chironomus (Chironomus) nuditarsis Keyl, 1961 - DW, E2, P1, V1, TL, O61, O62, RW, BN; 0-1280 m; 1, 2, 3; ? wp; Michailova 1982a, 1989; Polukonova et al. 2005; Kiknadze et al. 2006; Ihtimanska et al. 2018.

Chironomus (Chironomus) obtusidens Goetghebuer, 1921 - BN. BS; 0-50 m; 1; wces; Michailova 1989.

Chironomus (Chironomus) parathummi Keyl, 1961 - E2, BN, BS; 0-20 m; 1; ? e, ? wes; Michailova 1989.

Chironomus (Chironomus) piger Strenzke, 1956 - V5, S211, RW, BS; 0-1100 m; 1, 2, 3; wp; Michailova 1989, 2006; Ilkova et al. 2007; Michailova et al. 2012, 2015.

Chironomus (Chironomus) pilicornis (Fabricius, 1787) [Ch. niveipennis Fabricius, 1805] - V1; 600 m; 1, 2; wes; Nedelkov 1912.

Chironomus (Chironomus) plumosus (Linnaeus, 1758) [Ch. plumosus f. l. semireductus Lenz, 1924] - DW, DM, E1, E2, P1, P2, P3, B1, B2, B3, K9, V1, V4, S1, S21, S22, TL, T1, T31, O61, O62, R1, R2, R5, RW, RE, BN, BS; 0-2394 m; 1, 2, 3, 4, 5; hno; Nedelkov 1912; Thienemann 1936; Valkanov 1936, 1941b, 1957a; Arndt 1943; Caspers 1951a, 1951b; Strenzke 1951; Cvetkov 1955a, 1955b, 1957, 1958, 1962; Dimitrov 1957, 1960a, 1960b, 1962a, 1962b, 1962c, 1963a, 1966, 1981, 1982; Belcheva 1959; Russev 1959, 1961, 1962, 1964, 1966a, 1966b, 1978; Zaschev \& Angelov 1959; Mihailova-Neikova 1961; Michailova 1982a, 1989, 1998, 2006; Nachev 1983; Russev et al. 1984b, 1987, 1991, 1994; Islam et al. 1986; Janeva 1987; Stoichev 1994, 1996, 2001a; Kovachev \& Stoichev 1996; Janeva \& Russev 1989, 1997; Kovachev et al. 1999; Michailova \& Krastanov 2000; Uzunov et al. 2001, 2011; Janeva \& Bancheva 2002; Gunderina et al. 2008; Kenderov et al. 2008, 2012; Soufi \& Uzunov 2008; Varadinova et al. 2011, 2012, 2013; Borisova et al. 2013; Trichkova et al. 2013; Georgieva et al. 2017; Ihtimanska et al. 2018.

Chironomus (Chironomus) prasinus Meigen, 1804 - V1; 550-650 m; 1; e; Nedelkov 1912.

Chironomus (Chironomus) pseudothummi Strenzke, 1959 - TL, BN; 0-160 m; 1; ? wp, ? wes; Michailova 1989. 
Chironomus (Chironomus) riparius Meigen, 1804 [Ch. thummi Kieffer, 1911; ? Ch. anomalus (Kieffer, 1921)] - DW, DM, E1, E2, P1, P2, P3, B1, B2, K7, K8, K9, V1, V4, V5, S1, S21, S22, S211, TL, T1, T31, O61, O62, R1, R2, R5, RW, RE, BN, BS; 0-2535 m; 1, 2, 3, 4, 5, 6; hn; Nedelkov 1912; Thienemann 1936; Valkanov 1936, 1957a; Caspers 1951a; Strenzke 1951; Cvetkov 1955a; Belcheva 1959; Kolarov 1959; Russev 1959, 1961, 1964, 1966a, 1966b, 1977, 1978; Dimitrov 1960b, 1962a, 1962b, 1962d, 1963a, 1966, 1981; Russev \& Janeva 1975, 1986, 1989; Michailova 1982a, 1989, 1996, 2006; Janeva \& Russev 1985, 1997; Islam et al. 1986; Janeva 1987, 1989, 1991; Russev et al. 1987, 1991, 1994; Stoichev 1994, 1996, 1998, 2000a, 2000b, 2001a, 2002; Kovachev et al. 1999; Janeva et al. 2001; Stoichev \& Chernev 2001; Janeva \& Bancheva 2002; Ilkova et al. 2007; Kenderov et al. 2008, 2012; Sakelarieva et al. 2008; Soufi \& Uzunov 2008; Vidinova et al. 2008; Moskova \& Uzunov 2011; Uzunov et al. 2011; Varadinova et al. 2011, 2012, 2013; Michailova et al. 2012; Borisova et al. 2013; Trichkova et al. 2013; Ilkova et al. 2014; Georgieva et al. 2017; Ihtimanska et al. 2018. Chironomus (Chironomus) salinarius Kieffer, 1915 [Tendipes] - P2, S1, TL, BN, BS; 0-380 m, 1; tp; Thienemann 1936; Valkanov 1936, 1957a; Lenz 1950; Caspers 1951a, 1951b, 1952, 1957; Strenzke 1951; Cvetkov 1955a, 1958; Dimitrov 1957, 1960a, 1960b, 1962a, 1962b, 1963a; Zaschev \& Angelov 1959; Mihailova-Neikova 1961; Michailova 1980b, 1989, 1996; Stoichev 1996; Kovachev et al. 1999.

Chironomus (Chironomus) sororius Wuelker, 1973 - R5; 860 m; 2; e; Michailova 1982a.

Chironomus (Chironomus) uliginosus Keyl, 1960 - E1, P1, TL; 50-180 m; 1; e, ? wes; Michailova 1982.

Chironomus (Chironomus) usenicus Loginova \& Belyanina, 1994 - V1; 600 m; 1, 2; ? ee; Michailova 2004.

Chironomus (Chironomus) valkanovi Michailova, 1974 - BS; 0; 1; see; Michailova 1973, 1974a, 1980b, 1985b; Belcheva \& Michailova 1980.

Chironomus (Lobochironomus) dorsalis Meigen, 1818 [? Ch. dorsalis var. venustus Staeger, 1839; Ch. longipes Staeger, 1839; Einfeldia] - DW, P2, V1, TL, R1, R5, RW, BN; 0-2537 m; 1, 2, 3, 4, 5, 6; h; Nedelkov 1912; Michailova 1982, 1989, 2006; Russev et al. 1984, 1994; Stoichev 2000a; Trichkova et al. 2013; Varadinova et al. 2013.

Cladopelma fridmanae (Chernovskij, 1949) [Cryptochironomus; ? nomina dubia] - DW, DM, E1, S1, TL, R5, RW; 50-1550 m; 1, 2, 3, 4; des; Dimitrov 1960a, 1960b, 1962b, 1963a; Michailova 1982, 1989, 2006; Russev et al. 1994; Stoichev 1994, 1996; Janeva \& Russev 1997.

Cladopelma viridulum (Linnaeus, 1767) [Cryptochironomus] - TL, BS; 0-160 m; 1; ho; Mihailova-Neikova 1961; Cvetkov 1962; Dimitrov 1962b, 1963a.

? Cryptochironomus anomalus (Kieffer, 1918) [? nomen dubium] - BS; 0 m; 1; ? e; Mihailova-Neikova 1961; Dimitrov 1963a.

? Cryptochironomus conjugens (Kieffer, 1921) [? nomen dubium] - E1, P2, V5, S1, TL, RE, BN; 0-400 m; 1; ? wces; Cvetkov 1955a, 1955b, 1957, 1962; Valkanov 1957a; Dimitrov 1957, 1960a, 1960b, 1962b, 1962c, 1962d, 1963a; Russev 1959, 1966b.

Cryptochironomus (Cryptochironomus) defectus (Kieffer, 1913) - DW, DM, E1, E2, P1, P2, P3, B1, B2, B3, K8, K9, V1, V5, S1, S21, S22, S211, TL, T1, T31, O61, O62, R1, R2, RW, RE, BN, BS; 0-2545 m; 1, 2, 3, 4, 5, 6; pa; Cvetkov 1955a, 1955b, 1957, 1962; Valkanov 1957a; Dimitrov 1957, 1960a, 1960b, 1962b, 1962c, 1962d, 1963a, 1966, 1982; Mihailova-Neikova 1961; Russev 1962, 1963, 1966a, 1966b; Nachev 1983; Russev et al. 1984b, 1987, 1991, 1994; Janeva \& Russev 1985; Islam et al. 1986; Russev \& Janeva 1986; Janeva 1987, 1991; Janeva \& Russev 1989, 1997; Michailova 1989, 1996, 1998; Stoichev 1994, 1996, 1998, 2000a, 2000b, 2001, 2002; Kovachev \& Stoichev 1996; Kovachev et al. 1999; Janeva et al. 2001; Stoichev \& Chernev 2001; Uzunov et al. 2001, 2011; Janeva \& Bancheva 2002; Kenderov et al. 2008, 2012; Sakelarieva et al. 2008; Soufi \& Uzunov 2008; Vidinova et al. 2008; Moskova \& Uzunov 2011; Varadinova et al. 2011, 2012, 2013; Borisova et al. 2013; Trichkova et al. 2013; Dashinov 2017; Ihtimanska et al. 2018.

Cryptochironomus (Cryptochironomus) psittacinus (Meigen, 1830) - BS; 0-20 m; 1; h; Nedelkov 1912; Islam et al. 1986.

Cryptochironomus (Cryptochironomus) redekei (Kruseman, 1933) - TL; 150-180 m; 1; wces; Michailova 1982. Cryptochironomus (Cryptochironomus) rostratus Kieffer, 1921 - K8; 590-600 m; 1, 2; wpo; Islam et al. 1986.

Cryptochironomus (Cryptochironomus) supplicans (Meigen, 1830) - R5; 500-800 m; 1, 2; ? wp, ? wcp; Uzunov et al. 2011; Varadinova et al. 2013.

Demicryptochironomus (Demicryptochironomus) vulneratus (Zetterstedt, 1838) - DM, E1, P2, P3, B3, TL, T1, O61, O62, R1, R5, RW, RE, BN, BS; 0-2228 m; 1, 2, 3, 4, 5; po; Mihailova-Neikova 1961; Dimitrov 1963a; Nachev 1983; Islam et al. 1986; Janeva 1987; Michailova 1989; Russev et al. 1994; Stoichev 1994, 
1996, 2000a; Janeva \& Russev 1997; Stoichev \& Chernev 2001; Sakelarieva et al. 2008; Uzunov et al. 2011; Kenderov et al. 2008, 2012; Varadinova et al. 2013.

Dicrotendipes lobiger (Kieffer, 1921) - V1, V3, BN; 0-958 m; 1, 2; h; Michailova 1989; 2009.

Dicrotendipes modestus (Say, 1823) [Trichotanypus] - DW; 30 m; 1; h; Zilachi 1934.

Dicrotendipes nervosus (Staeger, 1839) [Limnochironomus] - DW, DM, E1, E2, P2, K3, V1, S1, TL, T1, O61, O62, R1, R5, RW, RE, BN, BS; 0-2440 m; 1, 2, 3, 4, 5; ho; Cvetkov 1955a, 1955b, 1957, 1962; Valkanov 1957a; Mihailova-Neikova 1961; Dimitrov 1962b, 1962c, 1963a, 1966; Russev 1966b; Michailova 1982, 1989, 1996; Russev et al. 1984, 1987, 1991, 1994; Janeva \& Russev 1985, 1989, 1997; Islam et al. 1986; Janeva 1987, 1991; Stoichev 1994, 1996, 1998, 2000a, 2002; Kovachev et al. 1999; Janeva et al. 2001; Stoichev \& Chernev 2001; Sakelarieva et al. 2008; Soufi \& Uzunov 2008; Vidinova et al. 2008; Uzunov et al. 2011; Kenderov et al. 2012; Varadinova et al. 2011, 2012, 2013; Borisova et al. 2013; Trichkova et al. 2013; Ihtimanska et al. 2018.

Dicrotendipes tritomus (Kieffer, 1916) [Limnochironomus] - DW, DM, E1, P1, BS; 0-250 m; 1; hn; Michailova 1982; Russev et al. 1994; Stoichev 1994, 1996; Janeva \& Russev 1997; Kenderov et al. 2012.

? Dicrotendipes dubia (Cure, 1973) [? nomen dubium] - DW, DM, P1, P2; 50-250 m; 1; see; Janeva 1987; Russev et al. 1994; Stoichev 1996.

Einfeldia pagana (Meigen, 1838) [Chironomus biappendiculatus Kruglova, 1940] - DW, DM, E1, E2, P1, P2, V1, V5. TL; 20-900 m; 1, 2; ho; Dimitrov 1963a; Russev 1966b; Michailova 1982; Janeva 1987; Russev et al. 1994; Stoichev 1994, 1996; Dashinov 2017.

Endochironomus albipennis (Meigen, 1830) - S1, RW; 1580 m; 3, 4; esca; Michailova \& Gercheva 1982; Russev et al. 1984b; Michailova 1987, 1989, 2006; Petrova \& Michailova 1989.

Endochironomus tendens (Fabricius, 1775) [? E. signaticornis (Kieffer, 1913) - nomina dubia; ? E. albidus (Konstantinov, 1956) - brackish] - DW, DM, E1, E2, P1, P2, V1, V5, S1, TL, O61, RW, BN, BS; 0-1550 m; 1, 2, 3, 4; tp; Cvetkov 1955a, 1955b, 1962; Valkanov 1957a; Dimitrov 1962b, 1963a; Russev \& Janeva 1975; Michailova 1982a, 1989, 1992, 1996, 2006; Michailova \& Gercheva 1982; Russev et al. 1984b, 1994; Janeva 1987; Stoichev 1994, 1996, 1998; Kovachev \& Stoichev 1996; Janeva \& Russev 1997; Kovachev et al. 1999; Soufi \& Uzunov 2008; Kenderov et al. 2012; Ihtimanska et al. 2018.

Synendotendipes dispar (Meigen, 1830) [Endochironomus lucidus (Zetterstedt, 1838); Trichocladius] - DW, DM, E1, P2, V1, R1, RW; 50-1200 m; 1, 2, 3; tes, ? hes; Nedelkov 1912; Dimitrov 1963a, 1966; Russev 1966a; Stoichev 1994, 1996; Janeva \& Russev 1997.

Synendotendipes impar (Walker, 1856) [Endochironomus] - E1, RW; 20-1550 m; 1, 2, 3, 4; tes, ? hoes; Michailova 1982, 1989, 1996; Michailova \& Gercheva 1982.

Glyptotendipes (Glyptotendipes) barbipes (Staeger, 1839) - R5, BN, BS; 0-800 m; 1, 2; ho; Michailova 1979a, 1979b, 1989; Varadinova et al. 2013.

Glyptotendipes (Glyptotendipes) cauliginellus (Kieffer, 1913) [G. gripekoveni Kieffer, 1913] - DW, DM, E1, E2, B1, TL, R5, RW, RE, BN, BS; 0-1550 m; 1, 2, 3, 4; po; Cvetkov 1955a, 1955b, 1962; Valkanov 1957a; Dimitrov 1960b, 1962a, 1962b, 1963a, 1963b, 1982; Mihailova-Neikova 1961; Russev 1966b; Michailova 1979a, 1982a, 1989, 1996, 1998, 2006; Russev et al. 1994; Stoichev 1994, 1996, 1998; Janeva \& Russev 1997; Kovachev et al. 1999; Janeva et al. 2001; Uzunov et al. 2001, 2011; Vidinova et al. 2008; Varadinova et al. 2011, 2012, 2013; Kenderov et al. 2012; Ihtimanska et al. 2018.

Glyptotendipes (Glyptotendipes) glaucus (Meigen, 1818) - DW, DM, E1, E2, K3, V1, TL, T1, O61, O62, RW, BN, BS; 0-1550 m; 1, 2, 3, 4; tes, ? wces; Michailova 1979a, 1982a, 1989, 1995, 1996, 1998, 2006; Islam et al. 1986; Janeva 1991; Russev et al. 1994; Stoichev 1994, 1996, 1998; Janeva \& Russev 1997; Kovachev et al. 1999; Uzunov et al. 2001; Varadinova et al. 2011, 2012; Kenderov et al. 2012; Michailova et al. 2012; Borisova et al. 2013; Ihtimanska et al. 2018.

Glyptotendipes (Glyptotendipes) pallens (Meigen, 1804) [G. polytomus Kieffer, 1909] - DW, DM, E1, E2, V1, TL, O61, R1, BN, BS; 0-1570 m; 1, 2, 3, 4; po; Cvetkov 1955a, 1962; Valkanov 1957a; Mihailova-Neikova 1961; Dimitrov 1962b, 1963, 1981, 1982; Nachev 1983; Islam et al. 1986; Russev et al. 1994; Stoichev 1994, 1996; Michailova 1995; Janeva \& Russev 1997; Michailova \& Todorova 1998; Stoichev \& Chernev 2001; Uzunov et al. 2001; Varadinova et al. 2011, 2012; Ihtimanska et al. 2018.

Glyptotendipes (Glyptotendipes) paripes (Edwards, 1929) - DW, E1, E2, P1, TL, O61, BS; 20-400 m; 1; h; Michailova 1979a, 1982a, 1989; Ihtimanska et al. 2018.

Glyptotendipes (Glyptotendipes) salinus Michailova, 1987 - BS; 0 m; 1; e; Michailova 1987b, 1989. 
Glyptotendipes (Caulochironomus) caulicola (Kieffer, 1913) - E2, RW, BN; 0-1200 m; 1, 2, 3, 4; e; Michailova 1979a, 1989, 1996, 2006; Stoichev 1994, 1996.

Glyptotendipes (Caulochironomus) scirpi (Kieffer, 1915) [G. mancunianus Edwards, 1929] - E1, E2, P1; 20-150 m; 1; ? e; Michailova 1982a, 1989.

? Harnischia burganadzeae (Chernovskij, 1949) [Cryptochironomus; ? nomen dubium] - TL; 150-170 m; 1; ? ee; Dimitrov 1962b, 1963a.

Harnischia curtilamellata (Malloch, 1915) - S1; 150 m; 1; hpta, sk; Russev et al. 1984b.

Harnischia fuscimanus Kieffer, 1921 [Cryptochironomus] - DM, TL, O61, O62, R1, RE; 150-1000 m; 1, 2; tp; Dimitrov 1962b, 1962c, 1963a, 1966; Russev 1966a; Islam et al. 1986; Janeva 1987; Russev et al. 1994.

Kiefferulus (Kiefferulus) tendipediformis (Goetghebuer, 1921) - E2, V1, RE, BN; 0-600 m; 1, 2; wp; Michailova 1996, 2006; Michailova \& White 2007; Ihtimanska et al. 2018.

? Microchironomus conjungens Lenz, 1926 [? nomen dubium] - DW, P1; ? ee; Russev et al. 1994.

Microtendipes chloris (Meigen, 1818) - DW, DM, E1, E2, P1, P2, V5, S211, TL, T3, R1, R5, RW, RE; 20-1200 m; 1, 2, 3; wpo; Dimitrov 1957, 1962d, 1963a; Russev 1959, 1962, 1966b; Janeva 1987; Russev et al. 1994; Stoichev 1994, 1996; Janeva \& Russev 1997; Sakelarieva et al. 2008; Soufi \& Uzunov 2008; Vidinova et al. 2008; Uzunov et al. 2011; Kenderov et al. 2012; Trichkova et al. 2013; Varadinova et al. 2013.

Microtendipes pedellus (De Geer, 1776) - DW, DM, E1, P1, P2, V5, S1, O61, RW; 20-1000 m; 1, 2; ho; Russev et al. 1984b, 1991, 1994; Michailova 1989, 2006; Stoichev 1994, 1996; Janeva \& Russev 1997; Kenderov et al. 2012; Dashinov 2017.

Microtendipes tarsalis (Walker, 1856) - DW, DM, P2; 50-450 m; 1; e; Janeva 1987; Russev et al. 1994; Stoichev 1994, 1996; Janeva \& Russev 1997.

Parachironomus frequens (Johannsen, 1905) [Cryptochironomus longiforceps Kieffer, 1921] - P1, TL; 150-200 m; 1; h; Michailova 1982.

Parachironomus gracilior (Kieffer, 1918) [P. arcuatus Goetghebuer, 1919; P. pararostratus (Harnisch, 1923); ? nomina dubia; Cryptochironomus pararostratus Lenz, 1938] - DW, DM, E1, E2, P1, P2, TL, T1, RW, RE, BN, BS; 0-1200 m; 1, 2, 3; po; Cvetkov 1955a, 1957, 1962; Belcheva 1959; Dimitrov 1960b, 1962a, 1962b, 1962c, 1963a, 1981, 1982; Mihailova-Neikova 1961; Russev et al. 1984b, 1994; Janeva 1987; Michailova 1989; Stoichev 1994; Janeva \& Russev 1997.

Parachironomus kuzini Shilova, 1969 - DW, DM, P1, P2, K3, O61; 50-600 m; 1; ee; Islam et al. 1986; Janeva 1991; Russev et al. 1991, 1994; Stoichev 1994, 1996; Janeva \& Russev 1997.

Parachironomus parilis (Walker, 1856) - RW; 1580 m; 4; h; Michailova 1989, 2006.

Parachironomus vitiosus (Goetghebuer, 1921) - DM, B1; 50-550 m; 1; esca, ? hoes; Janeva 1991; Russev et al. 1994.

Paracladopelma camptolabis (Kieffer, 1913) [Cryptochironomus] - P2, V5, S211, S1, TL, R1, RW; 150-1100 m; 1, 2, 3; h; Dimitrov 1957, 1960a, 1962a, 1962b, 1962d, 1963a, 1966; Russev 1966a; Russev et al. 1994.

Paratendipes albimanus (Meigen, 1818) - DW, DM, E1, P2, S1, TL, R1, RW, BS; 0-1200 m; 1, 2, 3; ho; Dimitrov 1957, 1960a, 1962a, 1962b, 1963a, 1966; Mihailova-Neikova 1961; Janeva \& Russev 1985; Janeva 1987; Russev et al. 1994; Stoichev 1994, 1996; Janeva \& Russev 1997.

? Paratendipes connectens Lipina, 1926 [? nomen dubium] - DW, DM, E1, E2; 20-30 m; 1; ? wces; Russev 1959, 1962, 1966b; Dimitrov 1963a.

? Paratendipes intermedius Chernovskij, 1949 [? nomen dubium] - DW, DM, E1, E2, V5; 20-900 m; 1, 2; ee; Russev 1962, 1966b; Dimitrov 1963a; Varadinova et al. 2013.

Paratendipes nudisquama (Edwards, 1929) [P. transcaucasicus Chernovskii, 1949; Limnophyes; Limnochironomus] - DW, DM, E1, E2, P2, V5, S211, TL, T1, O61, O62, R1, R5, RW, RE; 20-1200 m; 1, 2, 3; hno; Dimitrov 1962b, 1962d, 1963a, 1966; Russev 1966a, 1966b; Janeva 1987; Russev et al. 1994; Stoichev 1994, 1996; Janeva \& Russev 1997; Uzunov et al. 2011; Varadinova et al. 2013.

Paratendipes plebeius (Meigen, 1818) [P. fuscimanus Kieffer, 1921] - DW, DM, P1; e; Russev et al. 1994; Stoichev 1994, 1996.

Polypedilum (Pentapedilum) exsectum (Kieffer, 1916) [Pentapedilum] - DM, P2, K7, K8, K9, S1, O61, R1, R5, RW; 50-2200 m; 1, 2, 3, 4, 5; des, ? wces; Dimitrov 1963a; Nachev 1983; Islam et al. 1986; Michailova 1982, 1989, 2006; Russev et al. 1984b, 1994; Stoichev 1994; Uzunov et al. 2011; Varadinova et al. 2013.

Polypedilum (Pentapedilum) sordens (van der Wulp, 1875) [P. macrophthalma Chernovskij, 1949; Pentapedilum] - DW, DM, E2, P1, P2, P3, B1, K7, K8, K9, S1, TL, T1, O61, O62, R1, RW; 50-2250 m; 1, 2, 3, 4, 5; 
h; Dimitrov 1962b, 1963a, 1966; Russev 1966a; Islam et al. 1986; Janeva \& Russev 1989, 1997; Michailova 1982, 1989, 2006; Janeva 1991; Russev et al. 1991, 1994; Stoichev 1994; Ihtimanska et al. 2018.

Polypedilum (Uresipedilum) convictum (Walker, 1856) - DW, DM, E1, E2, P1, P2, K3, K8, K9, V5, S1, TL, T1, O61, O62, R1, R5, RW, RE; 20-1400 m; 1, 2, 3; ho; Dimitrov 1957, 1960a, 1960b, 1962b, 1962c, 1963a, 1966, 1981, 1982; Russev 1959, 1962, 1966a, 1966b; Cvetkov 1962; Michailova 1982; Nachev 1983; Russev et al. 1984a, 1984b, 1987, 1991, 1994; Janeva \& Russev 1985, 1989, 1997; Islam et al. 1986; Russev \& Janeva 1986; Janeva 1987, 1991; Stoichev 1994, 1996; Uzunov et al. 2011; Borisova et al. 2013; Varadinova et al. 2013; Dashinov 2017.

Polypedilum (Polypedilum) nubeculosum (Meigen, 1804) [Paratendipes] - DW, DM, E1, E2, P2, B2, K3, K7, K8, K9, S1, TL, T1, O61, O62, R1, RW, RE, BN, BS; 0-2535 m; 1, 2, 3, 4, 5, 6; h; Caspers 1951a, 1951b; Strenzke 1951; Cvetkov 1955a, 1955b, 1957, 1962; Dimitrov 1957, 1960a, 1960b, 1962a, 1962b, 1962c, 1963a, 1966; Valkanov 1957a; Mihailova-Neikova 1961; Russev 1964, 1966a; Michailova 1982, 1989, 1996; Russev et al. 1984a, 1984b, 1991, 1994; Islam et al. 1986; Janeva 1987; Janeva \& Russev 1989, 1997; Stoichev 1994, 1996, 1998, 2000a, 2001a, 2004; Kovachev et al 1999; Borisova et al. 2013; Trichkova et al. 2013.

Polypedilum (Polypedilum) nubifer (Skuse, 1889) [P. pharao Kieffer, 1925; P. aberrans Chernovskii, 1949] DW, DM, E1, E2, P1, P2, TL, T1, RW, RE, BN, BS; 0-1550 m; 1, 2, 3, 4; poa; Caspers 1951a; Strenzke 1951; Cvetkov 1955a, 1962; Valkanov 1957a; Dimitrov 1960b, 1962b, 1963a, 1966, 1982; Russev 1964, 1966a, 1966b; Russev et al. 1984b, 1991, 1994; Janeva 1989; Michailova 1988, 1989, 2006; Stoichev 1994, 1996.

Polypedilum (Polypedilum) pedestre (Meigen, 1830) - DM, E1, P2, S1, R1, R5, RW, RE; 200-1550 m; 1, 2, 3, 4; tp, ? h; Belcheva 1959; Dimitrov 1963a, 1966; Russev 1966a; Russev \& Janeva 1975; Russev et al. 1984b, 1994; Stoichev 1994, 1996; Janeva \& Russev 1997; Vidinova et al. 2008; Uzunov et al. 2011; Borisova et al. 2013; Varadinova et al. 2013; Dashinov 2017.

Polypedilum (Polypedilum) octopunctatum (Thunberg, 1784) [P. quadrimaculatum (Meigen, 1838)] - DW, P1; 220 m; 1; cse; Russev et al. 1991, 1994.

Polypedilum (Tripodura) bicrenatum Kieffer, 1921 - DW, K3, O61, O62; 107-600 m; 1, 2; tp; Islam et al. 1986; Russev et al. 1994; Stoichev 1996.

Polypedilum (Tripodura) scalaenum (Schrank, 1803) [P. breviantennatum Chernovskii, 1949; Diamesa] - DW, DM, E1, E2, P1, P2, K3, K8, K9, V5, S1, S211, TL, O61, O62, R1, R5, RW, RE, BN; 0-2003 m; 1, 2, 3, 4; ho; Cvetkov 1955a, 1955b, 1957; Valkanov 1957a; Dimitrov 1957, 1960a, 1962b, 1962c, 1963a, 1966; Russev 1962, 1964, 1966a, 1966b; Nachev 1983; Russev et al. 1984a, 1984b, 1994; Janeva \& Russev 1985, 1997; Islam et al. 1986; Russev \& Janeva 1986; Janeva 1987, 1991; Stoichev 1994, 1996; Stoichev \& Chernev 2001; Sakelarieva et al. 2008; Uzunov et al. 2011; Varadinova et al. 2013.

Polypedilum (Tripodura) tetracrenatum Hirvenoja, 1962 [Procladius] - DW, DM, E1, E2, K3; 20-600 m; 1, 2; dp; Islam et al. 1986; Janeva \& Russev 1989; Janeva 1991; Russev et al. 1994; Stoichev 1994, 1996; Janeva \& Russev 1997.

Sergentia coracina (Zetterstedt, 1850) [S. longiventris Kieffer, 1924] - S1; 380 m; 1; h; Dimitrov 1960a, 1963a. Stictochironomus crassiforceps (Kieffer, 1921) - BS; 0-5 m; 1; wcp; Michailova 1989.

Stictochironomus maculipennis (Meigen, 1818) - V1; 550-600 m; 1; wp; Nedelkov 1912.

Stictochironomus pictulus (Meigen, 1830) - B1, R2; 400-2394 m; 1, 2, 3, 4, 5; h; Janeva 1991; Stoichev 1996.

? Stictochironomus psammophilus Chernovskij, 1949 [? nomen dubium] - DW, P1, B1; 144-500 m; 1, 2; wces; Janeva 1991; Janeva \& Russev 1989; Russev et al. 1994.

Stictochironomus sticticus (Fabricius, 1781) [S. histrio (Fabricius, 1794)] - DW, DM, P1, P2, K3, K7, K8, V4, S1, S22, O61, O62, R5, RW; 50-1150 m; 1, 2, 3; h; Dimitrov 1957, 1963a; Belcheva 1959; Russev 1961; Nachev 1983; Islam et al. 1986; Russev \& Janeva 1986; Michailova 1989; Janeva 1991; Russev et al. 1991, 1994; Janeva \& Russev 1997; Uzunov et al. 2011; Varadinova et al. 2013.

Xenochironomus xenolabis (Kieffer, 1916) - BN; 0 m; 1; hn, ? hno; Cvetkov 1955a; Dimitrov 1963a.

Cladotanytarsus (Lenziella) bicornutus Kieffer, 1922 [C. wexionensis Brundin, 1947] - O61; 315 m; 1; des; Nachev 1983.

Cladotanytarsus (Cladotanytarsus) mancus (Walker, 1856) [Tanytarsus] - DW, DM, E1, E2, P1, P2, V1, V5, S1, S211, TL, R1, R5, RW, RE, BN; 0-1200 m; 1, 2, 3; wcp; Cvetkov 1955a, 1955b, 1957; Valkanov 1957a; Russev 1959, 1962, 1966a, 1966b; Dimitrov 1960b, 1962b, 1962c, 1962d, 1963a, 1966, 1982; Janeva 1987; Russev et al. 1994; Stoichev 1994, 1996, 2001a; Janeva \& Russev 1997; Uzunov et al. 2011; Trichkova et al. 2013; Varadinova et al. 2013. 
? Cladotanytarsus sexdentatus (Chernovskij 1949) [Tanytarsus; ? nomen dubium] - DW, DM, P2; 50-200 m; 1; wes; Russev 1962, 1966b; Stoichev 1994, 1996; Janeva \& Russev 1997.

Corynocera ambigua Zetterstedt, 1837 [Corynoneura; Tanytarsus pedicelliferus Birula, 1935; ? Tanytarsus sevanicus Chernovskij, 1949 (nomen dubium)] - DW, P1; 50-210 m; 1, 2; h; Janeva 1991; Russev et al. 1991, 1994.

Micropsectra acuta Goetghebuer, 1934 [Krenopsectra] - O61; 360 m; 1; ? e; Islam et al. 1986.

Micropsectra apposita (Walker, 1856) [M. trivialis (Kieffer, 1911)] - S1; 283 m; 1; h; Janeva \& Russev 1985.

Micropsectra atrofasciata (Kieffer, 1911) [Eukiefferiella] - DW, S1, RW; 200-1100 m; 1, 2, 3; wpo; Dimitrov1960a, 1962a, 1963a; ? Michailova 1982; Russev et al. 1991, 1994.

Micropsectra contracta Reiss, 1965 - K3, K7, K8, K9, O61, O62, R5; 100-820 m; 1, 2; des; Michailova 1982; Islam et al. 1986.

? Micropsectra curvicornis (Chernovskij, 1949) [? nomen dubium] - DW, DM, P1, P2, B1, O61; 150-530 m; 1, 2; wes; Nachev 1983; Islam et al. 1986; Janeva 1987, 1991; Russev et al. 1991, 1994.

Micropsectra junci (Meigen, 1818) [M. praecox Meigen, 1818; Microtendipes] - DW, DM, E1, E2, P1, P2, V1, S1, R1, R5, RW; 20-2368 m; 1, 2, 3, 4, 5; h; Dimitrov 1957, 1963a, 1966; Belcheva 1959; Russev 1959, 1962, 1966a, 1966b; Michailova 1982; Janeva 1987; Russev et al. 1994; Stoichev 1994, 1996, 2000a, 2004; Janeva \& Russev 1997.

Micropsectra lindrothi Goetghebuer, 1931 - R2; 1142 m; 3; h; Michailova 1989.

Micropsectra notescens (Walker, 1856) - R2; 1950 m; 4; wp; Michailova \& Petrova 1987; Michailova 1989.

Micropsectra radialis Goetghebuer, 1939 - R2; 2545-2709 m; 6; po; Stoichev \& Danova 2003.

Micropsectra recurvata Goetghebuer, 1928 - RW; 800-1550 m; 2, 3, 4; h; Michailova 1989, 2006.

Micropsectra pallidula (Meigen, 1830) [Microtendipes; M. viridiscutellata Goetghebuer, 1932] - DW, DM, E1, P1, P2, B1, K3, K9, O61, O62, R5; 50-1020 m; 1, 2, 3; wpo; Nachev 1983; Islam et al. 1986; Russev \& Janeva 1986; Janeva 1989, 1991; Michailova 1989; Russev et al. 1991, 1994; Stoichev 1994, 1996; Janeva \& Russev 1997; Uzunov et al. 2011; Varadinova et al. 2013.

Neozavrelia fuldensis Fitttkau, 1954 - DM, P2; 35-680 m; 1, 2; ? e, ? wes; Janeva 1987; Russe et al. 1994; Stoichev 1994, 1996.

Paratanytarsus dissimilis (Johannsen, 1905) [P. confusus Palmen, 1960] - DW, DM, P1, P2, K8, S1, T1, O61, R5; 50-800 m; 1, 2; h; Nachev 1983; Russev et al. 1984, 1994; Janeva \& Russev 1985, 1997; Islam et al. 1986; Russev \& Janeva 1986; Uzunov et al. 2011; Varadinova et al. 2013.

Paratanytarsus lauterborni (Kieffer, 1909) [Tanytarsus] - DW, DM, E1, E2, P1, B1, V5, S1, S211, TL, R1, RW, BS; 0-1550 m; 1, 2, 3, 4; h; Russev 1959, 1962, 1966b; Dimitrov 1960b, 1962a, 1962b, 1962d, 1963a, 1963b, 1966, 1981; Mihailova-Neikova 1961; Cvetkov 1962; Michailova 1982, 1989, 2006; Russev et al. 1991, 1994; Janeva \& Russev 1997.

Rheotanytarsus photophilus (Goetghebuer, 1921) - O61; 330 m; 1; dp; Nachev 1983; Islam et al. 1986.

? Rheotanytarsus exiguus (Johannsen 1905) [? North American species] - DW, DM, E1, E2, P1, P2, K3, K7, K8, K9, V4, O61, O62; 75-810 m; 1, 2; ? h; Russev 1978; Islam et al. 1986; Janeva 1987, 1991; Russev et al. 1991.

Stempellina bausei (Kieffer, 1911) - BN; 0 m; 1; wces; Cvetkov 1955a, 1957; Valkanov 1957a; Dimitrov 1963 a.

Stempellina cornuta Kieffer, 1922 [S. montivaga Goetghebuer, 1934] - BN; 0 m; 1; csee; Thienemann 1949; Dimitrov 1963a.

Stempellina subglabripennis (Brundin, 1947) - K7, K8, K9, O61, O62; 90-500 m; 1, 2; h; Nachev 1983; Islam et al. 1986.

Stempellinella brevis (Edwards, 1929) - DM, P2; 50-300 m; 1; wp, ? h; Russev et al. 1994; Janeva \& Russev 1997. Tanytarsus excavatus Edwards, 1929 - DW; 30-40 m; 1; e; Russev et al. 1991, 1994.

Tanytarsus gregarius Kieffer, 1909 [T. lobatifrons Kieffer, 1913] - DW, DM, E1, E2, P1, P2, V1, V5, S1, S21, S22, S211, TL, T1, T31, O61, O62, R1, R5, RW, RE, BN, BS; 0-2709 m; 1, 2, 3, 4, 5, 6; h; Caspers 1951b; Valkanov 1957a; Dimitrov 1960a, 1960b, 1962b, 1962c, 1962d, 1963a, 1963b, 1966; Russev 1964, 1966a, 1966b; Russev \& Janeva 1975; Russev et al. 1984b, 1991, 1994; Janeva 1987; Stoichev 1994, 1996, 2001a; Janeva \& Russev 1997; Stoichev \& Chernev 2001; Janeva \& Bancheva 2002; Stoichev \& Danova 2003; Kenderov et al. 2008, 2012; Sakelarieva et al. 2008; Soufi \& Uzunov 2008; Vidinova et al. 2008; Moskova \& Uzunov 2011; Uzunov et al. 2011; Varadinova et al. 2011, 2012, 1013; Borisova et al. 2013; Georgieva et al. 2017; Ihtimanska et al. 2018.

? Tanytarsus longipes Akhrorov, 1968 [? nomen dubium] - DW, DM; P1, B1, K3, K7, K8, K9, O61; 50-450 m; 1; ? ewca; Islam et al. 1986; Janeva 1991; Russev et al. 1994; Stoichev 1994, 1996; Janeva \& Russev 1997. 
Tanytarsus medius Reiss \& Fittkau, 1971 - DW, DM, E1, P1, P2, P3, B3, TL, T31, RE, BN, BS; 0-500 m; 1; ena; Russev et al. 1984, 1991, 1994; Russev \& Janeva 1986; Stoichev 1994, 1996; Janeva \& Russev 1997.

Tanytarsus mendax Kieffer, 1925 [T. holochlorus Edwards, 1929] - DW, DM, P1, P2, R5, BN; 0-820 m; 1, 2; h; Caspers 1951a; Strenzke 1951; Michailova 1982; Russev et al. 1984, 1991, 1994; Stoichev 1994, 1996; Janeva \& Russev 1997; Janeva 1991; Uzunov et al. 2011; Varadinova et al. 2013.

Tanytarsus occultus Brundin, 1949 - O61; 310 m; 1; des; Islam et al. 1986.

Tanytarsus pallidicornis (Walker, 1856) - DW, P1, P2, K3, K7, K8, K9, V4, O61; 50-810 m; 1, 2; h; Nachev 1983; Russev et al. 1984, 1991, 1994; Islam et al. 1986; Stoichev 1996; Janeva \& Russev 1997.

Tanytarsus usmaensis Pagast, 1931 [Cryptotendipes] - DW, DM, K7, K8, O62; 30-585 m; 1; h; Islam et al. 1986; Janeva 1991; Russev et al. 1991, 1994; Janeva \& Russev 1997.

Tanytarsus verralli Goetghebuer, 1928 - R5; 500-800 m; 1, 2; des; Uzunov et al. 2011; Varadinova et al. 2013. Virgatanytarsus arduennensis (Goetghebuer, 1922) [Tanytarsus] - DW, DM, P1, V4;30-810 m; 1, 2; ppt; Islam et al. 1986; Janeva 1991; Russev et al. 1991, 1994; Janeva \& Russev 1997.

Zavrelia pentatoma Kieffer \& Bause, 1913 - O61; 305 m; 1; des; Islam et al. 1986.

\section{BRACHYCERA}

\section{ORTHORRHAPHA}

\section{XYLOPHAGOMORPHA}

\section{Xylophagidae}

Xylophagus ater Meigen, 1804 - V1, V4; 550-800 m; 1, 2; e, ? des; Nedelkov 1910, 1912.

\section{Cenomyiidae}

Coenomyia ferruginea (Scopoli, 1763) - R1; 1150-1200 m; 3; h; Joakimoff 1899.

\section{STRATIOMYOMORPHA}

\section{Xylomyidae}

Solva marginata (Meigen, 1820) [Xylomyia] - DW, V1; 30-600 m; 1; hoes; Nedelkov 1912; Szilády 1934.

\section{Stratiomyidae}

Beris chalybata (Forster, 1771) [Actina nitens (Latreille, 1805)] - B1, S23, R2; 227-1800 m; 1, 2, 3, 4; e, ? wes; trogloxene; Dušek \& Rozkošný 1963; Beron 1972b, 1994, 2015, 2016; Beschovski 1972c; Rozkošný 19821983; Rozkošný \& Nartshuk 1988; Woodley 2001; Dvořák et al. 2021.

Beris clavipes (Linnaeus, 1767) - V4; 780-850 m; 2; e; Szilády 1934; Bankowska 1967a; Rozkošný 1982; Rozkošný \& Nartshuk 1988; Woodley 2001.

Beris morrisii Dale, 1841 - V4; 780-850 m; 2; e; Bankowska 1967a; Rozkošný 1982; Rozkošný \& Nartshuk 1988; Woodley 2001.

Chorisops tibialis (Meigen, 1820) - V1; 550 m; 1; ? eswa; Rozkošný 1982; Rozkošný \& Nartshuk 1988.

Chloromyia formosa (Scopoli, 1763) - \$ DW, DM, E1, E2, B1, B2, V1, V4, S1, S2, TL, T31, O1, O62, R1, R5, RW, RE, BN; 30-1700 m; 1, 2, 3, 4; wcp, h, i; Löw 1862; Joakimoff 1899; Kovachev 1905; Nedelkov 1910, 1912; Szilády 1934; Drenowsky 1936; Lindner 1936; Bankowska 1967a; Beschovski 1971a, 2004, 2006a; Rozkošný 1982; Woodley 2001. 
Chloromyia speciosa (Macquart, 1834) [Ch. melampogon (Zeller, 1842); Chrysomyia] - B1, B2, V1, S1, S23, TL, T31, O62, R2, RW, BN; 160-1326 m; 1, 2, 3; tp; Nedelkov 1910, 1912; Szilády 1934; Dušek \& Rozkošný 1963; Bankowska 1967a; Beschovski 1971a, 2006a; Rozkošný 1982-1983; Woodley 2001; Dvořák et al. 2021.

Microchrysa cyaneiventris (Zetterstedt, 1842) - V1; 550-600 m; 1; e; Rozkošný 1982; Rozkošný \& Nartshuk 1988; Woodley 2001.

Microchrysa flavicornis (Meigen, 1822) - V1; 550-600 m; 1; h (? i), hoes, ? tp; Rozkošný 1982; Rozkošný \& Nartshuk 1988; Woodley 2001.

Microchrysa polita (Linnaeus, 1758) - V1, S23, TL; 183-600 m; 1; h (? i), hoes, ? tp; Nedelkov 1910, 1912; Szilády 1934; Dušek \& Rozkošný 1963; Rozkošný 1982-1983; Woodley 2001; Dvořák et al. 2021.

Sargus bipunctatus (Scopoli, 1763) - V1; 550-600 m; 1; h (? i), ? wp; Szilády 1934; Rozkošný 1982; Woodley 2001.

Sargus cuprarius (Linnaeus, 1758) [S. nubeculosus Zetterstedt, 1842] - DM, V1, V4, TL; 20-800 m; 1, 2; h (? i), ? wcp; Meunier 1897; Nedelkov 1910, 1912; Szilády 1934; Rozkošný 1982; Rozkošný \& Nartshuk 1988; Woodley 2001.

Sargus flavipes Meigen, 1822 - V4; 650-750 m; 1, 2; tp; Szilády 1934; Rozkošný 1982; Rozkošný \& Nartshuk 1988; Woodley 2001.

Sargus iridatus (Scopoli, 1763) [S. infuscatus Meigen, 1822] - V1, V4, TL; 250-870 m; 1, 2; hoes, ? tp; Nedelkov 1910, 1912; Rozkošný 1982; Rozkošný \& Nartshuk 1988; Woodley 2001.

Odontomyia angulata (Panzer, 1798) [Eulalia hydroleon angulata (Panzer, 1798)] - \$; DW, DM, TL; 25-200 m; 1; tp; Nedelkov 1910, 1912; Szilády 1934; Lindner 1936; Dušek \& Rozkošný 1965; Rozkošný 1982; Woodley 2001.

Odontomyia annulata (Meigen, 1822) [Eulalia; Stratiomys septemguttata (Meigen, 1822)] - BN; 20 m; 1; cse; Löw 1862; Lindner 1936; Rozkošný 1982; Woodley 2001.

Odontomyia cephalonica Strobl, 1898 - BN; 0-20 m; 1; em; Beschovski 1973a; Rozkošný 1982; Rozkošný \& Nartshuk 1988; Woodley 2001.

Odontomyia flavissima (Rossi, 1790) [Eulalia] - B1, O62, RW, BN, BS; 0-465 m; 1; hom; Löw 1862; Nedelkov 1910, 1912; Szilády 1934; Lindner 1936; Bankowska 1967a; Rozkošný 1982; Woodley 2001.

Odontomyia hydroleon (Linnaeus, 1758) [Eulalia] - DW, B1, K9, V1, O1, O62; BN; 0-700 m; 1, 2; tp; Nedelkov 1910, 1912; Szilády 1934; Drenowsky 1936, 1939; Bankowska 1967a; Beschovski 1971a; Rozkošný 1982; Woodley 2001.

Odontomyia ornata (Meigen, 1822) [Eulalia] - BN; 0-20 m; 1; wcp; Szilády 1934; Rozkošný 1982; Rozkošný \& Nartshuk 1988; Woodley 2001.

Odontomyia tigrina (Fabricius, 1775) - V4; 750-850 m; 2; esca; Bankowska 1967a; Rozkošný 1982; Rozkošný \& Nartshuk 1988; Woodley 2001.

Oplodontha viridula (Fabricius, 1775) [Hoplodontha] - E2, V1, V4, S21, O62, BN, BS; 0-800 m; 1, 2; tp; Meunier 1897; Nedelkov 1910, 1912; Szilády 1934; Bankowska 1967a; Beschovski 1971a; Rozkošný 1982; Woodley 2001.

Stratiomys cenisia Meigen, 1822 [Stratiomyia] - DM, V1, BN; 0-660 m; 1, 2; wp; Nedelkov 1912; Szilády 1934; Bankowska 1967a; Rozkošný 1982; Woodley 2001.

Stratiomys chamaeleon (Linnaeus, 1758) [Stratiomyia] - DM, V1, S21, S211, TL, R2, BS; 0-1050 m; 1, 2, 3; esca; Meunier 1897; Nedelkov 1910, 1912; Szilády 1934; Lindner 1936; Bankowska 1967a; Rozkošný 1982; Woodley 2001.

Stratiomys equestris Meigen, 1835 [Stratiomyia] - E2; 50-100 m; 1; hoes, ? esca; Kovachev 1905; Rozkošný 1982; Rozkošný \& Nartshuk 1988; Woodley 2001.

Stratiomys longicornis (Scopoli, 1763) [Hirtea; S. strigata Fabricius, 1781; S. anubis Wiedemann, 1830] - *; RW, BN, BS; 0-1200 m; 1, 2, 3; hop; Löw 1862; Szilády 1934; Lindner 1936; Bankowska 1967a; Beschovski 1971a; Rozkošný 1982; Beschovski 2006a; Woodley 2001.

Stratiomys ruficornis (Macquart, 1838) [S. erythrocera Egger, 1859; Stratiomyia sublunata Löw, 1869] - B2, S1, TL, O62, R2, RW; 200-1300 m; 1, 2, 3; eswa; Nedelkov 1910, 1912; Szilády 1934; Rozkošný 1982; Rozkošný \& Nartshuk 1988; Woodley 2001.

Stratiomys singularior (Harris, 1776) [S. furcata Fabricius, 1794; S. riparia Meigen, 1822; S. ruficornis (Macquart, 1838); Stratiomyia] - V1, TL, T31, O61, R1, RW, BN, BS; 0-1450 m; 1, 2, 3; esca, ? tp; Joakimoff 1899; Nedelkov 1909, 1910, 1912; Szilády 1934; Drenowsky 1936; Dušek \& Rozkošný 1965; Rozkošný 1982; Woodley 2001; Beschovski 2006a. 
Clitellaria ephippium (Fabricius, 1775) [Potamida] - RW; 1180-1250 m; 3; e; Nedelkov 1912; Rozkošný 1982; Woodley 2001.

Clitellaria pontica (Lindner, 1936) [Taurocera] - BN; 0-10 m; 1; Ebg; Lindner 1936; Rozkošný 1982; Rozkošný \& Nartshuk 1988; Woodley 2001.

Lasiopa balius (Walker, 1849) [Cyclogaster tenuirostris Loew, 1854] - E1, B3, V1, V3, V4, S2, TL, O61, O62, R1, RW, BN, BS; 0-1200 m; 1, 2, 3; seean; Löw 1863; Szilády 1934; Bankowska 1967a; Dušek \& Rozkošný 1970; Beschovski 1971a, 2006a; Rozkošný 1982; Rozkošný \& Nartshuk 1988; Woodley 2001.

Lasiopa calva (Meigen, 1822) - E1, SB, B3, K4, V1, V4, S2, S21, R1, R3, RW; BN; 0-1450 m; 1, 2, 3; csee; Nedelkov 1910, 1912; Szilády 1934; Drenowsky 1939; Drensky 1939a; Bankowska 1967a; Dušek \& Rozkošný 1970; Rozkošný 1982; Rozkošný \& Nartshuk 1988; Woodley 2001; Beschovski 2006a.

Lasiopa villosa (Fabricius, 1794) - DM, SB, K4, S2, S23, TL, O62, BN, BS; 0-1100 m; 1, 2, 3; eswa; Nedelkov 1909, 1910, 1912; Lindner 1936; Bankowska 1967a; Dušek \& Rozkošný 1970; Rozkošný 1982-1983; Rozkošný \& Nartshuk 1988; Woodley 2001; Dvořák et al. 2021.

Nemotelus (Nemotelus) aerosus Gimmerthal, 1847 [N. lomnickii Mik, 1867] - E1, V1; 300-600 m; 1; ee; Szilády 1934; Rozkošný 1977, 1982; Rozkošný \& Nartshuk 1988; Woodley 2001.

Nemotelus (Nemotelus) argentifer Loew, 1846 - BS; 0-20 m; 1; mwca; Beschovski 1975b; Rozkošný 1977, 1982; Rozkošný \& Nartshuk 1988; Woodley 2001.

Nemotelus (Nemotelus) bipunctatus Loew, 1846 [Galeodioptriger] - BS; 0-20 m; 1; seean; Bankowska 1967a; Beschovski 1971a, 1975b; Rozkošný 1977, 1982; Rozkošný \& Nartshuk 1988; Woodley 2001.

Nemotelus (Nemotelus) brachystomus Loew, 1846 - BN; 0-10 m; 1; eanna; Bankowska 1967a; Beschovski 1971a, 1975b; Rozkošný 1977, 1982; Rozkošný \& Nartshuk 1988; Woodley 2001.

Nemotelus (Nemotelus) brevirostris Meigen, 1822 - BN; 0-10 m; 1; esca, ? wces; Beschovski 1971a; Rozkošný 1977, 1982; Rozkošný \& Nartshuk 1988; Woodley 2001.

Nemotelus (Nemotelus) crenatus Egger, 1859 - BN, BS; 0-20 m; 1; Ebs, ? se; Beschovski 1971a, 1975b; Rozkošný 1977, 1982; Rozkošný \& Nartshuk 1988; Woodley 2001.

Nemotelus (Nemotelus) obscuripes Loew, 1871 - BS; 0-20 m; 1; emit; Beschovski 1975b; Rozkošný 1977, 1982; Rozkošný \& Nartshuk 1988; Woodley 2001.

Nemotelus (Nemotelus) pantherinus (Linnaeus, 1758) - E1, V1, V4, TL, O62, BN, BS; 0-800 m; 1, 2; wcp; Meunier 1897; Nedelkov 1910, 1912; Szilády 1934; Bankowska 1967a; Dušek \& Rozkošný 1967; Beschovski 1973a; Rozkošný 1977, 1982; Rozkošný \& Nartshuk 1988; Woodley 2001.

Nemotelus (Nemotelus) rumelicus Beschovski \& Manassieva, 1996 - BS; 40 m; 1; Ebg; Beschovski \& Manassieva, 1996; Woodley 2001.

Nemotelus (Nemotelus) signatus Frivaldsky in Schiner, 1855 - BN; 0-20 m; 1; ? wcp; Dušek \& Rozkošný 1967; Rozkošný 1977, 1982; Rozkošný \& Nartshuk 1988; Woodley 2001.

Nemotelus (Camptopelta) nigrinus Fallén, 1817 - S21; 650-700 m; 2; hn; Rozkošný 1977, 1982; Woodley 2001. Oxycera meigenii Staeger, 1844 - BN; 0-20 m; 1; esanca; Beschovski 1971a; Rozkošný 1982; Woodley 2001.

Oxycera nigricornis Olivier, 1812 - O62, R2; 250-540 m; 1; e; Rozkošný 1982; Woodley 2001.

Oxycera trilineata (Linnaeus, 1767) - O62, BS; 0-300 m; 1; wcp; Meunier 1897; Bankowska 1967a; Rozkošný 1982; Woodley 2001.

? Hermetia illucens (Linnaeus, 1758) - ; k; Rozkošný 1982; Woodley 2001.

Eupachygaster tarsalis (Zetterstedt, 1842) - V4; 700-800 m; 2; et; Szilády 1934; Rozkošný 1982; Woodley 2001.

Pachygaster atra (Panzer, 1798) - E1, B2, O62, R1, R2, BN; 0-1700 m; 1, 2, 3, 4; ean; Szilády 1934; Bankowska 1967a; Beschovski 1971a; Rozkošný 1982; Rozkošný \& Nartshuk 1988; Woodley 2001.

Pachygaster leachii Curtis, 1824 - P2, BN; 0-200 m; 1; e; Szilády 1934; Bankowska 1967a; Rozkošný 1982; Rozkošný \& Nartshuk 1988; Woodley 2001.

\section{TABANOMORPHA}

\section{Rhagionidae}

Chrysopilus cristatus (Fabricius, 1775) [Ch. auratus (Fabricius, 1805); Ch. maerens Loew, 1873] - V1, V4, TL, R1, R2, BS; 50-1200 m; 1, 2, 3; e; Nedelkov 1909, 1910, 1912; Szilády 1934; Rozkošný \& Spitzer 1965; Spitzer 1978; Beschovski 2006a. 
Chrysopilus asiliformis (Preyssler, 1791) [Ch. aureus Meigen, 1804)] - E1, V1; 250-600 m; 1; e; Nedelkov 1912; Szilády 1934; Rozkošný \& Spitzer 1965; Spitzer 1978.

Chrysopilus erythrophthalmus Loew, 1840 - RW; 650 m; 1, 2; e; Spitzer 1978; Beschovski $2006 a$.

Chrysopilus luteolus (Fallen, 1814) - RW; 650 m; 1, 2; des; Spitzer 1978; Beschovski 2006a.

Chrysopilus maerens Loew, 1873 - R1; 1200-2500 m; 3, 4, 5; eswa; Szilády 1934; Rozkošný \& Spitzer 1965.

Chrysopilus nubecula (Fallen, 1814) - S22; 1500 m; 3, 4; wp; Nedelkov 1909, 1910, 1912; Rozkošný \& Spitzer 1965. Chrysopilus pullus Loew, 1869 - R2; 1100 m; 3; e; Spitzer 1978.

Chrysopilus splendidus (Meigen, 1820) - P1, B1, V1, V4, R2; 450-1100 m; 1, 2, 3; wes; Nedelkov 1912; Szilády 1934; Rozkošný \& Spitzer 1965; Spitzer 1978.

Ptiolina obscura (Fallen, 1814) - T31; 60 m; 1; wes; Spitzer 1978.

Rhagio annulatus (De Geer, 1776) - V1; 550 m; 1; dpo; Nedelkov 1912; Rozkošný \& Spitzer 1965; Spitzer 1978.

Rhagio cingulatus (Loew, 1856) - R2, RR; 1; e; Nedelkov 1910, 1912; Rozkošný \& Spitzer 1965; Spitzer 1978; Beschovski 2006a.

Rhagio conspicuus Meigen, 1804 - B2, V1, V2, R1, R2; 650-1800 m; 2, 3, 4; e; Nedelkov 1910, 1912; Szilády 1934; Spitzer 1978.

Rhagio lineola Fabricius, 1794 - R2; 1100-1200 m; 3; des; Spitzer 1978.

Rhagio maculatus (De Geer, 1776) [R. macedonicus Szilady, 1934] - \$; e; Szilády 1934; Spitzer 1978.

Rhagio maculipennis (Loew, 1854) [Leptis] - TL, T31, BN, BS; 0-350 m; 1; seean; Löw 1862; Rozkošný \& Spitzer 1965; Spitzer 1978.

Rhagio scolopaceus (Linnaeus, 1758) [Leptis] - B2, V1, V4, R1, R2; 600-1200 m; 2, 3; wes; Joakimoff 1899; Nedelkov 1910, 1912; Rozkošný \& Spitzer 1965; Spitzer 1978.

Rhagio tringarius (Linnaeus, 1758) [Leptis] - B3, V1, V4, S22, O62, R1, R2; 350-1500 m; 1, 2, 3; wes; Meunier 1897; Joakimoff 1899; Nedelkov 1909, 1910, 1912; Rozkošný \& Spitzer 1965; Spitzer 1978.

Rhagio vitripennis (Meigen, 1820) [Leptis] - B1, V1, V4, S1, S211, T31, R2, RW, BS; 0-1600 m; 1, 2, 3, 4; e; Meunier 1897; Nedelkov 1910, 1912; Rozkošný \& Spitzer 1965; Spitzer 1978; Beschovski 2006a.

\section{Athericidae}

Atherix ibis (Fabricus, 1798) - ; V4; 800 m; 2; h; Nedelkov 1910, 1912; Szilády 1934; Rozkošný \& Spitzer 1965. Ibisia marginata (Fabricius, 1781) [Atherix] - V4; 800 m; 2; e; Nedelkov 1910, 1912; Rozkošný \& Spitzer 1965.

\section{Tabanidae}

Pangonius (Pangonius) obscuratus Loew, 1859 [P. pyritosus var. hirsutipalpis (Kröber, 1921)] - DM, B3, V1, S1, TL, T31, O62, RW, BN, BS; 0-700 m; 1, 2; nem, ? seean; Nedelkov 1912; Kröber 1921, 1925, 1938; Drensky 1929a, 1942; Shannon \& Hadjinicolaou 1936; Chvála et al. 1972; Chvála 1988; Ganeva 2005b.

Pangonius (Pangonius) pyritosus Loew, 1859 [P. pyritosa var. hirsutipalpis (Kröber 1921)] - DM, V1, TL, T31, R3, RW, BN; 0-1450 m; 1, 2, 3; seean; Löw 1862; Nedelkov 1912; Kröber 1925, 1938; Shannon \& Hadjinicolaou 1936; Drenowsky 1939; Drensky 1929a, 1939a, 1942; Moucha. \& Chvála 1961; Chvála et al. 1972; Chvála 1988; Ganeva 2005b.

Pangonius (Melanopangonius) funebris Macquart, 1846 - •; seena; Chvála et al. 1972; Chvála 1988; Ganeva 2005b.

Pangonius (Melanopangonius) haustellatus Fabricius, 1781 [P. marginatulus (Fabricius, 1805] - DW, SB, TL, T31, RW, BN; 0-400 m; 1; sena; Nedelkov 1912; Drensky 1929a, 1942; Shannon \& Hadjinicolaou 1936; Kröber 1938; Leclercq 1957; Chvála et al. 1972; Chvála 1988; Ganeva 2005b.

Silvius algirus Meigen, 1830 - TL, RW, BN; 0-300 m; 1; hom; Drensky 1929a, 1942; Shannon \& Hadjinicolaou 1936; Kröber 1938; Chvála et al. 1972; Chvála 1988; Ganeva 2005b, 2006.

Silvius alpinus (Scopoli, 1763) [S. vituli Fabricius, 1805)] - B3, K9, S1, S23, TL, T31, O1, R1, R3, RW, BS; 0-2183 m; 1, 2, 3, 4; eanna; Nedelkov 1909, 1912; Drensky 1929a; Shannon \& Hadjinicolaou 1936; Kröber 1938; Drenowsky 1939; Leclercq 1957; Trifonov et al. 1964; Chvála et al. 1972; Chvála 1988; Ganeva 1993, 1995a, 1996, 1998, 1999b, 2005a, 2005b, 2006, 2011, 2017; Ganeva \& Penev 2002.

? Silvius variegatus (Fabricius, 1805) [Hematopota variegatus Fabricius, 1805; ? Chrysozona italica var. variegata Fabricius, 1805; ? = H. pandazisi (Kröber, 1936)] - V1, S211; 550-900 m; 1, 2; hom; Nedelkov 1912; 
Drensky 1929a; Kröber 1938; Leclercq 1966. According to Ganeva (2005b) the species probably is $H$. pandazisi (Kröber, 1936).

Nemorius vitripennis (Meigen, 1820) [Chrysops] - B1, B2, V1, R1, RW; 400-800 m; 1, 2; nmwca, ? nmca; Nedelkov 1912; Drensky 1929a; Shannon \& Hadjinicolaou 1936; Kröber 1938; Leclercq 1957; Chvála et al. 1972; Chvála 1988; Ganeva 2005b, 2006, 2017; Ganeva \& Ivanov 2015.

Chrysops (Chrysops) caecutiens (Linnaeus, 1758) [Ch. ludens Loew, 1858; Ch. caecutiens var. meridionalis Stobl, 1906] - $\boldsymbol{\Delta}$; ; DM, E2, P1, P2, B1, B2, B3, V1, V4, S1, S21, S211, S23, TL, O61, R1, R3, RW, BN, BS; 0-1668 m; 1, 2, 3, 4; hoes, ? tp; Löw 1862; Meunier 1897; Nedelkov 1912; Drensky 1929a, 1939a, 1955, 1960; Drenowsky 1936; Shannon \& Hadjinicolaou 1936; Buresch 1953a; Leclercq 1957; Chvála et al. 1972; Ganeva 1993, 1995a, 1995b, 1995c, 1996, 1999b, 2000, 2004, 2005a, 2005b, 2006, 2008a, 2009a, 2009c, 2011, 2017; Ganeva \& Penev 2002; Ganeva \& Kalmushka 2012, 2019; Ganeva \& Ivanov 2015.

Chrysops (Chrysops) flavipes Meigen, 1804 [Ch. punctifer Loew, 1856; Ch. perspicillaris Loew, 1856] - P2, B1, V1, S23, TL, RW, BS; 0-1800 m; 1, 2, 3, 4; spo; Nedelkov 1912; Drensky 1929a; Shannon \& Hadjinicolaou 1936; Kröber 1938; Leclercq 1957; Beschovski 1964a, Trifonov et al. 1964; Chvála et al. 1972; Chvála 1988; Ganeva 2005b, 2006, 2016; Dvořák et al. 2021.

Chrysops (Chrysops) italicus Meigen, 1804 [Ch. marmoratus (Rossi, 1790)] - $\mathbf{\Delta}$; B1, V1, RW, BN, BS; 0-750 m; 1, 2; mi; Nedelkov 1909, 1912; Drensky 1929a, 1942, 1960; Shannon \& Hadjinicolaou 1936; Kröber 1938; Leclercq 1957; Moucha. \& Chvála 1961; Trifonov et al. 1964; Beschovski 1971a; Chvála et al. 1972; Chvála 1988; Ganeva 2004, 2005b, 2006.

Chrysops (Chrysops) parallelogrammus Zeller, 1842 - P2, RW; 140-1260 m; 1, 2, 3; e; Drensky 1929a, 1929b; Shannon \& Hadjinicolaou 1936; Kröber 1938; Leclercq 1957; Chvála et al. 1972; Chvála 1988; Ganeva 2005b, 2006.

Chrysops (Chrysops) relictus Meigen, 1820 - $\mathbf{\Delta}$; DM, S23, TL, RW, BN; 0-800 m; 1, 2; hoes, ? esca; Nedelkov 1909, 1912; Drensky 1929a, 1929b; Shannon \& Hadjinicolaou 1936; Leclercq 1957; Chvála et al. 1972; Ganeva 2005b, 2006; Ganeva \& Kalmushka 2012.

Chrysops (Chrysops) viduatus (Fabricius, 1794) [Ch. quadratus Meigen, 1820; Ch. pictus Meigen, 1820] - B1, B2, V1, S21, S23, TL, T1, R1, BN, BS; 0-1274 m; 1, 2, 3; wesan, ? wp; Meunier 1897; Nedelkov 1909, 1912; Drensky 1929a, 1929b; Shannon \& Hadjinicolaou 1936; Leclercq 1957; Moucha. \& Chvála 1961; Trifonov et al. 1964; Beschovski 1971a; Chvála et al. 1972; Parvu 1983; Ganeva 1993, 1995a, 1995b, 1996, 1998, 1999b, 2000, 2005b, 2006, 2008b, 2011, 2016, 2017; Ganeva \& Penev 2002; Ganeva \& Ivanov 2015.

Chrysops (Petersenichrysops) hamatus Loew, 1858 - O62; 200-260 m; 1; ban; Moucha. \& Chvála 1961; Chvála et al. 1972; Chvála 1988; Ganeva 2005b.

Atylotus flavoguttatus (Szilady, 1915) - ム; S1, S23, TL, T2; 150-250 m; 1; mwca; Trifonov et al. 1964; Lavchiev \& Ganeva 1991; Ganeva 1993, 1995a, 1996, 1998, 1999b, 2000, 2002, 2004, 2005b, 2011; Ganeva \& Penev 2002.

Atylotus fulvus (Meigen, 1804) [Tabanus rufipes Meigen, 1820; T. fulvus var. rufipes Meigen, 1820] - B2, V1, V4, S23, TL, O5, O62, R1, RW, BN; 0-2620 m; 1, 2, 3, 4, 5, 6; tp; Nedelkov 1909, 1912; Drensky 1929a, 1929b, 1931a 1931b; Shannon \& Hadjinicolaou 1936; Buresch 1953a; Leclercq 1957; Moucha. \& Chvála 1961; Chvála et al. 1972; Ganeva 1999b, 2005b, 2006, 2009b, 2017; Ganeva \& Penev 2002; Ganeva \& Ivanov 2015.

Atylotus latistriatus Brauer, 1880 [A. nigrifacies Gobert, 1880] - RW; 2076 m; 4; ena; Drensky 1929a, 1929b; Shannon \& Hadjinicolaou 1936; Kröber 1938; Leclercq 1957, 1966; Chvála et al. 1972; Chvála 1988; Ganeva 2005b, 2006.

Atylotus loewianus (Villleneuve, 1920) [Ochrops fulvus var. loewianus Villleneuve, 1920] - B2, B3, S1, S23, TL, T1, R3, RW; 150-1630 m; 1, 2, 3, 4; ena; Drensky 1929a, 1929b; Kröber 1938; Leclercq 1966; Chvála et al. 1972; Chvála 1988; Ganeva 1993, 1995a, 1995b, 1996, 1998, 2000, 2002, 2006, 2008b, 2009c, 2011, 2017; Ganeva \& Penev 2002; Ganeva \& Kalmushka 2012, 2019; Ganeva \& Ivanov 2015.

Atylotus rusticus (Linnaeus, 1761) - P2, B1, B2, V1, S23, Tl, RW; 150-1130 m; 1, 2, 3; wp; Nedelkov 1912; Drensky 1929a, 1929b; Shannon \& Hadjinicolaou 1936; Kröber 1938; Leclercq 1957; Chvála et al. 1972; Ganeva 1993, 1999b, 2006, 2011; Ganeva \& Penev 2002; Ganeva \& Ivanov 2015.

Therioplectes gigas (Herbst, 1787) - DM, E1, B2, K9, V1, S1, S23, TL, O5, O62, RW, BN; 0-1100 m; 1, 2, 3; ? cseel; Nedelkov 1909, 1912; Enderlein 1925; Drensky 1929a, 1929b, 1931a, 1931b, 1942; Shannon \& Hadjinicolaou 1936; Kröber 1938; Leclercq 1957, 1966; Chvála et al. 1972; Parvu 1983; Chvála 1988; Ganeva 2005b, 2006, 2009b; Ganeva \& Kalmushka 2012, 2019; Ganeva \& Ivanov 2015. 
Therioplectes tricolor Zeller, 1842 [T. ruficauda (Enderlein, 1925); T. tricolor pallidicauda (Olsufjev, 1937); Atylotus gigas var. tricolor Zeller, 1842] - B3, S1, S23, TL, T1; 100-400 m; 1; nmi; Enderlein 1925; Drensky 1929b; Kröber 1938; Moucha. \& Chvála 1964; Leclercq 1966; Chvála et al. 1972; Chvála 1988; Ganeva 1993, 1995a, 1995b, 1995c, 1996, 1998, 1999b, 2000, 2005b, 2008b, 2011; Ganeva \& Penev 2002; Ganeva \& Kalmushka 2012, 2019.

Hybomitra aterrima (Meigen, 1820) [Sziladynus, Therioplectes, Tabanus] - E2, S211, TL, O5, O62, R1, R2, RW; 180-2654 m; 1, 2, 3, 4, 5, 6; cse, m; Nedelkov 1912; Drensky 1929a, 1931a, 1931b, 1955; Shannon \& Hadjinicolaou 1936; Leclercq 1957; Chvála et al. 1972; Ganeva 1996, 1998, 2000, 2005b, 2006, 2009b, 2017.

Hybomitra auripila (Meigen, 1820) [Tabanus aterrimus var. auripilus Meigen, 1820; T. aterrimus var. lugubris Zetterstedt, 1838; Sziladynus, Therioplectes] - S211, T31, O62, R1, R2, RW; 300-2550 m; 1, 2, 3, 4, 5, 6; e; Nedelkov 1912; Drensky 1929a, 1929b, 1931a, 1931b; Kröber 1932; Trifonov et al. 1964; Chvála et al. 1972; Chvála 1988; Ganeva 1998, 2000, 2006, 2009b, 2017.

Hybomitra bimaculata (Macquart, 1826) [Tabanus tropicus Panzer, 1794; ? = H. solstitialis sensu Lyneborg, 1959 (nec Meigen, 1820)] - B1, B2, B3; 250-1440 m; 1, 2, 3; hoes; Drensky 1929a, 1929b; Ganeva 2008a; Ganeva \& Ivanov 2015.

Hybomitra caucasi (Szilady, 1923) - S23, TL; 150-650 m; 1; seean; Ganeva 1993, 1995a, 1995d, 1996, 1998, 1999b, 2000, 2005b, 2011; Ganeva \& Penev 2002; Ganeva \& Kalmushka 2012, 2019.

Hybomitra caucasica (Enderlein, 1925) - R1; 1625-1324 m; 4, 5; csei, m, ? mm; Ganeva 2017.

Hybomitra ciureai (Séguy, 1937) [? = H. solstitialis (Meigen, 1820); Tabanus solstitialis Schiner,1862; Sziladynus, Therioplectes] - $\mathbf{\Delta}$; B3, S23, TL, T1, T11, T31, R1, RW, BN; 0-2000 m; 1, 2, 3, 4; esca; Drensky 1929a, 1929b, 1942; Shannon \& Hadjinicolaou 1936; Kröber 1938; Leclercq 1957; Trifonov et al. 1964; Chvála et al. 1972; Ganeva 1993, 1995a, 1995b, 1995c, 1996, 1998, 1999b, 2002, 2004, 2005a, 2005b, 2006, 2008a, 2008b,2009a, 2011, 20017; Ganeva \& Penev 2002; Ganeva \& Ivanov 2015; Ganeva \& Kalmushka 2012, 2019.

Hybomitra decora (Loew, 1858) [Sziladynus, Tabanus, Therioplectes] - B3, S1, S23, TL; 100-150 m; 1; mi; Drensky 1929a, 1929b; Shannon \& Hadjinicolaou 1936; Kröber 1938; Leclercq 1966; Chvála et al. 1972; Chvála 1988; Ganeva 1999b, 2000, 2005b, 2011; Ganeva \& Penev 2002.

Hybomitra distinguenda (Verrall, 1909) [Tabanus, Tylostypia] - B2, B3, V4, S23, TL, R1, BN; 0-2000 m; 1, 2, 3, 4; tp; Moucha. \& Chvála 1961; Leclercq 1966; Chvála et al. 1972; Ganeva 1993, 1995b, 1996, 1998, 1999b, 2000, 2005a, 2005b, 2011, 2017; Ganeva \& Penev 2002; Ganeva \& Ivanov 2015; Ganeva \& Kalmushka 2012, 2019.

Hybomitra micans (Meigen, 1804) [Tabanus] - R1, RW; 1150-2100 m; 3, 4; e; Joakimoff 1899; Shannon \& Hadjinicolaou 1936; Leclercq 1957; Chvála et al. 1972; Ganeva 1996, 1998, 2000, 2005b, 2006, 2017.

Hybomitra montana (Meigen, 1820) [Tabanus] - V4, O62, R1, RW; 160-1400 m; 1, 2, 3; tp; Drensky 1929a, 1929b, 1931a, 1931b; Shannon \& Hadjinicolaou 1936; Buresch 1953a; Ganeva 2017.

Hybomitra muehlfeldi (Brauer, 1880) - R1; 1526 m; 3, 4; hoes, ? tp; Ganeva 2017.

Hybomitra pilosa (Loew, 1858) [H. laterralis (Meigen, 1820); Sziladynus, Tabanus, Therioplectes] - B1, V1, V4, S211, S23, TL, RW, BN; 0-800 m; 1, 2; cse; Nedelkov 1912; Drensky 1929a, 1929b, 1942; Shannon \& Hadjinicolaou 1936; Kröber 1938; Buresch 1953a; Leclercq 1957, 1966; Ganeva 1995a, 1995b, 1996, 1998, 1999b, 2000, 2005b, 2006, 2011, 2016; Ganeva \& Penev 2002.

Hybomitra solstitialis (Meigen, 1820) [? = H. ciureai (Séguy, 1937); Tabanus] - V1, R1; 550-2036 m; 2, 3, 4; e, ? esca; Meunier 1897; Ganeva 2017.

Hybomitra tropica (Linnaeus, 1758) [Tabanus montanus var. fulvicornis; Tabanus] - V1, V4, R1, RW; 650-1540 m; 2, 3, 4; e; Meunier 1897; Drensky 1929a, 1929b; Shannon \& Hadjinicolaou 1936; Kröber 1932.

Tabanus autumnalis Linnaeus, 1761 - $\boldsymbol{\Delta}$; E2, B2, V1, V3, V4, S23, TL, T31, O62, BN; 0-1300 m; 1, 2, 3; wcp; Nedelkov 1912; Drensky 1929a, 1929b, 1931a, 1931b, 1942, 1955; Shannon \& Hadjinicolaou 1936; Kröber 1938; Leclercq 1957, 1966; Moucha. \& Chvála 1961; Trifonov et al. 1964; Chvála et al. 1972; Lavchiev \& Ganeva 1991; Ganeva 1993, 1995a, 1995b, 1995c, 1996, 1998, 1999b, 2002, 2004, 2005b, 2006, 2008b, 2009a, 2009b, 2011, 2017; Ganeva \& Penev 2002; Ganeva \& Kalmushka 2012, 2019; Ganeva \& Ivanov 2015.

Tabanus bifarius Loew, 1858 [Atylotus, Therioplectes] - B1, V1, S23, TL, T11, T31, O5, O62, RE, BN, BS; 0-1000 m; 1, 2; mi; Drensky 1929a, 1929b, 1931a, 1931b; Shannon \& Hadjinicolaou 1936; Kröber 1938; Leclercq 1957, 1966; Moucha. \& Chvála 1961; Trifonov et al. 1964; Chvála et al. 1972; Parvu 1983; Chvála 1988; Ganeva 1993, 1995a, 1995b, 1995c, 1996, 1998, 1999b, 2000, 2002, 2005b, 2006, 2008b, 2009a, 2009b, 2011, 2016; Ganeva \& Penev 2002; Ganeva \& Kalmushka 2012, 2019; Ganeva \& Ivanov 2015. 
Tabanus bovinus Linnaeus, 1758 - $\mathbf{\Delta}$; DM, E1, E2, B2, V1, V3, S23, TL, T31, R1, BS; 0-2000 m; 1, 2, 3, 4; wcp; Meunier 1897; Joakimoff 1899; Kovachev 1905; Nedelkov 1909, 1912; Drensky 1929a, 1929b, 1955, 1960; Shannon \& Hadjinicolaou 1936; Leclercq 1957, 1966; Moucha. \& Chvála 1961; Trifonov et al. 1964; Lavchiev \& Ganeva 1991; Ganeva 1993, 1995a, 1995b, 1995c, 1996, 1998, 1999b, 2000, 2004, 2005b, 2011, 2017; Ganeva \& Penev 2002; Ganeva \& Ivanov 2015.

Tabanus briani Leclercq, 1962 - S23, TL, RW; 230-596 m; 1; se, ? cse; Chvála et al. 1972; Chvála 1988; Ganeva 1999b, 2000, 2006; 2011, Ganeva \& Penev 2002.

Tabanus bromius Linnaeus, 1758 - ^ ; DM, E2, B1, B2, B3, K9, V1, V4, S1, S211, S23, TL, T1, T11, T31, O5, O61, R1, RW, BN, BS; 0-2000 m; 1, 2, 3, 4; wp; Meunier 1897; Nedelkov 1909, 1912; Drensky 1929a, 1942, 1955, 1960; Shannon \& Hadjinicolaou 1936; Buresch 1953a; Leclercq 1957, 1966; Moucha. \& Chvála 1961; Trifonov et al. 1964; Chvála et al. 1972; Lavchiev \& Ganeva 1991; Ganeva 1993, 1995a, 1995b, 1995c, 1996, 1998, 1999a, 1999b, 2001, 2002, 2004, 2005a, 2005b, 2006, 2008b, 2009a, 2009b, 2009c, 2011, 2016, 2017; Ganeva \& Penev 2002; Ganeva \& Kalmushka 2012, 2019; Ganeva \& Ivanov 2015.

Tabanus cordiger Meigen, 1820 [T. vicinus Egger, 1859] - B1, B3, V1, V4, S1, S23, TL, O5, O62, R1, RW, RE; 150-2082 m; 1, 2, 3, 4; wp; Nedelkov 1912; Drensky 1929a, 1929b, 1931a, 1931b; Shannon \& Hadjinicolaou 1936; Buresch 1953a; Leclercq 1957; Trifonov et al. 1964; Chvála et al. 1972; Ganeva 1993, 1995a, 1995b, 1995c, 1996, 1998, 1999b, 2005a, 2005b, 2009b, 2011, 2016, 2017; Ganeva \& Penev 2002; Ganeva \& Ivanov 2015.

Tabanus eggeri Schiner, 1868 [T. intermedius Egger, 1859] - B3, V1, S1, O62, RW, BS; 0-1545 m; 1, 2, 3, 4; hom; Drensky 1929a, 1929b, 1931a, 1931b; Shannon \& Hadjinicolaou 1936; Kröber 1938; Leclercq 1957; Olsifev et al. 1967; Chvála et al. 1972; Chvála 1988; Ganeva 2005b, 2006, 2009 b.

Tabanus exclusus Pandelle, 1883 - B1, B2, B3, V1, S1, S23, TL, T11, R1, R3, RE, BN, BS; 0-1221 m; 1, 2, 3; nm; Drensky 1929a, 1929b, 1931a, 1931b; Shannon \& Hadjinicolaou 1936; Leclercq 1957, 1966; Moucha. \& Chvála 1961; Chvála et al. 1972; Chvála 1988; Ganeva 1993, 1995a, 1995b, 1995c, 1996, 1998, 1999b, 2000, 2002, 2005a, 2005b, 2008b, 2009c, 2011, 2016, 2017; Ganeva \& Penev 2002; Ganeva \& Kalmushka 2012, 2019; Ganeva \& Ivanov 2015.

Tabanus fraseri Austen, 1925 - BN; 0-30 m; 1; ban; Moucha \& Chvála 1963; Chvála et al. 1972; Chvála 1988; Ganeva 2005b.

Tabanus glaucopis Meigen, 1820 [T. cognatus Loew, 1858] - P2, B2, B3, K9, S1, S23, TL, O5, O61, O62, R1, R3, RW, RE; 200-2080 m; 1, 2, 3, 4; esca; Drensky 1929a, 1929b, 1931a, 1931b; Shannon \& Hadjinicolaou 1936; Kröber 1938; Leclercq 1957, 1966; Moucha. \& Chvála 1961, 1967; Chvála et al. 1972; Chvála 1988; Lavchiev \& Ganeva 1991; Ganeva 1993, 1995a, 1995c, 1996, 1999b, 2002, 2005a, 2005b, 2006, 2008a, 2009b, 2009c, 2011, 2017; Ganeva \& Penev 2002; Ganeva \& Kalmushka 2012, 2019; Ganeva \& Ivanov 2015.

Tabanus indrae Hauser, 1939 - S1, S23, TL; 200-650 m; 1; nmwca; Olsifev 1970, 1977; Chvála et al. 1972; Moucha 1976; Chvála 1988; Lavchiev \& Ganeva 1991; Ganeva 1993, 1995a, 1995c, 1996, 1998, 1999b, 2000, 2005b, 2011; Ganeva \& Penev 2002.

Tabanus leleani Austen, 1920 - ; spo; Shannon \& Hadjinicolaou 1936.

Tabanus lunatus Fabricius, 1794 [Atylotus, Therioplectes] - V4, S23, TL, T31, BS; 0-880 m; 1, 2; mit; Nedelkov 1912; Drensky 1929a, 1929b; Shannon \& Hadjinicolaou 1936; Kröber 1938; Buresch 1953a; Leclercq 1957, 1966; Trifonov et al. 1964; Chvála et al. 1972; Chvála 1988; Ganeva 2005b, 2008b; Ganeva \& Kalmushka 2012.

Tabanus maculicornis Zetterstedt, 1842 - P3, B2, B3, V4, S23, TL, T31, R1, RW, RE; 100-2000 m; 1, 2, 3, 4; wes; Drensky 1929a, 1929b; Shannon \& Hadjinicolaou 1936; Kröber 1938; Buresch 1953a; Leclercq 1957, 1966; Trifonov et al. 1964; Chvála et al. 1972; Lavchiev \& Ganeva 1991; Ganeva 1993, 1995a, 1995c, 1996, 1998, 1999b, 2000, 2005a, 2005b, 2006, 2011, 2017; Ganeva \& Penev 2002; Ganeva \& Kalmushka 2012, 2019; Ganeva \& Ivanov 2015.

Tabanus miki Brauer, 1880 [T. velutinus Kröber, 1936; T. postvelutinus Moucha, 1962] - B1, B2, S1, S23, TL, O5, O62, R1, RW, BN; 0-2000 m; 1, 2, 3, 4; patn, ? esca; Drensky 1929a, 1929b; 1931a, 1931b; Shannon \& Hadjinicolaou 1936; Kröber 1938; Buresch 1953a; Leclercq 1957, 1966; Moucha. \& Chvála 1961; Chvála et al. 1972; Lavchiev \& Ganeva 1991; Ganeva 1993, 1995a, 1995b, 1995c, 1996, 1998, 1999b, 2000, 2005b, 2006, 2009b, 2011, 2016, 2017; Ganeva \& Penev 2002; Ganeva \& Kalmushka 2012, 2019; Ganeva \& Ivanov 2015.

Tabanus obsolescens Pandellé, 1883 - R1; 1049 m; 3; Eb; Ganeva 2017.

Tabanus paradoxus Jaennicke, 1866 - O5, R3; 650-760 m; 2; e; Ganeva 2009b, 2009c. 
Tabanus prometheus Szilady, 1923 - P2, B2, S23, TL, BN; 0-550 m; 1; bci, ? nemi; Olsifev et al. 1967; Chvála et al. 1972; Chvála 1988; Ganeva 1999b, 2000, 2002, 2005a, 2005b, 2011; Ganeva \& Penev 2002; Ganeva \& Ivanov 2015.

Tabanus quatuornotatus Meigen, 1820 [Atylotus, Therioplectes] - B2, B3, V4, S23, TL, T11, T31, R1, R3, RW, BN; 0-2076 m; 1, 2, 3, 4; ? wp; Löw 1862; Nedelkov 1912; Drensky 1929a, 1929b; Drenowsky 1936; Shannon \& Hadjinicolaou 1936; Kröber 1938; Buresch 1953a; Leclercq 1957, 1966; Moucha. \& Chvála 1961; Trifonov et al. 1964; Chvála et al. 1972; Chvála 1988; Ganeva 1993, 1995a, 1995b, 1995c, 1996, 1998, 1999b, 2002, 2004, 2005a, 2005b, 2006, 2008b, 2009a, 2011, 2017; Ganeva \& Penev 2002; Ganeva \& Kalmushka 2012, 2019; Ganeva \& Ivanov 2015.

Tabanus rectus Loew, 1858 [T. spodopterus Wied.; T. automnalis L.] - DM, B1, B3, V1, V4, S1, S211, TL, T31, RW, BN, BS; 0-950 m; 1, 2; nm; Nedelkov 1912; Drensky 1929a, 1929b; Shannon \& Hadjinicolaou 1936; Buresch 1953a.

Tabanus regularis Jaennicke, 1866 - S23, TL, RE, BN; 0-1490 m; 1, 2, 3; mi, ? hom; Moucha. \& Chvála 1961; Chvála et al. 1972; Chvála 1988; Ganeva 1999b, 2000, 2005b, 2006, 2009b, 2017; Ganeva \& Penev 2002.

Tabanus rupium (Brauer, 1880) - B2, S23, TL; 150-643 m; 1, 2; csean; Ganeva1993, 1995a, 1995b, 1995c, 1996, 1998, 1999b, 2000, 2005b, 2008b; Ganeva \& Penev 2002; Ganeva \& Ivanov 2015.

Tabanus shannonellus Krober, 1936 - B2, B3, S23, TL, O5, O62, R3, RE, BN, BS; 0-760 m; 1, 2; Eb; Moucha. \& Chvála 1961; Chvála et al. 1972; Chvála 1988; Ganeva1993, 1995a, 1995c, 1996, 1998, 1999b, 2000, 2002, 2005a, 2005b, 2006, 2009b, 2009c, 2011; Ganeva \& Penev 2002; Ganeva \& Ivanov 2015.

Tabanus smirnovi Olsufjev, 1962 - B2, B3, TL; 190-600 m; 1; see; Ganeva 2005a, 2005b; Ganeva \& Ivanov 2015.

Tabanus spectabilis Loew, 1858 [T. graecus F.] - E2, B3, V1, V4, S23, TL, BN, BS; 0-800 m; 1, 2; mit; Löw 1863; Nedelkov 1912; Drensky 1929a, 1929b; Shannon \& Hadjinicolaou 1936; Kröber 1938; Leclercq 1957, 1966; Moucha. \& Chvála 1961; Chvála et al. 1972; Chvála 1988; Lavchiev \& Ganeva 1991; Ganeva 1993, 1999b, 2002, 2005a, 2005b; Ganeva \& Penev 2002; Ganeva \& Kalmushka 2012.

Tabanus spodopterus Meigen, 1820 [T. spodopterus subsp. ponticus Olsufjev, Moucha \& Chvala, 1967] - E2, B1, B2, B3, V1, V4, S23, TL, T1, T11, T31, O5, O62, R1, R2, RW, RE, BN, BS; 0-2550 m; 1, 2, 3, 4, 5, 6; csean; Löw 1863; Drensky 1929a, 1929b, 1931a, 1931b, 1942, 1960; Shannon \& Hadjinicolaou 1936; Kröber 1938; Buresch 1953a; Leclercq 1957, 1966; Moucha. \& Chvála 1961; Beschovski 1964a; Petkov 1964; Olsifev et al. 1967; Chvála et al. 1972; Chvála 1988; Ganeva 1996, 1998, 1999b, 2000, 2002, 2005a, 2005b; 2006, 2009a, 2009b, 2011, 2016, 2017; Ganeva \& Penev 2002; Ganeva \& Kalmushka 2012, 2019; Ganeva \& Ivanov 2015.

Tabanus sudeticus Zeller, 1842 - B1, B2, B3, S23, TL, O5, O62, R3; 150-1526 m; 1, 2, 3; eanna, ? sk; Drensky 1929a, 1929b, 1931a, 1931b; Shannon \& Hadjinicolaou 1936; Leclercq 1957; Chvála et al. 1972; Chvála 1988; Ganeva 2005a, 2005b, 2009b, 2009c, 2016, 2017; Ganeva \& Kalmushka 2012, 2019; Ganeva \& Ivanov 2015.

Tabanus tergestinus Egger, 1859 - E2, P3, B1, B2, B3, K9, V1, V4, S1, S211, S23, TL, T1, T11, T31, O5, O62, R1, RW, RE, BN; 0-1668 m; 1, 2, 3, 4; eswa; Nedelkov 1912; Drensky 1929a, 1929b, 1931a, 1931b, 1942, 1955; Kröber 1938; Leclercq 1957, 1966; Moucha. \& Chvála 1961; Petkov 1964; Trifonov et al. 1964; Chvála et al. 1972; Chvála 1988; Lavchiev \& Ganeva 1991; Ganeva 1993, 1995a, 1995b, 1995c, 1996, 1998, 1999a, 1999b, 2000, 2001, 2002, 2005a, 2005b, 2006, 2008a, 2008b, 2009a, 2009b, 2011, 2016; Ganeva \& Penev 2002; Ganeva \& Kalmushka 2012, 2019; Ganeva \& Ivanov 2015.

Tabanus tinctus Walker, 1850 [T. mixus Szilády, 1914] - B1, B3, S23, TL, T11, T31, O5, RE, BN, BS; 0-1040 m; 1, 2, 3; hom; Szilády 1923; Kröber 1925, 1938; Drensky 1929a, 1929b; Shannon \& Hadjinicolaou 1936; Moucha \& Chvála 1961; Leclercq 1966; Olsifev et al. 1967; Chvála et al. 1972; Chvála 1988; Lavchiev \& Ganeva 1991; Ganeva 1993, 1995a, 1995b, 1995c, 1996, 1998, 1999b, 2000, 2002, 2005a, 2005b, 2006, 2009a, 2009b, 2011; Ganeva \& Penev 2002; Ganeva \& Kalmushka 2012, 2019.

Tabanus unifasciatus Loew, 1858 - B3, V1, S23, TL, T11, T31, O5, O62, R1; 150-1600 m; 1, 2, 3, 4; wp; Drensky 1929a, 1929b, 1931a, 1931b; Shannon \& Hadjinicolaou 1936; Kröber 1938; Leclercq 1957, 1966; Trifonov et al. 1964; Chvála et al. 1972; Chvála 1988; Lavchiev \& Ganeva 1991; Ganeva 1993, 1995a, 1995b, 1995c, 1996, 1998, 1999b, 2000, 2002, 2005a, 2005b, 2008b, 2009a, 2009b, 2011, 2017; Ganeva \& Penev 2002; Ganeva \& Kalmushka 2012, 2019; Ganeva \& Ivanov 2015.

Heptatoma pellucens (Fabricius, 1776) [Tabanus albipes Schrank, 1781] - B2, S23, TL; 150-710 m; 1, 2; wes; Chvála 1988; Ganeva 1993, 1995a, 1995b, 1995c, 1996, 1998, 2000, 2005b; Ganeva \& Penev 2002; Ganeva \& Ivanov 2015. 
Haematopota bigoti Gobert, 1880 [Chrysozona] - K9, V1, V4, S23, TL; 150-800 m; 1, 2; eanna; Drensky 1929a, 1929b; Kröber 1938; Buresch 1953a; Leclercq 1957; Ganeva 1995a, 1995b, 1995c, 1996, 1998, 1999b, 2000, 2005b, 2011; Ganeva \& Penev 2002.

Haematopota crassicornis Wahlberg, 1848 [Chrysozona] - K8, V3, V4; 750-860 m; 2; wes; Drensky 1929a, 1929b; Shannon \& Hadjinicolaou 1936; Buresch 1953a.

Haematopota csikii Szilady, 1922 [Chrysozona] - S23, TL; 150-350 m, 1; e, ? cse; Kröber 1938; Leclercq 1966; Moucha 1970; Pârvu 1981; Lehrer \& Lehrer 1985; Chvála 1988; Lavchiev \& Ganeva 1991; Ganeva 1993, 1995a, 1995c, 1999b, 2000, 2005b, 2011; Ganeva \& Penev 2002.

Haematopota grandis Meigen, 1820 [Chrysozona italica var. grande Macquart, 1834] -S23, TL, R1; 150-1400 m; 1, 2, 3; wp; Drensky 1929a, 1929b; Kröber 1938; Leclercq 1957; Moucha 1962; Chvála et al. 1972; Chvála 1988; Ganeva 1993, 1995a, 1995b, 1998, 1999b, 2000, 2005b, 2011, 2017; Ganeva \& Penev 2002; Ganeva \& Ivanov 2015.

Haematopota italica Meigen, 1804 [Chrysozona] - B2, B3, K9, V1, V4, S211, S23, TL, T11, T31, O1, O5, O62, R1, R3, RW, RE, BS; 0-1400 m; 1, 2, 3; eanna; Nedelkov 1909, 1912; Drensky 1929a, 1929b, 1931a, 1931b, 1960; Drenowsky 1936; Shannon \& Hadjinicolaou 1936; Buresch 1953a; Leclercq 1957; Trifonov et al. 1964; Chvála et al. 1972; Ganeva 1993, 1995a, 1995c, 1996, 1998, 1999b, 2000, 2005a, 2005b, 2006, 2009a, 2009b, 2009c, 2011, 2017; Ganeva \& Penev 2002; Ganeva \& Ivanov 2015.

Haematopota longeantennata (Olsufjev, 1937) - S23, TL, T2; 350-650 m; 1; ban; Ganeva 1995a, 1995c, 1996, 1998, 1999b, 2000, 2005b; Ganeva \& Penev 2002; Ganeva \& Ivanov 2015.

Haematopota ocelligera (Kröber, 1922) [H. hispanica Szilady, 1923] - S23, TL, BN, BS; 0-350 m; 1; hom; Moucha. \& Chvála 1961; Leclercq 1957; Chvála et al. 1972; Chvála 1988; Ganeva 1993, 1995c, 1999b, 2000, 2005b; Ganeva \& Penev 2002.

Haematopota pandazisi (Kröber, 1936) [H. variegata pandazisi Kröber, 1936; Chrysozona italica var. variegata Fabricius, 1805] - B2, B3, S211, S23, TL, BN, BS; 0-780 m; 1, 2; hom; Drensky 1929a, 1929b; Moucha \& Chvála 1961; Leclercq 1966; Chvála et al. 1972; Chvála 1988; Ganeva 1999b, 2000, 2005a; Ganeva \& Ivanov 2015.

Haematopota pluvialis (Linnaeus, 1758) [Chrysozona, Tabanus] - ; $\mathbf{\Delta}$; B1, B2, B3, K9, V1, V4, S23, TL, T11, T31, O5, O62, R1, RW, BN, BS; 0-2261 m; 1, 2, 3, 4, 5; pat, ? hop; Löw 1862; Meunier 1897; Joakimoff 1899; Nedelkov 1909, 1912; Drensky 1929a, 1929b, 1931a, 1931b, 1942, 1960; Shannon \& Hadjinicolaou 1936; Moucha \& Chvála 1961; Leclercq 1957, 1966; Beschovski 1964a; Petkov 1964; Trifonov et al. 1964; Chvála et al. 1972; Chvála 1988; Lavchiev \& Ganeva 1991; Ganeva 1993, 1995a, 1995b, 1995c, 1996, 1998, 1999a, 1999b, 2001, 2002, 2004, 2005a, 2005b, 2008b, 2009a, 2009b, 2011, 2017; Ganeva \& Penev 2002; Ganeva \& Kalmushka 2012, 2019; Ganeva \& Ivanov 2015.

Haematopota scutellata (Olsufjev, Moucha \& Chvala, 1964) - B3, S23, TL, T11, O5, R1, R3; 230-1625 m; 1, 2, 3, 4; e; Chvála et al. 1972; Chvála 1988; Ganeva 1993, 1995a, 1995c, 1996, 1998, 1999b, 2000, 2005a, 2005b, 2009a, 2017; Ganeva \& Penev 2002; Ganeva \& Ivanov 2015.

Haematopota subcylindrica Pandelle, 1883 [Chrysozona pluvialis var. subcylindrica Pandellé, 1883] - \$; S23, TL, R1; 150-1569 m; 1, 2, 3, 4; esca, ? esit; Drensky 1929a, 1929b; Kröber 1932; Leclercq 1966; Chvála 1988; Lavchiev \& Ganeva 1991; Ganeva 1993, 1995a, 1996, 1998, 1999b, 2000, 2017; Ganeva \& Penev 2002.

Haematopota turkestanica (Kröber, 1922) - TL; 230 m; 1; esca; Ganeva 1995a.

Dasyrhamphis anthracinus (Meigen, 1820) - TL, T11; 150-400 m; 1; sena; Chvála et al. 1972; Chvála 1988; Ganeva 2005b, 2009a.

Dasyrhamphis ater (Rossi, 1790) [Atylotus] - B2, S1, S23, TL, T31, O5, O62, BN, BS; 0-1526 m; 1, 2, 3; sena, ? hom; Löw 1863; Drensky 1929a, 1929b, 1931a, 1931b, 1942, 1960; Shannon \& Hadjinicolaou 1936; Leclercq 1957; Chvála et al. 1972; Chvála 1988; Ganeva 1993, 1995a, 1995c, 1996, 1999b, 2000, 2009b, 2017; Ganeva \& Penev 2002; Ganeva \& Kalmushka 2012, 2019; Ganeva \& Ivanov 2015.

Dasyrhamphis nigritus (Fabricius, 1794) [Tabanus] - S1, TL, BN, BS; 0-250 m; 1; hom; Drensky 1929a, 1929b, 1942; Shannon \& Hadjinicolaou 1936; Moucha \& Chvála 1961.

Dasyrhamphis umbrinus (Meigen, 1820) [Atylotus, Tabanus] - S23, TL, T11, T31, O5, O62, BN; 0-400 m; 1; nmi; Löw 1862; Nedelkov 1912; Drensky 1929a, 1929b, 1931a, 1931b; Shannon \& Hadjinicolaou 1936; Kröber 1938; Leclercq 1966; Trifonov et al. 1964; Chvála 1988; Ganeva 1993, 1995b, 1996, 1998, 1999b, 2002, 2005b, 2006, 2008b, 2009a, 2009b; Ganeva \& Penev 2002; Ganeva \& Kalmushka 2012. 
Philipomyia aprica (Meigen, 1820) [Tabanus; T. graecus apricus Meigen, 1820] - E2, B3, K9, S211, T31, O62, R1, R2, RW, RE, BN; 0-2082 m; 1, 2, 3, 4; eani; Drensky 1929a, 1929b, 1931a, 1931b, 1942, 1955; Drenowsky 1936; Shannon \& Hadjinicolaou 1936; Kröber 1938; Leclercq 1957, 1966; Moucha \& Chvála 1961; Trifonov et al. 1964; Chvála et al. 1972; Pârvu 1983; Chvála 1988; Ganeva 1988, 1996, 2000, 2005a, 2009a, 2009b, 2017.

Philipomyia graeca (Fabricius, 1794) [Tabanus; ? T. apricus graecus Meigen, 1820] - E2, P3, B1, B3, V3, V4, S1, S23, TL, T11, T31, O5, O62, R1, RW, RE, BN, BS; 0-2082 m; 1, 2, 3, 4; csean; Nedelkov 1912; Drensky 1929a, 1929b, 1931a, 1931b, 1960; Shannon \& Hadjinicolaou 1936; Kröber 1938; Leclercq 1957, 1966; Moucha \& Chvála 1961; Beschovski 1971a; Chvála et al. 1972; Chvála 1988; Lavchiev \& Ganeva 1991; Ganeva 1993, 1995a, 1995b, 1995c, 1996, 1998, 1999b, 2000, 2002, 2005b, 2006, 2008a, 2009a, 2009b, 2011, 2016, 2017; Ganeva \& Penev 2002; Ganeva \& Kalmushka 2012, 2019; Ganeva \& Ivanov 2015.

\section{Vermileonidae}

Vermileo vermileo (Linnaeus, 1758) [V. degeeri Macquart, 1834] - B1; 390 m; 1; hom; Popov 1968; Majer 1988.

\section{Nemestrinidae}

Nemestrinus caucasicus (Fischer, 1806) [Rhynchocephalus analis Olivier, 1810; R. albofasciatus Wiedemann, 1828] - S1; 245 m; 1; nemit; Löw 1863; Richter 1988.

\section{Acroceridae (Cyrtidae, Oncodidae, Ogcodidae)}

Ogcodes (Ogcodes) lautereri Chvala, 1980 - R2; 2000-2900 m; 4, 5, 6; sena, ? mm; Chvála 1980.

Ogcodes (Ogcodes) reginae (Trojan, 1956) - BS; 0-20 m; 1; esca; Bankowska 1967a; Nartshuk 1988.

\section{Bombyliidae}

Toxophora fasciculata (Villers, 1789) [T. maculata Rossi, 1790] - BN; 0-30 m; 1; swpat; Löw 1862; Zaitzev 1989; Katbeh-Bader \& Arabyat 2004; Evenhuis 2015.

Phthiria gaedii Wiedemann in Meigen, 1820 - swp, ? wp; Zaitzev 1989; Evenhuis 2015.

Usia (Usia) aenea (Rossi, 1794) - V3; 900-1000 m; 2; nm, ? hom; Drenowsky 1936, 1939; Zaitzev 1989; KatbehBader \& Arabyat 2004.

Geron gibbosus (Olivier, 1789) - TL; 150-200 m; 1; mca; Nedelkov 1909, 1912; Zaitzev 1989; Beschovski $2006 a$.

? Geron intonsus Bezzi, 1925 - ; ? mi; Zaitzev 1989. According to Evenhuis (2015) the species is not presented in Europe.

Geron krymensis Paramonov, 1929 - swp; Katbeh-Bader \& Arabyat 2004.

Chalcochiton holosericea (Fabricius, 1794) - V1, S21; 600-750 m; 2; mit; Nedelkov 1912.

Chalcochiton pallasii (Loew, 1856) [Mulio] - S1, BN; 0-300 m; 1; ? mit, ? wp; Löw 1862, 1863; Zaitzev 1989; Katbeh-Bader \& Arabyat 2004; Evenhuis 2015.

Cytherea obscura Fabricius, 1794 - ; mit, ? mwca; Zaitzev 1989; Katbeh-Bader \& Arabyat 2004; Evenhuis 2015.

Amictus pictus Loew, 1869 - mi; Zaitzev 1989; Katbeh-Bader \& Arabyat 2004; Evenhuis 2015.

Amictus validus Loew, 1869 - TL, RE, BS; 0-300 m; 1; mit; Nedelkov 1912; Beschovski 2004, 2006a; Zaitzev 1989; Katbeh-Bader \& Arabyat 2004; Evenhuis 2015.

Anastoechus nitidulus (Fabricius, 1794) - DM, P2, SB, V1, V4, TL, RW, BN, BS; 0-800 m; 1, 2; po; Nedelkov 1912; Zaitzev 1989; Beschovski 2006a; Evenhuis 2015.

Bombylisoma minimum (Scopoli, 1771) - B1, V1, TL; 250-700 m; 1, 2; nmi; Nedelkov 1912; Evenhuis 2015.

Bombylisoma nigriceps (Loew, 1855) [Dischistus] - BN; 0-20 m; 1; eani, ? nmi; Löw 1862; Zaitzev 1989; Evenhuis 2015.

Bombylisoma unicolor (Loew, 1855) - \$; mi; Zaitzev 1989; Evenhuis 2015.

Bombylella atra (Scopoli, 1763) [Bombylius] - E2, B1, V3, V4, S1, S2, TL, R1, BN; 0-2100 m; 1, 2, 3, 4; tp; Löw 1862; Joakimoff 1899; Nedelkov 1912; Drensky 1955; Evenhuis 2015. 
Bombomyia vertebralis Dufour, 1833 [Bombilius punctatus Fabricius, 1794] - E2, TL, BN; 0-200 m; 1; wp; Löw 1862; Nedelkov 1909, 1912.

Bombylius (Bombylius) canescens Mikan, 1796 [B. variabilis Loew, 1855] - DM, B1, K9, V1, S211; 30-780 m; 1, 2; wp; Nedelkov 1909, 1912; Katbeh-Bader \& Arabyat 2004; Evenhuis 2015.

Bombylius (Bombylius) cinerascens Mikan, 1796 - S211; 700-800 m; 2; eanna, ? wp; Nedelkov 1912; KatbehBader \& Arabyat 2004; Evenhuis 2015.

Bombylius (Bombylius) discolor Mikan, 1796 - V1, V3, V4, TL; 150-800 m; 1, 2; wpo; Meunier 1897; Nedelkov 1912.

Bombylius (Bombylius) fimbriatus Meigen, 1820 - V1, V4; 550-750 m; 2; wcp; Nedelkov 1912.

Bombylius (Bombylius) floccosus Loew, 1857 - S1; 250-300 m; 1; mi; Löw 1863; Zaitzev 1989; Evenhuis 2015.

Bombylius (Bombylius) fulvescens Wiedemann in Meigen, 1820 - P1, P2, B1, V1, V4, BN, BS; 0-800 m; 1, 2; wcp, ? wp; Nedelkov 1912; Beschovski 1971a; Katbeh-Bader \& Arabyat 2004; Evenhuis 2015.

Bombylius (Bombylius) major Linnaeus, 1758 - ; V1, V4, O61, R1; 300-2000 m; 1, 2, 3, 4; ho; Meunier 1897; Joakimoff 1899; 1933; Nedelkov 1912; Evenhuis 2015.

Bombylius (Bombylius) medius Linnaeus, 1758 - E2, V1, TL, O61, R1, RE; 20-2100 m; 1, 2, 3, 4; wcp; Joakimoff 1899; 1933; Nedelkov 1909, 1912; Beschovski 2004; Evenhuis 2015.

Bombylius (Bombylius) minor Linnaeus, 1758 - V1, S21, R1, BN; 0-1150 m; 1, 2, 3; esca; Joakimoff 1899; Nedelkov 1912; Evenhuis 2015.

Bombylius (Bombylius) niveus Meigen, 1804 - mi; Zaitzev 1989; Evenhuis 2015.

Bombylius (Bombylius) nubilus Mikan, 1796 - BN; 0-30 m; 1; ? swp; Löw 1862.

Bombylius (Bombylius) posticus Fabricius, 1805 [B. vulpinus Wiedemann in Meigen, 1820] - DM, P1, B1, V1, S21, S211, BN; 0-800 m; 1, 2; wp; Löw 1862; Nedelkov 1912; Evenhuis 2015.

Bombylius (Bombylius) trichurus Pallas, 1818 - wpat; Katbeh-Bader \& Arabyat 2004.

Bombylius (Bombylius) venosus Mikan, 1796 - V1, V4, S211; 550-800 m; 2; wp; Nedelkov 1912; Evenhuis 2015.

Bombylius (Zephyrectes) cruciatus Fabricius, 1798 - DM, TL, BN, BS; 0-200 m; 1; mi; Nedelkov 1909, 1912.

Bombylius (Zephyrectes) quadrifarius Loew, 1855 - emca; Zaitzev 1989; Katbeh-Bader \& Arabyat 2004; Evenhuis 2015.

Triplasius pictus (Panzer, 1794) [Bombylius] - E2, V1, V4, TL; 140-1000 m; 1, 2; eanna; Nedelkov 1912; Drensky 1955.

Conophorus glaucescens (Loew, 1863) [Ploas] - S1; 250-300 m; 1; em; Löw 1863; Zaitzev 1989; Katbeh-Bader \& Arabyat 2004; Evenhuis 2015.

Conophorus virescens (Fabricius, 1789) [Ploas] - V1, V4, S1, Tl, R3, RW, BN; 0-1500 m; 1, 2, 3; wcp; Löw 1862, 1863; Nedelkov 1912; Drenowsky 1939; Drensky 1939a; Beschovski 1971a, $2006 a$.

Prorachthes beckeri Paramonov, 1927 - ; seean, ? nm; Zaitzev 1989; Evenhuis 2015.

Systoechus autumnalis (Pallas, 1818) - \$; swp; Zaitzev 1989; Evenhuis 2015.

Systoechus ctenopterus (Mikan, 1796) [S. sulphureus Mikan, 1796] - DM, V1, S2, BN, BS; 0-1000 m; 1, 2; wcp; Meunier 1879; Nedelkov 1909, 1912; Evenhuis 2015.

Systoechus gradatus (Wiedemann in Meigen, 1820) [Bombylius leucophaeus Wiedemann in Meigen, 1820] - P1, B1, B2, V1, S1, S211, BN, BS; 0-1000 m; 1, 2; wcp; Löw 1863; Nedelkov 1912; Evenhuis 2015.

Systoechus longirostris Becker, 1916 - it; Zaitzev 1989; Katbeh-Bader \& Arabyat 2004; Evenhuis 2015.

Lomatia alecto Loew, 1846 - csei; Zaitzev 1989; Evenhuis 2015.

Lomatia atropos Egger, 1859 - P1, B1; 300-400 m; 1; Ebs; Nedelkov 1912.

Lomatia belzebul (Fabricius, 1794) - P1, B1, V1, S1; 250-600 m; 1; wp; Löw 1863; Nedelkov 1912.

Lomatia erinnys Loew, 1869 - swp, ? wp; Zaitzev 1989; Evenhuis 2015.

Lomatia tysiphone Loew in Schiner, 1860 [L. infernalis Schiner, 1868] - V1; 600 m; 1; mt; Nedelkov 1912; Zaitzev 1989; Katbeh-Bader \& Arabyat 2004; Evenhuis 2015.

Lomatia lachesis Egger, 1859 - R3; 1450 m; 3; wp; Drenowsky 1939; Drensky 1939a; Zaitzev 1989; Evenhuis 2015.

Lomatia lateralis (Meigen, 1820) - P1, B1; 300-400 m; 1; wp; Nedelkov 1912; Zaitzev 1989; Evenhuis 2015.

Lomatia sabaea (Schaeffer, 1766) - P2, B1, S21, O61, R1; 150-800 m; 1, 2; eanna, ? wp; Joakimoff 1899; Nedelkov 1912; Zaitzev 1989; Evenhuis 2015.

Anthrax aethiops (Fabricius, 1781) [Argyromoeba, Spodostylum] - Tl, RE; 150-200 m; 1; wp; Nedelkov 1912; Zaitzev 1989; Beschovski 2004; Katbeh-Bader \& Arabyat 2004; Evenhuis 2015.

Anthrax anthrax (Schrank, 1781) - ; tp; Joakimoff 1933; Evenhuis 2015. 
Anthrax binotatus Wiedemann in Meigen, 1820 [Argyromoeba] - V1, V4, 500-800 m, 1, 2; wp; Nedelkov 1912; Zaitzev 1989; Evenhuis 2015.

Anthrax trifasciatus Meigen, 1804 [Argyromoeba] - V1, TL, RE; 180-550 m; 1; wpo; Nedelkov 1909, 1912; Zaitzev 1989; Beschovski 2004; Evenhuis 2015.

Anthrax varius Fabricius, 1794 [Argyromoeba] - R1, BN; 0-1350 m; 1, 2, 3; wcp; Nedelkov 1912; Drensky 1942; Zaitzev 1989; Evenhuis 2015.

Anthrax virgo Egger, 1859 - \$; mi; Zaitzev 1989; Evenhuis 2015.

Desmatoneura nivea (Rossi, 1790) - ; sena; Zaitzev 1989; Evenhuis 2015.

Petrorossia hespera (Rossi, 1790) - ; mt, ? mit; Zaitzev 1989; Katbeh-Bader \& Arabyat 2004; Evenhuis 2015.

Petrorossia letho (Wiedemann, 1828) - swpat; Zaitzev 1989; Katbeh-Bader \& Arabyat 2004; Evenhuis 2015.

Spogostylum isis (Meigen, 1820) [Argyromoeba subnotata Walker, 1871] - V1, V4, S21, TL, RE; 150-900 m; 1, 2; mwca, ? mit, ? swpat; Nedelkov 1909, 1912; Beschovski 2004.

Spogostylum tripunctatum (Pallas in Wiedemann, 1818) - ; mwca; Zaitzev 1989; Evenhuis 2015.

Exhyalanthrax afer (Fabricius, 1794) [Anthrax] - S21, TL; 200-700 m; 1, 2; ppt; Nedelkov 1912; Zaitzev 1989; Katbeh-Bader \& Arabyat 2004; Evenhuis 2015.

Exoprosopa capucina (Fabricius, 1781) - V4, R1; 800-1000 m; 2; esanca; Nedelkov 1912; Evenhuis 2015.

Exoprosopa jacchus (Fabricius, 1805) [E. picta Wiedemann in Meigen, 1820] - BN, BS; 0-100 m; 1; ? wcp, ? eanca; Nedelkov 1912; Drensky 1942; Zaitzev 1989; Evenhuis 2015.

Exoprosopa rutila (Pallas \& Wiedemann, 1818) - mca; Zaitzev 1989.

Defilippia minos (Meigen, 1804) [Exoprosopa germari Wiedemann, 1818] - ; DW, DM, E2, SB, V1, S211, S2, TL, RW, RE, BN, BS; 0-1200 m; 1, 2, 3; wcp; Nedelkov 1909, 1912; Drensky 1942, 1955; Beschovski 1971a, 2004, 2006a.

Balaana grandis (Wiedemann in Meigen, 1820) [Exoprosopa fasciata Dufour, 1850] - \$; TL; 250; 1; mwca; Nedelkov 1912; Joakimoff 1933; Zaitzev 1989; Beschovski 2006a; Evenhuis 2015.

Hemipenthes hamifera (Loew, 1854) - nmca; Zaitzev 1989; Evenhuis 2015.

Hemipenthes maura (Linnaeus, 1758) [Anthrax] - V1, S21, R1, R3; 550-1700 m; 1, 2, 3, 4; esanca; Meunier 1897; Nedelkov 1912; Drenowsky 1936; Evenhuis 2015.

Hemipenthes morio (Linnaeus, 1758) [Anthrax] - DM, E1, E2, P1, SB, B1, V1, V4, S211, TL, R1, RW, BN, BS; 0-1000 m; 1, 2; h, ? ho; Meunier 1897; Nedelkov 1909, 1912; Joakimoff 1933; Drensky 1955; Evenhuis 2015.

Hemipenthes velutina (Meigen, 1820) [Anthrax] - P1, SB, B1, V1, V4, S1, S22, TL, BN; 0-800 m; 1, 2; spo; Nedelkov 1909, 1912; Drensky 1955; Zaitzev 1989; Katbeh-Bader \& Arabyat 2004; Evenhuis 2015.

Heteralonia (Zygodipla) aeaca (Meigen, 1804) [Exoprosopa] - BS; 0-10 m; 1; spat; Nedelkov 1912; Zaitzev 1989; Evenhuis 2015.

Heteralonia (Homolonia) megerlei (Meigen, 1820) [Exoprosopa vespertilio Wiedemann in Meigen, 1820] - S22, TL, RW, BN; 0-1000 m; 1, 2; sppt; Löw 1862; Nedelkov 1912; Drensky 1942; Zaitzev 1989; Katbeh-Bader \& Arabyat 2004; Beschovski 2006a; Evenhuis 2015.

Exoprosopa rivularis (Meigen, 1820) - mca; Zaitzev 1989.

Ligyra ferrea (Walker, 1849) - nm; Zaitzev 1989; Evenhuis 2015.

Micomitra iris (Loew, 1869) - nmwca; Zaitzev 1989; Katbeh-Bader \& Arabyat 2004; Evenhuis 2015.

Thyridanthrax elegans (Wiedemann in Meigen, 1820) - mca; Zaitzev 1989; Katbeh-Bader \& Arabyat 2004; Evenhuis 2015.

Thyridanthrax fenestratus (Fallén, 1814) [Anthrax] - V1, BN, BS; 0-600 m; 1, 2; wcp; Nedelkov 1909, 1912; Evenhuis 2015.

Thyridanthrax perspicillaris (Loew, 1869) - ppt; Katbeh-Bader \& Arabyat 2004.

Villa brunnea Becker, 1916 - nmi; Zaitzev 1989; Evenhuis 2015.

Villa cana (Meigen, 1804) [V. quinquefasciata Wiedemann in Meigen, 1820] - \$; wp, ? swp; Zaitzev 1989; Evenhuis 2015.

Villa clarissima (Loew, 1857) - ; hom; Zaitzev 1989; Evenhuis 2015.

Villa fasciata (Meigen, 1804) [Anthrax circumdata Meigen, 1820] - BN; 0-20 m; 1; wcp; Löw 1862; Zaitzev 1989; Evenhuis 2015.

Villa ixion (Fabricius, 1794) [Anthrax humilis Ruthe, 1831; V.pygarga (Loew, 1868)] - V1, S1, TL, BN; 0-600 m; 1, 2; wp; Nedelkov 1912; Drensky 1942; Zaitzev 1989; Katbeh-Bader \& Arabyat 2004; Evenhuis 2015.

Villa halteralis (Kowarz, 1883) - e; Zaitzev 1989; Evenhuis 2015. 
Villa hottentotta (Linnaeus, 1758) [Anthrax flava Meigen, 1820] - DM, E2, P1, B1, V1, S1, S21, S22, TL, R1, BN, BS; 0-1000 m; 1, 2; hop; Löw 1862; Nedelkov 1909, 1912; Joakimoff 1899, 1933; Drensky 1955; Evenhuis 2015.

Villa niphobleta (Loew, 1869) - wcp; Zaitzev 1989; Katbeh-Bader \& Arabyat 2004; Evenhuis 2015.

Villa occulta (Wiedemann in Meigen, 1820) - ess; Zaitzev 1989; Evenhuis 2015.

Villa paniscus (Rossi, 1790) [Anthrax] - V1, TL, RW; 150-800 m; 1, 2; po; Nedelkov 1909, 1912; Beschovski 2006a; Evenhuis 2015.

Villa ventruosa (Loew, 1869) - \$; mi; Zaitzev 1989; Evenhuis 2015.

\section{Mythicomyiidae}

Cyrtosia marginata Perris, 1839 - BS; 0-10 m; 1; se; Mielczarek 2018.

Platypygus ridibundus (Costa, 1863) - O62, R2; 250-550 m; 1; sena, ? hom; Zaitzev 1989; Evenhuis 2002; Gharali et al. 2013; Mielczarek 2018.

\section{Therevidae}

Acrosathe annulata (Fabricius, 1805) [Thereva] - BN; 0-20 m; 1; e; Löw 1862.

Cionophora kollari Egger, 1854 - BN; 0-20 m; 1; see; Löw 1862; Lyneborg 1989.

Cliorismia ardea (Fabricius, 1794) [Psilocephala] - B2, V1; 600-1300 m; 2, 3; e; Nedelkov 1912; Drensky 1934c.

Pandivirilia eximia (Meigen, 1820) [Psilocephala] - B2, R1; 1300-1400 m; 3; e; Nedelkov 1910, 1912; Drensky 1934c.

Thereva aurata (Loew, 1854) - DW; 130-140 m; 1; csean; Tsvetanov 2021.

Thereva cinifera Meigen, 1830 [T. subfasciata Schummel, 1839] - B2, V4; 800-1300 m; 2, 3; wes; Nedelkov 1910, 1912; Drensky 1934c.

Thereva marginula Meigen, 1820 - B2, V4; 800-1300 m; 2, 3; e; Nedelkov 1910, 1912; Drensky 1934c.

Thereva plebeja (Linnaeus, 1758) - BN; 0-30 m; 1; ? des; Drensky 1942.

Thereva praecox Egger, 1859 - TL, RW; 150-500 m; 1; e; Nedelkov 1910, 1912; Lyneborg 1989; Beschovski $2006 a$.

Thereva spinulosa Loew, 1847 - BN; 0-30 m; 1; hom; Löw 1862.

Thereva tuberculata Loew, 1847 - B2, R1, BN; 0-1300 m; 1, 2, 3; sena; Löw 1862; Drensky 1934c.

\section{Scenopinidae (Omphralidae)}

Scenopinus fenestralis (Linnaeus, 1758) - V1, S21, TL; 150-800 m; 1, 2; sk, ? k; Nedelkov 1910, 1912.

Scenopinus glabrifrons Meigen, 1824 - V1; 600-650 m; 1; sk, ? k; Nedelkov 1912.

\section{Asilidae}

Choerades dioctriaeformis (Meigen, 1820) [Laphria] - B1, TL; 150-370 m; 1; cse, ? e; Nedelkov 1910, 1912.

Choerades fimbriata (Meigen, 1820) [Laphria] - B1, V1, V4, R1, RW, BS; 0-1450 m; 1, 2, 3; wes; Nedelkov 1909, 1910, 1912; Szilady 1934; Hradský \& Moucha 1964, 1967; Jelesova 1971; Beschovski 2006a.

Choerades fuliginosa (Panzer, 1798) [Laphria] - P2, B1, B2, S1, S22, TL, T31, O62, R2, R5, BS; 0-1200 m; 1, 2, 3; wces; Nedelkov 1910, 1912; Drenowsky 1936; Hradský \& Moucha 1964, 1967; Lehr 1988.

Choerades fulva (Meigen, 1804) [Ch. proboscidea (Loew, 1847); Laphria] - V1, S1, TL, O62, RW; 150-1100 m; 1, 2, 3; des; Nedelkov 1910, 1912; Hradský \& Moucha 1967; Beschovski 2006a.

Choerades gilva (Linnaeus, 1758) [Laphria] - TL, RW; 150-1926 m; 1, 2, 3, 4; h; Hradský \& Moucha 1964, 1967; Jelesova 1975; Lehr 1988; Beschovski 2006a; Beron 2011.

Choerades ignea (Meigen, 1820) [Laphria] - S21, R1; 650-800 m; 2; wes; Nedelkov 1909, 1912.

Choerades marginata (Linnaeus, 1758) [Laphria] - S21, RE; 120-600 m; 1; e; Meunier, 1897; Nedelkov 1910, 1912; Jelesova 1975; Beschovski 2004; Beron 2011.

Laphria aurea (Fabricius, 1794) - ; eswa; Lehr 1988.

Laphria ephippium (Fabricius, 1781) - SB, V4, RW; 600-1000 m; 1, 2; e; Nedelkov 1910, 1912; Szilady 1934; Hradský \& Moucha 1964, 1967; Lehr 1988; Beschovski 2006a. 
Laphria flava (Linnaeus, 1761) - DW, P2, B1, B2, B3, V1, V4, S2, R1, R2, R3, RW, BS; 0-2500 m; 1, 2, 3, 4, 5; po; Meunier, 1897; Nedelkov 1909, 1910, 1912; Szilady 1934; Drenowsky 1936; Hradský \& Moucha 1964, 1967; Jelesova 1971, 1974a, 1975; Lehr 1988; Beschovski 2006a; Beron 2011.

Laphria gibbosa (Linnaeus, 1758) - B2, B3, R1, R3; 600-1400 m; 2, 3; hoes; Szilady 1934; Drenowsky 1936, 1939; Drensky 1939a; Hradský \& Moucha 1964, 1967; Jelesova 1974a.

Andrenosoma atrum (Linnaeus, 1758) - B1; 1450 m; 3; ena; Jelesova 1971.

Pogonosoma maroccanum (Fabricius, 1794) [Laphria] - DM, B3, K9, V1, S1, TL, BN, BS; 0-1100 m; 1, 2, 3; eanna; Löw 1862; Nedelkov 1909, 1910, 1912; Hradský \& Moucha 1964, 1967; Jelesova 1974a; Lehr 1988.

Dioctria atricapilla Meigen, 1804 - B1, V1, RW; 1000-1300 m; 3; tes; Hradský \& Moucha 1967; Jelesova 1971, 1975; Beschovski 2006a; Beron 2011.

Dioctria bulgarica Hradsky \& Moucha, 1964 - V4; 1500 m; 3, 4; Ebg, ? Er; Hradský \& Moucha 1964, 1967; Lehr 1988.

Dioctria cothurnata Meigen, 1820 [D. reinhardi Meigen, 1820] - V1, S1, TL, R1, RW; 150-1600 m; 1, 2, 3, 4; wces; Nedelkov 1910, 1912; Hradský \& Moucha 1964, 1967; Jelesova 1975; Lehr 1988; Beschovski 2006a; Beron 2011.

Dioctria flavipennis Meigen, 1820 [D. aurifrons Meigen, 1820] - B1, B3, V1, V4, T31; 300-1300 m; 1, 2, 3; esca; Nedelkov 1910, 1912; Hradský \& Moucha 1967; Jelesova 1971, 1974a; Lehr 1988.

Dioctria harcyniae Loew, 1844 - V4; ; e; Hradský \& Moucha 1967; Lehr 1988.

Dioctria humeralis Zeller, 1840 - B2, V1; 1070 m; 3; tes; Nedelkov 1912; Jelesova 1974a; Lehr 1988.

Dioctria hyalipennis (Fabricius, 1794) [? D. flavipes Meigen, 1804] - B2; 1070-1200 m; 3; h; ? Hradský \& Moucha 1964; Jelesova 1974a; Lehr 1988.

Dioctria lateralis Meigen, 1804 [Cyrtopogon] - B2, RW; 360-1000 m; 1, 2; ena; Nedelkov 1912; Jelesova 1974a; Lehr 1988; Beschovski 2006a.

Dioctria linearis (Fabricius, 1787) - P2, B2, O62, RW, BS; 0-1000 m; 1, 2; e; Hradský \& Moucha 1964, 1967; Lehr 1988; Beschovski 2006a.

Dioctria longicornis Meigen, 1820 - T31; 300 m; 1; e; Hradský \& Moucha 1967; Lehr 1988.

Dioctria oelandica (Linnaeus, 1758) - B1, B2, V1, V4, S211, TL, O61, R1, R2, R5, RW; 200-1700 m; 1, 2, 3, 4; e; Joakimoff 1899; Nedelkov 1910, 1912; Hradský \& Moucha 1964, 1967; Jelesova 1971, 1974a, 1975; Lehr 1988; Beschovski 2006a; Beron 2011.

Dioctria rufipes (De Geer, 1776) - B2, V1, TL, BN; 0-1300 m; 1, 2, 3; esca; Meunier, 1897; Drensky 1942; Hradský \& Moucha 1964, 1967; Jelesova 1974a; Beschovski 2006a.

Dioctria rufithorax Loew, 1853 - V1, BN; 0-600 m; 1; e, ? csee; Nedelkov 1912; Drensky 1942; Hradský \& Moucha 1964, 1967; Lehr 1988.

Molobratia teutonus (Linnaeus, 1767) [Dasypogon, Selidopogon] - P2, B1, B2, R2, RW, BS; 0-1110 m; 1, 2, 3; esanca; Drenowsky 1936; Hradský \& Moucha 1964, 1967; Jelesova 1971, 1974a, 1975; Lehr 1988; Beschovski 2006a; Beron 2011.

Lasiopogon cinctus (Fabricius, 1781) - B1, R1, RW; 300-2650 m; 1, 2, 3, 4, 5, 6; e; Nedelkov 1910, 1912; Jelesova 1974a, 1975; Lehr 1988; Beschovski 2006a; Beron 2011.

Lasiopogon montanus Schiner, 1862 - B2, R1, RW; 1400-2150 m; 3, 4; se, mm; Jelesova 1974a, 1975; Lehr 1988; Beschovski 2006a; Beron 2011.

Lasiopogon soffneri Hradsky \& Moucha, 1964 - TL, RW; 230-360 m; 1; Ebg; Hradský \& Moucha 1964, 1967; Lehr 1988; Beschovski 2006a.

Stichopogon albofasciatus (Meigen, 1820) - D, V1, BS; 0-600 m; 1; eanna; Nedelkov 1910, 1912; Hradský \& Moucha 1964, 1967; Lehr 1988.

Stichopogon elegantulus (Wiedemann, 1820) [S. tener Loew, 1847] - DM, V1, BN, BS; 0-600 m; 1; wp; Nedelkov 1909, 1910, 1912; Hradský \& Moucha 1964, 1967; Lehr 1988.

Stichopogon inaequalis Loew, 1847 - V1, BS; 0-600 m; 1; wpo; Nedelkov 1910, 1912; Hradský \& Moucha 1964, 1967; Lehr 1988.

Stichopogon scaliger Loew, 1847 - B1, V1, BN, BS; 0-600 m; 1; mi; Nedelkov 1910, 1912; Hradský \& Moucha 1967; Lehr 1988.

Stichopogon schineri Koch, 1872 [Asilus] - DM; 20-30 m; 1; ? wp; Nedelkov 1909, 1912.

Ancylorhynchus glaucius (Rossi, 1790) [Xiphocerus] - DM, V1, S1, BN; 0-600 m; 1; ? hom; Löw 1862; Nedelkov 1910, 1912. 
Cyrtopogon platycerus Villeneuve, 1913 [Cyclosocerus] - RW; 1000-2150 m; 3, 4; se, ? mm; Jelesova 1975; Beschovski 2006a; Tomasovic 2006; Beron 2011.

Cyrtopogon maculipennis (Macquart, 1834) - B3; 600-650 m; 1, 2; h; Jelesova 1975; Lehr 1988; Tomasovic 2006. Cyrtopogon ruficornis (Fabricius, 1794) - B1, S22, RW; 450-1926 m; 1, 2, 3, 4; e; Nedelkov 1909, 1912; Jelesova 1971, 1975; Beschovski 2006a; Tomasovic 2006; Beron 2011.

Heteropogon ornatipes Loew, 1851 - em; Lehr 1988.

Holopogon nigripennis (Meigen, 1820) - RW; 250-400 m; 1; esca; Hradský \& Moucha 1964, 1967; Lehr 1988; Beschovski 2006a.

Holopogon priscus (Meigen, 1820) [H. clavipes (Loew, 1840)] - V1; 600 m; 1, 2; west; Nedelkov 1912.

Pycnopogon fasciculatus (Loew, 1847) - O62; 250 m; 1; hom; Hradský \& Moucha 1967; Lehr 1988.

Stenopogon callosus (Pallas, 1818) - e; Lehr 1988.

Stenopogon coracinus (Loew, 1847) - B3, TL, O61, O62; 150-1000 m; 1, 2; nm; Nedelkov 1912; Hradský \& Moucha 1967; Jelesova 1974a; Lehr 1988.

Stenopogon elongatus (Meigen, 1804) - DM, E1, E2; 20-30 m; 1; atm; Nedelkov 1910, 1912.

Stenopogon milvus (Loew, 1847) [S. subaudus milvus (Loew, 1847)] - TL, R3, RW; 250-1500 m; 1, 2, 3, 4; ban; Drenowsky 1936; Hradský \& Moucha 1964, 1967; Lehr 1988.

Stenopogon sabaudus (Fabricius, 1794) - DM, B1, V1, S1, S2, TL, O62, RW, RE, BN, BS; 0-1000 m; 1, 2; nmi; Nedelkov 1909, 1910, 1912; Hradský \& Moucha 1964, 1967; Jelesova 1971; Lehr 1988; Beschovski 2004, 2006a; Beron 2011.

Leptarthrus brevirostris (Meigen, 1804) - V4, R2; 1000-1200 m; 2, 3; e; Hradský \& Moucha 1964, 1967; Lehr 1988.

Dasypogon diadema (Fabricius, 1781) [Selidopogon] - E1, B1, B2, V1, V3, S1, S211, TL, R1, RW, BS; 0-2370 m; 1, 2, 3, 4, 5; wp; Nedelkov 1909, 1910, 1912; Drenowsky 1936, 1939; Hradský \& Moucha 1964, 1967; Jelesova 1971, 1974a, 1975; Lehr 1988; Beschovski 2006a; Beron 2011.

Dasypogon melanopterus Loew, 1869 [D. diadema melanoptera Loew, 1869] - O62; 150 m; 1; se; Hradský \& Moucha 1967; Lehr 1988.

Saropogon luctuosus (Wiedemann, 1820) - DM, P2; 30-200 m; 1; se; Nedelkov 1910, 1912.

Leptogaster cylindrica (De Geer, 1776) [L. nigricornis Loew, 1847] - B1, B2, V1, V4, S2, O61, R1, RW; 2002150 m; 1, 2, 3, 4; tp; Joakimoff 1899; Nedelkov 1909, 1910, 1912; Jelesova 1971, 1974a, 1975; Lehr 1988; Beschovski 2006a; Beron 2011.

Leptogaster guttiventris Zetterstedt, 1842 - V1; 600 m; 2; wes; Nedelkov 1910, 1912.

Leptogaster pubicornis Loew, 1847 - P1, V1, V4, S1, S211, TL; 200-1000 m; 1, 2; ewca; Nedelkov 1910, 1912; Jelesova 1971; Lehr 1988.

Leptogaster stackelbergi Lehr, 1961 - RW; 700 m; 2; eeca; Jelesova 1975; Beschovski 2006a.

Promachus leoninus Loew, 1848 - TL, BS; 0-250 m; 1; oem; Nedelkov 1909, 1910, 1912.

Engelepogon goedli (Loew, 1854) [Acanthopleura] - em; Lehr 1988.

Antipalus reginae Moucha \& Hradsky, 1966 - O62; 150 m; 1; Eb; Hradský \& Moucha 1967; Lehr 1988.

Antipalus sinuatus (Loew, 1854) - RE; 90-100 m; 1; cee; Jelesova 1975; Beschovski 2004; Beron 2011.

Antipalus varipes (Meigen, 1820) - TL; 200-300 m; 1; e; Nedelkov 1910, 1912.

Antiphrisson trifarius (Loew, 1849) - E2, V1, S21, RE; 20-650 m; 1; wp; Nedelkov 1910, 1912; Jelesova 1975; Beschovski 2004; Beron 2011.

Asilus crabroniformis Linnaeus, 1758 - O62, RW; 150-1100 m; 1, 2, 3; dp; Nedelkov 1909, 1912; Hradský \& Moucha 1967; Lehr 1988; Beschovski 2006a.

Cerdistus denticulatus (Loew, 1849) [Cyclosocerus platicerus (Villeneuve, 1913)] - TL RW; 250-300 m; 1; seean; Hradský \& Moucha 1967; Lehr 1988; Beschovski 2006a.

Dysmachus antipai Weinberg, 1968 - B3, TL, RE; 160-650 m; 1, 2; see, Ebs; Jelesova 1974a, 1975; Beschovski 2004.

Dysmachus bifurcus (Loew, 1848) [Asilus] - P2, B1, B3, V1, S1; 200-1200 m; 1, 2, 3; eani; Nedelkov 1910, 1912; Jelesova 1974a.

Dysmachus bilobus Loew, 1871 - S1, TL; 150-250 m; 1; eeca, ? eanca; Hradský \& Moucha 1964, 1967; Lehr 1988.

Dysmachus bimucronatus (Loew, 1854) - P1, B3, V1, V4, R1, RW; 200-2000 m; 1, 2, 3, 4; wes; Nedelkov 1912; Hradský \& Moucha 1964, 1967; Jelesova 1971, 1974a, 1975; Lehr 1988; Beschovski 2006a.

Dysmachus cephalenus Loew, 1871 - S1, TL, RW, RE; 200-1450 m; 1, 2, 3; seean, ? ees; Hradský \& Moucha 1964, 1967; Jelesova 1974a, 1975; Lehr 1988; Beschovski 2006a. 
Dysmachus cochleatus (Loew, 1854) - B1, K9, RE; 150-600 m; 1; ena, ? wp; Nedelkov 1912; Jelesova 1971, 1975; Lehr 1988; Beschovski 2004

Dysmachus fuscipennis (Meigen, 1820) [Asilus spiniger Zeller, 1840] - P1, SB, B1, B2, B3, K9, V1, V3, V4, S1, S2, S211, TL, T31, O1, O62, R1, RR, RW, RE, BN, BS; 0-2370 m; 1, 2, 3, 4, 5; wesit; Löw 1862; Nedelkov 1909, 1910, 1912; Hradský \& Moucha 1964, 1967; Jelesova 1971, 1974a, 1975; Lehr 1988; Beschovski 2004, $2006 a$.

Dysmachus hiulcus (Pandelle, 1905) - se; Lehr 1988.

Dysmachus praemorsus (Loew, 1854) [Asilus, Lophonotus] - B1, B2, B3, V1, V4, RE, BS; 0-1550 m; 1, 2, 3, 4; ean; Nedelkov 1910, 1912; Hradský \& Moucha 1967; Jelesova 1971, 1974a, 1975; Lehr 1988; Beschovski 2004.

Dysmachus stylifer (Loew, 1854) [Asilus, Lophonotus] - E2, P1, P2, B1, B2, B3, V1, V4, S1, S211, TL, R1, R2, R5, RW, RE, BN, BS; 0-2370 m; 1, 2, 3, 4, 5; eani; Nedelkov 1910, 1912; Drensky 1942, 1955; Hradský \& Moucha 1964, 1967; Jelesova 1971, 1974a, 1975; Lehr 1988; Beschovski 2004, $2006 a$.

Didysmachus picipes (Meigen, 1820) [Asilus forcipula Zeller, 1840; Dysmachus; Lophonotus] - DM, E2, P1, SB, B1, B2, B3, V4, S2, S21, S22, TL, O61, O62, R1, R2, R5, RW, RE; 100-2190 m; 1, 2, 3, 4, 5; h, ? wesca; Joakimoff 1899; Nedelkov 1909, 1910, 1912; Drensky 1955; Hradský \& Moucha 1964, 1967; Jelesova 1971, 1974a, 1975; Lehr 1988; Beschovski 2004, 2006a; Beron 2011.

Echthistus cognatus (Loew, 1849) - S1, O62, RW, RE, BS; 0-400 m; 1; nm; Hradský \& Moucha 1964, 1967; Jelesova 1975; Lehr 1988; Beschovski 2004, 2006a; Beron 2011.

Echthistus rufinervis (Meigen, 1820) [Asilus] - P1, B1, B2, V1, V3, TL, R3, RE; 200-1550 m; 1, 2, 3, 4; wp; Nedelkov 1909, 1910, 1912; Drenowsky 1939; Hradský \& Moucha 1964, 1967; Jelesova 1971, 1974a, 1975; Lehr 1988; Beschovski 2004; Beron 2011.

Machimus arthriticus (Zeller, 1840) [Epitriptus] - e; Lehr 1988.

Tolmerus cingulatus (Fabricius, 1781) [Asilus, Epitriptus] - B2, B3, K9, V1, V3, V4, S21, TL, BS; 0-2370 m; 1, 2, 3, 4, 5; eswa; Nedelkov 1910, 1912; Hradský \& Moucha 1964, 1967; Jelesova 1974a; Lehr 1988.

Neoepitriptus setosulus (Zeller, 1840) [Asilus, Epitriptus] - B3, V1, V3, S1; 250-1100 m; 1, 2, 3; et, ? e; Nedelkov 1910, 1912; Hradský \& Moucha 1967; Jelesova 1974a; Lehr 1988.

Erax barbatus Scopoli, 1763 [Asilus, Lophonotus] - E2, P1, V1, V4, TL; 200-850 m; 1, 2; e; Nedelkov 1910, 1912; Jelesova 1971; Lehr 1988.

Erax crassicauda (Loew, 1862) [Protophanes] - BN; 0-30 m; 1; Ebg; Löw 1862; Hradský \& Moucha 1964, 1967; Lehr 1988; Tomsovic 2002.

Eutolmus bureschi Hradsky \& Moucha, 1964 - RW; 250-380 m; 1; Ebg; Hradský \& Moucha 1964, 1967; Lehr 1988; Beschovski 2006a.

Eutolmus lavcievi Jelesova, 1974 - RR; Ebg; Jelesova 1974b; Lehr 1988.

Eutolmus rufibarbis (Meigen, 1820) - B1, B2, B3, V1, V4, S2, TL, R1, RW, BN; 0-1550 m; 1, 2, 3, 4; tp; Nedelkov 1909, 1910, 1912; Hradský \& Moucha 1964, 1967; Jelesova 1971, 1974a, 1975; Lehr 1988; Beschovski 2006a.

Machimus annulipes (Brullé, 1832) - P1, P3, B3, S1, TL, RE, BS; 0-1190 m; 1, 2, 3; eswa; Hradský \& Moucha 1964, 1967; Jelesova 1971, 1974a, 1975; Lehr 1988; Beschovski 2004.

Machimus caliginosus (Meigen, 1820) - B1, B2, B3, S1, S211, TL, O62, R2, R5, RW, BS; 0-1300 m; 1, 2, 3; csean; Hradský \& Moucha 1964, 1967; Jelesova 1971, 1974a; Lehr 1988; Beschovski 2006a.

Machimus cyanopus (Loew, 1849) - P1, SB, B3, S2, TL, RW; 250-1100 m; 1, 2, 3; e; Nedelkov 1912; Hradský \& Moucha 1964, 1967; Jelesova 1971; Lehr 1988; Beschovski 2006a.

Machimus fimbriatus (Meigen, 1804) - S1, T2, RW, BS; 0-1550 m; 1, 2, 3, 4; eanna; Nedelkov 1910, 1912; Jelesova 1975; Beschovski 2006a; Beron 2011.

Machimus gonatistes (Zeller, 1840) - P1, B1, TL, O62, RE, BN, BS; 0-520 m; 1; wp; Nedelkov 1910, 1912; Hradský \& Moucha 1964, 1967; Jelesova 1971, 1975; Lehr 1988; Beschovski 2004.

Machimus modestus (Loew, 1849) - seean; Lehr 1988.

Machimus rusticus (Meigen, 1820) - DM, B1, B2, K9, V1, V4, S2, TL, RW, RE, BN; 0-2160 m; 1, 2, 3, 4; wp; Nedelkov 1909, 1910, 1912; Hradský \& Moucha 1967; Jelesova 1971, 1975; Lehr 1988; Beschovski 2004, 2006a; Beron 2011.

Machimus setibarbus (Loew, 1849) - P2; 420-460 m; 1; eanna; Jelesova 1974a; Lehr 1988.

Neoitamus cothurnatus (Meigen, 1820) [Asilus, Itamus] - B1, V1, R1; 460-1300 m; 1, 2, 3; tp; Nedelkov 1910, 1912.

Neoitamus cyanurus (Loew, 1849) [Asilus] - B1, B2, V1, R1, RW; 550-1510 m; 1, 2, 3, 4; po; Meunier 1897; Joakimoff 1899; Jelesova 1971, 1974a, 1975; Lehr 1988; Beschovski 2006a; Beron 2011.

Neoitamus dasymallus (Gerstaecker, 1862) - P2, B2; 300-1190 m; 1, 2, 3; ee; Jelesova 1971, 1974a; Lehr 1988. 
Neoitamus impudicus (Gerstaecker, 1862) - O62, R2, RW; 150-1000 m; 1, 2; Eb; Hradský \& Moucha 1964, 1967; Lehr 1988.

Neoitamus socius (Loew, 1871) - B2, T31, RW; 300-1920 m; 1, 2, 3, 4; tes; Hradský \& Moucha 1967; Jelesova 1974a, 1975; Lehr 1988; Beschovski 2006a; Beron 2011.

Aneomochtherus flavicornis (Ruthe, 1831) [Neomochtherus, Mochtherus, Heligmoneura, Asilus] - DM, TL, BN; 0-250 m; 1; cse, ? e; Nedelkov 1910, 1912; Hradský \& Moucha 1964, 1967; Lehr 1988.

Aneomochtherus flavipes (Meigen, 1820) [Mochtherus, Heligmoneura, Asilus] - TL; 190-250 m; 1; wes, ? sess; Nedelkov 1910, 1912.

Aneomochtherus granitis (Tsacas, 1963) [Neomochtherus] - RE; 100-130 m; 1; Eb; Jelesova 1975; Lehr 1988; Beschovski 2004; Beron 2011.

? Premochtherus fuscifemoratus (Macquart, 1838) [? Premochtherus striatipes (Loew, 1849); Asilus, Mochtherus, Neomochtherus, Heligmoneura] - DM, V1, BN, BS; 0-600 m; 1; se; Nedelkov 1909, 1910, 1912.

Premochtherus striatipes (Loew, 1849) [Neomochtherus] - P1; 200-230 m; 1; se; Jelesova 1971; Lehr 1988.

Neomochtherus geniculatus (Meigen, 1820) [Asilus, Itamus, Neoitamus, Paritamus] - SB, V1, V4, R1; 550-1300 m; 1, 2, 3; e; Nedelkov 1910, 1912; Hradský \& Moucha 1967; Lehr 1988.

Neomochtherus pallipes (Meigen, 1820) [Asilus, Mochtherus] - B3, V4, TL, RW; 200-1900 m; 1, 2, 3, 4; h; Nedelkov 1909; Hradský \& Moucha 1964, 1967; Jelesova 1974a, 1975; Lehr 1988; Beschovski 2006a; Beron 2011.

Neomochtherus schineri (Egger, 1855) [Heligmoneura] - DM, S2; 30-900 m; 1, 2; cse; Nedelkov 1912.

Philonicus albiceps (Meigen, 1820) [Asilus] - E2, P1, P2, B1, V1, V3, S21, S211, TL, O62, R2, RW, RE, BN, BS; 0-1100 m; 1, 2, 3; tp; Meunier 1897; Nedelkov 1909, 1910, 1912; Drensky 1942, 1955; Beschovski 1964a, 1965, 2004; Hradský \& Moucha 1964, 1967; Jelesova 1971, 1974a, 1975; Beschovski 2006a; Beron 2011.

Stilpnogaster aemula (Meigen, 1820) - R1, RW; 1300-1900 m; 3, 4; e; Nedelkov 1912; Jelesova 1975; Beschovski 2006a; Beron 2011.

Tolmerus atricapillus (Fallén, 1814) - B1, V1, R1, RW; 550-1450 m; 2, 3; hoes; Meunier 1897; Nedelkov 1912; Hradský \& Moucha 1967; Jelesova 1971; Lehr 1988; Beschovski 2006 a.

Tolmerus atripes Loew, 1854 - B1, B2, B3, V1, RE; 250-1300 m; 1, 2, 3; ean; Nedelkov 1912; Jelesova 1971, 1974a, 1975; Lehr 1988; Beschovski 2004.

Tolmerus bolgaricus Lehr, 1981 - R1; 990-1000 m; 2; Ebg; Lehr 1981, 1988.

Tolmerus poecilogaster (Loew, 1849) [Asilus] - B3, S1, S2, TL, BN; 0-1000 m; 1, 2; e; Nedelkov 1909; Hradský \& Moucha 1967; Lehr 1988.

Tolmerus pyragra (Zeller, 1840) - BN; 0-20 m; 1; e; Hradský \& Moucha 1964, 1967; Lehr 1988.

Tolmerus strymonicus (Tsacas, 1960) - R3, RW, RE; 140-1550 m; 1, 2, 3, 4; Eb; Hradský \& Moucha 1964, 1967; Jelesova 1975; Lehr 1988; Beschovski 2004, 2006a; Beron 2011.

\section{Empididae}

Hilara aeronetha Mik, 1892 - RW; 400-1420 m; 1, 2, 3; e; Beschovski \& Dzhambazov 1998; Dzhambazov 2000.

Hilara albitarsis von Roser, 1840 - RW; 1590-1600 m; 3, 4; e; Kanavalova et al. 2018.

Hilara albiventris von Roser, 1840 - S1; 480 m; 1; e; Kanavalova et al. 2018.

Hilara anglodanica Lundbeck, 1913 - B2, S1; 400-720 m; 1, 2; e; Kanavalova et al. 2018.

Hilara angustifrons Strobl, 1892 - RW; 1380-1412 m; 3; e; Dzhambazov 2000; Dzhambazov \& Beschovski 2000.

Hilara beckeri Strobl, 1892 - RW; 1320-1825 m; 3, 4; e; Dzhambazov 2000; Dzhambazov \& Beschovski 2000.

Hilara biseta Collin, 1927 - R3, RW; 770-1400 m; 2, 3; e; Beschovski \& Dzhambazov 1998; Dzhambazov 2000; Dzhambazov \& Teneva 2000.

Hilara brevipilosa Collin, 1966 [H. psammophytophilia Beschovski, 1973] - BN, BS; 0-10 m; 1; se; Beschovski, 1973b, 1976a; Chvála 2013.

Hilara brevistyla Collin, 1927 - E2, RW; 90-1050 m; 1, 2; e; Beschovski \& Dzhambazov 1998; Dzhambazov 2000.

Hilara brevivittata Macquart, 1827 - P2; 600 m; 1; e; Beschovski \& Dzhambazov 1998.

Hilara bulgarica Barták, 2018 - R2, RW; 1170-2000 m; 3, 4, 5; Ebg; Kanavalova et al. 2018.

Hilara canescens Zetterstedt, 1849 - RW; 1593-1712 m; 3, 4; e; Dzhambazov 2000.

Hilara chorica (Fallén, 1816) - B2, V4, RW; 800-1400 m; 2, 3; e; Nedelkov 1912; Drensky 1934c; Dzhambazov 1999, 2000; Dzhambazov \& Teneva 2000.

Hilara cilipes Meigen, 1822 - RW; 1200-1400 m; 3; e; Dzhambazov 2000; Dzhambazov \& Beschovski 2000. 
Hilara clypeata Meigen, 1822 - RW; 1000-1400 m; 3; e, ? ena; Beschovski \& Dzhambazov 1998; Dzhambazov 2000. Hilara coracina Oldenberg, 1916 - B2; 1160 m; 3; e; Kanavalova et al. 2018.

Hilara cornicula Loew, 1873 - RW; 1517 m; 3, 4; e; Dzhambazov 2000.

Hilara discalis Chvála, 1997 - B2, S1, R2, RW; 480-1600 m; 1, 2, 3; e; Dzhambazov 1999, 2000; Kanavalova et al. 2018.

Hilara discoidalis Lundbeck, 1910 - R2, RW; 400-2500 m; 1, 2, 3, 4, 5, 6; e; Beschovski \& Dzhambazov 1998; Dzhambazov 2000.

Hilara discolor Strobl, 1892 - RW; 1400-1517 m; 3, 4; e; Dzhambazov 1999, 2000.

Hilara diversipes Strobl, 1892 - RW, BS; 0-1600 m; 1, 2, 3, 4; e; Dzhambazov 1995a, 2000.

Hilara femorella Zetterstedt, 1842 - R2; 2000 m; 4; e; Kanavalova et al. 2018.

Hilara flavipes Meigen, 1822 [H. cingulata Dahlbon, 1850] - TL, RW; 150-1000 m; 1, 2; e; Dzhambazov 2000; Dzhambazov \& Beschovski 2000.

Hilara fulvibarba Strobl, 1899 - RW; 1320 m; 3; e; Dzhambazov 2000.

Hilara fuscipes (Fabricius, 1794) [H. quadrivittata Meigen, 1822] - B2, V4, RW; 720-1300 m; 2, 3; e; Nedelkov 1912; Drensky 1934c; Dzhambazov 2000; Kanavalova et al. 2018.

Hilara galactoptera Strobl, 1910 - RW; 1100-1590 m; 3, 4; e; Dzhambazov 1999, 2000; Kanavalova et al. 2018. Hilara gallica (Meigen, 1804) - RW; 580 m; 1; e; Dzhambazov 1999, 2000.

Hilara hirta Strobl, 1892 - RW; 1400-1720 m; 3, 4; e; Dzhambazov 2000; Dzhambazov \& Beschovski 2000.

Hilara intermedia (Fallén, 1816) - RW; 400 m; 1; e; Dzhambazov 2000.

Hilara interstincta (Fallén, 1816) - K2, RW; 700-1600 m; 2, 3, 4; h; Beschovski \& Dzhambazov 1998; Dzhambazov 2000.

Hilara litorea (Fallén, 1816) - B2, V4, RW; 500-1620 m; 1, 2, 3, 4; e; Nedelkov 1912; Drensky 1934c; Beschovski et al. 1995; Dzhambazov 1999, 2000.

Hilara longivittata Zetterstedt, 1842 - B2; 1240-1350 m; 3; e, ? tes; Kanavalova et al. 2018.

Hilara lugubris (Zetterstedt, 1819) - TL, RW; 150-400 m; 1; e; Beschovski \& Dzhambazov 1998; Dzhambazov 2000.

Hilara lurida (Fallén, 1816) - B2, RW; 720-1620 m; 2, 3, 4; e; Nedelkov 1912; Drensky 1934c; Dzhambazov 2000; Kanavalova et al. 2018.

Hilara macquarti Straka, 1984 - RW; 360 m; 1; e; Dzhambazov 1999.

Hilara lasiopa Strobl, 1892 - R2; 1300-1600 m; 3, 4; e; Kanavalova et al. 2018.

Hilara maura (Fabricius, 1776) - P2; 600 m; 1, 2; e; Beschovski \& Dzhambazov 1998.

Hilara medeteriformis Collin, 1961 - RW, RE; 400-1000 m; 1, 2; e; Beschovski \& Dzhambazov 1998; Dzhambazov 2000; Beschovski 2004.

Hilara media Collin, 1927 [H. bechevi Dzhambazov, 1998] - RW; 400 m; 1; e; Dzhambazov 1998, 1999, 2000.

Hilara monedula Collin, 1927 - RW; 400-700 m; 1; e; Beschovski \& Dzhambazov 1998; Dzhambazov 2000.

Hilara nigrina (Fallén, 1816) - BN; 0-10 m; 1; e; Beschovski \& Dzhambazov 1998.

Hilara nigrita Chvála, 2005 - B3; 800 m; 2; csee; Chvála 2005.

Hilara nigrocincta de Meijere, 1935 - S1; 480 m; 1; e; Kanavalova et al. 2018.

Hilara nitidorella Chvála, 1997 - B2, R2; 1300-1600 m; 3, 4; e; Kanavalova et al. 2018.

Hilara platyura Loew, 1873 - BN; 0-20 m; 1; e; Beschovski 1971a; Chvála \& Wagner 1989.

Hilara pruinosa Wiedemann in Meigen, 1822 - B2, V4; 800-1300 m; 2, 3; e, ? cse; Nedelkov 1912; Drensky 1934c. Hilara quadriseta Collin, 1927 - R2, RW; 400-1600 m; 1, 2, 3, 4; e; Dzhambazov 2000; Kanavalova et al. 2018. Hilara regneali Parvu, 1991 - RW; 500-1100 m; 1, 2, 3; see; Dzhambazov 1999, 2000.

Hilara scrobiculata Loew, 1873 - RW; 400-1620 m; 1, 2, 3, 4; e; Beschovski \& Dzhambazov 1998; Dzhambazov 2000.

Hilara setipes Straka, 1976 - R2; 1200-1300 m; 3; Ebg; Straka 1976; Chvála \& Wagner 1989.

Hilara splendida Straka, 1976 - B2, RW; 720-1600 m; 2, 3, 4; e; Kanavalova et al. 2018.

Hilara sturmii Wiedemann in Meigen, 1822 - B2; 720 m; 2; e; Kanavalova et al. 2018.

Hilara subpollinosa Collin, 1927 - V4; 1000-1200 m; 2; e; Beschovski et al. 1995.

Hilara thoracica Macquart, 1827 - RW; 800-1200 m; 2, 3; e; Dzhambazov 2000.

Hilara triseta Chvála, 2005 - B3; 700 m; 2; csee; Chvála, 2005.

Empis (Euempis) calcarata Bezzi, 1899 - B2, RW; 770-1600 m; 2, 3, 4; nm; Kanavalova et al. 2018.

Empis (Euempis) ciliata Fabricius, 1787 - V1, TL, R1; 200-1400 m; 1, 2, 3; des; Joakimoff 1899; Nedelkov 1912. 
Empis (Euempis) sericans Brullé, 1832 [E. nepticula Loew, 1869] - RW, BS; 0-330 m; 1; e; Beschovski 1971a; Chvála \& Wagner 1989; Dzhambazov 2000.

Empis (Euempis) tessellata Fabricius, 1794 - B2, V4, O61, R1, RW; 400-2200 m; 1, 2, 3, 4, 5; tp, ? hop; Joakimoff 1899; Nedelkov 1912; Drensky 1934c; Dzhambazov 1999, 2000.

Empis (Pachymeria) femorata Fabricius, 1798 - V1, RW, BS; 0-1593 m; 1, 2, 3, 4; e; Meunier 1897; Beschovski \& Dzhambazov 1998; Dzhambazov 2000.

Empis (Pachymeria) vikhrevi Shamshev \& Barták, 2019 - R2; 1300-1600 m; 3, 4; ban; Shamshev \& Barták 2019. Empis (Polyblepharis) crassa Nowicki, 1868 - \$; cee; Chvála \& Wagner 1989.

Empis (Polyblepharis) dedecor Loew, 1869 - RW; 700-800 m; 2; nm, ? hom; Dzhambazov 2000.

Empis (Polyblepharis) fallax Egger, 1860 - B2, V1, V4; 800-1300 m; 2, 3; tes; Nedelkov 1912; Drensky 1934c.

Empis (Polyblepharis) gravipes Loew, 1856 - B2, RW; 700-1620 m; 2, 3, 4; ess; Nedelkov 1912; Drensky 1934c; Dzhambazov 2000.

Empis (Polyblepharis) haemi Loew, 1862 - BN; 0-20 m; 1; see; Löw 1862; Chvála \& Wagner 1989.

Empis (Polyblepharis) nigerrima Loew, 1862 - BN; 0-20 m; 1; Eb; Löw 1862; Chvála \& Wagner 1989.

Empis (Polyblepharis) opaca Meigen, 1804 - B2, R1; 1300 m; 3; e; Nedelkov 1912; Drensky 1934c.

Empis (Kritempis) livida Linnaeus, 1758 - V1, V4; 550-900 m; 2; e; Nedelkov 1912.

Empis (Planempis) frauscheri Strobl, 1901 - RW; 1170 m; 3; csee; Kanavalova et al. 2018.

Empis (Leptempis) adusta Loew, 1869 - ; se; Chvála \& Wagner 1989.

Empis (Leptempis) confusa Loew, 1865 [E. maculata Fabricius, 1781] - RW, BN; 0-1050 m; 1, 2, 3; cse; Löw 1862; Dzhambazov 2000.

Empis (Leptempis) discolor Loew, 1856 - B2, R1, R2, RW; 1200-1600 m; 3, 4; e; Nedelkov 1912; Drensky 1934c; Dzhambazov 2000; Kanavalova et al. 2018.

Empis (Leptempis) divisa Loew, 1869 - RW; 332 m; 1; see; Chvála \& Wagner 1989; Dzhambazov 2000.

Empis (Leptempis) grisea Fallén, 1816 - V4, RW, RE; 250-1400 m; 1, 2, 3; e; Beschovski et al. 1995; Dzhambazov 1995a, 2000; Dzhambazov \& Teneva 2000.

Empis (Leptempis) maculata Fabricius, 1781 - SB, V4, RW, BN; 0-1200 m; 1, 2, 3; cse; Löw 1862; Frey 1956; Beschovski et al. 1995; Dzhambazov 2000.

Empis (Leptempis) meridionalis Meigen, 1822 - B2, V1, RW; 600-130 m; 2, 3; see; Nedelkov 1912; Drensky 1934c. Empis (Leptempis) nigricans Meigen, 1804 - RW; 1420 m; 3; e; Dzhambazov 2000.

Empis (Leptempis) rava Loew, 1862 - BN; 0-20 m; 1; Ebg; Löw 1862; Chvála \& Wagner 1989.

Empis (Leptempis) rhodopensis Barták, 2018 - RW; 1600-1900 m; 4; Ebg; Kanavalova et al. 2018.

Empis (Leptempis) rustica Fallén, 1816 - V1, S21, TL, RW; 150-1420 m; 1, 2, 3; e; Nedelkov 1909, 1912; Chvála 1977; Dzhambazov 2000; Dzhambazov \& Teneva 2000.

Empis (Leptempis) spitzeri Chvála, 1977 - RW; 1000-1620 m; 3, 4; Ebg, ? Er; Chvála 1977; Chvála \& Wagner 1989; Dzhambazov 2000.

Empis (Leptempis) variegata Meigen, 1804 - RW, RE; 200-1700 m; 1, 2, 3, 4; e; Beschovski \& Dzhambazov 1998; Dzhambazov 2000; Beschovski 2004.

Empis (Anacrostichus) bistortae Meigen, 1822 - V4, TL; 200-1200 m; 1, 2, 3; cse, ? e; Nedelkov 1912.

Empis (Empis) aestiva Loew, 1867 - V4, RW; 400-1300 m; 1, 2, 3; e; Beschovski et al. 1995; Dzhambazov 1995a, 2000.

Empis (Empis) caudatula Loew, 1867 - V4, RW; 580-1720 m; 2, 3, 4; e; Beschovski et al. 1995; Dzhambazov 2000.

Empis (Empis) chioptera Meigen, 1804 - V4, RW; 1070-1335; 3; e; Beschovski et al. 1995; Dzhambazov 2000; Dzhambazov \& Teneva 2000.

Empis (Empis) decora Meigen, 1822 - V4, RW; 1380-2078 m; 3, 4; csena; Nedelkov 1912; Beschovski et al. 1995; Dzhambazov 1999, 2000.

Empis (Empis) decorella Chvala, 1981 - RW; 1320-1400 m; 3; se; Dzhambazov 2000; Dzhambazov \& Beschovski 2000.

Empis (Empis) florisomna Loew, 1856 - RW; 332 m; 1; e; Chvála \& Wagner 1989; Dzhambazov 2000.

Empis (Empis) gymnopoda Bezzi, 1908 - S1; 550-690 m; 1, 2; ? e; Bezzi 1908; Chvála \& Wagner 1989.

Empis (Empis) lyneborgi Chvala, 1981 - RW; 1000-1300 m; 3; se; Dzhambazov \& Beschovski 2000.

Empis (Empis) nigripes Fabricius, 1794 - V1, V4, RW; 580-1700 m; 1, 2, 3, 4; wes; Nedelkov 1912; Beschovski et al. 1995; Dzhambazov 1999, 2000; Dzhambazov \& Teneva 2000.

Empis (Empis) nitidiventris Loew, 1873 - RW; 1600 m; 4; e; Dzhambazov 2000. 
Empis (Empis) nuntia Meigen, 1838 - RW, BS; 0-1600 m; 1, 2, 3, 4; e; Beschovski \& Dzhambazov 1998; Dzhambazov 2000; Dzhambazov \& Teneva 2000.

Empis (Empis) pennipes Linnaeus, 1758 - V4, RW; 920-2078 m; 2, 3, 4; e; Beschovski et al. 1995; Dzhambazov 2000.

Empis (Empis) pilosa Loew, 1867 - V4, RW; 970-1100 m; 2, 3; e; Dzhambazov 1995; Dzhambazov \& Teneva 2000. Empis (Empis) planetica Collin, 1927 - RW; 1600 m; 4; e; Dzhambazov 2000; Dzhambazov \& Beschovski 2000. Empis (Empis) praecox Loew, 1867 [Hilara] - B; 0-5 m; 1; Eb; Beschovski 1973c.

Empis (Empis) praevia Collin, 1927 - V5; RW; 580-1400 m; 1, 2, 3; ? e; Beschovski \& Dzhambazov 1998; Dzhambazov 2000.

Empis (Empis) procera Loew, 1873 - RW; 1320 m; 3; cse; Dzhambazov 2000.

Empis (Empis) prodromus Loew, 1867 - R2, RW; 1100-1720 m; 3, 4; e; Beschovski \& Dzhambazov 1998; Dzhambazov 2000.

Empis (Empis) rufiventris Meigen, 1838 - V4, RW; 900-2078 m; 2, 3, 4; e; Nedelkov 1912; Beschovski et al. 1995; Dzhambazov 1999, 2000; Dzhambazov \& Teneva 2000.

Empis (Empis) simulium (Nowicki, 1868) - RW; 1200 m, 3; e, m; Dzhambazov 2000.

Empis (Empis) tristis Loew, 1867 - csee; Chvála \& Wagner 1989.

Empis (Empis) woodi Collin, 1927 - V4, RW; 850-1420 m; 2, 3; cse, ? e; Beschovski et al. 1995; Dzhambazov 2000; Dzhambazov \& Teneva 2000.

Empis (Coptophlebia) albinervis Meigen, 1822 - RW; 1300-1400 m; 3; e; Dzhambazov 1995a, 2000.

Empis (Coptophlebia) hyalipennis Fallen, 1816 - RW; 400-1420 m; 1, 2, 3; e; Dzhambazov 1995a, 2000.

Empis (Coptophlebia) impennis Strobl, 1902 - V4, RW; 580-1825 m; 1, 2, 3, 4; e; Beschovski et al. 1995; Beschovski \& Dzhambazov 1998; Dzhambazov 2000.

Empis (Coptophlebia) vitripennis Meigen, 1822 - B2; 650 m; 1, 2; e; Dzhambazov \& Beschovski 2000.

Empis (Coptophlebia) volucris Wiedemann in Meigen, 1822 - V4, RW; 850-1200 m; 2, 3; e; Beschovski et al. 1995; Beschovski \& Dzhambazov 1998; Dzhambazov 2000.

Empis (Rhadinempis) bazini Collin, 1926 - RW; 580-1000 m; 2; csee; Dzhambazov 1999, 2000.

Empis (Xanthempis) digramma Meigen in Gistl, 1835 - V1, V4, RW; 550-1000 m; 1, 2; e; Nedelkov 1912; Dzhambazov 2000.

Empis (Xanthempis) laetabilis Collin, 1926 - BS; 0-15 m; 1; e; Dzhambazov \& Beschovski 2000.

Empis (Xanthempis) lutea Meigen, 1804 - B2; RW; 600-1600 m; 2, 3, 4; e; Dzhambazov 2000; Dzhambazov \& Beschovski 2000.

Empis (Xanthempis) punctata Meigen, 1804 - B2, V1, S211; 550-1300 m; 2, 3; e; Nedelkov 1912; Drensky 1934c.

Empis (Xanthempis) scutellata Curtis, 1835 - RW, BS; 0-1000 m; 1, 2; e; Dzhambazov 1995a, 2000.

Empis (Xanthempis) semicinerea Loew, 1867 - B2, R1, 1240-2220 m; 3, 4, 5; cse; Kanavalova et al. 2018.

Empis (Xanthempis) stercorea Linnaeus, 1761 - B2, V1, S211, RW; 550-1600 m; 2, 3, 4; tes; Nedelkov 1912; Drensky 1934c; Kanavalova et al. 2018.

Empis (Xanthempis) trigramma Wiedemann in Meigen, 1822 - V1, V4; 650-800 m; 2; e; Nedelkov 1912.

Empis (Lissempis) nigritarsis Meigen, 1804 - V4, RW; 700-1870 m; 2, 3, 4; e; Dzhambazov 1995a, 1999, 2000; Dzhambazov \& Teneva 2000.

Rhamphomyia (Aclonempis) longipes (Meigen, 1804) - RW; 1320 m; 3; e; Dzhambazov 2000; Dzhambazov \& Beschovski 2000.

Rhamphomyia (Aclonempis) umbripes Becker, 1887 - B2; 1350 m; 3; cse; Kanavalova et al. 2018.

Rhamphomyia (Rhamphomyia) argentata von Roder, 1887 - RW; 130-1600 m; 3, 4; csee; Dzhambazov 1995a, 2000.

Rhamphomyia (Rhamphomyia) cinerascens (Meigen, 1804) - V1; 550-600 m; 1, 2; wes; Nedelkov 1912.

Rhamphomyia (Rhamphomyia) crinita Becker, 1887 - R2; 2000 m; 4, 5; cse, m; Kanavalova et al. 2018.

Rhamphomyia (Rhamphomyia) dorsata Becker, 1915 - RW; 400 m; 1; des; Dzhambazov 2000; Dzhambazov \& Beschovski 2000 .

Rhamphomyia (Rhamphomyia) laevipes (Fallén, 1816) - RW, RE; 350-1380 m; 1, 2, 3; e; Dzhambazov 2000; Dzhambazov \& Beschovski 2000; Beschovski 2004.

Rhamphomyia (Rhamphomyia) lautereri Bartak, 1981 - ; csee; Barták 1981; Chvála \& Wagner 1989.

Rhamphomyia (Rhamphomyia) luridipennis Nowicki, 1868 - \$; e; Barták 1981; Chvála \& Wagner 1989.

Rhamphomyia (Rhamphomyia) montana Oldenberg, 1915 - \$; e; Barták 1981; Chvála \& Wagner 1989. 
Rhamphomyia (Rhamphomyia) morio Zetterstedt, 1838 - R2; 2000-2230 m; 4, 5; e; Dzhambazov \& Beschovski 2000.

Rhamphomyia (Rhamphomyia) paraleucoptera Frey, 1950 - RW; 1400-1450 m; 3; ees, ? wes; Dzhambazov 1995a, 2000.

Rhamphomyia (Rhamphomyia) siebecki Strobl, 1898 - V4, RW; 1100-1870 m; 3, 4; e; Dzhambazov 1995a, 2000; Dzhambazov \& Teneva 2000.

Rhamphomyia (Rhamphomyia) spinipes (Fallen, 1816) - \$; e; Barták 1981; Chvála \& Wagner 1989.

Rhamphomyia (Rhamphomyia) stigmosa Macquart, 1827 - B2, RW; 1320-1825 m; 3, 4; e; Barták 1981; Chvála \& Wagner 1989; Dzhambazov 2000.

Rhamphomyia (Rhamphomyia) sulcata (Meigen, 1804) - B2, V1, R1, RW; 515-1800 m; 1, 2, 3, 4; tes; Nedelkov 1912; Drensky 1934c; Beschovski et al. 1995; Dzhambazov 2000.

Rhamphomyia (Rhamphomyia) tibialis Meigen, 1822 - R1; 1200-1300 m; 3; des, ? tes; Joakimoff 899.

Rhamphomyia (Megacyttarus) maculipennis Zetterstedt, 1842 - RW; 400 m; 1; e; Dzhambazov 2000; Dzhambazov \& Beschovski 2000.

Rhamphomyia (Pararhamphomyia) aethiops Zetterstedt, 1838 - RW; 1050 m; 3; e; Dzhambazov 2000.

Rhamphomyia (Pararhamphomyia) atra Meigen, 1822 - V1, RW; 550-1412 m; 1, 2, 3; e; Nedelkov 1912; Dzhambazov 2000.

Rhamphomyia (Pararhamphomyia) curvula Frey, 1913 - RW; 580 m; 1; e; Dzhambazov 1999, 2000.

Rhamphomyia (Pararhamphomyia) lamelliseta Ringdahl, 1928 - RW; 1380-1825 m; 3, 4; ? e; Dzhambazov 2000; Dzhambazov \& Beschovski 2000.

Rhamphomyia (Pararhamphomyia) nudipes Oldenberg, 1927 - B2, RW; 1240-1590 m; 3, 4; cse, ? se; Kanavalova et al. 2018.

Rhamphomyia (Pararhamphomyia) simplex Zetterstedt, 1849 - V4, R2; 1800-1820 m; 4; e; Beschovski et al. 1995; Dzhambazov \& Beschovski 2000.

Rhamphomyia (Holoclera) caliginosa Collin, 1926 - RW; 1517 m; 4; e; Dzhambazov 1999, 2000.

Rhamphomyia (Holoclera) culicina (Fallén, 1816) - R1, RW; 1420 m; 3, 4; e; Barták 1981; Chvála \& Wagner 1989; Dzhambazov 1999, 2000.

Rhamphomyia (Holoclera) flava (Fallén, 1816) - e; Barták 1981; Chvála \& Wagner 1989.

Rhamphomyia (Holoclera) heterochroma Bezzi, 1898 - @; e; Barták 1981; Chvála \& Wagner 1989.

Rhamphomyia (Holoclera) lamellata Collin, 1926 - RW; 322 m; 1; e; Dzhambazov 2000.

Rhamphomyia (Holoclera) nigripennis (Fabricius, 1794) - RW; 700-1620 m; 2, 3, 4; wes; Dzhambazov 2000; Dzhambazov \& Beschovski 2000.

Rhamphomyia (Holoclera) trigemina Oldenberg, 1927 - R2, RW; 1100-1712 m; 3, 4; e; Dzhambazov 2000; Dzhambazov \& Beschovski 2000; Dzhambazov \& Teneva 2000.

Rhamphomyia (Holoclera) umbripennis Meigen, 1822 - R2, RW; 1420-2200 m; 3, 4, 5; e; Dzhambazov 1999, 2000; Kanavalova et al. 2018.

Rhamphomyia (Amydroneura) claripennis Oldenberg, 1922 - RW; 770-1590 m; 2, 3, 4; cse; Kanavalova et al. 2018.

Rhamphomyia (Amydroneura) stojanovae Barták, 2018 - RW; 1260 m; 3; Ebg, ? Er; Kanavalova et al. 2018.

Rhamphomyia (Lundstroemiella) dudai Oldenberg, 1927 - RW; 1260 m; 3; e; Kanavalova et al. 2018.

Rhamphomyia (Lundstroemiella) hybotina Zetterstedt, 1838 - RW; 700-1100 m; 2, 3; e; Dzhambazov 2000; Dzhambazov \& Beschovski 2000.

Rhamphomyia (Lundstroemiella) kerteszi Oldenberg, 1927 - s; csee; Barták 1985; Chvála \& Wagner 1989.

Rhamphomyia (Lundstroemiella) magellensis Frey, 1922 - R2; 1300-2600 m; 3, 4; cse, m; Kanavalova et al. 2018.

Rhamphomyia (Lundstroemiella) sphenoptera Loew, 1873 - R2; 2000 m; 4, 5; ? cse; Kanavalova et al. 2018.

Rhamphomyia (Lundstroemiella) strobli Barták, 1985 - ; csee; Chvála \& Wagner 1989.

Heleodromia (Heleodromia) immaculata Haliday, 1833 - RW; 1400-1760 m; 3, 4; e; Dzhambazov 2000; Dzhambazov \& Beschovski 2000.

Heleodromia (Heleodromia) schachti Wagner, 1985 - RW; 1420 m; 3; se; Dzhambazov 1999, 2000.

Chelifera precabunda Collin, 1961 - R1, RW; 1000 m; 2, 3; e; Joost 1982; Chvála \& Wagner 1989; Dzhambazov 2000.

Chelifera precatoria (Fallen, 1816) - RW; 1420 m; 3; h; Dzhambazov 2000.

Chelifera stigmatica (Schiner, 1862) - \$; e; Joost 1982; Chvála \& Wagner 1989. 
Chelifera trapezina (Zetterstedt, 1838) - RW; 1100 m; 3; e; Dzhambazov 2000.

Dolichocephala guttata (Haliday, 1833) - e; Joost 1982; Chvála \& Wagner 1989.

Wiedemannia (Wiedemannia) andreevi Joost, 1982 - B2; 1200 m; 3; Ebg; Joost 1982; Chvála \& Wagner 1989.

Wiedemannia (Chamaedipsia) lota Walker, 1851 - R1, RW; 1100 m; 3; eswa, ? ean; Joost 1982; Chvála \& Wagner 1989; Dzhambazov 2000.

Wiedemannia (Philolutra) fallaciosa (Loew, 1873) - \$; ena; Joost 1982; Chvála \& Wagner 1989.

Wiedemannia (Philolutra) hygrobia (Loew, 1858) - \&; Joost 1982; Chvála \& Wagner 1989.

Wiedemannia (Chamaedipsia) wachtli (Mik, 1880) - \$; cse; Joost 1982; Chvála \& Wagner 1989.

Wiedemannia (Eucelidia) zetterstedti (Fallén, 1826) [W. escheri (Zetterstedt, 1838)] - ^; e; Joost 1982; Chvála \& Wagner 1989.

Wiedemannia (Roederella) czernyi (Bezzi, 1905) - RW; 400 m; 1; se; Dzhambazov 2000.

Clinocera appendiculata (Zetterstedt, 1838) - e; Joost 1982; Chvála \& Wagner 1989.

Clinocera nigra Meigen, 1804 - ena; Joost 1982; Chvála \& Wagner 1989.

Clinocera stagnalis (Haliday, 1833) - \$; Chvála \& Wagner 1989.

Phaeobalia dimidiata (Loew, 1869) [Clinocera] - SB, R2, RW; 1000-1400 m; 3; e; Joost 1982; Chvála \& Wagner 1989; Dzhambazov 2000.

Kowarzia barbatula (Mik, 1880) [Clinocera] - cse; Joost 1982; Chvála \& Wagner 1989.

Kowarzia bipunctata (Haliday, 1833) [Clinocera] - P1; 150-190 m; 1; ena; Dzhambazov 2000.

Kowarzia plectrum (Mik, 1880) [Clinocera] - e; Joost 1982; Chvála \& Wagner 1989.

Kowarzia tenella (Wahlberg, 1844) [Clinocera] - ^; e; Joost 1982; Chvála \& Wagner 1989.

\section{Hybotidae}

Trichina bilobata Collin, 1926 - RW; 720-1160 m; 2, 3, 4; e; Dzhambazov 2000.

Trichina clavipes Meigen, 1830 - RW; 300-770 m; 1, 2; ? h; Dzhambazov 2000.

Trichina elongata Haliday, 1833 - RW; 1100-1450 m; 3; e; Dzhambazov 2000.

Bicellaria austriaca Tuomikoski, 1955 - R2, RW; 1400-1800 m; 3, 4; e; Chvála 1983; Chvála \& Kovalev 1989; Dzhambazov 1995b, 1999, 2000.

Bicellaria intermedia Lundbeck, 1910 - R2, RW; 770-1500 m; 2, 3, 4; e; Chvála 1983; Chvála \& Kovalev 1989; Dzhambazov 1995b, 2000.

Bicellaria longisetosa Chvala, 1991 - R1; 2000-2380 m; 4, 5; des, m; Chvála 1991; Dzhambazov 1995b; Barták \& Kubík 2013.

Bicellaria nigra (Meigen, 1824) - R1, RW; 900-1500 m; 2, 3, 4; e; Dzhambazov 1995b, 2000.

Bicellaria nigrita Collin, 1926 - \&; e; Chvála 1983; Chvála \& Kovalev 1989; Dzhambazov 1995b; Barták \& Kubík 2013.

Bicellaria pilosa Lundbeck, 1910 - RW; 520-1550 m; 1, 2, 3, 4; e; Dzhambazov 1995a, 2000.

Bicellaria spuria (Fallén, 1816) - V4, T31, RW; 200-1450 m; 1, 2, 3, ? 4; ? h; Chvála 1983; Chvála \& Kovalev 1989; Beschovski et al. 1995; Dzhambazov 1995b, 1999, 2000.

Bicellaria subpilosa Collin, 1926 - RW; 750-1420 m; 2, 4; e; Chvála \& Kovalev 1989; Beschovski et al. 1995; Dzhambazov 1995a, 1999, 2000; Barták \& Kubík 2013.

Bicellaria sulcata (Zetterstedt, 1842) - V4, R2, RW; 1100-1820 m; 3, 4; des; Chvála 1983; Chvála \& Kovalev 1989; Beschovski et al. 1995; Dzhambazov 1995b, 1999, 2000.

Bicellaria vana Collin, 1926 - RW; 1100-1820 m; 3, 4; e; Dzhambazov 2000.

Oedalea holmgreni Zetterstedt, 1852 - RW; 1100-1250 m; 3; e; Dzhambazov 2000.

Euthyneura gyllenhali (Zetterstedt, 1838) - e; Chvála 1983; Chvála \& Kovalev 1989; Dzhambazov 1995 b.

Leptopeza flavipes (Meigen, 1820) - RW; 300-500 m; 1; h; Dzhambazov 2000.

Ocydromia glabricula (Fallén, 1816) - V1, RW; 1000-1400 m; 2, 3; h; Nedelkov 1912; Dzhambazov 1995b, 1999, 2000.

Ocydromia melanopleura Loew, 1840 - RW; 1000-1100 m; 3; e; Dzhambazov 1999, 2000.

Oropezella sphenoptera (Loew, 1873) - RW, BS; 0-400 m; 1; ena; Dzhambazov 1995a, 1999, 2000.

Hybos culiciformis (Fabricius, 1775) - RW, BS; 0-1450 m; 1, 2, 3; ean; Dzhambazov 1995a, 2000.

Hybos femoratus (Muller, 1776) - RW; 1000-1450 m; 2, 3; ena; Dzhambazov 1995a, 2000.

Platypalpus albocapillatus (Fallen, 1815) - V5; 1000-1100 m; 3; e; Beschovski \& Dzhambazov 1997. 
Platypalpus alpinus Chvala, 1971 - T31; 300 m; 1; e; Beschovski \& Dzhambazov 1997.

Platypalpus analis (Meigen, 1830) - V1; 520-530 m; 1; e; Nedelkov 1912.

Platypalpus annulatus (Fallén, 1815) - BN; 0-5 m; 1; h; Beschovski 1973; Chvála \& Kovalev 1989; Dzhambazov 1995b.

Platypalpus annulipes (Meigen, 1822) - RW; 1250 m; 3; e; Dzhambazov 2000.

Platypalpus articulatoides (Frey, 1918) - RW; 300-1320 m; 1, 2, 3; e; Dzhambazov 2000.

Platypalpus brachystylus (Bezzi, 1892) - \$; e; Chvála 1989; Chvála \& Kovalev 1989; Dzhambazov 1995b.

Platypalpus calceatus (Meigen, 1822) - e; Chvála 1989; Chvála \& Kovalev 1989; Dzhambazov 1995b.

Platypalpus ciliaris (Fallén, 1816) - RW; 580-1200 m; 1, 2, 3; e; Dzhambazov 2000.

Platypalpus collini (Chvala, 1966) - RW; 1120-1490 m; 3, 4; e; Dzhambazov 2000.

Platypalpus cothurnatus Macquart, 1827 - S1, BN; 0-700 m; 1; e; Beschovski \& Dzhambazov 1997.

Platypalpus cryptospina (Frey, 1909) - BS; 0-20 m; 1; e; Beschovski \& Dzhambazov 1997.

Platypalpus cursitans (Fabricius, 1775) [P. bicolor (Meigen, 1804)] - V1; 550-600 m; 1, 2; e; Nedelkov 1912;

Dzhambazov 1995b.

Platypalpus ecalceatus (Zetterstedt, 1838) - BS; 0-5 m; 1; e; Beschovski \& Dzhambazov 1997.

Platypalpus excisus (Becker, 1907) - V4, RW; 580-1300 m; 1, 2, 3; e; Chvála 1983, 1989; Chvála \& Kovalev 1989; Dzhambazov 1995b, 2000.

Platypalpus exilis (Meigen, 1822) - RW; 780-1420 m; 2, 3; e; Dzhambazov 2000.

Platypalpus flavicornis (Meigen, 1822) - RW; 300-1400 m; 1, 2, 3; ? ena; Dzhambazov 1999, 2000.

Platypalpus infectus (Collin, 1926) - BS; 0-10 m; 1; e; Beschovski \& Dzhambazov 1997.

Platypalpus interstinctus (Collin, 1926) - RW; 720-1500 m; 2, 3, 4; wes; Beschovski \& Dzhambazov 1997;

Dzhambazov 2000.

Platypalpus laticinctus Walker, 1851 - E2, DM, BS; 150-200 m; 1; e; Beschovski \& Dzhambazov 1997.

Platypalpus longicornis (Meigen, 1822) - S211, R2; 1170-1430 m; 3, 4; e; Beschovski \& Dzhambazov 1997.

Platypalpus longiseta (Zetterstedt, 1842) - DW, DM, TL, RW; 120-600 m; 1; wp; Beschovski \& Dzhambazov

1997; Dzhambazov 2000.

Platypalpus luteicornis (Meigen, 1838) - B2, B3; 530-1400 m; 1, 2, 3; e; Beschovski \& Dzhambazov 1997.

Platypalpus luteus (Meigen, 1804) - e; Chvála 1989; Chvála \& Kovalev 1989; Dzhambazov 1995b.

Platypalpus maculipes (Meigen, 1822) - R2, RW; 450-1000 m; 1, 2; e; Chvála \& Kovalev 1989; Dzhambazov 1995b, 1999, 2000.

Platypalpus major (Zetterstedt, 1842) - V1, V4, RW; 550-1000 m; 1, 2; e; Nedelkov 1912; Beschovski et al. 1995; Dzhambazov 1995b, 2000.

Platypalpus melancholicus (Collin, 1961) - DW; 55-70 m; 1; e; Beschovski \& Dzhambazov 1997.

Platypalpus mikii (Becker, 1890) - \$; e; Chvála 1975; Chvála \& Kovalev 1989; Dzhambazov 1995b.

Platypalpus minutus (Meigen, 1804) - V1; 550-600 m; 1, 2; e; Nedelkov 1912; Chvála \& Kovalev 1989; Dzhambazov 1995b.

Platypalpus niger (Meigen, 1804) - RW; 890-1420 m; 2, 3; e; Dzhambazov 1995a, 2000.

Platypalpus nigritarsis (Fallén, 1816) - B3, R2, RW; 1000-1900 m; 3, 4; e; Beschovski \& Dzhambazov 1997; Dzhambazov 2000.

Platypalpus nigrosetosus (Strobl, 1893) - B2, S1; 700-1400 m; 2, 3; e; Beschovski \& Dzhambazov 1997.

Platypalpus niveiseta (Zetterstedt, 1842) - E2, BN; 90-180 m; 1; e; Beschovski \& Dzhambazov 1997.

Platypalpus pallidicornis (Collin, 1926) - V4, RW; 450-900 m; 1, 2; e; Beschovski et al. 1995; Dzhambazov 1999, 2000.

Platypalpus pallidiventris (Meigen, 1822) [Coryneta, Tachydromia] - V1, BN; 0-550 m; 1; ena; Nedelkov 1912; Beschovski 1971a; Dzhambazov 1995b.

Platypalpus pectoralis (Fallén, 1815) - e; Chvála 1989; Chvála \& Kovalev 1989; Dzhambazov 1995b.

Platypalpus pictitarsis (Becker, 1902) - BN; 0-10 m; 1; ena; Beschovski 1973a; Chvála \& Kovalev 1989; Dzhambazov 1995b.

Platypalpus pseudociliaris (Strobl, 1910) - RW; 390-1610 m; 1, 2, 3, 4; e; Dzhambazov 1999, 2000.

? Platypalpus pygialis Chvala, 1973 - \&; e; Chvála 1989 ?; Chvála \& Kovalev 1989 ?; Dzhambazov 1995b.

Platypalpus soosi Chvala, 1989 - \$; csee; Chvála 1989; Dzhambazov $1995 \mathrm{~b}$.

Platypalpus stabilis (Collin, 1961) - E2, RW; 90-1190 m; 1, 2, 3; e; Beschovski \& Dzhambazov 1997; Dzhambazov 1999, 2000. 
Platypalpus stigmatellus (Zetterstedt, 1842) - @ ; des; Chvála 1989; Chvála \& Kovalev 1989; Dzhambazov 1995b. Platypalpus tergestinus Egger, 1860 - RW; 1400-1500 m; 3, 4; sena; Dzhambazov 2000.

Tachypeza nubila (Meigen, 1804) - V1; 550-600 m; 1, 2; e; Nedelkov 1912; Dzhambazov 1995b.

Tachydromia annulimana Meigen, 1822 - RW; 580-1300 m; 2, 3; e; Dzhambazov 2000.

Tachydromia carpathica Chvala, 1966 - RW; 580 m; 1; csee; Dzhambazov 2000.

Tachydromia interrupta (Loew, 1864) - RW; 1100 m; 3; cse, ? m; Dzhambazov 2000.

Tachydromia productipes (Strobl, 1910) - cee; Chvála 1989; Chvála \& Kovalev 1989; Dzhambazov 1995b.

Tachydromia sabulosa Meigen, 1830 - V1; 550-600 m; 1, 2; e; Nedelkov 1912; ? Chvála \& Kovalev 1989; Dzhambazov 1995b.

Tachydromia woodi (Collin, 1926) - RW; 580 m; 1; e; Dzhambazov 2000.

Drapetis (Drapetis) assimilis (Fallén, 1815) - RW; 300-890 m; 1, 2; e, ? h; Dzhambazov 1999, 2000.

Drapetis (Drapetis) exilis Meigen, 1822 - RW, BN; 0-520 m; 1; e; Beschovski 1973; Dzhambazov 1995b, 2000.

Drapetis (Drapetis) flavipes Macquart, 1834 - P1; 180-230 m; 1; ena; Czerný 1930; Guéorguiev \& Beron 1962;

Beschovski 1972c; Beron 1994, 2015.

Crossopalpus aeneus (Walker, 1871) - RW, BN; 0-580 m; 1; ena; Beschovski 1973a; Dzhambazov 1995b, 2000.

Crossopalpus flexuosus (Loew, 1840) - BN; 0-5 m; 1; ? e, ? ena; Beschovski 1971a; Dzhambazov 1995b

Crossopalpus humilis (Frey, 1913) - R2, RW; 1200-1500 m; 3, 4; wces; Chvála 1975, 1983; Chvála \& Kovalev 1989; Dzhambazov 1995b, 2000.

Crossopalpus nigritellus (Zetterstedt, 1842) - P1, RW; 300-400 m; 1; eswa; Dzhambazov 1995a, 2000.

Crossopalpus pilipes (Loew, 1859) [Drapetis] - RW, BN; 0-400 m; 1; sena; Beschovski 1971; Chvála \& Kovalev 1989; Dzhambazov 1995b, 2000.

Crossopalpus setiger (Loew, 1859) - RW; 720-1450 m; 2, 3, 4; des, ? e; Chvála \& Kovalev 1989; Dzhambazov 1995b, 2000.

Chersodromia bureschi Beschovski, 1973 - BN; 0-10 m; 1; Ebg; Beschovski 1973d; Chvála \& Kovalev 1989; Dzhambazov 1995b.

? Chersodromia colliniana Frey, 1936 [? Ch. pontica Chvála 1970] - BS; 0-5 m; 1; ? se; Caspers 1951a, 1951b; Beschovski 1964b, 1972b, ? 1973; Dzhambazov 1995b.

Chersodromia cursitans (Zetterstedt, 1819) [Tachydromia] - V1; 550-600 m; 1, 2; e; Nedelkov 1912; Chvála \& Kovalev 1989; Dzhambazov 1995b.

Chersodromia curtipennis Collin, 1950 - BS; 0-5 m; 1; see; Collin 1950; Caspers 1951a, 1951b; Beschovski 1964b; Chvála \& Kovalev 1989; Dzhambazov 1995b.

Chersodromia milanchvalai Beschovski, 1973 - BN; 0-5 m; 1; Ebg; Beschovski 1973d; Chvála \& Kovalev 1989; Dzhambazov 1995b.

Chersodromia nigrosetosa Chvala, 1970 - \$ ? hom; Chvála 1970; Chvála \& Kovalev 1989; Dzhambazov 1995 b.

Chersodromia pontica Chvala, 1970 [Ch. colliniana Frey, 1936] - BN, BS; 0-50 m; 1; ? se; Chvála 1970; Beschovski 1973a; Chvála \& Kovalev 1989; Dzhambazov 1995b.

\section{Atelestidae}

Atelestus pulicarius (Fallén, 1816) - RW; 1100 m; 3; e; Dzhambazov 2000.

Nemedina alamirabilis Chandler, 1981 - B2; 1160 m; 3; see; Kanavalová et al. 2020.

\section{Microphoridae}

Microphor albopilosus (Becker, 1910) [Microphorus, Micromorphus] - BS; 0-5 m; 1; se; Beschovski 1971a; Chvála 1989; Chvála \& Kovalev 1989; Dzhambazov 1995b.

Microphor anomalus (Meigen, 1824) - V4, RW; 700-1300 m; 2, 3; des; Chvála 1983, 1989; Chvála \& Kovalev 1989; Dzhambazov 1995b, 2000.

Microphor crassipes (Macquart, 1827) - RW; 400-1400 m; 1, 2, 3; e; Dzhambazov 2000.

Microphor holosericeus (Meigen, 1804) - V4, RW; 700-1000 m; 2, 3; tes, ? des; Chvála 1983, 1989; Chvála \& Kovalev 1989; Dzhambazov 1995b, 2000.

Microphorella praecox (Loew, 1864) - RW; 750 m; 2; e; Dzhambazov 1995b, 2000. 


\section{Dolichopodidae}

Sciapus bellus (Loew, 1873) - RE; 354-473 m; 1; e; Kechev 2021b.

Sciapus contristans (Wiedemann, 1817) [S. vialis (Raddatz, 1873)] - E1, BN; 30 m; 1; ena; Beschovski 1967a; Negrobov 1991; Beschovski \& Dzhambazov 2002; Kechev 2005, 2015; Grichanov \& Negrobov 2014; Kechev et al. 2020.

Sciapus euchromus (Loew, 1857) - BN; 0-20 m; 1; em; Grichanov \& Negrobov 2014; Kechev et al. 2020.

Sciapus flavicinctus (Loew, 1857) - TL, RE, BS; 0-473; 1; csean; Beschovski 1971a; Negrobov 1991; Beschovski \& Dzhambazov 2002; Kechev 2005, 2014, 2015; Kechev et al. 2020; Kechev 2021 b.

Sciapus frater (Parent, 1927) - TL; 149 m; 1; ? e; Kechev 2012, 2015; Kechev \& Ivanova 2015; Kechev et al. 2020.

Sciapus glaucescens (Loew, 1856) - BN; 0-20 m; 1; sena; Beschovski 1967a; Negrobov 1991; Beschovski \&

Dzhambazov 2002; Kechev 2005, 2015; Kechev et al. 2020.

Sciapus heteropygus Parent, 1926 - E2; 218 m; 1; eswa; Kechev et al. 2020.

Sciapus longulus (Fallén, 1823) - TL; 150 m; 1; weswca; Beschovski 1967a; Negrobov 1991; Beschovski \&

Dzhambazov 2002; Kechev 2005, 2015; Kechev et al. 2020.

Sciapus maritimus Becker, 1918 - RW; 580-600 m; 1; e; Kechev 2007a.

Sciapus maurus Parent, 1930 - seeanna; Parent 1938; Kechev 2005; Grichanov \& Negrobov 2014.

Sciapus opacus (Loew, 1866) - BN, BS; 0-10 m; 1; sena; Beschovski 1971a; Negrobov 1991; Beschovski \&

Dzhambazov 2002; Kechev 2005, 2015; Grichanov \& Negrobov 2014; Kechev et al. 2020.

Sciapus pallens (Wiedemann, 1830) - BS; 0-10 m; 1; h; Beschovski 1967a; Negrobov 1991; Beschovski \&

Dzhambazov 2002; Kechev 2005, 2015; Grichanov \& Negrobov 2014; Kechev et al. 2020.

Sciapus palmipes Collin, 1966 - R2; 1760 m; 4; se; Kechev et al. 2020.

Sciapus platypterus (Fabricius, 1805) - DM, P2, TL, RW, RE, BN; 0-473 m; 1; e; Beschovski 1971a; Negrobov 1991; Kechev 2005, 2006, 2007a, 2012a, 2015, 2017, 2021b; Beschovski \& Dzhambazov 2002; Kechev et al. 2014, 2020; Grichanov \& Negrobov 2014.

Sciapus wiedemanni (Fallén, 1823) - TL; 140-190 m; 1; h; Grichanov \& Negrobov 2014; Kechev et al. 2014, 2020; Kechev 2015.

Xanthochlorus luridus Negrobov, 1978 - RW; 670-1200 m; 2, 3; ? e; Kechev 2007a, 2007b, 2015; Kechev et al. 2020.

Xanthochlorus tenellus (Wiedemann, 1817) - TL, RW; 150-1200 m; 1, 2, 3; ean; Negrobov 1991; Kechev 2005, 2006, 2007a, 2015; Beschovski \& Dzhambazov 2002; Kechev et al. 2014, 2020.

Rhaphium albifrons Zetterstedt, 1843 - RW; 400 m; 1; esca; Kechev 2007a, 2007b; Kechev et al. 2020.

Rhaphium antennatum (Carlier, 1835) - RE; 473 m; 1; ei; Kechev 2021b.

Rhaphium appendiculatum Zetterstedt, 1849 [R. macrocerum Meigen, 1824; Xyphandrium] - S23, RW, BN; 0-1000 m; 1, 2; pat; Beschovski 1971a; Negrobov 1991; Kechev 2005, 2006, 2007a, 2015, 2016; Beschovski \& Dzhambazov 2002; Kechev et al. 2020.

Rhaphium auctum Loew, 1857 - S23, TL, RW; 150-400 m; 1; wcp; Kechev 2005, 2007a, 2007b, 2015, 2016; Kechev et al. 2014, 2020; Kechev \& Ivanova 2015.

Rhaphium brevicorne Curtis, 1835 - S23, RW; 300-1350 m; 1, 2, 3; wp; Kechev 2007a, 2007b, 2015, 2016; Kechev et al. 2020.

Rhaphium caliginosum (Zetterstedt, 1843) [R. zetterstedti Parent, 1925; Xiphandrium] - DM, P1, P2, B3, S23, TL, RW; 120-850 m; 1, 2; tp; Beschovski 1967a; Negrobov 1991; Beschovski \& Dzhambazov 2002; Kechev 2005, 2006, 2007a, 2012a, 2012b, 2014, 2016, 2017, 2021b; Kechev et al. 2014, 2020; Kechev \& Ivanova 2015.

Rhaphium crassipes (Meigen, 1824) [Porphyrops] - R1; 1374-1400 m; 3; h; Kechev 2006; Kechev et al. 2020.

Rhaphium discigerum Stenhammar, 1851 - TL; 168-240 m; 1; eca; Kechev 2011a, 2014, 2015; Kechev et al. 2020.

Rhaphium fascipes (Meigen, 1824) - P2; 280-360 m; 1; h; Kechev 2005, 2015; Beschovski \& Dzhambazov 2002; Kechev et al. 2020.

Rhaphium fissum Loew, 1850 - S23, RW; 280-330 m; 1; wcp; Kechev 2007a, 2007b, 2016; Kechev et al. 2020.

Rhaphium laticorne (Fallén, 1823) [Porphyrops nemorum Meigen, 1824] - P1, P2, V1; 120-600 m; 1; wes; Nedelkov 1912; Negrobov 1991; Kechev 2005, 2015, 2017, 2021b; Beschovski \& Dzhambazov 2002; Kechev et al. 2020.

Rhaphium micans (Meigen, 1824) [Porphyrops] - V1; 550-600 m; 1; esca; Nedelkov 1912; Negrobov 1991; Beschovski \& Dzhambazov 2002; Kechev 2005, 2015; Kechev et al. 2020. 
Rhaphium monotrichum Loew, 1850 - TL, RW; 149-1400 m; 1, 2, 3; wces; Kechev 2007a, 2010, 2015; Kechev \& Ivanova 2015; Kechev et al. 2020.

? Rhaphium pectinatum (Loew, 1859) [R. brevicorne Curtis, 1835] - TL; 149 m; 1; e; Kechev 2015; Kechev \& Ivanova 2015.

Rhaphium penicillatum Loew, 1850 - S23, TL, RW; 150-300 m; 1; e; Kechev 2007a, 2010, 2016; Kechev et al. 2014, 2020.

Rhaphium riparium (Meigen, 1824) [R. praerosum Loew, 1850] - RW; 300 m; 1; e; Kechev 2007a, 2010; Kechev et al. 2020.

Chrysotimus flaviventris (von Roser, 1840) [Chrysotus concinnus Zetterstedt, 1843] - V4, RW; 1000-1350 m; 2, 3; e; Kechev 2007a, 2010, 2015, 2021b; Kechev et al. 2020.

Chrysotimus molliculus (Fallén, 1823) - S23, TL, RW; 150-1200 m; 1, 2, 3; e; Kechev 2005, 2007a, 2015, 2016, 2020; Kechev et al. 2014, 2020; Kechev \& Ivanova 2015.

Epithalassius caucasicus Becker, 1918 - BS; 0-5 m; 1; bc; Caspers 1951a; Beschovski \& Dzhambazov 2002; Kechev 2005, 2015; Kechev et al. 2020.

Epithalassius stackelbergi Beschovski, 1966 - BN; 0-5 m; 1; Eb; Beschovski 1966d, 1975a; Negrobov 1991; Beschovski \& Dzhambazov 2002; Kechev 2005, 2015; Kechev et al. 2020.

Micromorphus albipes (Zetterstedt, 1843) - TL, BN; 0-150 m; 1; ppta; Beschovski 1967a; Beschovski \& Dzhambazov 2002; Kechev 2005, 2015; Kechev \& Ivanova 2015; Kechev et al. 2020.

Peloropeodes acuticornis (Oldenberg, 1916) - S23; 350 m; 1; nm; Kechev 2016; Kechev et al. 2020.

Peloropeodes meridionalis Parent, 1928 [Anomalopyga] - DM; 120-130 m; 1; se; Beschovski 1967a; Negrobov 1991; Beschovski \& Dzhambazov 2002; Kechev 2005, 2015; Kechev et al. 2020.

Vetimicrotes mediterraneus (Becker, 1918) [Microtes] - BS; 0-5 m; 1; Eb; Beschovski 1975b; Negrobov 1991; Beschovski \& Dzhambazov 2002; Kechev 2005, 2015; Kechev et al. 2020.

Achalcus flavicollis (Meigen, 1824) - BN; 0-5 m; 1; e; Pollet 1996; Kechev 2005, 2015; Kechev et al. 2020.

Aphrosylus fuscipennis Strobl, 1909 - BN, BS; 0-5 m; 1; se; Beschovski 1973a; Negrobov 1991; Beschovski \& Dzhambazov 2002; Kechev 2005, 2015; Kechev et al. 2020.

Aphrosylus piscator Lichtwardt, 1902 - BN; 0-5 m; 1; Eb; Beschovski 1973a; Negrobov 1991; Beschovski \& Dzhambazov 2002; Kechev 2005, 2015; Kechev et al. 2020.

Aphrosylus venator Loew, 1857 - BN, BS; 0-5 m; 1; sena; Beschovski 1964a, 1964b, 1965, 1975a; Negrobov 1991; Beschovski \& Dzhambazov 2002; Kechev 2005, 2015; Kechev et al. 2020.

Thinophilusflavipalpis (Zetterstedt, 1843) - BN, BS; 0-5 m; 1; ppt; Nedelkov 1912; Beschovski 1971a; Negrobov 1991; Beschovski \& Dzhambazov 2002; Kechev 2005, 2015; Kechev et al. 2020.

Thinophilus ruficornis (Haliday, 1838) - BN, BS; 0-5 m; 1; po; Beschovski 1967a; Negrobov 1991; Beschovski \& Dzhambazov 2002; Kechev 2005, 2015; Kechev et al. 2020.

Schoenophilus versutus (Haliday, 1851) - TL; 55-65 m; 1; eanna; Beschovski 1967a; Negrobov 1991; Beschovski \& Dzhambazov 2002; Kechev 2005, 2015; Kechev et al. 2020.

Orthoceratium lacustre (Scopoli, 1763) - BS; 0-5 m; 1; eanna; Beschovski 1971a; Negrobov 1991; Beschovski \& Dzhambazov 2002; Kechev 2005, 2015; Kechev et al. 2020.

Liancalus virens (Scopoli, 1763) - P2, B1, B2, B3, V1, RE, BN, BS; 0-1000 m, 1, 2; wp; ? wcp; subtroglophile; Nedelkov 1912; Buresch et al. 1949; Guéorguiev \& Beron 1962; Beschovski 1964a, 1964b, 1965 1972c; Negrobov 1991; Beron 1994, 2015, 2016; Kechev 2005, 2015, 2017, 2021b; Kechev et al. 2020.

Hydrophorus balticus (Meigen, 1824) - B3, TL, R1, R2, RW; 170-2300 m; 1, 2, 3, 4, 5; ppt; Beschovski 1967a; Negrobov 1991; Beschovski \& Dzhambazov 2002; Kechev 2005, 2006, 2007a, 2012b, 2015, 2017; Kechev et al. 2020.

Hydrophorus praecox (Lehmann, 1822) - BN; 0-5 m; 1; k; Beschovski 1964a, 1964b; Beschovski \& Dzhambazov 2002; Kechev 2005, 2015; Kechev et al. 2020.

Hydrophorus viridis (Meigen, 1824) - BN; 0-10 m; 1; po; Beschovski 1967a; Negrobov 1991; Beschovski \& Dzhambazov 2002; Kechev 2005, 2015; Kechev et al. 2020.

Scellus notatus (Fabricius, 1781) - TL, RW; 65-1200 m; 1, 2, 3; wesan, wes ?; Beschovski 1967a; Beschovski \& Dzhambazov 2002; Kechev 2005, 2007a, 2015; Kechev \& Ivanova 2015; Kechev et al. 2020.

Oncopygius distans (Loew, 1857) - V4; 1650-1700 m; 4; csee; Beschovski 2012, Kechev 2015; Kechev et al. 2020.

Neurigona biflexa Strobl, 1909 - ; se; Grishanov 2010; Kechev et al. 2020.

Neurigona erichsoni (Zetterstedt, 1843) - S23; 287-560 m; 1; eani; Kechev 2016; Kechev et al. 2020. 
Neurigona nubifera (Loew, 1869) - RE; 354-473; 1; Eb; Kechev 2021 b.

Neurigona pallida (Fallén, 1823) - DM, RE, B2; 108-530 m; 1; wes; Kechev 2021a, 2021b.

Neurigona quadrifasciata (Fabricius, 1781) - P1, RW; 280-362 m; 1; wces, ? tes; Kechev 2007a, 2021.

Neurigona suturalis (Fallén, 1823) - RW, RE; 200-473 m; 1; e; Kechev 2007a, 2010, 2015, 2021b; Kechev et al. 2020.

Sympycnus brevimanus Loew, 1857 - RW; 680 m; 1, 2; cee; Kechev 2007a.

Sympycnus cirripes (Haliday, 1851) - R2; RW; 280-1650 m; 1, 2, 3, 4; e; Parent 1938; Negrobov 1991; Beschovski \& Dzhambazov 2002; Kechev 2005, 2007a, 2015; Kechev et al. 2020.

Sympycnus desoutteri Parent, 1925 [S. pulicarius (Fallén, 1823), S. annulipes (Meigen, 1824)] - B3, S23, TL, RW, RE; 150-1450 m; 1, 2, 3; h; Beschovski 1967a, 2004; Negrobov 1991; Beschovski \& Dzhambazov 2002; Kechev 2005, 2007a, 2015, 2016, 2017; Kechev et al. 2014; Kechev \& Ivanova 2015.

Sympycnus pulicarius (Fallén, 1823) [S. annulipes (Meigen, 1824)] - B3, S23, TL, R5, RW, RE; 250-900 m; 1, 2; h; Kechev 2006, 2015; Kechev et al. 2020; Kechev 2021b.

Sympycnus simplicipes Becker, 1908 - P2, RW, RE; 144-450 m; 1; e; Kechev 2007a; Kechev et al. 2020.

Syntormon aulicum (Meigen, 1824) [Eutarsus] - RW; 269-1000 m; 1, 2; wp; Beschovski 1967a; Negrobov 1991; Beschovski \& Dzhambazov 2002; Kechev 2005, 2007a, 2015; Kechev et al. 2020.

Syntormon bulgariensis Negrobov \& Kechev, 2012 - RW; 1620 m; 4; Er; Negrobov \& Kechev, 2012; Kechev 2015; Kechev et al. 2020.

Syntormon denticulatum (Zetterstedt, 1843) [S. rufipes (Meigen, 1824)] - TL, T11, R1, RW; 128-1400 m; 1, 2, 3; wp; Beschovski 1967a; Negrobov 1991; Beschovski \& Dzhambazov 2002; Kechev 2005, 2006; 2014; Kechev et al. 2014; Kechev \& Ivanova 2015; Kechev et al. 2020.

Syntormon filiger Verrall, 1912 - TL, BS; 0-200 m; 1; e; Beschovski 1971a; Negrobov 1991; Beschovski \& Dzhambazov 2002; Kechev 2005, 2015; Kechev et al. 2014, 2020.

Syntormon fuscipes (von Roser, 1840) [S. spicatum (Loew, 1857)] - TL, R1, RW, BN; 0-1400 m; 1, 2, 3; wpat; Beschovski 1971a; Negrobov 1991; Beschovski \& Dzhambazov 2002; Kechev 2005, 2007a, 2014, 2015; Kechev et al. 2014, 2020; Kechev \& Ivanova 2015.

Syntormon metathesis (Loew, 1850) - TL; 120-125 m; 1; wes; Kechev 2012a, 2015; Kechev et al. 2020.

Syntormon mikii Strobl, 1899 - TL; 120-150 m; 1; ena; Kechev 2012a, 2012b, 2015; Kechev \& Ivanova 2015; Kechev et al. 2020.

Syntormon monile (Haliday, 1851) - RW; 300-1400 m; 1, 2, 3; wp; Kechev 2005, 2006, 2015; Kechev et al. 2020. Syntormon pallipes (Fabricius, 1794) - B3, S23, TL, R1, R5, RW, RE, BN; 0-1400 m; 1, 2, 3; ppt; Beschovski 1964a, 1964b; Negrobov 1991; Beschovski \& Dzhambazov 2002; Kechev 2005, 2006, 2007a, 2012a, 2012b, 2012c, 2015, 2016, 2017, 2021b; Kechev et al. 2014, 2020; Kechev \& Ivanova 2015.

Syntormon pumilum (Meigen, 1824) - TL, R1, RW, BN; 0-1400 m; 1, 2, 3; wp; Beschovski 1967a; Beschovski \& Dzhambazov 2002; Kechev 2006, 2007a, 2012b, 2015; Kechev et al. 2020.

Syntormon pseudospicatum Strobl, 1899 - V1; 630-640 m; 1; cse; Kechev \& Glogov 2021.

Syntormon triangulipes Becker, 1902 - TL; 140-150 m; 1; mwca; Kechev 2014, 2015; Kechev et al. 2014, 2020.

Lamprochromus bifasciatus (Macquart, 1827) - TL; 170 m; 1; e; Kechev 2015; Kechev \& Ivanova 2015; Kechev et al. 2020.

Lamprochromus kowarzi Negrobov et Tshalaja, 1988 - V1; 630-640 m; 1; ? csel; Kechev \& Glogov 2021.

Lamprochromus speciosus (Loew, 1871) - TL; 60-75 m; 1; wp; Beschovski 1967a; Negrobov 1991; Beschovski \& Dzhambazov 2002; Kechev 2005, 2015; Kechev et al. 2020.

Lamprochromus semiflavus (Strobl, 1880) [L. strobli Parent, 1925] - TL, BN; 0-200 m; 1; e; Beschovski 1967a; Negrobov 1991; Beschovski \& Dzhambazov 2002; Kechev 2005, 2015; Kechev et al. 2014, 2020.

Campsicnemus curvipes (Fallén, 1823) - S23, TL, R1, RW, BS; 0-1400 m; 1, 2, 3; ena; Beschovski 1967a; Negrobov 1991; Beschovski \& Dzhambazov 2002; Kechev 2005, 2006, 2007a, 2012a, 2012c, 2015, 2016; Kechev et al. 2014, 2020; Kechev \& Ivanova 2015.

Campsicnemus filipes Loew, 1859 - BN; 0-5 m; 1; e; Beschovski 1971a; Negrobov 1991; Beschovski \& Dzhambazov 2002; Kechev 2005, 2015; Kechev et al. 2020.

Campsicnemus magius (Loew, 1845) - BN; 0-5 m; 1; wpat; Beschovski 1971a; Negrobov 1991; Beschovski \& Dzhambazov 2002; Kechev 2005, 2015; Kechev et al. 2020.

Campsicnemus scambus (Fallén, 1823) - BN; 25-30 m; 1; tes; Beschovski 1967a; Negrobov 1991; Beschovski \& Dzhambazov 2002; Kechev 2005, 2015; Kechev et al. 2020. 
Campsicnemus simplicissimus Strobl, 1906 - S23, TL, RW, BN; 0-600 m; 1; eanca; Beschovski 1973a; Negrobov 1991; Beschovski \& Dzhambazov 2002; Kechev 2005, 2007a, 2012a, 2014, 2015, 2016; Kechev et al. 2014, 2020; Kechev \& Ivanova 2015.

Campsicnemus umbripennis Loew, 1856 - S23, TL, RW; 200-1620 m; 1, 2, 3, 4; eanca; Parent 1938; Negrobov 1991; Beschovski \& Dzhambazov 2002; Kechev 2005, 2006, 2007a, 2012c, 2015, 2016; Kechev et al. 2020.

Campsicnemus varipes Loew, 1859 - RW; 770 m; 2; eca; Kechev et al. 2020.

Telmaturgus tumidulus (Raddatz, 1873) - V4; 850-900 m; 2; eanca; Beschovski 2012; Kechev 2015; Kechev et al. 2020.

Teuchophorus chaetifemoratus Pollet \& Kechev, 2007 - S23, TL, RW; 300-330 m; 1; ban; Pollet \& Kechev 2007; Kechev 2015, 2016; Kechev et al. 2020.

Teuchophorus cristulatus Meuffels \& Grootaert, 1992 - S23, TL, RW; 149-280 m; 1; nm; Kechev 2012, 2015, 2016; Kechev \& Ivanova 2015; Kechev et al. 2020.

Teuchophorus medovoensis Kechev, Negrobov \& Grichanov, 2014 - S23, TL; 277-405 m; 1; Ebg; Kechev et al. 2014, 2020; Kechev 2015, 2016, 2020.

Teuchophorus monacanthus Loew, 1859 - P2, S23, TL, RW, BN; 130-400 m; 1; e; Beschovski 1967a; Negrobov 1991; Beschovski \& Dzhambazov 2002; Kechev 2005, 2006, 2007a, 2012b, 2015, 2016, 2017; Kechev et al. 2014, 2020; Kechev \& Ivanova 2015.

Teuchophorus simplex Mik, 1880 - RW; 300 m; 1; e; Kechev 2007a, 2015; Pollet \& Kechev 2007; Kechev et al. 2020.

Teuchophorus spinigerellus (Zetterstedt, 1843) - S23, TL, BN; 140-300 m; 1; wp; Beschovski 1967a; Negrobov 1991; Beschovski \& Dzhambazov 2002; Kechev 2005, 2012a, 2012b, 2015, 2016; Kechev et al. 2014, 2020; Kechev \& Ivanova 2015.

Argyra argyria (Meigen, 1824) - TL; 150 m; 1; eanna; Kechev 2011b, 2012b, 2015; Kechev \& Ivanova 2015; Kechev et al. 2020.

Argyra atriceps Loew, 1857 - \$; e; Negrobov 1991; Beschovski \& Dzhambazov 2002; Kechev 2005, 2015; Kechev et al. 2020.

Argyra auricollis (Meigen, 1824) - R1, RW; 1400 m; 3; e; Kechev 2007a, 2007b, 2015; Kechev et al. 2020.

Argyra diaphana (Fabricius, 1775) - RW; 300 m; 1; ei; Kechev 2007a, 2010, 2015; Kechev et al. 2020.

Argyra ilonae Gosseries, 1988 [A. confinis Zetterstedt, 1849] - RW; 770 m; 2; e; Kechev et al. 2020.

Argyra leucocephala (Meigen, 1824) - S23, TL, T31, RW; 150-1050 m; 1, 2, 3; wp; Negrobov 1991; Beschovski \& Dzhambazov 2002; Kechev 2005, 2012a, 2012b, 2015, 2016, 2020; Kechev et al. 2014, 2020; Kechev \& Ivanova 2015.

Argyra vestita (Wiedemann, 1817) [Leucostola] - BN; 0-10 m; 1; e; Beschovski 1967a; Negrobov 1991; Beschovski \& Dzhambazov 2002; Kechev 2005, 2015; Kechev et al. 2020.

Melanostolus nigricilius (Loew, 1871) - BN; 0-5 m; 1; ppt; Beschovski 1967a; Negrobov 1991; Beschovski \& Dzhambazov 2002; Kechev 2005, 2015; Kechev et al. 2020.

Diaphorus hoffmannseggi Meigen, 1830 - RW; 300 m; 1; e; Kechev 2007a, 2010, 2015; Kechev et al. 2020.

Chrysotus angulicornis Kowarz, 1874 - R2, RW; 900-1760 m; 2, 3, 4; e; Kechev et al. 2020.

Chrysotus cilipes Meigen, 1824 - RW; 40-600 m; 1, 2; po; Kechev 2007a, 2007b, 2015; Kechev et al. 2020.

Chrysotus femoratus Zetterstedt, 1843 - P2, S23, RW; 130-300 m; 1; po; Kechev 2007a, 2016, 2017; Kechev \& Ivanova 2015; Kechev et al. 2020.

Chrysotus gramineus (Fallen, 1823) - P1, R1, RW; 260-1900 m; 1, 2, 3, 4; hop; Negrobov 1991; Beschovski \& Dzhambazov 2002; Kechev 2005, 2007a, 2015, 2021b; Kechev et al. 2020.

Chrysotus laesus (Wiedemann, 1817) - R1, RW, RE; 473-1750 m; 1, 2, 3, 4; po; Kechev 2007a, 2007b, 2015, 2021b; Kechev et al. 2020.

Chrysotus monochaetus Kowarz, 1874 [Ch. viridifemoratus von Roser, 1840] - RW; 400-1926 m; 1, 2, 3, 4; des; Kechev 2007b, 2015; Kechev et al. 2020.

Chrysotus neglectus (Wiedemann, 1817) - R2, RW; 900-1760 m; 2, 3, 4; esca; Kechev 2007a; Kechev et al. 2020.

Chrysotus obscuripes Zetterstedt, 1838 - R2, RW; 1550-2000 m; 3, 4; po; Kechev 2007a; Kechev et al. 2020.

Chrysotus palustris Verrall, 1876 - BN; 0-20 m; 1; e; Beschovski 1972a; Beschovski \& Dzhambazov 2002; Kechev 2005, 2015; Kechev et al. 2020.

Chrysotus pennatus Lichtwardt, 1902 - P2, TL, RW; 110-800 m; 1, 2; eani; Kechev 2005, 2006, 2007a, 2014, 2015, 2017; Kechev et al. 2020.

Chrysotus polleti Olejnicek, 1999 - BS; 0-10 m; 1; Ebg; Olejníček 1999; Kechev 2005, 2015; Kechev et al. 2020. 
Chrysotus pulchellus Kowarz, 1874 - R1, RW, RE; 100-1400 m; 1, 2, 3; po; Beschovski 1967a, 2004a; Beschovski \& Dzhambazov 2002; Kechev 2005, 2007a, 2015; Kechev et al. 2020.

Chrysotus suavis Loew, 1857 - DM, P1, RW, RE, BN; 0-300 m; 1; ppt; Beschovski 1967a, 2004a; Negrobov 1991; Beschovski \& Dzhambazov 2002; Kechev 2005, 2007a, 2015, 2021b; Kechev et al. 2020.

Asyndetus albipalpus Negrobov, 1973 [Dolichopus] - R2; 2200-2400 m; 5; seeca; Beschovski \& Dzhambazov 2002; Kechev 2005.

Asyndetus latifrons (Loew, 1857) - P1, TL, RW; 190-720 m; 1, 2; ppt; Kechev 2007a, 2007b, 2015; Kechev et al. 2014, 2020, Kechev 2021b.

Tachytrechus consobrinus (Haliday, 1851) - TL, RW, RE; 110-473 m; 1; wpat; Kechev 2007a, 2010, 2014, 2015, 2021b; Kechev et al. 2020.

Tachytrechus genualis Loew, 1857 - V1; 590-600 m; 1, 2; dpo; Nedelkov 1912; Beschovski \& Dzhambazov 2002; Kechev 2005, 2015; Kechev et al. 2020.

Tachytrechus insignis (Stannius, 1831) - BS; 0-5 m; 1; ena; Nedelkov 1912; Beschovski \& Dzhambazov 2002; Kechev 2005, 2015; Kechev et al. 2020.

Tachytrechus notatus (Stannius, 1831) - V1, TL, BN; 0-600 m; 1; wp; Nedelkov 1912; Beschovski 1964a, 1964b; Negrobov 1991; Beschovski \& Dzhambazov 2002; Kechev 2005, 2015; Kechev et al. 2014, 2020.

Tachytrechus ripicola Loew, 1857 - BN; 0-5 m; 1; wesan; Beschovski 1967a; Beschovski \& Dzhambazov 2002; Kechev 2005, 2015; Kechev et al. 2020.

Sybistroma crinipes Stæger, 1842 [Hypophyllus] - RW; 300-1100 m; 1, 2, 3; e; Beschovski \& Dzhambazov 2002; Kechev 2005, 2006, 2015; Kechev et al. 2020.

Sybistroma discipes (Germar, 1817) [Hypophyllus] - RW; 300 m; 1; eani; Kechev 2007a, 2010, 2015; Kechev et al. 2020.

Sybistroma impar Rondani, 1843 [Ludovicius] - S23, TL, BN; 30-328 m; 1; eswa; Olejníček \& Barták 1997; Kechev 2005, 2012b, 2012c, 2015, 2016; Kechev et al. 2014, 2020; Kechev \& Ivanova 2015; Kechev et al. 2020.

Sybistroma nodicornis Meigen, 1824 - TL, RW; 145-300 m; 1; ? mi, ? wp; Kechev 2007a, 2007b, 2012a, 2014, 2015, 2020; Kechev \& Ivanova 2015; Kechev et al. 2020.

Sybistroma obscurellum (Fallén, 1823) [Hypophyllus] - RW; 300-1000 m; 1, 2; e; Kechev 2007a, 2007b, 2015; Kechev et al. 2020.

Sybistroma setosa Schiner, 1862 - TL; 100 m; 1; csee; Kechev 2012a, 2015; Kechev et al. 2020.

Gymnopternus aerosus (Fallén, 1823) [Hercostomus] - S23, RW; 400-1360 m; 1, 2, 3; po; Beschovski \& Dzhambazov 2002; Kechev 2005, 2006, 2015, 2016, 2021b; Kechev et al. 2020.

Gymnopternus angustifrons (Stæger, 1842) [Hercostomus] - R1, RW; 1330-1400 m; 3; wes; Kechev 2007a; Kechev et al. 2020.

Gymnopternus brevicornis (Stæger, 1842) [Hercostomus] - R1, RW, RE; 473-1400 m; 1, 2, 3; e; Kechev 2007a, 2007b, 2015, 2021b; Kechev et al. 2020.

Gymnopternus celer (Meigen, 1824) [Hercostomus] - RW, RE; 300-1200 m; 1, 2, 3; wces; Beschovski 1967a; Negrobov 1991; Beschovski \& Dzhambazov 2002; Kechev 2005, 2006, 2015, 2021 b; Kechev et al. 2020.

Gymnopternus chalybeus (Wiedemann, 1817) [Ethiromyia, Hercostomus] - RW; 1200 m; 3; e; Kechev 2007a, 2007b, 2015; Kechev et al. 2020.

Gymnopternus metallicus (Stannius, 1831) - TL, RW; 150-1000 m; 1, 2; wesca; Kechev 2007a, 2007b, 2012a, 2015; Kechev \& Ivanova 2015; Kechev et al. 2020.

Hercostomus chetifer (Walker, 1849) - B2; 657 m; 1, 2; ho; Kechev 2021.

Hercostomus convergens Loew, 1857 - TL; 128-150 m; 1; eswa; Kechev 2012a, 2012b, 2014, 2015; Kechev \& Ivanova 2015; Kechev et al. 2020.

Hercostomus fugax (Loew, 1857) - R2, RW; 1200-2060 m; 3, 4, 5; wces; Parent 1938; Negrobov 1991; Beschovski \& Dzhambazov 2002; Kechev 2005, 2006, 2007a, 2015; Kechev et al. 2020.

Hercostomus fulvicaudis (Walker, 1851) - TL; 120-125 m; 1; po; Kechev 2012a, 2015; Kechev et al. 2020.

Hercostomus gavarniae Parent, 1928 - RW; 1200 m; 3; e, ? se; Kechev 2007a, 2007b, 2015; Kechev et al. 2020.

Hercostomus germanus (Wiedemann, 1817) - RW; 1100-1620 m; 3, 4; wces, ? wcp; Kechev 2007a, 2010, 2015; Kechev et al. 2020.

Hercostomus gracilis (Stannius, 1831) [H. bicolor Macquart, 1827] - E1, S23, TL; 172-420 m; 1; esca; Parent 1938; Negrobov 1991; Beschovski \& Dzhambazov 2002; Kechev 2005, 2015, 2016, 2020, 2021b; Kechev et al. 2014, 2020. 
Hercostomus labiatus (Loew, 1871) - R2; 1600-2230 m; 4, 5; e; Beschovski \& Dzhambazov 2002; Kechev 2005, 2015; Kechev et al. 2020.

Hercostomus longiventris (Loew, 1857) - RW; 1350 m; 3; wp; Kechev 2005, 2007a, 2015; Kechev et al. 2020.

Hercostomus nanus (Macquart, 1827) - TL, T31, BS; 0-150 m; 1; ean; Kechev 2005, 2015, 2020; Kechev \& Ivanova 2015; Kechev et al. 2020.

Hercostomus nigrilamellatus (Macquart, 1827) - RW; 300-1620 m; 1, 2, 3, 4; e; Kechev 2007a, 2010, 2015; Kechev et al. 2020.

Hercostomus nigriplantis (Stannius, 1831) - RW; 300 m; 1; des, ? wces; Kechev 2007a, 2010, 2015; Kechev et al. 2020 .

Hercostomus parvilamellatus (Macquart, 1827 - S23; 287 m; 1; e; Kechev 2016; Kechev et al. 2020.

Hercostomus plagiatus (Loew, 1857) - S23, TL; 150-405 m; 1; ena; Kechev 2015, 2016; Kechev \& Ivanova 2015; Kechev et al. 2020.

Hercostomus rusticus (Meigen, 1824) - V5, R2, RW; 450-2014 m; 1, 2, 3, 4; tes; Beschovski \& Dzhambazov 2002; Kechev 2005, 2006, 2007a, 2015, 2021b; Kechev et al. 2020.

Hercostomus sahlbergi (Zetterstedt, 1838) [Hydrophorus] - R1; 1600-1700 m; 3, 4; e; Nedelkov 1912; Negrobov 1991; Beschovski \& Dzhambazov 2002; Kechev 2005, 2015; Kechev et al. 2020.

Hercostomus stroblianus Becker, 1917 - RW; 300 m; 1; ? atm; Kechev 2007a, 2010, 2015; Kechev et al. 2020.

Hercostomus thraciensis Kechev \& Negrobov, 2015 - TL; 130-150 m; 1; em; Kechev 2015, 2020; Kechev \& Ivanova 2015; Kechev \& Negrobov 2015; Kechev et al. 2020.

Hercostomus vivax (Loew, 1857) - RW; 1926 m; 4; wces; Kechev 2007a, 2007b, 2015; Kechev et al. 2020.

Poecilobothrus chrysozygos (Wiedemann, 1817) [Hercostomus] - S23, TL, RW, RE; 130-400 m; 1; esanca; Negrobov 1991; Beschovski \& Dzhambazov 2002; Kechev 2005, 2007a, 2011a, 2012a, 2015, 2016, 2021b; Kechev et al. 2014, 2020.

Poecilobothrus comitialis (Kowarz, 1867) [Hercostomus] - T31, BS; 0-10 m; 1; eanca; Kechev 2005, 2011a, 2015; Kechev et al. 2020.

Poecilobothrus ducalis (Loew, 1857) [P. infuscatus Parent, 1938] - T31, BS; 0-10 m; 1; ena; Kechev 2011a, 2015; Kechev et al. 2020.

Poecilobothrus nobilitatus (Linnaeus, 1767) [Gymnopternus, Hercostomus] - V1, V4, TL, RW; 200-1000 m; 1, 2; e; Nedelkov 1909, 1912; Beschovski \& Dzhambazov 2002; Kechev 2005, 2007a, 2011a, 2015; Kechev et al. 2020.

Poecilobothrus principalis (Loew, 1861) [Hercostomus fumipennis Stannius, 1831] - BS; 0-10 m; 1; eswa; Beschovski 1967a; Beschovski \& Dzhambazov 2002; Kechev 2005, 2011a, 2015; Kechev et al. 2020.

Poecilobothrus regalis (Meigen, 1824) [Hercostomus] - S23, TL, BN, BS; 0-1050 m; 1, 2, 3; eanit; Parent 1938; Beschovski 1971a; Negrobov 1991; Beschovski \& Dzhambazov 2002; Kechev 2005, 2011a, 2012a, 2012b, 2012c, 2014, 2015, 2016, 2020, 2021b; Kechev et al. 2014, 2020; Kechev \& Ivanova 2015.

Ortochile nigrocoerulea Latreille, 1809 [O. unicolor Loew, 1850] - RW, BS; 0-1750 m; 1, 2, 3, 4; eanna; Kechev 2005, 2015; Kechev et al. 2020.

Dolichopus arbustorum Stannius, 1831 - V4; 950-1050 m; 2, 3; e; Beschovski 2013a; Kechev 2015; Kechev et al. 2020.

Dolichopus argyrotarsis Wahlberg, 1850 - V4, RW; 900-1700 m; 3, 4; e; Kechev 2007a, 2010, 2015; Beschovski 2013a; Kechev et al. 2020.

Dolichopus beschovskii Negrobov \& Kechev, 2010 - R2; 2020-2200 m; 5; Er; Negrobov \& Kechev 2010; Kechev 2015; Kechev et al. 2020.

Dolichopus campestris Meigen, 1824 - B2, V1, V4, RW; 600-1500 m; 2, 3; tp; Kechev 2007a, 2015; Beschovski 2013a; Kechev et al. 2020.

Dolichopus cilifemoratus Macquart, 1827 - RW; 1200 m; 3; tes, ? des; Kechev 2007a, 2007b, 2015; Kechev et al. 2020.

Dolichopus claviger Stannius, 1831 - RW; 280-1050 m; 1, 2, 3; wces, ? wes; Kechev 2007a, 2015; Kechev et al. 2020.

Dolichopus clavipes Haliday, 1832 - RW; 1620 m; 4; po; Kechev 2007a.

Dolichopus diadema Haliday, 1832 [Macrodolichopus] - RE, BN, BS; 0-440 m; 1; po; Beschovski 1967a, 2004a, 2013a; Kechev 2005, 2015, 2021b; Kechev et al. 2020.

Dolichopus excisus Loew, 1859 - DW, B3, S23, TL, O61, O62, BN; 0-350 m; 1; eanca; Beschovski 1967a, 2013a; Negrobov 1991; Beschovski \& Dzhambazov 2002; Kechev 2005, 2012b, 2015, 2016; Kechev et al. 2020. 
Dolichopus festivus Haliday, 1832 - RW; 1105 m; 2, 3; e; Kechev 2007a.

Dolichopus griseipennis Stannius, 1831 - E1, V1, S23, TL, T11, RW, RE; 120-1190 m; 1, 2, 3; wp; Nedelkov 1912; Negrobov 1991; Beschovski \& Dzhambazov 2002; Kechev 2005, 2007a, 2015, 2016, 2021b; Beschovski 2013a; Kechev et al. 2014, 2020; Kechev \& Ivanova 2015.

Dolichopus latilimbatus Macquart, 1827 - DW, S23, TL, BN, BS; 0-300 m; 1; po; Beschovski 1973a; Negrobov 1991; Beschovski \& Dzhambazov 2002; Kechev 2005, 2012a, 2015, 2016; Beschovski 2013a.

Dolichopus lepidus Stæger, 1842 - B2, V4, RW; 900-1500 m; 2, 3; po; Kechev 2007a, 2015; Beschovski 2013a; Kechev et al. 2020.

Dolichopus linearis Meigen, 1824 - TL; 170 m; 1; po; Kechev 2012b, 2015; Kechev et al. 2020.

Dolichopus litorellus Zetterstedt, 1852 - V4; 950-980 m; 2; po; Beschovski 2013a; Kechev 2015; Kechev et al. 2020.

Dolichopus longicornis Stannius, 1831 - B2; 530 m; 1; ho; Kechev 2021.

Dolichopus longisetosus Negrobov, 1973 - B1; 1450 m; 3; ees; Beschovski \& Dzhambazov 2002; Kechev 2005, 2015; Kechev et al. 2020.

Dolichopus longitarsis Stannius, 1831 - V5, R1; 1100-1150 m; 3; eanit; Beschovski 2013a; Kechev 2015, 2021b; Kechev et al. 2020.

Dolichopus nigricornis Meigen, 1824 [D. discifer Stannius, 1831] - TL, BN; 0-300 m; 1; h; Beschovski 1967a; Negrobov 1991; Beschovski \& Dzhambazov 2002; Kechev 2015, 2015; Kechev et al. 2014, 2020.

Dolichopus nitidus Fallén, 1823 - V1, TL, BN; 150-600 m; 1; po; Nedelkov 1912; Negrobov 1991; Beschovski \& Dzhambazov 2002; Kechev 2005, 2015; Kechev \& Ivanova 2015; Kechev et al. 2020.

Dolichopus nubilus Meigen, 1824 - V1, S23, TL, T11, BN; 0-550 m; 1; eanca, ? eanit; Beschovski 1967a, 1973a, 2013a; Negrobov 1991; Beschovski \& Dzhambazov 2002; Kechev 2005, 2012a, 2014, 2015, 2016; Kechev \& Ivanova 2015; Kechev et al. 2020.

Dolichopus pennatus Meigen, 1824 - V4, R1, RW; 1100-1700 m; 3, 4; po; Kechev 2005, 2006, 2007a, 2015; Beschovski 2013a; Kechev et al. 2020.

Dolichopus phaeopus Haliday, 1851 - R1, R2, RW; 1500-2200 m; 4, 5; e; Kechev 2007a, 2015; Beschovski 2013a; Kechev et al. 2020.

Dolichopus picipes Meigen, 1824 - B2, R1, R2, RW; 1200-2014 m; 3, 4; wesan; Kechev 2007a, 2010, 2015, 2021b; Beschovski 2013a; Kechev et al. 2020.

Dolichopus plumipes (Scopoli, 1763) - V4, R5, S22, R1, RW, BN; 0-1400 m; 1, 2, 3; hno; Beschovski 1971a, 2013a; Negrobov 1991; Beschovski \& Dzhambazov 2002; Kechev 2005, 2006, 2007a, 2015, 2021b; Kechev et al. 2020.

Dolichopus plumitarsis Fallen, 1823 - ; h; Negrobov 1991; Beschovski \& Dzhambazov 2002; Kechev 2015; Kechev et al. 2020.

Dolichopus popularis Wiedemann, 1817 - R1, RW; 300-1400 m; 1, 2, 3; wces; Kechev 2007a, 2007b, 2015, 2021b; Kechev et al. 2020.

Dolichopus pyrenaicus Parent, 1920 - R2; 1800-2200 m; 4, 5; wes; Beschovski \& Dzhambazov 2002; Kechev 2005, 2015; Kechev et al. 2020.

Dolichopus sabinus Haliday, 1838 - RW, BN; 0-300 m; 1; ean; Beschovski 1967a; Negrobov 1991; Beschovski \& Dzhambazov 2002; Kechev 2005, 2006, 2007a, 2015; Kechev et al. 2020.

Dolichopus salictorum Loew, 1871 - S23, TL, BN; 0-300 m; 1; eani; Beschovski 1971a; Negrobov 1991; Beschovski \& Dzhambazov 2002; Kechev 2005, 2012a, 2015, 2016, 2020; Kechev \& Ivanova 2015; Kechev et al. 2020.

Dolichopus siculus Loew, 1859 - O61; 300-350 m; 1; mi; Beschovski 2013a; Kechev 2015; Kechev et al. 2020.

Dolichopus signifer Haliday, 1838 - DW, K1, K2, S1, S23, TL, O5, O62, RW, BN; 0-1200 m; 1, 2, 3; wpat; Beschovski 1967a, 2013a; Negrobov 1991; Beschovski \& Dzhambazov 2002; Kechev 2005, 2007a, 2012a, 2014, 2015, 2016; Kechev et al. 2014, 2020.

Dolichopus simplex Meigen, 1824 - B1, V4, V5, R1, RW; 300-1400 m; 1, 2, 3; wces; Beschovski \& Dzhambazov 2002; Kechev 2005, 2006, 2007a, 2015; Beschovski 2013a; Kechev et al. 2020.

Dolichopus strigipes Verrall, 1875 - BN, BS; 0-5 m; 1; e; Beschovski 1967a; Beschovski \& Dzhambazov 2002; Kechev 2005, 2015; Kechev et al. 2020.

Dolichopus trivialis Haliday, 1832 - RW; 1300 m; 3; e; Kechev et al. 2020.

Dolichopus ungulatus (Linnaeus, 1758) - V4, R1, RW; 1000-1400 m; 3; h; Negrobov 1991; Beschovski \& Dzhambazov 2002; Kechev 2005, 2006, 2007a, 2015, 2021b; Beschovski 2013a; Kechev et al. 2020. 
Medetera diadema (Linnaeus, 1767) - P2, V1; 220-600 m; 1; h; Nedelkov 2012; Negrobov 1991; Beschovski \& Dzhambazov 2002; Kechev 2005, 2015; Kechev et al. 2020.

Medetera flavipes (Linnaeus, 1758) - TL; 174 m; 1; eanna; Kechev et al. 2020.

Medetera glauca Loew, 1869 - BN; 0-5 m; 1; e; Beschovski 1967a; Negrobov 1991; Beschovski \& Dzhambazov 2002; Kechev 2005, 2015; Kechev et al. 2020.

Medetera helvetica Naglis \& Negrobov, 2014 - R2; 2000 m; 4; csee; Kechev et al. 2020.

Medetera impigra Collin, 1941 - R2; 2000 m; 4; wces; Kechev et al. 2020.

Medetera jacula (Fallén, 1823) - E1, V4, RE, BN; 48-1350 m; 1, 2, 3; tp; Kechev et al. 2020; Kechev 2021b

Medetera micacea Loew, 1857 - E1, P1, O62, BN; 0-267 m; 1; po; Beschovski 1967a; Negrobov 1991; Beschovski \& Dzhambazov 2002; Kechev 2005, 2015, 2021b; Kechev et al. 2020.

Medetera muralis Meigen, 1824 - P1, B2; 260-530 m; 1; e; Kechev 2021a, 2021b

Medetera murina Becker, 1917 - DW, RW; 108-680 m; 1, 2; ? e, cse; Kechev 2007a, 2021b.

Medetera pallipes (Zetterstedt, 1843) - P1; 362 m; 1; e; Kechev $2021 \mathrm{a}$.

Medetera perfida Parent, 1932 - R2; 2000 m; 4; csee; Kechev et al. 2020.

Medetera petrophila Kowarz, 1877 - E2, RW, BN; 0-1620 m; 1, 2, 3, 4; ena; Beschovski 1967a; Beschovski \& Dzhambazov 2002; Kechev 2005, 2007a, 2015; Kechev et al. 2020.

Medetera petrophiloides Parent, 1932 - BN; 0-5 m; 1; e; Beschovski 1967a; Kechev et al. 2020.

Medetera pinicola Kowarz, 1877 - V4; 1550 m; 4; h; Doychev et al. 2016; Kechev et al. 2020.

Medetera truncorum Meigen, 1824 - E2; 218 m; 1; ena, ? wp; Kechev et al. 2020.

Medetera tumidula Negrobov, 1967 - RW; 270 m; 1; see; Kechev 2007a.

Systenus scholtzi (Loew, 1850) - O62; 267 m; 1; des; Kechev 2021b.

Thrypticus bellus Loew, 1869 - DM, RW, BN; 0-1200 m; 1, 2, 3; pat; Beschovski 1967a; Negrobov 1991; Beschovski \& Dzhambazov 2002; Kechev 2005, 2007a, 2015; Kechev et al. 2020.

\section{CYCLORRHAPHA}

\section{AschIZA \\ Platypezidae (Clythiidae)}

Callomyia saibhira Chandler, 1976 - R1; 930-1000 m; 2, 3; des; Chandler 1976; Beschovski 2004c.

Kesselimyia chandleri Vanhara, 1981 - S211; 950-1000 m; 2; csee; Beschovski 2004c.

\section{Lonchopteridae (Muscidoridae)}

Lonchoptera bifurcata (Fallén, 1810) [L. furcata (Fallén, 1823); L. lacustris Meigen, 1824] - DW, DM, E2, P2, B2, V1, V4, V5, S1, S211, S22, TL, T11, T2, T31, O4, O5, O61, O62, R1, R2, R3, R5, RW, RE, BN, BS; 0-2400 m; 1, 2, 3, 4, 5; k; Nedelkov 1912; Beschovski \& Georgieva 2000; Beschovski 2004a, 2006a.

Lonchoptera lutea Panzer, 1809 - DW, P2, B2, B3, V1, V2, V4, S1, T11, O61, O62, R2, R3, R4, R5, RW, BN; 0-1700 m; 1, 2, 3, 4; tp; Nedelkov 1912; Beschovski \& Georgieva 2000; Beschovski 2006a.

Lonchoptera strobli de Meijere, 1906 - V4, T31; 120-1400 m; 1, 2, 3; e, ? cee; Beschovski \& Georgieva 2000 ? Lonchoptera tristis Meigen, 1824 - V1; 600 m; 1; e; Nedelkov 1912.

\section{Phoridae}

Aenigmatias franzi Schmitz, 1950 - B2, V4, R2; 1000-2270 m; 3, 4, 5; eca; Langourov 2009.

Aenigmatias lubbockii (Verrall, 1877) - B2, V4, S1; 450-940 m; 1, 2; e; Langourov 2001a, 2009.

Anevrina curvinervis (Becker, 1901) - V4; $820 \mathrm{~m} ; 2$; h; Langourov 2001a.

Anevrina thoracica (Meigen, 1804) - V4, O1; 1000-2020 m; 3, 4, 5; h; Beschovski \& Langourov 1997; Langourov 2001a.

Anevrina unispinosa (Zetterstedt, 1860 - V4; 1270-1360 m; 3; des; Langourov 2001a.

Anevrina urbana (Meigen, 1830) - V1, V4, RW; 550-940 m; 1, 2; h; Langourov 2001a, 2004a, 2011. 
Beckerina umbrimargo (Becker, 1901) - V4, RW; 657-820 m; 2; e; Langourov 2001a, 2011.

Borophaga carinifrons (Zetterstedt, 1848) - V4; 1360 m; 3; e; Langourov 2001a.

Borophaga femorata (Meigen, 1830) - V1; 550 m; 1; dp; Langourov 2004a.

Borophaga germanica (Schmitz, 1918) - V1; 550 m; 1; e; Langourov 2004a.

Borophaga incrassata (Meigen, 1830) [Phora] - •; V4; 870-1270 m; 1, 2, 3; e; Drensky 1932b, 1934b, 1936, 1939a; Langourov 2001a.

Borophaga irregularis (Wood, 1912) - V4; 1200-1270 m; 3; e; Beschovski \& Langourov 1997; Langourov 2001 . Chaetopleurophora bohemanni (Becker, 1901) - V4; 820-1650 m; 2, 3, 4; e; Langourov 2001a.

Chaetopleurophora erythronota (Strobl, 1892) - V4, RW, BS; 0-1500 m; 1, 2, 3; h; Beschovski \& Langourov 1997; Langourov 2011.

Chaetopleurophora spinosior Schmitz, 1938 - V4; 1360 m; 3; e; Langourov $2001 \mathrm{a}$.

Chaetopleurophora spinosissima (Strobl, 1892) - V4; 820 m; 2; des; Langourov $2001 \mathrm{a}$.

Conicera dauci (Meigen, 1830) - V1, V4; 550-1600 m; 1, 2, 3, 4; h; Beschovski \& Langourov 1997; Langourov 2001a, 2004a.

Conicera floricola Schmitz, 1938 - V4; 940-1350 m; 2, 3; e; Langourov 2001a.

Conicera schnittmanni Schmitz, 1926 - V4; 1350 m; 3; des; Langourov 2001a.

Conicera similis (Haliday, 1833) - V4; $940 \mathrm{~m}$; 2; h; Langourov $2001 \mathrm{a}$.

Conicera tibialis Schmitz, 1925 - V4; 940-1350 m; 2, 3; h; Langourov 2001a.

Diplonevra amphichaeta (Schmitz, 1949) - V4; 1000 m; 2, 3; csee; Beschovski \& Langourov 1997; Langourov 2001a.

Diplonevra concinna (Meigen, 1830) - V1; 550-600 m; 1; e; Nedelkov 2012; Langourov 2004a.

Diplonevra crassicornis (Meigen, 1830) - V1, V4; 550-1350 m; 2, 3; e, ? cse; Langourov 2001a, 2004a.

Diplonevra florescens (Turton, 1801) [D. florea (Fabricius, 1794)] - V1, V4, RW; 550-1350 m; 2, 3; des; Beschovski \& Langourov 1997; Langourov 2001a, 2004a, 2011; Langourov et al. 2014.

Diplonevra funebris (Meigen, 1830) - DW, E2, V1, V4, S211, RE; 80-1360 m; 1, 2, 3; hn; Beschovski \& Langourov 1997; Langourov 2001a, 2004a, 2004b; Langourov et al. 2014.

Diplonevra glabra (Schmitz, 1927) - V1, V4; 550-1360 m; 2, 3; e; Langourov 2001a, 2004a.

Diplonevra lophochaeta (Schmitz, 1927) - V4; 1000 m; 2, 3; csee; Beschovski \& Langourov 1997.

Diplonevra nitidula (Meigen, 1830) - V1, V4, O4; 400-1360 m; 1, 2, 3; h; Beschovski \& Langourov 1997; Langourov 2001a, 2004a.

Diplonevra unisetalis (Schmitz, 1935) - BS; 0-10 m; 1; e; Beschovski \& Langourov 1997.

Dohrniphora cornuta (Bigot, 1857) - 0-550 m; 1; k; Beschovski \& Langourov 1997; Langourov 2004a.

Gymnophora arcuata (Meigen, 1830) - V4; 940-960 m; 2; e; Langourov 2001a; Langourov et al. 2014.

Gymnophora integralis Schmitz, 1920 - V4, RE; 160-1360 m; 1, 2, 3; des; Langourov 2001a, 2004b; Langourov et al. 2014.

Gymnophora quartomollis Schmitz, 1920 - V4; 1270-1550 m; 3, 4; e; Langourov 2001a.

Gymnoptera vitripennis (Meigen, 1830) - V4; 940-1360 m; 2, 3; e; Langourov 2001a.

Megaselia abdita Schmitz, 1959 - V1; 550 m; 1; h; Langourov 2004a.

Megaselia abernethae Disney, 1988 - V4; 1360 m; 3; e; Langourov 2001a.

Megaselia aculeata (Schmitz, 1919) - V4, RW; 450-2150 m; 1, 2, 3, 4, 5; e; Langourov 2001a, 2011.

Megaselia aequalis (Wood, 1909) - V4, RW; 450-2150 m; 1, 2, 3, 4, 5; h; Langourov 2001a, 2011.

Megaselia affinis (Wood, 1909) - V4; 940 m; 2; e; Langourov $2001 \mathrm{a}$.

Megaselia albicans (Wood, 1908) - V4; $1360 \mathrm{~m} ; 3$; e; Langourov 2001a.

Megaselia albicaudata (Wood, 1910) - V4, RW; 390-1470 m; 1, 2, 3, 4; h; Langourov 2001a, 2011.

Megaselia albiclava Schmitz, 1926 - V4; 1350-1360 m; 3; e; Langourov 2001a.

Megaselia albocingulata (Strobl, 1906) - V4; 940-1006 m; 2; e; Langourov 2001a; Langourov et al. 2014.

Megaselia alticolella (Wood, 1909) - V4; 2108 m; 5; e; Langourov 2001a.

Megaselia altifrons (Wood, 1909) - V1, V4, RW; 390-1550 m; 1, 2, 3, 4; h; Langourov 2001a, 2004a, 2011.

Megaselia angusta (Wood, 1909) - V4, RW; 450-1120 m; 1, 2, 3; h; Langourov 2011; Langourov et al. 2014.

Megaselia aquilonia Schmitz, 1958 - V4; 1900 m; 4, 5; e; Langourov 2001a.

Megaselia basispinata (Lundbeck, 1920) - V1, V4, RW, RE; 160-1900 m; 1, 2, 3, 4, ? 5; hn; Langourov 2001a, 2004a, 2004b, 2011.

Megaselia beckeri (Wood, 1909) - V4; 1360-1620 m; 3, 4; h; Langourov 2001a. 
Megaselia berndseni (Schmitz, 1919) - V1, RW, RE; 390-1900 m; 1, 2, 3, 4; h; Disney 1991; Langourov 2001a, 2004b, 2011.

Megaselia bovista (Gimmerthal, 1848) - V4; 1900 m; 4; eswa; Langourov 2001a.

Megaselia brevicostalis (Wood, 1910) - B1, V4, RW, RE; 382-2150 m; 1, 2, 3, 4, 5; hn; Schmitz 1953; Langourov 2001a, 2004b, 2011.

Megaselia brevior (Schmitz, 1924) - V4; 940-954 m; 2; e; Langourov 2001a; Langourov et al. 2014.

Megaselia breviterga (Lundbeck, 1920) - V4, RW; 390-1118 m; 1, 2, 3; h; Langourov 2011; Langourov et al. 2014.

Megaselia brunneipennis Costa, 1857 - V4; 940-1360 m; 2, 3; e; Langourov $2001 \mathrm{a}$.

Megaselia campestris (Wood, 1908) - V4; 940-1360 m; 2, 3; e; Langourov 2001a.

Megaselia ciliata (Zetterstedt, 1848) - V1, V4; 550-2150 m; 1, 2, 3, 4, 5; e; Langourov 2001a, 2004a.

Megaselia cirriventris Schmitz, 1929 - V4; 1350-1900 m; 3, 4, ? 5; h; Langourov 2001 a.

Megaselia coei Schmitz, 1938 - V4; 1360-2000 m; 3, 4, 5; e; Langourov 2001a.

Megaselia conformis (Wood, 1909) - V4; 1900 m; 5; e, ? aa; Langourov 2001a.

Megaselia crassicosta (Strobl, 1892) - V4; 1040-2150 m; 3, 4, 5; e; Langourov 2001a.

Megaselia crassipes (Wood, 1909) - V4; 1270 m; 3; h; Langourov $2001 \mathrm{a}$.

Megaselia curvicapilla Schmitz, 1947 - V4, RW; 940-2150 m; 2, 3, 4, 5; eswa; Langourov 2001a, 2011.

Megaselia dahli (Becker, 1901) - V4, RW; 940-1360 m; 2, 3; e; Langourov 2001a, 2011.

Megaselia differens Schmitz, 1948 - V4; 1900 m; 5; e; Langourov $2001 \mathrm{a}$.

Megaselia discreta (Wood, 1909) - V4; 1270 m; 3; e; Langourov 2001a.

Megaselia diversa (Wood, 1909) - V4, O62, RW, RE; 260-2280 m; 1, 2, 3, 4, 5; eswa; Langourov 2001a, 2004b, 2011; Langourov \& Sakalian 2001.

Megaselia dubitalis (Wood, 1908) - V4; 1350-1360 m; 3; e; Langourov 2001a.

Megaselia eccoptomera Schmitz, 1927 - V4; 1550-1970 m; 4, 5; e; Langourov 2001 a.

Megaselia elongata (Wood, 1914) - V4; $1350 \mathrm{~m}$; 3; eswa; Langourov 2001a.

Megaselia emarginata (Wood, 1908) - V1, V4; 550-1350 m; 1, 2, 3; e; Langourov 2001a, 2004a.

Megaselia errata (Wood, 1912) - V4; 820-1270 m; 2, 3; e; Langourov 2001a.

Megaselia fenestralis (Schmitz, 1919) - V1; 550 m; 1; des; Langourov 2004a.

Megaselia filamentosa Schmitz, 1958 - RW; 450 m; 1; se, ? cse; Langourov 2011.

Megaselia flava (Fallen, 1823) - V4, RW; 490-1400 m; 1, 2, 3; ho; Langourov 2001a, 2011.

Megaselia flavicans Schmitz, 1935 - V4, O62; 210-1360 m; 1, 2, 3; e; Langourov 2001a; Langourov \& Sakalian 2001.

Megaselia flavicoxa (Zetterstedt, 1848) - V4; 1270 m; 3; e; Langourov 2001a.

Megaselia frameata Schmitz, 1927 - V4; 1400 m; 3; e; Langourov 2001a.

Megaselia frontalis (Wood, 1909) - V4; $940 \mathrm{~m} ; 2$; e; Langourov 2001a.

Megaselia fungivora (Wood, 1909) - V4; 1270 m; 3; h; Langourov 2001a.

Megaselia fusca (Wood, 1909) - B1, V4, RE; 160-2150 m; 1, 2, 3, 4, 5; e; troglophile; Czerny 1930; Buresch 1936; Guéorguiev \& Beron 1962; Langourov 2001a, 2001b, 2004b; Beron 2015.

Megaselia fusciclava Schmitz, 1935 - RW, RE; 160-1200 m; 1, 2, 3; e; Langourov 2004b, 2011.

Megaselia fuscipalpis (Lundbeck, 1920) - V4; 1360 m; 3; e; Langourov $2001 \mathrm{a}$.

Megaselia fuscovariana Schmitz, 1933 - V4; 820 m; 2; e; Langourov 2001a.

Megaselia giraudii (Egger, 1862) - V1, V4, RW; 390-1900 m; 1, 2, 3, 4, 5; ho; Langourov 2001a, 2004a, 2011.

Megaselia glabrifrons (Wood, 1909) - V4; 1350-1900 m; 3, 4, 5; h; Langourov 2001a.

Megaselia gregaria (Wood, 1910) - V4; 940 m; 2; e; Langourov 2001a.

Megaselia halterata (Wood, 1910) - B1, V1, V4, RE; 280-940 m; 1, 2; h; trogloxene; Langourov 2001a, 2001b, 2004a, 2004b; Beron 2015.

Megaselia hirsuta (Wood, 1910) - V4; 940-1900 m; 2, 3, 4, 5; e; Langourov 2001a.

Megaselia hirticaudata (Wood, 1910) - RE; 160 m; 1; e; Langourov 2004b.

Megaselia hirtiventris (Wood, 1909) - V1, V4, RE; 160-1270 m; 1, 2, 3; e; Langourov 2001a, 2004a, 2004b.

Megaselia horsfieldi Disney, 1986 - V4; 1900 m; 4; e; Langourov 2001a.

Megaselia humeralis (Zetterstedt, 1838) - RW; 1200 m; 3; e; Langourov 2011.

Megaselia hyalipennis (Wood, 1912) - V4; 1550 m; 4; des; Langourov 2001a.

Megaselia hypopygialis (Lundbeck, 1920) - V4; 1270 m; 3; e; Langourov $2001 \mathrm{a}$.

Megaselia infraposita (Wood, 1909) - RW; 390 m; 1; e; Langourov 2011. 
Megaselia intonsa Schmitz, 1948 - V4; 1270-2200 m; 3, 4, 5; e, bm; Langourov 2001 a.

Megaselia involuta (Wood, 1910) - V4, O62, RW; 260-1550 m; 1, 2, 3, 4; e; Langourov 2001a, 2011; Langourov \& Sakalian 2001.

Megaselia largifrontalis Schmitz, 1939 - V4; 900 m; 2; e; Langourov et al. 2014.

Megaselia lata (Wood, 1910) - V1, RW; 390-2108 m; 1, 2, 3, 4, 5; e; Langourov 2001a, 2011.

Megaselia latifemorata (Becker, 1901) - V4; 820-2150 m; 2, 3, 4, 5; des; Langourov 2001a.

Megaselia latifrons (Wood, 1910) - V4, R1, RW; 390-1900 m; 1, 2, 3, 4, 5; eswa; Schmitz 1953; Schmitz \& Delage 1974; Disney 1991; Langourov 2001a, 2011.

Megaselia longicostalis (Wood, 1912) - P3, B2, RW, RE; 160-600 m; 1; eswa; trogloxene; Langourov 2001b, 2004b, 2004c, 2011; Beron 2015.

Megaselia longiseta (Wood, 1909) - V4; 1600 m; 4; e; Langourov $2001 \mathrm{a}$.

Megaselia lucifrons (Schmitz, 1918) - V1, RW; 1360-1620 m; 3, 4; e, ? bm; Langourov 2001a, 2011.

Megaselia lutea (Meigen, 1830) - V4, RW; 390-1850 m; 1, 2, 3, 4; h; Langourov 2001a, 2011.

Megaselia mallochi (Wood, 1909) - V4, RW; 1270-2150 m; 3, 4, 5; e; Langourov 2001a, 2011.

Megaselia manicata (Wood, 1910) - V4; 1360 m; 3; e, ? m; Langourov 2001a.

Megaselia meconicera (Speiser, 1925) - V4, RW, RE; 160-1550 m; 1, 2, 3, 4; h; Langourov 2001a, 2004b, 2011.

Megaselia melanocephala (von Roser, 1840) - V4; 940 m; 2; dp; Langourov 2001a.

Megaselia nigra (Meigen, 1830) - ; ; h; Disney 1991.

Megaselia nigriceps (Loew, 1866) - V4; 1260-1830 m; 3, 4, 5; h; Schmitz 1957; Disney 1991; Langourov 2001a, 2004c.

Megaselia nigripalpis (Lundbeck, 1920) - V4; 1260-1900 m; 3, 4, 5; e; Langourov 2001a.

Megaselia obscuripennis (Wood, 1909) - V4; 1150-1850 m; 3, 4; e; Langourov 2001a.

Megaselia oxybelorum Schmitz, 1928 - V4; 1340-1900 m; 3, 4, 5; hom, ? mm; Langourov $2001 \mathrm{a}$.

Megaselia parva (Wood, 1909) - RW; 1470 m; 3; e; Langourov 2011.

Megaselia palmeni (Becker, 1901) - V4; 1550-2041 m; 4, 5; e; Langourov 2001a.

Megaselia pectinifera Schmitz, 1926 - V4; 1900 m; 4, 5; csee; Langourov 2001a.

Megaselia pectoralis (Wood, 1910) - V1, V4, RW; 550-2150 m; 1, 2, 3, 4, 5; e; Langourov 2001a, 2004a, 2004c, 2011.

Megaselia pectorella Schmitz, 1929 - V4; 1550 m; 4; e; Langourov 2001a.

Megaselia perfusca Schmitz, 1935 - V4; 1550-2150 m; 4, 5; e, ? csee; Langourov 2001a.

Megaselia perdistans (Schmitz, 1924) - RW; 390 m; 1; e; Langourov 2011.

Megaselia pleuralis (Wood, 1909) - P1, B1, B2, V1, V4, T3, T31, RW, RE; 160-1926 m; 1, 2, 3, 4, 5; h; trogloxene; Czerny 1930; Buresch 1936; Guéorguiev \& Beron 1962; Disney 1991; Langourov 2001a, 2001b, 2004a, 2004b, 2004c, 2011; Langourov et al. 2014; Beron 2015.

Megaselia plurispinulosa (Zetterstedt, 1860) - V1, V4; 550-1240 m; 1, 2, 3; des; Langourov 2001a, 2004a.

Megaselia posticata (Strobl, 1898) - V4; 820-992 m; 2; e; Langourov 2001a; Langourov et al. 2014.

Megaselia producta (Schmitz, 1921) - V4, RW, RE; 160-2150 m; 1, 2, 3, 4, 5; e, ? aa; Langourov 2001a, 2004b, 2011.

Megaselia propinqua (Wood, 1909) - V4; 1360-2150 m; 3, 4, 5; e; Langourov 2001a.

Megaselia protarsalis Schmitz, 1927 - V4; 1360-1470 m; 3; e; Langourov 2001a.

Megaselia pseudogiraudii (Schmitz, 1920) - V4; 1240-1270 m; 3; e; Langourov 2001a.

Megaselia pulicaria (Fallén, 1823) [? M. angusta (Wood, 1909)] - V4; 1140 m; 3; h; Langourov 2001a.

Megaselia pumila (Meigen, 1830) - V4, O62, RW; 210-1950 m; 1, 2, 3, 4, 5; ena; Langourov 2001a, 2004c, 2011; Langourov \& Sakalian 2001.

Megaselia pusilla (Meigen, 1830) - V4, O62, RW, RE; 260-2150 m; 1, 2, 3, 4, 5; h; Langourov 2001a, 2004b, 2011; Langourov \& Sakalian 2001; Langourov et al. 2014.

Megaselia pygmaea (Zetterstedt, 1848) - V4; 1130-1270 m; 3; e; Langourov $2001 \mathrm{a}$.

Megaselia quadriseta (Schmitz, 1918) - TL; 210 m; 1; e; Georgiev \& Langurov 1997.

Megaselia robusta Schmitz, 1928 - V4; 1900 m; 4, 5; h, bm; Langourov 2001a.

Megaselia rubella (Schmitz, 1920) - V4, RW; 390-1400 m; 1, 2, 3; e; Langourov 2001a, 2011.

Megaselia ruficornis (Meigen, 1830) - V4, RW; 390-1270 m; 1, 2, 3; h; Langourov 2001a, 2011; Langourov et al. 2014. 
Megaselia rufipes (Meigen, 1804) [Aphiochaeta heracleellae (Bouché, 1834); Phora incrassata] - DW, P1, B2, V1, V3, V4, T31, RW, RE, BN; 30-1350 m; 1, 2, 3; sk, ? k; trogloxene; Nedelkov 1912; Langourov 2001a, 2001b, 2004a, 2004b, 2011; Beron 2015.

Megaselia rupestris Schmitz, 1934 - V4; 1270 m; 3; ? cse, ? e; Langourov 2001a.

Megaselia scalaris (Loew, 1866) - V1, V4; 550-900 m; 1, 2; k; Langourov 2001a, 2004a.

Megaselia scutellaris (Wood, 1909) [M. scutellariformis (Schmitz, 1926)] - V4, RW, RE; 160-2150 m; 1, 2, 3, 4, 5; e; Schmitz 1953; Langourov 2001a, 2004b, 2011.

Megaselia sepulchralis (Lundbeck, 1920) - V4; 1550 m; 4; e; Langourov 2001a.

Megaselia sericata Schmitz, 1935 - V4; 1360 m; 3; csee; Langourov 2001a.

Megaselia setulipalpis Schmitz, 1938 - V4; 1270 m; 3; e; Langourov $2001 \mathrm{a}$.

Megaselia simplex (Wood, 1910) - V4; 1200 m; 3; e; Langourov 2001a.

Megaselia simulans (Wood, 1912) - V4, RW; 1360-1400 m; 3; des; Langourov 2001a, 2011.

Megaselia sordida (Zetterstedt, 1838) - V4; RW; 1620-2226 m; 4, 5; h; Langourov 2001a, 2011.

Megaselia spinata (Wood, 1910) - V4; $1360 \mathrm{~m}$; 3; e; Langourov 2001a.

Megaselia spinigera (Wood, 1908) - V4; 820-1900 m; 2, 3, 4, 5; e, bm; Langourov 2001a.

Megaselia stichata (Lundbeck, 1920) - V1, V4; 550-1270 m; 1, 2, 3; eswa; Langourov 2001a, 2004a, 2004c.

Megaselia striolata Schmitz, 1940 - RW; 1350 m; 3, 4; e; Langourov 2011.

Megaselia styloprocta (Schmitz, 1921) - V4; 1200-1360 m; 3; e; Langourov $2001 \mathrm{a}$.

Megaselia subconvexa (Lundbeck, 1920) - V4; 940-1360 m; 2, 3; e; Langourov 2001a.

Megaselia subfuscipes Schmitz, 1935 [M. capronata Schmitz, 1940] - V4, RW; 390-1550 m; 1, 2, 3, 4; eswa; Langourov 2001a, 2011.

Megaselia subnudipennis (Schmitz, 1919) - RW; 1470 m; 3; e; Langourov 2011.

Megaselia subpleuralis (Wood, 1909) - V4; 1270-1550 m; 3, 4; h; Langourov $2001 \mathrm{a}$.

Megaselia subtumida (Wood, 1909) - V4, RW; 657-1400 m; 1, 2, 3; e; Langourov 2001a, 2011; Langourov et al. 2014.

Megaselia superciliata (Wood, 1910) - RW; 1200-1926 m; 3, 4; eswa; Langourov 2011.

Megaselia sylvatica (Wood, 1910) - V4; 1350-1360 m; 3; e; Langourov 2001a.

Megaselia tama (Schmitz, 1926) - V4; 820-940 m; 2; e; Langourov $2001 \mathrm{a}$.

Megaselia tarsalis (Wood, 1910) - V4; 820-2150 m; 2, 3, 4, 5; e; Langourov $2001 \mathrm{a}$.

Megaselia tarsella (Lundbeck, 1921) - V1, V4; 550-2150 m; 1, 2, 3, 4, 5; e; Langourov 2001a, 2004a.

Megaselia tarsicia Schmitz, 1926 - V4; 1270-2108 m; 3, 4, 5; e, ? bm; Langourov 2001a.

Megaselia tenebricola Schmitz, 1934 - P1, V4; 195-1120 m; 1, 2, 3; e; troglophile; Langourov 2001a, 2001b; Beron 2015.

Megaselia tergata (Lundbeck, 1920) - V1; 550 m; 1; e; Langourov 2004a.

Megaselia trichorrhoea (Schmitz, 1921) [M. smirnovi Naumov, 1979] - V4; 1360 m; 3; e; Langourov 2001a.

Megaselia tumida (Wood, 1909) - V4; 1270 m; 3; ena; Langourov 2001a.

Megaselia uliginosa (Wood, 1909) - V4; $1270 \mathrm{~m}$; 3; e; Langourov 2001a.

Megaselia unicolor (Schmitz, 1919) - V4, RE; 160-2150 m; 1, 2, 3, 4, 5; des, ? bm; Langourov 2001a, 2004b.

Megaselia variana Schmitz, 1926 - V4; 1270-1350 m; 3; e; Langourov 2001a.

Megaselia verna Schmitz, 1932 - V4, O62; 260-1900 m; 1, 2, 3, 4; e; Langourov 2001a; Langourov \& Sakalian 2001.

Megaselia vernalis (Wood, 1909) - V4, RE; 160-2150 m; 1, 2, 3, 4, 5; e; Langourov 2001a, 2004b.

Megaselia verralli (Wood, 1910) - V4, RE; 410-940 m; 1, 2; eswa; Langourov 2001a, 2004b.

Megaselia vestita (Wood, 1914) - V4, RW; 1200-1470 m; 3; e; Langourov 2001a, 2011.

Megaselia woodi (Lundbeck, 1922) - V4; 1240-2150 m; 3, 4, 5; e, bm; Langourov 2001a.

Megaselia xanthozona (Strobl, 1892) - V4, RW; 820-1926 m; 2, 3, 4, 5; ena; Langourov 2001a, 2011.

Menozziola schmitzi (Menozzi, 1921) - V4; 1350 m; 3; e; Langourov 2001a.

Metopina braueri (Strobl, 1880) - V4; 940 m; 2; e; Langourov 2001a.

Metopina crassinervis Schmitz, 1920 - V4; 940 m; 2; e; Langourov 2001a.

Metopina galeata (Haliday, 1833) - V4; 940-1006 m; 2; e; Langourov 2001a; Langourov et al. 2014.

Metopina heselhausi Schmitz, 1914 - V4; 940-1900 m; 2, 3, 4, 5; e; Langourov 2001a.

Metopina oligoneura (Mik, 1867) - V4; 940-2020 m; 2, 3, 4, 5; e; Langourov 2001a.

Metopina perpusilla (Six, 1878) - V4; 870-940 m; 2; e; Langourov 2001a.

Metopina pileata Schmitz, 1936 - V4; 850-1360 m; 2, 3; ena; Langourov 2001 a. 
Metopina ulrichi Disney, 1979 - V4; 940 m; 2; e; Langourov 2001a.

Phalacrotophora berolinensis Schmitz, 1920 - V1; 550 m; 1; e; Langourov 2004a.

Phalacrotophora fasciata (Fallen, 1823) - V1, V4; 550-940 m; 1, 2; dp; Langourov 2001a, 2004a.

Phalacrotophora pictofasciata Schmitz, 1919 - V1; 550 m; 1; see; Langourov 2004a.

Obscuriphora sheppardi Disney, 1986 - RW; 1000 m; 2, 3; e; Langourov 2011.

Phora atra (Meigen, 1804) - V1, V4, RE; 120-1090 m; 1, 2, 3; h; Langourov 2001a, 2004a, 2004b.

Phora dubia (Zetterstedt, 1848) - V1; 820-2100 m; 2, 3, 4, 5; dp, ? des; Langourov 2001a.

Phora edentata Schmitz, 1920 - V1, V4, RE; 300-940 m; 1, 2; dp; Langourov 2001a, 2004a, 2004b.

Phora hamata Schmitz, 1927 - V4, RE; 300-1270 m; 1, 2, 3; e; Langourov 2001a, 2004b.

Phora holosericea Schmitz, 1920 - V1, V4, RW; 550-1400 m; 1, 2, 3; h; Langourov 2001a, 2004a, 2011.

Phora horrida Schmitz, 1920 - V4; 1900 m; 4, 5; csee, ? e; Langourov $2001 \mathrm{a}$.

Phora penicillata Schmitz, 1920 - V4, RW; 1200-2050 m; 3, 4, 5; e; Langourov 2001a, 2011.

Phora pubipes Schmitz, 1920 - V4; 1200-1850 m; 3, 4; po; Langourov 2001 a.

Phora stictica Meigen, 1830 - V4, RW; 1200-2000 m; 3, 4, 5; hn; Langourov 2001a, 2011.

Phora tincta Schmitz, 1920 - V4, RW; 820-1750 m; 2, 3, 4; e; Langourov 2001a, 2011.

Plectanocnema nudipes (Becker, 1901) - V4; $940 \mathrm{~m}$; 2; h; Langourov 2001a.

Pseudacteon fennicus Schmitz, 1927 - RW; 390 m; 1; e; Langourov 2011.

Spiniphora bergenstammi (Mik, 1864) - V1, V4; 550-940 m; 1, 2; hna; Beschovski \& Langourov 1997; Langourov 2001a, 2004a.

Spiniphora dorsalis (Becker, 1901) - V4; 1360-1850 m; 3, 4; e; Langourov 2001a.

Spiniphora jugorum (Schmitz, 1924) - V4; 1550-2173 m; 4, 5; cse; Langourov 2001a.

Spiniphora maculata (Meigen, 1830) - V4, RE; 200-2150 m; 1, 2, 3, 4, 5; e; Langourov 2001a, 2004b.

Triphleba antricola (Schmitz, 1918) - DW, E1, P1, P2, B1, B2, B3, V4, T3, T31, O1, R2, RW, RE; 100-1650 m; 1, 2, 3, 4; e; troglophile; Hazelton 1970; Langourov 2001a, 2001b, 2004b, 2011; Beron et al. 2004, 2011; Langourov et al. 2014; Beron 2015.

Triphleba aptina (Schiner, 1853) - B1; 818 m; 2; cse; troglophile; Langourov 2001b; Beron 2015.

Triphleba autumnalis (Becker, 1901) - V4, RE; 160-1550 m; 1, 2, 3, 4; e; Beschovski \& Langourov 1997; Langourov 2001a, 2004b.

Triphleba bicornuta (Strobl, 1910) - V4, R1; 940-2200 m; 2, 3, 4, 5; e, bm; Beschovski \& Langourov 1997; Langourov 2001a.

Triphleba citreiformis (Becker, 1901) - V4; 1350 m; 3; e; Langourov 2001a.

Triphleba distinguenda (Strobl, 1892) - V1, V4, RW; 550-1650 m; 1, 2, 3, 4; des; Beschovski \& Langourov 1997; Langourov 2001a, 2004a, 2011; Langourov et al. 2014.

Triphleba dudai (Schmitz, 1918) - V4; 1080-1360 m; 2, 3; csee, ? e; Beschovski \& Langourov 1997; Langourov 2001a.

Triphleba gracilis (Wood, 1907) - V4; 820-1750 m; 2, 3, 4; e; Beschovski \& Langourov 1997; Langourov 2001 a.

Triphleba hyalinata (Meigen, 1830) - B2, V1, V4, RE; 75-1270 m; 1, 2, 3; e; Langourov 2001a, 2001b, 2004a, 2004b; Langourov et al. 2014.

Triphleba hypopygialis (Schmitz, 1918) - V4; 1550-1900 m; 4, 5; e, ? cse; Langourov $2001 \mathrm{a}$.

Triphleba inaequalis Schmitz, 1943 - V4; 900-1270 m; 2, 3; e; Langourov 2001a; Langourov et al. 2014.

Triphleba intermedia (Malloch, 1908) - V4, RW, RE; 160-2200 m; 1, 2, 3, 4, 5; e; Langourov 2001a, 2004b, 2011.

Triphleba longifurcata (Schmitz, 1922) - V4; 1360 m; 3; csee; Langourov 2001a.

Triphleba lugubris (Meigen, 1830) - V4; 940-1270 m; 2, 3; ho; Langourov 2001a.

Triphleba nudipalpis (Becker, 1901) - V4, O62; 200-940 m; 1, 2; des; Langourov 2001a; Langourov \& Sakalian 2001.

Triphleba opaca (Meigen, 1830) - V1, V4; 550-2280 m; 1, 2, 3, 4, 5; des, ? bm; Nedelkov 1912; Beschovski \& Langourov 1997; Langourov 2001a.

Triphleba pachyneurella (Schmitz, 1919) - V4; 1650-2280 m; 4, 5; e; Langourov 2001a.

Triphleba papillata (Wingate, 1906) - V4, RE; 200-1650 m; 1, 2, 3, 4; e; Langourov 2001a, 2004b; Langourov et al. 2014.

Triphleba trinervis (Becker, 1901) [T. opaca (Meigen, 1830)] - V1, V4, RE; 160-2150 m; 1, 2, 3, 4, 5; h; Beschovski \& Langourov 1997; Langourov 2001a, 2004a, 2004b.

Triphleba tumidula (Schmitz, 1918) - V4, RE; 160-1200 m; 1, 2, 3; e; Langourov 2001a, $2004 \mathrm{~b}$. 
Triphleba vitrea (Wood, 1906) - V4; 1930 m; 4, 5; e; Langourov $2001 \mathrm{a}$.

Triphleba withersi Disney, 1994 - V4; 820-940 m; 2; se; Langourov 2001a.

Tubicera lichtwardti Schmitz, 1920 - V4; 1240 m; 3; se, ? cse; Langourov 2001a.

\section{Pipunculidae (Dorylaidae)}

Verrallia aucta (Fallén, 1817) - O62, R2; 200-600 m; 1; h; Lauterer 1983.

Pipunculus campestris Latreille, 1802 [P. ater Meigen, 1824] - V4, R1; 770-1000 m; 2; ho; Bankowska 1967a. Pipunculus spinipes Meigen, 1830 - RW; 390-400 m; 1; wes; Bankowska 1967a; Beschovski 2006a.

Pipunculus thomsoni Becker, 1897 - V1, V4, S1; 300-800 m; 1, 2; e; Nedelkov 1910, 1912; Bankowska 1967a.

Cephalosphaera (Cephalosphaera) furcata (Egger, 1860) [Pipunculus] - V4; 800 m; 2; tp; Bankowska 1967a; Tanasijtshuk 1988.

Cephalops (Cephalops) aeneus Fallen, 1810 [C. pratorum Fallén, 1816; Pipunculus] - V1, V4, S1; 300-900 m; 1, 2; tp; Nedelkov 1910, 1912; Bankowska 1967a.

Dasydorylas horridus (Becker, 1897) [Eudorylas, Dorylas] - R2, BN; 0-300 m; 1; e; Beschovski 1972a; Lauterer 1983.

Eudorylas fuscipes (Zetterstedt, 1844) - e; Beschovski \& Manassieva 1995.

Eudorylas fusculus (Zetterstedt, 1844) - R2; 1500 m; 4; e; Lauterer 1983.

Eudorylas jenkinsoni Coe, 1966 - R1; 2729 m; 6; dp; Lauterer 1983.

Eudorylas pannonicus (Becker, 1897) - DW; 240 m; 1; e; Beschovski \& Manassieva 1995.

Eudorylas ruralis (Meigen, 1824) - E2, S1, R2, BS; 0-1750 m; 1, 2, 3, 4; wp, ? ena; Lauterer 1983; Beschovski \& Manassieva 1995.

Eudorylas subterminalis Collin, 1956 - R2; 300-1400 m; 1, 2, 3; ? e; Lauterer 1983.

Eudorylas terminalis (Thomson, 1870) - R2; 1250-1430 m; 3; e; Lauterer 1983.

Eudorylas unicolor (Zetterstedt, 1844) [Dorylus] - BN; 0-5 m; 1; e; Beschovski 1972a.

Eudorylas zonatus (Zetterstedt, 1849) [Pipunculus] - O62; 100-250 m; 1; e; Bankowska 1967a; Lauterer 1983; Tanasijtshuk 1988.

Eudorylas zonellus Collin, 1956 - R2; 400-950 m; 1, 2; e; Lauterer 1983.

Dorylomorpha (Dorylomorpha) confusa (Verrall, 1901) - R2; 1250-1900 m; 3, 4; dp; Lauterer 1983.

Dorylomorpha (Dorylomyza) haemorrhoidalis (Zetterstedt, 1838) [Dorylas] - BN; 0-5 m; 1; des; Beschovski 1972a.

Dorylomorpha (Dorylomyia) incognita (Verrall, 1901) - R2; 930-2000 m; 2, 3, 4; tes, ? des; Lauterer 1983; Tanasijtshuk 1988.

Tomosvaryella cilifemorata (Becker, 1907) - O62, R2; 200-600 m; 1; ena; Lauterer 1983.

Tomosvaryella coquilletti (Kertesz, 1907) - DM, P1, V4, V5, S1, TL, O4, O61, O62, R2, BN; 0-1900 m; 1, 2, 3, 4; ho; Lauterer 1983; Tanasijtshuk 1988; Beschovski \& Manassieva 1995.

Tomosvaryella geniculata (Meigen, 1824) [Pipunculus nigritula Zetterstedt, 1844] - V1, V4, R1, R2; 300-1475 m; 1, 2, 3, 4; ena, ? wp; Nedelkov 1910, 1012; Lauterer 1983.

Tomosvaryella kuthyi Aczel, 1944 - TL, R2; 120-950 m; 1, 2; ean; Lauterer 1983.

Tomosvaryella minima (Becker, 1897) - S1; 650-700 m; 1, 2; eanna, ? wp; Beschovski \& Manassieva 1995.

Tomosvaryella mutata (Becker, 1897) - R2; 600-650 m; 1; hom; Beschovski \& Manassieva 1995.

Tomosvaryella sylvatica (Meigen, 1824) - DM, V4, S1, R2; 250-2350 m; 1, 2, 3, 4, 5; ho; Bankowska 1967a; Lauterer 1983; Beschovski \& Manassieva 1995.

\section{Syrphidae (Microdontidae)}

Dasysyrphus albostriatus (Fallén, 1817) [Syrphus albostriatus var. confusus Egger, 1860] - E2, V1, V4, R1, RW, BN; 0-1400m; 1, 2, 3; tp; Nedelkov 1912; Drensky 1934a, 1942, 1955; Bankowska 1967b; Beschovski 2006a; Markova 2006.

Dasysyrphus friuliensis (van der Goot, 1960) [Dasysyrphus venustus var. friuliensis (van der Goot, 1960)] - V4; \$; hoes; Bankowska 1967b; Peck 1988.

Dasysyrphus tricinctus (Fallén, 1817) [Syrphus] - O62; 250-300 m; 1; tp; Drensky 1934a. 
Dasysyrphus venustus (Meigen, 1822) [Syrphus arcuatus (Fallén, 1817); S. lunulatus Meigen, 1822] - B1, K6, V1, V4, R1, R2, RW; 260-2000 m; 1, 2, 3, 4; h; Nedelkov 1912; Drensky 1934a; Szilády 1934; Bankowska 1967b; Markova 1997, 2006; Beschovski 2006a.

Didea alneti (Fallén, 1817) - R1, RW; 2076-2925 m; 5, 6; h; Drensky 1934a; Beschovski 2006a; Markova 2006. Didea fasciata Macquart, 1834 - RW; 2076 m; 4, 5; po; Drensky 1934a; Beschovski 2006a; Markova 2006.

Doros profuges (Harris, 1780) [D. conopseus (Fabricius, 1775); D. destillatorius Mik, 1885] - R1, BS; 0-1400 m; 1, 2, 3; hoes, ? tp; Drensky 1934a; Markova 2011.

Epistrophe diaphana (Zetterstedt, 1843) - R1; 1200-1400; 3; esca; Drensky 1934a.

Epistrophe eligans (Harris, 1780) [E. bifasciata (Fabricius, 1794); Syrphus] - V1, R1, R2; 550-1400 m; 1, 2, 3; et; Nedelkov 1912; Drensky 1934a; Bankowska 1967b; Peck 1988.

Epistrophe grossulariae (Meigen, 1822) [Syrphus] - B1, K9, V1, R1; 350-1100 m; 1, 2, 3; h; Nedelkov 1912; Drensky 1934a; Szilády 1934; Markova 1998.

Epistrophe melanostoma (Zetterstedt, 1843) [Syrphus] - B1, V1, RW; 350-600 m; 1, 2; tp, ? hoes; Nedelkov 1912; Drensky 1934a; Peck 1988; Beschovski 2006a; Markova 2006.

Epistrophe nitidicollis (Meigen, 1822) [Syrphus] - DM, P2, B1, K4, V1, R1; 100-1400 m; 1, 2, 3; h; Nedelkov 1912; Drensky 1934a; Markova 1994a, 1994c.

Epistrophe ochrostoma (Zetterstedt, 1849) [Syrphus] - V1, V4, O62; 250-1100 m; 1, 2, 3; hoes, ? h; Drensky 1934a; Bankowska 1967b; Peck 1988; Markova 1998.

Epistrophella euchroma (Kowarz, 1885) [Epistrophe] - R1; 1500-1550 m; 3, 4; hoes; Drensky 1934a.

Episyrphus balteatus (De Geer, 1776) [Syrphus, Epistrophe] - ; DW, DM, E2, P2, B1, B2, K2, K6, K9, V1, V4, S1, TL, T31, R1, O62, RW, RE, BS; 0-1350 m; 1, 2, 3; poa; Joakimoff 1899; Nedelkov 1912; Drensky 1928, 1934a; Bankowska 1967b; Kontev et al. 1991; Markova \& Tsonev 1993; Markova 1994a, 1994b, 1997, 1998, 1998a, 2003, 2006, 2011; Markova \& Dimcheva 1998; Markova \& Alexiev 2000.

Eriozona syrphoides (Fallén, 1817) - RW; 1400-1600 m; 3, 4; tp; Drensky 1934a; Beschovski 2006a; Markova 2006.

Leucozona glaucia (Linnaeus, 1758) [Ischyrosyrphus] - V1; 550-600 m; 1, 2; tp; Drensky 1934a.

Leucozona laternaria (Muller, 1776) [Ischyrosyrphus] - V1; 550-600 m; 1, 2; tp; Drensky 1934a.

Leucozona lucorum (Linnaeus, 1758) - R1; 1500-2300 m; 3, 4, 5; h; Drensky 1934a.

Eriozona erratica (Linnaeus, 1758) - [Megasyrphus annulipes (Zetterstedt, 1838); Syrphus] - B1; 260-380 m; 1; tp; Drensky 1934a.

Melangyna barbifrons (Fallén, 1817) [Epistrophe] - V1; 550-600 m; 1, 2; hoes; Drensky 1934a.

Melangyna compositarum (Verrall, 1873) - S1; 800-850 m; 2; h; Markova \& Tsonev 1993.

Melangyna lasiophthalma (Zetterstedt, 1843) [Epistrophe, Syrphus] - O62, R1, R3; 280-1800 m; 1, 2, 3, 4; h; Drenowsky 1936, 1939; Drensky 1939a; Bankowska 1967b; Peck 1988.

Melangyna quadrimaculata Verrall, 1873 - B1, V1; 700-720 m; 2; des; Drensky 1934a.

Melangyna umbellatarum (Fabricius, 1794) [Epistrophe] - BN; 0-5 m; 1; h; Drensky 1934a; Peck 1988.

Meligramma cincta (Fallen, 1817) [Epistrophe, Syrphus] - B3, V4, RW; 270-1000 m; 1, 2; dp; Nedelkov 1912; Drensky 1934a; Bankowska 1967b; Markova 2006.

Meligramma guttata (Fallen, 1817) [Epistrophe] - R1; 1200-1400 m; 3; h; Drensky 1934a.

Meligramma triangulifera (Zetterstedt, 1843) [Epistrophe] - S1, R1; 400-1400 m; 1, 2, 3; h; Drensky 1934a; Markova 2003.

Meliscaeva auricollis (Meigen, 1822) [Epistrophe, Syrphus] - E2, B2, V1, V4, T31, O62, R1, R3, RW, BS; 0-1500 m; 1, 2, 3, 4; wp; Nedelkov 1912; Drensky 1934a; Drenowsky 1936; Bankowska 1967b; Kontev et al. 1991; Markova \& Alexiev 2000; Beschovski 2006a; Markova 2006, 2011.

Meliscaeva cinctella (Zetterstedt, 1843) [Syrphus] - R1, R2; 1170-1200 m; 3; ho; Bankowska 1967b.

Eupeodes corollae (Fabricius, 1794) [Metasyrphus, Syrphus] - DM, E2, P2, B1, B2, K4, K6, K9, V1, V3, V4, S1, TL, T2, T31, O61, O62, R1, R2, RW, BN, BS; 0-1800 m; 1, 2, 3, 4; ppta; Nedelkov 1912; Drensky 1928, 1934a, 1942, 1955; Bankowska 1967b; Kontev et al. 1991; Markova \& Tsonev 1993; Markova 1994a, 1994b, 1994c 1997, 1998, 1998a, 2003, 2006, 2011; Markova \& Dimcheva 1998; Markova \& Alexiev 2000; Beschovski 2006a;

Eupeodes flaviceps (Rondani, 1857) [Syrphus braueri Egger, 1858; Metasyrphus] - B1; 260-270 m; 1; west; Drensky 1934a.

Eupeodes lapponicus (Zetterstedt, 1838) [Metasyrphus, Syrphus] - V4, T31, R1, R2, BS; 0-1400 m; 1, 2, 3; h; Drensky 1934a; Bankowska 1967b; Peck 1988; Markova 2011. 
Eupeodes latifasciatus (Macquart, 1829) [Metasyrphus, Syrphus] - DM, E1, P2, K6, V1, V4, S1, T31, R1, BS; 0-1400 m; 1, 2, 3; ho; Nedelkov 1912; Drensky 1934a; Szilády 1934; Markova 1994a, 1994b, 1994c 1997, 2003, 2011; Markova \& Alexiev 2000.

Eupeodes luniger (Meigen, 1822) [Syrphus bucculatus Rondan, 1857; Metasyrphus] - B2, V1, V4, S22, R1, R2, R3, RW, BS; 0-1450 m; 1, 2, 3, 4; ho; Nedelkov 1912; Drensky 1934a; Drenowsky 1936; Bankowska 1967b; Markova \& Tsonev 1993; Markova 1994a, 1994b, 1997, 1998, 2003, 2006; Beschovski 2006a.

Eupeodes nitens (Zetterstedt, 1843) [Syrphus] - V4, T31, R1, R2, RW, BS; 0-1500 m; 1, 2, 3; tp; Drensky 1934a; Tschorbadjiew 1939b; Bankowska 1967b; Markova2006, 2011.

Parasyrphus annulatus (Zetterstedt, 1838) [Epistrophe] - BN; 0-15 m; 1; tp; Drensky 1934a.

Parasyrphus lineolus (Zetterstedt, 1843) [Syrphus, Epistrophe] - V4, R1, R2; 1200-1800 m; 3, 4; h; Bankowska 1967b; Peck 1988.

Parasyrphus macularis (Zetterstedt, 1843) - V1; 550-600 m; 1, 2; h; Drensky 1934a.

Parasyrphus malinellus (Collin, 1952) [Syrphus] - V4, R1, R2; 1500-1800 m; 3, 4; des; Bankowska 1967b; Peck 1988.

Parasyrphus nigritarsis (Zetterstedt, 1843) - S1; 450-500 m; 1; h; Markova 2003.

Parasyrphus tarsatus (Zetterstedt, 1838) [Epistrophe] - V1; 550-600 m, 1, 2; h; Drensky 1934a.

Parasyrphus vittiger (Zetterstedt, 1843) [Epistrophe lineola vitigera (Zetterstedt, 1843)] - S1, R1; 450-1400 m; 1, 2, 3, 4; tes; Szilády 1934; Markova 2003.

Scaeva albomaculata (Macquart, 1842) [Lasiopthicus] - BS; 0-20 m; 1; wpo; Drensky 1934a; Markova 2011.

Scaeva pyrastri (Linnaeus, 1758) [Lasiopthicus] - E2, P1, B1, B3, V1, V4, S1, T31, R1, R2, R3, RW, RE, BS; 0-1500 m; 1, 2, 3, 4; ho; Meunier 1897; Nedelkov 1912; Drensky 1934a; Szilády 1934; Drenowsky 1936; Bankowska 1967b; Peck 1988; Kontev et al. 1991; Markova \& Tsonev 1993; Markova \& Dimcheva 1998; Markova 1998a, 2003, 2006, 2011; Markova \& Alexiev 2000; Beschovski 2006a.

Scaeva selenitica (Meigen, 1822) [Lasiopthicus, Syrphus] - - DM, P2, B1, V1, V4, S1, TL, T31, RW, BS; 0-1300 m; 1, 2, 3; ho, ? po; Löw 1862; Nedelkov 1912; Drensky 1934a; Bankowska 1967b; Peck 1988; Markova 1998a, 2003, 2006, 2011; Markova \& Alexiev 2000; Beschovski 2006a.

Sphaerophoria interrupta (Fabricius, 1805) [S. menthastri (Linnaeus, 1758); S. picta (Meigen, 1824)] - DM, P1, P2, K6, V1, R1, RW, RE; 30-1400 m; 1, 2, 3; hop; Nedelkov 1912; Drensky 1934a; Bankowska 1967b; Markova 1994a, 1994b, 2006.

Sphaerophoria philantha (Meigen, 1822) [S. dubia Zettersdedt, 1849] - DM, P2, K6, V1, V4, S1, T2, T31, R1, BS; 0-1000 m; 1, 2; h; Bankowska 1967b; Peck 1988; Markova 1994a, 1994b, 1994c, 1997, 1998, 2011; Markova \& Alexiev 2000.

Sphaerophoria rueppelli (Wiedemann, 1830) [S. flavicauda var. nitidicollis Zettersdedt, 1849; S. flavicauda Zettersdedt, 1843] - DM, P2, B3, K6, K9, V1, S1, T2, T31, O62, R3, RW, RE, BN, BS; 0-1500 m; 1, 2, 3, 4; hop; Nedelkov 1912; Drensky 1934a; Drenowsky 1936; Bankowska 1964, 1967b; Peck 1988; Markova \& Tsonev 1993; Markova 1994a, 1994b, 1994c, 1997, 1998, 2003, 2006, 2011; Markova \& Dimcheva 1998; Markova \& Alexiev 2000.

Sphaerophoria scripta (Linnaeus, 1758) [S. dispar (Loew, 1840); S. strigata Staeger, 1845; Melithreptus] - ; DM, E2, P2, B1, B2, B3, K2, K6, K9, V1, V4, S1, S21, S22, TL, T2, T31, R1, R2, R3, RW, RE, BN, BS; 0-1900 m; 1, 2, 3, 4; ho; Joakimoff 1899; Nedelkov 1909, 1912; Drensky 1934a 1942, 1955; Szilády 1934; Drenowsky 1936; Bankowska 1964, 1967b; Peck 1988; Kontev et al. 1991; Markova \& Tsonev 1993; Markova 1994a, 1994b, 1994c, 1997, 1998, 1998a, 2003, 2006, 2011; Markova \& Dimcheva 1998; Markova \& Alexiev 2000.

Sphaerophoria taeniata (Meigen, 1822) [S. menthastri var. taeniatus Meigen, 1822] - DM, P2, V1, V4, S21, S22, TL, BN, BS; 0-1000 m; 1, 2; tp; Nedelkov 1909, 1912; Drensky 1934a; Bankowska 1964, $1967 \mathrm{~b}$.

Syrphus ribesii (Linnaeus, 1758) - $\$$; 2, V1, V4, S1, S21, T31, R1, R2, RW, RE, BS; 0-1500 m; 1, 2, 3; h; Nedelkov 1912; Drensky 1934a; Bankowska 1967b; Peck 1988; Markova \& Tsonev 1993; Markova \& Dimcheva 1998; Markova \& Alexiev 2000; Beschovski 2004a, 2006a; Markova 2006, 2011.

Syrphus torvus Osten-Sacken, 1875 - E2, V4, S1, R2, RW, BN; 0-1500 m; 1, 2, 3; ho; Drensky 1934a; Bankowska 1967b; Peck 1988; Kontev et al. 1991; Markova \& Tsonev 1993; Markova 2003, 2006.

Syrphus vitripennis Meigen, 1822 - \$ E2, K9, V1, V4, S1, S22, TL, T31, R1, R2, R3, RW, RE, BS; 0-1500 m; 1, 2, 3; ho; Nedelkov 1909, 1912; Drensky 1934a; Szilády 1934; Drenowsky 1936; Bankowska 1967b; Peck 1988; Kontev et al. 1991; Markova \& Tsonev 1993; Markova 1997, 1998a, 2003, 2006, 2011; Markova \& Alexiev 2000; Beschovski 2006a. 
Xanthogramma citrofasciatum (De Geer, 1776) - V4; 870-900 m; 2; weswca; Drensky 1934a; Bankowska 1967b; Peck 1988.

Xanthogramma laetum (Fabricius, 1794) - R3; 1000-1500 m; 3; des; Drensky 1934a; Peck 1988.

Xanthogramma pedissequum (Harris, 1776) [X. ornatum (Meigen, 1822); X. ornatum var. dives (Rondani, 1857)] - DM, P2, B1, B2, V1, V4, O62, R1, RW; 30-1600 m; 1, 2, 3; tp; Nedelkov 1909, 1912; Drensky 1934a; Szilády 1934; Bankowska 1967b; Peck 1988; Beschovski 2006a; Markova 2006.

Baccha elongata (Fabricius, 1775) [B. obscuripennis Meigen, 1822] - P2, V1, TL, R1, R2, RW, BS; 0-1350 m; 1, 2, 3; h; Nedelkov 1912; Drensky 1934a; Szilády 1934; Bankowska 1967b; Peck 1988; Beschovski 2006a; Markova 2006.

Chrysotoxum arcuatum (Linnaeus, 1758) - R1, RW, RE; 230-1550 m; 1, 2, 3; po; Nedelkov 1912; Drensky 1934a; Peck 1988; Beschovski 2006a; Markova 2006.

Chrysotoxum bicinctum (Linnaeus, 1758) - DM, E1, P2, B1, V1, V4; 100-900 m; 1, 2; wcp, ? tp; Nedelkov 1912; Drensky 1934a; Peck 1988; Markova 1994a, 1998a.

Chrysotoxum cautum (Harris, 1776) - V1, V4, TL, RW, RE, BN; 0-1000 m; 1, 2; weswca; Löw 1862; Drensky 1934a; Bankowska 1967b; Peck 1988; Markova 1998a, 2006.

Chrysotoxum cisalpinum Rondani, 1845 - R3; set; Drensky 1934a.

Chrysotoxum elegans Loew, 1841 - P1, B1, V1, V4, S1, T31, R1, RW, BN; 0-1550 m; 1, 2, 3; et, ? e; Nedelkov 1912; Drensky 1934a, 1942; Bankowska 1967b; Peck 1988; Markova 1998a, 2003, 2006.

Chrysotoxum fasciolatum (De Geer, 1776) - V4, S211, R1; 770-1550 m; 2, 3; h; Drensky 1934a; Bankowska 1967b; Peck 1988.

Chrysotoxum festivum (Linnaeus, 1758) [Lasiopticus] - B1, B2, V1, V4, O61, R1, R2, R3, BN, BS; 0-1900 m; 1, 2, 3, 4; po; Löw 1862; Joakimoff 1899; Nedelkov 1912; Drensky 1934a; Drenowsky 1936; Bankowska 1967b; Peck 1988; Markova 1998a; Markova \& Alexiev 2000.

Chrysotoxum impressum Becker, 1921 - V4, T31, R3; 250-1500 m; 1, 2, 3; se; Drensky 1934a; Bankowska 1967b; Peck 1988; Markova 2011.

Chrysotoxum intermedium (Meigen, 1822) - TL, T31, R1, RW, BS; 0-1550 m; 1, 2, 3; wp; Drensky 1934a; Drenowsky 1936; Bankowska 1967b; Peck 1988; Markova \& Alexiev 2000; Beschovski 2006a; Markova 2006, 2011.

Chrysotoxum lineare (Zetterstedt, 1819) - V4; 800 m; 2; e; Drensky 1934a; Peck 1988.

Chrysotoxum octomaculatum Curtis, 1837 - V1, V4, R1; 600-1400 m; 2, 3; wes; Nedelkov 1912; Drensky 1934a; Bankowska 1967b; Peck 1988.

Chrysotoxum parmense Rondani, 1845 - T31, BS; 0-320 m; 1; set; Drensky 1934a; Peck 1988; Markova 2011.

Chrysotoxum rhodopense Drensky, 1934 - RW; 1000-1300 m; 3; Er; Drensky 1934a; Peck 1988; Beschovski 2006a; Markova 2006.

Chrysotoxum vernale Loew, 1841 - $\$$; B, V1, V4, TL, T31, R1, R2, RW, RE; 0-1500 m; 1, 2, 3; esca; Nedelkov 1912; Drensky 1934a; Drenowsky 1936; Bankowska 1967b; Peck 1988; Beschovski 2006a; Markova 2006, 2011.

Chrysotoxum verralli Collin, 1940 - K4, K6, V4; 650-1200 m; 2, 3; vces; Bankowska 1967b; Peck 1988; Markova 1998a, 1994c.

Melanostoma dubium (Zetterstedt, 1838) - O62; 200-260 m; 1; hoes, ? h; Drenowsky 1936; Peck 1988.

Melanostoma mellinum (Linnaeus, 1758) - DM, P2, B1, B2, K2, K6, K9, V1, V4, S1, S21, TL, T31, O62, R1, R2, R3, RW, RE, BS; 0-2100 m; 1, 2, 3, 4; h; Meunier 1897; Joakimoff 1899; Nedelkov 1912; Drensky 1934a; Szilády 1934; Bankowska 1967b; Markova \& Tsonev 1993; Markova 1994a, 1994b, 1994c, 1997, 1998, 1998a, 2003, 2006, 2011; Markova \& Alexiev 2000; Beschovski 2004a, 2006a;

Melanostoma scalare (Fabricius, 1794) - E1, V1, TL, O62, BN; 60-600 m; 1; ppt; Nedelkov 1909, 1912; Drensky 1934a; Bankowska 1967b; Peck 1988.

Xanthandrus comtus (Harris, 1780) - V1, T31, R1; 290-1550 m; 1, 2, 3, 4; po; Nedelkov 1912; Drensky 1934a; Bankowska 1967b; Peck 1988; Markova 1998a, 2011.

Platycheirus albimanus (Fabricius, 1781) - B2, V1, S1, R1, R2, RW; 550-1800 m; 2, 3, 4; ho; Nedelkov 1912; Drensky 1934a; Bankowska 1967b; Peck 1988; Markova \& Tsonev 1993; Beschovski 2006a; Markova 2006.

Platycheirus ambiguus (Fallén, 1817) - V4, R1; 1200-1400 m; 3; po, ? ho; Drensky 1934a; Bankowska 1967b; Peck 1988.

Platycheirus angustatus (Zetterstedt, 1843) - K6, S1; 660-750 m; 2, h; Markova \& Tsonev 1993; Markova 1997. 
Platycheirus clypeatus (Meigen, 1822) - V4, S1, R1, RW; 750-1400 m; 2, 3; h; Joakimoff 1899; Bankowska 1967b; Peck 1988; Markova \& Tsonev 1993; Markova 2006.

Platycheirus fulviventris (Macquart, 1829) - K6, V1, R1; 600-1400 m; 2, 3; esca; Nedelkov 1912; Drensky 1934a; Peck 1988; Markova 1994b, 1994c.

Platycheirus granditarsus (Forster, 1771) [Pyrophaena] - S22; h; Nedelkov 1909.

Platycheirus immarginatus (Zetterstedt, 1849) - K2, K6; 700-760 m; 2; h; Markova 1997.

Platycheirus manicatus (Meigen, 1822) - R1; 900-1400 m; 2, 3; h; Drensky 1934a; Peck 1988.

Platycheirus melanopsis Loew, 1856 - R1; 1800 m; 4; des; Bankowska 1967b; Peck 1988.

Platycheirus peltatus (Meigen, 1822) - R1; 1147-1550 m; 3; tp, ? h; Joakimoff 1899; Drensky 1934a.

Platycheirus perpallidus (Verrall, 1901) - V1; 550-600 m; 1; h; Drensky 1934a; Peck 1988.

Platycheirus podagratus (Zetterstedt, 1838) - K6, K9, V1, R1; 500-900 m; 1, 2; h; Nedelkov 1912; Drensky 1934a; Peck 1988; Markova 1994b, 1994c, 1997.

Platycheirus rosarum (Fabricius, 1787) [Pyrophaena] - V3, S1, RW; 350-1100 m; 1, 2, 3; h; Drensky 1934a; Bankowska 1967b; Peck 1988; Beschovski 2006a; Markova 2006.

Platycheirus scutatus (Meigen, 1822) - V1, V4, R1, R2; 600-1400 m; 1, 2, 3; h; Nedelkov 1912; Drensky 1934a; Bankowska 1967b; Peck 1988.

Platycheirus tarsalis (Schummel, 1836) - V4; 800-1300 m; 2, 3; ? hoes, ? tes; Bankowska 1967b; Peck 1988.

Paragus albifrons (Fallén, 1817) - DM, E1, P1, P2, B1, K6, V1, V4, TL, T31, O62, R1, BS; 0-1300 m; 1, 2, 3; tp; Nedelkov 1909, 1912; Drensky 1934a; Szilády 1934; Bankowska 1967b; Peck 1988; Markova 1994a, 1994c, 2011; Markova \& Alexiev 2000.

Paragus bicolor (Fabricius, 1794) [P. bicolor var. testaceus Meigen, 1822] - DM, E2, P1, P2, B1, B2, K6, V1, V3, V4, S1, S211, TL, T2, T31, R1, RW, RE, BN, BS; 0-1374 m; 1, 2, 3; hop, ? h; Löw 1862; Nedelkov 1912; Drensky 1934a; Szilády 1934; Drenowsky 1936; Bankowska 1967b; Markova 1994a, 1994b, 1997, 1998, 1998a, 2003, 2006, 2011; Markova \& Alexiev 2000; Beschovski 2006a.

Paragus cinctus Schiner \& Egger, 1853 - P3, B3, K9, V1, S21, R1, BS; 0-1200 m; 1, 2, 3; ? mt; Nedelkov 1912; Drensky 1934a; Peck 1988.

Paragus haemorrhous Meigen, 1822 [P. trianguliferus Zetterstedt, 1838] - V1, R1; 600-1400 m, 2, 3; hat; Nedelkov 1912; Drensky 1934a; Peck 1988.

Paragus quadrifasciatus Meigen, 1822 [P. concinnus (Wiedemann, 1822); P. pulcherrimus Strobl, 1893] - DM, P2, K6, V1, S1, O5, O62, R1, BN; 0-1550 m; 1, 2, 3, 4; tp; Nedelkov 1912; Drensky 1934a, 1942; Drenowsky 1936; Peck 1988; Markova 1994a, 1994c, 2003.

Paragus strigatus Meigen, 1822 - V1, TL, BS; 0-600 m; 1, 2; ? mca; Nedelkov 1912; Drensky 1934a; Peck 1988; Markova 2011.

Paragus tibialis (Fallén, 1817) [P. tibialis var. meridionalis Becker, 1921; P. tibialis var. obscurus Meigen,1822] - DM, E1, P1, P2, B1, B3, K2, K6, K9, V1, V4, S1, T31, O62, R1, R2, RW, RE, BN, BS; 0-1400 m; 1, 2, 3; ho, ? hpt; Nedelkov 1912; Drensky 1934a; Szilády 1934; Bankowska 1967b; Peck 1988; Markova 1994a, 1994b, 1994c, 1997, 1998, 1998a, 2003, 2006, 2011; Markova \& Alexiev 2000; Beschovski 2006a.

Heringia heringi (Zetterstedt, 1843) - B1, V1, V4; 300-800 m; 1, 2; wesca; Drensky 1934a; Peck 1988.

Heringia latitarsis (Egger, 1865) [Cnemodon, Neocnemodon] - B3; 950-1000 m; 2; e, ? h, i; Bankowska 1967b; Peck 1988.

Heringia pubescens (Delucchi \& Pschorn-Walcher, 1955) [Cnemodon, Neocnemodon] - V4, R2; $\$$; Bankowska 1967b; Peck 1988.

Heringia vitripennis (Meigen, 1822) [Cnemodon, Neocnemodon] - V4, S1, S211, TL, O62, RW, RE; 220-1070 m; 1, 2, 3; tp, ? hoes; Drensky 1934a; Peck 1988; Georgiev et al. 2004; Beschovski 2006a; Markova 2006.

Pipiza austriaca Meigen, 1822 - V1, RW; 550-1450 m; 1, 2, 3; hoes; Drensky 1934a; Peck 1988; Beschovski 2006a; Markova 2006.

Pipiza bimaculata Meigen, 1822 - DM, V1, V4; 100-800 m; 1, 2; esca; Nedelkov 1912; Drensky 1934a; Bankowska 1967b.

Pipiza carbonaria Meigen, 1822 [Penium, Trichopsomyia] - V1, BS; 0-600 m; 1; e; Nedelkov 1912; Drensky 1934a; Peck 1988.

Pipiza fasciata Meigen, 1822 [? = P. festiva Meigen, 1822] - V4; 1; des; Bankowska 1967b; Peck 1988.

Pipiza festiva Meigen, 1822 - B3, V1; 550-1000 m; 1, 2; esca; Drensky 1934a; Bankowska 1967b; Peck 1988. 
Pipiza lugubris (Fabricius, 1775) - K9, V1, V4; 500-950 m; 1, 2; dp, ? des; Nedelkov 1912; Drensky 1934a; Peck 1988; Markova 1998.

Pipiza noctiluca (Linnaeus, 1758) - V4, O62; 220-1050 m; 1, 2, 3; esca; Drensky 1934a; Peck 1988; Markova 1998a. Pipiza quadrimaculata (Panzer, 1804) - P2, V4, R1; 420-1200 m; 1, 2, 3; h; Drensky 1934a; Bankowska 1967b; Peck 1988.

Pipiza signata Meigen, 1822 - DM, V1; 100-550 m; 1; des, ? po; Drensky 1934a; Peck 1988.

Pipizella maculipennis (Meigen, 1822) - K6, S1; 290-700 m; 1, 2; wes; Markova 1994c, 2003.

Pipizella virens (Fabricius, 1805) [Heringia] - B1, B2, K6, V1, V4, TL, T31, O62, R1, R2, RW, RE, BN, BS; 0-1800 m; 1, 2, 3, 4; tp; Nedelkov 1912; Drensky 1934a; Szilády 1934; Bankowska 1967b; Peck 1988; Markova 1994c, 1997, 2006, 2011; Beschovski 2006a.

Trichopsomyia flavitarsis (Meigen, 1822) [Heringia] - R1, RW; 900-1150 m; 2, 3; hoes; Drensky 1934a; Peck 1988; Beschovski 2006a; Markova 2006.

Triglyphus primus Loew, 1840 - O61, R1; 350-1550 m; 1, 2, 3, 4; tp, ? hoes; Drensky 1934a; Peck 1988.

Chamaesyrphus scaevoides (Fallén, 1817) - R1, R2; 1200-1810 m; 3, 4; des; Bankowska 1967b; Peck 1988.

Cheilosia aerea Dufour, 1848 [Ch. bigoti (Becker, 1894); Ch. correcta (Becker, 1894); Ch. cetterstedti Becker, 1894] - B2, V4, S1, RW; 150-1440 m; 1, 2, 3; cse; Drensky 1934a; Bankowska 1967b; Peck 1988; Beschovski 2006a; Markova 2006.

Cheilosia albipila Meigen, 1838 - V1, V4; 550-880 m; 1, 2; wces; Drensky 1934a; Bankowska 1967b; Peck 1988.

Cheilosia albitarsis (Meigen, 1822) - B1, K9, V4, R1, R2, R5, RW; 550-1800 m; 1, 2, 3, 4; h; Joakimoff 1899; Drensky 1934a; Bankowska 1967b; Peck 1988; Beschovski 2006a; Markova 2006.

Cheilosia antiqua (Meigen, 1822) - $\$$, V1, V4, TL, T31, R2, BN, BS; 0-1800 m; 1, 2, 3, 4; e, ? cse; Nedelkov 1912; Drensky 1934a; Bankowska 1967b; Peck 1988; Beschovski 2004a; Markova 2011.

Cheilosia barbata Loew, 1857 - P2, B1, V1, V4, R1, R2, R5; 200-1000 m; 1, 2; e; Nedelkov 1912; Drensky 1934a; Bankowska 1967b; Peck 1988.

Cheilosia canicularis (Panzer, 1801) - B2, O62, R1; 200-1700 m; 1, 2, 3, 4; ? tes; Drensky 1934a, 1939a; Drenowsky 1936, 1939; Bankowska 1967b; Peck 1988.

Cheilosia carbonaria Egger, 1860 - R2; 1500 m; 3; wces; Bankowska 1967b; Peck 1988.

Cheilosia chloris (Meigen, 1822) - V1; 600 m; 1, 2; wes; Markova 1998.

Cheilosia cynocephala Loew, 1840 - V4; ; wes; Bankowska 1967b; Peck 1988.

Cheilosia fasciata Schiner \& Egger, 1853 - K9; 530-550 m; 1; e; Drensky 1934a.

Cheilosia flavipes (Panzer, 1798) - R2; 1500 m; 3; wces, ? esca; Bankowska 1967b; Peck 1988.

Cheilosia frontalis Loew, 1857 - V4, R2; 900-1000 m; 2; e; Bankowska 1967b; Peck 1988.

Cheilosia gagatea Loew, 1857 - V4, O62, R2; 150-1810 m; 1, 2, 3, 4; cse, ? e; Drensky 1934a; Bankowska 1967b; Peck 1988.

Cheilosia gigantea (Zetterstedt, 1838) - \$; hoes; Peck 1988.

Cheilosia grossa (Fallén, 1817) - DM, V1, V4; 100-1080 m; 1, 2, 3; po; Drensky 1934a; Peck 1988.

Cheilosia illustrata (Harris, 1780) [Ch. rupestris (Panzer, 1798)] - V4, R1; 1200-1550 m; 3; hoes; Nedelkov 1912; Drensky 1934a; Bankowska 1967b; Peck 1988.

Cheilosia impressa Loew, 1840 - E2, V4, R1, R2, BN; 0-1810 m; 1, 2, 3, 4; hoes, ? esca; Nedelkov 1912; Drensky 1934a, 1942, 1955; Bankowska 1967b; Peck 1988.

Cheilosia latifrons (Zetterstedt, 1843) [Ch. intonsa Loew, 1857] - R1; 1200-1400 m; 3; wp; Drensky 1934a; Peck 1988.

Cheilosia melanopa (Zetterstedt, 1843) - $\$$ B1, B3, V1, V3, V4, S211, T31, O62, R1, RW; 20-1550 m; 1, 2, 3; e; Nedelkov 1912; Drensky 1934a; Peck 1988; Beschovski 2006a; Markova 2006, 2011.

Cheilosia melanura (Becker, 1894) - V4, R1; 800-1800 m; 2, 3, 4; wces, ? des; Bankowska 1967b; Peck 1988.

Cheilosia montana Egger, 1860 - V4, R2, RW; 900-1500 m; 2, 3; wes; Bankowska 1967b; Peck 1988; Beschovski 2006a.

Cheilosia morio (Zetterstedt, 1838) - V4, O61, R1; 370-1550 m; 1, 2, 3; tes, ? wces; Drensky 1934a; Bankowska 1967b; Peck 1988.

Cheilosia mutabilis (Fallén, 1817) - V4, T31, O61, R1, R2; 330-2000 m; 1, 2, 3, 4; wcp; Drensky 1934a; Bankowska 1967b; Peck 1988; Markova 2011.

Cheilosia nebulosa (Verrall, 1871) [Ch. langhofferi (Becker, 1894)] - S21, O62, R2; 180-1800 m; 1, 2, 3, 4; e; Drensky 1934a; Bankowska 1967b; Peck 1988. 
Cheilosia nigripes (Meigen, 1822) - RW; 950-1100 m; 2, 3; hoes; Bankowska 1967b; Peck 1988; Beschovski 2006a; Markova 2006.

Cheilosia pagana (Meigen, 1822) - V4, R1; 870-1550 m; 2, 3; h; Nedelkov 1912; Drensky 1934a; Peck 1988.

Cheilosia pallipes Loew, 1863 [Ch. flavissima (Becker, 1894)] - R1; 1500-1550 m; 3; h; Drensky 1934a.

Cheilosia pictipennis Egger, 1860 [Ch. bureschi (Delkeskamp, 1942)] - R2; 900-1000 m; 2; wces; Delkeskamp, 1942; Josifov 1957.

Cheilosia proxima (Zetterstedt, 1843) - B1, V1, V4, S1, T31, R1, R2, RW, BS; 0-1400 m; 1, 2, 3; hoes, ? esca; Joakimoff 1899; Nedelkov 1912; Drensky 1934a; Bankowska 1967b; Peck 1988; Beschovski 2006a; Markova 2006, 2011.

Cheilosia pubera (Zetterstedt, 1838) - R1, R2; 1800 m; 4; e; Bankowska 1967b; Peck 1988.

Cheilosia rhynchops Egger, 1860 [Ch. drenowskii (Szilády, 1936)] - V4, R1, R3; 1500-1800 m; 3, 4; e, ? cse; Szilády 1936; Drenowsky 1936, 1939; Bankowska 1967b; Peck 1988.

Cheilosia ruficollis (Becker, 1894) - P1, V4; 400-1200 m; 1, 2, 3; ? e; Bankowska 1967b; Peck 1988.

Cheilosia rufimana (Becker, 1894) - V4, R2; 1000-1820 m; 3, 4; e; Bankowska 1967b; Peck 1988.

Cheilosia sahlbergi (Becker, 1894) - V4, R2; 1000-1820 m; 3, 4; e; Bankowska 1967b; Peck 1988.

Cheilosia schineri Egger, 1860 - R1, BS; 0-1550 m; 1, 2, 3; se; Drensky 1934a; Markova 2011.

Cheilosia scutellata (Fallen, 1817) - S1, T31, O62, RW, RE, BN, BS; 0-650 m; 1; esca; Löw 1862; Nedelkov 1912; Bankowska 1967b; Peck 1988; Beschovski 2006a; Markova 2006, 2011.

Cheilosia semifasciata (Becker, 1894) - V4, R1; 1000-1300 m; 3; e; Buhr 1941; Bankowska 1967b; Peck 1988.

Cheilosia soror (Zetterstedt, 1843) - P2, K9, V4, O62, R2; 180-1200 m; 1, 2, 3; tp; Nedelkov 1912; Drensky 1934a; Markova 1994a.

Cheilosia urbana (Meigen, 1822) [Ch. ruralis (Meigen, 1822); ? Ch. mutabilis Fallén, 1817; Ch. praecox (Zetterstedt, 1843)] - V1, V4, O62, R1, R2, BS; 0-1820 m; 1, 2, 3, 4; tp; Joakimoff 1899; Nedelkov 1912; Drensky 1934a; Bankowska 1967b; Peck 1988.

Cheilosia variabilis (Panzer, 1798) - V4, R1, BN; 0-1500 m; 1, 2, 3; wcp; Joakimoff 1899; Nedelkov 1912; Drensky 1934a; Bankowska 1967b; Peck 1988.

Cheilosia velutina Loew, 1840 - DW, B1, K9, V1, S21, R1, R2, RW; 30-1000 m; 1, 2; esca; Nedelkov 1912; Drensky 1934a; Bankowska 1967b; Peck 1988; Markova 1997, 2006.

Cheilosia vernalis (Fallén, 1817) - R1, RW; 400-1820 m; 1, 2, 3, 4; hoes; Drensky 1934a; Bankowska 1967b; Peck 1988; Markova 2006.

Cheilosia vulpina (Meigen, 1822) [Ch.pigra Loew, 1840] - ^; K9, V1, V4, S21, S22, TL, T31, O62, R1, R3, RW, BS; 0-1400 m; 1, 2, 3; wesit; Nedelkov 1909, 1912; Drensky 1934a; Bankowska 1967b; Peck 1988; Beschovski 2006a; Markova 2006, 2011.

Ferdinandea aurea Rondani, 1844 - S1, BS; 0-270 m; 1; se; Drensky 1934a; Peck 1988.

Ferdinandea cuprea (Scopoli, 1763) [F. euptera (Scopoli, 1763)] - B1, V1, V4, T31, R1, R3, RW, BS; 0-1500 m; 1, 2, 3; tp; Nedelkov 1912; Drensky 1934a; Drenowsky 1936; Peck 1988; Markova 2006, 2011.

Ferdinandea sziladyi Drensky, 1934 - S1, BS; 0-270 m; 1; see; Drensky 1934a; Peck 1988.

Pelecocera tricincta Meigen, 1822 - V1, R1; 550-1400 m; 1, 2, 3; des; Drensky 1934a; Szilády 1934; Peck 1988. Rhingia campestris Meigen, 1822 - R1, BS; 0-1480 m; 1, 2, 3; hoes; Joakimoff 1899; Drensky 1960.

Rhingia rostrata (Linnaeus, 1758) - V4, R1; 870-1550 m; 2, 3; wes; Nedelkov 1912; Drensky 1934a; Peck 1988. Callicera aenea (Fabricius, 1777) [Chrysogaster] - V1; 550-600 m; 1; po; Nedelkov 1912; Drensky 1934a.

Callicera rufa Schummel, 1842 - O62; 180 m; 1; ena; Drensky 1934a; Peck 1988.

Volucella bombylans (Linnaeus, 1758) [V.plumata (De Geer, 1776); V. terestriformis Drensky, 1934] - *; E2, B1, V1, V4, S1, O1, O62, R1; 150-2500 m; 1, 2, 3, 4, 5, 6; h; Löw 1863; Joakimoff 1899; Nedelkov 1912; Drensky 1934a, 1939a, 1955; Szilády 1934; Drenowsky 1936; Buresch 1953a; Bankowska 1967b; Peck 1988.

Volucella inanis (Linnaeus, 1758) - V1, O62, R1; 180-2000 m; 1, 2, 3, 4; tp; Meunier 1897; Drensky 1934a; Szilády 1934; Peck 1988.

Volucella inflata (Fabricius, 1794) - \$; P1, B1, TL, R1, RW, BN; 0-1300 m; 1, 2, 3; e; Löw 1862; Nedelkov 1912; Drensky 1934a; Peck 1988; Beschovski 2006a; Markova 2006.

Volucella pellucens (Linnaeus, 1758) - \$; E2, V1, V4, T31, R1, R3, RW, BS; 0-1700 m; 1, 2, 3, 4; po; Joakimoff 1899; Nedelkov 1912; Drensky 1934a, 1955; Drenowsky 1936; Bankowska 1967b; Peck 1988; Beschovski 2006a; Markova 2006, 2011. 
Volucella zonaria (Poda, 1761) - \$ DW, DM, E2, P1, P2, B1, B2, V1, V3, V4, S1, TL, T31, O62, R1, RW, BN, BS; 0-1400 m; 1, 2, 3; tp; Joakimoff 1899; Nedelkov 1912; Drensky 1934a, 1942, 1955; Szilády 1934; Drenowsky 1936; Bankowska 1967b; Peck 1988; Beschovski 2006a; Markova 2006, 2011.

Brachyopa bicolor (Fallén, 1817) - K9, R1; 670-1550 m; 2, 3; hoes; Nedelkov 1912; Drensky 1934a.

Brachyopa panzeri Goffe, 1945 - R1; 1140-1200 m; 3; des; Joakimoff 1899.

Chrysogaster coemiteriorum (Linnaeus, 1758) [Ch. chalybeata Meigen, 1822] - V1; 550-600 m; 1; tp; Drensky 1934a; Peck 1988.

Chrysogaster solstitialis (Fallen, 1817) - T31, O62, R1, RW, BN, BS; 0-1400 m; 1, 2, 3; ena; Drensky 1934a, 1939a; Drenowsky 1939; Bankowska 1967b; Peck 1988; Markova 2006, 2011.

Chrysogaster viduata (Linnaeus, 1758) [? Pipizella] - K6, V1, V4, S1, TL, T31, O62, R1, R2, R5, RW, BS; 0-1980 m; 1, 2, 3, 4, 5; e, ? ena; Joakimoff 1899; Nedelkov 1912; Drensky 1934a; Bankowska 1967b; Peck 1988; Markova \& Tsonev 1993; Markova 1994a, 1994b, 1994c, 1997, 2006, 2011; Beschovski 2006a.

Melanogaster aerosa (Loew, 1843) [Chrysogaster macquarti Loew, 1843] - R1; 1300-1400 m; 3; wces; Drensky 1934a.

Melanogaster nigricans (Stackelberg, 1922) [Lejogaster] - s seesfe; Peck 1988.

Hammerschmidtia ferruginea (Fallén, 1817) - V4; 800-1200 m; 2, 3; h; Bankowska 1967b.

Lejogaster metallina (Fabricius, 1781) [Chrysogaster] - DM, V1, R1, R2; 100-1500 m; 1, 2, 3; tp; Nedelkov 1912; Drensky 1934a; Bankowska 1967b; Markova 1994a, 1994b, 1997, 1998.

Lejogaster tarsata (Meigen, 1822) [L.splendida (Meigen, 1822); Orthoneura longior Becker, 1921; Chrysogaster] - DM, E2, V1, V4, T2, T31, O62, R1, RE, BS; 0-1550 m; 1; tp; Nedelkov 1912; Drensky 1934a; Bankowska 1967b; Peck 1988; Markova 1994a, 2006, 2011; Markova \& Alexiev 2000.

Myolepta dubia (Fabricius, 1805) [M. luteola (Gmelin, 1790)] - S1, T31, O62, BS; 0-300 m; 1; e; Bankowska 1967b; Peck 1988; Markova 2011.

Orthonevra brevicornis (Loew, 1843) [Chrysogaster] - V4, R1; 1000-1960 m; 3, 4, 5; wes; Bankowska 1967b; Peck 1988.

Orthonevra elegans (Meigen, 1822) - V1, R1; 550-1400 m; 1, 2, 3; hoes; Drensky 1934a; Peck 1988.

Orthonevra frontalis (Loew, 1843) - K6, BS; 0-750 m; 1, 2; eanit, ? eanca; Drensky 1934a; Peck 1988; Markova 1997, 2011.

Orthonevra fumipennis (Loew, 1843) - BS; 0-10 m; 1; seean; Drensky 1934a; Peck 1988.

Orthonevra geniculata (Meigen, 1830) - R1, RW; 1100-1550 m; 3, 4; hoes, ? tes; Drensky 1934a; Peck 1988; Beschovski 2006a; Markova 2006.

Orthonevra nobilis (Fallen, 1817) [Chrysogaster] - P2, V1, V4, R1, RW; 390-1400 m; 1, 2, 3; esca; Nedelkov 1912; Drensky 1934a; Bankowska 1967b; Peck 1988; Beschovski 2006a; Markova 2006.

Orthonevra plumbago (Loew, 1840) - O62; 200-260 m; 1; esca; Bankowska 1967b; Peck 1988.

Riponnensia longicornis (Loew, 1843) [Orthonevra] - V4, RW, BS; 20-950 m; 1, 2; hom; Drensky 1934a; Bankowska 1967b; Peck 1988; Markova \& Alexiev 2000; Markova 2006.

Riponnensia splendens (Meigen, 1822) [Chrysogaster] - S1, O62, BS; 0-450 m; 1; eanna; Drensky 1934a; Peck 1988; Markova \& Alexiev 2000; Markova 2003.

Neoascia geniculata (Meigen, 1822) - R1; 1800-1950 m; 4, 5; hoes; Bankowska 1967b.

Neoascia interrupta (Meigen, 1822) - T31, BS; 0-300 m; 1; wes; Bankowska 1967b; Peck 1988; Markova 2011.

Neoascia meticulosa (Scopoli, 1763) [N. aenea (Meigen, 1822); N. dispar (Meigen, 1822)] - V4, R1; 800-1950 m; 2, 3, 4, 5; esca; Bankowska 1967b; Peck 1988.

Neoascia podagrica (Fabricius, 1775) [N. floraqlis (Meigen, 1822); Eristalis] - P1, P2, B1, K6, V1, V4, S1, R1, BN; 0-1200 m; 1, 2, 3; wcp; Meunier 1897; Joakimoff 1899; Drensky 1934a; Szilády 1934; Bankowska 1967b; Peck 1988; Markova 1994b, 1994c, 1997.

Sphegina clunipes (Fallén, 1816) - V1, V4, R1; 550-1960 m; 2, 3, 4, 5; des; Meunier 1897; Nedelkov 1912; Drensky 1934a; Bankowska 1967b; Peck 1988.

Sphegina elegans Schummel, 1843 [S. kimakowiczi (Strobl, 1897)] - V4, T31; 290-1200 m; 1, 2, 3; e; Bankowska 1967b; Peck 1988; Markova 2011.

Sphegina latifrons Egger, 1865 - V4; 800-1200 m; 2, 3; e; Bankowska 1967b; Peck 1988.

Sphegina montana Becker, 1921 - R1; 950-1400 m; 3; e; Drensky 1934a; Peck 1988.

Sphegina verecunda Collin, 1937 - P2; 400-450 m; 1; e; Bankowska 1967b; Peck 1988.

Arctophila bequaerti Herve-Bazin, 1913 - R1; 900-1000 m; 2; seean; Drenowsky 1936; Peck 1988. 
Arctophila bombiforme (Fallén, 1810) - V4, R1, RW; 1000-2150 m; 3, 4, 5; e; Joakimoff 1899; Nedelkov 1912; Drensky 1934a; Peck 1988; Beschovski 2006a; Markova 2006.

Arctophila superbiens (Müller, 1776) [A. mussitans (Fabricius, 1776)] - O62, RW; 250-1100 m; 1, 2, 3; e; Nedelkov 1912; Drensky 1934a; Peck 1988; Beschovski 2006a; Markova 2006.

Sericomyia lappona (Linnaeus, 1758) - V4, R1; 1000-1960 m; 3, 4, 5; hoes; Bankowska 1967b; Peck 1988.

Sericomyia silentis (Harris, 1776) [Cinxia borealis (Fallén, 1816)] - R1; 1160-1400 m; 3; hoes; Drensky 1934a; Szilády 1934; Peck 1988.

Eumerus argyropus Loew, 1848 - O62; 180-250 m; 1; nm; Bankowska 1967b; Peck 1988.

Eumerus basalis Loew, 1848 - BS; 0-20 m; 1; mi; Drensky 1934a; Peck 1988.

Eumerus funeralis Meigen, 1822 [E. tuberculatus Rondani, 1857] - \$; h; Popoff \& Nikolova 1958.

Eumerus graecus Becker, 1921 - ; ? se; Peck 1988.

Eumerus olivaceus Loew, 1848 - V4; se; Bankowska 1967b; Peck 1988.

Eumerus ornatus Meigen, 1822 - P2; 190-200 m; 1; ena; Markova 1994a.

Eumerus ruficornis Meigen, 1822 - P2, RE, BS; 0-250 m; 1; ena; Bankowska 1967b; Peck 1988; Markova 2006, 2011.

Eumerus sogdianus Stackelberg, 1952 - DM; 30-120 m; 1; eca; Markova 1994a.

Eumerus strigatus (Fallen, 1817) - DM, P2, K6, K9, V1, V4, S1, TL, T31, R1, BS; 0-1500 m; 1, 2, 3, 4; ha, ? i; Nedelkov 1912; Drensky 1934a; Popoff \& Nikolova 1958; Bankowska 1967b; Peck 1988; Markova 1994a, 1994c, 1997, 1998a, 2003, 2011; Markova \& Dimcheva 1998; Markova \& Alexiev 2000.

Eumerus tricolor (Fabricius, 1798) - V1, O62; 180-600 m; 1; weswca; Nedelkov 1912; Drensky 1934a; Peck 1988.

Merodon aberrans Egger, 1860 [Lampetia] - P2, B2, V1, T31, R1, BS; 0-1440 m; 1, 2, 3; eanna; Nedelkov 1912; Drensky 1934a; Szilády 1934; Bankowska 1967b; Peck 1988; Markova 2011.

Merodon aeneus Meigen, 1822 [Lampetia] - V4, S22, TL, R1, RW; 260-1750 m; 1, 2, 3, 4; ena; Löw 1863; Nedelkov 1909, 1912; Drensky 1934a, 1939a; Szilády 1934; Drenowsky 1937, 1939; Buresch 1953a; Bankowska 1967b; Peck 1988; Beschovski 2006a; Markova 2006.

Merodon albifrons Meigen, 1822 [Lampetia] - V1, V4, T31, O62, BS; 0-1080 m; 1, 2, 3; ? hom; Nedelkov 1912; Drensky 1934a; Bankowska 1967b; Peck 1988; Markova 2011.

Merodon armipes Rondani, 1843 [Lampetia] - S1, TL, R3, RW, BS; 0-1500 m; 1, 2, 3; swp; Löw 1863; Nedelkov 1912; Drensky 1934a, 1939a; Drenowsky 1939; Peck 1988; Beschovski 2006a; Markova 2006.

Merodon avidus (Rossi, 1790) [M. spinipes (Fabricius, 1794)] - ; P1, P2, B1, B2, B3, V1, V4, S1, TL, T31, R1, R3, RW, BN, BS; 0-1400 m; 1, 2, 3; ena; Löw 1862; Joakimoff 1899; Nedelkov 1909, 1912; Drensky 1934a; Szilády 1934; Drenowsky 1936; Bankowska 1967b; Peck 1988; Beschovski 2006a; Markova 2006, 2011.

Merodon caerulescens Loew, 1869 [Lampetia] - V4, R3; 1500-1750 m; 3, 4; seena; Drenowsky 1936, 1937, 1939; Buresch 1953a; Drensky 1939a.

Merodon chalybeatus Sack, 1913 - R3; 900 m; 2; see; Bankowska 1967b; Peck 1988.

Merodon cinereus (Fabricius, 1794) [Lampetia] - V1, V4, S211, TL, R1, R3; 200-1700 m; 1, 2, 3, 4; cse, ? e; Nedelkov 1912; Drensky 1934a; Drenowsky 1936; Bankowska 1967b; Peck 1988.

Merodon clavipes (Fabricius, 1781) [Lampetia] - B1, R1; 260-1550 m; 1, 2, 3, 4; ena; Nedelkov 1912; Drensky 1934a.

Merodon clunipes Sack, 1913 [Lampetia] - BS; 0-20 m; 1; nm; Drensky 1934a; Bankowska 1967b; Peck 1988; Markova 2011.

Merodon constans (Rossi, 1794) [Lampetia] - B3, V1, R3; 900-1050 m; 2, 3; ena; Drensky 1934a; Bankowska 1967b; Peck 1988.

Merodon crymensis Paramonov, 1925 [Lampetia] - B2, T31, RW, BS; 0-1350 m; 1, 2, 3; csean, ? cseean; Drensky 1934a; Bankowska 1967b; Peck 1988; Markova 2006, 2011.

Merodon distinctus Palma, 1863 [Lampetia] - R3; 1; mt; Drensky 1934a; Peck 1988.

Merodon eques (Fabricius, 1805) [Lampetia] - S1, BS; 0-250 m; 1; sena; Drensky 1934a; Bankowska 1967b; Peck 1988.

Merodon equestris (Fabricius, 1794) [Lampetia] - O62, R1; 250-600 m; 1; ha, i; Joakimoff 1899; Drensky 1934a.

Merodon femoratus Sack, 1913 [Lampetia] - RW; 700-750 m; 2; nm; Drensky 1934a; Peck 1988; Beschovski 2006a; Markova 2006.

Merodon flavus Sack, 1913 [Lampetia] - K8; 760 m; 2; cse; Drensky 1934a; Peck 1988. 
Merodon funestus (Fabricius, 1794) [Lampetia] - K9, TL, RW; 250-550 m; 1; sena; Nedelkov 1909, 1912; Drensky 1934a; Peck 1988; Beschovski 2006a; Markova 2006.

Merodon geniculatus Strobl, 1909 [Lampetia] - BS; 0-10 m; 1; hom; Drensky 1934a; Peck 1988.

Merodon hamifer Sack, 1913 [Lampetia] - O62; 200-250 m; 1; ban; Drensky 1934a; Peck 1988.

Merodon loewi van der Goot, 1964 [M. graecus Loew, 1862; Lampetia] - R1, BN; 0-1550 m; 1, 2, 3; seean; Löw 1862; Drensky 1934a; Peck 1988.

? Merodon macedonica (Szilady, 1940) [Lampetia] - R3; 1500-1700 m; 3, 4; ? Ebg; Drenowsky 1939.

Merodon nanus Sack, 1931 - S1, BS; 0-1100 m; 1, 2, 3; nemi; Bankowska 1967b; Peck 1988; Markova 2011.

Merodon natans (Fabricius, 1794) [Lampetia] - O62; 150-250 m; 1; se; Drensky 1934a; Peck 1988.

Merodon pruni (Rossi, 1790) [Lampetia] - BS; 0-10 m; 1; hom; Drensky 1934a; Peck 1988.

Merodon ruficornis Meigen, 1822 [Lampetia] - V4, T31, O62, R1, R2, RW, BS; 0-1800 m; 1, 2, 3, 4; csena; Drensky 1934a; Bankowska 1967b; Beschovski 2006a; Markova 2006, 2011.

Merodon rufipes Sack, 1913 - S1; 230-300 m; 1; see; Bankowska 1967b; Peck 1988.

Merodon rufus Meigen, 1838 [Lampetia] - V4, S211, TL, O62, R1, RW, BS; 0-1100 m; 1, 2, 3; wp; Nedelkov 1912; Drensky 1934a; Peck 1988; Beschovski 2006a; Markova 2006, 2011.

Merodon segetum (Fabricius, 1794) [Lampetia] - *; O62, BN; 0-250 m; 1; sena; Drensky 1934a, 1942, 1955; Peck 1988.

Merodon tener Sack, 1913 [Lampetia] - R3; 1200-1500 m; 3; se; Drenowsky 1936; Drensky 1939a.

Merodon testaceus Sack, 1913 [Lampetia] - R1; 1500-1550 m; 3; nm; Drensky 1934a; Peck 1988.

Merodon tricinctus Sack, 1913 [Lampetia] - O62; 180-250 m; 1; hom; Drensky 1934a; Peck 1988.

Merodon velox Loew, 1869 [Lampetia] - BS; 0-10 m; 1; nm; Drensky 1934a; Peck 1988; Markova 2006, 2011.

Psilota atra (Fallén, 1817) - V1, R1; 400-1550 m; 1, 2, 3; e; Drensky 1934a.

Psarus abdominalis (Fabricius, 1794) - K9, V1, T31, O62, R1, RW; 180-1550 m; 1, 2, 3; e; Nedelkov 1912; Drensky 1934a; Szilady 1934; Bankowska 1967b; Peck 1988; Markova 2006, 2011.

Ceriana conopsoides (Linnaeus, 1758) [Cerioides] - V1, R1; 600-1400 m; 1, 2, 3; tp; Nedelkov 1912; Drensky 1934a; Peck 1988.

Sphiximorpha binominata (Verrall, 1901) [Cerioides] - T31, BS; 0-350 m; 1; see; Drensky 1934a; Markova 2011.

Sphiximorpha subsessilis (Illiger in Rossi, 1807) [Cerioides] - B1, O62; 180-460 m; 1; e; Nedelkov 1912; Drensky 1934a; Peck 1988.

Eristalinus aeneus (Scopoli, 1763) [Eristalodes, Eristalis, Lathyrophtalmus] - ; DM, E2, P1, P2, B1, V1, S1, S21, TL, T31, R1, RE, BN, BS; 0-600 m; 1, 2; hpta, ? sk; Meunier 1897; Joakimoff 1899; Nedelkov 1909, 1912; Drensky 1934a; Beschovski 1964a, 1965; Bankowska 1967b; Peck 1988; Markova 1994a, 2003, 2006, 2011; Markova \& Alexiev 2000.

Eristalinus quinquelineatus (Fabricius, 1781) [? = E. megacephalus (Rossi, 1794); Eristalodes] - O62; 180-250 m; 1; ppt; Drensky 1934a; Peck 1988.

Eristalinus sepulchralis (Linnaeus, 1758) [Eristalis] - P1, P2, B1, K6, V1, S1, S21, TL, T2, T3, T31, R1, RE, BS; 0-1150 m; 1, 2, 3; po; Joakimoff 1899; Drensky 1934a; Bankowska 1967b; Peck 1988; Markova 1994a, 1997, 2003, 2006, 2011; Markova \& Alexiev 2000.

Eristalinus taeniops(Wiedemann, 1818) [Eristalodes] - BS; 0-20 m; 1; ppt; Drensky 1934a; Peck 1988; Markova 2011.

Eristalis abusiva Collin, 1931 - P2, B1, K6, V1, S1, T31; 50-600 m; 1; hoes; Markova 1994a, 1994b, 1994c, 1998, 1998a, 2003, 2011.

Eristalis alpina (Panzer, 1798) - V4; ; esca; Bankowska 1967b; Peck 1988.

Eristalis arbustorum (Linnaeus, 1758) [E. bulgarica Szilády, 1934; E. nemorum (Linnaeus, 1758)] - ; DW, DM, E2, P1, P2, B1, B2, B3, K2, K6, K9, V1, V3, V4, S1, S21, S211, TL, T2, T31, R1, R2, R3, RW, RE, BS; 0-1960 m; 1, 2, 3, 4, 5; ho; Joakimoff 1899; Nedelkov 1909, 1912; Drensky 1934a, 1955; Szilády 1934; Drenowsky 1936, 1939; Bankowska 1967b; Peck 1988; Markova \& Tsonev 1993; Markova 1994a, 1994b, 1994c, 1997, 1998, 1998a, 2003, 2006, 2011; Markova \& Dimcheva 1998; Markova \& Alexiev 2000; Beschovski 2006a.

Eristalis cryptarum (Fabricius, 1794) [E. nigritarsis Macquart, 1834] - R1; 1200-1400 m; 3; des; Drensky 1934a; Peck 1988.

Eristalis lineata (Harris, 1776) [= E. horticola (De Geer, 1776)] - V1, V4, R1; 600-1900 m; 2, 3, 4, 5; po; Nedelkov 1912; Drensky 1934a; Bankowska 1967b; Peck 1988.

Eristalis intricaria (Linnaeus, 1758) - K6, V4, R1; 600-1200 m; 2, 3; hoes, ? esca; Joakimoff 1899; Drensky 1934a; Peck 1988; Markova 1994c. 
Eristalis jugorum Egger, 1858 - \$ V1, V4, RW; 550-1900 m; 1, 2, 3, 4; e, ? cse; Nedelkov 1912; Drensky 1934a; Bankowska 1967b; Peck 1988; Markova 2006.

Eristalis pertinax (Scopoli, 1763) - \$ E2; 200-230 m; 1; wp, ? h; Drensky 1934a, 1955; Szilády 1934; Peck 1988. Eristalis rupium Fabricius, 1805 - * DM, P2, B2, V4, S21, S22, TL, R1; 30-1900 m; 1, 2, 3, 4, 5; h; Nedelkov 1909; Drensky 1934a; Szilády 1934; Bankowska 1967b; Peck 1988; Markova 1994a.

Eristalis similis (Fallén, 1817) [E. pratorum Meigen, 1822] - *; E2, B2, B3, V4, R1, R3, RW, BS; 0-1960 m; 1, 2, 3, 4, 5; wcp, ? h; Drensky 1934a, 1955, 1960; Szilády 1934; Bankowska 1967b; Peck 1988; Beschovski 2006a; Markova 2006.

Eristalis tenax (Linnaeus, 1758) [E. tenax var. hortorum Meigen, 1822; Eristalomyia] - DM, E1, E2, P2, K6, V1, S1, S21, S22, TL, T2, T31, RW, RE, BN, BS; 0-1500 m; 1, 2, 3, 4; k; Joakimoff 1899; Kovachev 1905; Nedelkov 1909, 1912; Drensky 1934a, 1942, 1955, 1960; Szilády 1934; Beschovski 1964a, 1965, 2006a; Lavčiev 1965d; Bankowska 1967b; Lavchiev \& Jovčev 1978; Markova \& Tsonev 1993; Markova 1994a, 1994b, 1994c, 1997, 1998, 1998a, 2003, 2006, 2011; Markova \& Alexiev 2000;

Parhelophilus frutetorum (Fabricius, 1775) [Eurinomyia, Helophilus] - T31, BS; 0-330 m; 1; wces; Bankowska 1967b; Peck 1988; Markova 2011.

Parhelophilus versicolor (Fabricius, 1794) [Helophilus] - O62; 180-250 m; 1; wp; Drensky 1934a; Peck 1988.

Helophilus hybridus Loew, 1846 [Tubifera] - K8, TL, R1, RW, RE; 200-1430 m; 1, 2, 3; h; Drensky 1934a; Peck 1988; Beschovski 2006a; Markova 2006.

Helophilus pendulus (Linnaeus, 1758) [Tubifera] - V1, V4, O62, R1, RE, BS; 0-1100 m; 1, 2, 3; hoes; Nedelkov 1912; Drensky 1934a; Bankowska 1967b; Peck 1988; Markova 2006.

Helophilus trivittatus (Fabricius, 1805) [H. parallelus (Harris, 1776); Tubifera] - \$ DM, E2, P2, K9, V1, V3, V4, TL, T31, R1, RW, BN, BS; 0-1200 m; 1, 2, 3; tp, ? h; Joakimoff 1899; Nedelkov 1909, 1912; Drensky 1934a, 1955; Bankowska 1967b; Markova 1994a, 1998a, 2006, 2011; Markova \& Alexiev 2000.

Anasimyia lineata (Fabricius, 1787) [Eurinomyia, Helophilus] - V1; 550-600 m; 1; hoes; Drensky 1934a; Peck 1988.

Anasimyia lunulata (Meigen, 1822) [Eurinomyia, Helophilus] - RW; 1100 m; 3; h; Drensky 1934a; Peck 1988; Markova 2006.

Anasimyia transfuga (Linnaeus, 1758) [Eurinomyia, Helophilus] - V1; 550-600 m; 1, 2; wes; Drensky 1934a; Peck 1988.

Lejops vittata (Meigen, 1822) - P2, T31, BS; 0-300 m; 1; esca; Drensky 1934a; Peck 1988; Markova 2011.

Mesembrius peregrinus (Loew, 1846) - BS; 0-20 m; 1; tp; Drensky 1934a; Bankowska 1967b; Peck 1988; Markova 2011.

Myathropa florea (Linnaeus, 1758) [M. florea var. nigrotarsata (Schiner, 1862); Helophilus] - ; DM, E2, P2, B1, V1, S1, S22, TL, T31, O62, R2, R3, RW, BN, BS; 0-1400 m; 1, 2, 3; hop, ? h; Nedelkov 1909, 1912; Drensky 1934a, 1955, 1960; Szilády 1934; Drenowsky 1936; Bankowska 1967b; Peck 1988; Markova 1994a, 1998a, 2006, 2011; Markova \& Alexiev 2000; Beschovski 2006a.

Blera fallax (Linnaeus, 1758) [Cynorrhina] - V4, R1; 1200-1400 m; 3; hoes; Drensky 1934a; Bankowska 1967b; Peck 1988.

Caliprobola speciosa (Rossi, 1790) - BN; 0-20 m; 1; wces; Löw 1862.

Criorhina asilica (Fallén, 1816) - V4; e; Bankowska 1967b; Peck 1988.

Milesia crabroniformis (Fabricius, 1775) - O62, BS; 0-250 m; 1; sena, ? csena; Drensky 1934a; Bankowska 1967b; Peck 1988; Markova 2011.

Milesia semiluctifera (Villers, 1798) [M. splendida (Rossi, 1790)] - E1, E2, V3, S22, TL, RW, BN, BS; 0-800 m; 1, 2; cset; Nedelkov 1909, 1912; Drensky 1934a, 1942, 1955; Bankowska 1967b; Peck 1988; Beschovski 2006a; Markova 2006, 2011.

Palumbia eristaloides Portschinsky, 1887 [P. flavipes Paramonov, 1927] - O62; 180-250 m; 1; bc; Drensky 1939a. Spilomyia diophthalma (Linnaeus, 1758) - P2, V1; 320-600 m; 1; tp; Nedelkov 1912; Drensky 1934a; Peck 1988. Spilomyia saltuum (Fabricius, 1794) - S21, S22, T31, R1, BS; 0-140 m; 1, 2, 3; cse; Nedelkov 1912; Drensky 1934a; Bankowska 1967b; Peck 1988; Markova 2011.

Syritta flaviventris Macquart, 1842 - T31, O62, BS; 0-450 m; 1; swpnata, ? sk; Drensky 1934a; Bankowska 1967b; Peck 1988; Markova 2011.

Syritta pipiens (Linnaeus, 1758) - * DW, DM, E1, E2, P1, P2, B1, B2, K2, K6, K9, V1, V4, S1, S22, TL, T2, T31, O62, R1, R2, R3, RW, RE, BN, BS; 0-1320 m; 1, 2, 3; hno, ? sk; Meunier 1897; Nedelkov 1909, 1912; 
Drensky 1934a, 1942, 1955; Szilády 1934; Bankowska 1967b; Markova \& Tsonev 1993; Markova 1994a, 1994b, 1994c, 1997, 1998, 1998a, 2003, 2006, 2011; Markova \& Dimcheva 1998; Markova \& Alexiev 2000; Beschovski 2004a, 2006a.

Tropidia fasciata Meigen, 1822 - K6; 680 m; 1, 2; des; Markova 1994b.

Brachypalpus laphriformis (Fallen, 1816) [B. bimaculatus (Macquart, 1829)] - V4, R1; 780-1400 m; 2, 3; e; Nedelkov 1912; Drensky 1934a.

Brachypalpus valgus (Panzer, 1798) - TL, BS; 0-200 m; 1; e; Nedelkov 1912; Drensky $1934 \mathrm{a}$; Peck 1988.

Chalcosyrphus femoratus (Linnaeus, 1758) [Zelima, Xylota] - T31, R1; 280-2300 m; 1, 2, 3, 4, 5; esca, ? hoes; Drensky 1934a; Bankowska 1967b; Peck 1988; Beschovski 2006a; Beschovski 2006a; Markova $2006,2011$.

Chalcosyrphus pannonicus (Oldenberg, 1916) [Zelima] - RW; 1100 m; 3; see; Drensky 1934a; Peck 1988; Markova 2006.

Xylota ignava (Panzer, 1798) [Zelima] - V4, S1, R1, RW; 300-1400 m; 1, 2, 3; tp; Drensky 1934a; Bankowska 1967b; Peck 1988; Beschovski 2006a; Markova 2006.

Xylota segnis (Linnaeus, 1758) [Zelima] - V1, V4, R1, BN, BS; 0-1400 m; 1, 2, 3; h; Nedelkov 1912; Drensky 1934a, 1942; Szilády 1934; Bankowska 1967b; Peck 1988; Markova 2011.

Xylota sylvarum (Linnaeus, 1758) [Zelima] - V4, R1, BS; 0-1400 m; 1, 2, 3; hoes; Nedelkov 1912; Drensky 1934a; Bankowska 1967b; Peck 1988.

Microdon analis (Macquart, 1842) [M. latifrons Loew, 1856] - V4, S211; 700-800 m; 2; tp; Drensky $1934 \mathrm{a}$.

Microdon devius (Linnaeus, 1761) - K8, V4, T31, R1; 290-1470 m;1, 2, 3; tes, ? wces; Joakimoff 1899; Drensky 1934a; Bankowska 1967b; Peck 1988; Markova 2011.

Microdon mutabilis (Linnaeus, 1758) - K9, S211, V4, R2, RW; 500-1600 m; 2, 3; hoes; Nedelkov 1912; Drensky 1934a; Bankowska 1967b; Peck 1988; Beschovski 2006a; Markova 2006.

Microdon sophianus Drensky, 1934 - V1; 560 m; 1; Er; Drensky 1934a; Peck 1988.

\section{SCHIZOPHORA}

\section{ACALYPTRATA}

\section{Micropezidae (Tylidae)}

Micropeza corrigiolata (Linnaeus, 1767) - V1, V4; 550-980 m; 1, 2; h; Meunier 1897; Nedelkov 1912.

\section{Psilidae}

Oxypsila abdominalis (Schummel, 1844) [Psila] - V1; 600 m; 1, 2; csee; Nedelkov 1912.

Chamaepsila rosae (Fabricius, 1794) [Ch. hennigi Thompson \& Pont, 1994; Psila] - -; $\$$ E2; 150-160 m; 1; tp, ? ha, i; Byuletin No 2 1943; Buresch \& Lazarov 1956; Popoff \& Nikolova 1958; Kovachevski et al. 1959; Grigorov 1972.

Chamaepsila rufa (Meigen, 1826) [Psila] - K9, V1; 550-600 m; 1; des; Nedelkov 1912.

Chamaepsila villosula (Meigen, 1826) [Psila] - V1; 550-600 m; 1; csee; Nedelkov 1912.

Loxocera aristata (Panzer, 1801) [L. elongata Meigen, 1826] - V1; 550-600 m; 1; e; Nedelkov 1912.

Loxocera fulviventris Meigen, 1826 - RW; 1513 m; 3, 4; e; +++.

Chyliza annulipes Macquart, 1835 - K9; 560 m; 1; des, ? h; Nedelkov 1912.

\section{Diopsidae}

Sphyracephala europaea Papp \& Foldvari, 1997 - DM; 30 m; 1; see; Kutsarov \& Hubenov 2019.

\section{Conopidae}

Abrachyglossum capitatum (Loew, 1847) - V1; 550-600 m; 1; ewca; Drensky 1939 b. 
Leopoldius coronatus (Rondani, 1857) [Brachyglossum] - S1, O62, R2; 200-350 m; 1; ena; Drensky 1939b; Bankowska 1967a.

Conops (Asiconops) elegans Meigen, 1824 - R1; 900 m; 2; hom; Drensky 1939b; Beschovski 2006a.

Conops (Asiconops) flavifrons Meigen, 1824 - DM, O62, R3; 30-450 m; 1; wp; Nedelkov 1912; Drensky 1939b; Chvála \& Smith 1988.

Conops (Conops) flavipes Linnaeus, 1758 - V1, V4, S211, O62, RW; 220-1700 m; 1, 2, 3, 4; tp; Meunier 1897; Nedelkov 1912; Drensky 1939b; Buresch 1953a; Beschovski 2006a.

Conops (Conops) quadrifasciatus De Geer, 1776 - V1, O62, R1; 220-1400 m; 1, 2, 3; esanca; Nedelkov 1912; Szilady 1934; Drensky 1939b.

Conops (Conops) scutellatus Meigen, 1804 - O4, R1; 300-1550 m; 1, 2, 3; e; Szilady 1934; Drensky 1939b.

Conops (Conops) silaceus Wiedemann in Meigen, 1824 - DM, B1, V1, TL, R1; 30-1550 m; 1, 2, 3; se; Nedelkov 1909, 1912; Drensky 1939b.

Conops (Conops) strigatus Wiedemann in Meigen, 1824 - BN; 0-50 m; 1; des; Drensky 1942.

Conops (Conops) vesicularis Linnaeus, 1761 - R1; 1150-1200 m; 3; tp; Joakimoff 1899; Drensky 1939b.

Conops (Conops) vitellinus Loew, 1847 - DW, R1; 20-450 m; 1; nm; Nedelkov 1912; Drensky 1939b.

Physocephala chrysorrhoea (Meigen, 1824) - R1; 1200-1400 m; 3; tp, ? hop; Drensky 1939b.

Physocephala lacera (Meigen, 1824) - TL, O62, BS; 0-250 m; 1; tp; Nedelkov 1912; Drensky 1939b; Bankowska 1967a.

Physocephala nigra (De Geer, 1776) - R1; 900 m; 2; tp, ? hop; Drensky 1939b.

Physocephala pusilla (Meigen, 1824) - E1, R2, BN, BS; 0-400 m; 1; wcp; Nedelkov 1909, 1912; Drensky 1939b; Bankowska 1967a.

Physocephala rufipes (Fabricius, 1781) - P2, O62, R3, R5, RW; 130-1400 m; 1, 2, 3; tp; Nedelkov 1912; Drenowsky 1936; Drensky 1939b; Bankowska 1967a; Beschovski 2006a.

Physocephala variegata (Meigen, 1824) - R1, BN, BS; 0-1400 m; 1, 2, 3; sp; Nedelkov 1912; Drensky 1939b; Bankowska 1967a.

Physocephala vittata (Fabricius, 1794) - V4, S211, TL, O62, R3, R4, BS; 0-1100 m; 1, 2, 3; wcp; Nedelkov 1912; Drensky 1939b; Buresch 1953a; Bankowska 1967a.

Zodion cinereum (Fabricius, 1794) - V1, TL, R1, RE, BN, BS; 0-1500 m; 1, 2, 3; po; Nedelkov 1912; Szilady 1934; Drensky 1939b; Bankowska 1967a; Beschovski 2006a.

Zodion erythrurum Rondani, 1865 [Z. grande Kröber, 1915] - O62, R1; 220-1400 m; 1, 2, 3; tp, ? wcp, sp; Drensky 1939b.

Zodion notatum (Meigen, 1804) - B3, S1, R1, RW, BS; 0-1600 m; 1, 2, 3, 4; hop; Drensky 1939b; Bankowska 1967a; Beschovski 2006a.

Myopa buccata (Linnaeus, 1758) - V4, R1, R2, R4, RW, BN; 0-1950 m; 1, 2, 3, 4, 5; tp; Löw 1862; Joakimoff 1899; Drensky 1939b; Bankowska 1967a; Beschovski 2006a.

Myopa dorsalis Fabricius, 1794 - V1, R1, BN; 0-1400 m; 1, 2, 3; wpo; Nedelkov 1912; Drensky 1939b.

Myopa fasciata Meigen, 1804 - O62, R3; 430-1200 m; 1, 2, 3; tp; Drensky 1939 b.

Myopa occulta Wiedemann in Meigen, 1824 - V4; 770-830 m; 2; hop; Szilady 1934; Drensky 1939b; Buresch 1953a.

Myopa picta Panzer, 1798 - RW, BN; 0-1000 m; 1, 2; wpo; Drensky 1939b; Bankowska 1967a; Beschovski 2006a.

Myopa polystigma Rondani, 1857 - BN; 0-20 m; 1; ? des; Drensky 1939b.

Myopa testacea (Linnaeus, 1767) - V1, V4, TL, R1, BN; 0-1700 m; 1, 2, 3, 4; ho, ? hop; Nedelkov 1912; Drensky 1939b; Buresch 1953a; Bankowska 1967a.

Melanosoma bicolor (Meigen, 1824) - S211, O62, R3; 220-1600 m; 1, 2, 3, 4; wp; Drenowsky 1936, 1939; Drensky 1939b; Bankowska 1967a.

Melanosoma nigritarse Strobl, 1902 - V3; 900-1000 m; 2; Eb; Drensky 1939b.

Myopotta pallipes (Wiedemann in Meigen, 1824) - R1; 400 m; 1; wesanca; Drensky 1939b; Beschovski $2006 \mathrm{a}$.

Thecophora atra (Fabricius, 1775) [Occemyia] - E2, B1, V1, V4, TL, O5, R1, RW, BN; 0-1500 m; 1, 2, 3; po; Nedelkov 1912; Szilady 1934; Drensky 1939b, 1955; Buresch 1953a; Bankowska 1967a; Beschovski $2006 a$.

Thecophora distincta (Wiedemann in Meigen, 1824) [Occemyia] - O62; 220-300 m; 1; esanca; Drensky 1939b.

Thecophora fulvipes (Robineau-Desvoidy, 1830) [Occemyia sunderwalli (Zetterstedt, 1844)] - P1, B1, V3; 350900 m; 1, 2; tp, ? hop; Szilady 1934; Drensky 1939b.

Thecophora melanopa Rondani, 1857 [Occemyia] - O62; 90-100 m; 1; wcp; Drensky 1939b.

Thecophora pusilla (Meigen, 1824) [Occemyia] - V1, R1; 600-1400 m; 2, 3; hop; Nedelkov 1912; Drensky 1939b. 
Sicus ferrugineus (Linnaeus, 1761) - E2, B2, K9, V4, S22, R1, R3, RW; 220-1700 m; 1, 2, 3, 4; po; Joakimoff 1899; Nedelkov 1912; Szilady 1934; Drenowsky 1936; Drensky 1939b, 1955; Buresch 1953a; Bankowska 1967a; Beschovski 2006a.

Sicus fusenensis Ouchi, 1939 - dp; Chvála \& Smith 1988.

Dalmannia aculeata (Linnaeus, 1761) - E1, TL, O62, R3, BN; 0-1500 m; 1, 2, 3, 4; wp; Löw 1862; Szilady 1934; Drenowsky 1936, 1939; Drensky 1939b; Bankowska 1967a.

Dalmannia marginata (Meigen, 1824) - BS; 0-5 m; 1; eant; Drensky 1939 b.

Dalmannia punctata (Fabricius, 1794) - V1, V4, RW, BS; 0-1000 m; 1, 2; eanit; Drensky 1939b; Bankowska 1967a; Beschovski 2006a.

\section{Lonchaeidae}

Lonchaea chorea (Fabricius, 1781) - V1, V4; 550-980 m; 1, 2; h; Nedelkov 1912.

Lonchaea fugax Becker, 1895 - V4; 1550 m; 4; wp; Doychev et al. 2016.

Lonchaea peregrina Becker, 1895 - R2; 280 m; 1; e; Kechev \& Langourov 2019.

Silba fumosa Egger, 1864 [Setisquamalonchaea] - R2, RE; 280-300 m; 1; h; Kechev \& Langourov 2019.

Silba virescens Macquart, 1851 [Lonchaea aristella Becker, 1903] - -; TL, O62, BS; 0-300 m; 1; atm; Tsalev \& Krastev 1955 Byuletin 1957a, 1957b.

\section{Ulidiidae}

Physiphora alceae (Preyssler, 1791) [Ph. demandata (Fabricius, 1798); Chrysomyza] - E1, E2, TL, R3; 20-1700 m; 1, 2, 3, 4; k; Nedelkov 1909, 1912; Drenowsky 1936; Lavčiev 1965d; Lavchiev \& Jovčev 1978.

Euxesta pechumani Curran, 1938 - ; h, i; Tomov et al. 2009.

Callopistromyia annulipes (Macquart, 1855) - V1; 550-600 m; 1; h, i; +++; reported from Nataliya Popova.

\section{Otitidae (Ortalidae)}

Dorycera graminum (Fabricius, 1794) - V1, TL; 180-600 m; 1; ean; Nedelkov 1912.

Dorycera hybrida Loew, 1862 - BN; 0-20 m; 1; nm; Löw 1862; Soós 1984.

Dorycera maculipennis Macquart, 1843 - S1, BN; 0-300 m; 1; nm; Löw 1862, 1863; Soós 1984.

Otites formosa (Panzer, 1798) [O. ruficeps (Fabricius, 1805); Ortalis] - K9, S1, S23, TL, BN; 0-550 m; 1; e; Löw 1862, 1863; Nedelkov 1912; Beuk et al. 2007; Dvořák et al. 2021.

Otites lamed (Schrank, 1781) - S23; 215-227 m; 1; e; Beuk et al. 2007; Dvořák et al. 2021.

Otites levigata (Loew, 1873) - S23; 383 m; 1; cse; Beuk et al. 2007; Dvořák et al. 2021.

Tetanops (Tetanops) psammophila Loew, 1862 - BN; 0-20 m; 1; Ebg; Löw 1862; Soós 1984.

Tetanops (Tetanops) myopina Fallén, 1820 - BN; 0-20 m; 1; e; Drensky 1942.

Ceroxys fraudulosa (Loew, 1864) - BN; 0-20 m; 1; se; Löw 1862; Soós 1984.

Ceroxys hortulana (Rossi, 1790) [Ortalis] - DM, P1, BN; 0-190 m; 1; ess; Löw 1862; Nedelkov 1912.

Herina aartseni Merz, 2002 - BS; 20 m; 1; em; Merz 2002; Kameneva 2007.

Herina nigrina (Meigen, 1826) - B3, S23; 294-500 m; 1; e; Kameneva 2007; Dvořák et al. 2021.

Seioptera vibrans (Linnaeus, 1758) - V4; 800-1000 m; 2; h; Nedelkov 1912.

\section{Platystomatidae (Platystomidae)}

Platystoma lugubre (Robineau-Desvoidy, 1830) - O61; 270-380 m; 1; ean; Gregor \& Povolny 1959.

Platystoma seminationis (Fabricius, 1775) - B1, B2, V4; 800-1000 m; 2; ean; Nedelkov 1912.

Platystoma subfasciatum Loew, 1862 - S1, BN; 0-300 m; 1; ban; Löw 1862, 1863.

Platystoma tegularium Loew, 1859 - P2, V1, TL, RW; 130-600 m; 1; cse; Nedelkov 1909, 1912; Beschovski 2006.

\section{Tephritidae (Trypetidae)}

Acanthiophilus helianthi (Rossi, 1794) [A. eluta (Meigen, 1826)] - -; DM, V1, TL, T2, BN, BS; 0-700 m; 1, 2; po; Nedelkov 1912; Tschorbadjiew 1940; Drensky 1943; Buresch \& Lazarov 1956; Dirlbek \& Dirlbek 1963. 
Acidia cognata (Wiedemann, 1817) - R1; 1150-1300 m; 3; e; Buhr 1941.

Acinia corniculata (Zetterstedt, 1819) - K4; 700-1000 m; 2; e; Drensky 1943; Dirlbek \& Dirlbek 1963.

Aciura coryli (Rossi, 1794) [A. femoralis Robineau-Desvoidi, 1830] - -; B2, V1, S1, O62, R2, R3, RW, BN; 501700 m; 1, 2, 3, 4; mit; Löw 1863; Nedelkov 1912; Drenowsky 1931; Drensky 1931a, 1931b, 1940, 1943; Buresch \& Lazarov 1956; Beschovski 2006a.

Anomoia purmunda (Harris, 1780) [Phagocarpus] - V4; 770-830 m; 2; tp; Drensky 1940, 1943; Buresch 1953a; Buresch \& Lazarov 1956; Dirlbek \& Dirlbek 1963.

Euaresta bullans (Wiedemann, 1830) [Camaromyia] - BN; 0-5 m; 1; sk; Dirlbek \& Dirlbek 1963.

Carpomya schineri (Loew, 1856) - -; ४; V1; 650 m; 1, 2; tp; Drensky 1943; Buresch 1953a; Buresch \& Lazarov 1956; Dirlbek \& Dirlbek 1963; Grigorov 1972.

Carpomya vesuviana Costa, 1854 - BN; 0-10 m; 1; wpo; Drensky 1943.

Ceratitis capitata (Wiedemann, 1824) - BN, BS; 0-50 m; 1; sk, ? k; Tschorbadjiew 1939a; Statelov 1942; Drensky 1943; Byuletin 1957b; Dikov 1962; Momchilov 1962.

Chaetorellia australis Hering, 1940 - ; h, ? i; Foote, 1984.

Chaetorellia jaceae (Robineau-Desvoidy, 1830) - B2, V1, V4; 600-1600 m; 2, 3, 4; wp; Nedelkov 1912; Buresch 1953a; Dirlbek \& Dirlbek 1963.

Dithryca guttularis (Meigen, 1826) - B2, V1; 550-620 m; 1; des; Nedelkov 1912; Drensky 1943; Dirlbek \& Dirlbek 1963.

Ensina sonchi (Linnaeus, 1767) - V1, BN; 0-550 m; 1; ppt; Drensky 1940, 1943; Dirlbek \& Dirlbek 1963.

Euleia heraclei (Linnaeus, 1758) [Philopylla] - S1, R1, BN; 0-2200 m; 1, 2, 3, 4, 5; tp; Drensky 1940, 1943; Buhr 1941; Dirlbek \& Dirlbek 1963.

? Goniglossum wiedemanni (Meigen, 1826) - csena; Drensky 1943; Dirlbek \& Dirlbek 1963.

Philophylla caesio (Harris, 1780) [Myoleja] - B2; 1600 m; 4; e; Drensky 1943; Dirlbek \& Dirlbek 1963.

Myopites inulaedyssentericae Blot, 1927 - BN; 0-60 m; 1; wp; Drensky 1943; Dirlbek \& Dirlbek 1963.

Myopites stylatus (Fabricius, 1794) - E1, BN, BS; 0-50 m; 1; sena, ? hom; Dirlbek \& Dirlbek 1963.

Oedaspis sofiana Drensky, 1943 - V1; 550 m; 1; Ebg; Drensky 1943.

Terellia (Cerajocera) ceratocera (Hendel, 1913) - K8; 600-650 m; 1; wesan; Drensky 1943; Dirlbek \& Dirlbek 1963.

Terellia (Cerajocera) lappae (Cederhielm, 1798) - K9; 530-580 m; 1; ei; Drensky 1943; Dirlbek \& Dirlbek 1963.

Terellia (Cerajocera) tussilaginis (Fabricius, 1775) [Orellia] - TL; 75-80 m; 1; esan; Drensky 1940, 1943; Dirlbek \& Dirlbek 1963; Beschovski 2004a.

Terellia (Terellia) colon (Meigen, 1826) [Orellia, Trypeta] - V1, R1; 700-1400 m; 2, 3; wp; Nedelkov 1912; Drensky 1940, 1943; Dirlbek \& Dirlbek 1963.

Orellia falcata (Scopoli, 1763) - R3; 410-450 m; 1; ? wp; Drensky 1943; Dirlbek \& Dirlbek 1963.

Orellia stictica (Gmelin, 1790) [O.punctata (Schrank, 1781)] - V4; 1080 m; 3; dp; Drensky 1940, 1943; Buresch 1953a; Dirlbek \& Dirlbek 1963.

Oxyaciura tibialis (Robineau-Desvoidy, 1830) - S1, R3; 240-1000 m; 1, 2; swpat; Drensky 1940, 1943; Buresch \& Lazarov 1956; Dirlbek \& Dirlbek 1963.

Oxyna flavipennis (Loew, 1844) - P2, B1, B2, V1, V4, R1, R3, RW; 300-1400 m; 1, 2, 3; wces; Nedelkov 1912; Drensky 1943; Buresch 1953a; Dirlbek \& Dirlbek 1963; Beschovski 2006a.

Oxyna nebulosa (Wiedemann, 1817) - R2; 1810 m; 4; eswa; Drensky 1943; Dirlbek \& Dirlbek 1963.

Oxyna parietina (Linnaeus, 1758) - BN; 0-20 m; 1; e; Drensky 1940, 1943; Dirlbek \& Dirlbek 1963.

Paracanthella pavonina (Portschinsky, 1875) - BN; 0-20 m; 1; ees; Dirlbek \& Dirlbek 1963.

Campiglossa absinthii (Fabricius, 1805) [Paroxyna, Tephritis] - K4, V1, V4, TL, BN; 0-1000 m; 1, 2; tp; Nedelkov 1912; Drensky 1943; Buresch 1953a; Dirlbek \& Dirlbek 1963; Beschovski 1964a, 2004a.

Campiglossa difficilis (Hendel, 1927) [Paroxyna] - V1; 600 m; 1, 2; esca; Drensky 1940, 1943; Dirlbek \& Dirlbek 1963.

Campiglossa misella (Loew, 1869) [Paroxyna] - BN; 0-20 m; 1; po; Drensky 1940, 1943; Dirlbek \& Dirlbek 1963.

Campiglossa plantaginis (Haliday, 1833) [Paroxyna] - V1; 550-600 m; 1, e; Drensky 1943; Dirlbek \& Dirlbek 1963.

Campiglossa punctella (Fallén, 1814) [Paroxyna] - R3; 1000 m; 2; eca; Drensky 1940, 1943; Dirlbek \& Dirlbek 1963.

Campiglossa tessellata (Loew, 1844) [? = C. difficilis (Hendel, 1927); Paroxyna] - V1, V3, V4, R3, BN; 0-1100 m; 1, 2, 3; wcp; Nedelkov 1912; Drensky 1943; Buresch 1953a; Dirlbek \& Dirlbek 1963. 
Platyparea discoidea (Fabricius, 1787) - V1; 550-600 m; 1; e; Drensky 1940, 1943; Dirlbek \& Dirlbek 1963. Plioreocepta poeciloptera (Schrank, 1776) [Platyparea] - V1; 550-600 m; 1; ewca; Drensky 1943; Popoff \& Nikolova 1958; Dirlbek \& Dirlbek 1963.

Rhagoletis alternata (Fallén, 1814) - V4, S1, BN; 0-830 m; 1, 2; des; Drensky 1943; Buresch 1953a; Buresch \& Lazarov 1956; Dirlbek \& Dirlbek 1963.

Rhagoletis cerasi (Linnaeus, 1758) - -; $\$$ DW, E1, E2, P1, P2, B1, K9, V1, S1, TL, RW; 0-1000 m; 1, 2; eanit, h, i; Malkov 1903, 1907; Stamboliev 1907; Kozarov 1908; Nedelkov 1912; Tschorbadjiew 1924b, 1927, 1928a, 1928b, 1929a, 1929b, 1930a, 1930b, 1932, 1933, 1936, 1938, 1940; Popov 1928, 1954, 1958; Gaydarov 1930; Belov 1931; Spasov 1932; Zahov 1934; Stribarni 1934; Petkoff 1939; Drensky 1943; Balevski \& Felbinger 1943; Byuletin No 2 1943, No 3 1945; Hristov 1948; Zhelev 1948a; Lazarov 1949a, 1949b; Buresch \& Lazarov 1956; Popoff 1956; Byuletin 1956, 1957a, 1957b; Gospodinov 1958; Kovachevski et al. 1959; Lazarov et al. 1960, 1965; Dirlbek \& Dirlbek 1963; Grigorov 1972, 1976; Harizanov et al. 1996; Beschovski 2006a. Chaetostomella cylindrica (Robineau-Desvoidy, 1830) [Ch. anotrophes (Loew, 1846)] - E1, B2, V4, BN; 0-1880 m; 1, 2, 3, 4, 5; wp; Drensky 1940, 1943; Buresch 1953a; Dirlbek \& Dirlbek 1963.

Sphenella marginata (Fallen, 1814) - R3; 1000-1600 m; 3, 4; wpat; Drensky 1943; Dirlbek \& Dirlbek 1963.

Tephritis arnicae (Linnaeus, 1758) - B2; 550-600 m; 1; e; Drensky 1943; Dirlbek \& Dirlbek 1963.

Tephritis bardanae (Schrank, 1803) - B1, V1, TL, R1; 60-1300 m; 1, 2; 3; eit; Joakimoff 1899; Nedelkov 1912; Drensky 1943; Dirlbek \& Dirlbek 1963; Beschovski 2004a.

Tephritis cometa (Loew, 1840) - BN, BS; 0-50 m; 1; esca; Dirlbek \& Dirlbek 1963.

Tephritis conura (Loew, 1844) - B2; 1500-1600 m; 3; ? ean, ? e; Drensky 1940, 1943; Dirlbek \& Dirlbek 1963.

Tephritis crepidis Hendel, 1927 - P1, B1; 400 m; 1; wesca; Drensky 1943; Dirlbek \& Dirlbek 1963.

Tephritis dilacerata (Loew, 1846) - E1; 40 m; 1; h; Dirlbek \& Dirlbek 1963.

Tephritis dioscurea (Loew, 1856) - B2; 1900-2200 m; 4, 5; dp; Drensky 1943; Dirlbek \& Dirlbek 1963.

Tephritis fallax (Loew, 1844) - B2; 500-700 m, 2; e, bm; Drensky 1943; Dirlbek \& Dirlbek 1963.

Tephritis formosa (Loew, 1844) - TL; 60 m; 1; eswa; Drensky 1940, 1943; Dirlbek \& Dirlbek 1963; Beschovski 2004a.

Tephritis leontodontis (De Geer, 1776) - B2; 550-600 m; 1, 2; wp; Drensky 1940, 1943; Dirlbek \& Dirlbek 1963.

Tephritis matricariae (Loew, 1844) - TL; 60 m; 1; wp; Drensky 1940, 1943; Dirlbek \& Dirlbek 1963; Beschovski 2004a.

Tephritis neesii (Meigen, 1830) - R3; 1000 m; 2, 3; wes; Drensky 1943; Dirlbek \& Dirlbek 1963.

Tephritis nigricauda (Loew, 1856) - B1, TL, O62, R3; 60-800 m; 1, 2; wp; Nedelkov 1912; Drensky 1940, 1943; Dirlbek \& Dirlbek 1963; Beschovski 2004a.

Tephritis praecox (Loew, 1844) [T. poecilura Loew, 1869] - O62, R3, BN; 50-1000 m; 1, 2; wp; Drensky 1940, 1943; Dirlbek \& Dirlbek 1963.

Tephritis pulchra (Loew, 1844) - TL, R3; 60-1000 m; 1, 2; wp; Drensky 1940, 1943; Dirlbek \& Dirlbek 1963; Beschovski 2004a.

Tephritis ruralis (Loew, 1844) - BN; 0-10 m; 1; ena, ? wp; Dirlbek \& Dirlbek 1963.

Tephritis truncata (Loew, 1844) - O62, R3; 430-550 m; 1; ena; Drensky 1940, 1943; Dirlbek \& Dirlbek 1963.

Tephritis vespertina (Loew, 1844) - V4, R1; 780-1400 m; 2, 3; ena; Drensky 1940, 1943; Buresch 1953a; Dirlbek \& Dirlbek 1963.

Terellia (Terellia) serratulae (Linnaeus, 1758) - K9, BN; 0-650 m; 1; wcp; Drensky 1940, 1943; Dirlbek \& Dirlbek 1963.

Terellia (Terellia) virens (Loew, 1846) - BN, BS; 0-20 m; 1; h; Dirlbek \& Dirlbek 1963.

Trupanea amoena (Frauenfeld, 1857) - O62; 85-270 m; 1; pata; Drensky 1940, 1943; Buresch \& Lazarov 1956; Dirlbek \& Dirlbek 1963.

Trupanea stellata (Fuesslin, 1775) - E1, O62, BN; 0-250 m; 1; po; Drensky 1943; Dirlbek \& Dirlbek 1963; Beschovski 1964a.

Trypeta artemisiae (Fabricius, 1794) - P1, R1; 260-1150 m; 1, 2, 3; tp; Drensky 1940, 1943; Buhr 1941; Dirlbek \& Dirlbek 1963.

Trypeta zoe Meigen, 1826 - V1, BN; 0-600 m; 1; des; Drensky 1940, 1943; Dirlbek \& Dirlbek 1963.

Urophora affinis (Frauenfeld, 1857) - BN; 0-60 m; 1; h; Dirlbek \& Dirlbek 1963.

Urophora aprica (Fallen, 1814) [Euribia] - P1, B2, BN; 0-600 m; 1; ean; Drensky 1940, 1943; Dirlbek \& Dirlbek 1963. 
Urophora cardui (Linnaeus, 1758) [Euribia] - TL; 70-90 m; 1; h; Drensky 1940, 1943; Dirlbek \& Dirlbek 1963; Beschovski 2004a.

Urophora congrua Loew, 1862 [Euribia] - V1; 700 m; 1, 2; csean; Drensky 1943; Dirlbek \& Dirlbek 1963.

Urophora cuspidata (Meigen, 1826) [Euribia] - S23; 260-430 m; 1; des; Drensky 1943; Dirlbek \& Dirlbek 1963. Urophora mauritanica Macquart, 1851 [U. algira Macquart, 1843; U. macrura (Loew, 1851)] - TL, T31, BN; 50-500 m; 1; mwca; Dirlbek \& Dirlbek 1963; Beschovski 2004a.

Urophora quadrifasciata (Meigen, 1826) [Euribia] - E1, B2, BN, BS; 0-570 m; 1; h; Drensky 1940, 1943; Dirlbek \& Dirlbek 1963.

Urophora solstitialis (Linnaeus, 1758) - P1, B1, V1, S1, TL, BN; 0-700 m; 1, 2; ha; Nedelkov 1912; Drensky 1943; Dirlbek \& Dirlbek 1963; Beschovski 2004a.

Urophora stylata (Fabricius, 1775) [Euribia] - P2, B2, BN; 0-450 m; 1; hoa; Drensky 1943; Dirlbek \& Dirlbek 1963.

Urophora terebrans (Loew, 1850) [Euribia eriolepidis (Loew, 1856)] - V1; 550-600 m; 1; eani; Drensky 1943; Dirlbek \& Dirlbek 1963.

Inuromaesa maura (Frauenfeld, 1857) [Euribia] - BN; 60 m; 1; wesca; Drensky 1943.

Stemonocera cornuta (Scopoli, 1763) [Vidalia] - R1; 1150-1300 m; 3; tp; Buhr 1941.

Xyphosia miliaria (Schrank, 1781) [X. miliaris balcanica Drensky, 1943] - B2, V1, TL; 150-2100 m; 1, 2, 3, 4, 5; hoes, ? tp; Nedelkov 1912; Drensky, 1943; Dirlbek \& Dirlbek 1963; Foote 1984.

\section{Pallopteridae}

Toxoneura usta (Meigen, 1826) [Palloptera] - V1; 550-600 m; 1; e; Nedelkov 1912.

\section{Piophilidae}

Piophila casei (Linnaeus, 1758) - \; * V1, S1, T31, BN, BS; 0-700 m; 1, 2; k; Nedelkov 1912; Drenowsky 1921b; Tschorbadjiew 1928a, 1928b; Drensky 1928, 1960; Popoff 1939b, 1941, 1948; Buresch \& Lazarov 1956; Beschovski 1965.

Liopiophila varipes (Meigen, 1830) [Piophila affinis Meigen, 1830] - V1, R2; 550-1810 m; 1, 2, 3, 4; h; Nedelkov 1912; Drensky 1928; Gregor \& Povolny 1959.

Stearibia nigriceps (Meigen, 1826) [Piophila foveolata Meigen, 1826)] - \; V1; 550-600 m; 1; hn; Drensky 1928; Buresch \& Lazarov 1956.

\section{Lauxaniidae (Sapromyzidae)}

Homoneura christophi (Becker, 1895) - e; Papp 1984a; Pape \& Beuk 2017.

Homoneura limnea (Becker, 1895) - e; Papp 1984a; Pape \& Beuk 2017.

Homoneura notata (Fallén, 1820) - ei; Papp 1984a; Pape \& Beuk 2017.

Homoneura patelliformis (Becker, 1895) - \$; e; Papp 1984a; Pape \& Beuk 2017.

Minettia longipennis (Fabricius, 1794) - \$; h; Papp 1984a; Pape \& Beuk 2017.

Minettia bulgarica Papp, 1981 - BN; 0-20 m; 1; seei; Papp 1981, 1984a; Pape \& Beuk 2017.

Minettia fasciata (Fallén, 1820) - \$ h; Papp 1984a; Pape \& Beuk 2017.

Minettia flaviventris (Costa, 1844) - ; e; Papp 1984a; Pape \& Beuk 2017.

Minettia lupulina (Fabricius, 1787) - P1, TL. BS; 0-530 m; 1; h; Lavčiev 1965b; Beschovski 2004a.

Minettia plumicornis (Fallén, 1820) - V1, BN; 0-600 m; 1; e; Löw 1862; Nedelkov 1912.

Lyciella affinis (Zetterstedt, 1847) - e; Papp 1984a; Pape \& Beuk 2017.

Lyciella conjugata (Becker, 1895) - e; Papp 1984a; Pape \& Beuk 2017.

Lyciella decipiens (Loew, 1847) [Sapromyza] - V1; 600 m; 1, 2; e; Nedelkov 1912; Papp 1984a; Pape \& Beuk 2017.

Lyciella laeta (Zetterstedt, 1838) - e; Papp 1984a; Pape \& Beuk 2017.

Lyciella pallidiventris (Fallén, 1820) [Sapromyza] - V1; 600 m; 1; e; Nedelkov 1912.

Calliopum aeneum (Fallén, 1820) [Halidayella] - V1, R1; 550-1400 m; 1, 2, 3; e; Nedelkov 1912; Drenowsky 1939; Drensky 1939a.

Sapromyzosoma quadricincta (Becker, 1895) - e; Papp 1984a; Pape \& Beuk 2017. 
Sapromyzosoma quadripunctata (Linnaeus, 1758) [Sapromyza] - V1; 600 m; 1; ei; Nedelkov 1912. Sapromyza apicalis Loew, 1847 - V1; 600 m; 1; wesanca; Nedelkov 1912.

Sapromyza intonsa Loew, 1847 - ean; Papp 1984a; Pape \& Beuk 2017.

Sapromyza simplicior Hendel, 1908 [S. simplex Loew, 1847] - V1; 600 m; 1; eca; Nedelkov 1912.

\section{Cremifaniidae}

Cremifania bulgarica Papp, 2010 - R1; 2250 m; 5; Er; Papp 2010.

\section{Chamaemyiidae (Ochthiphilidae)}

Parochthiphila (Euestelia) coronata (Loew, 1858) - DW, P1, S1, S211, T3, T31, O4, R2, BN, BS; 0-2350 m; 1, 2, 3, 4, 5; tp; Beschovski 1966a, 1976a, 1998c.

Parochthiphila (Euestelia) nigripes (Strobl, 1900) - S1, S22, RE, BN, BS; 0-690 m; 1; nmwca; Beschovski 1998c.

Parochthiphila (Euestelia) transversa Hennig, 1938 - V4; 1070 m; 3; see; Beschovski 1998c.

Parochthiphila (Parochthiphila) kirilli Tanasijtshuk, 1986 - BN; 0-5 m; 1; see; Beschovski 1998c.

Parochthiphila (Parochthiphila) spectabilis (Loew, 1858) - TL, O62, BN, BS; 0-150 m; 1; wes; Beschovski 1998c. Chamaemyia aestiva Tanasijtshuk, 1970 - P1, B1, V4, V5, S211, R2, RW, BN; 150-2500 m; 1, 2, 3, 4, 5; tp, ? hop; Tanasijtshuk \& Beschovski 1991; Beschovski 1995b, 2006a.

Chamaemyia aridella (Fallén, 1823) [Ch. juncorum (Fallén, 1823)] - DW, B2, V4, V5, S1, O4, R2, RW, BS; 0-2200 m; 1, 2, 3, 4, 5; e, ? h; Beschovski 1966a, 1995b; Tanasijtshuk \& Beschovski 1991, 2006 a.

Chamaemyia bicolor Beschovski, 1994 [Ch. flavipalpis (Haliday, 1838)] - P1, B2, V5, O62, R2, BN, BS; 0-1450 m; 2, 3; Ebg; Tanasijtshuk \& Beschovski 1991; Beschovski 1994a, 1995b.

Chamaemyia emiliae Tanasijtshuk, 1970 - B2, V4, V5, S1, S211, O4, R2, RW; 600-1800 m; 1, 2, 3, 4; ceet; Tanasijtshuk \& Beschovski 1991; Beschovski 1995b, 2006a.

Chamaemyia flavipalpis (Haliday, 1838) - P1, B2, O62, R2, BN, BS; 0-1450 m; 1, 2, 3; h; Tanasijtshuk 1984; Tanasijtshuk \& Beschovski 1991.

Chamaemyia flavoantennata Beschovski, 1994 [Ch. flavipalpis (Haliday, 1838)] - P1, B2, O62; 50-1500 m; 1, 2, 3; ee; Tanasijtshuk \& Beschovski 1991; Beschovski 1994a, 1995b.

Chamaemyia geniculata (Zetterstedt, 1838) - S1, R3, R5; 680-1200 m; 2, 3; esca; Tanasijtshuk \& Beschovski 1991; Beschovski 1994a, 1995b.

Chamaemyia juncorum (Fallen, 1823) - DW, E1, E2, P1, B1, B2, V4, S22, T31, O4, O62, R2, R3, R5, RW, BN, BS; 0-2200 m; 1, 2, 3, 4, 5; tp, ? hop; Beschovski 1966a, 1995b; Tanasijtshuk \& Beschovski 1991, 2006a.

Chamaemyia polystigma (Meigen, 1830) - DW, E1, E2, P1, P2, B1, B2, B3, V4, S1, S211, T11, T31, O62, R2, R4, R5, RW, RE, BN, BS; 0-2000 m; 1, 2, 3, 4; tp, ? hop; Tanasijtshuk \& Beschovski 1991; Beschovski 1995b, 2004a, 2006a.

Chamaemyia subjuncorum Tanasijtshuk, 1970 - DW, P2, B1, B2, V4, S1, S22, R1, R2, R3, R5; 90-2500 m; 1, 2, 3, 4, 5, 6; dp, ? tp; Tanasijtshuk \& Beschovski 1991; Beschovski 1995b.

Chamaemyia submontana Beschovski, 1994 - V4; 1300-1600 m; 3, 4; csee, m; Beschovski 1994a, 1995b; Beschovski \& Merz 1998.

Chamaemyia sylvatica Collin, 1966 - B3, V4; 1000-1300 m; 2, 3; ean; Tanasijtshuk \& Beschovski 1991; Beschovski 1995b; Beschovski \& Merz 1998; Ebejer \& Barták 2019.

Leucopis (Leucopis) annulipes Zetterstedt, 1848 - ; h; Tanasijtshuk 1984; Beschovski 1998a.

Leucopis (Leucopis) aphidiperda Rondani, 1847 - P2, B2, RW, RE, BN; 0-730 m; 1, 2; tp; Beschovski 1998a, $2006 \mathrm{a}$. Leucopis (Leucopis) argentata Heeger, 1848 - BN, BS; 0-5 m; 1; hata; Beschovski 1998a.

Leucopis (Leucopis) atritarsis Tanasijtshuk, 1958 [L. griseola (Fallén, 1823)] - DW, DM, E2, P1, B2, V1, O62, R2, BN; 0-600 m; 1; h; Beschovski 1966a, 1998a.

Leucopis (Leucopis) compacta Tanasijtshuk, 1972 - BN; 0-15 m; 1; eanca; Beschovski 1998a; Ebejer \& Barták 2019.

Leucopis (Leucopis) dobrodginus Beschovski, 1998 - BN; 0-5 m; 1; Ebg; Beschovski 1998a.

Leucopis (Leucopis) glyphinivora Tanasijtshuk, 1958 - DW, E2, P2, V4, O62, R2, R3, R5, BN, BS; 0-2000 m; 1, 2, 3, 4; ho; Beschovski 1998a. 
Leucopis (Leucopis) ninae Tanasijtshuk, 1966 - BN; 0-10 m; 1; wcp, ? hat; Beschovski 1998a; Ebejer \& Barták 2019.

Leucopis (Leucopis) pallidolineata Tanasijtshuk, 1961 - R5; 500-550 m; 1; esanca; Beschovski 1998a.

Leucopis (Leucopis) pseudomelanopus Tanasijtshuk, 1961 - DW, DM, E2, S1, O62, R2, BN; 0-700 m; 1, 2; cset; Beschovski 1998a.

Leucopis (Leucopis) revisenda Tanasijtshuk, 1970 - R2; 350-450 m; 1; nmwca; Beschovski 1998a.

Leucopomyia silesiaca (Egger, 1862) [Leucopis] - p; tp; Tanasijtshuk 1984; Beschovski 1998a.

\section{Coelopidae}

Coelopa (Fucomyia) frigida (Fabricius, 1805) [C. eximia (Stenhammar, 1854)] - BN; 0-5 m; 1; h; Beschovski 164a, 1964b, 1965.

Coelopa (Coelopa) pilipes Haliday, 1838 - w wp; Gorodkov 1984b

Malacomyia sciomyzina (Haliday, 1833) - BN; 0-5 m; 1; e; Caspers 1951a; Remmert 1960; Beschovski 1964 b.

\section{Dryomyzidae}

Neuroctena anilis Fallén, 1820 [Dryomyza] - B1, TL, RW; 60-1400 m; 1, 2, 3; hoes; Lavčiev 1965b; Beschovski 2006a.

Dryomyza flaveola (Fabricius, 1794) - P1, B1, V4, S23, TL; 260-1650 m; 1, 2, 3, 4; e; Lavčiev 1965 b.

\section{Sciomyzidae (Tetanoceridae)}

Salticella fasciata (Meigen, 1830) - BN; 0-5 m; 1; ? swp; Beschovski 1972a.

Colobaea punctata (Lundbeck, 1923) - BN; 0-5 m; 1; ? tp; Beschovski 1973a.

Ditaeniella grisescens (Meigen, 1830) [Pherbellia] - BN; 0-5 m; 1; ho; Beschovski 1964a.

Pherbellia cinerella (Fallén, 1820) - R1, BN; 0-99 m; 1, 2; po; Rozkošny 1965; Beschovski 1973a.

Pherbellia czernyi (Hendel, 1902) - csee; Beschovski det.; +++.

Pherbellia dorsata (Zetterstedt, 1846) - V1; 600 m; 1, 2; wp; Nedelkov 1912.

Pherbellia dubia (Fallén, 1820) [Sciomyza] - V1; 600 m; 1, 2; hoes; Nedelkov 1912.

Pherbellia griseola (Fallén, 1820) - ; heschovski det.; +++.

Pherbellia scutellaris (von Roser, 1840) - esca; Beschovski det.; +++.

Pherbellia schoenherri (Fallén, 1826) [Ph. punctata (Fabricius, 1794)] - S21, S22, TL, BS; 0-900 m; 1, 2; hoes, ? esca; Nedelkov 1912.

Pherbellia ventralis (Fallen, 1820) [Sciomyza] - V1, BN; 0-600 m; 1; ean; Nedelkov 1912; Beschovski 1973a.

Coremacera amoena (Loew, 1853) [C. trivittata (Loew, 1860); C. manni (Schiner, 1864)] - V4; 770-830 m; 2; csean; Nedelkov 1912.

Coremacera catenata (Loew, 1847) [Limnia] - V1, S1, S21, S22, TL; 0-900 m; 1, 2; eani; Löw 1862, 1863; Nedelkov 1909, 1912; Beschovski 2004a.

Coremacera fabricii Rozko冈ný, 1981 [C. cincta (Fabricius, 1794)] - BN; 0-5 m; 1; e; Rozkošny 1987.

Coremacera marginata (Fabricius, 1775) [Tetanocera] - V1, TL, BN, BS; 0-600 m; 1; ean; Löw 1862; Nedelkov 1912.

Dichetophora finlandica Verbeke, 1964 - e; Rozkošny 1987; Greve et al. 2008.

Dictya umbrarum (Linnaeus, 1758) - V1; 600 m; 1; hoes; Nedelkov 1912.

Euthycera chaerophylli (Fabricius, 1798) - BN; 0-10 m; 1; ean; Löw 1862.

Euthycera fumigata (Scopoli, 1763) [Tetanocera rufifrons (Fabricius, 1781); Limnia recta (Loew, 1845)] - TL, BN; 0-250 m; 1; e; Löw 1862; Nedelkov 1912.

Euthycera stictica (Fabricius, 1805) [Limnia] - BS; 0-5 m; 1; eani; Beschovski 1972a.

Hydromya dorsalis (Fabricius, 1775) - V1, BN; 0-600 m; 1; pat; Nedelkov 1912; Beschovski 1972a.

Ilione (Knutsonia) albiseta (Scopoli, 1763) [Elgiva] - V1; 550-600 m; 1; wcp; Nedelkov 1912.

Limnia paludicola Elberg, 1965 - 1 ; hoes, ? tes; Pape \& Beuk 2017.

Limnia unguicornis (Scopoli, 1763) - V1, V4, BN; 0-870 m; 1, 2; esanca; Nedelkov 1912; Beschovski 1972a.

Pherbina coryleti (Scopoli, 1763) - V1, BN; 0-600 m; 1; tp; Nedelkov 1912; Beschovski 1972a. 
Psacadina verbekei Rozkošný in Knutson \& al., 1975 - \$; eani; Pape \& Beuk 2017.

Psacadina vittigera (Schiner, 1864) - V1; 600 m; 1; e; Nedelkov 1912.

Psacadina zernyi (Mayer, 1953) - BN; 0-5 m; 1; wp; Beschovski 1973a.

Sepedon (Sepedon) sphegea (Fabricius, 1775) - B1, V1, TL, BN; 0-700 m; 1, 2; po; Nedelkov 1912; Beschovski 1972a.

Sepedon (Sepedon) spinipes (Scopoli, 1763) - V1, TL, BN, BS; 0-700 m; 1, 2; tp; Nedelkov 1912; Beschovski $1972 \mathrm{a}$. Tetanocera arrogans Meigen, 1830 - $\mathbf{1}$; tp; Pape \& Beuk 2017.

Tetanocera elata (Fabricius, 1781) -

Tetanocera ferruginea Fallen, 1820 - V1, V4, R1; 600-1300 m; 2, 3; h; Joakimoff 1899; Nedelkov 1912.

Tetanocera punctifrons Rondani, 1868 - e; Pape \& Beuk 2017.

Tetanocera silvatica Meigen, 1830 - $\$$; Beschovski det.; +++.

Trypetoptera punctulata (Scopoli, 1763) - BN; 0-5 m; 1; tp; Beschovski 1973a.

\section{Phaeomyiidae}

Pelidnoptera nigripennis (Fabricius, 1794) - V3; 800-1000 m; 2; e; Nedelkov 1912.

\section{Helcomyzidae}

Helcomyza mediterranea (Loew, 1854) - BN; 0-5 m; 1; nm; Beschovski 1966a; Hubenov et al. 1993, 1998; Hubenov 2015d.

\section{Sepsidae}

Meroplius minutus (Wiedemann, 1830) [M. stercoraria (Robineau-Desvoidy, 1830); Nemopoda] - V1; 600 m; 1; ho; Nedelkov 1912.

Nemopoda nitidula (Fallén, 1820) [N. cylindrica (Fabricius, 1794)] - V1, TL; 170-600 m; 1, 2; hat; Nedelkov 1912. Sepsis cynipsea (Linnaeus, 1758) - V1, TL; 170-600 m; 1; tp; Nedelkov 1912.

Sepsis flavimana Meigen, 1826 - V1, V4, 550-1200 m; 1, 2, 3; ho; Nedelkov 1912.

Sepsis fulgens Meigen, 1826 [S. communis Frey, 1925] - E1, E2, R2; 30-1800 m; 1, 2, 3, 4; tp; Gregor \& Povolny 1959; Lavčiev 1965a, 1972.

Sepsis orthocnemis Frey, 1908 - ; po; Zuska \& Pont 1984; Pape \& Beuk 2017.

Sepsis punctum (Fabricius, 1794) - V1, BS; 0-600 m; 1; ho; Nedelkov 1912; Gregor \& Povolny 1959; Drensky 1960.

Sepsis thoracica (Robineau-Desvoidy, 1830) - ppta; Zuska \& Pont 1984; Pape \& Beuk 2017.

Sepsis violacea Meigen, 1826 - E1, E2, V1; 30-600 m; 1; po; Nedelkov 1912; Lavčiev 1965d.

Themira annulipes (Meigen, 1826) - ; h; Zuska \& Pont 1984; Pape \& Beuk 2017.

Themira minor (Haliday, 1833) - \$ ho; Zuska \& Pont 1984; Pape \& Beuk 2017.

\section{Acartophthalmidae}

Acartophthalmus bicolor Oldenberger, 1910 [A. nigrinus Beschovski nec Zetterstedt, 1848; A. pusio Beschovski nec Frey, 1947] - V4, O61; 300-1000 m; 1, 2; h, m; Beschovski 1976b, $2009 \mathrm{~b}$.

Acartophthalmus nigrinus (Zetterstedt, 1848) - R2; 2000 m; 4; h, bm; Beschovski 1976b; 2009b.

\section{Odiniidae}

Odinia boletina (Zetterstedt, 1848) - BS; 0-10 m; 1; h; Máca 1987; Beschovski 2009b.

Odinia meijerei Collin, 1952 - DM; 20-30 m; 1; h; Beschovski \& Georgiev 1993; Beschovski 2009b.

\section{Agromyzidae}

Agromyza abiens Zetterstedt, 1848 - RW; 900-1300 m; 2, 3; eanna, h; Buhr 1941; Beiger 1979a. Agromyza albitarsis Meigen, 1830 - P1, RW; 130-750 m; 1, 2; dp, ? des; Buhr 1941; Beiger 1979a. 
Agromyza alnibetulae Hendel, 1931 - R1; 1150-1300 m; 3; ei; Buhr 1941.

Agromyza alnivora Spencer, 1969 - RW; 750 m; 2; e; Buhr 1941; Beiger 1979a; Beschovski 2006a.

Agromyza anthracina Meigen, 1830 - RW; 370-1000 m; 1, 2; eanca, ? dp; Beiger 1979a; Beschovski 2006a.

Agromyza bromi Spencer, 1966 - RE; 160 m; 1; ean; Černý \& Merz 2006; Černý 2018.

Agromyza flaviceps Fallén, 1823 - TL, R1, RW; 150-760 m; 1, 2; e; Buhr 1941; Beiger 1979a; Beschovski $2006 a$. Agromyza flavipennis Hendel, 1920 - P2, S22, TL; 135-400 m; 1; wp; Beiger 1979a.

Agromyza frontella (Rondani, 1875) - DM, K9, V1, TL, R5; 100-600 m; 1; h; Grigorov 1962; Beiger 1979a.

Agromyza igniceps Hendel, 1920 - TL; 160-180 m; 1; e; Beiger 1979a.

Agromyza johannae de Meijere, 1924 - B1; 800-1100 m; 2, 3; e; Buhr 1941; Beiger 1979a.

Agromyza kolobowai Hendel, 1931 - DM, P1, K9, V1, TL; 100-600 m; 1; ? e; Grigorov 1962.

Agromyza lithospermi Spencer, 1963 - R2, RW; 350-1000 m; 1, 2; e; Beiger 1979a; Beschovski 2006 a.

Agromyza mobilis Meigen, 1830 - TL; 150-180 m; 1; dp; Makarov 1959; Beiger 1979a.

Agromyza myosotidis Kaltenbach, 1864 - RW; 700-800 m; 2; wpat; Beiger 1979a; Beschovski 2006a.

Agromyza nana Meigen, 1830 - V1, TL, R1, R5, RW; 150-1450 m; 1, 2, 3; po; Buhr 1941; Grigorov 1962; Beiger 1979a; Beschovski 2006a.

Agromyza nigrescens Hendel, 1920 [A. heringi de Meijere, 1925] - 1700 m; 4; m; wp; Buhr 1941.

Agromyza nigripes Meigen, 1830 - B2; 1400 m; 3; ho; Černý \& Merz 2006.

Agromyza idaeiana Hardy, 1853 [A. potentillae (Kaltenbach, 1864)] - V4, TL; 150-1200 m; 1, 2, 3; ho; Buhr 1941; Beiger 1979a.

Agromyza prespana Spencer, 1957 - TL; 150-170 m; 1; eant; Beiger 1979a; Černý 2018.

Agromyza pseudoreptans Nowakowski, 1967 - V4, TL, RW, BS; 0-1400 m; 1, 2, 3; h; Beiger 1979a; Beschovski 2006a.

Agromyza reptans Fallen, 1823 - V4, R1, R2, R5, RW; 360-1700 m; 1, 2, 3, 4; ho; Buhr 1941; Beiger 1979a; Beschovski 2006a.

Agromyza rondensis Strobl, 1900 - TL; 150-170 m; 1; dpo; Beiger 1979a.

Agromyza rufipes Meigen, 1830 - R1; 1700 m; 4; wpo; Buhr 1941.

Agromyza spenceri Griffiths, 1963 - BS; 0-10 m; 1; wp; Beiger 1979a; Černý 2013; Černý \& Bächli 2018.

Agromyza sulfuriceps Strobl, 1898 - R1; 1700 m; 4; h; Buhr 1941.

Agromyza viciae Kaltenbach, 1872 - TL, R5; 160-920 m; 1, 2; e; Beiger 1979a.

Hexomyza schineri (Giraud, 1861) - DM, B1, V4, V5; 30-1200 m; 1, 2, 3; h; Georgiev 1991, 2004; Tsankov et al. 1991.

Melanagromyza aeneoventris (Fallén, 1823) - B3, BS; 0-900 m; 1, 2; po; Černý \& Merz 2006; Černý 2018.

Melanagromyza albocilia Hendel, 1931 - P2; 130-170 m; 1; wpo; Černý \& Merz 2006; Černý 2013, 2018.

Melanagromyza cunctans (Meigen, 1830) - V1, O61, R5, BN, BS; 0-820 m; 1, 2; wcp, ? ppt; Černý \& Merz 2006; Černý 2018.

Ophiomyia cichorii Hering, 1949 - T31; 45-70 m; 1; e; Černý \& Merz 2006; Černý 2013.

Ophiomyia curvipalpis (Zetterstedt, 1848) - DM, B3, R5; 100-550 m; 1; dp; Černý \& Merz 2006; Černý 2018.

Ophiomyia galii Hering, 1937 - P2, R3; 130-1500 m; 1, 2, 3; e; Buhr 1941; Černý \& Merz 2006.

Ophiomyia heringi Stary, 1930 - R1; 1150-1300 m; 3; e; Buhr 1941.

Ophiomyia labiatarum Hering, 1937 - R1, RW; 350-1300 m; 1, 2, 3; h; Buhr 1941; Beschovski 2006a; Černý 2009, 2018.

Ophiomyia maura (Meigen, 1838) - R1, RW; 370-1100 m; 1, 2, 3; h; Buhr 1941; Beiger 1979a; Černý \& Bächli 2018.

Ophiomyia nasuta (Melander, 1913) - B3; 900 m; 2; h; Černý \& Merz 2006; Černý 2018.

Ophiomyia orbiculata (Hendel, 1931) - DW, DM, E2, P1; 100-260 m; 1; eanit; Černý \& Merz 2006; Černý 2013, 2018.

Ophiomyia pinguis (Fallén, 1820) - DW, E2, P1, K6, RE; 20-800 m; 1, 2; tp; Černý \& Merz 2006; Černý 2018.

Ophiomyia pulicaria (Meigen, 1830) - \$ hptn, ? pat; Spasić \& Spencer 1992; Černý 2013, 2018.

Ophiomyia rostrata (Hendel, 1920) - DW, E2; 130-220 m; 1; et; Černý \& Merz 2006; Dursun et al. 1915; Černý \& Bächli 2018.

Ophiomyia subheracleivora Černý, 1994 - T31; 60-70 m; 1; csee; Černý \& Merz 2006.

Amauromyza (Amauromyza) lamii (Kaltenbach, 1858) [Dizygomyza] - B3, V1, TL, R1, RW; 160-1700 m; 1, 2, 3, 4; dp; Buhr 1941; Beiger 1979a; Beschovski 2006a. 
Amauromyza (Amauromyza) morionella (Zetterstedt, 1848) [Dizygomyza] - P3, B1, B3, TL, R1, R2, RW, RE; 160-1300 m; 1, 2, 3; eanna; Buhr 1941; Beiger 1979a; Beron 2004; Beschovski 2006 .

Amauromyza (Amauromyza) fraxini (Beiger, 1980) [Aulagromyza, Paraphytomyza] - BS; 0-50 m; 1; see; Beiger 1980.

Amauromyza (Cephalomyza) flavifrons (Meigen, 1830) [Trilobomyza] - V1, R1, RE; 550-1300 m; 1, 2, 3; h; Buhr 1941; Beiger 1979a; Beron 2004.

Amauromyza (Cephalomyza) gyrans (Fallen, 1823) [Dizygomyza] - R1; 1150-1200 m; 3; ei; Buhr 1941.

Amauromyza (Cephalomyza) labiatarum (Hendel, 1920) [Dizygomyza; Trilobomyza] - P2, V1, TL, R1, R2, RW, RE; 160-1700 m; 1, 2, 3, 4; ean; Buhr 1941; Beiger 1979a; Beron 2004; Beschovski 2006a.

Amauromyza (Cephalomyza) verbasci (Bouché, 1847) [Dizygomyza] - P2, B3, R1; 180-2300 m; 1, 2, 3, 4, 5; ? e; Buhr 1941; Beiger 1979a.

Cerodontha (Icteromyza) geniculata (Fallen, 1823) - ppt; Papp 1984b; Dursun et al. 1915; Pape \& Beuk 2017.

Cerodontha (Xenophytomyza) biseta (Hendel, 1920) - \$; happ 1984b; Spasić 1996; Pape \& Beuk 2017; Černý 2018.

Cerodontha (Cerodontha) affinis (Fallén, 1823) - RW; 1200-1500 m; 3; e; Černý \& Merz 2006; Černý 2013.

Cerodontha (Cerodontha) caucasica Zlobin, 1979 - R1; 2150-2350 m; 4, 5; bc; Černý 2018

Cerodontha (Cerodontha) denticornis (Panzer, 1806) - DW; 110-120 m; 1; ppt; Zamfirov 1961a.

Cerodontha (Cerodontha) phragmitophila Hering, 1935 - w; wp; Papp 1984b; Dursun et al. 1915; Pape \& Beuk 2017; Černý \& Bächli 2018.

Cerodontha (Cerodontha) unguicornis Hendel, 1932 - R1, R2; 1810-2350 m; 4, 5; des; Černý 2013.

Cerodontha (Poemyza) atra (Meigen, 1830) - B3; 150-160 m; 1; ean; Černý \& Merz 2006.

Cerodontha (Poemyza) incisa (Meigen, 1830) [Dizygomyza] - \$; h; Buhr 1941; Beiger 1979a; Černý 2013.

Cerodontha (Poemyza) lateralis (Macquart, 1835) - TL, BN; 0-170 m; 1; h; Nowakowski 1973; Beiger 1979a.

Cerodontha (Poemyza) muscina (Meigen, 1830) - RW; 370-400 m; 1; h; Beiger 1979a; Beschovski $2006 \mathrm{a}$.

Cerodontha (Poemyza) pygmaea (Meigen, 1830) - B3, TL, R1, RW, RE; 160-1400 m; 1, 2, 3; h; Beiger 1979a; Beron 2004; Beschovski 2006a.

Cerodontha (Phytagromyza) flavocingulata (Strobl, 1909) - V4; 900 m; 2; h; Černý \& Merz 2006; Černý \& Bächli 2018.

Cerodontha (Dizygomyza) fasciata (Strobl, 1880) - R2; 1400 m; 3; h; Černý 2013; Dursun et al. 1915.

Cerodontha (Dizygomyza) iraeos (Robineau-Desvoidy, 1851) - BS; 0-20 m; 1; ho; Beiger 1979a.

Cerodontha (Dizygomyza) spinata (Groschke, 1954) - R2; 2400-2500 m; 5, 6; des; Černý 2013.

Cerodontha (Dizygomyza) suturalis (Hendel, 1931) - ; tp; Papp 1984b; Dursun et al. 1915; Pape \& Beuk 2017; Černý 2018.

Liriomyza amoena (Meigen, 1830) - P2, V1, TL, R1, RW, RE; 150-600 m; 1; wpo, dp; Buhr 1941; Beiger 1979a; Beron 2004; Beschovski 2004 2006a; Černý \& Bächli 2018.

Liriomyza approximata (Hendel, 1920) - RW; 1000-1500 m; 1, 2, 3, 4; h; Beiger 1979a; Beschovski 2006 .

Liriomyza artemisicola de Meijere, 1924 - R1; 1150-1200 m; 3; ho; Buhr 1941.

Liriomyza balcanica (Strobl, 1900) - TL, RW; 150-400 m; 1; pat; Buhr 1941; Beiger 1979a; Černý 2018.

Liriomyza brassicae (Riley, 1884) - TL, RW; 150-400 m; 1; k; Beiger 1979a; Beschovski 2006a.

Liriomyza bryoniae (Kaltenbach, 1858) - TL; 150-170 m; 1; po; Buhr 1941; Elenkov \& Hristova 1974; Beiger 1979a.

Liriomyza buhri Hering, 1937 - R1; 650-670 m; 1; dp; Buhr 1941.

Liriomyza bulgarica Beiger, 1979 - TL; 150-170 m; 1; ee; Beiger 1979a, 1979 b.

Liriomyza cannabis Hendel, 1931 - TL; 150-170 m; 1; ean; Beiger 1979a.

Liriomyza centaureae Hering, 1927 - V4, RW; 400-1200 m; 1, 2, 3; ean; Beiger 1979a; Beschovski 2006 a.

Liriomyza congesta (Becker, 1903) - DM, P2, K9, V1, TL, R1, RW, RE; 130-1400 m; 1, 2, 3; po; Buhr 1941; Grigorov 1962; Beiger 1979a; Beron 2004; Beschovski 2006a; Černý 2018.

Liriomyza demeijerei Hering, 1930 - B3, TL, R1, RW; 160-1300 m; 1, 2, 3; dp; Buhr 1941; Beiger 1979a; Beschovski 2006a.

Liriomyza endiviae Hering, 1955 - B3, TL, RW; 160-750 m; 1, 2; h; Beiger 1979a; Beschovski $2006 \mathrm{a}$.

Liriomyza eupatorii (Kaltenbach, 1873) - P2, R1, R2, RW, BS; 20-1300 m; 1, 2, 3; h; Buhr 1941; Beiger 1979a; Beschovski 2006a.

Liriomyza eupatoriana Spencer, 1954 - \$; e; Černý 2013. 
Liriomyza flaveola (Fallén, 1823) - TL, RW; 160-1650 m; 1, 2, 3, 4; ho; Buhr 1941; Beiger 1979a; Beschovski 2006a; Černý 2018.

Liriomyza huidobrensis (Blanchard, 1926) - -; TL, O62; 150-200 m; 1; k, i; Beschovski \& Karadjova 1996; Karadjova \& Beschovski 1996; Harizanova \& Pavlov 1999.

Liriomyza infuscata Hering, 1926 - R2; 2400-2500 m; 5, 6; wces; Černý 2013; Černý \& Bächli 2018.

Liriomyza intonsa Spencer, 1976 - BN; 20-30 m; 1; ena; Černý \& Merz 2006; Černý 2018.

Liriomyza myrsinitae Hering, 1957 - RW; 1700 m; 4; se; Hering, 1957; Papp 1984b; Beschovski 2006 a.

Liriomyza pascuum (Meigen, 1838) - P2, B1, RW; 150-1380 m; 1, 2, 3; ean; Buhr 1941; Beiger 1979a; Beschovski 2006a; Černý \& Bächli 2018.

Liriomyza puella (Meigen, 1830) - B3, V4, R1; 500-1300 m; 1, 2, 3; ean; Buhr 1941; Beiger 1979a.

Liriomyza pusilla (Meigen, 1830) [L. fasciola (Meigen, 1838)] - B3, V1, TL, RW; 160-600 m; 1; po; Buhr 1941; Beiger 1979a; Beschovski 2006a.

Liriomyza scorzonerae Ryden, 1951 [L. scariolae Hering, 1955] - TL; 160-170 m; 1; e; Beiger 1979a.

Liriomyza solivaga Spencer, 1971 - R1; 2150- 2350 m; 4, 5; e; Černý 2018.

Liriomyza sonchi Hendel, 1931 - P2, TL, R1, RW, RE; 297-1200 m; 1, 2, 3; ho; Buhr 1941; Beiger 1979a; Beron 2004; Beschovski 2004, 2006a; Černý \& Bächli 2018.

Liriomyza soror Hendel, 1931 - P2; TL, RE; 160-300 m; 1; e; Beiger 1979a; Beron 2004; Beschovski 2004, 2006a; Černý \& Bächli 2018.

Liriomyza strigata (Meigen, 1830) - P2, V1, TL, R1, RW, RE; 160-1200 m; 1, 2, 3; wpo; Buhr 1941; Beiger 1979a; Beron 2004; Beschovski 2004, 2006a.

Liriomyza taraxaci Hering, 1927 - TL, R1, RW; 160-2100 m; 1, 2, 3, 4, 5; h; Buhr 1941; Beiger 1979a; Beschovski 2006a.

Liriomyza thesii Hering, 1924 - csee; Buhr 1941.

Liriomyza valerianae Hendel, 1932 - \$; e; Buhr 1941.

Liriomyza xanthocera (Czerny in Czerny \& Strobl, 1909) [L.crucifericola Hering, 1951] - RW; 350-400 m; 1; ? ena, ? sk; Beiger 1979a; Beschovski 2006a.

Galiomyza morio (Brischke, 1880) - ; e; Buhr 1941.

Phytoliriomyza arctica (Lundbeck, 1901) - BN; 15-30 m; 1; k; Černý \& Merz 2006.

Phytoliriomyza hilarella (Zetterstedt, 1848) [Dizygomyza] - \$; hi Buhr 1941.

Phytoliriomyza melampyga (Loew, 1869) [Liriomyza impatientis (Brischke, 1880)] - R1; 1150-1200 m; 3; h; Buhr 1941.

Phytoliriomyza perpusilla (Meigen, 1830) - ; pat; Černý 2013.

Phytoliriomyza variegata (Meigen, 1830) [Liriomyza] - R1; 660-1200 m; 1, 2, 3; ? wpo; Buhr 1941.

Calycomyza artemisiae (Kaltenbach, 1856) - B1, TL, R5, RW; 150-1700 m; 1, 2, 3, 4; hno, ? ho; Buhr 1941; Beiger 1979a; Beschovski 2006; Černý 2018.

Calycomyza humeralis (von Roser, 1840) - TL, RW, RE; 270-400 m; 1; k; Buhr 1941; Beiger 1979a; Beron 2004; Beschovski 2004, 2006a.

Calycomyza solidaginis (Kaltenbach, 1869) [Dizygomyza] - 1300 m; 3; h; Buhr 1941.

Nemorimyza posticata (Meigen, 1830) [Dizygomyza] - RW; 350-400 m; 1; hno; Buhr 1941; Beiger 1979a; Beschovski 2006a.

Aulagromyza anteposita (Strobl, 1898) - P2, B3; 170-1000 m; 1, 2; e; Černý \& Merz 2006.

Aulagromyza fulvicornis (Hendel, 1935) [Paraphytomyza; Phytagromyza langei Hering, 1937] - \$; e; Buhr 1941. Aulagromyza luteoscutellata (de Meijere, 1924) [Paraphytomyza] - B2, TL, R5, RW, RE; 160-1300 m; 1, 2, 3; h; 1941; Beiger 1979a; Beron 2004; Beschovski 2004, 2006a.

Aulagromyza orphana (Hendel, 1920) - P2, B3; 180-1000 m; 1, 2; ean; Černý \& Merz 2006; Černý 2018.

Aulagromyza populi (Kaltenbach, 1864) [Paraphytomyza] - B1, V1, TL; 160-800 m; 1, 2; ean; Buhr 1941; Beiger 1979a; Georgiev 1998; Georgiev \& Boyadzhiev 2002.

Aulagromyza similis (Brischke, 1880) [Phytagromyza] - V4, R1; 1150-1200 m; 3; e; Buhr 1941; Beiger 1979a. Aulagromyza tridentata (Loew, 1858) [Phytagromyza] - B1, R1; 670-1200 m; 1, 2, 3; ? ewca; Buhr 1941.

Pseudonapomyza europaea Spencer, 1973 - B2, V4, V5, S1, R2; 460-18100 m; 1, 2, 3, 4; h; Černý \& Merz 2006; Černý 2018.

Pseudonapomyza palliditarsis Cerny, 1992 - BN; 0-5 m; 1; dp; Černý \& Merz 2006; Černý 2013, 2018.

Pseudonapomyza strobliana Spencer, 1973 - \$; ean; Černý 2018. 
Napomyza bellidis Griffiths, 1967 - tp; Černý 2018.

Phytomyza albipennis Fallén, 1823 [Napomyza] - V4; 1200 m; 3; dp; Černý \& Merz 2006.

Phytomyza actaeae Hendel, 1922 - ; po; Buhr 1941.

Phytomyza affinis Fallen, 1823 - R1; 660-670 m; 1, 2; ena, ? h; Buhr 1941.

Phytomyza agromyzina Meigen, 1830 - P2, RW; 350-480 m; 1; h; Beiger 1979a; Beschovski 2006a.

Phytomyza albiceps Meigen, 1830 - des; Buhr 1941.

Phytomyza alpina Groschke, 1957 - R1, R2, RW; 1374-2000 m; 3, 4, 5; e, ? h, m; Beiger 1979a; Beschovski 2006 . Phytomyza angelicae Kaltenbach, 1872 [Ph. aegopodii Hendel, 1924] - V4; 800-1300 m; 2, 3; h; Beiger 1979a. Phytomyza angelicastri Hering, 1932 - \$ e; Buhr 1941; Pape \& Beuk 2017; Černý \& Bächli 2018.

Phytomyza aquilegiae Hardy, 1849 - V1, TL, R5; 160-600 m; 1; ewca; Buhr 1941; Beiger 1979a; Černý 2018.

Phytomyza artemisivora Spencer, 1971 - P2, V1, TL, R2, RW, RE; 160-1200 m; 1, 2, 3; ewca, ? dp; Buhr 1941;

Grigorov 1962; Beiger 1979a; Beron 2004; Beschovski 2004, 2006 a.

Phytomyza bipunctata Loew, 1858 - B1; 900-1100 m; 2, 3; e; Buhr 1941.

Phytomyza calthophila Hering, 1931 - RW; 900-1700 m; 2, 3, 4; e; Buhr 1941; Beiger 1979a; Beschovski 2006 .

Phytomyza campanulae Hendel, 1920 - P2, RW, BS; 0-1090 m; 1, 2, 3; e; Buhr 1941; Beiger 1979a; Beschovski 2006a.

Phytomyza chaerophylli Kaltenbach, 1856 [Ph. anthrisci Hendel, 1924; Ph. tordylii Hendel, 1927] - P2, B3, V1,

V4, TL, R1, R2, RW, BS; 0-1400 m; 1, 2, 3; ean; Buhr 1941; Beiger 1979a; Beschovski 2006a.

Phytomyza cirsii Hendel, 1923 - P1, R1; 200-1200 m; 1, 2, 3; ? tp; Buhr 1941; Beiger 1979a.

Phytomyza continua Hendel, 1920 - P3, B3; 20-30 m; 1; dp; Černý \& Merz 2006.

Phytomyza conyzae Hendel, 1920 - P3, B3, TL, R2, R5, RW, RE, BS; 0-1000 m; 1, 2; wpo; Beiger 1979a; Beron 2004; Beschovski 2006a.

Phytomyza crassiseta Zetterstedt, 1860 - V1; 550-600 m; 1; hn; Buhr 1941; Černý 2018.

Phytomyza digitalis Hering, 1925 - B1; 900-1100 m; 2, 3; e; Buhr 1941.

Phytomyza doronici Hendel, 1923 - RW; 900-1650 m; 2, 3, 4; e; Buhr 1941; Beiger 1979a; Beschovski 2006 .

Phytomyza erigerophila Hering, 1927 - R1; 660-670 m; 1; h; Buhr 1941; Černý \& Bächli 2018.

Phytomyza eupatorii Hendel, 1927 - R1; 1150-1300 m; 3; wpo; Buhr 1941; Beiger 1979a.

Phytomyza fallaciosa Brischke, 1880 [Ph. auricomi Hering, 1924] - V4; 800-1200 m; 2, 3; h; Beiger 1979a.

Phytomyza farfarae Hendel, 1935 - R1, R2, R5, RW; 700-1400 m; 2, 3; e; Buhr 1941; Beiger 1979a; Černý \& Bächli 2018.

Phytomyza fulgens Hendel, 1920 - P2, B3, RW, RE; 200-600 m; 1; eo; Beiger 1979a; Beron 2004.

Phytomyza glechomae Kaltenbach, 1862 - P2, S22, TL; 160-550 m; 1; des; Beiger 1979a.

Phytomyza hellebori Kaltenbach, 1872 - P3; 480 m; 1; e; Beiger 1979a.

Phytomyza heracleana Hering, 1937 - P2, TL, R5, RW; 150-1000 m; 1, 2; e; Buhr 1941; Beiger 1979a.

Phytomyza kyffhusana Hering, 1928 - s; e; Buhr 1941.

Phytomyza lappae Goureau, 1851 [Ph. lappina Goureau, 1851] - P2, V1, R1, RW, RE, BS; 0-1400 m; 1, 2, 3; e; Buhr 1941; Beiger 1979a; Beron 2004.

Phytomyza lycopi Nowakowski, 1959 - e; Pape \& Beuk 2017; Černý 2018.

Phytomyza marginella Fallén, 1823 [Ph. hieracina Hering, 1932; Ph. prenanthidis Hering, 1932; Ph. sonchi Robineau-Desvoidy, 1851] - V4, R1, RW; 370-1570 m; 1, 2, 3, 4; e; Buhr 1941; Beiger 1979a; Pape \& Beuk 2017.

Phytomyza minuscula Goureau, 1851 - V4; 800-1300 m; 2, 3; des; Buhr 1941; Beiger 1979a;Černý 2018.

Phytomyza nigrifemur Hering, 1934 - R2; 1600 m; 4; e; Černý 2013; Černý \& Bächli 2018.

Phytomyza notata Meigen, 1830 - R1, R2, RW; 1150-2350 m; 3, 4, 5; e; Beiger 1979a; Beschovski 2006a; Černý 2013, 2018.

Phytomyza obscura Hendel, 1920 - P2, RW, RE, BS; 0-1400 m; 1, 2, 3; e, ? ena; Buhr 1941; Beiger 1979a Beron 2004; Beschovski 2006a.

Phytomyza obscurella Fallén, 1823 - P2, V4, R2, RW; 200-1100 m; 1, 2, 3; tp; Buhr 1941; Beiger 1979a.

Phytomyza origani Hering, 1931 - RW; 900-1000 m; 2; eanna; Buhr 1941; Beiger 1979a.

Phytomyza pastinacae Hendel, 1923 - B3, V4, R1, RW; 500-1650 m; 1, 2, 3, 4; h; Buhr 1941; Beiger 1979a; Beschovski 2006a.

Phytomyza petoei Hering, 1924 - P2, R1, R2, RW; 200-1400 m; 1, 2, 3; wpo; Buhr 1941; Beiger 1979a; Beschovski 2006a; Černý 2018. 
Phytomyza plantaginis Robineau-Desvoidy, 1851 - P1, P2, P3, B3, V1, BS; 0-800 m; 1, 2; sk, ? k; Buhr 1941; Beiger 1979a; Černý \& Merz 2006.

Phytomyza pubicornis Hendel, 1920 - RW; 350-400 m; 1; ean; Beiger 1979a; Beschovski 2006a.

Phytomyza pullula Zetterstedt, 1848 [Ph. matricariae Hendel, 1920] - P2, B2, R1; 520-1300 m; 1, 2, 3; h; Buhr 1941; Černý \& Merz 2006.

Phytomyza pulmonariae Nowakowski, 1959 - P2, RW, BS; 0-400 m; 1; e; Beiger 1979a; Beschovski 2006 a.

Phytomyza ranunculi (Schrank, 1803) - P2, P3, S22, TL, RW; 160-1080 m; 1, 2, 3; ho; Beiger 1979a; Beschovski 2006a; Černý 2013.

Phytomyza ranunculivora Hering, 1932 - B3, R1, RW, BS; 0-1400 m; 1, 2, 3; e; Beiger 1979a; Beschovski 2006 . Phytomyza rapunculi Hendel, 1927 - RW; 1350 m; 3; e; Beiger 1979a; Beschovski 2006a.

Phytomyza rhodopaea Beiger, 1979 - RW; 750 m; 2; Er, ? Ebg; Beiger 1979b; Beschovski 2006a.

Phytomyza salviae (Hering, 1924) - B3, R1, R2, RW; 500-1400 m; 1, 2, 3; e; Buhr 1941; Beiger 1979a.

Phytomyza senecionis Kaltenbach, 1869 - R1, RW; 1150-1950 m; 3, 4; dp; Buhr 1941; Beiger 1979a; Beschovski 2006a; Černý \& Bächli 2018.

Phytomyza solidaginis Hendel, 1920 - RW; 350-400 m; 1; e; Buhr 1941; Beiger 1979a; Beschovski 2006a.

Phytomyza spondylii Robineau-Desvoidy, 1851 [Ph. sphondylii Goureau, 1851] - P2, R1; 200-1300 m; 1, 2, 3; ho; Buhr 1941; Beiger 1979a.

Phytomyza sphondyliivora Spencer, 1957 - V4; RW; 800-1080 m; 2, 3; e; Beiger 1979a; Beschovski 2006a.

Phytomyza spinaciae Hendel, 1935 [Ph. autumnalis Griffiths, 1959] - P2, TL; 160-220 m; 1; ewca; Buhr 1941; Beiger 1979a.

Phytomyza tanaceti Hendel, 1923 - R5; 550-580 m; 1; des; Buhr 1941; Beiger 1979a.

Phytomyza tetrasticha Hendel, 1927 - P2, V4, TL, R1, RW, RE; 160-1400 m; 1, 2, 3; eswa; Buhr 1941; Beiger 1979a; Beron 2004; Beschovski 2004, 2006a.

Phytomyza tussilaginis Hendel, 1925 - V4, R1, R2, RW; 900-1400 m; 2, 3; h; Beiger 1979a; Beschovski 2006 .

Phytomyza virgaureae Hering, 1926 - RW; 360-400 m; 1; e; Beiger 1979a; Beschovski 2006a.

Phytomyza vitalbae Kaltenbach, 1872 - P2, B2, V1, RW, RE; 0-1000 m; 1, 2; ppta; Buhr 1941; Beiger 1979a; Beron 2004; Beschovski 2004, 2006a; Černý \& Merz 2006.

Pseudonapomyza palliditarsis Cerny, 1992 - \$; des; Černý 2013.

Chromatomyia aprilina (Goureau, 1851) [Phytomyza] - V1; 550-600 m; 1; ena; Buhr 1941.

Chromatomyia fuscula (Zetterstedt, 1838) [Phytomyza] - S22, R1; 370-1900 m; 1, 2, 3, 4; h; Beiger 1979a; Černý 2013.

Chromatomyia gentianae (Hendel, 1920) [Phytomyza veratri Hering, 1941] - \$; eo; Buhr 1941; Pape \& Beuk 2017.

Chromatomyia gentianella (Hendel, 1932) [Phytomyza] - \$; e; Buhr 1941.

Chromatomyia horticola (Goureau, 1851) [Phytomyza] - P2, TL, R5, RE; 150-550 m; 1; hpt; Beiger 1979a; Beron 2004.

Chromatomyia milii (Kaltenbach, 1864) [Phytomyza] - B3; 800 m; 2; ho; Černý \& Merz 2006; Černý 2018.

Chromatomyia nigra (Meigen, 1830) [Phytomyza] - DM; 260 m; 1; ho; Černý \& Merz 2006.

Chromatomyia opacella (Hendel, 1935) - R1; 2150-2350 m; 4, 5; e, ? h; Černý 2013.

Chromatomyia saxifragae (Hering, 1924) [Phytomyza] - R2; $2000 \mathrm{~m} ; 4$, 5; csee; Buhr 1941; Beiger 1979a.

Chromatomyia scolopendri (Robineau-Desvoidy, 1851) [Phytomyza] - ean; Buhr 1941.

\section{Opomyzidae}

Opomyza florum (Fabricius, 1794) - -; ; DW, DM, E2, P1, P2, B1, B2, V1, V4, S211, T2, T31, O5, O62, R1, R2, R3, R4, RW, BN, BS; 0-2389 m; 1, 2, 3, 4, 5; e; Nedelkov 1912; Popoff 1956; Lyubenov 1956; Zamfirov 1958, 1961a, 1962c, 1963b; Makarov 1959; Grigorov 1972; Beschovski \& Minkova 1991; Krasteva \& Beschovski 2001; Krasteva et al. 2012.

Opomyza germinationis (Linnaeus, 1758) - DW, DM, E2, P1, P2, K9, V1, V4, S1, S211, T2, T31, O5, O62, R2, R3, R4, R5, RW, BN, BS; 0-1500 m; 1, 2, 3, 4; h; Beschovski \& Minkova 1991.

Opomyza petrei Mesnil, 1934 - DW, P2, V4, S1, TL, T31, RW, RE; 30-1200 m; 1, 2, 3; h; Beschovski \& Minkova 1991.

Opomyza punctella Fallén, 1820 - V4, RW; 1860-2000 m; 4, 5; e; Beschovski \& Minkova 1991. 
Geomyza combinata (Linnaeus, 1767) [G. martineki Drake, 1992] - V4, BN; 0-1150 m; 1, 2, 3; e; Beschovski \& Minkova 1991.

Geomyza paganettii (Strobl, 1909) - R3, BN; 0-1300 m; 1, 2, 3; e; Beschovski \& Minkova 1991.

Geomyza tripunctata Fallén, 1823 - DM, B2, V4, T31, R1, R2, R3, R5, RW; 100-2575; 1, 2, 3, 4, 5, 6; h; Beschovski \& Minkova 1991.

\section{Anthomyzidae}

Anagnota major Rohacek \& Freidberg, 1993 - e; Roháček 2004; Beschovski 2009b.

Anthomyza anderssoni Rohacek, 1984 - e; Roháček 2004; Beschovski $2009 \mathrm{~b}$.

Anthomyza gracilis Fallén, 1823 [A. sordidella Zetterstedt, 1848] - T31, BN; 0-70 m; 1; h; Beschovski 1976b, 2009b; Roháček 1998.

Fungomyza albimana (Meigen, 1830) [Anthomyza] - P2; 200-450 m; 1; e; Beschovski 1976b, 2009b; Roháček 1998.

Paranthomyza nitida (Meigen, 1838) - O62; 160 m; 1; e; Roháček 2004; Beschovski 2009b.

\section{Aulacigastridae}

Aulacigaster leucopeza (Meigen, 1830) - V1; 550-600 m; 1; h; Beschovski 1976b, $2009 \mathrm{~b}$.

\section{Periscelididae}

Periscelis (Myodris) annulata (Fallén, 1813) - V4, V5, BS; 0-1240 m; 1, 2, 3; dp, ? h; Beschovski 2001, 2009b; Máca 1987.

\section{Asteiidae}

Asteia (Asteia) amoena Meigen, 1830 - DW, E1, E2, P2, V4, S1, T31, O62, R2, RE, BN, BS; 0-940 m; 1, 2; pat; Beschovski 1966a, 2009b.

Asteia (Asteia) concinna Meigen, 1830 - BS; 0-5 m; 1; des; Beschovski 1976a, $2009 \mathrm{~b}$.

Leiomyza laevigata (Meigen, 1830) - DW, V1; 100-600 m; 1; h; Beschovski $2009 \mathrm{~b}$.

\section{Braulidae}

Braula coeca Nitzsch, 1818 - $\Delta$; •; V1, TL; 160-600 m; 1; sk, ? k; Drensky 1928, 1931b, 1932b, 1934b, 1936, 1939a; Hennig 1938; Shopov 1965; Beschovski 2013b.

Braula orientalis Örösi Pál, 1963 - \$ dp; Papp 1984c, 1998; Sidorenko 2004; Beschovski 2013b; Pape \& Beuk 2017.

Braula schmitzi Örösi Pál, 1963 - \$; pn; Papp 1984c, 1998; Beschovski 2013b; Pape \& Beuk 2017.

\section{Carnidae}

Meoneura alpina Hennig, 1948 - V4, R2; 1000-2000 m; 3, 4; csee; Beschovski 2004b, 3013b.

Meoneura atoma Papp, 1981 - B2; 1900-2170 m; 4, 5; csee; Beschovski 2004b, 3013b.

Meoneura carpathica Papp, 1977 - B2, V4, RE, BS; 0-1800 m; 1, 2, 3, 4; e; Beschovski 2004b, 3013b.

Meoneura flavifacies Collin, 1930 - V4, R2; 700-2010 m; 2, 3, 4; h; Beschovski 2004b, 3013b.

Meoneura flavifrons Papp, 1981 - S22, R2, R5; 560-2700 m; 1, 2, 3, 4, 5, 6; cse; Beschovski 2004b, 3013b.

Meoneura glaberrima Becker, 1910 - E2, B2, B3, V5, S1, T2, T31, R2, BN; 0-1800 m; 1, 2, 3, 4; wp; Beschovski 1972a, 2004b, 3013b.

Meoneura graeca Hennig, 1972 [M. vagans (Fallén, 1823)] - R2, RE, BN, BS; 0-450 m; 1; Eb; Beschovski 2004b, 3013b.

Meoneura prima (Becker, 1903) - B2; 1000 m; 2; h; Beschovski 2004b, 3013b. 


\section{Tethinidae}

Pelomyiella mallochi (Sturtevant, 1923) [Pelomyia kuntzei (Czerny, 1928)] - O62, BN; 0-150 m; 1; h, ? hn; Hendel 1934; Beschovski 1972a, 1975b, 1994b, 2009, 2013; Munari 2002; Munari \& Mathis 2010.

Tethina albosetulosa (Strobl, 1900) [T. griseola auct. (censu Czerny, 1928)] - BN, BS; 0-5 m; 1; atm, ? wpat; Beschovski 1964a, 1964b, 1972b, 1973c, 1973e, 1975a, 1975b, 1976a, 1993b, 1994b, 2009, 2013b; Mathis \& Munari 1996; Munari 2002; Munari \& Mathis 2010.

Tethina czernyi (Hendel, 1934) - BN, BS; 0-10 m; 1; wp; Beschovski 1993b, 1994b, 2009, 2013b; Mathis \& Munari 1996; Munari 2002; Munari \& Mathis 2010.

Tethina flavigenis (Hendel, 1934) [T. grisea Fallén, 1823] - BN, BS; 0-5 m; 1; ena, Beschovski 1964a, 1964b, 1965, 1993b, 1994b, 2009, 2013b; Munari 2002; Munari \& Mathis 2010.

Tethina grisea (Fallén, 1823) [Rhicnoessa cinerea Loew, 1862] - BN, BS; 0-5 m; 1; wp; Löw 1862; Czerny, 1928; Beschovski 1964a, 1964b, 1972b, 1973c, 1973e, 1975a, 1975b, 1976a, 1993b, 1994b, 2009, 2013b; Mathis \& Munari 1996; Munari 1996, 2002; Munari \& Mathis 2010.

Tethina pallipes (Loew, 1865) [Rhicnoessa ochracea Hendel, 1913] - BN, BS; 0-5 m; 1; sk; Beschovski 1993b, 1994b, 2009, 2013; Munari 1991, 2002; Mathis \& Munari 1996; Munari \& Mathis 2010.

Tethina strobliana (Mercier, 1923) [Rhicnoessa pallipes Loew, 1865] - E2, O62, BN; 0-120 m; 1; wp, ? swp; Beschovski 1993b, 1994b, 2009, 2013; Munari 2002; Munari \& Mathis 2010.

\section{Canacidae (Canaceidae)}

Canace salonitana Strobl, 1900 - BN, BS; 0-5 m; 1; em; Beschovski 1966a, 1972b, 1973a, 1975a, 2009, 2013b; Canzoneri \& Meneghini 1983; Hubenov et al. 1998; Munari \& Mathis 2010.

\section{Milichiidae}

Desmometopa m-nigrum (Zetterstedt, 1848) - V1, V4, BS; 0-700 m; 1, 2; k; Beschovski 1966a, 2004b, $2013 \mathrm{~b}$.

Desmometopa sordida (Fallén, 1820) - DW, DM, P1, S1, TL, T31, O62, R2, R5; 30-1000 m; 1, 2; ho; Beschovski 2004b, 2013b.

Leptometopa niveipennis (Strobl, 1900) - DW, E2, V1, TL, T2, O62, R2, BN, BS; 0-650; 1; wcp; Beschovski 1966a, 2004b, 2013b.

Leptometopa rufifrons Becker, 1903 - DM, E2, R2, BN, BS; 0-650 m; 1; mca, ? mit; Beschovski 1976a, 2004b, $2013 \mathrm{~b}$.

Madiza glabra Fallén, 1820 - DM, E2, B2, V1, S1, T31, O62, R1, R2, R3, R5, BN, BS; 0-2100 m; 1, 2, 3, 4, 5; h; Beschovski 2004b, 2013b.

Neophyllomyza acyglossa (Villeneuve, 1920) - O4, O62; 200-500 m; 1; eca; Beschovski 2004b, $2013 \mathrm{~b}$.

Phyllomyza securicornis Fallén, 1823 - V4, RW; 800-1330 m; 2, 3; h; Beschovski 2004b, 2013b.

Milichia speciosa Meigen, 1830 - BS; 0-5 m; 1; csena; Beschovski 2004b, $2013 \mathrm{~b}$.

\section{Chloropidae}

Rhodesiella fedtschenkoi Nartshuk, 1978 - R2; 300 m; 1; seet, ? nemit; Beschovski 2008, 2013b.

Rhodesiella plumiger (Meigen, 1830) - DW, O62, RE; 120-500 m; 1; tp; Beschovski 1982a, 1985a, $2013 \mathrm{~b}$.

Arcuator sexstriatus (Becker, 1912) [Oscinella pectoralis Becker, 1910] - BS; 0-5 m; 1; see; Beschovski 1982a, 2008, 2013b; Hubenov et al. 1993, 1998.

Aphanotrigonum bicolor Nartshuk, 1964 - DW, DM, B2, S1, T2, RE, BN, BS; 0-500 m; 1; seewca; Beschovski 1982a, 1985a, 2013; Dely-Draskovits 1985.

Aphanotrigonum femorellum Collin, 1946 [A. cinctella (Zetterstedt, 1848); Conioscinella] - DW, DM, P1, P2, P3, B2, K9, S1, TL, T31, O62, R2, R5, RE, BN, BS; 0-1600 m; 1, 2, 3, 4; wp; Beschovski 1968a, 1973c, 1975a, 1975b, 1976a, 1977a, 1985a, 1996a, 2004a, 2006a, 2008, 2013b; Beschovski \& Georgiev 1993.

Aphanotrigonum inerme Collin, 1946 - DW, DM, RE, BN, BS; 0-500 m; 1; e; Beschovski 1982a, 1985a, 2008, 2013.

Aphanotrigonum meijerei (Duda, 1933) [Conioscinella] - B2, BN; 0-600 m; 1, 2; eca; Beschovski 1968a, 1973c, 1975a, 1975b, 1976a, 1985a, 2008, 2013; Hubenov et al. 1993, 1998. 
Aphanotrigonum nigripes (Zetterstedt, 1848) - V1, V4, V5, O62, BS; 0-1250 m; 1, 2, 3; wces; Beschovski 1977a, 1985a, 2013b; Dely-Draskovits 1981; Beschovski \& Dimitrova 1990; Krasteva \& Beschovski 2000, 2001.

Aphanotrigonum parahastatum Dely-Draskovits, 1981 [A. femorellum Collin, 1946] - DW, DM, E2, P1, P2, P3, B3, K6, S1, S211, T2, O4, O5, O62, R2, R5, BS; 0-1540 m; 1, 2, 3, 4; hom, ? mwca; Beschovski 1977a, 1985a, 2008, 2013b; Dely-Draskovits 1981, 1985.

Aphanotrigonum trilineatum (Meigen, 1830) [A. beschovskii Dely-Draskovits, 1981] - DW, DM, E2, P2, B2, B3, V1, TL, R1, R2, R5, BN; 0-1150 m; 1, 2, 3; wces; Szilády 1934; Zamfirov 1960a, 1961a; Dely-Draskovits 1981; Beschovski 1985a, 2008, 2013b; Krasteva \& Beschovski 2000, 2001.

Calamoncosis (Calamoncosis) duinensis (Strobl, 1909) - BN, BS; 0-5 m; 1; wcp; Beschovski 1968a, 1985a, 2013b.

Calamoncosis (Calamoncosis) minima (Strobl, 1893) - BN, BS; 0-5 m; 1; tp; Beschovski 1968a, 1985a, 2013b.

Calamoncosis (Rhaphiopyga) glyceriae Nartshuk, 1958 - E2; 220-500 m; 1; wes; Beschovski 1985a, 2013b.

Conioscinella frontella (Fallen, 1820) [C. frontella fulvifrons Duda, 1933; C. mimula Collin, 1946] - DW, P1, B2, V1, V4, V5, T31, O62, R1, R2, R5, RW, BN, BS; 0-2250 m; 1, 2, 3, 4, 5; hoes, ? tp; Beschovski 1968a, 1975b, 1976a, 1977a, 1985a, 1998d, 2008, 2013b; Krasteva \& Beschovski 1998, 2000; Hubenov et al. 1999, 2000a; Popov et al. 2000a, 2000b.

Conioscinella gallarum (Duda, 1933) - e; Nartshuk 2004; Beschovski $2013 \mathrm{~b}$.

Conioscinella sordidella (Zetterstedt, 1848) - DM, B2, V1, O62, R1, BN; 0-1400 m; 1, 2, 3, 4; e; Beschovski 1996a, 2013b.

Conioscinella zetterstedti Andersson, 1966 - S1, R2; 280-600 m; 1; h; Beschovski 1982a, 1985a, 2008, 2013b; Hubenov et al. 1993, 1998.

Dicraeus (Dicraeus) ingratus (Loew, 1866) - DW, E2, P1, P2, V4, T2, O62, R2; 30-1800 m; 1, 2, 3, 4; h; Beschovski 1982a, 1985a, 2008, 2013b; Beschovski \& Dimitrova 1990.

Dicraeus (Dicraeus) nigropilosus Becker, 1910 - \$ DM, E1, E2, P1, P2, V4, T2, O62, R2, R5, BN, BS; 0-1200 m; 1, 2, 3; cseit, ? eit, ? set; Beschovski 1977a, 1982a, 1985a, 2008, 2013b.

Dicraeus (Dicraeus) raptus (Haliday, 1838) - ; DM, E2, P2, V1, V4, S211, TL, T2, O62, R2, R5, BN, BS; 0-1200 m; 1, 2, 3; e; Beschovski 1982a, 1985a, 2008, 2013b; Beschovski \& Dimitrova 1990.

Dicraeus (Dicraeus) sabroskyi Beschovski, 1977 - V1, V4, TL; 150-1000 m; 1, 2; Ebg; Beschovski 1977b, 1985a, 2013b.

Dicraeus (Dicraeus) tibialis (Macquart, 1835) [D. pallidiventris (Macquart, 1835)] - ; DW, DM, E1, E2, P1, P2, K2, V1, V4, V5, S1, TL, T2, T31, O4, O62, R2, RE, BN, BS; 0-900 m; 1, 2; ha, ? sk; Beschovski 1968a, 1977a, 1985a, 2008, 2013b; Beschovski \& Dimitrova 1990.

Dicraeus (Dicraeus) valkanovi Beschovski, 1982 - BN, BS; 0-5 m; 1; Er; Beschovski 1982a, 1985a, 2013b.

Dicraeus (Oedesiella) discolor (Becker, 1910) - T2, BN, BS; 0-35 m; 1; seean, ? nem; Beschovski 1974, 1976a, 1985a, 1996a, 2008, 2013b.

Dicraeus (Oedesiella) fennicus Duda, 1933 - P2; 400 m; 1; h; Beschovski 2008, 2013b.

Dicraeus (Paroedesiella) vagans (Meigen, 1838) [D. xanthopygus Strobl, 1909] - P2, V4, T2, BN; 0-1000 m; 1, 2; e; Beschovski 1968a, 1985a, 2008, 2013b.

Elachiptera agricola Beschovsky \& Krasteva, 1998 - V1; 520-600 m; 1; Ebg; Beschovski \& Krasteva 1998, 2001; Beschovski 2008, 2013b.

Elachiptera bimaculata (Loew, 1845) - V1, V4, O61, O62, BN; 0-800 m; 1, 2; csena; Beschovski 1980a, 1985a, 2008, 2013b.

Elachiptera brevipennis (Meigen, 1830) - BN; 0-5 m; 1; e; Beschovski \& Krasteva 1998; Beschovski 2008, $2013 \mathrm{~b}$. Elachiptera cornuta (Fallén, 1820) [Crassiseta] - * DW, DM, E1, E2, P1, B1, B2, B3, V1, V4, V5, S1, TL, T11, T31, O4, O61, O62, R1, R2, R5, RW, RE, BN, BS; 0-2200 m; 1, 2, 3, 4, 5; hop, ? wcp; Nedelkov 1912; Szilady 1934; Beschovski 1975b, 1977a, 1980a, 1985a, 1996a, 1998d, 1998e, 2006a, 2008, 2013b; Beschovski \& Dimitrova 1990; Beschovski \& Krasteva 1997; Krasteva \& Beschovski 1998, 2000, 2001; Hubenov et al. 1999, 2000a; Popov et al. 2000a, 2000b.

Elachiptera diastema Collin, 1946 - B2, V4, R1; 1000-2300 m; 2, 3, 4, 5; ena; Beschovski 1998e, 2008, 2013b; Hubenov et al. 1999, 2000a; Popov et al. 2000a, 2000b.

Elachiptera megaspis (Loew, 1858) - BN; 0-5 m; 1; ena; Beschovski 1980a, 1985a, 1996a, 2008, $2013 \mathrm{~b}$.

Elachiptera rufifrons Duda, 1932 [E. cornuta var. rufifrons Duda, 1932] - B2, O61, BN, BS; 0-2050 m; 1, 3, 4, 5; ? wp; Beschovski 1968a, 1980a, 1985a, 1996a, 2008, 2013b; Popov et al. 2000a, 2000 b.

Elachiptera scrobiculata (Strobl, 1901) - V4; 700-800 m; 2; des; Beschovski 2008, 2013b. 
Elachiptera sibirica (Loew, 1858) - DM, E2, P1, B2, V1; 200-1600 m; 1, 2, 3, 4; tes; Beschovski 1980a, 1985a, 2008, 2013b; Hubenov et al. 1999, 2000a.

Elachiptera submediterranea Beschovski, 1980 - TL, T31, R2, RW; 200-1250 m; 1, 2, 3; Ebg; Beschovski 1980a, 1985a, 2006a, 2008, 2013b; Hubenov et al. 1993, 1998.

Elachiptera tuberculifera (Corti, 1909) - \$ DW, DM, E2, P1, B2, K6, V1, V4, S1, TL, T1, T31, O62, R2, R5, RE; 20-2070 m; 1, 2, 3, 4, 5; hop; Beschovski 1968a, 1977a, 1980a, 1985a, 1998e, 2006a, 2008, 2013b; Beschovski \& Dimitrova 1990; Hubenov et al. 1999, 2000a; Popov et al. 2000a, 2000b.

Gampsocera numerata (Heeger, 1858) - E1; 230-300 m; 1; tes; Beschovski 2008, $2013 \mathrm{~b}$.

Gaurax fascipes Becker, 1910 - R1; 1400 m; 3; e; Beschovski 2008, 2013b.

Hapleginella laevicollis (Zetterstedt, 1858) - R1, RW; 500-1400 m; 1, 2, 3; hoes; Nartshuk 2004a; Beschovski 2008, 2013b.

Incertella albipalpis (Meigen, 1830) - \$ DW, DM, E1, E2, P1, P2, B1, B2, K6, V1, V4, V5, S1, S211, TL, T2, T31, O62, R1, R2, R5, RE, BN, BS; 0-2150 m; 1, 2, 3, 4, 5; hoes; Beschovski 1981b, 1985a, 2008, 2013b; Beschovski \& Dimitrova 1990; Hubenov et al. 1999, 2000a; Popov et al. 2000a, $2000 \mathrm{~b}$.

Incertella kerteszi (Becker, 1910) - DW, DM, E2, P2, B2, T31, R1; 40-1500 m; 1, 2, 3, 4; hoes; Beschovski 2008, 2013 b.

Incertella nigrifrons (Duda, 1933) [Conioscinella] - E2, B1, B2, BN, BS; 0-1500 m; 1, 2, 3; des; Beschovski 1982a, 1985a, 2008, 2013b.

Incertella zuercheri (Collin, 1946) [Tropidoscinis] - TL, R1, BN, BS; 0-1400 m; 1, 2, 3; eca; Beschovski 1968a, 1998d, 2008, 2013b.

Lasiambia brevibucca (Duda, 1933) [Fiebrigella palposa Fallén, 1820] - DW, DM, P1, B1, TL, O3; 80-450 m; 1; e; Beschovski 1982a, 1985a, 2008, 2013b; Nartshuk 1984, 2004a; Beschovski \& Georgiev 1993; Georgiev et al. 2004.

Lasiambia palposa (Fallén, 1820) [Fiebrigella] - B2, R1, R3, RW; 1200-2200 m; 3, 4, 5; ? hoes; Beschovski 1982a, 1985a, 2008, 2013b.

Lipara lucens Meigen, 1830 - O61, O62, BN, BS; 0-550 m; 1; ? wp, h*; Beschovski 1982a, 1985a, 1993a, 1995a, 2008, 2013b.

Lipara pullitarsis Doskocil \& Chvala, 1971 - K4, K8, O1, O61, O62, BN, BS; 0-700 m; 1, 2; e; Beschovski 1993a, 1995a, 2008, 2013b.

Lipara rufitarsis Loew, 1858 - V1, O1, O62, BN, BS; 0-600 m; 1; ? tp, h*; Beschovski 1982a, 1985a, 1993a, 1995a, 2008, 2013b.

Lipara similis Schiner, 1854 - E2, K3, K4, K8, V1, O61, O62, R2, BN, BS; 0-750 m, 1, 2; eit, ? wp; Beschovski 1968a, 1985a, 1993a, 1995a, 2008, 2013b.

Melanochaeta pubescens (Thalhammer, 1898) [Elachiptera] - $\$$ DW, DM, E2, P1, B1, K8, V4, S1, TL, T11, T2, T31, O4, O62, BN, BS; 0-1200 m; 1, 2, 3; wp; Szilady 1934; Beschovski 1975b, 1976a, 1977a, 1985a, 1996a, 2008, 2013b.

Microcercis trigonella (Duda, 1933) [Incertella, Oscinella] - DW, P2, B2, V1, V4, S1, S22, R2, BN; 5-1800 m; 1, 2, 3, 4; hoes, ? tp; Beschovski 1978a, 1981b, 1985a, 1996a, 1998e, 2008, 2013b; Hubenov et al. 1999, 2000a; Popov et al. 2000a, 2000b.

Oscinella (Cyclocercula) nartshukiana Beschovski, 1978 - B3, V1, S1, O62, BN; 0-650 m; 1; sppt; Beschovski 1978a, 1985, 2008, 2013b.

Oscinella (Oscinella) alopecuri Balachovsky \& Mesnil, 1935 - DW, E1, E2, P2, V1, V4, S1, S22, O4, R2, R5, BN, BS; 0-1800 m; 1, 2, 3, 4; cse, e; Beschovski 2008, 2013b.

Oscinella (Oscinella) angularis Collin, 1946 [O. angustipennis Duda 1933] - BN; 0-10 m; 1; e; Beschovski 1978a, 1996a, 2008, 2013b.

Oscinella (Oscinella) cariciphila Collin, 1946 - P1, B2, V4, S22, O61, O62, R2, BN; 0-2700 m; 1, 2, 3, 4, 5, 6; wces; Beschovski 1978a, 1985a, 2008, 2013b.

Oscinella (Oscinella) frit (Linnaeus, 1758) [O. hortensis Collin, 1946; Oscinisoma; Oscinis; ? Sabroskyina szilady (Duda, 1933)] - -; DW, DM, E1, E2, P1, P2, B1, B2, K2, K7, V1, V4, V5, S1, S21, S211, S22, TL, T11, T2, T31, O4, O5, O62, R1, R2, R3, R5, RW, RE, BN, BS; 0-2700 m; 1, 2, 3, 4, 5, 6; k; Malkov 1901, 1902b, 1904b, 1905, 1906a, 1907, 1908; Dospevski 1908b, 1908c; Kozarov 1908; Hitilov 1912a, 1912b; Drenowsky 1922c, 1923a; Drenowsky \& Enderlein 1923; Tschorbadjiew 1925a, 1925c, 1925f, 1926b, 1927, 1929a, 1929b, 1930a, 1930b, 1931, 1932; Antonov 1927; Drensky 1930a, 1930b; Szilady 1934; Lazarov 1935b, 1942, 1943; 
Buresch \& Lazarov 1956; Popoff 1956; Lyubenov 1958, 1960, 1961; Gospodinov 1958; Zamfirov 1958, 1961a, 1961b, 1962a, 1962b, 1962c, 1962d, 1962e, 1963a, 1963b, 1963c; Kovachevski et al. 1959; Makarov 1959; Donchev 1961; Grigorov 1972, 1976; Beschovski 1973c, 1975a, 1975b, 1976a, 1977a, 1978a, 1985a, 1996a, 1998d, 1998e, 2006a, 2008, 2013b; Michailova \& Beschovski 1985; Beschovski \& Dimitrova 1990; Kontev et al. 1991; Harizanov et al. 1996; Beschovski \& Krusteva 1997, 1998; Krasteva \& Beschovski 1998, 2000; Hubenov et al. 1999, 2000a; Popov et al. 2000a, 2000b; Hristov et al. 2004.

Oscinella (Oscinella) maura (Fallén, 1820) - DM, E2, P2, B2, V4, S1, T31, O1, O62, R1, R2, RW, RE, BN; 0-1450 m; 1, 2, 3, 4; wes; Beschovski 1978a, 1985, 1985a, 1998e, 2008, 2013b; Beschovski \& Dimitrova 1990.

Oscinella (Oscinella) nigerrima (Macquart, 1935) - - DM, E1, E2, P2, B2, B3, V1, V4, V5, S1, TL, T2, T31, O4, O5, O61, O62, R1, R2, R3, R5, RW, RE, BN, BS; 0-2500 m; 1, 2, 3, 4, 5, 6; e; Beschovski 1977a, 1978a, 1985a, 1996a, 1998e, 2006a, 2013b; Michailova \& Beschovski 1985; Beschovski \& Dimitrova 1990; Hubenov et al. 1999, 2000a; Popov et al. 2000a, 2000b.

Oscinella (Oscinella) nitidigenis (Becker, 1908) - DW, DM, B3, S1, TL, O4, R2, R3, RE, BS; 0-1000 m; 1, 2; swpat; Beschovski 1978a, 1985a, 2008, 2013b.

Oscinella (Oscinella) nitidissima (Meigen, 1838) - \$ DW, DM, P2, B1, B2, V1, V4, S1, S211, TL, T11, T2, T31, O61, O62, R1, R2, RW, RE, BN; 0-2200 m; 1, 2, 3, 4, 5; h; Szilady 1934; Lazarov 1935b; Beschovski 1977a, 1978a, 1985a, 1996a, 1998e, 2006a, 2008, 2013b; Beschovski \& Dimitrova 1990; Hubenov et al. 1999, 2000a; Popov et al. 2000a, 2000b.

Oscinella (Oscinella) phlei Nartshuk, 1955 - DW, E2, P2, V1, V4, S22, TL, O62, R2, R3, R5, RW; 50-2500 m; 1, 2, 3, 4, 5, 6; ee; Beschovski 1978a, 1985a, 2008, 2013 b.

Oscinella (Oscinella) pusilla (Meigen, 1830) [O. frit var. pusilla Meig, 1830; Oscinis] - $\$$; DW, DM, E1, E2, P1, P2, B1, B2, V1, V4, V5, S1, S22, TL, T11, T2, O4, O5, O62, R1, R2, RW, RE, BN, BS; 0-2700 m; 1, 2, 3, 4, 5, 6; hop; Hitilov 1912a, 1912b; Tschorbadjiew 1932; Buresch \& Lazarov 1956; Beschovski 1972a, 1973c, 1975a, 1975b, 1976a, 1977a, 1978a, 1996a, 1998e, 2006a, 2013b; Michailova \& Beschovski 1985; Beschovski \& Dimitrova 1990; Krasteva \& Beschovski 1998, 2001; Hubenov et al. 1999, 2000a; Popov et al. 2000a, 2000b.

Oscinella (Oscinella) vastator (Curtis, 1845) - ; DW, DM, E2, P1, B2, B3, V1, V4, S1, S22, T2, O4, O5, R1, R2, R5, BN; 0-1740 m; 1, 2, 3, 4; e; Beschovski 1977a, 1978a, 1985a, 2008, 2013b; Beschovski \& Dimitrova 1990.

Oscinella (Oscinella) ventricosi Nartshuk, 1955 - DW, B2, V1, V4, S1, S211, TL, T31, O4, O61, O62, R2, RE, BN, BS; 0-2200 m; 1, 2, 3, 4, 5; wes; Beschovski 1978a, 1985a, 1996a, 2008, 2013b; Beschovski \& Dimitrova 1990.

Oscinimorpha albisetosa (Duda, 1932) [Conioscinella] - B2, BN, BS; 0-1750 m; 1, 2, 3; wesca; Beschovski 1968a, 1985a, 1998e, 2013b; Hubenov et al. 1999, 2000a; Popov et al. 2000a, 2000 b.

Oscinimorpha arcuata (Duda, 1932) - \$ DW, DM, E1, E2, P1, P2, V4, S1, T11, T31, O4, O5, O62, R2, RE, BN, BS; 0-2200 m; 1, 2, 3, 4, 5; wesca, ? eswa, esca; Beschovski 1977a, 1985a, 1996a, 2013b; Beschovski \& Dimitrova 1990.

Oscinimorpha minutissima (Strobl, 1900) - \$ DM, E1, E2, P1, P2, B1, B2, V4, TL, T2, T31, O4, O62, R1, R2, RE, BN, BS; 0-2050 m; 1, 2, 3, 4, 5; wp; Szilady 1934; Beschovski 1977a, 1985a, 1998d, 1998e, 2008, 2013b; Hubenov et al. 1999, 2000a; Popov et al. 2000a, 2000b.

Oscinimorpha novakii (Strobl, 1893) - P1, B2, V1, O62, BS; 0-1550 m; 1, 2, 3; eswa; Beschovski 1982a, 1985a, 2008, 2013b; Hubenov et al. 1993, 1998; Krasteva \& Beschovski 2000.

Oscinimorpha sordidissima (Strobl, 1893) - DW, E2, P2, B2, TL, R1, R2, BS; 0-1550 m; 1, 2, 3, 4; e; Beschovski 1982a, 1985a, 1998e, 2013b; Hubenov et al. 1999, 2000a; Popov et al. 2000a, 2000b.

Oscinisoma cognatum (Meigen, 1830) - B3, V4; 900-1000 m; 2, 3; hoes; Beschovski 1982a, 1985a, 2008, 2013b; Beschovski \& Dimitrova 1990.

Polyodaspis ruficornis (Macquart, 1835) [Goniopsita, Macrothorax] - DM, E2, P2, B2, T31, O61, O62, R2, RE, BS; 0-2150 m; 1, 2, 3, 4, 5; po; Sachtleben 1930; Szilady 1934; Lazarov 1940; Buresch 1953b; Beschovski 1977a, 1985a, 1998e, 2008, 2013b; Beschovski \& Georgiev 1993; Hubenov et al. 1999, 2000a; Popov et al. 2000a, 2000b.

Polyodaspis sulcicollis (Meigen, 1838) - \$ DM, E2, P1, B2, S1, T31, O62, R2, RE, BN, BS; 0-1800 m; 1, 2, 3, 4; wp; Beschovski 1976a, 1977a, 1982a, 1985a, 1996a, 1998e, 2008, 2013b; Hubenov et al. 1999, 2000; Popov et al. 2000a, 2000b.

Rhopalopterum anthracinum (Meigen, 1830) [Lioscinella] - DW, O62, R2; 30-500 m; 1; wces ?; Beschovski 1977a, 1985a, 2008, 2013b.

Rhopalopterum femorale (Collin, 1946) - DW, B2; 100-800 m; 1, 2; wces; Beschovski 2008, 2013b. 
Sabroskyina sziladyi (Duda, 1933) [Oscinella] - O62, BN; 0-250 m; 1; Ebg; Duda 1933; Szilady 1934; Lazarov 1935b; Nartshuk 1984; Beschovski 1985a, 1987a, 2008, 2013b.

Siphonella oscinina (Fallen, 1820) - R1; 1400 m; 3, 4; h; Nartshuk 1984; Beschovski 1998d, 2008, 2013b.

Speccafrons halophila (Duda, 1933) [Conioscinella] - BN; 0-5 m; 1; hoes, ? tp; Beschovski 1968a, 1985a, 2013b; Hubenov et al. 1993, 1998.

Trachysiphonella pygmaea (Meigen, 1838) - DW, DM, E1, E2, P1, P2, B2, V4, TL, T31, O4, O62, R2, R3, RW, BN, BS; 0-1000 m; 1, 2; wes; Nartshuk 1984; Beschovski 2008, 2013 b.

Trachysiphonella ruficeps (Macquart, 1835) - \$ DW, DM, E1, E2, P1, P2, B2, V1, V4, V5, S1, S211, S22, T31, O4, O62, R1, R2, R3, BN, BS; 0-1900; 1, 2, 3, 4; ena; Beschovski 1982a, 1985a, 1998d, 1998e, 2008, 2013b; Beschovski \& Dimitrova 1990; Hubenov et al. 1999, 2000a; Popov et al. 2000a, $2000 \mathrm{~b}$.

Trachysiphonella scutellata (von Roser, 1840) [Oscinella pumilio var. flaviola Zetterstedt, 1848; Conioscinella] - DW, DM, E1, E2, P1, P2, B1, B2, V1, V4, T31, O62, R2, R3, RE, BN; 0-1600 m; 1, 2, 3; eca; Szilady 1934; Lazarov 1935b; Beschovski 1976a, 1985a, 1977a, 1996a, 2008, 2013b; Beschovski \& Dimitrova 1990.

Tricimba (Nartshukiella) albiseta Dely-Draskovits, 1983 - RW; 300-800 m; 1, 2; e; Dely-Draskovits 1983; Nartshuk 2004a; Beschovski 2013b.

Tricimba (Nartshukiella) cincta (Meigen, 1830) [Notonaulax] - \$ DW, DM, E2, P1, P2, B1, B2, V1, V4, S1, S211, T2, O62, R2, R5, RW, RE, BN, BS; 0-1820 m; 1, 2, 3, 4; h; Nedelkov 1912; Szilady 1934; Beschovski 1976a, 1977a, 1981c, 1985a, 1996a, 2008, 2013b; Beschovski \& Dimitrova 1990; Popov et al. 2000a, 2000b.

Tricimba (Nartshukiella) humeralis (Loew, 1858) - DM, E2, V1, V4, T2, O62, R2, RW, RE, BN, BS; 0-1000 m; 1, 2; hop; Beschovski 1981c, 1982a, 1985a, 2008, 2013b.

Tricimba (Tricimba) lineella (Fallén, 1820) [Notonaulax] - DW, P1, B1, V1, BN; 0-600 m; 1; h, ? hat; Nedelkov 1912; Beschovski 1981c, 1985a, 2008, 2013b.

Assuania thalhammeri (Strobl, 1893) - DW, E2, T31, O62, RE, BN, BS; 0-280 m; 1; wp; Beschovski 1973c, 1975b, 1985a, 2013b.

Camarota curvipennis (Latreille, 1805) [C. flavitarsis Meigen, 1830] - ; DW, DM, E1, E2, P1, P2, B1, B2, B3, V1, V4, S1, S211, T2, T31, O5, RE, BN, BS; 0-2000 m; 1, 2, 3, 4; ena; Szilady 1934; Zamfirov 1960b, 1961a; Beschovski 1973c, 1975a, 1985a, 1998e, 2013b; Beschovski \& Dimitrova 1990; Beschovski \& Krusteva 1997; Krasteva \& Beschovski 1998, 2000; Hubenov et al. 1999, 2000a; Popov et al. 2000a, 2000b.

Cetema (Cetema) cereris (Fallen, 1820) [Centor] - DW, E2, P1, P2, V4, V5, S1, T11, T31, O62, R1, R2, BN; 0-1800 m; 1, 2, 3, 4; hoes; Nedelkov 1912; Beschovski 1977a, 1985a, 2013b; Beschovski \& Dimitrova 1990.

Cetema (Cetema) elongatum (Meigen, 1830) [C. obliqua Beschovski, 1984; C. simile Ismay, 1985] - P2, B2, V1, V4, V5, S211, T2, R1, R2, R5, RW, BN; 0-1920 m; 1, 2, 3, 4; h; Beschovski 1982a, 1984a, 1985a, 2013b; Beschovski \& Dimitrova 1990; Hubenov et al. 1999, 2000a; Nartshuk 2004.

Cetema (Cetema) myopinum (Loew, 1866) [Chlorops] - DW, P2, B2, V1, V4, R1, R2, RW, BN; 0-2160 m; 1, 2, 3, 4, 5; wesca; Beschovski 1968a, 1977a, 1985a, 1998e, 2013b; Beschovski \& Dimitrova 1990; Hubenov et al. 1999, 2000a; Popov et al. 2000a, 2000b.

Cetema (Cetema) neglectum Tonnoir, 1921 - DW, E1, B2, V4, T2, T31, R1, RW, BN, BS; 0-1860 m; 1, 2, 3, 4, 5; e; Beschovski 1982a, 1985a, 2013b; Beschovski \& Dimitrova 1990.

Chlorops (Chlorops) adjunctus Becker, 1910 - B3, V4; 800-1000 m; 2; e; Beschovski 1982a, 1985a, $2013 \mathrm{~b}$.

Chlorops (Chlorops) anthracophagoideus Strobl, 1901 - V1, V4, V5; 600-1000 m; 1, 2; e; Beschovski 1978b, 1980a, 1982a, 1985a, 2013b; Beschovski \& Dimitrova 1990; Hubenov et al. 1993, 1998.

Chlorops (Chlorops) calceatus Meigen, 1830 - DM, E2, P2, B1, B2, V4, V5, S1, O4, R1, R2, R3, RW; 100-1900 m; 1, 2, 3, 4; wces; Beschovski 1978b, 1985a, 1998e, 2013b; Beschovski \& Dimitrova 1990.

Chlorops (Chlorops) fasciatus Meigen, 1830 - P2, B2, V4, V5, S22, R2; 190-1900 m; 1, 2, 3, 4; wces; Beschovski 1978b, 1980b, 1985a, 2013b, 2013c; Beschovski \& Dimitrova 1990.

Chlorops (Chlorops) finitimus Becker, 1910 - DW, V4, S1, TL, R2; 120-2350 m; 1, 2, 3, 4, 5; ewca; Beschovski 1978b, 1980b, 1985a, 2013b; Beschovski \& Dimitrova 1990.

Chlorops (Chlorops) geminatus Meigen, 1830 - V4, S211, R1, R2, R4, RW, BN: 0-1900 m; 1, 2, 3, 4; wces; Beschovski 1978b, 1980b, 1985a, 2013b; Beschovski \& Dimitrova 1990.

Chlorops (Chlorops) gracilis Meigen, 1830 - V4, BS; 0-1000 m; 1, 2; wces; Beschovski 1978b, 1980b, 1985a, 2013b; Hubenov et al. 1993, 1998. 
Chlorops (Chlorops) hypostigma Meigen, 1830 - ; DW, E2, P2, B2, B3, V4, V5, S1, O1, O5, R2, RW, RE, BN; 0-1800 m; 1, 2, 3, 4; e; Szilady 1934; Lazarov 1935b; Beschovski 1977a, 1978b, 1980b, 1985a, 1998e, 2013b; Beschovski \& Dimitrova 1990.

Chlorops (Chlorops) interruptus Meigen, 1830 - P2, B2, R3, RE, BN, BS; 0-1500 m; 1, 2, 3; ena; Beschovski 1978b, 1980b, 1985a, 2013b; Hubenov et al. 1999, 2000a.

Chlorops (Chlorops) meigenii Loew, 1866 - DM, B2, V4, S211, R2, RW; 200-1800 m; 1, 2, 3, 4; hoes; Beschovski 1978b, 1980b, 1985a, 1998e, 2013b; Beschovski \& Dimitrova 1990; Hubenov et al. 1999, 2000a; Popov et al. 2000a, 2000b.

Chlorops (Chlorops) novakii Strobl, 1902 - E2, V4; 230-1650 m; 1, 2, 3; tes, ? hoes; Beschovski 1978b, 1980b, 1985a, 2013b; Beschovski \& Dimitrova 1990.

Chlorops (Chlorops) pumilionis (Bjerkander, 1778) [Ch. nasutus (Schrank, 1781); Ch. taeniopus Meigen, 1830; Oscinis pumilio Zetterstedt, 1848] - -; m; 1, 2, 3; wcp; Malkov 1902b, 1904b, 1905, 1907; Kozarov 1912; Nedelkov 1912; Drenowsky 1920a, 1921a, 1922b, 1922c, 1922d, 1923a, 1923b; Drenowsky \& Enderlein 1923; Tschorbadjiew 1924b, 1925a, 1925f, 1926b, 1927, 1928a, 1928b, 1929a, 1929b, 1930a, 1932; Botev 1925; Savov 1925; Antonov 1927; Szilady 1934; Lazarov 1935b, 1936a, 1942; Petkoff 1939; Buresch \& Lazarov 1956; Popoff 1956; Gospodinov 1958; Lyubenov 1958, 1960, 1961; Zamfirov 1958, 1960b, 1961a, 1962a, 1962b, 1962c, 1962e, 1963a, 1963b, 1963c; Kovachevski et al. 1959; Makarov 1959; Grigorov 1964, 1972, 1976; Beschovski 1977a, 1978b, 1985a, 1980b, 1996a, 2013b; Dely-Draskovits 1985; Beschovski \& Dimitrova 1990; Harizanov et al. 1996; Beschovski \& Krusteva 1997; Krasteva \& Beschovski 1998.

Chlorops (Chlorops) quercophila Beschovski, 1979 - B3, BS; 0-100 m; 1; Ebg; Beschovski 1979, 1980b, 1985a, 2013b; Hubenov et al. 1993, 1998.

Chlorops (Chlorops) ringens Loew, 1866 - DW, DM, B2, V4, V5, S211, S22, T2, R2, R3, RE, BN, BS; 0-1800 m; 1, 2, 3, 4; wces; Beschovski 1978b, 1980b, 1985a, 2013b; Hubenov et al. 1999, 2000a.

Chlorops (Chlorops) rufinus (Zetterstedt, 1848) - B1, B2, S22, R3; 390-2000 m; 1, 2, 3, 4, 5; hoes; Szilady 1934; Lazarov 1935b; Beschovski 1979, 1980b, 1985a, 1998e, 2013b; Hubenov et al. 1999, 2000a; Popov et al. 2000a, 2000b.

Chlorops (Chlorops) scalaris Meigen, 1830 - ; E1, E2, K2, V1, V4, V5, S1, R2, RW, BN, BS; 0-1900 m; 1, 2, 3, 4; wces, ? esca; Beschovski 1978b, 1980b, 1985a, 2013b; Beschovski \& Dimitrova 1990.

Chlorops (Chlorops) serenus Loew, 1866 - P2, TK, V1, V4, V5, S1, S211, RW, BN; 0-1900 m; 1, 2, 3, 4; wpn; Beschovski 1978b, 1980b, 1985a, 2006a, 2013b; Beschovski \& Dimitrova 1990.

Chlorops (Chlorops) speciosus Meigen, 1830 - B2, V1, V4, V5, S1, O1, R1, R2, RW, BN; 0-2500 m; 1, 2, 3, 4, 5, 6; wes; Beschovski 1978b, 1980b, 1985a, 1998e, 2013b; Beschovski \& Dimitrova 1990; Hubenov et al. 1999, 2000a; Popov et al. 2000a, 2000b.

Chlorops (Chlorops) strigulus (Fabricius, 1794) - B3, TL, RE, BN, BS; 0-1000 m; 1, 2; des; Beschovski 1979, 1980b, 1985a, 2013b.

Chlorops (Chlorops) troglodytes (Zetterstedt, 1848) - DM, P2, B2, V1, V4, V5, S211, R1, R2, R3, RW, RE, BS; 5-2100 m; 1, 2, 3, 4, 5; wces; Beschovski 1977a, 1978b, 1980b, 1985a, 1998e, 2006a, 2013b; Beschovski \& Dimitrova 1990; Hubenov et al. 1999, 2000a; Popov et al. 2000a, $2000 \mathrm{~b}$.

Chlorops (Sclerophallus) limbatus Meigen, 1830 [Ch. brevimanus Loew, 1866] - O61; 220-260 m; 1; tes; Beschovski 1980b, 1985a, 2013b, 2013c; Nartshuk 2004.

Chlorops (Sclerophallus) pallidiventris (Duda, 1933) - E2; 200-500 m; 1; cse; Beschovski 1978b, 1980b, 1985a, 2013b; Hubenov et al. 1993, 1998.

Chlorops (Sclerophallus) varsoviensis Becker, 1910 - DW, E2, P1, P2, TL; 100-300 m; 1; tes, ? hoes; Beschovski 1978b, 1980b, 1985a, 2013b.

Cryptonevra diadema (Meigen, 1830) - V1, O61, O62, BN, BS; 0-600 m; 1; wp; Beschovski 1976a, 1981a, 1985a, 2013b.

Cryptonevra flavitarsis (Meigen, 1830) [Haplegis consimilis Collin, 1923] - V1, TK, O61, O62, BN, BS; 0-600 m; 1; hop; Beschovski 1968a, 1976a, 1981a, 1985a, 2013b.

Neohaplegis glabra (Duda, 1933) [Cryptonevra] - BN, BS; 0-10 m; 1; e; Beschovski 1981a, 1982a, 1985a, 1996a, 2013b.

Neohaplegis obscuripennis (Loew, 1874) - RE; 100-200 m; 1; eeca; Beschovski 2013b, 2013c. 
Neohaplegis tarsata (Fallén, 1820) [Cryptonevra] - BN; 0-10 m; 1; wces; Beschovski 1968a, 1976a, 1981a, 1985a, 2013b; Hubenov et al. 1993, 1998.

Diplotoxa messoria (Fallén, 1820) - ; DW, DM, E1, E2, P1, B1, V1, V4, V5, S1, TL, T11, T31, O4, O62, R1, R2, RE, BN, BS; 0-1400 m; 1, 2, 3; h; Nedelkov 1912; Szilady 1934; Beschovski 1977a, 1985a, 1996a, 2013b; Beschovski \& Dimitrova 1990.

Elachiptereicus italicus Duda, 1933 - O2, O62, R2; 90-450 m; 1; se; Beschovski 2013b, 2013c.

Eurina ducalis A. Costa, 1885 - DW, P3, O62, BN, BS; 0-100 m; 1; csena; Beschovski 1982a, 1985a, 1996a, 2013b; Hubenov et al. 1993, 1998.

Eurina lurida Meigen, 1830 - O62, BN, BS; 0-150 m; 1; wp; Beschovski 1982a, 1985a, 1996a, 2013b; Hubenov et al. 1993, 1998.

Eutropha fulvifrons (Haliday, 1833) [Chlorops fulvifrons var. ruficornis Hend, 1931] - BN, BS; 0-5 m; 1; ena; Beschovski 1964a, 1968a, 1975a, 1976a, 1973c, 1985a, 1996a, 2013b.

Eutropha variegata Loew, 1866 - O62, BS; 0-200 m; 1; ewca, ? eit, et; Beschovski 1977a, 1985a, 2013b.

Homalura tarsata Meigen, 1826 - RE, BN; 0-600 m; 1; e; Beschovski 1968a, 1985a, 1996a, 2013b; Hubenov et al. 1993, 1998.

Lasiosina albipila (Loew, 1866) - O62, R2, BN, BS; 0-650 m; 1; des; Beschovski 1968a, 1976a, 1977a, 1985a, $2013 \mathrm{~b}$.

Lasiosina brevisurstylata Dely-Draskovits, 1977 - V1, V4, RE, BN; 0-1000 m; 1, 2; e; Beschovski 1982a, 1985a, 2013b; Beschovski \& Dimitrova 1990; Beschovski \& Krusteva 1995a, 1995b, 1997; Krasteva \& Beschovski 1998, 2000, 2001.

Lasiosina herpini (Guérin-Méneville, 1843) [L. cinctipes (Meigen, 1830)] - -; *; E1, E2, P1, P2, B1, B2, V1, V4, V5, S1, S211, T11, T2, T31, O5, O62, R1, R2, R3, R5, RE, BN, BS; 0-2200 m; 1, 2, 3, 4, 5; tp; Drenowsky 1921a, 1922d, 1923a, 1923b, 1923c; Drenowsky \& Enderlein 1923; Tschorbadjiew 1924b, 1924c, 1925a, 1925f, 1929b; Savov 1925; Szilady 1934; Buresch \& Lazarov 1956; Popoff 1956; Grigorov 1972, 1976; Beschovski 1976a, 1977a, 1985a, 1998e, 2013b; Dely-Draskovits 1985; Beschovski \& Dimitrova 1990; Beschovski \& Krusteva 1995a, 1995b, 1997; Krasteva \& Beschovski 1998, 2000, 2001; Hubenov et al. 1999, 2000a; Popov et al. 2000a, 2000b.

Lasiosina immaculata Becker, 1912 - O62; 150-200 m; 1; eswa; Beschovski 1982a, 1985a, 2013b; Hubenov et al. 1993, 1998.

Lasiosina intermedia Dely-Draskovits, 1977 - O62, BN; 0-160 m; 1; e; Beschovski 2013b, 2013c.

Lasiosina lindbergi (Duda, 1933) [Mepachymerus, Steleocerus] - TK, O62, RE; 200-800 m; 1, 2; sena; Beschovski 1982a, 1983, 1985a, 2004a, 2013b; Hubenov et al. 1993, 1998.

Melanum laterale (Haliday, 1833) [Chlorops] - BN, BS; 0-10 m; 1; hoes, ? tp; Beschovski 1968a, 1985a, 2013b.

Meromyza (Meromyza) athletica Fedoseeva, 1974 [M. variegata Meigen, 1830] - * DW, P1, P2, B2, V4, V5, S1, O4, R1, R2, R5, BS; 0-1840 m; 1, 2, 3, 4; csee; Beschovski 1973a, 1975a, 1977a, 1985a, 2013b, 2013c; Beschovski \& Dimitrova 1990; Nartshuk 1992.

Meromyza (Meromyza) balcanica Beschovski, 1996 - B2; 1500-2200 m; 3, 4, 5; Ebg; Beschovski 1996b, 1998e, 2013b; Hubenov et al. 1999, 2000a; Popov et al. 2000a, 2000 b.

Meromyza (Meromyza) bohemica Fedoseeva, 1962 - P2, B2, TK, V4, O4, R1, R2; 300-1600 m; 1, 2, 3, 4; e; Beschovski 1985a, 2013b; Beschovski \& Dimitrova 1990.

Meromyza (Meromyza) femorata Macquart, 1835 - P1, P2, B2, V1, V4, T2, T31, O62, R1, R2, RW, RE, BN, BS; 0-1550 m; 1, 2, 3; e; Beschovski 1982a, 1985a, 1998e, 2013b; Beschovski \& Dimitrova 1990.

Meromyza (Meromyza) hungarica Dely-Draskovits, 1978 - T2; 30-40 m; 1; csee, ? e; Beschovski 1982a, 1985a, 2013b; Hubenov et al. 1993, 1998.

Meromyza (Meromyza) meigeni Nartshuk, 2006 [M. laeta Meigen, 1830] - V4, R2, RW; 1100-1900 m; 3, 4; wes; Beschovski 1982a, 1985a, 2006a, 2013b, 2013c.

Meromyza (Meromyza) mosquensis Fedoseeva, 1960 - B2, R1, RW, BN; 20-1450 m; 1, 2, 3, 4; e; Beschovski 2013b, 2013c.

Meromyza (Meromyza) nigriseta Fedoseeva, 1960 - E1, B2, V4, R2, R5, RW, BN, BS; 0-1400 m; 1, 2, 3; wces; Beschovski 1975b, 1977a, 1996a, 2013b; Beschovski \& Dimitrova 1990.

Meromyza (Meromyza) nigriventris Macquart, 1835 [M. saltatrix nigriventris Maqcquart, 1835] - ; DW, DM, E2, P1, B2, V1, V4, V5, S1, TL, T2, T31, O4, O62, R1, R2, R5, RW, RE, BN, BS; 0-2200 m; 1, 2, 3, 4, 5; h; Beschovski 1968a, 1976a, 1977a, 1985a, 1996a, 1998e, 1913b; Beschovski \& Dimitrova 1990; Krasteva 
1995, 1996; Beschovski \& Krusteva 1997; Krasteva \& Beschovski 1998, 2000; Hubenov et al. 1999, 2000a; Popov et al. 2000a, 2000b.

Meromyza (Meromyza) obtusa Peterfi, 1961 - B2, V4, V5; 800-1650 m; 2, 3, 4; cee; Beschovski 1982a, 1985a, 2013b; Beschovski \& Dimitrova 1990; Hubenov et al. 1993, 2008.

Meromyza (Meromyza) ornata (Wiedemann, 1817) [M. sororcula Fedoseeva, 1962] - B2, V4; 680-1300 m; 2, 3; esca, ? hoes; Beschovski 2013b, 2013c.

Meromyza (Meromyza) pluriseta Peterfi, 1961 - TK, V4, V5, S1, R2; 650-1250 m; 2, 3; wces; Beschovski 1982a, 1985a, 2013b; Beschovski \& Dimitrova 1990; Hubenov et al. 1993, 2008.

Meromyza (Meromyza) pratorum Meigen, 1830 - E1, R1, BS; 0-1450 m; 1, 2, 3; h; Beschovski 1976a, 1985a, 2013b.

Meromyza (Meromyza) quadrimaculata Fedoseeva, 1961 - K6, RW, BN; 0-1650 m; 1, 2, 3, 4; ee; Beschovski 1982a, 1985a, 2013b.

Meromyza (Meromyza) rohdendorfi Fedoseeva, 1974 - V4, S1, R1, R2; 650-1500 m; 2, 3, 4; e; Beschovski 1982a, 1985a, 2013b; Beschovski \& Dimitrova 1990.

Meromyza (Meromyza) rufa Fedoseeva, 1962 - DW, DM, E2, P1, P2, B2, V4, S1, S211, T31, O1, O4, O5, O62, R1, R2, RW, RE; 120-2050 m; 1, 2, 3, 4; e; Beschovski 1985a, 1998e, 2013b; Beschovski \& Dimitrova 1990; Hubenov et al. 1999, 2000a; Popov et al. 2000a, 2000b.

Meromyza (Meromyza) saltatrix (Linnaeus, 1761) - - ; DW, DM, E1, E2, P1, P2, B1, B2, V1, V4, V5, S1, S211, TL, T31, O1, O4, O5, R1, R2, R3, R5, RW, RE, BN; 5-2200 m; 1, 2, 3, 4, 5; h; Szilady 1934; Popoff 1956; Lyubenov 1958; Zamfirov 1958, 1961a; Donchev 1961; Grigorov 1972, 1976; Beschovski 1975b, 1976a, 1985a, 1996a, 1998e, 2013b; Beschovski \& Dimitrova 1990; Kontev et al. 1991; Hubenov et al. 1999, 2000a; Popov et al. 2000a, 2000b.

Meromyza (Meromyza) triangulina Fedoseeva, 1960 - E1, B2, V4, V5, S211, T2, O4, R2, R5, RW; 30-2200 m; 1, 2, 3, 4, 5; e; Beschovski 1982a, 1985a, 1998e, 2013b; Beschovski \& Dimitrova 1990; Hubenov et al. 1999, 2000a; Popov et al. 2000a, 2000b.

Meromyza (Meromyza) variegata Meigen, 1830 [M. lidiae Nartshuk, 1992] - ; V1, R1, RW; 600-2000 m; 2, 3, 4, 5; e, ? ewca; Nedelkov 1912; Szilady 1934; Beschovski 1982a, 1985a, 2006a, 2013 b.

Meromyza (Meromyza) zachvatkini Fedoseeva, 1960 - B2, V4, V5, T31, O4, R1, R2; 180-2100 m; 1, 2, 3, 4, 5; des; Beschovski 1982a, 1985a, 1998e, 2013b; Beschovski \& Dimitrova 1990; Hubenov et al. 1999, 2000a; Popov et al. 2000a, 2000b.

Parectecephala longicornis (Fallén, 1820) - DW, V4, R2, BN, BS; 0-1000 m; 1, 2; eswa; Beschovski 1976a, 1985a, 2013b; Beschovski \& Dimitrova 1990.

Phyladelphus thalhammeri Becker, 1910 - $\$$ DW, DM, E1, E2, P2, B3, V1, S1, T2, T31, O2, O62, R2, RE, BN, BS; 0-1000 m; 1, 2; e, ? cse; Beschovski 1977a, 1985a, 1996a, 2013b.

Platycephala planifrons (Fabricius, 1798) - BN, BS; 0-50 m; 1; ? tp; Beschovski 1968a, 1985a, 1996a, $2013 \mathrm{~b}$.

Platycephala umbraculata (Fabricius, 1794) - BN; 0-10 m; 1; tp; Beschovski 1968a, 1985a, 2013b; Hubenov et al. 1993, 1998.

Pseudopachychaeta approximatonervis (Zetterstedt, 1848) - P1, P2, BN, BS; 0-700 m; 1, 2; h; Beschovski 1968a, 1975a, 1985a, 2013b.

Pseudopachychaeta pachycera Strobl, 1902 - BN; 0-10 m; 1; sena; Beschovski 1968a, 1985a, 2013b; Hubenov et al. 1993, 1998.

Thaumatomyia elongatula (Becker, 1910) - R2; 360-600 m; 1; e; Beschovski 2013b, 2013c.

Thaumatomyia glabra (Meigen, 1830) [Chloropisca] - DW, DM, E1, P1, P2, B1, B2, V1, V4, V5, S1, S22, T2, O4, O62, R1, R2, R5, RE, BN, BS; 0-2000 m; 1, 2, 3, 4; h; Szilady 1934; Beschovski 1977a, 1985a, 1996a, 1998e, 2013b; Beschovski \& Dimitrova 1990; Hubenov et al. 1999, 2000a; Popov et al. 2000a, 2000b.

Thaumatomyia hallandica Andersson, 1966 - ; DW, P2, B2, V1, V4, V5, S1, T31, R1, R2, BN, BS; 0-1900 m; 1, 2, 3, 4; wces; Beschovski 1975b, 1982a, 1985a, 1996a, 1998e, 2013b; Hubenov et al. 1999, 2000a; Popov et al. 2000a, 2000b.

Thaumatomyia notata (Meigen, 1830) [Th. notata var. circumdata (Meigen, 1830); Th. notata var. ornata (Meigen, 1830); Chlorops ] - \$ DW, P2, B2, K2, V1, V4, V5, S1, S22, T2, T31, O61, R1, R2, R3, R5, RW, RE, BN, BS; 0-2200 m; 1, 2, 3, 4, 5; ppt; Nedelkov 1912; Szilady 1934; Beschovski 1968a, 1985a, 1975b, 1976a, 1977a, 1996a, 1998a, 2013b; Beschovski \& Dimitrova 1990; Hubenov et al. 1999, 2000a; Popov et al. 2000a, 2000b. 
Thaumatomyia rufa (Macquart, 1835) - E1, P1, P2, B1, B2, V1, V4, S211, O4, R2, RE, BN, BS; 0-1500 m; 1, 2, 3; hop, ? po; Beschovski 1968a, 1985a, 1998e, 2013b; Hubenov et al. 1999, 2000a; Popov et al. 2000a, 2000b.

Thaumatomyia sulcifrons (Becker, 1907) - \$ DM, B2, V1, S1, R2, R5, RW, BN, BS; 0-1500 m; 1, 2, 3; wcp; Beschovski 1975b, 1985a, 1996a, 1998e, 2013b; Hubenov et al. 1999, 2000a; Popov et al. 2000a, 2000b.

Trichieurina pubescens (Meigen, 1830) - V1, V4; 600-950 m; 1, 2; et, ? ewca; Beschovski 1982a, 1985a, 2013b; Hubenov et al. 1993, 1998.

\section{Siphonellopsidae}

Apotropina longepilosa (Strobl, 1893) [Lasiopleura] - DM, P2, B2, T31, RE, BN; 0-600 m; 1, 2; tes; Beschovski 1968a, 1972a, 1975a, 1977a, 1985a, 2013b.

Siphonellopsis lacteibasis Strobl, 1906 - T31, BN; 0-200 m; 1; mt; Beschovski 1968a, 1985a, 2013b; Hubenov et al. 1993, 1998.

\section{Heleomyzidae (Helomyzidae)}

Orbellia borisregis Czerny, 1930 - R1; 2010 m; 4, 5; Ebg, ? Er; Czerný 1930; Kantardzhieva-Minkova 1957; Beschovski \& Gueorguieva 2001; Hubenov et al. 2000b.

Eccoptomera emarginata Loew, 1862 - P1, P2, B1, B2, B3, RW; 300-1200 m; 1, 2, 3; e; subtroglophile; Czerný 1930; Buresch 1936; Guéorguiev \& Beron 1962; Beron \& Guéorguiev 1967; Beschovski 1972c, 2006a; Beron 1994, 2015, 2016; Beschovski \& Gueorguieva 2001; Beron et al. 2011.

Eccoptomera infuscata Wahlgren, 1918 - V4; 1860-1890 m; 4; des; Beschovski \& Gueorguieva 2001.

Eccoptomera longiseta Loew, 1862 - V1, RW; 300-600 m; 1; e; Nedelkov 1912; Beschovski \& Gueorguieva 2001. Eccoptomera microps (Meigen, 1830) - 1; e; Gorodkov 1984c; Beschovski \& Gueorguieva 2001.

Eccoptomera obscura (Meigen, 1830) - B3, RW; 200-1330 m; 1, 2, 3; e; trogloxene; Czerný 1930; Guéorguiev \& Beron 1962; Beron 1994, 2015; Beschovski \& Gueorguieva 2001; Beron et al. 2011.

Eccoptomera pallescens (Meigen, 1830) - P1, B1; 650-1000 m; 2; e; subtroglophile; Burghele-Bălăcesko 1966; Beron \& Guéorguiev 1967; Beron 1994, 2015, 2016; Beschovski \& Gueorguieva 2001.

Neoleria flavicornis (Loew, 1862) - DW, V4, BS; 0-940 m; 1, 2; e; Beschovski 1973a; Beschovski \& Gueorguieva 2001.

Neoleria ruficeps (Zetterstedt, 1838) - ena; Gorodkov 1984c; Beschovski \& Gueorguieva 2001.

Acantholeria cineraria (Loew, 1862) - B1, RW, B; 0-1600 m; 1, 2, 3, 4; ewca; trogloxene; Beron \& Guéorguiev 1967; Beron 1972b, 1994, 2015, 2016; Beschovski 1972c, 1973c, 1975a; Beschovski \& Gueorguieva 2001; Beron et al. 2011.

Morpholeria (Spanoparea) innotata (Czerny, 1933) - BN, BS; 0-5 m; 1; ewca; Beschovski 1973a; Beschovski \& Gueorguieva 2001.

Morpholeria (Spanoparea) ruficornis (Meigen, 1830) - V4; 1350 m; 3; e; Gorodkov 1984c; Beschovski \& Gueorguieva 2001.

Morpholeria (Spanoparea) variabilis (Loew, 1862) - V4; 1350-1900 m; 3, 4; cse; Gorodkov 1984c; Beschovski \& Gueorguieva 2001.

Scoliocentra (Leriola) brachypterna (Loew, 1873) - B1; 445 m; 1; e; trogloxene; Beschovski 1972c; Beron 1994, 2015, 2016; Beschovski \& Gueorguieva 2001.

Scoliocentra (Leriola) nigrinervis (Wahlgren, 1918) [Helomyza] - R1; 2000 m; 4, 5; wcp; Czerný 1930; Beschovski \& Gueorguieva 2001.

Scoliocentra (Chaetomus) flavotestacea (Zetterstedt, 1838) - \$; h; Gorodkov 1984c; Beschovski \& Gueorguieva 2001.

Scoliocentra (Scoliocentra) villosa (Meigen, 1830) [S. villosa var. villosula Czerny, 1924] - P2, B1; 350-600 m; 1; wes; trogloxene; Czerný 1930; Buresch 1936; Guéorguiev \& Beron 1962; Beron 1994, 2015.

Gymnomus caesius (Meigen, 1830) [Amoeboleria, Blepharoptera, Scoliocentra] - P1, P2, B1, B2, B3, V1, RW; 300-1550 m; 1, 2, 3, 4; e; trogloxene; Nedelkov 1912; Czerný 1930; Buresch 1934; Guéorguiev \& Beron 1962; Burghele-Bălăcesko 1966; Beschovski 1972c, 2006a; Beron 1994, 2015; Beschovski \& Gueorguieva 2001; Beron et al. 2011. 
Gymnomus spectabilis (Loew, 1862) [Amoeboleria, Scoliocentra] - P2, B3; 400-800 m; 1, 2; e; troglophile; Czerný 1930; Buresch 1936; Guéorguiev \& Beron 1962; Beron 1994, 2015; Beschovski \& Gueorguieva 2001; Beschovski 2006a.

Gymnomus ventricosus (Becker, 1907) [Scoliocentra] - P2; 250-500 m; 1; dp; trogloxene; Czerný 1930; Buresch 1936; Guéorguiev \& Beron 1962; Beron 1994, 2015.

Heleomyza (Heleomyza) captiosa (Gorodkov, 1962) - P1, P2, B1, B3, RW, RE; 300-1400 m; 1, 2, 3; e; subtroglophile; Beron 1972b, 1994, 2015, 2016; Beschovski 1972c, 2006a; Beschovski \& Gueorguieva 2001; Beron et al. 2004, 2011.

Heleomyza (Heleomyza) modesta (Meigen, 1835) [Blepharoptera] - V1, RW; 550-990 m; 1, 2; e; trogloxene; Nedelkov 1912; Beschovski \& Gueorguieva 2001; Beschovski 2006a; Beron et al. 2011; Beron 2015.

Heleomyza (Heleomyza) serrata (Linnaeus, 1758) [Helomyza, Leria] - DW, P1, P2, B1, B3, V1, V4, TL, RW, RE; 100-1500 m; 1, 2, 3; h; subtroglophile; Nedelkov 1912; Czerný 1930; Buresch 1936; Guéorguiev \& Beron 1962; Burghele-Bălăcesko 1966; Beschovski 1972c; Lavchiev \& Jovčev 1978; Lavchiev \& Zhekov 1980; Beron 1994, 2015, 2016; Beschovski \& Gueorguieva 2001; Beron et al. 2011.

Suillia affinis (Meigen, 1830) - E2, B2, V4, S23, T31; 0-1400 m; 1, 2, 3; e; Gorodkov 1984c; Beschovski \& Gueorguieva 2001; Dvořák et al. 2021.

Suillia atricornis (Meigen, 1830) - V4; 1400 m; 3, 4; h; Gorodkov 1984c; Beschovski \& Gueorguieva 2001.

Suillia bicolor (Zetterstedt, 1838) [Helomyza zetterstedti Loew, 1862] - V1, T31; 350-600 m; 1; tp; Nedelkov 1912; Nikolova 1963; Beschovski \& Gueorguieva 2001.

Suillia crinimana (Czerny, 1904) [S. subdola Czerny, 1927] - ^; e; Gorodkov 1984c; Beschovski \& Gueorguieva 2001.

Suillia flava (Meigen, 1830) [Helomyza rufa Fallén, 1820] - BN; 0-10 m; 1; esca; Löw 1862; Beschovski \& Gueorguieva 2001.

Suillia flavifrons (Zetterstedt, 1838) - V4, BN; 0-10 m, 1240-1870 m; 1, 3, 4, 5; tes, ? hoes; Gorodkov 1984c; Beschovski \& Gueorguieva 2001.

Suillia fuscicornis (Zetterstedt, 1847) - B2, V4, R1; 1300-1880 m; 3, 4, 5; wces; Gorodkov 1984c; Beschovski \& Gueorguieva 2001.

Suillia gigantea (Meigen, 1830) - DM; 125-180 m; 1; e; Gorodkov 1984c; Beschovski \& Gueorguieva 2001.

Suillia humilis (Meigen, 1830) [S. inornata Loew, 1862] - T31, O5, R4; 65-1250 m; 1, 2, 3; tes; Gorodkov 1984c; Beschovski \& Gueorguieva 2001.

Suillia imberbis Czerny, 1924 - BS; 0-10 m; 1; e; Martinek 1987; Beschovski \& Gueorguieva 2001.

Suillia laevifrons (Loew, 1862) - B2, V5, S211, T31, R1, R2, R4; 350-1400 m; 1, 2, 3, 4; tes; Gorodkov 1984c; Beschovski \& Gueorguieva 2001.

Suillia lurida (Meigen, 1830) - -; DW, E1, E2, P1, P2, TK, V1, S1, TL, T1, T2, O62, R5; 30-800 m; 1, 2; e; Nedelkov 1912; Nikolova 1953, 1958, 1959, 1960, 1963; Popoff 1956; Gaspodinov 1958; Popoff \& Nikolova 1958; Kovachevski et al. 1959; Grigorov 1972, 1976; Harizanov et al. 1996; Beschovski \& Gueorguieva 2001.

Suillia nemorum (Meigen, 1830) - B2, R2, RW; 1300-2000 m; 3, 4, 5; h; Gorodkov 1984c; Beschovski \& Gueorguieva 2001; Beschovski 2006a.

Suillia notata (Meigen, 1830) - T31, RW; 350-1920 m; 1, 2, 3, 4; ena; Gorodkov 1984c; Beschovski \& Gueorguieva 2001.

Suillia oxyphora (Mik, 1900) - B2, V1, R2; 390-1400 m; 1, 2, 3; e; Gorodkov 1984c; Beschovski \& Gueorguieva 2001.

Suillia pallida (Fallén, 1820) [Helomyza olens (Meigen, 1830)] - B2, V1, R1, R4; 550-2300 m; 2, 3, 4, 5; e; Nedelkov 1912; Beschovski \& Gueorguieva 2001.

Suillia parva (Loew, 1862) - V4; 900-1240 m; 2, 3; wces; Gorodkov 1984c; Beschovski \& Gueorguieva 2001.

Suillia tuberiperda (Rondani, 1867) - V4; 940 m; 2; se; Beschovski \& Gueorguieva 2001.

Suillia umbratica (Meigen, 1835) - B2, V4; 1240-1300 m; 3; e; Beschovski \& Gueorguieva 2001.

Suillia ustulata (Meigen, 1830) - V4, R2; 740-940 m; 2; e; Beschovski \& Gueorguieva 2001.

Suillia variegata (Loew, 1862) - T31, O62, RE; 124-340 m; 1; wp; Gorodkov 1984c; Beschovski \& Gueorguieva 2001.

Suillia villeneuvei Czerny, 1924 - B2, T31, O62, R1, R4, BN; 0-2300 m; 1, 2, 3, 4, 5; e; Gorodkov 1984c; Beschovski \& Gueorguieva 2001. 
Tephrochlaena halterata (Meigen, 1830) [Tephroclamys] - RW; 1500-1600 m; 4; e; Beron \& Guéorguiev 1967; Beron 1994, 2015; Beschovski \& Gueorguieva 2001; Beron et al. 2011.

Heteromyza atricornis Meigen, 1830 [Thelida] - DM, E1, P1, P2, B1, B2, B3, V4, S21, RW; 40-1240 m; 1, 2, 3; e; troglophile; Czerný 1930; Buresch 1936; Guéorguiev \& Beron 1962; Burghele-Bălăcesko 1966; Beron \& Guéorguiev 1967; Beschovski 1972c, 2006a; Beron 1994, 2015; Beschovski \& Gueorguieva 2001; Beron et al. 2011.

Heteromyza commixta Collin, 1901 [Thelida] - DM, P1, B1; 130-1100 m; 1, 2, 3; e; trogloxene; Beron \& Guéorguiev 1967; Beron 1994, 2015, 2016; Beschovski \& Gueorguieva 2001.

Heteromyza rotundicornis (Zetterstedt, 1846) [Thelida] - P2, B1, V1, V4; 280-1100 m; 1, 2, 3; e; trogloxene; Beron \& Guéorguiev 1967; Beschovski 1972c; Beron 1994, 2015; Beschovski \& Gueorguieva 2001.

Tephrochlamys flavipes (Zetterstedt, 1838) - V1, TL, RW; 200-1000 m; 1, 2; e; Nedelkov 1912.

Tephrochlamys rufiventris (Meigen, 1830) - V1, V4, TL, R2, BN, BS; 0-1240 m; 1, 2, 3; h; Beschovski 1973a; Beschovski \& Gueorguieva 2001.

\section{Trixoscelididae (Trichoscelidae)}

Trixoscelis obscurella (Fallén, 1823) - BN; 0-5 m; 1; eca; Beschovski 1966a.

\section{Chyromyidae}

Chyromya flava (Linnaeus, 1758) - B1, V1; 600-800 m; 1, 2; h; trogloxene; Nedelkov 1912; Beron 1994, 2015.

\section{Sphaeroceridae (Borboridae)}

Sphaerocera curvipes Latreille, 1805 [S. subsultans] - V1; 550-600 m; 1; k; Nedelkov 1912.

Ischiolepta pusilla (Fallén, 1820) - RW; 850-1480 m; 2, 3, 4; hna; Beschovski 1967b, 2006a.

Lotobia pallidiventris (Meigen, 1830) - ppt; Papp 1984d.

Copromyza equina Fallén, 1820 [Borborus, Trichiaspis] - V1, R1; 550-1500 m; 1, 2, 3, 4; k; Nedelkov 1912; Lavčiev 1965a; Lavchiev et al. 1984.

Lotophila atra (Meigen, 1830) [Borborus, Olina geniculata (Macquart, 1835)] - V1, BS; 0-600 m; 1; ho; Nedelkov 1912; Gregor \& Povolny 1959.

Norrbomia hispanica (Duda, 1923) [Borborillus] - ewca; Papp 1984d.

Norrbomia nilotica (Becker, 1903) [N. nitidifrons Duda, 1923] - ena; Papp 1984d.

Borborillus vitripennis (Meigen, 1830) - \$; tes; Papp 1984d.

Crumomyia fimetaria (Meigen, 1830) - e; Papp 1984d.

Crumomyia glabrifrons (Meigen, 1830) [Borborus] - V1; 550-600 m; 1; ewca; Nedelkov 1912.

Crumomyia rohaceki Norrbom \& Kim, 1985 [C. glacialis Meigen, 1830] - R1; 2005 m; 4, 5; e; Czerný 1930.

Crumomyia nigra (Meigen, 1830) [Borborus, Copromyza, Cypsela, Sphaerocera] - B1, V1, RW; 460-1540 m; 1, 2, 3, 4; ho; trogloxene; Nedelkov 1912; Czerný 1930; Guéorguiev \& Beron 1962; Beron \& Guéorguiev 1967; Beron 1994, 2015, 2016; Beron et al. 2011.

Crumomyia nitida (Meigen, 1830) [Copromyza, Cypsela, Borborus, Stratioborborus] - B1, V1; 450-600 m; 1; wes; trogloxene; Nedelkov 1912; Beron \& Guéorguiev 1967; Beron 1994, 2015, 2016.

Crumomyia roserii (Rondani, 1880) [Copromyza] - T11; 100-300 m; 1; e; trogloxene; Beron 1994, 2015.

Coproica digitata (Duda, 1918) - BN; 0-5 m; 1; spo; Beschovski 1967b.

Coproica ferruginata (Stenhammar, 1855) [Limosina] - V1; 550-600 m; 1, 2; k; Nedelkov 1912.

Coproica hirticula Collin, 1956 - sk, i; Papp 1984d.

Coproica hirtula (Rondani, 1880) - BN; 0-10 m; 1; k; Beschovski 1967b.

Coproica pusio (Zetterstedt, 1847) - ppt; Papp 1984d.

Coproica vagans (Haliday, 1833) [C. vagans var. flava (Duda, 1918); Coprophila] - RW, BN; 0-1200 m; 1, 2, 3; k; Beschovski 1967b.

Elachisoma kerteszi (Duda, 1924) [Trachyopella] - BN; 0-5 m; 1; se; Beschovski 1967b.

Gonioneura spinipennis (Haliday, 1836) [Halidayiana] - R5, RW; 550-1490 m; 1, 2, 3, 4; ho; Beschovski 1967b, 2006a; Roháèek 1983. 
Thoracochaeta brachystoma (Stenhammar, 1855) [Leptocera] - BN, BS; 0-5 m; 1; k; Caspers 1951a, 1951b; Beschovski 1964a, 1964b, 1965.

Thoracochaeta zosterae (Haliday, 1833) - BS; 0-5 m; 1; hn; Beschovski $1967 \mathrm{~b}$.

Chaetopodella scutellaris (Haliday, 1836) - DM, RW; 156-1060 m; 1, 2, 3; pat; Beschovski 1967b, 2006a; Roháèek 1983.

Pteremis fenestralis (Fallén, 1820) - e; Papp 1984d.

Opacifrons coxata (Stenhammar, 1855) - P1, RW, BN, BS; 0-1000 m; 1, 2; hat; Beschovski 1967b, $2006 \mathrm{.}$.

Opacifrons maculifrons (Becker, 1907) [Limosina] - BN; 0-5 m; 1; ha; Beschovski 1967b; 1968b.

Pseudocollinella humida (Haliday, 1836) [Spinotarsella] - R2; 2220-2320 m; 5; pat; Beschovski 1967b.

Pseudocollinella septentrionalis (Stenhammar, 1855) - RW; 1320-1480 m; 3, 4; tes; Beschovski 1967b, $2006 \mathrm{a}$. Leptocera caenosa (Rondani, 1880) [Paracollinella] - DM, TL, BN; 0-230 m; 1; sk; Beschovski 1967b, 2006 .

Leptocera fontinalis (Fallén, 1826) [Paracollinella] - TL, RW; 250-900 m; 1, 2; hnat; Beschovski 1967b, 2006 a. Leptocera nigra Olivier, 1813 [Paracollinella curvinervis Stenhammar, 1855] - DM, P3, TL, R5, BN; 0-530 m; 1; ppt; Beschovski 1967b, 2004a, 2006a; Roháèek 1983.

Rachispoda breviceps (Stenhammar, 1855) [Collinellula] - P3, BN, BS; 0-30 m; 1; e; Beschovski 1967b.

Rachispoda cilifera (Rondani, 1880) [Collinellula] - P3, BN, BS; 0-30 m; 1; tes; Beschovski 1967b.

Rachispoda fuscipennis (Haliday, 1833) [Collinellula fuscipennis var. plurisetosa (Strobl, 1900); Limosina oelandica Stenhammar, 1854] - V1, BN, BS; 0-600 m; 1; k; Nedelkov 1912; Beschovski 1967b.

Rachispoda limosa (Fallén, 1820) [Collinellula] - V1; 550-600 m; 1; hat; Nedelkov 1912.

Rachispoda hostica (Villeneuve, 1917) [Collinellula] - P3; 20-30 m; 1; eanca; Beschovski 1967b.

Rachispoda lutosa (Stenhammar, 1855) [Collinellula; C. palustris (Collin, 1930)] - R2, RW; 1300-2300 m; 3, 4, 5; h; Beschovski 1967b, 2006a.

Rachispoda lutosoidea (Duda, 1938) [Collinellula] - BN; 0-5 m; 1; wcp; Beschovski 1964a, 1964b.

Rachispoda modesta (Duda, 1924) [Collinellula] - P3, BN, BS; 0-40 m; 1; wp; Beschovski 1967b.

Rachispoda varicornis (Strobl, 1900) [Collinellula] - P3, BN; 0-40 m; 1; sppt; Beschovski 1967b.

Paralimosina fucata (Rondani, 1880) - ena; Papp 1984d.

Opalimosina (Pappiella) liliputana (Rondani, 1880) - ; ; Papp 1984d.

Opalimosina (Opalimosina) mirabilis (Collin, 1902) [Limosina] - TL, RW; 80-1480 m; 1, 2, 3, 4; sk; Beschovski 1967b, 2004a, 2006a.

Paralimosina macedonica (Roháček, 1977) - s seei; Papp 1984d.

Spelobia (Spelobia) clunipes (Meigen, 1830) [Limosina crassimana Haliday, 1836] - RW, BS; 0-1480 m; 1, 2, 3, 4; h; Gregor \& Povolny 1959; Beschovski 1967b, 2006a.

Spelobia (Spelobia) luteilabris (Rondani, 1880) [Limosina] - 1; ha; Papp 1984d.

Spelobia (Eulimosina) ochripes (Meigen, 1830) [Limosina] - V1; 600 m; 1; ho; Nedelkov 1912.

Spelobia (Spelobia) palmata (Richards, 1927) [Limosina] - s; ena; Papp 1984d.

Spelobia (Spelobia) parapusio (Dahl, 1909) [Limosina] - tp; Papp 1984d.

Spelobia (Spelobia) pseudosetaria (Duda, 1918) [Limosina] - DM, BN; 0-150 m; 1; hata; Beschovski $1967 \mathrm{~b}$.

Spelobia (Spelobia) rufilabris (Stenhammar, 1855) [Limosina] - \$; po; Papp 1984d.

Spelobia (Spelobia) talparum (Richards, 1927) [Limosina] - ; e; Papp 1984d.

Telomerina flavipes (Meigen, 1830) [Limosina] - \$ k; Papp 1984d.

Pullimosina (Pullimosina) heteroneura (Haliday, 1836) [Limosina] - DM, BN, BS; 0-160 m; 1; k; Beschovski 1967b.

Phthitia (Kimosina) longisetosa (Dahl, 1909) [Limosina] - \$ des; Papp 1984d.

Minilimosina (Minilimosina) parvula (Stenhammar, 1855) [Limosina] - \$; Papp 1984d.

Terrilimosina racovitzai (Bezzi, 1911) [Limosina] - DM, P1, B1, RW; 60-1330; 1, 2, 3; h; trogloxene; Hazelton 1970; Beschovski 1972c; Papp 1984d; Beron 1994, 2015, 2016; Beron et al. 2011.

Limosina silvatica (Meigen, 1830) - P1, B1; 520-550 m; 1; ena, ? h; trogloxene; Beschovski 1972c; Beron 1994, 2015.

\section{Curtonotidae}

Curtonotum anus (Meigen, 1830) - V1, BN, BS; 0-600 m; 1; esca, ? tp; Nedelkov 1912; Beschovski 1976a, 2007. 


\section{Camillidae}

Camilla atrimana Strobl, 1910 [C. glabra (Fallen,1823)] - R2, BN, BS; 0-1000 m; 1, 2; eswa; Beschovski 1976b, 2007.

\section{Drosophilidae}

Phortica (Phortica) variegata (Fallén, 1823) [Amiota] - B1, R2, BS; 0-1000 m; 1; po; trogloxene; Beschovski 1972c; Máca 1987; Beron 1994, 2015, 2016.

Drosophila (Drosophila) funebris (Fabricius, 1787) [D. confusa Staeger, 1844] - B2, V1; 600-750 m; 1; k; trogloxene; Nedelkov 1912; Czerný 1930; Guéorguiev \& Beron 1962; Beron 2015.

Drosophila (Drosophila) phalerata Meigen, 1830 - DW, BS; 0-200 m; 1; tp; Kozarov 1908, 1911; Buresch \& Lazarov 1956; Máca 1987.

Drosophila (Drosophila) testacea von Roser, 1840 - R2; 950-1000 m; 2; po; Máca 1987.

Drosophila (Drosophila) transversa Fallén, 1823 - R2; 1800 m; 4; h; Máca 1987.

Drosophila (Sophophora) melanogaster Meigen, 1830 [D. approximata Zetterstedt, 1847; D. fasciata Meigen, 1930] - P1, B1, V1, R2, RW; 260-1340 m; 1, 2, 3; k; trogloxene; Nedelkov 1912; Beschovski 1972c; Máca 1987; Beron 1994, 2015, 2016; Beron et al. 2011.

Drosophila (Sophophora) obscura Fallén, 1823 - BS; 0-10 m; 1; wp; Máca 1987.

Drosophila (Sophophora) subobscura Collin, 1936 - S211, R2, BS; 0-800 m; 1, 2; hn; Máca 1987.

Drosophila suzukii (Matsumura, 1931) - -; K9, TL, O61, O62, BN; 0-700 m; 1; sk*; Karadjova et al. 2016.

Lordiphosa fenestrarum (Fallén, 1823) [Drosophila; D. melanogaster Meigen, 1830] - V1, BN; 0-600 m; 1; e; Nedelkov 1912; Vassileva-Dryanovska \& Gencheva 1965; Máca 1987.

Hirtodrosophila cameraria (Haliday, 1833) - R2; 950-1000 m; 2; wp; Bächli \& Teresa Rocha Pite 1984.

Hirtodrosophila trivittata (Strobl, 1893) [Drosophila] - BS; 0-10 m; 1; po; Máca 1987.

Scaptodrosophila deflexa (Duda, 1924) - BS; 0-10 m; 1; e; Máca 1987.

Mycodrosophila poecilogastra (Loew, 1874) - BS; 0-10 m; 1; tp; Máca 1987.

Scaptomyza (Parascaptomyza) pallida (Zetterstedt, 1847) - B2, K9, V4, S211, R1, R2, BN, BS; 0-2500 m; 1, 2, 3, 4, 5, 6; k; Máca 1987.

Scaptomyza (Scaptomyza) flava (Fallén, 1823) [S. apicalis Hardy, 1849] - V1, V4, R1; 550-1200 m; 1, 2, 3; h; Buhr 1941; Máca 1987.

Scaptomyza (Scaptomyza) graminum (Fallén, 1823) - V1, V4, R1, R2; 550-2500 m; 1; ho; Nedelkov 1912; Máca 1987.

\section{Campichoetidae}

Campichoeta obscuripennis (Meigen, 1830) - DW, K6, K9, BN; 0-800 m; 1, 2; po; Chandler 1987; Beschovski 2007. Campichoeta punctum (Meigen, 1830) - O62, RE; 90-300 m; 1; ean, ? po; Beschovski 2007.

Euthychaeta spectabilis (Loew, 1864) - RW; 360-4oo m; 1; e; Chandler 1987; Beschovski 2007.

\section{Diastatidae}

Diastata adusta Meigen, 1830 - B2; 500-600 m; 1; ena; Chandler 1987; Máca \& Roháček 1994; Beschovski 2007. Diastata cervinala Chandler, 1987 - BN; 0-5 m; 1; e; Chandler 1987; Máca \& Roháček 1994; Beschovski 2007. Diastata costata Meigen, 1830 [D. fiscula Fallén, 1823] - S22, O5, R1, R2; 700-1400 m; 2, 3; h; Beschovski 1976b, 2007; Máca \& Roháček 1994.

Diastata fuscula (Fallen, 1823) [D. inornata Loew, 1864] - T31, O5, R4, RE, BN; 0-1250 m; 1, 2, 3; ena; Beschovski 1976b, 2007; Chandler 1987; Máca \& Roháček 1994.

\section{Ephydridae}

Discomyza incurva (Fallén, 1823) - E2, BN, BS; 0-150 m; 1; tp, ? h; Zatwarnicki 1991; Beschovski 2009; Beschovski \& Zatwarnicki 2000. 
Clanoneurum cimiciforme (Haliday, 1855) [C. infumatum Becker, 1903] - O62, BN, BS; 0-150 m; 1; ena; ? h; Beschovski 1966e, 2009; Beschovski \& Zatwarnicki 2000.

Diasemocera glabricula (Fallén, 1813) [Psilopa nigritella Stenhammar, 1844; P. obscuripes Loew, 1860] - E2, P1, T11, T31, O62, RW, RE, BN, BS; 0-1300 m; 1, 2, 3; wcp; Beschovski 1966e, 2004a, 2006a, 2009; Beschovski \& Zatwarnicki 2000; Zatwarnicki 2018.

Diasemocera leucostoma (Meigen, 1830) [Psilopa] - BN, BS; 0-5 m; 1; h; Beschovski \& Zatwarnicki 2000; Beschovski 2009; Zatwarnicki 2018.

Diasemocera marginella Fallén, 1823 [Psilopa] - DW, B2, B3, V4, S211; 50-1550 m; 1, 2, 3; e; Beschovski \& Zatwarnicki 2000; Beschovski 2009; Zatwarnicki 2018.

Diasemocera maritima (Perris, 1847) [Psilopa] - BN, BS; 0-5 m; 1; se, ? hom; Beschovski 1966e, 2009; Beschovski \& Zatwarnicki 2000; Zatwarnicki 2018.

Diasemocera nana (Loew, 1860) [Psilopa nigritella Stenhammar, 1844] - RW, RE, BN, BS; 0-1100 m; 1, 2, 3; pat; Beschovski 1964a, 1964b, 1972b, 1973c, 1973e, 1975a, 1975b, 1976a, 1996a, 2004a, 2006a, 2009; Beschovski \& Zatwarnicki 2000; Zatwarnicki 2018.

Psilopa nitidula Fallén, 1813 - \$ DW, DM, E1, E2, P1, P2, B2, B3, K2, K6, V1, V4, V5, S1, S21, S211, S22, TL, T2, T31, O4, O5, O62, R2, R3, R5, RW, RE, BN, BS; 0-2570 m; 1, 2, 3, 4, 5, 6; pat; Beschovski 1966e, 1976a, 1996c, 1997b, 2004a, 2006a, 2009; Zatwarnicki 1991; Beschovski \& Zatwarnicki 2000.

Psilopa obscuripes Loew, 1860 [P. compta (Meigen, 1830)] - \$ DW, DM, E2, P1, P2, B2, V1, V4, V5, S1, S21, TL, T11, T2, T31, O5, O61, O62, R2, RE, BN, BS; 0-2300 m; 1, 2, 3, 4, 5; eanna, ? wp; Zamfirov 1960b; Beschovski 1966e, 1975b, 1976a, 1996c, 1997b, 2004a, 2009; Beschovski \& Zatwarnicki 2000.

Psilopa polita (Macquart, 1835) - DW, DM, E1, E2, P1, P2, B1, B2, B3, K2, K6, V1, V4, V5, S1, S211, S22, TL, T11, T2, T31, O4, O5, O62, R1, R2, R3, R5, RW, RE, BN, BS; 0-2300 m; 1, 2, 3, 4, 5; dp; Zatwarnicki 1991; Beschovski 1996c, 1997b, 2004a, 2006a, 2009; Beschovski \& Zatwarnicki 2000.

Psilopa roederi Girschner, 1889 - RE; 130-160 m; 1; e; Beschovski 2009.

Psilopa stackelbergi Nartshuk, 1970 - DW, DM, P1, P2, B2, V4; 100-1600 m; 1, 2, 3, 4; cse, ? e; Beschovski \& Zatwarnicki 2000; Beschovski 2009; Zatwarnicki 2018.

Trimerina madizans Fallén, 1813 [Madiza] - ; DW, E2, V5, S211, BN; 0-1000 m; 1, 2; h; Szilady 1934; Beschovski 1966e, 2009; Beschovski 2009; Zatwarnicki 2018.

Trimerina microchaeta Hendel, 1932 - B3; 800 m; 2; des, ? dp; Zatwarnicki 1996; Beschovski \& Zatwarnicki 2000; Beschovski 2009.

Asmeringa inermis Becker, 1903 - BS; 0-5 m; 1; atm; Beschovski 1973f, 2009; Beschovski \& Zatwarnicki 2001a.

Atissa hepaticoloris Becker, 1903 - BN, BS; 0-5 m; 1; sena; Beschovski \& Zatwarnicki 2001a; Beschovski 2009.

Atissa kerteszi Papp, 1974 [A.pygmaea Haliday, 1833] - BN, BS; 0-5 m; 1; csee; Beschovski 1964a, 1964b, 1972a, 1973c, 1973e, 1975a, 1975b, 2009; Beschovski \& Zatwarnicki 2001a.

Atissa limosina Becker, 1896 - BN, BS; 0-5 m; 1; ena; Beschovski 1966e, 1973c, 1973e, 1975a, 1975b, 1976a, 2009; Beschovski \& Zatwarnicki 2001a.

Atissa pygmaea (Haliday, 1833) - DW, BN, BS; 0-100 m; 1; wpat; Beschovski 1964a, 1964b, 1972a, 1973c, 1973e, 1975a, 1975b, 1976a, 1996c, 2009; Beschovski \& Zatwarnicki 2001a.

Ptilomyia orsovana (Enderlein, 1922) - DW, DM; 100-200 m; 1; sena, ? ee; Zatwarnicki 1996; Beschovski \& Zatwarnicki 2001a; Beschovski 2009.

Schema acrosticale (Becker, 1903) [Atissa] - BN, BS; 0-5 m; 1; ? wp; Beschovski 1966e, 2009; Beschovski \& Zatwarnicki 2001a.

Hydrellia albifrons Fallén, 1813 - RW; 1300-1400 m; 3, 4; wp; Beschovski 2009.

Hydrellia albilabris (Meigen, 1830) - V4, O62, BN; 0-900 m; 1, 2; ena; Beschovski 1966e, 2009; Beschovski \& Zatwarnicki 2001a.

Hydrellia cardamines Haliday, 1839 - BN; 0-5 m; 1; e; Beschovski 1966e, 2009; Beschovski \& Zatwarnicki 2001 a.

Hydrellia fascitibia (von Roser, 1840) - BN, BS; 0-10 m; 1; h; Beschovski 1966e, 2009; Beschovski \& Zatwarnicki $2001 \mathrm{a}$.

Hydrellia flaviceps (Meigen, 1830) - DM, E2, O62, BN; 0-200 m; 1; po; Beschovski \& Zatwarnicki 2001a; Beschovski 2009.

? Hydrellia flavicornis (Fallén, 1823) [? H. nigripes Zetterstedt, 1838; ? H. svecica Zatwarnicki, 1998] - TL, BN; 0-250 m; 1; e; Beschovski 1966e. 
Hydrellia fusca (Stenhammar, 1844) - RW; 1320-1450 m; 3, 4; e; Beschovski \& Zatwarnicki 2001a; Beschovski 2006a, 2009.

Hydrellia griseola (Fallén, 1813) [H. chrysostoma (Meigen, 1830); H. cochleariae Haliday, 1839; H. nigripes cochleariae Haliday, 1839] - -; ; DW, DM, E2, P1, P2, B1, B2, B3, K2, V1, V4, V5, S1, S22, TL, T11, T2, T31, O5, O61, O62, R1, R2, R3, R5, RW, RE, BN, BS; 0-2500 m; 1, 2, 3, 4, 5, 6; sk; Kozarov 1910-1911; Buresch \& Lazarov 1956; Lyubenov 1958; Beschovski 1966e, 2004a, 2006a, 2009; Beschovski \& Zatwarnicki 2001a.

Hydrellia ischiaca Loew, 1862 [H. appendiculata Collin, 1966] - DW, E2, P2, O5, O62, BN; 0-750 m; 1, 2; h; Beschovski \& Zatwarnicki 2001a; Beschovski 2009.

Hydrellia maculiventris Becker, 1896 - E2, B1; 150-700 m; 1; ena; Beschovski \& Zatwarnicki 2001a; Beschovski 2009.

Hydrellia maura Meigen, 1838 [H. modesta Loew, 1860] - E2, B2, V1, V4, S1, T31, R1, R2, RW, BN, BS; 0-2500 m; 1, 2, 3, 4, 5, 6; ena, ? wp; Beschovski 1966e, 2009; Beschovski \& Zatwarnicki 2001 .

Hydrellia mutata (Zetterstedt, 1846) - RW; 1270-1470 m; 3, 4; e; Beschovski 1966e.

Hydrellia pubescens Becker, 1926 [H. nasturtii Collin, 1928] - BN; 0-5 m; 1; ena; Beschovski 1966e.

Hydrellia obscura (Meigen, 1830) - DW, E2, B1, TL, R5, RW, BN; 0-1500 m; 1, 2, 3, 4; wcp; Beschovski 1966e, 2009; Beschovski \& Zatwarnicki 2001a.

Hydrellia parafrontosa Papp, 1983 - E2, BN; 0-150 m; 1; see; Beschovski \& Zatwarnicki 2001a; Beschovski 2009.

Hydrellia ranunculi Haliday, 1839 [H. albiceps Meigen, 1830; H. flavicornis Fallén, 1823; H. pilitarsis Stenhammar,1984] - B2, BN, BS; 0-1500 m; 1, 2, 3, 4; ena; Beschovski 1966e, 1972a, 1975a, 2009; Beschovski \& Zatwarnicki 2001a.

Hydrellia subalbiceps Collin, 1966 - DW, V4, RW; 110-1570 m; 1, 2, 3, 4; ena; Beschovski \& Zatwarnicki 2001a; Beschovski 2006a, 2009.

Hydrellia tarsata Haliday, 1839 [H. flavicornis Fallén, 1823] - T31, BN; 0-200 m; 1; e; Beschovski \& Zatwarnicki 2001a; Beschovski 2009.

Hydrellia thoracica Haliday, 1839 - E2, BN; 0-150 m; 1; e; Beschovski \& Zatwarnicki 2001a; Beschovski 2009.

Dichaeta caudata (Fallén, 1813) - TK, V1, T2, O61, BN; 0-700 m; 1, 2; h; Beschovski 1996c, 2009; Beschovski \& Zatwarnicki 2001a.

Notiphila (Agrolimna) venusta Loew, 1856 - DW, V1, T2, O62, R2; 37-1250 m; 1, 2, 3; des; Beschovski 1966e, 2009; Beschovski \& Zatwarnicki 2001a.

Notiphila (Notiphila) annulipes Stenhammar, 1844 - DW, B2, T2, O61, RW, BN; 0-1380 m; 1, 2, 3, 4; dp; Beschovski \& Zatwarnicki 2001a; Beschovski 2006a, 2009.

Notiphila (Notiphila) brunipes (Robineau-Desvoidy, 1830) - TL, BN; 0-250 m; 1; e; Beschovski 1966e, 2009; Beschovski \& Zatwarnicki 2001a.

Notiphila (Notiphila) cinerea Fallén, 1813 - DW, DM, P1, P2, V1, O62, R1, RW, BN, BS; 0-1400 m; 1, 2, 3, 4; tp; Nedelkov 1912; Beschovski 1964a, 1964b, 2006a, 2009; Beschovski \& Zatwarnicki 2001a.

Notiphila (Notiphila) dorsata Stenhammar, 1844 - O61, BN, BS; 0-400 m; 1; tp, ? dp; Beschovski \& Zatwarnicki 2001a; Beschovski 2009.

Notiphila (Notiphila) graecula Becker, 1926 - DW, E2, P1, B2, T31, O62, R1, R2, RW, RE, BN, BS; 0-1400 m; 1, 2, 3, 4; ewca; Beschovski 1996c, 2004a, 2006a, 2009; Beschovski \& Zatwarnicki 2001a.

Notiphila (Notiphila) maculata Stenhammar, 1844 - B1, B2; 500-700 m; 1, 2; ena; Beschovski \& Zatwarnicki 2001a; Beschovski 2009.

Notiphila (Notiphila) nigricornis Stenhammar, 1844 [N. uliginosa Haliday, 1839] - DW, E2, P1, B1, B2, V1, V4, TL, O62, R1, R2, BN, BS; 0-1500 m; 1, 2, 3, 4; esca; Nedelkov 2012; Beschovski 1997b, 2009; Beschovski \& Zatwarnicki 2001a.

Notiphila (Notiphila) riparia Meigen, 1830 - DW, P1, V1, BN, BS; 0-600 m; 1; wp, ? h; Beschovski \& Zatwarnicki 2001a; Beschovski 2009.

Notiphila (Notiphila) stagnicola (Robineau-Desvoidy, 1830) [N. australis Loew, 1860] - V1, O62; 150-600 m; 1; wp; Nedelkov 2012; Beschovski \& Zatwarnicki 2001a; Beschovski 2009.

Notiphila (Notiphila) subnigra Krivosheina, 1998 - P1, B2, V1; 400-800 m; 1, 2; e; Beschovski \& Zatwarnicki 2001a; Beschovski 2009.

Athyroglossa (Athyroglossa) glabra (Meigen, 1830) - DW, DM, P2, B2, T31, RE, BN, BS; 0-700 m; 1, 2; h; Beschovski 1966e, 2004a, 2009; Beschovski \& Zatwarnicki 2002. 
Athyroglossa (Athyroglossa) nudiuscula Loew, 1860 - P3, S1, T31, R2; 45-650 m; 1; swp; Zatwarnicki 1996; Beschovski \& Zatwarnicki 2002; Beschovski 2009.

Athyroglossa (Parathyroglossa) ordinata Becker, 1896 [Discocerina brevipectinata (Becker, 1896)] - DW, O62, R2, RE, BN; 0-660 m; 1; wp; Beschovski 1966e, 2009; Beschovski \& Zatwarnicki 2002.

Chlorichaeta albipennis (Loew, 1848) [Gymnopa] - O62, BN, BS; 0-200 m; 1; pata; Caspers 1951a; Beschovski 1966e, 2009; Beschovski \& Zatwarnicki 2002.

Mosillus subsultans (Fabricius, 1794) - B2, V1, O5, O62, BN, BS; 0-750 m; 1, 2; wp; Beschovski 1966e, 2009; Beschovski \& Zatwarnicki 2002.

Ochthera mantis (De Geer, 1776) - V1, BN, BS; 0-600 m; 1; h; Nedelkov 2012; Beschovski 1964a, 2009; Beschovski \& Zatwarnicki 2002.

Ochthera schembrii Rondani, 1847 [O. setigera Czerny, 1909] - BN, BS; 0-5 m; 1; wp; Beschovski 1966e, 2009; Beschovski \& Zatwarnicki 2002.

Allotrichoma bezzii Becker, 1896 - BN, BS; 0-5 m; 1; ewca; Zatwarnicki 1991; Beschovski \& Zatwarnicki 2002; Beschovski 2009.

Allotrichoma bifidum Papp, 1974 - DW, DM, O62, BN, BS; 0-150 m; 1; csee; Beschovski \& Zatwarnicki 2002.

Allotrichoma laterale (Loew, 1860) [A. valkanovi Beschovski, 1966] - DW, E2, B2, TK, K8, V1, S1, TL, T2, T31, O62, R2, BN, BS; 0-660 m; 1; h; Beschovski 1966b, 2009; Beschovski \& Zatwarnicki 2002.

Allotrichoma simplex Loew, 1861 [A. filiforme Becker, 1896; A. dahli Beschovski, 1966] - DW, E2, P2, V1, TL, T31, O62, BN; 0-550 m; 1; ena, ? wp; Beschovski 1966d, 2009; Beschovski \& Zatwarnicki 2002.

Allotrichoma quadripectinatum (Becker, 1903) - O62, BS; 0-150 m; 1; atm; Beschovski \& Zatwarnicki 2002; Beschovski 2009.

Hecamede albicans (Meigen, 1830) - BN, BS; 0-5 m; 1; hat; Caspers 1951a, 1951b; Beschovski 1964a, 1964b, 1965, 2009; Beschovski \& Zatwarnicki 2002.

Glenanthe nigripes Czerny, 1909 [G. ripicola (Haliday, 1839)] - BN, BS; 0-5 m; 1; se; Beschovski 1964a, 1964b, 1972a, 1973c, 1975a, 1975b, 1976a, 1996c, 2009; Beschovski \& Zatwarnicki 2002.

Glenanthe ripicola (Haliday, 1839) - BN; 0-5 m; 1; wcp; Beschovski \& Zatwarnicki 2002; Beschovski 2009.

Diclasiopa niveipennis (Becker, 1896) [Discocerina obscurella (Fallén, 1813)] - DW, P2, B2, V1, TL, T31, O62, RE, BN, BS; 0-1500 m; 1, 2, 3; wpat; Beschovski \& Zatwarnicki 2002; Beschovski 2004a, 2009.

Discocerina obscurella (Fallén, 1813) - DW, DM, E2, P2, B1, B2, V1, T31, O61, O62, R2, R5, RE, BN, BS; 0-800 m; 1, 2; hnat; Beschovski 1966e, 2004a, 2009; Beschovski \& Zatwarnicki 2002.

Diclasiopa lacteipennis Loew, 1862 [D. xanthocera (Loew 1848)] - DW, DM, P2, B2, V1, T31, O62, RE, BN; 0-800 m; 1, 2; hat; Beschovski 1966e, 2004a, 2009; Beschovski \& Zatwarnicki 2002.

Ditrichophora calceata (Meigen, 1830) - E2, TL, T31, O5, R2, RW, BN; 0-1350 m; 1, 2, 3; ena; Beschovski \& Zatwarnicki 2002; Beschovski 2006a, 2009.

Ditrichophora canzonerii (Rampini, 1980) - T31; 150 m; 1; se; Beschovski \& Zatwarnicki 2002; Beschovski 2009.

Ditrichophora fuscella (Stenhammar, 1844) - DW, O62, R2, RE; 100-1250 m; 1, 2, 3; des; Beschovski \& Zatwarnicki 2002; Beschovski 2004a, 2009.

Ditrichophora graeca (Canzoneri \& Meneghini, 1985) - DW; 100-120 m; 1; Eb; Beschovski \& Zatwarnicki 2002; Beschovski 2009.

Ditrichophora palliditarsis (Becker, 1896) - O62; 150-200 m; 1; des; Beschovski \& Zatwarnicki 2002; Beschovski 2009.

Gymnoclasiopa nigerrima (Strobl, 1893) [Discocerina plumosa Fallén, 1823] - O62, RW; 200-1450 m; 1, 2, 3, 4; ? wes; Beschovski 1966e, 2006a, 2009; Beschovski \& Zatwarnicki 2002.

Gymnoclasiopa pulchella (Meigen, 1830) - DW, K6, V1, TL, O62, RW, BN; 0-1200 m; 1, 2, 3; ena, ? h; Beschovski \& Zatwarnicki 2002; Beschovski 2006a, 2009.

Hecamedoides costatus (Loew, 1860) - BN, BS; 0-5 m; 1; wpat; Beschovski 1966e, 2009; Beschovski \& Zatwarnicki 2002.

Hecamedoides glaucellus (Stenhammar, 1844) - DW, RE, BN, BS; 0-230 m; 1; wpat; Beschovski 1966e, 2004a, 2009; Beschovski \& Zatwarnicki 2002.

Hecamedoides unispinosus (Collin, 1943) - DW, B1, V1, V4, T31, O62, R2, RE, BN; 0-1300 m; 1, 2, 3; hnat; Zatwarnicki 1996; Beschovski \& Zatwarnicki 2002; Beschovski 2004a, 2009.

Polytrichophora duplosetosa (Becker, 1896) - DW, P2, O62, BN, BS; 0-300 m; 1; wpat; Zatwarnicki 1996; Beschovski \& Zatwarnicki 2002; Beschovski 2009. 
Ilythea spilota Curtis, 1832 - B2, T31, O4, RE; 150-1500 m; 1, 2, 3; h; Beschovski \& Zatwarnicki 2002; Beschovski 2004a, 2009.

Nostima picta (Fallén, 1813) - DM, E2, P1, B2, B3, V4, S1, T2, T31, O61, O62, R1, R2, RW, RE, BN; 0-2400 m; 1, 2, 3, 4, 5; hn, ? h; Mathis \& Zatwarnicki 1995; Beschovski \& Zatwarnicki 2001b; Beschovski 2004a, 2006a, 2009.

Philygria femorata (Stenhammar, 1844) - V4; 1000 m; 2; e; Hollmann-Schirrmacher 1998; Beschovski 2009.

Philygria interstincta (Fallén, 1813) - DW, E2, B1, V4, RW; 100-1400 m; 1, 2, 3; e; Beschovski \& Zatwarnicki 2001b; Beschovski 2006a, 2009.

Philygria obtecta Becker, 1896 - DW, DM, O62, RR, RW; 120-1000 m; 1, 2; et; Beschovski \& Zatwarnicki 2001b; Beschovski 2006a, 2009.

Philygria posticata (Meigen, 1830) - DW, DM, E2, P2, B2, V4, S1, R2, R5, RE; 100-1200 m; 1, 2, 3; des; Beschovski \& Zatwarnicki 2001b; Beschovski 2004a, 2009.

Philygria stictica (Meigen, 1830) - \$ DW, DM, E2, P2, B2, V4, V5, S1, TL, T11, T2, T31, O5, O62, R1, R2, R3, R5, RE, BN, BS; 0-2400 m; 1, 2, 3, 4, 5; e; Beschovski 1997b, 2004a, 2009; Beschovski \& Zatwarnicki 2001b.

Philygria vittipennis (Zetterstedt, 1838) - E2, V4, S1, R2, R5, RW; 230-2400 m; 1, 2, 3, 4, 5; h; Beschovski \& Zatwarnicki 2001b; Beschovski 2009.

Hyadina guttata (Fallén, 1813) - DW, E1, E2, P1, B2, V1, V4, V5, S1, T2, O62, R1, R2, R5, RE, BN, BS; 0-2320 m; 1, 2, 3, 4, 5; tp; Beschovski 1975b, 2004a, 2009; Beschovski \& Zatwarnicki 2001 b.

Hyadina rufipes (Meigen, 1830) - B2, O62; 200-1500 m; 1, 3; des; Beschovski \& Zatwarnicki 2001b; Beschovski 2009.

Hyadina scutellata (Haliday, 1839) - DW; 110-120 m; 1; e; Beschovski \& Zatwarnicki 2001b; Beschovski 2009.

Pelina aenea (Fallén, 1813) - B2, O62, BS; 0-1300 m ; 1, 2, 3; tp; Beschovski \& Zatwarnicki 2001b; Beschovski 2009.

Pelina similis Papp, 1974 [P. nitens Loew, 1873; P. subpunctata Becker, 1896] - DM, V4, R4, RE, BN, BS; 0-1800 m; 1, 2, 3, 4; et; Beschovski 1966e, 1996c, 2004a, 2009; Zatwarnicki 1991; Mathis \& Zatwarnicki 1995; Beschovski \& Zatwarnicki 2001b.

Parydra (Chaetoapnaea) fossarum (Haliday, 1833) [Napaea] - DW, DM, E2, P1, P2, B1, B2, B3, TK, V1, V4, S1, TL, T11, T2, T31, O4, O61, O62, R1, R2, RW, RE, BN, BS; 0-2000 m; 1, 2, 3, 4, 5; h; Nedelkov 1912; Beschovski 1972b, 1975b, 1996c, 2009; Beschovski \& Zatwarnicki 2002.

Parydra (Chaetoapnaea) hecate (Haliday, 1833) [P. fossarum (Haliday, 1833)] - P1, B2, V1, RW, BN; 0-1400 m; 1, 2, 3; ena; Nedelkov 1912; Beschovski \& Zatwarnicki 2004; Beschovski 2006a, 2009.

Parydra (Chaetoapnaea) obliterata Duda, 1942 - DW, P1, K6, V4, BN; 0-1100 m; 1, 2, 3; e; Beschovski \& Zatwarnicki 2004; Beschovski 2009.

Parydra (Chaetoapnaea) pusilla (Meigen, 1830) - RW, BN; 0-1500 m; 1, 2, 3, 4; tes; Beschovski \& Zatwarnicki 2004; Beschovski 2006a, 2009.

Parydra (Parydra) aquila (Fallén, 1813) [Napaea] - DW, P1, P2, B1, B2, V1, V5, TL, BN, BS; 0-1000 m; 1, 2; h; Nedelkov 1912; Beschovski 1972b, 1975b, 1996c, 2009; Beschovski \& Zatwarnicki 2004.

Parydra (Parydra) coarctata (Fallén, 1813) [P. litoralis (Meigen 1830); Napaea cognata Loew, 1860] - DW, P1, B1, B2, B3, V1, V4, T11, T2, T31, O4, O5, O62, R1, R2, RW, RE, BN, BS; 0-2160 m; 1, 2, 3, 4, 5; tp, ? hop; Nedelkov 1912; Beschovski 1966e, 1976a, 1996c, 1997b, 2006a, 2009; Beschovski \& Zatwarnicki 2004.

Parydra (Parydra) cognata Loew, 1860 [P. aquila (Fallén, 1813); Napaea] - DW, P2, B1, B2, V1, S1, TL, T31, O4, O61, O62, R1, R2, RW, RE, BN, BS; 0-2160 m; 1, 2, 3, 4, 5; ena, ? wp; Nedelkov 1912; Beschovski 1966e, 1976a, 1996c, 1997b, 2004a, 2006a, 2009; Beschovski \& Zatwarnicki 2004.

Parydra (Parydra) littoralis (Meigen, 1830) [P. quadripunctata (Meigen, 1830); Napaea] - DW, B2, V1, V4, V5, T31, R1, RW, RE; 100-2000 m; 1, 2, 3, 4; ena, ? wp; Nedelkov 1912; Krivosheina 1989a; Beschovski 1997b, 2006a, 2009; Beschovski \& Zatwarnicki 2004.

Ephydra afghanica Dahl, 1961 - V1, RE, BS; 0-530 m; 1; emca; Beschovski \& Zatwarnicki 2004; Beschovski 2009.

Ephydra attica Becker, 1896 [E. macellaria Egger, 1862] - RE, BN, BS; 0-220 m; 1; see, ? se; Wirth 1975; Mathis \& Zatwarnicki 1995; Beschovski 1996c, 2009; Beschovski \& Zatwarnicki 2004.

Ephydra bivittata Loew, 1860 - BN, BS; 0-5 m; 1; hom; Beschovski 1966e, 2009; Wirth 1975; Beschovski \& Zatwarnicki 2004.

Ephydra flavipes (Macquart, 1843) [E. macellaria Egger, 1862] - BS; 0-5 m; 1; swpat; Beschovski \& Zatwarnicki 2004; Beschovski 2009. 
Ephydra glauca Meigen, 1830 - RE, BN; 0-220 m; 1; wcp; Wirth 1975; Beschovski \& Zatwarnicki 2004; Beschovski 2009.

? Ephydra macellaria Egger, 1862 [according to Beschovski \& Zatwarnicki (2004) not presented in Bulgaria; confused with E. murina, E. flavipes, E. attica) - V1, BN, BS; 0-600 m; 1; ena, ? wp; Nedelkov 1912; Caspers 1951a, 1952, 1957; Valkanov 1957a; Beschovski 1964a, 1964b, 1966e, 1972c,1973c, 1973e, 1976a; Beschovski \& Zatwarnicki 2004; Pape \& Beuk 2017.

Ephydra murina Wirth, 1975 [E. macellaria Egger, 1862] - T31, BN, BS; 0-62 m; 1; eanit; Beschovski \& Zatwarnicki 2004; Beschovski 2009.

Ephydra riparia Fallén, 1813 - T31, BN, BS; 0-65 m; 1; h; Drensky 1959, 1960; Beschovski 1964b, 1965, 1996c, 2009; Wirth 1975; Beschovski \& Zatwarnicki 2004.

Ephydra scholtzi Becker, 1896 - T31; 60-65 m; 1; des; Beschovski \& Zatwarnicki 2004; Beschovski 2009.

Setacera breviventris (Loew, 1860) [Ephydra micans Haliday, 1833] - V1, TL, T31, RW, BN, BS; 0-1380 m; 1, 2, 3; ppta; Drensky 1942; Beschovski 1972b, 1973e, 1975a, 1996c, 2006a, 2009; Beschovski \& Zatwarnicki 2004.

Paracoenia fumosa (Stenhammar, 1844) [Coenia] - DW, V1, BN, BS; 0-550 m; 1; tp, ? hop; Becker 1926; Beschovski 1966e, 1973e, 1996c, 2009; Beschovski \& Zatwarnicki 2004.

Coenia palustris (Fallén, 1823) - DW, T31, BN, BS; 0-110 m; 1; wces, ? wcp; Beschovski 1966e, 1975b, 1976a, 1996c, 2009; Beschovski \& Zatwarnicki 2004.

Halmopota septentrionalis Canzoneri \& Meneghini, 1974 - RE; 80 m; 1; nmwca; Beschovski \& Zatwarnicki 2004; Beschovski 2009.

Philotelma defectum (Haliday, 1833) - BN; 0-5 m; 1; h; Beschovski \& Zatwarnicki 2004; Beschovski 2009.

Scatophila caviceps (Stenhammar, 1844) - DW, DM, E2, TK, V1, TL, O61, O62, R2, BN; 0-2500 m; 1, 2, 3, 4, 5, 6; hop; Beschovski \& Zatwarnicki 2004; Beschovski 2009.

Scatophila despecta (Haliday, 1839) - DM, K8, V1, R2, BN; 0-2500 m; 1, 2, 3, 4, 5, 6; h; Beschovski 1996c, 2009; Beschovski \& Zatwarnicki 2004.

Scatophila farinae Becker, 1903 - DW, R2; 100-350 m; 1; sena; Beschovski \& Zatwarnicki 2004; Beschovski 2009.

Scatophila modesta Becker, 1908 - TK; 620-630 m; 1; se; Beschovski \& Zatwarnicki 2004; Beschovski 2009.

Scatophila signata (Loew, 1860) - O62; 80-100 m; 1; e; Beschovski \& Zatwarnicki 2004; Beschovski 2009.

Scatophila unicornis Czerny, 1900 - DW, B3, S1; 100-350 m; 1; h; Zatwarnicki 1996; Beschovski \& Zatwarnicki 2004; Beschovski 2009.

Limnellia quadrata (Fallén, 1813) - DW, B2, V1, V4, S1, S211, TL, O1, O62, R1, R2, R5, RW, RE; 70-2400 m; 1, 2, 3, 4, 5, 6; dp ? h; Beschovski \& Zatwarnicki 2004; Beschovski 2006a, 2009.

Lamproscatella bimaculata Hendel, 1933 - R1, R2; 2000-2500 m; 4, 5, 6; h; Beschovski \& Zatwarnicki 2004; Beschovski 2009.

Lamproscatella sibilans (Haliday, 1833) [Scatella pilosigenis Becker, 1896] - DW, E1, B2, V1, V4, V5, S1, O1, O4, R1, R2, R5, RW, RE, BN, BS; 0-2300 m; 1, 2, 3, 4, 5, 6; ? h; Beschovski 1966e, 1975b, 1976a, 1996c, 1997b, 2006a, 2009; Beschovski \& Zatwarnicki 2004.

Lamproscatella unipunctata (Becker, 1907) - B2, TL, R1, R2, R5, RW, RE; 70-2560 m; 1, 2, 3, 4, 5, 6; mca; Beschovski \& Zatwarnicki 2004; Beschovski 2006a, 2009.

Haloscatella dichaeta Loew, 1860 [Lamproscatella] - TK, V1, BN, BS; 0-650 m; 1; pat, ? hat; Beschovski 1964a, 1964b, 1975b, 1976a, 1996c, 2009; Beschovski \& Zatwarnicki 2004.

Scatella (Neoscatella) subguttata (Meigen, 1830) - DW, V1, T31, R1, BN, BS; 0-2000 m; 1, 2, 3, 4; ena, ? sk; Beschovski 1964a, 1964b, 1972b, 1973c, 1973e, 1975a, 1975b, 1976a, 1997b, 2009; Beschovski \& Zatwarnicki 2004.

Scatella (Scatella) ciliata Collin, 1930 - BN, BS; 0-5 m; 1; sena, ? ena; Beschovski \& Zatwarnicki 2004; Beschovski 2009.

Scatella (Scatella) lutosa (Haliday, 1833) - BN, BS; 0-5 m; 1; wcp; Beschovski 1966e, 1972b, 1973c, 1973e, 1975b, 2009; Beschovski \& Zatwarnicki 2004.

Scatella (Scatella) obsoleta Loew, 1861 - DW, T31, RE; 56-170 m; 1; h; Beschovski \& Zatwarnicki 2004; Beschovski 2009.

Scatella (Scatella) paludum (Meigen, 1830) - DW, P2, B2, TK, V1, V4, S1, T31, O1, R1, R3, RW, RE, BN, BS; 0-2000 m; 1, 2, 3, 4; hptn, ? sk; Beschovski 1972b, 1973c, 1973e, 1975a, 1975b, 1996c, 1997b, 2006a, 2009; Beschovski \& Zatwarnicki 2004.

Scatella (Scatella) rufipes Strobl, 1905 - BS; 0-5 m; 1; mi; Beschovski \& Zatwarnicki 2004; Beschovski 2009. 
Scatella (Scatella) stagnalis (Fallén, 1813) - O4, R1, R2, RW; 1000-2200 m; 3, 4; hpta, sk; Beschovski \& Zatwarnicki 2004; Beschovski 2009.

Scatella (Scatella) tenuicosta Collin, 1930 - DW, DM, E1, E2, P1, B1, B2, B3, TK, K6, V1, V4, S1, TL, T2, T31, O4, O61, O62, R1, R2, R5, RW, RE, BN, BS; 0-2500 m; 1, 2, 3, 4, 5, 6; hat; Nedelkov 1912; Caspers 1951a; Beschovski 1964a, 1964b, 1972b, 1973c, 1973e, 1975a, 1975b, 1996a, 1996a, 1997a, 2006a, 2009; Beschovski \& Zatwarnicki 2004.

\section{CALYPTRATA}

\section{Hippoboscidae}

Ornithoica turdi (Olivier in Latreille, 1811) - B3, S1; 500-550 m; 1; swpat; Beron 1972a.

Ornithomya avicularia (Linnaeus, 1758) - P1, P2, B1, B3, S1, TL, O62, R2, RW, BS; 0-2340 m; 1, 2, 3, 4, 5; ppt; Nedelkov 1912; Drensky 1926b; Beron 1972a, 2002, 2011.

Ornithomya fringillina Curtis, 1836 - S1; 500-550 m; 1; hn; Beron 1972a.

Crataerina pallida (Olivier in Latreille, 1811) [Oxypterum] - RW; 770 m; 2; ena; Drensky 1926b; Beron 1972a, 2011.

Stenepteryx hirundinis (Linnaeus, 1758) - V1; 550-600 m; 1; po; Nedelkov 1912; Drensky 1926b; Beron 1972a.

Icosta ardeae (Macquart, 1835) [Lynchia albipennis (Say, 1823); Olfersia] - P2, V1; 390-650 m; 1; hpta; Drensky 1926b; Buresch 1939; Beron 1972a.

Pseudolynchia canariensis (Macquart in Webb \& Berthelot, 1839) [P. rufipes (Macquart, 1847)] - TL; 80-85 m; 1; k; Beron 1972a.

Hippobosca equina Linnaeus, 1758 - А ; ; P2, B2, BS; 0-2376 m; 1, 2, 3, 4, 5; ppta; Joakimoff 1899; Nedelkov 1912; Drensky 1926b, 1934c; Chalupský 1956; Valerianov 1961; Beron 1972a, 2011.

Hippobosca longipennis Fabricius, 1805 [H. equina canina Drensky 1926] - V1, R1, RW; 570-1300 m; 1, 2, 3; hpt; Drensky 1926b; Buresch 1939; Beron 1972a, 2011.

Lipoptena cervi (Linnaeus, 1758) - $\mathbf{\Delta}$; S1, TL, BS, T31, +++; 30-260 m; 1; pat, hat, i; Drensky 1926a, 1926b; Beron 1972a.

Lipoptena fortisetosa Maa, 1965 - S23, +++; 306 m; 1; dp; Dvořák et al. 2021.

Melophagus ovinus (Linnaeus, 1758) [? M. caprae Drensky, 1939] - A ; B B1, V1, S21, R2, R3, RW; 380-1810 m; 1, 2, 3, 4; k, i; Nedelkov 1909, 1912; Drensky 1926b; Beron 1972a, 2011.

\section{Streblidae}

Brachytarsina flavipennis Macquart, 1851 - K8, RE; 400-1000 m; 1, 2; swp; Ivanova et al. 1995; Beron et al. 2004; Beron 2004, 2015.

\section{Nycteribiidae}

Nycteribia (Nycteribia) latreillii (Leach, 1817) [Listropodia] - E2, B3, V1, RW, BS; 0-1000 m; 1, 2; wpo; Karamann 1939; Kantardzhieva-Minkova 1957; Hůrka 1958; Buresch 1960; Kock 1974; Nowosad et al. 1987; Beron 1994, 2004, 2011, 2015; Beron et al. 2011.

Nycteribia (Nycteribia) pedicularia Latreille, 1805 - RW, BS; 0-950 m; 1, 2; swp; Hůrka 1958, 1972; Buresch 1960; Kock 1974; Nowosad et al. 1987; Beron 1994, 2004, 2011, 2015; Beron et al. 2011.

Nycteribia (Nycteribia) schmidlii Schiner, 1853 - E2, B1, B3, T31, RW, RE, BS; 0-1100 m; 1, 2, 3; wpat; Hůrka 1958, 1962, 1972; Buresch 1960; Guéorguiev \& Beron 1962; Beron \& Guéorguiev 1967; Skuratowicz 1970; Kock 1974; Nowosad et al. 1987; Beron 1994, 2011, 2015, 2016; Beron et al. 2004, 2011.

Nycteribia (Acrocholidia) vexata Westwood, 1835 - E1, B1, B3, T31, RW, RE, BS; 0-1050 m; 1, 2, 3; wp; Karamann 1939; Kantardzhieva-Minkova 1957; Hůrka 1958, 1972, 1984; Buresch 1960; Guéorguiev \& Beron 1962; Beron \& Guéorguiev 1967; Kock 1974; Nowosad et al. 1987; Beron 1994, 2004, 2015, 2016; Beron et al. 2004, 2011. 
Phthiridium biarticulatum Hermann, 1804 [Celeripes, Stylidia] - B1, B3, S21, RW, RE, BS; 0-900 m; 1, 2; wp; Karamann 1939; Kantardzhieva-Minkova 1957; Hůrka 1958, 1962, 1984; Buresch 1960; Guéorguiev \& Beron 1962; Nowosad et al. 1987; Beron 1994, 2004, 2011, 2015, 2016; Beron et al. 2004, 2011.

Penicillidia conspicua Speiser, 1901 [Neopenicillidia] - E1, B1, B3, T31, RW, RE, BS; 0-1100 m; 1, 2, 3; om; Hůrka 1958, 1962; Buresch 1960; Guéorguiev \& Beron 1962; Beron \& Guéorguiev 1967; Skuratowicz 1970; Nowosad et al. 1987; Beron 1994, 2004, 2011, 2015, 2016; Beron et al. 2004, 2011.

Penicillidia dufourii (Westwood, 1835) - P1, P2, B1, B3, S21, RW, RE, BS; 0-1100 m; 1, 2, 3; po; Karamann 1939; Kantardzhieva-Minkova 1957; Hůrka 1958; Buresch 1960; Guéorguiev \& Beron 1962; Beron \& Guéorguiev 1967; Hazelton 1970; Nowosad et al. 1987; Beron 1994, 2004, 2011, 2015, 2016; Beron et al. 2004, 2011.

\section{Scathophagidae (Cordyluridae, Scatomyzidae, Scopeumatidae)}

Norellisoma armipes (Meigen, 1826) [Norellia] - R1; 2700 m; 6; e; Nedelkov 1912.

Norellisoma seguyi Šifner, 1972 - R1; 1200 m; 3; csee; Šifner 2018.

Norellisoma spinimanum (Fallén, 1819) [Norellia] - V1; 600 m; 1; h; Nedelkov 1912.

Cordilura (Cordilurina) albipes Fallen, 1819 [Parallelomma] - V1; 600 m; 1; e; Nedelkov 1912.

Nanna flavipes (Fallén, 1819) [Amaurosoma] - wces; Lyubenov 1958.

Phrosia albilabris (Fabricius, 1805) - V1, V4, TL; 200-800 m; 1, 2; e; Nedelkov 1912.

Scathophaga furcata (Say, 1823) [Scopeuma] - B1; 1400 m; 3; h; Lavčiev 1965b.

Scathophaga inquinata Meigen, 1826 [Scopeuma] - B1; 1270 m; 3; e; Lavčiev 1965 b.

Scathophaga lutaria (Fabricius, 1794) [Scopeuma] - B1; 1270 m; 3; wp; Lavčiev 1965b.

Scathophaga stercoraria (Linnaeus, 1758) [Scopeuma] - E2, SB, V1, V4, S22, TL, R1, R3, BN, BS; 0-2100 m; 1, 2, 3, 4; hat, ? hnat; Löw 1862; Meunier 1897; Joakimoff 1899; Kovachev 1905; Nedelkov 1909, 1912; Drenowsky 1936; Drensky 1955, 1960; Valerianov 1961; Lavčiev 1965a, 1965d, 1980; Lavchiev \& Jovčev 1978; Lavchiev \& Tsankova 1980; Lavchiev et al. 1984.

Scathophaga suilla (Fabricius, 1794) [S. taeniopa Rondani, 1867; Scopeuma] - B1; 860-880 m; 2; hat; Lavčiev 1965 b.

Anthomyiidae

Adia cinerella (Fallén, 1825) [Paregle] - ; ho; Lavchiev \& Jovčev 1978; Lavčiev 1980.

Paregle coerulescens (Strobl, 1893) [Adia] - P1, B1, V4, RW, BS; 0-1700 m; 1, 2, 3, 4; cse; Lavčiev 1965b; Beschovski 2006a.

Anthomyia pluvialis (Linnaeus, 1758) - \; ; E2, B1, V1, V3, V4, S1, RW, BS; 0-1300 m; 1, 2, 3; hoa, poa; Löw 1863; Meunier 1897; Nedelkov 1912; Gregor \& Povolny 1959; Drensky 1960; Lavčiev 1965a, 1965d, 1972, 1980; Lavchiev \& Jovčev 1978; Beschovski 2006a.

Botanophila discreta (Meigen, 1826) [Chortophila] - V1; 550-600 m; 1, 2; tp; Nedelkov 1912.

Botanophila striolata (Fallén, 1824) [Chortophila] - V1; 550-600 m; 1, 2; tes, ? tp; Nedelkov 1912.

Calythea nigricans (Robineau-Desvoidy, 1830) [C. albicincta (Fallén, 1825)] - V1, V4, RW; 550-1200 m; 1, 2, 3; wcp; Nedelkov 1912; Lavčiev 1972; Lavchiev \& Jovčev 1978; Beschovski 2006 .

Calythea pratincola (Panzer, 1809) - B1; 450 m; 1; h; Lavčiev 1965b.

Chirosia cinerosa (Zetterstedt, 1845) [Pycnoglossa] - dp; Buhr 1941.

Delia albula (Fallén, 1825) [Chortophila, Crinura] - BS; 0-5 m; 1; h; Lavčiev 1965b.

Delia antiqua (Meigen, 1826) [Anthomyia ceparum (Meigen, 1830), Chortophila, Crinura, Hylemyia] - -; DW, DM, E1, P1, P2, K9, V1, S1, TL, T1, O62, RR, RW; 0-1100 m; 1, 2, 3; hn, ? k, i; Stribarni 1898; Malkov 1906a, 1907; Nedelkov 1912; Kozarov 1912; Ipatov 1921; Tschorbadjiew 1927, 1928a, 1928b, 1929a, 1929b, 1930a, 1930b, 1932; Lazarov 1936b; Buresch \& Lazarov 1956; Popoff 1956; Popoff \& Nikolova 1958; Kovachevski et al. 1959; Gospodinov 1958; Grigorov 1972, 1976; Harizanov et al. 1996; Beschovski 2006a. Delia cardui (Meigen, 1826) [Chortophila] - B1, V4, RW; 600-1400 m; 2, 3; h; Lavčiev 1965b; Beschovski 2006a. Delia coarctata (Fallén, 1825) [Leptohylemya, Hylemyia] - V1; 520-600 m; 1; h; Hitilov 1912b; Drenowsky 1920b; Buresch \& Lazarov 1956; Lyubenov 1958; Krasteva \& Beschovski 2001.

Delia floralis (Fallen, 1824) [Chortophila, Hylemyia] - -; ; h; Popoff \& Nikolova 1958; Grigorov 1972.

Deliaflorilega (Zetterstedt, 1845) [Chortophila trichodactyla (Rondani, 1866); Crinura] - V1, V4, RW, BS; 0-2000 m; 1, 2, 3, 4; h; Nedelkov 1912; Drenowsky \& Enderlein 1923; Popoff \& Nikolova 1958; Kovachevski et al. 1959; Nikolova 1964; Lavčiev 1965b; Beschovski $2006 a$. 
Delia lineariventris (Zetterstedt, 1845) [Chortophila bicalcarea (Pandellé, 1900)] - P1, B1; 500-550 m; 1; h; Lavčiev 1965b.

Delia platura (Meigen, 1826) [Phorbia; Chortophila cilicrura Rondani, 1866] - DW; 120 m; 1; k; Zamfirov 1960b, 1961a; Nachev 1976.

Delia radicum (Linnaeus, 1758) [Chortophila brassicae Wiedemann, 1817; Anthomyia brassicae Bouché, 1833; Hylemyia; Paregle] - -; P2, V1, S1, S21, S22, TL, O61, O62, R1, R2, RW; 100-1800 m; 1, 2, 3, 4; h; Meunier 1897; Malkov 1903; Grigoriev 1922; Tschorbadjiew 1926a, 1926b, 1927, 1928a, 1928b, 1929a, 1929b, 1930a, 1930b, 1932; Vasilev 1934; Gomon 1940; Nikolova 1945, 1948, 1949b; Buresch \& Lazarov 1956; Popoff 1956; Nikolova \& Popoff 1957; Popoff \& Nikolova 1958; Gregor \& Povolny 1959; Kovachevski et al. 1959; Gospodinov 1958; Grigorov 1972, 1976; Harizanov et al. 1996.

Egle ciliata (Walker, 1849) [E. muscaria Malloch 1920] - P1, B1, RW; 400-2000 m; 1, 2, 3, 4; h; Lavčiev 1965b; Beschovski 2006a.

Egle concomitans (Pandellé, 1900) - B1; 600 m; 1, 2; h; Lavčiev 1965b.

Egle parva Robineau-Desvoidy, 1830 - P1, B1, V4, T31, O61, R1, RW; 200-1700 m; 1, 2, 3, 4; des; Lavčiev 1965b; Lavčiev et al. 1984; Beschovski 2006a.

Emmesomyia socia (Fallén, 1825) [Pegomya] - P1; 450 m; 1; h; Lavčiev $1965 b$.

Eustalomyia hilaris (Fallén, 1823) - B1, V1; 550-1060 m; 1, 2, 3; tp; Nedelkov 1912; Georgiev et al. 2004.

Eustalomyia histrio (Zetterstedt, 1838) - B1, RW; 1100-1900 m; 3, 4; des; Lavčiev 1965b; Beschovski 2006a.

Fucellia maritima (Haliday, 1838) - BN, BS; 0-5 m; 1; ena; Caspers 1951a, 1951b; Beschovski 1964a, 1965.

Fucellia tergina (Zetterstedt, 1845) - \$; sk; Dely-Draskovits 1993; Beuk et al. 2017.

Heterostylodes macrura (Schnabl in Schnabl \& Dziedzicki, 1911) - B1; 1200-1900 m; 3, 4; e; Lavčiev 1965b.

Heterostylodes pratensis (Meigen, 1826) - B1, RE; 200-1900 m; 1, 2, 3, 4; wces; Lavčiev 1965b; Beschovski 2004a.

Hydrophoria lancifer (Harris, 1780) [H. conica (Wiedemann, 1817)] - V4; 870-880 m; 2; h; Nedelkov 1912.

Hydrophoria linogrisea (Meigen, 1826) - B1, V4; 1300-1900 m; 3, 4; des; Lavčiev 1965b.

Hylemya nigrimana (Meigen, 1826) - V1; 600 m; 1; po, ? dp; Nedelkov 1912.

Hylemya urbica Van der Wulp, 1896 [H. latifrons (Schnabl, 1911)] - ho; Dely-Draskovits 1993; Beuk et al. 2017.

Hylemya vagans (Panzer, 1798) [H. strigosa (Fabricius, 1794)] - * V1, R1, RR, RW; 550-1500 m; 1, 2, 3; wcp;

Nedelkov 1912; Lavčiev 1965a, 1972, 1980; Lavchiev \& Jovčev 1978; Lavčiev et al. 1984; Beschovski 2006a.

Hylemya variata (Fallén, 1823) - V1, V4; 530-1000 m; 1, 2; wes; Nedelkov 1912; Drenowsky \& Enderlein 1923.

Lasiomma picipes (Meigen, 1826) [L. octoguttata (Zetterstedt, 1845)] - P1, B1, RW; 400-2000 m; 1, 2, 3, 4; h; Lavčiev 1965b; Beschovski 2006a.

Lasiomma strigilatum (Zetterstedt, 1838) [Opsolasia eriophthalma (Zetterstedt, 1860)] - B1; 850 m; 2; des; Lavčiev $1965 b$.

Leucophora grisella Hennig, 1967 [Hammomyia] - B1, RW; 800-1550 m; 2, 3, 4; tp; Lavčiev 1965b; Beschovski 2006a.

Eutrichota schineri (Schnabl, 1910) [Pegomya, Parapegomyia] - RE; 280 m; 1; des; Lavčiev 1965b; Beschovski 2004a.

Paregle vetula (Zetterstedt, 1838) - dp, ? des; Dely-Draskovits 1993; Beuk et al. 2017.

Pegomya bicolor (Wiedemann, 1817) - V1; 550-600 m; 1, 2; h; Nedelkov 1912.

? Pegomya conformis (Fallén, 1825) - E1, E2, S1, TL; 150-450 m; 1; h; Kozarov 1907; Tschorbadjiew 1939a; Buresch \& Lazarov 1956.

Pegomya flavifrons (Walker, 1849) [P. albimargo Pandellé, 1901] - \$; h; Buchr 1941.

Pegomya hyoscyami (Panzer, 1809) [Anthomyia conformis (Fallén, 1825); P. hyoscyami chenopodii (Rondani, 1866); P. silenis (Hering, 1924)] - -; ; E1, E2, S1, TL; 150-450 m; 1; h; Kozarov 1907; Tschorbadjiew 1932, 1939a; Buchr 1941; Buresch \& Lazarov 1956; Popoff 1956; Popoff \& Nikolova 1958; Kovachevski et al. 1959; Gospodinov 1958; Grigorov 1972, 1976; Harizanov et al. 1996.

Pegomya nigrisquama (Stein, 1888) - e; Buchr 1941.

Pegomya setaria (Meigen, 1826) - B1, RW; 410-1900 m; 1, 2, 3, 4; h; Lavčiev 1965b; Beschovski 2006a.

Pegomya testacea (De Geer, 1776) [P. silacea (Meigen, 1830)] - B1; 850 m; 2; ena; Lavčiev 1965b.

Pegomya solennis (Meigen, 1826) [P. nigritarsis (Zetterstedt, 1838)] - V1; 550-600 m; 1; h; Nedelkov 1912.

Pegomya steini Hendel, 1925 - R1; 1180-1200 m; 3; e; Buchr 1941.

Pegomya ulmaria (Rondani, 1866) - B1; 900-1900 m; 2, 3, 4; wp; Lavčiev $1965 b$.

Pegoplata aestiva (Meigen, 1826) [Paregle] - B1, RW, BS; 0-1400 m; 1, 2, 3; ho; Lavčiev 1965b; Beschovski $2006 a$. 
Phorbia fumigata (Meigen, 1826) [Ph. securis Tiensuu, 1936] - -; $\$$ DW, DM, E2, V1, S1, T1; 50-550 m; 1; wp; Zamfirov 1961a, 1962a, 1962c, 1962f, 1962a, 1963b, 1963d, 1963e; Grigorov 1972; Kontev et al. 1991; Beschovski \& Krusteva 2001.

Phorbia genitalis (Schnabl in Schnabl \& Dziedzicki, 1911) - * DW, DM, E1, E2, P1, P2, V1, TL,T2; 30-600 m; 1; h; Popoff 1956; Zamfirov 1958, 1960b, 1962f; Kovachevski et al. 1959; Makarov 1959; Lyubenov 1960; Donchev 1961.

Phorbia haberlandti (Schiner, 1865) [Ph. pecillifera Jermy, 1853] - -; DW, DM, E2, P1, V1, S1, T1; 50-550 m; 1; e, ? csee; Zamfirov 1960b, 1961a, 1962c, 1962f, 1963b, 1963d, 1963e; Grigorov 1972; Kontev et al. 1991; Beschovski \& Krusteva 2001; Krasteva \& Beschovski 2001.

Subhylemyia longula (Fallén, 1824) [Chortophila] - RW; 1200-1400 m; 3; ho; Lavčiev 1965b; Beschovski $2006 a$.

\section{Fanniidae}

Euryomma peregrinum (Meigen, 1826) - RE; 300 m; 1; k; Lavčiev 2003.

Piezura graminicola (Zetterstedt, 1846) [P. boletorum (Rondani, 1866)] - RE; 500 m; 1; h; Lavčiev 2003.

Fannia armata (Meigen, 1826) - E2, P1, P2, B1, TL; 200-1000 m; 1, 2; e; Lavčiev 1965b, 1966, 1970a, 1974, 1980, 2003; Lavčiev \& Karastoyanov 1970; Mirčeva 1981.

Fannia barbata (Stein, 1892) - P1, B1, B3, RR; 0-800 m; 1, 2; tes, ? tp; Lavčiev 1970a, 1974, 2003.

Fannia canicularis (Linnaeus, 1761) - $\mathbf{\Delta}$; ; DW, E1, E2, P1, B1, K8, V1, S211, TL, O61, O62, R1, R2, R5, RW, RE, BN, BS; 0-2300 m; 1, 2, 3, 4, 5; k; trogloxene; Löw 1862; Kozarov 1912; Nedelkov 1912; Czerný 1930; Popoff 1941; Drensky 1955, 1960; Vesselinov \& Gabev 1956; Gregor \& Povolny 1959; Valerianov 1961; Guéorguiev \& Beron 1962; Lavčiev 1964a, 1964b, 1965a, 1965d, 1969, 1970a, 1974, 1980, 2003; Surbova 1965; Beron \& Guéorguiev 1967; Lavčiev \& Nestorova 1967; Tahirov 1969, 1970, 1973; Tsanev 1969; Lavčiev \& Karastoyanov 1970; Lavčiev \& Tahirov 1970; Stoicheva 1970; Beschovski \& Lăvčiev 1971; Lavčiev \& Lateva 1973, 1976; Lavčiev et al. 1974, 1992; Mirčeva 1974, 1977, 1979, 1981; Lavčiev \& Jovčev 1978; Lavčiev \& Mirčeva 1980; Lavčiev \& Tsankova 1980, 1982; Lavčiev \& Zhekov 1980; Beron 1994, 2015; Kirin \& Buchvarov 1999.

Fannia difficilis (Stein, 1895) - B1; 450-620 m; 1; h; Lâvčiev 1970a, 2003.

Fannia fuscula (Fallén, 1825) - B1, B2, R4; 560-1350 m; 1, 2, 3; ho; Lavčiev 1966, 1974, 2003.

Fannia genualis (Stein, 1895) - B1, RW; 1100-1300 m; 3; h; trogloxene; Beron \& Guéorguiev 1967; Beron 1994, 2015.

Fannia incisurata (Zetterstedt, 1838) - $\mathbf{\Delta}$; E2, B1, B2, B3, V1, TL, O61, O62, R2, BN, BS; 0-2000 m; 1, 2, 3, 4; hn; Nedelkov 1912; Szilády 1934; Gregor \& Povolny 1959; Drensky 1960; Lavčiev 1964a, 1965c, 1965a, 1965d, 1969, 1970a, 1974, 1980, 2003; Lavčiev \& Nestorova 1967; Lavčiev \& Karastoyanov 1970; Stoicheva 1970; Beschovski \& Lăvčiev 1971; Smilova 1971; Lavčiev \& Lateva 1973, 1976, 1977; Lavčiev et al. 1973, 1974, 1981, 1992; Tahirov 1973; Mirčeva 1977, 1981; Lavčiev \& Jovčev 1978; Lavčiev \& Mirčeva 1980; Lavčiev \& Zhekov 1980; Lavčiev \& Tsankova 1982.

Fannia latipalpis (Stein, 1892) - B1, B2; 400-1500 m; 1, 2, 3; e; Lavčiev 1970a, 1974, 2003.

Fannia lepida (Wiedemann, 1817) [F. mutica (Zetterstedt, 1845)] - P1, P2, B1, R2; 500-2000 m; 1, 2, 3, 4; ho; Gregor \& Povolny 1959; Lavčiev 1965c, 1970a, 1974, 1980, 2003; Lavčiev \& Tahirov 1970.

Fannia lucidula (Zetterstedt, 1860) [F. glaucescens Zetterstedt, 1845] - DW, B1, B2, RW; 200-1300 m; 1, 2, 3; h; trogloxene; Beron \& Guéorguiev 1967; Lavčiev 1970a, 1974, 2003; Beron 1994, 2015.

Fannia leucosticta (Meigen, 1838) - E1, E2, P1, B1, B3, O62, BS; 0-600 m; 1; hpta, ? sk; Lavčiev 1965b, 1969, 1970a, 1974, 1980, 2003; Lavčiev \& Tahirov 1970; Lavčiev \& Tsankova 1980; Lavčiev \& Zhekov 1980; Lavčiev et al. 1981; Mirčeva 1981.

Fannia lineata (Stein, 1895) - BS; 0-10 m; 1; e; Lavčiev 1965d, 1969, 1974, 2003; Lavčiev \& Karastoyanov 1970; Lavčiev et al. 1974.

Fannia manicata (Meigen, 1826) [F. armillata (Zetterstedt, 1837); Amaurosoma] - B1, B3, V1, R2; 200-2000 m; 1, 2, 3, 4; ho; Nedelkov 1912; Lyubenov 1958; Gregor \& Povolny 1959; Lavčiev 1967, 1970a, 1974, 1980, 2003; Lavčiev \& Karastoyanov 1970; Lavčiev \& Tahirov 1970; Mirčeva 1981.

Fannia metallipennis (Zetterstedt, 1838) [F. kowarzi Verral, 1892] - P1, B1, B3; 300-800 m; 1, 2; h; Lavčiev 1965b, 1970a, 1974, 2003.

Fannia minutipalpis (Stein, 1895) - B3, BN; 180-1070 m; 1, 2, 3; ho; Lavčiev 1974, 2003. 
Fannia monilis (Haliday, 1838) - P2, B1, R2, BS; 0-1810 m; 1, 2, 3, 4; wcp; Gregor \& Povolny 1959; Lavčiev 1969, 1970a, 1974, 2003.

Fannia pallitibia (Rondani, 1866) - B2, R4; 400-1180 m; 1, 2, 3; e; Lavčiev 1966, 1970a, 2003.

Fannia parva (Stein, 1895) - BS; 0-10 m; 1; wes; Lavčiev 1969, 2003; Lavčiev \& Karastoyanov 1970.

Fannia polychaeta (Stein, 1895) - B1, BS; 0-1500 m; 1, 2, 3; e; Lavčiev 1969, 1970a, 2003.

Fannia postica (Stein, 1895) - B1; 850-1500 m; 2, 3; h; Lavčiev 1965b, 1970a, 2003.

Fannia rondanii (Strobl, 1893) - B1, B2; 750-2000 m; 2, 3, 4; h; Lavčiev 1965c, 1967, 1970a, 1974, 2003.

Fannia scalaris (Fabricius, 1794) - $\mathbf{\Delta}$; DW, E1, E2, B1, B3, K8, K9, V1, V4, TL, O61, O62, R1, R2, R5, RW, RE, BN, BS; 0-2700 m; 1, 2, 3, 4, 5, 6; k; trogloxene; Nedelkov 1912; Vesselinov \& Gabev 1956; Gregor \& Povolny 1959; Drensky 1960; Lavčiev 1964a, 1965a, 1965d, 1969, 1970a, 1974, 1980, 2003; Surbova 1965; Beron \& Guéorguiev 1967; Lavčiev \& Nestorova 1967; Lavčiev \& Karastoyanov 1970; Lavčiev \& Tahirov 1970; Beschovski \& Lăvčiev 1971; Lavčiev \& Lateva 1973, 1976, 1977; Lavčiev et al. 1974, 1981, 1983, 1984; Lavchiev \& Jovčev 1978; Mirčeva 1979, 1981; Lavčiev \& Mirčeva 1980; Lavčiev \& Zhekov 1980; Beron 1994, 2015; Kirin \& Buchvarov 1999.

Fannia serena (Fallén, 1825) - P1, P2, B1, B2; 200-1800 m; 1, 2, 3, 4; h; Lavčiev 1965b, 1965c, 1967, 1970a, 1974, 2003; Lavčiev \& Karastoyanov 1970; Mirčeva 1974, 1981.

Fannia sociella (Zetterstedt, 1845) - B2; 1240 m; 3; h; Lavčiev \& Karastoyanov 1970; Lavčiev 2003.

Fannia tuberculata (Zetterstedt, 1849) - B2; 1240 m; 3; h; Lavčiev 1974, 2003.

\section{Muscidae}

Achanthiptera rohrelliformis (Robineau-Desvoidy, 1830) - V1; 550-600 m; 1; dp, ? tp; Zielke 2018b, 2019a.

Muscina levida (Harris, 1780) [M. assimilis (Fallén, 1823)] - $\mathbf{\Delta}$; DW, E1, E2, P1, B1, B2, K8, K9, V1, V4, S1, S211, TL, T31, O61, O62, R1, R2, R5, RW, RE, BN, BS; 0-2500 m; 1, 2, 3, 4, 5, 6; h; Szilády 1934; Vesselinov \& Gabev 1956; Gregor \& Povolny 1959; Lavčiev 1964a, 1965a, 1965d, 1969, 1970a, 1972, 1974, 1980, 2003; Lavčiev \& Nestorova 1967; Lavčiev \& Tahirov 1970; Beschovski \& Lăvčiev 1971; Lavčiev \& Lateva 1973; Lavčiev et al. 1973, 1974, 1981, 1984; Lavčiev \& Jovčev 1978; Lavčiev \& Mirčeva 1980; Lavčiev \& Zhekov 1980; Mirčeva 1981; Zielke 2019a.

Muscina pascuorum (Meigen, 1826) - E2, B1, B2, V1, TL, R2, RW; 200-1990 m; 1, 2, 3, 4; ho; Nedelkov 1912; Lavčiev 1970a, 2003; Mirčeva 1974, 1981; Zielke 2019a.

Muscina prolapsa (Harris, 1780) [M. pabulorum (Fallén, 1817)] - \$; E2, P1, P3, B1, B3, V1, S1, T31, O61, O62, RW, RE, BN, BS; 0-1600 m; 1, 2, 3, 4; h, ? hat; Nedelkov 1912; Gregor \& Povolny 1959; Lavčiev 1964a, 1965d, 1968, 1970a, 1974, 2003; Lavčiev \& Nestorova 1967; Lavčiev \& Karastoyanov 1970; Lavčiev \& Tahirov 1970; Mirčeva 1974, 1981; Lavčiev \& Zhekov 1980; Zielke 2019a.

Muscina stabulans (Fallén, 1817) - \; ; DW, E1, E2, P1, P2, B1, B2, K8, K9, V1, V3, V4, S1, TL, T11, O61, O62, R1, R2, R5, RW, RE, BN, BS; 0-2500 m; 1, 2, 3, 4, 5, 6; k; Nedelkov 1912; Szilády 1934; Popoff 1941; Drensky 1955, 1960; Vesselinov \& Gabev 1956; Gregor \& Povolny 1959; Valerianov 1961; Lavčiev 1964a, 1965a, 1965d, 1969, 1970a, 1974, 1980, 2003; Surbova 1965; Lavčiev \& Nestorova 1967; Lavčiev \& Karastoyanov 1970; Lavčiev \& Tahirov 1970; Stoicheva 1970; Beschovski \& Lăvčiev 1971; Lavčiev \& Lateva 1973; Lavčiev et al. 1973, 1974, 1981, 1984; Mirčeva 1974, 1977, 1979, 1981; Lavčiev \& Jovčev 1978; Lavčiev \& Mirčeva 1980; Lavčiev \& Zhekov 1980; Kirin \& Buchvarov 1999; Zielke 2019a.

Azelia aterrima (Meigen, 1826) - S23; 350-450 m; 1; eca; Lavčiev \& Karastoyanov 1970; Lavchiev 2003; Zielke 2019a.

Azelia cilipes (Haliday, 1838) - E1, B1, V1, RW, BS; 0-1100 m; 1, 2, 3; h; Nedelkov 1912; Lăvčiev 2003; Beschovski 2006a; Zielke 2019a.

Azelia gibbera (Meigen, 1826) - ; h; Pont 1986, 2017; Zielke 2019a.

Azelia monodactyla Loew, 1874 - \$ e, ? dp; Pont 2017; Zielke 2019a.

Azelia nebulosa Robineau-Desvoidy, 1830 - E1, P2, B1, V4, R2; 400-1300 m; 1, 2, 3; e; Zielke 2019a.

Azelia triquetra (Wiedemann, 1817) - B1, V1, V4, S23; 500-1400 m; 1, 2, 3; h; Lăvčiev 1964b, 2003; Lavčiev \& Karastoyanov 1970; Zielke 2019a.

Thricops aculeipes (Zetterstedt, 1838) - V4, R1, R2; 1200-2400 m; 3, 4, 5; wes, bm; Kozuharova 1994; Lavčiev 2003; Kozuharova et al. 2005; Zielke 2019a.

Thricops beckeri (Pokorny, 1892) - R1; 1300-2400 m; 3, 4, 5; e; Zielke 2019a. 
Thricops bukowskii (Ringdahl, 1934) - B1, B2; 800-1200 m; 2, 3; seeani; Lavčiev 1970a, 1974, 2003; Zielke 2019a. Thricops culminum (Pokorny, 1889) - 1 ; e, ? cse; Pont 2017; Zielke 2019a.

Thricops cunctans (Meigen, 1826) [T. hirsutula (Zetterstedt, 1837); Rhynchotrichops subrostratus (Zetterstedt, 1845)] - P2, B2, V1, V4, T31, R1, R2, RW, RE; 70-2150 m; 1, 2, 3, 4; hoes, ? tp; Lavčiev 1964b, 1974, 2003; Kozuharova 1994; Kozuharova et al. 2005; Beschovski 2006a; Zielke 2019a.

Thricops diaphanus (Wiedemann, 1817) - R1; 1350 m; 3, 4; ho; Zielke 2019a.

Thricops furcatus (Stein, 1916) [Alloeostylus] - R2; 1200 m; 3; h, ? bm; Lavčiev \& Karastoyanov 1970; Lavčiev 2003; Zielke 2019a.

Thricops genarum (Zetterstedt, 1838) [Alloeostylus sundewalli (Zetterstedt, 1845)] - V4, R2, RW; 1230-1850 m; 3, 4; des, ? wces; Lavčiev 1965c, 2003; Lavčiev \& Karastoyanov 1970; Beschovski 2006a; Zielke 2019a.

Thricops innocuus (Zetterstedt, 1838) - B1, V4, RW; 620-1800 m; 2, 3, 4; h, ? bm; Lavčiev 1970a, 2003; Zielke 2019a.

Thricops longipes (Zetterstedt, 1845) - V4, R1, R2, RW; 230-2400 m; 1, 2, 3, 4, 5; hoes; Kozuharova 1994; Lavčiev 2003; Kozuharova et al. 2005; Beschovski 2006a; Zielke 2019a.

Thricops nigrifrons (Robineau-Desvoidy, 1830) [Hera; Trichopticus variabilis (Fallén, 1823)] - B1, B2, B3, V4, R1, R2, R4, RW, BS; 0-2400 m; 1, 2, 3, 4, 5; wesani; Nedelkov 1912; Lavčiev 1964b, 1966, 1974, 2003; Lavčiev \& Karastoyanov 1970; Beschovski 2006a; Zielke 2019a.

Thricops nigritellus (Zetterstedt, 1838) [Trichopticus] - B2, V4, R1, R2, RW; 600-2400 m; 2, 3, 4, 5; esan; Nedelkov 1912; Hennig 1961; Beron 1969; Lavčiev 1974, 2003; Kozuharova 1994; Kozuharova et al. 2005; Zielke 2019a.

Thricops semicinereus (Wiedemann, 1817) [Lasiops] - B1, B2, V4, R1, R2, RW, RE; 250-2000 m; 1, 2, 3, 4; wesanca; Lavčiev 1964b, 1970a, 2003; Kozuharova 1994; Kozuharova et al. 2005; Zielke 2019a.

Thricops simplex (Wiedemann, 1817) [Alloeostylus] - E1, E2, B1, B2, B3, V1, V4, R1, R2, R4, RW; 200-1990 m; 1, 2, 3, 4; wp; Gregor \& Povolny 1959; Lavčiev 1964b, 1966, 1970a, 1974, 2003; Lavčiev \& Tahirov 1970; Mirčeva 1981; Zielke 2019a.

Thricops sudeticus (Schnabl, 1888) [Alloeostylus] - B1, V4, R1, RW; 800-2250 m; 2, 3, 4, 5; ean; Lavčiev 1970a, 2003; Beschovski 2006a; Zielke 2019a.

Drymeia alpicola (Rondani, 1871) [Pogonomyia] - B1, B2, V4, RW; 700-2360 m; 2, 3, 4, 5; h; Lavčiev 1964b, 1970a, 1974, 2003; Beschovski 2006a; Zielke 2019a.

Drymeia cinerea (Meigen, 1826) [Eriphia; Pogonomyia] - RW; 1240-1700 m; 3, 4; e; Lavčiev 1964b, 2003; Beschovski 2006a; Zielke 2019a.

Drymeia fasciculata (Stein, 1916) [Pogonomyia] - V4, R1; 1500-2000 m; 3, 4; se, ? mm; Lavčiev 1964b, 2003; Zielke 2019a.

Drymeia hamata (Fallén, 1823) - B2, V4, R5; 850-2370 m; 2, 3, 4, 5; e; Lavčiev 1964a, 2003; Pont 1986, 2017; Zielke 2019a.

Drymeia vicana (Harris, 1780) [Pogonomyia decolor (Fallén, 1824)] - B1, B2, V4, R1, RW; 600-2400 m; 2, 3, 4, 5; esanca; Lavčiev 1964b, 1970a, 1974, 2003; Zielke 2019a.

Hydrotaea aenescens (Wiedemann, 1830) - B1; 465 m; 1; hna; Zielke $2018 \mathrm{~b}$.

Hydrotaea albipuncta (Zetterstedt, 1845) - B1, B2, RW; 600-2000 m; 2, 3, 4; tp; Lavčiev 1970a, 1974, 2003; Lavčiev et al. 1981.

Hydrotaea armipes (Fallén, 1825) [H. occulta (Meigen, 1826)] - \; ; E2, P1, B1, B2, R2, RW, BN, BS; 20-2200 m; 1, 2, 3, 4, 5; ho; Nedelkov 1912; Caspers 1951b; Gregor \& Povolny 1959; Lavčiev 1964a, 1965d, 1967, 1969, 1970a, 1972, 1974, 1980, 2003; Lavčiev \& Tahirov 1977; Mirčeva 1981; Beschovski 2006a.

Hydrotaea borussica Stein, 1899 - B1, B3, V4, RW; 800-2000 m; 2, 3, 4; ei; Lavčiev 1965b, 1970, 1974, 2003; Lavčiev \& Karastoyanov 1970; Beschovski 2006a.

Hydrotaea capensis (Wiedemann, 1818) [Ophyra anthrax (Meigen, 1826)] - E2, V1; 200-600 m; 1; ppt, i, sk; Lavčiev 1964a, 1965d, 2003; Surbova 1965; Lavčiev \& Nestorova 1967; Mirčeva 1981; Tsankova \& Lavčiev 1992; Lavčiev \& Tsankova 1994.

Hydrotaea cyrtoneurina (Zetterstedt, 1845) - E1, B1, V1, RW, BS; 0-1900 m; 1, 2, 3, 4; po; Lavčiev 1964b, 1969, 1970a, 1972, 1974, 2003; Lavčiev \& Nestorova 1967; Lavčiev \& Karastoyanov 1970; Beschovski \& Lăvčiev 1971; Mirčeva 1974; Lavčiev et al. 1974; Lavčiev \& Lateva 1977; Lavčiev \& Tahirov 1977; Beschovski $2006 a$.

Hydrotaea dentipes (Fabricius, 1805) - $\Delta$; E2, B1, V1, V4, R2, BN, BS; 0-2200 m; 1, 2, 3, 4, 5; ho, ? hno; Nedelkov 1912; Gregor \& Povolny 1959; Drensky 1960; Lavčiev 1964a, 1965a, 1965d, 1969, 1970a, 1974, 
1980, 2003; Lavčiev \& Nestorova 1967; Stoicheva 1970; Lavčiev \& Karastoyanov 1970; Lavčiev \& Tahirov 1970, 1977 ; Beschovski \& Lăvčiev 1971; Lavčiev et al. 1974, 1981; Lavčiev \& Lateva 1973, 1976; Mirčeva 1974, 1981; Lavčiev \& Jovčev 1978; Lavčiev \& Tsankova 1980; Lavčiev \& Zhekov 1980.

Hydrotaea floccosa Macquart, 1835 - ; ho; Pont 1986, 2017.

Hydrotaea glabricula (Fallén, 1825) - E2, B1, O61, R2; 150-900 m; 1, 2; hop; Gregor \& Povolny 1959; Lavčiev 1970a, 1974, 2003; Lavčiev \& Karastoyanov 1970; Mirčeva 1981.

Hydrotaea hennigi Pont, 1986 [H. spinigera Hennig, 1962] - B1; 550 m; 1; see; Lavčiev 1970a.

Hydrotaea hirticeps (Fallén, 1824) [H. bezzii Stein, 1899] - RW; 1000-1190 m; 3; e; Lavčiev 1964b, 2003; Beschovski 2006a.

Hydrotaea ignava (Harris, 1780) [H. leucostoma (Wiedemann, 1817); Ophyra] - \$ DW, E2, B1, V1, O61, O62, R5; BN, BS; 0-2000 m; 1, 2, 3, 4; ho, ? sk; Nedelkov 1912; Vesselinov \& Gabev 1956; Gregor \& Povolny 1959; Lavčiev 1964a, 1965a, 1965d, 1969, 1970a, 1974, 2003; Surbova 1965; Lavčiev \& Nestorova 1967; Lavčiev \& Karastoyanov 1970; Lavčiev \& Tahirov 1970, 1977; Stoicheva 1970; Lavčiev \& Lateva 1973; Lavčiev et al. 1974; Lavčiev \& Jelesova 1978; Lavchiev \& Jovčev 1978; Lavčiev \& Mirčeva 1980; Lavčiev \& Zhekov 1980; Lavčiev \& Tsankova 1982.

Hydrotaea irritans (Fallén, 1823) - \$; E1, E2, B1, V1, R1, R2, R5, BS; 0-2500 m; 1, 2, 3, 4, 5, 6; tp, ? po; Gregor \& Povolny 1959; Lavčiev 1964a, 1965a, 1965d, 1967, 1969, 1970a, 1972, 1974, 1980, 2003; Lavčiev \& Nestorova 1967; Mirčeva 1974; Lavčiev \& Tahirov 1977; Lavchiev \& Jovčev 1978; Lavčiev \& Zhekov 1980; Lavčiev et al. 1981, 1984.

Hydrotaea meridionalis Porchinskiy, 1882 - B2, B3, T11, R2, RE; 180-1700 m; 1, 2, 3, 4; wesan; Lavčiev 1964b, 1970a, 1974, 2003.

Hydrotaea meteorica (Linnaeus, 1758) - P1, B1, B3, V1, V4, R1, R2; 300-2000 m; 1, 2, 3, 4; ho; Nedelkov 1912; Gregor \& Povolny 1959; Lavčiev 1967, 1970a, 1972, 1974, 1980, 2003; Lavchiev \& Tsankova 1982; Lavčiev et al. 1984.

Hydrotaea militaris (Meigen, 1826) - B1, RW; 780-2000 m; 2, 3, 4; h; Lavčiev 1970a, 1974, 2003; Beschovski 2006a.

Hydrotaea palaestrica (Meigen, 1826) - B1, RW; 400-1960 m; 1, 2, 3, 4; h; Lavčiev 1965c, 1970a, 2003; Beschovski 2006a.

Hydrotaea pandellei Stein, 1899 - P1, B1, K4, V4, RW; 300-1960 m; 1, 2, 3, 4; tp; Lavčiev 1965c, 1967, 1970a, 1972, 1974, 2003; Lavchiev \& Zhekov 1980; Beschovski 2006a.

Hydrotaea parva Meade, 1889 - RW; 1600 m; 3, 4; eca; Lavčiev 2003; Beschovski 2006a.

Hydrotaea pellucens Porchinskiy, 1879 - RW; 1000-1200 m; 3, 4; wp, ? wes, bm; Lavčiev 2003; Beschovski 2006a.

Hydrotaea penicillata (Rondani, 1866) - B1, V1, R3, RW, RE, BS; 0-1900 m; 1, 2, 3, 4; ean; Lavčiev 1964b, 1967, 1969, 1972, 2003; Lavčiev \& Nestorova 1967; Lavčiev \& Karastoyanov 1970; Lavčiev et al. 1974; Lavčiev \& Jelesova 1978; Beschovski 2006a.

Hydrotaea pilipes Stein, 1903 - B1; 400 m; 1; h; Lavčiev 1965b, 1970a, 2003.

Hydrotaea similis Meade, 1887 - B2, V4, R1, R2, R4, RW; 500-2000 m; 1, 2, 3, 4; tp; Gregor \& Povolny 1959; Lavčiev 1966, 1970a, 1974, 2003; Lavčiev \& Nestorova 1967; Lavčiev \& Karastoyanov 1970; Beschovski 2006 .

Hydrotaea tuberculata Rondani, 1866 - P1, P2, B1, B2, R1; 700-2000 m; 2, 3, 4; h; Lavčiev 1967, 1970a, 1974, 2003; Lavčiev et al. 1984.

Hydrotaea velutina Robineau-Desvoidy, 1830 - DW, P1, B3, V1; 50-800 m; 1, 2; tp, ? hop; Nedelkov 1912; Lavčiev 1970a, 1974, 2003.

Potamia littoralis Robineau-Desvoidy, 1830 [Phaonia querceti (Bouché, 1834)] - B1, V4, R1; 1300-1800 m; 3, 4; ho; Lavčiev 1964b, 1966, 2003; Zielke 2019a.

Mesembrina intermedia Zetterstedt, 1849 - B1, S211, R1, RW; 700-1800 m; 2, 3, 4; tes; Lavčiev 1965c, 1966, 1970a, 2003.

Mesembrina meridiana (Linnaeus, 1758) [? Scopeuma] - B1, B2, B3, V1, V3, V4, R3, RW, BS; 0-2000 m; 1, 2, 3, 4; tp; Nedelkov 1909, 1912; Drensky 1960; Lavčiev 1966, 1967, 1970a, 1972, 1974, 2003; Lavčiev \& Nestorova 1967; Lavčiev \& Karastoyanov 1970; Lavčiev \& Tahirov 1977; Lavčiev \& Tsankova 1980; Beschovski $2006 a$.

Mesembrina mystacea (Linnaeus, 1758) - B1, RW; 1500-1900 m; 3, 4; esanca; Lavčiev 1970a, 2003; Beschovski 2006a.

Mesembrina resplendens Wahlberg, 1844 - R1, RW; 1300-2000 m; 3, 4; tes; Drensky 1939a; Lavčiev 1966, 2003; Beschovski 2006a. 
Polietes domitor (Harris, 1780) [P. albolineatus (Fallén, 1823)] - \$; E1, E2, P2, B1, B2, B3, S1, R5; 220-1600 m; 1, 2, 3; tp; Lavčiev 1964a, 1970a, 1974, 1980, 2003; Lavčiev \& Tahirov 1977; Mirčeva 1981.

Polietes lardarius (Fabricius, 1781) - * E1, B1, B2, V1, TL, T31, R1, RW, BS; 0-2000 m; 1, 2, 3, 4; pat; Lavčiev 1964b, 1969, 1070a, 1972, 1974, 1977, 1980, 2003; Lavčiev \& Nestorova 1967; Lavčiev \& Tahirov 1970; Stoicheva 1970; Lavchiev \& Jovčev 1978; Lavčiev \& Zhekov 1980; Lavčiev \& Tsankova 1982; Beschovski 2006a; Zielke 2016c.

Polietes meridionalis Peris \& Llorente, 1963 - E1, O62, RE; 90-600 m; 1; eanna; Lavčiev \& Tahirov 1977; Lavčiev \& Jelesova 1978; Lavčiev 2003; Zielke 2016c.

? Musca amita Hennig, 1964 [M. amica Zimin, 1951; ? = M. autumnalis De Geer, 1776] - DW, E1, E2, B1, K8, K9, V1, S1, TL, O61, O62, R1, R2, R5, RW, RE, BN, BS; 0-2500 m; 1, 2, 3, 4, 5, 6; esca, ? tp; Lavčiev 1964a, $1965 \mathrm{~d}$.

Musca autumnalis De Geer, 1776 [M. corvina Fabricius, 1781; ? M. amica Zimin, 1951] - \ ; ; DW, E1, E2, B1, K9, V1, V3, V4, S1, TL, O61, O62, R1, R2, R5, RW, RE, BN, BS; 0-2400 m; 1, 2, 3, 4, 5; ho, ? hpt; Nedelkov 1909, 1912; Szilády 1934; Drensky 1955, 1960; Vesselinov \& Gabev 1956; Gregor \& Povolny 1959; Lavčiev 1964a, 1965a, 1965d, 1967, 1969, 1970a, 1972, 1974, 1980, 2003; Lavčiev \& Nestorova 1967; Lavčiev \& Karastoyanov 1970; Beschovski \& Lăvčiev 1971; Jovčev \& Lavčiev 1972; Lavčiev \& Tahirov 1977; Lavčiev \& Jelesova 1978; Lavčiev \& Jovčev 1978; Mirčeva 1979; Lavčiev \& Zhekov 1980; Lavčiev et al. 1984.

Musca domestica Linnaeus, 1758 [M. domestica domestica Linnaeus, 1758; M. domestica vicina Macquart, 1843] - $\boldsymbol{\Delta}$; ; DW, DM, E1, E2, B1, B2, V1, V4, S22, TL, O61, O62, R1, R2, R5, RW, BN, BS; 0-2300 m; 1, 2, 3, 4, 5; k; trogloxene; Meunier 1897; Joakimoff 1899; Kovachev 1905; Nedelkov 1909, 1912; Drensky 1926; Czerný 1930; Szilády 1934; Popoff 1941; Drensky 1955, 1960; Surbova \& Lavčiev 1956; Vesselinov \& Gabev 1956; Gregor \& Povolny 1959; Kozarov et al. 1959; Surbova \& Avramov 1959; Valerianov 1961; Guéorguiev \& Beron 1962; Lavčiev 1964a, 1965a, 1969, 1970a, 1974, 1980, 2003; Surbova 1965; Lavčiev \& Nestorova 1967; Lavčiev \& Jelesova 1968, 1971; Lavčiev \& Karastoyanov 1970; Lavčiev \& Tahirov 1970; Stoicheva 1970; Tahirov 1970, 1973, 1977; Beschovski \& Lăvčiev 1971; Lavčiev \& Lateva 1973, 1976; Lavčiev et al. 1973, 1974, 1981; Mirčeva 1974, 1977, 1979, 1981; Lavčiev \& Jovčev 1978; Lavčiev \& Mirčeva 1980; Lavčiev \& Tsankova 1980, 1994; Lavčiev \& Zhekov 1980; Beron 1994, 2015; Kirin \& Buchvarov 1999; Beschovski 2006 .

Musca larvipara Porchinskiy, 1910 [M. convexifrons Thompson 1868; M. bezzii Patton \& Cragg, 1913] - $\mathbf{\Delta}$; E1, E2, V1, O61, O62, R2, R5; 20-1800 m; 1, 2, 3, 4; wcp; Gregor \& Povolny 1959; Lavčiev 1964a, 1965a, 1965d, 1967, 1970a, 1972, 1977, 1980, 2003; Lavčiev \& Karastoyanov 1970; Lavčiev \& Tahirov 1977; Lavčiev \& Jelesova 1978; Lavchiev \& Jovčev 1978.

Musca osiris Wiedemann, 1830 - E1, E2; 30-300 m; 1; wp; Lavčiev 1964a, 2003.

Musca sorbens Wiedemann, 1830 - DM, V1, BS; 0-600 m; 1; sppt; Vesselinov \& Gabev 1956.

Musca tempestiva Fallén, 1817 - E1, E2, B1, K9, V1, V4, R1, R2, BS; 0-1200 m; 1, 2, 3; ppt; Szilády 1934; Drensky 1960; Lavčiev 1964a, 1965a, 1965d, 1967, 1969, 1970a, 1972, 1974, 1980, 2003; Lavčiev \& Karastoyanov 1970; Stoicheva 1970; Beschovski \& Lăvčiev 1971; Lavčiev et al. 1974; Lavčiev \& Tahirov 1977; Lavčiev \& Jelesova 1978; Lavčiev \& Jovčev 1978; Lavčiev \& Zhekov 1980; Mirčeva 1981.

Musca vitripennis Meigen, 1826 - $\mathbf{\Delta}$; ; DW, E1, E2, B1, B2, B3, K8, K9, V1, S1, TL, O61, O62, R1, R2, R5, RW, RE, BN, BS; 0-2500 m; 1, 2, 3, 4, 5, 6; ppt; Nedelkov 1912; Szilády 1934; Lavčiev 1964a, 1965a, 1965d, 1967, 1969, 1970a, 1972, 1974, 1977, 1980, 2003; Lavčiev \& Nestorova 1967; Lavčiev \& Karastoyanov 1970; Stoicheva 1970; Beschovski \& Lăvčiev 1971; Jovčev \& Lavčiev 1972; Lavčiev \& Tahirov 1977; Lavčiev \& Jelesova 1978; Lavčiev \& Jovčev 1978; Mirčeva 1979, 1981; Kirin \& Buchvarov 1999.

Morellia aenescens Robineau-Desvoidy, 1830 - E1, B2, B3, S1, O61, O62, R1, R2; 150-1620 m; 1, 2, 3; tp; Lavčiev 1964a, 1974, 1980, 2003; Lavčiev et al. 1981; Lavčiev \& Tahirov 1977; Lavčiev \& Jovčev 1978.

Morellia hortorum (Fallén, 1817) - E1, P1, B1, B2, B3, R5, BS; 0-2000 m; 1, 2, 3, 4; po; Drensky 1960; Lavčiev 1964a, 1965a, 1965d, 1970a, 1974, 1980, 2003; Lavčiev \& Karastoyanov 1970; Lavčiev et al. 1974, 1981; Lavčiev \& Jovčev 1978; Lavčiev \& Zhekov 1980; Lavchiev \& Tsankova 1982.

Morellia podagrica (Loew, 1857) - B1, B2, O61, R1, R2, R5; 480-2000 m; 1, 2, 3, 4; h; Gregor \& Povolny 1959; Lavčiev 1964a, 1967, 1970a, 1974, 1980, 2003; Lavchiev \& Jovčev 1978.

Morellia simplex (Loew, 1857) - \$ DW, E1, E2, B1, B2, B3, V1, V4, TL, O61, R1, BS; 0-2200 m; 1, 2, 3, 4, 5; tp; Nedelkov 1912; Szilády 1934; Drensky 1960; Lavčiev 1964a, 1965a, 1965d, 1967, 1969, 1970a, 1972, 1974, 1980, 2003; Lavčiev \& Nestorova 1967; Lavčiev \& Karastoyanov 1970; Stoicheva 1970; Beschovski \& Lăvčiev 
1971; Jovčev \& Lavčiev 1972; Lavčiev \& Lateva 1973; Lavčiev et al. 1974, 1981; Lavčiev \& Tahirov 1977; Lavčiev \& Jelesova 1978; Lavčiev \& Jovčev 1978; Mirčeva 1979, 1981; Lavčiev \& Zhekov 1980.

Neomyia cornicina (Fabricius, 1781) [Cryptolucilia caesarion (Meigen, 1826); Orthellia] - ; E1, E2, B1, B2, B3, V1, S22, TL, O61, R1, R5, BN, BS; 0-2200 m; 1, 2, 3, 4, 5; sk; Nedelkov 1909, 1912; Szilády 1934; Lavčiev 1964a, 1965a, 1965d, 1967, 1969, 1970a, 1972, 1974, 1980, 2003; Lavčiev \& Nestorova 1967; Lavčiev \& Karastoyanov 1970; Lavčiev \& Tahirov 1970, 1977; Stoicheva 1970; Beschovski \& Lăvčiev 1971; Lavčiev \& Jelesova 1972, 1978; Lavčiev \& Lateva 1973; Mirčeva 1974, 1981; Lavčiev \& Jovčev 1978; Lavčiev \& Zhekov 1980; Lavčiev et al. 1984.

Neomyia viridescens (Robineau-Desvoidy, 1830) - t tp; Pont 1986, 2017.

Pyrellia rapax (Harris, 1780) [P. aenea (Zetterstedt, 1838); P. ignita Robineau-Desvoidy, 1830; Dasyphora serena (Meigen, 1826)] - \$; E1; 0-1400 m; 1, 2, 3; tp; Nedelkov 1912; Lavčiev \& Karastoyanov 1970; Lavčiev \& Tahirov 1977; Lavčiev 2003.

Pyrellia vivida Robineau-Desvoidy, 1830 [P. cadaverina] - \; ; DW, E1, E2, B1, K8, V1, V4, S1, TL, O61, O62, R2, R5, RW, RE, BN, BS; 0-2500 m; 1, 2, 3, 4, 5, 6; hno, po; Nedelkov 1909, 1912; Szilády 1934; Gregor \& Povolny 1959; Lavčiev 1964a, 1965a, 1965d, 1969, 1970a, 1972, 1974, 1980, 2003; Lavčiev \& Nestorova 1967; Lavčiev \& Karastoyanov 1970; Lavčiev \& Tahirov 1970, 1977; Beschovski \& Lăvčiev 1971; Lavčiev et al. 1974; Mirčeva 1974, 1981; Lavčiev \& Lateva 1976; Lavčiev \& Jovčev 1978.

Eudasyphora cyanella (Meigen, 1826) [Dasyphora eriophthalma (Macquart, 1834)] - E1, P1, B1, BS; 0-1500 m; 1, 2, 3; wpat; Lavčiev 1969, 1970a, 2003; Lavčiev et al. 1974; Lavčiev \& Tahirov 1977.

Eudasyphora cyanicolor (Zetterstedt, 1845) - B1, B2, B3, RW; 600-1400 m; 2, 3; hptn, ? ho; Lavčiev 1965c, 1970a, 1974, 2003.

Eudasyphora zimini (Hennig, 1963) - B1, RW; 750-1000 m; 2; e; Lavčiev 1974, 2003; Beschovski $2006 a$.

Dasyphora albofasciata (Macquart, 1839) [D. saltum Rondani, 1862] - \$; B1, B2, B3, TL, BN, BS; 0-1500 m; 1, 2, 3; swp; Szilády 1934; Gregor \& Povolny 1959; Lavčiev 1967, 1969, 1970a, 1972, 1974, 1980, 2003; Lavčiev \& Karastoyanov 1970; Stoicheva 1970; Beschovski \& Lăvčiev 1971; Lavčiev \& Jelesova 1978; Lavčiev \& Jovčev 1978.

Dasyphora gussakovskii Zimin, 1947 - R1; 1800-2200 m; 4, 5; po; Lavčiev 1964a, 2003.

Dasyphora penicillata (Egger, 1865) [D. versicolor (Meigen, 1826)] - DW, E1, E2, B1, B2, K8, K9, V1, V4, S1, TL, O61, O62, R1, R2, R5, RW, RE, BS; 0-2400 m; 1, 2, 3, 4, 5; wp, ? mm; Nedelkov 1912; Gregor \& Povolny 1959; Drensky 1960; Lavčiev 1964a, 1970a, 1974, 2003; Lavčiev \& Nestorova 1967.

Dasyphora pratorum (Meigen, 1826) - $\$$ DW, E1, E2, B1, B2, B3, K8, K9, V1, V4, S1, TL, O61, R1, R2, R5, RW, BN, BS; 0-2400 m, 1, 2, 3, 4, 5; wp; Nedelkov 1909, 1912; Szilády 1934; Gregor \& Povolny 1959; Lavčiev 1964a, 1967, 1969, 1970a, 1972, 1974, 1980, 2003; Lavčiev \& Nestorova 1967; Lavčiev \& Karastoyanov 1970; Stoicheva 1970; Lavčiev \& Lateva 1976; Lavčiev \& Jelesova 1978; Lavčiev \& Jovčev 1978; Lavčiev et al. 1981, 1984; Mirčeva 1981; Lavchiev \& Tsankova 1982; Beschovski 2006a.

Dasyphora similis Zimin, 1951 - E2; 200-300 m; 1; seewca; Mirčeva 1981; Lavchiev 2003.

Stomoxys calcitrans (Linnaeus, 1758) - $\mathbf{\Delta}$; DM, E2, P2, B2, S22, TL, BN, BS; 0-1600 m; 1, 2, 3; k; Nedelkov 1909, 1910, 1912; Drensky 1934, 1955, 1957a, 1960; Szilády 1934; Popoff 1941; Valerianov 1961; Lavčiev 1965a, 1965d, 1967, 1980, 2003; Surbova 1965; Lavčiev \& Nestorova 1967; Stoicheva 1970; Lavčiev et al. 1974; Lavčiev \& Lateva 1973; Lavčiev \& Jovčev 1978; Mirčeva 1979, 1981; Darlenski et al. 2020.

Haematobia irritans (Linnaeus, 1758) [Lyperosia, Siphona] - E2, TL, R1;30-1700 m; 1, 2, 3, 4; hn, ? hna; Szilády 1934; Drensky 1957a; Lavčiev 1967, 1972, 1980, 2003; Lavčiev \& Nestorova 1967; Lavčiev \& Tahirov 1977; Lavčiev \& Jelesova 1978; Lavčiev \& Jovčev 1978; Lavčiev \& Zhekov 1980.

Haematobia titillans (Bezzi, 1907) - E1, S1, TL, BS; 0-800 m; 1, 2; po; Szilády 1934; Drensky 1957a, 1960; Lavčiev 1967, 1980, 2003; Lavčiev \& Tahirov 1977.

Haematobosca atripalpis (Bezzi, 1895) [Haematobia, Lyperosia, Siphona] - ४; E2, BN; 0-2000 m; 1, 2, 3, 4; eanca; Szilády 1934; Lavčiev 1967, 1972, 1980, 2003; Mirčeva 1981.

Haematobosca stimulans (Meigen, 1824) [Haematobia, Siphona] - \; * E1, SB, BS; 0-1900 m; 1, 2, 3, 4; po; Szilády 1934; Drensky 1955, 1957a, 1960; Lavčiev 1967, 1970a, 1972, 1980, 2003; Lavčiev \& Tahirov 1977; Lavčiev \& Jovčev 1978; Lavčiev \& Tsankova 1982.

Eginia ocypterata (Meigen, 1826) - TL; $60 \mathrm{~m}$; 1; e; Zielke 2018a.

Atherigona varia (Meigen, 1826) - P2, B2, BN, BS; 0-1000 m; 1, 2; tp; Beschovski \& Lăvčiev 1971; Lavčiev 2003; Zielke 2018a. 
Phaonia alpicola (Zetterstedt, 1845) - S22, RW; 1500-1590 m; 3, 4; h; Lavčiev 2003; Beschovski 2006a; Zielke $2016 \mathrm{a}$.

Phaonia angelicae (Scopoli, 1763) [Ph. basalis (Zetterstedt, 1837)] - P2, B1, B2, V1, V4, R1, RW; 500-1800 m; 1, 2, 3, 4; tp; Lavčiev 1964b, 1965c, 1966, 1970a, 1974, 2003; Beschovski 2006a; Zielke 2016a, 2018a.

Phaonia bitincta (Rondani, 1866) - B1, B2, S1, R1, RW; 500-1800 m; 1, 2, 3, 4; e; Lavčiev 1964b, 1970a, 1974, 2003; Lavčiev \& Karastoyanov 1970; Lavčiev et al. 1984; Beschovski 2006a.

Phaonia boleticola (Rondani, 1866) - E1, B2; 450-800 m; 1, 2; e; Lavčiev 1974, 2003.

Phaonia consobrina (Zetterstedt, 1838) [Midaea marmorata (Zetterstedt, 1860)] - RW; 1100-1200 m; 3; h; Nedelkov 1912; Lavčiev 2003; Beschovski 2006a.

Phaonia errans (Meigen, 1826) [Ph. erratica (Fallén, 1825); Ph. tinctipennis (Rondani, 1866); Aricia] - P1, B1, B3, V1, V4, TL, R2, R3, RW, BN, BS; 0-1450 m; 1, 2, 3, 4; h; Nedelkov 1912; Drenowsky 1939; Drensky 1939a, 1942; Lavčiev 1964b, 1969, 1970a, 1974, 2003; Lavčiev \& Karastoyanov 1970; Beschovski 2006a; Zielke 2016a, 2018a.

Phaonia erronea (Schnabl, 1887) - B1, RW; 780-800 m; 2; e; Lavčiev 1964b, 1970a, 1974, 2003.

Phaonia exoleta (Meigen, 1826) - B1, RW; 770-1440 m; 2, 3; ewca; Lavčiev \& Karastoyanov 1970; Lavčiev 2003; Zielke 2016a.

Phaonia falleni Michelsen, 1977 [Ph. vagans (Fallén, 1825)] - V1, V4, TL, RW; 200-1900 m; 1, 2, 3, 4; hoes; Nedelkov 1912; Lavčiev 2003; Beschovski 2006a.

Phaonia fuscata Fallén, 1825 - P2, B2, B3, V1, R2, R5, RW; 300-2200 m; 1, 2, 3, 4; po; Lavčiev 1964a, 1974, 2003; Beschovski 2006a; Zielke 2016a.

Phaonia gobertii (Mik, 1881) - E2; 230-300 m; 1; dp; Lavčiev et al. 1981; Lavčiev 2003.

Phaonia hybrida (Schnabl, 1888) - B2, V4, R1; 1800-2900 m; 4, 5, 6; h, bm; Pont 1986, 2017; Zielke 2016 a.

Phaonia impura Zinoviev, 1987 - BS; 0-5 m; 1; e; Zielke 2016a.

Phaonia incana (Wiedemann, 1817) - V1, V4, RW; 700-2000 m; 2, 3, 4; po; Nedelkov 1912; Lavčiev 2003; Beschovski 2006a; Zielke 2016a.

Phaonia laeta (Fallén, 1823) - RE; 430 m; 1; ? wp; Lavčiev 2003; Zielke 2016a.

Phaonia lavcievi Zielke 2016 - RE; 270 m; 1; Ebg; Zielke 2016a.

Phaonia lugubris (Meigen, 1826) [Ph. morio (Zetterstedt, 1845)] - B1, B2, V1, V4, S1, S21, TL, R1, RW; 230 2100 m; 1, 2, 3, 4; h, ? bm; Nedelkov 1912; Lavčiev 1966, 1970a, 1974, 2003; Beschovski 2006a.

Phaonia mediterranea Hennig, 1963 - P1, B1, O61, O62, RE; 200-1800 m; 1, 2, 3, 4; ena, ? wp; Lavčiev 1970a, 2003; Zielke 2016a, 2018a.

Phaonia meigeni Pont, 1986 - B1, V4, RW; 770-2100 m; 2, 3, 4, 5; tes; Zielke 2016a, 2018a.

Phaonia mystica (Meigen, 1826) - RW; 2000 m; 4; e; Lavčiev 1964b, 1965a, 2003; Beschovski 2006a.

Phaonia pallida (Fabricius, 1787) - E1, P1, P2, B1, B2, B3, V1, V3, V4, S211, S22, TL, RW, RE, BN, BS; 0-1630 m; 1, 2, 3, 4; wp; Nedelkov 1912; Lavčiev 1965a, 1969, 1970a, 1972, 1974, 1980, 2003; Lavčiev \& Nestorova 1967; Lavčiev \& Karastoyanov 1970; Lavčiev \& Tahirov 1970; Beschovski \& Lăvčiev 1971; Lăvčiev et al. 1974; Lavčiev \& Jovčev 1978; Lavčiev \& Zhekov 1980; Mirčeva 1981; Zielke 2016a, 2018a.

Phaonia palpata (Stein, 1897) - P2, B1, B3, V1; 500-800 m; 1, 2; eo; Lavčiev 1964b, 1970a, 1974, 2003; Zielke 2016a.

Phaonia perdita (Meigen, 1830) - V1, V3, R2, RW; 700-1600 m; 2, 3, 4; wp; Lavčiev \& Karastoyanov 1970; Lăvčiev 2003; Zielke 2016a, 2018a.

Phaonia pratensis (Robineau-Desvoidy, 1830) [Ph. laeta (Fallén, 1823)] - B3, RE; 430-800 m; 1, 2; e; Lavčiev 1965c, 1970a, 1974, 2003; Zielke 2016a, 2018a.

Phaonia profugax (Pandelle, 1899) - RW; 1000-1050 m; 3; tes; Zielke 2018a.

Phaonia pura (Loew, 1873) [Ph. candicans (Pandellé, 1898)] - B1, R1, R2, RW; 530-1550 m; 1, 2, 3, 4; cse, ? e; Lavčiev 1964b, 19656, 1970a, 1974, 2003; Beschovski 2006a; Zielke 2016a.

Phaonia regalis (Stein, 1900) - P1, B3, R1, RE; 180-800 m; 1, 2; cseean; Lavčiev 1974, 2003; Zielke 2016a, 2018 a.

Phaonia rufipalpis (Macquart, 1835) - O62, BS; 0-300 m; 1; wp; Lavčiev 1969, 2003; Zielke 2016a.

Phaonia rufiventris (Scopoli, 1763) [Ph. populi (Meigen, 1826)] - B1, B2, B3, S1, R1, RW; 300-1900 m; 1, 2, 3, 4; wp; Lavčiev 1970a, 1974, 2003; Lavčiev et al. 1984; Georgiev et al. 1998; Zielke 2016a.

Phaonia sandanskii Zielke, 2017 - O62; 260 m; 1; Ebg; Zielke 2017b, 2018a.

Phaonia scutellata (Zetterstedt, 1845) - B1, B3, S1, R1, R2, RW, BS; 0-1800 m; 1, 2, 3, 4; ena; Lavčiev 1969, 1970a, 2003; Lavčiev \& Karastoyanov 1970; Zielke 2016a, 2018 a. 
Phaonia serva (Meigen, 1826) - P1, B1, B2, V4, R1, R2, R4, R5, RW; 400-2000 m; 1, 2, 3, 4; h; Lavčiev 1964a, 1966, 1970a, 1974, 2003; Lavčiev \& Karastoyanov 1970; Zielke 2016a, 2018 a.

Phaonia siebecki Schnabl, 1911 - B1, B2; 480-800 m; 1, 2; e; Lavčiev 1970a, 1974, 2003; Zielke 2016a.

Phaonia subventa (Harris, 1780) [Ph. variegata (Meigen, 1826)] - E1, B1, B2, B3, K4, V1, V3, V4, S1, TL, O62, R2, R4, RW, BS; 0-1900 m; 1, 2, 3, 4; ena; Lavčiev 1964b, 1969, 1970a, 1974, 2003; Lavčiev \& Nestorova 1967; Lavčiev \& Karastoyanov 1970; Lavčiev \& Zhekov 1980; Beschovski 2006a; Zielke 2016a, 2018a.

Phaonia tiefii (Schnabl, 1888) - P2, B2, R1, RW; 700-2390 m; 2, 3, 4, 5; ? e; Lavčiev 1974, 2003; Zielke 2016a, 2018a.

Phaonia trimaculata (Bouché, 1834) - P2, V1, V3, R1, RW; 550-1700 m; 1, 2, 3, 4; ? wp; Lavčiev 2003; Beschovski 2006a; Zielke 2016a, 2018a.

Phaonia tuguriorum (Scopoli, 1763) [Ph. signata (Meigen, 1826)] - P1, B1, B2, V1, V3, TL, R2, RE; 160-1200 m; 1, 2, 3; wcp; Nedelkov 1912; Lavčiev 1970a, 1974, 2003; Lavčiev \& Karastoyanov 1970; Zielke 2016a, 2018 a.

Phaonia valida (Harris, 1780) - E1, P1, B1, V1, V4, S22, TL, O62, R1, RE, BN; 0-1270 m; 1, 2, 3; wp, ? eanna; Pont, 1986, 2013; Zielke 2016a, 2018a.

Phaonia villana Robineau-Desvoidy, 1830 - d dpo; Pont, 1986, 2013.

Phaonia zugmayeriae (Schnabl, 1888) - R1, RW; 1300-1950 m; 3, 4; des; Zielke 2016a, 2018a.

Helina allotalla (Meigen, 1830) - B1, V4, RW; 1200-1630 m; 3, 4; e; Lavčiev 1965c, 1970a, 2003; Beschovski 2006a; Zielke 2018a.

Helina annosa (Zetterstedt, 1838) [H. multisetosa (Strobl, 1898)] - B1, B2, B3, V1, TL, R1, RW; 200-2100 m; 1, 2, 3, 4; h, ? ho; Lavčiev 1964b, 1965c, 1970a, 1974, 2003; Lavčiev et al. 1984; Zielke 2018a.

Helina arctata Collin, 1953 - V1, RW; 530-1800 m; 1, 4; wes; Pont 1986; Zielke 2018a.

Helina atricolor (Fallén, 1825) - R1, RW; 1300-1750 m; 3, 4; e; Zielke 2018a.

Helina ciliatocosta (Zetterstedt, 1845) [Enoplopteryx] - E1, B1, B2, V1, R1, RW; 200-1800 m; 1, 2, 3, 4; e; Lavčiev 1964b, 1970a, 1974, 2003; Lavčiev \& Tahirov 1977; Zielke 2018a.

Helina cilipes (Schnabl, 1902) - B3, RW; 600-1400 m; 1, 2, 3; e; Lavčiev 1974; Beschovski 2006a; Zielke 2018a.

Helina cinerella (van der Wulp, 1867) [H. vanderwulpi (Schnabl, 1888); H. tuleskovi Lavčiev 1968] - B1, V5, R1, RW; 380-2400 m; 1, 2, 3, 4, 5; h; Lavčiev 1965c, 1968, 1970a, 1974, 2003; Beschovski 2006a; Zielke 2018a.

Helina concolor (Czerny, 1900) - B1, B3, RW; 600-1500 m; 2, 3, 4; e; Lavčiev 1970a, 1974, 2003; Zielke 2018a.

Helina confinis (Fallén, 1825) [H. anceps (Zetterstedt, 1837); Mydaea (Spilogaster)] - E2, P1, B1, B3, S211, R2, R4, RW, RE, BN; 170-1800 m; 1, 2, 3, 4; des, ? wces; Lavčiev 1964b, 1966, 1970a, 1974, 2003; Lavčiev \& Karastoyanov 1970; Mirčeva 1974, 1981; Beschovski 2006a; Zielke 2018a.

Helina dabovetsa Zielke, 2017 - RE; 180-190 m; 1; Ebg; Zielke 2017b, 2018a.

Helina decipiens Mihalyi, 1974 - P3, B3, V1, V5, RW; 370-1020 m; 1, 2, 3; esan, ? wces; Zielke 2018a.

Helina deleta (Stein, 1914) - B2, RW; 1000-1600 m; 3, 4; des; Zielke $2018 \mathrm{a}$.

Helina depuncta (Fallén, 1825) - E1, E2, B3, V1, R1, R2, RW; 30-1500 m; 1, 2, 3, 4; tes; Gregor \& Povolny 1959; Lavčiev 1964a, 1965d, 1966, 1974, 2003; Lavčiev \& Lateva 1973, 1976; Lavčiev et al. 1984; Zielke 2018a.

Helina evecta (Harris, 1780) [H. laetifica (Robineau-Desvoidy, 1830); H. lucorum (Fallén, 1823); E1, P2, B1, B2, V1, V4, S1, S23, TL, R1, R2, R3, R4, RW, BS; 0-2390 m; 1, 2, 3, 4, 5; hptn; Nedelkov 1912; Lavčiev 1966, 1970a, 1974, 2003; Stoicheva 1970; Lavčiev \& Jelesova 1978; Lavčiev et al. 1984; Beschovski 2006a; Zielke 2018a.

Helina fratercula (Zetterstedt, 1845) - B2, R2, RW; 900-1400 m; 2, 3; e; Lavčiev \& Karastoyanov 1970; Lavčiev 1974, 2003; Beschovski 2006a; Zielke 2018a.

Helina impuncta (Fallén, 1825) - E1, B2, V1, O62, RW, RE; 250-1000 m; 1, 2; tp; Lavčiev 1974, 2003; Beschovski 2006a; Zielke 2018a.

Helina interfusa (Pandellé, 1899) - P1, B1, TL; 200-400 m; 1; e; Lavčiev 1970a, 2003; Stoicheva 1970; Zielke 2018a, 2018c.

Helina intermedia (Villeneuve, 1899) - B1, RW; 1270-1550 m; 3, 4; e; Zielke 2018a.

Helina lasiophthalma (Macquart, 1835) - B1, B2, B3, V1, V3, V4, TL, T31, R1, R3, RE; 60-1300 m; 1, 2, 3; ean; Lavčiev 1965b, 1970a, 1974, 2003; Zielke 2018a.

Helina latitarsis Ringdahl, 1924 - DW, B1, B2, B3, R1, R4, RW; 50-1900 m; 1, 2, 3, 4; esanca; Lavčiev 1966, 1970a, 1974, 2003; Lavčiev \& Karastoyanov 1970; Kozuharova 1994; Zielke 2018 a.

Helina laxifrons (Zetterstedt, 1860) - B1, V1, V4, R2, R4, BS; 0-1800 m; 1, 2, 3, 4; h; Lavčiev 1969, 2003; Lavčiev \& Karastoyanov 1970; Zielke 2018a. 
Helina maculipennis (Zetterstedt, 1845) - B3; 630-700 m; 1, 2; h; Zielke 2018a.

Helina moedlingensis (Schnabl, 1911) - B1, B2, V5, R1, R2, RW; 600-2400 m; 1, 2, 3, 4, 5; ho; Lavčiev 1965c, 1970a, 1974, 2003; Beschovski 2006a; Zielke 2018a.

Helina momchili Zielke, 2016 - RE; 450 m; 1; Ebg; Zielke 2016d, 2018a.

Helina montana (Rondani, 1866) - V1, V4, R1, RW; 500-1500 m; 1, 2, 3; csei; Nedelkov 1912; Lavčiev 1966, 2003; Beschovski 2006a; Zielke 2018a.

Helina obscurata (Meigen, 1826) - B1, B2, V4, R1, RW; 420-2000 m; 1, 2, 3, 4; h; Lavčiev 1965c, 1966, 1970a, 1974, 2003; Lavčiev \& Karastoyanov 1970; Lavčiev et al. 1984; Beschovski 2006a; Zielke 2018a.

Helina obscuratoides (Schnabl, 1887) - B1; 1300-1600 m; 3, 4; h; Lavčiev 1965b, 2003; Zielke 2018a.

Helina obtusipennis (Fallén, 1823) - B3, RW; 600-1850 m; 2, 3, 4; ean; Lavčiev 1965c, 1974, 2003; Beschovski 2006a; Zielke 2018a.

Helina pandellei (Villeneuve, 1922) - RW; 1270-1400 m; 3; e; Lavčiev 1965b, 2003; Beschovski 2006a; Zielke 2018a.

Helina parcepilosa (Stein, 1907) [H. calceata Schnabl, 1911; Mydaea (Spilogaster)] - B1, B3, V1, V4, R4, RE, BN, BS; 0-1700 m; 1, 2, 3, 4; wcp; Lavčiev 1964b, 1966, 1970a, 1974, 2003; Beschovski \& Lăvčiev 1971; Lavčiev et al. 1974; Zielke 2018a.

Helina protuberans (Zetterstedt, 1845) - RW; 1380 m; 3; ? e; Zielke $2018 \mathrm{a}$.

Helina pubescens (Stein, 1893) - V4, S22, O62, R4, RW, RE; 260-1960 m; 1, 2, 3, 4; ean; Lavčiev 1966, 2003; Beschovski 2006a; Zielke 2018a.

Helina pubiseta (Zetterstedt, 1845) - P1, B1, R1, RW; 320-1900 m; 1, 2, 3, 4; e; Lavčiev 1966, 1970a, 2003; Lavčiev \& Karastoyanov 1970; Beschovski 2006a; Zielke 2018a.

Helina quadrinotata (Meigen, 1826) - O62, RW; 260-1900 m; 1, 3, 4; e; Lavčiev 2003; Beschovski 2006a; Zielke 2018a.

Helina quadrum (Fabricius, 1805) - V1, RW, RE; 75-1800 m; 1, 2, 3, 4; tp; Nedelkov 1912; Lavčiev 1965c, 2003; Beschovski 2006a; Zielke 2018a.

Helina reversio (Harris, 1780) [H. duplicata (Meigen, 1826); H. prospinosa (Pandellé, 1898); Mydaea (Spilogaster)] - P1, P2, B1, B2, B3, V1, V4, V5, S1, S211, TL, T1, O61, R1, R2, R3, R4, RW, RE, BS; 0-2230 m; 1, 2, 3, 4, 5; ho; Nedelkov 1912; Gregor \& Povolny 1959; Lavčiev 1966, 1969, 1970a, 1974, 2003; Lavčiev \& Nestorova 1967; Mirčeva 1974, 1981; Lavčiev \& Jelesova 1978; Lavčiev \& Zhekov 1980; Zielke $2018 \mathrm{a}$.

Helina richardi Pont, 2012 - R4; 1240 m; 3; sena, ? mm; Zielke 2018a.

Helina rilae Zielke, 2017 - R1; 1800 m; 4; Ebg; Zielke 2017b, 2018a.

Helina setiventris Ringdahl, 1924 - B1, V1, RW; 350- 1700 m; 1, 2, 3, 4; wesant; Lavchiev 1965c, 1970a, 2003; Beschovski 2006a; Zielke 2018a.

Helina sexmaculata (Preyssler, 1791) [H. punctata (Robineau-Desvoidy, 1830); H. uliginosa (Fallén, 1825)] - E1, B1, B2, V1, S1, O62, RE, BN; 0-1100 m; 1, 2, 3; hoa; Nedelkov 1912; Lavčiev 1966, 1974, 2003; Lavčiev \& Tahirov 1977; Lavčiev \& Jelesova 1978; Zielke 2018a.

Helina siutkae Zielke, 2017 - RW; 1700-1800 m; 4; Ebg; Zielke 2017b, $2018 \mathrm{a}$.

Helina spinicosta (Zetterstedt, 1845) - B2, S1, RW; 360-1400 m; 1, 2, 3; h; Lavčiev 1974, 2003; Beschovski 2006a; Zielke 2018a.

Helina subvittata (Seguy, 1923) [H. rothi Ringdahl, 1939] - B2, R1, RW; 1000-1400 m; 2, 3; h; Lavčiev 1974, 2003; Beschovski 2006a; Zielke 2018a.

Helina syracusana Hennig, 1957 - B1, RW; 1400-1960 m; 3, 4; ? hom, ? mm; Zielke 2018a, 1919b.

Helina tetrastigma (Meigen, 1826) - B1, O62, BS; 0-1000 m; 1, 2; eswa; Gregor \& Povolny 1959; Lavčiev 1969, 2003; Zielke 2018a.

Helina trivittata (Zetterstedt, 1860) [H. atripes (Meade, 1889)] - P1, B1, RW; 500-1400 m; 1, 2, 3; wces; Lavčiev 1965c, 1970a, 2003; Beschovski 2006a; Zielke 2018a.

Gymnodia eremophila (Brauer \& Bergenstamm, 1894) [G. impedita Pandellé, 1898; Brontaea] - V1, RW, BS; 0-1560 m; 1, 2, 3, 4; wpat; Lavčiev 1967, 1969, 1972, 1980, 2003; Lavčiev et al. 1974; Lavčiev \& Jelesova 1978; Lavčiev \& Jovčev 1978; Beschovski 2006a; Zielke 2016b.

Gymnodia genurufa (Pandellé, 1899) [Brontaea] - sena; Pont, 1986, 2013; Zielke 2016b.

Gymnodia humilis (Zetterstedt, 1860) - B1, R1; 520-1350 m; 1, 2, 3; h; Zielke 2016b.

Gymnodia polystigma (Meigen, 1826) - B3; 630 m; 1, 2; ? ess, ? sess; Zielke $2016 \mathrm{~b}$. 
Mydaea ancilla (Meigen, 1826) [Spilogaster bicincta (Villeneuvé, 1899)] - E1, P2, B1, B2, B3, V1, V3, S1, R1, R4, RW, RE, BS; 0-1800 m; 1, 2, 3, 4; tp; Lavčiev 1966, 1969, 1970a, 1974, 2003; Lavčiev \& Karastoyanov 1970; Beschovski \& Lăvčiev 1971; Lăvčiev et al. 1974; Lavčiev \& Tahirov 1977; Zielke 2016b.

Mydaea anicula (Zetterstedt, 1860) - P1, B1, R1, R4, RW, BS; 0-1800 m; 1, 2, 3, 4; wes; Lavčiev 1966, 1969, 1970a, 2003; Lavčiev \& Karastoyanov 1970; Lăvčiev et al. 1974; Lavčiev \& Tahirov 1977; Zielke 2016b.

Mydaea corni (Scopoli, 1763) [M. pagana (Fabricius, 1794); M. scutellaris (Robineau-Desvoidy, 1830)] - E1, E2, B1, B2, V4, S1, O62, R1, R2, RW, BS; 0-1900 m; 1, 2, 3, 4; tp, ? hop; Lavčiev 1964a, 1970a, 1974, 2003; Lavčiev \& Nestorova 1967; Lavčiev \& Karastoyanov 1970; Lavčiev \& Tahirov 1977; Lavčiev \& Jovčev 1978; Lavčiev \& Zhekov 1980; Mirčeva 1981; Lavchiev et al. 1984; Zielke 2016b.

Mydaea deserta (Zetterstedt, 1845) - R4; 1240 m; 3; ? e; Lavchiev 1966.

Mydaea detrita (Zetterstedt, 1845) [M. electa (Zetterstedt, 1860)] - P1, B1, B2, B3, R2, R4, RW, BS; 0-1200 m; 1, 2, 3; h; Lavčiev 1969, 1970a, 1974, 2003; Lavčiev \& Karastoyanov 1970; Beschovski \& Lăvčiev 1971; Lavčiev et al. 1974; Zielke 2016b.

Mydaea humeralis Robineau-Desvoidy, 1830 [M. tincta (Zetterstedt, 1845)] - B1, B2, B3, V1, V3, S1, TL, R2; 300-1000 m; 1, 2; tp, ? esca; Lavčiev 1964b, 1970a, 1974, 2003; Lavčiev \& Karastoyanov 1970; Stoicheva 1970; Zielke 2016b.

Mydaea lateritia (Rondani, 1866) - R4, BS; 0-1240 m; 1, 2, 3; eswa; Lavčiev 1969, 2003; Zielke $2016 \mathrm{~b}$.

Mydaea maculiventris (Zetterstedt, 1846) [M. spinipes Karl, 1929] - B1, B2; 700-800 m; 2; e; Zielke $2016 \mathrm{~b}$.

Mydaea nebulosa (Stein, 1893) - P2, B1, B2, B3, V4; 470-2000 m; 1, 2, 3, 4; des; Lavčiev 1965c, 1970a, 1974, 2003; Zielke 2016b.

Mydaea nebulosa (Stein, 1893) - P1, B1, TL; 230-500 m; 1; h; Lavčiev 1965c, 1970a, 2003; Zielke 2016b.

Mydaea orthonevra (Macquart, 1835) [M. detrita (Zetterstedt, 1845)] - P1, B1, B3, S1, RW, BS; 0-1600 m; 1, 2, 3, 4; hoes; Lavčiev 1969, 1970a, 1974, 2003; Lavčiev \& Karastoyanov 1970; Lavčiev et al. 1974; Lavčiev \& Zhekov 1980; Zielke 2016b.

Mydaea setifemur Ringdahl, 1924 - B2; hoes; Zielke 2016b.

Mydaea urbana (Meigen, 1826) - \$; E1, E2, P1, P2, B1, B2, B3, V1, V3, V4, S1, R1, R2, R4, RW, RE, BS; 0-1800 m; 1, 2, 3, 4; h; Lavčiev 1964b, 1966, 1969, 1970a, 1972, 1974, 1980, 2003; Lavčiev \& Karastoyanov 1970; Lavčiev \& Tahirov 1970; Beschovski \& Lăvčiev 1971; Lavčiev et al. 1974; Mirčeva 1974; Lavčiev \& Jelesova 1978; Lavčiev \& Jovčev 1978; Lavčiev \& Zhekov 1980; Zielke 2016b.

Myospila bimaculata (Macquart, 1834) [M. hennigi Gregor \& Povolný, 1959] - E2, B1, B3, R1, RW, BS; 0-1800 m; 1, 2, 3, 4; eca; Lavčiev 1969, 2003; Lavčiev \& Karastoyanov 1970; Lavčiev et al. 1974; Mirčeva 1974, 1981; Zielke 2016b.

Myospila meditabunda (Fabricius, 1781) [Mydaea] - ^; E1, E2, P1, B1, B2, B3, V1, V3, V4, V5, TL, T2, T31, O61, R1, R2, R4, RW, RE, BN, BS; 0-2200 m; 1, 2, 3, 4, 5; hno; Nedelkov 1912; Gregor \& Povolny 1959; Lavčiev 1964a, 1965a, 1965d, 1969, 1970a, 1972, 1974, 1980, 2003; Lavčiev \& Nestorova 1967; Lavčiev \& Karastoyanov 1970; Lavčiev \& Tahirov 1970, 1977; Beschovski \& Lăvčiev 1971; Jovčev \& Lavčiev 1972; Lavčiev et al. 1974, 1981; Mirčeva 1974, 1981; Lavčiev \& Jelesova 1978, 1979; Lavčiev \& Tsankova 1980; Lavčiev \& Zhekov 1980; Kozuharova 1994; Zielke 2016b.

Hebecnema fumosa (Meigen, 1826) - P1, P2, B1, B2, B3, V1, V4, S1, O62, R1, RW, BN, BS; 0-2000 m; 1, 2, 3, 4; po; Nedelkov 1912; Lavčiev 1970a, 1974, 2003; Lavčiev \& Karastoyanov 1970; Lavčiev \& Zhekov 1980; Kozuharova 1994; Zielke 2016b.

Hebecnema nigra (Robineau-Desvoidy, 1830) [H. vespertina nec (Fallén, 1823)] - BS; 0-5 m; 1; h; Pont 1986; Zielke 2016b.

Hebecnema nigricolor (Fallén, 1825) - E1, B1, B2; 200-1400 m; 1, 2, 3; ho; Lavčiev 1970a, 1974, 1977, 2003; Zielke 2016b.

Hebecnema umbratica (Meigen, 1826) - E1, E2, P1, P2, B1, B2, B3, V1, S1, TL, R1, BS; 0-1800 m; 1, 2, 3, 4; ho; Nedelkov 1912; Lavčiev 1967, 1969, 1970a, 1972, 1974, 1980, 2003; Lavčiev \& Nestorova 1967; Lavčiev \& Karastoyanov 1970; Lavčiev \& Tahirov 1970, 1977; Stoicheva 1970; Lavčiev et al. 1974, 1984; Lavčiev \& Zhekov 1980; Lavčiev \& Tsankova 1982; Mirčeva 1981; Zielke 2016b.

Hebecnema vespertina (Fallén, 1823) [H. affinis Malloch, 1921] - E2, P1, B2, V1, V4, R1, R2, RW, BS; 0-1900 m; 1, 2, 3, 4; h; Nedelkov 1912; Lavčiev 1964a, 1969, 1970a, 1972, 1974, 1980, 2003; Lavčiev et al. 1974, 1984; Lavčiev \& Karastoyanov 1970; Mirčeva 1974, 1981; Lavčiev \& Zhekov 1980; Pont 1986, 2013; Beschovski 2006a; Zielke 2016b. 
Graphomya maculata (Scopoli, 1763) - - DW, DM, E1, E2, P1, P2, B1, B2, B3, V1, V4, S1, TL, R1, R2, R5, RW, BN, BS; 0-1300 m; 1, 2, 3; po, ? ppta, ? sk; Nedelkov 1909, 1912; Szilády 1934; Drensky 1960; Lavčiev 1964a, 1969, 1970a, 1974, 1980, 2003; Lavčiev \& Nestorova 1967; Lavčiev \& Karastoyanov 1970; Stoicheva 1970; Lavčiev et al. 1974; Mirčeva 1974, 1981; Lavčiev \& Tahirov 1977; Lavčiev \& Jovčev 1978; Zielke 2016b.

Spilogona acrostichalis (Stein, 1916) - B3; 800-1000 m; 2; e; Lavčiev 1974, 2003.

Spilogona baltica (Ringdahl, 1918) - V4, BN; 0-1500 m; 1, 3, 4; h; Beschovski \& Lăvčiev 1971; Kozuharova 1994; Lăvčiev 2003.

Spilogona biseriata (Stein, 1916) - BN; 0-5 m; 1; ean; Beschovski \& Lăvčiev 1971; Beschovski 1975b; Lăvčiev 2003. Spilogona carbonella (Zetterstedt, 1845) - V4, S22, R1, BN; 0-1500 m; 1, 2, 3; ean; Nedelkov 1909, 1912; Lavčiev 2003.

Spilogona denigrata (Meigen, 1826) - R1, RW; 1400-1500 m; 3, 4; e; Lavčiev 1966, 2003; Beschovski $2006 a$.

Spilogona dispar (Fallén, 1823) [S. funeralis (Rondani, 1866); Limnophora] - P1, B1, B2, V4, R1, R2; 20-2100 m; 1, 2, 3, 4; wesan; Lavčiev 1964b, 1970a, 1974, 2003; Lavčiev \& Karastoyanov 1970; Lavčiev et al. 1984. Spilogona meadei (Schnabl, 1915) - B2; 1100-1300 m; 3; e; Lavčiev 1974, 2003.

Spilogona ocularia (Villeneuve, 1922) - RE; 400-450 m; 1; se; Lavčiev 1964b, 2003.

Spilogona placida (Huckett, 1932) [S. vana (Zetterstedt, 1845); Coenosia] - V1; 600 m; 1, 2; h; Nedelkov 1912; Lavčiev 2003.

Neolimnophora maritima (Roder, 1887) [Fuselia, Limnophora] - BS; 0-5 m; 1; ena; Beschovski 1964a; Lavčiev 1969, 2003.

Limnophora exuta (Kowarz, 1893) - B1; 450-620 m; 1, 2; ena; Lavčiev 1970a, 2003.

Limnophora ljubomirovi Zielke, 2017 - RE; 239 m; 1; Ebg; Zielke 2017.

Limnophora maculosa (Meigen, 1826) - P1, B1, B3, R2, BN, BS; 0-1800 m; 1, 2, 3, 4; eca; Lavčiev 1969, 1970a, 1974, 2003; Lavčiev \& Karastoyanov 1970; Beschovski \& Lavčiev 1971; Lavčiev et al. 1974.

Limnophora nigripes (Robineau-Desvoidy, 1830) - B1; 450 m; 1; h; Lavčiev 1970a, 2003.

Limnophora obsignata (Rondani, 1866) - R2, BS; 0-900 m; 1, 2; atm; Lavčiev 1969, 2003; Lavčiev \& Karastoyanov 1970.

Limnophora orbitalis Stein, 1907 - B2, BN; 0-800 m; 1, 2; tp; Beschovski \& Lavčiev 1971; Lăvčiev 1974, 2003.

Limnophora pandellei Seguy, 1923 - is ena; Pont 1986, 2013.

Limnophora pollinifrons Stein, 1916 - BN; 0-5 m; 1; wcp; Beschovski 1964a, 1964b; Beschovski \& Lavčiev 1971; Lavčiev 2003.

Limnophora pulchriceps (Loew, 1860) - P2; 600 m; 1, 2; cseean; Lavčiev 1974, 2003.

Limnophora riparia (Fallén, 1824) - B1, B2, V1, V4; 600-2000 m; 2, 3, 4; wp; Nedelkov 1912; Arndt 1943; Lavčiev 1970a, 1974, 2003.

Limnophora rufimana (Strobl, 1893) - mca; Pont 1986, 2013.

Limnophora setinerva Schnabl, 1911 - BN; 0-5 m; 1; dp; Beschovski \& Lavčiev 1971; Lavčiev 2003.

Limnophora tigrina (Am Stein, 1860) - V1; 600 m; 1, 2; wcp; Nedelkov 1912; Lavčiev 2003.

Limnophora triangula (Fallén, 1825) - \$; tp; Pont 1986, 2013.

Lispe caesia Meigen, 1826 - V1, BS; 0-600 m; 1; wp; Nedelkov 1912; Beschovski \& Lavčiev 1971; Beschovski 1975b; Lavčiev 2003.

Lispe consanguinea Loew, 1858 - P1, B1, V1, V4, RW, BN; 0-1700 m; 1, 2, 3, 4; tp; Nedelkov 1912; Beschovski 1972a, 1972b, 1973e; Lavčiev 1970a, 2003; Beschovski 2006a.

Lispe flavicincta Loew, 1847 - BS; 0-5 m; 1; ena; Beschovski \& Lavčiev 1971; Lavčiev 2003.

Lispe litorea Fallén, 1825 - BN; 0-5 m; 1; e; Beschovski \& Lavčiev 1971; Lavčiev 2003.

Lispe loewi Ringdahl, 1922 - BS; 0-5 m; 1; wpat; Beschovski \& Lavčiev 1971; Beschovski 1975b; Lavčiev 2003.

Lispe longicollis Meigen, 1826 - RW, BS; 0-1100 m; 1, 2, 3; po; Beschovski \& Lavčiev 1971; Beschovski 1972a; Lavčiev 2003; Beschovski 2006a.

Lispe melaleuca Loew, 1847 - BN; 0-5 m; 1; po; Beschovski \& Lavčiev 1971; Beschovski 1972a, 1972b; Lavčiev et al. 1974; Lavčiev 2003.

Lispe nana Macquart, 1835 - BN, BS; 0-5 m; 1; ppt; Lavčiev 1969, 2003; Beschovski \& Lavčiev 1971.

Lispe pygmaea Fallén, 1825 - BN, BS; 0-5 m; 1; ppt; Beschovski \& Lavčiev 1971; Beschovski 1973e, 1975b; Lavčiev 2003.

Lispe tentaculata (De Geer, 1776) - E1, E2, P2, B1, V1, BN, BS; 0-1000 m; 1, 2; hno; Löw 1862; Nedelkov 1912; Lavčiev 1965d, 1969, 1970a, 1974, 2003; Lavčiev \& Nestorova 1967; Beschovski \& Lavčiev 1971; Lavčiev 
\& Karastoyanov 1970; Beschovski 1972a, 1972b; Lavčiev et al. 1974; Lavčiev \& Tahirov 1977; Lavčiev \& Zhekov 1980; Mirčeva 1981.

Lispe uliginosa Fallén, 1825 - V1, BN; 0-600 m; 1, 2; h; Nedelkov 1912; Drensky 1960; Lavčiev 2003.

Lispocephala brachialis (Rondani, 1877) [Caricea] - B1, S23; 400-600 m; 1; ena; Lavčiev 1970a, 2003; Lavčiev \& Karastoyanov 1970.

Lispocephala erythrocera (Robineau-Desvoidy, 1830) [Caricea] - BN; 0-5 m; 1; ho; Beschovski \& Lavčiev 1971; Lavčiev 2003.

Orchisia costata (Meigen, 1826) [Sapromyza] - BS; 0-5 m; 1; ppta; Caspers 1951a; Beschovski 1964a; Lavčiev 2003. Schoenomyza litorella (Fallén, 1823) - BN; 0-5 m; 1; hpt; Beschovski \& Lavčiev 1971; Lavčiev 2003.

Macrorchis meditata (Fallén, 1825) - \$; E2, P2, B1, B2, TL, BN, BS; 0-1620 m; 1, 2, 3, 4; wcp; Lavčiev 1969, 1974, 2003; Lavčiev \& Karastoyanov 1970; Stoicheva 1970; Beschovski \& Lavčiev 1971; Mirčeva 1974, 1981.

Dexiopsis lacteipennis (Zetterstedt, 1845) [Coenosia] - B1, BN; 0-600 m; 1; e; Lavčiev 1970a, 2003; Beschovski \& Lavčiev 1971.

Dexiopsis ruficornis Macquart, 1835 [D. litoralis (Zetterstedt, 1845); Coenosia] - RW, BN, BS; 0-1700 m; 1, 2, 3, 4; e; Beschovski \& Lavčiev 1971; Lavčiev 2003; Beschovski 2006a.

Coenosia agromyzina (Fallén, 1825) - wesan; Pont 1986, 2013.

Coenosia albicornis Meigen, 1826 - V1; 600 m; 1, 2; e; Lavčiev \& Lateva 1973; Lavčiev 2003.

Coenosia ambulans Meigen, 1826 - BN; 0-5 m; 1; wcp; Beschovski \& Lavčiev 1971; Lavčiev 2003.

Coenosia antennata (Zetterstedt, 1849) - BN, BS; 0-5 m; 1; e; Beschovski \& Lavčiev 1971; Lavčiev 2003.

Coenosia atra Meigen, 1830 - wp; Pont 1986, 2013.

Coenosia beschovskii Lavciev, 1970 - BN, BS; 0-5 m; 1; Ebg; Lavčiev 1970b, 2003; Beschovski \& Lavčiev 1971.

Coenosia bilineella (Zetterstedt, 1838) - P2, B2, RW; 600-1200 m; 1, 2, 3; tes; Lavčiev 1974, 2003; Beschovski 2006a.

Coenosia flavimana (Zetterstedt, 1845) - BN, BS; 0-5 m; 1; e; Beschovski \& Lavčiev 1971; Lavčiev 2003.

Coenosia humilis Meigen, 1826 - RW, BN; 0-1500 m; 1, 4; hpt; Beschovski \& Lavčiev 1971; Lavčiev 2003.

Coenosia infantula Rondani, 1866 - BN; 100-200 m; 1; e; Lavčiev 1974, 2003.

Coenosia intermedia (Fallén, 1825) - P1, B1, V1, RW; 320-1700 m; 1, 2, 3, 4; tes, ? wces; Nedelkov 1912; Lavčiev 1970a, 2003; Beschovski 2006a.

Coenosia mixta Schnabl, 1911 - sena; Pont 1986, 2013.

Coenosia mollicula (Fallen, 1825) - h; Pont 1986, 2013.

Coenosia pygmaea (Zetterstedt, 1845) - BN; 0-5 m; 1; tes, ? wces; Beschovski \& Lavčiev 1971; Lavčiev 2003.

Coenosia rufipalpis Meigen, 1826 - BN; 0-5 m; 1; tes; Beschovski \& Lavčiev 1971; Beschovski 1976a.

Coenosia strigipes Stein, 1916 - BS; 0-5 m; 1; ppt; Beschovski \& Lavčiev 1971; Lavčiev 2003.

Coenosia testacea (Robineau-Desvoidy, 1830) [C. tricolor Zetterstedt, 1845] - V1, BN; 0-600 m; 1; wcp; Nedelkov 1912; Beschovski \& Lavčiev 1971; Lavčiev 2003.

Coenosia tigrina (Fabricius, 1775) - \$; E1, E2, P2, B1, B2, B3, V1, V4, BN, BS; 0-1800 m; 1, 2, 3, 4; h; Nedelkov 1912; Lavčiev 1969, 1979a, 1964, 2003; Lavčiev \& Karastoyanov 1970; Lavčiev \& Tahirov 1977; Mirčeva 1981.

\section{Calliphoridae}

Bellardia bayeri (Jacentkovsky, 1937) [Onesia] - RW; 400 m; 1; h; Jacentkovsky 1937.

Bellardia pandia (Walker 1849) [Onesia biseta Kramer, 1917] - R1; 1600 m; 3, 4; e; Jacentkovsky 1936, 1937.

Bellardia siciliensis (Villeneuve, 1926) [Onesia] - E1, RW; 150-370 m; 1; se; Jacentkovsky 1936, 1937; Beschovski 2006a.

Bellardia viarum (Robineau-Desvoidy, 1830) [Onesia pusilla (Meigen, 1826)] - E2; 230 m; 1; des; Drensky 1958.

Bellardia vulgaris (Robineau-Desvoidy, 1830) [Onesia agilis (Meigen, 1826)] - V1, V4; 550-850 m; 1, 2; h; Jacentkovsky 1936, 1937; Drensky 1958.

Calliphora genarum (Zetterstedt, 1838) [Acrophaga alpina (Zetterstedt, 1838)] - R1; 1600 m; 4; h; Jacentkovsky 1936, 1937.

Calliphora vicina Robineau-Desvoidy, 1830 [C. erythrocephala (Meigen, 1826)] - $\mathbf{\Delta}$; ; DM, E2, V1, S22, TL, RW, BN, BS; 0-1810 m; 1, 2, 3, 4; k, i; Nedelkov 1909, 1912; Jacentkovsky 1936, 1937; Popoff 1941; Drensky 1955, 1958, 1960; Vesselinov \& Gabev 1956; Gregor \& Povolny 1959; Valerianov 1961; Lavčiev 1965a, 1965d, 1980; Surbova 1965; Lavčiev \& Jovčev 1978; Beschovski 2006a. 
Calliphora vomitoria (Linnaeus, 1758) - $\mathbf{\Delta}$; ; DM, E2, B1, V1, V4, S22, R1, R2, BN, BS; 0-1900 m; 1, 2, 3, 4; sk, i; Meunier 1897; Kovachev 1905; Nedelkov 1909, 1912; Jacentkovsky 1936, 1937; Popoff 1941; Drensky 1955, 1958; Vesselinov \& Gabev 1956; Gregor \& Povolny 1959; Lavčiev 1965a, 1980; Lavčiev \& Jovčev 1978.

Cynomya mortuorum (Linnaeus, 1761) - E2, V1, V4, RW, BN, BS; 0-1300 m; 1, 2, 3; ho; Nedelkov 1912; Drensky 1958, 1960; Lavčiev 1965d; Surbova 1965.

Lucilia caesar (Linnaeus, 1758) [? L. ruficeps (Meigen, 1826)] - $\mathbf{\Delta}$; ; E1, E2, V1, TL, O61, R1, R2, BN, BS; 0-1810 m; 1, 2, 3, 4; hno, ? sk, i; Meunier 1897; Kovachev 1905; Nedelkov 1909, 1912; Szilády 1934; Jacentkovsky 1936, 1937; Popoff 1941; Drensky 1942, 1958, 1960; Vesselinov \& Gabev 1956; Gregor \& Povolny 1959; Lavčiev 1965a, 1965d, 1980; Lavčiev \& Jovčev 1978.

Lucilia ilustris (Meigen, 1826) - E2, V1, BS; 0-600 m; 1, 2; hoa; Vesselinov \& Gabev 1956; Drensky 1958; Lavčiev 1965d.

Lucilia richardsi Collin, 1926 - B3, V4, S1, O61, R1; 250-1100 m; 1, 2, 3; e; Jacentkovsky 1936, 1937.

Lucilia sericata (Meigen, 1826) [L. nobilis (Meigen, 1826)] - \; \$ DM, E2, B1, V1, V4, S1, S22, TL, O61, R1, BN, BS; 0-2100 m; 1, 2, 3, 4, 5; k; Joakimoff 1899; Nedelkov 1909, 1912; Szilády 1934; Jacentkovsky 1936, 1937; Popoff 1941; Drensky 1955, 1958, 1960; Vesselinov \& Gabev 1956; Gregor \& Povolny 1959; Valerianov 1961; Zhecheva 1961; Beschovski 1964a; Lavčiev 1965d, 1980; Surbova 1965; Lavčiev \& Jovčev 1978.

Lucilia silvarum (Meigen, 1826) - ; E2, S1, T31, R1, RW, BS; 0-400 m; 1; hn; Joakimoff 1899; Nedelkov 1912; Jacentkovsky 1936, 1937; Drensky 1958, 1960; Beschovski 1964a, 1965, 2006a.

? Lucilia ruficeps (Meigen, 1826) [? = L. caesar (Linnaeus, 1758)] - V1; 550-600 m; 1; e; Nedelkov 1912.

? Melinda cognata (Meigen, 1830) [Onesia] - E2; 200-250 m; 1; ena; Drensky 1958.

Melinda gentilis Robineau-Desvoidy, 1830 [Onesia; ? M. cognata (Meigen, 1830)] - DM, E2, V1, V4; 140-800 m; 1, 2; h; Drensky 1958.

Onesia austriaca Villeneuve, 1920 [O. macrophallus Stein, 1924] - V4; 770-1000 m; 2; cse; Jacentkovsky 1936, 1937; Drensky 1958.

Onesia sepulcralis (Meigen, 1826) [O. floralis Robineau-Desvoidy, 1830] - V1, V4, TL; 200-1820 m; 1, 2, 3, 4; e; Nedelkov 1909, 1912; Drensky 1958.

Chrysomya albiceps (Wiedemann, 1819) [Compsomyia] - B3, S1, TL, BS; 0-500 m; 1; pptn; Jacentkovsky 1936, 1937; Drensky 1958, 1960; Lavčiev \& Jovčev 1978; Lavčiev 1980; Beschovski 2004a.

Phormia regina (Meigen, 1826) [Lucilia thalassina Meigen, 1826] - V1, V4, TL, T31, O61, BS; 0-1000 m; 1, 2; ha; Nedelkov 1909, 1912; Jacentkovsky 1936, 1937; Drensky 1958, 1960; Gregor \& Povolny 1959; Zhecheva 1961; Lavčiev 1965a, 1965d, 1980; Surbova 1965; Stoicheva 1970.

Protocalliphora azurea (Fallén, 1817) [P. caerulea (Robineau-Desvoidy, 1830)] - E2; 20-30 m; 1; po; Lavčiev $1965 \mathrm{~d}$.

Protophormia terraenovae (Robineau-Desvoidy, 1830) - E2, V1, O61, R1; 30-1500 m; 1, 2, 3; hno; Vesselinov \& Gabev 1956; Gregor \& Povolny 1959; Lavčiev 1965d, 1980; Surbova 1965; Lavčiev et al. 1984.

Pollenia amentaria (Scopoli, 1763) [P. vespillo (Fabricius, 1794)] - \$; DW, DM, V1, V3, V4, S1, TL, BS; 0-1000 m; 1, 2; wp; Nedelkov 1912; Szilady 1934; Jacentkovsky 1936, 1937; Drensky 1958; Beschovski 2004a.

Pollenia atramentaria (Meigen, 1826) [? P. nigrina (Meigen, 1838)] - E1, BS; 0-350 m; 1; ena; Drensky 1958.

Pollenia bulgarica Jacentkovsky, 1939 - S1, RW; 300-400 m; 1; cseei; Jacentkovsky 1937, 1939; Beschovski 2006 a.

Pollenia dasypoda Portschinsky, 1881 - R1; 900-1100 m; 2, 3; wpo; Jacentkovsky 1939.

Pollenia fulvipalpis Macquart, 1835 [P. bisulca Pandelle, 1896] - S1, TL, RW; 150-370 m; 1; csena; Jacentkovsky 1936, 1937; Drensky 1958; Beschovski 2006a.

Pollenia labialis Robineau-Desvoidy, 1863 [P. excarinata Wainwright, 1940; ? P. depressa (Meigen, 1826)] DW, E2, V1, V4, TL, BS; 0-800 m; 1, 2; h; Drensky 1958, 1960.

Pollenia pediculata Macquart, 1834 [P. obscura Bigot, 1888] - BS; 0-5 m; 1; hoa; Drensky 1958.

Pollenia rudis (Fabricius, 1794) [P. varia (Meigen, 1826); ? P. depressa (Meigen, 1826)] - D DM, E2, V1, V4, S22, TL, O61, O62, R1, BN, BS; 0-1600 m; 1, 2, 3, 4; sk, hpta, ? hoa; Joakimoff 1899; Nedelkov 1909, 1912; Szilády 1934; Jacentkovsky 1936, 1937; Vesselinov \& Gabev 1956; Drensky 1958, 1960; Gregor \& Povolny 1959; Lavčiev 1965d, 1980; Surbova 1965; Lavčiev \& Jovčev 1978; Beschovski 2004a.

Pollenia tenuiforceps Séguy, 1928 [P. angustifrons (Jacentkovsky, 1941)] - TL, R1; 80-1400 m; 1, 2, 3; ena; Szilády 1934; Drensky 1958; Beschovski 2004a.

Pollenia vagabunda (Meigen, 1826) - K9, V1, S1, TL, RW; 80-600 m; 1; h; Drensky 1958; Beschovski 2004a, $2006 a$. Pollenia vera Jacentkovsky, 1936 - B3, V1; 500-750 m; 1, 2; e; Jacentkovsky 1936, 1937. 
Pollenia viatica Robineau-Desvoidy, 1830 [P.pallida Rohdendorf, 1926] - R1; 1600 m; 3, 4; ewca; Jacentkovsky 1939.

Pollenia pruinosa (Macquart, 1835) [? nomina dubia] - BS; 0-5 m; 1; ? se; Drensky 1958, 1960.

Rhyncomya columbina (Meigen, 1824) - TL, BS; 0-250 m; 1; sena; Nedelkov 1909.

Rhyncomya cyanescens (Loew, 1844) - DM, E1, B2, V4, S1, TL, O61, BN, BS; 0-1400 m; 1, 2, 3; hom; Löw 1862, 1863; Nedelkov 1909, 1912; Jacentkovsky 1936, 1937; Drensky 1958, 1960; Beschovski 2004a.

Rhyncomya impavida (Rossi, 1790) [R. columbina Schiner, 1861] - E1, S1, TL, RW, BN, BS; 0-500 m; 1; sena; Nedelkov 1909, 1912; Jacentkovsky 1936, 1937; Drensky 1942, 1958, 1960; Beschovski 2006a.

Rhyncomya speciosa (Loew, 1844) - DW, O62, R5, RW, BN, BS; 0-550 m; 1; ? nm; Nedelkov 1909, 1912; Drensky 1942, 1958, 1960; Beschovski 2006a.

Stomorhina lunata (Fabricius, 1805) [S. cinerea (Robineau-Desvoidy, 1830)] - V1; 550-600 m; 1; hpt; Nedelkov 2012.

\section{Sarcophagidae}

Amobia signata (Meigen, 1824) [Pachyophthalma] - S1; 250-300 m; 1; ppt; Jacentkovsky 1936, 1937; Verves 1986; Pape \& Beuk 2017.

Senotainia albifrons (Rondani, 1859) - S1, O61, RW; 250-320 m; 1; ppt; Jacentkovsky 1936, 1937; Beschovski 2006a; Pape \& Beuk 2017.

Senotainia conica (Fallén, 1810) - esca; Verves 1986; Pape \& Beuk 2017.

Senotainia tricuspis (Meigen, 1838) - S1, O61, RW; 250-320 m; 1; tp; Jacentkovsky 1936, 1937; Verves 1986; Beschovski 2006a; Pape \& Beuk 2017.

Taxigramma heteroneura (Meigen, 1830) - ho; Verves 1986; Pape \& Beuk 2017.

Apodacra seriemaculata Macquart, 1854 - V1; 600 m; 1; cseit; Nedelkov 1912.

Miltogramma germari Meigen, 1824 - V1, TL, O61; 170-600 m; 1; wcp; Nedelkov 1912; Jacentkovsky 1936, 1937; Verves 1986; Pape \& Beuk 2017.

Miltogramma murina Meigen, 1824 - V1; 600 m; 1; ena; Nedelkov 1912.

Miltogramma oestracea (Fallén, 1820) - V1; 600 m; 1; tp; Nedelkov 1912.

Miltogramma punctata Meigen, 1824 - \$ tp; Verves 1986; Pape \& Beuk 2017.

Miltogramma taeniata Meigen, 1824 - O61; 315 m; 1; wpo; Jacentkovsky 1936, 1937; Verves 1986; Pape \& Beuk 2017.

Miltogramma testaceifrons (von Roser, 1840) [M. pilitarse Rondani, 1859] - B1, V1, S22, BN; 0-700 m; 1, 2; po; Nedelkov 1909, 1912.

Pterella grisea (Meigen, 1824) [Setula] - O61; 315 m; 1; ? wcp; Jacentkovsky 1936, 1937; Verves 1986; Pape \& Beuk 2017.

Taxigramma stictica (Meigen, 1830) [Hilarella dura (Robineau-Desvoidy, 1830)] - O61, R1; 450 m; 1; wcp; Jacentkovsky 1936, 1937; Verves 1986; Pape \& Beuk 2017.

Mesomelena mesomelaena (Loew, 1848) - esca; Verves 1986; Pape \& Beuk 2017.

Metopia argentata Macquart, 1850 - V1; 600 m; 1; po; Nedelkov 1912.

Metopia argyrocephala (Meigen, 1824) [M. leucocephala (Rossi, 1790)] - V1, O61, R1; 450-1600 m; 1, 2, 3, 4; hno; Nedelkov 1912; Jacentkovsky 1936, 1937; Verves 1986; Pape \& Beuk 2017.

Metopia campestris (Fallén, 1810) - V4, O61, R1; 450-1600 m; 1, 2, 3, 4; ho; Jacentkovsky 1936, 1937; Verves 1986; Pape \& Beuk 2017.

Taxigramma multipunctata (Rondani, 1859) [Heteroneura; Paragusia] - V3, S1; 270-1000 m; 1, 2; wcp; Jacentkovsky 1936, 1937; Verves 1986; Pape \& Beuk 2017.

Phylloteles pictipennis Loew, 1844 - V1, O61; 315-600 m; 1; wesant; Nedelkov 1912; Jacentkovsky 1936, 1937; Verves 1986; Pape \& Beuk 2017.

Agria affinis (Fallén, 1817) [A. punctata Robineau-Desvoidy, 1830] - \$; tp; Verves 1986; Pape 1996; Pape \& Beuk 2017.

Agria mamillata (Pandellé, 1896) [Pseudosarcophaga] - ; TL; 200-230 m; 1; esca; Popoff 1956; Trenchev 1979; Georgiev 1996b; Georgiev \& Langurov 1997; Verves 1986; Pape 1996; Pape \& Beuk 2017.

Angiometopa falleni Pape, 1986 [A. ruralis (Fallén, 1817)] - esca; Verves 1986; Pape \& Beuk 2017. 
Brachicoma devia (Fallén, 1820) - V4, R1; 750-1800 m; 2, 3, 4; ho; Jacentkovsky 1936, 1937; Drensky 1957b; Verves 1986; Pape \& Beuk 2017.

Eurychaeta muscaria (Meigen, 1826) [Helicobosca] - R1, RW; 380-1800 m; 1, 2, 3, 4; ena; Jacentkovsky 1936, 1937; Drensky 1957b; Beschovski 2006a; Pape \& Beuk 2017.

Eurychaeta palpalis (Robineau-Desvoidy, 1830) [Helicobosca] - O61; 310-340 m; 1; wces; Gregor \& Povolny 1959; Verves 1986.

Nyctia halterata (Panzer, 1798) [Anthracia caminaria (Meigen, 1826)] - V4, BN; 0-1100 m; 1, 2, 3; ena; Löw 1862; Jacentkovsky 1936, 1937; Drensky 1957b; Verves 1986; Pape \& Beuk 2017.

Sarcophila latifrons (Fallén, 1817) - E2, V4, S1, TL, RW, RE, BN, BS; 0-400 m; 1; esca; Nedelkov 1909, 1912; Jacentkovsky 1936, 1937; Drensky 1957; Lavčiev 1965d; Beschovski 2004a; Pape \& Beuk 2017.

Wohlfahrtia magnifica (Schiner, 1862) [Sarcophila] - ৯; V1, O61, O62, BN; 0-600 m; 1; wcp; Nedelkov 1909, 1912; Jacentkovsky 1936, 1937; Drensky 1957; Gregor \& Povolny 1959; Verves 1986; Pape \& Beuk 2017.

Wohlfahrtia vigil (Walker, 1849) [W. meigeni (Schiner, 1862); Sarcophila] - М ; V4, BN; 0-1100 m; 1, 2, 3; h, ? ho; Nedelkov 1909, 1912; Verves 1986; Pape \& Beuk 2017.

Blaesoxipha (Blaesoxipha) cochlearis (Pandellé, 1896) - O62; 90-100 m; 1; tp; Drensky 1957; Verves 1986; Povolný \& Verves 1990; Pape \& Beuk 2017.

Blaesoxipha (Blaesoxipha) laticornis (Meigen, 1826) [B. grylloctona Löw, 1861] - S1; 276 m; 1; tp; Jacentkovsky 1936, 1937; Verves 1986; Pape \& Beuk 2017.

Blaesoxipha (Blaesoxipha) litoralis (Villeneuve, 1911) - S1, O62; 90-270 m; 1; tp; Jacentkovsky 1939; Drensky 1957; Verves 1986; Povolný \& Verves 1990; Pape \& Beuk 2017.

Blaesoxipha (Blaesoxipha) plumicornis (Zetterstedt, 1859) [B. gladiatrix (Pandellé, 1896)] - DM, B3, V1, V4, S1, TL, T31, RE; 80-1100 m; 1, 2, 3; tp, ? hop; Jacentkovsky 1936, 1937; Drensky 1957; Verves 1986; Povolný \& Verves 1990; Beschovski 2004a; Pape \& Beuk 2017.

Blaesoxipha (Blaesoxipha) lapidosa Pape, 1994 [B. lineata Fallén, 1817; B. campestris (Robineau-Desvoidy, 1863); B. redemta (Pandellé, 1896); Gesneroides] - E1, E2, S1, TL, T31, O61, RW, RE, BS; 0-500 m; 1; ppta; Petkoff 1921; Jacentkovsky 1936, 1937; Drensky 1955, 1957; Verves 1986; Povolný \& Verves 1990; Pape \& Beuk 2017.

Blaesoxipha (Blaesoxipha) ungulata (Pandellé, 1896) - R1; 1600 m; 3, 4; ena; Jacentkovsky 1936, 1937; Drensky 1957; Verves 1986; Povolný \& Verves 1990; Pape \& Beuk 2017.

Blaesoxipha (Blaesoxipha) unicolor (Villeneuve, 1912) - BN, BS; 0-10 m; 1; po; Jacentkovsky 1937, 1939; Drensky 1957; Povolný \& Verves 1990; Pape \& Beuk 2017.

Blaesoxipha (Servaisia) erythrura (Meigen, 1826) [Sarcophaga strenua (Robineau-Desvoidy, 1863)] - DM, B1, V1, V4, TL, T31, R1, RW, BN; 0-1500 m; 1, 2, 3, 4; esca; Nedelkov 1909, 1912; Drensky 1957; Verves 1986; Povolný \& Verves 1990; Pape \& Beuk 2017.

Blaesoxipha (Servaisia) rossica Villeneuve, 1912 - S1, O61, R1; 320-1200 m; 1, 2, 3; esca; Jacentkovsky 1936, 1937; Drensky 1957; Verves 1986; Povolný \& Verves 1990; Pape \& Beuk 2017.

Blaesoxipha (Tephromyia) grisea (Meigen, 1826) - E1, P2, R4, BN, BS; 0-1000 m; 1, 2; tes; Drensky 1957; Verves 1986; Povolný \& Verves 1990; Pape \& Beuk 2017.

Ravinia pernix (Harris, 1780) [R. striata (Fabricius, 1794); R. haematodes (Meigen, 1826); Sarcophaga] - $\mathbf{\Delta}$; ; DM, E2, P1, V4, S1, TL, O61, R1, R2, RW, BN, BS; 0-2000 m; 1, 2, 3, 4; ppt; Joakimoff 1899; Jacentkovsky 1936, 1937; Drensky 1957, 1960; Karnožitzky 1957; Gregor \& Povolny 1959; Stefanov \& Keremidchiev 1961; Lavčiev 1965a, 1965d, 1980; Surbova 1965; Stoicheva 1970; Lavčiev \& Jovčev 1978; Verves 1986; Povolný \& Verves 1990; Pape \& Beuk 2017.

Sarcophaga (Bellieriomima) subulata Pandellé, 1896 [S. laciniata Pandellé, 1896] - B2, K9, R2, R5; 520-1200 m; 1, 2, 3; des; Drensky 1957; Verves 1986; Povolný \& Verves 1990; Pape \& Beuk 2017.

Sarcophaga (Helicophagella) agnata Rondani, 1860 [Bellieria] - K9, V4, R1; 670-1500 m; 2, 3, 4; e; Drensky 1957; Verves 1986; Povolný \& Verves 1990; Pape \& Beuk 2017.

Sarcophaga (Helicophagella) crassimargo Pandellé, 1896 [Bellieria] - E1, E2, P2, O62, R2; 20-2000 m; 1, 2, 3, 4; wesca; Drensky 1957; Verves 1986; Povolný \& Verves 1990; Pape \& Beuk 2017.

Sarcophaga (Helicophagella) hirticrus Pandelle, 1896 [Bellieria] - E1, P2, S1, BS; 0-300 m; 1; ena; Jacentkovsky 1936, 1937; Drensky 1955, 1957; Povolný \& Verves 1990; Pape \& Beuk 2017.

Sarcophaga (Helicophagella) macrura (Rohdendorf, 1937) - E2; 20-30 m; 1; ees; ? tes; Povolný \& Verves 1990; Pape \& Beuk 2017. 
Sarcophaga (Helicophagella) maculata Meigen, 1835 [Bellieria] - O61, R2, BN, BS; 0-1800 m; 1, 2, 3, 4; ? wcp, ? sp; Gregor \& Povolny 1959; Verves 1986; Lavčiev \& Jovčev 1978; Povolný \& Verves 1990.

Sarcophaga (Helicophagella) melanura Meigen, 1826 [Bellieria] - $\Delta$; ; DW, DM, E2, B3, V1, V3, V4, S1, TL, O61, R1, RW, RE, BN, BS; 0-1200 m; 1, 2, 3; hpt; Jacentkovsky 1936, 1937; Drensky 1955, 1957; Valerianov 1961; Lavčiev 1965a, 1965d, 1980; Lavčiev \& Jovčev 1978; Povolný \& Verves 1990; Beschovski 2004a; Pape \& Beuk 2017.

Sarcophaga (Helicophagella) noverca Rondani, 1860 [Bellieria] - V4, R1, R4; 600-1600 m; 2, 3, 4; e; Jacentkovsky 1936, 1937; Drensky 1957; Verves 1986; Povolný \& Verves 1990; Pape \& Beuk 2017.

Sarcophaga (Helicophagella) novercoides Bottcher, 1913 [Bellieria] - O62, R2; 90 m, 1200-2200 m; 1, 3, 4, 5; ena; Drensky 1957; Verves 1986; Povolný \& Verves 1990; Pape \& Beuk 2017.

Sarcophaga (Helicophagella) rosellei Bottcher, 1912 [Bellieria] - B1, V4, R1; 400-1700 m; 1, 2, 3, 4; des; Drensky 1957; Verves 1986; Povolný \& Verves 1990; Pape \& Beuk 2017.

Sarcophaga (Krameromyia) anaces Walker, 1849 - R1; 530-670 m; 1, 2; ena; Povolný \& Verves 1990; Pape \& Beuk 2017.

Sarcophaga (Mehria) sexpunctata (Fabricius, 1805) [Thyrsocnema clathrata Meigen, 1826] - E2, K4; 30-1000 m; 1, 2; tp; Drensky 1957; Verves 1986; Povolný \& Verves 1990; Pape \& Beuk 2017.

Sarcophaga (Mehria) nemoralis Kramer, 1908 [Pierretia] - P1; 570-590 m; 1; tes, ? des; Povolný \& Verves 1990; Pape \& Beuk 2017.

Sarcophaga (Myorhina) nigriventris Meigen, 1826 [Pierretia, Thyrsocnema] - DM, B2, S1, R2, BS; 0-2400 m; 1, 2, 3, 4, 5; dp; Nedelkov 1912; Drensky 1957; Verves 1986; Povolný \& Verves 1990; Pape \& Beuk 2017.

Sarcophaga (Myorhina) socrus Rondani, 1860 [S. rostrata Pandellé, 1896; Pierretia; Thyrsocnema] - E1, S1, TL, R2, RW, BS; 0-2000 m; 1, 2, 3, 4; e; Jacentkovsky 1936, 1937; Drensky 1957; Verves 1986; Povolný \& Verves 1990; Beschovski 2006a; Pape \& Beuk 2017.

Sarcophaga (Myorhina) soror Rondani, 1860 [Thyrsocnema] - K9, R2; 500-2200 m; 1, 2, 3, 4, 5; e; Drensky 1957; Verves 1986; Povolný \& Verves 1990; Pape \& Beuk 2017.

Sarcophaga (Myorhina) villeneuvei Bottcher, 1912 [Thyrsocnema] - DW, E2; 50-140 m; 1; des; Drensky 1957; Verves 1986; Povolný \& Verves 1990; Pape \& Beuk 2017.

Sarcophaga (Kramerella) granulata Kramer, 1908 [Thyrsocnema] - E2, B1; 50-400 m; 1; e; Drensky 1957; Verves 1986; Povolný \& Verves 1990; Pape \& Beuk 2017.

Sarcophaga (Sarcotachinella) sinuata Meigen, 1826 [Arhopocnemis] - E2, R2; 50-1810; 1, 2, 3, 4; h; Drensky 1957; Verves 1986; Povolný \& Verves 1990; Pape \& Beuk 2017.

Sarcophaga (Thyrsocnema) incisilobata Pandellé, 1896 [? S. striata Fabricius, 1794] - ; E2, B3, V1, V3, V4, S1, TL, R1, RW, RE, BS; 0-1000 m; 1, 2; tp; ? Joakimoff 1899; Jacentkovsky 1936, 1937; Drensky 1957; Verves 1986; Povolný \& Verves 1990; Beschovski 2004a, 2006a; Pape \& Beuk 2017.

Sarcophaga (Thyrsocnema) kentejana (Rohdendorf, 1937) - V4; 1800-1900 m; 4; ho, bm; Povolný \& Verves 1990; Pape \& Beuk 2017.

Sarcophaga (Discachaeta) arcipes Pandellé, 1896 [Pierretia] - E1, K9; 165-680 m; 1; e; Drensky 1957; Verves 1986; Povolný \& Verves 1990; Pape \& Beuk 2017.

Sarcophaga (Discachaeta) cucullans Pandellé, 1896 [D. jacentkovskyi Enderlein, 1936; Pierretia] - E1, O62; 160-190 m; 1; csean; Enderlein, 1936; Jacentkovsky 1936, 1937; Drensky 1957; Verves 1986; Povolný \& Verves 1990; Pape \& Beuk 2017.

Sarcophaga (Discachaeta) pumila Meigen, 1826 [Pierretia] - O62, R3; 420 m; 1; e; Drensky 1957; Verves 1986; Povolný \& Verves 1990; Pape \& Beuk 2017.

Sarcophaga (Heteronychia) ancilla Rondani, 1865 [Pierretia] - DW, BN; 0-50 m; 1; cse; Drensky 1957; Verves 1986; Povolný \& Verves 1990; Pape \& Beuk 2017.

Sarcophaga (Heteronychia) balanina Pandellé, 1896 [Heteronychia, Pierretia] - K9, O5, O62; 380-670 m; 1; se; Drensky 1957; Povolný \& Verves 1990.

Sarcophaga (Heteronychia) benaci Bottcher, 1913 [Pierretia] - V4, S1, O61, R1, RW; 500-1600 m; 1, 2, 3, 4; cse; Jacentkovsky 1936, 1937; Drensky 1957; Povolný \& Verves 1990; Beschovski 2006a; Pape \& Beuk 2017.

Sarcophaga (Heteronychia) bulgarica (Enderlein, 1936) [Helicobia; Pierretia (Pierretia) boettcheriana Rohdendorf, 1937;] - DM, E1, E2, V4, S1, BN; 0-1200 m; 1, 2, 3; e; Enderlein 1936; Jacentkovsky 1936, 1937, 1939; Buresch 1953a; Drensky 1957; Verves 1986; Povolný \& Verves 1990; Pape \& Beuk 2017. 
Sarcophaga (Heteronychia) consanguinea Rondani, 1860 [Heteronychia (Eupierretia) atanassovi Lehrer, 1977; Pierretia (Eupierretia) portschinskyana Rohdendorf, 1937] - E1, R3; 160-1700 m; 1, 2, 3, 4; sena; Lehrer, 1977; Verves 1986; Povolný \& Verves 1990; Whitmore 2011; Pape \& Beuk 2017.

Sarcophaga (Heteronychia) depressifrons Zetterstedt, 1845 [S. arvorum (Rondani, 1860); Pierretia (s.str.) obscurata Rohdendorf, 1937; ? S. rondaniana (Rohdendorf, 1937)] - DW, O61; 30-320 m; 1; po; Jacentkovsky 1936, 1937; Drensky 1957; Verves 1986; Povolný \& Verves 1990; Pape \& Beuk 2017.

Sarcophaga (Heteronychia) dissimilis Meigen, 1826 [S. offuscata Meigen, 1826; Pierretia] - DW, E2, K4, V4, O62, R1, RW; 30-1600 m; 1, 2, 3, 4; des; Nedelkov 1912; Jacentkovsky 1936, 1937; Drensky 1957; Verves 1986; Povolný \& Verves 1990; Pape \& Beuk 2017.

Sarcophaga (Heteronychia) enderleini Jacentkovský, 1937 - RW; 360-400 m; 1; se; Jacentkovsky 1937; Verves 1986; Povolný \& Verves 1990; Pape \& Beuk 2017.

Sarcophaga (Heteronychia) filia Rondani, 1860 [Pierretia] - DW, E1, K9, O61, RW, BS; 0-600 m; 1; e; Jacentkovsky 1936, 1937; Drensky 1957; Povolný \& Verves 1990; Beschovski 2006a; Whitmore 2011; Pape \& Beuk 2017.

Sarcophaga (Heteronychia) haemorrhoa Meigen, 1826 [Pierretia] - B1, V1, S1; 270-900 m; 1, 2; e; Nedelkov 1912; Jacentkovsky 1936, 1937; Drensky 1957; Verves 1986; Povolný \& Verves 1990; Pape \& Beuk 2017.

Sarcophaga (Heteronychia) haemorrhoides Böttcher, 1913 - E1, RW, BN; 0-120 m; 1; wesan; Drensky 1957; Verves 1986; Povolný \& Verves 1990; Beschovski 2006a; Pape \& Beuk 2017.

Sarcophaga (Heteronychia) infantilis Bottcher, 1913 [S. bezziana Böttcher, 1913; ? Heteronychia (Heteronychia) drenskiana Lehrer, 1977] - V4, R1, R2; 600-2200 m; 1, 2, 3, 4, 5; e; Verves 1986; Povolný \& Verves 1990; Pape \& Beuk 2017.

Sarcophaga (Heteronychia) lacrymans Villeneuve, 1912 [Sarcophaga (Heteronychia) thalhammeri Böttcher, 1913; Pierretia] - E1, RW, BN; 0-400 m; 1; em; Jacentkovsky 1936, 1937; Drensky 1957; Povolný \& Verves 1990; Whitmore 2011; Pape \& Beuk 2017.

Sarcophaga (Heteronychia) minima Rondani, 1862 [Sarcophaga (Heteronychia) fertoni Villeneuve, 1911; Pierretia (Bercaea) graeca Rohdendorf, 193] - O62, R3; 420-2180 m; 1, 4, 5; csena; Drensky 1957; Povolný \& Verves 1990; Pape \& Beuk 2017.

Sarcophaga (Heteronychia) mutila Villeneuve, 1912 [Heteronychia (Boettcherella) nedelkoffi Lehrer, 1977; S. setinervis var. mutila Villeneuve, 1912] - E1, B1, V1, S1, R2; 130-2400 m; 1, 2, 3, 4, 5; see; Jacentkovsky 1936, 1937; Drensky 1957; Lehrer, 1977; Verves 1986; Povolný \& Verves 1990; Whitmore 2011; Pape \& Beuk 2017.

Sarcophaga (Heteronychia) pauciseta Pandellé, 1896 - RW; 360-400 m; 1; wces; Jacentkovsky 1936, 1937; Drensky 1957; Verves 1986; Povolný \& Verves 1990; Pape \& Beuk 2017.

Sarcophaga (Heteronychia) pontica (Rohdendorf, 1937) [Pierretia] - BS; 0-5 m; 1; ee; Drensky 1957, 1960; Verves 1986; Povolný \& Verves 1990; Pape \& Beuk 2017.

Sarcophaga (Heteronychia) porrecta Bottcher, 1913 [Heteronychia (Eupierretia) bulgariensis Lehrer, 1977] - R1, R2; 520-2200 m; 1, 2, 3, 4, 5; se, m; Lehrer, 1977; Verves 1986; Povolný \& Verves 1990; Whitmore 2011; Pape \& Beuk 2017.

Sarcophaga (Heteronychia) proxima Rondani, 1860 [Pierretia] - DW, P2, K4, O61, O62; 30-1000 m; 1, 2; wesca; Drensky 1957; Verves 1986; Povolný \& Verves 1990; Pape \& Beuk 2017.

Sarcophaga (Heteronychia) pseudobenaci (Baranov, 1942) [Heteronychia (Heteronychia) drenskiana Lehrer, 1977] - V4; Eb; Lehrer 1977; Whitmore 2011.

? Sarcophaga (Heteronychia) rondaniana (Rohdendorf, 1937) [Pierretia] - O5, O62; 180-460 m; 1; e; Drensky 1957; Verves 1986; Povolný \& Verves 1990; Pape \& Beuk 2017.

Sarcophaga (Heteronychia) schineri Bezzi, 1891 [Pierretia] - P2, B1, R1; 200-1600 m; 1, 3, 4; e; Jacentkovsky 1936, 1937; Drensky 1957; Verves 1986; Povolný \& Verves 1990; Pape \& Beuk 2017.

Sarcophaga (Heteronychia) setinervis Rondani, 1860 - E1, S1; 130-270 m; 1; mwca; Jacentkovsky 1936, 1937; Verves 1986; Povolný \& Verves 1990; Pape \& Beuk 2017.

Sarcophaga (Heteronychia) siciliensis Böttcher, 1913 [Pierretia] - BS; 0-5 m; 1; sena; Drensky 1957; Verves 1986; Povolný \& Verves 1990; Pape \& Beuk 2017.

? Sarcophaga (Heteronychia) tenuiforceps Böttcher, 1913 [S. benaci var. tenuiforceps Böttcher, 1913; ? = Sarcophaga (Heteronychia) benaci Böttcher, 1913] - RW; 370-400 m; 1; se; Jacentkovsky 1936, 1937; Drensky 1957; Whitmore 2011. 
Sarcophaga (Heteronychia) vagans Meigen, 1826 [S. frenata Pandellé, 1896; Pierretia] - DW, DM, P1, P2, V1, TL, R1, RW, BN, BS; 0-1800 m; 1, 2, 3, 4; hoes; Nedelkov 1909, 1912; Jacentkovsky 1936, 1937; Drensky 1957; Verves 1986; Povolný \& Verves 1990; Beschovski 2006a; Pape \& Beuk 2017.

Sarcophaga (Heteronychia) vicina Macquart, 1835 [S. ebrachiata Pandellé, 1896; Pierretia] - K9, R2; 670-2400 m; 2, 5; e; Drensky 1957; Verves 1986; Povolný \& Verves 1990; Pape \& Beuk 2017.

Sarcophaga (Bercaea) africa (Wiedemann, 1824) [Coprosarcophaga haemorrhoidalis Fallén, 1817; Bercaea cruentata (Meigen, 1826); Pierretia] - $\mathbf{\Delta}$; ; DW, DM, E2, P2, B1, B3, K9, V1, V3, V4, S1, TL, O61, O62, R5, RW, RE, BN, BS; 0-1500 m; 1, 2, 3, 4; sk; Nedelkov 1909, 1912; Jacentkovsky 1936, 1937; Drensky 1955, 1957, 1960; Vesselinov \& Gabev 1956; Gregor \& Povolny 1959; Valerianov 1961; Lavčiev 1965a, 1965d, 1980; Lavčiev \& Jovčev 1978; Verves 1986; Povolný \& Verves 1990; Beschovski 2006a; Pape \& Beuk 2017. Sarcophaga (Kramerea) schuetzei Kramer, 1909 - E2; 10-30 m; 1; po; Drensky 1957; Verves 1986; Povolný \& Verves 1990; Pape \& Beuk 2017.

Sarcophaga (Pandelleana) protuberans Pandellé, 1896 - O62, R2, R3; 400-2200 m; 1, 2, 3, 4, 5; wesca; Drensky 1957; Verves 1986; Povolný \& Verves 1990; Pape \& Beuk 2017.

Sarcophaga (Liopygia) argyrostoma (Robineau-Desvoidy, 1830) [Parasarcophaga barbata (Thomson, 1869)] - V1, O62, R5, BN, BS; 0-600 m; 1; sk; Drensky 1957; Surbova 1965; Lavčiev 1980; Verves 1986; Povolný \& Verves 1990; Pape \& Beuk 2017.

Sarcophaga (Liopygia) crassipalpis Macquart, 1839 [Parasarcophaga securifera (Villeneuve, 1908)] - B2, O62, BN, BS; 0-1100 m; 1, 2, 3; k; Drensky 1957; Verves 1986; Povolný \& Verves 1990; Pape \& Beuk 2017.

Sarcophaga (Varirosellea) uliginosa Kramer, 1908 [Parasarcophaga] - E1, BN; $40-50$ m; 1; h, i; Drensky 1957; Verves 1986; Povolný \& Verves 1990; Pape \& Beuk 2017.

Sarcophaga (Liosarcophaga) aegyptica Salem, 1935 [Parasarcophaga parkeri Rohdendorf, 1937] - E1, P2, BN, BS; 0-250 m; 1; sppt; Drensky 1957; Verves 1986; Povolný \& Verves 1990; Pape \& Beuk 2017.

Sarcophaga (Liosarcophaga) emdeni (Rohdendorf, 1969) - O61; 400 m; 1; weswca; Gregor \& Povolny 1959; Verves 1986; Povolný \& Verves 1990; Pape 1996; Pape \& Beuk 2017.

Sarcophaga (Liosarcophaga) dux Thomson, 1869 [Parasarcophaga exuberans Pandellé, 1896; ? S. tuberosa var. exuberans Pandellé, 1896] - TL, O61, BN, BS; 0-400 m; 1; sppta; Jacentkovsky 1936, 1937; Drensky 1957; Povolný \& Verves 1990; Pape \& Beuk 2017.

Sarcophaga (Liosarcophaga) harpax Pandellé, 1896 [? S. tuberosa var. harpax Pandellé, 1896; Parasarcophaga] - DM, V1, S1, TL, R1, RW, BN; 0-1600 m; 1, 2, 3, 4; po; Jacentkovsky 1936, 1937; Drensky 1957; Verves 1986; Povolný \& Verves 1990; Pape \& Beuk 2017.

Sarcophaga (Liosarcophaga) jacobsoni (Rohdendorf, 1937) [S. tuberosa var. exuberans Pandellé, 1896; Parasarcophaga] - S1, O61, O62, R1, R3, R5, RW, BN; 0-400 m; 1; tp; Jacentkovsky 1936, 1937, 1939; Drensky 1957; Gregor \& Povolny 1959; Verves 1986; Povolný \& Verves 1990; Beschovski 2006a; Pape \& Beuk 2017.

Sarcophaga (Liosarcophaga) portschinskyi (Rohdendorf, 1937) [S. tuberosa var. harpax Pandellé, 1896; Parasarcophaga] - E1, S1, O61, BN, BS; 0-400 m; 1; po; Jacentkovsky 1939; Drensky 1957, 1960; Gregor \& Povolny 1959; Verves 1986; Povolný \& Verves 1990; Pape 1996; Pape \& Beuk 2017.

Sarcophaga (Liosarcophaga) teretirostris Pandellé, 1896 - E1, S1, RW; 120-500 m, 1; ena; Jacentkovsky 1936, 1937; Verves 1986; Pape 1996; Beschovski 2004a.

Sarcophaga (Liosarcophaga) tibialis Macquart, 1851 [Parasarcophaga beckeri (Villeneuve, 1908)] - TL, RW, RE, BN, BS; 0-400 m; 1; sppta; Jacentkovsky 1936, 1937; Drensky 1957; Povolný \& Verves 1990; Beschovski 2004a, 2006a.

Sarcophaga (Liosarcophaga) tuberosa Pandellé, 1896 - DW, DM, E1, E2, P1, B1, B3, S1, TL, O61, BS; 0-500 m; 1; ho; Jacentkovsky 1936, 1937; Drensky 1957, 1960; Verves 1986; Povolný \& Verves 1990; Pape 1996; Pape \& Beuk 2017.

Sarcophaga (Pandelleisca) similis Meade, 1876 [Parasarcophaga] - TL, T31, BS; 0-200 m; 1; po; Jacentkovsky 1936, 1937; Drensky 1957; Povolný \& Verves 1990; Pape 1996; Pape \& Beuk 2017.

Sarcophaga (Parasarcophaga) albiceps Meigen, 1826 - DW, DM, E1, E2, B1, B3, K8, K9, V1, V3, V4, S1, TL, T1, O61, R1, RW, RE, BN, BS; 0-1400 m; 1, 2, 3; hoa, ? poa; Nedelkov 1909, 1912; Jacentkovsky 1936, 1937; Drensky 1955, 1957; Gregor \& Povolny 1959; Povolný \& Verves 1990; Pape 1996; Beschovski 2004a; Pape \& Beuk 2017.

Sarcophaga (Parasarcophaga) hirtipes Wiedemann, 1830 - S1, R2; 360-450 m; 1; sppt, ? swppt; Povolný \& Verves 1990; Pape 1996; Pape \& Beuk 2017. 
Sarcophaga (Rosellea) aratrix Pandellé, 1896 [Parasarcophaga] - E1, V1, T31, BN, BS; 0-600 m; 1; h; Jacentkovsky 1936, 1937; Drensky 1957; Povolný \& Verves 1990; Pape \& Beuk 2017.

Sarcophaga (Robineauella) caerulescens Zetterstedt, 1838 [S. scoparia Pandellé, 1896; Parasarcophaga] - V4, R1; 520-1600 m; 1, 2, 3, 4; ho; Jacentkovsky 1936, 1937; Drensky 1957; Povolný \& Verves 1990; Pape \& Beuk 2017.

Sarcophaga (Sarcophaga) baranoffi Rohdendorf, 1937 [S. subvicina baranoffi Rohdendorf, 1937] - K9; se, ? Ebs; Drensky 1957; Povolný \& Verves 1990; Pape \& Beuk 2017.

Sarcophaga (Sarcophaga) bergi Rohdendorf, 1937 [S. subvicina ssp. bergi Rohdendorf, 1937] - S1; 270-370 m; 1; em; Jacentkovsky 1936, 1937; Drensky 1957; Povolný \& Verves 1990; Pape \& Beuk 2017.

Sarcophaga (Sarcophaga) carnaria (Linnaeus, 1758) [S. subvicina vulgaris Rohdendorf, 1937; S. carnaria var. latipennis Jacentkovsky, 1937] - \$ DM, E2, P1, P2, B1, V1, V4, S21, S22, TL, RW, BS; 0-1480 m; 1, 2, 3, 4; esca, ? hno; Kovachev 1905; Nedelkov 1909, 1912; Jacentkovsky 1936, 1937, 1939; Drensky 1942, 1955, 1957, 1960; Buresch 1953a; Vesselinov \& Gabev 1956; Gregor \& Povolny 1959; Gospodinov 1963; Lavčiev 1965a, 1965d, 1980; Surbova 1965; Lavčiev \& Jovčev 1978; Verves 1986; Povolný \& Verves 1990; Pape 1996; Pape \& Beuk 2017.

Sarcophaga (Sarcophaga) lehmanni Müller, 1922 [S. carnaria meridionalis Rohdendorf, 1937] - DW, E2, TL, T31; 30-160 m; 1; wp; Jacentkovsky 1939; Drensky 1957; Verves 1986; Povolný \& Verves 1990; Pape \& Beuk 2017.

Sarcophaga (Sarcophaga) serbica Baranov, 1929 - S1; 260-300 m; 1; see; Jacentkovsky 1939; Drensky 1957; Verves 1986; Povolný \& Verves 1990; Pape \& Beuk 2017.

Sarcophaga (Sarcophaga) subvicina Rohdendorf, 1937 [S. vicina Villeneuve, 1899] - DM, E2, V4; 100-850 m; 1, 2; ewca; Jacentkovsky 1936, 1937; Drensky 1955, 1957; Verves 1986; Povolný \& Verves 1990; Pape \& Beuk 2017.

Sarcophaga (Sarcophaga) ukrainica Rohdendorf, 1937 - P1; 580-600 m; 1; see; Povolný \& Verves 1990.

Sarcophaga (Sarcophaga) variegata (Scopoli, 1763) - R2; 600 m; 1; tp; Gregor \& Povolny 1959; Verves 1986; Povolný \& Verves 1990; Pape \& Beuk 2017.

Sarcophaga (Sarcophaga) vitoschana (Enderlein, 1936) [Sarcotachinella] - V4; 750-800 m; 2; Ebg; Enderlein 1936; Jacentkovsky 1936, 1937; Buresch 1953a; Drensky 1957; Pape 1996.

Sarcophaga balcanica (Enderlein, 1936) [Jacentkovskya] - S1; 276 m; 1; Ebg; Enderlein, 1936; Jacentkovsky 1936, 1937; Drensky 1957.

\section{Rhinophoridae}

Morinia doronici (Scopoli, 1763) [Anthracomyia melanoptera Fallén, 1817] - V1; 600 m; 1; e; Nedelkov 1912. Stevenia atramentaria (Meigen, 1824) [S. melania Meigen 1826] - V1, V4, TL, BN; 0-1000 m; 1, 2; ean; Löw 1862; Nedelkov 1912; Cerretti et al. 2020.

Stevenia lateralis (Macquart, 1849) - RW; 350-400 m; 1; sena; Jacentkovsky 1936, 1937.

Stevenia pannonica Villeneuve, 1919 - RW; 400 m; 1; see; Jacentkovsky 1937; Beschovski 2006a.

Stevenia signata (Mik, 1886) [S. femoralis Stein, 1924; not Rondani, 1862] - O61; 300-320 m; 1; nm, ? nem; Jacentkovsky 1937.

Stevenia umbratica (Fallén, 1820) - R1; 900-1100 m; 2, 3; ena; Jacentkovsky 1936, 1937.

Rhinophora lepida (Meigen, 1824) - e; Verves et al. 2019; Cerretti et al. 2020.

Phyto melanocephala (Meigen, 1824) - O61, RW; 300-400 m; 1; ena; Jacentkovsky 1937.

\section{Oestridae}

Oestrus ovis Linnaeus, 1758 - \; ; V1, TL, RE, BS; 0-1000 m; 1, 2; k; Nedelkov 1910, 1912; Drensky 1928, 1933, 1939a, 1960; Paschev 1937; Beschovski 2004a.

Rhinoestrus purpureus (Brauer, 1859) - A; V1, TL, BN, BS; 0-600 m; 1; ppt; Drensky 1933.

\section{Hypodermatidae}

Hypoderma actaeon Brauer, 1858 - А ; E1; 20-30 m; 1; csee; Drensky 1933. 
Hypoderma bovis (Linnaeus, 1758) - $\mathbf{\Delta}$; E2, V1, S1, R1, BS; 0-1300 m; 1, 2, 3; k; Raykov 1908; Nedelkov 1910, 1912; Drensky 1928, 1933, 1934c, 1955, 1960; Kitanov 1943; Zenginov 1949; Popov \& Bankov 1961. Hypoderma diana Brauer, 1858 - А ; E1, RW; 20-750 m; 1, 2; esca; Drensky 1933.

Hypoderma lineatum (Villers, 1789) - А; K9, S1; 200-600 m; 1; ho; Löw 1863; Drensky 1933; Zenginov 1949.

\section{Gasterophilidae}

Gasterophilus haemorrhoidalis (Linnaeus, 1758) - ৯; ; V1, O62, R1; 100-1400 m; 1, 2, 3; sk, ? k; Drensky 1932c, 1933.

Gasterophilus inermis (Brauer, 1858) - \ ; ; hat, ? pat; Drensky 1933.

Gasterophilus intestinalis (De Geer, 1776) - $\mathbf{\Delta}$; ; E2, V1, O62, R1; 20-1400 m; 1, 2, 3; k; Joakimoff 1899; Kovachev 1905; Nedelkov 1910, 1912; Drensky 1928, 1932c, 1933, 1934c, 1955.

Gasterophilus pecorum (Fabricius, 1794) - А; ; V1, O62, R1; 100-1400 m; 1, 2, 3; ppt; Drensky 1932c, 1933.

\section{Tachinidae}

Exorista (Exorista) larvarum (Linnaeus, 1758) [Tachina] - DW, DM, E2, P1, P2, B1, B3, V1, V4, S1, TL, T31, O5, R1, R2, R3, RW, RE, BN, BS; 0-1250 m; 1, 2, 3; ho; Tschorbadjiew 1925g, 1933; Burgess \& Crossman 1929; Drensky \& Zacharieva-Stoilova 1951; Drensky 1955; Karnožitzky 1957; Stefanov 1959; Dochkova 1971; Ganchev 1972, 1975, 1980; Mamoon 1978a, 1978b; Trenchev 1980d; Hubenov 1980b, 1983a, 1985a, 1988c, 1990, 1992a, 1992b, 1993a, 1993b, 1995a, 1995b, 2001b, 2004, 2006, 2008a, 2008b, 2015b, 2015c, 2016, 2017, 2018, 2019a; Zaharieva 1982, 1983; Tsankov 1985; Ziegler 1989; Georgiev 1996b; ZaharievaPentcheva \& Georgiev 1997.

Exorista (Exorista) segregata (Rondani, 1859) [E. fasciata (Fallén, 1820); Trycholyga] - DW, DM, E1, P1, P2, B1, B3, S1, TL, RE, BS, +++; 0-800 m; 1, 2; mca; Burgess \& Crossman 1929; Russkoff 1929-1930; Tschorbadjiew 1933; Karnožitzky 1957; Ganchev 1972, 1975; Mamoon 1978b; Hubenov 1985a, 1992b, 1993a, 1993b, 2008a, 2008b.

Exorista (Podotachina) grandis (Zetterstedt, 1844) [E. sorbillans (Wiedemann, 1830); Trycholyga] - B1, V1, V4, S1, O5, O62, R2, R3; 150-1100 m; 1, 2, 3; ei, ? wp, ess; Tschorbadjiew 1925g, 1928c; Keremidchiev 1954; Ganchev 1975; Mamoon 1978b; Hubenov 1985a, 1985b, 1992b, 1993a, 1993b, 1995a, 2008a, 2008b, 2015c, 2018, 2019a; Beschovski \& Hubenov 1986.

Exorista (Podotachina) sorbillans (Wiedemann, 1830) [Trycholyga grandis (Zetterstedt, 1844)] - V1, O5, O62, R2, R3; 200-1200 m; 1, 2, 3; sppta; Hubenov 1980b, 1985a, 1985b, 1988a, 1988b, 1988c, 1992a, 1992b, 1993a, 1993b, 1995a, 1996a, 2008a, 2008b, 2015b, 2015c, 2017; Beschovski \& Hubenov 1986.

Exorista (Thrycholyga) nova (Rondani, 1859) - V1, V4, BS; 550-1120 m; 1, 2, 3; mwca, ? wp; Lavčiev et al. 1977; Hubenov 1985a, 1992b, 1993a, 1993b, 2008a, 2008b, 2018.

Exorista (Ptilotachina) civilis (Rondani, 1859) - E1, V1, BS; 0-600 m; 1; spo, ? po; Hubenov 1977, 1980b, 1985a, 1992b, 1993a, 1993b, 2001b, 2008a, 2008b; Ziegler 1989.

Exorista (Ptilotachina) deligata Pandelle, 1896 [E. aberrans (Strobl, 1893); Trycholyga] - V1, S1; 260-600 m; 1; wp; Jacentkovsky 1936, 1937; Lavčiev et al. 1977; Hubenov 1985a, 1992b, 1993a, 1993b, 2008a, 2008b.

Exorista (Ptilotachina) xanthaspis (Wiedemann, 1830) [E. fallax Meigen 1824] - E2, V1, S1; 30-750 m; 1, 2; ppta; Nikolova 1972; Hubenov 1980b, 1985a, 1992b, 1993a, 1993b, 2008a, 2008b; Trenchev 1980d.

Exorista (Adenia) mimula (Meigen, 1824) [E. erucarum (Rondani, 1859); E nigricans (Egger, 1861); E. pratensis (Robineau-Desvoidy, 1830); Eutachina, Microtachina, Tachina] - B1, V1, V4, S1, TL, O5, O61, O62, R1, R2, R3, RW, RE, BS; 0-1300 m; 1, 2, 3; ho; Nedelkov 1912; Drensky 1931c; Tschorbadjiew 1933; Jacentkovsky 1936, 1937; Lavčiev et al. 1977; Zaharieva 1982, 1983; Hubenov 1983a, 1985a. 1985b, 1988a, 1988b, 1988c, 1992a, 1992b, 1993a, 1993b, 1995a, 2001b, 2004, 2006, 2008a, 2008b, 2015b, 2015c, 2016, 2017, 2018, 2019a; Beschovski \& Hubenov 1986.

Exorista (Adenia) rustica (Fallén, 1810) [Eutachina, Tachina] - E2, B1, V1, V4, S1, O3, O5, O61, O62, R1, R2, R3, RW, RE, BN, BS; 0-1600 m; 1, 2, 3; po; Jacentkovsky 1936, 1937; Belanovsky 1953; Drensky 1955; Nikolova 1946, 1972; Popov 1978; Hubenov 1985a, 1985b, 1988a, 1988b, 1988c, 1992a, 1992b, 1993a, 1993b, 1995a, 1995b, 1999, 2001a, 2004, 2006, 2008a, 2008b, 2015b, 2015c, 2016, 2017, 2018, 2019a; Beschovski \& Hubenov 1986. 
Neophryxe vallina (Rondani, 1861) - S23; 500 m; 1; csena; Tschorsnig et al. 2005, 2009; Hubenov 2008a, 2008b; Lutovinovas et al. 2022.

Chetogena acuminata Rondani, 1859 [Spoggosia, Stomatomyia, Stomatomyiopsis] - B2, S1, O61, R1, RW; 2701350 m; 1, 2, 3; ppt; Jacentkovsky 1936, 1937; Belanovsky 1953; Hubenov 1992b, 1993a, 1993b, 1996b, 2006, 2008a, 2008b, 2016, 2017.

Chetogena fasciata (Egger, 1856) [Spoggosia] - S211; 750-900 m; 2; des, ? ess; Trenchev 1980d; Hubenov 1992b, 1993a, 1993b, 2008a, 2008b.

Chetogena filipalpis Rondani, 1859 [Stomatomyia] - V4, O5, R1, R2, R3, RW; 450-1600 m; 1, 2, 3; csewca; Jacentkovsky 1936, 1937; Hubenov 1988c, 1990, 1992a, 1992b, 1993a, 1993b, 1995a, 2006, 2008a, 2008b, 2015b, 2015c, 2016, 2017, 2018.

Chetogena nigrofasciata (Strobl, 1902) - swpat; O'Hara et al. 2020.

Chetogena obliquata (Fallén, 1810) [Spoggosia echinura (Robineau-Desvoidy, 1830)] - B1, B2, V4, S211, O5, R2, R3, R4, RW, RE; 500-1200 m; 1, 2, 3; wcp, ? tp; Hubenov 1980a, 1988c, 1992a, 1992b, 1993a, 1993b, 1995a, 2004, 2006, 2008a, 2008b, 2015b, 2015c, 2017, 2018; Trenchev 1980d.

Diplostichus janitrix (Hartig, 1838) [Chetogena] - TL, RE; 200 m; 1; des; Georgiev 1996a; Hubenov 2001b, 2004, 2008a, 2008b, 2018; O'Hara et al. 2020.

Parasetigena silvestris (Robineau-Desvoidy, 1863) [P. agilis (Stein, 1924), not Robineau-Desvpidy, 1830; $P$. segregata Brauer and Bergenstamm, 1891, not Rondani, 1859; Phorocera] - DW, E1, P1, P2, B1, B3, V4, S1, O5, R1, R2, BS; 0-1400 m; 1, 2, 3, 4; h; Nedelkov 1912; Burgess \& Crossman 1929; Tschorbadjiew 1933; Keremidchiev 1951; Karnožitzky 1957; Stefanov \& Keremidchiev 1961; Ganchev 1972, 1975; Mamoon 1978a, 1978b; Trenchev 1980d; Hubenov 1985a, 1992a, 1992b, 1993a, 1993b, 1995a, 2008a, 2008b, 2015b, 2015c, 2016, 2017, 2018, 2019a.

Phorocera assimilis (Fallén, 1810) - DM, B1, V4, S211, O1, O5, O61, R1, R2, RW, BN; 0-1400 m; 1, 2, 3, 4; tp; Nedelkov 1912; Dochkova 1971; Zonati 1978; Trenchev 1980d; Hubenov 1985a, 1990, 1992a, 1992b, 1993a, 1993b, 1995a, 2001b, 2006, 2008a, 2008b, 2015b, 2015c, 2016, 2017, 2018, 2019a.

Phorocera grandis (Rondani, 1859) - V4, O5, R1, R2, RW; 350-1000 m; 1, 2; dpo; Hubenov 1980b, 1983a, 1985a, 1992a, 1992b, 1993a, 1993b, 1995a, 1996a, 2006, 2008a, 2008b, 2015b, 2015c, 2016, 2017, 2018.

Phorocera obscura (Fallén, 1810) - B1, V4, S211, O1, O5, O62, R1, R2, R3, R4, RW, RE; 200-1300 m; 1, 2, 3; des, ? dp; Lavčiev et al. 1977; Mamoon 1978b; Hubenov 1985a, 1985b, 1988a, 1988b, 1988c, 1992a, 1992b, 1993a, 1993b, 1995a, 1999, 2001a, 2004, 2006, 2008a, 2008b, 2015b, 2015c, 2016, 2017, 2018, 2019a; Beschovski \& Hubenov 1986.

Phorinia aurifrons Robineau-Desvoidy, 1830 - s; dpo; Tschorsnig et al. 2005, 2009; Hubenov 2008a, 2008b; O'Hara et al. 2020.

Bessa parallela (Meigen, 1824) [B. fugax (Rondani, 1861)] - E1, V1, S211, O5; 280-850 m; 1, 2; po; Ganchev 1977; Mamoon 1978b; Trenchev 1979, 1980d; Hubenov 1985a, 1992b, 1993a, 1993b, 1995a, 2001b, 2008a, 2008b, 2015c.

Bessa selecta (Meigen, 1824) - DW, V4, S211, O62, R2; 200-1300 m; 1, 2, 3; hoes; Popov 1956; Trenchev 1979, 1980d; Hubenov 1985a, 1985b, 1988a, 1988b, 1992a, 1992b, 1993a, 1993b, 2001b, 2008a, 2008b, 2015b, 2017; Beschovski \& Hubenov 1986.

Belida angelicae (Meigen, 1824) [Aporotachina] - V4, R2, R3; 1250 m; 3; tp; Hubenov 1980a, 1988c, 1992a, 1992b, 1993a, 1993b, 2008a, 2008b, 2015b, 2015c, 2017, 2018.

Meigenia dorsalis (Meigen, 1824) [M. pilosa Baranov, 1926] - B1, V1, V4, S1, S211, O5, O61, O62, R1, R2, R3, R5, RW, BN, BS; 0-1600 m; 1, 2, 3, 4; po; Jacentkovsky 1936, 1937; Trenchev 1980d; Hubenov 1985b, 1988a, 1988b, 1988c, 1990, 1992a, 1992b, 1993a, 1993b, 1995a, 2006, 2008a, 2008b, 2015b, 2015c, 2016, 2017, 2018, 2019a; Beschovski \& Hubenov 1986.

Meigenia incana (Fallén, 1810) - T31; 70-100 m; 1; po; Jacentkovsky 1936, 1937; Hubenov 1992b, 1993a, 1993b, 2008a, 2008b.

Meigenia majuscula (Rondani, 1859) - R1; 1100 m; 3; po; Jacentkovsky 1936, 1937; Nikolova 1972; Hubenov 1992b, 1993a, 1993b, 2008a, 2008b, 2016, 2017.

Meigenia mutabilis (Fallén, 1810) [M. bisignata (Meigen, 1824)] - B1, V1, V4, S1, S211, TL, O5, O61, O62, R1, R2, R3, RW, RE, BN, +++; 50-2400 m; 1, 2, 3, 4, 5; wcp; Jacentkovsky 1936, 1937; Gruev 1969, 1972, 1973; Kaytazov 1971b; Tomov 1974; Lavchiev et al. 1977; Trenchev 1980d; Hubenov 1985a, 1988c, 1990, 
1992a, 1992b, 1993a, 1993b, 1995a, 1995b, 1996a, 1996b, 2004, 2006, 2008a, 2008b, 2015b, 2015c, 2016, 2017, 2018, 2019a.

Meigenia uncinata Mesnil, 1967 - O61, O62; 200-400 m; 1; wces; Tomov 1971, 1974; Hubenov 1985a, 1985b, 1988a, 1988b, 1992b, 1993a, 1993b, 1999, 2001a, 2008a, 2008b; Beschovski \& Hubenov 1986.

Conogaster pruinosa (Meigen, 1824) - B1; 740-1400 m; 2, 3; csess, ? esca; Hubenov 1977, 1992b, 1993a, 1993b, 2008a, 2008b.

Zaira cinerea (Fallén, 1810) [Viviania] - P1, B1, V4, S1, O5, O62, R2, R3, RW, RE, BN; 100-1400 m; 1, 2, 3; tp; Jacentkovsky 1936, 1937; Trenchev 1980d; Hubenov 1985b, 1988a, 1988b, 1988c, 1990, 1992a, 1992b, 1993a, 1993b, 1995a, 2004, 2006, 2008a, 2008b, 2015b, 2015c, 2017, 2018, 2019a; Beschovski \& Hubenov 1986.

Gastrolepta anthracina (Meigen, 1826) [Medoria] - S1;260-300 m; 1; wp; Jacentkovsky 1936, 1937; Belanovsky 1953; Hubenov 1992b, 1993a, 1993b, 2008a, 2008b.

Steleoneura czernyi Stein, 1924 [Villeneuvenia elegans Jacentkovsky, 1937] - R1; 350-500 m; 1; mca; Jacentkovsky 1937; Hubenov 1992b, 1993a, 1993b, 2008a, 2008b, 2016, 2017.

Medina collaris (Fallén, 1820) - V4, R2; 700-900 m; 2; po; Hubenov 1983a, 1992a, 1992b, 1993a, 1993b, 2008a, 2008b, 2015b, 2017, 2018.

Medina luctuosa (Meigen, 1824) [Degeeria] - V4, O5, R1, R2; 700-1300 m; 1, 3; po; Jacentkovsky 1936, 1937; Belanovsky 1953; Hubenov 1992a, 1992b, 1993a, 1993b, 1995a, 2008a, 2008b, 2015b, 2015c, 2016, 2017, 2018.

Medina separata (Meigen, 1824) - tes; Tschorsnig et al. 2005, 2009; Hubenov 2008a, 2008b; O'Hara et al. 2020.

Istocheta cinerea (Macquart, 1850) - c csena, ? hom, swp; Tschorsnig et al. 2005, 2009; Hubenov 2008a, 2008b; O'Hara et al. 2020.

Lecanipa bicincta (Meigen, 1824) - V4, O5, R2; 800-1400 m; 2, 3; wces; Hubenov 1982a, 1992a, 1992b, 1993a, 1993b, 1995a, 2008a, 2008b, 2015b, 2015c, 2017, 2018.

Lecanipa leucomelas (Meigen, 1824) - O5; 800-1300 m; 2, 3; e; Hubenov 1982a, 1992b, 1993a, 1993b, 1995a, 2008a, 2008b, 2015c.

Admontia grandicornis (Zetterstedt, 1849) - B2, R2; 730-2000 m; 2, 3, 4; ho; Lutovinovas et al. 2022.

Admontia maculisquama (Zetterstedt, 1859) [Trichoparia] - V4, O5; 720-1900 m; 2, 3, 4; des; Hubenov 1982a, 1992b, 1993a, 1993b, 1995a, 2008a, 2008b, 2015c, 2018.

Admontia podomyia Brauer \& Bergenstamm, 1889 [Trichoparia] - B2, O5, R1, R2; 720-2300 m; 2, 3, 4, 5; dpo, bm; Hubenov 1982a, 1992a, 1992b, 1993a, 1993b, 1995a, 1996a, 1996b, 2008a, 2008b, 2015b, 2015c, 2016, 2017.

Oswaldia muscaria (Fallén, 1810) - B1, V4, R2; 1100-1250 m; 3; dpo; Hubenov 1990, 1992a, 1992b, 1993a, 1993b, 2008a, 2008b, 2015b, 2017, 2018, 2019a.

Oswaldia spectabilis (Meigen, 1824) [O. albisquama (Zetterstedt, 1844)] - B1, V1, V4, O5, R1, R2, RW; 8501400 m; 2, 3; wes; Hubenov 1977, 1990, 1992a, 1992b, 1993a, 1993b, 1995a, 2006, 2008a, 2008b, 2015b, 2015c, 2016, 2017, 2018, 2019a.

Lomachantha parra Rondani, 1859 - V4, R2, R3; 1100-1400 m; 3; ? wp; Hubenov 1980a, 1988c, 1992a, 1992b, 1993a, 1993b, 2008a, 2008b, 2015b, 2015c, 2017, 2018.

Ligeria angusticornis (Loew, 1847) - \$; des; Tschorsnig et al. 2005, 2009; Hubenov 2008a, 2008b; O’Hara et al. 2020.

Picconia incurva (Zetterstedt, 1844) - \$; wcp; Tschorsnig et al. 2005, 2009; Hubenov 2008a, 2008b; O’Hara et al. 2020.

Erynniopsis antennata (Rondani, 1861) [E. rondanii Townsend, 1926] - B1, V1, V4, TL, O5, O62, R1, R2, RW, RE; 150-1200 m; 1, 2, 3; mt, h*, i; Tomov 1971, 1974; Lavchiev et al. 1977; Hubenov 1985a, 1985b, 1988a, 1988b, 1992a, 1992b, 1993a, 1993b, 1995a, 1999, 2001a, 2001b, 2004, 2006, 2008a, 2008b, 2015b, 2015c, 2016, 2017, 2018, 2019a; Beschovski \& Hubenov 1986; Tomov \& Mitov 1987.

Ligeriella aristata (Villeneuve, 1911) - V1, O61; 330-700 m; 1, 2; eca; Lavchiev et al. 1977; Hubenov 1985a, 1992b, 1993a, 1993b, 2008a, 2008b.

Blondelia inclusa (Hartig, 1838) - DW, P1; 40-250 m; 1; po, ? dpo; Mamoon 1978b; Trenchev 1980d; Hubenov 1985a, 1992b, 1993a, 1993b, 2008a, 2008b.

Blondelia nigripes (Fallén, 1810) [Ceromasia] - \$ DW, DM, E1, E2, P1, P2, B1, B2, B3, V1, V3, V4, S1, S211, O5, O61, O62, R1, R2, R3, RW, RE, BN; 0-2400 m; 1, 2, 3, 4, 5; po, h*, i; Jacentkovsky 1936, 1937; Ganchev 1972, 1975, 1977, 1980; Nikolova 1972; Mamoon 1978a, 1978b; Zonati 1978; Trenchev 1980d; Hubenov 
1983a, 1985a, 1985b, 1988a, 1988b, 1988c, 1990, 1992a, 1992b, 1993a, 1993b, 1995a, 1995b, 1996a, 1996b, 1999, 2001a, 2001b, 2004, 2006, 2008a, 2008b, 2015b, 2015c, 2016, 2017, 2018, 2019a; Beschovski \& Hubenov 1986; Mirchev et al. 1999.

Compsilura concinnata (Meigen, 1824) - $\$$ DW, E1, E2, P1, P2, B1, B2, B3, V1, V4, S1, TL, O5, O61, O62, R1, R2, R3, RW, RE, BN, BS; 0-1400 m; 1, 2, 3; hpta, sk, i; Russkoff 1929-1930; Tschorbadjiew 1933; Jacentkovsky 1936, 1937; Keremidchiev 1954, 1965; Karnožitzky 1957; Stefanov et al. 1958; Stefanov 1959; Ganchev 1972, 1975, 1980; Mamoon 1978a, 1978b; Zonati 1978; Hubenov 1980b, 1983a, 1985a, 1985b, 1990, 1988a, 1988b, 1988c, 1992a, 1992b, 1993a, 1993b, 1995a, 1995b, 1999, 2001a, 2001b, 2004, 2006, 2008a, 2008b, 2015b, 2015c, 2016, 2017, 2018, 2019a; Trenchev 1980d; Zaharieva 1982, 1983; Tsankov 1985; Beschovski \& Hubenov 1986; Georgiev 1996b; Zaharieva-Pencheva \& Georgiev 1997; Mirchev et al. 1999.

Vibrissina debilitata (Pandellé, 1896) - B2; 1160 m; 3; dpo; Tschorsnig et al. 2005, 2009; Hubenov 2008a, 2008b; O'Hara et al. 2020; Lutovinovas et al. 2022.

Vibrissina turrita (Meigen, 1824) - V4, S1, R2; 700-1400 m; 2, 3; dpo; Nikolova 1972; Hubenov 1985a, 1990, 1992a, 1992b, 1993a, 1993b, 1996a, 2008a, 2008b, 2015b, 2017, 2018.

Acemya acuticornis (Meigen, 1824) - V4, R2, R3; 900-1350 m; 2, 3; tp; Hubenov 1980a, 1988c, 1992a, 1992b, 1993a, 1993b, 2008a, 2008b, 2015b, 2015c, 2017, 2018.

Acemya rufitibia (von Roser, 1840) - s; des; Tschorsnig et al. 2005, 2009; Hubenov 2008a, $2008 \mathrm{~b}$.

Ceracia mucronifera Rondani, 1865 - s; ptm; Tschorsnig et al. 2005, 2009; Hubenov 2008a, 2008 b.

Ethilla aemula (Meigen, 1824) [Aplomyia] - B3; 500 m; 1; wcp; Jacentkovsky 1936, 1937; Hubenov 1992b, 1993a, 1993b, 2008a, 2008b.

Paratryphera barbatula (Rondani, 1859) [P. hirtipilis (Pandelé, 1896); Chaetina, Exorista] - B3, S1; 250-300 m; 1; po; Jacentkovsky 1936, 1937; Belanovsky 1953; Hubenov 1992b, 1993a, 1993b, 2008a, 2008 b.

Atylomyia loewii Brauer, 1898 - s; tp; Tschorsnig et al. 2005, 2009; Hubenov 2008a, 2008b; O’Hara et al. 2020.

Smidtia amoena (Meigen, 1824) [Nemosturmia, Timavia] - V1, V4, O5, O62, R1, R2, R3, RW, BS; 0-1230 m; 1, 2, 3; po; Lavchiev et al. 1977; Hubenov 1985b, 1988a, 1988b, 1988c, 1992a, 1992b, 1993a, 1993b, 1995a, 1999, 2001a, 2006, 2008a, 2008b, 2015b, 2015c, 2016, 2017, 2018.

Smidtia conspersa (Meigen, 1824) - dp; Tschorsnig et al. 2005, 2009; Hubenov 2008a, 2008b; O’Hara et al. 2020.

Winthemia cruentata (Rondani, 1859) [Tachina] - V1, V4, RW; 600-1200 m; 1, 2, 3; dp, ? tp; Nedelkov 1912; Hubenov 1990, 1992b, 1993a, 1993b, 2006, 2008a, 2008b, 2018.

Winthemia erythrura (Meigen, 1838) - B1, V1, V4; 300-900 m; 1, 2; wces; Nedelkov 1912; Trenchev 1980d; Hubenov 1990, 1992b, 1993a, 1993b, 2008a, 2008b, 2018.

Winthemia quadripustulata (Fabricius, 1794) - DW, B1, B3, V1, V4, S1, O5, R1, R2; 30-1400 m; 1, 2, 3; ho; Popoff 1934; Jacentkovsky 1936, 1937; Dochkova 1871, 1972; Nikolova 1972; Mamoon 1978b; Trenchev 1980d; Hubenov 1985a, 1990, 1992a, 1992b, 1993a, 1993b, 1995a, 1996a, 2008a, 2008b, 2015b, 2015c, 2016, 2017, 2018, 2019a.

Winthemia variegata (Meigen, 1824) - K4; 800-1000 m; 2; e; Hubenov 1980b, 1992b, 1993a, 1993b, $2008 \mathrm{a}, 2008 \mathrm{~b}$.

Nemorilla floralis (Fallén, 1810) - V1, V4, S1, TL, O5, O62, R2, RE, BN; 0-1200 m; 1, 2, 3; pat, ? hat; Jacentkovsky 1936, 1937; Nikolova 1967, 1972; Dochkova 1871, 1972; Mamoon 1978b; Trenchev 1980d; Ivanov \& Savov 1982; Hubenov 1985a, 1985b, 1988a, 1988b, 1992a, 1992b, 1993a, 1993b, 1995a, 2001b, 2004, 2008a, 2008b, 2015b, 2015c, 2017, 2018; Beschovski \& Hubenov 1986; Velcheva et al. 2010.

Nemorilla maculosa (Meigen, 1824) - s; po; Ganchev 1977; Mamoon 1978b; Hubenov 1992b, 1993a, 1993b, 2008a, 2008b; O'Hara et al. 2020.

Aplomya confinis (Fallén, 1820) - E1, P1, B1, B2, V1, V4, S1, O5, O61, O62, R1, R2, R3, RW, RE; $100-1800$ m; 1, 2, 3, 4; ppt; Nedelkov 1912; Jacentkovsky 1936, 1937; Lavchiev et al. 1977; Mamoon 1978b; Hubenov 1985a, 1985b, 1988a, 1988b, 1988c, 1990, 1992a, 1992b, 1993a, 1993b, 1995a, 1996b, 1999, 2001a, 2004, 2006, 2008a, 2008b, 2015b, 2015c, 2016, 2017, 2018, 2019a; Beschovski \& Hubenov 1986.

Phebellia nigripalpis (Robineau-Desvoidy, 1847) [Exorista agnata Rondani, 1859; Prooppia] - V4, S22, TL, O5, R2, R3, RW, RE; 200-1400 m; 1, 2, 3; h; Nedelkov 1912; Hubenov 1988c, 1990, 1992a, 1992b, 1993a, 1993b, 1995a, 2004, 2006, 2008a, 2008b, 2015b, 2015c, 2017, 2018; O’Hara et al. 2020.

Phebellia pauciseta (Villeneuve, 1908) - e; Tschorsnig et al. 2005, 2009; Hubenov 2008a, 2008b; O’Hara et al. 2020.

Ptesiomyia alacris (Meigen, 1824) [Exorista crinita Rondani, 1859] - S22, R3, R4; 1150-1500 m; 3; e; Nedelkov 1909; Hubenov 1988c, 1992b, 1993a, 1993b, 2008a, 2008b, 2015c. 
Nilea hortulana (Meigen, 1824) [Platymyia] - B1, V1, R1, RW, BS; 0-1000 m; 1, 2; hoes; Lavchiev et al. 1977; Hubenov 1985a, 1992b, 1993a, 1993b, 2006, 2008a, 2008b.

Nilea innoxia Robineau-Desvoidy, 1863 - S1; 480 m; 1; dp; Lutovinovas et al. 2022.

Tlephusa cincinna (Rondani, 1859) [Hubneria] - V1, V4, R2, R3, RW; 600-1600 m; 1, 2, 3, 4; ess; Jacentkovsky 1936, 1937; Hubenov 1988c, 1990, 1992a, 1992b, 1993a, 1993b, 2008a, 2008b, 2015b, 2015c, 2017, 2018.

Epicampocera succincta (Meigen, 1824) - B1, V1, V4, O5, R1, R2, R3, 600-1500 m; 2, 3; po; Jacentkovsky 1936, 1937; Belanovsky 1953; Hubenov 1988c, 1990, 1992a, 1992b, 1993a, 1993b, 1995a, 2008a, 2008b, 2015b, 2015c, 2016, 2017, 2018, 2019a.

Buquetia musca Robineau-Desvoidy, 1847 - BN; 0-10 m; 1; po; Hubenov 1980b, 1985a, 1992b, 1993a, 1993b, 2008a, 2008b.

Phryxe erythrostoma (Hartig, 1838) - RW; 1240 m; 3; wes; Hubenov 1980b, 1992b, 1993a, 1993b, 2006, 2008a, $2008 \mathrm{~b}$.

Phryxe magnicornis (Zetterstedt, 1838) [Ph. longicauda Wainwright, 1940] - DW, TL, O61; 70-380 m; 1; hoes; Mamoon 1978b; Trenchev 1980d; Hubenov 1985a, 1992b, 1993a, 1993b, 2008a, 2008b.

Phryxe nemea (Meigen, 1824) - B1, V1, V4, V5, TL, O1, O5, O62, R1, R2, R3, R4, RW, RE, BN; 150-1950 m; 1, 2, 3, 4; tp; Jacentkovsky 1936, 1937; Dochkova 1971, 1972; Mamoon 1978b; Trenchev 1980d; Hubenov 1985a, 1985b, 1988a, 1988b, 1988c, 1990, 1992a, 1992b, 1993a, 1993b, 1995a, 1995b, 1999, 2001a, 2001b, 2004, 2006, 2008a, 2008b, 2015b, 2015c, 2016, 2017, 2018, 2019a; Beschovski \& Hubenov 1986.

Phryxe prima (Brauer \& Bergenstamm, 1889) - V4, O5, R2, R3, RE; 700-1120 m; 2, 3; mwca; Hubenov 1980a, 1988c, 1992a, 1992b, 1993a, 1993b, 1995a, 2004, 2008a, 2008b, 2015b, 2015c, 2017, 2018.

Phryxe unicolor (Villeneuve, 1908) - e; O'Hara et al. 2020.

Phryxe vulgaris (Fallén, 1810) - E2, B1, B2, V1, V4, S211, TL, O5, O62, R1, R2, R3, RW, RE, BN; 100-2000 m; 1, 2, 3, 4; ho; Nedelkov 1912; Drensky 1955; Ganchev 1977; Mamoon 1978b; Trenchev 1980d; Hubenov 1983a, 1985a, 1985b, 1988a, 1988b, 1988c, 1990, 1992a, 1992b, 1993a, 1993b, 1995a, 1995b, 1996a, 1996b, 1999, 2001a, 2001b, 2004, 2006, 2008a, 2008b, 2015b, 2015c, 2016, 2017, 2018, 2019a; Beschovski \& Hubenov 1986.

Periarchiclops scutellaris (Fallén, 1820) [Prosopaea] - R1, R3; 500-1100 m; 2, 3; wces; Jacentkovsky 1936, 1937; Belanovsky 1953; Mamoon 1978b; Hubenov 1988c, 1992b, 1993a, 1993b, 2008a, 2008b, 2015c, 2016, 2017.

Bactromyia aurulenta (Meigen, 1824) - S211; 720-900 m; 2; dp, ? des; Mamoon 1978b; Trenchev 1980d; Hubenov 1992b, 1993a, 1993b, 2008a, 2008b.

Pseudoperichaeta nigrolineata (Walker, 1853) [P. insidiosa (Robineau-Desvoidy, 1863); P. roseanae Brauer et Bergenstamm, 1891; P. major Brauer \& Bergenstamm, 1891; Zenillia] - DW, E1, B1, B3, V1, V4, R1, R2, R3, RW, RE; 40-1600 m; 1, 2, 3; po; Jacentkovsky 1937; Tsankov 1968, 1972, 1977, 1979, 1985; Hubenov 1980a, 1988c, 1992a, 1992b, 1993a, 1993b, 2001b, 2004, 2006, 2008a, 2008b, 2015b, 2015c, 2016, 2017, 2018, 2019a.

Pseudoperichaeta palesoidea (Robineau-Desvoidy, 1830) [P. trizonata (Zetterstedt, 1844); P. hirta (Bigot, 1880); Anoxycampta] - S1, O62, RW; 75-300 m; 1; wcp; Jacentkovsky 1936, 1937; Belanovsky 1953; Hubenov 1992b, 1993a, 1993b, 2001b, 2006, 2008a, 2008b.

Catagonia aberrans (Rondani, 1859) [Sisyropa] - O62; 250-300 m; 1; dpo; Hubenov 1982a, 1988a, 1988b, 1992b, 1993a, 1993b, 2008a, 2008b.

Lydella grisescens Robineau-Desvoidy, 1830 [L. senilis (Rondani, 1861), not Meigen, 1838] - P2, B2, V4, S1, BS; 0-850 m; 1, 2; po; Nedelkov 1912; Tschorbadjiew 1930b, 1931, 1939b; Hubenov 1985a, 1990, 1992b, 1993a, 1993b, 2001b, 2008a, 2008b.

Lydella stabulans (Meigen, 1824) [Tachina doris Meigena, 1824] - B1, V4, O5, R2, RW, RE; 350-1100 m; 1, 2, 3; esca; Nedelkov 1912; Hubenov 1983a, 1992a, 1992b, 1993a, 1993b, 1995a, 2004, 2006, 2008a, 2008b, 2015b, 2015c, 2017, 2018, 2019a.

Lydella thompsoni Herting, 1959 - DW, DM, O62; 100-150 m; 1; h, i; Trenchev 1980d; Hubenov 1985a, 1985b, 1988a, 1988b, 1992b, 1993a, 1993b, 2008a, 2008b; Beschovski \& Hubenov 1986.

Chetina setigena Rondani, 1856 [Ch. ambivius (Walker, 1849)] - B3, S1, O62; 100-500 m; 1; mwca; Jacentkovsky 1936, 1937; Hubenov 1985b, 1988a, 1988b, 1992b, 1993a, 1993b, 2008a, 2008b; Beschovski \& Hubenov 1986.

Cadurciella tritaeniata (Rondani, 1859) - V4, O5, O62, R2, RW, RE; 100-1450 m; 1, 2, 3, 4; dp; Hubenov 1983a, 1985b, 1988a, 1988b, 1992a, 1992b, 1993a, 1993b, 1995a, 1999, 2001a, 2004, 2006, 2008a, 2008b, 2015b, 2015c, 2017, 2018; Beschovski \& Hubenov 1986. 
Drino atropivora (Robineau-Desvoidy, 1830) - B1, V1, V4, O3, O5, O62, R1, R2, R3, RW, RE; 100-1300 m; 1, 2, 3; sppta; Lavchiev et al. 1977; Hubenov 1980b, 1983a, 1985a, 1985b, 1988a, 1988b, 1988c, 1992a, 1992b, 1993a, 1993b, 1995a, 1999, 2001a, 2004, 2006, 2008a, 2008b, 2015b, 2015c, 2016, 2017, 2018, 2019a; Beschovski \& Hubenov 1986.

Drino gilva (Hartig, 1838) [D. inconspicua (Meigen, 1830); Sturmia] - E1, S1, BS; 10-300 m; 1; dp, ? dpat; Tschorbadjiew 1925g, 1928c; Keremidchiev 1954; Karnožitzky 1957; Ganchev 1975; Mamoon 1978b; Hubenov 1985a, 1992b, 1993a, 1993b, 2008a, 2008b.

Drino inconspicua (Meigen, 1830) [D. bimaculata (Hartig, 1837); Sturmia] - DW, E1, P1, P2, B1, B2, B3, V4, TL, T1, T11, R1, R2, R3, R4, RW, RE, BN, BS; 0-1320 m; 1, 2, 3; po, ? ppt; Tschorbadjiew 1928c, 1928d; Keremidchiev 1954; Karnožitzky 1957; Stefanov \& Keremidchiev 1961; Ganchev 1972, 1975; Mamoon 1978b; Hubenov 1985a, 1988c, 1992a, 1992b, 1993a, 1993b, 2001b, 2004, 2006, 2008a, 2008b, 2015b, 2015c, 2016, 2017, 2018, 2019a; Tsankov 1985; Georgiev \& Bochev 1996.

Drino lota (Meigen, 1824) [Carcelia] - V1, V4, TL, O5, R2, R3, RE; 180-1300 m; 1, 2, 3; ppt; Nedelkov 1912; Hubenov 1988c, 1992a, 1992b, 1993a, 1993b, 1995a, 2004, 2008a, 2008b, 2015b, 2015c, 2017, 2018.

Drino vicina (Zetterstedt, 1849) [D. gyrovaga (Rondani, 1861); Hemimasicera, Sturmia] - V4, S1, O5, O61, R1, R2, R3; 200-1300 m; 1, 2, 3; wcp; Jacentkovsky 1936, 1937; Belanovsky 1953; Hubenov 1988c, 1992a, 1992b, 1993a, 1993b, 1995a, 2008a, 2008b, 2015b, 2015c, 2016, 2017, 2018.

Thelyconychia solivaga (Rondani, 1861) - DW, V1, BN; 30-600 m; 1; ppt; Lavchiev et al. 1977; Trenchev 1980d; Hubenov 1985a, 1992b, 1993a, 1993b, 2008a, 2008b.

Amelibaea tultschensis (Brauer \& Bergenstamm, 1891) - d dp; O'Hara et al. 2020.

Huebneria affinis (Fallén, 1810) [Exorista] - E2, B1, B2, V1, V4, S1, T31, O5, O62, R1, R2, R3, RW, RE, BN; 0-2400 m; 1, 2, 3, 4, 5; ess, ? wces; Nedelkov 1912; Jacentkovsky 1936, 1937; Mamoon 1978b; Popov 1978; Trenchev 1980d; Hubenov 1985a, 1985b, 1988a, 1988b, 1988c, 1992b, 1993a, 1993b, 1995a, 1996a, 1996b, 1999, 2001a, 2004, 2006, 2008a, 2008b, 2015b, 2015c, 2016, 2017, 2018, 2019a; Beschovski \& Hubenov 1986.

Tryphera lugubris (Meigen, 1824) - V1; 600-700 m; 1, 2; wcp; Hubenov 1993b, 2008a, 2008b.

Carcelia (Carcelia) bombylans Robineau-Desvoidy, 1830 - V4, R2; 1150-1800 m; 3, 4; po; Hubenov 1990, 1992a, 1992b, 1993a, 1993b, 2008a, 2008b, 2015b, 2017, 2018.

Carcelia (Carcelia) dubia (Brauer \& Bergenstamm, 1891) - BS; 0-10 m; 1; spo; Trenchev 1980d; Hubenov 1992b, 1993a, 1993b, 2008a, 2008b.

Carcelia (Carcelia) gnava (Meigen, 1824) [C. excavata (Zetterstedt, 1844)] - DW, E1, E2, P2, B1, B3, V1, V4, V5, S1, O5, R1, R2, RW, RE, BS; 0-1350 m; 1, 2, 3; dpo, h*; Tschorbadjiew 1925g, 1928c; Burgess \& Crossman 1929; Karnožitzky 1957; Ganchev 1972, 1975, 1980; Mamoon 1978b; Nikolova 1972; Zaharieva 1982, 1983; Hubenov 1983a, 1985a, 1992a, 1992b, 1993a, 1993b, 1995a, 2001b, 2004, 2006, 2008a, 2008b, 2015b, 2015c, 2016, 2017, 2018, 2019a.

Carcelia (Carcelia) iliaca (Ratzeburg, 1840) - S21, +++; 650-700 m; 2; cse; Tschorsnig et al. 2005, 2009; Hubenov 2008a, 2008b.

Carcelia (Carcelia) laxifrons Villeneuve, 1912 - DW, P1, V1; 40-600 m; 1; ho, h*; Tschorbadjiew 1924d, 1925g, 1933; Lavchiev et al. 1977; Mamoon 1978b; Trenchev 1980d; Hubenov 1985a, 1992b, 1993a, 1993b, 2008a, 2008b.

Carcelia (Carcelia) lucorum (Meigen, 1824) [C. cheloniae (Rondani, 1859)] - E2, P2, B1, K9, V1, V4, S1, O5, R1, R2, R3, RW, BN; 0-1400 m; 1, 2, 3; po; Jacentkovsky 1936, 1937; Drensky 1955; Lavchiev et al. 1977; Trenchev 1980d; Hubenov 1983a, 1985a, 1988c, 1992a, 1992b, 1993a, 1993b, 1995a, 2006, 2008a, 2008b, 2015b, 2015c, 2016, 2017, 2018, 2019a.

Carcelia (Carcelia) puberula Mesnil, 1941 - B2; 730 m; 2; dpo; Lutovinovas et al. 2022.

Carcelia rasa (Macquart, 1849) [C. amphion Robineau-Desvoidy, 1863] - \$; po; Popov 1956; Hubenov 1985a, 1992b, 1993a, 1993b, 2008a, 2008b.

Carcelia rasella Baranov, 1931 - dpo; Tschorsnig et al. 2005, 2009; Hubenov 2008a, 2008b; O’Hara et al. 2020.

Carcelia (Euryclea) falenaria (Rondani, 1859) - S1; 250-300 m; 1; swp; Jacentkovsky 1936, 1937; Belanovsky 1953; Hubenov 1992b, 1993a, 1993b, 2008a, 2008b.

Carcelia (Euryclea) tibialis (Robineau-Desvoidy, 1863) - V4, S211, O61; 350-1000 m; 1, 2; dpo; Dochkova 1971, 1972; Lavchiev et al. 1977; Trenchev 1980d; Hubenov 1980b, 1985a, 1990, 1992b, 1993a, 1993b, 2008a, 2008b, 2018. 
Carcelia (Carcelina) stackelbergi Mesnil, 1963 - DW, S1; 80-700 m; 1, 2; ee; Hubenov 1977, 1985a, 1992b, 1993a, 1993b, 2008a, 2008b; Trenchev 1980d.

Senometopia confundens (Rondani, 1859) [Carcelia, Eucarcelia] - DW, E1, E2, P2, B3; 40-350 m; 1; dpo; Ganchev 1972, 1975; Mamoon 1978b; Hubenov 1985a, 1992b, 1993a, 1993b, 2008a, 2008b.

Senometopia excisa (Fallén, 1820) - po; O'Hara et al. 2020.

Senometopia separata (Rondani, 1859) [Eucarcelia excisa var. separata (Rondani, 1859); Carcelia gnava (Meigen, 1824)] - E1, E2, S1, TL, T31, RW, BS; 20-380 m; 1; po; Tschorbadjiew 1933; Karnožitzky 1957; Ganchev 1975; Mamoon 1978b; Hubenov 1985a, 1992b, 1993a, 1993b, 2001b, 2006, 2008a, 2008b; Tsankov 1985.

Thecocarcelia acutangulata (Macquart, 1850) [T. incedens (Rondani, 1861)] - O5, O62, R3; 100-1100 m; 1, 2, 3; spat, ? dpat; Hubenov 1980b, 1985b, 1988a, 1988b, 1988c, 1992b, 1993a, 1993b, 1995a, 1996a, 2008a, 2008b, 2015c; Beschovski \& Hubenov 1986.

Erycia fatua (Meigen, 1824) - tes; Tschorsnig et al. 2005, 2009; Hubenov 2008a, 2008b.

Erycia fasciata Villeneuve, 1924 - B2; 1160 m; 3; wcp; Lutovinovas et al. 2022.

Erycia festinans (Meigen, 1824) - R2, R3; 800-1300 m; 2, 3; wces; Hubenov 1982a, 1988c, 1992a, 1992b, 1993a, 1993b, 2008a, 2008b, 2015b, 2015c, 2017.

Xylotachina diluta (Meigen, 1824) [X. ambulans (Rondani, 1861); X. ligniperdae Brauer et Bergenstamm, 1891; Lidella, Sturmia] - S211, TL, BS; 150-800 m; 1, 2; des; Dirimanov \& Sengalevich 1969; Sengalevich 1972; Trenchev 1980d; Hubenov 1985a, 1992b, 1993a, 1993b, 2008a, 2008b; Lutovinovas et al. 2022.

Alsomyia capillata (Rondani, 1859) [Platymyia] - B1, V4, O5, R2, R3, RW, RE; 400-1000 m; 1, 2; mwca, ? swp; Hubenov 1982a, 1988c, 1992a, 1992b, 1993a, 1993b, 1995a, 2004, 2006, 2008a, 2008b, 2015b, 2015c, 2017, 2018, 2019a.

Townsendiellomyia nidicola (Townsend, 1908) [Sturmia] - B1, V1; 550-800 m; 1, 2; nmwca, ? nmsws, swp, h*; Tschorbadjiew 1924d, 1925g, 1933; Lavchiev et al. 1977; Hubenov 1985a, 1992b, 1993a, 1993b, 2008a, 2008 b.

Platymya antennata (Brauer \& Bergenstamm, 1891) - V1, R3; 550-1100 m; 1, 2, 3; wcp; Hubenov 1980a, 1988c, 1992b, 1993a, 1993b, 2008a, 2008b, 2015c.

Platymya fimbriata (Meigen, 1824) [P. nemestrina (Meigen, 1824)] - DW, B1, B2, V4, O1, O5, R1, R2, RW; 80-2500 m; 1, 2, 3, 4, 5, 6; po, ? bm; Trenchev 1980d; Hubenov 1982a, 1993a, 1993b, 1985a, 1992a, 1992b, 1995a, 1996b, 2001b, 2006, 2008a, 2008b, 2015b, 2015c, 2016, 2017, 2018.

Eumea linearicornis (Zetterstedt, 1844) [E. westermanni (Zetterstedt, 1844); Platymyia] - DW, P1, B1, V1, V4, O5, R1, R2, R3, RW, RE; 70-1550 m; 1, 2, 3, 4; po; Mamoon 1978a, 1978b; Hubenov 1980a, 1985a, 1988c, 1992a, 1992b, 1993a, 1993b, 1995a, 2001b, 2004, 2006, 2008a, 2008b, 2015b, 2015c, 2016, 2017, 2018, 2019a; Trenchev 1980d.

Eumea mitis (Meigen, 1824) [Platymyia] - V1, R1; 530-1300 m; 1, 2, 3; hoes; Jacentkovsky 1936, 1937; Mamoon 1978b; Trenchev 1980d; Hubenov 1985a, 1992b, 1993a, 1993b, 2001b, 2008a, 2008b, 2016, 2017.

Myxexoristops blondeli (Robineau-Desvoidy, 1830) - DW, V1, V4; 80-800 m; 1, 2; po; Trenchev 1980d; Hubenov 1985a, 1992b, 1993a, 1993b, 2001b, 2008a, 2008b, 2018.

Myxexoristops stolida (Stein, 1924) - B2; 730; 2; des; Tschorsnig et al. 2005, 2009; Hubenov 2008a, 2008b; O'Hara et al. 2020; Lutovinovas et al. 2022.

Zenillia dolosa (Meigen, 1824) - V4, R1; 700-850 m; 2; po; Lavchiev et al. 1977; Hubenov 1985a, 1992b, 1993a, 1993b, 2001b, 2008a, 2008b, 2018.

Zenillia libatrix (Panzer, 1798) [Exorista] - DW, E1, E2, P1, B1, V1, V4, R1, RW, RE, BN; 0-1600 m; 1, 2, 3, 4; po; Nedelkov 1912; Tschorbadjiew 1924d, 1924g, 1933; Jacentkovsky 1936, 1937; Belanovsky 1953; Drensky 1955; Lavchiev et al. 1977; Mamoon 1978b; Zonati 1978; Trenchev 1980d; Hubenov 1985a, 1992b, 1993a, 1993b, 2001b, 2004, 2006, 2008a, 2008b, 2016, 2017, 2018, 2019a.

Calozenillia tamara (Portshinsky, 1884) [Tamaromyia] - BS; 0-5 m; 1; dp; Hubenov 1983a, 1992b, 1993a, 1993b, 1996a, 2008a, 2008b.

Clemelis pullata (Meigen, 1824) [Zenillia] - B1, B2, B3, V4, S1, O61, R1, R2; 150-2400 m; 1, 2, 3, 4, 5; wcp; Jacentkovsky 1936, 1937; Mamoon 1978b; Hubenov 1992a, 1992b, 1993a, 1993b, 1996a, 1996b, 2008a, 2008b, 2015b, 2016, 2017, 2018, 2019a.

Pales pavida (Meigen, 1824) [Ctenophorocera] - DW, DM, E1, P2, B1, V1, V4, S1, TL, O5, O61, O62, R1, R2, R3, RW, RE, BN, BS; 0-1400 m; 1, 2, 3; po; Tschorbadjiew 1924d, 1924g; Jacentkovsky 1936, 1937; Ganchev 1972, 1975; 1977, 1980; Nikolova 1972; Lavchiev et al. 1977; Mamoon 1978b; Trenchev 1980d; Zaharieva 1982, 1983; Hubenov 1983a, 1985a, 1985b, 1988a, 1988b, 1988c, 1990, 1992a, 1992b, 1993a, 1993b, 1995a, 
1995b, 1999, 2001a, 2001b, 2004, 2006, 2008a, 2008b, 2015b, 2015c, 2016, 2017, 2018, 2019a; Beschovski \& Hubenov 1986; Georgiev 1996b.

Pales pumicata (Meigen, 1824) - S1, O5, O62, R1, R2, RW, RE, BS; 0-1600 m; 1, 2, 3; nm, ? se; Jacentkovsky 1936, 1937; Hubenov 1985b, 1988a, 1988b, 1992a, 1992b, 1993a, 1993b, 1995a, 2004, 2008a, 2008b, 2015b, 2015c, 2016, 2017; Beschovski \& Hubenov 1986.

Phryno vetula (Meigen, 1824) - O1, O5, R2; 450-700 m; 1, 2; dpo; Hubenov 1982a, 1992a, 1992b, 1993a, 1993b, 1995a, 2001b, 2008a, 2008b, 2015b, 2015c, 2017.

Bothria frontosa (Meigen, 1824) [B. pascuorum (Rondani, 1859); B. obliquata Fallén, 1810] - B1, V1, V4, TL, R2; 200-1300 m; 1, 2, 3; tp, ? hoes; Nedelkov 1912; Hubenov 1990, 1992a, 1992b, 1993a, 1993b, 2008a, 2008b, 2015b, 2017, 2018, 2019a.

Ceromasia rubrifrons (Macquart, 1834) [C. florum (Macquart, 1850); Edesia, Lydella] - V4, R1, BS; 0-1400 m; 1, 2, 3; tp; Jacentkovsky 1936, 1937; Belanovsky 1953; Hubenov 1990, 1992b, 1993a, 1993b, 2008a, 2008b, 2016, 2017, 2018.

Allophorocera ferruginea (Meigen, 1824) [Erycilla] - B1, V1, V4, O5, R1, R2, RW; 600-1950 m; 2, 3, 4; des; Lavchiev et al. 1977; Trenchev 1980d; Hubenov 1990, 1992a, 1992b, 1993a, 1993b, 1995a, 2008a, 2008b, 2015b, 2015c, 2016, 2017, 2018, 2019a.

Allophorocera pachystyla (Macquart, 1850) [A. auripilla (Brauer et Bergenstamm, 1891)] - B2, V4, R1, R2; 1800-2000 m; 4; e, m; Hubenov 1980a, 1992a, 1992b, 1993a, 1993b, 1996a, 1996b, 2008a, 2008b, 2015b, 2016, 2017, 2018.

Ocytata pallipes (Fallén, 1820) [Rhacodineura] - V1; 550-600 m; 1; dp; Trenchev 1980d; Hubenov 1992b, 1993a, 1993b, 2008a, 2008b.

Erythrocera nigripes (Robineau-Desvoidy, 1830) [E. rubrifrons (Perris, 1852)] - B3, V1; 500-600 m; 2; des; Jacentkovsky 1936, 1937; Hubenov 1992b, 1993a, 1993b, 2008a, 2008b.

Eurysthaea scutellaris (Robineau-Desvoidy, 1848) [Discochaeta evonymellae Ratzeburg, 1848] - DW, E1, B1, V1, V4, S1, S211, TL, T1, O5, R1, R2, RW, RE, BN; 0-1100 m; 1, 2, 3; dpo; Tschorbadjiew 1925g, 1926c, 1933; Ganchev 1977; Lavchiev et al. 1977; Mamoon 1978b; Trenchev 1979, 1980d; Hubenov 1980b, 1083a, 1985a, 1992a, 1992b, 1993a, 1993b, 1995a, 1995b, 2001b, 2004, 2006, 2008a, 2008b, 2015b, 2015c, 2016, 2017, 2018, 2019a.

Erynnia ocypterata (Fallén, 1810) - dp; Tschorsnig et al. 2005, 2009; Hubenov 2008a, 2008b; O’Hara et al. 2020.

Elodia ambulatoria (Meigen, 1824) [E. convexifrons (Zetterstedt, 1844)] - O5, R2, BS; 0-1300 m; 1, 2, 3; tp, ? dp; Hubenov 1977, 1992a, 1992b, 1993a, 1993b, 1995a, 2008a, 2008b, 2015b, 2015c, 2017.

Elodia morio (Fallén, 1820) [E. tragica (Meigen, 1824)] - V1, V4, S211, TL; 200-1200 m; 1, 2, 3; tp; Ganchev 1977; Mamoon 1978b; Trenchev 1980d; Hubenov 1992b, 1993a, 1993b, 2008a, 2008b, 2018; Velcheva et al. 2012.

Sturmia bella (Meigen, 1824) - B1, V1, V4, O61, R1, R2, R3, RW, RE, BN; 20-1400 m; 1, 2, 3; poa; Jacentkovsky 1936, 1937; Belanovsky 1953; Mamoon 1978b; Hubenov 1980b, 1983a, 1985a, 1988c, 1992a, 1992b, 1993a, 1993b, 1996a, 2004, 2006, 2008a, 2008b, 2015b, 2015c, 2016, 2017, 2018, 2019a.

Blepharipa pratensis (Meigen, 1824) [B. scutellata (Robineau-Desvoidy, 1830); Sturmia; Blepharipoda, Crossocosmia] - DW, DM, E1, E2, P1, P2, B1, B3, V4, S1, O5, O62, R1, R2, RW, RE, BN, BS; 0-1400 m; 1, 2, 3; pat, hat, h*, i; Burgess \& Crosman 1929; Tschorbadjiew 1933; Keremidchiev 1951, 1965; Karnožitzky 1957; Keremidchiev 1959; Stefanov \& Keremidchiev 1961; Gospodinov 1963; Ganchev 1972, 1975; Mamoon 1978a, 1978b; Hubenov 1985a, 1985b, 1988a, 1988b, 1992a, 1992b, 1993a, 1993b, 1995a, 1995b, 1999, 2001a, 2001b, 2004, 2006, 2008a, 2008b, 2015b, 2015c, 2016, 2017, 2018, 2019a; Tsankov 1985; Beschovski \& Hubenov 1986.

Blepharipa schineri (Mesnil, 1939) - po; Tschorsnig et al. 2005, 2009; Hubenov 2008a, 2008b; O’Hara et al. 2020.

Masicera pavoniae (Robineau-Desvoidy, 1830) [M. pratensis Meigen, 1824] - B3, V1, S1, O3, R1, R2, R3; 3501000 m; 1, 2; wp; Lavchiev et al. 1977; Hubenov 1985a, 1988c, 1992a, 1992b, 1993a, 1993b, 2008a, 2008b, 2015b, 2015c, 2016, 2017.

Masicera silvatica (Fallén, 1810) - B1, V1, V4, O5, O62, R1, R2, RE; 100-1250 m; 1, 2, 3; wes; Jacentkovsky 1936, 1937; Hubenov 1985b, 1988a, 1988b, 1990, 1992a, 1992b, 1993a, 1993b, 1995a, 2004, 2008a, 2008b, 2015b, 2015c, 2016, 2017, 2018, 2019a; Beschovski \& Hubenov 1986.

Masicera sphingivora (Robineau-Desvoidy, 1830) [M. silvatica (Fallén, 1810)]-S1, BN; 0-500 m; 1; tp; Nikolova 1972; Hubenov 1985a, 1992b, 1993a, 1993b, 2008a, 2008b. 
Prosopea nigricans (Egger, 1861) - B1, V4, O5, O61, O62, R1, R2, R3, RW, RE; 100-1300 m; 1, 2, 3; tp; Jacentkovsky 1936, 1937; Belanovsky 1953; Hubenov 1983a, 1985a, 1985b, 1988a, 1988b, 1988c, 1992a, 1992b, 1993a, 1993b, 1995a, 2004, 2006, 2008a, 2008b, 2015b, 2015c, 2016, 2017, 2018, 2019a; Beschovski \& Hubenov 1986.

Gaedia connexa (Meigen, 1824) - V4, R2, R3; 700-1400 m; 2, 3; e; Nedelkov 1912; Hubenov 1988c, 1990, 1992a, 1992b, 1993a, 1993b, 2008a, 2008b, 2015b, 2015c, 2017, 2018.

Gaedia distincta Egger, 1861 - B1, V1, R2, R3, RW; 600-1350 m; 2, 3; wp, ? wes; Nedelkov 1912; Hubenov 1988c, 1992a, 1992b, 1993a, 1993b, 2006, 2008a, 2008b, 2015b, 2015c, 2017, 2019a.

Hebia flavipes Robineau-Desvoidy, 1830 - d dp, ? des; Tschorsnig et al. 2005, 2009; Hubenov 2008a, 2008b; O'Hara et al. 2020.

Thelymorpha marmorata (Fabricius, 1805) [Histochaeta] - V1, V4; 600-1000 m; 2; esca; Nedelkov 1912; Jacentkovsky 1936, 1937; Mamoon 1978b; Hubenov 1990, 1992b, 1993a, 1993b, 2008a, 2008b, 2018.

Baumhaueria goniaeformis (Meigen, 1824) - V1, V4, S1, R2; 290-1350 m; 1, 2, 3; wp; Nedelkov 1912; Tschorbadjiew 1928c; Zonati 1978; Trenchev 1980d; Hubenov 1985a, 1990, 1992a, 1992b, 1993a, 1993b, 2008a, 2008b, 2015b, 2017, 2018.

Gonia atra Meigen, 1826 [Redia] - O62; 150-200 m; 1; ho, ? spo; Hubenov 1980b, 1985b, 1988a, 1988b, 1992b, 1993a, 1993b, 2008a, 2008b; Beschovski \& Hubenov 1986.

Gonia bimaculata Wiedemann, 1819 [G. cilipeda Rondani, 1859; Salmacia] - P2, B1, B3, V1, V4, S1, O5, O61, O62, R1, R2, BN, BS; 0-1200 m; 1, 2, 3; sppt; Jacentkovsky 1936, 1937; Drensky 1942; Nikolova 1961; Hubenov 1985a, 1985b, 1988a, 1988b, 1992a, 1992b, 1993a, 1993b, 1995a, 1999, 2001a, 2008a, 2008b, 2015b, 2015c, 2016, 2017, 2018, 2019a; Beschovski \& Hubenov 1986.

Gonia capitata (De Geer, 1776) [G. trifaria Zeller, 1842] - B1, B2, V1, V4, S211, S22, O3, O5, R1, R2, R3, R5, RW, RE, BN; 500-2000 m; 1, 2, 3, 4; wcp, tp; Nedelkov 1909, 1912; Jacentkovsky 1936, 1937; Belanovsky 1953; Hubenov 1980b, 1985a, 1988c, 1990, 1992a, 1992b, 1993a, 1993b, 1995a, 1995b, 1996b, 2004, 2006, 2008a, 2008b, 2015b, 2015c, 2016, 2017, 2018, 2019a; Trenchev 1980d.

Gonia ornata Meigen, 1826 - V4, O62, R2, RW; 250-800 m; 1, 2; tp; Hubenov 1980a, 1985b, 1988a, 1988b, 1992a, 1992b, 1993a, 1993b, 2006, 2008a, 2008b, 2015b, 2017, 2018; Beschovski \& Hubenov 1986.

Gonia picea (Robineau-Desvoidy, 1830) - B1, V3, R2; 350-700 m; 1, 2; po; Hubenov 1977, 1992a, 1992b, 1993a, 1993b, 2008a, 2008b, 2015b, 2017, 2019a.

Onychogonia flaviceps (Zetterstedt, 1838) [Gonia] - S22; 600-1200; 1, 2, 3; h; Nedelkov 1909; Hubenov 1992b, 1993a, 1993b, 2008a, 2008b.

Pseudogonia parisiaca (Robineau-Desvoidy, 1851) [Isomera blondeli Robineau-Desvoidy, 1830; Gonia cognata Rongani, 1859] - B1, B3, V1, S1, S211, R2, R3, R5, BS; 0-1350 m; 1, 2, 3; ess, ? tes; Jacentkovsky 1936, 1937; Dochkova 1971, 1972; Trenchev 1980d; Hubenov 1985a, 1988c, 1992a, 1992b, 1993a, 1993b, 2008a, 2008b, 2015b, 2015c, 2017, 2019a.

Pseudogonia rufifrons (Wiedemann, 1830) [Gonia cinerascens Rongani, 1859; Isomera] - B1, V4, S1, O5, O62, R2, RE, BS; 0-1200 m; 1, 2, 3; ppta; Jacentkovsky 1936, 1937; Belanovsky 1953; Hubenov 1985b, 1988a, 1988b, 1992a, 1992b, 1993a, 1993b, 1995a, 2004, 2008a, 2008b, 2015b, 2015c, 2017, 2018, 2019a; Beschovski \& Hubenov 1986.

Spallanzania hebes (Fallen, 1820) [Cnephalia bucephala (Meigen, 1824)] - B1, V1, V4, S1, S211, S22, TL, O5, O61, O62, R1, R2, BN; 30-1000 m; 1, 2; hno; Nedelkov 1909, 1912; Jacentkovsky 1936, 1937; Dochkova 1971, 1972; Trenchev 1980d; Hubenov 1985a, 1985b, 1988a, 1988b, 1990, 1992a, 1992b, 1993a, 1993b, 1995a, 2008a, 2008b, 2015b, 2015c, 2016, 2017, 2019a; Beschovski \& Hubenov 1986.

Tachina (Eudoromyia) casta (Rondani, 1859) [T. lefebvrei (Robineau-Desvoidy, 1830) - S211, O5, O62, R1, R2, RE; 150-850 m; 1, 2; nm; Trenchev 1980a; Hubenov 1985b, 1988a, 1988b, 1992a, 1992b, 1993a, 1993b, 1995a, 2004, 2008a, 2008b, 2015b, 2015c, 2016, 2017, 2018; Beschovski \& Hubenov 1986.

Tachina (Tachina) corsicana (Villeneuve, 1931) [Eudoromyia] - O62; 180-200 m; 1; mca, ? sp; Hubenov 1982a, 1985b, 1988a, 1988b, 1992b, 1993a, 1993b, 1999, 2001a, 2008a, 2008b; Beschovski \& Hubenov 1986.

Tachina (Tachina) fera (Linnaeus, 1761) [Echinomyia, Eudoromyia] - ४; B1, K8, V1, V4, S1, S211, T31, O3, O5, O62, R1, R2, R3, RW, RE, BN; 100-1900 m; 1, 2, 3, 4; hop; Nedelkov 1909, 1912; Drenowsky 1936; Jacentkovsky 1936, 1937; Drensky 1942, 1955; Drensky \& Zacharieva-Stoilova 1951; Keremidchiev 1951; Nikolova 1972; Mamoon 1978b; Trenchev 1980a; Hubenov 1985a, 1985b, 1988a, 1988b, 1988c, 1990, 
1992a, 1992b, 1993a, 1993b, 1995a, 1995b, 1999, 2001a, 2004, 2006, 2008a, 2008b, 2015b, 2015c, 2016, 2017, 2018, 2019a; Beschovski \& Hubenov 1986.

Tachina (Tachina) grossa (Linnaeus, 1758) [Echinomyia] - E2, B1, V4, V5, S211, S22, T31, O5, R1, R2, RE, BN, +++; 0-1450 m; 1, 2, 3; tp; Löw 1862; Kovachev 1905; Jacentkovsky 1936, 1937; Belanovsky 1951; Mamoon 1978b; Hubenov 1990, 1992a, 1992b, 1993a, 1993b, 1995a, 2004, 2008a, 2008b, 2015b, 2015c, 2016, 2017, 2018, 2019a.

Tachina (Tachina) magna (Giglio-Tos, 1890) - S211, O62, R2; 100-850 m; 1, 2; sess; Trenchev 1980a; Hubenov 1985b, 1988a, 1988b, 1992a, 1992b, 1993a, 1993b, 2008a, 2008b, 2015b, 2017; Beschovski \& Hubenov 1986.

Tachina (Tachina) magnicornis (Zetterstedt, 1844) [T. tessellata (Fabricius, 1794); T. errans (Robineau-Desvoidy, 1830); T. vernalis (Robineau-Desvoidy, 1830); Echinomyia, Eudoromyia] - ; DM, E1, P1, P2, B1, B2, V1, V3, V4, S1, S21, S211, S22, TL, T31, O5, O61, O62, R1, R2, R3, RW, RE, BN, BS; 0-1900 m; 1, 2, 3, 4; hop; Joakimoff 1899; Nedelkov 1909, 1912; Ilchev 1923; Tschorbadjiew 1925g; Drensky 1930a; Jacentkovsky 1936, 1937; Drensky \& Zacharieva-Stoilova 1951; Lavchiev et al. 1977; Mamoon 1878b; Trenchev 1980a; Hubenov 1985a, 1985b, 1988a, 1988b, 1988c, 1990, 1992a, 1992b, 1993a, 1993b, 1995a, 1995b, 1999, 2001a, 2004, 2006, 2008a, 2008b, 2015b, 2015c, 2016, 2017, 2018, 2019a; Beschovski \& Hubenov 1986.

Tachina (Tachina) nupta (Rondani, 1859) [T. orientalis Zimin, 1967; Eudoromyia] - B1, V1, V3, V4, S211, O5, O61, O62, R1, R2, R3; 150-1750 m; 1, 2, 3, 4; po; Hubenov 1977, 1985b, 1988a, 1988b, 1992a, 1992b, 1993a, 1993b, 1995a, 1999, 2001a, 2008a, 2008b, 2015b, 2015c, 2016, 2017, 2018, 2019a; Trenchev 1980a; Beschovski \& Hubenov 1986.

Tachina (Tachina) praeceps Meigen, 1824 (Echinogaster, Echinomyia) - B1, V1, V4, S1, T31, O61, O62, R1, R2, R3, RW, RE, BS; 90-1300 m; 1, 2, 3; tp; Jacentkovsky 1936, 1937; Mamoon 1878b; Hubenov 1983a, 1985a, 1985b, 1988a, 1988b, 1988c, 1992a, 1992b, 1993a, 1993b, 2004, 2006, 2008a, 2008b, 2015b, 2015c, 2016, 2017, 2018, 2019a; Beschovski \& Hubenov 1986.

Tachina (Servillia) lurida (Fabricius, 1781) - B1, V4, S211, O62, R1, R2, RW; 100-1150 m; 1, 2, 3; wcp; Trenchev 1980a; Hubenov 1985b, 1988a, 1988b, 1992a, 1992b, 1993a, 1993b, 2006, 2008a, 2008b, 2015b, 2016, 2017, 2018, 2019a; Beschovski \& Hubenov 1986.

Schineria tergestina Rondani, 1859 - P1, B1; 300-400 m; 1; po; Nedelkov 1912; Hubenov 1992b, 1993a, 1993b, 2008a, 2008b.

Nowickia (Nowickia) marklini (Zetterstedt, 1838) [Tachina] - B2, V4, O5, R1, R2, R3; 1200-2300 m; 3, 4, 5; h, ? bm; Hubenov 1980a, 1988c, 1992a, 1992b, 1993a, 1993b, 1995a, 1996a, 1996b, 2008a, 2008b, 2015b, 2015c, 2016, 2017, 2018.

Nowickia (Fabriciella) atripalpis (Robineau-Desvoidy, 1863) [Fabriciella, Tachina] - B2, V4, O5, R1, R2, RW; 1150-2250 m; 3, 4, 5; po, ? bm; Hubenov 1980a, 1992a, 1992b, 1993a, 1993b, 1995a, 1996a, 1996b, 2006, 2008a, 2008b, 2015b, 2015c, 2017, 2018.

Nowickia (Fabriciella) ferox (Panzer, 1809) [Tachina] - B1, B3, V4, O5, R1, R2, R3, RW; 900-2300 m; 2, 3, 4, 5; wces; Nedelkov 1912; Jacentkovsky 1936, 1937; Hubenov 1988c, 1990, 1992a, 1992b, 1993a, 1993b, 1995a, 2006, 2008a, 2008b, 2015b, 2015c, 2016, 2017, 2018, 2019a.

Nowickia (Fabriciella) rondanii (Giglio-Tos, 1890) [F. nigricornis Robineau-Desvoidy, 1863; Tachina] - O5, R1, R2, R3; 700-1600 m; 2, 3, 4; spo; Hubenov 1980a, 1988c, 1992a, 1992b, 1993a, 1993b, 1995a, 2008a, 2008b, 2015b, 2015c, 2016, 2017.

Cnephaotachina danilevskyi (Portshinsky, 1882) [Nowickia, Tachina] - R2, R3; 350-1000 m; 1, 2; mca; Hubenov 1982a, 1985b, 1988a, 1988b, 1988c, 1992a, 1992b, 1993a, 1993b, 2008a, 2008b, 2015b, 2015c, 2017; Beschovski \& Hubenov 1986.

Peleteria abdominalis Robineau-Desvoidy, 1830 - B2, O5, O62, R2, R3; 200-1400 m; 1, 2, 3; nmi; Hubenov 1980a, 1985b, 1988a, 1988b, 1988c, 1992a, 1992b, 1993a, 1993b, 1995a, 1996b, 1999, 2001a, 2008a, 2008b, 2015b, 2015c, 2017; Beschovski \& Hubenov 1986.

Peleteria ferina (Zetterstedt, 1844) - B1, V1, V4, TL, O3, O5, O62, R2, R3, RW; 180-1300 m; 1, 2, 3; tes; Nedelkov 1912; Hubenov 1985b, 1988a, 1988b, 1988c, 1992a, 1992b, 1993a, 1993b, 1995a, 1999, 2001a, 2006, 2008a, 2008b, 2015b, 2015c, 2017, 2018, 2019a; Beschovski \& Hubenov 1986.

Peleteria iavana (Wiedemann, 1819) - p pta; O'Hara et al. 2020.

Peleteria prompta (Meigen, 1824) [Echinomyia] - S1; 250-300 m; 1; ess, ? m; Löw 1863; Hubenov 1992b, 1993a, 1993b, 1996a, 2008a, 2008b. 
Peleteria rubescens (Robineau-Desvoidy, 1830) [P. nigricornis (Meigen, 1838)] - E1, B1, V1, V4, S1, S21, S211, TL, T31, O3, O5, O61, O62, R1, R2, R3, RW, RE, BN; 150-2000 m; 1, 2, 3, 4; tp; Jacentkovsky 1936, 1937; Drensky 1942; Hubenov 1980a, 1980b, 1985a, 1985b, 1988a, 1988b, 1988c, 1990, 1992a, 1992b, 1993a, 1993b, 1995a, 1995b, 1999, 2001a, 2004, 2006, 2008a, 2008b, 2015b, 2015c, 2016, 2017, 2018, 2019a; Beschovski \& Hubenov 1986.

Peleteria ruficornis (Macquart, 1835) [Cuphocera] - V4, S211, TL, O61, R1, R2; 80-850 m; 1, 2; wpat; Jacentkovsky 1936, 1937; Trenchev 1980a; Hubenov 1992a, 1992b, 1993a, 1993b, 2008a, 2008b, 2015b, 2016, 2017, 2018.

Peleteria varia (Fabricius, 1794) [P. pyrrogaster (Rondani, 1859); Cuphocera] - E1, B1, V4, S1, S211, TL, T31, O5, O61, O62, R1, R2, R3, RW, RE, BN, BS; 0-1450 m; 1, 2, 3; ppta; Nedelkov 1909, 1912; Jacentkovsky 1936, 1937; Trenchev 1980a; Hubenov 1985b, 1988a, 1988b, 1988c, 1990, 1992a, 1992b, 1993a, 1993b, 1995a, 1995b, 1999, 2001a, 2004, 2006, 2008a, 2008b, 2015b, 2015c, 2016, 2017, 2018, 2019a; Beschovski \& Hubenov 1986.

Germaria ruficeps (Fallén, 1820) - V4, R2; 700-1300 m; 2, 3; wcp; Hubenov 1977, 1990, 1992a, 1992b, 1993a, 1993b, 2008a, 2008b, 2015b, 2017, 2018.

Nemoraea pellucida (Meigen, 1824) - B1, V4, O5, O62, R2, RW; 150-1500 m; 1, 2, 3; po; Jacentkovsky 1936, 1937; Belanovsky 1953; Trenchev 1980a; Hubenov 1985b, 1988a, 1988b, 1992a, 1992b, 1993a, 1993b, 1995a, 2006, 2008a, 2008b, 2015b, 2015c, 2017, 2018, 2019a; Beschovski \& Hubenov 1986.

Linnaemya (Linnaemya) comta (Fallén, 1810) - DM, E1, B1, V1, V4, S1, S211, O5, O62, R1, R2, R3, RW, RE, BN, BS; 0-1400 m; 1, 2, 3; hno, sk; Nedelkov 1912; Jacentkovsky 1936, 1937; Belanovsky 1953; Nikolova 1961; Trenchev 1980a; Hubenov 1983a, 1985a, 1985b, 1988a, 1988b, 1988c, 1990, 1992a, 1992b, 1993a, 1993b, 1995a, 1995b, 1996a, 1999, 2001a, 2004, 2006, 2008a, 2008b, 2015b, 2015c, 2016, 2017, 2018, 2019a; Beschovski \& Hubenov 1986.

Linnaemya (Linnaemya) vulpina (Fallén, 1810) [Micropalpus] - V1, T31; 100-600 m; 1; po; Nedelkov 1912; Jacentkovsky 1936, 1937; Belanovsky 1953; Mamoon 1978b; Hubenov 1992b, 1993a, 1993b, 2008a, 2008 b.

Linnaemya (Bonellimyia) impudica (Rondani, 1859) [Micropalpus] - B1, V4, S1, O5, O61, O62, R1, R2, R3, RW, RE; 100-1300 m; 1, 2, 3; e; Jacentkovsky 1936, 1937; Belanovsky 1953; Trenchev 1980a; Hubenov 1985b, 1988a, 1988b, 1988c, 1992a, 1992b, 1993a, 1993b, 1995a, 2004, 2006, 2008a, 2008b, 2015b, 2015c, 2016, 2017, 2018, 2019a; Beschovski \& Hubenov 1986.

Linnaemya (Bonellimyia) tessellans (Robineau-Desvoidy, 1830) - po; Tschorsnig et al. 2005, 2009; Hubenov 2008a, 2008b; O'Hara et al. 2020.

Linnaemya (Ophina) haemorrhoidalis (Fallén, 1810) - B1, B2, V1, V4, O5, O62, R1, R2, R3, RW; 200-1900 m; 1, 2, 3, 4; hoes, bm; Hubenov 1985b, 1988a, 1988b, 1988c, 1990, 1992a, 1992b, 1993a, 1993b, 1995a, 1996a, 1996b, 1999, 2001a, 2006, 2008a, 2008b, 2015b, 2015c, 2016, 2017, 2018, 2019a; Beschovski \& Hubenov 1986.

Linnaemya (Ophina) helvetica Herting, 1963 - V4, R1, R2, R3; 500-1000 m; 1, 2, 3; cse; Hubenov 1992a, 1992b, 1993a, 1993b, 2008a, 2008b, 2015b, 2016, 2017, 2018.

Linnaemya (Ophina) olsufjevi Zimin, 1954 - O5, R2; 700-1000 m; 2; esca, ? hoes; Hubenov 1983a, 1992a, 1992b, 1993a, 1993b, 1995a, 2008a, 2008b, 2015b, 2015c, 2017.

Linnaemya (Ophina) perinealis Pandelle, 1895 - O61, R1; 350-400 m; 1; po; Trenchev 1980a; Hubenov 1992b, 1993a, 1993b, 2008a, 2008b, 2016, 2017.

Linnaemya (Ophina) picta (Meigen, 1824) [L. retroflexa Pandellé, 1895; Micropalpus] - B1, V4, S1, S211, O5, R1, R2, RW; 270-1300 m; 1, 2, 3; po; Joakimoff 1899; Jacentkovsky 1936, 1937; Trenchev 1980a; Hubenov 1985a, 1990, 1992a, 1992b, 1993a, 1993b, 1995a, 2006, 2008a, 2008b, 2015b, 2015c, 2016, 2017, 2018, 2019a.

Linnaemya (Ophina) rossica Zimin, 1954 - V4, S1, S211, O5, O62, R2, R3, RW, BN; 140-1800 m; 1, 2, 3, 4; po, ? hoes, bm; Hubenov 1977, 1985b, 1988a, 1988b, 1988c, 1992a, 1992b, 1993a, 1993b, 1995a, 1995b, 1996a, 2008a, 2008b, 2015b, 2015c, 2017, 2018; Beschovski \& Hubenov 1986.

Linnaemya (Homoeonychia) frater (Rondani, 1859) - V4, S1, O61, R1; 260-800 m; 1, 2; mi; Jacentkovsky 1936, 1937; Hubenov 1990, 1992b, 1993a, 1993b, 2008a, 2008b, 2016, 2017, 2018.

Linnaemya (Homoeonychia) lithosiophaga (Rondani, 1859) - B1, V4, S1, O5, O62, R2, RE; 100-920 m; 1, 2; hom; Jacentkovsky 1936, 1937; Hubenov 1985b, 1988a, 1988b, 1992a, 1992b, 1993a, 1993b, 1995a, 2004, 2008a, 2008b, 2015b, 2015c, 2017, 2018, 2019a; Beschovski \& Hubenov 1986.

Gymnoglossa transsylvanica Mik, 1898 - V4; 1800 m; 4; ees; Hubenov 1980b, 1990, 1992b, 1993a, 1993b, 2008a, 2008b, 2018. 
Chrysosomopsis aurata (Fallén, 1820) [Chrysocosmius, Chrysosoma] - V4, O61, R1; 450-900 m; 1, 2; po; Nedelkov 1912; Jacentkovsky 1936, 1937; Belanovsky 1953; Hubenov 1990, 1992b, 1993a, 1993b, 2008a, 2008b, 2016, 2017, 2018.

Lydina aenea (Meigen, 1824) - S1; 300-500 m; 1; esca; Nikolova 1972; Mamoon 1978b; Hubenov 1992b, 1993a, 1993b, 2008a, 2008b.

Lypha dubia (Fallén, 1810) - V1; 600 m, +++; 2; hoes; Tschorsnig et al. 2005, 2009; Hubenov 2008a, 2008b.

Petagnia subpetiolata Rondani, 1859 [P. petiolata Rnd.; P. occlusa Rondani, 1856] - V4; 750 m; 2; cse; Jacentkovsky 1937; Hubenov 1992b, 1993a, 1993b, 2008a, 2008b, 2018.

Ernestia laevigata (Meigen, 1838) [Panzeria] - V4, O1, R2; 900-1450 m; 2, 3; hoes; Hubenov 1982a; 1990, 1992a, 1992b, 1993a, 1993b, 2008a, 2008b, 2015b, 2017, 2018.

Ernestia puparum (Fabricius, 1794) [Meriania, Nemoraea, Panzeria] - V4, R1; 800-1250 m; 2, 3; hoes, ? tp; Joakimoff 1899; Nedelkov 1912; Hubenov 1990, 1992b, 1993a, 1993b, 2008a, 2008b, 2016, 2017, 2018.

Ernestia rudis (Fallén, 1810) [Panzeria, ? Phaonia] - B1, V4, O5, R1, R2, R3, RW; 700-1800 m; 2, 3, 4; tp; ? Drenowsky 1939; ? Drensky 1939; Lavčiev et al. 1977; Hubenov 1990, 1992a, 1992b, 1993a, 1993b, 1995a, 2006, 2008a, 2008b, 2015b, 2015c, 2016, 2017, 2018, 2019a.

Eurithia anthophila (Robineau-Desvoidy, 1830) [E. radicum (Fabricius, 1794); Ernestia, Panzeria, Platychira] - B2, V1, V4, S211, R2; 520-1500 m; 1, 2, 3, 4; po; Nedelkov 1912; Trenchev 1980a; Hubenov 1990, 1992a, 1992b, 1993a, 1993b, 2008a, 2008b, 2015b, 2017, 2018.

Eurithia caesia (Fallén, 1810) [Panzeria] - B1, V4, O5, R1, R2, R3, RW; 850-1700 m; 2, 3, 4; po; Hubenov 1980b, 1988c, 1990, 1992a, 1992b, 1993a, 1993b, 1995a, 2006, 2008a, 2008b, 2015b, 2015c, 2016, 2017, 2018, 2019a.

Eurithia consobrina (Meigen, 1824) [Panzeria] - R2, R3; 1200-1400 m; 3; esca; Hubenov 1988c, 1992a, 1992b, 1993a, 1993b, 2008a, 2008b, 2015b, 2015c, 2017.

Eurithia gemina (Mesnil, 1972) - P2, B2, R2, RW; 1170-2000 m; 3, 4; e, m; Lutovinovas et al. 2022.

Hyalurgus lucidus (Meigen, 1824) - B1, B2, V4, R1, R2; 1400-2000 m; 3, 4; po, bm; Hubenov 1977, 1990, 1992a, 1992b, 1993a, 1993b, 1996a, 1996b, 2008a, 2008b, 2015b, 2016, 2017, 2018, 2019a.

Gymnocheta viridis (Fallén, 1810) - B1, V4, +++; 850-950 m; 2; tp; Tschorsnig et al. 2005, 2009; Hubenov 2008a, 2008b, 2018, 2019a.

Zophomyia temula (Scopoli, 1763) - E2, B1, B2, V3, V4, O5, O62, R1, R2, BN; 250-2370 m; 1, 2, 3, 4, 5; tp; Joakimoff 1899; Nedelkov 1912; Hubenov 1985b, 1988a, 1988b, 1992a, 1992b, 1993a, 1993b, 1995a, 1995b, 1996a, 1996b, 1999, 2001a, 2008a, 2008b, 2015b, 2015c, 2016, 2017, 2018, 2019a; Beschovski \& Hubenov 1986.

Cleonice callida (Meigen, 1824) [Steiniella] - B1, V4, R2, R3, RW; 400-1250 m; 1, 2, 3; des; Trenchev 1980a; Hubenov 1985a, 1988c, 1992a, 1992b, 1993a, 1993b, 2008a, 2008b, 2015b, 2015c, 2017, 2018, 2019a.

Cleonice nitidiuscula (Zetterstedt, 1859) [Steiniella] - V4; 700-900 m; 2; wes; Trenchev 1980a; Hubenov 1992b, 1993a, 1993b, 2008a, 2008b, 2018.

Loewia brevifrons (Rondani, 1856) - B1, V4, S211, O61, O62, R1, R2, R3, RW, RE, BN; 0-1300 m; 1, 2, 3; csei; Jacentkovsky 1936, 1937; Trenchev 1980a; Hubenov 1985b, 1988a, 1988b, 1988c, 1990, 1992a, 1992b, 1993a, 1993b, 2004, 2006, 2008a, 2008b, 2015b, 2015c, 2016, 2017, 2018, 2019a; Beschovski \& Hubenov 1986.

Loewia phaeoptera (Meigen, 1824) - V4, O5, O62, R2, RE; 300-1300 m; 1, 2, 3; wes; Hubenov 1982a, 1985b, 1988a, 1988b, 1992a, 1992b, 1993a, 1993b, 1995a, 1999, 2001a, 2004, 2008a, 2008b, 2015b, 2015c, 2017, 2018; Beschovski \& Hubenov 1986.

Loewia piligena Mesnil, 1973 - R3, +++; 1300 m; 3; e, ? cse; Tschorsnig et al. 2005, 2009; Hubenov 1993b, 2008a, 2008b.

Loewia setibarba Egger, 1856 - V1, V4; 150-1200 m; 1, 2, 3; e; Nedelkov 1912; Hubenov 1990, 1992b, 1993a, 1993b, 2008a, 2008b, 2018.

Loewia submetallica (Macquart, 1855) [L. petiolata (Pandellé, 1895)] - S211; 750-900 m; 2; e; Trenchev 1980a; Hubenov 1992b, 1993a, 1993b, 2008a, 2008b.

Pseudopachystylum gonioides (Zetterstedt, 1838) - R1, R2, RW; 900-1800 m; 2, 3, 4; hoes; Hubenov 1980a, 1992a, 1992b, 1993a, 1993b, 2006, 2008a, 2008b, 2015b, 2016, 2017.

Pelatachina tibialis (Fallén, 1810) - V4, O62, R2, R3; 150-1300 m; 1, 2, 3; hoes; Dirimanov \& Sengalevich 1969; Hubenov 1982a, 1985a, 1985b, 1988a, 1988b, 1988c, 1992a, 1992b, 1993a, 1993b, 2008a, 2008b, 2015b, 2015c, 2017, 2018; Beschovski \& Hubenov 1986. 
Macquartia chalconota (Meigen, 1824) - B1, B2, V1, V4, S211, O5, R1, R2, RW; 500-1500 m; 1, 2, 3, 4; esca; Nedelkov 1912; Trenchev 1980a; Hubenov 1990, 1992a, 1992b, 1993a, 1993b, 1995a, 2006, 2008a, 2008b, 2015b, 2015c, 2016, 2017, 2018, 2019a.

Macquartia dispar (Fallén, 1820) [M. flavipes (Meigen, 1824)] - B1,V4, R1, R2, R3, RW; 300-1400 m; 1, 2, 3; esca; Nedelkov 1912; Gruev 1968, 1972; Hubenov 1985a, 1985b, 1988a, 1988b, 1988c, 1990, 1992a, 1992b, 1993a, 1993b, 2006, 2008a, 2008b, 2015b, 2015c, 2016, 2017, 2018, 2019a; Beschovski \& Hubenov 1986.

Macquartia grisea (Fallén, 1810) - V4, O5, R1, R2; 450-950 m; 1, 2; wesca; Hubenov 1982a, 1992a, 1992b, 1993a, 1993b, 1995a, 2008a, 2008b, 2015b, 2015c, 2016, 2017, 2018.

Macquartia praefica (Meigen, 1824) [Bebricia] - B1, V4, O62, R1, R2, RW, RE; 150-1260 m; 1, 2, 3; ? wp; Hubenov 1982a, 1985b, 1988a, 1988b, 1992a, 1992b, 1993a, 1993b, 1999, 2001a, 2004, 2006, 2008a, 2008b, 2015b, 2016, 2017, 2018, 2019a; Beschovski \& Hubenov 1986.

Macquartia tenebricosa (Meigen, 1824) [M. nitida (Zetterstedt, 1838)] - B1, B2, V1, V4, S1, TL, O5, O61, R1, R2, RW, RE, BN, BS; 0-1800 m; 1, 2, 3, 4; wcp; Nedelkov 1912; Jacentkovsky 1936, 1937; Trenchev 1980a; Hubenov 1985b, 1988a, 1988b, 1990, 1992a, 1992b, 1993a, 1993b, 1995a, 1995b, 1996b, 2004, 2006, 2008a, 2008b, 2015b, 2015c, 2016, 2017, 2018, 2019a; Beschovski \& Hubenov 1986.

Macquartia tessellum (Meigen, 1824) [M. brevicornis (Macquart, 1839); M. occlusa Rondani, 1859] - O62, R1, R2, RW; 300-1400 m; 1, 2, 3; spo; Jacentkovsky 1936, 1937; Gruev 1972, 1973; Hubenov 1985a, 1992b, 1993a, 1993b, 2006, 2008a, 2008b, 2016, 2017.

Macquartia viridana Robineau-Desvoidy, 1863 - V4; 1100-1200 m; 3; dpo; Hubenov 1990, 1992b, 1993a, 1993b, 2008a, 2008b, 2018.

Macroprosopa atrata (Fallén, 1810) - S1; 350-500 m; 1; ess; Nikolova 1972; Hubenov 1992b, 1993a, 1993b, 2008a, 2008b.

Triarthria setipennis (Fallén, 1810) - V1, V5, TL, +++; 90-720 m; 1, 2; h; Georgiev \& Hubenov 2000; Hubenov 2008a, 2008b.

Neaera atra Robineau-Desvoidy, 1850 - nm, ? hom; Tschorsnig et al. 2005, 2009; Hubenov 2008a, 2008b; O'Hara et al. 2020.

Phytomyptera abnormis (Stein, 1924) [Elfia, Phytomyzoneura] - R1, R2; 1600-2000 m; 3, 4; cse; Jacentkovsky 1936, 1937; Hubenov 1992b, 1993a, 1993b, 2008a, 2008b, 2016, 2017.

Phytomyptera cingulata (Robineau-Desvoidy, 1830) [Elfia] - O62, R2; 150-400 m; 1; e; Hubenov 1982a, 1985b, 1988a, 1988b, 1992a, 1992b, 1993a, 1993b, 2008a, 2008b, 2015b, 2017; Beschovski \& Hubenov 1986.

Phytomyptera nigrina (Meigen, 1824) - V1, V4; 700-900 m; 2; wcp; Hubenov \& Georgiev 1996; Georgiev \& Delkov 1997; Georgiev 2000a, 2000b, 2000c; Hubenov 2001b, 2008a, 2008b, 2018.

Graphogaster brunnescens Villeneuve, 1907 - B1, V1, V4, S1, O61, R1, R3, BS; 0-1160 m; 1, 2, 3; ess; Hubenov 1977, 1988c, 1992b, 1993a, 1993b, 2008a, 2008b, 2015c, 2016, 2017, 2018, 2019a; Trenchev 1980a.

Graphogaster vestita Rondani, 1868 - ; hom, ? mi; Tschorsnig et al. 2005, 2009; Hubenov 2008a, 2008b; O'Hara et al. 2020.

Ceromya dilecta Herting, 1977 - cse, ? se; Tschorsnig et al. 2005, 2009; Hubenov 2008a, 2008b; O'Hara et al. 2020.

Ceromya flaviseta (Villeneuve, 1921) - O62; 100-250 m; 1; eo; Hubenov 1982a, 1985b, 1988a, 1988b, 1992b, 1993a, 1993b, 1999, 2001a, 2008a, 2008b; Beschovski \& Hubenov 1986.

Actia crassicornis (Meigen, 1824) - B1, B3, V4, S1, S21, S211, O5, R1, R2, R3, RW, RE; 200-1500 m; 1, 2, 3; po; Jacentkovsky 1936, 1937; Belanovsky 1953; Mamoon 1978b; Trenchev 1980d; Hubenov 1985a, 1988c, 1990, 1992a, 1992b, 1993a, 1993b, 1995a, 2001b, 2004, 2006, 2008a, 2008b, 2015b, 2015c, 2016, 2017, 2018, 2019a.

Actia infantula (Zetterstedt, 1844) [A. antennalis Rondani, 1859; A. aristalis Rondani, 1865] - B3, S1, O61, R1; 200-1100 m; 1, 2, 3; wcp; Jacentkovsky 1936, 1937; Hubenov 1992b, 1993a, 1993b, 2008a, 2008b, 2016, 2017.

Actia lamia (Meigen, 1838) - hoes, ? se; Tschorsnig et al. 2005, 2009; Hubenov 2008a, 2008b; O'Hara et al. 2020.

Actia nigroscutellata Lundbeck, 1927 - V1, S211; 600-700 m; 1, 2; dpo; Trenchev 1980d; Hubenov 1985a, 1992b, 1993a, 1993b, 2008a, 2008b.

Actia pilipennis (Fallén, 1810) - DW, P1, V1, V4, R2; 40-1300 m; 1, 2, 3; po; Ganchev 1977; Lavchiev et al. 1977; Mamoon 1978b; Trenchev 1980d; Hubenov 1985a, 1992a, 1992b, 1993a, 1993b, 2001b, 2008a, 2008b, 2015b, 2017, 2018.

Peribaea apicalis Robineau-Desvoidy, 1863 - E1, RW, RE, BN; 100-120 m; 1; tes; Mirchev et al. 2000; Hubenov 2001b, 2004, 2006, 2008a, 2008b. 
Peribaea tibialis (Robineau-Desvoidy, 1851) [Actia, Strobliomyia] - DW, B1, V4, S211, O5, O62, R1, R2, RW, RE, BN; 0-1160 m; 1, 2, 3; ppt; Nedelkov 1912; Mamoon 1978b; Trenchev 1980d; Hubenov 1985a, 1985b, 1988a, 1988b, 1992a, 1992b, 1993a, 1993b, 1995a, 1999, 2001a, 2004, 2006, 2008a, 2008b, 2015b, 2015c, 2016, 2017, 2018, 2019a; Beschovski \& Hubenov 1986.

Ceranthia lichtwardtiana (Villeneuve, 1931) - V4; 1900 m; 4, 5; des; Ziegler 1989; Hubenov 1993b, 2001b, 2008a, 2008b, 2018.

Siphona collini Mesnil, 1960 - ; hoes, ? des; Tschorsnig et al. 2005, 2009; Hubenov 2008a, 2008b; O’Hara et al. 2020.

Siphona cristata (Fabricius, 1805) [Bucentes] - E1, B1, V1, V4, S1, T31, O5, R1, R2, RE; 70-1150 m; 1, 2, 3; ho; Nedelkov 1912; Jacentkovsky 1936, 1937; Mamoon 1978b; Hubenov 1985b, 1988a, 1988b, 1992a, 1992b, 1993a, 1993b, 1995a, 2004, 2008a, 2008b, 2015b, 2015c, 2016, 2017, 2018, 2019a; Beschovski \& Hubenov 1986.

Siphona flavifrons Staeger, 1849 [Bucentes] - V1, V4, R2; 600-1900 m; 2, 3, 4; wces, ? des, h*; Nedelkov 1912; Hubenov 1990, 1992a, 1992b, 1993a, 1993b, 2008a, 2008b, 2015b, 2017, 2018.

Siphona geniculata (De Geer, 1776) [Bucentes] - B3, V1, V4, S1, S211, T31, O3, R2, RW; 150-1300 m; 1, 2, 3; ho, h*; Nedelkov 1912; Jacentkovsky 1936, 1937; Trenchev 1980d; Hubenov 1990, 1992a, 1992b, 1993a, 1993b, 2006, 2008a, 2008b, 2015b, 2017, 2018.

Siphona hokkaidensis Mesnil, 1957 - R2; 1760 m; 4; h; Lutovinovas et al., 2022.

Siphona hungarica Andersen, 1984 - e, ? csee; Tschorsnig et al. 2005, 2009; Hubenov 2008a, 2008b; O'Hara et al. 2020.

Siphona maculata Stæger, 1849 [Bucentes] - B3, S1; 380-400 m; 1; h; Jacentkovsky 1936, 1937; Hubenov 1992b, 1993a, 1993b, 2008a, 2008b.

Siphona pauciseta Rondani, 1865 - \$; po; Tschorsnig et al. 2005, 2009; Hubenov 2008a, 2008b; O’Hara et al. 2020.

Siphona setosa Mesnil, 1960 - RW; 1300 m; 3; wces, ? tes; Lutovinovas et al., 2022.

Siphona variata Andersen, 1982 - wp; Tschorsnig et al. 2005, 2009; Hubenov 2008a, 2008b; O'Hara et al. 2020.

Aphantorhaphopsis siphonoides (Strobl, 1898) - R2, RW; 1260-2000 m; 3, 4; e; Lutovinovas et al. 2022.

Aphria latifrons Villeneuve, 1908 - R1; 900 m; 2; mss,? mca; Jacentkovsky 1936, 1937; Hubenov 1992b, 1993a, 1993b, 2008a, 2008b, 2016, 2017.

Aphria longirostris (Meigen, 1824) - B1, B2, B3, V4, S1, O1, O3, O5, O62, R1, R2, R3, RW, RE; 200-2300 m; 1, 2, 3, 4, 5; wcp; Jacentkovsky 1936, 1937; Hubenov 1985b, 1988a, 1988b, 1988c, 1992a, 1992b, 1993a, 1993b, 1995a, 1996a, 1996b, 1999, 2001a, 2004, 2006, 2008a, 2008b, 2015b, 2015c, 2016, 2017, 2018, 2019a; Beschovski \& Hubenov 1986.

Aphria xyphias Pandellé, 1896 - esca; Tschorsnig et al. 2005, 2009; Hubenov 2008a, 2008b; O’Hara et al. 2020.

Demoticus plebejus (Fallén, 1810) - P1, B1, V1, V4, R1, R2, R3; 360-1950 m; 1, 2, 3, 4; wces; Nedelkov 1912; Jacentkovsky 1936, 1937; Belanovsky 1953; Hubenov 1988c, 1990, 1992a, 1992b, 1993a, 1993b, 2008a, 2008b, 2015b, 2015c, 2016, 2017, 2018, 2019a.

Bithia demotica (Egger, 1861) - des; Tschorsnig et al. 2005, 2009; Hubenov 2008a, 2008b; O'Hara et al. 2020.

Bithia geniculata (Zetterstedt, 1844) [Pseudodemoticus] - BS; 0-5 m; 1; wces; Hubenov 1980b, 1992b, 1993a, 1993b, 2008a, 2008b.

Bithia glirina (Rondani, 1861) [Rhinotachina] - B1, V1, V4, T2, O3, O5, R1, R2, R3, RW, RE, BS; 0-1500 m; 1, 2, 3, 4; wes; Hubenov 1977, 1985b; Trenchev 1980a; Hubenov 1985b, 1988a, 1988b, 1988c, 1992a, 1992b, 1993a, 1993b, 1995a, 2004, 2006, 2008a, 2008b, 2015b, 2015c, 2016, 2017, 2018, 2019a; Beschovski \& Hubenov 1986.

Bithia immaculata (Herting, 1971) - \$; cse, ? se; Tschorsnig et al. 2005, 2009; Hubenov 2008a, 2008b; O’Hara et al. 2020.

Bithia jacentkovskyi (Villeneuve, 1937) [B. incerta (Belanovsky, 1953); Pseudodemoticus, Rhinotachinopsis] S1; 250-300 m; 1; eca; Villeneuve 1937; Belanovsky 1953; Herting 1984; Herting \& Dely-Draskovits 1993; Hubenov 1992b, 1993a, 1993b, 2008a, 2008b.

Bithia modesta (Meigen, 1824) [Rhinotachina] - P1, B1, V1, V3, V4, T31, O5, R1, R2, R3, RW, RE, BS; 0-1200 m; 1, 2, 3; mca; Jacentkovsky 1936, 1937; Trenchev 1980a; Hubenov 1985b, 1988a, 1988b, 1988c, 1992a, 1992b, 1993a, 1993b, 1995a, 2004, 2006, 2008a, 2008b, 2015b, 2015c, 2016, 2017, 2018, 2019a; Beschovski \& Hubenov 1986. 
Bithia spreta (Meigen, 1824) - V4; 800-900 m; 2; wes; Trenchev 1980a; Hubenov 1990, 1992b, 1993a, 1993b, 2008a, 2008b, 2018.

Leskia aurea (Fallén, 1820) - P2, B1, B2, B3, V1, V4, S1, TL, O5, R1, R2, RW, RE, +++; 200-1100 m; 1, 2, 3; hoes; Nedelkov 1912; Jacentkovsky 1936, 1937; Dirimanov \& Sengalevich 1969; Trenchev 1980a; Hubenov 1985a, 1992a, 1992b, 1993a, 1993b, 1995a, 2001b, 2004, 2006, 2008a, 2008b, 2015b, 2015c, 2016, 2017, 2018, 2019a.

Solieria fenestrata (Meigen, 1824) [S. fuscana Robineau-Desvoidy, 1848; Myobia] - V4; 800-1000 m; 2; wes; Jacentkovsky 1936, 1937; Belanovsky 1953; Hubenov 1990, 1992b, 1993a, 1993b, 2008a, 2008b, 2018.

Solieria pacifica (Meigen, 1824) [Anthoica tibialis (von Roser, 1840)] - V4, S211; 700-900 m; 2; des; Trenchev 1980a; Hubenov 1990, 1992b, 1993a, 1993b, 2008a, 2008b, 2018.

Solieria vacua (Rondani, 1861) - e, e, cse; Tschorsnig et al. 2005, 2009; Hubenov 2008a, 2008b; O'Hara et al. 2020.

Mintho compressa (Fabricius, 1787) [M.praeceps (Scopoli, 1763)] - V1, S22, T31; 70-1000 m; 1, 2; atm; Nedelkov 1912; Jacentkovsky 1936, 1937; Hubenov 1992b, 1993a, 1993b, 2008a, 2008b.

? Mintho praeceps (Scopoli, 1763) - V1, S21; 520-800 m; 1, 2; hom; Nedelkov 1912; O'Hara et al. 2020.

Mintho rufiventris (Fallén, 1817) [M. praeceps (Scopoli, 1763)] - B1, V1, V3, V4, S1, S21, S211, S22, O5, O62, R1, R2, RW, RE, BN, BS; 0-1200 m; 1, 2, 3; tp; Nedelkov 1912; Jacentkovsky 1936, 1937; Trenchev 1980a; Hubenov 1990, 1992a, 1992b, 1993a, 1993b, 1995a, 2004, 2006, 2008a, 2008b, 2015b, 2015c, 2016, 2017, 2018, 2019a.

Minthodes picta (Zetterstedt, 1844) [Myxominthodes monticola Villeneuve, 1910] - V4; 900-1700 m; 2, 3, 4; wes, bm; Jacentkovsky 1937; Hubenov 1990, 1992b, 1993a, 1993b, 1996a, 2008a, 2008b, 2018.

Minthodes pictipennis Brauer \& Bergenstamm, 1889 - S1; 160-280 m; 1; emi; Jacentkovsky 1937; Hubenov 1992b, 1993a, 1993b, 2008a, 2008b.

Palmonia hermonensis Kugler, 1972 - em; Tschorsnig et al. 2005, 2009; Hubenov 2008a, 2008b; O’Hara et al. 2020.

Ziminia masiceraeformis (Portshinsky, 1881) - mwca; Tschorsnig et al. 2005, 2009; Hubenov 2008a, 2008b; O'Hara et al. 2020.

Mesnilomyia longicornis Kugler, 1972 [Rossimyiops] - ^; em; Tschorsnig et al. 2005, 2009; Hubenov 2008a, 2008b; O'Hara et al. 2020.

Melisoneura leucoptera (Meigen, 1824) - RW; 1300 m; 3; des; Lutovinovas et al. 2022.

Microphthalma europaea Egger, 1860 - DM, E1, E2, B1, V4, TL, O5, R1, R2, BN; 0-1160 m; 1, 2, 3; wpo, ? wppt; Nedelkov 1912; Hubenov 1992a, 1992b, 1993a, 1993b, 1995a, 2008a, 2008b, 2015b, 2015c, 2016, 2017, 2018, 2019a.

Dexiosoma caninum (Fabricius, 1781) - V4, O5, R2; 1100-1300 m; 3; des; Hubenov 1990, 1992a, 1992b, 1993a, 1993b, 1995a, 2008a, 2008b, 2015b, 2015c, 2017, 2018.

Trixa caerulescens Meigen, 1824 - O61, R1; 320-400 m; 1; wes; Trenchev 1980c; Hubenov 1992b, 1993a, 1993b, 2008a, 2008b, 2017.

Trixa conspersa (Harris, 1776) [T. oestroidea (Robineau-Desvoidy, 1830); T. variegata Meigen, 1824] - V3, O61, T31, R1, RW, BS; 0-1200 m; 1, 2, 3; po; Lavchiev et al. 1977; Zonati 1978; Trenchev 1980c; Hubenov 1992b, 1993a, 1993b, 2006, 2008a, 2008b, 2016, 2017, 2018.

Billaea adelpha (Loew, 1873) [B. subrotundata (Rondani, 1862)] - V4, S1; 400-1100 m; 1, 2, 3; eswa; Jacentkovsky 1936, 1937; Belanovsky 1951; Hubenov 1990, 1992b, 1993a, 1993b, 2008a, 2008b, 2018.

Billaea fortis (Rondani, 1862) - B1, V4, O5, O62, R1, R2, RE; 150-1200 m; 1, 2, 3; po; Hubenov 1983a, 1992a, 1992b, 1993a, 1993b, 1995a, 2004, 2008a, 2008b, 2015b, 2015c, 2016, 2017, 2018, 2019a.

Billaea irrorata (Meigen, 1826) - DW, DM, P1, B1, V1, V4, V5, S1, S21, S22, O5, R1, RW, RE; 30-1850 m; 1, 2, 3, 4; e; Tsankov et al. 1989; Hubenov 1992b, 1993a, 1993b, 1995a, 2001b, 2004, 2006, 2008a, 2008b, 2015c, 2016, 2017, 2018, 2019a; Georgiev 2001; Georgiev et al. 2004.

Billaea maritima (Schiner, 1862) - \$ mfe, dp; Tschorsnig et al. 2005, 2009; Hubenov 2008a, 2008b; O'Hara et al. 2020.

Billaea pectinata (Meigen, 1826) - B1, B2, V1, V4, S1, S21, O3, O5, O62, R1, R2, R3, RW, RE; 100-1300 m; 1, 2, 3; swp, ? wp, ? mca; Jacentkovsky 1936, 1937; Hubenov 1985b, 1988a, 1988b, 1988c, 1990, 1992a, 1992b, 1993a, 1993b, 1995a, 1999, 2001a, 2004, 2006, 2008a, 2008b, 2015b, 2015c, 2016, 2017, 2018, 2019a; Beschovski \& Hubenov 1986. 
Billaea steini (Brauer \& Bergenstamm, 1891) - R3; 1000-1100 m; 3; des; Hubenov 1980a, 1988c, 1992b, 1993a, 1993b, 2008a, 2008b, 2015c.

Billaea triangulifera (Zetterstedt, 1844) - B1, B2, V4, V5, R1, R2, RW; 820-1700 m; 2, 3, 4; tp, ? hoes; Hubenov 1977, 1990, 1992a, 1992b, 1993a, 1993b, 1996b, 2001b, 2006, 2008a, 2008b, 2015b, 2016, 2017, 2018, 2019a.

Dinera carinifrons (Fallén, 1817) [Myiocera] - E1, B1, B2, V1, V3, V4, S1, S211, O5, O61, O62, R1, R2, R3, R5, RW, RE, BN; 100-2550 m; 1, 2, 3, 4, 5, 6; hoes; Nedelkov 1912; Jacentkovsky 1936, 1937; Hubenov 1985b, 1988a, 1988b, 1988c, 1990, 1992a, 1992b, 1993a, 1993b, 1995a, 1995b, 1996a, 1996b, 1999, 2001a, 2004, 2006, 2008a, 2008b, 2015b, 2015c, 2016, 2017, 2018, 2019a; Beschovski \& Hubenov 1986.

Dinera ferina (Fallén, 1817) [Dexia, Myiocera] - B1, B2, V1, V4, S1, S211, O3, O5, O61, O62, R1, R2, R3, RW, RE, BN; 0-1600 m; 1, 2, 3, 4; wces; Jacentkovsky 1936, 1937; Drensky 1942; Hubenov 1988c, 1990, 1992a, 1992b, 1993a, 1993b, 1995a, 1995b, 2004, 2006, 2008a, 2008b, 2015b, 2015c, 2017, 2018, 2019a.

Dinera fuscata Zhang \& Shima, 2006 [D. fuscata occidentalis Ziegler, 2016] - \$; po, wpo; O'Hara et al. 2020.

Dinera grisescens (Fallén, 1817) - V4, S211, O61, R1, RW; 300-900 m; 1, 2; h; Jacentkovsky 1936, 1937; Trenchev 1980c; Hubenov 1990, 1992b, 1993a, 1993b, 2006, 2008a, 2008b, 2016, 2017, 2018.

Estheria bohemani (Rondani, 1862) - V4, R2, RW; 1000-1850 m; 3, 4; e; Hubenov 1980a, 1992a, 1992b, 1993a, 1993b, 2006, 2008a, 2008b, 2015b, 2017, 2018.

Estheria cristata (Meigen, 1826) - S21, R1, RW; 1000-1400 m; 3; eca; Hubenov 1980a, 1992b, 1993a, 1993b, 2006, 2008a, 2008b, 2016, 2017, 2018.

Estheria petiolata (Bonsdorff, 1866) [Dexiomorpha] - B1, B2, V4, S21, S22, O3, O5, O62, R1, R2, R3, RW, RE; 150-1900 m; 1, 2, 3, 4; esca; Jacentkovsky 1936, 1937; Hubenov 1985b, 1988a, 1988b, 1988c, 1990, 1992a, 1992b, 1993a, 1993b, 1995a, 1996b, 1999, 2001a, 2004, 2006, 2008a, 2008b, 2015b, 2015c, 2016, 2017, 2018, 2019a; Beschovski \& Hubenov 1986.

Estheria picta (Meigen, 1826) [Dexiomorpha] - B1, V1, V4, S1, O4, O5, O61, O62, R1, R2, RW; 150-1800 m; 1, 2, 3, 4; esca; Jacentkovsky 1936, 1937; Hubenov 1985b, 1988a, 1988b, 1990, 1992a, 1992b, 1993a, 1993b, 1995a, 1999, 2001a, 2006, 2008a, 2008b, 2015b, 2015c, 2016, 2017, 2018, 2019a; Beschovski \& Hubenov 1986.

Dexia rustica (Fabricius, 1775) - B1, V4, S1, S22, O5, O62, R1, R2, R3, RW, RE, BN; 150-1200 m; 1, 2, 3; tp; Nedelkov 1912; Jacentkovsky 1936, 1937; Trenchev 1980c; Hubenov 1985b, 1988a, 1988b, 1988c, 1990, 1992a, 1992b, 1993a, 1993b, 1995a, 1995b, 1999, 2001a, 2004, 2006, 2008a, 2008b, 2015b, 2015c, 2016, 2017, 2018, 2019a; Beschovski \& Hubenov 1986.

Prosena siberita (Fabricius, 1775) - B1, B2, V1, V3, V4, O3, O5, O61, R1, R2, R3, RW, RE, BN, BS; 0-1850 m; 1, 2, 3, 4; hpta, sk, i; Nedelkov 1912; Jacentkovsky 1936, 1937; Drensky 1942; Hubenov 1988c, 1990, 1992a, 1992b, 1993a, 1993b, 1995a, 1996a, 1996b, 2004, 2006, 2008a, 2008b, 2015b, 2015c, 2016, 2017, 2018, 2019a.

Zeuxia brevicornis (Egger, 1860) [Ptilozeuxia brachycera Brauer et Bergenstamm, 1891)] - V1, O61; 300-600 m; 1; esca; Nedelkov 1912; Trenchev 1980c; Hubenov 1992b, 1993a, 1993b, 2008a, 2008b, 2016, 2017.

Zeuxia cinerea Meigen, 1826 - B1, V1, V3, V4, S1, O5, O61, O62, R1, R2, R3, RW, BS; 0-2000 m; 1, 2, 3, 4; wp; Nedelkov 1912; Jacentkovsky 1936, 1937; Trenchev 1980c; Hubenov 1985b, 1988a, 1988b, 1988c, 1990, 1992a, 1992b, 1993a, 1993b, 1995a, 1996b, 1999, 2001a, 2006, 2008a, 2008b, 2015b, 2015c, 2016, 2017, 2018, 2019a; Beschovski \& Hubenov 1986.

Zeuxia erythraea (Egger, 1856) [Z. nigricornis (Egger, 860)] - O61, R1; 300-400 m; 1; nmca; Trenchev 1980c; Hubenov 1992b, 1993a, 1993b, 2008a, 2008b, 2016, 2017.

Zeuxia tricolor (Portshinsky, 1881) - \$; em; Tschorsnig et al. 2005, 2009; Hubenov 2008a, 2008b; O'Hara et al. 2020.

Eriothrix apenninus (Rondani, 1862) [E. latifrons Brauer, 1898] - B2, V1, V3, V4, S211, R1, R2, R3, RW; 7502450 m; 2, 3, 4, 5, 6; wcp; Jacentkovsky 1936, 1937; Belanovsky 1951; Hubenov 1980a, 1988c, 1992a, 1992b, 1993a, 1993b, 1996b, 2006, 2008a, 2008b, 2015b, 2015c, 2016, 2017, 2018; Trenchev 1980c.

Eriothrix argyreatus (Meigen, 1824) - wcp; Tschorsnig et al. 2005, 2009; Hubenov 2008a, 2008b; O'Hara et al. 2020.

Eriothrix inflatus Kolomiets, 1967 [E. chrysanthes Kolomiets, 1967] - S211; 750-900 m; 2; ee; Trenchev 1980c; Hubenov 1992b, 1993a, 1993b, 2008a, 2008b.

Eriothrix prolixa (Meigen, 1824) - esca; Tschorsnig et al. 2005, 2009; Hubenov 2008a, 2008b; O'Hara et al. 2020.

Eriothrix rufomaculatus (De Geer, 1776) [E. lateralis (Fabricius, 1775); E. monochaeta Wainwright, 1928] - B2, B3, V1, V3, V4, S1, S211, TL, T31, O5, R1, R2, R3, RW, RE, BN; 200-2450 m; 1, 2, 3, 4, 5, 6; tp; Nedelkov 1912; Jacentkovsky 1936, 1937; Trenchev 1980c; Hubenov 1985b, 1988a, 1988b, 1988c, 1990, 1992a, 1992b, 
1993a, 1993b, 1995a, 1995b, 1996a, 1996b, 2004, 2006, 2008a, 2008b, 2015b, 2015c, 2016, 2017, 2018, 2019a; Beschovski \& Hubenov 1986.

Trafoia monticola Brauer \& Bergenstamm, 1893 - S211; 750-900 m; 2; e; Trenchev 1980c; Hubenov 1992b, 1993a, 1993b, 2008a, 2008b.

Campylocheta inepta (Meigen, 1824) - RW; 1240-1260 m; 3; wcp; Lutovinovas et al. 2022.

? Campylocheta latigena Mesnil, 1974 - e; e; Tschorsnig et al. 2005, 2009; Hubenov 2008a, 2008b; O'Hara et al. 2020.

Campylocheta similis Ziegler \& Shima, 1996 - des; Tschorsnig et al. 2005, 2009; Hubenov 2008a, 2008b; O'Hara et al. 2020.

Blepharomyia pagana (Meigen, 1824) [B. amplicornis (Zetterstedt, 1844)] - DW, P1, S211; 40-1000 m; 1, 2; des; Ganchev 1977; Lavchiev et al. 1977; Mamoon 1978a, 1978b; Hubenov 1985a, 1992b, 1993a, 1993b, 2008a, 2008b.

Blepharomyia piliceps (Zetterstedt, 1859) - O62, R2; 300-400 m; 1; e, bm; Mamoon 1978b; Trenchev 1980c; Hubenov 1985a, 1985b, 1988a, 1988b, 1992b, 1993a, 1993b, 1996a, 2008a, 2008b; Beschovski \& Hubenov 1986.

Peteina erinaceus (Fabricius, 1794) [Petina] - S211; 700-900 m; 2; esca; Zonati 1978; Trenchev 1980c; Hubenov 1992b, 1993a, 1993b, 2008a, 2008b.

Ramonda prunaria (Rondani, 1861) [Wagneria] - O3; 400-450 m; 1; esca, ? wces; Trenchev 1980c; Hubenov 1992b, 1993a, 1993b, 2008a, 2008b.

Ramonda spathulata (Fallén, 1820) [Wagneria] - DW, P1, V4, O5, O62, S211, R1, R2, R3; 50-1450 m; 1, 2, 3, 4; tp; Mamoon 1978a, 1978b; Trenchev 1980c; Hubenov 1982a, 1985a, 1985b, 1988a, 1988b, 1988c, 1992a, 1992b, 1993a, 1993b, 1995a, 2008a, 2008b, 2015b, 2015c, 2016, 2017, 2018; Beschovski \& Hubenov 1986.

Periscepsia carbonaria (Panzer, 1798) [Wagneria] - P1, V1, V4, TL, O3, R1, R2, RW, BN; 0-1300 m; 1, 2, 3; Nedelkov 1912; Trenchev 1980c; Hubenov 1992a, 1992b, 1993a, 1993b, 2006, 2008a, 2008b, 2015b, 2016, 2017, 2018.

Wagneria costata (Fallen, 1815) - V1, TL; 170-600 m; 1; e; Nedelkov 1912; Hubenov 1992b, 1993a, 1993b, 2008a, 2008b.

Wagneria cunctans (Meigen, 1824) - csena; Tschorsnig et al. 2005, 2009; Hubenov 2008a, 2008b.

Wagneria gagatea Robineau-Desvoidy, 1830 - des; Tschorsnig et al. 2005, 2009; Hubenov 2008a, 2008 b.

Athrycia curvinervis (Zetterstedt, 1844) - RW; 1170 m; 3; hoes; Lutovinovas et al. 2022.

Athrycia impressa (van der Wulp, 1869) - R2, R3; 1100-1300 m; 3; esca; Hubenov 1980a, 1988c, 1992a, 1992b, 1993a, 1993b, 2008a, 2008b, 2015b, 2015c, 2017.

Athrycia trepida (Meigen, 1824) [A. flavipalpis Robineau-Desvoidy, 1863; Blepharigena] - B1, V4, O5, O62, R1, R2, RW, RE; 100-1350 m; 1, 2, 3; tp; Lavchiev et al. 1977; Hubenov 1982a, 1985b, 1988a, 1988b, 1990, 1992a, 1992b, 1993a, 1993b, 1995a, 1999, 2001a, 2004, 2006, 2008a, 2008b, 2015b, 2015c, 2016, 2017, 2018 , 2019a; Beschovski \& Hubenov 1986.

Voria ruralis (Fallén, 1810) [Plagia] - B1, B2, V1, V4, TL, O5, O61, R1, R2, RW; 200-1800 m; 1, 2, 3, 4; k; Nedelkov 1912; Jacentkovsky 1936, 1937; Nikolova 1972; Trenchev 1980c; Hubenov 1985a, 1990, 1992a, 1992b, 1993a, 1993b, 1995a, 1996a, 1996b, 2006, 2008a, 2008b, 2015b, 2015c, 2016, 2017, 2018, 2019a.

Cyrtophloeba ruricola (Meigen, 1824) - 200-1200 m; 1, 2, 3; tp; Dochkova 1971, 1972; Trenchev 1980c; Hubenov 1985a, 1992b, 1993a, 1993b, 2008a, 2008b; O’Hara et al. 2020.

Hyleorus elatus (Meigen, 1838) [Plagia, Steiniomyia] - V1, O62, R2, R3; 300-1300 m; 1, 2, 3; po; Lavchiev et al. 1977; Trenchev 1980c; Hubenov 1985b, 1988a, 1988b, 1988c, 1992a, 1992b, 1993a, 1993b, 2008a, 2008b, 2015b, 2015c, 2017; Beschovski \& Hubenov 1986.

Klugia marginata (Meigen, 1824) - S211; 750-900 m; 2; esca; Trenchev 1980c; Hubenov 1992b, 1993a, 1993b, 2008a, 2008b.

Phyllomya volvulus (Fabricius, 1794) - B1, V4, O3, O5, O61, O62, R1, R2, RE; 120-1750 m; 1, 2, 3, 4; tp; Jacentkovsky 1936, 1937; Belanovsky 1951; Trenchev 1980c; Hubenov 1985b, 1988a, 1988b, 1992a, 1992b, 1993a, 1993b, 1995a, 2004, 2008a, 2008b, 2015b, 2015c, 2016, 2017, 2018, 2019a; Beschovski \& Hubenov 1986.

Thelaira leucozona (Panzer, 1809) - V1; 600 m; 1, 2; po; Nedelkov 1912; Hubenov 1992b, 1993a, 1993b, 2008a, 2008 b.

Thelaira nigripes (Fabricius, 1794) - B1, V4, O4, O5, O61, O62, R1, R2, R3, RW, RE; 120-1300 m; 1, 2, 3; po; Jacentkovsky 1936, 1937; Mamoon 1878b; Trenchev 1980c; Hubenov 1985b, 1988a, 1988b, 1988c, 1990, 
1992a, 1992b, 1993a, 1993b, 1995a, 2004, 2006, 2008a, 2008b, 2015b, 2015c, 2016, 2017, 2018, 2019a; Beschovski \& Hubenov 1986.

Thelaira solivaga (Harris, 1780) - po; Tschorsnig et al. 2005, 2009; Hubenov 2008a, 2008b; O'Hara et al. 2020. Halidaya aurea Egger, 1856 - V1, V4, O5, O62, R1, R2, R3, BN; 300-1100 m; 1, 2, 3; po; Lavchiev et al. 1977; Hubenov 1985a, 1985b, 1988a, 1988b, 1988c, 1992a, 1992b, 1993a, 1993b, 1995a, 1995b, 2008a, 2008b, 2015b, 2015c, 2016, 2017, 2018; Beschovski \& Hubenov 1986.

Stomina caliendrata (Rondani, 1862) [Morphomyia] - B1, V1, V4, S1, O5, O62, R1, R2, RE; 350-1100 m; 1, 2, 3; swp, ? mwca; Jacentkovsky 1936, 1937; Hubenov 1985b, 1988a, 1988b, 1990, 1992a, 1992b, 1993a, 1993b, 1995a, 2004, 2008a, 2008b, 2015b, 2015c, 2016, 2017, 2018, 2019a; Beschovski \& Hubenov 1986.

Stomina iners (Meigen, 1838) [S. varians Villeneuve, 1930] - P1, V1, S1, O61, R1, R2, RW; 120-1100 m; 1, 2, 3; mit; Jacentkovsky 1936, 1937; Trenchev 1980c; Hubenov 1992b, 1993a, 1993b, 2006, 2008a, 2008b, 2016, 2017.

Stomina tachinoides (Fallén, 1817) - V1, V4, S1, O5, O61, R1, R2, RE; 350-1200 m; 1, 2, 3; wcp; Jacentkovsky 1936, 1937; Trenchev 1980c; Hubenov 1985b, 1988a, 1988b, 1992a, 1992b, 1993a, 1993b, 1995a, 2004, 2008a, 2008b, 2015b, 2015c, 2016, 2017, 2018; Beschovski \& Hubenov 1986.

Rhamphina pedemontana (Meigen, 1824) - B2, V4, O5, R1, R2; 800-1800 m; 2, 3, 4; se; Hubenov 1992a, 1992b, 1993a, 1993b, 1995a, 1996a, 1996b, 2008a, 2008b, 2015b, 2015b, 2015c, 2016, 2017, 2018; Hubenov et al. 2000a; Tschorsnig et al. 2005, 2009.

Dufouria chalybeata (Meigen, 1824) [Minella] - V4, O62, R2, R3, RE; 140-1300 m; 1, 2, 3; tp; Nedelkov 1912; Hubenov 1985b, 1988a, 1988b, 1988c, 1990, 1992a, 1992b, 1993a, 1993b, 1996a, 2004, 2008a, 2008b, 2015b, 2015c, 2017, 2018; Beschovski \& Hubenov 1986.

Dufouria nigrita (Fallén, 1810) - V4, S211, O5, O62, R1, R2, R3, RW; 150-1300 m; 1, 2, 3; wcp; Hubenov 1980a, 1985a, 1985b, 1988a, 1988b, 1988c, 1992a, 1992b, 1993a, 1993b, 1995a, 2006, 2008a, 2008b, 2015b, 2015c, 2016, 2017, 2018; Trenchev 1980c; Pavlov 1981; Beschovski \& Hubenov 1986.

Dufouria occlusa (Robineau-Desvoidy, 1863) [D. nitida Brauer et Bergenstamm, 1891] - V1; 550-600 m; 1; e; Lavchiev et al. 1977; Hubenov 1992b, 1993a, 1993b, 2008a, 2008b.

Chetoptilia puella (Rondani, 1862) - O5, R2, RW; 150-800 m; 1, 2; des; Hubenov 1983a, 1992a, 1992b, 1993a, 1993b, 1995a, 2006, 2008a, 2008b, 2015b, 2015c, 2017.

Rondania fasciata (Macquart, 1834) - R2, RW; 1260-1500 m; 3, 4; wes; Lutovinovas et al. 2022.

Rondania dimidiata (Meigen, 1824) - S211; 750-850 m; 2; wces; Trenchev 1980c; Hubenov 1992b, 1993a, 1993b, 2008a, 2008b.

Microsoma exiguum (Meigen, 1824) [Campogaster] - V4; 880 m; 2; dp; Trenchev 1980c; Hubenov 1992b, 1993a, 1993b, 1996a, 2008a, 2008b, 2018.

Freraea gagatea Robineau-Desvoidy, 1830 - \$; wcp, ? wces; Tschorsnig et al. 2005, 2009; Hubenov 2008a, 2008b; O'Hara et al. 2020.

Redtenbacheria insignis Egger, 1861 - R1; 1300-1350 m; 3; tp; Hubenov 1980a; 1992b, 1993a, 1993b, 1996a, 2008a, 2008b.

Euthera fascipennis (Loew, 1854) - S23; 231 m; 1; ptm; Dvořák et al. 2021.

Eliozeta helluo (Fabricius, 1805) [Clytiomyia, Heliozeta] - DW, B1, V1, V4, S211, O5, R1, R2, R3, RW, RE, BN, BS; 0-950 m; 1, 2; tp; Grigorov 1959; Lazarov et al. 1969; Kaytazov 1971a; Trenchev 1980b; Hubenov 1983b, 1985a, 1988c, 1992a, 1992b, 1993a, 1993b, 1995a, 1995b, 2001b, 2004, 2006, 2008a, 2008b, 2015b, 2015c, 2016, 2017, 2018, 2019a; Harizanov \& Harizanova 1997.

Eliozeta pellucens (Fallén, 1820) [Clytiomyia, Heliozeta] - DW, B1, B3, V4, S1, O3, O5, O62, R1, R2, R3, RW, RE; 55-1000 m; 1, 2; dp; Jacentkovsky 1936, 1937; Trenchev 1980b; Hubenov 1985a, 1985b, 1988a, 1988b, 1988c, 1992a, 1992b, 1993a, 1993b, 1995a, 2004, 2006, 2008a, 2008b, 2015b, 2015c, 2016, 2017, 2018, 2019a; Beschovski \& Hubenov 1986.

Clytiomya continua (Panzer, 1798) [Clytiomyia dalmatica (Robineau-Desvoidy, 1830] - B1, V4, S1, S211, O5, O62, R1, R2, R3, RW, RE, BS; 5-1350 m; 1, 2, 3; tp; Jacentkovsky 1936, 1937; Trenchev 1980b; Hubenov 1985a, 1985b, 1988a, 1988b, 1988c, 1992a, 1992b, 1993a, 1993b, 1995a, 2004, 2006, 2008a, 2008b, 2015b, 2015c, 2016, 2017, 2018, 2019a; Beschovski \& Hubenov 1986.

Clytiomya sola (Rondani, 1861) - hom; Tschorsnig et al. 2005, 2009; Hubenov 2008a, 2008b; O'Hara et al. 2020.

Ectophasia crassipennis (Fabricius, 1794) [Phasia] - \$ DM, E2, P1, B1, B2, B3, V1, V4, S211, T31, O4, O5, O61, O62, R1, R2, R3, RW, RE, BN, BS; 0-1650 m; 1, 2, 3, 4; 0-1700 m; tp; Löw 1862, 1863; Nedelkov 1912; 
Jacentkovsky 1936, 1937; Lazarov et al. 1969; Kaytazov 1971a; Trenchev 1980b; Hubenov 1982b, 1983b, 1985a, 1985b, 1988a, 1988b, 1988c, 1990, 1992a, 1992b, 1993a, 1993b, 1995a, 1995b, 1999, 2001a, 2004, 2006, 2008a, 2008b, 2015b, 2015c, 2016, 2017, 2018, 2019a; Beschovski \& Hubenov 1986; Kontev et al. 1991.

Ectophasia leucoptera (Rondani, 1865) [? Phasia nigerrima Hubenov, 1982] - O62, R2, RE, BS; 0-400 m; 1; nmwca; Hubenov 1982c, 1985a, 1985b, 1988a, 1988b, 1992a, 1992b, 1993a, 1993b, 1999, 2001a, 2004, 2008a, 2008b, 2015b, 2017; Herting 1984; Beschovski \& Hubenov 1986; Herting \& Dely-Draskovits 1993.

Ectophasia oblonga (Robineau-Desvoidy, 1830) [E. rubra (Girschner, 1888; Phasia] - B1, V4, O5, O62, R1, R2, R3, RW, RE, BN; 100-1400 m; 1, 2, 3; wp; Hubenov 1982a, 1982b, 1983b, 1985a, 1985b, 1988a, 1988b, 1988c, 1992a, 1992b, 1993a, 1993b, 1995a, 1995b, 1999, 2001a, 2004, 2006, 2008a, 2008b, 2015b, 2015c, 2016, 2017, 2018, 2019a; Beschovski \& Hubenov 1986.

Subclytia rotundiventris (Fallén, 1820) - \$; tp; Tschorsnig et al. 2005, 2009; Hubenov 2008a, 2008b; O’Hara et al. 2020.

Gymnosoma clavatum (Rohdendorf, 1947) [G. verbekei (Mesnil, 1952)] - B1, V4, O5, O62, R1, R2, R3, RW, RE, BN, BS; 0-1700 m; 1, 2, 3, 4; tp; Hubenov 1977, 1985b, 1988a, 1988b, 1988c, 1992a, 1992b, 1993a, 1993b, 1995a, 2004, 2006, 2008a, 2008b, 2015b, 2015c, 2016, 2017, 2018, 2019a; Lavchiev et al. 1977; Beschovski \& Hubenov 1986.

Gymnosoma costata (Panzer, 1800) - V4, O62, R1, R2, RE; 100-950 m; 1, 2; wcp, ? tp, ? e; Hubenov 1983a, 1985b, 1988a, 1988b, 1990, 1992a, 1992b, 1993a, 1993b, 1999, 2001a, 2004, 2008a, 2008b, 2015b, 2016, 2017, 2018; Beschovski \& Hubenov 1986.

Gymnosoma desertorum (Rohdendorf, 1947) - DW, S211, TL, O5, O62, R2, R3, RE; 60-1250 m; 1, 2, 3; po; Hubenov 1980b, 1985a, 1985b, 1988a, 1988b, 1988c, 1992a, 1992b, 1993a, 1993b, 1995a, 1999, 2001a, 2004, 2008a, 2008b, 2015b, 2015c, 2017; Trenchev 1980b; Beschovski \& Hubenov 1986.

Gymnosoma dolycoridis Dupuis, 1961 - B1, B2, V1, V4, S21, S211, O5, O62, R1, R2, R3, RW, RE, BS; 0-1360 m; 1, 2, 3; po; Lavchiev et al. 1977; Trenchev 1980b; Beschovski \& Hubenov 1986; Hubenov 1988a, 1988b, 1988c, 1992a, 1992b, 1993a, 1993b, 1995a, 1996b, 2004, 2006, 2008a, 2008b, 2015b, 2015c, 2016, 2017, 2018, 2019a.

Gymnosoma inornatum Zimin, 1966 - DW, V4, O5, O62, R1, R2, R3, RE, BN; 0-1250 m; 1, 2, 3; po; Hubenov 1977, 1985b, 1988a, 1988b, 1988c, 1990, 1992a, 1992b, 1993a, 1993b, 1995a, 1999, 2001a, 2004, 2008a, 2008b, 2015b, 2015c, 2016, 2017, 2018; Trenchev 1980b; Beschovski \& Hubenov 1986.

Gymnosoma nitens Meigen, 1824 - B1, V3, V4, S1, O5, R1, R2, R3, R4, RW; 550-1400 m; 1, 2, 3; esca; Jacentkovsky 1936, 1937; Hubenov 1988c, 1992a, 1992b, 1993a, 1993b, 1995a, 1996a, 2006, 2008a, 2008b, 2015b, 2015c, 2016, 2017, 2018, 2019a.

Gymnosoma nudifrons Herting, 1966 - O5, R2, R3; 580-1300 m; 1, 2, 3; tp; Hubenov 1980a, 1988c, 1992a, 1992b, 1993a, 1993b, 1995a, 2008a, 2008b, 2015b, 2015c, 2017.

Gymnosoma rotundatum (Linnaeus, 1758) - DW, DM, E1, P1, P2, B1, B2, B3, V1, V4, S1, S21, S211, TL, O3, O5, O61, O62, R1, R2, R3, RW, RE, BN, BS; 0-1800 m; 1, 2, 3, 4; po; Joakimoff 1899; Nedelkov 1912; Jacentkovsky 1936, 1937; Trenchev 1980b; Hubenov 1985b, 1988a, 1988b, 1988c, 1990, 1992a, 1992b, 1993a, 1993b, 1995a, 1995b, 1996b, 1999, 2001a, 2004, 2006, 2008a, 2008b, 2015b, 2015c, 2016, 2017, 2018, 2019a; Beschovski \& Hubenov 1986.

Gymnosoma rungsi (Mesnil, 1952) - swp, mit, ? mwca; Tschorsnig et al. 2005, 2009; Hubenov 2008a, 2008b; O'Hara et al. 2020.

Cistogaster globosa (Fabricius, 1775) - V1, S211, R2, BS; 0-800 m; 1, 2; ess; Nedelkov 1912; Trenchev 1980b; Hubenov 1992a, 1992b, 1993a, 1993b, 2008a, 2008b, 2015b, 2017.

Opesia cana (Meigen, 1824) [Xysta] - V4, R2, RE; 350-1120 m; 1, 2, 3; tp; Hubenov 1983a, 1992a, 1992b, 1993a, 1993b, 2004, 2008a, 2008b, 2015b, 2017, 2018.

Opesia grandis (Egger, 1860) [Xysta] - BS; 0-10 m; 1; tp; Hubenov 1977, 1992b, 1993a, 1993b, 2008a, 2008b.

Elomya lateralis (Meigen, 1824) [Ananta, Helomyia] - B1, V1, V4, S1, S211, TL, O5, O62, R1, R2, R3, RW, RE; 90-1400 m; 1, 2, 3; tp; Nedelkov 1912; Grigorov 1959; Lazarov et al. 1969; Kaytazov 1971a; Trenchev 1980b; Hubenov 1985a, 1985b, 1988a, 1988b, 1988c, 1992a, 1992b, 1993a, 1993b, 1995a, 1999, 2001a, 2004, 2006, 2008a, 2008b, 2015b, 2015c, 2016, 2017, 2018, 2019a; Beschovski \& Hubenov 1986.

Phasia (Phasia) aurigera (Egger, 1860) [Alophora, Hyalomyia] - S1; 260-720 m; 1, 2; dp; Draber-Mońko 1965; Nikolova 1972; Hubenov 1992b, 1993a, 1993b, 2008a, 2008b. 
Phasia (Phasia) aurulans Meigen, 1824 [Alophora] - V1, S211, O61, R1; 320-900 m; 1, 2; h; Lavchiev et al. 1977; Trenchev 1980b; Hubenov 1992b, 1993a, 1993b, 2008a, 2008b, 2016, 2017.

Phasia (Phasia) hemiptera (Fabricius, 1794) [Alophora] - O61, R1, R3; 320-1000 m; 1, 2; tp, ? hoes; Nedelkov 1912; Trenchev 1980b; Hubenov 1992b, 1993a, 1993b, 2008a, 2008b, 2016, 2017.

Phasia (Phasia) obesa (Fabricius, 1798) [Alophora, Alophorella, Hyalomyia] - B1, V4, S1, S211, O5, O61, O62, R1, R2, R3, RW, RE, BS; 0-1700 m; 1, 2, 3, 4; tp, ? hop; Jacentkovsky 1936, 1937; Belanovsky 1951; Draber-Mońko 1965; Trenchev 1980b; Hubenov 1983a, 1985a, 1985b, 1988a, 1988b, 1988c, 1990, 1992a, 1992b, 1993a, 1993b, 1995a, 1999, 2001a, 2004, 2006, 2008a, 2008b, 2015b, 2015c, 2016, 2017, 2018, 2019a; Beschovski \& Hubenov 1986.

Phasia (Phasia) subcoleoptrata (Linnaeus, 1767) [Alophora] - DW, P1, B1, B2, V4, O5, O61, R1, R2, RW, RE; 30-1400 m; 1, 2, 3; tp; Nedelkov 1912; Lazarov et al. 1969; Kaytazov 1971a; Trenchev 1980b; Hubenov 1985a, 1992a, 1992b, 1993a, 1993b, 1995a, 1996b, 2004, 2006, 2008a, 2008b, 2015b, 2015c, 2016, 2017, 2018, 2019a; Harizanov \& Harizanova 1997.

Phasia (Hyalomyia) pandellei (Dupuis, 1957) [Alophora] - DW; 290 m; 1; e; Hubenov 1980b, 1992b, 1993a, 1993b, 2008a, 2008b.

Phasia (Hyalomyia) pusilla Meigen, 1824 [Alophora, Parallophora, Hyalomyia] - E1, E2, B1, V1, V4, S1, TL, O5, O61, O62, R1, R2, RW, RE; 85-1200 m; 1, 2, 3; po; Jacentkovsky 1936, 1937; Draber-Mońko 1965; Trenchev 1980b; Hubenov 1985b, 1988a, 1988b, 1992a, 1992b, 1993a, 1993b, 1995a, 2004, 2006, 2008a, 2008b, 2015b, 2015c, 2016, 2017, 2018, 2019a; Beschovski \& Hubenov 1986.

Xysta holosericea (Fabricius, 1805) [Kiritshenkia] - V1; 600 m; 1, 2; mi; Lavchiev et al. 1977; Hubenov 1992b, 1993a, 1993b, 2008a, 2008b.

Catharosia pygmaea (Fallén, 1815) - DM, S211, BS; 0-800 m; 1, 2; wcp; Hubenov 1977, 1992b, 1993a, 1993b, 2008a, 2008b; Trenchev 1980c.

Litophasia hyalipennis (Fallén, 1815) - ^; e; Tschorsnig et al. 2005, 2009; Hubenov 2008a, 2008b; O’Hara et al. 2020.

Strongygaster globula (Meigen, 1824) [Tamiclea] - S21, S211, TL, R2; 20-850 m; 1, 2; hoes, ? tes; Hubenov 1980a, 1992a, 1992b, 1993a, 1993b, 2008a, 2008b, 2015b, 2017; Trenchev 1980b.

Dionaea aurifrons (Meigen, 1824) - B1, V4, S211, O5, O62, R1, R2, R3, RW, RE, BS; 0-1550 m; 1, 2, 3, 4; tp, ? dp; Hubenov 1977, 1985b, 1988a, 1988b, 1988c, 1990, 1992a, 1992b, 1993a, 1993b, 1995a, 2004, 2006, 2008a, 2008b, 2015b, 2015c, 2016, 2017, 2018, 2019a; Trenchev 1980b; Beschovski \& Hubenov 1986.

Eulabidogaster setifacies (Rondani, 1861) - RW; 400 m; 1; wcp; Jacentkovsky 1936, 1937; Hubenov 1992b, 1993a, 1993b, 2006, 2008a, 2008b.

Leucostoma abbreviatum Herting, 1971 - \$; mit; Tschorsnig et al. 2005, 2009; Hubenov 2008a, 2008b; O’Hara et al. 2020.

Leucostoma anthracinum (Meigen, 1824) - O62, R2; 250-300 m; 1; ? wcp; Hubenov 1982a, 1985b, 1988a, 1988b, 1992a, 1992b, 1993a, 1993b, 2008a, 2008b, 2015b, 2017; Beschovski \& Hubenov 1986.

Leucostoma engeddense Kugler, 1966 - atm; Tschorsnig et al. 2005, 2009; Hubenov 2008a, 2008b; O’Hara et al. 2020.

Leucostoma nudifacies Tschorsnig, 1991 - sesfe; Tschorsnig et al. 2005, 2009; Hubenov 2008a, 2008b; O’Hara et al. 2020.

Leucostoma simplex (Fallén, 1815) - S1; 280 m; 1; sk, i; Jacentkovsky 1936, 1937; Hubenov 1992b, 1993a, 1993b, 2008a, 2008b.

Leucostoma tetraptera (Meigen, 1824) [L. anale (Meigen, 1824); Pseudoleucostoma slivense Jacentkovsky, 1937; P. buresi Jacentkovsky, 1938] - E1, S1, V4, O5, O61, R1, R2, RW, BS; 0-1300 m; 1, 2, 3; pat; Jacentkovsky 1936, 1937, 1938; Belanovsky 1951; Josifov 1957; Herting 1984; Hubenov 1985b, 1988a, 1988b, 1992a, 1992b, 1993a, 1993b, 1995a, 2006, 2008a, 2008b, 2015b, 2015c, 2016, 2017, 2018; Beschovski \& Hubenov 1986; Herting \& Dely-Draskovits 1993; Tschorsnig et al. 2005, 2009.

Leucostoma turonicum Dupuis, 1964 - csei; Tschorsnig et al. 2005, 2009; Hubenov 2008a, 2008b; O’Hara et al. 2020.

Clairvillia biguttata (Meigen, 1824) - B1, V3, V4, O5, O62, R1, R2, RW, RE, BS; 0-1130 m; 1, 2; dp; Jacentkovsky 1936, 1937; Hubenov 1985b, 1988a, 1988b, 1992a, 1992b, 1993a, 1993b, 1995a, 1996a, 1999, 2001a, 2004, 2006, 2008a, 2008b, 2015b, 2015c, 2016, 2017, 2018, 2019a; Beschovski \& Hubenov 1986.

Clairvillia pninae Kugler, 1971 - hom; Tschorsnig et al. 2005, 2009; Hubenov 2008a, 2008b; O’Hara et al. 2020. 
Labigastera forcipata (Meigen, 1824) [Dionaea] - V4, O5, R1, R2, RW; 800-1300 m; 2, 3; wes; Hubenov 1982a; 1992a, 1992b, 1993a, 1993b, 1995a, 2006, 2008a, 2008b, 2015b, 2015c, 2016, 2017, 2018.

Labigastera nitidula (Meigen, 1824) - swp, mt; Tschorsnig et al. 2005, 2009; Hubenov 2008a, 2008b; O'Hara et al. 2020.

Labigastera pauciseta (Rondani, 1861) [Dionaea] - V4, O5, R2, BS; 0-1300 m; 1, 2, 3; e, csei; Hubenov 1977, 1992a, 1992b, 1993a, 1993b, 1995a, 2008a, 2008b, 2015b, 2015c, 2017, 2018.

Weberia digramma (Meigen, 1824) - mt; Tschorsnig et al. 2005, 2009; Hubenov 2008a, 2008b; O’Hara et al. 2020.

Lophosia (Lophosia) fasciata Meigen, 1824 - S211; 700-900 m; 2; po; Trenchev 1980b; Hubenov 1992b, 1993a, 1993b, 2008a, 2008b.

Cylindromyia (Exogaster) rufifrons (Loew, 1844) - TL; 200 m; 1; nmwca, ? mwca; Nedelkov 1912; Hubenov 1992b, 1993a, 1993b, 2008a, 2008b.

Cylindromyia (Cylindromyia) bicolor (Olivier, 1812) [Ocyptera] - B1, V1, V4, S1, S211, TL, O5, O62, R1, R2, R3, RW, RE, BN, BS; 0-1300 m; 1, 2, 3; cseit; Nedelkov 1912; Trenchev 1980b; Hubenov 1985b, 1988a, 1988b, 1988c, 1992a, 1992b, 1993a, 1993b, 1995a, 1995b, 1999, 2001a, 2004, 2006, 2008a, 2008b, 2015b, 2015c, 2016, 2017, 2018, 2019a; Beschovski \& Hubenov 1986.

Cylindromyia (Cylindromyia) brassicaria (Fabricius, 1775) [Ocyptera] - DW, E1, P1, P2, B1, B2, V1, V3, V4, S1, TL, O3, O5, O61, O62, R1, R2, R3, RW, RE, BN; 30-1800 m; 1, 2, 3, 4; po, ? hop; Joakimoff 1899; Nedelkov 1909, 1912; Jacentkovsky 1936, 1937; Dupuis 1963; Trenchev 1980b; Hubenov 1985b, 1988a, 1988b, 1988c, 1990, 1992a, 1992b, 1993a, 1993b, 1995a, 1995b, 1996b, 1999, 2001a, 2004, 2006, 2008a, 2008b, 2015b, 2015c, 2016, 2017, 2018, 2019a; Beschovski \& Hubenov 1986.

Cylindromyia (Cylindromyia) brevicornis (Loew, 1844) [Ocyptera] - E1, V4, O5, O61, R1, R2; 100-1300 m; 1, 2, 3; des; Jacentkovsky 1936, 1937; Hubenov 1992a, 1992b, 1993a, 1993b, 1995a, 2008a, 2008b, 2015b, 2016, 2017, 2018.

Cylindromyia (Cylindromyia) crassa (Loew, 1845) [Dupuisia] - V3, V4, S1, S211, R1, R3, RW, BS; 0-1100 m; 1, 2, 3; mss; Lavchiev et al. 1977; Trenchev 1980b; Hubenov 1988c, 1990, 1992a, 1992b, 1993a, 1993b, 2006, 2008a, 2008b, 2015b, 2015c, 2016, 2017, 2018.

Cylindromyia (Cylindromyia) pilipes (Loew, 1844) - DM, V4, S1, TL, O62, R1, R2, RW, BS; 0-1200 m; 1, 2, 3; wcp; Nedelkov 1912; Jacentkovsky 1936, 1937; Hubenov 1985b, 1988a, 1988b, 1990, 1992a, 1992b, 1993a, 1993b, 1999, 2001a, 2006, 2008a, 2008b, 2015b, 2016, 2017, 2018; Beschovski \& Hubenov 1986.

Cylindromyia (Cylindromyia) rubida (Loew, 1854) [Exogaster, Plesiocyptera] - V4; 800-900 m; 2; swppt; Nedelkov 1912; Hubenov 1992b, 1993a, 1993b, 2008a, 2008b, 2018.

Cylindromyia (Cylindromyia) xylotina (Egger, 1860) - \$; cse; Tschorsnig et al. 2005, 2009; Hubenov 2008a, 2008b; O'Hara et al. 2020.

Cylindromyia (Ocypterula) pusilla (Meigen, 1824) - V1, R1; 600-1300 m; 2, 3; tp; Nedelkov 1912; Hubenov 1992b, 1993a, 1993b, 2008a, 2008b, 2016, 2017.

Cylindromyia (Conopisoma) rufipes (Meigen, 1824) - swppt; Tschorsnig et al. 2005, 2009; Hubenov 2008a, 2008b; O'Hara et al. 2020.

Cylindromyia (Calocyptera) intermedia (Meigen, 1824) [C. scalaris (Loew, 1844); Ocyptera cylindrica (Meigen, 1824); O. boscii Bezzi \& Stein, 1907] - E1, V4, S1, S211, O5, O61, O62, R1, R2, RW, RE, BN, BS; 0-1300 m; 1, 2, 3; hn; Nedelkov 1909, 1912; Jacentkovsky 1936, 1937; Drensky 1942; Trenchev 1980b; Hubenov 1985b, 1988a, 1988b, 1990, 1992a, 1992b, 1993a, 1993b, 1995a, 2004, 2006, 2008a, 2008b, 2015b, 2015c, 2016, 2017, 2018; Beschovski \& Hubenov 1986.

Cylindromyia (Neocyptera) auriceps (Meigen, 1838) [Ocyptera] - B1, V4, S1, T31, R2, RW, BS; 0-1200 m; 1, 2, 3; tp; Jacentkovsky 1936, 1937; Trenchev 1980b; Hubenov 1992a, 1992b, 1993a, 1993b, 2006, 2008a, 2008b, 2015b, 2017, 2018, 2019a.

Cylindromyia (Neocyptera) hermonensis Kugler, 1974 - R2; 350-370 m; 1; ? hom; Hubenov 1982a, 1985b, 1988a, 1988b, 1992b, 1993a, 1993b, 2008a, 2008b; Beschovski \& Hubenov 1986.

Cylindromyia (Neocyptera) interrupta (Meigen, 1824) - V4, O5, R2; 700-1300 m; 2, 3; h; Hubenov 1982a, 1992a, 1992b, 1993a, 1993b, 1995a, 2008a, 2008b, 2015b, 2015c, 2017, 2018.

Hemyda obscuripennis (Meigen, 1824) - dpo; Tschorsnig et al. 2005, 2009; Hubenov 2008a, 2008b; O'Hara et al. 2020. 
Hemyda vittata (Meigen, 1824) [Phania] - V4, O5, R2, RE, BN; 700-1100 m; 2, 3; hoes, ? tp; Hubenov 1983a, 1992a, 1992b, 1993a, 1993b, 1995a, 1995b, 2004, 2008a, 2008b, 2015b, 2015c, 2017, 2018.

Besseria anthophila (Loew, 1871) [Apostrophus] - B2, R1; 1300-1400 m; 3; h; Jacentkovsky 1936, 1937; Hubenov 1992b, 1992b, 1993a, 1993b, 1996a, 1996b, 2008a, 2008b, 2016, 2017.

Besseria dimidiata (Zetterstedt, 1844) - V4; O3, O62, R2; 300-1100 m; 1, 2, 3; cse; Belanovsky 1951; Trenchev 1980b; Hubenov 1985b, 1988a, 1988b, 1990, 1992a, 1992b, 1993a, 1993b, 1999, 2001a, 2008a, 2008b, 2015b, 2017, 2018; Beschovski \& Hubenov 1986.

Besseria lateritia (Meigen, 1824) [Phaniosoma] - O5, O62, R2, R3, BS; 0-600 m; 1; mit; Hubenov 1977, 1985b, 1988a, 1988b, 1988c, 1992a, 1992b, 1993a, 1993b, 2008a, 2008b, 2015b, 2015c, 2017; Beschovski \& Hubenov 1986.

Besseria melanura (Meigen, 1824) - V1, S211; 600-850 m; 1, 2; wcp; Nedelkov 1912; Trenchev 1980b; Hubenov 1992b, 1993a, 1993b, 2008a, 2008b.

Besseria reflexa Robineau-Desvoidy, 1830 [Wahlbergia appendiculata (Perris, 1852)] - V3, V4, S1; 300-800 m; 1, 2; cse; Jacentkovsky 1936, 1937; Tschorsnig et al. 2005, 2009; Hubenov 1993a, 1993b, 2008a, 2008 b.

Phania curvicauda (Fallén, 1820) [Weberia] - V1, S211, R3, RW; 600-1100 m; 2, 3; ei; Nedelkov 1912; Trenchev 1980b; Hubenov 1988c, 1992b, 1993a, 1993b, 2006, 2008a, 2008b, 2015c.

Phania funesta (Meigen, 1824) [Weberia pseudofunesta (Villeneuve, 1931)] - B1, V3, V4, O5, O62, R1, R2, RE, BS; 0-850 m; 1, 2; ei; Jacentkovsky 1936, 1937; Belanovsky 1951; Hubenov 1985b, 1988a, 1988b, 1992a, 1992b, 1993a, 1993b, 1995a, 1999, 2001a, 2004, 2008a, 2008b, 2015b, 2015c, 2016, 2017, 2018, 2019a; Beschovski \& Hubenov 1986.

Phania incrassata Pandellé, 1894 - cse; Tschorsnig et al. 2005, 2009; Hubenov 2008a, 2008b; O’Hara et al. 2020. Phania speculifrons (Villeneuve, 1919) [Weberia] - V1; 600 m; 1; cse, ? e; Lavchiev et al. 1977; Hubenov 1992b, 1993a, 1993b, 2008a, 2008b.

\section{Conclusion}

A total of 5038 species of order Diptera (Nematocera - 1672 species, Brachycera - 3366 species), belonging to 110 families, has been established in Bulgaria so far. The families Chironomidae ( 327 species - 6.5\%), Mycetophilidae (263 species - 5.2\%) and Cecidomyiidae (262 species - 5.2\%) of Nematocera and Tachinidae (425 species $-8.4 \%$ ), Syrphidae (303 species $-6.0 \%$ ) and Muscidae (267 species - 5.3\%) of Brachycera are the most numerous. Of the established species, 61 are pests on the forestry or agriculture and 75 species have a human or veterinary medical significance. The distribution of dipterans in the different regions of Bulgaria is presented. The vertical distribution of the families is considered. The greatest number of species has been found in the zone of the xerothermic oak forests (3362 species $-66.7 \%$ ). The dipterous fauna can be divided into 2 main groups: 1) species with Mediterranean type of distribution (515 species - 10.2\%) - more thermophilic and distributed mainly in the southern parts of the Palaearctic and the lower parts of the mountains. The species of the southern type, distributed in the Palaearctic and beyond it, can be formally related to this group as well; 2) species with Palaearctic and Eurosiberian type of distribution (4377 species - 87.2\%) - more eurybiontic and widely distributed in the Palaearctic. The species of the northern type, distributed in the Palaearctic and beyond it, can be formally related to this group as well. The zoogeographical character of the Diptera fauna is determined by the second group. The South European and Southeast European forms are the most numerous in the first group. The European and Holarctic taxa prevail in the second group. A total of 128 (2.5\%) endemics has been found. The endemism is better presented in Nematocera (60 species - 3.6\%) than in Brachycera (68 species - 2.0\%). The distribution of the zoogeographical categories in the separate vegetation belts of Bulgaria is scrutinized. The variety of areographical categories (150) decreases with altitude. The bibliography contains 1372 publications.

Xerothermic oak forests (3362 species or 66.7\%). Of the species with Mediterranean type of distribution (372 species or 11.1\%) the Southeast European, South European, Holomediterranean and South European-North African taxa are the most numerous. Of the species with Palaearctic and Eurosiberian type of distribution (2918 species or 86.8\%) - the European, Holarctic, Transpalaearctic, West Palaearctic, Palaearctic-Oriental, West and Central Palaearctic, Holarctic-Oriental and Disjunct Eurosiberian taxa are best represented. This belt includes the greatest number of areographical categories -143 . The main part of the endemic forms has been established 
in this belt -72 species (2.1\%). The Balkan and Bulgarian endemics prevail. Most Mediterranean species (324 species or $9.6 \%)$ are represented.

Xeromesophyllic and mesophyllic mixed (oak-hornbeam) forests (2598 species or $51.8 \%)$. Of the species with Mediterranean type of distribution (153 species or 5.9\%) the Holomediterranean, North Mediterranean and South European taxa are the most numerous. Of the species with Palaearctic and Eurosiberian type of distribution (2421 species or 93.2\%) the European, Holarctic, Transpalaearctic, West Palaearctic, Disjunct Eurosiberian and Palaearctic-Oriental taxa are best represented. The percentage of the Mediterranean species (133 species or $5.1 \%$ ) and endemics (24 species or 0.9\%) decreases. The Bulgarian endemics prevail.

Beech forests (2272 species or 45.3\%). Of the species with Mediterranean type of distribution (103 species or 4.5\%) the South European, Southeast European and Holomediterranean taxa are the most numerous. Of the species with Palaearctic and Eurosiberian type of distribution (2134 species or 93.9\%) the European, Holarctic, Transpalaearctic, Disjunct Eurosiberian, West Palaearctic, Palaearctic-Oriental and Holoeurosiberian taxa are best represented. The number of the areographical categories decreases significantly. The percentage of the endemics increases (35 species or 1.5\%). The Bulgarian endemics prevail.

Coniferous forests (1123 species or 22.4\%). Of the species with Mediterranean type of distribution (36 species or 3.2\%) two Super-Palaearctic forms of southern type (South Palaearctic-Oriental) are represented. The South European, Holomediterranean, Balkan-Anatolian and Central and South European-North African taxa are the most numerous. Of the species with Palaearctic and Eurosiberian type of distribution (1065 species or $94.8 \%$ ) the European, Holarctic, Transpalaearctic and Disjunct Eurosiberian taxa prevail. The number of the areographical categories and the percentage of the Mediterranean forms decrease significantly (34 species or 3.0\%). The Bulgarian endemics prevail.

Subalpine vegetation (348 species or 6.9\%). Of the species with Mediterranean type of distribution (12 species or 3.4\%) the Super-Palaearctic forms are not presented. All areographical categories are presented with one species each. Of the species with Palaearctic and Eurosiberian type of distribution (330 species or 94.8\%) the European and Holarctic taxa prevail. The percentage of the Mediterranean forms is considerably decreased. The areographical categories are decreased. Four Bulgarian and two regional endemics are established. Forty families are represented. It is of interest that the species Cremifania bulgarica Papp, 2010 (family Cremifaniidae, recorded from the Rila Mts. at $2250 \mathrm{~m}$ a.s.l.) is found and described - third Palaearctic species of the family).

Alpine vegetation (57 species or 1.1\%). This zone is best presented in the Rila and Pirin Mts. Two species with Mediterranean type of distribution and forty-one species with Palaearctic and Eurosiberian type of distribution (28 areographical categories, of which 15 are represented by one species each) have been established. The Holarctic, European and Cosmopolitan forms are represented by more species. Twenty-one families (4 of Nematocera and 17 of Brachycera) have been established. In the alpine zone, one Bulgarian endemic (Molophilus lautereri Stary, 1974 of the family Limoniidae) is known, reported from the Rila Mts. In the alpine zone, there is no a faunistic similarity between the Rila and Pirin Mts. With the exception of 4-5 families, there is almost no studies on the two-winged insects in this zone of the high Bulgarian mountains.

\section{Acknowledgements}

The author is grateful to Dr. Venelin Beschovski and Dr. Valentin Lavchiev for the help, advices and literature on the Bulgarian Diptera fauna. The author also would like to thank Dr. Pjotr Oosterbroek for the provided data referring to the families Tipulidae, Limoniidae and Pediciidae; Dr. Paraskeva Michailova for the advices and literature on the family Chironomidae; Dr. Dimitar Bechev for the advices and literature on the families Hesperinidae, Mycetophilidae, Ditomyiidae, Bolitophilidae, Diadocidiidae, Keroplatidae and Sciaridae; Dr. Mario Langourov for some information about the family Phoridae; Dr. Mihail Kechev for the literature and data on the family Dolichopodidae; Dr. Jan Ježek and Dr. Diana Ganeva for the literature on the Bulgarian Psychodidae and Tabanidae; Dr. Georgi Georgiev and Dr. Anelia Pencheva for the data and literature on the some forests pests; Dr. Petar Beron for the advices and literature on the high mountains, parasitic and cave dipterans; Dr. Alexi Popov for the advices and literature on the separate families; Dr. Nyonka Velcheva and Dr. Georgi Trenchev for the data on some hosts of the family Tachinidae. 


\section{References}

Adler P. \& Crosskey R. 2018. World Blackflies (Diptera: Simuliidae): a comprehensive revision of the taxonomic and geographical inventory [2018]. 134 p. https://biomia.sites.clemson.edu/pdfs/blackflyinventory.pdf

Agushev T. 2012. Preliminary study of species composition, ecology, and health importance of family Culicidae in Plovdiv City, Bulgaria. Science \& Techynologies 2 (1): 204-207. (In Bulgarian with English summary).

Agushev T. 2014. Preliminary results for the species composition and dynamics of mosquito family (Culicidae) in the city of Plovdiv. Ecologica Balkanica 5 (Special edition): 123-131.

Agushev T. 2015. Vidov sastav, ekologiya i znachenie na semeystvo komari (Culicidae) v grad Plovdiv [Species composition, ecology and importance of the mosquito family (Culicidae) in the Plovdiv Town]. Ph.D. thesis, Plovdiv, Agricultural University. 172 p. (In Bulgarian).

Agushev T. 2018. Prouchvane na vidoviya sastav na komarite ot semeystvo Culicidae v grad Plovdiv. [Study of the species composition of the mosquitoes family (Culicidae) in the Plovdiv Town]. Mollov I., Georgiev D. \& Todorov O. (Eds.). Faunistic diversity of the city of Plovdiv (Bulgaria), Volume 1 - Invertebrates. Bulletin of the Natural History Museum - Plovdiv, Supplement 1: 49-55.

Agushev T. \& Bileva T. 2014. Prouchvane na vidoviya sastav na komarite ot semeystvo Culicidae v grad Plovdiv [Study of the species composition of the mosquitoes of the Culicidae family in the Plovdiv Town]. Natural \& Mathematical science 4 (3): 79-83.

Angelov A. 1956 Hydrologische und hydrobiologische Untersuchungen über den Rabischa-See. Annuaire de l’Université de Sofia, Faculté de Biologie, Géologie et Géographie 59 (1 - Zoology): 1-3 (In Bulgarian, Russian and German summaries).

Antonov C. 1927. Maladies et enemies des cultures dans le champ experimental pour l'année économique 19241925. Compte-rendu de la Station agronomique déxpérimentation et de contrôle à l'Obrazcov Čiflik près de Roussé pour en 1925. p. 86.

Arndt W. 1943. Beiträge zur Kenntnis der Süsswasserfauna Bulgariens. (Ergebnisse eines Aufenthaltes in Bulgarien im Juli - August 1924). Mitteilungen aus den Königl Naturwissenschaftlichen Instituten in Sofia (Bulgarien) 16: 189-206.

Ashe P. \& Cranston P. S. 1990. Family Chironomidae. In: Soós Á. \& Papp L. (Eds.). Catalogue of Palaearctic Diptera. 2. Budapest, Akadémiai Kiadó, Hungarian Natural History Museum. pp. 113-355.

Ashe P. \& O'Connor J. P. 2009. A World catalogue of Chironomidae. Part 1. Biochonomyiinae, Chilenomyiinae, Podonominae, Aphroteniinae, Tanypodinae, Usambaromyiinae, Diamesinae, Prodiamesinae and Telmatogetoninae. Dublin, Irish Biogeographical Society \& National Museum of Ireland. 445 p.

Ashe P. \& O'Connor J. P. 2012. A World catalogue of Chironomidae. Part 2. Orthocladiinae. Dublin, Irish Biogeographical Society \& National Museum of Ireland. 968 p.

Atanassov D. \& Christova T. 1960. The results from the investigation of the resistance of Anopheles maculipennis to the chloric insecticides in some region of the country. Trudy naucho-issledovatelskogo instituta epidemologii i mikrobiologii 7: 279-287. (In Russian, English summary).

Atanassov D. \& Petrov B. 1961. Prouchvane anofeliynostta ns nyakoi napoitelni sistemi v Narodna Republika Bulgariya [Study of the Anaphelian fauna of some irrigation systems in the Republic of Bulgaria]. Higiene 4 (3): 51-55. (In Bulgarian).

Atanassof D., Avlavidopv T., Kovchazov G., Hristova T. \& Deanov I. 1962. Sastoyanie na chuvstvitelnostta na An. maculipennis Meig. i An. sacharovi Favre. po otnoshenie na DDT i dieldrin v nyakoi rayoni na Bulgaria [Sensibility state of An. maculipennis Meig. and An. sacharovi Favre. with regard to DDT and dieldrin in some regions of Bulgaria]. Byuletin na NIEM 3: 121-128. (In Bulgarian).

Avlavidov T. 1947. Anofeliynata fauna v Dolniya Kamchiyski kray [The Anaphelian fauna in the Lower Kamchia region]. Lekarski pregled 9 (9/10): 127-129. (In Bulgarian).

Avlavidov T. 1948. Za kasnata esenna malariya prez 1947 godina v Severna Bulgaria [For the late autumn malaria in 1947 in northern Bulgaria]. Lekarski pregled 10 (9/10): 134-136. (In Bulgarian).

Avlavidov T. 1958. Po vaprosa za izkorenyavane na malariyata vav Varnenski okrag [To the question for the malaria eradication in the Varna district]. Hygiene, Epidemology and Microbiology 2 (2): 16-19. (In Bulgarian).

Avlavidov T. 1959. To the question for the sensibility of the anophelian mosqutos against DDT in the region of Varna. Hygiene, Epidemology and Microbiology 3 (5): 40-47. (In Bulgarian). 
Avlavidov T. 1961. Prichini za vaznikvane na malariynite zabolyavaniya v ozdravena veche teritoriya [Causes of malaria disease rising in already recovered territory]. Hygiene 4 (4): 27-30. (In Bulgarian).

Avlavidov T. 1970. Fenologichni osobenosti na anofeliynite komari v severiztochniya kray na nashata strana do likvidiraneto na malariyata [Fenological characteristics of the anopheline mosquitoes in the northeastern part of Bulgaria until the malaria eradication]. Letopisi na HEI 4 (26): 70-74. (In Bulgarian).

Bächli G. \& Teresa Rocha Pite M. 1984. Family Drosophilidae. In: Soós Á. \& Papp L. (Eds.). Catalogue of Palaearctic Diptera. 10. Budapest, Akadémiai Kiadó, Hungarian Natural History Museum. pp. 186-220.

Balevski A. \& Felbinger H. 1943. Varhu masovoto poyavyavane na nyakoi ovoshtni nepriyateli v Bulgaria prez 1943 godina [On the mass appearance of some fruit pests in Bulgaria in 1943]. Spisanie na zemedelskite izpitatelni instituti v Bulgaria 13 (3/4): 125-132. (In Bulgarian).

Bankowska R. 1964. Die Palaearctische Arten der Gattung Sphaerophoria St. et Sery. Annales Zoologici 22 (15): 285-353.

Bankowska R. 1967a. Materiaux pour l'etude des dipteres de Bulgarie (Stratiomyidae, Conopidae, Pipunculidae et Acroceridae). Fragmenta faunistica (Warszawa) 13 (17): 303-314.

Bankowska R. 1967b. Materiaux pour l'etude des Syrphidae (Diptera) de Bulgarie. Fragmenta faunistica (Warszawa), 13 (21): 345-389.

Barták M. 1981. A revision of the Rhamphomyia (Diptera, Empididae), with descriptions of new species. Acta Universitatis Carolinae Biologica, 361-407.

Barták M. 1985. A revision of the Rhamphomyia subgenus Lundstromiella (Diptera, Empididae), with description of a new species. Acta Universitatis Carolinae Biologica, 9-46.

Barták M. \& Kubík Š. 2013. Species of Bicellaria Macquart (Diptera: Hybotidae) of Europe, with descriptions of four new species. Zootaxa 3647 (2): 251-278.

Bechev D. 1985a. The species of Superfamily Mycetophiloidea (Diptera) Distributed in Bulgaria. Acta zoologica bulgarica 27: 36-40. (In Bulgarian, English summary).

Bechev D. 1985b. Acnemia vratzatica n. sp. from Bulgaria (Diptera, Mycetophilidae). Reichenbachia Staatliches Museum für Tierkunde Dresden 23 (8): 35-36.

Bechev D. 1986a. New species from Superfamily Mycetophiloidea (Diptera) for Bulgarian Fauna. Acta zoologica bulgarica 30: 72-74. (In Bulgarian, English summary).

Bechev D. 1986b. New species from the Superfamily Mycetophiloidea (Diptera) for Bulgarian Fauna. II. Acta zoologica bulgarica 32: 56-59. (in Bulgarian, English summary).

Bechev D. 1986c. Sciophila rufa Meigen (Diptera, Mycetophilidae) as a Host for Orthocentrus stigmaticus Holmgren (Hymenoptera, Ichneumonidae). Acta zoologica bulgarica 32: 60-61 (In Bulgarian, English summary).

Bechev D. 1988a. Two new species of Mycetophilidae from Bulgaria (Insecta, Diptera). Reichenbachia 25 (38): 185-186.

Bechev D. 1988b. Sciophila zaitzevi n. sp. from Bulgaria (Insecta, Diptera, Mycetophilidae). Reichenbachia 25 (39): 187-188.

Bechev D. 1989a. New species Mycetophiloidea (Diptera) for Bulgarian fauna. III. Travaux Scientifiques Universite de Plovdiv, Biologie - Animalia 27 (6): 153-159. (In Bulgarian, English summary).

Bechev D.1989b. Larvae of Mycetophiloidea (Diptera) established in fungi in Bulgaria. Travaux Scientifiques Universite de Plovdiv, Biologie - Animalia 27: 161-163. (In Bulgarian, English summary).

Bechev D. 1989c. Monocentrota matilei n. sp. from Bulgaria and Algeria (Insecta, Diptera: Keroplatidae). Reichenbachia 26 (30): 173-174.

Bechev D. 1990a. New species Mycetophiloidea (Diptera) to the Bulgarian fauna. IV. Travaux Scientifiques Universite de Plovdiv, Biologie - Animalia 28: 85-88. (In Bulgarian, English summary).

Bechev D. 1990b. Recent Holarctic species of the genus Anaclileia Meunier (Insecta, Diptera: Mycetophilidae). Reichenbachia 28 (13): 67-71.

Bechev D. 1990c. Review of the Holarctic Species of Genus Polylepta Winnertz (Insecta, Diptera: Mycetophilidae). Entomologische Abhandlungen Staatliches Museum für Tierkunde Dresden 53 (12): 179-184.

Bechev D. 1991a. New species Mycetophiloidea (Diptera) to the Bulgarian fauna. V. Travaux Scientifiques Universite de Plovdiv, Biologie - Animalia 29: 27-30. (In Bulgarian, English summary).

Bechev D. 1991b Ptychopteridae and Hesperinidae - new families to the Bulgarian fauna. Travaux Scientifiques Universite de Plovdiv, Biologie - Animalia 29, (6): 31-32. (In Bulgarian, English summary). 
Bechev D. 1991c. Macrocera gemagea sp. n. from Bulgaria (Insecta, Diptera: Mycetophiloidea). Reichenbachia 28 (35): 185-186.

Bechev D. 1992a. On the type specimens of Macrocera nigricoxa Winnertz 1863 and Macrorrhyncha exempla (Plassmann 1978) (Insecta: Diptera: Mycetophiloidea). Senckenbergiana Biologica 72 (4/6): 317-320.

Bechev D. 1992b. A new species of Genus Macrorrhyncha (Diptera: Keroplatidae) from Bulgaria. Acta zoologica bulgarica 45: 61-62.

Bechev D. 1994. New species Mycetophiloidea (Diptera) to the fauna of Bulgaria. VI. Travaux Scientifiques Universite de Plovdiv, Biologie - Animalia 30 (6): 25-28.

Bechev D. 1995 The palearctic species of the genus Scetonia Winnertz (Diptera: Mycetophilidae). Travaux Scientifiques Universite de Plovdiv, Biologie - Animalia 31 (6): 7-21.

Bechev D. 1996a. New species of fungus gnats (Diptera: Sciaroidea, excluding Sciaridae) to the fauna of Bulgaria. VII. Travaux Scientifiques Universite de Plovdiv, Biologie - Animalia 32 (6): 19-21.

Bechev D. 1997. Check-list of the fungus gnats (Diptera: Sciaroidea, excluding Sciaridae) of the Balkan Peninsula. Travaux Scientifiques Universite de Plovdiv, Biologie - Animalia 33: 9-22.

Bechev D. 1998. The species of Allodia Winnertz on the Balkan Peninsula (Diptera, Mycetophilidae). Acta zoologica bulgarica 50 (1): 61-63.

Bechev D. 1999a. Biotic connections of fungus gnats (Diptera: Sciaroidea, excluding Sciaridae) in forest habitats of Mount Pirin. Travaux Scientifiques Universite de Plovdiv, Biologie - Animalia 35: 37-40. (In Bulgarian, English summary).

Bechev D. 1999b. Species of the Genus Phronia Winnertz in Bulgaria (Diptera: Mycetophilidae). Acta zoologica bulgarica 51 (2/3): 39-42.

Bechev D. 2000. Seasonal activity and vertical distribution of fungus gnats (Diptera: Sciaroidea, excluding Sciaridae) in Western and Central parts of Star Planina Ridge (Bulgaria). Travaux Scientifiques Universite de Plovdiv, Biologie - Animalia 36: 51-66.

Bechev D. 2001. New species of fungus gnats (Diptera: Sciaroidea, excluding Sciaridae) to the fauna of Bulgaria. VIII. Travaux Scientifiques Universite de Plovdiv, Biologie - Animalia 37: 5-8.

Bechev D. 2002a. Check-list of the fungus gnats of the families Bolitophilidae, Diadocidiidae, Ditomyiidae, Keroplatidae and Mycetophilidae in Bulgaria (Diptera: Nematocera). Travaux Scientifiques Universite de Plovdiv, Biologie - Animalia 38: 81-112.

Bechev D. 2002b. Orfelia gruevi spec. nov. from Bulgaria (Diptera: Sciaroidea: Keroplatidae). Acta entomologica slovenica 10 (2): 199-201.

Bechev D. 2003. Corrections and additions to the check list of the fungus gnats in Bulgaria (Diptera: Sciaroidea). - Travaux Scientifiques Universite de Plovdiv, Biologie - Animalia 39: 97-99.

Bechev D. 2004a. Fungus Gnats (Diptera: Bolitophilidae, Diadocidiidae, Ditomyiidae, Keroplatidae, Mycetophilidae) of the Eastern Rhodopes (Bulgaria). In: Beron P. \& Popov A. (Eds.). Biodiversity of Bulgaria. 2. Biodiversity of Eastern Rhodopes (Bulgaria and Greece). Sofia, National Museum of Natural History \& Pensoft. pp. 735-739.

Bechev D. 2004b. New distributional data about the fungus gnats of the families Bolitophilidae, Diadocidiidae, Ditomyiidae and Keroplatidae in Bulgaria (Diptera: Nematocera: Sciaroidea). Travaux Scientifiques Universite de Plovdiv, Biologie - Animalia 40: 129-136.

Bechev D. 2006a. New data about Keroplatidae (Diptera: Nematocera: Sciaroidea) in Bulgaria. Historia naturalis bulgarica 17: 93-94.

Bechev D. 2006b. Fungus Gnats (Diptera: Bolitophilidae, Diadocidiidae, Ditomyiidae, Keroplatidae, Mycetophilidae) of the Western Rhodopes (Bulgaria). In: Beron P. (Eds.). Biodiversity of Bulgaria. 3. Biodiversity of Western Rhodopes (Bulgaria and Greece) I. Sofia, National Museum of Natural History \& Pensoft. pp. 701-710.

Bechev D. 2006c. The fungus gnats of the families Bolitophilidae, Diadocidiidae, Ditomyiidae and Keroplatidae (Diptera: Sciaroidea ) of Bulgaria. Scientific Studies of the University of Plovdiv, Biology, Animalia 42:21-83.

Bechev D. 2007. Семейства Bolitophilidae, Diadocidiidae, Ditomyiidae, Keroplatidae и Mycetophilidae (Diptera: Sciaroidea) на Балканския полуостров и Източното Средиземноморие. [Families Bolitophilidae, Diadocidiidae, Ditomyiidae, Keroplatidae and Mycetophilidae (Diptera: Sciaroidea) in the Balkan Peninsula and East Mediterranean]. DSc thesis, Sofia, Bulgarian Academy of Sciences. 412 p. (In Bulgarian). 
Bechev D. 2009. The species of Ctenophorinae (Diptera: Tipulidae) in the fauna of Bulgaria. ZooNotes 1: 1-3. (In Bulgarian with English summary).

Bechev D. 2010. On the family Mycetophilidae (Insecta: Diptera) in Bulgaria. ZooNotes, Supplement 1, 1-72.

Bechev D. \& Chandler P. 2011. Catalogue of the Bolitophilidae and Diadocidiidae of the World. Zootaxa 2741: 38-58.

Bechev D. \& Pavlova A. 2012. Greenomyia tomovi sp. n. from Bulgaria (Diptera: Mycetophilidae: Leiinae) and a key to the Palaearctic species of the genus. Acta zoologica bulgariaca 64 (2): 109-111.

Bechev D. \& Pavlova A. 2016. Fungus gnats (Diptera: Bolitophilidae, Diadocidiidae, Ditomyiidae, Keroplatidae and Mycetophiliade) of Vrachanska Planina Mountains. In: Bechev D. \& Georgiev D. (Eds.). Faunistic diversity of Vrachanski Balkan Nature Park. ZooNotes, Supplement 3: 169-179.

Becker T. 1926. Ephydridae. In: Lindner E. Die Fliegen de Palaearktischen Region 6. Stuttgart, Schweicerbart'sche Verlagsbuchhandlung. $115 \mathrm{p}$.

Beiger M. 1979a. Materials to the knowledge of mining insects of Bulgaria. Polskie Pismo Entomologiczne 49: 485-534.

Beiger M. 1979b. Liriomyza bulgarica sp. n. and Phytomyza rhodopaea sp. n. (Diptera, Agromyzidae), two new mining flies from Bulgaria. Bulletin of the Polish Academy of Sciences, Biology 26 (10): 687-693.

Beiger M. 1980. Paraphytomyza fraxini sp. n. (Diptera, Agromyzidae), a new mining fly from Eastern Bulgaria. Bulletin de la Société des amis des sciences et des lettres de Poznań D20: 145-148.

Belanovsky I. 1951. Tahinidi Ukrainskoy SSR [Tachinidae of the Ukrainian SSR]. 1. Kiev, AN USSR. 191 p. (in Russian)

Belanovsky I. 1953. Tahinidi Ukrainskoy SSR [Tachinidae of the Ukrainian SSR]. 2. Kiev, AN USSR. 239 p. (in Russian)

Belcheva R. 1959. Prouchvaniya varhu yazovir "V. Kolarov" s ogled ribostopanskoto mu izpolzvane [Studies on the Vasil Kolarov Dam with a view to fish-breeding]. Annuaire de l'Université de Sofia, BGGF 51(1): 302-338. (In Bulgarian).

Belcheva R. \& Michailova P. 1980. Quinacrine fluorescent patterns of the chromosomes of Chironomus valkanovi Michailova (Diptera, Chironomidae). Zoologischer Anzeiger 204 (5/6): 362-370.

Belov Y. 1931. Nay-vazhnite zhivotinski nepriyateli na chereshovoto darvo [The most important animal pests of the cherry tree]. Zemedelsko stopanstvo 5 (5/6): 152-153. (In Bulgarian).

Beron P. 1969. Sur les éléments boréo-alpins de la faune Bulgare. Bulletin de l'Institut de zoologique et Musée 30: 115-132.

Beron P. 1972a. Revue des mouches de la famille des Hippoboscidés en Bulgarie et notes sur leurs Acariens - Hyperparasites. Bulletin de l'Institut de zoologique et Musée 34: 189-195. (in Bulgarian with French summary).

Beron P. 1972b. Essai sur la faune cavernicole de Bulgarie III. Résultats des recherches biospéléologiques de 1966 à 1970. International Journal of Speleology 4: 285-349.

Beron P. 1994. Résultats des recherhes biospéléologiques en Bulgarie de 1971 à 1994 et liste des animaux cavernicoles bulgares. Tranteeva 1. Sofia, Fédération bulgare de spéléologie. $137 \mathrm{p}$.

Beron P. 2002. On some owls (Aves: Strigidae) in the high parts of Pirin (SW Bulgaria) and their parasitic flies (Dptera: Hippobodcidae). Historia naturalis bulgarica 15: 147-149.

Beron P. 2004. Contributions of various authors to the faunistics of the Eastern Rhodopes (Bulgaria and Greece). In: Beron P., Popov A. (Eds.). Biodiversity of Bulgaria. 2. Biodiversity of Eastern Rhodopes (Bulgaria and Greece). Sofia, Pensoft \& National Museum of Natural History, 823-848.

Beron P. 2011. Various contributions ti the faunistics of the Western Rhodopes (Bulgaria and Greece) - Tardigrada, Crustacea, Diplura, Collembola, Phthiraptera, Coleoptera, Homoptera, Hymenoptera, Siphonaptera, Diptera. In: Beron P. (Ed.). Biodiversity of Bulgaria. 4. Biodiversity of Western Rhodopes (Bulgaria and Greece) II. Sofia, Pensoft \& National Museum of Natural History, 499-524.

Beron P. 2015. Cave fauna of Bulgaria. Sofia, East-Wst. 440 p.

Beron P. 2016. Terrestrial cave Invertebrates of the Vrachanska Planina mountains. In: Bechev D. \& Georgiev D. (Eds.). Faunistic diversity of Vrachanski Balkan Nature Park. ZooNotes, Supplement 3: 185-230.

Beron P. \& Guéorguiev V. 1967. Essai sur la faune cavernicole de Bulgarie II. Résultats des recherches biospéléologiques de 1961 à 1965. Bulletin de l'Institut de zoologie et musée 24: 151-212. 
Berom P., Petrov B. \& Stoev P. 2004. The ninvertebrate cave fauna of the Eastern Rhodopes (Bulgaria and Greece). In: Beron P. \& Popov A. (Eds.). Biodiversity of Bulgaria. 2. Biodiversity of Eastern Rhodopes (Bulgaria and Greece) II. Sofia, Pensoft \& National Museum of Natural History, 791-822.

Berom P., Petrov B. \& Stoev P. 2011. The ninvertebrate cave fauna of the Western Rhodopes (Bulgaria and Greece). In: Beron P. (Ed.). Biodiversity of Bulgaria. 4. Biodiversity of Western Rhodopes (Bulgaria and Greece) II. Sofia, Pensoft \& National Museum of Natural History, 583-662.

Beschovski V. 1964a. Diptera aus dem litoralen Gebiet der bulgarischen Schwarzmeerküste. Bulletin de l'Institut de pisciculture et de pêcherie (Varna) 4: 91-98. (In Bulgarian with German sumarry).

Beschovski V. 1964b. Beitrag zum Studium der halobionten und halophilen Diptera Brachycera vom Bulgarischen Küstenbereich des Schwarzen Meeres. Zoologischer Anzeiger 172 (4): 261-264.

Beschovski V. 1965. The Insects of the Bulgarian Black Sea Coast. Varna, Darzhavno izdatelstvo. 96 p. (In Bulgarian).

Beshovski V. 1966a. Several families of Diptera unknown to Bulgarian fauna: Dryomyzidae, Chamaemyiidae, Trichoscelidae, Astiidae, Canaceidae and Milichiidae. Bulletin de L'Institut de Zoologie et Musée 21: 1113. (In Bulgarian, English summary).

Beschovski, V. 1966b. Allotrichoma valcanovi n. sp. - eine neue Art von (Diptera, Ephydridae) aus Bulgarien. Comptes rendus de l'Académie bulgare des Sciences 19 (9): 851-853.

Beschovski V. 1966c. Epithalassius stackelbergi n. sp. une nouvelle espece de Diptera (Dolichopodidae) du littoral bulgare de la Mer Noire. Comtes rendus de l'Academie bulgare des Sciences 19 (11): 1079-1082.

Beschovski V. 1966d. Allotrichoma dahli n. sp. - eine neue Art Diptera (Ephydridae) aus Bulgarien. Comptes rendus de l'Académie bulgare des Sciences 19 (10): 937-939.

Beschovski V. 1966e. Unbekannte Ephydridae (Dipt.) in der Fauna Bulgariens. Zoologischer Anzeiger 176 (4): 269-276.

Beschovski V. 1967a. Für die Fauna Bulgariens bislang unbekannte Dolichopodidae (Dipt.). Zoologischer Anzeiger 178 (3/4): 219-224.

Beschovski V. 1967b. Unknown Sphaeroceridae (Dipt.) to Bulgaria's fauna. Bulletin de L'Institut de Zoologie et Musée 23: 217-228. (In Bulgarian, English summary).

Beschovski, V. 1968a. Chloropidae (Diptera) unknown in the fauna of Bulgaria. Bulletin de l'Institut de Zoologie et Musée 26: 33-38. (In Bulgarian, English summary).

Beschovski, V. 1968b. Sur la taxonomie des mâles de l'espèce Limosina (Opacifrons) maculifrons Becker (1907) (Diptera: Sphaeroceridae). Proceedings of the Royal entomological Societi of London 37 (3-4): 41-42.

Beschovski V. 1971a. A Contribution to the dipter fauna (Diptera Brachycera) at the Bulgarian Black Sea Coast. Bulletin de l'Institut de zoologie et musée 33: 5-11. (In Bulgarian, English summary).

Beschovski V. 1972a. A Contribution to the dipteral Fauna (Diptera, Brachycera) in the Bulgarian Black Sea Coast. II. Bulletin de l'Institut de zoologie et musée 34: 5-14. (In Bulgarian, English summary).

Beschovski V. 1972b. Ecologic Investigations on the representatives of Diptera-Brachycera from the salt biotops along the Bulgarian Seaside.(Sum.). I. Communication: Dipteral Fauna from the Cliffs. Bulletin de l'Institut de zoologie et musée 35: 5-21. (In Bulgarian, English summary).

Beschovski V. 1972c. Predstaviteli na Diptera-Brachycera ot peshterite v Bulgaria [Representatives of Diptera Brachycera in the caves of Bulgaria]. Bulletin de l'Institut de Zoologie et Musée 35: 23-29. (In Bulgarian with English summary).

Beschovski V. 1973a. Contribution to the Dipterous Fauna (Diptera-Brachycera) of the Bulgarian Black Sea Coast. III. Bulletin de l'Institut de Zoologie et Musée 37: 33-41. (In Bulgarian with English summary)

Beschovski V. 1973b. Hilara psammophytophila n. sp. (Diptera: Empididae) - a new species for the Bulgarian Black Sea Coast. Comtes rendus de l'Academie bulgare des Sciences 26 (8): 1061-1063.

Beschovski V. 1973c. Ökologische Untersuchungen über Diptera, Brachycera Vertreter aus den salzigen Biotopen des bulgarischen Schwarzmeerstrands. III. Die Diptera-Fauna des von Wellen überfluteten sandigen Strandes. Bulletin de l'Institut de Zoologie et Musée 38: 195-230. (In Bulgarian with German summary).

Beschovski V. 1973d. Deux nouvelles espèces du genre Chersodromia (Diptera: Empididae): Chersodromia milanchvalai sp.n. et Chersodromia bureschi sp. n. du littoral bulgare de la Mer Noire. Zoologischer Anzeiger 191 (5/6): 337-342. 
Beschovski V. 1973e. Ecologic Investigations on the representatives of Diptera-Brachycera from the salt biotops along the Bulgarian Black Sea coast. II. The Dipteral fauna from the seaweeds cas. Bulletin de l'Institut de zoologie et musée 38: 5-20. (In Bulgarian, English summary).

Beschovski, V., 1973f. Sur la taxonomie et la distribution d'Asmeringa inermis Becker, 1903 (Diptera, Ephydridae). Nouvelle Revue d'Entomologie 3 (1): 49-52.

Beschovski V. 1974. Sur la position taxonomique de Dicraeus discolor (Becker, 191) (Diptera: Chloropidae) et Tetanops psammophila Loew, 1862 (Diptera: Otitidae) du littoral bulgare de la Mer Noire. Nouvelle revue d'entomologie 4 (2): 137-143.

Beschovski V. 1975a. The Black Sea Coast inundated by waves and its Dipterous fauna (Diptera-Brachicera). Hydrobiology 2: 3-18. (In Bulgarian with English summary).

Beschovski V. 1975b. Ökologische Untersuchunden über Vertreter von Diptera-Brachicera von den Salzbiotopen der bulgarischen Schwarzmeerküste. IV. Diptera-Brachycera from the salt grounds. Ecology 1: 64-74. (In Bulgarian with German summary).

Beschovski V. 1976a. Diptera-Brachycera in the dunes along the Bulgarian Black Sea coast. In: Terrestrial fauna of Bulgaria. Materials. Sofia, Bugarian Academy of Sciences. pp. 35-59. (In Bulgarian with English summary).

Beschovski V. 1976b. Acartophthalmidae, Anthomyzidae, Aulacigastridae, Camilidae and Diastatidae - new Diptera-Brachycera for the Bulgarian fauna. Acta zoologica bulgarica 5: 93-96. (In Bulgarian with English summary).

Beschovski V. 1977a. Investigations on the Chloropid Flies (Diptera, Chloropidae) of Southwestern Bulgaria. Acta Zoologica Bulgarica 6: 40-49. (In Bulgarian with English summary).

Beschovski V. 1977b. Dicraeus sabroskyi sp. n. (Diptera, Chloropidae ) - a new species from Bulgaria. Acta Zoologica Bulgarica 6: 54-57.

Beschovski V. 1978a. Subdivision of the genus Oscinella Becker, 1909 (Diptera, Chloropidae) with description of a new srecies. Acta zoologica bulgarica 10: 21-29. (In Bulgarian with English summary).

Beschovski V. 1978b. Faunistic and taxonomic investigations on the genus Chlorops Megien, 1803 (Diptera, Chloropidae) from Bulgaria with description of a new subgenus. Nouv. Rev. Entomol. 8: 397-402.

Beschovski V. 1979. Contribution to the taxonomy of the genus Chlorops Megien, 1803 (Diptera, Chloropidae). Reichenbachia 17 (26): 219-223.

Beschovski V. 1980a. Elachiptera submediterranea sp. n. and a review of the species of the genus Elachiptera (Diptera, Chloropidae) from Bulgaria. Acta zoologica bulgarica 16: 57-62.

Beschovski V. 1980b. On the taxonomic structure of the genus Chlorops Megien, 1803 (Diptera, Chloropidae). Acta zoologica bulgarica 14: 19-31.

Beschovski V. 1981a. On the taxonomy of the genus Cryptonerva Lioy, 1864 (Diptera, Chloropidae). Reichenbachia 19 (9): 51-54.

Beschovski V. 1981b. On the systematic status of Oscinella (Microcercis) trigonella Duda, 1933 (Diptera, Chloropidae). Reichenbachia 19 (11): 59-61.

Beschovski V. 1981c. On the systematic of the genus Tricimba Lioy, 1864 (Diptera, Chloropidae). Reichenbachia 19 (21): 119-122.

Beshovski V. 1982a. Chloropidae (Diptera) - new to the Bulgarian fauna. Acta Zoologica Bulgarica 20: 89-95. (In Bulgarian with English summary).

Beschovski V. 1982b. Dicraeus valkanovi sp. n., a new species of Chloropidae (Diptera) from Bulgaria. Reichenbachia 20 (3): 31-32.

Beschovski V. 1983. On the systematic status of Steleocerus lindbergi Duda, 1933 (Diptera, Chloropidae ). Acta zoologica bulgarica 21: 78-82.

Beschovski V. 1984a. Cetema obliqua sp. n., a new species of Chloropidae (Diptera) from Southeast Europe. Reichenbachia 22 (30): 213-214.

Beschovski V. 1985a. Fauna bulgarica. 14. Grass flies (Diptera, Chloropidae). Sofia, Editio Academica. 220 p. (In Bulgarian).

Beschovski V. 1985b. On the origin and formation of Palaearctic-Palaeotropical randing genera of family Chloropidae (Diptera) in the Palaearctic. Acta Zoologica Bulgarica 29: 36-49.

Beschovski V.1987a. Description of Sabroskyina genus novum (Diptera, Cloropidae) and taxonomic remarks on the species included. Acta Zoologica Bulgarica 34: 36-44. 
Beschovsky V. 1993a. The species of the genus Lipara Meigen (Diptera, Cloropidae), and its galls on the common reed along the Bulgarian Blask Sea coast. Second National Scientific Conference of Entomology, Sofia, 25-27 october, 1993: 14-18. (In Bulgarian with English summary).

Beschovsky V. 1993b. Taxonomic and systematic notes on the genera Tethina Haliday, 1838, and Rhicnoessa Loew. 1862 (Insecta: Diptera: Tethinidae). Rechenbachia 30 (16): 103-107.

Beschovski V. 1993c. Review of the families of Diptera - Nematocera (Diptera) from Bulgaria. Second National Scientific Conference of Entomology, Sofia, 25-27 october, 1993: 19-23. (In Bulgarian with English summary).

Beschovsky V. 1994a. Three new species of genus Chamaemyia Meigen, 1803 from Europe (Insecta: Diptera: Chamaemyiidae). Reichenbachia, 30 (35): 203-209.

Beschovsky V. 1994b. Contribution to the study of the West Palaearctic Tethinidae (Diptera). Acta zoologica bulgarica, 47: 16-29.

Beschovski V. 1995a. Key of determinatoin the Lipara - species (Diptera, Chloropidae) from Bulgaria by the adults, mature larval stage and galls. Acta Entologica Bulgarica 2: 5-10. (In Bulgarian with English summary).

Beschovsky V. 1995b. Contribution to the knowledge of the taxonommy and distribution of the Chamaemyia species established in Bulgaria (Insecta, Diptera, Chamaemyiidae). Acta zoologica bulgarica, 48: 34-47.

Beschovski V. 1996a. Qualitative composition and distribution of the Chloropidae species (Diptera) on the moist zones of the Shablenska like system. Acta Entomologica Bulgarica 2 (2): 16-20. (In Bulgarian with English summary).

Beschovski V. 1996b. Meromyza balcanica spec. nov. - a new chloropid species from Bulgaria (Insecta: Diptera : Chloropidae). Reichenbachia 31 (42): 233-235.

Beschovski V. 1996c. Qualitative composition and distribution of the Ephydridae species (Diptera) on the moist zones of the Shablenska like system. Acta Entomologica Bulgarica 2 (1): 16-20. (Bulgarian; Englesh summary).

Beschovski V. 1997a. Review of the Ephydridae species (Insecta: Diptera), established in Bulgaria up to the present. Acta Entologica Bulgarica 3 (1/2): 7-9. (In Bulgarian with English summary).

Beschovski V. 1997b. Ephidrids species from the woodless zone of the National park „Central Balkan” (Insecta: Diptera: Ephydridae). Acta Entologica Bulgarica 3 (1/2): 25-28.

Beschovsky V. 1998a. Contribution to the study of the genus Leucopis Meigen, 1830 in Bulgaria (Diptera: Chamaemyiidae) with description of a new species. Acta zoologica bulgarica 50 (2/3): 9-18.

Beschovsky V. 1998b. Two new Elachiptera species from Bulgaria (Insecta: Diptera: Chloropidae). Reichenbachia, 32 (50): 329-332.

Beschovski V. 1998c. Contribution to the study of the distribution of the Parochthiphila species in Bulgaria (Diptera: Chamaemyidae). Acta zoologica bulgarica 50 (1): 3-6.

Beschovski V. 1998d. Species from families Opomyzidae and Chloropidae (Diptera) from Rila Mountain. Acta Entomologica Bulgarica 4 (1): 16-18.

Beschovski V. 1998e. The species from families Opomyzidae and Chloropidae (Diptera) established in the woodles zone of the National Park "Central Balkan" - Stara Planina Mountains. Acta zoologica bulgarica 50 (1): 11-19.

Bheschovski V. 2001. Periscelididae - a new family to the Bulgarian fauna (Insecta: Diptera). Acta zoologica bulgarica, 53 (1): 39-40.

Beschovski V. 2004a. Diptera Brachycera recorded for the Eastern Rhodopes (Bulgaria). In: Beron P. \& Popov A. (Eds.). Biodiversity of Bulgaria. 2. Biodiversity of Eastern Rhodopes (Bulgaria and Greece). Sofia, Pensoft \& National Museum of Natural History. pp. 741-757.

Beschovsky V. 2004b. Review of families Milichiidae and Carnidae from Bulgaria (Insecta: Diptera). - Acta zoologica bulgarica 56 (2): 129-135.

Beschovski V. 2004c. Genus Kesselimyia, K. Chandleri and subfamily Platypezinae (Insecta: Diptera, Platypezidae): new taxa to the Bulgarian fauna. - Acta zoologica bulgarica 56 (2): 237-238.

Beschovski V. 2006a. Diptera Brachycera (Insecta) establisched in the Western Rhodopes Mountains (Bulgaria). In: Beron P. (Ed.). Biodiversity of Bulgaria. 3. Biodiversity of Western Rhodopes (Bulgaria and Greece) 1. Sofia, Pensoft \& National Museum of Natural History. pp. 617-674. 
Beschovski V. 2006b. Faunistic review of some Nematocera families (Diptera) establisched in the Western Rhodopes (Bulgaria). In: Beron P. (Ed.). Biodiversity of Bulgaria. 3. Biodiversity of Western Rhodopes (Bulgaria and Greece) 1. Sofia, Pensoft \& National Museum of Natural History. pp. 729-752.

Beschovski V. 2007. Review of the small Ephydroidea families (Diptera: Brachycera) in Bulgaria. Acta zoologica bulgarica 59 (1): 29-32.

Beschovski V. 2008. Additional record and corrections on the Oscinellinae fauna (Diptera: Chloropidae) of Bulgaria. Acta zoologica bulgarica 60 (1): 51-59.

Beschovski V. 2009. Fauna bulgarica. 28. Insecta: Diptera: Ephydridae, Tethinidae, Canacidae. Sofia, Editio Academica "Professor Marin Drinov". 423 p. (In Bulgarian).

Beschovski V. 2009b. Review of the species from some Opomyzoidea families (Insecta: Diptera: Acalyptrata) established in Bulgaria. Acta zoologica bulgarica 61 (2): 205-208.

Beschovski V. 2012. Two genera of long-legged flies (Diptera: Dolichopodidae) new for the fauna of Bulgaria. ZooNotes 29: 1-2.

Beschovski V. 2013a. Dolichopus species (Diptera: Dolichopodidae) in the entomological collection of the Institute of Biodiversity and Ecosystem Research at the Bulgarian Academi of Sciences. ZooNotes 38: 1-4.

Beschovski V. 2013b. Catalogus faunae bulgaricae. 8. Carnoidea. Insecta: Diptera Brachycera Acaliptratae, Sofia, Editio Academica "Professor Marin Drinov". 152 p.

Beschovski V. 2013c. Additions and corrections to the species list of the Bulgarian Chloropinae (Diptera: Chloropidae). ZooNotes 40: 1-4

Beschovski V. \& Dimitrova B. 1990. Frit flies (Insecta, Diptera, Chloropidae) from Vitosha. In: Fauna of Southwestern Bulgaria. 3. Sofia, Bulgarian Academy of Sciences, 154-160. (In Bulgarian with English summary).

Beschovski V. \& Dzhambazov B. 1997. New records of the genus Platypalpus Macquart, 1827 (Diptera: Empididae, Hybotidae) from the fauna of Bulgaria. Acta zoologica bulgarica 49: 94-96.

Beschovski V. \& Dzhambazov B. 1998. New species from the family Empididae for the Bulgarian fauna (Diptera, Empidoidea, Empididae). Acta zoologica bulgarica 50: 19-26.

Beschovski V. \& Dzhambazov B. 2002. Dolichopodidae taxa known a new to the Bulgarian fauna (Diptera: Empidoidea). Acta zoologica bulgarica 54 (1): 19-26.

Beschovski V., Dzhambazov B. \& Gajdarova A. 1995. Contribution to the study of Empidoidea fauna from Vitosha Mountain (Insecta: Diptera: Empidoidea). Acta entomologica bulgarica 1 (2): 13-15.

Beschovski V. \& Georgiev G. 1993. Three species of Diptera-Acalyptrata (Diptera) dwelling galls of Parathrene tabaniformis Rott. (Lepidoptera, Aegeridae). Acta Zoologica Bulgarica 46: 44-49.

Beschovski V. \& Gueorguieva R. 2000. Contribution to the study of the Bulgarian Lonchopteridae (Insecta: Diptera) with data for some localities for other geographic regions. Acta zoologica bulgarica 52 (1): 41-44.

Beschovski V. \& Gueorguieva R. 2001. Review of the bulgarian Helomyzidae species (Insecta: Diptera). Acta Zoologica Bulgarica 53 (1): 29-38.

Beschovski V. \& Hubenov Z.. 1986. Die Insekten von der Familie Tachinidae (Diptera) in den Submediterranbiotopen Südwestbulgariens. I. Artenbestand und zoogeographische Charakteristik der Tachinidae im Tal zwischen Sandanski und Petrič. In: Fauna of Southwestern Bulgaria. 1. Sofia, Bulgarian Academy of Sciences, 118-129. (In Bulgarian with German summary).

Beschovski V. \& Karadjova O. 1996. The South American leafminer Liriomyza huidobrensis Blanch. (Diptera: Agromyzidae) - a new pest in Bulgarian greenhouses. Acta Entologica Bulgarica 2 (2): 5-11. (In Bulgarian with English summary).

Beschovski V. \& Krusteva C. 1995a. Lasiosina brevisurstylata Dely-Draskovits, 1977- an unknown enamy on the spring barley and wheat (Diptera: Chloropidae). Acta Entologica Bulgarica 2: 32-36.

Beschovski V. \& Krusteva C. 1995b. Lasiosina brevisurstylata nepoznat dosega nepriyatel po proletniya echemik i pshenitsata [Lasiosina brevisurstylata unknown enemy of the spring barley and wheat so far]. Rastitelna zshtita 10, p. 19.

Beschovski V. \& Krusteva H. 1997. Contribution to the study of the pupa and bioligy of Lasiosina brevisurstylata and L. cinctipes (Insecta: Diptera: Chloropidae). Acta Zoologica Bulgarica 49: 32-42.

Beschovski V. \& Krusteva C. 1998. Two new Elachiptera species from Bulgaria (Insecta: Diptera: Chloropidae). Reichenbachia 32 (50): 329-332.

Beschovski V. \& Krusteva C. 2001. Taksonomichni belezi na chernite pshenichni muhi [Taxonomic features of the black wheat flies]. Rastitelna zashtita 8-9, p.16. (In Bulgarian). 
Beschovski V. \& Langourov M. 1997. Contribution to the study of the distribution of Phoridae species in the Balkan Peninsula (Diptera, Phoridae). Acta zoologica bulgarica, 49: 43-47.

Beschovski (Bešovski) V. \& Lăvčiev V. 1971. Muscides (Diptera-Brachycera) du littoral Bulgare de la Mer Noire. Bulletin de l'Institut de zoologie et musée 33: 85-98. (In Bulgarian, French summary).

Beschovski V. \& Manassieva E. 1995. Review of the Pipunculidae species in Bulgaria. In: Third National scientific conference of entomology, Sofia, 18-20 September, 66-69. (In Bulgarian with English summary).

Beschovski V. \& Manassieva E. 1996. Contribution to the study of the Stratiomyidae species in the Balkan Peninsula, with description of Nemotelus rumelicus spec. nov. (Insecta: Diptera). Rechenbachia 31 (39): 217-223.

Beschovski V. \& Merz B.1998. Contribution to the knowledge of the Chamaemyiidae (Diptera), with particularreference to the fauna of Switzerland. Mitteilungen der Schweizerischen Entomologischen Gesellschaft 71: 83-106.

Beschovski V. \& Minkova V. 1991. Species from the family Opomyzidae (Diptera) in Bulgaria. Acta Zoologica Bulgarica. 42: 62-65. (In Bulgarian with English summary).

Beshovski V. \& Zatwarnicki T. 2000. Faunistic review of the subfamily Discomyzinae in Bulgaria (Insecta: Diptera: Ephydridae) with some data from other palaearctic countries. Acta zoologica bulgarica 52:17-28.

Beshovski V. \& Zatwarnicki T. 2001a. Faunistic review of the subfamily Hydrelliinae in Bulgaria (Insecta: Diptera: Ephydridae) with some data from other European countries. Acta zoologica bulgarica 53 (3): 3-18.

Beshovski V. \& Zatwarnicki T. 2001b. Faunistic review of the subfamily Ilytheinae (Insecta: Diptera, Ephydridae) in Bulgaria with some data from other European countries. Acta zoologica bulgarica 53 (3): 19-26.

Beshovski V. \& Zatwarnicki T. 2002. Faunistic review of the Subfamily Gymnomyzinae (Insecta: Diptera: Ephydridae) in Bulgaria with some data from other European countries. Acta zoologica bulgarica 54 (1): 3-17.

Beshovski V. \& Zatwarnicki T. 2004. Faunistic review of the subfamily Ephydrinae in Bulgaria (Insecta: Diptera, Ephydridae) with some data from other Palaearctic countries. Acta zoologica bulgarica 56 (1): 31-55.

Beuk P., Pare T., Bystrowski C., Gree P., Kameneva E. \& Richter W. 2017. Otitidae (Diptera). In: Pape T., Beuk P. (Eds.), Fauna Europaea: Diptera Brachycera. Fauna Europaea version, 1.2-2.4. http://www.faunaeur.org.

Bezzi M. 1908. Rhagionidae et Empididae Palaearticae novae ex Museo nationali Hungarico. Annales historiconaturales Musei nationalis hungarici 3: 362-365.

Bobeva A. 2014. Molecular studies of the specificity of avian malaria parasites (Haemosporidia) to the vectors of the genus Culicoides Latreille, 1809 (Diptera: Ceratopogonidae). Ph.D. thesis, Sofia, Bulgarian Academy of Sciences. 81 p. (In Bulgarian).

Bobeva A., Zehtindjiev P., Bensch S., \& Radrova J. 2013. A survey of biting midges of the genus Culicoides Latreille, 1809 (Diptera: Ceratopogonidae) in NE Bulgaria, with respect to transmission of avian haemosporidians. Acta Parasitologica 58 (4): 585-591. DOI: 10.2478/s11686-013-0185-Z

Bobeva A., Ilieva M., Dimitrov D. \& Zehtindjiev P. 2014. Degree of associations among vectors of the genus Culicoides (Diptera: Ceratopogonidae) and host bird species with respect to haemosporidian parasites in NE Bulgaria. Parasitology Research 113 (12). DOI: 10.1007/s00436-014-4140-1

Bobeva A., Zehtindjiev P., Ilieva M., Dimitrov D., Mathis A. \& Bensch S. 2015. Host preferences of ornithophilic biting midges of the genus Culicoides in Eastern Balkans with respect to transmission of haemosporidian parasites. Medical and Veterinary Entomology 29 (3): 290-296. DOI: 10.1111/mve.12108

Bondev I. 1991. The vegetation of Bulgaria. Sofia: Kliment Ohridski University Press. 184 p. (In Bulgarian with English summary).

Bondev I. 1997. Geobotanic regioning. In: Yordanova M., D. Donchev (Ed.). Geography of Bulgaria. Sofia: Bulgarian Academy of Sciences, 269-305. (In Bulgarian with English summary).

Bondev I. 2002. Geobotanic regioning. In: Geography of Bulgaria. Sofia: ForCom, 336-352. (In Bulgarian with English summary).

Borisova P., Varadinova E. \& Uzunov Y. 2013. Contemporary state of the bottom invertebrate communities of the Tundzha River basin (South-East Bulgaria. Acta zoologica bulgarica 65 (1): 75-87.

Botev S. 1925. The stem fly (Chlorops taeniopus Meig.). Zemedelie 29 (8): 117-118. (In Bulgarian).

Boychev D. 1950a. Prinos kam rasprostranenieto na roda Phlebotomus (Diptera) v Bulgaria [Contribution to the distribution of the genus Phlebotomus (Diptera) in Bulgaria]. Godishnik na Selskostopanskata akademiya "G. Dimitrov". Zootehnicheski fakultet 3: 101-107. (In Bulgarian). 
Boychev D. 1950b. Podvidovete na Anopheles maculipennis po nasheto dunavsko kraybrezhie [Subspecies of Anopheles maculipennis on the Bulgarian Danubian coast]. Godishnik na Selskostopanskata akademiya "G. Dimitrov". Zootehnicheski fakultet 3: 109-112. (In Bulgarian).

Bozhkov (Božkov) D. 1953. Können die Larven der Anopheles maculipennis Eier ihrer eigenen Art aufessen ?. Bulletin de l'Institut zoologque de l'Academie Bulgare des Sciences 2: 163-188. (In Bulgarian with German summary).

Bozhkov D. 1957. Malariynite komari kato obekt za izuchvane ot uchenicheskite zoologicheski krazhotsi [The malaria mosquitoes, as a subject of study by the pupil's zoological groups]. Priroda i znanie 10 (2): 20-22. (In Bulgarian).

Bozhkov D. 1958. Eine für die Fauna Bulgariens neue Art blutsaugende Stechmücke. Comptes rendus de l'Academie bulgare des Sciences 11 (6): 501-502.

Bozhkov D. 1959. Beitrag zur Erforschung der Stechmückenfauna im Rhodopen- und Rilagebirge. Bulletin de l'Institut zoologque de l'Academie Bulgare des Sciences 8: 109-119. (In Bulgarian with German summary).

Bozhkov D. 1961. Mückenlarven aus dem Gebiet von Burgas und dem Strandžagebirge. In: Paspalev G. (Ed.). Prirodni ognishta na zaraza v Strandzha planina. Sofia, Bulgarian Academy of Sciences. pp. 147-153. (In Bulgarian with German summary).

Bozhkov D. 1962. Komarni larvi ot Petrichko i Gotse Delchevsko [Mosquitoes larvae from Petrich and Gotse Delchev]. Prirodni ognishta na zaraza v Petrichko i Gotse Delchevsko. Sofia, Bulgarian Academy of Sciences. pp. 219-224. (In Bulgarian).

Bozhkov D. 1965. Stechmückenlarven aus Thrakien. In: Paspalev G. (Ed.). Die Fauna Thrakiens 2. Sofia, Bulgarischen Akademie der Wissenschaften. pp. 255-264. (In Bulgarian with German summary).

Bozhkov D. 1966a. Krovososushtie komary Bolgarii [Blood-sucking mosquitoes of Bulgaria]. Entomologicheskoe obozrenie 45 (3): 570-574. (In Russian).

Bozhkov D. 1966b. Beitrag zum studium der Steichmücken im Vitoscha-Gebirge. Bulletin de l'Institut de zoologie et musée 21: 65-67. (In Bulgarian with German summary).

Bozhkov D. 1967. Stechmücken aus dem westlichen Balkangebirge. Bulletin de l'Institut de zoologie et musée 25: 41-64. (In Bulgarian with German summary).

Bozhkov D. 1974a. Stechmücken aus dem mittleren und dem östlichen Balkangebirge (Mit einigen Notizen über die Stechmücken des Balkangebirges überhaupt). Bulletin de l'Institut de zoologie et musée 41: 161-175. (In Bulgarian with German summary).

Bozhkov D. 1974b. Kravosmucheshtite komari na balgarskite planini [The blood-sucking mosquitoes of the Bulgarian mountains]. Priroda 23 (3): 40-42. (In Bulgarian).

Bozhkov D. 1991. Kravosmucheshtite komari [The blood-sucking mosquitoes]. Sofia, Bulgarian Academy of Sciences. 223 p. (In Bulgarian).

Bozhkov D. \& Christova T. 1965. Kravosmucheshtite komari kato prenositeli na virusi v Evropa [The bloodsucking mosquitoes as virus carriers in Europe]. Priroda 14 (4): 71-74. (In Bulgarian).

Bozhkov D., Christova T. \& Canev I. 1969. Stechmücken an der bulgarischen Schwarzmeerküste. Bulletin de l'Institut de zoologie et musée 29: 151-166. (In Bulgarian with German summary).

Buhr H. 1941. Dipteren - insbesondere Agromyziden - Minen aus Sudeuropa. Stettiner Entomologische Zeitung 102 (1): 73-119.

Buresch I. 1924. Referate und Berichte. Mitteilungen der Bulgarischen Entomologischen Gesellschaft in Sofia 1: 16-20. (In Bulgarian).

Buresch I. 1926a. Untersuchungen über die Höhlenfauna Bulgariens. II. Travaux de la Sociéte Bulgare de Sciences naturelles 12: 17-56. (In Bulgarian with German summary).

Buresch I. 1926b. Referate und Berichte im Jahre 1925. Mitteilungen der Bulgarischen Entomologischen Gesellschaft in Sofia 3: 22-27. (In Bulgarian).

Buresch I. 1928. Referate und Berichte im Jahre 1926-1927. Mitteilungen der Bulgarischen Entomologischen Gesellschaft in Sofia 4: 12-17. (In Bulgarian).

Buresch I. 1930. Ausländische Literatur über die Fauna Bulgariens, Thraciens und Mazedoniens. II. Mitteilungen aus den Königl Naturwissenschaftlichen Instituten in Sofia 3: 253-256. (In Bulgarian).

Buresch I. 1934. Referate und Berichte. Mitteilungen der Bulgarischen Entomologischen Gesellschaft in Sofia 8: 208-214. (In Bulgarian). 
Buresch I. 1936. Ubersicht der bisherigen Kentnisse und Erforschungen der rezenten Höhlen - Fauna Bulgariens. Sofia, Bulgarian Speleological Society 1: 13-43. (In Bulgarian with German summary).

Buresch I. 1938. Ausländische Literatur über die Fauna Bulgariens, Thraciens und Mazedoniens. VI. Mitteilungen aus den Königl Naturwissenschaftlichen Instituten in Sofia 11: 182-184. (In Bulgarian).

Buresch I. 1939. Referate und Mitteilungen. Mitteilungen der Bulgarischen Entomologischen Gesellschaft in Sofia 10: 140-156. (In Bulgarian).

Buresch I. 1953a. Bibliography of the fauna of the Vitosha Mt. In: Bibliografiya po prirodata na Viitosha planina. Planinska prirodonauchna stantsiya Vitosha. 2. Sofia, Bulgarian Academy of Sciences. pp. 75-120. (In Bulgarian).

Buresch I. 1953b. Ausländische Literatur über die Fauna Bulgariens. Bulletin de l'Institut zoologque de l'Academie Bulgare des Sciences 2: 401-412.

Buresch I. 1960. Ausländische Literatur über die Fauna Bulgariens. 10. Bulletin de l'Institut zoologque de l'Academie Bulgare des Sciences 9: 461-472. (In Bulgarian with German summary).

Buresch I. \& Arndt W. 1926. Die Glazialrelicte stellenden Tierarten Bulgariens und Mazedoniens. Zeitshrift für Morphologie und Ökologie der Tiere 5 (3): 381-405.

Buresch I. \& Lazarov A. 1956. Harmful insects for agriculture and forestry enterprise in Bulgaria. Bibliography and catalogue. Trudove na instituta po zoologiya 5. Sofia, Bulgarian Academy of Sciences. 291 p. (In Bulgarian).

Buresch I., Tranteev P. \& Alexandrov I. 1949. Peshterata „Temnata dupka” v Iskarskiya prolom pri gara Lakatnik i neynata fauna [Temnata Dupka Cave in the Iskar Gorge near Lakatnik Railway Station and its fauna]. Priroda i znanie (Nature and Knowledge) 2 (5): 7-9; (6): 16-20. (In Bulgarian).

Burgess A. \& Crossman S. 1929. Imported insect enemies of the gipsy moth and the brow-tail moth. Technical Bulletins, United States Department of Agriculture 86, p. 147.

Burghele-Bălăcesko A. 1966. Diptéres cavernicoles recueillis en Bulgarie. International Journal of Speleology 2 (3): 303-308.

Burghele-Bălăcesko A. 1969. Révision des genres Chionea et Niphadobata en Europe centrale et méridionale (Dipt., Tipulidae). Annales de la Société Entomologique de France (N. S.) 5: 983-1000.

Byuletin No 2 Za poyavilite se bolesti i nepriyateli v stranata prez mesetsite yuli, avgust i septemvri 1943 godina. [Diseases and pests appeared on the cultivated plants in Bulgaria in July, August and September, 1943] Sofia, Tsiklostilno izdanie. 10 p. (In Bulgarian).

Byuletin No $3 \mathrm{Za}$ konstatiranite bolesti i nepriyateli po rasteniyata $\mathrm{v}$ stranata prez 1945 godina. [Diseases and pests appeared on the cultivated plants in Bulgaria in 1945] Sofia, Tsiklostilno izdanie. 18 p. (In Bulgarian).

Byuletin po rastitelna zaashtita. 1956. Bolesti i nepriyateli poyavili se po kulturnite rasteniya u nas prez 1954 godina [Diseases and pests appeared on the cultivated plants in Bulgaria in 1954] 4 (6/7): 3-74. (In Bulgarian).

Byuletin po rastitelna zaashtita. 1957a. Bolesti i nepriyateli poyavili se po kulturnite rasteniya u nas prez 1955 godina [Diseases and pests appeared on the cultivated plants in Bulgaria in 1955] 5 (1/8):3-83. (In Bulgarian).

Byuletin po rastitelna zaashtita. 1957b. Bolesti i nepriyateli poyavili se po kulturnite rasteniya v Bulgaria prez 1956 godina [Diseases and pests appeared on the cultivated plants in Bulgaria in 1956] 6 (2/10): 7-100. (In Bulgarian).

Canzoneri S. \& Meneghini D. 1983. Ephydridae et Canaceidae. Fauna d'Italia. 76. Bologna, Edicione Calderoni. $312 \mathrm{p}$.

Caspers H. 1951a. Biozönotische Untersuchungen über die Strandarthropoden im bulgarischen Küstenbereich des Schwarzen Meeres. Hydrobiologia, den Haag 3 (2): 131-193.

Caspers H. 1951b. Quantitative Untersuchungen über die Bodentierwelt des Schwarzen Meeres im bulgarischen Küstenbereich. Archiv für Hydrobiologie 45 (1-2): 1-192.

Caspers H. 1951c. Rhytmische Erscheinungen in der Fortpflanzung von Clunio marinus (Dipt., Chironomidae) und das Problem der lunaren PeriodizitAt bei Organismen. Archiv für Hydrobiologie, Supl. 18 (3): 415-594.

Caspers H. 1952. Untrrsuchung Uber der bulgarischen Kuste des Schwarzen Meeres. Zoologisher Anzeiger 148 (5/8): 243-259.

Caspers H. 1957. Black Sea and Sea of Azov. Treafise on Marine Ecology and Palaeoecology 1: 801-889.

Černý M. 2009. New faunistic data on the Agromyzidae (Diptera) from the West Palaearctic Region. Klapalekiana 45: 9-21.

Černý M. 2013. Additional records of Agromyzidae (Diptera) from the West Palaearctic Region. Časopis Slezského zemského muzea. Série A, 62: 281-288. 
Černý M. 2018. Additional new records of Agromyzidae (Diptera) from the Palaearctic Region. Acta Musei Silesiae, Scientiae Naturales 67: 117-137

Černý M. \& Bächli G. 2018. New records of Agromyzidae (Diptera) from Switzerland and an updated checklist. Alpine Entomology 2: 115-137. DOI 10.3897/alpento.2.28973

Černý M. \& Merz B. 2006. New records of Agromyzidae (Diptera) from the Palaearctic Region. Mitteilungen der Schweizerischen Entomologischen Gesellschaft 79 (1): 77-106.

Cerretti P., Badano D., Gisondi S., Lo Giudice G. \& Pape T. 2020. The world woodlouse flies (Diptera, Rhinophoridae). ZooKeys 903: 1-130. https://doi.org/10.3897/zookeys.903.37775

Chandler P. 1976. Anew species Callomyia Meigen (Diptera, Platypezidae) from Bulgaria. Entomologist's Gazette 27: 257-261.

Chandler P. 1987. The families Diastatidae and Campichoetidae (Diptera, Drosophiloidea) with a revision of Palaearctic and Nepalese species of Diastata Meigen. Entomologica scandinavica 18: 1-50.

Chalupský J. 1956. Pupipara (Diptera) ze sběrů prof. Jaroslava Štorkána. Věstník Československé zoologické společnosti v Praze 20 (2): 143-146.

Chichkoff G. \& Konsuloff S. 1914. Izuchavaniya varhu komarite (Culicidae) v Bulgaria i tehnite larvi [Studies on the mosquitoes (Culicidae) in Bulgaria and their larvae]. Annuaire de l'Université de Sofia. Faculté physico-mathématique, 8/9: 1-51. (In Bulgarian).

Choleva B. 1964. Vredni muhi po pechurkite [Harmful flies on the field mushrooms]. Rastitelna zhashtita 6: (in Bulgarian).

Christova T. 1980. Epidemiologichno znachenie i geografsko rasprostranenie na anofeliynite komari v Bulgariya [Epidemiological significance and geographical distribution of the Anopheles mosquitoes in Bulgaria]. DDD periodichen byuletin 11 (1): 3-6. (In Bulgarian).

Christova T., Bachvarov T. \& Kirin D. 2000. [Vidov sastav na kravosmucheshtite komari ot semeystvo Culicidae na teritoriyata na grad Plovdiv [Species composition of the blood-sucking mosquitoes of the family Culicidae on the territory of the Plovdiv Town]. DDD byuletim, 1-2: 56-63.

Christova T. \& Bozhkov D. 1966. Neue Arten blutsaugender Mücken für die Fauna Bulgariens. Bulletin de l'Institut de zoologie et musée 21: 147-148. (In Bulgarian with German summary).

Christova T. \& Bozhkov D. 1977. Kravosmucheshti komari ot Kresnenskiya prolom [Blood-sucking mosquitoes from the Kresna gorge]. Desinfektsiya, desinsektsiya i deratizatsiya (byuletin na MNZ) 8 (3-4): 55-57. (In Bulgarian).

Christova T., Canev I. \& Smilova D. 1971. Promeni vav vidoviya sastav na kravosmucheshtite komari ot bulgarskoto Chernomorsko kraybrezhie [Changes in the species composition of the blood-sucking mosquitoes from the Bulgarian Black Sea coast]. Letopisi na HEI 5 (31): 173-183. (In Bulgarian).

Christova T. \& Dorovski D. 1972. Razprostranenie na komarni larvi ot sem. Culicidae v Plevenski okrag [Distribution of mosquito larvae from the Culicidae family in Pleven district]. Epidemiologiya, mikrobiologiya i infektsiozni bolesti 9 (2): 185-192. (In Bulgarian).

Christova T., Simeonov T. \& Balukova Yu. 1972. Opiti za borba s komarni larvi v orizishtata [Attempts to control mosquito larvae in the rice fields]. Epidemiologiya, mikrobiologiya i infektsiozni bolesti 9 (4): 371-377. (In Bulgarian).

Christova T. \& Todorova M. 1969. Komarite v grad Sofiya i borbata sreshtu tyah: 1 . Vidov sastav i razprostranenie na kravosmucheshtite komari v Sofiya [The mosquitoes in Sofia and the fight against them: 1. Species composition and distribution of the mosquitoes in Sofia]. Letopisi na HEI 3 (16): 88-92. (In Bulgarian).

Chvála M. 1970. Descriptions of nine new species of Palaearctic Chersodromia Walk. (Diptera, Empididae), with notes on the genus. Acta entomologica Bohemoslovaca 67: 384-407.

Chvála M. 1975. The Tachydromiinae (Dipt., Empididae) of Fenoscandia and Denemark. Fauna Entomologica Scandinavica 3. Brill, Dutch academic publishing house. $336 \mathrm{p}$.

Chvála M. 1977. Revision of the Empis rustica Fall. species-group of the subgenus Leptempis Coll. in Europe, with the discription of a new species (Diptera, Empididae). Acta Entomologica Bohemoslovaka 74: 41-55.

Chvála M. 1980. Two new european Ogcodes species (Diptera, Acroceridae). Acta entomologica bohemoslovaca, 77 (2): 131-137.

Chvála M. 1983. The Empidoidea (Diptera) of Fenoscandia and Denmark. 2. General Part; Families Hybotidae, Atelestidae and Macrophoridae. Fauna entomologica Scandinavica. 12. Klampenborg, Scandinavian science pres LTD. 279 p. 
Chvála M. 1988. Family Tabanidae. In: Soós Á. \& Papp L. (Eds.). Catalogue of Palaearctic Diptera. 5. Budapest, Akadémiai Kiadó, Hungarian Natural History Museum. pp. 97-191.

Chvála M. 1989. Monograph of northern and central European species of Platypalpus Macq. (Diptera, Hybotidae), with data on the occurrence in Czechoslovakia. Acta Universitatis Carolinae Biologica 32, 209-376.

Chvála M. 1991. Two new mountain species of Bicellaria (Diptera, Hybotidae) from Central and Eastern Europe. Acta Universitatis Carolinae - Biologica 35: 9-18.

Chvála M. 2005. Descriptions of three new Hilara species (Diptera: Empididae) from the Central European and Balkan mountains. Acta Universitatis Carolinae Biologica 49: 99-110.

Chvála M. 2013. Mediterranean species of the genus Hilara (Diptera: Empididae) - Part 2. Studia dipterologica 20 (2): 233-238.

Chvála M. \& Kovalev V. 1989. Family Hybotidae. In: Soós Á. \& Papp L. (Eds.). Catalogue of Palaearctic Diptera. 6. Budapest, Akadémiai Kiadó, Hungarian Natural History Museum. pp. 174-227.

Chvála M., Lineborg L. \& Moucha J. 1972. The horse flies of Europe (Diptera, Tabanidae). Copenhagen, Entomological Society of Copenhagen. 499 p.

Chvála M. \& Smith K. 1988. Family Conopidae. In: Soós Á. \& Papp L. (Eds.). Catalogue of Palaearctic Diptera. 8. Budapest, Akadémiai Kiadó, Hungarian Natural History Museum. pp. 245-272.

Chvála M. \& Wagner R. 1989. Family Empididae. In: Soós Á. \& Papp L. (Eds.). Catalogue of Palaearctic Diptera. 6. Budapest, Akadémiai Kiadó, Hungarian Natural History Museum. pp. 228-336.

Coher E. 1995. A contribution to a revision of the genus Azana Walker, 1856 (Insecta: Diptera: Mycetophilidae: Sciophilinae). Reichenbachia 31(17): 83-91.

Collin J. 1950. A New Palaearctic species of Chersodromia with very short wings (Diptera: Empididae). Proceedings of the Royal Entomological Society of London (B) 19 (5/6): 68-79.

Crosskey R. \& White G. 1977. The Afrotropical Region. A recommendet term in zoogeography. Journal of Natural History, 11: 541-544.

Cvetkov (Zvetkov) L. 1955a. Die Chironimidenfauna der Bulgarischen Schwarzenmeerseen. Bulletin de l'Institut zoologque de l'Academie des Sciences de Bulgarie 4/5: 215-249. (In Bulgarian with German summary).

Cvetkov L. 1955b. Untersuchungen über das Futter der Fische im Beloslavsko-See. Bulletin de l'Institut zoologque de l'Academie des Sciences de Bulgarie 4/5: 329-352. (In Bulgarian with German summary).

Cvetkov L. 1957. Zoobenthos des Beloslav-Sees. Bulletin de l'Institut zoologque de l'Academie des Sciences de Bulgarie 6: 381-439. (In Bulgarian with German summary).

Cvetkov (Tsvetkov) L. 1958. Investigations into the microbenthos of the Bulgarian Black Sea lakes. Bulletin de l'Institut zoologique de l'Academie des sciences de Bulgarie, 7: 219-250. (In Bulgarian with English summary).

Cvetkov L. 1962. Sur la prolifération quantitative des Chironomidés et la productivité des bassins de pisciculture. Bulletin de l'Institut de zoologie et musée 12: 173-196. (In Bulgarian with French summary).

Czekanowski J. 1909. Zur differential Diagnose der Neandertalgruppe. Korrespondenzblatt der deutschen Gesellschaft für Anthropologie, Ethnologie und Urgeschichte 40: 44-47.

Czerný L. 1930. Dipteren auf Schnee und Höhlen. Mitteilungen königl naturwissenschaftlichen Instituten in Sofia (Bulgarien) 3: 113-118.

Dahl Ch. 1992. Family Trichoceridae (Petauristidae). In: Soós Á., Papp L. \& Oosterbroek P. (Eds.). Catalogue of Palaearctic Diptera. 1. Budapest, Akadémiai Kiadó, Hungarian Natural History Museum. pp. 31-37.

Darlenski R., Bogdanov I., Langourov M. \& Manuelyan K. 2020. An outbreak of cellulitis-like insect dermatitis from black flies (Simuliidae) and treatment algorithm. Dermatology and venerology, 59 (2): 19-25. (In Bulgarian with English summary).

Darlington P. 1957. Zoogeography. New York, Wiley. 675 p.

Dashinov D. 2017. New data on the Chironomidae (Diptera) fauna from the Upper Iskar River catchment (Bulgaria) - spatial distribution and ecological notes. In: Ecological Engineering and Environment Protection 9, 16-21.

Dashinov D. \& Vidinova Y. 2018 (2017). First records of the parasite Symbiocladius rhithrogenae (Zavrel, 1924) (Diptera: Chironomidae) in several streams of Rila Mountain, Bulgaria. Aquatic Insects, 38 (4): 255-259. https://doi.org/10.1080/01650424.2017.1392023

Delkeskamp K. 1942. Eine neue Art der Gattung Chilosia Meigen aus Bulgarien (Dipt., Syrph.). Mitteilungen der Deutschen Entomologischen Gesellschaft 11: 11-13. 
Dely-Draskovits A. 1981. Revision der palaearktischen Arten der Gattung Aphanotrigonum Duda, 1932, und Aphanotrigonella Nartshuk, 1964 (Diptera: Chloropidae). Acta Zoologica Hungarica 27 (3-4): 265-271.

Dely-Draskovits A. 1983. Revision der Typen der Paläarktischen Arten der Gattung Tricimba Lioy, 1864 (Diptera: Chloropidae). Acta Zoologica Academiae Scientarum Hungaricae 29 (4): 327-355.

Dely-Draskovits A. 1985. Contribution to the knowledge of the Chloropid fauna of Bulgaria (Diptera: Chloropidae). Folia Entomologica Hungarica 46 (2): 27-29.

Dely-Draskovits A. 1993. Family Anthomyiidae. In: Soós Á. \& Papp L. (Eds.). Catalogue of Palaearctic Diptera. 13. Budapest, Akadémiai Kiadó, Hungarian Natural History Museum. pp. 11-102.

Dice L. 1945. Measures of the Amount of Ecologic Association Between Species. Ecology 26 (3): 297-302.

Dikov I. 1962. Da predotvratim razprostranenieto na sredizemnomorskata plodova muha na nasha teritoriya [To prevent the distribution of the Mediterranean fruit fly in Bulgaria]. Rastitelna zashtita 10 (3): 7-9. (In Bulgarian).

Dimitrov M. 1957. Hidrologichna i hidrobiologichna harakteristika na yazovir "Al. Stamboliyski” [Hydrological and hydrobiological characteristic of the Aleksandar Stamboliiski Dam]. Travaux de l'Institut de recherches scientifiques sur la pêche et les industries s'y rattachant (Varna) 1: 159-197. (In Bulgarian).

Dimitrov M. 1960a. Hidrologichna i hidrobiologichna harakteristika na yazovir "G. Dimitrov" [Hydrological and hydrobiological characteristic of the Georgi Dimitrov Dam]. Bulletin de l'Institut central de recherche scientifique de pisciculture et de pêcherie (Varna), 1: 87-122. (In Bulgarian with German summary).

Dimitrov M. 1960b. Bentosat na nyakoi malki yazoviri v Plovdivski okrag [The benthos of some small dams in the Plovdiv district]. Bulletin de l'Institut central de recherche scientifique de pisciculture et de pêcherie (Varna), 1: 123-138. (In Bulgarian).

Dimitrov M. 1962a. The Benthos in the „Batak” Dam. Bulletin of the test station of Freshwater Pisciculture 1: 59-80. (In Bulgarian with German summary).

Dimitrov M. 1962b. Bentosat na ribovadnite stopanstva v Plovdivski okrag [The benthos of the fish-farms in the Plovdiv district]. Bulletin of the test station of Freshwater Pisciculture 1: 21-57. (In Bulgarian).

Dimitrov M. 1962c. The Benthos in the "Studen Kladenets” Dam. Bulletin of the test station of Freshwater Pisciculture 1: 81-94. (In Bulgarian with German summary).

Dimitrov M. 1962d. Materiali po hironomidnata fauna (larvae) na r. Iskar [Materials on the Chironomidae fauna (larvae) of the Iskar River]. Bulletin of the test station of Freshwater Pisciculture 1: 129-135. (In Bulgarian).

Dimitrov M. 1963a. Studies of Chironomid fauna (Larvae) in Bulgaria. Bulletin of the test station of Freshwater Pisciculture 2: 5-24. (In Bulgarian with German summary).

Dimitrov M. 1963b. Biologicheski osnovi i efektivnost na zelenoto torene v ribovadnite baseyni [Biological basics and efficiency of the green fertilization in the fish-farms]. Priroda 12 (6): 74-77. (In Bulgarian).

Dimitrov M. 1966. Chironomidae larvae (Family Chironomidae, Diptera) and Oligachaeta (Order Oligochaeta) of the Maritsa River and some of its tributaries. In: Die Fauna Thrakiens 3. Sofia, Bulgarische Akademie der Wissenschaften. pp. 307-318. (In Bulgarian, Russian and English summaries).

Dimitrov M. 1969. The benthos of the Pysachnik Dam. Proceedings of the freshwater fishery research station (Plovdiv). 6: 31-39. (In Bulgarian, Russian and English summaries).

Dimitrov M. 1970. Das Benthos des Stausees “Ivailovgrad”. Proceedings of the freshwater fishery research station (Plovdiv). 7: 35-43. (In Bulgarian, Russian and German summaries).

Dimitrov M. 1972. Das Benthos im Stausee "Ovtschariza". Proceedings of the freshwater fishery research station (Plovdiv). 9: 83-96. (In Bulgarian, Russian and German summaries).

Dimitrov M. 1981. Periphytic Chironomid larvae found on oats planted as green fertilizer of carp-breeding ponds. Hyrdobiology 14: 81-87. (In Bulgarian, Russian and English summaries).

Dimitrov M. 1982. Chironomid larvae development under the conditions of intensive carp raising. Hydrobiology 16: 50-57. (In Bulgarian, Russian and English summaries).

Dimitrov T. 1934. Lesoohrana [Forest protection]. Sofia, Universitetska biblioteka 153. (In Bulgarian).

Dimitrov T. 1935. Prinos kam izuchvane nasekomnite i gabnite vrediteli v nashite gorski kulturi [Contribution to the study of the insect and fungal pests in the Bulgarian forests and forest crops]. Godishnik na sofiyskiya universitet. Agronomo-lesovadski fakultet 13 (2): 220-252. (In Bulgarian).

Dimitrova B. 1987. A Contribution to the fauna of the subfamily Cecidomyiinae (Diptera, Cecidomyiidae) in Vitosha, Bulgaria. In: Savremenni postizheniya na balgarskata zoologiya. Sofia, Bulgarian Academy of Sciences. pp. 25-29. (In Bulgarian). 
Dimitrova B. 1989. Faunistic, zoogeographical and environmental studies of the subfamily Cecidomyiinae (Diptera, Cecidomyiidae) of the Vitosha Mt. Ph.D. thesis, Sofia, Bulgarian Academy of Sciences. 174 p. (In Bulgarian).

Dimitrova B. 1990. New data about the species from the subfamily Cecidomyiinae (Diptera, Cecidomyiidae) from Vitosha. In: Fauna of Southwestern Bulgaria. 3. Sofia, Bulgarian Academy of Sciences. pp. 146-149. (In Bulgarian with English summary).

Dimitrova B. 1992. Coniophora nijveldti sp. n. (Diptera, Cecidomyiidae) from Bulgaria. Acta zoologica bulgarica 44: 79-81.

Dimitrova B., Mamaev B. 1993. New Gall midges Lestremiinae and Porricondylinae (Diptera, Cecidomyiidae) from Vitosha (Bulgaria). Acta zoologica bulgarica 46: 97-99.

Dimitrova B., Mohrig W. 1993. Beitrag zur Trauermückenfauna Bulgariens (Diptera, Sciaridae). II. Acta zoologica bulgarica 46: 89-96.

Dimitrova B., Pencheva A. 2004. Dasineura gleditchiae (Diptera: Cecidomyiidae) - anew pest of honeylocust in Bulgaria. Forestry Ideas 10 (3): 64-69. (In Bulgarian with English summary).

Dimov I. 1957. Zimuvane i diapauza na Anopheles maculipennis typicus v Sofiysko [Wintering and diapause of Anopheles maculipennis typicus in the Sofia district]. Higiena, Epidemologiya i Mikrobiologiya 1 (2): 36-41. (In Bulgarian).

Dimov I. 1959. Des nouveaux mosquites pour la faune de Bulgarie. Comptes rendus de l'Academie bulgare des Sciences 12 (2): 168-172.

Dimtchev D., Stefanov S., Kovtchazov G. \& Christova T. 1960. Sur la composition des especes de la faune des Anopheles et des varietes de A. maculipennis Meigen sur le littoral bulgare du Danube. Folia medica 2 (1): 25-31.

Dimtchev D., Stefanov S., Kovtchazov G. \& Christova T. 1962. Prinos kam prouchvane na anofeliynata fauna po dunavskoto kraybrezhie s ogled epidemiologiyata na malariyata $v$ tozi rayon [Contribution to the study of the Anopheles fauna along the Danubian coast in view of the epidemic of malaria in this region]. Sbornik trudove na Visshiya meditsinski institut „I. P. Pavlov“v Plovdiv 16: 245-262. (In Bulgarian).

Dirimanov M. \& Sengalevich G. 1969. Muhi tahini paraziti po gasenitsite na nyakoi staklenki i darvesinoyadi u nas [Tachinidae flies parasites on the caterpillars of Sesiidae and Cossidae in Bulgaria]. Rastitelna zashtita 11: 17-20. (In Bulgarian).

Dirlbek J. \& Dirlbek I. 1963. K faune muh-pestrokrylok Diptera (Tripetidae) Bolgarii [To the fauna of Diptera (Trypetidae) Bulgaria]. Entomologicheskoe Obozrenie 42 (4): 845-854. (In Russian with English summary).

Disney R. 1991. Family Phoridae. In: Soós Á. \& Papp L. (Eds.). Catalogue of Palaearctic Diptera. 7. Budapest, Akadémiai Kiadó, Hungarian Natural History Museum. pp. 143-204.

Dochkova B. 1971. Vredni noshtenki (Lepidoptera, Noctuidae) po zaharnoto tsveklo v Severna Bulgaria [Harmful Lepidoptera, Noctuidae on the sugar beet in the Northern Bulgaria]. Ph.D. thesis, Kostinbrod, Institut po zashtita na rasteniyata, 108-109. (In Bulgarian).

Dochkova B. 1972. Nyakoi biologichni i ekologichni prouchvaniya varhu gamoznachnata noshtenka [Some biological and ecological studies on the gamma nightingale]. Rastenievadni nauki 10: 141-149. (In Bulgarian).

Dominiak P. \& Szadziewski R. 2010. Distribution and new synonymy in European biting midges of the genus Dasyhelea Kieffer (Diptera: Ceratopogonidae). Zootaxa 2437: 1-37.

Donchev K. 1968. Vredni galitsi po lyutsernata [Harmful Cecidomyiidae on the alfalfa]. Rastitelna zashtita 8: 19-21. (In Bulgarian).

Donchev K. 1969. Entomologicheski problemi na furazhnite kulturi [Entomological problems of the fodder crops]. Vnedreni novosti 5: 9-19. (In Bulgarian).

Donchev K. 1978. Prouchvaniya varhu entomofaunata po eksparzetata i vazmozhnosti za borba s ikonomicheski nay vrednite vidove [Studies on the entomofauna of the sainfoin and possibilities for a fight against the most economically harmful species]. Ph.D. thesis, Sofia, Agricultural Academy. 204 p. (In Bulgarian).

Donchev N. 1961. Prouchvane na zavisimostta mezhdu srokovete za zasyavane na pshenitsa, echemik, oves i povredite, koito nanasyat varhu tyah rarlichni vidove zhitni muhi [Study of the relationship between the timing of sowing wheat, barley, oats and the damage caused to them by different species of cereal flies]. Izvestiya na Dobrudzhanskiya selskostopanski nauchnoizsledovatelski institut 1: 167-183. (In Bulgarian). 
Dorovski D. 1976. Prouchvaniya varhu vidoviya sastav, razprostranenieto i borbata s komarite v Plevenski okrag [Studies on the species composition, distribution and mosquito control in the Pleven district]. Ph.D. thesis, Sofia, National Center of Infectious and Parasitic Diseases. 155 p. (In Bulgarian).

Dospevski S. 1908a. Bolesti i nepriyateli po kulturnite rasteniya - nasekomi, koito povrezhdat lozata [Diseases and pests on the cultivated plants - insects that damage the vineyards]. Sadovo 11 (9/10): 313-318. (In Bulgarian).

Dospevski S. 1908b. Bolesti i povredi po kulturnite rasteniya, izprateni za izsledvane v Sadovskata opitna stantsiya prez 1906-1907 godina [Diseases and damages on the cultivated plants, sent for research in the Sadovsky experimental station in 1906-1907]. Otchet na Darzhavnata opitna stantsiya v Sadovo za 1907 godina 5: 207-307. (In Bulgarian).

Dospevski S. 1908c. Izsledvaniya i nablyudeniya varhu razni bolesti i nepriyateli po kulturnite rasteniya [Research and observations on the various diseases and pests on the cultivated plants]. Plovdiv, Izdanie na Ministerstvoto na targoviyata i zemedelieto. 107 p. (In Bulgarian).

Doychev D., Kechev M., Todorov I., Mirchev P., Bencheva S. \& Georgiev G. 2016. New entomophagous enemies of Ips typographus (Linnaeus, 1758) (Coleoptera: Curculionidae) from Bulgaria. Acta zoologica bulgarica 68 (1): 131-134.

Doychev D., Zaemdzhikova G., Topalov P., Hubenov Z. \& Georgiev G. 2019. New Parasitoids of Longhorn Beetles (Coleoptera: Cerambycidae) in Bulgaria. Acta zoologica bulgarica 71 (2): 175-182.

Draber-Mońko A. 1965. Monographie der paläarktischen Arten der Gattung Allophora R.-D. (Diptera, Larvaevoridae). Annales Zoologici 23 (6): 70-194.

Drenowsky A. 1920a. Saobshtenie varhu Chlorops taeniopus [Message on Chlorops taeniopus]. Estestvoznanie i geographiya 4 (7): p. 336. (In Bulgarian).

Drenowsky A. 1920b. Saobshtenie varhu nyakoi vredni nasekomi [Message on some harmful insects]. Estestvoznanie i geographiya 4 (9/10): p. 454-455. (In Bulgarian).

Drenowsky A. 1921a. Saobshtenie varhu nyakoi vredni nasekomi [Message on some harmful insects]. Estestvoznanie i geografiya 5 (3/4): 156-158. (In Bulgarian).

Drenowsky A. 1921b. Iz prirodoizpitatelnite druzhestva [From nature societies]. Estestvoznanie i geographiya 5 (7/8): 316-319. (In Bulgarian).

Drenowsky A. 1922a. Saobshtenie varhu vredni nasekomi [Message on the harmful insects]. Estestvoznanie i geografiya 6 (1): 44-48. (In Bulgarian).

Drenowsky A. 1922b. Chlorops taeniopus Meig. (Dipt.). Zhitna steblena muha, nanasyanite povredi i borbata s neya v Sofiysko [Chlorops taeniopus Meig. (Dipt.). Wheat stalk fly, the damages applied and the fight against it in the Sofia region]. Arhiv na Ministerstvoto na zemedelieto i darzhavnite imoti 2: 1-32. (In Bulgarian).

Drenowsky A. 1922c. Nepoznati vredni muhi na zhitnite rasteniya u nas, novi vidove za naukata [Unknown harmful flies of the cereals in Bulgaria, new species for science]. Svedeniya po zemedelieto 3 (10): 12-15. (In Bulgarian).

Drenowsky A. 1922d. Nepoznat vreden vid peperuda po zhitnite rasteniya u nas [Unknown harmful butterfly species on the cereals in Bulgaria]. Estestvoznanie i geographiya 6 (1): 44-45. (In Bulgarian).

Drenowsky A. 1923a. Lasiosina cinctipes (Dipt.), muhata po klasovete na echemika, nanasyanite povredi ot neya v Sofiysko i vazmozhnosti za borba [Lasiosina cinctipes (Dipt.), the fly on the barley classes, the damage caused by it in the Sofia region and opportunities for fight]. Svedeniya po zemedelieto 4 (3/4): 29-62. (In Bulgarian).

Drenowsky A. 1923b. Lasiosina cinctipes Meig. die Gerstenfliege, ihre Schäden und Bekämpfung in der Umgegend von Sofia, Bulgarien. Zeitschrift für wissenschaft Insektenbiologie 18 (10/11): 284-290.

Drenowsky A. 1923c. Nepoznati vredni muhi na zhitnite rasteniya u nas, novi vidove za naukata [Unknown harmful flies of the cereals in Bulgaria, new species for science]. Estestvoznanie i geographiya 7 (7): 240242. (In Bulgarian). ?

Drenowsky A. 1929a. I. Anatomo-morfologichni i biologichni belezhki varhu malarichniya komar i negovata larva. II. Kratka sistematika na petrichkata komarna fauna [I. Anatomic-morphological and biological notes on the malarial mosquito and its larva. II. A brief systematics of the Petrich mosquitos fauna]. Izvestiya na Direktsiyata na narodnoto zdrave 14 (priturka) 2: 118-137. (In Bulgarian).

Drenowsky A. 1929b. Deynostta na petrichkata protivomalarichna opitna stantsiya prez 1928 godina [The activity of the Petrich Antimalarial Experimental Station in 1928]. Bulgarska klinika 1 (10): 745-777. (In Bulgarian). 
Drenowsky A. 1931. Referate und Mitteilungen. Mitteilungen der Bulgarischen Entomologischen Gesellschaft in Sofia 6: 13-19. (In Bulgarian).

Drenowsky A. 1936. Beitrag zur Insektenfauna Bulgariens u. Mazedoniens. II. Mitteilungen der Bulgarischen Entomologischen Gesellschaft in Sofia 9: 237-256. (In Bulgarian with German summary).

Drenowsky A. 1937. Entomologicheski prinosi [Entomological contributions]. Sofia, Sobstveno izdanie na avtora. 16 p. (In Bulgarian).

Drenowsky A. 1939. Referate und Mitteilungen. Mitteilungen der Bulgarischen Entomologischen Gesellschaft in Sofia 10: 156-163. (In Bulgarian).

Drenowsky A. \& Enderlein G. 1923. Novi vredni muhi za zhitnite rasteniya v Bulgariya [New harmful flies on the cereal plants in Bulgaria]. Arhiv na Ministerstvoto na zemedelieto i darzhavnite imoti 3: 1-20. (In Bulgarian).

Drensky K. 1926. Sanitary significans of the Insekts. Mitteilungen der Bulgarischen Entomologischen Gesellschaft in Sofia 3: 59-70. (In Bulgarian with English summary).

Drensky K. 1939. The varieties of Anopheles maculipennis Meigen and their relation to the distribution of malaria. Mitteilungen der Bulgarischen Entomologischen Gesellschaft in Sofia 10: 31-44. (In Bulgarian with English summary).

Drensky K. 1949. Malariynite komari u nas [Malaria mosquitoes in Bulgaria]. Izvestiya na institutite po eksperimentalna meditsina, sotsialna meditsina, obshta biologiya i veterinarno-eksperimentalna meditsina 1: 246-279. (In Bulgarian).

Drensky K. 1950. Malariynite komari u nas [Malaria mosquitoes in Bulgaria]. In: II nauchen sabor na balgarskite lekari, chast II, malariya. Sofiya. pp. 27-48. (In Bulgarian).

Drensky K. 1958. Anofeliynite komari i borbata protiv malariyata [Anopheline mosquitoes and the fight against the malaria]. Priroda 7 (3): 43-48. (In Bulgarian).

Drensky P. 1926a. Referate und Berichte. Mitteilungen der Bulgarischen Entomologischen Gesellschaft in Sofia 3: 27-29. (In Bulgarian).

Drensky P. 1926b. Die Parasitär Lebenden Fliegen der Fam. Pupiparae (Diptera) in Bulgarien. Mitteilungen der Bulgarischen Entomologischen Gesellschaft in Sofia 3: 89-104. (In Bulgarian with German summary).

Drensky P. 1928. Referate und Berichte im Jahre 1926-1927. Mitteilungen der Bulgarischen Entomologischen Gesellschaft in Sofia 4: 18-21. (In Bulgarian with German summary).

Drensky P. 1929a. Blutsaugende Fliegen aus der Familie der Tabanidae (Bremsen) in Bulgarien. Mitteilungen aus den Königl. Naturwissenschaftlichen Instituten in Sofia 2: 55-128. (In Bulgarian with German summary).

Drensky P. 1929b. Die bisher aus Bulgarien, Thrazien und Mazedonien bekannt gevordenen Tabaniden. Sitzunsberichte der Geselschaftnaturforschender Freunde. 233-235.

Drensky P. 1930a. Bolesti i nepriyateli po zhitnite rasteniya v Bulgaria [Diseases and pests on the cereals in Bulgaria]. Svedeniya po zemedelieto 11 (7/8): 3-54. (In Bulgarian).

Drensky P. 1930b. Saobshtenie varhu vredni nasekomi [Message on the harmful insects]. Mitteilungen der Bulgarischen Entomologischen Gesellschaft in Sofia 5: 25-27. (In Bulgarian).

Drensky P. 1931a. Kleine entomologische Mitteilungen. Mitteilungen der Bulgarischen Entomologischen Gesellschaft in Sofia 6: 123-141. (In Bulgarian).

Drensky P. 1931b. Referate und Berichte im Jahre 1930. Mitteilungen der Bulgarischen Entomologischen Gesellschaft in Sofia 6: 19-23. (In Bulgarian).

Drensky P. 1931c. Uber die Biologie des Wiesenzünslers - Logsostege (Phyctaenodes) sticticalis L. in Bulgarien wahrend der Jahre 1929-1930. Mitteilungen der Bulgarischen Entomologischen Gesellschaft in Sofia 6: 31-48. (In Bulgarian with German summary).

Drensky P. 1932a. Referate und Berichte im Jahre 1931. Mitteilungen der Bulgarischen Entomologischen Gesellschaft in Sofia 7: 26-28. (In Bulgarian).

Drensky P. 1932b. Izsledvani bolesti i nepriyateli na pchelite prez 1932 g. v Entomologichnata stantsiya v Sofia [Researched diseases and pests of the bees in 1932 at the Entomological Station in Sofia]. Pchela 15 (12): 379-382. (In Bulgarian).

Drensky P. 1932c. Kleine entomologische Mitteilungen. II. Mitteilungen der Bulgarischen Entomologischen Gesellschaft in Sofia 7: 62-77. (In Bulgarian).

Drensky P. 1933. Die parasitären Fliegen der Familie Oestridae in Bulgarien. Mitteilungen aus den Königl. Naturwissenschaftlichen Instituten in Sofia 6: 125-1149. (In Bulgarian with German summary). 
Drensky P. 1934a. Die Fliegen der Familie Syrphidae (Dipt.) in Bulgarien. Mitteilungen der Bulgarischen Entomologischen Gesellschaft in Sofia 8: 109-131. (In Bulgarian with German sumarry).

Drensky P. 1934b. Bolesti i nepriyateli po pchelite prez 1933 g. u nas [Diseases and enemies of the bees in 1933 in Bulgaria]. 20 p.

Drensky P. 1934c. Fauna na Lovchansko i Troyansko [The fauna of Lovchansko and Troyansko]. Lovech i Lovchansko 6: 107-125.

Drensky P. 1936. Krankheiten und Schädlinge der Bienen in Bulgarien. Mitteilungen der Bulgarischen Entomologischen Gesellschaft in Sofia 9: 47-66. (In Bulgarian with German summary).

Drensky P. 1939a. Referate und Mitteilungen. Mitteilungen der Bulgarischen Entomologischen Gesellschaft in Sofia 10: 163-168. (In Bulgarian).

Drensky P. 1939b. Die parasitären Fliegen der Famillie Conopidae in Bulgarien. Travaux de la Société Bulgare de Sciences Natureles 18: 70-80. (In Bulgarian with German summary).

Drensky P. 1940. Referate und Mitteilungen. Mitteilungen der Bulgarischen Entomologischen Gesellschaft in Sofia 11: 252-253. (In Bulgarian).

Drensky P. 1942. Über die Insektenfauna des Küstengebietes nördlich von Warna. Mitteilungen der Bulgarischen Entomologischen Gesellschaft in Sofia 12: 15-44. (In Bulgarian with German summary).

Drensky P. 1943. Muhi ot semeystvo Trypetidae v Bulgaria [Flies of the family Trypetidae (Dipt.) in Bulgaria]. Annuaire de l'Université de Sofia 39: 69-126. (In Bulgarian with German summary).

Drensky P. 1955. Fliegen und Arachnoideen aus den Wäldern und den Feldschutzwaldstreifen der Süd-Dobrudscha. In: Sbornik na ekspeditsiyata po polezashtitnite poyasi v Dobrudzha prez 1952 godina, Sofia, Bulgarian Academy of Sciences. pp. 385-397. (In Bulgarian with German summary).

Drensky P. 1957a. Die Familie der Stechfliegen (Stomoxydidae) in Bulgarien. Bulletin de l'Institut zoologique de l'Academie des sciences de Bulgarie 6: 441-452. (In Bulgarian with German summary).

Drensky P. 1957b. Bestand und Verbreitung der grauen Fliegen aus der Familie Sarcophagidae in Bulgarien. Bulletin de l'Institut zoologique de l'Academie des sciences de Bulgarie 6: 199-232. (In Bulgarian with German sumarry).

Drensky P. 1958. Bestand, Verbreitung und Bedeutung der Schmeissfliegen aus der Familie Calliphoridae in Bulgarien. Bulletin de l'Institut zoologique de l'Academie des sciences de Bulgarie 7: 115-131. (In Bulgarian with German sumarry).

Drensky P. 1959. Ephydra riparia Fall. (Dipt.) als Halobiont vom Schwarzen Meer. Bulletin de l'Institut zoologique de l'Academie des sciences de Bulgarie 8: 181-184. (In Bulgarian with German summary).

Drensky P. 1960. Beitrag zur Untersuchung der sinanthropischen Mücken im Bezirk Burgas. Bulletin de l'Institut zoologique de l'Academie des sciences de Bulgarie 9: 339-345. (In Bulgarian with German summary).

Drensky P. \& Drensky K. 1928. Beitrag zur Erforschung von Genus Phlebotomus (Dipt.) und des Dreitägigen Fiebers in Bulgariaen. Mitteilungen der Bulgarischen Entomologischen Gesellschaft in Sofia 4: 31-56. (In Bulgarian with German summary).

Drensky P. \& Stefanoff At. 1939. Das erste fossile Insekt aus Bulgarien: Mycetophila aff. pulchella Heer. (Dipt.). Mitteilungen der Bulgarischen Entomologischen Gesellschaft in Sofia 10: 51-54. (In Bulgarian with German summary).

Drensky P. \& Zacharieva-Stoilova B. 1951. Rapacious and parasite enemies of Agrotis temera caterpillars as observed in 1948 and 1949. Bulletin de l'Institut zoologique de l'Academie des sciences de Bulgarie 1: 298-304. (In Bulgarian with English summary).

Duda O. 1933. Chloropidae. In: Lindner E. (Ed.). Die Fliegen der palaearktischen Region 6. Stuttgart, E. Schweizerbart'sche Verlagsbuchhandlung. 248 p.

Đuknić J., Jovanović V., Popović N., Živić I., Raković M., Čerba D., \& Paunović M. 2019. Phylogeography of Simulium Subgenus Wilhelmia (Diptera: Simuliidae) - Insights From Balkan Populations. Journal of Medical Entomology, 56(4): 967-978. doi: 10.1093/jme/tjz034

Dupuis C. 1963. Essai monographique sur les Phasiinae (Diptera Tachinaires parassites d'Hétéroptères). Mémoires du Museum national d'histoire naturelle, Sér. A, Zool. 26:7-461.

Dursun O., Civelek H., Barták M., Kubík Š., Yildirim E. \& Černý M. 2015. Contributions to leafminer (Diptera: Agromyzidae) fauna and new records of plant pests and weeds in Turkey. Turkish Journal of Entomology 39 (2): 159-169. 
Dušek J. \& Rozkošný R. 1963. Revision mitteleuropäischer Arten der Familie Stratiomyidae (Diptera) mit besonderer Berücksichtigung der Fauna der ČSSR I. Časopis České společnosti entomologické 60: 202-231.

Dušek J. \& Rozkošný R. 1965. Revision mitteleuropäischer Arten der Familie Stratiomyidae (Diptera) mit besonderer Berücksichtigung der Fauna der ČSSR III. Acta entomologica bohemoslovaca 62: 24-60.

Dušek J. \& Rozkošný R. 1967. Revision mitteleuropäischer Arten der Familie Stratiomyidae (Diptera) mit besonderer Berücksichtigung der Fauna der ČSSR IV. Acta entomologica bohemoslovaca 64: 140-165.

Dušek J. \& Rozkošný R. 1970. Revision der palaearktischen Arten der Gattung Lasiopa Brullé, 1832 (Diptera: Stratiomyidae). Beiträge Zur Entomologie 20 (1-2): 19-41.

Dvořák L., Kolsár L.-P., Georgiev D. \& Mariychuk R. 2019. New and interesting records of window-gnats (Diptera: Anisopodidae) from Europe. Klapalekiana 55: 187-192.

Dzhambazov B. 1995a. New species Empidoidea (Diptera) to the fauna of Bulgaria. Travaux Scientifiques Universite de Plovdiv, Biologie - Animalia 31 (6): 31-33.

Dvořák L., Schneppat U., Georgiev D. \& Dvořáková K. 2021. Some new records of Diptera species from Sarnena Gora Mts. In: Fauna of Sarnena Sredna Gora Mts. 2. Zoonotes, Supplement 10: 8-11.

Dzhambazov B. 1995b. Species superfamily Empidoidea (Diptera) known to the fauna of Bulgaria till 1995. Travaux Scientifiques Universite de Plovdiv, Biologie - Animalia 31 (6):35-39. (In Bulgarian with English sumarry).

Dzhambazov B. 1998. Hilara bechevi spec. nov. from Bulgaria (Insecta: Diptera: Empididae). Reichenbachia 32 (2): 327-328.

Dzhambazov B. 1999. Faunistical studi of the Microphoridae, Hybotidae and Empididae (Diptera, Eempidoidea) from the Bulgarian Rhodope Mts. Travaux Scientifiques Universite de Plovdiv, Biologie - Animalia 35 (6): 41-48.

Dzhambazov B. 2000. Faunistic, zoogeographical and ecological investigations on Empidoidea (Diptera) in Western Rhodopes. Ph.D. thesis, Sofia, Bulgarian Academy of Sciences. 168 p. (In Bulgarian).

Dzhambazov B. \& Beschovski V. 2000. New records of Empidoidea fauna from Bulgaria (Deiptera: Atelestidae, Microphoridae, Hybotidae, Empididae. Acta zoologica bulgarica 52 (1): 3-7.

Dzhambazov B. \& Teneva I. 2000. Beobachtungen zum Blütenbesuch von Fliegen der Überfamilie Empidoidea (Hybotidae, Empididae). Studia dipterologica 7 (2): 553-557.

Ebejer M. \& Barták M. 2019. An annotated list of the Chamaemyiidae (Diptera, Acalyptrata) of Turkey with new records and additional data. ZooKeys 838: 35-48. https://doi.org/10.3897/zookeys.838.33027

Elenkov E. \& Hristova E. 1974. Bolesti i nepriyateli po zelenchukovite kulturi v oranzheriite [Diseases and pests of the vegetable crops in the greenhouses]. Sofia, Zemizdat. 211p. (In Bulgarian).

Emeljanov A. F. 2018. Sectorial attribution of the range types proposed by K. B. Gorodkov for northern palaearctic. Entomologicheskoe obozrenie 97 (1): 47-59. ( In Russian with English summary).

Enderlein G. 1921. Neue palaearctische Simuliden. Sitzunsberichte der Geselschaftnaturforschender Freunde 8/10: 211-224.

Enderlein G. 1924. Die Simuliden Bulgariens und ihre Schäden im Jahre 1923. Zoologischer Anzeiger, B. 61 (11/12): 280-288.

Enderlein G. 1925. Studien am blutsaugenden Insecten. I. Grundlagen eines neuen Systems der Tabaniden. Mitteilungen aus dem Zoologische Museum in Berlin 11: 255-410.

Enderlein G. 1930. Beitrag zur Kenntnis der Prosimuliinen und Hellichiinen. Stzungsberichte der Geselschaftnaturforschender Freunde, 222-224.

Enderlein G. 1936. Eine neue von Herrn D. Jacentkovsky in Balkan gesammelte Sarcophagiden (Dipt.). Mitteilungen königl naturwissenschaftlichen Instituten in Sofia (Bulgarien) 9: 98-100.

Evenhuis N. L. 2002. Catalog of the Mythicomyiidae of the World (Insecta: Diptera). Honolulu, Bishop Museum Press. 85 p.

Evenhuis N. L. 2006. Catalog of the Keroplatidae of the World (Insecta: Diptera). Honolulu, Bishop Museum Press. 179 p.

Evenhuis N. L. 2015. World catalog of bee flies (Diptera: Bombyliidae) web site. [http://hbs.bishopmuseum.org/ bombcat/].

Evenhuis N. L. \& Greathead D. J. 1999. World catalog of bee flies (Diptera: Bombyliidae). Backhuys Publishers, Leiden. xlviii $+756 \mathrm{p}$. 
Ficetola G., Mazel F. \& Thuiller W. 2017. Global determinants of zoogeographical boundaries. Nature Ecology \& Evolution 1 (0089): 1-7.

Foote R. 1984. Family Tephritidae. In: Soós Á. \& Papp L. (Eds.). Catalogue of Palaearctic Diptera. 9. Budapest, Akadémiai Kiadó, Hungarian Natural History Museum. pp. 66-149.

Frey R. 1954-1956. Empididae. In: Lindner E. (Ed.). Die Fliegen der palaearktischen Region 4, 28. Stuttgart, E. Schweizerbart'sche Verlagsbuchhandlung. pp. 400-639.

Gagné R. \& Jaschhof M. 2014. A Catalog of the Cecidomyiidae (Diptera) of the World. 3rd Edition. Digital version $2.493 \mathrm{p}$.

Ganchev G. 1972. Vidov sastav i rolya na parazitite po gabotvorkata (Lymantria dispar L.) v gorite [Species composition and role of the fungal parasites (Lymantria dispar L.) in the forests]. Ph.D. thesis, Sofia, Forsttechnische Hochschule (Sofia), 17-18. (In Bulgarian).

Ganchev G. 1975. Artenzusammenstand und Verbreitung der parasiten auf den Schammsptner von der Familie Tachinidae (Dipt.) und ihre Hiperparasiten. Forsttechnische Hochschule (Sofia) 20: 97-103. (In Bulgarian with German sumarry).

Ganchev G. 1977. Beitrag zur Untersuchung der Artenzusammensetzung und Verbreitung der Parasiten einiger Arten Spanner und Blattwicjler in der Wäldern. Forsttechnische Hochschule (Sofia) 22: 111-118. (In Bulgarian with German sumarry).

Ganchev G. 1980. Ein Beitrag bei forschung Arten und Verbreitung der Parasiten bei Ringebspiner (M. neustria L.) im Wälder. Forsttechnische Hochschule (Sofia) 21: 129-134. (In Bulgarian with German sumarry).

Ganchev G. 1981. Lesozashtita. Sofia, Zemizdat. 295 p. (In Bulgarian).

Ganeva D. 1993. Species composition and phenology of Tabanidae (Diptera) in the Stara Zagora district. In: Tsankov G., Beschovski V. \& Popov A. (Eds.). Second national scientific conference of entomology. Sofia, Union of the Scientists in Bulgaria \& Bulgarian societi of entomology, 28-33. (In Bulgarian with English summary).

Ganeva D. 1995a. Seasonal prevalence and daily activity of Tabanids (Tabanidae, Diptera) in the Stara Zagora district. Acta entomologica bulgarica 1: 6-14. (In Bulgarian with English summary).

Ganeva D. 1995b. The tabanids fauna (Tabanidae, Diptera) of Stara Planina (Bulgaria). I. In: Gruev B., Nikolova M. \& Donev A. (Eds.). Proceedings of the Balkan Scientific conference of biology in Plovdiv (Bulgaria), 397-403.

Ganeva D. 1995c. Blood-sucking Tabanids (Tabanidae, Diptera) on cattle in the Stara Zagora district. Vaterinary medicine 2: 228-232 (In Bulgarien).

Ganeva D. 1995d. Seasonal prevalence of tabanids (Tabanidae, Diptera) in the Stara Zagora district. In: Tsankov G., Pelov V, Beschovski V. \& Popov A. (Eds.). Third national scientific conference of entomology. Sofia, Union of the Scientists in Bulgaria \& Bulgarian societi of entomology, 7-12. (In Bulgarian with English summary).

Ganeva D. 1996. Faunal composition of tabanids (Tabanidae, Diptera) in cattle pastures of different types in Bulgaria. Acta Entomologica Bulgarica, 2 (2): 21-27. (In Bulgarian with English sumarry).

Ganeva D. 1998. Faunal composition of Tabanids (Tabanidae, Diptera) in cattle pastures of different types in Bulgaria. Acta Entomologica Bulgarica, 1: 10-15. (In Bulgarian with English sumarry).

Ganeva D. 1999a. Daily activity of Tabanus bromius L., Tabanus tergestinus Egg. and Haematopota pluvialis L. (Tabanidae, Diptera) in the Stara Sagora district. Periodicum Biologorum 101 (3): 215-220.

Ganeva D. 1999b. Fauna, phenology and activity of blood sucking tabanids (Tabanidae, Diptera) in Stara Zagora Region. Ph.D. thesis, Sofia, Bulgarian Academy of Sciences. 215 p. (In Bulgarian).

Ganeva D. 2000. New data about the Tabanids (Diptera: Tabanidae) from Bulgaria. Travaux du Muséum National d'Histoire naturelle „Grigore Antipa” 42: 133-142.

Ganeva D. 2001. Seasonal activity of Tabanus bromius L., Tabanus tergestinus Egg. and Haematopota pluvialis L. (Tabanidae, Diptera) in Stara Sagora district, Bulgaria. Acta zoologica bulgarica 53 (1): 89-96.

Ganeva D. 2002. New localities of tabanids (Tabanidae, Diptera) in Bulgaria. Stara Zagora Union of scientists, Veterinary medicine, Human medicine 3: 41-44. (In Bulgarian).

Ganeva D. 2004. Analysis of the Bulgarian tabanid fauna with regard to its potential for epidemiological involvement. Bulgarian Journal of Veterinary Medicine 7 (1): 1-8. 
Ganeva D. 2005a. The tabanids fauna (Tabanidae, Diptera) of Stara Planina (Bulgaria). I. In: Gruev B, Nikolova M. \& Donev A. (Eds.). Proceedings of the Balkan Scientific conference of biology in Plovdiv (Bulgaria), 397-403.

Ganeva D. 2005b. Review of the Tabanids (Diptera: Tabanidae) from Bulgaria. Acta zoologica bulgarica 57 (3): 279-298.

Ganeva D. 2006. Tabanids (Tabanidae, Diptera) of the Bulgarian pert of the Rhodopes. In: Beron P. (Ed.). Biodiversity of Bulgaria. 3. Biodiversity of Western Rhodopes (Bulgaria and Greece) 1. Sofia, Pensoft \& National Museum of Natural History. pp. 719-728.

Ganeva D. 2008a. A contribution to the study of the Bulgarian tabanid fauna (Diptera, Tabanidae). In: Velcheva I. \& Tsekov A. (Eds.). Proceedings of the Anniversary Scientific conference of ecology. Plovdiv, 96-100.

Ganeva D. 2008b. The tabanid fauna (Diptera: Tabanidae) of the Saint Ilijski Eminences (Bulgaria). Acta zoologica bulgarica Supplement 2: 19-24.

Ganeva D. 2009a. New data on the Tabanids fauna (Diptera, Tabanidae) of the Sakar Mountain, Bulgaria. Trakia Journal of Sciences 7 (1): 17-21.

Ganeva D. 2009b. The tabanids (Diptera: Tabanidae) from the Belassitsa Mountain, Bulgaria. Agricultural Science and Technology 1:30-32.

Ganeva D. 2009c. New data on tabanids (Diptera: Tabanidae) from Slavyanka Mts., Bulgaria. Comptes rendus de l'Académie bulgare des sciences 62 (3): 365-366.

Ganeva D. 2011. Horse flies (Diptera, Tabanidae) of Surnena Sredna Gora Mountain, Bulgaria. Trakia Journal of Sciences 9 (3): 13-16.

Ganeva D. 2016. Horse flies (Diptera: Tabanidae) of Vrachanska Planina Mountains. In: Bechev D. \& Georgiev D. (Eds.). Faunistic diversity of Vrachanski Balkan Nature Park. ZooNotes, Supplement 3: 181-184.

Ganeva D. 2017. Horse flies (Diptera: Tabanidae) in the Rila Mts., Bulgaria. Acta zoologica bulgarica Supplement 8: 131-138.

Ganeva D. \& Ivanov I. 2015. Species composition and zoogeographical aspects of the Horsefly fauna (Diptera: Tabanidae) in the Central Balkan Mountains. Acta zoologica bulgarica 67 (2): 215-222.

Ganeva D. \& Kalmushka M. 2012. The tabanid fauna (Diptera: Tabanidae) of the Chirpan Eminences (Bulgaria). Entomologica Hellenica 21: 45-53.

Ganeva D. \& Kalmushka M. 2019. Seasonal abundance of Horse flies (Diptera, Tabanidae) in the Chirpan Eminences, Bulgaria. Trakia Journal of Sciences 17 (1): 34-41.

Ganeva D. \& Penev L. 2002. Structure and diversity of tsbsnid assemblages from three pastures in South Bulgaria (Diptera, Tabanidae). Studia dipterologica 9 (2): 743-755.

Ganov V. 1949. Klinika na papatatsievata treska u nas [The clinical picture of the Phlebotomidae fever in Bulgaria]. Izvestiya na Instituta za eksperimentalna meditsina, sotsialna meditsina, obshta biologiya i veterinarna eksperimentalna meditsina 1: 452-464. (In Bulgarian).

Gaydarov I. 1930. Chereshova muha, Spilographa cerasi [The cherry fly, Spilographa cerasi]. Balgarsko ovoshtarstvo i gradinarstvo 9 (4): 91-92. (In Bulgarian).

Georgiev B., Nikolov P. 2010. Invertebrates in the Pomorie Wetland. - In: Collection with reports Pomorie Lake region (Protected Site "Pomorie Lake", Ramsar Site, NATURA 2000 sites "Pomorie" BG 0000620 and "Pomorie Lake" BG 0000152), 72-87. (In Bulgarian).

Georgiev G. 1991. Nov darvesinoatakuvasht vreditel po topolite v nashata strana [A new wood attack pest on the poplars in Bulgaria]. Gorsko stopanstvo 4: 22-23. (In Bulgarian).

Georgiev G. 1996a. Diplostichus janitrix Hart. (Diptera, Tachinidae) - parazitoid po Gilpinia sp. (Hymenoptera: Diprionidae) i nov vid za faunata na Bulgaria [Diplostichus janitrix Hart. (Diptera, Tachinidae) - parasitoid on Gilpinia sp. (Hymenoptera: Diprionidae) and a new species for the fauna of Bulgaria]. Lesovadska misal 2 (7): 103-105. (In Bulgarian).

Georgiev G. 1996b. Bioecological peculiarities of the parasitoids on the late-stage larvae and pupae of the satin moth (Stilpnotia salicis L., Lepidoptera: Lymantridae) in Bulgaria. Forest Science 3: 57-64. (In Bulgarian with English summary).

Georgiev G. 1998. New and little-known phytophagous insects on the willows (Salix spp.) in Bulgaria. In: Proceedings scientific papers Jubilee Scientific conference with international participation "70-th anniversary of the Forest Research Institute", 6-7 october 1998, Sofia, 2: 196-199. (In Bulgarian with English summary). 
Georgiev G. 2000a. Parazitoidi na Paranthrene tabaniformes (Rott.) (Lepidoptera, Sesiidae) v Bulgaria [Parasitoids on Paranthrene tabaniformes (Rott.) (Lepidoptera, Sesiidae) in Bulgaria]. Annuaire de l'Université de Sofia „Kliment Ohridski“, Faculté de Biologie 92 (Zoology): 121-126. (In Bulgarian with English summary).

Georgiev G. 2000b. New and rare Tachinidae (Diptera) parasitoids of insect pests on the poplars (Populus spp.) in Bulgaria. Forest Science 1 (1): 49-56. (In Bulgarian with English summary).

Georgiev G. 2000c. Studies on larval parasitoids of Paranthrene tabaniformis (Rott.) (Lepidoptera: Sesiidae) on urban poplars (Populus spp.) in Sofia, Bulgaria. Annals of Forest Science 57: 181-186.

Georgiev G. 2001. Parasitoids of Saperda populnea (L.) (Coleoptera: Cerambycidae) on aspen (Populus tremula L.) in Bulgaria. Journal of Pest Sciemce 74: 155-158.

Georgiev G. 2004. Two new Chalcidoidea (Hymenoptera) parasitoids of the poplar twiggall fly, Hexomyza schineri (Gir.) (Diptera: Agromyzidae) in Bulgaria. Silva Balcanica 5 (2): 57-60.

Georgiev G. \& Bochev N. 1996. Bioecological features of parasitoids on the pine sawfly (Diprion pini L., Hymenoptera: Diprionidae). Forestry ideas 2 (7): 86-92. (In Bulgarian with English summary).

Georgiev G. \& Boyadzhiev P. 2002. New parasitoids of Paraphytomyza populi (Kltb.) (Diptera, Agromyzidae) in Bulgaria. Journal of Pest Sciemce 75: 69-71.

Georgiev G. \& Delkov A. 1997. Phytophagous insects and their parasitoids on poplar trees in Sofia. Acta entomologica bulgarica 3 (1-2): 61-65. (In Bulgarian with English summary).

Georgiev G. \& Hubenov Z.. 2000. Triarthria setipennis (Fal.) (Diptera: Tachinidae) - A new species for the fauna of Bulgaria. Forest Science 1 (1): 69-71. (In Bulgarian with English summary).

Georgiev G., Hubenov Z., Georgieva M., Mirchev P., Matova M., Solter L., Pilarska D. \& Pilarski P. 2012 (2013). Interactions between the introduced fungal pathogen Entomophaga maimaiga and indigenous tachinid parasitoids of gypsy moth Lymantria dispar in Bulgaria. Phytoparasitica, 41: 125-131. DOI 10.1007/ s12600-012-0269-6, ISSN 0334-2123.

Georgiev G. \& Langurov M. 1997. Megaselia quadriseta Schmitz (Diptera: Phoridae) - hiperparasitoid on Pseudosarcophaga mamillata Pandele (Diptera: Sarcophagidae) in pupae of the satin moth (Stilpnotia salicis L., Lepidoptera: Lymantridae). Forest science 1/2: 136-138. (In Bulgarian with English summary).

Georgiev G., Ljubomirov T., Raikova M., Ivanov K. \& Sakalian V. 2004. Insect inhabitants of old larval galleries of Saperda populnea (L.) (Coleoptera: Cerambycidae) in Bulgaria. Journal of Pest Sciemce 77: 235-243.

Georgiev G., Luvchiev V., Ljubomirov T., Markova E. \& Bochev N. 1998; Species of Sphecidae, Syrphidae and Muscidae dwelling larval galleries of poplar clearing moth (Paranthrene tabaniformis Rott.), (Lepidoptera, Sesiidae) in Bulgaria. Acta zoologica bulgarica 50 (1): 19-22.

Georgiev G., Mirchev P., Hubenov Z. \& Beshkov S. 2002. Pseudoperichaeta nigrolineata (Walk.) and Zenillia libatrix Panz. (Diptera: Tachinidae) - new parasitoids of Acrobasis consociella (Hbn.) (Lepidoptera: Pyralidae) in Bulgaria. Forest Science 2: 87-90.

Georgiev G., Sakalian V., Ivanov K. \& Boyadzhiev P. 2004. Insects reared from stems and branches of goat willow (Salix caprea L.) in Bulgaria. Journal of Pest Sciemce 77: 151-153. Journal of Pest Sciemce

Georgiev G. \& Stojanova A. 2003. New Chalcidoidea (Hymenoptera) parasitoids of Dasineura saliciperda (Dufour) (Diptera, Cecidomyiidae) in Bulgaria. Journal of Pest Sciemce 76: 161-162.

Georgiev G., Zaemdzhikova G. \& Topalov P. 2017. Impact of Chorebus gedanensis (Ratz.) on Hexomyza schineri (Gir.) (Diptera: Agromyzidae) at low host population density in Vitosha mountain (Bulgaria). Silva Balcanica 18 (2): 39-41.

Georgieva G., Radeva K. \& Uzunov Y. 2017. New data of botton Invertebrates of the Negovan Marshes and the adjacent Lesnovska River. Acta zoologica bulgarica 69 (1): 89-94.

Geptner V. 1936. Obshtaya zoogeografiya. Moscow, Biomedgiz. 548 p. (In Russian).

Gharali B., Evenhuis N. L. \& Almeida J. 2013. World synopsis of described species of the genus Platypygus LOEW (Diptera: Mythicomyiidae: Platypyginae). Zootaxa 3745 (2): 199-242.

Gomon G. 1940. Rakovodstvo za borba s bolestite i nepriyatelite po zelenchukovite kulturi [A guide to a fight with diseases and pests in the vegetable crops]. Plovdiv. $103 \mathrm{p}$.

Gorodkov K. B. 1984a. Ranges types of insects of tundra and forests zones of European Part of USSR. In: Provisional Atlas of the insects of the European Part of USSSR. Leningrad, Nauka, 3-20. (In Russian).

Gorodkov K. B. 1984b. Family Coelopidae. In: Soós Á. \& Papp L. (Eds.). Catalogue of Palaearctic Diptera. 9. Budapest, Akadémiai Kiadó, Hungarian Natural History Museum. pp. 151-152. 
Gorodkov K. B. 1984c. Family Heleomyzidae. In: Soós Á. \& Papp L. (Eds.). Catalogue of Palaearctic Diptera. 10. Budapest, Akadémiai Kiadó, Hungarian Natural History Museum. pp. 15-33.

Gospodinov G. 1958. Ikonomicheski po-vazhni bolesti i nepriyateli, poyavili se po selskostopanskite kulturi prez 1952 g. v Bulgaria [Economically more important diseases and pests that appeared on crops in 1952 in Bulgaria]. Byuletin po rastitelna zashtita 6 (3/11): 3-43. (In Bulgarian).

Gospodinov G. 1963. Novi vidove paraziti po nyakoi vredni nasekomi u nas [New types of parasites on some harmful insects in Bulgaria]. Rastitelna zshtita 11 (11): 10-11. (In Bulgarian).

Gospodinov G. 1968. Borba s nepriyatelite na malinata i kasisa [Fight against pests on the raspberries and blackcurrants]. Ovoshtarstvo 11: 31-36. (In Bulgarian).

Gregor F. \& Povolny D. 1959. Beitrag zur Kenntnis synantroper Fliegen Bulgariens. Prace Brnenske ČAV, 31 (7): $377-384$.

Grehan J. 1988. The natural biogeographic regions. Revista di Biologia - Biology Forum, 81 (4): 569-575.

Greve L., Jonassen T. \& Olsen T. J.. 2008. New records of Phaeomyiidae and Sciomyzidae (Diptera) in Norway. Norwegian Journal of Entomology 55: 49-52.

Grehan J. 1993. Conservation biogeography and the biodiversity crisis: a global problem in space/time. Biodiversity Letters 1: 134-140.

Grichanov I. 2010. West Palaearctic species of the genus Neurigona Rondani (Diptera: Dolichopodidae). Russian Entomological Journal 19 (3): 249-296.

Grichanov I. \& Negrobov O. 2014. Palaearctic species of the genus Sciapus Zeller (Diptera: Dolichopodidae). Plant Protection News, Supplements 13: 4-84; http://www.vestnik.iczr.ru/

Grigoriev V. 1922. Bolesti i vrediteli po zeleto nablyudavani v okolnostite na Sofia prez proletta na 1922 godina [Diseases and pests on the cabbage observed in the vicinity of Sofia in the spring of 1922]. Zemedelie 26 (9): 136-139. (In Bulgarian).

Grigorov S. 1959. Prouchvane biologiyata na zhitnite darvenitsi ot rod Eurygaster Lap. i rod Aelia F. v Bulgaria i borbata s tyah [Study of the biology of wheat bedbugs of the genus Eurygaster Lap. and the genus Aelia F. in Bulgaria and the fight against them]. Nauchni trudove na SA "G. Dimitrov", 6: 339-363. (In Bulgarian).

Grigorov S. 1962. Prinos kam entomofaunata na Bulgariya [Contribution to the entomofauna of Bulgaria]. Rastitelna zashtita 10 (1): 48-54. (In Bulgarian).

Grigorov S. 1964. Prouchvane na vredata ot zhitnata stablena muha v Sofiysko [Study of the damage from the wheat stalk fly in the Sofia region]. Rastenievadni nauki 1 (5): 145-152. (In Bulgarian).

Grigorov S. 1972. Spetsialna entomologiya [Special entomology]. Sofia, Zemizdat. 592 p. (In Bulgarian).

Grigorov S. 1976. Spetsialna entomologiya [Special entomology]. Sofia, Zemizdat. 545 p. (In Bulgarian).

Grigorov S. 1982. Listnite vashki po kulturnite rasteniya i borbata s tyah [Aphids on the cultivated plants and their control]. DSc thesis, Plovdiv, VSI. 535 p. (In Bulgarian).

Gruev B. 1968. Ein neuer Dipterenparasit Macquartia flavipes (Meigen,1824) in der Larve Colaphellus sophiae Schall. (Coleoptera, Chrysomelidae). Mitteilungen der Deutschen Entomologischen Gesellschaft 5/6, p. 54.

Gruev B. 1969. Beitrag zur Forschung der Familie Chrysomelidae (Col.) in Bulgarien in Bezug auf die Dipterenparasiten. Mitteilungen der Deutschen Entomologischen Gesellschaft 28 (5/6: p. 54.

Gruev B. 1972. Dipterni paraziti po nyakoi vidove listoyadi u nas [Diptera parasites on some species of the deciduous trees in Bulgaria]. Rastitelna zashtita 9: 10-11. (In Bulgarian).

Gruev B. 1973. Untersuchungen über einige Blattkäfer (Col., Chrysomelidae) in Bulgarien zur Bestimmung von Dipterenparasiten. Travaux Scientifiques Universite de Plovdiv, Biologie - Animalia 11 (5): 149-151.

Guéorguiev V. \& Beron P. 1962. Essai sur la faune cavernicole de Bulgarie. Annales de Spéléologie 17 (2/3): 285-411.

Gulička J. 1966. Die Blepharoceriden (Diptera) Mittel-und Südeuropas und des Kaukasus. Ac. rer. natur. Mus. nat. Slov. (Bratislava) 12 (2): 45-150.

Gunderina L., Kiknadze I., Michailova P. \& Krastanov B. 2008. Variation and divergence of genomic DNA in Chironomus plumosus L. and Ch. balatonicus Dévai, Wülker et Scholl (Diptera, Chironomidae) of natural populations in Russia and Bulgaria. Euroasian Entomological Journal 7 (2): 167-174. (In Russian with English summary).

Harizanov A. 1964. Krushova listna muha [Pear leaf fly]. Ovoshtarstvo 6: (In Bulgarian).

Harizanov A., Angelova R. \& Babrikova T. 1996. Entomology. Sofia, Zemizdat. 416 p. (In Bulgarian). 
Harizanov A. \& Harizanova V. 1997. Extend of parasitization of the eggs and adults of Eurygaster integriceps Put. (Hemiptera: Scutelleridae) in non-sprayed fields. Acta Entomologica Bulgarica 3 (1-2): 104-108. (In Bulgarian with English summary).

Harizanova V. \& Pavlov A. 1999. Degree of damage caused by Liriomyza huidobrensis Blanchard (Agromyzidae) on different glasshouse crops and cultivars. Acta Entomologica Bulgarica 5 (2, 3, 4): 18-22.

Hazelton M. 1970. Fauna from some caves in Bulgaria and one in Yugoslavia. The Transactions of the Cave Research Group of Great Britain, Ledbury 12 (1): 33-37.

Hendel F. 1934. Revision der Tethiniden (Dipt. Muscid. Acal.). Tijdschrift vor Entomologie 77: 37-54.

Hennig W. 1938. Braulidae. In: Lindner E. (Ed.): Die Fliegen der paläarktischen Region. 60c (Lieferung 122). Stuttgart, Schweizerbartsche Verlagsbuchhandlung, $14 \mathrm{p}$.

Hennig W. 191961. Muscidae. In: Lindner E. (Ed.): Die Fliegen der paläarktischen Region. 63b (Lieferung 215, 218). Stuttgart, Schweizerbartsche Verlagsbuchhandlung, 481-576.

Hering M. 1957. Die Larven der Agromysiden (Diptera) III. Tijdschrift voor entomologie 100: 73-94.

Herting B. 1984. Catalogue of Palearctic Tachinidae (Diptera). Stuttgarter Beiträge zur Naturkunde, A: 1-228

Herting B. \& Dely-Draskovits A. 1993. Family Tachinidae. In: Soós Á. \& Papp L. (eds.). Catalogue of Palearctic Diptera. 13. Amsterdam, Elsevier, 118-624.

Hitilov I. 1912a. Shvedskata i hesenskata muhi [The Swedish and Hessian flies]. Zemedelie 18 (1): 3-5. (In Bulgarian).

Hitilov I. 1912b. Rolyata na navremennoto zaoravane na starnishteto za unishtozhavane na nepriyatelite na zhitnite rasteniya [The role of the ploughing on time of the stubble for destroying of the pests on the cereal plants]. Zemedelie 18 (11/12): 171-173. (In Bulgarian).

Hollmann-Schirrmacher V. 1998. Phylogeny of the subfamily Ilytheinae (Diptera, Ephydridae) with special reference to the genus Philygria. Studia Dipterologica, Supplement 5: 1-144.

Holt B., Lessard J.-P., Borregaard M., Fritz S., Araújo M., Dimitrov D., Fabre P.-H., Graham C., Graves G., Jønsson K., Nogués-Bravo D., Wang Z., Whittaker R., Fjeldså J. \& Rahbek C. 2013. An ubdate of Wallace’s zoogeographic regions of the world. Science 339: 74-78.

Hradský M. \& Moucha J. 1964. Raubfliegen (Diptera, Asilidae) Bulgariens. Acta faunistica entomologica Musei nationalis Pragae 10 (89): 23-30.

Hradský M. \& Moucha J. 1967. Raubfliegen-Fauna (Diptera, Asilidae) von Bulgarien. Fragmenta Faunistica 13 (18): 315-323.

Hristov A. 1948. Borba s bolestite i nepriyatelite po ovoshtnite darveta [Fight with diseases and pests on the fruit trees]. Sofia, Zemsnab. 211 p.

Hristov H., Krusteva H. \& Nikolova G. 2004. Susceptibility of spring malting barley varieties to frit fly Oscinella frit L. (Diptera: Chloropidae). Acta Entomologica Bulgarica 10 (1): 15-22. (In Bulgarian with English summary).

Hristova E. 1959. Lyutsernov tsvetov komar (Contarinia medicaginis Kieff.) i borbata s nego [Alfalfa blossom mosquito (Contarinia medicaginis Kieff.) and the fight against it]. Nauchni trudove na nauchno-izsledovatelskiya institut po furazhite v Pleven 1: 55-57. (In Bulgarian).

Hristova K. 1954. Lyutsernov tsvetov komar - Contarinia medicaginis i borbata s nego [Alfalfa blossom mosquito - Contarinia medicaginis and the fight against it]. Byuletin po rastitelna zashtita 3 (1): 67-69. (In Bulgarian).

Hubenov Z. 1977. Ein Beitrag zu der Fauna fon Tachinidae (Diptera) in Bulgarien. Acta zoologica bulgarica 8: 50-53. (In Bulgarian with German summary).

Hubenov Z. 1980a. Tachinid species Tachinidae (Diptera), new for the fauna of Bulgaria. Acta zoologica bulgarica 14: 79-82.

Hubenov Z. 1980b. A contribution to the studies on the fauna and biology of the species of the family Tachinidae (Diptera) in Bulgaria. Acta zoologica bulgarica 15: 77-80. (In Bulgarian with English summary).

Hubenov Z. 1982a. New Tachinidae species (Diptera) to the Bulgarian fauna from the Blagoevgrad District. Acta zoologica bulgarica 19: 84-87. (In Bulgarian with English summary).

Hubenov Z. 1982b. Morphologische Untersuchungen über den Artenkomplex Phasia crassipennis F. Entomologische Abhandlungen Staatliches Museum für Tierkunde Dresden 45 (5): 91-98.

Hubenov Z. 1982c. Eine neue Phasia-Art aus Bulgarien (Diptera, Tachinidae). Reichenbachia 20 (19): 163-166.

Hubenov Z. 1983a. A contribution to the studies on family Tachinidae (Diptera). Acta zoologica bulgarica 23: 57-61. (In Bulgarian with English summary). 
Hubenov Z. 1983b. Studies on the significance of some species of family Tachinidae (Diptera) for limiting the number of harmful insects of genus Eurygaster (Heteroptera, Scutelleridae). Ecology 11: 84-91. (In Bulgarian with English summary).

Hubenov Z. 1985a. On hosts of family Tachinidae (Diptera) species in Bulgaria. Acta zoologica bulgarica 27: 27-35. (In Bulgarian with English summary).

Hubenov Z. 1985b. Faunistichni, zoogeografski i ekologichni izsledvaniya varhu semeystvo Tachinidae (Diptera) ot Sandansko-Petrichkata kotlovina [Faunistic, zoogeographical and ecological studies on the family Tachinidae (Diptera) from the Sandanski-Petrich Valley]. Ph.D. thesis, Sofia, Bulgarian Academy of Sciences. 163 p. (In Bulgarian).

Hubenov Z. 1988a. Insects from the family Tachinidae (Diptera) in Submediterranean biotopes from Southwestern Bulgaria. II. Phenology and activity of the imaginal forms from the Sandanski-Petrič Valley. In: Fauna of Southwestern Bulgaria. 2. Sofia, Bulgarian Academy of Sciences, 30-50. (In Bulgarian with English summary).

Hubenov Z. 1988b. Insects from the family Tachinidae (Diptera) in Submediterranean biotopes from Southwestern Bulgaria. III. Distribution for stations, trophic relations and frequency of the species in the SandanskiPetrič Valley. In: Fauna of Southwestern Bulgaria. 2. Sofia, Bulgarian Academy of Sciences, 51-73. (In Bulgarian with English summary).

Hubenov Z. 1988c. Species composition and zoogeographical characteristics of the family Tachinidae (Diptera) from the Slavjanka Mountain. Acta zoologica bulgarica, 36: 17-30. (In Bulgarian with English summary).

Hubenov Z. 1990. Tachina flies (Insecta, Diptera, Tachinidae) from Vitoša. In: Fauna of Southwestern Bulgaria. 3. Sofia, Bulgarian Academy of Sciences, 161-166. (In Bulgarian with English summary).

Hubenov Z. 1992a. Artenbestand, Höhenferbreitung und zoogeographische Charakteristik der Familie Tachinidae (Diptera) aus dem Pirin-gebirge. Acta zoologica bulgarica 44: 3-18.

Hubenov Z. 1992b. Systematische Liste der bulgarischen Raupenfliegen (Diptera, Tachinidae). Acta zoologica bulgarica 45: 63-71.

Hubenov Z. 1993a. Übersicht der in Bulgarien festgestellten Arten der Familie Tachinidae (Diptera). Annuaire de l'Université de Sofia „Kliment Ohridski“, Faculté de Biologie 82 (Zoology): 147-162.

Hubenov Z. 1993b. Höhenverbreitung der Familie Tachinidae (Diptera) in Bulgarien. Acta zoologica bulgarica 46: $24-38$.

Hubenov Z. 1993c. Zoogeographical characteristics of Tachinidae (Diptera) with Mediterranean type of distribution in Bulgaria. In: Second national scientific conference of entomology. Sofia, 24-27. (In Bulgarian with English summary).

Hubenov Z. 1995a. Artenbestand, Vertikalverbreitung und zoogeographische Characteristik der Familie Tachinidae (Diptera) aus dem Belasiza-Gebirge. Acta zoologica bulgarica 48: 48-61.

Hubenov Z. 1995b. The insects from the family Tachinidae (Diptera) of the damp zones around the ShablaEzerets Lake complex. In: Third national scientific conference of entomology. Sofia, 31-34. (In Bulgarian with English summary).

Hubenov Z. 1996a. Zoogeographische Charakteristik der bulgarischen Raupenfliegen (Diptera, Tachinidae). Historia naturalis bulgarica, 6: 49-58.

Hubenov Z. 1996b. A contribution to the Tachinidae fauna (Diptera: Tachinidae) of the woodless zone of the National Park Central Balkan. Acta Entomologica Bulgarica, 2 (2): 12-15. (In Bulgarian with English summary).

Hubenov Z. 1997. Possibilities for using of a system from the really defined natural territories for the faunistic researches in Bulgaria. Acta zoologica bulgarica 49: 5-9.

Hubenov Z. 1999. Species composition and zoogeographical characteristic of Tachinidae (Diptera) in the Kresna gorge (South-Western Bulgaria). Acta zoologica bulgarica 51 (1): 15-20.

Hubenov Z. 2001a. Tachimidae (Diptera) from Kresna Gorge (SW Bulgaria). In: Beron P. (Ed.). Biodiversity of Kresna Gorge. Sofia, National Museum of Natural History, 211-224. (In Bulgarian with English summary).

Hubenov Z. 2001b. Addition to the list of hosts of the Bulgarian Tachinidae (Diptera). Acta Entomologica Bulgarica, 7 (3/4): 51-56. (In Bulgarian with English summary).

Hubenov Z. 2004. Tachinidae (Diptera) from the Eastern Rhodopes (Bulgaria). In: Beron P., A. Popov (Eds.). Biodiversity of Bulgaria. 2. Biodiversity of Eastern Rhodopes (Bulgarian and Greece). Sofia, National Museum of Natural History \& Pensoft, 769-775. 
Hubenov Z. 2006. Tachinidae (Insecta: Diptera) from the Western Rhodopes (Bulgaria). In: Beron P. (Ed). Biodiversity of Bulgaria. 3. Biodiversity of Western Rhodopes (Bulgaria and Greece) I. Sofia, National Museum of Natural History \& Pensoft, 689-697.

Hubenov Z. 2008a. Composition and zoogeographical characteristics of the family Tachinidae (Insecta: Diptera) in the Balkan countries. Acta zoologica bulgarica 60 (3): 243-265.

Hubenov Z. 2008b. Composition and zoogeographical characteristics of the family Tachinidae (Diptera: Insecta) in Serbia and Bulgaria. - Advances in Arachnology and Developmental Biology. Papers dedicated to Prof. Dr. Božidar Ćurčić. S. E. Makarov \& R. N. Dimitrijević (Eds.). Inst. Zool., Belgrade; BAS, Sofia; Fac. Life Sci., Viena; SASA, Belgrade \& UNESCO MAB Committee, Serbia. Viena-Belgrade-Sofia, Monographs, 12: 375-394.

Hubenov Z. 2015a. Areographical structure of the Bulgarian non-marine invertebrate fauna (Metazoa: Invertebrata). Acta zoologica bulgarica 67 (2): 203-213.

Hubenov Z. 2015b. Two-winged insects (Insecta: Diptera) of Pirin Mountain. Historia naturalis bulgarica 21: 215-256.

Hubenov Z. 2015c. Comparative zoogeographical review of the tachinid fauna (Diptera: Tachinidae) of the Belasitsa and Slavyanka mountains. Acta zoologica bulgarica 67 (3): 351-364.

Hubenov Z. 2015d. Species composition of the free living multucellular invertebrate animals (Metazoa: Invertebrata) from the Bulgarian sector of the Black Sea and the coastal brackish basins. Historia naturalis bulgarica 21: 49-168.

Hubenov Z. 2016. The Dipterans (Insecta: Diptera) of the Rila Mountains. Historia naturalis bulgarica 23: 37-99. Hubenov Z. 2017. Vertical distribution and comparative zoogeographical characteristic of dipteran fauna (Insecta: Diptera) according to the vegetation belts of the Pirin and Rila Mountains. Historia naturalis bulgarica 24: 61-119.

Hubenov Z. 2018. The Dipterans (Insecta: Diptera) of the Vitosha Mountains. Historia naturalis bulgarica 26: 1-66. Hubenov Z. 2019a. The Dipterans (Insecta: Diptera) of the Vrachanska Planina Mountains. In: Bechev D. \& Georgiev D. (Eds.). Faunistic diversity of Vrachanski Balkan Nature Park. Part 2. ZooNotes, Supplement 7: $107-165$.

Hubenov Z. 2019b. Distribution and Comparative Characteristics of the Dipteran Fauna (Insecta: Diptera) of the Vrachanska Planina, Vitosha, Rila and Pirin Mountains, Bulgaria. Acta zoologica bulgarica 71 (4): 2019: 501-518.

Hubenov Z. 2021. Review of the Nematocera families (Insecta: Diptera) of Bulgaria. Acta zoologica bulgarica 73

Hubenov Z., Beschovski V., Beshkow S., Kolarov J., Kumanski K., Popov A. \& Vassileva E. 1993. Insecta. 2. Blattodea, Mantodea, Isoptera, Orthoptera, Dermaptera, Embioptera, Megaloptera, Raphidioptera, Neuroptera, Mecoptera, Hymenoptera, Trichoptera, Lepidoptera, Diptera. In: Sakalian M. (Ed.). Bulgaria’s biological diversity: Conservation status and Needs Assessment. 1. Sofia, Pensoft, 323-404. (In Bulgarian with English summary).

Hubenov Z., Beschovski V., Beshkow S., Kolarov J., Kumanski K., Popov A. \& Vassileva E. 1998. Insects of Bulgaria, Part 2: Blattodea, Mantodea, Isoptera, Orthoptera, Dermaptera, Embioptera, Megaloptera, Raphidioptera, Neuroptera, Mecoptera, Hymenoptera, Trichoptera, Lepidoptera and Diptera. In: Meine C. (Ed.). Bulgaria’s biological diversity: Conservation status and Needs Assessment. 1-2. Washington-Sofia, Pensoft, 211-318.

Hubenov Z., Beshovski V., Josifov M., Popov A., Kumanski K., Sakalian V., Abadjiev S., Vidinova Y. \& Lyubomirov T. 1999a. Entomofaunistic diversity of the Central Balkan National Park. In: Biological diversity of the Central Balkan National Park, Sofia Pensoft, 333-377, 511-532, 558-606. (In Bulgarian with English summary).

Hubenov Z., V. Beshovski, M. Josifov, A. Popov, K. Kumanski, V. Sakalian, S. Abadjiev, Y. Vidinova, T. Lyubomirov. 1999b. Entomofaunistic diversity of the Rila National Park. In: Biological diversity of the Rila National Park, Sofia Pensoft, 307-353, 461-496, 557-651. (In Bulgarian with English summary).

Hubenov Z., Beshovski V., Josifov M., Popov A., Kumanski K., Sakalian V., Abadjiev S., Vidinova Y. \& Lyubomirov T. 2000a. Entomofaunistic diversity of the Central Balkan National Park. In: Biological diversity of the Central Balkan National Park, Sofia Pensoft, 319-362, 491-512, 538-586.

Hubenov Z., V. Beshovski, M. Josifov, A. Popov, K. Kumanski, V. Sakalian, S. Abadjiev, Y. Vidinova, T. Lyubomirov. 2000b. Entomofaunistic diversity of the Rila National Park. In: Biological diversity of the Rila National Park, Sofia Pensoft, 285-331, 429-464, 525-619. 
Hubenov Z. \& Georgiev G. 1996. Phytomyptera nigrina (Meig.) (Diptera, Tachinidae) - new parasitoid on Poplar clearwing moth (Paranthrene tabaniformes Rott.) (Lepidoptera, Sesiidae). Forest Science 4: 87-89. (In Bulgarian with English summary).

Hubenov Z., Georgiev G., Mirchev P. \& Naidenov J. 2001. Acanthocynus griseus (F.) (Coleoptera: Cerambycidae) - a new host of Billaea triangulifera (Zett.) (Diptera: Tachinidae) in Bulgaria. Forest Science, 1/2: 87-89. (In Bulgarian with English summary).

Hůrka K. 1958. Prispèvek k faune muchuli Bulharska (Nycteribidae, Diptera). Zoologickè Listy, Brno 7 (3): 231-234.

Hůrka K. 1962. Beitrag zur Nycteribien - und Streblidenfauna Albanies nebst bemerkungen zur Fauna von Bulgarien, Ungarn und UdSSR. Acta Societatis Entomologicae Cechoslovakiae, Praha, 59 (2): 156-164.

Hůrka K. 1972. Basilia mongolensis nudior subsp. n. nebst Bemerkungen zur Nycteribiden- und Streblidenfauna Thrakiens (Diptera: Pupipara). Annalen des Naturhistorischen Museums in Wien 76: 709-713.

Hůrka K. 1984. New taxa and new records of Palaearctic Nicteribiidae and Streblidae (Diptera: Pupipara). Věstník Československé společnosti zoologické 48: 90-101.

Ihtimanska M., Ilkova J. \& Michailova P. 2018. Biodiversity of family Chironomidae (Diptera) in Srebarna Lake (North-East Bulgaria) and genome instability of some species from genus Chironomus Meigen, 1803. Ecologica Balkanica 10 (2): 41-53.

Ilchev D. 1923. Siviyat chervey kato nepriyatel na lozyata [The gray worm as an pest on the vineyards]. Svedeniya po zemedelieto 4 (2): 14-27. (In Bulgarian).

Ilkova J., Hankeln T., Schmidt E., Michailova P., Petrova N., Sella G. \& White K. 2007. Genome instability of Chironomus riparius Mg. and Chironomus piger Strenzke (Diptera, Chironomidae). Caryologia 60 (4): 299-308.

Ilkova J., Michailova P., Thomas A. \& White K. 2014. Genome instability of Chironomus riparius Mg. (Diptera, Chironomidae) from polluted water basins in Bulgaria. Ekologia Balkanica 5: 1-8.

Islam S., Uzunov Y. \& Kovachev S. 1986. Composition and distribution of the macrozoobenthos of the river Struma. Hydrobiology 28: 15-35. (In Bulgarian with English summary).

Ipatov F. 1921. Vrednite nasekomi v selskoto stopanstvo [Harmful insects in the agriculture]. Selskostopanska biblioteka 23 p. (In Bulgarian).

Ivanov S. 1981. Esenno-zimni grizhi za traynite nasazhdeniya [Autumn-winter care for plantings]. Rastitelna zashtita 9: 41-44. (In Bulgarian).

Ivanov S. \& Savov N. 1982. Po-vazhni paraziti po listozavivachkite v Plovdivski okrag [More important parasites on the leafhoppers in the Plovdiv district]. III Natsionalna nauchna konferentsiya po biologichna borba s vreditelite po rasteniyata, 25-30. X.1982, Sofia, 15-16. (In Bulgarian).

Ivanova T., Stoev P. \& Petrov B. 1995. Brachytarsina flavipes Macquart, 1851 (Diptera, Streblidae) member of a new family for the Bulgarian fauna. Historia naturalis bulgarica 5: 35-36.

Jacentkovsky D. 1936. Beitrag zur Kentnis der Raupenfliegen (Tachinariae, Diptera) Bulgariens. Mitteilungen aus dem Konigl. Naturwissenschaftlichen Instituten in Sofia 9: 109-134.

Jacentkovsky D. 1937. Př́spěvek k studiu biologické obrany proti lesním škůdcům. Sborník vysoké Školy zemĕdĕlské v Brnĕ, ČSR., Fakulta Lesnická, D24, 54 p.

Jacentkovsky D. 1938. Sur deux Tachinaires nouvelles de la subfam. Phasiinae (Dipt.). Acta societatis scientiarum naturalium Moravicae 11 (5), F 105: 1-5.

Jacentkovsky D. 1939. Uber einige interessante Sarcophagiden (Tachinariae, Diptera) aus Bulgarien. Mitteilungen aus den Königlichen Naturwissenschaftlichen Instituten in Sofia 12: 188-192.

Janeva I. 1987. The zoobenthos of the river Vit. I. Composition, structure and dynamics of the zoocoenoses. Hydrobiology 31: 37-64. (In Bulgarian with English summary).

Janeva I. 1989. Changes in the saprobiological state of the Černa river and their effect on the composition and structure of the benthic organisms. Hydrobiology 34: 20-29. (In Bulgarian with English summary).

Janeva I. 1991. Saprobiological state of the river Ogosta in various periods of study. Hydrobiology 36: 32-48. (In Bulgarian with English summary).

Janeva I. \& Bancheva N. 2002. Ecological characteristics of the Topolnitsa River. Acta zoologtica bulgarica 54 (3): 83-108.

Janeva I. \& Russev B. 1985. Trends in changes of the hydrobiological and saprobiological state of the Tundža River. II. May-November, 1981. Hydrobiology 26: 15-36. (In Bulgarian with English summary). 
Janeva I. \& Russev B. 1989. Saprobiological state of the Isk冈r river in the initial years following the putting into operation of the Sofia purifyng station. Hydrobiology 34: 3-19. (In Bulgarian with English summary)

Janeva I. \& Russev B. 1997. Veränderungen der Artenzusammensetzung und Güteklasse des bulgarischen Donauzuflusses Jantra nach dem Makrozoobenthon. Lauterbornia 31: 1-16.

Janeva I., Vidinova Y. \& Tyufekchieva V. 2001. Contemporary saprobiological characteristics of Arda River in the section of future "Gorna Arda" cascade building. Acta zoologica bulgarica 53 (2): 37-46.

Jelesova M. 1971. Asilidae (Diptera) aus der Westlichen Stara Planina. Bulletin de l'Institut de zoologique et Musée, 33: 71-74. (in Bulgarian with German summary).

Jelesova M. 1974a. Die Asiliden (Diptera) in dem Mittleren und dem Östlichen Balkangebirge. Bulletin de l'Institut de zoologique et Musée, 41: 199-206. (in Bulgarian with German summary).

Jelesova M. 1974b. Eine neue Raubfliegen aus Bulgarien (Diptera, Asilidae). Reichenbachia 15 (16): 105-107.

Jelesova M. 1975. Raubfliegen (Diptera: Asilidae) aus den Rhodopen. In: La faune des Rhodopes. Materiaux. Sofia, Bulgarian Academy of Sciences. pp. 197-204. (In Bulgarian with German summary).

Ježek J. 1990a. Redescriptions of nine common Palaearctic and Holarctic species of Psychodini end. (Diptera: Psychodidae). Acta Entomologica Musei Nationalis Pragae 43: 33-83.

Ježek J. 1990b. Descriptions of new Sycoracine and Trichomyine moth flies (Diptera, Psychodidae) from the Palaearctic region. Acta Entomologica Musei Nationalis Pragae 43: 203-214.

Ježek J. 2004. New faunistic data of non-phlebotomine moth flies (Diptera, Psychodidae) from the Palaearctic region. In: Kubík Š. \& Barták M. (Eds.). Dipterologica Bohemoslovaca. Folia Facultatis Scientiarum Naturalium Universitatis Masarykianae Brunensis, Biologia 11: 141-151.

Ježek J., Chvojka P., Manko P. \& Oboňa J. 2017. Faunistic and bibliographical inventory of moth flies from Ukraine (Diptera, Psychodidae). ZooKeys 693: 109-128.

Ježek J. \& Goutner V. 1995. Psychodidae (Diptera) of Greece. Acta Musei Nationalis Pragae, B, Historia Naturalis 50 (1-4): 107-124.

Ježek J., Manko P. \& Oboňa J. 2018. Checklist of known moth flies and sand flies (Diptera, Psychodidae) from Armenia and Azerbaijan. ZooKeys 798: 109-133.

Ježek J., Manko P. \& Oboňa J. 2020. Synopsis of the Psychodidae (Diptera) fauna of Bulgaria. Zootaxa 4877 (2): 201-240. doi: https://doi.org/10.11646/zootaxa.4877.2.1

Ježek J. \& Oboňa J. 2019. Pneumia mladeni sp. nov. (Diptera: Psychodidae: Psychodinae) from Bulgaria. Klapalekiana, 55: 199-205.

Ježek J. \& Oboňa J., Přikryl I. \& Mikátová B. 2019. Moth flies (Diptera: Psychodidae) of the western Hercynian mountains, Sokolov open-cast coal mines and dumps (Czech Republic). Acta Mus. Siles. Sci. Natur. 67: 193-292. DOI: 10.2478/cszma-2018-0015.

Joakimoff D. 1899. Prinos kam faunata ot nasekomi na Rila planina [Contribution to the insect fauna of the Rila Mountain]. Periodichesko spisanie na Balgarskoto knizhovno druzhestvo (Periodical Journal of the Bulgarian Literary Society), 60: 858-884. (In Bulgarian).

Joakimoff D. 1933. Muhite Bombyliini i Anthracini [Bombyliini and Anthracini flies]. Priroda 34 (4): c. 7. (In Bulgarian).

Josifov M. 1957. Tierarten und Pflanzen, die auf den Namen des Herrn Akademiken Bureš benannt sind. Bulletin de l'Institut zoologique de l'Academie des sciences de Bulgarie 6: 107-142. (In Bulgarian).

Joost W. 1978. Beitrag zur Dunkelmucken-Fauna Bulgariens (Diptera, Thaumaleidae). Entomologische Nachrichten, 22 (7-8): 118-121.

Joost W. 1982. Beitrag zur Kenntnis der Hemerodromiinae Bulgariens (Insecta, Diptera, Empididae). Faunistische Abhandlungen Staatliches Museum für Tierkunde Dresden 9: 121-124.

Jovčev E. \& Lavčiev V. 1972. Über die epizootologische Bedeutung einiger synboviner koprophiler Fliegenarten. Monatshefte für Veterinärmedizin 24: 932-934.

Kalaydzhiev L., Bozhkov D., Stefanov S. \& Mihaylova L. 1960. Varhu izpolzvaneto na komarnite larvi kato hrana na mladi dagovi pastarvi [On the use of the mosquito larvae as a food for young rainbow trouts]. Izvestiya na instituta po fiziologiya 4: 235-239. (In Bulgarian).

Kameneva E. P. 2007. Anew species of Herina (Diptera, Ulidiidae) from Switzerland, with a key to european species and notes on nomenclature and distribution. Vestnik zoologii 41 (5): 405-421.

Kanavalová L., Kubík Š. \& Barták M. 2018. Empidinae (Diptera, Empididae) from Bulgaria with new records and descriptions of three new spwcies. ZooKeys 785: 145-161. 
Kanavalová L., Kubík Š. \& Barták M. 2020. Two new West Palaearctic species of Atelestus Walker (Diptera, Atelestidae) and new distributional records of the family. ZooKeys 955: 147-158.

Kantardzhieva-Minkova S. 1957. Wissenschaftliche Arbeiten von ausländischen Gelehrten, die auf Grund des von Herrn Akademiker Bureš gesammelten zoologischen Materials gearbeitet wurden. Bulletin de l'Institut zoologique de l'Academie des sciences de Bulgarie 6: 143-173. (In Bulgarian).

Karadjova O. \& Beschovski V. 1996. Neveroyatnite vazmozhnosti na edin nov za stranata vid listominirashta muha Liriomyza huidobrensis [The incredible possibilities of a new species for the country - leaf-mining fly Liriomyza huidobrensis]. Rastitelna zashtita 6: 20-21. (In Bulgarian)

Karadjova O., Ilieva Zh., Petrova E. \& Laginova M. 2016. Predicted and actual distribution of the invasive species Drosophila suzukii (Diptera: Drosophilidae) in Bulgaria. Agricultural sciences 8 (19): 45-52.

Karamann Z. 1939. Beitrag zur Kenntnis der Nycteribien. Annales Musei Serbiae Meridionalis 1 (3) : 31-44.

Karnožitzky N. 1949. Nashite solenolyubivi (halobiontni) nasekomi [Our salt-loving (halobiont) insects]. Priroda 1 (6): 63-68. (In Bulgarian).

Karnožitzky N. 1957. Die Massenvermehrungen des Schwammspinners im Gebiet um Varna und möglichkeiten der biologischen Bekämpfung. Proceedings of the Institute of Zoology 4: 3-102. (In Bulgarian with German summary).

Katbeh-Bader A. \& Arabyat S. 2004. The bee flies (Diptera: Bombyliidae) of Jordan. Denisia 14, zugleich Kataloge der OÖ. Landesmuseen Neue Serie 2: 353-384

Kaytazov A. 1971a. Paraziti po zhitnite darvenitsi [Wheat bedbug parasites]. Rastitelna zashtita 1: 15-16. (In bBulgarian).

Kaytazov A. 1971b. Muha tahina, poseshtavashta larvite na koloradskiya brambar [A Tachinidae fly visiting the larvae of the Colorado potato beetle]. Rastitelna zashtita 8: 19-20. (In Bulgarian).

Kechev M. 2005. Check-list of Dolichopodidae (Diptera, Brachycera) species in Bulgaria. Animalia 41: 47-62.

Kechev M. 2006. Dolichopodidae (Diptera: Brachycera) of the Western Rhodopes (Bulgaria). In: Beron P. (ed.). Biodiversity of Bulgaria. 3. Biodiversity of Western Rhodopes (Bulgaria and Greece) I. Sofia, Pensoft \& National Museum of Natural History. pp. 613-616.

Kechev M. 2007a. The Dolichopodidae fauna (Insecta: Diptera: Dolichopodidae) of Western Rhodopes. Ph.D. thesis, Sofia, Bulgarian Academy of Sciences. 124 p. (In Bulgarian).

Kechev M. 2007b. New long-legged flies (Diptera: Brachycera: Dolichopodidae) to the fauna of Bulgaria collected in the West Rhodopes. Acta zoologica bulgarica 59 (1): 105-108.

Kechev M. 2010. New Dolichopodid flies (Diptera, Dolichopodidae) to the fauna of Bulgaria collected in the West Rhodopes (Bulgaria). Distribution and habitat preferences. International Lournal of Dipterological Research 21 (3): 179-182.

Kechev M. 2011a. Two new dolichopodid flies (Diptera, Dolichopodidae) to the fauna of Bulgaria. Ecological notes to the species of genus Poecilobothrus known for the country. In: International Scientific and Applied Conference "Opportunites and problems for alternative forms of tourism development in small villages", 14-16 march 2011, Plovdiv, Bulgaria. pp. 293-297.

Kechev M. 2011b. An improved chek-list of Dolichopodidae (Diptera, Brachycera) to the fauna of Bulgaria. In: Negrobov O., Golub V., Gaponov S. \& Logvinovskii V. (Eds.). Current problems of entomology, Voronezh State University. pp. 73-78.

Kechev M. 2012a. Diversity of long-legged flies (Diptera: Dolichopodidae) in Gradina floodplain forest, Bulgaria. Acta zoologica bulgarica, Suppl. 4: 43-46.

Kechev M. 2012b. Long-legged flies (Diptera: Dolichopodidae) from the Upper Thracian Plain new to Bulgaria: habitats and distribution. Acta zoologica bulgarica 64 (2): 205-208.

Kechev M. 2012c. Dolichopodid flies (Diptera: Dolichopodidae) from the Upper Thracian Plain. Ecology. New Knowledge Journal of Science 1 (1): 46-48. (In Bulgarian).

Kechev M. 2014. Long-legged flies (Diptera: Brachycera: Dolichopodidae) from the middle course of the Maritsa River and some its tributaries in Bulgaria. New Knowledge Journal of Science 3 (4): 50-56.

Kechev M. 2015. Vidovo raznoobrazie i ekologiya na hishtni dolihopodidni muhi (Diptera: Brachycera: Dolichopodidae) v Bulgaria [Species diversity and ecology of the predatory dolichopod flies (Diptera: Brachycera: Dolichopodidae) in Bulgaria]. Plovdiv. 119 p. (In Bulgarian).

Kechev M. 2016. Forestry and riparian long-legged flies (Diptera: Dolichopodidae) from Sarnena Sredna Gora Mountain with new records from Bulgaria. Silva Balcanica 17 (2): 45-55. 
Kechev M. 2017. Predatory long-legged flies (Diptera, Empidoidea, Dolichopodidae) from the Yantra River and „Sinite Kamani” Natural Park, Bulgaria. New Knowledge Journal of Science 6 (4): 115-118.

Kechev M. 2020. Forest predatory flies of the family Dolichopodidae (Diptera: Empidoidea) from the Upper Thracian Plain and Sarnena Sredna Gora Montain. Forest Science, Special Issue, 99-102.

Kechev M. 2021a. Diversity of long-legged flies (Diptera, Dolichopodidae) of the Balkan Mountains (Bulgaria and Serbia). Historia naturalis bulgarica 42: 15-24.

Kechev M. 2021b. Data on the distribution of Dolichopodidae (Diptera: Empidoidea) in Bulgaria, with first records for the coutry. Historia naturalis bulgarica 42: 95-102.

Kechev M. \& Glogov P. 2021. Forest Long-legged flies (Diptera: Dolichopodidae) from the park of the Forest Research Institute with new records for Bulgaria and notes on habitat affinity. Silva Balcanica 22 (1): 67-75.

Kechev M. \& Ivanova M. 2015. New records of Dolichopodidae (Diptera) from Bulgaria. Comparison of Dolichopodid diversity on river banks and in some forests of the Upper Thracian plain. Silva Balcanica 16 (1): 87-104.

Kechev M. \& Langourov M. 2019. New records of lance flies (Diptera: Lonchaeidae) to Bulgaria and Montenegro with notes on the distribution of the family Lonchaeidae for the Balkan Peninsula. Silva Balcanica 20 (1): 85-88.

Kechev M. \& Negrobov O. 2015. A new species of the genus Hercostomus Loew, 1857 (Diptera: Dolichopodidae) from Bulgaria. Acta zoologica bulgarica 67 (2): 199-202.

Kechev M., Negrobov O. \& Grichanov I. 1914. Diversity of long-legged flies (Diptera: Dolichopodidae) along the banks of the Omurovska River (Bulgaria), with the description of a new species of the genus Teuchophorus Loew. Acta zoologica bulgarica 66 (3): 317-323.

Kechev M., Naglis S., Tonguç \& Pollet M. 2020. Checklist of the Dolichopodidae (Diptera, Empidoidea) of the Balkan Peninsula, with first records for Bulgaria, Montenegro, North Macedonia, and for the European part of Turkey. Zootaxa 4819 (3): 436-472.

Kenderov L., Stoichev S. \& Yaneva I. 2012. Comparative studies of water Invertebrate fauna from benthal and hyporheic habitats in Iskar River catchment. I. Infauna: Free-living Nematodes (Nematoda) and Chironomid larvae (Diptera: Chironomidae). Acta zoologica bulgarica 64 (3): 273-281.

Kenderov L., Yaneva I. \& Pavlova M. 2008. Ecological assessment of the upper stretch of the Iskar River based on selected biological parameters in conformity with the Water Frame Directie 2000/60/EU. Acta zoologica bulgarica, Supplementum $2: 255-268$.

Keremidchiev M. 1951. Biologichna borba s vreditelite po gorskite poyasi [Biological pest control in the forest belts]. Gorsko stopanstvo 7 (7): 319-323. (In Bulgarian).

Keremidchiev M. 1954. Vredi ot gabotvorkata (Ocneria dispar L.) po nashite gori i merki za borba sreshtu neya [Damages from the fungus (Ocneria dispar L.) in the Bulgarian forests and measures to fight it]. Nauchni trudove NIIGGS 2: 243-296. (In Bulgarian).

Keremidchiev M. 1965. Biologichna borba s nasekomnite vrediteli po gorite [Biological control of the insect pests in the forests]. Priroda 14 (4): 37-42. (In Bulgarian).

Kiknadze I., Michailova P., Istomina A., Goligina V., Panis L. \& Krastanov B. 2006. The chromosomal polymorphism and divergence of populations in Chironomus nuditarsis Str. (Diptera, Chironomidae). Tsitologia 48 (7): 595-609. (In Russian with English summary).

Kircheva M. 1991. A study of the relationship between seed yield of winter rape (Brassica napus L. var. oleifera) and attack level of Dasineura brassicae Winn. (Diptera, Cecidomyiidae) and Ceuthorrhynchus assimilis Payk. (Coleoptera, Curculionidae). In: First National Conference of Entomology, Sofia, 28-30 october 1991, 60-65. (In Bulgarian, English summary).

Kirin D. \& Buchvarov G. 1999. Species composition and season numeration sinantrope flies in agricultural objects in villiage Archar (Vidinsko). Travaux Scientifiques Universite de Plovdiv, Biologie - Animalia 35 (6): 49-54. (In Bulgarian, English summary).

Kitanov B. 1943. Materiali po upotrebata na rasteniyata v narodnite sredstva za borba sreshtu parazitite i stopanski vrednite zhivotni [Materials on the plants use in the people means of fight against parasites and pests]. Spisanie na zemedelskite ispitatelni instituti v Balgariya 13 (1/2): 29-37. (In Bulgarian).

Kock D. 1974. Pupipare Dipteren von Säugentieren des nordöstlichen Mittelmeerraumes (Ins.: Diptera). Senckenbergiana Biologica 55: 87-104. 
Kolarov P. 1959. Materiali varhu hranata na chigata ot bulgarskoto kraybrezhie na r. Dunav [Materials on the food of the chiga fish from the Bulgarian coast of the Danube River]. Priroda 8 (4): 62-65. (In Bulgarian).

Kolarov J. \& Bechev D. 1995. Hymenopterenparasiten (Hymenoptera) auf Pilzmücken (Mycetophiloidea, Diptera). Acta entomologica bulgarica 2: 18-20.

Kolcsár L.-P., Ivkovic M. \& Ternjej I. 2015. New records of Limoniidae and Pediciidae (Diptera) from Croatia. ZooKeys 5: 23-37.

Kolcsár L.-P., Torok E. \& Keresztes L. 2012. An annotated list of Pediciidae (Insecta, Diptera) from Romania with a revision of the literature data. Entomologica Romanica 17: 21-27.

Kolcsár L.-P., Petrašiūnas A., Torok E. \& Keresztes L. 2018 (2017). A new species of Trichocera Meigen with further records of Metatrichocera Dahl from Bulgaria, Romania, and Serbia (Diptera, Trichoceridae). Turkish Journal of Zoology 42: 172-178.

Komárek J. \& Vimmer A. 1921. O larvach evropskych druhu čelede Blepharoceridae. Časopis Československe společnostr entomologicke 13: 37-53.

Komárek J. \& Vimmer A. 1922. The Larvae of the europaean Blepharoceridae. Annales de Biologie lacustre 11 (1): 63-77.

Komárek J. \& Vimmer A. 1934. Blepharoceridae Balkanicae. Mitteilungen aus den Königl Naturwissenschaftlichen Instituten in Sofia 7: 1-35.

Konsuloff S. 1921a. Die Lebensbedingungen der Anophelinen in Bulgarien im Zusammenhang mit der Malariaverbreitung. Archif fur Schiffs- und Tropen-Hygiene 25: 227-240.

Konsuloff S. 1921b. Orizoseyaneto i malariyata [Rice fields and malaria]. Spisanie na zemedelskite izpitatelni instituti v Balgaria 2 (1/2): 86-120. (In Bulgarian).

Konsuloff S. 1922a. Biologichni usloviya za malariyata u nas [Biological conditions for malaria in Bulgaria]. Annuaire de l'Université de Sofia 17: 1-32. (In Bulgarian).

Konsuloff S. 1922b. Uber die Doppeleatmung der Muckenlarven. Biologischen Zentralblatt 42 (4): 188-192.

Konsuloff S. 1922c. Die Bekämpfung der Malariamucken in den Reisfeldern. Zeitschrift für angewandte Entomologie 8 (2): 283-297.

Konsuloff S. 1923a. Biologichni belezhki po borbata s malariyata u nas [Biological notes on the fight against malaria in Bulgaria]. Annuaire de l'Université de Sofia 19 (1a): 139-150. (In Bulgarian).

Konsuloff S. 1923b. Zla muha [Evil flies]. Priroda, 23 (10): 150-152. (In Bulgarian).

Konsuloff S. \& Paspalev G. 1924. Predvaritelni izuchvaniya varhu zlata muha [Preliminary studies on the evil fly]. Trudove na Balgarskiya nauchen zemedelsko-stopanski institut, 10: 3-13. (In Bulgarian).

Konsuloff S. \& Paspalev G. 1925. Izuchvaniya varhu zlata muha (Simuliidae) [Studies on the evil fly (Simuliidae)]. Annuaire de l'Université de Sofia 21: 59-66. (In Bulgarian).

Kontev Ch., Dimitrova D. \& D. Peycheva. 1991. Studies of the triticale entomofauna under the conditions of Dobroudja region. In: First National Conference of Entomology, Sofia 28-30 October, 1991, 76-82. (In Bulgarian with English summary).

Kovachev S. 1969. Beitrag zur erforschung der Simulliden-Fauna von Bulgarien (Diptera, Simullidae). Bulletin de l'Institut de zoologique et Musée 30: 191-195. (In Bulgarian with German summary).

Kovachev S. 1973. Zur Simuliidefauna Bulgariens (Diptera). Faunistische Abhandlungen Staatliches Museum für Tierkunde in Dresden 4 (18):

Kovachev S. 1975. Materialien über die Simuliidenfauna (Diptera, Simuliidae) der Rhodopen. In: La Faune des Rhodopes. Matériaux. Sofia, Bulgarian Academy of Sciences. pp. 205-208. (In Bulgarian with German summary).

Kovachev S. 1976. Simulidae (Diptera) from the Strouma and Mesta River systems. Hydrpbiology 3: 67-77. (In Bulgarian with English summary).

Kovachev S. 1979. Bemerkungen über die Zusammensetzung, Verbreitung und Ökologie der Simuliiden (Diptera, Simuliidae) in der Bulgarischen Donauzuflüssen. In: XXI Arbeitstag IAD (Novi Sad). pp 392-396.

Kovachev S. 1985a. The Simuliid complex of the Maritsa River as an example of a hyghly destructed taxocenosis. Hydrobiology 26: 53-58. (In Bulgarian with English summary).

Kovachev S. 1985b. Metacnephia uzunovi sp. n. - a new black fly (Diptera, Simuliidae) from Bulgaria, with notes on its bionomy. Acta zoologica aulgarica 28: 63-65.

Kovachev S. 1985c. Formation of the qualitative composition of simuliid fauna in the Mesta River as an illustration of the concept of saprobic successions. Hydrobiology 26: 49-52. (In Bulgarian with English summary). 
Kovachev S. 1990. Black-flies (Insecta, Diptera, Simulidae) from Vitosha. In: Fauna of Southwestern Bulgaria. 3. Sofia, Bulgarian Academy of Sciences. pp. 150-153. (In Bulgarian with English summary).

Kovachev S. 2000. Qualitative structure and distribution of Black-flies (Diptera: Simuliidae) from high mountain streams of glacial origin in the Rila Mountains. In: Golemsnski V. \& Naidenov W (Eds.). Biodiversity and evolution of glacial water ecosystems in the Rila Mountains. Sofia, Institute of Zoology, BAS. pp. 67-74.

Kovachev S. \& Stoichev S. 1996. The zoobenthos from three sand-pit lakes at different stages of their development. Hydrobiology 40: 33-42.

Kovachev S., Stoichev S. \& Hainadjieva V. 1999. The zoobenthos of several lakes along the Northern Bulgarian Black Sea coast. Lauterbornia 35: 33-38.

Kovachev V. 1905. A Contribution to the study of the entomological fauna of Bulgaria. Godishnik na Rusenskata gimnaziya za uchebnata godina 1904-1905: 3-12. (In Bulgarian).

Kovachevski I., Lazarov A., Bogdanov V., Hristov A., Balevski A. \& Martinov S. 1949. Spravochnik po zashtita na rasteniyata ot bolesti i nepriyateli [A guide of plant protection from diseases and pests]. Sofia, Zemizdat. 424 p. (In Bulgarian).

Kovachevski I., Lazarov A., Bogdanov V., Hristov A., Balevski A., Nikolova V., Dodov D. \& Martinov S. 1959. Spravochnik po zashtita na rasteniyata ot bolesti i nepriyateli [A guide of plant protection from diseases and pests]. Sofia, Zemizdat. 424 p. (In Bulgarian).

Kovchazov T. 1958. Rezultati ot prouchvaniyata za rezistentnost na Anopheles maculipennis spryamo DDT v Rusenski okrag [Results of the resistance studies of Anopheles maculipennis vs. DDT in Ruse District]. Higiena, epidemiologiya i mikrobiologiya 2 (2): 20-25. (In Bulgarian).

Kovchazov T. 1961. Otnosno izkorenyavaneto na malariyata v Rusenski okrag [On the eradication of malaria in the Ruse District]. Epidemiologiya, microbiologiya i virusologiya 4 (4): 23-26. (In Bulgarian).

Kovchazov T. 1976. Prouchvaniya varhu chuvstvitelnostta na Anopheles maculipennis spryamo insektitsidi vav Varnenski okrag [Studies on the sensitivity of Anopheles maculipennis towards insecticides in the Varna District]. Letopisi na HEI 9 (6): 161-163. (In Bulgarian).

Kozarov D., Benevisti I. \& Dobreva E. 1959. Prouchvaniya varhu aerozolniya metod za poleva dezinsektsiya s pomoshta na heksahloranovi dimki [Studies on the aerosol method for field disinsection using hexachlorane fumes]. Higiena, epidemiologiya i mikrobiologiya 3 (5): 29-36. (In Bulgarian).

Kozarov G. 1949. Malarichnite komari prez zimata [Malaria mosquitoes in winter]. Priroda i znanie 2 (7): 8-11. (In Bulgarian).

Kozarov G. \& Bozhkov D. 1953. Izuchavane varhu diapauzata i zimuvaneto na Anopheles maculipennis maculipennis Meig. v Bulgariya [Study on the diapause and wintering of Anopheles maculipennis maculipennis Meig. in Bulgaria]. Annuaire de l'Université de Sofia 47: 157-175. (In Bulgarian).

Kozarov P. 1907. Statistika na bolestite i nepriyatelite po kulturnite rasteniya v Severna Bulgariya prez $1906 \mathrm{~g}$. spored postapilite $\mathrm{v}$ stantsiyata saobshteniya i zapitvaniya [Statistics of the diseases and pests on the cultivated plants in Northern Bulgaria in 1906 according to the messages and inquiries received at the station]. Trudove na Zemedelskata opitna stantsiya v Obraztsov Chiflik 1 (1): 37-79. (In Bulgarian).

Kozarov P. 1908. Statistika na bolestite i nepriyatelite po kulturnite rasteniya v Severna Bulgariya prez 1907 g. spored postapilite $\mathrm{v}$ stantsiyata saobshteniya i zapitvaniya [Statistics of the diseases and pests on the cultivated plants in Northern Bulgaria in 1907 according to the messages and inquiries received at the station]. Trudove na Darzhavnata zemedelska opitna stantsiya kray gr. Ruse 1 (2): 209-264. (In Bulgarian).

Kozarov P. 1910-1911. Bolesti i nepriyateli po kulturnite rasteniya v Severna Bulgariya prez 1808 godina [Diseases and pests of the cultivated plants in Northern Bulgaria in 1908]. Trudove na Darzhavnata zemedelska opitna stantsiya kray gr. Ruse 2 (1): 68-175; 2 (2): 73-102. (In Bulgarian).

Kozarov P. 1912. Spisak na nablyudavanite bolesti, nepriyateli i povredi po kulturnite rasteniya v Severna Bulgaria prez 1909 god. [List of the observed diseases, pests and damages on the cultivated plants in Northern Bulgaria in 1909]. Godishen otchet na Darzhavnata zemedelska opitna stantsiya v Obraztsov Chiflik za 1909 god., 6-20. (In Bulgarian).

Kozuharova E. 1994. Biologiya i razmnozhavane na vidovete ot rod Gentiana L. (Gentianaceae), predstaveni v balgarskata flora [Biology of reproduction of the species of the genus Gentiana L. (Gentianaceae), presented in the Bulgarian flora]. Ph.D. thesis, Sofia, Bulgarian Academy of Sciences. 183 p. (In Bulgarian).

Kozuharova E., Lavchiev V. \& Lennartsson T. 2005. Flies of the genus Thricops (Muscidae, Diptera) as pollinators plants with bell-shaped flowers in the mountains of Bulgaria. Studia dipterologica 12 (2): 409-418. 
Krasteva H. 1995. Study of density dynamics, phenology and damage of Meromyza nigriventris Macquart, 1835 (Diptera: Chloropidae). Acta Entomologica Bulgarica 1 (2): 41-47. (In Bulgarian with English summary). Krasteva H. 1996. Harmfulness of Meromyza nigriventris Macquart, 1833 (Diptera: Chloropidae) in spring barley fields. Acta Entomologica Bulgarica 2 (1): 29-37. (In Bulgarian with English summary).

Krasteva H. \& Beschovski V. 1998. Studies of the species composition of Diptera order internal stem pests and damages caused by them on spring barley. Acta zoologica bulgarica 50 (1): 43-50.

Krasteva H. \& Beschovski V. 2000. Studies of the species composition of the stem-boring dipterous pests and damage caused by their larvae on oats. Acta zoologica bulgarica 52 (3): 29-36.

Krasteva H. \& Beschovski V. 2001. Studies on the species composition of stem-boring dipterous pests and damage caused by their larvae on winter wheat. Acta Entomologica Bulgarica 3/4: 19-25. (In Bulgarian with English summary).

Krasteva (Krusteva) H., Karadjova O. \& Ventsislavov V. 2012. Monitoring methods and prognosis of Opomyza florum Fabr. (Diptera: Opomyzidae) - a pest of wheat in Bulgaria. Journal of plant protection research 52 (4): 472-478. DOI: 10.2478/v10045-012-0076-6

Krivosheina N. P. 1986. Family Bibionidae. In: Soós Á. \& Papp L. (Eds.). Catalogue of Palaearctic Diptera. 4. Budapest, Akadémiai Kiadó, Hungarian Natural History Museum. pp. 319-330.

Krivosheina N. P. 1989a. A review of the flies of the genus Parydra Stph. [sic] (Diptera, Ephydridae) of the fauna of the USSR. Revue d'Entomologie de l'URSS 68 (1): 209-2020. (In Russian with English summary).

Kröber O. 1921. Die paläarktischen Arten der Gattung Pangonia Latr. (Versuch einer Auseinandersetzung.) Archiv für Naturgeschichte, 87A (1): 1-67.

Kröber O. 1925. Tabanidae. In: Lindner E. (ed.). Die Fliegen der palaearktischen Region 4 (1). Stuttgart, E. Schweizerbart'sche Verlagsbuchhandlung. 146 p.

Kröber O. 1932. Familie Tabanidae (Bremsen). In: Dahl F. (Ed.). Tierwelt Deutschlands 26. Jena, Gustaf Fischer. pp. 55-99.

Kröber O. 1938. Katalog der palaearktischen Tabaniden nebst Bestimmungstabelle und Zusätzen zu einzelnen Arten so wie Neubeschreibungen. Acta Instituti et Musei Zoologici Universitatis Atheniensis 2: 57-245.

Kryzhanovsky O. 1965. Composition and origin of the terrestrial fauna of Central Asia (based chiefly on the beetles material). Moscow-Leningrad, Nauka. 430 p. (In Russian).

Kryzhanovsky O. 1976. On the principles of zoogeographical regionalization of land. Zoologichesky Zhurnal, 55 (7): 965-975. (In Russian with English summary).

Kryzhanovsky O. 2002. Composition and distribution of entomofauna of the globe. Moscow, KMK. 237 p. (In Russian).

Krzemiński W. 1984. Limoniidae of Bulgaria (Diptera, Nematocera). I. Acta zoologica bulgarica 24: 27-34.

Krzemiński W. \& Starý J. 1989. Limoniidae of Bulgaria (Diptera, Nematocera). II. Bulletin Entomologique de Pologne 59 (1): 253-279.

Kurina O. 2003. Notes on the Palaearctic species of the genus Polylepta Winertz (Diptera: Micetophilidae) with a new synonymization. Entomologica Fenica 14: 91-97.

Kurina O. \& Chandler P. 2018. New European records of Ditomyia macroptera Winnertz (Diptera: Ditomyiidae) with notes on its distribution. Biodiversity Data Journal 6: e24857. https://doi.org/10.3897/BDJ.6.e24857

Kutsarov Y. 2006. Komarite (Culicidae) po porechieto na reka Dunav i savremenni metodi za borba s tyah [The Mosquitoes (Culicidae) along the Danube River and modern methods to control them]. Tutrakan, Protected site "Kalimok - Brashlen". 32 p. (In Bulgarian).

Kutsarov J. \& Hubenov Z. 2019. Diopsidae (Schizophora: Acalyptrata) - a New Family for the Bulgarian Recent Dipterous Fauna. Acta zoologica bulgarica 71 (1): 145-147.

Lackschewitz P. 1940a. Die paläarktischen Rhamphidiinen und Eriopterinen (Diptera) des Wiener Naturhistorischen Museums. Annalen des Naturhistorischen Museums in Wien 50 (1939): 1-67.

Lackschewitz P. 1940b. Die paläarktischen Limnophilinen, Anisomerinen und Pediciinen (Diptera) des Wiener Naturhistorischen Museums. Annalen des Naturhistorischen Museums in Wien 50 (1939): 68-122.

Langourov M. 2001a. Faunistic, ecological and zoogeographical studies of the family Phoridae (Diptera, Brachycera) of the Vitosha Mt. Ph.D. thesis, Sofia, Bulgarian Academy of Sciences. Institute of Zoology. 176 p. (In Bulgarian).

Langourov M. 2001b. Scutle Flies from caves on the Balkan Peninsula (Diptera: Phoridae). Acta zoologica bulgarica 53 (3): 33-40. 
Langourov M. 2004a. Scuttle Flies (Diptera: Phoridae) from urban and suburban areas in the Sofia Plain. In: Penev L., Niemelä J., Kotze J. \& Chipev N. 2004 (Eds.). Ecology of the city of Sofia. Species and communities in an urban environment. Sofia, Pensoft, 429-436.

Langourov M. 2004b. Scuttle flies (Diptera: Phoridae) from Eastern Rhodopes (Bulgaria) with special consideration of winter-active species. In: Beron P. \& Popov A. (Eds.). Biodiversity of Bulgaria. 2. Biodiversity of Eastern Rhodopes (Bulgaria and Greece). Sofia, Pensoft \& National Museum of Natural History, 759-768.

Langourov M. 2004c. Scuttle flies (Diptera: Phoridae) from Biogradska Gora National Park - Montenegro. In: Biodiversity of the Biogradska Gora National Park. Monographies 1. Podgorica, Department of Biology University of Montenegro, 104-112.

Langourov M. 2009. A New to Southeastern Europe Subfamily of Scuttle Flies (Diptera: Phoridae). Acta zoologica bulgarica 61 (1): 97-100.

Langourov M. 2011. Some scuttle-flies (Diptera, Phoridae) frm the Western Rhodopes. In: Beron P. (Ed.). Biodiversity of Bulgaria. 4. Biodiversity of Western Rhodopes (Bulgaria and Greece) II. Sofia, Pensoft \& National Museum of Natural History, 485-498.

Langourov M., Lazarov S., Stoev P., Guéorguiev B., Deltshev Ch., Petrov B., Andreev S., Simov N., Bekchiev R., Antonova V., Ljubomirov T., Dedov I. Georgiev D. 2014. New and interesting records of the MSS and cave fauna of Vitosha Mt., Bulgaria. In: Zhalov A., Ivanov I., Petrov I. (Eds.): Proceedings of Balkan speleological conference „Sofia 2014”, Sofia, Bulgaria, 38-30 March 2014. Special issue of Caving Club „Helictite” - Sofia, 66-76.

Langourov M., Pavlova A., Hubenov Z., Bozukov V. \& Simov N. 2021. First record of fossil Diptera (Insecta) in Miocene deposits in Bulgaria. Comptes rendus de l'Académie bulgare des Sciences 74 (2): 233-240.

Langourov M., Sakalian V. 2001. Scuttle flies (Diptera: Phoridae) from Kresna Gorge (SW Bulgaria). In: Beron (Ed.). Biodiversity of Kresna Gorge (SW Bulgaria). National Museum of Natural History \& Institute of Zoology, Sofia, BAS, 217-218. (In Bulgarian with English summary).

Lattin de G. 1967. Grundriss der Zoogeographie. Hochschullehrbücher für Biologie. 12. Jena, VEB Gustav Fischer Verlag. 602 p.

Lauterer P. 1983. Contribution to the knowledge of distribution and bionomics of somerepresentatives of the family Pipunculidae in Central and Southern Europe (Diptera). Acta Musei Moraviae 68: 131-138.

Lavčiev (Lavchiev, Lâvčiev, Lavtschiev, Lawtschiev, Luvchiev) V. 1964a. Contribution à létude des espèces synanthropes de la famille des Muscidés en Bulgarie. Bulletin de l'Institut de zoologie et musée 15: 105-113. (In Bulgarian, French summary).

Lavčiev V. 1964b. Mitteilung über Dipteren. Bulletin de l'Institut de zoologie et musée 16: 157-160. (In Bulgarian, French summary).

Lavčiev V. 1965a. Sinantropni muhi [Synanthropic flies]. Priroda 14 (5): 98-102. (In Bulgarian).

Lavčiev V. 1965b. Neue Kotfliegen aus der Fauna Bulgariens (Diptera - Brachycera). Bulletin de l'Institut de zoologie et musée 19: 119-127. (In Bulgarian, German summary).

Lavčiev V. 1965c. Neue Muscidenarten (Diptera) für die Fauna Bulgariens. Bulletin de l'Institut de zoologie et musée 18: 137-140. (In Bulgarian, German summary).

Lavčiev V. 1965d. Prinos kam izuchavaneto na sinantropnite muhi v Rusenski okrag s ogled na epidemiologichnoto im znachenie [Contribution to the study of synanthropic flies in Ruse district in view of their epidemiological significance]. Epidemiologiya, mikrobiologiya i infektsiozni bolesti 2 (1): 61-68. (In Bulgarian).

Lavčiev V. 1966. Neue und seltene für die Fauna Bulgariens Fliegen (Muscidae - Dipt.). Bulletin de l'Institut de zoologie et musée 21: 77-80. (In Bulgarian, German summary).

Lavčiev V. 1967. Untersuchungen über die synbovilen Fliegen der Fam. Muscidae (Dipt.) in Bulgarien. Bulletin de l'Institut de zoologie et musée 25: 23-39. (In Bulgarian, German summary).

Lavčiev V. 1968. Eine neue Art der Gattung Helina R.-D. aus Bulgarien (Diptera, Muscidae). Reichenbachia 10 (8): 63-64.

Lavčiev V. 1969. Forschungen über die echten Fliegen (Muscidae, Diptera) im Reservat Ropotamo. Bulletin de l'Institut de zoologie et musée 30: 59-70. (In Bulgarian, German summary).

Lâvčiev V. 1970a. Die Fliegen (Fam. Muscidae, Diptera) im Westlichen Balkangebirge. Bulletin de l'Institut de zoologie et Musée 30: 119-159. (In Bulgarian with German summary).

Lavčiev V. 1970b. Coenosia beschovskii n. sp. aus Bulgarien (Muscidae, Diptera). Reichenbachia 13 (22): 219-221. 
Lavčiev V. 1972. Contribution à létude des mouches en Bulgarie à partir de larves copribiontiques. Bulletin de l'Institut de zoologie et musée 36: 103-108. (In Russian, French summary).

Lavčiev V. 1974. Beitrag zur erforschung der eigentlichen Fliegen (Muscidae, Diptera) in dem Mittleren und dem Östlichen Balkangebirge. Bulletin de l'Institut de zoologie et Musée 41: 141-159. (In Bulgarian with German summary).

Lavčiev V. 1977. Ecological and etological notes on synbovilous flies in Bulgaria. In: Terrestrial fauna of Bulgaria, Sofia, Bulgarian Academy of Sciences. 97-120. (In Russian with English summary).

Lavčiev V. 1980. Muhite okolo nas i borbata s tyah [The flies around us and the fight against them]. Sofia, Bulgarian Academy of Sciences. 205 p. (In Bulgarian).

Lavčiev V. 2003. Catalogus faunae bulgaricae. 5. Diptera: Fannidae, Muscidae, Stomoxydidae. Sofia, Editio Academica "Professor Marin Drinov". 78 p.

Lavchiev V. \& Ganeva D. 1991. A contribution to a study of Tabanidae (Diptera) which infest cattle in the Stara Zagora District. Second international School „Parasite-Host-Environment“, Sofia

Lavčiev V. \& Jelesova M. 1968. Experimentelle Untersuchungen über die morphologische Veränderlicken von Musca domestica L. (Dipt. Muscidae. Zoologischer Anzeiger 181 (5/6): 411-421.

Lavčiev (Lawtschiev) V. Jelesova M. 1971. Untersuchungen über den Unterartenbestand der Hausfliege Musca domestica L. (Muscidae) in Bulgarien. Bulletin de l'Institut de zoologie et Musée 33: 75-83. (In Bulgarian with German summary).

Lavčiev V. \& Jelesova M. 1972. Spread and biology of Orthellia caesarionNg. (Muscidae, Diptera) in Bulgaria. Bulletin de l'Institut de zoologie et musée 34: 109-118. (In Bulgarian, English summary).

Lavčiev V. \& Jelesova M. 1978. Ecological investigations on the coprobiont dipterans (Diptera) in a pasture ecosystem. Ecology 4: 20-31. (In Bulgarian with English summary).

Lavchiev (Luvchiev) V., Zhelyazova M., Tachirov B. \& Mircheva. 1981. On the altitude distribution of exophylous synanthropic and coprophylous flies. Ecology 8: 29-33. (In Bulgarian with English summary).

Lavchiev V., Jelesova M., Stamenova M. \& Tsankova R. 1984. Activities of some coprophilous and necrophilous flies in Rila Mountain. Ecology 14: 53-62. (In Bulgarian with English summary).

Lavčiev V. \& Karastoyanov H. 1970. Prinos v izuchavaneto na hemisinantropnite koprofilni muhi (Diptera - Brachycera) v Bulgaria [Contribution to the study of hemisinanthropic coprophilous flies (Diptera Brachycera) in Bulgaria]. Letopisi HEI 23: 121-132. (In Bulgarian).

Lavčiev V., Karastoyanov H., Hristova T., Balukova Y., Dimitrasheva V., Tahirov B., Popova E., Smilova D. \& Ficheva D. 1973. Sezonna dinamika na nyakoi sinantropni muhi u nas [Seasonal dynamics of some synanthropic flies in Bulgaria]. Letopisi HEI 39: 129-136. (In Bulgarian).

Lavčiev V. \& Lateva A. 1973. Prinos kam prouchvaniyata na sinantropnite muhi v grad Dragoman [Contribution to the research of synanthropic flies in the town of Dragoman]. Byuletin DDD (3-4), $55 \mathrm{p}$.

Lavčiev V. \& Lateva A. 1976. Sravnitelen pregled na sinantropnite muhi v Sofiyski okrag s ogled epidemiologichnoto im znachenie [Comparative review of the synanthropic flies in the Sofia district in view of their epidemiological significance]. In: IV congres po zarazni i parasitni bolesti $2.31 \mathrm{p}$.

Lavčiev V. \& Lateva A. 1977. Nyakoi problemi na borbata sas sinantropnite muhi v planinskite kurorti [Some problems in the fight against the synanthropic flies in the mountain resorts]. Letopisi HEI 6: 157-159. (In Bulgarian).

Lavčiev V. \& Mirčeva M. 1980. A contribution to the phenology of some synanthropic flies in the Tolbuhin district. Ecology 7: 39-52. (In Bulgarian, English summary).

Lavčiev V. \& Mirčeva M. 1989. Effect of the preparation dimilin (TH 6040) on the reproduction of the housefly (Musca domestica L.). Ecology 22: 50-55. (In Bulgarian, English summary).

Lavčiev V. \& Nestorova E. 1967. Recherches sur les mouches synanthropes de la région de Sofia eu égard à leur importance du point de vue épidémiologique. Bulletin de l'Institut de zoologie et musée 23: 5-31. (In Bulgarian, French summary).

Lavčiev V., Shishiniova M. \& Lateva A. 1992. Sezonna chislenost na muhite v Sofiyski rayon s ogled na epidemiologichnoto im znachenie [Seasonal number of the flies in the Sofia region in view of their epidemiological significance]. Annual of Sofia University “St. Kliment Ohridski”. Faculty of Biology. Zoology 83 (1): 83-91. (In Bulgarian, English summary).

Lavčiev V. \& Tahirov B. 1970. Otnosno biotopite na nyakoi sinantropni muhi v grad Shumen [Regarding the biotopes of some synanthropic flies in the town of Shumen]. Letopisi HEI 21: 60-68. (In Bulgarian). 
Lavčiev V. \& Tahirov B. 1977. Composition, structure and distribution of populations of synanthrope flies in town of Shumen. Ecology 3: 60-69. (In Bulgarian, English summary).

Lavčiev (Luvchiev) V., Tsanev I. \& Smilova D. 1974. A contribution to the investigation into the synanthropic flies along the Bulgarian Black Sea coast. Bulletin de l'Institut de zoologie et musée 40: 133-151. (In Bulgarian, English summary).

Lavčiev (Luvchiev) V. \& Tsankova R. 1980. Ecological studies on coprobiont Dipterans in a dunghill near Sofia. Ecology 7: 3-14. (In Bulgarian with English summary).

Lavchiev V. \& Tsankova R. 1982. Oh the hibernation of some coprobiont flies in Bulgaria. Ecology 10: 59-64. (In Bulgarian with English summary).

Lavchiev V. \& Tsankova R. 1994. Ophyra capensis Wd. in Bulgaria and the possibilities for its using as an antagonist of Musca domestica L. Ecology 25: 117-123.

Lavchiev V. \& Yovchev E. 1978. Epizootichnata rolya na muhite v zhivotnovadstvoto [The epizootic role of flies in the animal husbandry]. Sofia, Zemizdat. 151 p. (In Bulgarian).

Lavchiev V. \& Zhekov N. 1980. A contribution to the studying of fliesin enlarged cattle farms. Ecology 6: 26-36. (In Bulgarian with English summary).

Lavchiev V., Zhelyazova M., Hubenov Z. 1977. Contribution to the fauna and biology of Tachinidae (Diptera) in Sofia area. Acta zoologica bulgarica 8: 23-31. (In Bulgarian with German summary).

Lazarov A. 1935a. Hesenska muha, Mayetiola destructor Mg. [Hessian fly, Mayetiola destructor Mg.]. Zemedelie 39 (7): p. 55. (In Bulgarian).

Lazarov A. 1935b. Shvedskata muha, Oscinis frit L., kato nepriyatel na zhitnite rasteniya u nas i sredstvata za borba s neya [The Swedish fly, Oscinis frit L., as a pest on the cereals in Bulgaria and the means to control it]. Sofia, Izdanie na Ministerstvoto na narodnoto stopanstvo - Sluzhba za zashtita na rasteniyata. 58 p. (In Bulgarian).

Lazarov A. 1936a. Kurzer Beitrag zur kenntnis de Schädlichkeit der Gelben Halmfliege Chlorops taeniopus Meig. Mitteilungen der Bulgarischen Entomologischen Gesellschaft in Sofia 9: 1-10. (In Bulgarian with German summary).

Lazarov A. 1936b. Lukovata muha, Hylemyia antiqua Mg. [The onion fly, Hylemyia antiqua Mg.]. Zemedelie 40 (5): p. 75. (In Bulgarian).

Lazarov A. 1939. Referate u. Mitteilungen. Mitteilungen der Bulgarischen Entomologischen Gesellschaft in Sofia 10: 169-170. (In Bulgarian with German summary).

Lazarov A. 1940. Saobshtenie varhu nyakoi vredni nasekomi [Message on some harmful insects]. Referate u. Mitteilungen. Mitteilungen der Bulgarischen Entomologischen Gesellschaft in Sofia 11: 253-254. (In Bulgarian with German summary).

Lazarov A. 1942. Nay-vazhnite vredni muhi, nepriyateli na zhitnite rasteniya u nas, i sredstva za borba s tyah [The most important harmful flies, pests on the cereals in Bulgaria and means to control them]. Sofia, Upatvane No 50 na Instituta za zashtita na rasteniyata. 8 p. (In Bulgarian).

Lazarov A. 1943. Dve vazhni vredni zhitni muhi [Two important harmful wheat flies]. Zemedelie 47 (4): 57-60.

Lazarov A. 1949a. Vrednite nasekomi po ovoshtnite kulturi v Bulgariya i borbata s tyah [Harmful insects on the fruit trees in Bulgaria and the fight against them]. Sofia, Bulgarian Academy of Sciences. 328 p. (In Bulgarian).

Lazarov A. 1949b. Borbata s chereshovata muha [Fight against the cherry fly]. Gradinarstvo 30 (4): 100-101. (In Bulgarian).

Lazarov A., Grigorov S., Arabadzhiev D., Kontev H., Kaytazov A., Popov B., Gospodinov G., Bogdanov V., Furtunov D. \& Duchevski B. 1969. Zhitnite darvenitsi v Bulgaria i borbata s tyah [Wheat bedbugs in Bulgaria and the fight against them]. Sofia, Bulgarian Academy of Sciences. 147 p. (In Bulgarian).

Lazarov A., Kovachevski I. \& Balevski A. 1960. Bolesti i nepriyateli po ovoshtnite kulturi [Diseases and pests on the fruit crops]. Sofia, Zemisdat. 295 p. (In Bulgarian).

Lazarov A., Kovachevski I., Balevski A. \& Ivanov. 1965. Bolesti i nepriyateli po ovoshtnite kulturi [Diseases and pests on the fruit crops]. Sofia, Zemisdat. 364 p. (In Bulgarian).

Leclercq M. 1957. Révision systématique et biogéographique des Tabanidae (Dipt.) de France. II. Annales de Parasitologie Humaine et Compare 32 (4) : 398-425.

Leclercq M. 1966. Révision systématique et biogéographique des Tabanidae (Diptera) Paléartiques. 2. - Mémoires Institut Royal des Sciences Naturelles de Belgique 80: 1-237. 
Lehr A. 1977. Espèces nouvelles du genre Heteronychia B. B. de la faune de la Bulgarie (Diptera, Sarcophagidae). - Acta zoologica bulgarica 7: 27-36.

Lehr P. 1981. Review of robber-flies subtribe Machimina Lehr (Diptera, Asilidae). I. In: Pauki i nasekomiye Dalnego Vostoka. pp. 90-128. (In Russian).

Lehr P. 1988. Family Asilidae. In: Soós Á. \& Papp L. (Eds.). Catalogue of Palaearctic Diptera. 5. Budapest, Akadémiai Kiadó, Hungarian Natural History Museum. pp. 197-326.

Lehrer A. \& Lehrer M. 1985. La presence de Haematopota csikii Szilady en Romanie. Bulletin de la Societe Entomologie de Mulhouse, jan.-mars: 11-12.

Lenz F. 1950. Die halobionten Jugendstadien der Chironomidengattung Halliella Kieff. Deutsche Zoologische Zeitschrift 1 (1): 3-14.

Lindner E. 1936. Über die von Gerd Heinrich im Jahre 1935 in Bulgarien gesammelten Diptera-Stratiomyiidae. Mitteilungen aus den Naturwissenschaftlichen Instituten in Sofia-Bulgarien 9: 91-92.

Löw H. 1862. Uber enige bei Varna gefangene Dipteren. Wienner Entomologische Monatschrift 6 (6): 161-175.

Löw H. 1863. Uber bei Sliwno im Balkan gefangene Dipteren. Wienner Entomologische Monatschrift 7 (2): 33-35.

Lutovinovas E., Kanavalova L., Barták M. \& Kokan B. 2022. New or rare records for the Balkan Tachinidae (Diptera: Oestroidea). Acta zoologica bulgarica 74

Lyneborg L. 1989. Family Therevidae. In: Soós Á. \& Papp L. (Eds.). Catalogue of Palaearctic Diptera. 6. Budapest, Akadémiai Kiadó, Hungarian Natural History Museum. pp. 11-35.

Lyubenov Ya. 1956. Znachenie na agrotehnikata v borba s zhitnite muhi [The importance of the agrotechnics in the fight with the wheat flies]. Celskostopanska misal 4 (6/7): 87-88. (In Bulgarian).

Lyubenov Ya. 1958. Po-vazhni nepriyateli po divata zhitna rastitelnost, koyato sluzhi kato postoyanen iztochnik na zaraza po kulturnite zhitni rasteniya [Important pests on the wild wheat crops which serve as a constant source of infection on the cultivated wheat crops]. Byuletin po rastitelna zashtita 6 (4/12): 56-60. (In Bulgarian).

Lyubenov Ya. 1960. Prouchvaniya varhu znachenieto na agrotehnicheskite meropriyatiya v borbata s nay-vazhnite vidove zhitni muhi (Oscinosoma frit L., Chlorops pumilionis Bjerk., Phorbia genitalis Schabl. и Mayetiola destructur Say) v Bulgaria [Studies on the importance of the agro-technical measures in the fight against the most important kinds of cereal flies (Oscinosoma frit L., Chlorops pumilionis Bjerk., Phorbia genitalis Schabl. and Mayetiola destructur Say) in Bulgaria]. Nauchni trudove na tsentralnia nauchnoizsledovatelski institut za zashtita na rasteniyata 3: 99-142. (In Bulgarian).

Lyubenov Ya. 1961. Opiti za kombinirano izpolzvane na herbitsidi i insektitsidi pri borbata s nyakoi pleveli i nepriyateli po zarneno-zhitnite kulturi [Attempts to combine the use of herbicides and insecticides in the fight against some pests on the wheat crops]. Izvestiya na tsentralnia nauchnoizsledovatelski institut za zashtita na rasteniyata 1: 65-82. (In Bulgarian).

Majer J. 1988. Family Vermileonidae. In: Soós Á. \& Papp L. (Eds.). Catalogue of Palaearctic Diptera. 5. Budapest, Akadémiai Kiadó, Hungarian Natural History Museum. pp. 34-35.

Máca J. 1987. Amiota (Phortica) goetzi sp. n. (Diptera, Drosophilidae) with faunistic notes to Drosophilidae, Odiniidae and Periscelididae from southeastern Europe and Turkey. Acta Entomologica Musei Nationalis Pragae 42: 311-320.

Máca J. \& Roháček J. 1994. The genus Diastata Meigen (Diptera, Diastatidae) of Czech and Slovak Republics. Cas. Časopis Slezského zemského muzea Opava, Série A, 43: 97-111.

Makarov M. 1959. Novi vidove muhi, nepriyateli po pshenitsata v Bulgariya [New species of flies, pests on the wheat in Bulgaria]. Nauchni trudove VSI Georgi Dimitrov 6 (34): 365-384. (In Bulgarian).

Malicky H., Ant H., Aspöck H., de Jong R., Thaler K. \& Varga Z. 1983. Argumente zur Existenz und Chorologie mitteleuropäischer (extramediterran-europäischer) Faunen-Elemente. Entomologia Generalis 9: 101-119.

Malkov K. 1901. Nepriyateli i bolesti po zemedelskite rasteniya pri opitnoto pole na Sadovskoto zemedelsko uchilishte prez 1900/1901 godina [Enemies and diseases on the agricultural plants in the experimental field of the Sadovo Agricultural School in years 1900-1901]. Sadovo 4 (9/10): 276-283. (In Bulgarian).

Malkov K. 1902a. Vrediteli nasekomi po ovoshtnite darveta [Insect pests of on the fruit trees]. Oralo 9 (10): 147-149. (In Bulgarian).

Malkov K. 1902b. Po-vazhni bolesti i povredi po zhitnite rasteniya [More important diseases and damages on the cereals]. Oralo 9 (16): 250-251; 9 (17): 262-264. (In Bulgarian). 
Malkov K. 1902c. Stradat li ovoshtnite darveta, zelenchutsite i zemedelskite rasteniya ot razni bolesti i kakvo mozhe da se napravi za tyahnoto unishtozhenie [Do fruit trees, vegetables and agricultural plants suffer from various diseases and what can be done to destroy them]. Sadovo 5 (6/7): 66-69. (In Bulgarian).

Malkov K. 1903. Bolesti i povredi po rapitsata i sredstva za tyahnoto unishtozhavane [Diseases and damages to the cole and means of destroying them]. Plovdiv. 54 p. (In Bulgarian).

Malkov K. 1904a. Kakvi bolesti i nepriyateli na zemedelskite rasteniya, ovoshtnite darveta i dr. sa se poyavili v stranata prez 1903 godina [What diseases and pests of agricultural plants, fruit trees and others. appeared in the country in 1903]. Godishen otchet na Darzhavnata zemedelska opitna stantsiya v Sadovo za 1903 godina 1: 209-221. (In Bulgarian).

Malkov K. 1904b. Po-glavnite bolesti i povredi po zemedelskite rasteniya, ovoshtnite darveta i zelenchutsite, koito sa se poyavili prez 1903 godina v Sadovo i okolnostite mu [The main diseases and damages on the agricultural plants, fruit trees and vegetables, which appeared in 1903 in Sadovo and its surroundings]. Godishen otchet na Darzhavnata zemedelska opitna stantsiya v Sadovo za 1903 godina 1: 201-208. (In Bulgarian).

Malkov K. 1905. Bolesti i povredi, poyaveni po zemedelskite rasteniya i ovoshtnite darveta u nas prez 1904 godina [Diseases and damages that appeared on the agricultural plants and fruit trees in the country in 1904]. Godishen otchet na Darzhavnata zemedelska opitna stantsiya v Sadovo za 1904 godina 2: 183-233. (In Bulgarian).

Malkov K. 1906a. Nay-razprostranenite bolesti i povredi po zemedelskite rasteniya i ovoshtnite darveta prez 1905 godina $v$ Knyazhestvo Bulgariya [The most common diseases and damages on the agricultural plants and fruit trees in 1905 in Bulgaria]. Godishen otchet na Darzhavnata zemedelska opitna stantsiya v Sadovo za 1905 godina 3: 120-140. (In Bulgarian).

Malkov K. 1906b. Nay-rasprostranenite bolesti i povredi, poyaveni po zemedelskite rasteniya i ovoshtni darveta prez 1906 godina $v$ Knyazhestvo Bulgariya [The most common diseases and damages occurring on the agricultural plants and fruit trees in 1906 in Bulgaria]. Godishen otchet na Darzhavnata zemedelska opitna stantsiya v Sadovo za 1906 godina 4: 156-176. (In Bulgarian).

Malkov K. 1907. Edin prinos kam prouchvane vrednite nasekomi po kulturnite rasteniya u nas [A contribution to the study of harmful insects on crops in Bulgaria]. Trudove na Darzhavnata zemedelska opitna stantsiya v Sadovo 2: 47-54. (In Bulgarian).

Malkov K. 1908. Bolesti i povredi po kulturnitr rasteniya, izprateni za izsledvane v Sadovskata opitna stantsiya prez 1907 godina [Diseases and damages on the cultivated plants, sent for examination to the Garden Experimental Station in 1907]. Godishen otchet na Darzhavnata zemedelska opitna stantsiya v Sadovo za 1907 godina 5: 207-274. (In Bulgarian).

Mamaev B. \& Dimitrova B. 1992. A new species of gall-midges from Bulgaria (Insecta, Diptera, Cecidomyiidae: Porricondylinae). Reichenbachia 29 (28): 173-175.

Mamoon A. 1978a. Varhu vidoviya sastav i rolyata na vidovete ot semeystva Braconidae i Tachinidae paraziti po listogrizeshti vrediteli $\mathrm{v}$ dabovite gori [On the species composition and the role of species from the families Braconidae and Tachinidae parasites on leaf-eating pests in the oak forests]. Gorsko stopanstvo. (In Bulgarian).

Mamoon A. 1978b. Kinds and role of some parasitic insects of the Braconidae (Hymenoptera) and Tachinidae (Diptera) families on tne folivorous forest insects. Ph.D. thesis, Sofia, Forsttechnische Hochschule, 6-9. (In Bulgarian).

Manolov S. 1907. Malariyata i komarite v Burgas i okolnostite [Malaria and mosquitoes in Burgas and the surroundings]. Letopis na lekarskiya sayuz v Balgariya 5 (3): 115-128. (In Bulgarian).

Manolov S. 1909a. Malariyata i komarite v gr. Tutrakan [Malaria and mosquitoes in the Tutrakan Town]. Letopis na lekarskiya sayuz v Balgariya 7 (1): 19-20. (In Bulgarian).

Manolov S. 1909b. Po sistematikata i biologiyata na komarite v Burgasko [On the systematics and biology of mosquitoes in Burgas]. Letopis na lekarskiya sayuz v Balgariya 7 (10): 644-648. (In Bulgarian).

Marinov T. 1957. Über die vertikale verbreitung von Anopheles maculipennis Mg. Bulletin de l'Institut zoologique de l'Academie des sciences de Bulgarie 6: 599-601. (In Bulgarian with German summary).

Markov K. 1923. Golubashkata muha [Simulia columbascense (Sch.)] [The Simulia columbascense (Sch.)]. Svedeniya po zemedelieto, 4 (3/4): 13-18. (In Bulgarian). 
Markov K. 1925a. Malariyata i borbata s neya [Malaria and the fight against it]. Sofia, Direktsiya na narodnoto zdrave, Inspektorat po malariyata. 52 p. (In Bulgarian).

Markov K. 1925b. Materiali za izuchavaneto na malariyata v Bulgariya [Materials for studying the malaria in Bulgaria]. Glavna direktsiya na narodnoto zdrave 2. Sofia, Darzhavna pechatnitsa. 95 p. (In Bulgarian).

Markov K. 1929. Epidemiologiya i borba s malariyata v Bulgariya [Epidemiology and the fight against malaria in Bulgaria]. Sbornik na BAN 25, Prirodomatematichski klon 11: 3-48. (In Bulgarian).

Markov K. 1937. (1920-1935) Malariyata v Bulgariya prez poslednite petnadeset godini (1920-1935) [The malaria in Bulgaria in the last fifteen years (1920-1935)]. Sofia, Druzhestvo za narodno zdrave. 47 p. (In Bulgarian).

Markov K. 1950. Epidemiologiya na malariyata v Bulgariya [Epidemiology of the malaria in Bulgaria]. In: II Nauchen sabor na balgarskite lekari. Chast II, Malariya. pp. 3-27. (In Bulgarian).

Markov K. \& Morov T. 1929. Nyakoi novi danni varhu raprostranenieto na malarichnite komari v Bulgariya [Some new data on the distribution of the malaria mosquitoes in Bulgaria]. Bulgarska klinika 1 (9): 669680. (In Bulgarian).

Markova E. 1994a. Beitrag zur erforschung der Struktur der Syrphidenzönosen (Syrphidae, Diptera) in der natürlichen Grassökosystemen und einige grundliche type Agroökosysteme der Mittleren Donauebene und der nordlichen teile des Mittleren Vorbalkans. Annuaire de l'Universite de Sofia "St. Kliment Ohridski", Faculte de Biologie (1 - Zoologie) 84: 43-57. (In Bulgarian with German summary).

Markova E. 1994b. Hover-flies (Syrphidae, Diptera) in base type agroecosystems in Radomir Valley. Annuaire de l'Universite de Sofia “St. Kliment Ohridski”, Faculte de Biologie (1 - Zoologie) 85: 53-67. (In Bulgarian with English summary).

Markova E. 1994c. Hover-flies (Syrphidae, Diptera) in natural grass ecosystems in Radomir Valley. Annuaire de l'Universite de Sofia "St. Kliment Ohridski", Faculte de Biologie (1 - Zoologie) 85: 69-83. (In Bulgarian with English summary).

Markova E. 1997. Syrphid flies in natural grass ecosystems of some areas of the Kraishte region (Bulgaria). Spixiana 20 (1): 73-79.

Markova E. 1998. Syrphid flies (Syrphidae, Diptera) in agroecosystems of the Sofia Valley. Annuaire de l'Universite de Sofia "St. Kliment Ohridski", Faculte de Biologie (1 - Zoologie) 88-90: 65-74.

Markova E. 1998a. Structure of the syrphid coenosis (Syrphidae, Diptera) in conditions of increased natural radioactive background. Acta Entomologica Bulgarica 4 (2, 3, 4): 44-50.

Markova E. 2003. Structure of the syrphid communities (Syrphidae, Diptera) in natural grass ecosystems and some types of agroecosystems of the Kazanlak Valley. Annuaire de l'Universite de Sofia "St. Kliment Ohridski”, Faculte de Biologie (1 - Zoologie) 93-94: 5-18.

Markova E. 2006. Hoverflies (Syrphidae, Diptera) from the Rhodopes, Bulgaria. In: Beron P. (Ed.). Biodiversity of Bulgaria. 3. Biodiversity of Western Rhodopes (Bulgaria and Greece) I. Pensoft \& National Museum of Natural History, Sofia, 675-688.

Markova E. 2011. Hover flies (Syrphidae, Diptera) from Strandzha Mountain, Bulgaria. Silva Balcanica 12 (1): 81-90.

Markova E. \& Alexiev A. 2000. Structure of the syrphid communities (Syrphidae Diptera) in natural grass- and wheat ecosystems in the Burgas Valley. Annuaire de l'Universite de Sofia "St. Kliment Ohridski", Faculte de Biologie (1 - Zoologie) 91: 5-18.

Markova E. \& Dimcheva P. 1998. Influence of the phosphore-organic insecticide chlorpyrifos on the structure of the syrphid coenosis (Syrphidae, Diptera) in a cabbage ecosystem. Acta Entomologica Bulgarica 4 (2, 3, 4): 37-43. (In Bulgarian with English summary).

Markova E. \& Tsonev I. 1993. Beitrag zur kenntnis der Schwebfliegen (Syrphidae, Diptera) in den Agroökosystemen des Talkessels von Zlatitza. Annuaire de l'Universite de Sofia "St. Kliment Ohridski", Faculte de Biologie (1 - Zoologie) 82: 197-209. (In Bulgarian with German summary).

Martinek V. 1987. Prehled druhu celedi Heleomyzidae (Diptera-Acalyptrata) ve sbirce hmyzu Narodniho Muzea v Praze. Casopis Narodniho Muzea, Rada prirodovedna 256 (1-4): 36-42.

Mathis W. \& Munari L. 1996. World catalog of the family Thethinidae (Diptera). Smithsonian contributions to zoology 527, p. 27.

Mathis W. \& Zatwarnicki T. 1995. World catalogue of shore flies (Diptera: Ephydridae). Memoirs on Entomology International $4.439 \mathrm{p}$. 
McAlpine J. F. 1989. Phylogeny and classification of the Muscomorpha. In: McAlpine J. F. \& Wood D. M. (Eds.). Manual of Nearctic Diptera 3. Ottawa, Research Branch Agriculture Canada. pp. 1397-1518.

Medlock J., Hansford K., Versteirt V., Cull B., Kampen H., Fontenille D., Hendrickx G., Zeller H., Bortel W. \& Schaffner F. 2015. An entomological review of invasive mosquitoes in Europe. Bulletin of Entomological Research 105 (6): 637-663.

Mendl H. 1986. Stelzmücken (Limoniidae) aus Bulgarien (Diptera, Nematocera). Spixiana 9 (3): 275-282.

Merz B. 2002. A revision of the Herina lugubris species group (Diptera, Ulidiidae, Otitinae), with the description of two new species. Revue Suisse de Zoologie 109 (2) : 407-431.

Meunier F. 1897. Quelques mots sur les faunes Hyménoptérologiques et Diptérologiques de la Bulgarie septentrionale. Annales de la Societe des Sciences de Bruxelles 22: 348-350.

Mihailova-Neikova M. 1961. Hydrobiological research of the Mandra Lake with regard to its importance as a fishing ground. Annuaire de l'Université de Sofia, Faculté de Biologie, Géologie et Géographie 53 (1 Zoology): 57-123. (In Bulgarian with English summary).

Michailova P. 1973a. Triploidy in Clunio marinus Haliday from the Bulgarian Black Sea coast. Comptes redus de l'Académie bulgare des Sciences 26 (11) : 1541-1543.

Michailova P. 1973b. Untersuchungen über den Chromosomenpolymorphismus bei Chironomus salinarius Kieff., Chironomus valkanovi Michailova und Chironomus anchialicus Michailova (Diptera, Chironomidae) von der bulgarischen Schwarzmeerküste. Zoologischer Anzeiger 191 (5/6): 348-364.

Michailova P. 1974. Zwei neue Arten der Gattung Chironomus (Diptera, Chironomidae) von der bulgarischen Schwarzmeerküste. Zoologische Beiträge 20 (2): 339-357.

Michailova P. 1975. Karyological characteristics of Cricotopus varians Staeg. and Cricotopus vitripennis Meig. (Diptera, Chironomidae) from the Bulgaria Black Sea coast. Acta zoologica bulgarica 2: 25-37. (In Bulgarian with English summary).

Michailova P. 1976a. Cytotaxonomical diagnostics of species from the genus Cricotopus (Chironomidae, Diptera). Caryologia 29 (3): 291-306.

Michailova P. 1976b. An additional b chromosome in Thalassomyia frauenfeldi Schiner (Chironomidae, Diptera) from Bulgaria’s Black Sea coast. Comptes rendus de $1 \mathbb{\text { }}$ Académie bulgare des Sciences 27 (2): 267-269.

Michailova P. 1977. Karyotaxonomische Charakteristik der Prodiamesa olivacea Meigen und Prodiamesa bureschi sp. n. (Diptera, Chironomidae). Zoologische Beiträge 23: 387-403.

Michailova P. 1978. Caryological characteristics of Cricotopus algarum Kieffer (Diptera, Chironomidae). Cytologia 43: 187-196.

Michailova P. 1979a. Comparative karyological analysis of the species of the genus Glyptotendipes Kieff. (Diptera, Chironomidae). Caryologia 32 (1) :23-44.

Michailova P. 1979b. The karyotypic variation og Glyptotendipes barbipes (Diptera, Chironomidae) from various localities. Tsitologia 21 (8): 959-964. (In Russian with English summary).

Michailova P. 1980a. A review of the European species of genus Clunio Haliday, 1855 (Diptera, Chironomidae). Zoologischer Anzeiger 205 (5/6): 417-432.

Michailova P. 1980b. The chromosomal polymorphism of some species of the family Chironomidae, Diptera. Acta Universitatis Carolinae - Biologica 1978: 141-149.

Michailova P. 1982a. Chironomids (Diptera, Chironomidae) in fish raising pools in Bulgaria. Acta zoologica bulgarica 20: 51-68. (In Bulgarian with English summary).

Michailova P. 1982b. External morphological and karyological characteristics of Orthocladius bipunctellus Zett., 1850 (Diptera, Chironomidae). Zoologischer Anzeiger 208 (1/2): 82-91.

Michailova P. 1985a. Cytotaxonomic review of some species of the genus Orthocladius Van der Vulp (Diptera, Chironomidae). Entomologische Abhandlungen Staatliches Museum für Tierkunde Dresden 48 (9): 149-166.

Michailova P. 1985b. Tendencies in the karyotype evolution of species of the family Chironomidae (Diptera). Acta zoologica bulgarica 26: 3-22.

Michailova P. 1987a. Comparative karyological analysis of species of the genus Endochironomus Kieffer (Diptera: Chironomidae. Entomologica scandinavica, Suppl. 29: 105-111.

Michailova P. 1987b. Comparative karyological studies of three species of the genus Glyptotendipes Kieff. (Diptera, Chironomidae) from Hungary and Bulgaria and Glyptotendipes salinus sp.n. from Bulgaria. Folia Biologica (Kraków) 1-2: 43-56. 
Michailova P. 1988. A revision of the genus Polypedilum Kieffer the cytotaxonomy of Polypedilum aberrans Tshernovskji. Spixiana, Supplement 14: 239-246.

Michailova P. 1989. The polytene chromosomes and their significance to the systematics and phylogeny of the family Chironomidae, Diptera. Acta Zoologica Fenica 186: 1-107.

Michailova P. 1992. Endochironomus tendens (F.) (Chironomidae, Diptera) an example of stasipatric speciacion. Netherlands journal of aquatic ecology 26 (2-4): 173-180.

Michailova P. 1994. Chironomus bonus Shilova et Dyvarsheishvili (Diptera, Chironomidae) from Bulgaria; karyotype and morphology. Studia dipterologica 1 (2): 186-194.

Michailova P. 1995. Contribution to the knowledge of Glyptotendipes pallens (Meigen, 1804) and Glyptotendipes glaucus (Meigen, 1818) (Insecta: Diptera: Chironomidae). Annalen des Naturhistorischen Museums in Wien 97B: 395-410.

Michailova P. 1996. Cytotaxonomy of Chironomidae (Diptera) from Lake Shabla (Bulgaria): cytogenetic evidence for introgressive hybridization. Hydrobiologia 318: 25-42.

Michailova P. 1998a. Order Diptera. Family Chironomidae. In: Michev T., Georgiev B., Petrova A. \& Stoyneva M. (Editors). Biodiversity of the Srebarna Biosphere Reserve. Checklist and bibliography. Sofia, Context \& Pensoft. p. 80.

Michailova P. 2004. Chironomus usenicus (Diptera: Chironomidae) from Bulgaria - external morphologi and karyotype. Acta zoologica bulgarica 56 (1): 3-14.

Michailova P. 2006. Family Chironomidae (Diptera) from the Rhodopes Mountains. In: Beron P. (Ed.). Biodiversity of Bulgaria. 3. Biodiversity of Western Rhodepes (Bulgaria and Greece) 1. Sofia, National Museum of Natural History \& Pensoft. pp. 711-718.

Michailova P. 2009. Karyotype and external morphology of Dicrotendipes lobiger Kieffer, 1921 (Diptera: Chironomidae) from some regions in Bulgaria. Acta zoologica bulgarica 61 (2): 115-121.

Michailova P. \& Beschovski V. 1985. Karyotaxonomical studies on genus Oscinella (Diptera, Chloropidae). Acta Zoologica Bulgarica 29: 25-35.

Michailova P. \& Dimitrova B. 1984. Caryological characteristics of Acricotopus lucens (Zett.) (Diptera, Chironomidae) from Bulgaria. Comptes rendus de $\mathbb{1}$ A Académie bulgare des Sciences 37 (8): 1081-1084.

Michailova P. \& Gercheva P. 1982. Cytotaxonomical characteristics of the species of genus Endochironomus Kieff. (Diptera, Chironomidae). Caryologia 35 (1): 33-56.

Michailova P., Ilkova J., Dean A. \& White K. 2015. Cytogenetic index and functional genome alterations in Chironomus piger Strenzke (Diptera, Chironomidae) in the assessment of sediment pollution: A case study of Bulgarian and UK rivers. Ecotoxicology and Environmental Safety 111: 220-227.

Michailova P., Ilkova J. \& White K. 2016. Implications of genome alterations in Chironomus bernensis Klötzli (Diptera) for assessment of trace metal pollution in two Bulgarian rivers. River research and applications, 32: 914-924.

Michailova P., Ilkova J., Szarek-Gwiazda E., Kownacki A. \& Ciszewski D. 2018. Genome instability in Chironomus annularius sensu Strenzke (Diptera, Chironomidae): A biomarker for assessment of the heavy metal contaminants in Poland. Journal of Limnology 77(s1): 15-24; DOI: 10.4081/jlimnol.2018.1710

Michailova P., Kownacki A., Woźnicka O., White K., Dean A. \& Szarek-Gwiazda E. 2014. Micropelopia nebulosa group (Diptera, Chironomidae, Tanypodinae) - karyotype and morphology of larvae and pupae. Zootaxa 3852 (1): 83-100.

Michailova P. \& Krastanov B. 2000. Cytotaxonomical differentiation oh Chironomus plumosus group (Diptera: Chironomidae) from fish pools near Plovdiv, Bulgaria. Acta zoologica bulgarica 52 (1): 2940.

Michailova P. \& Petrova N. A. 1987. The peculiarities of the karyotype of Micropsectra gr. notescens (Diptera, Chironomidae) taken from different populations. Tsitologia 24 (9): 1056-1061. (In Russian with English summary).

Michailova P. \& Petrova N. A. 1989. Microevolution differentiation of Pseudodiamesa gr. branickii Nowicki (Chironomidae, Diptera). Tsitologia 31 (7): 824-830. (In Russian with English summary).

Michailova P. \& Sella G. \& Petrova N. 2012. Polytene chromosomes of Chironomidae (Diptera) as a bioassay of tracemetal-induced genome instability. Proceedings of the $18^{\text {th }}$ International Symposium - Fauna norvegica 31: 227-234.

Michailova P. \& Todorova J. 1998. Cytogenetic characteristics of Glyptotendipes pallens (Diptera, Chironomidae) reared in standard laboratory conditions. Cytobios 94: 151-160. 
Michailova P \& White K. 2007. Morphology of the ventromental plates of two cytotypes of Kiefferulus tendipediformis (Goethgebuer, 1921) (Diptera, Chironomidae). Comptes rendus de $1 \mathbb{\nabla}$ Académie bulgare des Sciences 60 (7): 775-778.

Mielczarek Ł. 2018. New records of Mythicomyiidae and Bombyliidae (Usiinae) for Poland, Bulgaria, Ukraine and Iran. Dipteron 34: 68-79.

Mikov O. 2005. History of investigations of malaria mosquitoes in Bulgaria. Infectology 42 (4): 39-47. (In Bulgarian).

Mikov O. 2008a. Biologichni, ekologichni i epidemiologichni prouchvaniya na anofeliyni komari v nyakoi regioni na Bulgariya [Biological, ecological and epidemiological studies of the anopheline mosquitoes in some regions of Bulgaria]. Ph.D. thesis, Sofia, National Center of Infectious and Parasitic Diseases. 155 p. (In Bulgarian).

Mikov O. 2008b. Determination of the season of possible malaria transmission by anopheline mosquitos in South-Western Bulgaria. Meditsinskaya Parazitologiya i Parazitarnye Bolezni 1: 31-33.

Mikov O. 2011. Razprostranenie na komarite, potentsialni prenositeli na virusa Zapaden Nil v Bulgariya [Distribution of the mosquitoes, potential carriers of the West Nile virus in Bulgaria]. 9 Nationalen kongres po klinichna mikrobiologiya i infektsiya na Balgarskata asotsiatsiya na mikrobiolozite. Plovdiv, 28-30. IV.2011. In: Sbornik nauchni trudove. Plovdiv. pp. 45-46.

Mikov O., Vuchev O. \& Lalkovski N. 2011. Razprostranenie na komarite, potentsialni prenositeli na dinofilariozi po horata i zhivotnite $\mathrm{v}$ Bulgariya [Distribution of the mosquitoes, potential carriers of human and animal dinofilariasis in Bulgaria]. In: Sbornik dokladi i posteri ot yubileynata nauchna sesiya po sluchay 110 godini NDNIVMI. Sofia, Bulgarian Food Safety Agency. pp 152-156.

Mikov O., Nikolov G., Schaffner F. \& Mathis A. 2013. First record and establishment of Aedes albopictus in Bulgaria. In: VBORNET-EMCA Joint Meeting "Invasive mosquitoes and public health in the European context", Antwerp, Belgium, 28-29 November 2013 (Conference paper).

Minář J. 1990. Family Culicidae. In: Soós Á. \& Papp L. (Eds.). Catalogue of Palaearctic Diptera. 2. Budapest, Akadémiai Kiadó, Hungarian Natural History Museum. pp. 74-113.

Minarzh Y. \& Christova T. 1971. Materialy po izucheniyu fauny moshek i komarov v Bolgarii (Diptera: Simulidae, Culicidae) [Materials on the study of the fauna of black flies and mosquitoes in Bulgaria (Diptera: Simulidae, Culicidae)]. Trudy nauchno-issledovatelskogo instituta epidemiologii i mikrobiologii 15: 245-247. (In Russian).

Mirchev P., Georgiev G. \& Hubenov Z. 2000. Peribaea apicalis R.-D. (Diptera, Tachinidae) - A new species for the fauna of Bulgaria and new parasitoid of Operophthera brumata (L.) (Lepidoptera: Geometridae). Forest Science 4: 89-90.

Mirchev P., Georgiev G. \& Tsankov G. 1999. Parasitoids of noxious lepidopterian (Lepidoptera) insect pests in oak forests in Bulgaria. 3. Tachinidae (Diptera). Forestry Ideas 1 (18): 74-79. (In Bulgarian with English summary).

Minchev B. \& Mincheva P. 1967. Brezovata galitsa - opasen nasekomen vreditel [Birch gall - a dangerous insect pest]. Gorsko stopanstvo 23 (8): 21-24. (In Bulgarian).

Mirčeva M. 1974. Prouchvaniya varhu ekzofilnite sinantropni muhi v rayona na grad Tolbuhin [Studies on the exophilic synanthropic flies in the area of Tolbuhin Town]. Letopisi HEI 44: 88-97. (In Bulgarian).

Mirčeva M. 1977. Biologichni i ekologichni osobenosti na nay-chesto sreshtanite sinantropni muhi v razlichni naseleni mesta [Biological and ecological features of the most common synanthropic flies in the different places]. Letopisi HEI 5: 123-133. (In Bulgarian).

Mirčeva M. 1978. Produktivnost na populatsiite na sinantropnite muhi v nyakoi biotopi [Productivity of the synanthropic flies populations in some biotopes]. Letopisi HEI 6: 153-161. (In Bulgarian).

Mirčeva M. 1979. Harakteristika na endofilnite muhi, obitavashti razlichni tipove obekti [Characteristic of the endophilic flies inhabiting different types of objects]. Letopisi HEI 4: 164-170. (In Bulgarian).

Mirčeva M. 1981. Prouchvaniya varhu ekologichnite osobenosti, epidemiologichnoto znachenie na muhite i borbata s tyah $\mathrm{v}$ Tolbuhinski okrag [Studies on the ecological features, epidemiological significance of the flies and their control in the Tolbukhin district]. Ph.D. thesis, Sofia, National Center of Infectious and Parasitic Diseases. 198 p. (in Bulgarian).

Mirchev P. 1993. Of the ecology of Thecodiplosis brachyntera (Schwägr) (Diptera, Cecidomyidae). Nauka za gorata 2: 83-88. (In Bulgarian). 
Mondchadskiy A. 1951. Lichinki krovososushtih komarov SSSR i sopredelnyh stran (podsem. Culicidae) [Larvae of blood-sucking mosquitoes of the USSR and neighboring countries (subfamily Culicidae)]. Opredeliteli po faune SSSR. Leningrad, Zoologicheskim institutom Akademii nayk USSR. 376 p.

Mohrig W. \& Dimitrova B. 1992. Neue Arten der Gattung Corynoptera Winnertz aus Bulgarien (Insecta, Diptera: Sciaridae). Reichenbachia 29 (29): 177-185.

Mohrig W., Dimitrova B. \& Mamaev B. 1992. Beitrag zur Tauermücken-Fauna (Dipt., Sciaridae). Entomologische Nachrichten und Berichte 36 (3): 197-202.

Mohrig W. \& Dimitrova B. 1993. Zwei neue Arten der Gattung Epidapus Hal. Aus Bulgarien (Insecta: Diptera: Sciaridae). Reichenbachia 30 (15): 99-102.

Momchilov A. 1962. Rolyata na karantinnata inspektsiya vav Varna po opazvaneto na stranata ot karantinni obekti [The role of the quarantine inspection in Varna in the protection of the country from quarantine sites]. Rastitelna zashtita 10 (3): 9-11. (In Bulgarian).

Moskova G. \& Uzunov Y. 2011. The macrozoobenthos of the Rilska River, Southwest Bulgaria. Annuaire de l'Université de Sofia „St. Kliment Ohridski”, Faculte de Biologie 99 (1 - Zoology): 53-70.

Moucha J. 1962. Tabanidae und Asilidae (Diptera) aus Albanien. Acta faunistica entomologica Musei nationalis Prague 8: 21-35.

Moucha J. 1970. Die Tabaniden Fauna Osterreichs (Diptera, Tabanidae). Annalen des Naturhistorischen Museums Wien 74: 211-219.

Moucha J. 1976. Horse-flies (Diptera, Tabanidae) of the world synoptic catalogue. Acta Entomologica Musei Nationalis Prague, Suppl. 7: 1-319.

Moucha J. \& Chvála M. 1961. A contribution to knowledge of the Tabanidae (Diptera) of Bulgaria. Acta faunistica entomologica Musei nationalis Prague 7: 31-41.

Moucha J. \& Chvála M. 1963. Ergebnisse der Albanien-Expedition 1961 des Deutschen Entomologischen Institutes. 5. Beitrag: Diptera, Tabanidae. Beiträge zur Entomologie 13 (1/2): 25-39.

Moucha J. \& Chvála M. 1964. Notes on the genus Therioplectes Zeller, 1842 (Diptera, Tabanidae). Casopis Ceskoslovenske Spolenosti Entomologicke 61 (2): 100-105.

Müller P. 1974. Aspects of zoogeography. The Hague, W. Junk Publishers. 208 p.

Müller P. 1980. Biogeographie. Stuttgart, Ulmer. 414 p.

Munari, L. 1991. Contributo alla conoscenza dei Tethinidae afrotropicali. V. Aggiornamenti sistematici e biogeografici (Diptera Acalyptratae). Bollettino della Società Entomologica Italiana, Genova 123 (2): 165-170.

Munari L. 1996. Tethinidae (Diptera) in the Museum of Zoology, Lund University. Strobaeana 6: 1-11.

Munari L. 2002. Beach flies (Diptera: Tethinidae) of the Palaearctic Region: an annotated checklist, including world distribution. Sosieta Veneziana di Sciance Naturali 27: 17-25.

Munari L. \& Mathis W. 2010. World Catalogue of the family Canacidae (including Tethinidae) (Diptera), with keys to the supraspecific taxa. Zootaxa 2471: 1-184.

Nachev N. 1983. The distribution of Chironomid larvae as affected by the saprobic conditions in the lower course of the Blagoevgradska Bistritza and in the Struma Rivers at the point of their infusion. Hydrobiology 19: 40-48. (In Bulgarian with English summary)

Nachev P. 1964. Materiali po morfologiyata i biologiyata na slivovata papkova shikalkotvorka Ischnonyx (Asphondilia) prunorum Wachtl. (Diptera) [Materials on the morphology and biology of the plum bud Ischnonyx (Asphondilia) prunorum Wachtl. (Diptera)]. Gradinarska nauka i lozarska nauka 1 (6): 39-46. (In Bulgarian).

Nachev P. 1976. Entomologiya na tropika i subtropika [Entomology of the tropics and subtropics]. Sofia, Zemizdat. 308 p. (In Bulgarian).

Nagatomi. A. 1996. An essay on phylogeny of the orthorraphous Brachycera (Diptera). Entomologist's Monthly Magazine 132: 95-148.

Naidenov W. 1962. Bulgarische Itonidinae (Diptera, Itonididae) und ihre Gallbildungen. Bulletin de l'Institut de zoologie et musée 11: 141-165. (In Bulgarian with German summary)

Naidenov W. 1963. Shikalkoobrasuvane [Gallnuts formation]. Priroda i znanie 16 (1): 21-23. (In Bulgarian).

Nartshuk E. P. 1984. Family Chloropidae. In: Soós Á. \& Papp L. (Eds.). Catalogue of Palaearctic Diptera. 10. Budapest, Akadémiai Kiadó, Hungarian Natural History Museum. pp. 222-299. 
Nartshuk E. P. 1988. Family Acroceridae. In: Soós Á. \& Papp L. (Eds.). Catalogue of Palaearctic Diptera. 5. Budapest, Akadémiai Kiadó, Hungarian Natural History Museum. pp. 186-197.

Nartshuk E. P. 1992. Revision on the species of Meromyza Meigen (Diptera, Chloropidae) from Finland. Entomologica Fennica 3: 121-138,

Nartshuk E. P. 2003. Key to families of Diptera (Insecta) of the fauna of Russian and adjacent countries. St. Petersburg, Proceedings of the Zoological Institute 294, Russian Academy of Sciences. 251 p. (In Russian).

Nartshuk E. P. 2004a. Chloropidae. In Papp T. (Ed.). Fauna Europaea: Diptera Brachycera, version 1.3. http:// www.faunaeur.org, last ubdate 2007.

Nedelchev N. 2013. Kulikoidite v Bulgariya [Culicidae in Bulgaria]. Sofia, NTS. 211 p. (In Bulgarian).

Nedelkov N. 1909. Nashata entomologichna fauna [Our entomological fauna]. Arhiv na Ministerstvoto na narodnoto prosveshtenie 1 (3): 83-135. (In Bulgarian).

Nedelkov N. 1910. Betrag zu Diptera Bulgariens. Internationale Entomologische Zeitschrift 4 (7): 36-37.

Nedelkov N. 1912. Sexthe contribution to the entomlogical fauna of Bulgaria. Revue Academy Sciences de Bulgarie 2: 177-218. (In Bulgarian).

Negrobov O. 1991. Family Dolichopodidae. In: Soós Á. \& Papp L. (Eds.). Catalogue of Palaearctic Diptera. 7. Budapest, Akadémiai Kiadó, Hungarian Natural History Museum. pp. 11-139.

Negrobov O. \& Kechev M. 2010. A new species of the genus Dolichopus Latreille, 1796 (Dolichopodidae: Diptera) from Pirin Mountain, Bulgaria. Journal of the Entomological Research Society 12 (3): 71-73.

Negrobov O. \& Kechev M. 2012. A new species of the genus Syntormon Loew from Bulgaria (Diptera, Dolichopodidae). Dipterists Digest 19: 93-96.

Nikolova W. 1943. Zeleviyat komar Contarinia torquens Meig. i borbata sreshtu nego [Contarinia torquens Meig. and the fight against it]. Gradinarstvo 24 (2): 27-29. (In Bulgarian).

Nikolova W. 1945. Po-vazhni nepriyateli po zeleto u nas i borbata s tyah [More important pests of the cabbage in the country and the fight against them]. Gradinarstvo 26 (10): 12-15. (In Bulgarian).

Nikolova W. 1946. Zelevata noshtenka Mamestra (Barathra) brassicae L. kato nepriyatel na zeleto v Bulgaria i borbata sreshtu neya [Cabbage nightshade Mamestra (Barathra) brassicae L. as an pest of cabbage in Bulgaria and the fight against it]. Zemedelska nauka 1 (1): 123-156. (In Bulgarian).

Nikolova W. 1948. Esennata oran i unishtozhavaneto na rastitelnite ostatatsi kato myarka za borba s nepriyatelite i bolestite po zelenchutsite [Autumn ploughing and destruction of crop residues as a measure to fight against pests and diseases of the vegetables]. Gradinarstvo 29 (9): 273-274. (In Bulgarian).

Nikolova W. 1949a. Zelevata dalgonozhka kato nepriyatel na zelenchukovite kulturi u nas [Tipula oleracea as an enemy of the vegetable crops in Bulgaria]. Gradinarstvo 30 (7): 207-210. (In Bulgarian).

Nikolova W. 1949b. Zeleva muha, Chortophila (Hylemyia) brassicae Buché [Cabbage fly, Chortophila (Hylemyia) brassicae Buché]. Gradinarstvo 30 (4): 117-120. (In Bulgarian).

Nikolova W. 1950. Raspberry gall fly Lasioptera rubi and the fight against it. Gradinarstvo i lozarstvo 31 (3): 86-88.

Nikolova W. 1953. Edin nov nepriyatel po chesana - chesnovata muha Suillia lurida Meig. (predvaritelno saobshtenie) [A new pest of garlic - the garlic fly Suillia lurida Meig. (advance notice)]. Byuletin po rastitelna zshtita 2 (2): 17-20. (In Bulgarian).

Nikolova W. 1958. Suillia lurida Meig. (Dipt., Helomyzidae). Ein neuer Schädling der Kulturpflanzen. Comptes rendus de l'Academie bulgare des Sciences 11(4): 301-304.

Nikolova W. 1959. Prouchvaniya varhu Suillia (Helomyza) lurida Meig. (Dipt.) - nepoznat dosega nepriyatel na kulturnite rasteniya [Studies on the Suillia (Helomyza) lurida Meig. (Dipt.) - unknown so far pest of the cultivated plants]. Bulletin de l'Institut zoologique de l'Academie des sciences de Bulgarie 8: 205-234. (In Bulgarian).

Nikolova W. 1960. Opiti za borba sreshtu chesnovata muha Suillia (Helomyza) lurida Meig. (Dipt.) [Attempts to control the garlic fly Suillia (Helomyza) lurida Meig. (Dipt.)]. Nauchni trudove na Tsentralniya nauchnoizsledovatelski institut za zashtita na rasteniyata 3: 191-200. (In Bulgarian).

Nikolova W. 1961. Danni varhu biologiyata na ipsilonovata noshtenka (Agrotis ypsilon Roh. $=$ Feltia, Rhyacia ypsilon Roh.) i opiti za borba [Data on the biology of the Agrotis ypsilon Roh. = Feltia, Rhyacia ypsilon Roh. and attempts to control]. Izvestiya na IZR 1: 83-108. (In Bulgarian).

Nikolova W. 1962. Pochvoobrazuvashti nasekomi i tyahnoto znachenie za selskoto stopanstvo [Soil-forming insects and their importance for agriculture]. Priroda 11 (2): 92-96. (In Bulgarian). 
Nikolova W. 1963. Prouchvaniya varhu chesnovata muha Suillia (Helomyza) lurida Meig. (Dipt.: Helomyzidae) [Studies on the garlic fly Suillia (Helomyza) lurida Meig. (Dipt.: Helomyzidae)]. Ph.D. thesis, Sofia, Institut po zashtita na rasteniata. 100 p. (In Bulgarian).

Nikolova W. 1964. Pochveni nasekomi - nepriyateli na tsarevitsata v Bulgaria [Soil insects - pests of corn in Bulgaria]. Priroda 13 (6): 85-89. (In Bulgarian).

Nikolova W. 1967. Entomocenologic and biologic studies of Rosa damascena Mill. Plantations. 1. Lepidoptera. Bulletin de l'Institut de zoologie et musée 25: 101-128. (In Bulgarian with English summary).

Nikolova W. 1972. Entomocenologic and biologic studies of Rosa damascena Mill. Plantations. 4. Hymenoptera and Diptera. Bulletin de l'Institut de zoologie et musée 35: 107-138. (In Bulgarian with English summary).

Nikolova W. \& Natskova V. 1965. Vredni i polezni za selskoto stopanstvo kosmati komari - sem. Bibionidae (Diptera, Nematocera) [Harmful and useful for the agriculture march flies - Bibionidae (Diptera, Nematocera)]. Priroda 14 (5): 87-80. (In Bulgarian).

Nikolova W. \& Popoff W. 1957. Novi vazmozhnosti za borba sreshtu zelevata muha [New opportunities of fight against the cabbage fly]. Byuletin po rastitelna zashtita 6 (1/9): 29-32. (In Bulgarian).

Nowakowski J. 1073. Monographie der europäischen Arten der Gattung Cerodonta Rond. (Diptera, Agromyzi9dae). Annales Zoologici, Warszawa 31 (1): 1-327.

Nowosad A., Batchvarov G. \& Petrov P. 1987. Bat flies (Nycteribiidae, Diptera) of bats collected in Bulgaria. Polskie Pismo Entomologiczne 57: 673-694.

O'Hara J. E., Henderson S. J. \& Wood D. M. 2020. Preliminary checklist of the Tachinidae of the world. Version 2.0. PDF document, 1039 pages. Available at: http://www.nadsdiptera.org/Tach/WorldTachs/Checklist/ Worldchecklist.html (accessed [insert date accessed]).

Olejníček J. 1999. Chrysotus polleti sp.n. (Dolichopodidae) from Bulgaria and a note on the morphology of the Diaphorini, Biologia, Bratislava 54 (2): 159-164.

Olejníček J. \& Barták M. 1997. Some faunistically interesting Dolichopodidae (Diptera) from Europa. In: Vanfara J. \& Rozkosny R. (Eds.). Dipterologica bohemoslovaca. 8. Folia Fac. Sci. Nat. Univ. Masaryk. Brun., Biol., 95: 137-139.

Olsufjev N. 1970. New and little known Tabanidae (Diptera) from fauna of the USSR and neighbouring contries. Entomological Reviev USSR 49 (3): 683-687. (In Russian with French summary).

Olsufjev N. 1977. Tabanidae. In: Fauna USSR 7 (2): 1-434. (In Russian)

Olsufjev N., Moucha M \& Chvála M. 1967. Zur Taxonomie und Verbreitung der europäischen und kleinasiatischen Arten der Tabanus bovinus - Gruppe (Diptera, Tabanidae). Acta Entomologica Bohemoslovaca 64: 303-313.

Oosterbroek P. 2006. The European families of the Diptera. Identification, diagnosis, biology. KNNV Publishing. 209 p. DOI: $10.1163 / 9789004278066$

Oosterbroek P. 2009a. Catalogue of the Craneflies of the World (Diptera, Tipuloidea: Pediciidae, Limoniidae, Cylindrotomidae, Tipulidae). Available at: http://nlbif.eti.uva.nl/ccw/ (version 03 Feb 2009), (Accessed on 29 March 2009).

Oosterbroek P. 2009b. New distributional records for Palaearctic Limoniidae and Tipulidae(Diptera: Craneflies), mainly from the collection of the Zoological Museum, Amsterdam. In: Lantsov V. (Ed.). Crane flies. History, taxonomy and ecology (Diptera: Tipulidae, Limoniidae, Pediciidae, Trichoceridae, Ptychopteridae, Tanyderidae). Memorial volume dedicated to Dr. Charles Paul Alexander (1889-1981), Dr. Bernhard Mannheims (1909-1971) and Dr. Evgeniy Nikolaevich Savchenko (1909-1994). Zoosymposia 3: 179-197.

Oosterbroek P. 2017. Catalogue of the Craneflies of the World (Diptera, Tipuloidea: Pediciidae, Limoniidae, Cylindrotomidae, Tipulidae). ccw.naturalis.nl/ (Last update: 01 Nov 2017).

Oosterbroek P. \& Lantsov V. I. 2011. Review of the western Palaearctic species of Dolichopeza Curtis (Diptera, Tipulidae). Tijdschrift voor Entomologie 154: 269-281.

Oosterbroek P. \& Reusch H. 2008. Review of the European species of the genus Chionea (Dalman, 1816) (Diptera, Limoniidae). Braunschweiger Naturkundliche Schriften 8: 173-220.

Oosterbroek P. \& Theowald Br. 1992. Family Tipulidae. In: Soós Á., Papp L. \& Oosterbroek P. (Eds.). Catalogue of Palaearctic Diptera. 1. Budapest, Akadémiai Kiadó, Hungarian Natural History Museum. pp. 56-178.

Pape T. 1996. Catalogue of the Sarcophagidae of the World (Insecta: Diptera). Memoirs on Entomology, International, 8: 1-568.

Pape T. \& Beuk P. 2017. Diptera. Fauna Europaea version 2017.06, https://fauna-eu.org 
Pape T., Blagoderov V. \& Mostovski M. 2011. Order Diptera Linnaeus, 1758. In: Zhang Z.-Q. (Ed.). Animal biodiversity: An outline of higher-level classification and survey of taxonomic richness. Zootaxa 3148: 222-229.

Papp L. 1981. New species and taxonomical data of the Palaearctic Lauxaniidae and Carnidae. Acta Zoologica Hungarica 27 (1-2): 159-186.

Papp L. 1984a. Family Lauxaniidae. In: Soós Á. \& Papp L. (Eds.). Catalogue of Palaearctic Diptera. 9. Budapest, Akadémiai Kiadó, Hungarian Natural History Museum. pp. 193-217.

Papp L. 1984b. Family Agromyzidae. In: Soós Á. \& Papp L. (Eds.). Catalogue of Palaearctic Diptera. 9. Budapest, Akadémiai Kiadó, Hungarian Natural History Museum. pp. 263-343.

Papp L. 1984c. Family Braulidae. In: Soós Á. \& Papp L. (Eds.). Catalogue of Palaearctic Diptera. 10. Budapest, Akadémiai Kiadó, Hungarian Natural History Museum. pp. 178-180.

Papp L. 1984d. Family Sphaeroceridae. In: Soós Á. \& Papp L. (Eds.). Catalogue of Palaearctic Diptera. 10. Budapest, Akadémiai Kiadó, Hungarian Natural History Museum. pp. 68-107.

Papp L. 1998. Family Braulidae. In: Papp L. \& Darvas B. (Eds.). Contributions to a Manual of Palaearctic Diptera 3. Higher Brachycera. Budapest, Science Herald. pp. 325-330.

Papp L. 2010. A new Cremifania species from Bulgaria (Diptera, Cremifaniidae), with a proposal for wing venation terms in higher Diptera. Annales historico-naturales Musei nationalis hungarici 102: 193-204.

Papp L. \& Darvas B. (Eds.). 1997. Contributions to a Manual of Palaearctic Diptera 2. Nematocera and Lower Brachycera. Budapest, Science Herald. 592 p.

Papp L. \& Darvas B. (Eds.). 1998. Contributions to a Manual of Palaearctic Diptera 3. Higher Brachycera. Budapest, Science Herald. 880 p.

Papp L. \& Darvas B. (Eds.). 2000a. Contributions to a Manual of Palaearctic Diptera 4: Appendix. Budapest, Science Herald. 604 p.

Papp L. \& Darvas B. (Eds.). 2000b. Contributions to a Manual of Palaearctic Diptera 1: General and Applied Dipterology. Budapest, Science Herald. 978 p.

Parent O. 1938. Dipteres Dolichopodides. Fauna de France 35. Paris, Lechevalier. 720 p.

Pârvu C. 1981. Data on the occurrence and distribution of some Tabanids (Diptera) in Romania. Travaux du Museum National d'Histoire Naturelle "Gr. Antipa" 23: 155-162.

Pârvu C. 1983. Tabanidae (Diptera) din colectiile muzeuliu de istorie naturala din Sibiu. Studii si Communicari-Stünt Naturala Muzeul Brukenthal 25: 303-313.

Paschev K. 1937. Oftalmomiazis externa Oestris ovis (prinos kam miazisite u nas) [Ophthalmomyiasis externa Oestris ovis (Contribution to myiasis in the country)]. Bulgarska klinika 2: 65-70.

Paspalev G. 1950. Izuchavane na malariynite komari v Iztochnite Rodopi [Study of the malaria mosquitoes in the Eastern Rhodopes]. II nauchen sabor na balgarskite lekari. II. Malariya. 92-100. (In Bulgarian).

Paspalev G. 1951. Recherches sur les musquets malariens dans la région des Rhdopes orientales. Buletin de l'Institut zoologique de l'Academie Bulgare des sciences 1: 200-233. (In Bulgarian with French summary).

Pavlov A. 1981. Paraziti po zhitnite piyavitsi (Coleoptera: Chrysomelidae) [Wheat leech parasites (Coleoptera: Chrysomelidae)]. Rastenievadni nauki 18 (4): 116-123. (In Bulgarian).

Pavlova A. 2020a. First study of fungus gnats (Insecta: Diptera: Sciaroidea) in Tisata Reserve (SW Bulgaria). ZooNotes 154: 1-4.

Pavlova A. 2020b. Izsledvaniya na gabni komari ot semeystvata Bolitophilidae, Diadocidiidae, Ditomyiidae, Keroplatidae, Mycetophilidae v kritichno zastrasheni mediteranski i submediteranski mestoobitaniya $\mathrm{v}$ Bulgariya [Studies of the fungal mosquitoes of the families Bolitophilidae, Diadocidiidae, Ditomyiidae, Keroplatidae, Mycetophilidae in the critically endangered Mediterranean and sub-Mediterranean habitats in Bulgaria]. Ph.D. thesis, Plovdiv, Plovdiv University „Paisii Hilendarski”. Faculty of Biology. 177 p. (In Bulgarian).

Pavlova A. 2020c. New and rare fungus gnats for the fauna of Bulgaria (Diptera: Nematocera: Mycetophilidae). Historia naturalis bulgarica $41: 27-31$.

Pavlova A. \& Stojanova A. 2020. Winter Activity of Fungus Gnats (Diptera: Mycetophilidae) in Criticallyendangered Mediterranean Habitats in Bulgaria. Acta zoologica bulgarica, Supplement 15: 129-140.

Pavlova M., Pehlivanov L., Kazakov S., Varadinova E., Vidinova Y., Tyufekchieva \& Uzunov Y. 2012. Changes in the aquatic communities in the Rhodopes Mountain landslide lakes (South Bulgaria) for the last 40 years. I. Taxonomic composition of macrozoobenthos, zooplancton and fish communities. Acta zoologica bulgarica, Supplementum $4:$ 187-195. 
Peck L. 1988. Family Syrphidae. In: Soós Á. \& Papp L. (Eds.). Catalogue of Palaearctic Diptera. 8. Budapest, Akadémiai Kiadó, Hungarian Natural History Museum. pp. 11-230.

Pelov V. 1999. Parasitoids on burch seed gall midge (Semudobia betulae (Winn.) Diptera, Cecidomyiidae) in Bulgaria. Acta Entomologica Bulgarica 5 (1): 72-76. (In Bulgarian with English summary)

Petkoff P. 1921. Masovo izmirane na chervenokriliya skakalets Caloptenus italicus през 1919 [The mass extinction of the red-winged grasshopper Caloptenus italicus in 1919]. Spisanie na zemedelskite izpitatelni instituti 2 (1/2): 48-60. (In Bulgarian).

Petkoff P. 1939. Predpazvane na posevite i gradinite ot vredni nasekomi [Protection of the crops and gardens from harmful insects]. Sofia, Izvori na znanieto 13. 112 p. (In Bulgarian).

Petkov P. 1964. Prouchvaniya varhu morfologichnite osobenosti na nyakoi shtraklitsi ot sem. Tabanidae ot Primorska i Iztochna Stara planina [Study on the morphological features of some Tabanidae from the seaside and Eastern Stara Planina Mts.]. Nauchni trudove na VSSI G. Dimitrov 15: 393-403.

Petrova N. A. \& Michailova P. 1989. Chromoseme polymorphism of natural populations of Endochironomus albipennis Meig. (Diptera, Chironomidae). Tsitologia 31 (10): 1200-1208. (In Russian with English summary).

Plassmann E. 1978.Neue Pilzmücken aus Schveden und Bulgarien. Senckenbergiana Biologica 59: 205-214.

Plassmann E. 1988. Family Bolitophilidae. In: Soós Á. \& Papp L. (Eds.). Catalogue of Palaearctic Diptera. 3. Budapest, Akadémiai Kiadó, Hungarian Natural History Museum. pp. 193-196.

Pollet M. 1996. Systematic revision and phylogeny of the Palaearctic species of the genus Achalcus Loew (Diptera: Dolichopodidae) with the decription of four new species. Systematic Entomology 21: 353-386.

Pollet M. \& Kechev M. 2007. A rewiew of Palaearctic Teuchophorus, with a new species from Bulgaria (Diptera: Dolichopodidae). Zootaxa 1592: 45-56.

Polukonova N., Belyanina S. \& Michailova P. \& Goligina V. 2005. Comparative analysis of the midges Chironomus nuditarsis and Ch. curabilis, karyoforms and karyofunds (Chironomidae, Diptera). Zoologicheskii zhurnal 84 (2): 195-206. (In Russian with English summary).

Pont A. 1986. Family Muscidae. In: Soós Á. \& Papp L. (Eds.). Catalogue of Palaearctic Diptera. 11. Budapest, Akadémiai Kiadó, Hungarian Natural History Museum. pp. 57-215.

Pont A. 2017. Fauna Europaea: Muscidae. In: Pape T. \& Beuk P. Diptera: Brachycera, version 2017.06; http// www.fauna-eu.org/ (accessed: 20.11.2019).

Popoff W. 1934. Nablyudeniya varhu tsarevichnata buhlatka [Heliotis obsoleta Fab. (H. armigera Hübn.)] v Obraztsov chiflik [Observations on the [Heliotis obsoleta Fab. (H. armigera Hübn.)] in Obraztsov Chiflik]. Spisanie na zemedelskite opitni instituti 6 (3/4): 53-73. (In Bulgarian).

Popoff W. 1939a. Referate u. Mitteilungen. Mitteilungen der Bulgarischen Entomologischen Gesellschaft in Sofia 10: 170-175. (In Bulgarian).

Popoff W. 1939b. Skladovi nasekomi v Bulgariya i borbata s tyah [Storage insects in Bulgaria and their control]. Sofia, Institut za zshtita na rasteniyata v Sofia. 184 p. (In Bulgarian).

Popoff W. 1941. Zhilishtnite nasekomi v Bulgariya I borbata s tyah [Dwelling insects in Bulgaria and their control]. Sofia, Izvori na znanieto 17. 166 p. (In Bulgarian).

Popoff W. 1948. Nepriyateli po skladiranite produkti i materiali v Bulgariya i borbata s tyah [Pests on the stored products and materials in Bulgaria and the fight against them]. Sofia, Bulgarian Academy of Sciences. 414 p. (In Bulgarian).

Popoff W. 1956. Zemedelska entomologia. Sofia, Zemizdat. 464 p. (In Bulgarian).

Popoff W. \& Nikolova W. 1958. Nepriyateli po zelenchukovite rasteniya v Balgariya i borbata s tyah. Sofia, Izdatelstvo na BAN. 225 p. (In Bulgarian).

Popov A. 1968. A fly new to Bulgarian fauna and its peculiar way of life. Priroda 4: 55-57. (In Bulgarian).

Popov A. \& Bankov D. 1961. Prouchvane na hipodermatozata po govedata v stranata [Study on the bovine hypodermatosis in the country]. Izvestiya na tsentralniya veterinaren institut za zarazni i parazitni bolesti 1: 397-403. (In Bulgarian).

Popov A., Deltshev C., Hubenov Z., Beschovski V., Dobrev D., Gueorguiev B. 2000a. Invertebrate fauna. In: Popov A., Meshinev T. (Eds). High mountain treeless zone of the Central Balkan National Park. Biological diversity and problems of its conservation. Sofia, BSBCP, Pensoft, 351-431. (In Bulgarian with English summary).

Popov A., Deltshev C., Hubenov Z., Beschovski V., Dobrev D., Gueorguiev B.. 2000b. Invertebrate fauna. In: Popov A., Meshinev T. (Eds). High mountain treeless zone of the Central Balkan National Park. Biological diversity and problems of its conservation. Sofia, BSBCP, Pensoft, 339-416. 
Popov P. 1928. Chereshovata muha i vredata ot neya [The cherry fly and the damage from it]. Balgarsko ovoshtarstvo 9 (4): 81-82. (In Bulgarian).

Popov P. 1954. Rezultati ot nablyudeniyata varhu poyavata na chereshovata muha (Rhagoletis cerasi L.) v Sofiysko prez 1953 god. [Results from the observations on the appearance of the cherry fly (Rhagoletis cerasi L.) in the Sofia region in 1953]. Byuletin po rastitelna zashtita 3 (1): 80-83.

Popov P. 1956. Prinos varhu biologiyata, ekologiyata i prognozata na yabalkobiya molets Hyponomeuta malinella Z.v Bulgariya [Contribution to the biology, ecology and prognosis of the apple moth Hyponomeuta malinella Z. in Bulgaria]. Nauchni trudove na Ministerstvoto na zemedelieto (Rastenievadstvo) 1 (4): 21-35. (In Bulgarian).

Popov P. 1958. Kak da ustanovim nachaloto na letezha na chereshovata muha (Rhagoletis cerasi L.) [How to determine the beginning of the flight of the cherry fly (Rhagoletis cerasi $\mathrm{L}$.)]. Byuletin po rastitelna zashtita 6 (3/11): 76-79.

Popov P. 1978. Prinos kam vidoviya sastav na poleznata entomofauna u nas [Contribution to the species composition of the useful entomofauna in the country]. Rastenievadni nauki 15 (2): 132-136. (In Bulgarian).

Popov S. 1999. Species of family Tipulidae (Diptera) known to the fauna of Bulgaria till 1998. Travaux Scientifiques Universite de Plovdiv, Biologie - Animalia 35 (6): 33-36. (In Bulgarian with English summary).

Popova I. 2006. Species of the families Anisopodidae, Hesperinidae and Ptychopteridae (Diptera: Nematocera) in the Western Rhodopes (Bulgaria). In: Beron P. (Ed.). Biodiversity of Bulgaria. 3. Biodiversity of Western Rhodopes (Bulgaria and Greece) I. Sofia, Pensoft \& National Museum of Natural History, Bulgarian Academy of Sciences. pp. 699-700.

Povolný D. \& Verves Y. 1990. A preliminary list of Bulgarian Sarcophaginae (Diptera). Acta Entomologica Musei Nationalis Pragae 43: 283-329.

Procheş Ş. \& Ramdhani S. 2012. The World's zoogeographical regions confirmed by cross-taxon analyses. BioScience 62 (3): 260-270.

Pudar D., Petrić D., Allène X., Alten B., Ayhan N., Cvetkovikj A., Garros C., Goletić T., Gunay F., Hlavackova K., Ćupina A. I. , Kavran M., Lestinova T., Mathieu B., Mikov O., Pajović I., Rakotoarivony I., Stefanovska J., Vaselek S., Zuko A. \& Balenghien T. 2018. An update of the Culicoides (Diptera: Ceratopogonidae) checklist for the Balkans. Parasites \& Vectors 11: 468. https://doi.org/10.1186/s13071-018-3051-x

Raykov I. 1908. Kak da zapazim govezhdiya dobitak ot vagartsite, komarite, ovodite i pr. [How to keep cattle from the mosquitoes, bot-flies, warble-flies and so on.] Sadovo 11 (5): 156-157. (In Bulgarian).

Remmert H. 1960. Der Strandanwurf als Lebensraum. Zeitschrift für Morphologie und Ökologie der Tiere 48: 461-516.

Richter V. A. 1988. Family Nemestrinidae. In: Soós Á. \& Papp L. (Eds.). Catalogue of Palaearctic Diptera. 5. Budapest, Akadémiai Kiadó, Hungarian Natural History Museum. pp. 171-181.

Roháèek J. 1983. A monograph and re-classification of the previous genus Limosina Macquart (Diptera, Sphaeroceridae) of Europe. Beiträge zur entomologie 2: 3-195; 3: 203-255.

Roháček J. 1998. Taxonomic limits, phylogeny and higher classification of Anthomyzidae (Diptera), with special regard to fossil record. European Journal of Entomology 95: 141-177.

Roháček J. 2004. Fauna Europaea: Anthomyzidae. In Papp T.(Ed.): Fauna Europaea: Diptera Brachycera. Fauna Europaea version 1.3. http://www.faunaeur.org

Rozkošny R. 1965. Revision der Sciomyziden (Diptera) aus der Sammlungen des National Museum in Praha. Acta Entomologica Musei Nationalis in Pragae 36: 633-649.

Rozkošný R. 1977. The West Palaearctic species of Nemotelus Geoffroy (Diptera, Stratiomyidae). Folia Facultatis Scientiarum Naturalium Universitatis Purkynianae Brunensis 17, Biologia 51 (3): 1-105.

Rozkošný R. 1982. A biosystematic study of the European Stratiomyidae (Diptera). 1. Dr. W. Junk, The Hague, Boston, London. I-VIII, $401 \mathrm{p}$.

Rozkošný R. 1983. A biosystematic study of the European Stratiomyidae (Diptera). 2. Dr. W. Junk, The Hague, Boston, London. I-VIII, $431 \mathrm{p}$.

Rozkošný R. 1987. A review of the Palaearctic Sciomyzidae (Diptera). Brně, Univerzita Jana Evangelisty Purkyně. $100 \mathrm{p}$.

Rozkošný R. \& E. Nartshuk. 1988. Family Tipulidae. In: Soós Á. \& Papp L. (Eds.). Catalogue of Palaearctic Diptera. 5. Budapest, Akadémiai Kiadó, Hungarian Natural History Museum. pp. 42-96. 
Rozkošný R. \& Spitzer K. 1965. Ergebnisse der Albanien-Expedition 1961 des Deutschen Entomologischen Institutes. Diptera: Rhagionidae. Beiträge zur Entomologie 15 (5/6): 735-742.

Rubtsov I. 1956. Simuliidae. Fauna SSSR - Dvukrylye. 6 (6). Moskva - Leningrad, Akademii Nauk SSSR. 860 p. Rubtsov I. \& Yankovsky A. 1988. Family Simuliidae. In: Soós Á. \& Papp L. (Eds.). Catalogue of Palaearctic Diptera. 3. Budapest, Akadémiai Kiadó, Hungarian Natural History Museum. pp. 114-186.

Russev B. 1959. Beitrag zur Erforschung des Macrobenthos der Donau am bulgarischen Ufer. Comptes rendus de l'Academie bulgare des Sciences 12 (4): 345-348.

Russev B. 1961. Hydrobiologische Untersuchungen an einigen Bächen des Vitoša-Gebirges. Bulletin de l'Institut de zoologie et musée 10: 211-265. (In Bulgarian with German summary).

Russev B. 1962. Die Insektenfauna der Donau vor dem bulgarischen Ufer. Izvestiya na opitnata stantsiya po sladkovodno ribarstvo v Plovdiv 1: 115-128. (In Bulgarian with German summary).

Russev B. 1963. Anthropogene lithoreophile Biozönose in der Donau vor dem bulgarischen Ufer. Comptes rendus de l'Academie bulgare des Sciences 16 (4): 545-548.

Russev B. 1964. Hydrobiologischen Untersuchungen der Arda und einiger ihrer Nebenflüsse. Bulletin de l'Institut de zoologie et musée 17: 5-49. (In Bulgarian, Russian and German summaries).

Russev B. 1966a. Hydrobiologische untersuchungen der Marica. I. In: Die Fauna Thrakiens 3. Sofia, Bulgarische Akademie der Wissenschaften. pp. 231-291. (In Bulgarian, Russian and German summaries).

Russev B. 1966b. Das Zoobenthos der Donau zwischen dem 845. und 357. Flusskilometer. I. Zusammensetzung, Verteilung und Ökologie. Bulletin de l’Institut de zoologie et musée 20: 55-131. (In Bulgarian, Russian and German summaries).

Russev B. 1977. Die Verunreinigung und Selbstreinigung des Ossam nach den strukturellen Änderugen seiner Benthosfauna. Hydrobiology 6: 3-22. (In Bulgarian with German summary).

Russev B. 1978. Besonderheiten und Bedeutung des Zoobenthos der Donau zwischen Strom- km 845 und 357. In: Russev B. \& Naidenow W. (Eds.). Limnologie des Bulgarischen Donauabschnitts. Sofia, Bulgarische Akademie der Wissenschaften, pp. 145-200. (In Bulgarian with German summary).

Russev B. \& Yaneva I. 1975. Hydrofaunistische Erforschungen einiger rhodopischer Gewässer. In: La Faune des Rhodopes. Materiaux. Sofia, Academie Bulgare des Sciences, pp. 11-39. (In Bulgarian with German summary)

Russev B. \& Janeva I. 1986. Hydrobiological review of the right tributary of the Danube - the River Cibrica. Hydrobiology 28: 36-45. (In Bulgarian with English summary).

Russev B., Janeva I. \& Detcheva R. 1984a. Einige Besonderheiten in der Selbstreinigung des Donauzuflusses Ossam. Hydrobiology 21: 14-28.

Russev B., Kovachev S., Janeva I., Karapetkova M., Uzunov J. \& Detcheva R. 1976. Vertreter der bulgarischen Flussfauna als limnosaprobe Bioindikatoren. Hydrobiology 4:60-66.

Russev B., Nikolova M \& Dimitrova M. 1984b. Hydrobiological and saprobiological alterations in the Tundja River. I. 1955-1967. Hydrobiology 22: 59-73. (In Bulgarian with English summary).

Russev B., Nikolova M. \& Yaneva I. 1987. Tendencies in the changes of the hydrobiological state in the Rusenski Lom river valley. Hydrobiology 31: 65-82. (In Bulgarian with English summary).

Russev B., Yaneva I. \& Nikolova M. 1991. Hydrobiological state of the river valley of the river Lom. Hydrobiology 36: 13-31. (In Bulgarian with English summary).

Russev B., Yaneva I., Detcheva R. \& Karapetkova M. 1994. Zusammensetzung der Hydrofauna. In: Russev B. (Ed.) Limnologie der bulgarischen Donauzuflüsse. Sofia, Knizhen Tigar. pp. 130-174.

Russkoff M. 1928. Referate und Berichte im Jahre 1926-1927. Mitteilungen der Bulgarischen Entomologischen Gesellschaft in Sofia 4: p. 22. (In Bulgarian).

Russkoff M. 1929-1930. Beitrag zum Studium der Biologie und Ökologie des Pinienprozessionsspinners (Thaumatopoea pityocampa Schiff.) in Bulgarien. Jahrbuch der Universität in Sofia, Land- und Forstwirtschaft Fakultät 7: 262-284. (In Bulgarian with German summary)

Sachtleben H. 1930. Notes on Pyrausta nubilaris Hb. and its parasites in Bulgaria and Roumania. International Corn. Borer Investigation, Scientifique Reports 3: 42-47.

Sakelarieva L., Yaneva I., Uzunov Y., Kumanski K., Stoichev S., Vidinova Y. \& Tyufekchieva V. 2008. Taxonomic composition and dominant structure of macrozoobenthos in the Blagoevgradska Bistritsa River. Acta zoologica bulgarica, Supplementum 2: 201-214. 
Savchenko E. N., Oosterbroek P. \& Starý J. 1992. Family Limoniidae. In: Soós Á., Papp L. \& Oosterbroek P. (Eds.). Catalogue of Palaearctic Diptera. 1. Budapest, Akadémiai Kiadó, Hungarian Natural History Museum. pp. 183-369.

Savchenko E. \& Tomov V. 1975. A new species of Ormosia (Diptera, Limoniidae) from Bulgaria. Dopovidi AN URSR, ser. B, (No 10): 948-950. (In Ukrainian with English summary).

Savov H. 1925. The results from the open field experiments conducted on the experimental field of the Agricultural Institute in Sofia during 1919-1924. Bulletin of the Agricultural Experimental Institute 3 (4/6): 429-489. (In Bulgarian).

Schaffner F., Angel G., Geoffroy B., Hervy J. \& Rhaiem A. 2001. The mosquitoes of Europe (CD ROM). Montpellier, France: IRD Edition and EID Méditerranée.

Schmitz H. 1953. Ungarische und andere paläarktische Phoriden des Ungarischen Nationalmuseums (Diptera). Annales historico-naturales Musei nationalis hungarici 3: 203-211.

Schmitz H. 1957. Phoridae. In: Lindner E. (Ed.). Die Fliegen der palaearktischen Region 4 (7), Lief. 196: 417-464.

Schmitz H. \& Delage A. 1974. Phoridae. In: Lindner E. (Ed.). Die Fliegen der palaearktischen Region 4 (7), Lief. 301: 609-664.

Sengalevich G. 1964. Nepriyateli po krushovite plodove [Pear fruits pests]. Ovoshtarstvo 11 (8): 30-33. (In Bulgarian).

Sengalevich G. 1972. Vredni peperudi darvesinoyadi v Bulgariya i borbata s tyah [Harmful goat moths in Bulgaria and the fight against them]. Plovdiv, Hristo Danov. 84 p. (In Bulgarian).

Shamshev I. \& Barták M. 2019. New and little-known species of Empis (Diptera: Empididae) from Bulgaria, Israel and Turkey, with keys to the Palaearctic Pachymeria and Mediterranean Xanthempis. Zootaxa 4555 (1): 91-100.

Shannon R. \& Hadjinicolaou J. 1936. List of Tabanidae (Dipt.) of Greece. Acta Instituti et Musei Zoologici Universitatis Atheniensis 1: 160-172.

Shopov G. 1965. Distribution of the bee-wolf. Pchelarstvo 8: 24-25. (In Bulgarian).

Skuhrava M., Skuhravy V., Dončev K. \& Dimitrova B. 1991. Gall midges (Cecidomyiidae, Diptera) of Bulgaria. I. Faunistic researches in the 1978-1987 period. Acta zoologica bulgarica 42: 3-26.

Skuhrava M., Skuhravy V., Dončev K. \& Dimitrova B. 1992. Gall midges (Cecidomyiidae, Diptera) of Bulgaria. II. Host plant relations and economic importance. Acta zoologica bulgarica 43: 23-42.

Skuratowicz W. 1970. Nycteribidae (Diptera, Pupipara) we zbiorach Instytuta Zoologicznego Polskej Akademii Nauk w Warszawe. Polish journal of entomology 40: 745-748.

Šifner F. 2018. Annotated checklist of the family Scathophagidae (Diptera) in Central Europe, with new faunistics data on some species. Linzer Biologische Beiträge 50 (2): 1635-1655.

Sidorenko V. 2004. Family Braulidae. In: Ler P. (Ed.). Key to the insects of Russian Far East. 4 (3). Vladivostok, Dalnauka. pp. 398-399. (In Russian).

Slípka J. 1959. Symplectomorpha stictica Meig. (Diptera, Nematocera) als fakultativer Halobiont. Bulletin de l'Institut de zoologique et Musee (Academie Bulgare des sciences) 8: 105-108.

Slivenski M. 1935. Prinos kam izuchvaneto na malariyata v Bulgariya [Contribution to the study of malaria in Bulgaria]. Sofia, Pridvorna pechatnitsa. 230 p. (In Bulgarian).

Slivenski M. 1940. Malariyata u nas. Prinos kam iuchavaneto i [Malaria in Bulgaria. Contribution to its study]. Sofia, Pridvorna pechatnitsa. 250 p. (In Bulgarian).

Slivenski M. 1946. Malariyata. Prinos kam izuchavaneto i u nas, Zapadna Trakiya (Belomorieto) i Makedoniya [Malaria. Contribution to its study in Bulgaria, Western Thrace (Belomorie) and Macedonia]. Sofia, Voenno-izdatelski fond. 256 p. (In Bulgarian).

Smilova D. 1971. Sezonna dinamika na nyakoi vidove sinantropni muhi v grad Varna [Seasonal dynamics of some species of synanthropic flies in the city of Varna]. Letopisi HEI 33:198-202.

Soós A. 1984. Family Otitidae. In: Soós Á. \& Papp L. (Eds.). Catalogue of Palaearctic Diptera. 9. Budapest, Akadémiai Kiadó, Hungarian Natural History Museum. pp. 45-59.

Soós A. \& Pap L. (Eds.).1984-1993. Catalogue of Palaearctic Diptera 1-13. Budapest, Hungarian Natural History Museum.

Sørensen T. 1948. A method of establishing groups of equal amplitude in plant sociology based on similarity of species content. Kongelige Danske Videnskabernes Selskab. Biologiske Skrifter 4: 1-34. 
Soufi R. \& Uzunov Y. 2008. Data on the ecological status of the Kamchia River as assessed by invertebrate communities parameters. Acta zoologica bulgarica, Supplementum 2: 215-224.

Spasić R. 1996. Agromyzidae (Insecta, Diptera). The fauna of Durmitor, 5. Agromysidae (Insecta, Diptera). Podgorica, the Montenegrin Academy of Sciences and Art. Special Editions 32, Section of Natural Sciences 18: 107-135.

Spasić R. \& Spencer K. 1992. Agromyzidae (Diptera) in the collection of the Croatian Natural Hystory Museum, Zagreb. Bulletin of natural Hystory Museum in Belgrade 47: 139-143. (In Serbocroatian).

Spasov Ts. 1932. Varhu stopanskoto znachenie na bolestite i nepriyatelite v ovoshtarstvoto i borbata s tyah [On the economic importance of the diseases and pests in the pomology and the fight against them]. Zemedelie 36 (9): 155-161. (In Bulgarian).

Spitzer K. 1978. A Review of Bulgarian Rhagionidae (Diptera). Acta zoologica bulgarica 10: 30-36.

Stamboliev H. 1907. Chereshovata muha [The cherry fly]. Gradinar 3 (9): 369-372.

Starý J. 1970. Stand der faunistischen Forschung der Unterfamilie Limoniinae in der Tschechoslowakei (Tipulidae, Diptera). Infber. Landwirtschaft. Hochschule Nitra - Biolog. Grundlagen Landwirtschaft 1970: 29-53.

Starý J. 1971a. Ormosia pirinensis sp. n. und Molophilus priapoides sp. n. - zwei neue europäische LimoniinenArten (Diptera, Tipulidae). Acta Entomologica Bohemoslovaca 68: 95-99.

Starý J. 1971b. Eine neue oder wenig bekannte Arten der Gattung Molophilus Curt. (Diptera, Tipulidae). Annotationes Zoologicae et Botanicae, Bratislava 70: 1-12.

Starý J. 1972. Zwei neue Arten aus der Unterfamilie Limoniinae (Diptera, Tipulidae). Annotationes Zoologicae et Botanicae, Bratislava 81: 1-5.

Starý J. 1973. Boreoalpinni a alpinský prvek ve faune podčeledi Limoniinae (Tipulidae, Diptera) Jeseniků, Zprávy Vlastivěd. In: Ústavu v Olomouci, 163: 21-32. (in Czech with German summary).

Starý J. 1974a. Neue europäische Arten aus der Unterfamilie Limoniinae (Diptera, Tipulidae). Annotationes Zoologicae et Botanicae Bratislava 99: 1-9.

Starý J. 1974b. Beitrag zur Kenntnis der Limoniinen Bulgariens (Diptera, Tipulidae). Acta Musei Moraviae (Scientiae naturales) 58: 113-120.

Starý J. 1976a. Neue europäische Arten aus der Unterfamilie Limoniinae (Diptera, Tipulidae). II. Annotationes Zoologicae et Botanicae, Bratislava 112: 1-7.

Starý J. 1976b. Erioconopa gen. n., with the description of a new species from Central Europe (Diptera, Limonidae). Acta Entomologica Bohemoslovaca 73: 420-429.

Starý J. 1978. Zwei neue europäische Arten aus der Verwandtschaft von Molophilus undulatus (Diptera, Limonidae). Acta Entomologica Bohemoslovaca 75: 344-348.

Starý J. 1982. New species of Limoniidae from the Mediterranean (Diptera). Bulletin Zoölogisch Museum Universiteit van Amsterdam 9: 25-30.

Starý J. 1987. Revision of European species of the genus Cheilotrichia, subgenus Empeda (Diptera, Limoniidae). Acta Entomologica Musei Nationalis Pragae 42: 249-276.

Starý J. 1993. Two new European species of Dicranomyia Stephens, 1829, related to D. (s. str.) horea (Meigen, 1818) (Diptera, Limoniidae). Bulletin Zoölogisch Museum Universiteit van Amsterdam 13 (16): 175-181.

Starý J. 2003. Revision of European species of the genus Rhabdomastix (Diptera: Limoniidae). Part 1: Introduction and subgenus Lurdia subgen. n. European Journal of Entomology 100: 587-608.

Starý J. 2004a. Revision of European species of the genus Rhabdomastix (Diptera: Limoniidae). Part 2: Subgenus Rhabdomastix s. str. European Journal of Entomology 101: 657-687.

Starý J. 2004b. Dicranota (Paradicranota) cinerascens Lackschewitz, 1940, a valid species (Diptera: Pedicidae). Aquatic Insects, 26 (3/4): 273-279.

Starý J. 2006a. Nomenclatural changes in West Palaearctic Limoniidae and Pediciidae (Diptera). Acta Universitatis Carolinae, Biologica 49: 175-186.

Starý J. 2006b. Hoplolabis (Parilisia) species related to H. (P.) punctigera (Lackschewitz, 1940) and H. (P.) spinosa (Nielsen, 1953) with the description of a new species (Diptera, Limoniidae). Studia Dipterologica 13: 115-125.

Starý J. 2007. Nomenclatural changes in West Palaearctic Limoniidae and Pediciidae (Diptera), II. Casopis Slezskeho Musea v Opava (A) 56: 23-36.

Starý J. 2008. Three new European species of the genus Scleroprocta Edwards, 1938 (Diptera: Limoniidae). Biologia 63: 120-126. 
Starý J. 2009a. The identity of Dicranomyia (Dicranomyia) luteipennis Goetghebuer (Diptera, Limoniidae). Zootaxa 2155: 55-68.

Starý J. 2009b. West Palaearctic species of the genus Eloeophila (Diptera:Limoniidae). European Journal of Entomology 106: 425-440.

Starý J. 2011. Gonomyia (Gonomyia) lucidula de Meijere and allies (Diptera: Limoniidae). Entomologica Fennica 21: $232-242$.

Starý J. \& Krzemiński W. 1993a. A new Dicranota from Bulgaria (Diptera, Pediciidae). Acta Zoologica Cracoviensia 35: 565-567.

Starý J. \& Krzemiński W. 1993b. Additions to the list of Bulgarian Limoniidae and Pediciidae (Diptera). Acta Zoologica Cracoviensia 35: 569-572.

Starý J. \& Reusch H. 2009. European species of the subgenus Brachylimnophila (Diptera: Limoniidae). Entomologica Fennica 19: 207-217.

Starý J. \& Stubbs A. E. 2015. Five species under Dicranomyia (Dicranomyia) mitis (Meigen, 1830) (Diptera, Limoniidae). Zootaxa 3964: 321-334.

Statelov N. 1942. Sredizemnomorskata plodova muha Ceratitis capitata Wied. [The Mediterranean fruit fly Ceratitis capitata Wied.]. Mitteilungen der Bulgarischen Entomologischen Gesellschaft in Sofia 12: 45-63. (In Bulgarian with German summary).

Stefanov S. 1955. Prekratyavane na diapauzata i nachaloto na polovata aktivnost pri Anopheles maculipennis i Anopheles superpictus $\mathrm{v}$ Blagoevgradsko [Stopping of the diapause and beginning of the sexual activity in Anopheles maculipennis and Anopheles superpictus in Blagoevgrad region]. Annuaire de l'Université de Sofia, BGGF 48 (1): 157-175. (In Bulgarian with English summary).

Stefanov S. 1956. Sezonni izmeneniya na nyakoi morfologichni belezi na yaytsata pri Anopheles maculipennis maculipennis и Anopheles maculipennis messae v Sofiya [Seasonal changes of some morphological features of eggs in Anopheles maculipennis maculipennis and Anopheles maculipennis messae in Sofia]. Annuaire de l'Université de Sofia, BGGF 49 (1): 161-198. (In Bulgarian with English summary).

Stefanov S. 1959. Varhu nyakoi biologichni faktori, ogranichavashti masovoto rasprostranenie na gabotvorkata (Lymsntria dispar L.) $\mathrm{v}$ nashite gori [On some biological factors limiting the mass distribution of the fungus (Lymsntria dispar L.) in the forests]. Nauchni trudove na VLTI 7: 7-17. (In Bulgarian).

Stefanov D. \& Keremidchiev M. 1961. Vazmozhnosti za izpolzvaneto na nyakoi hishtni i parazitni nasekomi (entomofagi) v biologichnata borba s gabotvorkata (Lymsntria dispar L.) u nas [Possibilities for the use of some predatory and parasitic insects (entomophages) in the biological control of the fungus (Lymsntria dispar L.) in the country]. Nauchni trudove na VLTI 9: 157-168. (In Bulgarian).

Stefanov D., Keremidchiev M. \& Vutov V. 1958. Izsledvane gradatsiite na gabotvorkata (Lymantria dispar L.) i prastenotvorkata (Malacosoma neustria L.) u nas i ustanovyavane na tehnite prichini [Study of the gradations of Lymantria dispar L. and Malacosoma neustria L. in the country and establishment of their reasons]. Hauchni trudove na VLTI 6: 135-172. (In Bulgarian).

Stoichev S. 1994. Contribution to the study of the chironomid fauna (Diptera, Chironomidae) in the Bulgarian stretch of the Danube River and its tributaries. Frequency of occurrence and dominant analysis of the species found. Hydrobiology 39: 91-101. (In Bulgarian with English summary).

Stoichev S. 1996. On the Chironomid fauna from Bulgarian inland waters. Lauterbornia 25: 117-123.

Stoichev S. 1998. The zoobenthos from the lakes Schabla-Ezerets (northern Black Sea coast of Bulgaria). In: Golemansky V., Naidenov W. (Eds.). Biodiversity of Schabla Lake System, Sofia, "Prof. Marin Drinov" Academic Publishing House. pp. 91-99.

Stoichev S. 2000a. The zoobenthos from several glacial lakes in the Rila Mountains, Bulgaria. In: Golemsnski V, Naidenov W (eds.). Biodiversity and evolution of glacial water ecosystems in the Rila Mountains. Sofia, Institute of Zoology, BAS. pp. 155-162.

Stoichev S. 2000b. Zoobenthos in outflows of some glacial lakes in the Rila Mountains. In: Golemsnski V., Naidenov W. (eds.). Biodiversity and evolution of glacial water ecosystems in the Rila Mountains. Sofia, Institute of Zoology, BAS. pp. 163-168.

Stoichev S. 2001a. The zoobenthos of the Koprinka reservoir, Central Bulgaria. Lauterbornia 40: 39-41.

Stoichev S. 2001b. Of the Chironomid Fauna from Blagoevgradska Bistritsa River (Rila Mountain, Southwest Bulgaria).. Lauterbornia 40: 119-121. 
Stoichev S. 2002. Hydrofaunistic investigation of the Urdini Ezera Glacial Lakes, Northwestern Rila Mountains, West Bulgaria. Acta zoologica bulgarica 54 (1): 63-68.

Stoichev S., Danova E. 2003. Hydrofaunistic investigation of the Mussalenski Ezera Glacial Lakes, Eastern Rila Mountains, South-Wewst Bulgaria. Acta zoologica bulgarica, 55 (2): 75-80.

Stoichev S. 2004. The benthic Invertebrates in the Marichini Ezera Glacial Lakes, Eastern Rila Mountains, SouthWewst Bulgaria. Acta zoologica bulgarica 56 (1): 277-282.

Stoichev S., Chernev N. 2001. On the Chironomid fauna from Blagoevgradska Bistritsa River (Rila Mountain, Southwest Bulgaria. Lauterbornia 40: 119-121.

Stoicheva R. 1970. Prouchvaniya varhu sezonnata dinamika na preobladavashtite vidove sinantropni muhi v Starozagorski okrag [Studies on the seasonal dynamics of the predominant species of synanthropic flies in the Stara Zagora district]. Letopisi HEI 20: 132-133. (In Bulgarian).

Stojanov N. 1966. Rastitelna pokrivka. In: Beshkov A. (Ed.). Geography of Bulgaria. 1. Sofia, Bulgarian Academy of Sciences, 447-482. (In Bulgarian).

Stoyanov D. 1957. Bolesti i nepriyateli, poyavili se po kulturnite rasteniya v Bulgariya prez 1956. [Diseases and pests that appeared on the cultivated plants in Bulgaria in 1956]. Byuletin po rastitelna zashtita $4(2 / 10)$ : 7-100. (In Bulgarian).

Stoyanova T., Traykov I., Bogoev V., Yaneva I., Vidinova Y., Tyufekchieva V. \& Kenderov L. 2013. Composition of the macrozoobenthos in semi-mountainous river in South-Western Bulgaria. Natura Montenegrina 12 (3-4): 803-811.

Straka V. 1976. Description of new European species of the genus Hilara Meigen (Diptera, Empididae). Annotationes Zoologicae et Botanicae 116: 1-36.

Strenzke K. 1951a. Chironomiden von der bulgarischen Küste des Schwarzen Meeres. Archiv für Hydrobiologie, Supl. 18 (4): 678-691.

Strenzke K. 1951b. Eine südosteuropäische Chironomiden-Gattung Halliella (Diptera). Deutsche Zoologische Zeitschrift 1 (1): 15-23.

Stribarni V. 1898. Kak se predpazva kromid luk ot larvite na lukovata tsvetna muha, Anthomyia caparum [How to protect onions from the larvae of the onion flower fly, Anthomyia caparum]. Oralo 5 (16): 253-254. (In Bulgarian).

Stribarni V. 1909. Kromid (cherven luk) [Onion (red onion)]. Sadovo 12 (6): 219-222.

Stribarni V. 1934. Chervivost na chereshovite plodove [Worms of the cherries]. Lozarski pregled 19 (4): 116-117. (In Bulgarian).

Surbova S. 1965. Sezonnaya aktivnost sinantropnyh muh v nekotoryh rayonah gor. Sofii i ustoychivost $M$. domestica $\mathrm{k}$ deystviyu DDT i lindana [Seasonal activity of the synanthropic flies in some areas of Sofia and the resistance of $M$. domestica to DDT and lindane]. Trudove na nauchnoizsledovatelskiya institut po epidemiologiya i mikrobiologiya 10: 170-173. (In Russian).

Surbova S. \& Avramov S. 1959. Himichni i biologichni izsledvaniya na preparati ot livadna (99\%-gama izomer na heksahlorheksana) [Chemical and biological studies of the meadow preparations (99\% gamma isomer of hexachlorohexane)]. Higiena, epidemiologiya i mikrolbiologiya 3 (5): 23-29. (In Bulgarian).

Surbova S. \& Lavčiev V. 1956. Laboratorni opiti za izprobvane na nyakoi vidove preparati na DDT spryamo domashnata muha [Laboratory experiments to test some types of DDT preparations against houseflies]. Zdravno delo 9 (6): 22-29. (In Bulgarian).

Szadziewski R., Filatov S. \& Dominiak P. 2016. A redescription of Culicoides griseidorsum Kieffer, 1918, with comments on subgeneric position of some European taxa (Diptera: Ceratopogonidae). Zootaxa 4107 (3): 413-422. http://www.mapress.com/j/zt/

Szilády Z. 1923. New or little known horseflies (Tabanidae). Biologica Hungarica 1 (1): 1-39.

Szilády Z. 1934. Beiträge zur Dipterenfauna Bulgariens. Bulletin de la société entomologique de Bulgarie 8: 145-151.

Tahirov B. 1969. Prouchvaniya na sinantropnite muhi v grad Shumen [Studies on the synanthropic flies in Shumen Town]. Letopisi HEI 10: 33-36. (In Bulgarian).

Tahirov B. 1970. Sezonna dinamika na po-chesto sreshtanite sinantropni muhi v grad Shumen [Seasonal dynamics of the most common synanthropic flies in Shumen Town]. Letopisi HEI 25: 51-55. (In Bulgarian). 
Tahirov B. 1973. Prouchvaniya na sinantropnite muhi v Shumenski okrag s ogled epidemiologichnoto im znachenie [Studies of the synanthropic flies in Shumen district in view of their epidemiological significance]. Ph.D. thesis, Sofia, Bulgarian Academy of Sciences. 184 p. (In Bulgarian).

Tanasijtshuk V. 1984. Family Chamaemyiidae. In: Soós Á. \& Papp L. (Eds.). Catalogue of Palaearctic Diptera. 8. Budapest, Akadémiai Kiadó, Hungarian Natural History Museum. pp. 220-232.

Tanasijtshuk V. 1988. Family Pipunculidae. In: Soós Á. \& Papp L. (Eds.). Catalogue of Palaearctic Diptera. 8. Budapest, Akadémiai Kiadó, Hungarian Natural History Museum. pp. 230-245.

Tanasijtshuk V. \& Beschovski V. 1991. A contribution of the study of the Chamaemyia species (Diptera, Chamaemyiidae) from Bulgaria and some East European countries. Acta Zoologica Bulgarica 41: 18-25.

Theobald F. 1907. A monograph of the Culicidae or mosquitoes. 4. London. 639 p.

Theobald F. 1910. A monograph of the Culicidae or mosquitoes. 5. London. 646 p.

Theowald Br. \& Oosterbroek P. 1986. Zur Zoogeographie der westpalaearktischen Tipuliden. VII. Die Tipuliden der Balkanhalbinsel (Diptera, Tipulidae). Tijdschrift voor entomologie 129: 1-13.

Thienemann A. 1936. Haffmücken und andere Salzwasserchironomiden. Kieler Meeresforschungen 1: 167-178. Thienemann A. 1949. Die Metamorphose von Stempelina montivaga Goetgh. (Chironomiden aus dm Lunzer Seengebiet IX). Entomologisk tidskrift 70 (1-2): 12-18.

Tomasovic G. 2002. Etude sur matériaux typiques du complexe génital mâle de sept espèces du genre Erax Scopoli, 1763 (Diptera Asilidae) avec la description de trois espèces nouvelles. Notes fauniques de Gembloux 46: 27-37.

Tomasovic G. 2006. Distribution des espèces de Cyrtopogon Loew, 1847 en Europe avec note sur Cyrtopogon lateralis (Fällen, 1814), espèce également sibérienne. Notes fauniques de Gembloux 59 (3): 149-153.

Tomov R. \& Dimitrov S. 2009. Occurrence of alien insects on black locust (Robinia pseudoacacia L.) in Bulgaria. Acta Entomologica Bulgarica.

Tomov R., Trencheva K., Trenchev G., Çota E., Ramafhi A., Ivanov B., Nacheski S., Papazova-Anakieva I. \& Kenis M. 2009. Chuzhdozemnite nasekomi i zaplahata ot tyah za bioraznoobrazieto na Albania, Bulgaria i Macedonia [Foreign insects and their threat to the biodiversity of Albania, Bulgaria and Macedonia]. Sofia, Pensoft. 114 p.

Tomov V. 1971. Izsledvaniya varhu hrizomelidnata fauna v porechieto na Struma [Studies on the Chrysomelidae fauna in the Struma Valley]. Ph.D. thesis, Université de Plovdiv „Paissi Hilendarski”, 10-11. (In Bulgarian).

Tomov V. 1974. Zwei neue Diptereparasiten (Dipt., Tachinidae) auf Larven von Diorhabda elongata Brullé und Agelastica alni L. (Col. Chrysomelidae). Université de Plovdiv „Paissi Hilendarski”, Natura 7 (1): 105-106.

Tomov V. 1975. New species of subgenus Lunatipula Edw. (Dipt., Tipulidae) for the fauna of Bulgaria. Travaux Scientifiques Universite de Plovdiv, Biologie 13 (4): 127-131. (In Bulgarian with English summary).

Tomov V. \& Mitov P. 1987. Hishtnitsi i paraziti po bryastoviya listoyad Xanthogaleruca luteola Muell. (Col. Chrysomelidae) [Predators and parasites on the Xanthogaleruca luteola Muell. (Col. Chrysomelidae)]. Savremenni postizheniya na balgarskata zoologiya. Sofia, BAS, 297-299. (In Bulgarian).

Trenchev G. 1979. Investigation on the biology of Pseudosarcophaga mamillata Pandelle (Diptera, Sarcophagidae). Nauchni trudove na Vish selskostopanski institut Vasil Kolarov - Plovdiv 24 (3): 161-164. (In Bulgarian with English summary).

Trenchev G. 1980a. Parasite species of the subfamily Tachininae (Diptera, Tachinidae) in Bulgaria. Plant Science 17 (1): 132-135. (In Bulgarian with English summary).

Trenchev G. 1980b. Parasite species of subfamily Phasiinae (Diptera, Tachinidae) in Bulgaria. Plant Science 17 (2): 98-101. (In Bulgarian with English summary).

Trenchev G. 1980c. Parasite species of the subfamily Dexiinae (Diptera, Tachinidae) in Bulgaria. Plant Science 17 (3): 121-126. (In Bulgarian with English summary).

Trenchev G. 1980d. Parasite species of the subfamily Exoristinae (Diptera, Tachinidae) in Bulgaria. Plant Science, 17 (5): 92-95. (In Bulgarian with English summary).

Trichkova T., Tyufekchieva V., Kenderov L., Vidinova Y., Botev I., Kozuharov D., Hubenov Z., Uzunov Y., Stoichev S. \& Cheshmedjiev S.. 2013. Benthic macroinvertebrate diversity in relation to environmental parameters, and ecological potential of reservoirs, Danube River Basin, North-West Bulgaria. Acta zoologica bulgarica, 65 (3): 337-348.

Trifonov T., Pachev S. \& Meshkov S. 1964. Species composition, seasonal dynamics and distribution of Tabanidae in South-Eastern Bulgaria. Veterinary Medicine (Sofia) 1 (3): 47-60. (In Bulgarian with English summary). 
Tsalev M. \& Krastev L. 1955. Edin nov nepriyatel po smokinyata u nas [A new pest on the fig tree in the country]. Byuletin po rastitelna zashtita 3 (2): 85-86.

Tsanev K. 1969. Sezonna dinamika na sinantropnite muhi v grad Burgas [Seasonal dynamics of the synanthropic flies in the city of Burgas]. Letopisi HEI 16: 107-109. (In Bulgarian).

Tsankov G. 1968. Vazmozhnosti za izpolzvaneto na entomofagite v biologichnata borba sreshtu nasekomnite vrediteli v gorskoto stopanstvo [Possibilities for the use of the entomophages in the biological control of insect pests in forestry]. Zbornik ot dokladi i referati izneseni na saveshtanieto po lesozashtita, Sofia, 5-6. IV.1968. pp. 105-110. (In Bulgarian).

Tsankov G. 1972. Varhu parazitite na zimnata letoraslozavivachka (Ryacionia buoliana Schiff., Olethreutinae, Lepidoptera) v Bulgariya [On the parasites (Ryacionia buoliana Schiff., Olethreutinae, Lepidoptera) in Bulgaria]. In: Izsledvaniya po biologichnata borba s vreditelite na rasteniyata, IZR - Kostinbrod 1: 95105. (In Bulgarian).

Tsankov G. 1977. Vnutrilichinochnye parazity zimuyuchego pobegovyuna v Bolgarii [Intralarval parasites of Ryacionia buoliana in Bulgaria]. In: Sbornik SEV, Problemy biologicheskoy borby s vreditelyami lesa. Zvolen, 47-53. (In Russian).

Tsankov G. 1979. Prouchvane na parazitnite nasekomi po zimnata letoraslozavivachka (Ryacionia buoliana Schiff.) i borovata protsesionka (Thaumetopoea pityocampa Schiff.) s ogled izpolzvaneto im v biologichnata borba [Study of parasitic insects on Ryacionia buoliana Schiff. and Thaumetopoea pityocampa Schiff. with a view to their use in the biological control]. Habilitation work, Sofia, Bulgarian Academy of Sciences, 80-81, 103-104. (In Bulgarian).

Tsankov G. 1985. Annotirovannye spiski entomofagov neparnogo shelkopryada (Lymantria dispar L.) i zimuyushchego pobegovyuna (Rhyacionia buoliana Den. et Schiff.) v Bolgarii [Annotated lists of entomophages of Lymantria dispar L. and Rhyacionia buoliana Den. et Schiff. in Bulgaria]. Information bulletin EPS IOBS 10: 25-31. (In Russian with English sumarry).

Tsankov G., Georgiev G. \& Bochev N. 1989. New parasites of poplar longhorn beetle (Saperda populnea L., Coleoptera, Cerambycidae) in Northern Bulgaria. In: International organization for biological control of noxious animals and plants, East Palaearctic section. Biologicheskaya i integrirovannaya borba s vreditelyami v lesnyh biotsenozah, Moscow, 163-168. (In Russian with English sumarry).

Tsankov G., Georgiev G., Pelov V. \& Trenchev G. 1991. Parasitoids of Hexomyza schineri Gir. (Diptera, Agromyzidae) in Bulgaria. In: First National Conference of Entomology, Sofia, 28-30 October 1991, 207-212. (In Bulgarian with English sumarry).

Tsankova R. \& Luvchiev V. 1992. Laboratory investigations on the larval zoophagy of Ophyra capensis - an antagonist of Musca domestica. - Applied parasitology 34: 221-228.

Tschorbadjiew P. 1924a. Nyakolko neizvestni i malko poznati nepriyateli u nas [Several unknown and little known pests in the country]. Spisanie na zemedelskite ispitatelni instituti v Balgariya 3 (2/3): 169-175. (In Bulgarian).

Tschorbadjiew P. 1924b. Konstatirani vredni nasekomi i drugi zhivotinski nepriyateli po kulturnite rasteniya v Bulgariya prez 1923 god. [Identified harmful insects and other animal pests on the cultivated plants in Bulgaria in 1923]. Svedeniya po zemedelieto 5 (3/4): 2-26. (In Bulgarian).

Tschorbadjiew P. 1924c. Saobshtenie varhu nyakoi vredni nasekomi [Message on some harmful insects]. Mitteilungen der Bulgarischen Entomologischen Gesellschaft in Sofia 1: 24-27. (In Bulgarian with German summary).

Tschorbadjiew P. 1924d. Belezhki varhu vatreshnite parasiti na vrednite nasekomi i tyahnoto izpolzvane v praktikata [Notes on the internal parasites of the harmful insects and their use in practice]. Spisanie na zemedelskite izpitatelni instituti v Bulgaria 3 (1): 84-88. (In Bulgarian).

Tschorbadjiew P. 1925a. Konstatirani zhivotinski nepriyateli po kulturnite rastenia v Bulgariya prez $1924 \mathrm{~g}$. [Established animal pests on the cultivated plants in Bulgaria in 1924]. Svedeniya po zemedelieto $6(4 / 6)$ : 4-19. (In Bulgarian).

Tschorbadjiew P. 1925b. Messages on some harmful insects. Mitteilungen der Bulgarischen Entomologischen Gesellschaft in Sofia 2: 41-44. (In Bulgarian with German summary).

Tschorbadjiew P. 1925c. Otchet na entomologicheskata sektsiya pri Zemedelskiya izpitatelen institut v Sofiya za 1924 [Report of the Entomological Section at the Agricultural Testing Institute in Sofia in 1924]. Sofia. pp. 167-175. (In Bulgarian). 
Tschorbadjiew P. 1925d. Nyakolko neizvestni i malko izvestni nepriyateli u nas [Some unknown and little known pests in the country]. Spisanie na zemedelskite ispitatelni instituti v Balgariya 3 (2/3): 169-174. (In Bulgarian).

Tschorbadjiew P. 1925e. Resultat d'une enquête rur les Simuliidae nuisibles en Bulgarie. Svedeniya po zemedelieto 6 (9/19): 3-16. (In Bulgarian with French summary).

Tschorbadjiew P. 1925f. Otchet na entomologicheskiya otdel pri Zemedelskiya izpitatelen institut v Sofiya za 1923 [Report of the Entomological Department at the Agricultural Testing Institute in Sofia for 1923]. Godishen otchet na Zemedelskiya izpitatelen institut v Sofia za 1923 g. Sofia. pp. 99-109. (In Bulgarian).

Tschorbadjiew P. 1925g. Betrag zur Erforschung der Parasiten der schädlichen Insekten in Bulgarien. Mitteilungen der Bulgarischen Entomologischen Gesellschaft in Sofia 2: 84-89.

Tschorbadjiew P. 1926a. Saobshtenie varhu nyakoi vredni nasekomi [Message on some harmful insects] (Referate und Berichte). Mitteilungen der Bulgarischen Entomologischen Gesellschaft in Sofia 3: 32-35. (In Bulgarian).

Tschorbadjiew P. 1926b. Nepriyateli po kulturnite rasteniya v Bulgariya prez $1925 \mathrm{~g}$. [Pests on the cultivated plants in Bulgaria in 1925]. Svedeniya po zemedelieto 7 (2): 20-68. (In Bulgarian).

Tschorbadjiew P. 1926c. Bemerkungen über einige Tierische Feinde der Kulturpflanzen Bulgarien im 1925. Mitteilungen der Bulgarischen Entomologischen Gesellschaft in Sofia 3: 115-134. (In Bulgarian with German summary).

Tschorbadjiew P. 1927. Nepriyateli po kulturnite rasteniya v Bulgariya prez $1925 \mathrm{~g}$. [Pests on the cultivated plants in Bulgaria in 1925] Godishen otchet na Darzhavnata zemedelska opita stantsiya v Sofia za 1925 g. Sofia. pp. 207-256. (In Bulgarian).

Tschorbadjiew P. 1928a. Nepriyateli po kulturnite rasteniya v Bulgariya prez 1926 g. [Pests on the cultivated plants in Bulgaria in 1926] Svedeniya po zemedelieto 9 (3/4): 1-51. (In Bulgarian).

Tschorbadjiew P. 1928b. Nepriyateli po kulturnite rasteniya v Bulgariya prez 1926 g. [Pests on the cultivated plants in Bulgaria in 1926] Godishen otchet na Darzhavnata zemedelska opita stantsiya v Sofia za 1926 g. Sofia. pp. 175-241. (In Bulgarian)

Tschorbadjiew P. 1928c. Referate und Berichte im Jahre 1926-1927. Mitteilungen der Bulgarischen Entomologischen Gesellschaft in Sofia 4: 22-24. (In Bulgarian).

Tschorbadjiew P. 1928d. Bemerkungen über einige schädliche Insekten in Bulgarien beobachtet während der Jahren 1926 und 1927. Mitteilungen der Bulgarischen Entomologischen Gesellschaft in Sofia 4: 125-134. (In Bulgarian).

Tschorbadjiew P. 1929a. Nepriyateli po kulturnite rasteniya v Bulgariya prez $1927 \mathrm{~g}$. [Pests on the cultivated plants in Bulgaria in 1927]. Svedeniya po zemedelieto 10 (3/4): 3-59. (In Bulgarian)

Tschorbadjiew P. 1929b. L'Entomologie agricole et les isectes nuisibles aux plantes de culture en Bulgarie. Memoires de IV Congress international d'entomologie. Ithaca, August 1928. 2. Naumburg, pp. 746-756.

Tschorbadjiew P. 1930a. Otchet na entomologicheskata sektsiya pri Darzhavnata zemedelska opitna stantsiya v Sofiya za 1927 i 1928. [Report of the entomological section of the State Agricultural Experimental Station in Sofia in 1927 and 1928] Godishen otchet na Darzhavnata zemedelska opita stantsiya v Sofia za 1927 i 1928 g. Sofia. pp. 207-277. (In Bulgarian).

Tschorbadjiew P. 1930b. Bemerkungen über einige schädlichen Insekten auf den Kulturpflanzen in Bulgarien während der Jahre 1928-1929. Mitteilungen der Bulgarischen Entomologischen Gesellschaft in Sofia 5: 63-106. (In Bulgarian with German summary).

Tschorbadjiew P. 1931. Notizen über die schädlichen Insekten Fauna in Bulgarien während des Jahre 1930. Mitteilungen der Bulgarischen Entomologischen Gesellschaft in Sofia 6: 179-188. (In Bulgarian with German summary).

Tschorbadjiew P. 1932. Nepriyateli po kulturnite rasteniya v Bulgariya prez 1928 i 1929 godini [Pests on the cultivated plants in Bulgaria in 1928 and 1929]. Svedeniya po zemedelieto 13 (3/4): 3-48. (In Bulgarian).

Tschorbadjiew P. 1933. Harmful insects on the fruit trees in Bulgaria. Ministerstvo na zemedelieto i darzhavnite imoti (Ministry of Agriculture and State Property), Sofia, Semestopanska biblioteka 59. 240 p. (In Bulgarian).

Tschorbadjiew P. 1936. Chereshovata muha i borbata s neya [The cherry fly and the fight against it]. Upatvane 28 na Instituta za zashtita na rasteniyata. Sofia. 8 p. (In Bulgarian). 
Tschorbadjiew P. 1938. Chereshovata muha i borbata s neya [The cherry fly and the fight against it]. Gradinarstvo 19 (6): 185-188.

Tschorbadjiew P. 1939a. Materialien über die schädlichen Insekten und anderen Feinde der Kulturpflanzen in Bulgarien. Mitteilungen der Bulgarischen Entomologischen Gesellschaft in Sofia 10: 55-72. (In Bulgarian with German summary).

Tschorbadjiew P. 1939b. Referate und Mitteilungen. Mitteilungen der Bulgarischen Entomologischen Gesellschaft in Sofia 10: 177-180. (In Bulgarian).

Tschorbadjiew P. 1940. Materialien über die schädlichen Insekten und anderen Feinde der Kulturpflanzen in Bulgarien. (Fortsetzung III). Mitteilungen der Bulgarischen Entomologischen Gesellschaft in Sofia 11: 43-64. (In Bulgarian with German summary).

Tschorsnig H.-P., Richter V. A., Cerretti P., Zeegers T., Bergström C., Vaňhara J., Van de Weyer G., Bystrowski C., Raper C., Ziegler J. \& Hubenov Z. 2005. Fauna Europaea: Tachinidae. In: Pape, T., ed., Fauna Europaea: Diptera, Brachycera. Fauna Europaea version 1.2. Available online: http://www.faunaeur.org

Tschorsnig H.-P., Bergström C., Cerretti P., Hubenov Z., Raper C., Van de Weyer G., Vaňhara J., Zeegers T. \& Ziegler J. 2009. Fauna Europaea: Tachinidae. In: Pape T. (Ed.) Diptera: Brachycera. Fauna Europaea. Version 2.1 (22 December 2009). Available online: http://www.faunaeur.org

Tsolova E. \& Koleva L. 2018. Bibio marci L. (Diptera: Bibionidae): a new insect pest in organic raspberry production in West Bulgaria. Journal of Mountain Agriculture on the Balkans 21 (6): 178-184.

Tsvetanov T. 2021. First rocord of Thereva aurata (Loew, 1854) in Bulgaria (Insecta: Diptera: Therevidae). ZooNotes

Udvardi M. 1975. A classification of the Biogeographical Provinces of the World. Morges, IUCN Paper 18. 49 p.

Ujvarosi L. 2005. Additions to the Cylindrotomidae, Limoniidae and Pediciidae species (Diptera) in Romania and Bulgaria. Entomologica Romanica 10: 65-70.

Ujvarosi L. \& Balint M. 2012. Discovery of the second European Amalopis species: an integrative survey of the widespread Pedicia (Amalopis) occulta (Meigen, 1830) (Insecta, Diptera, Pediciidae). Zootaxa 3189: 1-28.

Ujvarosi L., Balint M., Meszaros N. \& Popescu O. 2009. Genetic diversity with morphological imprints among Pedicia (Amalopis) occulta (Meigen, 1830) (Diptera, Pediciidae) populations in the Carpathian area: Preliminary results. Lauterbornia 68: 47-58.

Ujvarosi L., Balint M., Schmitt T., Meszaros N., Ujvarosi T. \& Popescu O. 2010. Divergence and speciation in the Carpathians are: patterns of morphological and genetic diversity of the crane fly Pedicia occula (Diptera: Pediciidae). Journal of the North American Benthological Society 29: 1075-1088.

Ujvarosi L., Kolcsar L.-P. \& Vaida R. 2011. Additions to the Cylindrotomidae (Insecta, Diptera) fauna of Bulgaria and Romania. Entomologica Romanica 16: 47-50.

Uzunov Y., Russev B., Kovachev S. \& Yaneva I. 1981. Species composition and distribution of the Macrozoobenthos of he Maritsa River. Hydrobiology 14: 3-15. (In Bulgarian with English summary).

Uzunov Y., Tsankova V., Todorov I. \& Varadinova E. 2001. The macrozoobenthic fauna of the Biospher reserve Srebarna Lake in North-Eastern Bulgaria. Lauterbornia 40: 43-51.

Uzunov Y., Varadinova E.Yaneva I., Stoichev S., Vidinova Y. \& Kumanski K. 2011. Long-term changes of the bottom Invertebrate fauna of the Mesta River in Southwestern Bulgaria. Annuaire de l'Université de Sofia „St. Kliment Ohridski”, Faculte de Biologie 99 (1 - Zoology): 33-52.

Vaillant F. \& Joost W. 1983. On a small collection of Diptera Psychodidae from Caucasus (USSR) and Bulgaria. Reichenbachia 21 (15): 95-106.

Valerianov A. 1961. Varhu vidoviya sastav na muhite mezhdinni gostopriemnitsi na spiruridite na konya v Burgasko [On the species composition of the flies intermediate hosts of the horse spirurides in the Burgas region]. Bulletin of the Central helminthological laboratory 6: 101-107. (In Bulgarian).

Valkanov A. 1936. Notizen über die Brackwässer Bulgariens. II. Versuch einer hydrographischen und biologischen Erforschung derselben. Annuaire de l'Université de Sofia. Faculté physico-mathématique 32 (3): 209-341. (In Bulgarian with German summary).

Valkanov A. 1941a. Über das Auffinden von anabiotischen Dipteren in Bulgarien. Jarbuch der Universität Sveti Climent Ochridski in Sofia, Physico-Matematische Facultät 37 (3): 201-205. (In Bulgarian with German summary).

Valkanov A. 1941b. Nashite dobrudzhanski ezera [Bulgarian Dobrudzha Lakes]. Ribarski pregled 11 (6): 6-9. (In Bulgarian). 
Valkanov A. 1948. Thalassomyia frauenfeldi Schiner vom Schwarzen Meer. Arbeiten aus der Biologischen Meeresstation in Varna 14: 103-112. (In Bulgarian with German summary).

Valkanov A. 1954. Betrag zur Kenntnis unserer Schwarzmeerfauna. Arbeiten aus der Biologischen Meeresstation in Varna 18: 49-53. (In Bulgarian with German summary).

Valkanov A. 1957a. Katalog unserer Schwarzmeerfauna. Arbeiten aus der Biologischen Meeresstation in Varna 19: 1-62. (In Bulgarian with German summary).

Väisänen R. 1984. A monograph of the genus Mycomya Rondani in the Holarctic region. Acta Zoologica Fenica 177: 1-346.

Varadinova E., Borisova P., Pehlivanov L. \& Uzunov Y. 2012. Macroinvertebrate communities of the Srebarna Lake Biosphere Reserve: species diversity, abundance and modelling of the ecological status. In: Uzunov Y. Georgiev B., Varadinova E., Ivanova N., Pehlivanov L. \& Vasilev V. (Eds.). Ecosystems of the Biosphere Reserve Srebarna Lake. Sofia, Professor Marin Drinov Academic Publishing House. pp 93-113.

Varadinova E., Kerakova M., Alexandrova M., Soufi R., Stoichev S., Vidinova Y., Tyufekchieva V. \& Uzunov Y. 2013. Botton Invertebrate communites (the macrozoobenthos): Key biological quality element for ecological classification of the Mesta River basin. In: Uzunov Y., Pehlivanov L., Georgiev B. \& Varadinova E. (Eds.). Mesta River: Biological quality elements and ecological status. Sofia, Professor Marin Drinov Academic Publishing House. pp 61-96.

Varadinova E., Pechlivanov L., Stoichev S. \& Uzunov Y. 2011. Recovereing and succession of the species diversity of macrozoobenthos in Srabarna Biosphere Reserve (North-East Bulgaria). Acta zoologica bulgarica 63 (1): 85-95.

Vasilev P. 1931. Krushoviyat plodov komar, Contarinia pirivora [Pear fruit mosquito, Contarinia pirivora]. Lozarski pregled 16 (4/5): 112-113. (In Bulgarian).

Vasilev P. 1934. Zeleva muha [Cabbage fly]. Balgarsko ovoshtarstvo i gradinarstvo 15 (8): 150-152. (In Bulgarian).

Vassileva-Dryanovska O. \& Gencheva E. 1965. Changes in the sex correlation depending on the age of parents in Drosophila melanogaster. Comptes rendus de l'Academie bulgare des Sciences 18 (1): 59-61.

Velchev V. 1997. Features and regularities of distribution of contemporary vegetation. In: Yordanova M., Donchev D. (Ed.). Geography of Bulgaria. Sofia: Bulgarian Academy of Sciences, 265-269. (In Bulgarian with English sumarry)

Velchev V. 2002. Characteristic features and regularities in the distribution of the present-day vegetation. In: Copralev I. et al. (Ed.). Geography of Bulgaria. Sofia: ForKom, 321-324. (In Bulgarian with English sumarry)

Velchev V., Ganchev S. \& Bondev I. 1982. Rastitelni poyasi. In: Galabov Zh. (Ed.). Geography of Bulgaria. 1. Sofia: Bulgarian Academy of Sciences, 439-443. (In Bulgarian with English sumarry)

Velchev V., Bondev I., Kochev H., Russakova V., Vassilev P., Meshinev T., Nikolov V., Georgiev N. \& Valchev V. 1989. Vegetation. In: Mishev K. (Ed.). Natural and economic potential of the mountains in Bulgaria. Sofia: Bulgarian Academy of Sciences, 273-337. (In Bulgarian with English sumarry).

Velchev V. \& S. Tonkov. 1986. Vegetation and flora of Southwest Bulgaria. In: Botev B. (Ed.). Fauna of Southwestern Bulgaria. Sofia: Bulgarian Academy of Sciences, 20-43. (In Bulgarian with English sumarry)

Velcheva N., Atanassov A., Peeva P., Balevski N., Karadjova O., Kolarov Y., Pelov V. \& Z. Hubenov. 2010. Parasitoid assemblage of external-feeding in Lepidoptera in abandoned plum orchard in West Bulgaria. Journal of Balkan Ekology 13 (4): 405-416.

Velcheva N., Atanassov A., Karadjova O. \& Hubenov Z. 2012. Parasitoid assemblages isolated from externally feeding lepidopterans and Codling Moth (Cydia pomonella L., Tortricidae) in a young apple orchard in West Bulgaria. Bulgarian Journal of Agricultural Science 18 (5): 675-681.

Vermoolen D. 1983. The Tipula (Acutipula) maxima group (Insecta, Diptera, Tipulideae). I. Taxonomy and distribution. Bijdragen tot de Dierkunde 53 (1): 49-81.

Verves Yu. 1986. Family Sarcophagidae. In: Soós Á., Papp L. (Eds). Catalogue of Palaearctic Diptera. 12. Budapest, Akadémiai Kiadó, Hungarian Natural History Museum. pp. 58-193.

Verves Y., Zeegers T., Barták M., Kanavalová L. 2019. The family Rhinophoridae (Diptera) in the Czech Republic and new records from Jordan and Bulgaria. In: Kubík Š., Barták M. (Eds). 11th Workshop on biodiversity, Jevany. Česká Zemědělská Univerzita v Praze, Fakulta Agrobiologie, Potravinových a Př́rodních Zdrojů, 163-173. 
Vesselinov V. \& Gabev E. 1956. Sinantropnite muhi kato epidemiologichen faktor pri chrevnite infektsii [The synanthropic flies as an epidemiological factor in the intestinal infections]. Nauchni trudove na visshiya meditsinski institut 2 (4): 29-42. (In Bulgarian).

Vidinova Y., Tyufekchieva V., Yaneva I., Zadneprovski B., Stoichev S. \& Kumsnski K. 2008. Species composition and structure of macroinvertebrate communities in Arda River. Acta zoologica bulgarica 60 (3): 317-330.

Vigna Taglianti A., Audisio P., Biondi M., Bologna M., Carpaneto G., Biase A., Fattorini S., Piattella E., Sindaco R. 1999. A proposal for a chorotype classification of the Near East fauna, in the framework of the Western Palaearctic Region. Biogeographia 20: 31-59.

Villeneuve J. 1937. Myodaires superieurs inedits de la region Palëarctique. Musée royal d'Histoire naturelle de Belgique 13 (13): 1-4.

Vimmer A. 1916. O třech nonych druzich a některych staršich rodu Liponeura (Dipt.). Přispěvek k muši Bulharska. Rozpravy Čecke akademie pro vědu a uměni 25 (2, № 5): 1-18.

Wagner R. 1990. Family Psychodidae. In: Soós Á., Papp L. (Eds). Catalogue of Palaearctic Diptera. 2. Budapest, Akadémiai Kiadó, Hungarian Natural History Museum. pp. 11-65.

Wagner R. 2013. Psychodidae. In: Pape P. \& Beuk T. Fauna Europaea: Diptera: Nematocera. Fauna Europaea version 2017.06, https://fauna-eu.org

Wagner R. 2018. Psychodidae. In: de Jong H. (Ed.). Fauna Europaea: Diptera: Nematocera. https://fauna-eu.org (accessed 30 October 2018)

Wagner R. \& Joost W. 1988. Psychodidae aus Bulgarien (Insecta, Diptera). Faunistische Abhandlungen Staatliches Museum für Tierkunde Dresden B, 16 (3): 29-34.

Wirth W. 1975. A revision of the brine flies of the genus Ephydra of the Old World (Diptera: Ephydridae). Entomologica Scandinavica 6: 11-44.

Whitmore D. 2011. New taxonomic and nomenclatural data on Sarcophaga (Heteronychia) (Diptera: Sarcophagidae), with description of six new species. Zootaxa 2778: 1-57.

Wood D. M. \& Borkent A. 1989. Phylogeny and classification of the Nematocera. In: McAlpine J. F. \& Wood D. M. (Eds.). Manual of Nearctic Diptera 3. Ottawa, Research Branch Agriculture Canada. pp. 1333-1370.

Woodley N. E. 1989. Phylogeny and classification of the "Orthorraphous" Brachycera In: McAlpine J.F. \& Wood D. M. (Eds). Manual of Nearctic Diptera Volume 3. Ottawa, Resarch Branch Agriculture Canada. pp. 1371-1395.

Woodley N. E. 2001. A World Catalog of the Stratiomyidae (Insecta: Diptera). North American Dipterists' Society, Leiden, Backhuys Publishers. 483 p.

Yeates D. \& Wiegmann B. 1999. Congruence and Controversy: Toward a Higher-Level Phylogeny of Diptera. Annual Review of Entomology 44 (1):397-428. DOI: 10.1146/annurev.ento.44.1.397 · Source: PubMed

Yeates D., Wiegmann B., Courtney G., Meier R., Lambkin Ch. \& Pape T. 2007. Phylogeny and systematics of Diptera: Two decades of progress and prospects. Zootaxa 1668: 565-590.

Zaharieva A. 1982. Untersuchungen uber die Biologie und der Ökologie des Pappelspinners und das Erarbeiten von maßnahmen fur ihre bekampfung. Ph.D. thesis, Sofia, Forsttechnische Hochschule. 202 p. (In Bulgarian with German summary).

Zaharieva A. 1983. Vidov sastav i rolya na ustanovenite paraziti po Stilpnotia salicis L. [Species composition and role of the identified parasites on the Stilpnotia salicis L.] Nauchni trudove na VLTI 27/28: 107-113. (In Bulgarian).

Zaharieva-Pentcheva A. \& Georgiev G. 1997. Parasitoids of the satin moth Stilpnotia salicis (L.) (Lepidoptera, Lymantriidae) in Bulgaria. Bolletino di Zoologia agraria e di Bachicoltura, Ser. II, 29 (1): 81-90.

Zahov T. 1934. Chereshovata muha ot ikonomichno i biologichno gledishte [The cherry fly from an economic and biological point of view]. Balgarsko ovoshtarstvo i gradinarstvo 15 (4): 54-58. (In Bulgarian).

Zaitzev V. F. 1989. Family Bombyliidae. In: Soós Á. \& Papp L. (Eds.). Catalogue of Palaearctic Diptera. 6. Budapest, Akadémiai Kiadó, Hungarian Natural History Museum. pp. 43-169.

Zamfirov Ts. (Samfirov Z.) 1958. Nay-blagopriyatnite srokove za seitba na pshenitsa i oves v rayona na Instituta v Knezha, kato sredstvo za borba s zhitnite muhi [Most favorable sowing times for wheat and oats in the Knezha Institute region as a means of fight against wheat flies]. Nauchni trudove na kompleksniya selskostopsnski naucho-izsledovatelski institut v Knezha 1: 163-172. (In Bulgarian).

Zamfirov Ts. 1960a. Des travaux de recherhe sciantifique d'entomologie. Collection jubilaire de l'institut de recherché sciantifique d’agronomie a Kneza 1: 199-225. (In Bulgarian with German summary). 
Zamfirov Ts. 1960b. Nauchnoizsledovatelskata rabota po entomologiya [Entomology research work]. In: Yubileen sbornik na Kompleksniya selskostopanski nauchnoizsledovatelski institut v Knezha. pp. 133-137.

Zamfirov Ts. 1961a. Vidov sastav i nyakoi biologichni osobenosti na zhitnite muhi v rayona na Instituta v Kneha [Species composition and some biological features of the wheat flies in the region of the Knezha Institute]. Izvestiya na instituta po tsarevitsata 1: 199-225. (In Bulgarian).

Zamfirov Ts. 1961b. Srokat na seitbata na ovesa kato sredstvo za borba s shvedskata muha Oscinosoma frit L. [The term of sowing of the oat as a means of fight against the Swedish fly Oscinosoma frit L.]. Izvestiya na Kompleksniya selskostopanski nauchnoizsledovatelski institut v Knezha 2: 217-239. (In Bulgarian).

Zamfirov Ts. 1962a. Prouchvaniya varhu vazmozhnostite za borba s zhitnite muhi chrez predposevno tretirane na semenata na zhitnite kuturi s insektitsidi [Studied on the possibilities of fighting against wheat flies by treating the wheat seeds with insecticides before sowing]. Izvestiya na instituta po tsarevitsata 3: 181-192. (In Bulgarian).

Zamfirov Ts. 1962b. Vazmozhnosti za borba s zhitnite muhi pri pshenitsata Oscinosoma frit L., Chlorops pumilionis Bjerk. i Mayetiola destructor Say chrez naprashvane na poseva s insektitsidi [Possibilities for controlling wheat flies Oscinosoma frit L., Chlorops pumilionis Bjerk. and Mayetiola destructor Say in wheat by sowing treatment with insecticides]. Izvestiya na instituta po tsarevitsata 4: 179-183. (In Bulgarian).

Zamfirov Ts. 1962c. Srokat na seitbata na pshenitsata i echemika kato sredstvo za borba s zhitnite muhi [The time of sowing of the wheat and barley as a means of fighting against wheat flies]. Rastitelna zashtita 10 (4): 29-38. (In Bulgarian).

Zamfirov Ts. 1962d. Borba s shvedskata muha (O. frit L.) pri ovesa posredstvom naprashvaneto na poseva s insectitsidi [Control of the Swedish fly (O. frit L.) in oats by pollination of the crops with insecticides]. Izvestiya na Kompleksniya selskostopanski nauchnoizsledovatelski institut v Knezha 3: 171-180. (In Bulgarian).

Zamfirov Ts. 1962e. Deystvieto na heksahlorana pri vnasyane v pochvata za borba s shvedskata, zhitnata stablena i hesenskata muha (Oscinosoma frit L., Chlorops pumilionis Bjerk. i Mayetiola destructor Say) [The action of hexachlorane in the soil against Oscinosoma frit L., Chlorops pumilionis Bjerk. and Mayetiola destructor Say]. Rastitelna zashtita 10 (5): 47-52. (In Bulgarian).

Zamfirov Ts. 1962f. Vidov sastav na chernite zhitni muhi ot rod Phorbia (Diptera, Muscidae) v Severozapadna Bulgariya [Species composition of the black wheat flies of the genus Phorbia (Diptera, Muscidae) in the Northwestern Bulgaria]. Rastitelna zashtita 10 (2): 56-57. (In Bulgarian).

Zamfirov Ts. 1963a. Kam baprosa za sroka na seitbata i naprashvaneto na poseva s heksahloran v borbata s zhitnite muhi pri pshenitsata i ovesa [On the question of the terms of sowing and pollination of the crops with hexachlorane in the fight against wheat flies in the wheat and oats]. Izvestiya na instituta po tsarevitsata 6: 119-125. (In Bulgarian).

Zamfirov Ts. 1963b. Razmera na povredite ot zhitnite muhi pri pshenitsata vav vrazka s fenologichnite razlichiya $\mathrm{v}$ razvitieto na sortovete [The extent of damages from the wheat flies on the wheat in connection with the phenological differences in the development of the sorts]. Rastitelna zashtita 11 (10): 23-27. (In Bulgarian).

Zamfirov Ts. 1963c. Znachenie na borbata s zhitnite muhi za povishavane dobiva ot pshenitsata [Importance of fighting against wheat flies to increase the wheat yield]. Rastitelna zashtita 11 (12): 18-19. (In Bulgarian).

Zamfirov Ts. 1963d. Prouchvane varhu chernite pshenichni muhi Phorbia securis T. i Ph. penicillifera Jerm. (Diptera: Muscidae) i vazmozhnostite za borba s tyah [Study on the black wheat flies Phorbia securis T. and Ph. penicillifera Jerm. (Diptera: Muscidae) and the possibilities to fight with them]. Izvestiya na instituta po tsarevitsata 5: 139-148. (In Bulgarian).

Zamfirov Ts. 1963e. Chernite pshenichni muhi i borbata s tyah [The black wheat flies and the fight against them]. Rastitelna zashtita 11 (3): 15-18. (In Bulgarian).

Zamfirov Ts. 1966. Prouchvaniya varhu biologiyata i sredstvata za borba s zhitnata trevna muha [Studies on the biology and means of fight with the wheat grass fly]. Rastitelna zashtita 10/11: (In Bulgarian).

Zaschev G. \& Angelov A. 1959. Untersuchungen über den Burgas-See (Waja) in Beziehung zur Verbesserung seiner fischwirtschaftlichen Ausbeutung. Annuaire de l'Université de Sofia, Faculté de Biologie, Géologie et Géographie 51 (1 - Biologie): 161-210 (In Bulgarian with German summary).

Zatwarnicki T. 1991. Changes in nomenclature and synonymes of some genera and species of Ephyrdidae (Diptera). Deutsche entomologische Zeitschrift 38 (4-5): 285-333.

Zatwarnicki T. 1996. New records of Western Palaearctic shore flies (Diptera: Ephydridae). Annales of the Upper Silesian Museum, Entomology 6-7: 107-125. 
Zatwarnicki T. 2018. Solving the puzzle of taxonomic position of the petroleum fly by resurrection of Diasemocera Bezzi from Psilopa Fallén (Diptera: Ephydridae) with proposed specific and generic synonymies. Annales zoologici (Warszawa) 68 (3): 527-552.

Zenginov H. 1949. Vagartsite i vagarchivosta po govedata [Hypodermatossis on the cattle]. Nauka i iskustvo, Sofia, (In Bulgarian).

Zhecheva Zh. 1961. Selektsionna rabota s luka (Alium cepa L.) v Nauchnoizsledovatelskiya institut po zelenchukovite kulturi "Maritsa" Plovdiv (1937-1960) [Selection work with onions (Alium sepa L.) at the Research Institute of Vegetable Crops "Maritsa” Plovdiv (1937-1960)]. Izvestiya na nauchnoizsledovatelskiya institut po zelenchukovite kulturi "Maritsa" - Plovdiv 1: 5-25. (In Bulgarian).

Zhelev I. 1948a. Chereshova muha, Rhagoletis cerasi L. [Cherry fly, Rhagoletis cerasi L.]. Gradinarstvo 29 (6): 156-160. (In Bulgarian).

Zhelev I. 1948b. Plodovi hobotnitsi Rhynchites sp., i krushovo komarche, Contarinia pirivora [Fruit weevils, Rhynchites sp., and Pear mosquito, Contarinia pirivora]. Gradinarstvo 29 (8): 238-242. (In Bulgarian).

Ziegler J. 1989. Zur Kenntnis der Wirtsbeziehungen paläarktischer Raupenfliegen (Dipt., Tachinidae). 6. Beitrag. Parasiten-Wirts-Liste. Entomologische Nachrichten und Berichte 33 (6): 283-284.

Ziegler J. 2003. Ordnung Diptera, Zweiflügler (Fliegen und Mücken). In: Dathe H. (Ed.). Lehrbuch der Speziellen Zoologie, Band I: Wirbellose Tiere, 5. Teil: Insecta. Heidelberg, Spektrum. pp. 756-860.

Zielke E. 2016a. Update of distribution records of Phaonia Robineau-Desvoidy (Diptera: Muscidae) from Bulgaria with the description of a new species. Journal of Entomology and Zoology Studies 4 (4): 626-632.

Zielke E. 2016b. Update of distribution records of Mydaeinae (Diptera: Muscidae) from Bulgaria. Journal of Entomology and Zoology Studies 4 (6): 310-315.

Zielke E. 2016c. Observation on distinctions between Polietes lardarius (Fabricius) and Polietes meridionalis Peris \& Liorente (Diptera: Muscidae). Journal of Entomology and Zoology Studies 4 (6): 780-783.

Zielke E. 2016d. Description of a new species of Helina Robineau-Desvoidy (Diptera, Muscidae) from the Rhodope Mountains of Bulgaria. Contribution to Entomology (Beiträge zur Entomologie) 66 (2): 321-324.

Zielke E. 2017a. Description of a new Limnophora species from Bulgaria (Diptera: Muscidae). Contribution to Entomology (Beiträge zur Entomologie) 67 (2): 319-323.

Zielke E. 2017b. Four new species of the subfamily Phaoniinae (Diptera: Muscidae) from Bulgaria. Journal of Entomology and Zoology Studies 5 (6): 901-908.

Zielke E. 2018a. An update of distribution records of Helina R.-D. and other genera of the subfamily Phaoniinae (Diptera: Muscidae) from Bulgaria. Acta Musei Moraviae, Scientiae biologicae 103 (2): 249-267.

Zielke E. 2018b. On two remarkable species of Azeliinae (Diptera: Muscidae), previously unknown from the Balkans, but collected from Bulgaria already in the $20^{\text {th }}$ century. Historia naturalis bulgarica 30: 1-5.

Zielke E. 2018c. More records of Muscidae (Diptera) fron Croatia with a short comment on findings of Helina interfusa (Pandellé) reported to date in Europe. Acta Musei Moraviae, Scientiae biologicae 103 (2): 281-285.

Zielke E. 2019a. Update of distribution records of Thricops Rondani and other genera of the subfamily Azeliinae (Diptera: Muscidae) from Bulgaria. Journal of Entomology and Zoology Studies 7 (3): 316-323.

Zielke E. 2019b. Notes on the little known species of Helina syracusana Hennig, 1957 and Helina chaetopyga (Malloch, 1921) (Diptera: Muscidae). Entomologische Zeitschrift 129 (4): 205-210.

Zilahi G. 1934. Beiträge zur Fliegenfauna Bulgariens. I. Chironomidae. Mitteilungen der Bulgarischen Entomologischen Gesellschaft in Sofia 8: 152-158.

Zlatanov S. 1971. Insektenschädlinge der Eiche in Bulgarien. Sofia, Verlag der Bulgarischen Akademie der Wissenschaften. 250 p. (In Bulgarian with German summary).

Zonati E. 1978. Parazitni nasekomi po prastenotvorkata (Malacosoma neustria L.) v Bulgaria [Parasitic insects on the ringworm (Malacosoma neustria L.) in Bulgaria]. Ph.D. thesis, Sofia, Forsttechnische Hochschule., 14-15. (In Bulgarian).

Zuska J. \& Pont A. 1984. Family Sepsidae. In: Soós Á. \& Papp L. (Eds). Catalogue of Palaearctic Diptera. 9. Budapest, Akadémiai Kiadó, Hungarian Natural History Museum. pp. 154-167.

Zwick P. 1992. Blephariceridae. In: Soós Á., Papp L. \& Oosterbroek P. (Eds.). Catalogue of Palaearctic Diptera, 1. Budapest, Akadémiai Kiadó, Hungarian Natural History Museum. pp. 39-54.

Zwick P. 2007. The first record of net-winged midges (Diptera: Blephariceridae) from Hungary, and a corrected name in the genus Liponeura. Folia Historico Naturalia Musei Matraensis 31: 153-155. 